


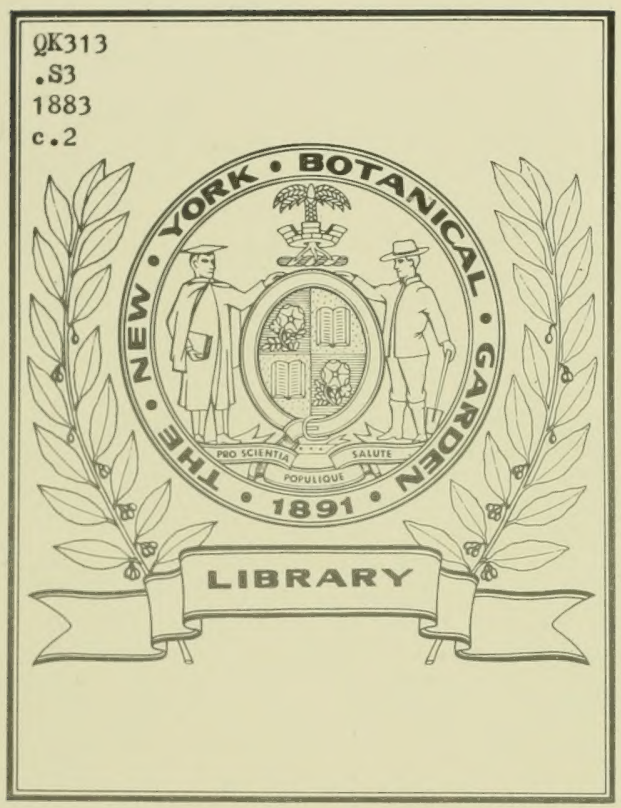







\section{GATALOGUE}

DES PLANTES VASCULAIRES

DE I,A

\section{FLORE DU BASSIN DU RHONE}

PAR LE

\section{SAINT-LAGER}

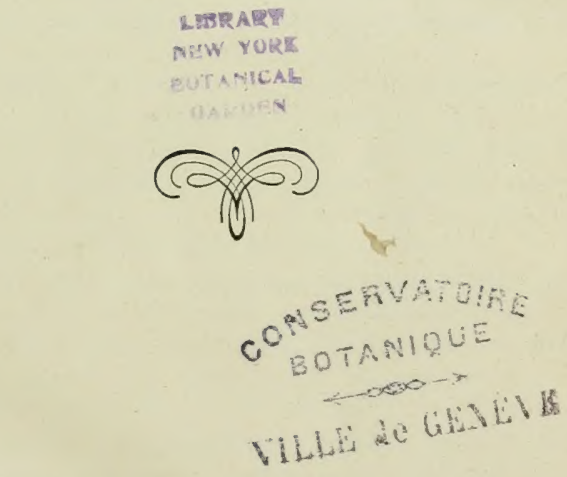

LIBRAIRIE H. GEORG

LYON. - GENEVE. - BALE

1883 
.53

1883

C. 2

LYON, ASSOCIATION TYPOGRAPHIQUE

Th. Giraud, rue de la Barre, 12

$-$ 


\section{PRÉFACE}

Le Calalogue des plantes vasculaires du bassin du Rhône est le fruit de recherches poursuivies pendant douze années consécutives dans l'Est et le Sud-Est de la France, ainsi que dans la région rhodanienne de la Suisse.

Comme il eût été impossible à un seul homme de visiter toutes les parties d'un aussi vaste domaine, nous avons fait appel à l'expérience de plusieurs naturalistes, membres de la Société de botanique de Lyon, qui ont bien voulu apporter à notre œuvre de statistique le précieux tribut de leurs observations personnelles. Ces collaborateurs dévoués sont MM. Borel, Chevalier, Dr Fabre, Gaston Gautier, Dr Gillot, Hanry, Lannes, $D^{r}$ Lesourd, Loret, Ven. Payot, Reverchon et Viallannes.

Enfin, nous avons extrait des ouvrages antérieurement imprimés tous les renseignements phytostatiques relatifs à la Flore du bassin du Rhône et des régions voisines.

Tel quel, et malgré les lacunes qui restent encore à combler, le Catalogue des plantes vasculaires du bassin du Rhône contient un ensemble de documents utiles à consulter pour la connaissance de la distribution géographique de chaque espèce végétale, considérée, soit en elle-mème, soit dans les rapports qu'elle a avec le climat et la nature du sol.

Désireux d'énumérer toutes les formes végétales décrites par les bons observateurs, et ne croyant pas cependant devoir les placer toutes sur le même rang, nous établissons entre elles une hiérarchie fondée sur l'importance des caractères, en partant de ce principe que les plantes sont sujettes à éprouver des modifications dont l'amplitude varie selon la nature propre rde chacune d'elles, et quelquefois aussi suivant les conditions D̄extérieures de sol et de climat. L'expérience nous apprend, 
d'ailleurs, que, parmi ces modifications, les unes se perpétuent héréditairement dans les stations naturelles et même dans nos cultures, les autres semblent plus ou moins éphémères et ne se reproduisent pas constamment par le semis. En conséquence, nous admettons dans la hiérarchie des plantes trois degrés: $1^{\circ}$ les types principaux, c'est-à-dire les formes qui sont le plus communément répandues; $2^{\circ}$ les races locales issues de ces types, sans que nous connaissions historiquement leur généalogie; $3^{\circ}$ les variétés instables. Soit, par exemple, la Brunelle vulgaire bien connue de tous les botanistes. Le type commun, souche probable des autres Brunelles, est le B. vulgaris $L$. A cette espèce principale se rattachent deux formes secondaires, $B$. alba Pall. et $B$. grandiflora Jacq., qui, par leur permanence, méritent le nom de races, en donnant à ce mot le sens d'espèces de seconde formation. Les noms de celles-ci sont imprimés en lettres italiques dans notre Catalogue. Enfin, chacune de ces deux formes, et le type lui-même, présentent parfois des déviations affectant des caractères de faible importance et non indéfiniment transmissibles par hérédité; de là, les variétés appelées integrifolia, pinnatifida, hastifolia.

Quant à ce qui regarde les changements apportés à plusieurs noms génériques et spécifiques dans le but de donner à la Nomenclature la correction et l'homogénéité qui souvent lui manquent, nous prions le lecteur de consulter l'avertissement placé en tête de la table. 


\section{THALAMIIFLORES}

\section{RENONCULAGÉES.}

CLEMATIS rectu L. - Ilaies et buissons. - Valais: Saillon, entre St-Pierre et Ardon, Vétroz, Plan-Conthey, St-Séverin, Erdes, Riddes, Biédron, Drône, Turtmann. - H.-Alp. et Dr. : Combe-Noire de Manteyer, Oze, Veynes, Gap, Tallard, Nyons, indiqué vaguement par Villars entre Iontélimar et Orange. B.-Alp.: St-Donat près Sisteron, bords du Buech. — Gard et Hér. : bords du Gardon près d'Alais, Aigues-Mortes, bois de Sérignan près St-Pons. - Pyr.-0r. : plaine de Rivesaltes près du Ilas de la Garrigue, bords de la Mlassane près Laval.

C. Aammula L. - Haies de la rếgion des Oliviers.

G. marilima DC., variété à folioles linẻaires qui se trouve çà et là sur les dunes sablonneuses du littoral.

C. vitalba L. - Haies des plaines et des montagnes.

ATIB fGE alpinn L. - Rochers des Alpes et des montagnes subalpines. - H.-Sav. et Sav. : Salève au-dessus d'Archamp, escarpements d'Andey au-dessus de Bonneville, mont Gargan sur Moutiers, vallées des Allues et de St-Martin de Belleville, Bas du Mottet au-dessus d'Aime, entre Tignes et Laval, forêt de Lans-le-Bourg, rochers au-dessus de la rive occidentale du lac du mont Cenis. - Is. : St-Nizier, la Pra de Belledonne, bords du lac Lovitel en Oisans, mont Chamoux près de la Salette. II.-Alp. : la Grave aux glaciers du Bec, Lautaret, Nẻvache, Monestier-de-Briançnn, Cerrières, les Hayes, Aiguilles en Queyras, Aluries, les Rousses, chalets de Ruines, Charance et Cote Giélive près Gap, col de Glaise, Manteyer, mont Séuse, le Noyer en Champsaur, Boscodon, Valgaudemar. - B.-Alp. : coteau des Catal. Bassin du Rhonce. 
Maures près de la Condamine, bois de Tournoux, la Blachière près Maurin, Colmars, forêt de Faillefeu près Prads. - Alp.Mar. : Entraunes à la Combe de Bourdoux.

C'est à tort que la Clématite des Alpes a été indiquée dans les Pyrénées par Pourret et par les auteurs qui l'ont copié.

T直A montagnes. - Jura helvétique et français: Chasseral, Creuxdu-Van, Suchet, Mont-d'Or, Aiguillon, Cótes du Doubs et du Dessoubre, montagnes de Salins, d'Arbois, de Poligny et de Champagnole, la Dòle, le Reculet, les Rousses, Sorgiaz, Cluses de Nantua, Retord et le Colombier du Bugey. - Vaud : Pélerin, Puidoux, Forel, Vernex, Boiron, Promenthoux, Avenches, Moudon, Payerne, Palezieux, St-Loup, Aubonne. - Valais : gorges de Durnant, Bourg-St-Pierre, St-Bernard, Thyon, Méribé, Novelli de Nendaz, Giétroz, vallées de Resclıy et de Saas, Simplon. - H.-Sav. : Salève, Voirons, Parmelan, Tournette, Charvin, mont Forchat au-dessus d'Habère-Poche, Cornettesde-Bise, mont Laouet près Reyvroz, mont des Granges, montagnes de Sixt, Clamonix, Brizon. - Sav. : mont Nivolet, mont du Chat, St-MIartin-de-Belleville. - Is. el H.-Alp. : Grande-Chartreuse, Grande-Vache, la Houcherolle, Gavet, Lautaret, Arcines, vallée du Guil en Queyras, col de Vars, StVéran, Boscodon, col Bayard, bois de Rabou, Chaudun, mont Séuse, forêt de Durbon. - B.--tlp. et Alp.-Mar. : la Condamine à Ste-Anne, Lauzanier, Fouillouse, lac d'Allos, sources du Var, le Breil, Clans, St-Marlin-Lantosque. - Gard : prés de Gourdouze, Valat de Longues-Fcuilles près Concoule. - Pyr.-0r. et Ariège : Canigou, Prats-de Mollo, Jont-Louis, Bolyuère, vallées d'Eyne et de Llo, Tarbézou, cirques d'Artounant et des Aiguettes, vallon de Boutaliol. - En dehors de nos limites dans les Pyr--Centr., l'Aubrac, Aveyron, Lozère, Haute-Loira.

Le Pigamon à feuilles d'Ancolie descend sur les rives de l'Ain justue près du Pont de Clazey.

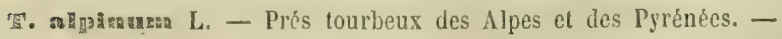
- I1.-Alp. et Alp.-Mar. : Lauzanier, Grange-Commune, Malemort, Crouis, Enchastraye au Grand-Qnartier, Fours, lac d'Allos, 
col de la Maddalena, mont Tenibre, sources du Var, col de Jallorgues, lac de Rabuons. - Pyr.-Or. : Nuria, Puigmal. - Pyr.Centrales.

T. Poetidum L. - Lieux pierreux des Alpes. - Vaud et Valais : Bovonnaz, Fully, Bagnes, plateau de Pradaz au St-Bernard, Evolena, Comba d'Arbaz, Zermatt, Zenschmieden, Sas, Mattmark, montagne de la Saxe sur Courmaycur en Italie. - Une variété inodorum croit sur les coteaux bien exposés du Bas-Valais à Folatères, Saxon, Alontorge, Tourbillon et jusqu’à Viège. Une variété glabrum existe au-dessous du village de Simplon cntre Algaby et Casermetta, dans le val Binn et la vallée de de Saas à Maltmark. - Is. : St-Christophe en Oisans, la Bérarde. - II.Alp. : Villard d'Arène, la Grave, Lautaret, Monestier-de-Briançon, Fort des Trois-Tètes à Briançon, cols de Vars et Agnel, mont Viso à la Traversette, la Taillante, Côte Gélive el col de Glaise près Gap, Chaudun, col du Noyer et des Bau. melles. - B.Alp. et Alp.-Mar. : la Condamine, Maurin, Estenc, Entraunes à la Combe de Bourdoux, St-Dalmas-le-Sauvage, versant nord du Tournairet près Venanson.

Une variété olidum Jord., qui a les feuilles simplement pu. bescentes, la tige striée, a été trouvée au Lautaret et à la Côte Gêlive près Gap.

T. Ouloratam G. G. - Lieux pierreux. - Is. et H.-Alp. : le Freney, la Salette, Laularet, Clıarance, col de Glaise, mont Séuse, mont Morgon. - Vaucl. : mont Ventoux.

T. mians L., majus L., maedium Jaç. - Ces trois formes principales constituent un groupe dont l'étude est extrêmement difficile. En attendant qu'un travail de révision ait été accompli, je me borne à énumérer les formes secondaires suivantes:

T. montanum Wallr, T. calcareum Jord. - Assez commun dans la chaine jurassique des dép. du Doubs, du Jara et de l'Ain. Dans les massifs calcaires du mont Rachais, du Grand Som, de Chalais et de la Moucherolle (Is.). - Sur les rochers jurassiques du Mont-d'Or' lyonnais à Couzon et à Pommiers près Villefranche (Rh.). - Collines jurassiqques de l'Ilérault à la Sérane, St-Martin de Londres, Lodeve. 
T. monticolum Jord. - Lautaret (H.-Alp.).

T. collinum Wallr. - Coteaux jurassiques de la C.-d'Or et de S.-et-L.

à Beaune, Nuits, Puligny, Cluny, Dezize. - Montagnes calc. des envir. de Grenoble à Chalais, St-Eynard, Néron, gorges d'Engins, St-Nizier, Vif.

T. expansum Jord. - Rh. : Lyon aux Brolleaux et à la Mouche, Pommiers, Pic de Seveletle. - Is. : bords du Rh. à Feyzin. - Hèr. : Montpellier à Lavaletle, Castelnau, Jords du Lez et de la Mosson, la Gardiole, les Matelles, St-Loup.

T. elegans Jord. - Lyon à la Têtedd'Or. - Tournon (Ard.).

T. praecox Jord, - H.-Alp. : Charance, Embrun, Briançon.

T. Laggerianum Jord. - Vallée de Saas en Valais.

T. praeflorens Jord. - Col de Vars, mont Viso (H.-Aip.).

T. oreitum Jord. - H.-Sav. : Saleve. - Mont Viso.

T. obscuratum Jord. - Vic (Hér.).

T. glaucescens Willd. - Mollard de Décines(Is.).

T. prevepubens Jord. - Vic (Gard).

T. puradoxum Jord. (Jordanianum Schl.). - Envir. de Lyon.

II. Jordan a trouvé autour de Lyon plusieurs autres formes de Thalictron qu'il a décrites sous les noms de : fruteticolum, thamnoplilum, virgulticolum, arrigens, dumulosum, propendens. eminens, affine, tortuosum, ambigens, nothum, silaifolium, stipellatum, Timeroyianum, laelum, procerulum, rhodanense; subspicalum, nitidulum, medianum, spurium.

T. angustifolium L., T. Bauhinimnum Crantz. - Doubs, Jura et Ain : plateaux de Goux, de Villard-sous-Ecot, Ecurcey, Mandeure, Audincourt, Arbouans, entre Baume et Ornans, plateau de Mamirolle et de Nancray près Besançon, la Châtelaine et les Planches près Arbois, Dole, Choisey, Molay, Peseux, cours de l'Ain jusqu'à Thoirelle, Valromey, Bugey. - Descend quelquefois jusque dans les îles du Rhône. - Vaud et Valais : Bex, entre Lavey et Châtel, Montet, Lausanne, Pierrettes, St-Sulpice, Morges, Bière, Duiller, Bonmont, Clarens, Crels sur Gingins, Cheserex, entre le Guercet et Martigny, Alpes de Bagnes à Gétroz, Champsec de Sion.

Espece polymorphe ayant tantot les folioles étroites, parfois 
filiformes (galioideum Pers.), tantôt plus larges ( $T$. laserpitifolium ou mieux laserophyllum Willı.).

Le T. alpicolum Jord. (simplex L.) est une variété à panicule pauciflore, à folioles trifides accompagnées de feuilles indivises, qui a été observée à Villard d'Arène, au Lautaret, à Fournet et 1a Tronchée au pied du mont Viso, au Bourget près Cervières (H.-Alp.); dans les Alp.-Mar. à St-Martin-d'Entraunes au Désert de Barnabé; dans les Pyr.-0r. dans les paturages humides du Capcir, de Carlite et du col de la Perche.

'w', Arum L. - Lieux humiłes, - Espece polymorphe comprenant les formes décrites par M. Jordan sous les noms de riparium, udum, prorepens, capitatum, mediterraneum.

La forme glaucum Desf., qui a une tige très-glauque, une taille élevée, des feuilles plus larges a été observéc dans les Pyr.-Or. sur les bords du ruisseau de Château-Roussillon à Canet, entre la Tet et l'étang de Canet. - Assez répandue en Espagne, en Portugal et en Algérie.

'. Anberosam L. - Pâturages humides de l'Aude et des Pyr.Or., au mont Alaric, sur les hauteurs de Nuria et de CostaBona. - En Espagne sur les collines rocheuses de la Catalogne, de l'Aragon et de la Navarre.

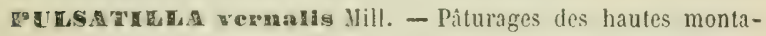
gnes sur les roches siliceuses. - Vaud et Valais: les Martinets,

- Anzendaz, Boulaire, Paneyrossaz, Lavarraz, Javernaz, la Sciaz, Naye, Aï, Tornettaz, Dorenaz, Fully, St-Bernard, Thyon, Zan, Zermatt, Saas, Simplon. - H.-Sav, et Sav. : Dents d'Oche, Morzine, Nautau, mont Chalune, Buet, col de Balme, mont Blanc à Leschaux, Mer-de-Glace, mont Lachat, col de Voza, Prarion, Méri, Tournette, cols du Bonhomme, de la Seigne et de la Madeleine, mont Jovet, les Allues, mont Iseran, mont Cenis à Pattes-Creuses, le Cramont, sur le versant italien. Is. : Belledonne, Champrousse, Piéméyan au mont de Lans, mont Sénèpe. - H.-Alp. : Lautaret, Névache, mont Genẻvre, Gondran, cols Isoard et des Ayes, col de Vars, mont Viso depuis la Cóte-Ronde au vallon des Brun jusqu'à la Traversette, StVéran à Serène, Guillestre au Mélezet, Chaillol-le-Vieil, mont 
Queyrel, Orcières. - B. Alp. : Fouillouse, Parpaillon, Scrennes, Croués, Plate-Lombarde, Malemort, Horonaye. - Ard. et H.-L. : Mezenc. - Pyr.-Or. et Ariège : Canigou, Cambres d'Aze, vallées d'Eyne et de Llo, lac du Llaurenti, Valbonne sous le Roc de la Trune. - En dehors de nos limites dans les Pyr.-Centr., Plomb du Cantal, grès vosgien entre Bitche et Obersteinbach en Alsace. Nul dans la chaine jurassique et dans les massifs calcaires exempts de bancs de grès ou autres roches siliceuses.

P. aerieen, P. Halleriana All. - Pâturages et rocailles des Alpes.

- Valais : Zermätt à Findelen, Augstkummen, Zmutt. - Sav. et Piémont : mont Cenis. - Is. et Dr. : col de l'Are, montagne de Gresse, mont de Brette près Die, mont des Lussettes près la Croix-Haute. - H.-Alp. : N.-D.-des-Neiges près Briançon, mont Viso à la Taillante, vallon Agnel, col de Glaise, Charance, Durbonnas, Valgaudemar. - B.-Alp. : Riou-German et vallon du Châtelet près Serennes, Horonaye, mont de Courchon près StAndré. - Alp.-Mar. : St-Martin d'Entraunes au col des Champs et à la montagne des Trottes.

Fo. vulgaris Lobel. - Coteaux calcaires de C.-d'Or et S.-et-L. H.-Sa@ne : entre Delain et Courtesoult, - Jura helvétique et français jusque dans le Bugey. - Collines granitiques du Lyonnais, de la Loire et de l'Ardèche. - Gard et Hér. : Salbous près Campestre, l'Éspérou, plateau du Jarzac à St-MIichaldes-Sers, le Caylar, la Vacquerie. - Is. ef Dr. : Mollard de Décines, Vernas, Crémieu, Morestel, Charvicu, Seyssuel près Vienne, Andancette, St-Vallier.

Espèce polymorphe dont la fleur a une couleur tantôt violette-lilacée ou rosée, comme c'est le cas dans la forme vulgaris de la Côte-d'Or et du centre de lit France, tantôt d'un violet foncé comme dans la forme montana Hoppe des H.-Alpes, tantôt rouge-brune dans la forme rubra Lam. du Valais, du Lyonnais et des parties voisines du Dauphiné, du Bugey, du Forez, du Vivarais et des Cévennes. Les styles, bruns et arqués dans les formes rubra et rigella Jord. Eont blanchâtres dans la forme propera Jnrd. 
[2. algotan Lois. - Pàturages des montagnes, - Jura liclvétique ct français: Chasseral, Chasscron, Creux-du-Van, Suchet, Aiguil. Ion, Prê de Biẻre, Ėmbernats, Mont-d’Or, Montendre, la Dôle, Colombier du Bugey. - Vaud et Valais : 1)ent de Jaman, Bevonnaz, Anzendaz, Lavarraz, Cheville, Croumaclire, St-Bernard à la Baux, Forclaz, Thyon, Schwarzsce de Zermatt, Saas à Fee, Simplon, Albrun, Binner et Aernergalen. - II.-Sar. : le Mole, Brizon, Tournette, col des Aravis, montagnes de Bonnevaux et de Morzine, Dents d'Oche, Cornettes de Bise, montagnes de Vacheresse, Roc d'Enfer, plateau des Gets, Charvin, mont Lachat, cols de Balme et du Bonhomme. - Sav. : mont Iseran, les Avanchers, les Allues, mont Cormet au-dessus d'Aime, col de la Iadeleine, mont Nivolet el d'Arith, mont Cenis. - Is. : Chamechaude, col de l'Arc, la Noucherolle, Lautaret, mont Genèvre, Cervières, montagnes du Queyras, Pic de Chabrières, Charance, Côte Gélive et col de Glaise près Gap, Sćuse, Aurouse, Orcières. - B.-Alp. et Alp.-Mar. : la Condaminc, Larche, Parpaillon, forêt de Faillefeu près Prads, sommités des vallées du Var, de la Vesubia, Tinea, Roja. - Var : cimes de Morgès a Ampus, bois de Vérignon. - Pyr.-0r. et Ariége : Nohẻdes, Pla Guilhem, vallées d'Eyne et de Llo, Llaurenti près des lacs, Boutadiol, cirques des Aigueltes et d'Artounant, Val do Paillères à Soucarrat. - En dehors de nos limites dans les Pyr.-Centr. et sur les Ballons de la chaine des Vosges.

Le type à fleurs blanches est quelquefois remplacẻ par la rariétẻ sulfurea à fleurs d'un jaune de soufre. - Vaul et Valais : Moveran, Lavarraz, Javernaz, Alesse, Forclaz de Martigny, St-Bernard à la Pierraz et à la Baux, Thyon, mont Nuoble, Zan, Vercorin, Zermatt, Simplon. - H.-Sav. ct Sav. : chalets de Balme, de Charamillon, d'Argentière, de la Joux, du Laranclıer, de Blaitière, le Mont près Chamonix, Alléc Blanche, les Ayers sur Serroz, Corneltes de Bise, Nautau, mont Pétetod, le Mole, les Allues, Beaupré, col du Cormet, la Sauce près du Bonhomme. - Is. : Champrousse, Grand Galbert. - H. et B.Alp. : Briançon aux Éduits, Château-Queyras, mont d'Avançon, Lauzanier, Fouillouse. - Gard : Hort-de-Diou, Arbous, Espérou. 
- Pyr.-Or. et Ariège: Nohèdes, vallées d'Eyne et de Llo, val de Paillères à Soucarrat, cirques des Aiguettles et d'Artounant, lacs du Llaurenti, de Rabassolès et de Valbonne, vallon de Boutadiol.

Une forme remarquable par les poils blancs-soyeux qui recouvrent sa tige, par ses feuilles peu profondément découpées, par ses sépales grands et largement ovales, ayant les fleurs tantôt blanches, plus souvent jaunes, le $P$. myrrhidophylla Vill, se trouve dans les montagnes d'Allevard à l'Haut-du-Pont, à St-Hugon, Marcieu près la Nire (Is.), Orcières, mont Genèvre, St-Véran (H.-Alp.); St-Paul, Maurin, Lauzanier, Fouillouse (B.-Alp.), flanc sud de la Croix-de-Javernaz (Vaud). - Cette même forme existe en grande abonànce sur les pàturages des sommités du Plomb du Cantal, du col de Cabre, du Puy Mary, du mont Dore et probablement aussi dans la chaine des Pyrénées.

ANEHON fragafera Wulf, baldensis L. - Débris rocheux des Alpes. - Vaud et Valais: Chaux-de-Mont, Alpes de Leysin, pied de la Tour d’Aï, Prapioz, les Outans, les Plans, Martincts, Javernaz, Grandvire, Fully, Lens, Gemmi, arête de Triqueud, Pépinet, St-Bernard à l'Ardifagoz, Orsera, Méribé, Cotter, Seilon, Zan, Zermatt au Hoernli, au Simplon, vallée de Binn, Guggerhubel, Val Reschy, Bellalui. - IJ.-Sav. et Sav. : Méri, Vergy, mont Saxonnet, Tournette, versant italien du mont Blanc dans l'Allée Blanche, vallon de Chapi sous Courmayeur, Combe de la Hyoulaz au Cramont, la Vanoise, mont Jovet, Saut-des-Allues, mont Cenis. - Is. et H.-Alp. : la Moucherolle, Fiémeyan au mont de Lans, Lautaret, Galibier, Arcines, Gondran, cols Isoard et des Hayes, mont Lopet en Champsaur, Chaillol-le-Vieil, Orcières, pâturages du Queyras, du col de Glaise, du mont Aurouse et du Pic de Chabrières, mont Obiou. - Dr. : mont Glandasse. - B.-Alp. : Crouès, Parpaillon, Riou-German, vallon du Châtelet, Bérard, col de Mirandole, Longet, sommet des Boules au-dessus de Prads, - Alp.-Nar. : Entraunes au Pas Robinouse ct à Roche-Grande, Val de Strop, le Garet, Alpes de Fenestre et de Tende. 
C'esı par erreur que celle espèce a été indiquée par Lapeyrouse et par Companyo dans la chaine des Pyrénées.

A. nemoress L. - Bois dans tout le bassin. - Une variété à fleurs rosées est quelquefois mélée au type à fleurs blanches.

A. sllvestrim L. - Bois. - Celle espice, qui se trouve en Alsace ct en Lorraine, existe peut-être dans les parties de notre bassin limitrophes des susdites provinces.

A. Ronuncullager L. - Prairies et bois. - Envir. de Belfort aux bois du Mont, des Fourches, de la Miotte et de Damjoutin. - H.-Saône : Frotey, Arbecey, bois de la Paume à Chaux, StAndoche, Colombe. - Doubs et Jura : Coles du Doubs, du Dessoubre et de la Barbèche, Roches, Audincourt, Belchamp, Héricourt, Montbéliard, Baume-les-Dames, Besançon, entre Gennes et Fontain, bois de la Ratte, Salins, Arbois, Auxange près Dole, St-Laurent-en-Grandvaux, Mirebel, Lons-le-Saunier. - Ain: Arvières, Tenay, Chartreuse de Portes, bois de Rothonne près Belley, Peyrieu, Drom en Revermont, entre Loyes et Villieux. - Genève, Vaud et Valais : bords de l'Arre, du Rhone et de l'Aïre, Payerne, Olleyre, Moudon, Yverdon, Palézieux, mont de Bons sur Daviaz, Roche, les Devens près de Bex, entre Massongex ei Monthey, la Combe près Martigny. H.-Sav, et Sav. : Vougy, Pringy, St-Julien, Evian, Thonon, Annecy, Aix-les-Bains, Tresserve, - Rh. et Is. : Écully, Rochecardon, Gleyzé, Liergues, Bourgoin, bords de la Bourbre jusqu'à Pont-de Chérui, Grande-Chartreuse, le Sappey, Sassenage. - Dr. et H.-Alp. : Aucelon, Charance. - Alp.-Mar. : Tende, col de Tanarello, mont Massiabo près Menton. - Gard : bois de Salbous, Alzon, l'Espérou.

A. marcissophylia L. - Prairies et pâturages des montagnes calcaires des Alpes et des Pyrénées. - Jura helvétirue et français : Chasseral, Creux-du-Van, Suchet, Aiguillon, Pré de Bière, Cluses de la Birse, Hont-d'Or, Montendre, la Dôle, Colombier, Reculet. - Vaud et Valais : Anzendaz, Bovonnaz, Fully, Chavallon de Vouvry, Val d'Illiez, Catogne, Bagnes, Branche dans le Val Ferret, Arête de Thyon, Nendaz, Comba d'Arbaz, Leukerbad, entre Bavon et Vichère de Liddes, Crou- 
maclire.-H.-Sav. et Sav. : Salève, le Mole, Brizon, Vergy, Méri, Dents d'Oche, Hautigny, mont des Granges, mont Pétetod, Haut de Lin, Abondance aux Essets, Signal des Agneaux, mont d'Ubine, Dent de Lanfond, Tournette, mont de Lauvenaz dans la vallée du Fier, entre le mont Jovet et le col du Bonhomme, le Chapiu, la Gitaz, la Sauce, Iauteluce, Beaupré, d'Aimo au col du Cormet, les Allues, col de la Madeleine, mont Cenis. Is. et H.-Alp. : prairies Dufour entre St-Ange et le col de l'Arc, mont Sénèpe, Lautaret, vallons de l'Alp et d'Arcines, mont Viso à la Traversette, St-Véran, Fontgillarde, Rocheronde, col de Vars, Orcières. - B.-Alp. : Lauzanier, Horonaye, Malemort, Fouillouse, St-Paul, Ste-Anne près de la Condamine. Alp.-Mar. : Entraunes dans la prairie des Tourres, lac d'Entrecoulpes, forêt de Clans, vallée de la Gordolasca, cols ds l'Abisso et de Tende, l'Authion. - Pyr.-Or. et Ariège : vallées d'Eyne et de Llo, lac du Llaurenti, cirque des Aiguelles, vallons de Boutadiol et de l'Estagnet, Valbonne sous le Roc de la Trune. - En dehors de nos limites dans les Pyr. centr., dans les Hautes Vosges au Hohneck et au Rotabac.

A. coronaria L. - Lieux cultivés de la région mérid. - Espére polymorphe comprenant les formes suivantes :

A. rosea Hanry : Toulon, au Pommet, le Luc, Nice. - Gard et Hér. : Sommieres, Montpellier au Plan des Quatre-Seigneurs, vers l'aqueduc en face de Bon-Secours, la Gaillarde, Croix-d'Argent, Figairolles, la Madeleine, Vendargues, Castries, Lansargues, St-Geniès, Fabrègues, Mèze. - Pyr.-0r. : prairies de Vivier, de Fosse et de St-Antoine-de-Galamus. - La forme à fleurs rosées est incomparablement la plus commune de toutes.

A. ryanea Risso : B.-du-Rh.: Marseille à Endoume, St-Henry, les Caillols, vallon des Belons à la Treille, Cassis, Aix, Martigues. - Var et Alp.-Mar. : St-Tropez à la Pampalaure, le Luc, Draguignan, Antibes, Nice. - Fleurs bleues.

A. rariegnta ou Rissoana: Grasse, Nice. - Fleurs pleines, panachées de rose, de blanc et de verdâtre.

A. Mouansiana Hanry : Carqueiranne, St-Jacques près Grasse, Rocavignon, le Luc, Mouans. - Fleurs d'un rose violacé. 
A. Ventrima IIanry: Inuans pris Grasse. - Fleurs llun blane jaunitre teinté de rouge à la base.

A. punicen Boiss. : Toulon, Ale Ste-IIargucrite, Grasse, Nice. - Fleurs pourpres.

A. alba Goaty : Rocarignon au-dessus de Grasse. - Fleurs blanclics.

A. Iortenais L. - Champs de la Provence et des Alp.-Nar. Espèce polymorphe comprenant les formes suivantes :

A. stellati Lam., la plus commune de toutes, ayant des fleurs petites, compose̊es de 12-1ə̈ pétales linéaires. - B.-du-Rh. : envir. d'Aix au Cacavéou au N.-0. de la Tour de Keirié, vallons de la Gérarde vers Carpiagne, Morgiou, la Nerthe, Arles, vallon ds St-Pierre d Martigues. - Var et Alp.-Mar. : Toulon, Abbaye du Thoronet, col!ines de Pipière près St-Nazaire, le Luc, Fréjus, Grasse, Auribeau, Menton.

A. pavonina Lam. - Fleurs grandes, à 8-9 pétales obovales, d'un rouge vif, marqués au centre d'une tache jaune; dans les lieux fertiles des localités ci-dessus énumérées.

A. purpurata Jord. - Fleurs pourpres. - Grasse.

A. versicolor Jord. - Fleurs roses, rouges, lilacées ou blanches; est peut-être une forme hybride. - Mouans près Grasse.

A. lepida Jord. - Fleurs intẻrieurement d'un rouge ponceau, exlêrieurement violettes, pourvues au centre d'une couronne blanche. - Grasse, Mouans, Nice.

A. palmata L. - Lieux cultivés. - B.-du-Rh. el Var : Aix dans le vallon qui s'ouvre derrière la Tour de Keirié, le Jasson et la Londe près Hyères, chemin du Château de Léoube.

IER TrCana Chaix. - Lisière des hois. - Envir. de Belfort à la Niotte et à la Justice. - H.-Saône, Doubs et Jura : Nans, Rougemont, Mancenans, Pont-de-Raide, Vermondans. Ain : Pierre-Châtel, Chemillon. - Vaud et Genève: Payerne, Villeneuve, Bavois, bords du lac près de la pointe de Bellerive, Bex. - Valais : Martigny, Val Ferret, Forclaz de Trient, Zan, Sion, Zermatt, Brieg, Naters. - H.-Sav, et Sav. : pied du Salève, Habère-lullin, Arthaz, Arenthon, Reignier, de Morzine aux Gets, Maglan, Passy, Sallanche, Servoz, Chamonix à la cascade de Folly, l'Argentière au Lavancher, Chozalet, Brides, 
St-Jean-de-IIaurienne, Lans-le-Bourg à la Ramasse. - Is. et Dr. : Morestel, mont de Lans au Pénail, Garde-Crosse près Nyons. - H.-Alp. : Villard d’Arène, Névache, la Vachette, mont Prorel près Briançon, granges d'Isoard, bois de Queyras, Devès de Rabou près Gap. - B.-Alp.: la Condamine, Tournoux, St-Paul, Larche, Sisteron à la Baume et au Mollard. Rh. : Cogny: - Vaucl. : le Luberon, Roquesalières, la Garde, mont Ventoux, St-Amans, Sault, Sivorgues, - B.-du-Rh. : SteVictoire, Auriol, Pic de Bretagne, Charleval, pied de TresFourcade près Roquevaire. - Var et Alp.-Mar. : la Ste-Baume, Châteaudouble près Draguignan, Rians, Aups, Fox-Amphoux, Cagnes, Nice, Menton. - Gard et Hér. : le Vigan, chaine de I'Espérou, la Sérane, Viols aux Cambrettes, St-Loup, Bédarieux à St-Raphaël, Avène, Ceillıes, St-Amand-de-Ilounis, Pégayrolles-de-l'Escalette, le Caylar. - Pyr.-0r, et Ariège : Vernetles-Bains, Trencada d'Ambulla, Nolèdes, Mont-Louis, vallée de Llo, col des Ares, Rouze, Roc de Mascaras, vallons de Councq et de Canals, lacs de Quérigut, de Llaurenti et de Rabassolès, Escale de Valbonne, vallon de Boutadiol.

ADONEs autumenalis L. - Moissons. - Commun dans la région mérid. d'oì il remonte jusque vers Gap, Briançon, Tienne, Lyon; Dijon, Flavigny, Lucenay, Ruffey dans la C.-d'Or; les plaines de l'Ain, du Jura el du Doubs; le Valais à Folatères, Sembrancher, Bagnes, Conthey, St-Pierre, Vétroz, Sion.

A. aestivalis $\mathrm{L}_{\text {. }}$ - Moissons. - Doubs et Jura : Audincourt, Ar bouans, Dôle, Peseux, Longwy, Annone, Petit-Noir. - Ain, Rh. et Is. : Ambronay, les Charpennes, Vaux-en-Velin, Décines, entre Prunières et La Mure, Ste-Luce en Beaumont. - Sav. et H.-Alp. : Aime, St-Jean-de-Maurienne, Briançon. - B.-duRh. : envir. d'Aix à Puyricard, à Collongue el à la Pioline. Var et Alp.-Mar. : Fox-Amphoux, Gourdon, Menton, SanRemo sur la côte ligurienne. - Gard et Hér. : Villeneuve en face d’Avignon, envir. de Montpellier, de Béziers, - Pyr.-Or. : Trencada d'Àmbulla, Oletle, Villefranche.

La couleur des fleurs est tantôt rouge (A. miniata Jacq.), tantot jaune (A. flava Vill.). 
A. liammen L. - Moissons, - G.ed'Or et S.-el-L. : Dijon, StRemy, Asnières en Montagne, Laignes, Lucenay, les Laumes, lluffey, Bourberain, la Canche, Curgy, Couches, Cluny. H.-Saone, Doubs et Jura : Andelarre, Charriez, Villers-Poz, Ruz-de-Vellcmoz, Chissey, Cramans, alluvions du Doubs et de la Loue près de Chaussin, Chemin, Montbarrey, Villers-Farlay. - Ain et Rh. : plaine d'Ambronay et de Leyment, Vaux-enVelin, les Clıarpennes. - H.Sav. et Sav. : Évian, Aime. Is., Dr. et H.-Alp. : Décines, la Motte Chalancon, Séuse, Rometle et les envir. de Gap. - Vaucl. et B.-du-Rh. : l'Isle, Vedene, Mazan, envir. d'Aix, entre Roquefavour et les Milles, vallon de Parouvier sous Venelles, Simiane. - Var et Alp.Mar. : Plan d'Aups, le Luc, Ampus, Draguignan, Mas près StAuban. - Gard et Hér. : Trèves, Lanuéjols, envir. de Montpellier, de Béziers et de Lodève.

Espèce polymorphe dans laquelle la forme des pétales est sujette à plusieurs variations.

A. vernalis L. - Coteaux secs. - Valais : les Folatères, Charrat, Saxon, Bramois sur Turtmann, Visp. - Gard: les Mazes, Lanuéjols, l'Espérou. - En dehors de nos limites sur les plateaux jurassiques de la Lozère à la Causse de Sauveterre et de Míjean près de Villaret.

A. pyrenatea DC. - Pelouses des Pyr.-Or, à Carença, Cambres d'Aze, valléc d'Eyne. - Pyr.-Centr. à la Piquette d'Endretlis, Castanèse, vallée de Gabas.

MrDEUges aninimers $L$. - Terres argileuses ou sablonneuses humides. - C.-d'Or : Citeaux, Seurre, Dijon, Genay, Rouvray. - La Bresse louhanaise et jurassienne, la Dombes, terrains granitiques des envir. de Lyon à Dardilly, la Tour-de-Salvagny, le Beaujolais. - Loire : Rive-de-Gier, St-Genis-Terre-Noire. Gard et Hér. : bois de Broussan, entre Nimes et St-Gilles, Aigues-Mlortes, Laltes, Palavas, Grammont, Agde, Pérols, Pézenas, Portiragnes. - Terres sablonn. du Roussillon.

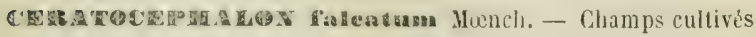
des plaines du Gard, Hér., Var, b.-du-Rh., Vaucl., B. et H.-Alp.

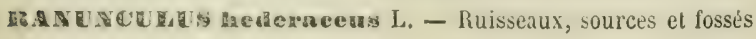


dans les terrains siliceux; nul dans les terrains calcaires. H.-Saône : Champagney, Melisey. - La Bresse jurassienne à Tassenières, Pleurre, Fays, Neublans; forêt granitique de la Serre entre Vriange et Serre, Bresse de l'Ain autour de Bourg, Neuville-les-Dames. - G.-d'Or et S.-et-L. : Morvan granitique is Saulieu, Champeaux, Roche en Brenil, Rourray, Arnay-le-Duc, Semur, Morvan autunois. - Coteaux granitiques du Beaujolais, du Lyonnais, de Tarare, du Pilat, du Vivarais, des Cévennes du Gard et de l'Hérault. - Aude et Tarn : les deux versants de la Montagne-Noire.

La forme coenosus Guss, à pétales plus longs, à carpelles munis d'un bec, et à feuilles crénelées a été signalée à St-Jeand'Ardière en Beaujolais.

P. aquatilis L. - Fossés el mares. - Espèce polymorphe. - Ordinairement les feuilles flottantes sont réniformes, les feuilles submergées ont des segments capillaires. Lorsque toutes les feuilles sont immergées dans l'eau on n'observe que des segments capillaires. Au contraire lorsque l'eau se retire, toutes les feuilles sont réniformes. C'est assez dire qu'il faut bien se garder de la tentation qu'on pourrait avoir d'établir des différences spécifiques entre les Renoncules aquatiques d'après la considération des organes végétatifs. C'est ainsi que le $R$. fluitans Lam. a des tiges tantôt glabres, tantôt velues; son réceptacle, ordinairement glabre, est quelqueíois hérissé de poils, comme on le voit dans les autres Batrachion.

Les $R$. trichophyllus Chaix et divaricatus Schrank ont, le plus souvent, des feuilles toutes divisées en lanières capillaires, ne se réunissant pas en pinceau lorsqu'on les tire hors de l'eau. La longueur du pédoncule n'est pas moins variable : celui-ci, court dans le $R$. trichophyllus, est allongé dans le $R$. divaricatus, dans le $R$. confusus Godr, ainsi que dans le R. marinus Fries (Bandotianus Gien.) qu'on observe dans les lieux arrosés par les eaux salées.

E3. rentrolings, $R$. Thore L. - Rocailles des montagnes calcaires. - Chaine jurassique : la Dóle, Colombier, Reculet, la Faucille, du côlé de Mijoux. - Vaud et Valais : Anzendaz, Chaudes, les 
Recourbes sur Nayes, la Montagnetle au pied lu rocher du Midi, Alpes d'Evionnaz et du Val d'llliez. - II.-Sav. : Salève au sommet de la Grande Gorge, le Hole, Brizon, Glacière de Solaison, Parmelan, Dent de Lanfond, Tournette, col des Aravis, chaine des Cornettes à la montagne de Fontaine et de Pelloua, Signal des Agneaux, Abondance aux Esssets, - Is. : Charmant-Som, St-Nizier, mont Sénèpe. - Pyr.-Or. et Ariège : Font-de-Comps, Nohèdes, Val de Paillères sous le Roc de Lorry, Valbonne à l'Escale et sous le Roc de la Trune. - Pyr.-Centr.

[x. sagestris L. - Paturages el rochers des montagnes calcaires.

- Jura helvétique et français: Hasenmatt, Chasseral, Chasseron, Creux-du-Yan, Mont-d'Or, Suchet, Montendre, Colombier, Reculet. - Vaud et Valais: Jaman, Anzendaz, Bovonnaz, Arête de Triqueud, Catogne, Comba d'Arbaz, Donin, Guggerlubel, Lens, Zan, Val Reschy, Mainghorn, Gemmi, val de Binn entre Tschampigen et Schinnern, Grimsel. - H..Sav. : le Môle, Vergy, Méri, Tournette, Dents d'Oche, Cornettes de Bise, mont des Granges, Abondance aux Essets, Nautau, col de Golèse, Buel, Sixt, Entre-les-Eaux, vallon du Vieux-Emousson, mont Jovet, cols du Bonhomme, de la Seigne et Joly, les Mottets, Allée-Blanche sur le versant italien. - Is. : Grand-Som, Cuarmant-Som, la Moucherolle. - H.-Alp. : mont Viso, la Taillante, col Agnel. - Alp.-Mar.

3i. glaciallis L. - Pâturages et rocailles des terrains siliceux des Alpes et des Pyrénées près des neiges et glaces fondantes. Vaud et Valais : Dent-de-Morcles, Martinets, Javernaz, Paneyrossaz, Surchamp, Anzendaz, les Diablerets, Chattillon, Prapioz, St-Bernard, Bagnes, col de Fenêtre, Thyon, Orsera, Diez, Ferpecloz, mout Nuoble, Laemmeralp, Torrenthorn, vallée de Saas à l'Offenthal, à Mattmark et au Schwarzberg, Zermatt au Hoernli, au Gornergratt et à Zmult, mont Cervin, col St-Théodule, Gries, Sirwolten, Simplon. - II.-Sav. et Sav. : chaine du mont Blanc à la Mer-de-Glace, au Jardin, aux Grands-Mulets, sous les Aiguilles du Gouté, du Nidi, des Charmoz et du Tour, Tré-la-Tête, cols du Géant et ảe Balme, Aiguilles-kouges, Buet, cols du Bonhomme, des Fours et de la Seigne, les Essets près 
Abondance, Nautau, mont Chalune, col de Golèse, les Aliues, mont Jovet, Iseran et Cenis. - Is. et H.-Alp. : chaines des SeptLaux, de Belledonne, des Grandes-Rousses, de Taillefer, du Mont de Lans et du Pelvoux, Cervières à Venton, col des Ayes, Galibier, mont Viso aux cols de la Traversette et de Ruines, St-Yéran, le Noyer en Champsaur, Orcières, Chaillol, mont Aurouse, mont Obiou. - B.-Alp. : Crouès, Lauzanier, Vallonnet, Parpaillon, Riou-German, Bérard, lac d'Allos, Colmars. Alp.-Mar. : Roche-Grande, col de Jallorgues, Alpes de Carlin et de Tende. - Vaucl. : mont Ventoux. - Pyr.-Or. : Canigou, vallẻe d'Eyne, Cambres d'Aze, Fosse-du-Géant, Carença. Fyr.-Centr. - Les fleurs, ordinairement blanches, sont quelquefois rosées.

Rz. dasyenups, $R$. Seguieriunus Vill. - Rocailles des montagnes calcaires. - Is. et Dr. : C'llamechaude, St-Nizier, col de l'Arc, lą Moucherolle, mont Glandasse. - H.-Alp. : Charance, cols de Glaise et du Noyer, Chaudun, mont Aurouse, - B.-Alp. et Alp.Mar. : Vallonnet, Bachasse, Parpaillon, Soleil-Buou, Serennes, Mont Grand-Couyer sur Annot, sources du Var à RocheGrande, Alpes de Tende, le Garret. - Vaucl. : mont Ventoux.

E. Rutifolius L. - Rocailles des Alpes près des neiges fondantes.

- Valais : Zermatt au Riffel, au Schwarzsee et au Staffel, mont Horo, Val Binn près du glacier de Langthal, Gries. - Is. et H.-Alp. : Piéméyan au mont de Lans, Taillefer, Galibier, Lautaret, mont Viso à la Traversette, St-Yéran sous la Chapelle de Clausis, mont Aurouse. - Sav. : Laval de Tignes, mont Iseran, col du Palet, mont Cenis à Cornerousse. - Alp.-Mlar. : Carlin, mont Bego.

R. aconitophyllus L. - Lieux humides des montagnes. Chaine des Vosges jusqu'aux Ballons de Giromagny et de Servance dans la H.-Saône, vallées du Rahin, de Miélin, du Fray, du Puy, de la Fonderie, Champagney, Bélonchamp, Ternuay, lisière vosgienne de l'arrond. de Belfort. - Région des Sapins du Jura helvétique et français jusqu'au Colombier du Bugey, à Retord et au Vély près IIauteville. - Vaud, Valais, Savoie, Dauphiné, B.-Alp., Alp.-Har. - C.-d'Or, S.-ct-L., Rh. et 
Loire: le Morvan autour de Saulieu et d'Autun, St-Léger, StSernin-du-Bois, Auxy, Antully, Marmague, Broye, sommités de la chalne beaujolaise, St lionnet-le-Froid, Sivelette, l'ilat. - Ard.et II.-L. : chaine du Virarais et du Mezenc. - Gard et Hér. : l'Espérou, Concoule, St-Pons. - Aude, Pyr.-Or. et Ariége: Montagne-Noire, Canigou, Nohèdes, Prats-de-Balaguer, vallées d'Eyne et de Carença, massif du Llaurenti. - En dehors de nos limites dans les Pyr.Centr., Aveyron, Tarn, Lozère, Cantal, mont Dore, Forez à Pierre-sur-Haute, Vosges.

Une forme naine se présente quelịuefois dans les montagnes. R. plutanophyllus L. - Forme du précédent à lobes des feuilles plus profondément divisés et plus aigus, à pédoncules ordinairement glabres, plante de taille plus laute croissant dans les mêmes rếgions que la Renoncule à feuilles d'Aconit, mais sur des terrains non constamment mouillés.

Le R. lacerus Bell., probablement hybride des $R$. platanophyllus et $R$. pyrenacus, avait étê signalé à Champrousse au-dessus d'Uriage et à la Grangetle près Gap, mais il n'a pas été retrouvé dans lés susdites localités.

8s. parnassogonghas L. - Débris pierreux calcaires des hauts pâturages des Alpes et des Pyrénées. - Vaud et Valais : Anzendaz, Paneyrossaz, Boulaire, Javernaz, Dorenaz, Arêtes de la Levraz, la Vauveresse, Fully, Dent du Midi, St-Bernard, Sánetscl, Pépinet, Laemmeralp, Guggerhubel. - II.-Sav. : Combe de Sixt à la Vauzalle, Samoëns, Vergy, Méri au Château. - Is. et II.-Alp. : Piéméyan au mont de Lans, Villard-d'Arène aux Trois-Évêchés et au Glacier du Bec, Goléon, Côte-Ronde, Galibier. - B.-Aip. : crêtes de Pancyron au S-E de l'Hospice de Vars, vallon de Peyres-Rousse, Riou-German près Serennes. PJr.-0r et Ariège: Collada de Nuria, Coma de la Bảca, vallons de Llaurenti et de Barbouillère sous les escarpements du Roc Blanc. - Pyr.-Centrales.

Il amgustifolius DC. - Forme du précédent à pédoncule glabre et à fcuilles linéaires. - Prés marécageux des Pyr.-Or. et de l'Ariege à Mont-Louis, la Llagone, Font-Romen, Coma du 'Tech, lacs du Llaurenti, de Yalbonne, de l'Estagnet ct de Rabassolès, Catal. Bassin du Rhône. 
vallons de Barbouillere et de Boutadiol, Val de Paillères à Soucarrat.

Mb. porrenacas L. - Piturages des Alpes et des Pyrénées. - Vaud el Valais : Javernaz, Martinets, Moveran, Lavarraz, Paneyrossaz, Anzendaz, Cheville, Prapioz, Alesse, Fully, Bagnes, Val Ferret, St-Bernard au Plan-des-Dames et de Jupiter, la Baux, Thyon, Guggerhubel, mont Nuoble, Cotter, Zan, Torrentalp sur Leukerbad, Val de Turtmann, Zermalt, Saas, Binner et Aernergalen, Furca. - H.-Sav. : Vergy, Méri, Cornettes de Bise, Signal des Agneaux, Pormenaz, col de Balme, du Bonhomme, et de la Seigne, les Mottets, la Saxe au-dessus de Courmayeur sur le versant italien. - Sav. : mont Cormet entre Roselein et le Chapili, Montagne de Tours près Albertville, les Allues, mont Jovet, Beaupré, mont Iseran, mont Cenis. - Is. et H.-Alp. : Champrousse, Alpe du mont de Lans, mont St-Nichel près la Mure, St-Nizier, la Salette, Lautaret, mont Viso à la Taillante et au col Agnel, Mélezct de Guillestre, cols Nalrif, des IIayes et de Glaise. - B.Alp. et Alp.-IIar. : Lauzanier, Parpaillon, Crouès, Maison-Méane, forêt de la Maïris. - Pyr.-Or. : Canigou, Nohèdes, Mont-Louis, vallẻes de Carença, d'Eyne et de Llo. - Pyr.-Centr. - Espèce polymorphe ayant des fleurs 1-5 de grandeur variable, des pédoncules plus ou moins hérissés, des feuilles plus ou moins étroites. Dans la variété plantagineus $\mathrm{DC}$., les feuilles sont ovales-lancéolées; dans la variété bupleurophyllis DG., elles sont lancéolées-linẻaires.

R. gramineasa L. - Pàturages. - C.ed'Or' et S.et-L. : Marsannayla.Côte, Gevrey, entre Buxy el Culles, Ñantoux près Bouzeron. - Valais : Plâtrières de St-Léonard, - Rh., Ain et Is. : Vauxen-Velin, Meximicu à Giron, St-Jean-de-Niost, Blyes, Balan au Content, Château-Gaillard, $\Lambda$ mbronay, Décines. - H. ct B.-Alp. : Rambaud, mont Bayard et Vescal près Gap, la BâtieNeuve, Annot, Sisteron. - Vaucl. : pente nord du St-Amans. - B.du-Rh. et Var : Monastère de Ste-Victoire, Vauvenargues, Tète-de-Carpiagne, Pic de Bretagne, Ste-Baume. - Alp.Mar. : montagnes de Cheiron el de Caussols. — Gard et Hér. : Alzon, Pouls, bords du Gardon à la Beaume, les Capouladoux, 
Ia Serane, pied de St-Loup, St-Pons, Avene, Pardailhan, Pontguiraud, le Larzac à Sorbs. - Pyr.-Or. : Noliedes, Mont-Louis.

I8. Inaceolatus C. Bauhin, R. Flammula L. - Fossés, étangs, prés humides des plaines argileuses. - Espèce polymorphe variant quant à la forme et la grandeur des feuilles, la direction des tiges, lesquuelles sont quelquefoiz couchées et radicantes aux nouds, comme il arrive dans la variétó reptans Thuill.

E8. Nongifollus C. Bauhin, R. Lingua L. - Mares et fossés. Elang de la Maiche près Belfort. - II.-Sacne: Vy-le-Ferroux, Vauchoux, Ferrières, - Doubs: maráis de Fesches, Seigne de Jorteau, marais de Saone pris Besançon. - Jura et Ain: la Bresse à Pleurre, Sergenon, Chaumergy, Bletterans, la Crose, et le lac de Bar pris Belley, Viricu-le-Grand, Nantua, Cormaranche. - Vaud et Valais : Orbe, Yverdon, Payerne, Yronand, Duillier, Vidy, Gourze, Vervey, Noville, Villeneuve, Roche, Aigle, Fully, Visp. - II.-Sav. : Pontchy près Bonncville, Epagny. - C.-d’Or et S.-et-L. : Saulieu, Limpré, Saulon, Hagnysur-Tille, Satenay, Laignes, Vertaut, Fontaine-Française, Beauregard entre Verdun et Chagny. - Rh. et Is. : Bourdelans près Anse, Salvinet près Panissières, Yaux-en-Velin, Décines, Janeyjiat, Crémicu, Frontonas, St-Quentin. - B.-du-Rh. : fossés de Raphèle, Arles en Cousticro, Montmayour, Mas Thibert, étang du Mas d'Icard, la Crau et la Camargue. - Gard et Hér. : Beaucaire, Mas-de-Bourry près le Caylar, prairies de Beauregard près de Marsillargues, étang de Capestang. - Pyr.-Or. : Nolièdes, Mont-Louis.

דE. nuricenaus L. - Prés el lieux ombragés dans les parties septentr. et moyenne du bassin; rare dans la région mérid. Gard el Hér. : le Vigan, Alzon, l'Espérou, St-Amand-de-Mounis, versant nord de la Serane.

EE. aม on helvétique ct français : Cliasseral, la Tourne, Aiguillon, Suchet, mont de Stc-Croix, Montendre, la Dolc, Colombier, Reculet, Sorgiaz, calc. jurass. des envir. de Montbéliard, de Pontarlier, Salins, Arbois, Nozeroy, Champagnole, Retord, Colombier du Bugey. - Vaud el Valais : Lioson, les Mosses, Jaman, Grand- 
vire, Anzendaz, Cheville, Sembrancher, Catogne, val Cliampé, Loze, Comba d'Arbaz, Tlıyon, Cleuson, val Nendaz, Leukerbad. - II.-Sav. et Sav. : Salève, Mốle, Brizon, Vergy, Méri, Platets, Ayers-sul-Servoz, cols de Balme et du Bonhomme, Dents d'Oche, Signal des Agneaux, Haut-de-Lin, Dent de Lanfond près Annecy, la Tournetle, la Madeleine, Dent du Chat, les Bauges. - Is. et H.-Alp. : Bovinant au pied du Grand Som, Charmant Som, Chamechaude, Sappey, St-Eynard, St-Nizier, col de l'Arc, Côte Gélive et col de Glaise, mont Séuse, Chaillol, Champoléon, Combe de Segure. - B.-Alp. el Alp.Mlar. : Lauzanier, Fouillouse, Parpaillon, St-Mlartin-Lantosque, col de Tende. - Vaucl. : mont Ventoux. - Aude, Pyr.-Or. et Ariege : col de l'Hommenet entre Nontfort et Ste-Colomibe, Carença, Mont-Louis, vallée d'Eyne, Barbouillère en montant au Pas du Roc Blanc, Pic de la Camisette, Jassettes du L!aurenti, Valbonne sous le Roc de ta Trune, lac de l'Estagnet, Port de Paillères, vallons de Canals et de Councr.

Espèce polymorphe à laquelle se rattachent :

$R$. gracilis Schl, ayant les lobes des feuilles plus écartés, plus étroits et plus aigus, des fleurs moins grances, une taille plus petite; se trouve dans les mêmes localités que le type montamus ou le remplace.

R. aduncus G. G., R. Villursiunus DC. - Forme alpine à grande fleur, à tige et feuilles velues, à bec des carpelles plus recourbé. Vaul et Valais : Bovonnaz, Anzendaz aux Essets, St-Bernard près de l'Hospice, Grimsel. - II.-Sav. et Sav. : Cornettes de Bise, mont des Granges, mont Pétetod, mont Ardin, Nautau, Sixt à la Vauzalle, Aiguilles-Rouges, Brévent, Allée-Blanche, Héri, Parmelan, Tournette, Charvin, montagne des Avanchers, mont Cenis, - Is. et Dr. : Pariset, St-Nizier, Prémol, la Motteles-Bains, la Chapelie-en-Valjoutfrey, mont Glandasse près Die. - H.-Alp. : Monestier de Briançon, Cervières, mont Genèvre, col de Vars, mont Viso, St-Véran à la Cote Grimaud, mont Séuse ct Aurouse, N.-B.-du-Laus, Boscodon, bois de Rabou et de Chaudun, Ribiers. - B.-Alp.: Ste-Anne press de la Condamine, Parpaillon, Lauzanier, Horonaye, Tournoux, Se- 
rennes, Colmars, Couss an it les Dourlues près Digne. - Alp.Mar.: sommités des vallées du Var, de la Vesubia, Tinea, Roja. - Vaucl. : mont Ventoux. - Pyr.-0r. : vallées d'Eyne et de Llo, Nohèdes.

R. geraniophyllus Pourr. - Pyr.-Or. et Aude : Bolquère près MontLouis, col de l'Hommenet entre Ionfort et Ste-Colombe.

R. villosissimus, R. Gouanianus Willd. - Forme très-velue, à feuilles caulinaires embrassantes, assez commune dans la cliaine des Pyr.-0r. el Ariège, dans les vallées d'Eyne et de Nohèles, valIons du Llaurenti, de Boutadiol et de Barbouillere, lac de Valbonne, Roc de l'Ermite. - Pyr. centrales.

re. neris $L$. - Clamps et prairies des plaines et des montagnes. Espece polymorplie dans laquelle on observe de nombreuses variations quant à la forme des feuilles, la direclion du bec des carpelles, la villosité des tiges. Sous ce dernier rapport les poils sont tantôt appliqués sur la tige, comme dans les $R$. vulgatus Jord. et Boraeanus Jord., tantôt étalés et roussâtres, comme dans le R. Friesianus Jord, - Quelquefois on rencontre des individus presque glabres et présentant les autres attributs qui appartiennent à ces trois formes.

Les feuilles profondément palmatiséquées, à subdivisions lancéolées-ćtroiles dans la forme Boracanus, ont des segments larges se recouvrant l'un l'autre dans la forme Fvicsiamus, et plus larges encore, mais écartés, dans la forme Stevenianus Andrz.

Et. In uaginosers L.. - Bois du Jura helvétique et français jusque dans le Bugey, Vaud, Valais, Savoie, Dauphiné el B.-Alp. Gard : l'Espérou, Salbous à Banahu.

15. Inolustris Sm. - Espece de Corse trouvée à Toulon sur les talus du fort Malbouscquet.

E. velutinus Ten, - Lieux frais et ombragés, - Var et Alp.Mar. : Toulon au Fort Malbousquet, Draguignan, St-Tropez à Berthaud, Cannes, Antibes, bords de la Brague.

m. silvaticas Thuill, - Bois des collines et des montagnes.

1R. repens L. - Prés el champs humides, fossés, - Espèce polymorplıe comprenant les formes décrites par II. Jordan sous les noms de $R$. reptabundus, glaber et villosus. 
re. bulbosus L. - Prés, champs, bords des chemins. - Espèce polymorphe comprenant les formes suivantes décrites par II. Jordan : bulbifer, albinacvus, valdepubens, sparsipilus.

Ix. neapolitanus Ten. - Forme à souche terminée par des fibres napiformes et non renflée en bulbe. - Var : Toulon, Hyères' iles d'Hyères, Porquerolles, Bormes, St-Tropez, Fréjus.

Hi. amosngpeldacus L. - Champs, vignes et pâturages de la rígion mérid. d'où il remonte jusque près de Lyon. - Espèce polymorphe se subdivisant en deux formes principales ci-après énumérées.

$R$. cyclophyllus Jord., R. rolundifolius DC., $R$, saxalilis Balbis. Forme particulière aux terrains siliceux, ayant des capitules ovales-oblongs, les fcuilles inférieures arrondies en cœur, à lobes courts. - Terrains granitiques du Lyonnais à Beaunant, Francheville, St-Genis-Laval, Chaponost sur les collines du Garon, Irigny, Grigny, Givors. - Dr. : granite de St-Vallier. - B.-du-Rh. : bords de l'Huveâume à la Pomme, Roquevaire, vallon de Vèdes à Auriol, Roussargues, Simiane, Mimet. Var: granite et gneiss des Maures aux Mayons. - Gard: le Vigan, Salbous, Alzon, Valleraugue. - Hér. : St-Guilhem-leDésert, la Sérane, St-Martin-d’Orb, St-Etienne-de-Illursan, vallée de la Mare à Andabre, Pardailhan-Pontguiraud, Lodèvo. - Aude : Trèbes.

$R$, albicans Jord. - Forme des terrains calcaires, de coulcur soycuse-blanchâtre, dont les feuilles inférieures ont des segments oblongs-cunéiformes, des carpelles en capitule ovale arrondi. - B.-Alp. : Castellane. - Vaucl. et B.-du-Rh. : envir. d'Apt, d'Avignon et d'Orange, envir. d'Aix à la colline des Pauvres, au vallon du Coq, à la Ste-Victoire, bords de l'lluveaume. - Var : les Calles à Ampus. - Dr. et Ard. : la côte du. Rhône depuis Montélimar et Viviers jusqu'à Valence et Crussol. - Rh. : collines caillouteuses de Grigny, Chassagny, StGenis-Iaval. - Gard : Nìmes, Tresques, bords du Gardon près de la Beaune, St-Nicolas, Remoulins, Vic. - Hér. : envir. de de Montpellier à Montplaisir, Sauret, Lavalette, Castelnau au bord du Lcz, Fabrègues, Montferrier, Lunel, Marsillargucs, 
Ganges, Lolève, Beicrs. -- P'yr.-Or. : Collioures, Banyulssur-Mer.

La plante décrite par II. Jordan sous le nom de R. lugdunensis a des fleurs plus grandes, des feuilles de couleur plus verte et moins cendrée. - Le $k$. Gonnetianus Jord., trouvé dans le Gard, a les feuilles primordiales d'un vert clair, ondulée sur les bords, à lobes se recouvrant un peu.

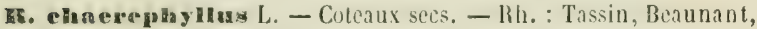
Charbonnières, Chaponost. Brindas, St-Alban, Vassieux, plaine de Royes. - Ain : la Valbonne, Meximieu, le Revermont au hois de Rosy. - Is. et Dr. : Crémieu au mont d'Annoisin, Charvieu, la Cóte-St-André, St-Vallier. - Vaucl, et B.-du-Rh. : les Barbiers près $\Lambda$ pt, Aix au N.-0. de la Tour de Keirié et sur les bords de l'Arc, St-Tronc, bords du Jarret vers St-Just, la CroixRouge, Pian de Carpiagne, Luminy, la Nerthe, en Coustiero, la Crau. - Var et Alp.-Mar. : Hyères, le Luc, Rians, St-Tropez, Fréjus, l'Esterel, Biot, la Roquette, Nice au Vinaigrier, Villefranche au cap Ferrat. - Gard et Hér. : Nìmes, Manduel dans les bois de Campagne, St-Guiral près Alzon, l'Espérou, envir. de Montpellier, de Béziers, de St-Pons et de Lodève au Caylar, aux Rives, au Cros, à St-Mlichel et à Pertus. - Aude et Pyr.Or. : Narbonne, vallée du Réart.

f. chacrphylloideus Jorl. - Forme à lige hérissie de nombreux poils étalés, à capitules fructifẻres plus allongés, à carpelles terminés par un bec plus courbé. - Var : Hyères, les Jaures du Luc. Gaujac près du Vigan (Gard).

re. philonotus Ehrh. - Lieux humides des terrains siliceux.

R. trilobus Desf. - Forme glabre, à fleurs plus petites, à carpelles hérissés de tubercules plus nombreux et plus forts. - B.-duRh., Var ct Alp.-Mar. : Narseille aux Catalans et à la Belle-deMai, Sablettes de la Seyne, Castigneaux, Antibes. - Pyr.-Or. : Argelès, Collioure, Port-Vendres, Banyuls-sur-Mer.

15. marvagrus L. - Champs humides de la région mérid, et moyenne. - Var et Alp.-Mar. : Hyères, île de Porquerolle, forêt des Maures, l'Esterel, la Roquette, Villars, Levens, Nice, Menton. - Gard et Hér. : envir. de Nimes et du Vigan, Argelliers, 
le Pous près du Pic St-Loun, Capouladoux, Bẻziers, Lamalou, Pardailhan-Pontguiraud, Lolève. - Aude et Pyr.-Or. : Narbonne, bois de Céleiran, Ste-Lucie, Argelès, pied des Albères, Collioure, Banyuls. - Rh. et Ain : Vernaison, Millery, Vaugneray, Chaponost, Pollionay, Quincié, Corcelles, Beaujeu, la Valbonne, St-Maurice, Charnoz, embouchure de l'Ain, Meximieu. - Is. : Bouvesse, Villette d'Anthon. - Dans cette espèce les pétales sont quelquefois plus courts que le calice.

T6. ophloglogsoghyltas L. - Lieux humides, fnssís de la région mérid. - B.-du-Rh. et Var: Arles en Coustiero, la Crau, la Camargue, bords de l'étang de Marignane, Hyères, forêt des, Maures, Fréjus. - Gard et Hér. : envir. de Nìmes, Montpezat, Campagne, Montpellier à Lamoure et Grammont, Mauguio à St-Marcel, Mas de Marot, la Planchude, Pérols.

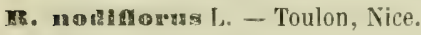

FE. Interdatoma DC. - Hér. : mares de Roquehaute à Portiragnes près Agrle.

R. sxvensis $L_{\text {. }}$ - Champs cultivẻs. - Espèce polymorphe prósentant quelrnefois tles carpelles inermes.

Es. maracatans L. - Lieux huiniđes de la région mérid. - Vaucl. : Rorquesalières près Apt. - B.-du-Rh, Var et Alp.-Nar. : envir. d'Aix, bords de l'étang de Marignane, de Martigues à la Couronne, Toulon, Six-Fours, Ollioules, St-Nazaire, la Garde. IIyères, Draguignan, St-Tropez, le Luc, Fréjus, Cannes, Antibes, Nice, Menton. - Gard et Hér. : envir. de Nimes, St-Gilles, Manduel, Andıze, Alais, Montpellier, Béziers, Lodève. - Aude et Pyr.-0r. : Narbonne, Perpignan, Port-Vendres, Banyuls. Remonte quelquefois dans la partie moyenne de la vallée du Rhône.

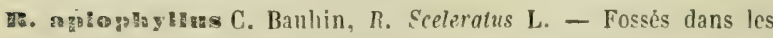
parties septentr. et moyenne de notre bassin; plus rare dans la région mérid.

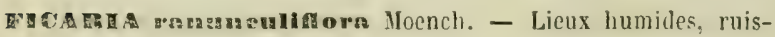
seaux, fossés dans tout le bassin. - Espèce polymorphe.

F. granliffora Robert. - Forme à grandes flcur's assez commune dans la région méridionale. 
Cartra polustria L. - Ruisenux et prés liumides des plaines et des montagnes. - Espèce polymorphe.

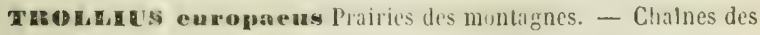
Vosges, du Jura, Alpes helvéliques el françaises, Pyrénẻes. Massifs du Pilat, du llezenc, de Concoule. - En dehors de nos limites dars les Pyr. centr., Lozère, Aubrac, Cantal, mont Dore, chaîne du Pay-de-Dôme, Forez à Tierre-sur-Ilaute et au pic Pìlé de Noirétable.

Fraxdraso hiemule Salisb. - Doubs : Montbéliard au coteau un Cimetière. - Vaud : Bex, Villa d'Cex, Ilorges, Lonay, Trey. covagnes, Bonvillard, Châtcau de Mathod, Champagne. - Is., II. et B.-Alp. : Monesticr-de-Clermont, Briançon, Castellane. Gard: bois de l'Agre près de Dourbie. - En dehors de nos limites, a êté naturalisé autour du château de Landsberg près Barr (Alsace), à St-Denis-en-Val près d'Orléans (Loirel), au bois de la Queue-en-Brie, au bois de la Boische et au parc de Nainvilliers près de Pilliviers, Malesherbes, pares du Trianon et du Raincy, Asnières près Paris; dans la Sarthe à Ivrc-l'Évêque, champs de Charreau; dans la Seine-Infér. à Quévilly près Rouen. - Il est probable que dans toutes les susdites localités l'E. hicmale est échappé des jardins ou naturalisé par' les soins de rquelques botanistes. Nous savons, en effet, que J. Bauhin et Thomas ont semé autour de Montbéliard et de Bex des graines de cetle plante si remarquable par la précocité de sa floraison.

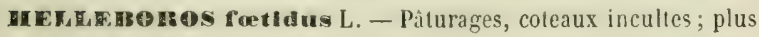
fréquent dans les terrains calcaires. - Espèce polymorphe préscntant de nombreuses variations dans la forme des feuilles et comprenant les $H$. rhodanicus, beugesiacus, deflexifolius et nemo. ralis décrits par MII. Jordan et Fourreau.

15. viridis L. - Bois et buissons. - Jura : Balanod près de StAmour, Dole. - Vaud et Valais: Salins, Plambuit, Chillon, entre Belmont et Gourze, Penthalaz, Lussy, la Vraconnaz, entre Martigny et le pont sur la Dranse. - H.-Sav. : St-Laurent près la Roche, Naves près Annecy, Dessy, Vougy, St-Roch près Sallanches, Mígère, Rumilly. - Is. el Dr. : St-Chef près Bourgoin, mont Rachais, la Fretle près de Quaix, St-Harcellin, St- 
Thomas près St-Jean-en-Royans, - H.-Alp. : Clarance près Gap. - Alp.-Har. : entre Lupega, Carlin el Tende. - Gard et llér. : bois de Lengas et de l'Aigual, St-Amand-de-Ilounis. Pyr.-Or. et Ariége: vallées do Vernet-les-Bains et de Fillols, Motte de Planès, Fctges, vallées d'Eynes et de Llo, le Capcir, col des Ares, Roc de Mascaras, vallon de Councr et de Canals, Couillade du Prat-Lastou, val de Paillères à Soucarrat, Baouzeilles de Tarbézou, Bose-Négré. - En dehors de nos limites dans les Pyr. centr., Tarn, Aveyron, Lot, Cantal, Puy-de-Dome, bassin parisien.

Reuter et Personat estiment que l'Hellebore vert de France est distinct de la plante orientale décrite par Linné sous le nom de Helleboros viridis, et, par consérquent, ils proposent de l'appeler II. occidentalis.

Une forme trouvée à St-Chef et à St-MIarcellin (Is.) a étê nommée II. brevicaulis par MII. Jordan et Fourreau.

Ir. niger L. - Souvent cultivẻ et quelıuefois échappé des jardins.

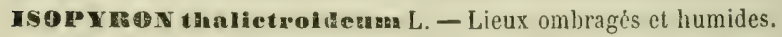
- C.-d'Or et S.-et-L. : bois de Barbirey, Combe d'Arcey près Pont-de-Pany, bois de Canada près Autun et jusqu'au Pont-duRoi entre Auxy et Tintry, garenne de Niou près Couches. Doubs et Jura : entre Quingey et Courtefontaine, Balanod près St-Amour, lisière du bois de Joux près de Chancy. - H.-Sav. et Sav. : Vulbens, Mandallaz près Annecy, Naglan, Mailand en face de la cascade d'Arpennaz, Seytenex, Tamié, Bromine, rive gauche du Rhone en aval de l'embouchure du Fier, le Tremblay, Chambéry. - Ain : St-Rambert, Bellcy, Heximieu, Reyrieu. - Rh. : Rochecardon, Ecully, Francheville, Tassin, Charbonnières, Ste-Foy aux Razes, Alix, Liergues, Panissières. - Is. : Crémieu, Bourgoin, Balmes de Fontaine. - Pyr.-Or. et Ariège : Salvanère, Boucheville, vallon de Quérigut à la forêt de la Limouse, Roc de Mascaras, cirques des Aiguettes et d'Artounant, val de Failleres à Soucarrat. - En dehors de nos limites dans les Pyr.-centr., Aubrac, Cantal, mont Dore, Puyde-Dôme, Allier, Creuse, Nièvre, centre ct ouest de la France.

GATHELA nigeniformis L. - Coteaux cultivés de la Pro- 
vence et des Alp.-Mar. : Yaucl. et B.-du-Rh. : Carpentras, Aix à la Tour de Keirié et au pré de Magnan, Montaiguez vers le bas du Chicalon, Marseille au vallon de la Panouse, St-I.ouis, Châtcau-Gombert, route d'Aubagne à St-Jean-de-Garguier, la Ciolat dans le vallon du Sémaphore, Berre près de la Gare, les Milles, Hognac, la Crau. - Var et Mlp.-Mar. : cap Brun près Toulon, Fort-Rouge, Dardennes, le Luc, C'annes, autrefois à Nice. - Remonte dans les B.-Alp. et la Dr. vers Gréoulx, les Baronies et Montélimar.

VIGELE damascena $L$. - Champs incultes et le long des chemins de la région mérid. - Remonte dans la partie mérid. de la Drôme vers St-Paul-Trois-Châteaux, Crest. - S'étend.dans l'Aveyron et le Tarn, l'Ouest depuis les Landes jusqu’à la Vendéc, et apparait parfois accidentellement dans les parties centrales et septentr. du bassin du Rhône; je l'ai observé à Lyon sur les talus du chemin de Bonde entre le fort des Brotteaux et celui de Villeurbanne.

x. sativa L. - Var et Alp.-Mar. : Ie Luc, Grasse.

N. arvensia L. - Moissons de la partie septentr. et moyenne du bassin, surtout dans les terrains calcaires, ou sur les terrains de transport.

N. galliea Jorl., $N$. hispranica G. G. non L. - Champs et bords des chemins de la rẻgion mérid. - Vaucl., B.-du-Rh. et Var: Orange, Bédoin, l'isle-sur-Sorgue, ile de la Barthelasse, Avignon, le Luc. - Gard et Hér. : Manduel, Bellegarde, Blauzac, Coudoulet, Caunelle, Mauguio au Mas de Marot, Florensac, Villeveyrac, Mèze, St-Félix-de-Lodez. - Aude et Pyr-Or. : Guiole près Quillan, Prades, Céret, Villefranche. - S'étend dans le Tarn, le Gers et la H.-Garonne, puis dans les Landes jusque dans la Charente-Infér. et les Deux-Sèvres.

AQUTCEGI vulgarls L. - Bois et lieux ombragés des collines et des montagnes, - Espèce polymorphe.

La variété atrata Koch a des fleurs plus petites, d'un violet noirâtre, des pédoncules visqueux; se montre çà et là dans les montagnes de la chaiue jurassique jusque dans le Bugey, ainsi que dans les massifs calcaires de la Grande-Chartreuse, des montagnes de Vaud, Valais, B.-Alp. el Alp.-Mar. 
La variçté viscosa Gouan, à folioles courtes, à tige liérisséc le poils glanduleux-risıucur, existe dans l'Hérault, à St-1lichel près Lodève, au sommet de la Sérane; dans les Pyr.-Or. à la Font de Comps; dans les Alp.-Mar. et le Var à Entraunes el près des sources du Var, sur les rochers de Morgès à Aiguines.

L'A. ruscinonensis décrit par MII. Timbal-Lagrave et Jeanbernat labite les Albères à la Tour de Massane et dans les bois de Valbonne (Pyr.-Or.).

La distribution des A. nemoralis et collina Jord. n'est pas bien conmue.

A. alpina L. - Forme à éperons droits ou un peu arqués, à élamines courtes, qui crolt dans les hauts pâturages des Alpes. - Vaud et Yalais: Alpes d'Aigle et de Bex, Javernaz, Lavarraz, Bovonnaz, Anzendaz aux Essets, Corbassieres de Bagnes, val Ferret, StBernard à la Baux el à Pradaz, Mazéria, Val d'Illiez, entre Esserz ct Orsera, Pas-du-Loup, Zinal, Zermatt, Galen de Fée, Mattmark, Simplon, Gries, Mainghorn. - H.-Sav. et Sav. : col de Golèse, Morzine, Bostan sur Samoëns, Signal des Agneaux, Nautau, Méri, Chartreuse du Reposoir, chaine du mont Blanc au Chapeau, à la Mer-de-Glace, à Tré-la-Têle et dans l'Allée Blanche vers le lac Combal, Aiguille à Bochard, l'Échelle, Pierre-Pointue, les Rassaches, Bas du Mottet au dessus d'Aime, les Allues, mont Cenis. - Is. : Belledonne vers le lac du Crouzet, Champrousse, Taillefer, la Moucherolle, la Salette au mont Chamoux. - H.-Alp. : Villard d'Arène, Jautaret, Monêtier, mont Genèvre, Gondran, mont Viso, Chalp-Ronde, St-Véran, mont Aurouse, col de Chaudun, Durbonnas, - B.-Alp. et Alp.Mar. : la Condamine aux Tardées, Fouillouse, St-Paul, vallon du Chátelet, Grange-Commune, Larche, haute vallée du Var à Strop, Alpes de Tende, Val de Cairos. - Vaucl. : nont Ventoux à Font de la Grave.

Une variété Stembergianı Rchb., à fleurs plus petites, se trouve au mont Ventoux; dans les B.-Alp. à Faillefeu près Prads, Serennes; dans les Alp.-Har. à St-Martin-Lantosque, lac d'Entrecoulpes, Roubion, Tende, Grammont au-dessus de Jenton. 
Une variêté mollis Timb.-Lagr. el Jeanbernat habite les bois de Salvanère el les prairics de Montfort (Aude).

Une forme pyrenaica DC., de petite taille, à éperons droits et courts, à fleurs petites, se rencontre dans toute la chaine des Pyrẻnées, et particulièrement dans les Pyr.-Or. et l'Ariẻge, au Cambres d'Aze, vallée d'Eyne, lacs du Llaurenti el de l'Estagnet, cirques des Aiguettes el d'Aitounant, Valbonne, Barbouillère, vallons de Canals et de Boutadiol, Forge de Mijanès, Roc de Nascaras. La plante de l'Ariége a été décrite par MII. Timbal Lagrave et Jeanbernat sous le nom d'A. cyclophylla, à cause de la forne arrondie du pourtour de ses feuilles inférieures.

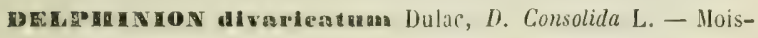
sons des terrains calcaires ou des terrains de transport contenant des cailloux calcaires. - Çà et là dans les parties septentr. et moyenne du bassin; plus rare dans la région mérid.

19. pualuescenn DC. - Champs cultivés des départements méridionaux. - Commun dans Vaucl. et B.-du-Bh. près d'Avignon, Orange, Carpentras, Flassan, Apt, Aix, Marseille à StJulien, les Martégaux, St-Loup, Marignane, Berre, les Martigues, Roquefavour, Rognac, le Pourrat. - B.-Alp. et Dr.: Gréoulx, Montélimar. - Var et Alp.-Mlar. : Cannet du Luc, Fréjus, entre Roccabigliera el Lantosque. - Gard et Hér. : Nìmes à Campuget, Manduel à Roquecourbe, Condoulet, Seynes, Serre-de-Bouquet, Montpellier à Lavalette, au chemin de Lavérune, Grabels, sources du Lez, le Triadoa, Montferrier, Villeneuve, le Caylar, Campagnan. - Aude et Pyr.-Or. : envir. de Narbonne à St Crescent, Donos, Raounel, Cuxac.

D. Iaxiflorum, D, Ajacis L. - Hoissons.

Le D. orientale Gay, souvent cultivé, s'échappe quelqucfois hors des jardins l'agrément.

b. peregrinam L. - Champs cullivés dans les torrains calcaires de la région mérid. - B.-du-Rh. et Var: le Bec de l'Aigle el N.-D. de la Garde à la Ciotat, dans le vallon qui remonte vers le Sémaphore, cliemin des Lèques, St Cyr, Toulon. - Aude, Pyr.-Or. el Ariege : Fontanes, vallons de Prades, d'Arles jusqu'à Prals-dc-Nlollo, Olette, Fontpédrouse, Hont-Louis, Bourg- 
Madame, Mijanès, Rouze, Artigues. - S'étend dans le Tarn, Aveyron, H.-Garonne, Lot-et-Garonne, l'Ouest jusque dans la Chrarente-Infér., les Deux-Sèvres et la Vendée. - Espèce polymorphe très variable sous le rapport de la taille, de l'inflorescence, de la longueur des pétales et de l'éperon, tantót pubescente-soyeuse $(D$. ambiguum L.), tantôt glabre, à pélales en cœur (D. cardiopetalum $\mathrm{DG}$.).

๑. ครฐนи W. Kit. - Coteaux rocailleux de la région mérid. B.-du-Rh, : les Alpines près d'Eyguières, vallon des Crides à St-Pons-de-Gémenos, St-Cassien sous le sommet des Béguines à la Ste-Baume, escarpements du Verdon près Ampus. - Alp.Mar. : mont Chier près Bezandun. - Gard: Serre de Bouquet près Uzès. - Remonte dans les II. ct B.-Alpes à Cliarance près Gap, Laragne à la Corniche de Chabre, Ribiers au mont Rognouse.

ED. elsaum L. - Prairies des hautes montagnes dans les Alpes et. les Pyrénées. - Vaud et Valais : Lioson, la Pierreuse, la Plane, la Montagnette, Vanil des Bimmis, Jaman, Lavarraz, Boulaire, les Martinets, Solalex, Taveyanaz, Prapioz, - H.-Alp. : du Lautaret au Galibier, mont Genèvre, dans les Saurẻots et à Gondran, Guillestre à Ressas, mont Viso aux chalets de la Tronchée, St-Véran vers les Chalanches. - B.-Alp. et Alp.-Mar. : St-Paul, Bachasse, Lauzanier, Soltron, la Portiolette, la Manche, sources du Var à Roche-Grande, Strop, Estenc, St-Dalmasle-Sauvage, Isola, Fenestre, Tende. - Pyr.-Or. : vallées d'Eyne et de Llo, Mont-Louis. - Espèce polymorphe ayant les pédicelles et ovaires tantôt glabres (D. glabrum ou intermedium $\mathrm{DC}$.), tantôt velus (D. villosum ou montanumi DC.).

D. Dzequienisan DC. - Var : île de Poriuerolles. - Corse.

D. staphidium, D. Staphis agria L. - Terres incultes. - Vaucl., Var et Alp.-Mar. : Flassans, Toulon au nord de Coudon et du mont Faron, Hyères, Évenos, Bormes, Monaco. - Gard et Hêr. : Cabrière, parc de Tresques, Pézenas, St-Mlartin-de-Tréviers, commun autrefois près de Montpellier oủ il a élé détruit soit par les défrichements, soit par la rapacité des collectionneurs. 
ACONTCON caberosums, $A$. Anthoru L. - Pùturages rocailleux des montagnes. - Chaine jurassique : Noirmont, la Dôle, Colombier, Reculet, Sorgiaz, Credo, le Bôle, descend à Champagnole, à Château-Vilain et jusqu'à Thoirette, puis se montre dans le Bugey à Mont-d'Ain, Colombier lu Bugey, Cote d'Hostiaz, St-Rambert, Pierre-Châtel, St-Benoit. - II.-Sav. et Sav. : St-Jean-d'Aulph, le Vuache, les Bauges. - Is. : Grande-Chartreuse, Desert de Beauregard, St Christophe en Oisans, la Salette. - II.Alp. : Lautaret, mont Viso, vallon de Ruines, Rabou près Gap, Charance, mont Séuse, Clıaillol. - B.Alp. et Alp.Mar, : entre St-Paul et le col de Var's, Parpaillon, Bérard, Lauzanier, Serennes, sommet du Cousson près Digne, col de Fenestre. - Vaucl. : mont Ventoux. - Pyr.-Or. et Ariège : Canigou, Font-de-Comps, Carença, Mont-Louis, vallée d'Eyne et de Llo, val de Carol, cirques d'Artounant et des Aiguettes, Barbouillère, Valbonne, val de Paillèes à Soucarrat, Roc de l'Ermite. - Pyr. centrales.

A. Iycoetonam L. - Bois et prís humides des montagnes. Hautes Vosges jusque dans la H.-Saône aux Ballons de Giromagny et de Servance, Plancher-les-Mines, Cliampagney, Champey, Chavanne. - Chatne du Jura helvétique el français jusque dans lo Bugey et le Revermont. - Vaud, Valais, Savoic, Dauphiné, B.-Alp. et Alp.-Nar. - C.-d’Or et S.-et-L. : Gevrey, Changey, Antheuil, Val-Suzon, Curtil près Ste-Foix, Morvan autunois, Auxy, Antully, Marmagne, Broye, St-Sernin du-Bois, Brisecou prìs Autun, bords du ruisseau de Canada. - Rh. et Loire : St-Rigaud en Beaujolais, Pilat. - Montagnes du Vivarais, Mezenc. - Cévennes du Gard et de l'Hér. à Salbous, Alzon, Concoule, St-Imand-de-Mounis, le Caylar. - Pyr.-Or. et Ariège : Canigou, Prats-de-Mollo, Conat, Madrès, Mont-Louis, le Capirr, le Llacrenti. - En dehors de nos limites dans les Pyr. centrales, la Lozcre, l'Aubrac, Tarn, Cantal, mont Dore, H.-Loire, Monts-Dômes, Forez à Pierre-sur-Haute et au Monton. celle, Vosges.

La variété pyrenaicum Seringe, hérissẻe de poils jaunâtres, se montre dans toute la chaine des Pyrénées. 
A. napeltum L. - Licux ombragis des montagnes. - Hautes Vosges jusque dans la H.-Saône aus. Ballons de Servance et de Giromagny, vallée du Rahin, de Miélin et du Fray, BeulotteSt-Laurent. - Chaine jurassique jusque dans le Bugey où il descend sur les rives de l'Ain à Martinaz près du Pont de Chazey. - Vaud, Valais, Savoie, Dauphiné, B.-Alp. el Alp.-Mar. - C.d'Or et S.-et-L. : Messigny, Orgeux, Flavigny, St-Remy, Val des Choues, Moloy, Avot, Selongey, valion du Suzon, Arcelot, Orgeux, Lusigny, bords de la Cure entre Anost et Hontsauge. - Rh. et L. : haut Beaujolais, Pilat. - Gard : bois de de Longues-Feuilles à Concoule, Valinières, l'Espérou. - Pyr.Or. et Ariège: Canigou, Hont-Louis, col de la Perclie, vallée d'Eyne, la Cerdagne, le Capcir, col des Ares, salvanère, le Llaurenti. - En dehors de nos limites dans les Pyr. centr., Aubrac, Cantal, mont Dore, Forez à Pierre-sur-Haute et à la Richarde, Morvan nivernais à Nataloux et à Gouloux, Morvan de l'Yonne sur les bords de la Cure vers Railly, Pierre-Perthuis, Maine-et-L, Indre-et-L., Sarthe, Oise, Vosges.

A. pantealatum Lam. - Bois humides des hautes montagnes. Ain : Colombier de Gex au-dessus du clialet de Platière, versant sud du col de la Faucille, Sorgiaz, Crèt de Chalam. - Vaud et Valais : Jaman, Leysin, la Croix, Bovonnaz, Lavarraz, le Lavanchy, pied des Jumelles, Alpes de Vouvry, St-Bernard à la Baux, sous le nont Cubil, entre les Places et le sommet de Proz, St-Barthẻlemy d'Hérémence, Vercorin, Gemmi, Mayens de Leukerbad, Stalden. - H.-Sav. et Sav. : col de Golèse, Cornetles de Bise, mont des Granges, le Brévent, bords de la Dioza, vallées de Sixt et du Reposoir, Méri, Semnoz, Beaufort. - Is. : Grande-Chartreuse au pied du Grand-Som, entre Vallombrey et le Collet, Belledonne, Prémol, Livet, Taillefer. - II.-Alp. : Lautaret au bois de la Madeleine, Risoul près Guillestre, Boscodon près Embrun, Morgon, Rabou près Gap, Combe-Noire de Manteycr, Durbon. - B.-Alp. et Alp.-Mar. : cascade du Lauzanier, bois de la Silve près Meyronnes, Barcelonnette, col de Fenestre, Roccabiglicra.

ACT aperata L. - Bois humides des parties septentr. et 
moyenne du Bassin. - Chaîne vosgienne jusque dans la II.Saône, chaîne jurassique jus que dans le bugey et le Revermont, Vaud, Valais, Savoie et Dauphiné. - C.-d'Or et S.-et-L. : Messigny, Gevrey, Couchey, St-Remy, Fontenay-les-Montbard, Flavigny, Recey, Moloy, Val Suzon, Blaisy-IBas, mont Afrique, Lusigny, Cuiseaux. - Rh. et L. : vallon de Sathonay, Dénicé prìs Villefranche, Pilat, Forez. - B.-Alp. et Alp.-Bar. : forêt de Faillcfeu prés Prads, col de Fenestre, Tende, mont Mulacé sur Menton. - Gard : Salbous, St-Guiral, Concoule. - Pyr.Or. et Ariege: Vernet-les-Bains, Canigou, Mont-Louis, cirque des Aiguettes, bois du Riplaou, Roc de Mascaras, val de Paillères à Soucarrat. - Manque dans la région des Oliviers.

PAOVA comallizare Retz. - Rochers. - C.-d'Or: bois de Corcelle-les-IIonts, Savigny-sur-Beaune, mont Afrique, entre Gevrey et Chambeuf, Bouilland, Chaignay, Val-Suzon. - En dehors de nos limites dans le Loir-et-Cher près Blois et Cheverni; dans le Loiret prìs Olivet; dans la Vienne à Quinçay.

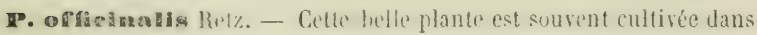
les jardins. On a prétendu qu'elle existe à l'état sauvage dans la Drôme el les Hautes-Alpes à Barnave, Die, au-dessus du lac Siguret près St-André d'Embrun, entre Brunechard et Barbein près Risoul; mais il est probable que la Piroine indiquée dans ces localités est le $P$. peregrina, et que, comme l'a dit Grenier (Flore de France, t. I, p. 53), la Pivoine officinale n'est nulle part spontanée en France.

P. peregrina Mill. - Bois. - I, ct B.-Alp. : Séguret près Embrun, Ribiers, Barrême, Annot à Allous et à Vergons, Digne à la monlagne de Dourbes. - Var et Alp.-Mar. : Vérignon, bois de Lagne à Ampus, montagnes au-dessus de Si-Auban, Caussols, Chàteaudouble, mont Cheiron au-dessus de Grasse, St-Vallier, Nice, Menton. - Gard et Hér. : bois de Luf près Broussan, Alais, Serre-de-Bouquet, bois du sommet de la Tessonne audessus d'Avèze près du Vigan, Anduze, Pic St-Loup, la Sérane, pic de Liausson près Clermont, Bédarieux à St-Raplaël, Lunas, Joncels, Pardailhan-Pontguiraud, St-Pons.

Lapeyrouse et, à sa suite Companyo, ont signalé le $P$. pereCatal. Bassin du Rhonc. 
grina dans les Pyrénées-0rientales au bois des Abeilles au fond du valion de Banyuls-sur-Ner, puis dans les environs de la Tour-de-Madaloch, enfin sur le plateau supérieur de ForçaRéal et de Caladroy. Ces indications, de mème que toutes celles qui ont la même origine, ne pourront être définitivement admises quaprès vérilication ullérieure.

\section{BERBÉRIDEES.}

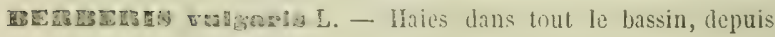
les plaines juscu'aux montagnes.

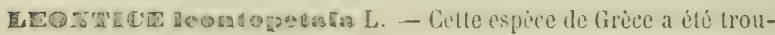
vée par M. Briard dans un champ cultivé près des Milles (B.-du-Rh.).

Epimedion alpinum L., a été naturalisé par Schleicher dans le bois de Bévieu près Bex, par Valot dans le Parc de Dijon, par Liotard à St-Hugon près de la Rochette (Sav.), où je ne l'ai pas trouvé, par Lindern près de Bâle, et au Haulenberg près Mundolshein en Alsace. - Celle plante est spontanée en Toscane, Lombardie, Vénétie, Littoral autrichicn, Tyrol, Carniole, Croatie, Slavonie, Styrie.

\section{NYMPHÉACÉES.}

Now fleurs est tris-variable dans cette espéce.

Ne alba.

La forme naine, N. pumilum Sm., n'a pas été trouvée dans notre bassin; elle existe dans les lacs vosgiens de Gerardmer, Longemer, Retournemer, Blanchemer, Noir, de la Maix, Fondromeix, eaux mortes de la Jloselle en amont de Remiremont, - En Auvergne aux lacs de Bourdouze et de Chambedaze près Besse.

\section{PAPAVÉRACÉES.}

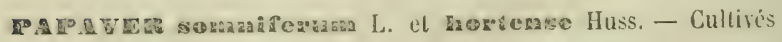
et quelquefois subspontanes. 
L. seligerum DC.-- Forme velue du $P$. somniferum, qui se montre sur quelques points de la Provence, des Alp.-Mar., du Languedoc et du Roussillon. - B.-du-Rh. : les Martigues, Arles à Montmajour. Var et Alp,-Mar. : St-Mandrier près Toulon, IIjères, ilte de Porquerolle et de Porteros, St-Iropez à Cavalaire, St-Raphaël, Nice, Monaco, Menton. - Gard: St-Nicolas, Châlcau de StRoman. - Aude et Pyr.-Or. : lle de Laute près de Narbonne. Cases de Pena, de Prades à la Trencada d'Ambulla, Collioure à Consolation, Paulille, Banyuls-sur-Micr.

P. rhacoundenan, P. Phoeas L. - Joissons des plaines et des montagnes. - Espice polymorphe dans laquelle les dimensions et la forme de la tige, des feuilles et des fleurs sont sujettes à varier, comme on le voit dans les formes décrites par M. Jordan sous tes noms de $P$. erraticum, agrivagum, cereale, crucirtum, rusticum, et par II. Timbal-Lagrave sous les noms de Dodonaeanum, Fuchsianum.

l'. dubium L. - Forme à capsule oblongue, beaucoup moins répanduc que le typo rhocoideum, et comprenant les variêtés depressum, vagum, luteorubrum, modestum, improperum décrites par M. Jordan, et en outre P. Lecoquianum Lamotte, Lamottianum Bor., collinum Bogenh., pinnatifidum Moris. Cette dernière torme a des feuilles simplement dentées et a été observée à Nice et à llenton.

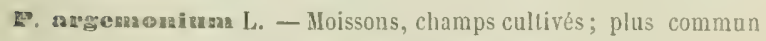
dans les terrains siliceux que dans les terrains calcaircs. Une variété a la capsule glabre.

P. Hağuréchuean L. - Moissons et champs cultivés de la région inérid.; se montre quelquefois accidentellement dans les parties mojenne et septentr. du bassin.

P. Rį̣àaum L. - Rocailles des hauts pâturages des Alpes et des I'yrénées. - Vaud et Valais : Château d'Oex dans la vallée du Grand Crau, la Pierreuse, ravin de la Potse di Gaulès, vallée de l'Hongrin aux Charbonnières, montagne de Vouvry, sommet de la Plane, Vanil du Plan-des-Eaux. - II.-Sav. : Jalouvre, Vergy, Héri au pied du col de Balafrasse, mont Saxonnet audessus du Lac, mont de Mieussy. - Is. et II.-Alp. : Taillefer, 
Grandes-Rousses, la Mouclierolle, l'Obiou, la Grangette près Gap, mont Aurouse. - Alp.-Mar. : St-Martin-d'Entraunes aux Aiguilles, Roche-Grande au col de Pale, lac Giugali, mont Bego. Vaucl. : mont Ventoux. - Pyr.-0r. : Canigou, Carença, pentes du Cambres d'Aze, Collada de Nuria.

Le Pavot des Alpes présente deux variẻtés, l'une, beaucoup plus commune, (faviflorum ou aurantiacum) a des fleurs de couleur orangẻe; l'autre (albiflorum), observée au mont Obiou du côté de Corps (Is.), a des fleurs blanches tachées de jaune à la base.

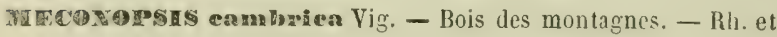
Loire : St-Rigaud en Beaujolais au bois de la Tour Valbenoite sur la lisière du Bois Noir. - Hér., alluvions de l'Agout, l'Espinouse à Fraisse. - Pyr.-0r. : entre la Font de Comps et la vallée d'Evol. - En dehors de nos limites dans les Pyr. centr., Cantal, mont Dore, Puy-de-Dôme près de Volvic et de PontgiJaud, forêt de la Madeleine (Loire), Nataloux (Nièvre), forêt de Laz dans les Montagnes-Noires (Finist.). - Catalogne, Aragon, Navarre, Biscaye, Asturies, Galice.

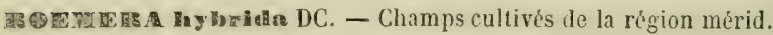
B.-Alp. et Vaucl. : entre Digne et Seynes, Avignon, Orange, Vacqueyras, - B.-du-Rlı. : Aix, Salon, les Martigues, Miramas, Eyguieres, Fos, Cassis, Roussargues. - Var : Gonfaron, Vidauban, le Luc, Draguignan, Fréjus. - Gard et Hér. : Manduel, Bouillargue, Montpellier près de l'Arfueduc, Mas-le-IIarot, Fontfroide, Castelnau, Doscares, Montferrier, Prades, Lattes, Mauguio, Béziers. - Aude et Pyr.-Or. : Pech de l'Agnèle prés de Narbonne, envir. de Carcassonne, Salses, Cases-de-Pena, Perpignan à Château-Roussillon. - Remonte dans la Drôme vers St-Paul-Trois-Châteaux; dons les H.-Alp. à Ribiers, Embrun.

A4⿴囗大 la région mérid.; remonte dans les H. et B.-Alp. à Gap, Ribiers, Tallard, Sisteron; dans la Dròme vers Nyons, Montẻlimar, Crest; dans l'Ardèche le long de la Côte du Rhône jusqu'au Pouzin el à la Voulte. - Il s'est même naturalisé à Lyon autour de la gare d'eau de Perrache, et sur les talus du chemin de 
Rondé près du Fort des Brotteaux; on l'a vu aussi dans le département de l'Ain à Virieu-le-Grand et jusque près de Thoirelto.

- En dehors de nos limites il s'étend dans la vallée de la Garonne, dlans l'Ouest depuis les Landes jusque sur les côtes de la Bretagne et de la Normandie.

G. comiculatum Curt. - Forme plus grêle, à lige velue, à siliques scabres et hispides. - Moissons. - Vaucl. et B.-du-Rh. : le Pontet près Avignon, Carpentras, Marseille à l'Estaque, la Valentine et Séon-St-Henry, entre Istres et St-Chamas, du Pas-des-Lanciers à Marignane et à Châteauneuf, les Milles, Nartigues. - Var et Alp.-Jar. : Carqueiranne, les Imbiers près Toulon, Pierrefeu, Fréjus, Roquebrune. - Ard. : le Pouzin. Gard et Hêr. : entre Aigues-\$Iortes et Candillac, Mas de Vianès, Bellegarde, St-Gilles, Castelnau, Clapiers, Lattes, Aniane, Pézenas, Béziers, Montpellier au Polygone et au-dessus de Figairolle, St-Guilhem-le-Désert, Vendres. - Aude et Pyr.-Or. : Crabit près Nas bonne, Junquières, Villeneuve, Cases-de-Pena, Trencada d'Ambulla. - Dans celte forme, la corolle est quelquefois panachée de jaune, de rose et de violet.

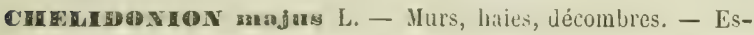
pèce polymorphe présentant quelquefois des feuilles lacinic̉es.

HIXECOON proeumbens L. - Champs de la rigion mérid. B.-du-Rlı. : Marseille à Bonnevaine, vallon de la Nerthe, StLouis, Cassis, la Ciotat, la Mède près Martigues. - Var et Alp.Jar. : le Revest près Toulon, Salins d'Hyères, Fréjus, St-Raphaël, Menton. - Gard el Hér. : Manduel, Comps, Candillac, Cette aux Salins de Villeroi, Balaruc, plage de Sérignan à Valras. - Aude et Pyr.-Or. : Narbonne, Molitg, Mosset, Prats-deBalaguer, les Escaldas, Fontpédrouse et Ur en Cerdagne.

H. grandiflorum Benth. - Forme à fleurs plus grandes ayant des sépales lancéolés-aigus. - Champs cullivés des Pyr.-0r. à la Porte Canet de Perpignan, Molitg, Tarerach, Ur en Cerdagne.

IX. pendulum L. - Champs cultivés. - Taucl. : Avignon, Carpentras, Gadagne, de l'Isle à Vaucluse. - B.-du-Rh. : Roquefavour, Roque-Martine entre Eyguière et Morgon, Port-de-Bouc, Berre, Aix près de l'Hopital et du Prégnon, pied des Alpines Catal. Bassin du Rhone. 
près de St-Remy. - Gard et Hẻr. : Manduel, Béziers, Villeneuve. - Aude et Pyr.-Or. : Narbonne, St-Crescent, l'Estagnol, entre le Pech de l'Agnèle et l'Aqueduc.

COI W Cos cava Schw. et Koert (1). - Haies et buissons. Bulfort à la Niotte; à la Justice, la Perche, Auxelles, alluvions de la Savoureuse au Valdoie. - H.-Saône : Chariez, Thieffans, Tête-des-Fougères, Planche des Belles-Filles. - Doubs et Jura : toute la vallée du Doubs depuis Montbíliard jusqu'au-delà de Dole; existe aussi sur le versant helvélique des cantons de Berne, de Neuchâtel et de Vaud; s'étend dans le Valais vers Fully, St-Maurice, Martigny à la Combe et au Brocard. H.-Sav. : Salève, Thonon, Vougy, Annecy, entre Cluse et la cascade d'Arpennaz jusqu'au Fayet. - Ain : le pays de Gex vers Thoiry, les Echenevox et Ferney. - Is. : S:-Quentin, Voreppe.

C. solida Sm. - Bois et haies dans tout le bassin. - Dans les départements mérid. il occupe exclusivement la région montagneuse du Gard, de l'Hér. et des Pyr.-Or. à l'Espérou, Pertus de Iodève, le Larzac, St-Pons, la Salvetat, Fraisse, vallées d'Eyne et de Llo, Mont-Louis, le Llaurenti; dans le Var et les Alp.-Mar. il se trouve à la Ste-Baume, à Caussols, bois de Lagnes près Ampus, entre Vence et Coursegoules au-dessus de Grasse, Alpes de Tende, col de Tanarello.

C. Concea Pers. - Bois, pâturages des montagnes. - Vaud et Valais : entre Eslex et Collonges, Nant, Ayerne, pied du Berthex, Lavarraz, Bovonnaz, Solalex, Bretaye, Leysin, Mazeria, val de Bagnes, Val Reschy et val Ferret au-dessus d'Essert, Gueuroz près Salvan. - H.-Sav. et Sav. : Ayers sur Servoz, chalets de la Charbonnière sous le Platet, entre Pontchy et la Roche, col entre Balafaux et Tinar, derrière St-Laurent, Dessy près Pontchy, les Voirons, mont Nivolet, Villarlurin près Moutiers. - Is. : Grande-Chartreuse à Bovinant - Rh. : Poulle à la Roche d'Ajoux ? - H.-Alp.: Lautaret à la Madeleine, bois entre l'Échelle et le vallon des Thures près Névache, Manteyer.

(1) On trouve dans les écrits des auteur's grecs les variantes corydallis, corydos, corydalos, corydallos et corydalion. 
C. Iuten, D. C. - Vieux mur's. - Saone-et-Loire: Autun. - IIauleSavoic: Saleve. - Environs de Lyon à Vaise, Saint-Cyr, SaintFortunat. - Isère : Meyland près Grenoble. - Drôme : SaintVallier.

C. emueaplnyin, D. C. - Fentes des rochers. - Montagnes de l'Ardeche.

C. clnviculata, D. C. - Isire : Crémieu, - Vaucluse : Arignon. - Le Languedoc.

FUMA IT capreolata, L. - Espèce mérilionale remontant de la Provence, par Avignon, Lyon, jusqu'à Genève, Vevey. - Lyonnais : Sainte-Foy-les-Lyon, Saint-Genis-Laval, Vernaison, Oullins. - Ain : Néron.

F. pallidiflora, Jord. - llêmes localités.

F. speciosa, Jord. - Rhone: Sainte-Foy, Saint-Genis-Laval, - Ain :

Néron. - Alpes-Nlaritimes.

F. officinalis, L. - Champs dans tout le Bassin.

F. agrearia, Lag. - Licux cultivés. - Vaucluse : Avignon. - Var : Toulon, Iyyères. - Bouches-du-Rhône : Marseille. - Gard : Manducl, le Caylard, route de Caissargues. - Hérault : Montpellier.

Faillantii, Lois, - Moissons. - Haute-Saone : Chariez, Echenoz, Chassey-les-Montbozon, Villersexel, Vellechevreux, Gouhenans. - Doubs : Besançon, environs de Montbéliard, Ecurcey, Mandeure, Mallhay, Audincourt. - Jura : Quingey, Salins, Dole, Lons-le-Saunier, vallée du Doubs et de la Loue. - Rhône: mont Cindre, sur la digue au Grand-Camp. - Hautes-Alpes: la Garde près Gap, mont Aurouse, la Grave. - Drôme : Ponsas près Saint-Vallier, Tain, Grest, - Vaucluse : Avignon. - Gard : Nimes, Manduel, le Vigan. - Bouches-du-Rhone: Arles.

F. Laggeri, Jord. - Bassin du Léman et Valais, - Hautes-Alpes : Villar's-d'Arène sur la route.

F. parriftora. L. - Champs des départements miridionaux. Alpes-Maritimes. - Var. - Bouches-du-Rhône. - Vaucluse. Gard. - Ardèche. - Drômo. - Beaucoup plus rare au nord de 
Lyon où on ne le rencontre qu'accidentellement dans les cultures de trèfle et de luzerne ensemencies de graines du Midi.Jura : Chaussin. - Ain : Néron, au dessus du pont de la Cadette, sur le bord du Rhône. - Rhône : Sainte-Foy-les-Lyon, Rochecardon, Saint-Alban, Saint-Genis-Laval. - Drôme : Romans, Crest, Saint-Vallier. - Hautes-Alpes : Château-Queyras, Villevieille.

F. spierata, L. - Alpes-Maritimes. - Bouches-du-Rhône. - Var. Gard. - Hérault : Cette, Aresquiès. - Drôme : Montélimar, le Buis, Crest.

\section{CRUCIFERES}

RARHAUS sativus, L. - Subspontané.

T. raphanistaum, L. - Commun dans les champs de tout le bassin. Présente dans les montagnes du Dauphiné une variẻté à fleurs violettes.

R. Iandra, Moretti. - Champs de la Provence et des Alpes-Maritimes; a été quelquefois trouvé accidentellement à Lyon, aux Brotteaux.

STMATs arvensis, L. - Commun dans les champs do tout le bassin.

S. Schkuriana, Rchb. - Champs. - Saône-et-Loire. - Haute-Savoie :

Thonon. - Drôme: Montmaur près Die. - Isère: Mens. (D'après Grenier, il n'est qu'une déformation de l'arvensis.)

s. cheirpathus, Koch. - Terrains siliceux de la zone vosgienne

de Haute-Saòne, de Saône-et-Loire, du Beaujọlais, du Lyonnais, du Pilat et de toute la chaine des Cévennes.

S. glareosa, Jord, - Isère : graviers du Bourg-d'Oisans.

S. arenosa, Jord. - Lyon.

S. montana, D. C. - Débris des rocher's des montagnes granitiques. — Isère: Allemont, Bellecionne vers le lac Crouzet, Séchilienne, le Fréney. - Hautes-Alpes : Lautaret.

8. alba, L. - Commun dans les moissons surtout des départements méridionaux; subspontané dans les localités oủ il a été cultivé. - Côte-dOr. - Doujss : Montbéliard. - Haute-Saóne: Ande- 
larre - Jura : Dôle. - Ain : Pont-d'Ain, etc. - Rhône: environs de Lyon, les Brotteaux, Oullins. - Loire: Alontbrison. Dròme : Crest.

EITUC saviva, Lam. - Subspontané.

maksSic oleracen, L. - Subspontané.

B. Hobertiana, Gay. Toulon au mont Faron et au Coudon. Ile Sainte-lIarguerite.

B. mapuas, L. - Cette espece présente deux variétís: l'une, a. oleifora, cultivée sous le nom de colza pour ses graines oléagineuses; l'autre, $\beta$.esculenta (navet) donne des produits qui varient avec les qualité cuu sol; les terrains siliceux en général, ceux du Morvan vers Saulieu, du Beaujolais à Chiroubles, en particulier, produisent des navets de qualité supérieure.

B. Tieherii, Vill. - Prairies des hautes montagnes. - Isère: Ja Lérarde. - Ilautes-Alpes: Lautaret, mont Genèvre, mont Viso.

B. nigra, Koch. - subspontané.

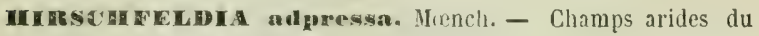
midi. - Alpes-Maritimes. - Var: Toulon, le Luc, Frẻjus. Bouches-du-Rhône. - Gard : environs de Nìmes, Manduel, Aigues-Hortes. - Drôme: Saint-Vallier, Crest, Remollon, Nyons. - Plus rare dans le nord du bassin où il est fugace et peut-être introduit par des graines du midi. - Jura: Rahon, Saint-Baraing, Chaussin. -Saône-et-Loire : llâcon, Tournus.Ain : Pont-d'Ain. - Environs de Genève. - Rhơne: Oullins, le Moulin-à-Vent, Vassieux. — Isère: Meyzieu, le Mollard de Décines, Feyzin, Saint-Rambert.

DIPLot Axus repanda, G. G. - Débris de rochers des montagnes. - Alpes du Dauphiné. - Isère : Grandes-Rousses audessus de Brandes. - IIautes-Alpes: le Lautarel, le Galibier, le mont Viso, le col d'Isoire, col Agnel, Marcel près Saint-Véran, la Cluse en Devoluy, mont Morgon, prè̀ Boscodon d'Embrun. - Parfois amené par le Drac jusqu’au Polygone de Grenoble.

D. saxatilis, D. C. - Rochers. - Basses-Alpes : Digne. -Bouchesdu-Rhóne: mont Sainte-Victoire. - Var : Garde-Freynet.

D. tenuifolis, D. C. - Bords des chemins. - Commun le long du 
Rhône depuis Genève jusqu'à Lyon et dans le bassin méridional, - Plus rare dans le Doubs: Besançon; le Jura : Saint-Ylie près Dole, Pesmes et la Saone-et-Loire.

D. muralis, D. C. - Champs. - Commun le long du khône de Genève à Lyon,-Alpes-llarıtimes, - Bouches-du-Rhône. - Var. - Gard. - Rare dans Saone-et-Loire: Châlon, Tournus, Brancion. - Jura : Chaussin.

D. viminea, D. C. - Vıgnes dans Saône-et-Loire à Dezize, Cluny. - Côte-d'Or: Talant, — (Nul dans Jura et Doubs.) - Drôme : Crest, Saint-Paul-Trois-Châteaux.-Assez répandu dans le Gard, l'Hérault, les Bouches-du-Rhône, le Var, les Mlpes-Maritimes.

D. erucoüdes, D. C. - Très-commun dans les champs du Gard et de la Provence. - Ne parait qu'accidentellement dans le reste du bassin. - Nous l'avons vu à Lyon sur le talus des fossés d'enceinte entre le fort des Brotteaux et celui de Villeurbanne.

ERUCAste Un Holliehii, Schimper et Spenner. - Bords du Rhone et de ses amuents. - Alluvions du Doubs et de la Loue, vallée de la Scille à Voiteur, Domblans. - Bords de la Saône, ça et là dans la Bresse. - Bords de l'Ain et du lac de Nantua. Bords de l'Arve et du Fier dans la Haute-Savoie. - Bords du Rhône à partir de Lyon; de l'Isère, à Houtiers (Savoie). - Nul dans le Gard et la Provence.

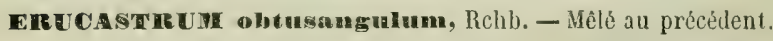
- Très-rare dans le Doubs, Jura et Ain. — Commun dans les terrains granitiques de la Côte-d'Or; sur les graviers du bord de l'Arve dans Haute-Savoie; de l'Are dans la Savoie; assez commun le long du Rhone. - Bouches-du-Rhone : Marseille, Aix, Istres, Miramas. — Gard : Bellegarde et Manduel. - Var : Fréjus, Hyères. - Alpes-llaritimes : l'Estérel, Grasse, Entraunes. $\boldsymbol{E}$. intermedium, Jord. - Isère: Chasse. - Ardèche: Tournon.

Morrchide arvensis. D. C. - Narseille.

Hesperers gylvestris, Clus. - Haies, bois, bords des ruisseaux. - Doubs : 'côtes du Doubs, Maiche, Grande-Combe-des-Bois, Noël-Cerneux. - Jura : roches de Baume près Lons-le-Saunier, Champagnole, Sirod, Dournon près Salins. - Ain : Hauteville, bords de l'Albarine, Cormaranche, Portes, le Molard de Don, 
Colombier du Bugey, Pierre-Châtel, - Rhône: Limonest, SaintJulien-sur-IIontmelas, bords du Garon. - Ilaute-Savoie: vallée du Reposoir. - Isère: Décines, Saint-Ismier près Grenoble. — Gard: Tresques. - Bouches-du-Rhone : le long de la Durance, Peyrolles, Roquefeuille, - Var : Sainte-Baume.

E. Ineininta, $\Lambda$ ll. - Rochers. - Dròme : Saou pris Crest. - BassesAlpes : Digne, Cabasse, Sisteron, Castellane. - Bouches-duRhône : rochers sous le pic de Bretagne. - Var : Bau-de-QuatreHeures près Toulon.

IIA LCOLnL africana, R. Br- - Champs de la région méridionale. - Basses-Alpes : Digne, — Gard : Beaucaire, Teziès, SilveRéal. - Hérault : Montpellier. - Vaucluse: Avignon. - Bouches-du-Rhône: Miramas, Istres entre Couvent et les Crottes.

M. parvigora, D. C. - Sables des côtes de la Méditerranée.-Var : Toulon, Hyères, Saint-Tropez, Fréjus.

II. Iittorea, R. Br. - Sables des bords de la mer, - Bouches-duRhône: Foz, les Martigues. - Gard : Aigues-Mlortes.-Hérault: Maguelonne, de Cette à Agde.

M. maritima, $\mathrm{R}$. $\mathrm{Br}$. - Sables des côtes de la Méditerranée. - Bouches-du-Rhône: Marseille. — Var : Sablettes près Toulon. — Gard:Aigues-Mortes. - Hérault : près de Montpellier.

ILATIIOLA incann, $\mathrm{R}$. Br. - Bords de la Mféditerranée : Marseille, Cassis, ile de Porteros, lles d'Ilyères, Fréjus, tle Sainte-Marguerite.

M. sinuata, R. Br. - Sables maritimes. - Bouches-du-Rhône : Bonneveine près Marseille, Foz, les Marligues. - Alpes-Mlaritimes et Var: Pesquiers près Hyères, la Napoule, Cannes. Gard : Grau-d'Orgon près Sainte-Marie. - Hérault : Cette.

M. tricuspiuata, R. Br. - Sables maritimes, - Var : Isthme de Giens, iles d'Hyères.

II. tristis, R. Br. - Lieux pierreux. - Bouchis-du-Rhóne: Pilondu-Roi, Pont-Royal, commun entre Barbentane et Tarascon, Orgon. - Vaucluse: Avignon, Bonpas, environs d'Apt à Roussillon, chemin de Buaux. - Gard: îles du Rhône à Vallabrègues. - Hérault : environs de Montpellier.

II. varia, D. C. - Rochers calcaires et gypseux de Villarodin (Savoie). 


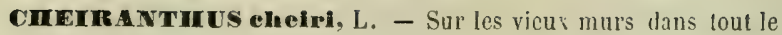
bassin.

ERYSTRUM cheiranthoïdes, L. - Champs cultivés, décnmbres. - Doubs : Montbéliard, Besançon.-Haute-Saône: Selles, Chariez, Pontcey, Chassey-les-Montbozon. - Jura : allurions du Doubs et de la Loue, Salins, Arbois, - Cole-d'Or. - Ain : bords de la Saône à Trévoux, Thoissey, Saint-Laurent-lesIlâcon, Sathonay. - Saône-et-Loire : bords de la Saône, Tournus, Cluny, Changes, Dezize. - Rhône : bords de la Saône et du Rhône, l'Ile-Barbe, Perrache, la Mouche, Sainte-Colombe.

- Loire : Saint-Pierre-de-Bøuf. -Isère : Eybens, - Gard: Saint-Gilles.

E. virgatun, Roth. - Lieux incultes. - - IJautes-Alpes: Guillestre, le Queyras, mont Dauphin, Embrun, la Grave, Monestier de Briançon. - Valais : entre Charax et Saxon.

E. confertum, Jord. - Hautes-Alpes : Briançon.

E. densiflorum, Jord. - Hautes-Alpes : Villevieille.

E. delpluinense, Jord. - Hautes-Alpes : Briançon.

E. glareosum, Jord. - Ain : Tenay, - Isère: Vertrieu.

E. confine, Jord. - Ardèche : Crussol.

E. nustrale, Gay. - Lieux pierreux. - IIaules-Alpes : Aiguilles et Abriès en Queyras, mont Aurouse, la Garde, Rabou. Drôme: Tain, Valence.- Basses-Alp̊s : Gréoux, Saint-Geniès près de Sisteron. - Vaucluse. - Bouches-du-Rhône : SainteVictoire, tête de Carpiagne, pic de Bretagne. - Var : le Luc, Cannet.

E. Inelvetieum, D. C. - Pelouses. - Hautes-Alpes : Lautaret, Briançon. - Savoie : d'Avrieux au forl de l'Esseillon.

E. monticolum, Jord. (hicracifolium, Villars). - Isère : mont Aiguille, col de la Limar près Gresse. - Hautes-Alpes : Charance près Gap, Ie Qucyras.

E. petrophilum, Jord. - Rochers calcaires de Châtcaubourğ (Ardèche).

E. curvifalium, Jord. - Nyons (Drôme).

E. leucophoum, Jord. - Avignon.

E. Aurosicum. Jord. - mont Aurouse près Gap.

E. ochroleucum, D. C. - Débris rocheux dans les hautes monta- 
gnes. - Doubs : creux du Van, Chasseral, Montfaucon près Besançon. - Jura : Rocailles calcaires de la Dole, du Colombier de Gex, vallée de Saint-flaude près Flamen, Poupet, fort Belin près Salins, - Ain : de Tenay à la Burbanche, mont d'Ain près Nantua. - Savoie : mont Cenis à Ronche. - Isère : Chamecliaude, col de l'Arc, la Moucherolle, l'Obiou-Hautes-Alpes: mont Viso, - Vaucluse : mont Ventoux.

E. consimile, Jord. - Mont Glandaz près Die (Drôme).

E. ascendens, Jord. - mont Aurouse près Gap.

E. pumilum, Gaud.-Dhbis de rochers dans les hautes montagnes.

- Savoic : mont Cenis à Ronches. - Alpes-Maritimes.

E. alpestre, Jord. - Ilautes-Alpes : mont Viso, Saint.Véran.

$E$. brevicaule, Jord. - Saint-Véran (Haules-Alpes).

E. parvulum, Jord. - Col Malrif (IIautes-Alpes).

E. oreiles, Jord. - Rabou près Gap (Hautes-Alpes).

E. orientale, R. Br. - Champs cultivés. - Côte-d'Or. - Saone-etLoire : champs calcaires, Saisy, Cheilly, Couches. - Rhone : Saint-Cyr-au-Mont-d'Or. - Ain : fort de l'Ecluse. - Isère: les Portes-en-Trièves, Roissard. - Haules-Alpes : Rosans, Saint-Didier-en-Devoluy. - Drôme : Crest. - Gard: Tresques, IIieuzet, Campestre. - Hérault. - Bouches du-Khône : Fonscolombe. - Var: le Luc, dups, Sainte-Baume.-Alpes-IIaritimes.

BAEIBATEA rulgaris, R. Br. - Bords des fossés. - Doubs, Haute-Słône, Jura, Słône-et-Loire, Ain, Savoie, Dauphiné. Gard, Bouclies-du-Rhône, Var, Alpes-Maritimes.

B. rivularis, Martr. - Isère : Grenoble, Grande-Chartreuse près le couvent, entre Génas et Meyzicu.

B. longisiliqua, Jord. - Lieux humides.

B. brevistyla, Jord. - Lieux humides.

B. arcualu, Rchb. - Bords des chemins. - Jura : Chaussin. - Saôneet-Loire: Saint-Pierre près Autun, sables de l'Arroux à Orney.Haute-Savoie: Allonzier, Pringy.- Rhôno : Mouche, la Tassin. B. stricta, Andr. - Champs. - Haute-Savoie: Allonzier, Pringy. Rhône: Bourdelans près Anse, Alix. - Isère.

B. intermedia, Bor. - Lieux humiles. - Jura : trouvé une fois près de Dôle par Michalet. - Sađne-et-Loire : Autun, Curgy, 
Antully. - Loire: Noirétable, route de Saint-Etienne à Annonay près de Planfoy. - Isère : Saint-Nizier, Prémol, la Salette. Gard : prairies de l'Espérou.

B. proecox, Rob. Brown. - Champs sablonneux. - Douls : Montbęliard, Belfort. - Jura : Sellières, Panessières près Lons-le-Saunier, Saint-Amour. - Ain : Langrin, entre Bellegarde et le fort de l'Ecluse, bords de la rivière de Glandieu. - Rhône : Vaulx-en-Velin, Charbonnières, Dardilly, Alix. - Isère : Uriage, Vaulnaveys, Saint-Nizier, Décines. - Hautes-Alpes : Gapà la Garde. - Drôme : Anneyron près Saint-Vallier, - Gard : Concoule, Anduze, Camprieux, bords du Gardon à la Beaume, l'Espérou. - Alpes-Maritimes et Var: le Pradet et la Garde près Toulon, le Luc, Frẻjus, Saint-Raphaël, Maures et Estérel.

SISYMinturr officinare, Scop. - Commun le long des chemins dans tout le bassin.

S. polyecratium, L. - Lieux incultes, décombres des départements méridionaux. - Hérault. - Gard. - Bouches-du-Rhone. Var. - Vaucluse : Barbiers, Saint-Martin-de-Castillon.

s. supinum, L. - Lieux sablonneux, bords des rivières. - HauteSaône: Authoison. - Doubs : Audincourt et de Montbéliard à la Saône, Besançon, etc. - Jura : bords du Doubs et de la Loue, Dole, Choisey, Molay, Montbarrey. - Cotc-d'Or : bords de la Tille, à Til-Châtel, Is-sur-Tille. - Saone-et-Loire : bords de la Saône, - Ain : bords de la Saône, Saint-Laurent-lès-llácon, Vésine, Pont-le-Vaux, Thoissey. - Rhone : sables à SainteColombe. - Isère : Vienne. — Hautes-Alpes : mont Bayard près Gap.

s. asperum, L. - Sables des rivières. - Côte-d'Or : Nuits, Arcelot. -Isẻre : Crémieu, Montalieu, Monestier de Clermont. - HautesAlpes : Gap. - Gard : Vaqueiroles près Nimes, Saint-Marcel, bords de l'étang de Jonquières. - Hérault : Saint-Loup. Bouches-du-Rhône: Puyricard, les Milles, plan d'Aups. - Vaucluse : Gadagne près Avignon, les Barbiers près Apt. - Var: Sainte-Baume.

S. Columna, Jacụ. - Champs et vignes de la région méridionale. - Drôme : Crest, Saint-Paul-Trois-Châteaux. -Vaucluse : envi- 
rons d'Apt. - Gard: Nimes, Bagnols, Pujau, Comps, Serre-deBouquet, Blauzac, - Hérault : Saint-Guilhem, de Saint-Bauzile à Ganges. - Alpes-Narilimes. - Bouches-du-Rlione. - Var : Toulon, Gonfaron, le Luc, Eréjus.

9. allinria, Scop. - Commun le long des haies dans tout le bassin.

s. Irto, r. - Vieux murs et décombres. - Ain : le mont prìs Nantua. - Cote-d'Or: Ari ay, Saulieu. - Saone-et-Loire : naturalisé à Autun.-Isère: Grenoble, le Freney en 0isans.-Savoie : mont Cenis. - Hautes-Alpes : Lautaret, Briançon. - Vaucluse : environs d'Apt au chemin de Rustrel, Saint-Martin-de-Castillon. - Hêrault, - Gard. - Bouches-du-Rhône. - Var. - AlyesMaritimes.

8. austriacum, Jacq. - Jura: rochers de Gily près Arbois, de Baume près Lons-le-Saunier. - Haule-Savoic: fentes des rochers du Salève, rochers du Coin et du Pas-de-l'Echelle, mont des Cornettes au-dessus de la Chapelle, Maggériaz près le lac d'Annecy, mont Tournette, vallon des Usses, mont Thobert près de Faverges. - Savoie : mont Cenis, Tignes, Laval, Pesey. - Ain : Saint-Rambert à la Craz-du-Reclus, Pierre-Chåtel, Parves. Isère: Vienne. - Hautes-Alpes :'la Grave, Lautaret, Viso, abondant dans le Queyras. - Drôme: Saint-Vallier.

S. rupestricolum, Jord. - Ain : Serrières-de-Briord, Rossillon. Isère: Dent d'Hyères près Grémicu.

s. strietigsimum, L. - Bords des champs, dans les montagnes. Isẻre: le Freney en montant à l'alpe du mont de Lans, Huez.Hautes-Alpes : le Queyras. - Savoie : la Chambre, Villette, Bourg-Saint-Maurice.

GHSYM BRI UI sophin. L.- Champs sablonneux humides, bords des rivières, décombres. - Haute-Saône : vallée de l’Ognon à Pesmes, Gray. - Côte-d'Or. - Doubs : Montbéliard. - Jura : Gily près Arbois, Chaussin, Pesmes. - Saône-et-Loire. - Ain : Trévoux, Misérieux, Ars, le Revermont, Nantua. - Savoie. Haute-Savoie: Salève. - Isère: Saint-Fons, Grenoble, SaintEynard. - Hautes-Alpes : Briançonnais, Queyras, Gapençais. - Rhône: Couzon, bords de l'Azergue, - Hèrault. - Gard. - 
Bouches-du-Rhône. - Var. - Vaucluse : vallon de Sivergues, Bastide-Basse près Apt.

s. pinnathatame, D. C - Díbris de rochers dés muntarnes à sol siliceux, - Hautc-Saroie: le Brévent, le Buet, mont Méry à la Pointe-Percée, col du Bonhoinme, Tri-les-Chosal près le glacice de Tré-la-Tête el près de tous les glaciers du mont Blane. Savoic: mont Cenis, le Srut prẻs des Allues. - Isère : chaines do Belledonne, des Sept-Laux et des Grandes-Rousses. - HautesAlpes : Lalutaret, Viso, Claallol-le-Vieil.

IIUGUNONA tamacetifolia, Rchb. -- Dits: is th rochers. - Haute-Savoie: Tré-les-Chosal.- Savoie : mont Cenis, mont Iseran du côté de Laval-de-Tignes. - Haules-Alpes : Laularet entre le mont Genèvre et Gondran, mont Viso à Pra-llichel, SaintVéran au-dessus de Clausis. - Isìre : entre Besse et les Valettes.

NASTURTI offeinate, R. Br. - Commun dans les ruisseaux de tout le bassin.

N. sylvestre, $R$. Br. - Commun dans les lieux humides de tout le bassin.

N. anceps, D. C - Mèmes lieux que le précédent. Espèco douteuse, Michalet pense que cetle forme est un hybride des $N$. sylvestre et $N$. amphibium; il a trouvé tous les intermédiaires qui la relient à ses deux parents.

ARAR brassicaformis, Wallr. - Bois et rocailles des monlagnes. - Jura : escarpements de la Dôle, du Reculet et du vallon d'Ardran,-Haute-Savoie: mont Tournelte, Saint-Germainsur-Talloire, mont Méry, Cornette-de-Bise, Nautau au-dessus de Montriond, roc d'Enfer au-dessus de Saint-Jean-d'Aulph. Ain : de Nantua au lac de Silan. - Isère : mont Rachais, Sassenage, la Pra-de-Belledonne, Saint-Christophe. - Hautes-Alpes: Charance, Boscodon. - Vaucluse : mont Ventoux. - Gard : bois de Salbous près Alzon. - Hérault : ravins de la Sérane. - Var : Sainte-Baume. - Alpes-Marilimes : montagnes au-dessus de Grasse et de Saint-Auban.

A. saxatilis, All. - Ilontagnes calcailes. - Haute-Savoie : PetilBornand, entre Cenise et Solaison, Saint-Germain-sur-Taltoire, Veyrier, Haggériaz près Annecy-le-Vieux; le Coin au Salève, 
mont Tournette, mont Mẻry. - Ain : Collonges. fort l'Ecluse, Saint-Rambert-en-Bugey à la Craz-lu-Reclus. - Isère : SaintEynard. - Hautes-Alpes : mont Viso, rochers calcaires le long de la Combe, - Jrôme: montagnes de Die.

A It Ars verna, $\mathrm{R}$. Br. - Coteaux des provinces méridionales. Bouches-du-Rhone. - Gard : Serre-de-Bouquet. - Fontaine de Vaucluse. - Var : coteaux calcaires, le Revest pris Toulon, te Cannet-du-Luc.

A. auriculata, Lam. - Rocailles dans les montagnes calcaires. Haule-Savoie: Salève, le Coin, le Pas-de-l'Echelle; Haggériaz près Annecy-le-Vieux, vallon des Usses, abbaye de Vallon. Valais: Branson. - Ain : fort de l'Ecluse, Belley, PierreChâtel, la Balme, Serrières, - Isère : Crémieu, Hyères, Vernaz, Saint-Eynard, Saint-Nizier. - Gard : rochers dolomitiques à Campestre, bois de Salbous près Alzon. - Hérault : bords de la Vis à Navacelle. - Bouches-du-Rhône: Saint-Loup.

A. stricta, Huds. - Rocailles calcaires. -- Haute-Saroie : Salève an Pas-de-l'Echelle, au Coin, mont Tournette, mont Méry, Dents-d'Oche, rochers de Saint-Clair dans la vallée du Fier. Ain: Thoiry, de Gex à la Faucille, fort de l'Ecluse. - Isère : la Bastille, Comboire, Pariset, Saint-Eynard. - Ilérault: SaintLoup, Capouladou. - Fontaine de Yaucluse.

A. serpilufolia, Vill. - Rochers. - Jura : les Rousses près du lac, Saint-Georges, la Dôle. - Haute-Savoie: rochers des pitons du Salève, grès numulitique du sommet des Voirons, Brizon, Vergy, mont Tournette, Parmelan, Dent-d'Oche. - Savoie : mont Cenis. - Ain : auldessus de Gex. - Isère : Bovinant (Grande-Chartreuse), col de l'Arc, la Houcherolle. - HautesAlpes : mont Aurouse, la Garde près Gap.

A. hiresuta, L. - Rochers et murs. - Très-commun dans la HauteSaone, le Doubs, le Jura, le Bugey, la Côte.d'Or, Srône-et-Loire, le Dauphiné et le Lyonnais. - Assez commun dans la Provence et le Languedoc.

A. procera, Jord. - Lyon.

A. pubigera, Jord. - Lyon.

A. accederis, Joid. - Lyon.
A. collisparsa, Jord. - Villeurbanne.

A. idanensis, Jord. - le Bugey. 
A. propera, Jord. - Nantua.

A. hirlella, Jord. - Le Salève.

A. propinqua, Jord. - Guillestre, Villevieille en Queyras.
A. subnitens, Jord. -.- Saint-Nizier, Giande-Chartreuse, Villars-d'Arène, mont Aurouse.

A. vesula, Jord. - Viso.

A. gracilescens, Jord. - Evian.

AR ARs Alienii, D. C. - Rare, - Jont Viso, mont Cenis.

A. muralis, Bertol. - Vieux murs, rochers. - Jura : vallées de l'Ain, de la Bienne et toutes les vallées autour de Saint-Claude; de là s'élève à Morez, aux Rousses et vallée de Joux. - Ain : très-commun à Nantua, dans les montagnes du Bugey, Villebois, Belley, Parres,- Haute-Savoie : Salive au Pas-dc-l'Echelle, mont de Pelloua, Colonies près Annecy, crêt du Mlaure, roc de Chère près Talloires, Allonzicr, Semncz, mont Charvin. Valais : Saint-Maurice, le Trient. - Isère : commun dans les montagnes de Crémieu, Grenoble, Saint-Eynard, Sassenage, Saint-Nizier, Villars-de-Lans. - Dròme : Crest. - Gard : bords du Gardon à Saint-Nicolas, bois de Salbous. - Bouches-duRhône : Aix, Montredon. - Var: Bau-de-Quatre-Heures près Toulon, Sainte-Baume.

A. roselle, Jord. - Digne.

A. saxigena, Jorl. - Chambéry, Grenoble.

A. alpestris, Sclıleich. - Pâturages des sommets du Jura, Doubs, Bugey. - Haute-Savoie: Salève, mont Parmélan, mont Hẻry, mont Darbon près Vacheresse, Hautigny près de Bonnevaux, Rocd'Enfer, Dents-d'uche. - Isère : Saint-Nizier, Chamechaude, alpe du mont de Lans, - Hautes-Alpes : Lautaret, montagnes de Gap.

A. cenisiu, Reuter. - Savoie : mont Cenis. - Jura : Colombier audessus de Saint-Genis.

A. Cerandil, Bess. - Prés. - Gard : le Vigan, le Pont du Gard, Uzès. - Héraull : Montpellier. - Bouches-du-Rhône : Isontredon près Marseille. - Var: Mauros-du-Luc.

A. perfolfata, Lam. (turritis glabra, L.). - Coteaux secs dans la Haute-Saône, le Doubs, Jura, Ain, Rhône, Côte-d'Or, Dauphiné, Savoie et Haute-Savoie. - Plus rare dans les provinces méri- 
dionales. - Bonches-du-Rhone : Arles. - Var: Notre-Dame des Anges près Pignans. - Alpes-Marilimes. - Gard : Alzon.

A. cebennensis, D. C. - Bois. - Ardèche : mont Mézenc. Gard : Banahu près l'Espérou, aux rochers du Valat de la Dauphine.

A. Thaliana, L. - Commun dans tout le bassin.

A. nremosa, Scop. - Très-commun sur les débris de rochers dans la partie septentrionale du bassin. - Haute-Saône : mont de Vannes, Plancher-Bas, Plancher-les-Mines, vallées de Miélin, Tétc-du-Creuzot, Chauves-Roches, Champagney, Sabot-de-Frotey, Conflandey, Echenoz, Fouvent, Roche, Chavanne, SaintGermain, Lure, Roye, la Cote, Recologne, la Miotte et la Justice près Belfort. - Doubs et Jura : plaines de Montbẻliard, đe Besançon et de tout le Jura jusqu'à Lons-le-Saunier, environs de Dole. - Saone-et-Loire : vignes des coteaux calcaires à SaintScrnin, Couches, Dezize, Cluny, Vergisson, Bruailles près Louhans. - Nul dans les régions moyennes et méridionales du bassin.

A. alplna, L. - Rochers calcaires du Doubs, Jura, Ain, Bugey, Revermont, Valais, Haute-Savoie, Savoie. - Isère : Vertrieu. Crémieu, Grande-Chartreuse et les autres montagnes du Dauphiné. - Hérault : Saint-Loul, Capouladou. - Gard : l'Espérou. - Provence: mont Ventoux, Sainte-Baume.

A suxeticola, Jord. - Ain : Saint-Rambert.

A. corulen, Jacq. - Rochers. - Haute-Savoie : col du Bonhomme, Sixt à la Vauzalle, Croix de fer du mont Méry, - Savoie: mont Cenis à Cornc-Rousse. - Isère : Grandes-Rousses. - HautesAlpes : le Galibier, le Viso en descendant du col de Ruine.

A. bellidifolla, Jacq. - Bords des sources el des ruisseaux. Haute-Savoie : mont Tournette, Cornetle-de-Bise, Sixt à la Vauzalle. - Savoie: mont-Cenis à paltes crcuses, Laval-de-Tignes, col du Bonhomme, montagne des Allues, Horteret. - Isère: la Pra-de-Belledonne, Piémeyan au mont de Lans.-Hautes-Alpes: Lautaret, mont Viso, Saint-Vèran aux Serènes.

A. pumila, Wulf. - Fentes des rochers, - Haute-Savoie : glacière du mont Brizon, mont Vergy, mont Tournette, montagnes de 
Samoëns, Cornette-de-Bise, Nautau au-dessus de Montriond, Roc* d'Enfer.-Isère : grande et pelite Moucherolle, Grand-Yeymont.

A. turmita, L. - Commun dans les rochers calcaires. - Doubs. Cồte-dOr. - Jura.-Ain : Revermont, Bugey.- Haute-Savoie : Salève, Voirons, environs d'Annecy, roc de Chère, Maggériaz, Meillerie. - Valais : porte du Scex, Saint-Maurice. - Rhône : entre Givors et Sainte-Colombe. - Isère: Crémicu, la GrandeChartreuse, Grenoble, Sassenage, Vienne.-Hautes-Alpes: Gap, Loubet près Rabou, - Vaucluse : mont Ventoux. - Garl : Vigan, Olas, Serre-de-Bouquet. - Var : Sainte-Baume, Dardenne près Toulon.

CATH DIN astifolia, L. - Basses-Alpes : vallée de l'Archo jusqu'au lac dı Lauzannier. - Vaucluse : environs d'Apt. Alpes-Maritimes.

C. Pumieri, Villars. - Rochers des alpes granitiques, - Isère : chaîne de Belledonne au chalet de Jassevieille, bords du lac Robert. - Hautes-Alpes : mont Viso à la Traversctte. - Saroie: petit mont Cenis à la Combe-d'Ambin.

C. protensis, L. - P’és dans tout le bassin.

C. praticola, Jordan. - Environs de Lyon.

C. herbivaga, Jordan. - Environs de Lyon.

C. udicola, Jordan. - Environs de Lyon.

C. Matholi, Moretti. - Prairies humiles. - Haule-Savoie : la Roche.

C. ammara. L.-Prés humides, bord des eal:x.-Haute-Saone: Auxon, Chassey-les-llontbozon, Lure, Corravillers, Champagney. Toute la chaine du Jura; monte depuis la plaine jusque dans les hautes vallées de Joux et des Rousses. - Ain : vallées de l'Albarine, chalne du Colombier, montagnes d'Hauteville et de Nantua. - Saone-et-Loire : Renaudiots, Champs-Chanoux près Autun, Selle-d'Auxy, Canada. - Cote-d'Or: Roghe-en-Brenil, Saulieu. - Rhone : Saint-Bonnet-le-Froid. - Loire : Pilat, Pierre-sur-Haute. - Isère : T'encin, gorges de Sarène au-dessus de Clavans. - Drôme : Saint-Vallier. - Gard : hords des ruisscaux de l'Aigual près de l'Espérou. - Alpes-Maritimes.

C. impatiens, L. - Licux frais et ombragés. - Toute la shaine du 
Doubs, Jura, Bugey, - Haute-Saone: vallée du P'uix, Plancher. les-Mines, Chemilly, Chargey, Thieflrans, - Cote-d'Or: Hlavignerot, vallon Sainte-Foy, Cîteaux. - Saone-ct-Loire: bords de l'Arroux, Canada, Paurret, Bourbon, Cluny. - Dauphiné. Saroie. - Herault. - Gard : Alzon, Vigan. - Alpes-Harilimes.

C. In Irsuta, L. - Champs et bois dans tout le bassin.

C. syloadiea, Link. - Bois des montagnes. - Doubs d Jura : foréts de Joux, de Champagnole, de Pontarlier, du Russey, du Rizoux, de la Dóle, de la Faucille; se montre aussi sur les sols siliccux de la plaine, nolammint dans la forét de la Serre et la bresse. - Zone vosgienne de la Haute-Saône: Chauves-Roches près Servance, montagne de Ternuay, vallée de Miélin, Plancher-les-Mines, Champagney, Plancher-Bas. - Ain : sous Pierre-Châtel, forêt de Ilazières an-dessus de l'Hallériat. Haute-Saroie : mont Semnoz, Tournette, Voilons. - Rhône: entre Givors et Sainte-Colombe. - Loire: Pilat. - Isere : Cornboire, Saint-Eynard, Grande-Ghartreuse, chaine de Belledonne.

- Hautes-Alpes : environs de Gap à Derez-de-Rabou.-Dròme : Saint-Vallier. - Gard : Aulas, Signe-Diou près Saint-Guiral. - Hérault.

C. parvifiorn, L. - Prés hunides des provinces méridionales. Gard: l'Espérou. - Bouches-du-Rhône: environs de Marseille. - Drôme: Montélimar.

C. alpina, Willd. - Pelouses des Alpes granitiques. - Haute-Saroie : chaîne du mont Blanc aux Grands-llulets à $3,060^{\mathrm{m}} \mathrm{et}$ au jardin de la Ifer de glace, Brévent, montagnes de Sixt et de Samoëns, mont Mery, Cornette-de-Bise, col du Bonhomme. - Savoie : vallẻe de Tignes, mont Iseran, mont Cenis à Cornerousse, montagnes des Ailues. - Isère : chaine de Belledonne, des SeptLaux, des Grandes-Rousses. - Hautes-Alpes : Galibier, Chaillolle-Vieil, mont Viso.

C. resedifolia, L. - Pelouses des hautes montagnes à sol siliceux. - Haute-Savoie : chaine du mont Blanc jusqu'aux grands Mulets à $3,060 \mathrm{~m}$, Brévent, Cornettes-de-Bise, col de Golèse, mont Tournette, grẻs du Vergy et du Méry. - Savoie: Tignes, mont Iseran, mont Cenis, col du Bonhonme, montagnes des Allues. 
- Sommités des Alpes-ßłaritimes. — Gard: l'Espérou à l'Horlde-Diou. - Ardèche et Haute-Loire : mont Mezenc.

DENTA RIA digitate, Lam. - Bois, - Doubs : cotes lu Doubs à Mauron, au Vallanvron, Morteau. - Jura et Ain : toute la chalne depuis le Suchet, Rizoux, Noirmont, Dỏle, Reculet jusı̨u'à la Faucille, au Gralet et au Sorgiaz. - Haute-Savoie : le Salève, les Voirons, chalets de Colone, Tournette, Veyrier près Annecy, montagnes de Bonnevaux. - Savoie. - Isère : Saint-Nizier, Grande-Chartreuse. - Hautes-Alpes : environs de Gap, chartreuse de Durbon. - Drome : Aucelon. - Gard : le long du Valat de la Cereirède, à Banahu, sur l'Espérou. - AlpesMaritimes.

D. pinnata, Lam.-Bois. Toute la chatne jurassique dans le Doubs, Haute-Saône, Jura, Bugey, Revermont. - Montagnes calcaires de la Savoie, du Dauphiné, de Vaucluse. - Gard : Bramabioou, Saint-Sauveur, l'Aigual. - Alpes-Naritimes.

D. bulbıfera, L. - Bois. - Isère : parc de Vizitle, Gavet. - AlpesMaritimes.

LUNA a rediviva, $L$. - Bois. - Doubs : cotes du Doubs et du Desscubre, Besançon, Nans, Gondenans. - Haule-Saône: vallées du Puix el du Rahin, Ballon-de-Giromagny, Thieffrans.-Jura : Salins, Lons-le-Saunier, Saint-Glaude, Dóle, le Reculet, SaintCergue. - Ain : Saint-Rambert, Tenay, Rufficu, la Fouge près Poncin, Nantua, Colombier du Bugey. - Saonc-et-Loire: Cuiseaux. - Cote-d'Or : bois de Savigny, Combe-d'Arcey. - HauteSavoie : montagnes de Bonnevaux. - Savoie : mont du Chat. Isère : Sassenage, Grande-Chartreuse, Saint-Gervais, Prémol._ Alpes-H aritimes.

vESICARA utrieulate, Lam. - Débris de rochers des montagnes. - Côte-l'Or : bords de l'Armançon à Semur, Bordes près Montbard. - Savoie : environs de Moutiers, Apremont. — Isére : Séchilienne, Bourg-d'Oisans, le Fréney, la Saletle. - HautesAlpes: la Grave, Monestier de Briançon, Villars-d'Arène.

AxYssUñ marltimum, Lam. - Coteaux du littoral de la Méditerranée; remonte quelquefois assez loin des bords de la mer jusque dans le département de la Drome vers Crest. 
A. halimifollum, L. - Montagnes de la Provence a Sigale et Gars dans la vallée de l'Esteron, Saint-Vallier près de Grasse, la Caille, Saint-Auban, confluent du Var et do la Tinéa, etc.

A. macroearpun, D. C. - Rocher's calcaires des Civennes, Serrede-Bouquet près d'Uzès. - Ardèche : vallée de l'Ouvèze. IJérault: de Saint Chinian à Saint-Pons.

A. apinosum, L. - Rochers calcaires des environs de Toulon, à Coudon et au mont Faron. - Bouches-du-khône: Aix. - Gard : le Vigan, Montdardier près d'Alais, bords du Gardon à SaintNicolas, la Beaume. - Hérault : de Saint-Chinian à Saint-Pons, P'ic-Saint-Loup, Granges, Saint-Guilhem-le-Disert.

A. surigenum, Jord. et Fourr.-Rochers calcarres.-Gard : Saint-Nicolas, la Beaume, Serre-de-Bouquet. - Drôme : Donzèré.

A. calyeinum, L. - Champs dans tout le bassin.

A. vagum, Jord. - Villeurbanne près Lyon.

A. sabulosum, Jord. - Le Bugey.

A. muderale, Jord. - Environs de Genève.

A. arvaticum, Jord. - La Grave (Hautes-Alpes).

A. compestre, L. - Champs des provinces miridionales. Provence, Languedoc; remonte dans les IIautes-Alpes vers Gap et dans la Drôme vers Crest.

A. montanum, L. - Pelouses et rochers. - Jura : Arbois, Poligny, Champagnole. - Côte-d'Or : vallée de Gourville, Gevrey, Marsannay. - Saône-et-Loire: Vergisson, Solutré. - Isère : gorges d'Engins. - Savoie : mont Cenis, mont Iseran, mont Galise. Hautes-Alnes : mont Viso. - Drôme : mont d'Ambel, Ratives, Crest. - Vaucluse : mont Ventoux. - Alpes-Maritimes : les Laltes près Saint-Auban. - Gard : environs de Nimes.

A. beugesiacum, Jord, et Fourr. - Rochers, - Ain : Saint-Sorlin entre Serrières et Villebois, Ambronay, Château-Gaillard, Loyettes.

A. psammerm, J. F. - Isère : Chasse près de Vienne.

A. brevifolium, J. F. - Isère: Charette près de Crẻmieu.

A. rhodanense, J. F. - Drome: Tain.

A. orophilum, J. F. - Hautes-Alpes : Briançon.

A. brigantiacum. J. F. - Hautes-Alpes: mont Gondran près Briançon. 
A. cunelfollum, Tenore. - Hautes-Alpes : mont Genèvre.

A. Iexicaule, Jord. - Mont Ventoux (Vaucluse).

A. alpestre, L. - Rochers des Hautes-Alpes : Villard-d'Arène, Galibier, environs de Briançon, mont Viso, Saint-Vẻran aux Sérènes. - Alpes-Maritimes.

A. Incanum, L. - Rochers. - Hautes-Alpes : Guillestre. - Var : remparts de Toulon.-Ain : d'Ambronay à Pont-d'Ain.-Isère : Chamagnieu.

CLYPEOLA jonthospI, L. - Vieux murs et rochers. - Ain : Saint-Rambert sous le rocher de la Craz-du-Reclus, Muzin, Serrières-de-Briord. - Haute-Savoie : sous les rochers du Parmelan au-dessus de Dingy. - Isère : Saint-Eynard, Comboire.

- Drôme : Nyons. - Vaucluse : Apt, Gargas. - Gard : Nìmes, Chusclan. - Hérault : mont de Saint-Clair près Celte-AlpesMaritimes : Antibes, Nice, Menton. - Var : Toulon, le Luc, Gonfaron, Fréjus. - Buuches-du-Rlıône : environs d'Arles. Se montre aussi dans le bassin supérieur du Rhòne près de Sion-en-Valais, à Tourbillon et Valère.

Cl. psiloca'pa, J. F. - Serrieres, Mluzin (Ain).

Cl. petrœa, J. F. - Donzère (Dı ôme).

Cl. semiglabru, J. F.-Vaucluse : Saint-Didier. - Bouches-du-Rhone :

Saint-Rémy. - Gard : Remoulins.

Cl. hispidulı, J. F. - Les Alpines (Bouches-du-Rhône).

Cl. lovigata, J. F. - Pinède d'Aigues-Mortes.

Ct. microcarpa, Moris. - La Provence.

Cl. cyclocarpa, J, F. - La Provence.

Cl. lomatotricha, J. F. - La Provence.

DRABA pyrenalea, L. - Rochers des Alpes. - Isère: Chamechaude, Colon-de-Belledunne, Grand-Veymont. - Hautes-Alpes : mont Aurouse, col Isoire, Saint-Veran, mont Viso au col des Ruines sous le rocher de la Traversette. - Savoie : mont Cenis à Ronches.

D. aizoides, L. - Rochers des montagnes. - Toute la chaine jurassique du Doubs, Jura, Ain: côtes du Doubs et du Dessoubre, Lomont; tout le Jura du vignoble aux sommités; le Colombier du Bugey, Torcieu, Dortan, mont d'Ain, le Revermont. - 
Cote-d'Or : rockers calcaires des combes de Gerrey, Bouilland, vallée de la Funtine-Froide. - Iaute-Savoie : rochers calcaires de Salive, Mole, Dents-d'Oche, montagnıs d'Abondance el de Saint-Jean-d'Aulph, Parmelan, Tournetle, chalets de Flaine, col des Aravis, Brévent, la Sauce près du Bontiomme, montagnes de Sixt. - Savoie : mont du Chat. - Isère : Saint-Nizier, Clamechaude, Grand-Som. - Hautes-Alpes : Lautarel, col du Golénn, mont Aurouse, mont Viso, Saint-Véran. - AlpesMaritimes. - Gard : rochers de Grailles près Campestre.

D. saxigena, Jord. - Rochers. - Isère: Vertrieu, Hyères, Crẻmieu.

D. chrysanthr, J. F. - Ain à Tenay.

D. ochroleucu, J. F. - Ain à Tenay.

D. trichocarpa, J. F. - Ain à Matafelon.

D. bengesinca, J. F. - Le Colombier-du-Bugey.

D. alpestris, J. - Briançon au Chabas (Hautes-Alpes).

D. Aadnizensis, Wulfen. - Rochers des Alpes granitiques. Haute-Savoie : cliaine du mont Blanc, Grands-Mulets, - Isère: chaine de Belledonne, des Grandes-Rousses, du Pelvoux. Hautes-Alpes : pic du Bec près de Villard-d'Arène, col du Goléon, mont Viso.

D. tomentosa, Wahlenb. - Rochers siliceux des Alpes. - HauteSavoie: mont Blanc, Brévent, montagnes de Samoëns, le Bonhomme, Tournette, col des Aravis, mont d'Etale près de la Clusaz, mont Méry à la Pointe-Pcrcée, Cornette-de-Bise, mont Dṛison sur Tamié. - Savoie: vallée de Tignes, mont Iseran, mont Cenis, le Saut près des Allues. - Isère : chalne de Belledonne à la Grande-Lance. - Hautes-Alpes : au pied des glaciers de la Grave, mont Viso à la Traversette, de Saint-Véran au col Agnel.

D. Priglda, Sauter. - Rochers des sommités alpines. - HauteSavoie: chaine du mont Blanc, Grands-Mulets, pied du glacier de Tré-la-Tête, montagnes de Sixt, Trelod, Vergy, Drizon, monts de Véron, Dents-d'Oche. - Savoie: mont Jovet, mont Cenis, col de la Madeleine, Hauteluce entre la Grande-Pariras et la Cyclaz, vallée de Tignes entre le pont de la Balme et Laval, mont Iseran. - Isère: Grandes-Rousses autour du lac Blanc, la Salette. - Hautes.Alpes : glacier du Bec au-dessus de Vil- 
lard-d'Arène, Galibier, mont Viso, Saint-Véran, Blanchette et la Noire.

D. carinthiace, Hoppe. - Prairies et rochers des hautes montagnes. - Haute-Savoie: Bueł, mont Vergy, Cornette-de-Bise.Savoie: mont Cenis, petit mont Cenis, mont Iseran. - Isère: chaine de Belledonne, Champrouse, Taillefer, Grandes-Rousses.

- Hautes-Alpes : Lautaret, Galibier, mont Chabrière-enChorges.

\#. maxalis, L. - Vicux murs, lieux arides. - Saône-et-Loire : Cluny, Tournus, Cormatin, Cortembert. - Rhône : Vallun du Mornantet entre Mornant et Givors, environs de Condrieu. Ain : Virignin, environs de Belley, à Muzin, Saint-Germain, Virieu-le-Grand, Saint-Rambert. - Isère : Vienne, Vaulnaveys, la Motte-les-Bains. - Drome: Saint-Vallier, Tain, Aucelon.-Bouches-du-Rhône: Aix à la Laouvo, Puyricard.-Gard: l'Arche près d'Anduze. - Var : Fréjus, le Luc. - Alpes-Nlaritimes: Houans, Biot.

D. ineana. L. - Prairies des montagnes. - Hautes-Alpes : Lautaret à Prime-Messe.

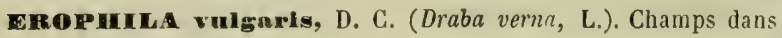
tout le bassin.

Les formes suivantes sont des démembrements de cette espèce :

E. virescens, Jord. - Environs de

Lyon.

E. vivariensis, Jord. - Ardèche :

Celles.

E. medioxima, Jord. - Lyon.

E. micrantha, Jord. - Lyon.

E. oblongata, Jord. - Lyon.

E. rubella, Jord. - Lyon.

E. chlorotica, Jord. - Lyon.

E. hirlella, Jord. - Villeurbanne près Lyon.

E. brachycarpa, Jord. - Villeurbanne. - Isère : St-Ismier.

- Annecy, Genève.
$E$. decipiens, Jord. - Lyon.

E. Lugdunensis, Jord. - Lyon.

E. furcipila, Jord. - Lyon.

E. stenocarpa, Jord. - Lyon. Polygone de Grenoble. - Genève.

E. majuscula, Jort. - Lyon. Isère : Saint-Nizier, Vaulnaveys, Tullins.-Haute-Savoie: Annecy, entre Evian et Thonon.

E. glabrescens, Jord. - Environs d'Annecy et de Genève. 
RorefPa nasturtoides, Spach. - Lieux humides dans tout le bassin; s'élève jusque sur les plateaux tourbeux du Jura et des Alpes.

R. pyrenaien, Spach, - Sables siliceux, - Doubs : Charmont près Monthéliard, sables de la Savoureuse. - Haute-Saône : Champagney, Rccologne. - Saônc-et-Loire: Autun, Auxy, Bourbon, bords de l'Arroux. - Assez comınun dans la région granilique du Lyonnais. - Ardèche : le Pouzin. - Garù : le Vigan, Trèves.

R. amphibia, Besser. - Bords des ruisscaux dans tout le bassin.

R. rustieana, Godron. - Prés lıumides, - Hautes-Alpes : Romette prìs Gap, Rosans.-Savoie : Belleville près Hauteluce.-Gard : entre Alais et la Grande-Combe. - Quelquefois subspontané près des habitations.

COCWA LA IEA glastifolia, L. - Gard : Aigues-Nortes.

KEENFEA saxatilis, Reichb. - Espéce caracléristique des rochers calcaires du Doubs, Jura français et helvétique, Revermont, Bagey, Savoie, Valais, Dauphiné, Basses-Alpes, AlpesMaritimes._Gard : environs du Vigan.-Hérault : Saint-Jeande-Fos.

MYAGEUX perfoliatum, L. - Moissons. - Bouches-duRhơne: Aix, Narseille, - Drôme: le Buis, Châteauneuf. Hérault : Montpellier, Aigues-Morles. — Gard : Nìmes, Manduel, Bellegarde, Saint-Gilles. - Rare dans la partie moyenne et septentrionale du bassin.

CAM E.LINA sautiva, Crantz. - Noissons; plante cul livêe et quelquefois subspontanće. - Haute-Savoie : Moneticr. - Rhône : Mont-Chat, digue du Grand-Camp, - Ain : Meximieux, entre Sainte-Croix et Saint-André-de-Corcy. - Drôme : Saint-Vallier. -Var: Fréjus, Aups. — Bouches-du-Rhône : Aix. — Gard : Campestre près d'Alzon. - Var. sylvestris. - Mloissons. - Avec le précédent.

C. Potida. Fries. - Champs de lin.

NESLIA paniculata, Desv. - Moissons des terrains calcaires. Doubs et Jura : depuis les plaines (excepté en Bresse) jusque dans les vallées les plus élevées. - Côte-d'Or. — Saône-et-Loire. 
-Rhône. - Ain. - Dauphiné, - Savoie. - Provence. - AlpesMaritimes, - Linguedor.

CALEPINA Corvini, Desv, - Rhône : environs de Lyon.-Isçre : Saint-Martin-le-Vinoux, Vizille. Hautes-Alpes: Gap.-Drôme: Saint-Vallier. - Gard : Candiac, Alais - Var : Fréjus, le Luc. - Bouches-du-Rhône: Aix, bords de l'Arc; Marseille, - AlpesMaritimes. - Valais à Branson, Saint-Maurice.

BUVias erucago, L. - Moissons des provinces méridionales, remonte à Lyon, Trévoux, Belley et dans le bassin du Léman.

$B$. arvensis, Jord. (silicules à angles non borủés d'une crête dentée). - Environs de Lyon et de Saint-Vallier.

B. brachyptera, Jord. - Environs de Lyon et la Provence.

B. macroptera, Reichb. - La Provence.

B. orientalis, L. - Quelquefois subspontané à Lyon sur la digue du Grand-Camp et autour de Bourg-d'Uisans (Isère).

ISATIS tinctoria, L. - Coteanx secs, bords les routes, quelquefois subspontané. - Saône-et-Loire: Charollıs, Gluny, Chàlon. - Côte-d'Or : environs de Nuits. - Jura : vallée de Joux près du village de l'Abbaye, - Ain : Tenay. - Isẻre : près du pelit sẻminaire de Grenoble, Trésane près du mont Aiguille. - Drôme: Crest, bassin du Léman et Valais. - Savoie : environs de Moutiers. - Plus répandu et moins fugace dans la Provence et le Languedoc.

Variété canescens, D. C. - Bouches-du-Rhơne: mont de SaintLoup près Marseille, la Treille. - Var : Toulon, la Valette, le Luc, Draguignan. - Alpes-Maritimes: Gourdon près de Grasse. Variété hirsuta, D. C.Villarsii, Gaudin.-Hautes-Alpes : la Grave, Lautaret, Briançon.

I. Alpina, All. - Pâturages du mont Viso à la Gaffe, Taillante audessus du lac Sgourgeau, passage de la brèche des Ruines, vallon des Brun.

BISCUTELC I Ievigata, L. - Rocailles des montagnes. Manque dans Côte-d'Or, Saone-et-Loire, Jura, Doubs. - Commence à paraltre dans le Valais aux Folateires, au Trient entre Martigny et Saint-Haurice, puis apparait dans la Haute-Savoie aux Dents-d'Oche, mont de Pelloua, Brizon, Vergy, mont Char- 
vin, crêt du Maure, Parmelan, Tournette, Se:mnoz, col du Bonlomme. - Savoie: Mont-Cenis, montagnes des Allues el des Avanchers. - Ain : Serrières-de-Briord, montagnes d'Ambronay.

- Isère : Belledonne vers le lac Crouzet, mont de Lans. Hautes-Alpes : Ia Grave, Lautaret. - Ardèche : Rochemaure. Drôme: Loriol, Livron. - Très-commun dans la Provenze, les Alpes-Maritimes et le Languedoc. - Cette plante présente un grand nombre de formes, qui se distinguent suivant que les feuilles radicales sont entières, dentelées ou pinnatifides. Les silicules sont tantôt lisses, tantôt scabres.

b. intricala, Jord. - Ardèche: Tournon.

B. collina, J. - Isère : Vernas, Hyêres près Crémieu.

B. lima, J. - Dróme : Loriol.

B. medilerranea, J. - Gard : Nimes.

B. polyclada, J. - Drôme: Nyons, Saint-Vallier.

B. picroïdes, J. - Gard : Lussan.

B. mollis, Lois. - Gurd : Nimes.

B. coronopifolia, Vill. - Débris de rochers. - Isère : la Moucherolle, Grand-Veymont. - Hautes-Alpes : mont Aurouse. - Hérault : entre Madière et Navacelle, environs de Béziers. - Vaucluse: mont Ventoux. - Bouches-du-Rhône. - Var : Saintc-Baume.

H. ciehorifolla, Lois. - Rochers. - Ain : Culoz, Serrièresde-Briord. - Isère : mont Rachais, Comboire. - Hautes-Alpes: Charance près Gap. - Basses-Alpes : Sisteron. - Var : Bormes, Collobrières, Fréjus. - Alpes-Maritimes : Grasse, Cagnes, Nice, Monaco, Menton.

TEERIS Cundolleana, Jord. - Débris de rochers calcaires; mont de Glandaz près Die.

I. stricta, Jord. - Pierrailles calcaires. - Hautes-Alpes : Gap.

I. Villar sii, Jord. - Pierrailles calcaires. - Drôme: Nyons. - HautesAlpes : Notre-Dame du Laus près Gap, bois de Lescout près Habou. - Ardeche: la Voulte, Cruas.

1. Timeroyi, Jord. - Collines calcaires. - Isère : entre Crémieu et la Balme.

I. delphinensis, S. - Pierrailles calcaires, - Drôme : Die. 
1. aurosica, Chaix. - Pierres calcaires. - Hautes-Alpes; mont Aurouse à Fontalibao, la Cluse en Devoluy. - Vaucluse : mont Ventoux.

I. collina, Jord. - Ain : Serrières, Lhuis.

I. leptophylla, Jord. - Bouches-du-Rhône.

I. Lamolti, J. - Ain : Virieu-le-Grand.

I. Violetti, Soy.-Vill. - Gard : Jonquières près Bagnols, - Ain : Chapelle du Mont près Nantua.

I. intermedia, Guers. - Coteaux calcaires. - Cote-d'0r : Chambolle, Sainte-Foy, Marsannay. - Gard : Alzon, Alais, le Vigan.

I. Prostii, Soy-Will. - Gard : Anduze, bois de Salbous près Campestre. - Ardèche : entre Thueyts et Montpezat, cratire de Jaujac.

V. clliata, All. - Rochers. - Vaucluse : Mornas. - Bouches-duRhône : Clâteau-Gombert près Marseille, Saint-Antoine. - Var : Brignoles, Toulon. - Alpes-Marilimes: Grasse.

I. Inifolia, L. - Coteaux calcaires de la Provence. - Bouches-duRhône: Aix, Marseille. - Var: Toulon, le Luc, Fox-Amphoux, Barjols. - Alpes-Haritimes : Grasse, le Chaudan, Valbonne, Nice. - Vaucluse : Orange. - Drôme : Nyons. - Ardèche : vallée d'Ouvéze, le Pouzin, mont Charray, - Je l'ai trouvé à Lyon sur les talus du chemin qui relie le fort des Brotteaux à celui de Villeurbanne.

๕. amara, L. - Hoissons. - Commun dans la partie septentrionale du bassin français; beaucoup plus rare dans les provinces méridionales. - Gard : Camprieux, bords dn ruisseau de Brama-Bioou.

I. affinis, Jord. - Environs de Lyon : le Vernay, la Mouche.- HautesAlpes : Gap.

I. pinnata, L. - Champs cultivés. - Rare dans la partie septentrionale du bassin. - Jura : Lons-le-Saunier, Pannessières. Saône-et-Loire : Solutré. - Est très-répandu dans les terrains graveleux du Lyonnais et du Bugey. - Isère : Sassenage, le Sappey, Clelles. - Drôme : Crest. - Hautes-Alpes : environs de Gap. - Savoie. - Haute-Sávoie. — Gard : Nimes, Blandas près du Vigan. - Var : Fréjus, le Muy, le Luc, Aups, FoxAmphoux. - Buches-du-Rhone. - Alpes-Haritimes.

T. ๙axatilis, L. - Collines calcaires des provinces méridionales. - 
Bouclies-du-Rlione: sommet de Sainte-Victoire, pic de Bretagne.

- Var: Sainte-Baume. - Vaucluse : mont Ventoux, - AlpesMaritimes: montagnes de Caussols et du Cheiron au-dessus de Grasse. - Basses-Alpes : Sisteron, - Dróme : Ie Buis, Brette. Gard : Serre-de-Bouçuet. - Hérault: Saint-Jean-de-Fos. N'est connu dans la partie septentrionale du bassin que sur le Crêt des roches, à Pont-de-Roide (Doubs).

1. Garrexiuna, All. - Fentes des rochers, - Basses-Alpes: l'Arche.

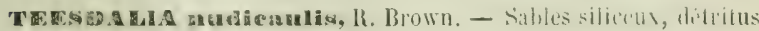
sablonneux sur la lisiere vosgienne de la llaute-Saône et le larrondissement de Montbéliard. Manque dans toute la chaine jurassique. - Côte-d'Or: terrains graniliques du Morvan autour d'Arnay, Saulieu, Semur, Tournesac, Roche-en-Brenil. - Se retrouve sur le même terrain dans la partie voisine de Sacneet-Loire, puis au sud sur les terrains siliceux du Beaujolais, du Lyonnais et du Forez jusqu'au Pilat et aux Cévennes, - Gard : le Vigan, bois de Campagne, près Nimes sur les cailloux quartzeux. - Var: porphyres et granits des Maures. - Isère : sables de la Mollasse ả Saint-Nizier, Pont-de-Beauvoisin, la Tourdu-Pin.

T. Iephlium, D. C, - Mêlé au précédent mais plus rare, si ce n'est dans la région méditerranćenne.

XTh caires; descend très-bas dans les vallées. - Manque dans le bassin suisse, le Doubs et le Jura. - Apparait dans l'Ain au fort de l'Ecluse, pied du Colombier vers Culoz, Muzin, Pierre-Châtel, Rossillon, Serrières, Portes. - Savoie : le mont du Chat, les deux rives du lac du Bourget. - Haute-Savoie: vallée du Fier, Thônes, rives du lac d'Annecy, Talloires, roc de Chè̀e, Veyrier, le Vuache. - Isère : la Bastille de Grenoble, la Balme. - Hautes-Alpes : Gap, Briançon. - Drôme : Crest, Nyons. Ardèche : Chàteaubourọ. - Bouches-du-Rhône: les Alpines à Saint-Rémy. - Var : Bagnols, Aups. - Alpes-IIaritimes : Gourdon, Grasse, Vence, Touët-de-Beuil, le Chaudan, Nice. Gard: Marguerite, Uzès, Serre-de-Bouquet, Sablons, Camprieux. 


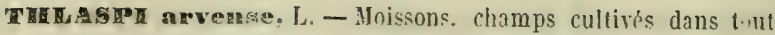
le bassin.

'. momandan, L. - Rochers calcaires des hautes montagnes. Doubs : très-répandu dans l'arrondissement de Montbéliard. Lomont, côtes du Doubset đa Dessoubre, environs de Besançon, Chaudanne, Rosemont. - Jura : Arbois, Salins, Lons-le-Saunier. - Ain : Saint-Rambert, côte d'Evoges, de Tenay à RossilIon, Nantua, - Cóte-d’Or : Route de Plombières, CombedeSairit-Joseph. - Saône-et-Loire: Solutré, Vergisson, Dezize. Isère: Chalais au Grand.Pic, rochers du Pas-de-l'Ane et des Bannelles, Pas-de-l'Echelle entre Saint-Gervais et Rencurel. Ardèche: col de l'Escrinet. - Les Cévennes.

T. Villarsianum. Jord. - Isère : col de l’Arc, la Houcherolle, mont Aiguille.

r. Trero RoRatuan, L. - Champs des terrains calcaires dans tout le bassin.

T. marliale, Jord. - Environs de Lyon : Grand-Camp, Charpennes.

T. erraticum, Jord. - Le Bugey. - Isère : Crémieu.

T. improperum, Jord. - Provinces méridionales.

T. alfpegta'e, L. - T. Gaudinianum, Jord. - Pâturages des montagnes. - Région supérieure du Jura: vallées des Verrières, de Joux, de la Brevine, des Rousses, des Dappes, de Mijoux, le Noirmont, la Dóle, la Trélasse, la Faucille, Saint-Claude, Belleydoux, Goumois. - Ain : montagnes de Hauteville, Colombier du Bugey, environs de Nantua. - Haute-Savoie : montagnes de Sixt et de Saint-Jean-d'Aulph. - Savoie : mont Nivolet.

T. Lereschii, Reuter. - Saint-Jean près de Thoiry (Ain). - Jura : prés tourbeux de la vallée de Joux.

T. brochypetalum, Jord. - Bois et prairics des hautes montagnes. - Haute-Savoie : Dents-d'Oche, Nautau, Parmelan, Tournette, Semnoz. - Savoie : Feissons-sur-Briançon, Hautecour, Galopaz, Sainte-Foy. - Isère : Pre̊mol, Champrousse, Chamechaude, mont Rachais. - Hautes-Alpes : Charance, mont Séuse près Gap. - Ardèche : mont Mezenc. - Gard : l'Espẻrou dans la gorge de la Cereirèdes, les Pises.

T. Verloti, Jord. - Prairies. - Isère : col de la Coche. 
T. sallicolum, Jord. - Prairies des hautes montagnes. - Isère : mont de Lans, Allemont, Saint-Christophe-en-0isans, la Chapelle-enValjouliray. - Ilautes-Alpes : mont Viso, vallon des Vaches.

T. benyesiacum, Jord. - Ain : Tenay, llauteville, Saint-Rambert.

T. vircns, Jord. - Rochers siliceux des montagnes. - Loire : mont

Pilat, Pierre-sur-IIaute, - Ardèche: mont Mézenc,-Cévennes.

- Gard : rochers à Concoule. Je ne sais s'il faut rapporter à celle forme ou à la suivante le T. alpestre des Ballons de Servance el de Giromagny.

T. sylvestre, Jord. - Rochers granitiques du Lyonnais à Chaponost, Soucieu, Brignais, Mornant; le Beaujolais. - Les Cévennes.

T. aIpia ana, L. - P'elouses des hautes montagnes, - Hautes-Alpes : mont Viso entre le chalet de Ruines et le col de la Traversette.

2r. rotonatifoliann, Gaudin. - Débris de rochers des hautes montagnes. - Haute-Savoie : mont Buet, le Bonhomme, Tournetle, Vergy, mont Charvin, Cornettes-de-Bise, roc d'Enfer, col de Golèse. - Savoie : mont Cenis à Ronche, col des Fours, Beaupré. - Isére : chaine de Belledonne vers le lac du Crouzet, lac Robert, Grandes-Rousses, haute tête de Raclia au-dessus du mont de Lans, Chamechaude. - Irautes-Alpes : mont Aurouse, mont Viso à la Traversette, Ubac-du-Mouth, Saint-Veran à Marcel.

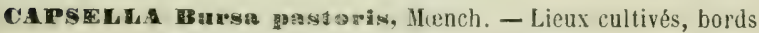
des chemins dans tout le bassin. - Espèce très-polymorphe.

C. agrestis, Jord. - Environs de Lyon.

C. virgata, Jord.-Environs de Lyon.

C. precox, Jord. - Environs de Lyon.

C. ruderalis, Jord. - Environs de Lyon.

C. sabulosı, Jord. - Rhône : les Chères.

C. rubellu, Reuter.- Lyonnais, Daupliné, Savoie, environs de Genève.

Hutchinsta alpina, R. Brown. - Débris de rochers des montagnes. Chaine jurassique: vallée des Dappes entre la Faucille et le Reculet, crêt des Neiges.-Haute-Savoic: mont Charvin, chalels de la Balme près du Bonhomme, Parmelan, Tournette, Buet, mont d'Etale près de la Clusaz, les Platets, mont Vergy, Corneltes-de-Bise, Dents-d'Oche, mont Ardin, Inont des Grauges 
dans les montagnes d'Abondance, montagnes de Sixt.-Savoie. - Isère: Grand-Som, Saint-Nizier, chaine de Belledonne ct des Sept-Laux, - Hautes-Alpes: la Grave, le Lautaret, mont Viso, Taillante près des lacs, col Agnel, environs de Gap. - Dròme: Glandaz près Die.

1F. petrea, R. Brown, - Rochers et graviers calcaires. - Doubs: Pont-de-Roide, Besançon, Ornans, Baume-les-Dames. - Jura : Poupet près Saline, Arbois à la source de la Cuisance, Baumeles-Messieurs, Saint-Glaude an vallon de Flumen. - Bassin du Léman et Valais. - Côle-d'or : Combe-Saint-Joseph. - Saôneet-Loire: Dezize, Vergisson, Solutré. - Haute-Savoie : Salève au Pas-de-l'Echelle, vallon des Usses pris de la Caille, mont Semnoz, Tournelte, Dents-d'Oche. - Ain : Nantua, Pierre-Chàtel, Parves, Belley, la Pape, lieximieux aux Peupliers, SaintRambert, Charabotte près Chaley. - Isère : balmes de Fontaine, Vienne, Revantin. - Hautes-Alpes : Gap. - Dròme : Saint-Vallier, Nyons, Barnave. - Bouches-du-Rhônc. - Var : Fréjus, lo Luc, le Cannet, Toulon. - Alpes-Haritimes. - Gard : Nìmes, bords du Brama-Bioou. - IIérault.

耳. preceana région méditerranéenne. - Bouches-du-Rhıonne: Marseille, bords de la mer. - Var: istlime de Giens. — Gard : bords des salines à Aigues-Mortes. - Hérault : borús de la mer près de Montpellier.

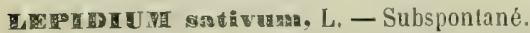

d. cam mestare, L. - Champs, bord des chemins dans tout le bassin.

L. campicolum, Jord. - Villeurbanne près Lyon.

L. errabundum, Jord, - Lyon.

L. accedens, Jord. - Ain : Viricu-le-Grand.

C. Roeterosphylluma, Bentham.-Prairies des montagnes.-HautesAlpes: col de Vars, la roche des Arnauds, mont Séuse, col de Glaise.

I. Hairtum, Smith. - Lieux incuites. - Drôme : Crest. - Bouchesdu-Rhônc. - Var : Fréjus, Touris, le Luc, Gonfaron, - Vaucluse:Apt. - Gard: Nimes, Tresques, Uzès, bois de Sallous près d'Alzon._Hirauli: pic de Saint-Loup près de IIontpollier. 
L. ratiera lo, I. - Lienx slipiles, dicombres. - Environs de Montbéliard et de Belfort. - Jura : bords du canal entre Dole et Saint-Jean-de-Losne. - Saône-et-Loire : Couches, Saint-Maurice, Iâcon, Cluny. - Côte-d'Or : Dijon, Ladoix. - Rhône: les Charpennes, Vaise. - Iscere : Crémieu, Grenoble. - HaulesAlpes : de Briançon à Embrun. - Valais : Martigny, Sion. Savoie : environs de Moutiers. - Bouches-du-Rhône: Peyrolles Marseille. - Gard : Aigues-Hortes, Bellegarde.

E. Frazan inifolium, L. - Bords des routes, dicombres. Dans tout le bassin.

L. polycladum, Jord. - Environs de Lyon.

L. dretsa, L. - Bords des champs, décombres. - Très-rare dans le boubs et le Jura à Neuchatey près Nans-les-Rougemont, pont de Baume-les-Dames, Mouchard, Dole le long des talus du chemin de fer. (Nul dans Côte-d'Or et Saòne-et-Loire). - Valais près de Sion. - Devient surtout commun à partir de Lyon, autour de la ville, Villeurbanne, Vaulx, Charbonnières, entre Hornant et Givors. - Loire: vallée du Gier. - Très-répandu dans la partie méridionale du bassin.

L. Iatifoliama, L. - Trẻs-rare dans la partie sepientrionale du bassin; assez répandu au sud de Lyon dans toute la partie méridionale du bassin.

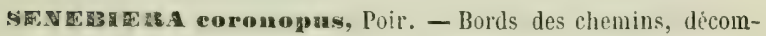
bres. Dans la plus grande partie du bassin.

s. pìaratifiala, D. C. - Espèce améı ìaine naturalisée dans les Bouches-du-Rhône prẻs Harseille à Saint-Just, bords du Jarret. - Var: près de Toulon à la Grosse-Tour, la Garde-Herault : Montpellier. - Isère : près du jardin de botanique de Grenoble.

CA

Th dans le bassin du Léman; manque dans la partie septentrionale. llu bassin français ; apparait dans l'Ain autour de Belley, Trévoux, Saint-Laurent-les-Mácon, la Pape. - Rłı̂ne : Villeurbanne, Saint-Alban. - Isère: Grenoble, les Portes-en-Trièves, Chichiliane. - Hautes-Alpes : Romette près Gap. - Drôme: Saint-Vallier. - Commun dans la région méditerranéenne. - 
Bouches-du-Rhône. - Var: Hyères, Fréjus, le Luc. - AlpesMlaritimes. - Hérault. — Gard : Nîmes, Manduel.

R. microcarpum, Jord. - Autour de Lyon à Villeurbanne, la CroixRousse, Dardilly.

\section{CAPPARIDÉES.}

CA RPATS spinosn, L. - Le Câprier est cultivé dans plusieurs parties de la Provence et devient souvent subspontané.

\section{CISTINÉES.}

Crstrus umbellntus, L. - Coteaux secs. - Gard: les bois de pins du Chanet près de Bourdezach, la Grand-Combe.

C. Iaurifolius, L. - Collines sèches de la région méridionale. Gard : terrains schisteux entre le Coulet et Trèves le long de la route de Sumène au Vigan, à Saint-Martial, Saint-Jean-duBruel. - Drôme: Nyons. - Ardèche: la Voulte. - Vaucluse: Avignon. - Hérault: Murviels près Montpellier, bois de Fabrègue près Roquebrun.

C. Iadaniferus, L. - Terrains siliceux de l'Hérault : de SaintChinian à Saint-Pons. - Var: granites et porphyres des Maures et de l'Estérel, Fréjus, Bagnols, Roquebrune.

c. albidus, L. - Collines calcaires de la région méridionale; plus rare sur les terrains siliceux. - Ardèche: Viviers. Drôme: Nyons, - Vaucluse : Orange, Avignon. - Gard : environs de Nìmes, bords du Gardon. - Hérault: environs de Montpellier. - Très-commun dans les Bouches-du-Rhône, Var, Alpes-Naritimes.

C. allido-crispus, Delile. - Collines pierreuses de la région méridionale. - Hérault: Montpellier. - Aude : Narbonne.

C. crispus, L. - Terrains siliceux de la région méridionale, -Hérault : graviers quartzeux de Montarnaud près Montpellier, Grammont, Lamoure, garrigue de Prègnes, entre Béziers et Bédarrieux, Roquehaute près Agrde. - Aude : Fontfroide près Narbonne. - Var: îles d'Hyères, Sablettes près Toulon, Fréjus. - 
Alpes-Maritimes: Grasse, île Sainte-Marguerite. - Vaucluse: Avignon.

C. Pouzotzil, Delile. - Ilérault: Montpellicr. - Gard: Peyremale près Alais, Quinti près le Vigan, Bourdezac, bois des Fourières, Ifontdardier, Saint-Laurent-le-llinier.

C. salviafolius, L. - Collines sè hes des terrains siliceux dans ba région méridionale; remonte jusqune vers les collines granitiques de Sainte-Colombe et d'Ampuis dans ie Rhône et de Vienne et Châsse dans l'Isère. - Drơme: Saint-Vallier, Serves, Tain, Montélimar, Crest. - Ardèche : granite de Tournon, Celle. Gard : terrains diluviens, cailloux siliceux autour de Nimes et Manduel. - Herault: graviers quartzeux de Taillade de Gignac, bois de Murviel entre la Planchude ct les bois de Moure, bois de Grammont, garrigue de Prègnes près de Béziers. - Alpes-Maritimes: Cannes sur le granite. - Var: micaschistes des environs de Toulon, granites et porphyres des Naures et de l'Estérel.

C. Rhodanensis, Jord, et Fourr. - Ain : Néron.

C. populitolius, L. - Environs de Narbonne à Font-Froide, forêt de Cascatel près Sigean, Font-Laurier.

C. corbariensis, Pourr. - Narbonne.

C. Iongifolius, Lam. - Donos et Font-Froide près Narbonne.

C. ledon. Lam. - Terrains siliceux de la région méridionale. Hérault : graviers quartzeux de Murviel, Grammont et Lavalette près Ilontpellier. - Bouches-du-Rhône : Jarseille. — Aude: bois de Cascatel près Narbonne.

C. monspeliensis, L. - Licux picrreux des terrains siliceux dans la région méridionale. - Vaucluse: Avignon, Orange. - Bouches-du-Rhône. - Var: environs de Toulon, Maures, Estèrel. - Alpes-Mlaritimes: Cannes. - Aude : Narbonne. - Hérault: environs de Montpellier, graviers quartzeux de Grammont, entre la Planchude et les bois de Moure, Nurviel, garrigue de Prègnes près de Béziers, de Caylus près Agde. — Gard : Nîmes.

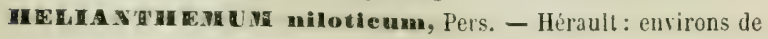
Montpellier, Pézenas, Gignac. - Gard : bois de Broussan. Bouches-du-Rhone: Aix près les Milles, la liède près les Martigues, Istres. - Aude : Narlonne. 
I. salleifolium, Pers. - Champs arides de ła région méridionale; remonte jusque vers Lyon. - Rhone: Saint-Alban, Irigny, Saint-Jean-des-Vignes près Charnay. - Ain : Néron, pont de la Cadelte, Balan. - Manque dans le Jura, Doubs, Côte-d’Or, Saôneet-Loire. - Apparaîl dans le Valais vers Branson près llartigny. - Isère : Vienne, Jonage, pic de Sírezin. - Drôme: Laveyron près Saint-Vallier,-Vaucluse : Avignon.-Bouches-du-Rhone : Aix, Marseille. - Var : Fréjus, Aups. - Aipes-Mlaritimes: la Malle au-dessus de Grasse, Saint-Vallier, Cannes, Nice. Hérault: Montpellier, Béziers. - Gard: Nìmes, Saint-Gilles, Villeneuve, pont du Gard, Uzès, Manduel, Corconne, Alzon, Brama-Bioou sur l'Espérou.

If. intermedium, Thib. - Lieux secs. - Hérault : Montpellier.

I. Iavandulofolium, D. C. - Coteaux secs. - Vaucluse : Viens près Apt. - Bouches-du-Rhòne : Marseille. - Var : Toulon.

II. Infrtum, Pers. - Coteaux arides de la région méridionale. Drôme, Nyons, Die. - Vaucluse : Avignon, Roussillon près Apt. - Bouches-du-Rhône : Marseille, Aix. - Gard : bois de Cygnan et de Broussan, Nimes, Aigues-Mortes, Beaucaire. Hérault : Montpellier, Saint-loup.

II. vulgare, Gaertı. - Espèce polymorphe très-lépandue sur les collines de tout le bassin. Les variations portent surtout sur la dimension des feuilles et des fleurs. Celles-ci, habituellement jaunes, sont quelquefois blanches ou roses. Les feuilles sont tantôt vertes sur les deux faces, d'autres fois blanches-tomenleuses en dessous. Les quatre formes ci-après indiquées sont décrites par la plupart des auteurs comme de simples variétés; par d'autres, au contraire, comme des especces.

H. tomentosum, Dun. - Feuilles blanches-tomenteuses en dessous. Assez commun dans la région méridionale. - Gard : collines du Vigan, l'Espèrou, Campestre, Genoulhac, Aigues-Mortes. Hérault. - Bouches-du-Rhône. - Hautes-Alpes : col de Glaise près Gap.

H. obscurum, Pers. - Feuilles vertes sur les deux faces. - Trìsrépandu dans tout le bassin. 
II. nummulurium, Irill. - Feuilles courtes, hérissées de poils. Drôme : cntre Cirest et Montélimar. - Hautes-1̇lpes : la Garde près Gap.

1. grandiforum, D. C. - Pétales trois fois plus grands que les sépales.-Très-commun dans les montagnes ilu Doubs, Jura, Bugey, Savoie, Dauphiné, Pilat et Cévennes.

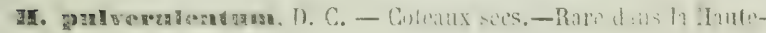
Saône au Sabot-de-Frotcy; dans le Jura ì Pagno\% près Salins; devient très-abondant à partir du mont Vuache (Haute-Savoie), puis dans l'Ain au fort de l'Ecluse, Pierre-Châtel, plaine de la Valbonne, Meximieu, Balan, Jiribel et la Pape. - Coteaux calcaires de Cóte-d'Or et de Saûne-et-Loire. - Isère : la Bastille de Grenoble, Pariset. - Hautes-Alpes : Gap. - Dròme : SaintVallier, Crest. - Vauclusc. - Arlèche : mont Combier près d'Alissas, le Pouzin. - Hérault : Montpellier, Cette - Gard: Nìmes, Saint-Nicolas, Serre-de-Bouqquet, Anduze, Alzon. Var : Mourières près Toulon, Cannet-du-Luc. - Bouches-duRhône : Marseille, la Crau. - Alpes-Maritimes.

II. vclutinum, Jord. - Débris de rochıers. - Ain : Serrières, Villebois, Saint-Sorlin. - Isère : Comboire. - Drôme : Claveyson près Saint-Vallier. - Hautes-Alpes : Briançon.

II. calcareum, Jord. - Jiontagnes calcaires des Hautes-Alpes : BrameBuou près Serres, Laragne.

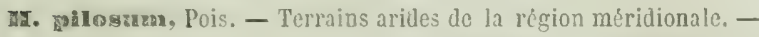
Vaucluse : Avignon, - Bouclıes-du-Bliône. - Var : Mourières près Toulon. - Alpes-IIaritimes : le Chaudun, montagne du Cheiron. - Gard : Pont-lu-Gard, Tresques, Uzès, Valbonne, Campestre. - IIérault : Mradières, Navacelle.

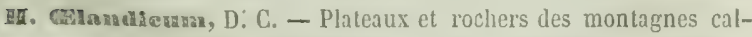
caires. - Isère : Chamechaude, col de la Ruchère,"la Mloucherolle. - Hérault. - Alpes-IIaritimes. - Cette espèce est réunie à la suivante par la plupart des auteurs.

II. alpestre, D. C.- Rochers calcaires des montagnes. - Haute-Savoie: mont Tournette, Vergy, Méry, Brizon, Cornette-de-Bise, mont Ardin, mont Chalune, Buet.-Savoie : mont Cenis à la Combe-leCatal. Bassin du Rhóne. 
Riverse. - Ain : Colombier-du-Bugey, Sorgiaz, fort de l'Ecluse.

- Isère : Néron, Saint-Eynarı, Saint-Nizier, Grande-Clartreuse. - Hautes-Alpes : le Quegras, la Garde pris Gap. -

Drôme : Crest.- Vaucluse : mont Ventoux. - Alpes-Maritimes.

H. penicilılum, Thib. - Bouches-du-Rhone : Saint-Rémy, Barbentane, Graveson. - Gard: Villeneuve, Beaucaire, bois des Espèces près de Nìmes, Blauzac. - Ardèche : Rochemaure, Viviers.

10. camuan, Dun. - Lieux jicrreux. - Doubs : Lomont, Pont-deRoide, mont d'Or. - Jura : Arbois, Salins, Lons-le-Saunier, Saint-Claude; s'èlève jusqu'aux sommilés clu Noirmont, de la Dôle et du lieculet. - Saunc-et-Loire: Dezize, Vergisson, Solutré. - Ain : bords de l'Ain, Balan, Heximieu, Ambronay, Pierre-Châtel, le Mont près Kantua. - Haute-Savoie : PetitPiton du Saleve, lo Mole, Parmelan, Toumette, Calvaire-deThônes. - Isère : Crémieu, Villette-d'Anthon, Jonage. Rhône: Vaulx-en-Velin, la Mlouche et les îles du Rhône près de Lyon. - Gard : le Vigan, Alzon, Montlardier, Brama-Bioou, Salbous. - Hérault: rochers de l'Abeille et du Pertus, SaintMichel-des-Sers, Pas-de-l'Escalette, Cette, Viols, Sérane, SaintLoup, Capouladou. Montpeyroux.

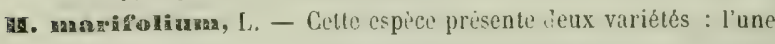
(tomentosum) ì feuilles blanchàtres-colonneuses en dessous, l'autre (virens) à feuilles non blanchâtres-tomenteuses. - Bouches-du-Rhône: les Pennes, Saint-Chamas, Montaud, SaintMitre, lisière de la Crau.

1x. guttatum, Hill. - Coleaux des terrains sablonneux. - Isère: Décines, Vienne. - Ain : la Pape. - Drôme : Saint-Vallier, Valence. - Vaucluse : Avignon. - Var : Toulon, forêts des Maures el de l'Estérel. - Alpes-Mlarilimes: Cannes, la Roquette, Antibes. - Gard: Nimes, Uzès, le Vigan. - Hérault: graviers quartzeux de Montarnaud, bois de Grammont, Murviel, Castelnau. - Bouches-du-Rhone : Aix, la Ciolat au Bec-de-l'Aigle.

耳. tuberasoin, Mill. - Terrains suliceux de la rigion meridionale. - Gard: Nimes en descendant à Saint-Gilles, Lois de Broussan près Loube, - Hérault : environs de Mont pellier, Grammont, 
Pérols. - Var: Toulon, Ilyères; Maures et Estérel. - AlpesMaritimes: Cannes, la Roquette, Berre, Contes.

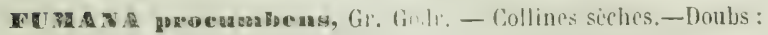
Ornans. - Jura : Salins, Lons-le-Saunier, Saint-Amour, mont d'Authume, Dólc. - Plus' commun dans le bassin du Léman et dans le Valais. - Côte-d'Ur, - SaOne-et-Loire. - Ain : collines du Bugey, Belley, Culoz, Pont-d'Ain, Nantua et jusque prés de Lyon, - Haute-Savoie : environs de T'honon.,-Savoie: mont du Calıat, environs de Moutiers. - Isère : la Bastille de Grenoble, Comboire, Décínes. - Hautes-Alpes : Gap. - Drôme : Saint-Yallier. - Bouches-du-Rhône: Arłes, bords de l'Arc aux Milles, - Var : Toulon, le Luc, Roq̣uelorune. - Alpes-Haritimes, - Vaucluse : Bedoin, Apt, Croignes. - Héraull : Roquehaule, environs de IIontpellier.-Gard: Aigues-diortes, Alais.

F. Sprehni, Gr. et Godr. - Rochers. - Savoie: Brizon près Ais, Saint-Innocent, Arbin au-dessus de la route de la Thuile. Isère : Pont-de-Claix, Comboire, la Bastille de Grenoble. Ardecho: mont Charray. - Gard : garrigues des environs de Nìmes, lo long du chemiri de la Beaume.

F. Ioevipes, Spach. - Coteaux secs de la rígion méridionale. Var: 'Toulon, Draguignan, le Luc, Fréjus, Aups. - Boucliesdu-Rhone : environs de Marseille. - Alpes-Haritimes. Hérault : environs de Mlontpellier, bois de Grammont, Murviel, Montpeyrnux.

F. viscida, Spacil. - Coteaur secs de la Provinee et du Languedoc. - Var: Toulon, Fréjus. - Bouchej-du-Rhóe. - Alpes-Maritimes. - Hérault : environs de Montpellier à Murviel. — Vaucluse: Orange, Avignon. - Drôme: Nyons.

Variété Thymifolia, Pers, - Drôme : Nyons. - Vaucluse : Orange. - Bouches-du-R tıône. - Gard : Villeneuve, Broussan, Nìmes. - Hérault: Grammont, Cette, Celleneive, Foncaude, Caunelle, Saint-Loup. 


\section{VIOLARIEESS.}

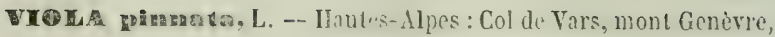
mont Cuculet près Guillestre.

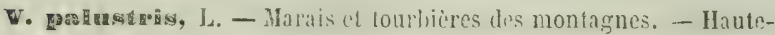
Saône: zône vosgiennc, Annegray, Ecromagny, vallée de Mansvillers, monts de Vannes et de Ternuay, vallée de Miélin, Plancher-les-Hines, Ballon de Giromagny, Fabier, Champey, Etobon, Belverne, Genechier, gres birarre de Magnirray, Giands-Bois pres Melisey, - Dou's: Morteau, Bilieu, Chenalotte, Mémont, liussey, Guinots, Pontarlier. - Versant helvétique des montagnes. Jura: la Trélasse près la Dôle. - Côte-d'Or et Saone-et-Loire: terrains granitiques du Morvan à Szulieu, Saint-Léger, la Rocheen-Brenil, l'Autunois. - Ain : marais de Colliart, Malbronde, Retord, vallée de Lélex. - Haute-Savoie: Brizon à Solaison, Montanvert, Barberine. - Savoie : Beaupré. - Isère : la Pra-deBelledonne, Prémol, Taillefer. - Hautes-Alpes : Lautaret, mont Viso, - Loire : Pilat, les Salles, Planfoy. - Drôme: Villeneuve près Saint-Vallier. - Alpes-Marilimes, _ Gard : l'Aigual, SaintGuiral, la Grandès-Haute.

V. Hineta, L. - Bois et coteaux dans tout le bassin.

r. Foudrasi, Jord. - Loire: Pilat.

V. Thomasima, Perr. et Sont. - Isire : Champrouse vers le iac Robert.

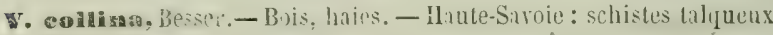
de la dent du Corbeau et toute la chaîne qui s'étend du mont Mirantin au col de la Louse. - Rhône: Rochecarlon, rives de l'Ardière, Saint-Jean-d'Ardière. - Isère : Saint-Nizier, Vif, Corenc. - Gard: bois de Salbous.

V. propera, Jord, - Environs de Lyon.

V. permicta, Jorù. - Haies. - Haute-Savoie: environs de Thonon, pied du Salève, base du Môle entre Contamines et Marignier, Cran près Annecy, Pringy, pied du Semnoz entre Annecy et Vovray, Crêt-du-Maure. - Savoie: Conflans. - Environs de Genève et de Lyon.-Isère: Comboire, Bastille de Grenoble. 
V. abortiva, Jord. - Ilaies et bois. - Environs de Genève, de Thonon et de Lyon.

V. sciaphylla, Jord, - Licux ombragés. - Jura : Cret-du-Niroir. Haute-Savoie : rocheres depuis la Tournette jusłu'à Arclusan. Savoie: Mont-Cenis, - Valais : au-dessus de Branson en montant à Joux-Brûlec, iliontorge, Tourbillon. - Isère : Comboire, mont Rachais, Saint-Eynard, Bourg-d'0isans.

V. Delphinensis, Jord. - Bois. - Hautes-Alpes : Rabou prèsGap, PuyMaure.

V. sulphurea, Cariot. - Loire: Cervieres.

V. Berandii, Jord, - Loire : Chalmaze!le.

w. virescene, Jord., V' alba, Besser. - Bois. - Calcaire jurassique de Ilaute-Sıone, Donbs, Jura, environs de Crémieu (Isère) el mont d'Or lyonnais. - Haute-Savoie: Voirons, pied du Salève, environs de Thonon, Armoy, Allinges. Arenthon, revers occidental du Crêt-du-XIaure près d'Annecy. - Environs de Genève. V. scotophylla, Jord. - Bois. - Huute-Savoie : environs de Thonon et d'Annecy. - Environs de Genève, de Lyon, de Grenoble, de Crest. - Provence.

v. otiorata, L. - Haies et bois dans tout le bassin. - Les neuf formes suivantes sont démembrées de celle-ri :

V. sepincola. Jord.-Haies. - Haute-Savoie : environs de Thonon. Arenthon. - Savoie: Aime, Moutiers, Conflans, de Cruet a Saint.Geoire. - Environs de Lyon. - Isère : Bron près Lyon, Vif, Comboire.

V. mullicaulis, Jord. - Haies et buissons. - Jura : environs de Dole et de Lons-le-Saunicr. - Haule-Savoie : environs de Thonon, Allinges, - Environș de Genève et rives du Leman dans le canton de Vaud. - Rhône: le Vernay, Saint-Cyr, Saint-Didier-ausMont-d'Or, d'Irigny à Saint-Genis-Laval, Vernaison. - Isère : Charvieu.

I. dlumetorum, Jord. - Haies. - Haute-Savoie : environs de Thonon.

- Rhône: bords de la Saone à Collonges. - Loire: Champoly. V. flor ibundu, Jorl. - Haies, broussailles. - Drôme : Crest.

V. propinqua, Jord. - Environs de Lyon.

V. consimilis, Jord. - Enviroris de Lyon. 
V. jucundn, Jord. - Environs de Lyon. de Thonon (Haute-Savoie).

V. suavissimn, Jord. - Environs de Lyon, de Thonon.

V. subcarnea, Jord. - Environs de Lyon, Sainte-Foy.

v. sylvatiea, Fries - Espece polymorphe répandue dans les bois de tout le bassin; les trois espèces suivantes en sont des démembrements :

V. Reichenbuchian, Jord. - Haies et bois. - Haute-Savoie : Chavanod, bois du Barioz près Pringy. - Environs de Genève. de Lyon, de Grenoble et de Gap.

r. Ritiniana, Rehb. - Bois raillis, - Plaine et collines du Jura, du Doubs et de l'Ain. - Bassin du Léman. - Haute-Savoie : Voirons, pied du Saleve, Chavanod, Reignier, bois de Barioz près Pringy, Cran près d'Annecy. - Isère : Polygone de Grenoble, Fontaine. - Haules-Alpes : enrirons de Gap. - Alpes-Maritimes. - Les Cévennes. - Comme les deux espèces précédentes ont été confundues sous le nom de $V$. syluatica. Frips, par un grand nombres d'auteurs, il y a lieu d'étulier de nouveau lear dispersion.

V. nemoralis, Jord.-Haute-Savoie: Salive au Grand-Piton, Vacheresse, mont Pétetod. - Savoie : Conflans, - Loire : les Salles, Chalmazelle. - Rhône: environs de Lyon. - Ain : bris des Volières aux Echeyx. - Rive gauche du R'ıơne près de Genève.

v. arenaria, D. C. - Lieux sablonneux et grareleux. - Jura : pelouses rocailleuses du Colombier. - Haute-Savoie : environs de Thonon, entre Annecy et Vovray, clêt du Ilaure, Servoz. Savoie : Moutiers, Champoulel, bois de Champion, Tignes, Lans-le-Bourg. - Isère : Belledonne vers le lac Crouzet. Hautes-Alpes : la Grave, Lautaret, Briançon, col des Hayes, mont Visı), Saint-Yéran aux Sérènes, col Ignel, Taillante, mont Bayard, mont Siuse. - Vaucluse : mont Ventoux. - Alpes-Maritimes : montạnes du Cheiron, de Caussols, du Bar cl de Śŕranon.

v. canina, L. - Pelouses sèches, bruyères des terrains silice $u x$. Morvan granitique dans Cỏte-d Or et Saônc et-Loire. - HauteSaône : zônes vosgienne et sous-vosgienne composées de schistes carbonifères travers's par les granites et porphyres : vallée du Rahin, Sigle de Ternuay, Ronchamp, Champagney, Lure, la 
Cote, Plancher-Bas, Clairegoutte, Belverne, Etobon, Frahier, Chenebier, Chagry, Chilonvillars. - Jura : granile de la furêt de la Serre, argiles tertiaires de la forct de Chaux, de la Chênée et de Pleurre près Chaussin. - Haute-Savoie : grès nummulitique des Voirons. - Valais : Ies Folaterres. - Isère : Charvieu, le Sappey, Vaulnareys, Prémol, Revel. - Drôme : Saint-Vallier, Crest. - Provence et Languedoc.

v. pumila, Chaix.-Prés humides. - - Haute-Savoie : pied du Salève dans les mares de Crévin, marais d'Allonzier. - Ain : Belley. - Hautes-Alpes: mont Bayard, Gap.

v. stagnina, Kıtaib.-Narais.-Roellebot et Sionnet près de Genève; Villeneuve el Aigle dans le canton de Vaud.-Haute-Savoie. Ain: marais des Echeyx. - Rhône : Bourdelans près Villefranche. - Isère: Charvieu, Brangues.

v. elatior, Fries. - Prés humides. - Environs de Genève et bassin du Léman. - Haute-Savoie. - Ain : Montluel, Pont-de-Vaux, Asnière, lle Bène près Trévoux. - Isère : Feyzin, Charvieu, Pont-de-Chérui, la Terrasse. - Rlıone: les Charpennes, Vaulxen-Velin, Anse, Villefranche, Bourdelans. - Var et AlpesMarilimes.

v. mirabilin, L. - Bois taillis des montagnes calcaires. - Cette belle espèce, indiquée comme fréquente à Belfort, à Grand-Villard près Monttéliard, sur I^s calcaires jurassiyues de Colombe et Frotey dans la Haute-Saône et, en dehors de nos limites, à Bâle, Schaffouse, Neuchâtel, ainsi que dans le Valais à Riddes, Branson, Porte-du-Scex, n'est pas signalée par Michalet, Reuter et Grenier, dans la chaine jurassique, quoique Babey prétende qu'elle existe au Montendre, au Colombier et au Reculet. Existe aux environs de Genève dans le ravin de Lagnon au-dessous de Bernex, Campagne-llifvernois pıès d'Aïre. - HauteSavoie: pied ılu Salẻve entre Veyrier et Crevin, Rrignier, près de Thunon entre la Dranse et le pont de Bioge. - Saroie: bois de Charapion près Moutiers. montagne de la Thuile. — Isère: mont Rachais, Saint-Eynard, - Hautes-Alpes: Clarance près Gap. - Côte-l'0r' : Coinbe-de-Flavignerot, Nuits, Savigaysous-Beaume. 
v. strieta, Horn. - Bois. - Environs de Genève dans les hois de Bey, de Veissy, de la Bâtic, de Meyrin. - Ain : marais des Echeyx, Colliard, les monts d'Ain.-Loire: Saint-Julien-MllinMolette au Grand-Pré.-Cévennes à Saint-Guiral et à l'Espérou.

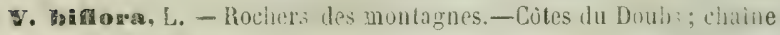
du Jura depuis le Montenure jusqu'au Reculet. - Toutes les sommités de la Savoie et de Haute-Savoie : le Mole, monts Brizoi, Vergy, Méry, Charvin, Buet, massif ủu mont Blanc. Savoie: vallée de Tignes, mont Iscran, mont Cenis, montagne des Allues, - Isère : chaine d'Allevard, de Belledonne, de l'0isans et de la Chartreuse. - Haules-Alpes : Lautaret, Viso, etc.

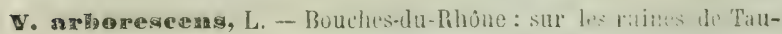
rentum dars le golfe des Lèques vis-à-vis la Ciotat. - Aude: Narbonne, Sainte-Lucie.

v. enlearata, L. - Pelouses des montagnes. - Jura : chinue du Colombier et du Reculet, crêt de Chalam.-Haute-Savoic : montagnes du Chablais, du Faucigny, inonts Charvin, Méry, Vergy, Tournette. - Savoie : mont Cenis, mont Iseran, mont Galise, Beaupré et toutes les hautes montagnes de la Maurienne et de la Tarantaise.-Isère et Hautes-Alpes : toute la chaine d'Allovard, de Belledonne, de la Grande-Chartreuse, de l'Oisans, di BrianÇonnais, du Queyras, montagnes de Gap. - Alpes-Iraritimes.

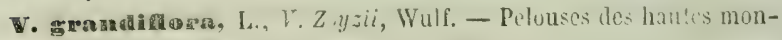
tagnes. - IIaute-Savoie : mont Charvin, chalets de Flaine, Tournette, Cornetles-le-Bise. - Hiutes-Llpes : Galibicr, col de l'Echauda, la Rochette, Avançon.

ซ. sudetiea, Willd. - Prairies des montagnes granitiques. Haute-SaOne: massif granitique čt porphyrique des bałlons de Servance el de Giromagny, Arrachies, Planche-des-Belles-killes.

- Loire : châne granilique du Pilat et de Pierre-sur-Hante.

y. cenalsia, L. - D bris de rochers des hautes montagnes.-HauteSaroie : chaino did mont Blanc, moraines de la iler de glace, le Buet, la Giltiz. - Savoic : mont Cenis vers le lac Clail. Hautes-Alpes : Granil-Villard près Briançon, col Isoire, col de l'Echauda, mont Viso. - Basses-Alpes: Barcēlonnette, l'Arche, Vars. - Vaucluse : mont Ventoux. - Alpes- Maritimes. 
v. trteolor, L. - Espece polymorphe disséminie dans tout le bassin et qui a été divisie en un grand nombre de formes dont l'ẻnumération suit :

V. segetalis, Jord. - Champs cultivés. - Doubs, Jura, Saône-et-Loire, Cólc-d’Or.-llaute-Savoie : Voirons, Thonon, Vacheresse, Pringy. - Environs de Genèvo et rives du Léman; Valais ả Branson. Ain : la Bresse. - Rhône: Charbonnières, Quincieu, - Isère : Vaulnaveys, Saint-Nizier. - Drôme: Saint-Vallier. — Provence ct Languedoc.

V. agreslis, Jord. - Champs cultivés. - Plaines du Doubs, Jura, Saône-et loire, Côte-d'0r. - Haute-Savoie : Voirons, Pringy, Thonon. - Bassin du Léman. - Rhone. - Languedoc. - Provence.

I. obtusifolia, Jord. - Environs de Lyon.

Y. contempta, Jord. - Loire: Sainl-Etienne, les Salles.

V. variata, Jord. - Environs de Lyon.

v. subtilis, Jord. - Champs cultivés. - Isère : Sassenage, Pont-deClaix.

V. alpestris, Jord, - Pelouses des montagnes. - Jura : Reculel, vallon d'Ardran. - Haute-Savoie: Onzon près le Biot, dents d'Oche audessus de Bonne dans les Voirons, Brizon, Saxonnet, abbaye de Vallon, Charvin, Tournette, environs du mont Blanc. - Savoie : vallée de Tignes, Conflans, mont Cenis. - Ain : Chartreuses d'Arvières et de Portes, Colombier, Retord. - Isère : Chartreuse de Prémol près Uriage, Saint-Eynard, Gavet, la Salelte, SainteLuce. - Hautes-Alpes : Gap. - Cévennes : Espérou, GrandèsHaute. - Alpes-Illaritimes.

V. Carioli, Jord. - Colombier-du-Bugey.

V. nemousensis, Jord. - Moissons. - Rhone : Chaponost. - Gard : Nìnes, Pont-du-Gard, Jonquière, Bellegarde, dunes de la pinède des Quatre-Maries près le Grau-d'Orgon, pinède d'Aigues-Mortes. -Var: Gonfaron, le Luc.

V. Sagołi, Jord. - Bois et lieux humides, - Haute-Savoie : Salève près des Treize-Arbres. - Gard : bois de l'Espérou, cap de Coste. Hérault : la Salvetat-Fraisse. 
V. Paillouxi, Jord. - Les Cévennes, le Beaujolais.

V. grarilescens, Jord. - Tourbières de la région supérieure du Jura,

la Brévine, etc. - Haute-Savoie : Pringy. - Compesières près

Genive. - Saône-el-Loire : Autun, Broye. - Rhône : Givors. -

Ain: Tramoye, Saint-André-de-Corcy. - Cévennes el Lozère.

$\boldsymbol{V}$. vivariensis, Jord, - Le Vivarais au-dessus de Burzet et d'Entrai-

gues. — Gard : champs cultivés de l'Espérou, de Concoule.

V. pallescens, Jord. - Rhone : Lyon. - Var : Bormes, - Alpes-Maritimes : cap Croisette près Cannes.-Gard : Capellier près Alzon.

\section{RESÉDACEES}

RESEA phyteuma, L. - Champs cultivés. - Très-commun dans la partie méridionale et moyenne du bassin; devient plus rare dans le Jura, où on le signale dans la vallie de l'Ain; plus rare encore dans Cóte-d'Or et Saône-et-Loire.

R. Iutea, L. - Terres sablonneuses, fentes des vieux murs. - Commun dans tout le bassin.

R. Iuteola, L. - Terrains inculles, murs. - Assez commun dans tout le bassin. - Cultivé sous le nom de Guıde.

E. Jrequini, Reichb. - Les Cévennes, Vigan, Bonpérier, Peiremale. - La Lozère.

a. anffrutlealoss, L. - Terrains sablonneux et graveleux. Bouches-du-Rhone: île de Maüré, la Ciolat. - Var : isthme de Giens, les Pesquiers près Hyères. - Gard : les dunes d'AiguesMortes. - S'est naturalisé à Lyon sur les talus du chemin de ronde.

AsTroCARUS sesamolden, Gay. - Sables granitiques et schisteux de l'Espérou et de la Lozère. - Isère : forêt de SaintSéverin prẻs Vertrieu?? (Indication à vérifier).

\section{DROSERACEES.}

Dromera rotundifolia, L. - Marais tourbeux des plaines et des montagnes. - Haute-Saone : terrains siliceux de la zone vosgienne. - Doubs et Jura. - Cote-d'Or: marais du Morvan gra- 
nitique à Saulieu, Semur; même ferrain dans Soône-et-Loire. Bugey, Savoie, Dauphiné, - Valais : marais de Salvan. Chaine granitique des montagnes du Lyonnais et du Pilat. - Isère: lac de Luitel au-dessus de Prémol, la Bérarde, Bressieu, forêt de Chambaran. - Cévennes : l'Espérou, Saint-Guiral. - Nal dans la Provence et les Alpes-llaritimes.

D. obovalt, M. K. - Marais tourbeux. - Haule-Savoie : marais de Lossy au pied des Voirons, Crevin, Bossey au pied du Salève. - Isìre: Renage, Eydoche. - Suivant Schiede, Godron et Grenier, le Dr. obotata est un hybride du Dr. rotundifolia et du Di. longifolia.

D. Ionglfolla, L. - Lieux tourbeux. - Zòne rosgienne de HauteSaơne: Fresse, Belfahy, Ballon de Giromagny.-Doubs et Jura : Pontariier, le Boulu, Prémanon, les Rousses. - Haute-Savoie: marais de Crevin, de Lossy, de Cernex près le mont Sion, d'Arention sur les bords de l'Arve. - Ain : Sainte-Croix près Montluel, environs de Belley à Premeysel, Magnieu. - Isère : Décines, Meyzieu, Frontonas, la Fullie près Saint-Quentin, Crímieu, environs de la Tour-du-Pin. - Nul dans la rẻgion méridionale.

D. Intermedia, Hayn. - Lieux tourbeux. - Haute-Siône : zône rosgienne, Fresse, Belfahy, les Guiủons, la Marcelle, zône sousvosgienne, Franchevelle, Saint-Germain, Lantenot, les Gi'andsBois près Mélisey. - Cóte-d'Or : bois d'Ausonne, Etang-Horin près Saulieu. - Saone-et-Loire : Cuisery. - Ain : étang Genoux à Pont-de-Veyle.

PARNASSI palustri*, L. $_{4}-$ Lieux tourbeux des plaines et des montagnes dans tout le bassin.

\section{POLYGALËES.}

POTTCA LA rosea, Desf. - Bruyères et bois de pins. - Var: Estérel, Bagnols, rochers des Lions près Saint-Raphaël. Alpes-Ilaritimes: Caussols, Yaucluse, Auribeau, Nice, Ilenton. P. comosa, Schkulı. - Pelouses des montagnes calcaires. - Calcaire jurassiyuc de la région du vignoble jurassien et des basses mon- 
tagnes du Dauphiné, du Bugey et de la Saroie. - Isère : mont Rachais, Comboire, Crénieu, Bourgoin. - Hautes-Alpes: Charance près Gap. - Savoie : entre Saint-Jean-d'Arvey et les Desserts, Monterminod, mont du Chat.-Haute-Savoie : Ie Salève. - Rhône : calcaire jurassique dı mont d'Or à Couzon, mont Toux. - Thurmann prétend que le $P$. comosa est une variété du $P$. vulgaris. Ce dernier serait, d'apris lui, la forme des sols frais et détritiques; le premier, la forme des collines sèches. La longueur des bractées ne lui paraît pas un caractère spécifique suffisant; il ajoute que les deux formes extrêmes sont reliées l'une à l'autre par une série d'intermédiaires.

P. rulgaris, L. - Prairies et bois des plaines et des montagnes dans tout le bassin. - Espèce très-polymorphe.

Variélé oxyplera, Reichenb. - Bois et prairies des terrains siliceux. - Haute-Saône: Champagney, Mont-de-Plancher, Chagey, Genechier, Chenebier, Frahier, Etobon, Belverne, FrédéricFontaine, Clairegoutte, Plancher-Bas. - Jura: Chêne-Bernard, Pleurre, Neublans, forêts de Clıaux et de la Serre. - Saône-etLoire: Bragny près Verdun, environs d'Autun. - Rhỏne : environs d'Alix. - Loire : Pierre-sur-Haute. - Isère : environs de Grenoble, le Sappey, Parisct.

Variété Lejennei, Boreau. - Ne diffère de la précédente que par la forme plus obtuse des ailes. - Bruyères des montagnes. Isère : Saint-Nizier, Revel, Prẻmol.

Variétẻ alpestris, Koch, - Ain : Colombier-du-Bugey. - Isère : Grande-Chartreuse à la Ruchère, Prémol. - Loire : Pierre-surHaute.

P. calcarea, Schultz. - Pelouses des terrains calcaires. De la plaine aux sommités de la chaîne jurassique. - Coteaux calcaires de Saône-et-Loire : Dezize, Clıamilly. - Isère : mont Rachais, Pariset, Grande-Chartreuse, Crémieu, Vertrieu, Chalais. - Savoie: Apremont, la Thuile. - Hautes-Alpes : environs de'Gap. Drome : Crest. - Gard : prairies de toute la chaine de l'Espérou, d'Alais et d'Anduze.

P. alpestris, Reiclienb. - Bois, pelouses des montagnes. Toutes les sommités du Jura : Noirmont, Montendre, Dôle, Suchet, Recu- 
let. - Ain : le Colombier-du-Bugey. - Haute-Savoie : mont Charvin, mont Châtillon, Haut de Véron près des chalets de Flaine, Brizon, Salève aux Pitons, montagnes d'Abondance et de Montriond, Cornettes-de-Bise, Dents-d'Oche. - Savoie : Nivolet, Margériaz, la Lavoire-sur-Arbine, Arclusan, mont Drison, la Gitaz près Beaufort, montagnes de Belleville près Hauteluce, Tignes, mont Iseran, mont Cenis,-Isère : IIaute-têle-de-Racha près du mont de Lans, - Hautes-Alpes : Lautaret, Villardd'Arène, environs de Gap. - Alpes-Maritimes.

P. depregs , Wender. - Pelouse, bruyies des ierrains silicenx.

- Irate-Saônè : grès des cantons d'Héricourt et Champagney, Chagey, vallée du Rahin vers la Grande-Goutte, Ballon de Giromagny, Château-Lambert; grès bigarrẻ et diluvium de Franchevelle, Linexert, Oxfordien, grès du lias à Auxon, Chariez, Grattery. - Doubs : la lisière vosgienne, tourbières du Mémont Jura : forêt de Chaux, bois de Gatey, de la Chénée, de Pleurre. -Morvan dans Côte-d'0r et Saône-et-Loire.-Ain : Colombier-duBugey. - Isère : mollasse de Saint-Nizier. - Rhone: Vaugneray aux Jumeaux, Larajasse. - Loire : Pilat, les Salles, Pierresur-Haute. - Haute-Savoic : roc de Chère sur les bords du lac d'Annecy, monts Voirons du côté de Boẻge--Savoie: montagnes de Saint-Hugon et loute la chaine qui s'étend du col de la Louse au mont Mirantin, vallées de Bàmont et de Hauteluce. — Gard : prairies de l'Espérou.

P. nmnra, Jacq. - Prés humides et tourbeux. - Doubs, Jura et Ain : depuis les plaines jusqu'aux sommités. - Haute-Savoie: marais de Glaisins près Annecy-le-Vieux, Pringy. - Savoie: mont Nivolet, Margériaz, bois Champion près Houtiers, Tignes, mont Cenis. - Isère : environs de Grenoble, Charraz près de Pusignan. - Hautes-Alpes : Briançon, environs de Molines. Vauclise : mont Ventoux, — Gard : l'Espérou. - La forme suivante est considérée par la plupart des auteurs comme une variété du $P$. amara.

r. austriaca, Crantz. - Prés marécageux, - Commun dans le Doubs el Jura. - Cote-d'Or. - Haute-Saroie : marais de Troinex et de Bossey au pied du Salève, Pringy, bords du Chéran près du clıa- 
teau des Balmes, montagnes de Jorzine, plateau des Gets. Ain : les Echeyx, Sainte-Croix près de Montluel, Hauteville. Isère : Décines, Meyzieu, Charvaz près Pusignan, Villars-deLans, environs de Grenoble. - Hautcs-Alpes : Lautaret, col de Glaise. - Alpes-Maritimes : Colmiane.

P. pedemontana, Perrier. - Savoie: mont Cenis à Ronches.

I. alpina, Perr. et Song. - Pàturages des Hautes-Alpes entre le col de la Croix et Abriés.

P. exillis, D. C. - Prairies marécageuses, pelouses humides. Ain : hords de l'Ain sous Chazey, Château-Gaillard et environs; entre diexinnieu et Balan. - Isère : Décines, derrière le moulin de Platacul, pàturages à Jonage entre Saint-Roman et Jonsac.Bouches-lu-Rhône: Pas-des-Lanciers. — Gard : Aigues-lfortes. - Hérault: dunes de Maguelonne.

P. chamobuxus, L. - Bois des montagnes. - Haute-Savoie : Calvaire de Thônes, Bluffy près Saint-Germain-sur-Talloires, bois du Barioz près Pringy, crête du peiil Salève entre Reignier et la Roche, mont Brizon, de Chède à Servoz, frêtes de Magland, bords de la Dranse près Armoy. - Savoie : entre Avrieux et le fort de l'Esseillon. — Isère : Saint-Nizier, la Gardette au-dessus de Bourg-d'Oisans, entre le Sappey et Sarcenas. - Hautes-Alpes: mont Genèvre, Briançon, Boscodon. - Alpes-Maritimes : mont Cheiron, Thorenc, les Lattes près Saint-Auban, Aiglun, Gillette, Clans, Colmiane.

P. rupestris, Pourr. - Coteaux pierreux, - Environs de Marseille.

F. monspeliaca, L. - Prés et collines de la région méridionale. - Vaucluse : Avignon. - Var : environs de Toulon à Coudon, forèt des Maures, - Alpes-Maritimes : Grasse. - Bouches-duRhône : Marseille, Pas-des-Lanciers. — Gard : garrigues du mas de Seine près Saint-Nicolas. - Hérault: Nissan près de Béziers. environs de Montpellier.

ALDFota va vesiculosa, L. - Signalé à Arles dans les eaux du Trébon, oủ il ne se trouve plus; bords du Rhơneà Orange? - Environs de Montpellier.

Frankenia pulverulenta, $L$. - Bords de la Méditerranée.

F. loevis, L. - Bords de la Méditerranèe.

F. Intermedia, L. - Bords de la Méditerranée. 


\section{SILENEES.}

CUCUnA LUs breetrerus. L. - Haies. - Dans tout le bassin.

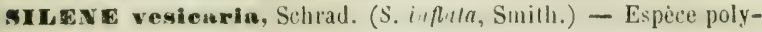
morphe commune dans tout le bassin. - Les quatre formes suivantes sont démembrécs de celle-ci :

S. brachyata, Jord. - Envitons de Lyon, de Thonon, elc.

S. puberula, Jord. - Haute-Savoie: Thonon, Evian, Lyand, Dentd'Oche, mont des Granges. - Isère: Saint-Martin-le-Vinoux, Pariset. - Drôme: Nyons.

S. glireosa, Jord. - Rocailles des montagnes du Doulss, Jura, Ain :

Nantua el tout lo haut Bugey. - Isère: Saint-Nizier, Chamechaude, col de l'Arc. - Haules-Alpes : environs de Gap.

S. ulpinu, Tlionas. - Débris de rochers schisteux des hautes montagnes.

- Haute-Savoie : haut de Véron au-dessus des chalets de Flaine.

- Savoie: mont Cenis. - Isère: mont de Lans, - HautesAlpes: la Grave, Villard-d'Arène entre Briançon ct Cervières, mont Viso, mont Aurouse. - Basses-Alpes : montagnes de Barcelonnelte. - Vaucluse : mont Ventoux. - Alpes-Ilaritimes.

s. conlea, L. - Lieux sablonneux. - Toute la régrion méridionale jusçu'à Lyon, Montluel et Meximieu. - N'est pas signalé dans le bassin du Léman, dans le Valais, ni dans le Jura, le Doubs, Saône-et-Loire, Côte-d'Or.

5. conohlen, L. - Rare dans les moissons. - Isère : Vienne au-delà duquel cette plante ne remonte pas. - Drôme: Montélimar. Basses-Alfes: Castellane. - Var : le Revest.- Alpes-Haritimes: Nice. - Hérault : environs de Montpellier.

\$. callica, L.-Espèce polymorphe commune ilans les cha nps sablonneux de la région méridionale, Prorence, Alpes-Maritimes, Languedoc, Drôme.-Plus rare dans la région moyenne.-Rhơne : Limonest, Marcilly, bords de l'Azergue et de l'Ardière, SaintAlban. - Isère: Décines, Bron. - Ain: Bourg, Thoissey, Garnerans. - Jura: champs sablonneux de la plaine, Arbois, Villersfarlay, Chaussin. - Haute-Saone: Champagney, Mansvillers, Ronchamp, Plancher-Bas, Franchevelle, Saint-Germain, 
Lure, Esprels, Chargey-les-Ports. - Manque vers Genève, le bassin du Léman, le Valais ainsi que dans Saone-et-Loire et Cote-d'Or.- Les deux formes suivantes sont des démembrements de celle-ci :

S. agreslina, Jord. et F. - Rhône : Quincieu.

S. quinquevilneru, L. - Champs sablenneux de la région méridionale. - Dròme: Nyons, Dieu-le-Fit. - Provence et Languedoc.

s. noeturana, L. - Champs des Provinces méridionales. - Provence. - Alpes-Maritimes. - Languedoc. - Remonte jusque dans la partie méridionale de la Drôme à Grignan, le Buis, Tain. - Nul dans la partie moyenne et supérieure du bassin.

S. brachypetala, Robert et Castagne. - Bouches-du-Rhone: Marseille, Montredon, Endoume. - Var: Fréjus. - Alpes-Maritimes: Cannes, Nice, Menton.

s. nlereensls, All. - Sables maritimes depuis Menton jusqu'à Toulon.

\$. vallesin, L. - Rochers siliceux des montagnes. - Massif granitique de l'Oisans dans les vallées de la Romanche, du Vénéon, de l'eau d'Olle, du torrent de Clavans. - Savoie: pelit mont Cenis à la Combe d'Ambin, mont Cenis. - Hautes-Alpes : massif granitique de Champoléon et du mont Viso au vallen des Brun, Malrif. - Une variété graminea, Vis., à feuilles plus étroites, existe sur les calcaires siliceux du mont Ventoux.

s. portensis, L. - Lieux sablonneux de la région méridionale. Drôme: Saint-Paul-trois-Châteaux. - Vaucluse : Mornas, Orange. — Gard: Tre:ques, la Capelle, Bagnols.

s. armeria, L. - Rochers de grìs et de granite. - Rhône : granite des bords du Garon et du Mornantet, Brignais, Chaponost, Givors, Lavore. - Loire: grès houiller et granite de la vallée du fier et du versant méridional du Pilat, Saint-Jodard, Clıampoly au bois des Gouttes, Chalmazelle. - Isère: Vertrieu, Bourg-d'Oisans, Séchilienne, Allemont. - Savoie: Venose, Calvaire de la Roche-Cévins, entre Petit-Cœur et Naves, Fontaines. - Valais : rochers granitiques de Salvan, Branson, Folateires. - Drôme : rochers granitiques de Tain, Saint-Vallier. - Ardèche : le Pouzin. - Gard : le Vigan, Concoules.-Bouches-du-Rhone : bords 
do ha Durance.-Alpes-Slaritimes: Berre, Tende, Saint-Sauveur, Saint-Étienne et Saint-Dalmas-le-Sauvage. - Manque sur toutes les chaines calcaires.

s. inaperıa, L. - Champs de la région méridionalc. - Uróme: Nyons, lc Buis, Saint-Paul-trois-Chateaux, - Gard : bords du Garion à Montfrin, le Vigan, l'Espérou, Alais, - Var : Hyères Cannet-du-Luc, Fréjus. - Hérault : Montpeyroux.

s. sed oides, Jaciuin. - Rochers maritimes. - Bouches-du-Rhơne : Montredon, île Ratoneau près Marscille, la Ciotat.

*. waxiframa. 1. - Rochers calcaires des collines et des montagnes. - Ain : fort de Pierre-Châtel et sur l'autre rive du Bhône à la Balme (Savoie): - Isère: Vertrieu. - Hautes-Alpes : mont Viso, col le la Traversette, Château-Queyras.-Drôme : Donzère, Nyons, Crest, Die, Luz-la-Croix-Haute. - Ardeche : Tournon, Viviers, - Vaucluse : fort de Buoux près d'Apt. - Var : mont Faron, Sainte-Baume. - Alpes-Marilimes. - Gard: Serre-deBouquet, Vialat, bords du Gardon. - Herault : pic Saint-Loup, Capouladou, Saint-Guilhem.

๑. quadrifiala, L. - Rochers des montagnes. - Jura : Reculet dans le vallon d'Ardran, creux de Pranciaux, crevasse d'Allemogne. - Haute-Sarole : Brizon près de la glacière, T'ournette, col des Aravis, Cornettes-de-Bise, mont Pétetod, mont d'Etale près de la Clusaz, Hautigny en face de Bellevaux.-Isère : Clıamechaude, la Moucherolle, Saint-Christophe-en-Oisans. - Hautcs-Alpes: Chaillol. - Alpes-Haritimes.

s. rupestris, L. - Rochers siliceux des montagnes. - Granite, porphyre et schistes carbonifëres de la zone vosgienne de HauteSaône: Hélisey, Plancher-Bas, Ecromagny, valıée de Mansvillers, Fresse, Saint-Bresson, mont de Vannes, Chaures-Roches, Servance, Belfahy, Ballons de Servance et de Giromagny; sur grès vosgien aux Guidons. - Haute-Savoie : toute la chaine granitique du mont Blanc, mont Brévent, grẻs nummulitique du sommet des Voirons, du mont Méry, du Haut-de-Véron et de la Tournelte. - Savoie : toute la chaine granitique qui confine au Piémont. - Isère : toute la chalne granitique qui s'étend depuis le grand Charnier d'Allevard jusqu'au mont Pelvoux et au-dela 
dans les Hautes-Alpes et les Alpes-Maritimes. - Ardeche : massif volcanique du Gerbier-de-Jonc. Terrains siliceux de la chalne des Cévennes à Saint-Guiral, l'Aigual près de l'Espérou. - Celte espèce manque dans les terrains calcaires.

s. acaulis, L. - Rochers et pelouses des hautes montagnes. Haute-Savoie: Brizon, Vergy, Méry, Charvin, col des Aravis, - col de Balme, Buet, mont. Blanc jusqu'aux Grands-Mulets $\left(3,060^{\mathrm{m}}\right)$. - Non moins ripandu dans toutes les hautes montagnes de la Savoie, du Dauphiné et dés Alpes-Maritimes. - Nul dans les Vosges et la clıaine jurassique. - Les deux formes suivantes sont des demembrements de celle-ci.

S. bryoides, Jord. - Haule-Savoie : Brizon, Miry, Tournelte, Dentsd'Oche, Drizon. - Savoie : mont Trélod, mont Grenier, mont Cenis. - Isère : la Moucherolle, Grand-Som, la Pra-de-Belledonne. - Hautes-Alpes : montagnes des environs de Gap, mont Viso.

S. exscapa, All. - Rochers des Montagnes. - Haule-Savoie : chaine du mont Blanc, Tré-la-Tête, col du Bonhomme, Brizon, Brévent, col de Balme. - Savoie : mont Mirantin, mont Iseran, mont Cenis à Ronche. - Hautes-Alpes : Lautaret, Galibier, mont Viso à la Traversette. - Alpes-Maritimes.

s. eretiea, L. - Champs cultivés de la Provence el du Languedoc. - Var: Frẻjus, Grasse. - Gard : entre le Vigan et le pont de l'Hẻrault.

s. museipula, L. - Moissons de la région méridıonale. - Drôme : Montélimar. - Vaucluse : Morières près d'Avignon. - Bouchesdu-Rhône: Marseille, Vauvenargues, Miramas, les Milles. Var: Toulon, le Lue, Draguignan. - Alpes-Maritimes: Antibes, Cannes._Gard at Hèrault : environs du Vigan et de Montpellier.

s. noctifora, L. - Champs cultivés. - Haute-Saône: Hẻricourt, Champey, Chassey-les-Iontbozon. - Doubs et Jura : Montbéliard, al!uvions du Doubs et de la Loue, Salins, Arbois, Bletterans, Chassey-les-Montboson, - Cote-d'Or : Meursault, bois d'Arcelot, Saint-Aubin. - Hautes-Alpes : le Champsaur et les environs de Rosans. - Var : Mourières près Toulon. - Gard : bois de Salbous près Alzon. - Valais à Martigny. 
๑. pratensis, Godron, Lycluivi dinica, D. C. - Lieux incultes dans tout le bassin.

s. dlurna, Goutron, Lychnis sylvestiix, Hoppe. - Bois dans tout le bassin.

\$. nutans, L. - Pelouses et rocher's dans tout le bassin.

S. spathulafolia, Jord. - Rocaillıs. - Isère : Saint-Christoplıe-enOisans. - Haules-Alpes : Briançon.

s. Itallea, Pers. - Coteaux pierreux. - Rare dans la partie septentrionale et moyenue du bassin. - Saonc-et-Loire: Brancion, Culles, Tournus. - Rhône: Oullius, les Etroits, Ecully, SaintAlban. - Ain : la Pape, environs de Belley. - Loire: vallée de Malleval. - Isère : Décines, Vienne. - Hautes-Alpes : environs de Gap. - Plus répandu dans la partie méridionale du bassin. - Drôme: Tain, Saint-Vallier, Crest, Nyons. - Vaucluse. Bouches-du-Rhône. - Var. - Alpes-Maritimes. - Gard. Hérault.

A. paradoxa, L., - Rochers. - Drome: pont de Serre, Crest. Hautes-Alpes : environs de Gap à Charance, la Roche-desArnauds.

s. otltes, Smith. - Coteaur secs et sablonneux. - Rare dans Saóneet-Loire et Cote-d’Or. - Nul dans Jura et Doubs. - HauteSavoie : Sciez près de Thonon. - Environs de Genève à la colline de Coudray, Sézegnien prés de Cartigny, colline des Crêts près de Chancy. - Valais : Martigny, Branson, Fouly. - Ain : la Pape-_Saroie: Salins près Moutiers, Pas-llu-Roc près SaintMichel. - Isère : Décines, - Rho̊ne : Villeurbanne, SaintAlban, Vernaison. - Bouches-du-Rhone : Notre-Dame-desAnges, Aix, Miramas, vallon de Toulouse. - Var : environs de Toulon. - Gard : bords du Gardon.

S. pseudo-oliles, Besser. - Coteaux secs et vieilles murailles. - Isère : Grenoble à Saint-Laurent, lat Tronehe, Comboire, Sassenage.

5. coli-rosa, Godron. - Les Sableltes près Toulon, Hyères (Var). visc a Rı purpurea, Wimmer. - Bois et rochers siliceux. Saone et-Loire: Cluny, Cotte, Buffères. - Rhône: Charbonnières. - Loire : chaine du Forez. - Valais : Gueuroz, val de Salvan. - Drôme : Tain. - Hautes-Alpes : Ubac-en-Valgaudemar, 
mont Viso, Penniı près Aiguilles, Saint-Víran. - Bouches-duRhône: Jouques, - Gard : Camprieux, Dourbie. - Alpes-Maritimes : entre Saint-Etienne-le-Sanvage et l'Enchastraye, val de Pesio.

v. alpolata, Fries, - Rochers siliceux des hautes montagnes. Savoie: col du mont Iseran, col de la Seigne, montagne entre le col des Fours et col de l'Enclave, mont Cenis, à Frassières-Dessus. - Isère : rochers granitiques des Grandes-Rousses audessus de Brandes, Taillefer, Pímeyan au Hont-de-Lans.

Lyehnis dos cuenit, L. - Prairies dans tout la bassin.

L. Hos Jovis, Lam. - Dibris de rochers h's hautes montagnes granitiques. - Haute-Savoie : mont Btiel. - Valais : Mayensde-Fouly. - Savoie: petit mont Cenis à lit Combe-d'Ambin et dans la vallẻe de Villette, Pralognan, près du col de la Louse en face de Couard. - Isére : chaîne granitique des Sept-Laux et de Belledonne, la grande Vaudaine, le Valjoufray près de la Salette. - Hautes-Alpes : Lautaret, mont Viso, Saint-Véran, la Grangeane. - Alpes-Haritimes.

1. coronaria. Lam. - bébris de rocher's. - Haute-Savoie : cháteau de la Motte à Aysc au pied du Mole. - Savoie : chapelle de la Madière près de Conflans, - Valais : au-dessus de Branson et de Fouly.

AGROSTerre githage, L. - Hoissons dans tout le bassin.

SAPOVA IB A Affeimalis, L. - Haics, bords des champs. Dans tout le bassin.

s. oeynoides, L. - Cuteanx pierreux. - Doubs: vallées die Doubs et du Dessoubre. - Jura : collines de Lons-le-Saunier, Arbois, Saint-Claude, toute la vállée de l'Ain juisqu’à Thoirelte.-Ain : toutes les basses montagnes du Bugey. - Haute-Savoie : pied du Salève, vallée du Fier, Pringy, les Allinges, Thonon. - Savoie : montagnes du bassin de Chambéry, vallées de l'Isère et de l'Arc. - Isère: environs de Vienne, de Crémieu et de Grenoble. Rhône : Condrieu. - Loire : Saint-Pierre-de-Boeuf, Malleval, Thélis-la-Combe.-Hautes-Alpes : Charance près Gap.-Drôme : Saint-Vallier, Tain, Crest.-Var: Toulon, Barjols, Aups, Vence. - Alpes-lifaritimes : Grasse, Nice, Menton. - Collines des Bou- 
ches-du-Rhône. - Gard : bords du Gardon, Sallous, Marguerile. - Hérault : Saint-Loup. - Excepté Vienne, Condrieu, les localités de la Loire, Tain et Saint-Vallier, dont le terrain est granitique, toutes les autres stations de cette espèce sont sur le calcaire jurassique ou crélacé. Il m'a semblé que la présence du S. ocymoides sur te granite pouvait s'expliquer par l'existence d'un dépôt diluvien superposé à lá roche granitique.

s. Iutea, L. - Savoie : mont Cenis à Pattes-Creuses, petit mont Cenis dans lc vallon de Villette, la Chapelle-Blanelie.

S. vacearia, L. - Champs cultivés dans tout le bassin.

ATresormiLa repens, L. - Sables, rocailles. - Rhỏne: Lyon à la Nouche, iles du Rhòne à Vaulx. - Ain : Seyssel, - Massigneu-de-Rives, Virignin, Cordon. - Jura : Colombier, Reculet au vallon d'Ardran, la Dôle. - Isère : la GrandeChartreuse, Chalais, Revel, mont Aiguille. - Haute-Savoie : Brizon, Vergy, Méry, mont Charvin, Platets, Tournette. Entraínée par l'Arve au-dessous de Genève, par le Rhône jusqu'à Lyon, par le Drac jusqu'à Grenoble, par le Fier aux environs d'Annecy. - Hautes-Alpes : col Isoire, col de Glaise, mont Aurcuse. - Alpes-Mlaritimes. - Ardèche : le Pouzin, vallée d'0uvèze.

G. alpestris, Jord. - Graviers à la Grave (Hautes-Alpes).

G. muralis, L. - Champs sablonneux. - Haute-Saône : région vosgienne dans la vallée de Mansvillers, Champagney, grès bigarré et diluvium à Franchevelle, Linexert, Lure, Saint-Germain, oxfordien siliceux et grès du lias à Grattery, Lambrey, Chariez, Bougnon. - Lisière vosgienne du Doubs et plaines du Douls, Jura, Ain jusque dans la Bresse et dans la Dombes. Terrains siliceux de Cote-d'or et Saone-et-Loire. - Environs de Genève à Aïre, Penex. - Rhône: Charbonnières, Tassin et loutc la région granitique du Lyonnais et du Pilat. - Sables de la molasse du Dauphiné et de la Savoie. - Nul dans la région méridionale.

TCNYCA saxifraga, Scop. - Graviers, rocailles. - Indiqué par Lorey dans Cóte-d'Or à la Roche-en-Brenil, où on l'a vainement cherché. - Nul dans Haute-Saône, Doubs, Jura, Saone-el-Loire. 
- Très-commun dans le bassin du Léman et dans la vallée du Rhione depuis Genère jusquä Mrles, puis dans le Var ct les Alpes-Maritimes, et, d'un autre côté, dans le Gard et l'Hérault.

D. prollfer, L. - Bords des chemins. terres incultes dans tout le bassin.

D. armeris, L. - Bois et pelouses des terraius siliceux el argilosiliceux. - Haule-Saône : Grattery, Chariez, Arbecy, mont de Vannes, Champagney. - Doubs: mollasse et lisière vosgienne de l'arrondissement de IIontbéliard. - Jura : terrains marneux et argileux de la plaine et des collines. - Côte-d'Or. - SaOne-etLoire. - Haute-Savoie : pied du Salève, Pringy, bassin du Léman. - Régions granitiques du Lyonnais. - Isère : Revel, Vaulnaveys, Villard-de-Lans, — Drôme: Crest. — Var : forêt des Maures, Notre-Dame-des-Anges, Fréjus. - Alpes-Maritimes : Grasse, Nice, Menton. — Gard : Barjac, Alzon, bois de Broussan, bords du Gardon. - Hérault : environs de Montpellier.

D. carthusianonoun, L. -- Pelouses sèches dans toute la partie septentrionale et moyenne du bassin; plus rare dans la région méridionale où il cst remplacé par le suivant.

D. liburnicus, Bartling et Wendl. - Collines pierreuses, - Bouchesdu-Rhone : entre Aubagne et Cujes, vallon de la Gérade. Var : Toulon, le Luc, Saint-Quenis près Camp, Fréjus: - AlpesMaritimes: Grasse, Antibes, Nice, Menton.

D. vaginatus, Chaix. - Prairies - Isère : Saint-Nizier, Corançon. Hautes-Alpes : Laularet, mont Séuse, Furmeyer.

D. atro-rubens, All. - Pâturages des Alpes-Haritimes.

s. Seguneri, Chaix. - Pelouses des montagnes. - Hautes-Alpes : environs de Gap, mont Bayard, mont Chaillol, la Bàtie-Neuve, mont Genève, Embrun.

D. sylvaticus, Hoppe. - Pelouses et bruyères des montagnes. Loire: Saint-Sauveur au-dessus des bois de Taillard, chaine de Pierre-sur-Haute, Pilat.

D. Mnotus, Vill. - Rochers calcaires dans ta région inérudionale. Bouches-du-Rhône: la Bourdonnière, Sainte-Victoire, SainteBaume. - Var: Montrieux, route de Draguignan à Castellane. - Hautes-Alpes : Serres. - Basses-Alpes : Digne, Gréoux, Col- 
mars, Sisteron. - Vaucluse : Avignon. - Alpes-Maritimes : montagnes de Caussols et des Claps au-dessus de Grasse.

1). graniticus, Jord. - l'orme silicicole dímembríe du D. lirtus, lequel est, au contraire, calcicole. - Loire : rochers granitiques des environs de Saint-ktienne et de Firminy. - Ardeche et Cévennes. - Gard : Saint-Paul-de-la-Coste, rochers granitiques de Valleraugue, l'Lspérou, Saint-Jean-lle-Gard.

8. wulowera Iis, Vill, - Rochers des montagnes, - Drome: llousset près Nyons, le Buis, montagne d'Angèle. - Hautes-Alpes: mont Aurouse. - Vaucluse : mont Ventoux.

B). meglectas, Lois. - Pelouses des hautes montagnes. - Savoie: mont Cenis, - Hautes-Alpes : la Grave, Lantaret, Galibier, nont Genèvre, mont Viso au col de Malrif, vallon des Vaches, val Agnel. - Basses-Alpes: Digne, col de l'Arche, vallée de BarceIonnette, - Vaucluse: mont Ventoux.

w. deltablew, L. - Prairies des montagnes, - Colc-l'Or: le Intran granitique à Saulieu. - Saône-ct-Loire: Millery pris Autun. Haute-Saône: zône vosgienne à Plancher-les-arines, le Thillot. - Nul sur les chaines calcarres du Doubs, Jura, Bugey, Savoie. - Région granitique du Lyonnais à Charbonnières, Tassin, Limonest; de la Loireà Pilal, Pierre-sur-Haute, mont Sémiuure; du Dauphiné à Prémol, Brandes et Huez-en-Oisans, Lautaret, mont Viso. - Calcaire crétacé à rognons siliceux du mont Aurouse; sur quelle couche se trouve-t-il au plateau jurassique du mont Bayard près Gap? - Ardèche: chaîne granitique et basaltique du Coyron. - Cévennes : massif granitique de l'Espérou à Concoule. - Alpes-Maritimes.

D. eresius, Smith. - Rochers et pelouses iles montagnes. - Doubs : coleaux jurassiques de l'arrondissement de Montbéliard, cótes du Doubs et du Dessoubre, Lomont, citadelle de Besançon, sur le versant helvétique au Chasseron, Suchet. - Jura : Baume, la Châtelaine, Salins, le Reculet. - Haute-Savoie : montagnes du Chablais, mont Laouet près Reyvroz, mont Tournette près du lac d'Annecy. - Isère : Grande-Chartreuse, Sassenage, gorges d'Engins, col de l'Arc, Moucherolle.

D. Gyvestris. Wulf. - Coteaux a ides. - Espèce polymorphe très- 
répandue dans le Jura, Bugey, la Savoie, lrs environs de Lyon et de Crémieu, les côtes du Ihlıône. - Côtc-d'Or. - Saône-elLoire : Solutré, Vergisson, Tournus, Culles. — Cóte du Bhône. - Ardèche: Chàteaubourg. - Var. - Alpes-Maritimes. - Les huit formes suivantes sont démembrées de cette espéce.

D. orophilus, Jord. - Pelouses des montagnes. - Isère : de Villard-deLans au col de l'Arc. - Hautes-Alpes : Lautaret, mont Séuse.

D. saxicola, Jord. - Rochers des basses montagnes. - Jura : région du vignoble. - Ain : montagnes inférieures du Bugey. - HauteSavoie : pied du Salève, vallon des Usses, Maggériaz, roc de Chère, Colonies près Annecy, base du Mole entre Marignier et Contamines, mont Darbon près Vacheresse, les Platets. - Isère : la Bastille de Grenoble, Comboire, gorges d'Engins, la Molteles-Bains. - Hautes-Alpes : environs de Gap.

D. juratensis, Jord. - Sommités du Colombier. du mont-d'Or, de la Dôle et du Reculet dans le Jura.

D. collivagus, Jord. - Coteaux des environs de Lyon à Néron.

D. aggericolus, Jord. - Le Reculet.

D. Godronianus, Jord. - Pelouses rocailleuses. - Environs de Gap à Charance, la Garde, Rabou. - Partie méridionale de la Dróme à Granne, Loriol, Montélimar, Donzère, Nyons. - Ardèche: le Pouzin, mont Combier, Rochemaure, Viviers.

D. Guyelani, Jord. - Coleaux secs. - Isère : Feysin, Crẻmieu.

D. consimilis, Jord. - Alpes de l'Oisans. - Pont de Sarrazin près Gap.

D. virgineus, L. - Coteaux de la région méridionale. - Provence, Nlpes-1laritimes et Languedoc.

D. monspessulanus, L. - Pâturages et rocher's des montagnes. - Jura et Ain : pentes du Colombier, du Reculet et de toute la chaine depuis la Faucille jusqu'au Credoz: - Savoie : mont du Chat. - Isère : mont Rachais, Saint-Nizier, Cliamechaude, col de la Ruchère, la Chapelle-en-Valjoufrey, Grande-Chartreuse. - Hautes-Alpes : mont Séuse, col de Glaise. - Loire : Pierresur-Haule. - Bouches-du-Hhône : Saintr.Victoire. - Alpes-Mlaritimes.-Gard : Aigual, Espérou, Concoule, Salbous — Hérault : assez rare dans les environs de Hontpellier ; beaucoup plus commun dans les environs de Saint-Pons. 
D. superbus, L. - Prés marécageux. - Haute-Saône: bois de la Dréole, Mollans, - Tourbières de la région des sapins du Doubs et du Jura : Bélicux, Noël-Cerneux, Chenalotte, Pontarlier, le Boulu, Prẻmanon, Saint-Laurent-en-Grand̀vaux, Nans près Salins, vallée de Joux. - Cóte-d'Or: bois d'Essarois. - Ain: bois du Chenavaret, Saint-Germain-de-Joux, Evoges, Divonne, Abergement-de-Varey. - Environs de Genève dans les bois de la Bàtie, des Frères, ciu Vangeron. - Haute-Savoie : pied des Voirons el du Saleve, Allonzier, Pringy, Thonon, - Isère : Meyzieu, Janeyriat, Charvieu. - Dròme : Saint-Barthélemy-de-Vals. — Gard : environs de l'Espérou.

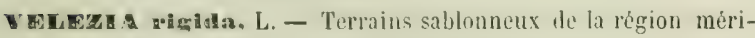
dionale. -Drôme: Tain, les Baronies. - Vaucluse : Avignon. - Bouches-lu-Rhone : Marseille, Aix, Miramas, - Var: Fréjus, la Lauzade et la Rouisse près le Luc, forêt des Naures, Evenos. Alpes-Haritimes : environs de Grasse, Baus-Rous _ Gard : bords du Gardon, mas Charlot, bois de Broussan, Loubes, Générac. Hérault : environs de Iontpellier et d'Agde.

\section{ALSINÉES.}

GATNA procumbsas. L. - Lieux sablonneux lumides dans tout le bassin, depuis les plaines jusqu'aux sommités des montagnes. - Nul sur les calcaires.

S. muscosa, Jord. - Pâturages tourbeux. - Loire : Pilat, pré Lager. - Montagnes de l'Ardèche.

s. apetals, L. - Meme habitat que le procumbens, mais moins cotumun.

\$. ciliatn, Fries, patula, Jord. - Mêlé aux précédents. Des expériences de culture, dans les conditions les plus variées, seraient nécessaires pour juger définitivement de la valeur spécifique des trois précédentes formes.

5. senaritima, Don. - Littoral de la Méditerranéc.

s. subulata, Wimm, - Lieux siblonneux. - Isère : Saint-Baudille près Crẻmicu, Bouvesse. - Alpes-Maritimes: Grasse, Cannes. 
\$. Linnaei. Prosl. - Pelouses des montagnes. - Summilis du Douhs, Jura, Ain: mont Suc, mont d'Or, les Ronsses, Primanon, le Noirmont, la Dôle, la Faucille, le Reculet, le Colombies'-de-Gex, Retord, le Colombier-du-Bugey. - Haute-Saroie : pilons du Salive, les Voirous, montagnes du Chablais, mont Laouct pres de Reyvroz, Nautau au-dessous de Montriond, Haut-de-Lin, Dentsd'Oche, mont Darbon près Vacheresse, la Flegire, Brévent, chinine du mont Blane, le jardin de la Her de glace, col de Balme. - Savoie : mont Cenis vers le lac Blanc. - Isère: chaine de Belledonne à la Combe de la Lance, Chamechaude, Saint-Nizicr. - Hautes-Alpes: la Graye, mont Aurousi, mont Chaillol, le Qinyras an bois Foran pròs Villevieille. - Drome: mont Glan1.tz. - Alpes-Maritimes.

s. Flabra, Willd. - Pelouses des montagnes, - IIaute-Savoie: rocliers siliceux du Brévent, des montagnes de Sixt et du Bonhomme, grés nummulitique du Haut-de-Véron el du col des Aravis. Savoie : mont Cenis. - Isère : châne g'anilique des Sept-Laux i) Pinté; de Belledonne à la Pra. - Hautes-Alpes : granite des sommités du Lautaret, Galibier, mont Viso, Villevieille en Queyras, collsoire, mont Chaillol, pic de Bure, - Alpes-Maritimes.

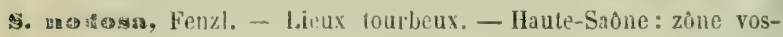
girnne à Eto on, Frahier, Clagey, Faucogney, - Doubs et Jura : lígion ders sapins, fourbières de Bélieu, Russey, Mèmont, Houthe, Pontarlier, Boujailles, Champagnole, vallée de Joux, li Trilasse. - Ain: Tourbières de Viry pris Oyonnax, Belley au lac de Bar. - Rhône: Vaulx-en-Velin et les iles voisines. Loire: Chalmazelle. - Isère : Heyzieu.

E2. Dijon, Nolai. - Sauncel-Loire : Vergisson, Solutrú, Dezize. Ain : lit Pape - Valais : Charraz, Ardon, la Horge, Sion, Sierre, Varone, - Rhone : le mont Cindre, le mont Toux, - Isère: Jords du Drae vers Seyssinet. - IIaules-Alpes : environs de Gap: le Queyras. - Drime : bords de l'Isere au Chapetier près Romare, Crest, le Glandiz. - Vaucluse: Avignon. - BouchesAln-Rlıônc. - Val': environs de Toulon et de Fréjus. - Gard: Campestre - Hératil : cnvirons de ifontpellier. 
B. cenuiforia, 1. - Cliamps de la Provence ef du Languetoc.

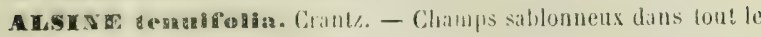
bassin. - I ess trois formes suivantes sont démembrées de cette espiece.

A. laxi, Joril. - Champs sablonieux des environs de Lyon, de Grenoble, de Saint-Vallier et de Geneve. - Hérault : Madière sur la Vis.

A. viscosn, Schreber, - Champs sablonneux. - Environs de tyon à

Villeurbane, Saint-A!ban, Décines. - Environs de Grenoble, de Saint-Vallier, du Crest, de Thonon et de Genève.

A. hybridn, Jord. - Graviers des rivières. - Environs de Lyon, de Grenoble et de Saint-Vallier. - Ain : plaines de Balan, d'Ambronay ef de Chàtea-Gaillardi. - Haute-Savoie : grèves de la Dranse, - Environs de Geneve.

A. Jacquint, Koch, - Terains sablonneux el graveleux. - Jura : environs de Saint-Clande, Moirans, Oyonnax, les Rousses, foret du Rizoux. - Còte-d’or : Dijon, Nolai. - Saône-et-Loire : Dezize, Alonlaigu, Buxy, - Ain : les Balmes de Balan, la Pape, Rullicu, Viricu-le-Grand, Artemare, Hottone. - Haute-Saroie : Pas-del'Echelle au Sulove, grèves de la Dranse près d'Armoy, iles du Ficr près d'Annecy, - Savole : mont Cenis, - Valais, Martigny, Charraz, Siou, Sierre. - Isère : environs de Crémieu 't de Grenoble au Polygone, Sassenage

A. mueronatgo, L. - Débris de rocher's des montagnes. - HauteSivoie : calvaire de Thônes, - Isère : Sassenage, Parisel, Bourgd'Uisans. - Drôme : Solaure près Die. - Hautes-Alpes : Laularel, Briancon, Cerviêres, Abriès, mont Viso.-Ardèclie : Cirussol, Sojons, ia Voulte, Rochemaure, Viviers - Vaucluse: Orange, mont Ventoux, Lançon près Apt. - Bouches-du-Rhône: les Alpines, - Var : Sainte-Baume. - Gard : Serre-de-Bouquet, Vigan, Campestre, Alzor, Montdardier, le long du Gardon.

A. petrua, Jord, - Débris de rochers des montagnes calcaires. Hautes-Alpes: Gap, Serres.

A. brevifo'ia, Jord. - Rocailles des montagnes. - Briançon.

A. setacea. Hert. et Koch.. - Coteaux secs. - Cote-dor : SiantRomaiı, Auxey, la Rochepot, roclies de Vauchignon à Nolay.- 
Sanne-et-Loire : Dezize, Saint-Cernin, Cluny, Vergisson. Bouches-du-Rhone: la Verdière.-Var : Sainte-Baume, Toulon.

A. verma, Bartl. - Pelouses des montagnes, - Doubs et Jura: le Noirmont, les Rousses, Prémanon, Saint-Laurent, la Dôle, le Colombier, le Reculet, Lélex, Thoiry; descend jusqu’à Durnon au-ilessus de Salins et entre Aubonne et le val de la Loue.

- Haute-Savoie : montagnes du Chablais, Cornettes-de-Bise, mont de Pelloua, mont Ardin, roc d'Enfer au-dessus de SaintJean-d Aulph, montagnes de Samoëns et de Sist, Haut-de-Véron, Brizon, mont Vergy, col du Bonhomme, mont Tournette. Savoie: mont Cenis, mont du Chat. - Isère : chaine des SeptLaux et de Belledonne, Champrousse, Chamechaude, col de l'Arc. - Hautes-Alpes: Lautaret, Galibier, col du Noyer, mont Aurouse, granges d'Isoire.

A. recurvar, Walılenb. - Pelouses. - Savoie: mont Cenis à l'EauBlanche. - Basses-Alpes et Alpes-Maritimes.

A. Villarsin. Mert. et Koch. - Rochers les montagnes, - HauteSavoie: la Vauzalle près Sixt, Haut-de-Véron, mont Tournette. - Savoie : mont Cenis, petit mont Cenis dans le vallon de Villette.-Isère : mont Aiguille - Hautes-Alpes : mont Séuse, mont Aurouse, mont Viso au chalet des Ruines, la Traversette, Saint-Véran, Blanchclte. - Basses-Alpes : Barcelonnette. Vaucluse : mont Ventoux. - Alpes-Haritimes.

A. striata, Gren. - Pelouses et rocailles des inontagnes granitiuues. - Savoie : entre les Brévières et Tignes, entre le Chapiu et le Moltet, chalet du Saut dans la vallée des Allues, - Isère : entre Gavet et Livet, vallée de la Bérarde, la Salette. - HautesAlpes: la Grave sous les glaciers, mont Chaillol, Vallon près Abriès-en-Queyras. - Alpes-Haritimes, — Gard : massif granilique de l'Espérou.

A. Baulnitaoram, Gay.-Débris de rocher's des hautes montagnes. - Jura : la Dôle, le Colombier, le Reculet. - Ain: Hollone, le Retord, Ambronay. - Haute-Savoie : Dents-d'0che, montagnes de Samoëns, mont Brévent. - Savoie: mont Cenis, petit mont Cenis à la Combe-d'Ambin. - Isère : Néron, la Moucherolle, mont Aiguille. - Hautes-Alpes : Abriès. Brame-Buou près 
Serres. - Basses-Alpes : Castellane, Sisteron. - Drome : environ de Luc-en-Diois. - Vaucluse : mont Ventoux, - Ardèche : Etheise. - Gard : Blandas, Saint-Michel-dei-Sers, Alzon, Montdardier. - Hérault : le Caylard.

A. Cherleri, Fenzl, - Pelouses des hautes montagnes, - llauteSavoie : chaine du mont Blane, jardin de la .ler de glace, Brévent, Buet, col du Bonhomme, Dents-d'Oche, Brizon, Méry, Vergy, Ilaut-de-Véron. - Savoie: mont Cenis à Cornerousse, montagnes des Allues. - Isère : Belledonne, Champrousse, 'Taillefer. - Hautes-Alpes : Galibier, col d'Isoire, mont Viso, Chaillol-le-Vieil. - Alpes-llaritimes.

A. wreicta, Wahlenb. - Tourbières de la chaine jurassique: la Chenalotte, les Ponts, la Brévine, Pontarlier, Sainte-Croix, val de Joux.

A. Manceolata, Mert. et Kioch.- Rochers des unontagnes.-HautesAlpes : mont Viso au col Agnel, pâturages du Selar, SaintVéran à Clausis, col des Tourettes entre Châteauroux et Orcières, mont Chabrière, mont Morgon, le Sapey de la Bâtie-Neuve.

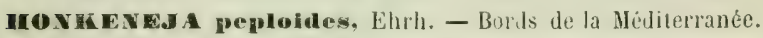

MOEHIE YGIA museosa, L. - Rocher's humides des montagnes. - Haute-Saône, Doulıs, Jura, Revermon I, Bugey, Savoie, Dauphiné, Pilat, Cévennes, Aipes-Maritimes.

I. dasyphilla, Bruno. - Rocliers des monlagnes. - Alpes-Maritimes.

M. Bolygomoides, Mert. et Koili. - Débris de rochers des montagnes. - Haute-Saroie : col de Golèse, Corneites-de-Bise, roc d'Enfer, montagnes de Samoëns, mont Tournette, Vergy, Brẻvent. - Isère : Ia lloucherolle. - Hautes-Alpes : mont Viso au-dessus du Grand-Chalet. - Vaucluse : mont Ventoux. Gard: Banahu près de l'Espérou.

Br. trimervia, Clairv. - Lieux ombragés des basses montagnes dans tout le bassin.

A. pentandrs, Gay. - Lieux pierreux de la rígion méridionale. - Var : Fréjus. - Alpes-Maritimes : l'Estérel et Maures de Tanneron. - Gard : l'Espérou, la Tessonne.

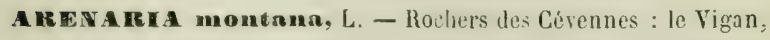
Aulas, Espérou, la Grandès. - Hérault : environs de St-Pons. 
A. Jidora, L. - Dibris de rochers des montignes granitipues. Haute-Savoie : clıaine du mont Blane, jardin de la Mer de glace, Brévent, col du Bonhomme. - Isère: Ies Sept-Laux, claîne de Belledonne, bords des lacs Doménon, Grandes-Rousses. Hautes-Alpes: Galibier, col de Valestresse, la Grave, mont Chaillol, mont Viso, vallon des Ruines près l'Asty, Saint-Véran sous le rocher des Etoiles, col Isoire.

A. ciliata, L. - Rocailles des montagnes calcaires, - La cliaine jurassique : Chasseral, Dole, Colombier, Reculet, bords du lac de Joux. - Ain : Colombier-du-Bugey. - Haute-Savoie: les montagnes du Chablais, Dents-d'Oche, Cornettes de Bise, roc d'Enfer au-dessus Saint-Jean-d'Aulph, Nautau au-dessus de Montriond, col de Golèse, Vergy, Héry, Tournette. - Savoie : mont Cenis, mont du Chat. - Isère : Saint-Nizier, col de l'Arc, SaintEynard, Grand-Som, Obiou. - Haules-Alpes : mont Aurouse, Briançon, le Queyras, mont Viso à la Traversette, Saint-Véran au col de Blanchetle. - Drôme: Glandaz. - Alpes-Maritimes. - Gard : l'Espérou.

A. serpylifolia, L - Terrains sablonneux et graveleux depuis les plaines jusqu'aux sommités alpines dans tout le bassin. Espèce polymorphe.

Variété Marschlinsii, Koch. - Chaine du mont Blanc, jarlin de la mer de glace.

Variété leptoclados, Guss. - Bords des chemins. - Environs de Genève, Thonon, Annecy, Grenoble, Sassenage, Revel, Lyon.

- Saône-et-Loire. - Ardèche : le Pouzin, mont Combier.

Variẻtẻ viscida, Loisel. - Hêmes stations que la précédente.

A. cinerea, D. C. - Basses-Alpes : Castellane. - Alpes-Maritines: Sigale, Mas près Saint-Auban.

A. hispiata, L. - Débris de rochers dans la région méridionale. Gard : Vigan, Montdardier, Campestre, Alais. - Hérault : environs de Montpellier, Cafouladou, Saint-Guilhem.

A. moalesta, Duf. - Bouches-du-Rlıône : in nntagnes des environs de Marseille, Notre-Dame-des-Anges, Sainte-Victoire. - Gard : sables dolomitiques de Campestre.

A. grandifora, All, - Débris de rochers des montagnes. - Chane 
jurassique : Chasseron, Chasseral, Suchet. - IIaute-Savoie: montagues du Chablais, Dents-d'Oche, mont Laouct près de Reyrroz, chaine du mont Blanc, moraines de la Her de glace, petit Saleve, - Savoie : mont Cenis, - Ain : fort de l'Ecluse, Isère : la SaJchle, - Hautes-Alpes : Briançon sous te fort des Tétes, mont Durbonnas, le Queyras. - Vaucluse : mont Ventoux. - Alpes-Maritimes. — Gard: Saint-Guiral près d'Alzon. Varicté stolonifera, Vill. - Détris de rocliers. - Isère: SaintNizis', Seyssins, mont Aiguille, - Didme : Glandaz.

A. tetraquetra, L. - Lieux arides des montagnes de la région mirilionale. - Vaucluse : mont Léberon et mont Ventoux. Bouches-1lu-Rhône, - Var : Sainte-Baume, Barjols. - AlpesMaritimes: Tende, l'Aiguille au-dessus de Nenton, mont Chaure et mont Ferrion au-dessus de Nire, mont Lachen au-dessus de Séranon. - Gard: sables dolomitiques de Campestre, le Vigan, Anduze. - Ilćrault : pic Saint-Loup, Capouladou, la Sérane près Saint-Guilhem, Saint-Jean-de-Fos, Nontpeyroux.

A. masmiliensiø, Fenzl. - Collines rocailleuses des environs de Marseille ct de Toulon.

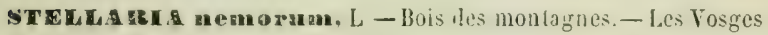
et tolite la chaine jurassique du Doulss, Jura, Bugiy, Savoie, Dauphiné. - Morvan dan; Côte-1’0r et Saône-cl-Loire. - Beaujolais, Pilat, Forez et Cérennes. - Alpes-Haritimes.

B. media, Vill. - Bords des champs cultivés et des chemins, environs des habitations et partout ou le sol est riche en matières azoties. - Les deux formes suivantes sont démembrées de celle-ci.

S. neglecta, Weihe. - Lieux cultivés autour de Lyon et de Saint-Vallier (Orome).

S. Borceana, Jord. - Le long des murailles dans les environs de Genève, de Lyon et de Grenoble. - Hérault : autour de Béziers

8. holostea, L. - Haies et bois. - "Très-commun sur les coteaux de la partie septentrionale et moyenne du bassin; plus rare dans la région méridionale oủ on ne l'observe que dans les montagnes des Alpes-Maritimes et des Cévennes. - Chose singulière! cette espèce est très-rare en Suisse. 
5. slauea, With. - Lieux humides dans les terrains siliceux ou argilo-siliceux. - Doubs : Lourbières de Pontarlier, Lomont-deRoche-d'Or, Montferney près Rougemont. - Haute-Saône : Scey-sur-Saône, alluvions du Val à Lure. - Jura : Dole, Pleurre, Neublans, Chaussin. - Côte-d'Or : étangs du Morvan granilique à Saulieu et Roche-en-Brenil, Citeaux, - Saône-et-Loire: Verdun, Ecuelles. - Ain : dans la Dombes à Marlieu, Beaumont, Saint-Didier-sur-Chalaronne. - Isère : Saint-Chef, Crẻmieu. Cette espèce manque dans la région méridionale.

8. graminea, L. - Haies et bois dans tout le bassin; plus rare dans la région méridionale.

s. uliginosa, Murr. - Lieux humides des terrains siliceux et argilo-siliceux. - Haute-Saone, zỏne vosgienne à Ruz-Jeannot, vallée de Mansvillers, Chauves-Roches, vallée de Hiélin à la Ravalle, Goulte-Radere, Champagney, Ballon-de-Giromagny; diluvium el grès bigarré à Grands-Bois près Melisey, Franchevelle; alluvions quartzeuses à Port-d'Atelier, Villersexel. Doubs : tourbières des arrondissements de Montbéliard et de Pontarlier. - Jura : la Bresse, granite de la forêt de la Serre, descente de la Dôle à Gex. - Granite du Morvan, du Beaujolais, du Lyonnais, du Pilat et des Cévennes. - Haute-Savoie : grès nummulitiques des Voirons. - Isère: Saint-Geoirs, bords du lac Luitel près de Prémol, Revel, col du Merdaret, les Fauges du Villard-de-Lans. - Bouches-du-Rhône : Sainte-Baume. Alpes-Maritimes : Saint-Martin-de-Lantosque.

HoLosteU umbellatum, L. - Champs sablonneux et graveleux dans tout le bassin.

CERAstudn trigynum, Vill. - Rochers humides. - HauteSavoie: montagnes du Chablais, Cornettes-de-Bise, roc d'Enfer, Buet, Brévent, col de Balme, chaine du mont Blanc, le jardin de la Mer de glace, Couvercle, Tré-la-Tête, Brizon, Vergy, Haut-de-Véron, mınt Charvin. - Savoie : mont Cenis à Cornerousse. - Isère : la Pra-de-Belledonne, au-dessus de l'Oursière, les Sept-Laux. - Hautes-Alpes : Lautaret, col de Valestresse, Chaillul-le-Vieil, mont Viso près la Traversette, Taillante près 
lo passage des Ruines. - Cévennes : entre Brama-Bioou et ileyrueis.

c. shiaseanas, Gr., variétí qunternellum, Fenzl. Sugina erecta, L. Chanips sablonneux des terrains siliceux et argilo-siliceux. Jura : Foucherans, Chaussin, liahon, Gatey, environs de Salins clil'Arbois, Cramans. - Cû́ced'or: Saulieu, Cîteaux. - Saònect-loire : le Morvan. - Ain : la Dombes, Thoissey, Treroux. Region granilique du Lyonnais a Chaponost, Hornant, Craponne, Charbonnières. - Loire: rallece du Gier. - Isire : sables de la Molasse entre la Tout-du-Pin et to Pont-de-Beauroisin, Saint-Michel-de-Geoirs, environs de Crémicu.-Drôme: SaintVallier, Laveyron. - Hautes-Alpes : Hontmót près de Gap. Var: IIyères, forit des Maures, - Gard: Alzon, le Vigau, bois de Campagne près de Nimes.

Varićté ortandrum, Godr. - Var : Frẻjus, Ilyères. - AlpesMaritimes: l'Estérel, Cannes, Biot.

6. Wiscoskgka, L. - Champs sablonneux ot graveleux dans lout le bassin.

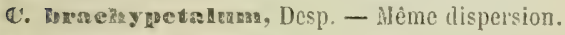

C: serso sin; plus rare que les deux précédents.

C. Egltutiamsama, Fries. - Mème dispersion.

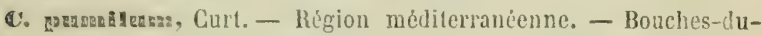
Rlıône: Marseille, Miramas, - Var. - Alpes-Naritimes : Biot, Nice, Menton.

C. aggregatasa, Dur - Cliamps, - Toulon, Hyères.

c. E⿱一𫝀口: Desmoulins. - Champs cultivés du Gard à Trèves et sur l'Espérou.

C. valyantaran, L. - Bords des champs dans tout le bassin.

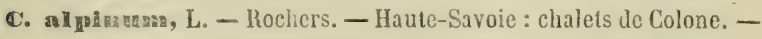
Isère : chaine de Bellchonne près des lacs Doménon, Champrousse, Haute-Tète-de-Racha dans l'Oisans, Taillefer, la Salette. - Hautes-Alpes : Galibier, col des Hayes près Arvieux, mont Pullevas près du village de la Monta au mont Viso. - AlpesMaritimes.

Gatal. Bassin du Rhône. 
Variétẻ Innatum, Lam., squalidum, Ram. - Rochers des montagnes granitiques. - Hautes-Alpes : pic du Bec au-dessus de Villard-d'Arène, Champoléon à la montagne de l'ours.

C. anvense, L. - Espèce polymorpie qui existe dans les lieux incultes de tout le bassin.

Variété strictum, L. - Commune sur les sommités de la claine jurassique du Doubs, Jura, haut Bugey, Savoie, Dauphiné.

Variété laricifolium, Villars. - Hontagnes de la partie méridionale de la Drôme et de la Provence.

C. Getgeradiagaa, L. - Débris de rochers. - Haute-Saroie: monlagnes de Samoëns et de Sixt, chaine du mont Blanc, le Jardin, Leschaux, moraines de la Mer de glace, col du Bonliomme, Tournette. - Savoie : mont Cenis vers le lac Clair et le lac Noir.

- Isère : bords des lacs Doménon, du lac Blanc dans les Grandes-Rousses, Haule-Tête-du-Racha au-dessus du mont te Lans, Taillefer, col de la Mluzelle, mont Obiou.-Hautes-Alpes: munt Aurouse, Chaillol-Je-Vieil, col Isoire, mont Viso sous les rochers de la Traversette, vallon des Brun. - Alpes-Haritimes. - Cévennes: entre Brama-Bioou et Mleyrueis.

C. andarieum, L. - Lieux humides dans tout le bassin.

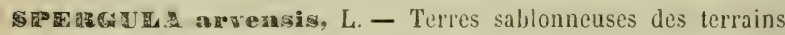
siliceux et argilo-siliceux dans tout le bassin.

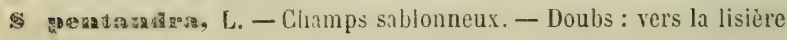
vosgienne (nul dans le reste du Doubs et Jura). - Terrains granitigues de Saòne-et-Loire; de Côte-d'Or vers Saulieu et Rouvray; du Lyonnais à Beaunant, Brignais. - Ain : Garnerans, Thoissey, Saint-Didier. - Isère: Décines, Clarvieu, Leyrieu.Drôme : granite de Szint-Vallier. - Var : Pignans, Fréjus, le Luc. - Alpes-IIaritimes: Berre. - Gard.

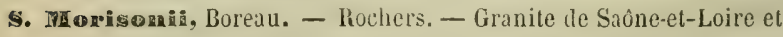
Côte-d’Or dans le Morvan. - Kégion granitique du Lyonnais à Vaugneray, Saint-Bonnet-le-Froid, Chaponost, bords du Garon; de la Loire à Pilat, la Valla, Ruthiange, Saint-Julien-MolinMolette, Chalmazelle, le Puy-Morand; des Cévennes dans la chaine de l'Espérou,

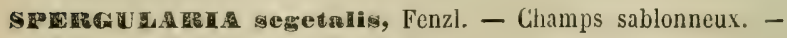




\section{- 99 -}

Haute-Sione : Doufss et Jura : lisière vosgienne de l'arrondissement de .Iontbéliard, grès keupriques des bords de l’ognon autour de Cubrial et sur le même terrain ver: Villersexel (IFaute-Saone), Dole, Baverans, Chatenois, Salins, Arbois, Poligny, Sellieres. - Cote-d'Or et Saône-et-Loire. - Ain : commun sur les terrains argilo-siliceux de la Bresse et de la Dombes. Région granitiq̨ue du Lyonnais à Dardilly, Charbonnières. Isère: Charvaz près Pusignan. - Rare dans la région méridionale ct dans le bassin du Léman.

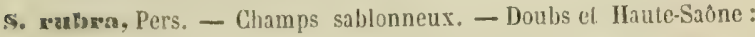
commun sur la lisière rosgienne de l'arrondissement de Mentbéliard, sur les grès keupriques des rives de l'ognon, les grès rouges et porphyres du canton de Champagney, - Jura : granite de la forêt de la Scrre; commun en Bresse. - Côte-d'Or et Saône-et-Loire; région granitique du Lyonnais et du Pilat; rare sur les sables de la mollasse dans le bassin du Léman; Savoie et Dauphiné. - Isẻre : sables granitiques de la Bẻrardeen-Oisans. - Hautes-Alpes : Névache, Lautaret. - Provence et Langleedoc.

5. enculia, - Pers, - Bords de la Méditerranéc où cette espéce polymorphe est très-commune.

Variété marginata, Fenzl. - Jêlẻe à la précédente; se trouve aussi sur les terrains imprégnés d'eau salée prés des sources du Plan-de-Phazy (Hautes-Alpes).

\section{ELATINÉES}

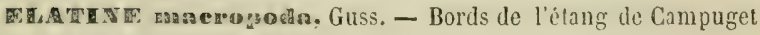
près Manduel (Gard).

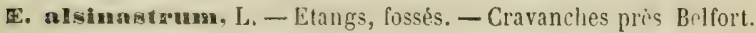
- Jura : ètang de Saint-Seine près Dóle. - Côte-d'or : Citteaux, forêt de Saint-Nicolas, Saint-Seine-en-Bâche, Longway.-Saonect-Loire: Saint-Pierre près Autun, Navilly. - Ain : Reyrieu, élangs de la Bresse, Montribloud, Saint-André-de-Corcy, environs de Bourg. - Isère: Iontceau près Bourgoin, Artas près Saint-Jean-de-Bournay. 
E. Lexamelas. D. C. - Etangs. - Environs de Bilfort, Eloye, Roppes, Bourogne. - Haute-Saône : étang du Grand-Mlorveau et d'Essouaivre. - Le Jlorvan dans Saone-el-looire et Colcd'Or. - Jura : aulour des élangs de la Bresse, Chaussin, Claa mergy, Sellieres, Poligny, mont Sous-Vaudrey. - Bolds du Léman entre Genthod et Versoix.

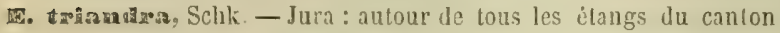
de Chaussin, Sainl-Baraing, Serrotte, Balaisseaux, Fort-Clos, Tassenières, Gatey, la Chênée, Pleurre, Chênc-Bernard, Ahergement-Saint-Jean, Neublans.

\section{LINEES.}

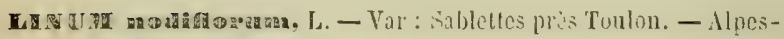
Maritimes: Cannes, Grasse, mont Cliauve et Ferrion au-dessus de Nice.

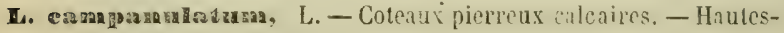
Alpes: Laragne, Ventavon, pont de Lagrand près Orpières. Drôme: Condillac. - Ardèche: Rochemaure, Cruas, Baix, vallée d'Ouvèse. - Collines des Bouches-du-Rhone et de Vaucluse. - Var : Bagnols, Vidauban, le Luc, Fox-Amphoux - AlpesMaritimes: de la Trinité à l'Escarène. - Gard : Vaqueirole près Nimes, Saint-Nicolas, Anduze, Alais, Saltous. - Hérault : bords de la Mosson, Saint-Loup, Cambous, Viols, Valène, SaintGuilhem, de Saint-Bauzile à Ganges, Capouladou, sources du Lez. - Celte espèce présente une variété furvum à sépales denticulés-ciliés.

E. garlieasn, L. - Champs sablonneux des terrains siliceux. Haute-Saòne: terrain minier d'Oyrière près Gray. - Jura: la Bresse, Chaumergy, Bois-do-Gand, les Abergements, Growon.Nul dans le Jassin du Lẻman. - Côte-d'Or : le Pays-Bas, Auxomne. - Saône-el-Loire: Chinay près Brancion, ChâteauRenaud près Loulıans, Ratle, Saint-Bonnet-en-Bresse. - Ain : Muzin, Meximieu, Balan, la Pape. - Isère: Décines, environs de Crémieu, Seyssuel, Viennc. - Drôme : Saint-Vallier. Bouches-du-Rhône; Var; Alpes-Maritimes. - Gard: Nìmes, 
Alais, Saint-Ambroix, Anduze, Manduel.- Hérault: Murviel, Palavas, Pérols, Vauguiores, Castelnau.

L. streterna, L. - Coteaux sces. - Très-commun dans la Provence ct les Alpes-Yaritimes.-Gard : Aigues-morles, Tresgues, SaintNicolas. - IIérault. : environs de Montpellier, de Celte, de Biziers. Sa limite septentrionale est d'une part Livron dans la Dròme, d'autre part Comboire et Beauregard près Grenoble.

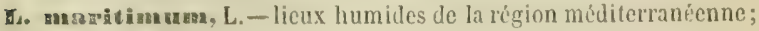
remonte jusque dans la partie méridionale de la Dróme vers Nyons, Montélimar.

L. tenuifolium, L. - Lieux arides dans tout te bassin.

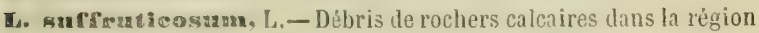
méridionale.-Bonches-lu-Rhône : Estaque, chaine de l'Etoile. - Var : Touris près Toulon, Nans. - Alpes-Mlaritimes. Gard : Nîmes, Uzès, Campestre, Montdardier. - Hérault : environs de Montpellier et de Bézicrs. Remonte dans l'Ardèche jusqu'au mont Clıarray; dans Vaucluse et Drome jusqu'à Nyons, Crest; dans Basses et Hautes-Alpes jusqu'à Esparon, Tallard, mont Síusc; dans l'Isère jusqu’à Saint-Andéol et au col de l'Arc.

L. narbonense, L. - Lieux arides de la région méridionale. Commun dans la Provence, les Alpes-Mlaritimes et le Languedoc ; remonte dans l'Ardèche à Rochcmaure et Viviers; dans la Drome à Nyons, Crest, Montélimar, Condillac; dans les HautesAlpes à Charance, Puy-Maure près Gap.

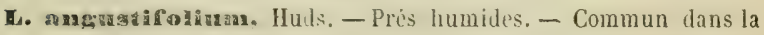
Provence, les Alpes-Ilaritimes et le Languedoc; remonte jusque ver's Grenoble et Lyon.

g. usicarissanuman, L. - Subspontané et fugace.

L. alpinum, L. - Rochers et pelouses des montagnes, - Jura : mont d'Or, Montenàre, Dôle, Colombier, Reculet. - HauteSavoie : mont Châtillon, Vergy, Tournette, col de Balme. Saroie: mont Cenis, montagnes des Avanchers. - Isère : chaine de Belledonne et de la Grande-Chartreuse. - Hautes-Alpes : Lautaret, mont Viso, mont Séuse, Boscodon. - Alpes-llaritimes. 
L. saxicolum, Jord. - Rochers - IIautes-Alpes: Ia Garde, Charance, Brame-Buou près Serres.

L. montanum, D. C., L. Loreyi, Jord. - Coteaux calcaires bien exposés. - Côte-d’or: Marsannay, Gevrey, Savigny. - Saône-etLoire : Dezize, Solutré, Vergisson.

L. austriacum, L. - Coteaux calcaires de la Cote-d'Or. - Le groupe qui comprend les quatre formes ci-dessus énumérées a besoin d'une révision sérieuse. Les auteurs ne sont point d'accord sur la synonymie, non plus que sur les caractères spécifiques.

L. cathroficun, L. - Prés humides des plaines et des montagnes dans tout le bassin.

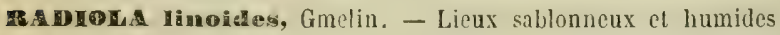
des terrains siliceux et argilo-siliceux. - Jura : la Bresse, forêt granitique de la Serre. - Côte-d'Or : la Roche-en-Brenil, Saulieu, Rouvray dans le Morvan granitique. - Saône-et-Loire: Tavernay, Autun, Issy-l'Erêque.-Ain : la Bresse et la Dombes, les Euheyx, Montribloud, Reyrieu, Bâgé, Chevroux, étang Genoux à Pont-de-Yeyle. - Isère : sables de la mollasse autour de Voiron, Bourgoin, la Côte-Saint-André, Saint-Geoirs, Charette. - Bouches-du-Rlıône: Aix. - Var: granites et porphyres de la forêt des Maures, Hyères, Antibes. - Alpes-Maritimes: sables tertiaires des collines de Menton. - Gard: terrains granitiques autour du Vigan et d'Alzon.

\section{TILIACEEES.}

TICXA greandirolin, Ehrh. - Assez rare dans les bois.

T. intersuedira, D. C. - Méme habitat.

T. parvifolia, Ehrl. - Jême habitat.

\section{MALVACEES.}

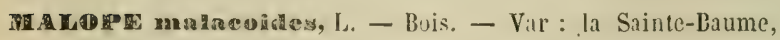
Aiglun, - Alpes-Maritimes : la Paoule près de Grasse, la Roquette, Nice au Var.

MAYV alezer, L. - Bois ct coteaux calcaires dans tout le bassin. 
Les feuilles caulinaires sont divisées jusquu'à la base en cinq lobes rhomboidaux dans la forme genuina, divisces en lanières étroites dans la forme multidentata et jusqu'au milieu dans la forme fastigiata.

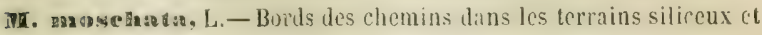
argilo-siliceux. - Les formes des feuilles sont, comme dans l'espèce précédente, sujettes à varier. - Haute-Saone : zône rosgienne jusqu'au Ballon de Servance. - Jura, Doubs et Ain : la Bresse et la Dombes, çà et là dans la région des sapins, lisiëre vosgienne de l'arrondissement de Montbéliard. - Sables de la mollasse dans le bassin du Liman. - Cote-d'Or : terrains granitiques du Morvan, - Même terrain dans Saone-et-Loire. Régrion granitique du Lyonnais. - Sables de la mollasse du Dauphiné. - Var : granites et porphyres de foret des Maures.Alpes-Marilimes. - Région granitique des Cévennes : l'Espérou, Dourbie, Alzon, le Vigan.

II. Tomanefortiana, L. - Lieux sablonneux. - Var : forêt des Maures, Notre-Uame-des-Anges près Pignans, Fréjus, l'Estérel. — Bouches-du-Rhône: Marignane, d'Auriol à Saint-Zacharie.

R. ๙ylvestrois, L. - Lieux riches en matiẻres azotécs, tels que dẻcombres, environs des habitations, dans tout le bassin.Forme ambigua, Guss. - Bords des chemins autour de Montpellier, Cette, Antibes, Menton.

Pr. niceernis, All. - Bords des chemins, décombres dans la Provence ct le Languedoc.

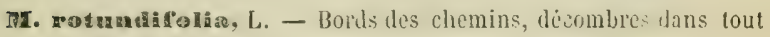
le bassin.

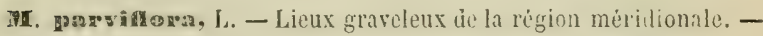
Bouches-du-Rhône : Marseille, Saint-Tronc. - Var : Iles d'Hyères, - Alpes-Maritimes: Courmes, le Bar, Nice, Menton. Gard : Aigues-1Iortes, Palavas.

Ir. mierocarzoa, Desf. - Lieux pierreux. - Marscille, Túlon, Hyères, Nice. - Gard : mas de Campagne prìs Nîmes, AiguesMortes.

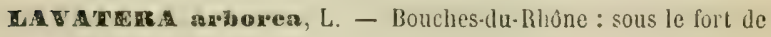


Ratonneau. - Var : Fréjus. - Alpes-Jiaritimes : golfe Joun, rochers des Lions, ile Sainte-Ilarguerite, Nice, Menton.

¿. Cretica, L. - Var : les Pesquiers près Hyères.

4. Odbsa, L. - Bonches-du-Rlione: Artes, la Crau, en Coustiero. Var et Alpes-llaritimes : Hyòres, Fréjus, Saint-Haxime, Estérel, ille Sainte-Marguerite.

1. zmaritisana, Gouan. - Rochers. - Bouches-du-Rlsone : Arles, un Crau, en Cousticro. - Var : Toulon au mont Faron. -- AlpreMaritimes: Villefranche, Monaco, Menton, le Bar, Saint-Arnoux. - Hérault : près la grotte de la Madeleine.

L. מunedata, All. - Champs de la région méditerranécnne. Bouches-du-khône aulour te Harseille - Var: Toulon, Fréjus. - Alpes-Maritimes : Cannes, Grasse, Nice.

L. toimestzoํㅗ, L. - Bouches-du-Rhône: Marseille. - Var: Toulon an fort Rouge, le Pradet. - Alpes-Haritimes: la Napoule, Nice à Mont-Boron et au Fabron.

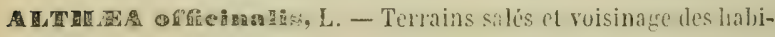
tations, - Jura : autour des salines de Lons-le-Saunier, Grozon, hords du Doubs et de la Loue au-dessous de Dôle. - Pays has de la Côte-d’Or. - Sxônc-et-Loire : près des bords de la Saône à Verdun, Châlon, de la Seille à Louhans, de la Grosne à Cluny. - Ain : bords de la Ressouse à Pont-de-Vaux, Vésine, Asniëres, bords de la Chalaronne. — Rhône: d'Anse à Villefranche. Isere : environs de Grenolile. - Drôme : Valence, Crest - Bouches-du-Rhône: environs de Marseille, Camargue, marais de Fos, Coustiero. - Var et Alpes-Mlaritimes: Antibes, Grasse, la Napoule, Nice au Var. - Garủ : Jonquiẻres, Bellegarde, Manduel - Hẻrault.

A. ernuabirna, L. - Bords des haies et des bois dans la Provence, les Alpes-Yarilimes el le Langueloc; remonte dans la Drûme à Montélimar, Valence el jusqu'à Vienue (Isère) vers Seyssucl, Cluzelle.

A. mar.hanezasis, Pourt. - Bords des haies el des bois dans la Pro vence cl le Languedoc.

A. Inirsuaa, L. - Coteaux graveleux et calcaires dans tout le bassin. 
AmUTIMON Avieenna, Presl. - Garl : marais de Jonquières près Beaucaire, bords du contre-canal à Bellegarde.

\section{GÉRANIÉES。}

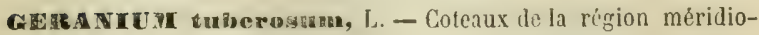
nale. - Bouches-du-Rhône: environs de Marseille, Plan-deCuques, Rouet, Chåteau-Gombert. - Var: le Revest, Toulon. - Alpes-Naritimes : Cannes, Antibes, Nice au mont Chauve.Hêrault: Agde.

G. prntense, L. - Haies et prés humides. - Doubs : environs de Pontarlier, marais de Saône. - Gard : Anduze, Lanuejols.

c. wylvatieum, L. - Prairies et bois des montagnes. - HauteSaone, Doubs, Jura, Bugey, Savoie, Dauphiné, Forez, Pilat, Cévennes, Alpes-Maritimes.

G. seonitifolama, Iher, - Prairies humides des hautes montagnes. - Hautes-Alpes: Lautaret, mont Genèvre à Gondran, vallée de Névache, mont Viso, Aiguilles, Ubac-le-Villevieille, Abriès, mont Séuse, col de Glaise. - Alpes-Maritimes.

G. bohemieum, L. - Bois et prairies sur terrains siliceux. Var et Alpes-Marilimes: Fréjus, la Napoule, l'Estẻrel, Maures de Tanneron.

G. nodesara, L. - Bois des montagnes. - Nul dans la chaine jurassique de Haute-Saône, Doubs, Jura, Bugey. - Rare dans la Haute-Saroie au pied du Môle au-dessus de Marignier, aux Voirons au-dessus de Saint-Cergues, Serraval, Sainte-Colombe, vallon d'Entrevernes. - Saône-et-Loire: Semur-en-Brionnais. - Isère : très-commun à Uriage, Eybens, Revel, côtẻ nord dé Saint-Eynard, Saint-Nizier, Ia Motte-les-Bains, la Salette. Hautes-Alpes: Manteyer, Morgon prẻs Gap. - Rhône: Givors, Collonges, bords de l'Azergue. - Loire : Pilat. - Ardèche: massif volcanique du Coyron et du Gerbier-de-Jonc. - Gard : Alais, Anủuze, le Vigan, Alzon. - Alpes-Maritimes.

G. phoeam, L. - Prairies des montagnes. - Jura : Reculet au valIon d'Ardran et en descendant vers Chẻzery. - Haute-Savoie : chaine des Cornetles, mont Charvin, Tournetle, valléc du RepoCatal. Bassin du Rhóne. 
soir. - Savoie : montagne des Avaanchers. - Isère: la GrandeChartreuse à Bovinant, entre Tenaison et Pont-Pérant, le Sappey. - Alpes-llaritimes.

G. palustre, L. - Prairies humides. - Doubs : Pontarlier, Etupes, Exincourt. - Ilaute-Sãone: Plancher-Bas, Plancher-les-Mlines.

- Jura: Ie long du ruisseau de Saint-Joseph près de Salins. Bassin du Léman. - Haute-Savoie: bords de l'Arve près de Sallanche, Allonzier.

G. argenteum, L. - Rochers des hautes montagnes. - HautesAlpes: Chaillol-le-Vieil, mont Quimpel près Saint-Bonnet. Basses-Alpes : montagnes de Digne à la forêt de Faye-Feu.

C. mangư̊neam, L. - Collines pierreuses. - Haute-Saône : Chariez. - Doubs : Lomont, Blamont, environs de Besançon, à Rosemont et à la Citadelle. - Jura : Salins, Lons-le-Saunier, Champagnole, Thoirette, le Reculet. - Côte-l'Or et Saône-etLoire. - Bassin du Léman et Valais. - Ain : le Revermont, le Bugey, Charnoz, Pierre-Châtel, Sathonay, la Pape. - Rhône: Saint-Alban, Beaunant. - Isère: environs de Grenoble, Sassenage, base du Saint-Eynard. - Ifautes-Alpes : mont Séuse. Drôme : Crest. - Provence, Alpes-Maritimes et Languedoc.

G. coluan bo an ana, L, - Haies, borids des chemins dans tout le bassin.

G. dingectum, L. - Mêmes lieux.

G. pyreanieam, L. - Bois, prés, bords des chemins dans tout le bassin.

G. mollar, L. - Bords des chemins, vignes, champs dans tout le bassin.

A. pasallum, L. - Mènes lieux.

G. rotundifolieam. L. - Mèmes lieux.

G. Iucidum, L. - Rochers ombragés, haies. - Doubs et Jura : Besançon, Baume-les-Dames, de Salius à Saint-Amour, SaintClaude, Thoirette. - Côte-l'Or: Virrey-sous-Salmaise, ronte de Chaume, vallee de Savigny-sous-Beaune, Saint-Aubin, Fussry. - Saône-et-Loire: Dezize, Chanilly, Cuiseaux. - Valais : entre Martigny et Pissevache. - Haulp-Savoie : banc supérieur du haut Sulève, fontaine du Pas-de-l'Echelle, mont Gosse, Chaumont-sur-le-Vuache, col du Corbier entre le Biot et Bonnevaux. - Ain: le Revermont à Ramasse, environs de Belley, Pierre- 
Châtcl, Iancrans, Confort. - Isère: Crémicu, Pont-de-Beauvoisin, la Molte-les-Bains, Pariset, balmes de Fontaine. - Drôme : Romans, Chanvasse près Sainl-Andri-de-Romans, Aucelon, Crest. - Ardèche : Rochemare. - Vaucluse. - Bouches-duRhone : Pilon-lu-Roi. - Var : Roquebrune, le Lis. - AlpesNaritines, - Gard: le Vigan, Anduze, Espérou, Alzon.

C. Itofrestianam, L. - Hities dans tout le bassin. - Espèce polymorphe.

G. semiglabrum, Jord. - Ain : Tenay, Rossillon.

G. purpureum, Vill.-Débris de rochers.-Isère: Seyssuøl près Vienne, Grenoble à Guy-Pape, Vouillant, Rochefort près du Pont-deClaix. - Drome : Saint-Vallier, Serves, Tain. - Ardèche: vallèe d'Ouvèze.

$G$ simile, Jord. - Gard.

G. modestum, Jord. - Buissons. - Isère : Crémieu.

G. minuliflorum, Jord. - Ain : Serrières.

G. Villarsianum, Jord. - Basses-Alpes.

EISOMUMI malacoides, Wild. - Bords des champs et des chemins dans la région méridionale.

E. elnium, Willd. - Murs et décombres, - Var : Fréjus.

E. pallidiriorum, Jord. - Pelouses, - Bouches-du-Rhone: SaintRémy. - Var : forêt des Maures.

D. Iitton'eum, Léman. - Bouches-lu-Rhơne: Montredon, Endoume.

E. Iaciniatum, Cav. - Sabies maritimes. - Var : salines d'Hyères. - Alpes-Maritimes: Nice, Menton.

E. botrys, Bertol. - Terrains granitiques du Var et des AlpesMaritimes, à Hyères, Roquebrune, le Luc, Cannes.

S. clconium, Willd. - Lieux secs de la région méridionale; remonte dans la Drôme jusque vers Montélimar et Aucelon, dans les Hautes-Alpes jusque vers Gap; a été observé accidentellement en Savoie entre Houtiers et Brides, et aussi à Lyon.

r. mosehatum, Willd. - Lieux sablonneux de la région méridionale. Bouches-du-Rhone: environs de Marseille. - Var : Frejus, le Luc, Hyères, Toulon. - Alpes-Maritimes. - Gard: bois de Campagne près Nimes.

C. cieutarium, Lhérit. - Espèce polymorphe répandue dans les 
champs et sur le bord des routes dans tout le bassin. - Les formes suivantes sont démembrées de celle-ci.

E. Iriviale, Jord. - Environs de Lyon, de Grenoble et de Gap.

E. protermissum, Jord. - Environs de Lyon.

E. commixtum, Jord. - Pelouses. - Haute-Saône: Champagney. -

Sấne-et-Loire : environs de Verdun. — Jura : forêtt de la Serre.

- Rhône: Chaponost. - Gard : montagnes du Vigan.

E. subalbidum, Jord. - Euvirons de Lyon à Villeurbanne, la Pape.Drôme : Saint-Vallier.

E. bicolor, Jord. - Ardèche: Viviers.

E. fallax, Jord. - Gard : Uzis.

E. pilosum, Bor. - Isère : entre Jonage et Anthon.

2. Tomassans. Willd. - Pelouses et bords des chemins dans la région méridionale.-Provener, Alpes-Haritimes et Languedoc.

ש. petranasn, Willd. - Rochers. - Hérault : pic Saint-Loup. Plus commun dans le Roussillon.

\section{HYPÉRICINÉES.}

מ耳 - Espèce polymorphe.

H. microphyllum, Jord. - Nême dispersion.

H. lineolntum, Jord. - Saone-et-Loire : Bugey, Pilat, Briançonnais. H. medianum, Jord. - Ain : Retord.

7. quadranganlazan. L. - Bois des montagnes. - Haute-Saone : zône vosgienne. - Région des sapins du Doubs, Jura, Bugey, Dauphiné, Savoie. - Saone-et-Loire : l'Autunois, bois du Boursier près Cluny, — Gard : Lanuejols. — Var et Alpes-Itaritimes.

H. ted ragateran, Fries. - Lieux liumides dans tout le bassin.

M. hunateresusta, L. - Champs sablonneux des terrains siliceux et argilo-siliceux. - Haute-Saòne : zône vosgienne, vallée de Mansvillers, Ecromagny, Raddon, forêt d'Arobert, vallée du Fray, Château-Lambert; diluvium et grès bigarré à Franchevelte, Linexert, Saint-Germain, Granges-đu-Bois-Béni ; oxfordien siliceux à Grattery, Chariez. - Doubs : lisière vosgienne et mollasse de l'arrondissement de Montbéliard. - Jura : ter- 
rains sablonneux de la plaine. - Pays bas de la Cote-d'Or. Saône-et-Loire. - Haute-Savoie : Veyrier. - Isère : SaintNizier, Revel, Jarrie, Brié, la Chapelle-en-Vercors. - Rhơne et Loire : région granitique du Lyonnais et du Pilat. — Gard : environs du Vigan.

f. australe, Tenore. - Coteaux. - Var: Toulon, Hyères, le Luc, Fréjus. - Alpes-Haritimes : Antibes, Cannes, la Roquette, l'Estérel.

Ix. tomentosum, L. - Prairies humides de la récrion méridionale. - Vaucluse Avignon. - Bouches-du-Rhône: environs de Marseille el d'Aix. - Var: Fréjus, le Revest, le Luc. - Alpes-IIaritimes: Auribeau, Grasse, Cannes, Saint-André, Blausasc, l'Escarène, entre Levens et Coarazza. - Gard : Nimes, Manduel, Corconne, Pujau, Aigues-lIortes. - Hérault : Montpellier, Caunelle, Peyrols, Lattes.

H. Coris, L. - Collines de la région méridionale. - Basses-Alpes: Saint-Arnoux. - Alpes-Maritimes : de Nice à llenton.

H. Hyssopifollum, Vill. - Débris de rochers. - Isère : la Saletle. Hautes-Alpes : Embrun, mont Aurouse, Charance, Loubet. Drôme: Aucelon, Lus au-dessus de Durbon. - Basses-Alpes : Digne. - Bouches-du-Rhône: Sainte-Victoire, pic de Bretagne, Sainte-Baume. - Alpes-IIaritimes : Sigale, sommet de l'Agel au-dessus de la Turbie. - Gard : d'Avèze à Montdardier, Salbous.

X. pulchrum, L. - Bois humides dans les terrains siliceux argilo-siliceux. - Haute-Saône : zône vosgienne, Raddon, tourbière dArfin près Saint-Hilaire; grès bigarré de la Grange-duBois-Béni ; diluvium de Franchevelle, Linexert, Saint-Germain; oxfordien à chailles siliceuses à Montigny, Ćlıriez, Lambrcy, Grattery. - Doubs et Jura : lisiẻre vosgienne et mollasse, terrains sablonneux des plaines. - Côte-d'Or : forêt de SaintNicolas, Gerland. — Saône-et-Loire : le Morvan. — Région granitique du Beaujolais, du Lyonnais, du Pilat et du Forez. Haule-Savoie: hois de Saint-Julien et de Viry. — Ain : environs de Belley, Bourg au bois de Seillon. - Isère : terrains sablonneux des Terres-Froides à Chambaran, Saint-Geoirs, Ville près Vienne, Meyrieu. - Drôme : la Chapelle-en-Vercors. — Gard : 
bois de pins du Chanet près Bourdezach. - Nul dans la Provence et les Alpes-Maritimes.

T. nummatarium, L. - Fentes des rochers des montagnes. Savoie: munt du Chat. - Isère : Grande-Chartreuse à Fourvoirie et Bovinant, le Collet, Bellefont, Deut-de-Crolles. - AlpesMarilimes.

II. Hirstum, L. - Bois des basses montagnes dans toule la partie septentrionale et moyenne du bassin; plus rare dans la région méridionale, - Alpes-Maritimes : le Chaudan, les Mujoulx dans la vallée de l'Esteron. - Gard : Vigan, Camprieux, Aumessas.

I. montanum, L.- Bois des basses montagnes dans tout le bassin.

Ir. Ibichear, Vill. - Prairies des hautes montagnes. - Toute la chaîne jurassique helvétique et française : Chasscral, Chasseron, Aiguillon, Suchet, Montendre, Noirmont, Dôle, Colombier, Reculet, la Faucille. - Ain : le Colombier-du-Bugey. Savoie: mont du Chat. - Haute-Savoie: mont Vergy, Méry, Tournette, Brévent vers le chalet de Bellevue près Cıımonix. - Savoie. - Isère: Grande-Chartreuse à Bovinant, chaine de Belledonne, Alpe du mont de Lans. - Hautes-Alpes : Ia Grave, le Lautaret, mont Viso, montagnes du Gapençais, Chaillul. Drôme : Glandaz, - Alpes-llaritimes.

Ir. ciliatum, Lam. - Prẻs lıumides. - Var : Hyères, îles d'Hyères.

1r. anshrosamum, L. - Buis et prés.- Saòne-et-Loire : bois de Prodhun, de Chizeuil, du Borsier, la Molte-Saint-Jean. - Jura : Condal près Saint-Amour, - Ain : forêt de Seillon, Saint-Rambert. - Rhône : Propières, entre Sainte-Colombe et Ampuis. - Isère: Morestel, Parmilieu, Chandicu, Combe-de-Malafossan près le Pont-de-Beauroisin, Saint-Geoirs, Saint-Laurent-du-Pont, Saint-Georges-d'Esperanche, Vienne, balmes de Voreppe. Bouches-du-Rhone: Arles. - Var : les Maures, Chartreuse de la Verne, I'Estérel. - Alpes-Maritimes: le Revest, Gourdon, Nice, Menton. - Gard: Anduze, Valleraugue, Gaujac.

ELODES paIustris, Spach. - Marais tourbeux dans les terrains siliceux. - Cote-d'Or: Morvan granitique, la Roche-en-Brenil, étang Champeau près Saulieu, ètang de Tournesac, Pré-Carré. 
Saone-et-Loire: ablme de Poizot à Tavernay. - Isère : Charette près Crémieu. - Gard : la Grandes-Haute, le Lengas, l'Espérou.

\section{ACÉRINÉES.}

ACER pseudoplatanus, L. - Rare dans les bois. - Zône vosgienno de Haute-Saône. - Doubs. - Jura : forêts de Chaux et de la Serre. - Saône-et-Loire: Cluny, Autun, Louhans, Ain. - Rhorre: Poleymieu. - Loire: mont Pilat. - Isère: Revel, Prémol, Clavans, Saint-Eynard, Saint-Nizier, Pont-deBeauvoisin. - Savoie: environs de Moutiers, détroit du Ciex. - Drôme : Morgon, Crest. - Hautes-Alpes : Loubet. - Gard : Nimes, Bagnols. - Bouches-du-Rhône : bords de l'Huveaume à la Moulte.

A. opulifolium, Vill.-Bois.-Basses montagnes calcaires.-Coted'Or: Bouillant, Arcenant, forêt de Mantuan.-Saune-et-Loire: Cortembert. - Doubs: cotes du Dessoubre, environs de Besançon. - Versant helvétique dans le Jura Bernois, Soleurois et Neuchâtelois. - Jura : toute la chaîne de Salins à Saint-Amour, Champagnole, Saint-Claude. - Ain: Colliard, le Colombier, Charabotte, Ruffieu, Hotonnes, Parves, Ceyseriat, Saint-Rambert, Cerdon, fort de l'Écluse. - Haute-Savoie: le Saléve, mont de Mandallaz près Bromine, vallon des Usses. - Rhône : Couzon.- Savoie: mont du Chat, Châtelard. - Isère: Crémieu, Chambaran, Sassenage, Comboire, Saint-Eynard, Grande-Chartreuse, - Haules-Alpes : Charance, Rabou. - Var : SainteBaume. - Alpes-Maritimes : Menton, Saorgio, l'Authion. Gard : l'Espérou, Campestre, Montdardier, à la Roque, près Tresques.

A. monspessulanum, L. - Bois, - Manque dans les départements de Haute-Saòne, Doubs, Jura, Côte-d'Or, Saône-ct-Loire. - Sa limite septentrionale est dans I'Ain au fort de l'Echuse, Pierre-Clıatel, mont de Parres, le Thuy, Muzin près Belley, Charabotte. - Haute-Savoie: Val-de-Fier, Bromine. - Savoie: mont du Chat, Chátillon, Chambolte, Moutiers. - Rhône: Couzon, Albigny, rives du Garon. - Isère: la Balme, Crémieu, 
Vienne, la Bastille de Grenoble, Rochefort près le Pont-deClaix. - Hautes-Alpes: la Grave, Charance près Gap. — Drơme : Saint-Uze près Saint-Vallier. — Ardèche : Crussol. - Bouchesdu-Rhône. - Var: Coudon près Toulon. - Hẻrault. - Gard : bords du Gardon, Saint-Nicolas, l'Espérou.

A. Martini, Jord. - Couzon près Lyon.

A. esmpestre, L. - Cotcaux et taillis dans tout le bassin.

A. platanoldes, L. - B is. - Haute-Saone: zone rosgienne. Doubs. - Jura helvétique et français. - Ain : Lélex, Thur. Savoie: mont du Chat. - Rhone : Limonest. — Isère : GrandeChartreuse près de la chapelle Saint-Bruno, le Champsaur. Gard: les Cévennes. l'Espérou. - N'est pas signalé dans la Provence ni dans les Alpes-IIaritimes.

\section{AMPÉLIDÉES.}

WITIS vinufera, L - - Cullivé et souvent subspontané.

\section{MÉLIACÉES.}

FreLta azedarach, L. - Plante orientale naturalisée autour de Nice et sur plusieurs points de la Provence, notamment près Cannet-du-Luc, au Bouillidou; en divers lieux du Languedoc.

\section{BALSAMINÉES.}

CMPATIST nolintsagere, L. - Bois des montagnes, - Commun dans les Vosges, la chaîne jurassiıjue française et lıelvétique, le Valais, le Bugey, la Savoie, le Dauphiné, le Morvan autunois, la chaine des montagnes du Beaujolais, du Lyonnais, du Forez et du Pilat. - Manque dans la Provence ct le Languedoc; rare dans les Alpes-Ilaritimes: val de Pesio, Tende, SainteAnne de Vinaï.

6. parvidlora, D. C. - Plante originaire de la Russie laquelle s'était naluralisée à Lyon dans le voisinage de l'ancien Jardindes-Plantes et à l'ile-Barbe. - Environs de Genève sur la route de Plain-Palais à Carouge, Rolle. 


\section{OXALIDÉES.}

DXAus ncetoselIn, I. - Bois lumides, - Toute la chaine vosgienne et jurassique, Valais, Savoie, Bugey, Dauphiné, le Morvin, Beaujolais, Forez, Pilat, Vivarais et Cévennes, - Manyue dans la région inférieure de la Provence; se retrouve dans la région montagneuse des Alpes-Mlaritimes.

- Iybiea, Viv. - Plante du Cap de Bonne-Espérance qui s'est nituralisie à Cannes, Nice, Villefranche, Monaco et Menton.

0. merictar. L. - Champs cultivés. - Haute-Saône et Doabs : Fougerolles, Fouvent, Larret, vallée de l'Ognon à Pesmes, Vy-lesLure, Montigny, Rougemont, environs de Besançon. - Jura: forêt de Chaux, la Vieille-Loye. - Côte-d'Or: Auxonne, Dijon. - Saône-et-Loire: bords de la Seille à Louhans, de la Saône à Mâron. - Lyonnais, environs de Genève et bassin du Léman. - Isère : environs de Grenoble et de Bourg-d'Oisans. - Var et Alpes-Haritimes : Fréjus êt Nice.

D. corniculata, L. - Licux cultivés. - Nul dans la partie septentrionale du bassin. - Rhóne: l'Argentière, environs de Lyon, la Mouche, les Étroits, la Croix-Rousse. - Isère: environs de Grenoble. - Commun dans la Provence, les Alpes-Maritimes et le Languedoc.

\section{ZYGOPHYLLÉES.}

TrI BuLUs terrestris, L. - Champs cultivés du Languedoc, de la Provence et des Alpes-Maritimes. - Dróme: Saint-PaulTrois-Lhâteaux, Saint-IIaurice-d'Exil, Romans. - Trouvé accidentellement à Lyon à Perrache et Pierre-Bénite.

\section{RUTACÉES.}

IXTA montana, L. - Coteaux secs et pierreux. - La Provence et le Languedoc. 
R. angustifolla, Pers. - Coteaux secs. - Drôme : Nyon: . schers de la Citadelle, Donzère, Saint-Paul-Trois-Châteaux, - Ardèche: Viviers, le Pouzin. - Gard: Nimes, Broussan, la Baume. Commun dans la Provence et les Alpes-Maritimes.

m. bracteosa, D. C.-Coteaux secs._Bouches-du-Bhone : Marseille, Sainte-IIargerite, la Belle-de-Hai, Saint-Jean-du-Désert, Raphẻle près Arles. - Var : Hyères, Fréjus, Grimaud. - Très-commun sur le littoral des Alpes-Maritimes : de Nice à Menton.

h. ฐraveolens, L. - Souvent cultivé; parfois subspontané sur les rochers. - Valais : Saint-Maurice. - Ain: entre Béon et Talissieu. - Savoie: au-dessus de Centron près Aime. - Isère : Varces. - Drôme: Livron, Saint-Hay près de Rémusat, Venterol. - Ardéche : Châteaubourg. - Alpes-Maritines : Saint-Martinde-Lantosque. - Gard : Anduze.

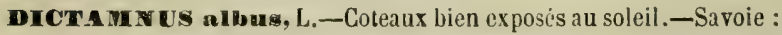
rive gauche du Doron entre $\$$ loutiers et Salins. - Isere : de Vif aux Saillans, Nantoin près la Côte-Saint-André. - HautesAlpes : mont Gauvi près Guillestre. - Drôme: le Buis. - Vaucluse: Orange. - Bouches-du-Rhone: Rognes, Beaulieu, les Alpines entre Saint-Remy et Eyguières. - Alpes-Ml aritimes : environs de Nice et de Grasse, Saint-Antoine, Utelle, SaintDalmas-de-Tende et Malaussène près du Villars. - Gard: environs de Nìmes à Roquecourbe, la Beaume, Pont-du-Gard, Coucol près Bagnols, Boussargues.

\section{CORIARIÉES.}

Coiry aera myrtifolia, L. - Coteaux et bords des bois dans la Provence et le Languedoc. - Var: Cannes, Bagnols à Curebesace, Seillans. - Alpes-Maritimes : Auribeau, Grasse, très-commun de Nice à Menton. - Gard : bords du Gardon, la Beaume, d'Alais à Saint-Ambroix, Anduze. — Ardèche: Jovyac près de Rochemaure. 


\section{CALICIFLORES}

\section{CÉLASTRINÉES.}

Evorrmus europaens, L. - Haies et bois dans tout le bassin du Rhône.

E. Intifolius, Scop. - Bois des montagnes. - H,-Sav. : pied du Salève entre Allonzier et Avregny, colonies d'Annecy, Vovray, Crêt-du-Maure, Semnoz. - Sav. : mont du Chat, environs de Chambéry, Saint-Cassin, Cascade de Couz, vallée de l'Arly entre Ugine et Flumet, Tamis, environs de Moutiers, de Saint-Jeande-Maurienne et de Saint-Michel. - Ain : Colombier du Bugey. - Is. : mont Rachais, Saint-Eynard. - H.-Alp. : environs de Gap à Charance, Devez-de-Rabou et Pleyne de Chaudun. Dr. : Aucelon. - B.-Alp. : Sisteron. - Vaucl. : Saint-Amans. - Var : Sainte-Baume. - Alp.-Mar. : La Briga, val de Pesio, mont Cheiron. - Gard : Serre de Bouquet.

\section{STAPHYLÉACÉES.}

STAPIYYEA pinnenta, L. - Bois. - Doubs : bois de Montfort à Clerval, côte du Moulin-de-Bélieu, Morvillars, Méziré, Vaudoncourt. - C.-d'Or : Abbaye de Sainte-Marguerite, vallẻe de Bouilland. - Pyr.-Or. : escarpements du bois de Saint-Antoine de Galamus. - Cette plante, fréquemment plantée dans les jardins d'agrément, est fort rare en France à l'état spontané.

\section{ILICINÉES.}

ILFX aquifolium, L. - Bois dans tout le bassin. 


\section{RHAMNĖES.}

ZLZYPYU vulgaris, Lam. - Le Jujubier est cultivé dans quelques parties de la Provence, du Roussillon et du Languedoc et se montre quelquefois à l'état subspontané.

PALruteus australis, Roen. et Schult. - Très-commun dans les haies de la région méridionale. - Vaucl. - B.-du-Rh. Var : Toulon, Saint-Tropez, le Luc, Fréjus. - Alp.-Nar. : Auribeau, Grasse, vallée de l'Estéron, comté de Nice. - Gard : NImes, Manuluel, Marguerittr, Beaucaire, Bellegarde. - Toute la partie basse des départements de IHér., de l'Aude et des Pyr.-0r. - Remonte dans la Drôme à Saint-Paul-Trois-Châteaux, Pierrelatte, Mirabel près Nyons et jusqu'à Valence. - B.-Alp. : route de Sisteron à Digne. - Is. : la Balme. - Rh. : près des acqueducs de Chaponost. - Probablement planté dans les deux dernières localités citées.

RHAwNus cathartiea, $L$. - Bois. - Très-rare dans la partio basse de la Provence el du comté de Nice; se montre dans la partie montagneuse à la Sainte-Baume, montagnes de Lévens, Molinet, Entraunes, Saint-Etienne et Saint-Dalmas-le-Sauvage. - Vaucl, : mont Ventoux, Saint-Amans. - Plus répandu dans le reste du bassin, mais non abondant.

R. sylvatiea, Serres. - Bois des environs de Gap à Rabou, la Roche-des-Arnauds.

R. saxatilis, L. - Rochers et pâturages. - Ain : la Pape et les iles voisines, Balan, Meximicux aux Peupliers, bords de l'Ain et de la Bienne, Loyette, Oyonnax, Dortan. - Is. : Ie Mollardde-Décines, Charvieu, Crémieu. - H.Alp. : col de Glaise, Charance, Montmorin, Lagrand, Villarrobert, la Rochette, mont Rognouse.-Dr. : Nyons. - B.-Alp. : Sisteron, la Baume. - Var : Sainte-Baume. - Alp.-Nar. : monta gnes de Caussols et de Défens, Saint-Dalmas-le-Sauvage. - Hér. : le Caylar, Mas-de-Houriẻs, la Sérane.-Pyr.-Or. : Font-de-Comps, Roc del Mouis, environs de Villefranche.

R. Villarsii, Jord. - Coteaux secs. - Is. : Vernas ct Leyrieu près Crémieu, Charvieu. - Ain : Néron près Lyon. 
It. infectorin, L. - Rochers. - Dr. : Montélimar, Crest, le Buis, Romeyer près Die. - Vaucl. : environs d'Orange et d'Avignon. - B.-du-Rh. : les Alpines, environs d'Aix, Tholonet, Puyloubier, Pourrière, Sainte-Victoire. - Gard : Nimes, Alais, Margueritte, Alzon, Serre-de-Bouquet, Aigues-Mortes. - Hér. : cnvirons de Montpellier, Saint-Loup, Viols, Capouladou, Colombière, Monmau, vallée de la Mosson, la Sérane, SaintGuilhem-le-Désert, mont Saint-Clair près Cette, Roquehaute, Béziers, Frontignan, Saint-Guiraud, Madières, le Caylar. Aude : Pech-de-l'Agnel. - Pyr.-Orientales. - L'absence de ce Rhamnus dans le Var el les Alpes-Maritimes est un fait digne de remarque.

18. mIpina, L. - Rochers des monlagnes calcaires. - Nul dans les Vosges. - Doubs et Jura : calcaire jurass. du Clos-du-Doubs, côtes du Doubs et du Dessoubre, Lomont et toute la chaine du Jura français et helvétique jusçu'au Reculet, puis dans l'Ain au Colombier du Bugey, les montagnes des environs de Nantua, le IIont, le Poizat, Cerdon, la vallée de l'Albarine, Rossillon, les côtes du Rhone à Saint-Benoit, Serrières, Villebois. - C.-d'Or: chaîne jurassique de Gevrey à Beaune. - S.-et-L. : roch. calc. de Dezize, Saint-Cernin, Rumigny, Cuiseaux. - Valais : roch. calc. de l'Ermitage près Saint-Haurice, et dans la vallée de Bagnes à la Monaya. - H.-Sav. : roch. calc. Salève, vallon des Usses près la Caille, Magériaz et mont Tournette près du lac d'Annecy, montagnes de Vacheresse. - Sav. : roch. calc. mont du Chat, les Bauges au Galopaz et mont Drizon. - Is. : calc. jurass. mont Rachais, Saint-Eynard, Grande-Chartreuse, Pariset, Crémieu. - H.-Alp. : calc. jurass. de Charance, col de Glaise, Rabou près Gap, fort des Têtes à Briançon, pic de Chabrières, bois de Loubel, mont Séuse, mont Morgon, Boscodon, montagne de Faye, pont de Corbière sur le Drac. - B.-Alp. : roch. calc. Condamine, Saint-Paul. - Dr. : calc. jur. des environs de Lucen-Diois, Barnave. - Vaucl. : roch. calc. du mont Ventoux, Saint-Amans. - Var : roch. calc. de la Sainte-Baume. - Alp.Mar. : chaînes calc. du mont Chciron, d'Estenc, Saint-MartinLantosque, de Saint-Dalmas à Tende. - Gard : Alzon, Cam- 
pestre, Montdardier, Saint-Saureur près Camprieux. - Hẻr. : roch. calc. du pic Saint-Loup, Saint-Jean-de-Fos, le Caylar, Mas-de-Mouriès, Pas-de-l'Escalette, le Pertus, Roqueredonde, Sorbs. - Pyr.-Or. : environs de mont Louis, la Preste, escarpement du Canigou. - Cette espèce est exclusivement calcicole.

R. pumila, L. - Rochers. - N'est connu dans la chaine jurassique que sur la cime du Mont-d'Or. - Valais : Valére et Tourbillon près de Sion, mont d'Orge, Pierre-à-Voir sur Saxon, Evolena, Findelen, Leulierbad. - H.-Sav. : le Môle près Bonneville, Vergy, Grand-Bornand, vallèe du Fier à la Balme de Dingy, chaîne des Aravis, montagnes du Chablais au mont Héribelle près Bonnevaux, dents d'Oche, mont des Cornettes au-dessus de la Chapelle, chaine du mont Blanc, moraines de la Mer de Glace. - Sav. : montagnes des Bauges, Margériaz, Nivolet, Arclusan, Drizon, montagnes de Tignes et mont Cenis. - Is. : col de la Ruchère, la Pinéa au-dessus de Sarcenas, la Moucherolle, de Gresse au mont Aiguille. - H.-Alp.: Villard-d'Arène, col Isoire, Brunissard, le Queyras, mont Aurouse, mont Chaillol, mont Chabrières, col de Glaise, col du Noyer au prẻ de l'Aigle, col de la Palette près Chaudun. - B.-Alp. : Grande-Serène, Châtelard, la Condamine, Saint-Paul. - Alp.-Mar. : col de Tende, la Briga, Saint-Sauveur, Estenc, Bouziejo, Salsamorena. Pyr.-Or. : Font de Comps.

R. alaternus, L. - Rochers. - Commun dans la Provence, les Alpes-Naritimes, le Roussillon, l'Aude, l'Hérault, le Gard. Remonte dans J'Ardèche vers Viviers, la Voulte, le Pouzin, vallée d'Ouvèze; dans la Drôme autour de Nyons el de Buis; dans les Basses et Hautes-Alpes vers Sisteron, la Saulse, Ribiers, et dans l'Isère jusque vers Vienne et les environs de Grenoble à la Bastille, Rabot, Saint-Martin-le-Vinoux, Comboire, Pontde-Claix.

R. Clusii, Willd. - Ile de Porquerolles.

I. frangula, L. - Bois et licux humides de la partie septentrionale et moyenne du bassin. Plus rare dans la région méridionale où on l'indjque sur quelques points des Alpes-Maritimes et du Var, notamment à Nice au Var, à Fréjus ; puis dans l'Hérault à la 
Salvetat, Fraïsse ; dans le Gard a Alais, Anduze, Chartreuse de Valbonne, et enfin dans le Roussillon, à la montagne de Céret, Arles, Baus-de-l'Aze, bois de Saint-Martin du Canigou.

\section{TÉRËBINTHACËES.}

PIstaci lentiseus, L. - Bois el rochers des Alpes-Maritimes, de la région littorale de la Provence, du Languedoc et du Roussillon.

P. terebinthus, L. - Rochers de la région méridionale. Remonte dans le Vivarais, le Dauphiné et jusqque dans le Bugey et la Savoie. - Drôme : Saint-Vallier, Tain, Saou près Crest, Nyons, Saint-May, Saliune, - Ardèche : Viviers, la Voulte, le Pouzin, Andance. - B.-Alp. : Sisteron, - H.-Alp. : Tallard. - Is. : Vienne, Crémieu, Rochefort près du Pont-de-Claix, Comboire, la Bastille de Grenoble. - Sav. : autour du lac du Bourget, à la base du mont du Chat et de Saint-Innocent à Chindrieux. Ain : Lavours, Muzin, Saint-Benoit, Serrières, Saint-Sorlin.

P. vera, L. - Cultivé dans la Provence, le Roussillon, et quelquefois subspontané.

RHus coriaria, L. - Coteaux rocailleux de la parlie littorale de la Provence. - Gard : bords du Gardon, Pont-du-Gard, la Baume, Saint-Nicolas. - Hér. : environs de Montpellier, de Béziers, Pézenas, Lodève. - Aude et Pyr.-Or.

r. cotinus, L. - Coteaux secs de la région méridionale. - Vaucl. : Avignon, en tre Cassagne et Vedenes, Gadagne, Caumont, Orange, Sérignan, Carpentras. - B.-du-Rh. : Notre-Dame-des-Anges, vallon du Rouet, crête de la Trévaresse près Aix. - Var : Montrieux, Fox-Amphoux, le Luc. - Alp.-Mar. : Grasse, l'E'stérel, de Nice à Menton. - Gard : bords du Gardon, SaintNicolas, Anduze, Tresques, Nimes. - Pyr.-0r. : vallée du Réart, de Thuir à Corbère. - Remonte dans l'Ardèche à Rochemaure, le Combier, Pouzin, vallée d'Ouvèze, la Voulte, Crussol ; dans la Drôme à Nyons, Condillac, Barnave, Grannes, Crest ; dans les B.-A!p. à Sisteron; dans les H.-Alp. aux environs de Gap à Charance, la Garde, Béotard, Eymeyères, Saint-André-de- 
Rosans, Montjai ; lans l'Isère a Var's, Comboire, mont Rachais, Ia Balme près Crémieu; dans l'Ain à Villebois, Serrières, Lhuis, Groslée, Belley; dans la Savoie au pied du mont du Chat vers Hautecombe et sur l'autre rive du lac du Bourget à Chindrieux près Saint-Innocent; dans la Haute-Savoie: environs de Thonon à Reyvroz, Féterne ; enfin dans le Valais aux Marques près Martigny, au-dessus du ponl de Rossetan et entre Campel et Leukerbad.

CNEAre U1 triedecsm, L. - Coteaux pierreux de la région méridionale jusque dans les Pyrénées-0rientales. - B.-du-Rh. : environs d'Arles. - Alp.-Mar. : Antibes, Nice, Villefranche, Monaco, Menton. - Gard : bois de Paris près Sommièrcs. Hér. : Villeneuve, Maguelonne, Balaruc, Saint-Mathieu-deTréviès, mont Saint-Clair près Cette. - Aude : garrigues des environs de Narbonne. - Pyr.-Or. : plateau de la Trencada d'Ambulla, garrigues de Baixas, de Cases-de-Pena jusqu'à SaintPaul, Salses.

\section{PAPILIONACÉES.}

ANAGyens foedie, $L_{\text {. }}$ - Coteaux arides de la région méridionale. - B.-du-Rh. : Iontmajour près Arles, colline des Pauvres près Aix, les Martigues, Saint-Jean-dc-Garguier. - Var : 0llioules, Touris et Sainte-Marguerite près Toulon. - Alp.-Mar. : Château de Nice. - Gard : environs de Nìmes. - Hér. : le Moulin-du-Trou aux bords de la Mosson, Pas-du-Loup près Nišan, collines de Marennes entre Pézenas et Montagnac, Saut de-l'Eau près de l'abloayc de Vallemagne. - Pyr.-Or. : entre Oms et Saint-Marsal, vallée de Valmanya, revers de SaintMarsal.

UICX europmens, Smith, - Lieux arides des terrains siliceux. - H.-Saône : grès rouge et vosgien à Ecromagny, Fouillies audessus de Champagney, grès bigaré à Fougucrolles, diluvium à Menoux, Lure, oxfordien à chailles siliceuses à Grattcry, Grandvelle, alluvions de la Saone entre Beaujeu et Mercey. Versant méridional du Salbert près Bclfort. - Douba el Jura : 
Boulot près Besançon, granite de Ifenotey, argiles à cailloux siliceux de la forêt de Chaux près Dôle, sables bressans du bois de Balaisscaux prìs Chaussin, grè's heuprique de Cernans près Salins. - Ain : argiles tertiaires de la Dombes et de la Bresse entre Montluel et Sainte-Croix, les Echeyx, le Plantay, SaintDenis, Châtillon-les-Dombes, environs de Bourg. - Nul dans le bassin helvétique. - Région granitique du Morvan dans Cote-d’Or el Sâne-et-L., Vrilly, le Moulin-à-Vent près Saulieu, Bcuvray; se trouve aussi à Ouges près Dijon. - Rh. : granite d'Alix, Grézieu-la-Varenne, Francheville - Loire: grès houiller ct granite des environs de Saint-Chamond, Grand-Croix, SaiıtEtienne. - Is. : granite des bords du Rhône entre Vienne et Revantin, sables sıliceux miocènes de Chambaran. - Drôme : granite des environs de Saint-Vallier. - Vaucluse : environs d'Apt en face des Beaumes, - Var : Toulon, ille de Porquerolles, Ampus. - Hér. : bains de Rieumajou près la Salvetat. - Pyr.Or. : vallon de Villefranche, vallée de Conat.

v. pnrviftorus, Pourr. - Licux arides des terrains siliceux de la région méridionale. - Vaucl, : environs d'Apt, Lourmarin. B.-tu-Rh. : cailloux quartzeux de la Crau, environs d'Aix. Gard : cailloux quartzeux du bois de Campagne près Nimes. Hêr. : garrigues de Roquehaute près Agde. - Pyr.-Or. - Remonte dans l'Ardèche vers Baix.

v. provincialis, Lois. - Var : Saintc-Mlarguerite près Toulon, Pourcieux, Esparron, Saint-Cyr.

v. manus, Smith. - Lreux stériles des terrains siliceux. - H.Saone : terrains quartzeux de Chassey-les-Scey, Menoux, Ferrières. - Rh. : granite d'Ecully, Dardilly, Alix. - Loire: granite des environs de Saint-Étienne et de Montbrison, Changy, Chavanay près du Rhône.

CAтусотолI spinosa, Link. - Coteaux arides de la Provence méridionale, des Alpes-Naritimes, du Languedoc et du Roussillon.

SPARTIUM junceum, L. - Coteaux secs de la région méridionale. Remonte dans la Drome de Saint-Paul-Trois-Châteaux à Donzère, Montélimar, Crest, Laveyron près Saint-Vallier, 
Sahune; dans l'Ardèche à Viviers, Rochemaure, Baix, Cruas. - Rh. : déblais des carrières du mont Cindre, de Couzon et de Limonest, Fleurie en Beaujolais. - S.-et-L. : Romanèche.

SA rotinamus scoparius, Wimm. - Lieux incultes des terrains siliceux et argilo-siliceux. - H.-Saône et arrondissement de Belfort : granites et porphyres de la zône vosgienne. Doubs, Jura et Ain : lisière vosgienne de l'arrondissement de Montbéliard, bois sablonneux des plaines; ne se montre dans la chaîne jurassique que sur les sables sidérolitiques ou sur les roches siliceuses transportées. - Très-commun dans toute la chaine granitique du Morvan, Beaujolais, Lyonnais, Forez, Pilat, Vivarais et jusque dans les Cévennes et leurs contreforts, dans la partie montagneuse du Gard et de l'Hérault; nul dans la partie basse de ces départements. - Is. : parties basses des chaines granitiques d'Allevard et de Belledonne, sables de la mollasse du Dauphiné et de la Savoie. - Drôme : granite de Saint-Vallier.-Pyr.-Or. : tous les coleaux de la partie centrale du département d'où il remonte très-haut dans les trois vallées. - Aude : Pinède de Fontfroide, Rennes-les-Bains. - Rare dans Ia Provence et les Alpes-Maritimes, sur les terrains sablonneux des environs d'Arles, Fox-Amphoux, Gréoulx, Saint-Vallier et Gourdon près Grasse, monts de Lachen. - Cette espèce, si commune sur les terrains siliceux de la plus grande partie de la France, est fort rare dans le bassin helvétique; on ne la signale que dans les bois de Prangins et de Ferney près Genève et vers Allaman, Buchillon, Signal d'Aumont dans le canton de Vaud.

s. nxhoreus, Webb. - Coleaux des Pyr.-Or. entre Céret et SaintMarsal, vallée du Réart, montagnes de Salses, les Corbières, Case-de-Pena, Força-Real.

5. purgans, Godr. Gren. - Lieux arides des terrains siliceux. Loire : commun dans le massif granitique du Pilat, de Pierresur-Haute el des montagnes du Forez. - Ardèche : toutes les montagnes granitiques et les collines de même nature qui bordent le Rlıone à Andance, Tournon, Celles, Baix, Chomérac, Rochemaure. Monte dans l'intéricur à Vals, Mayres et jusqu'au 
Gerbier-des-Joncs, la Roche-Gourdon, le Mẻzayon. - Drôme : coteaux granitiques entre Saint-Vallier ct Tain. - Alp.-Mar. : Contes. - Gard: dans les Cévennes à l'Espẻrou, Genolhac, Alzon, Concoule. - Hér. : massif granitique et schisteux de l'Espinouse à Avène, Saint-Amand-de-Nounis, Fraïsse.-Pyr.Or. : Montlouis, le Canigou, Costa-Bona, Font-de-Comps. - En dehors de nos limites, on le trouve dans les Pyrénées centrales, dans la Lozère et au mont Dore.

GEVIsTA argltalis, L. - Pelouses sèches dans tout le bassin depuis les plaines jusuu'aux sommités des montagnes; toutefois il est plus rare dans les rígions méridionales où il ne se montre que dans les montagnes du Garù à l'Espérou, Alzon, Salbous, Concoule, Genolhas et dans l'Hérault sur les montagnes de l'Escandorgue et du Larzac et dans les montagnes des PyrénéesOrientales. - Var : rare dans les Maures de Pignans et du Luc. - Alp.-Mar. : montagnes des environs de Berre, Bollena, forêt de la Maïris, bois de Gourdon, Vence.

G. Melphinensis, Verlot. - Montagnes de la Drôme, mont Toulau près Bouvante, mont Embel.

G. pilosn, L. - Coteaux secs. - Manque dans le Valais. - Disséminé et très-inégalement réparti dans le reste du bassin.

G. Viunrsinna, Jord. - G. humifusa, Vill. - Lieux secs et arides des montagnes.-Dr. : montagne d'Angèle.-H.-Alp. : BrameBuou près Montrond, montagne de Clialcauneuf-de-Chabre, entre Serres et Laragne. - Vaucl. : mont Ventoux. - Var : escarpements du Verdon près d'Ampus, montagnes de Margès près d'Aiguines.

G. tinetoria, L. - Prairies humides dans tout le bassin.

Variété lasiocarpa. - H.-Sav. : Pringy, Tuvat. - Sav. : Cruet, Rhonne près Albertville. - Alp.-Har. : Thorenc près Grasse.

G. ovata, Waldst. Kit. - Valais : la Crotta entre Saint-Maurice et Outre-Rhône. - Sav. : Grignon frès Albertville, la Maurienne. - Cette espèce doit être ajoutée à la Flore de France.

C. cluerea, D. C. - Licux secs des montagnes. - H.-Alp. : Charance et la Garde près Gap, Manteyer, Rosans, Serres, Ribiers. Dl. : entre Bouvante et Embel, entre Durbon et Saint-Julien- 
en-Beauchêne, Aucelon, graviers de la Drôme. - Is. : iles de Champagnier. - B.-Alp. : Digne, Sisteron, Gréoux, Signes, Manosque. - Ard. : roch. basaltiques du mont Combier, la Bastide de Suvinas. - Vaucl. : environs d'Apt, d'Orange, alluvions de l'Aigues. - B.-du-Rh. : environs d'Aix, Roussargue, Peyrolle, Jouque. - Var : la Roque-Broussane, Sainte-Baume. - Alp.Mar. : Contes, Grasse, Saint-Vallier, Caussols, Revest, Coursegoules, de Tende à Saint-Etienne-le-Saurage et jusqu'à Menton et Nice.

cr. aspalathoides, Lam. - Licux pierreux des montagnes. Vaucl. : mont Ventoux. - B.-du-Rh. : sommets de Saint-Cyr et de Carpiagne, Sainte-Victoire, Cujes. - Var : monts Faron et Coudon près Toulon.

G. Lobelii, D. C. - Sainte-Victoire et Sainte-Baume.

G. scorpius, D. C. - Lieux stériles de la région méridionale. Manque dars le Var et les Alpes-Maritimes. - Vaucl. : trèscommun dans les environs d'Orange et d'Avignon. - B.-duRh. : environs d'Aix, crête de la Trévaresse, Barbentane, Graveson, les Alpines. - Hér. et Aude : environs de Montpellier, de Béziers et de Narbonne.-Gard : environs de Nimes, Anduze, Saint-Ambroix, le Vigan, Alzon.- Remonte dans la Drôme vers Pierrelatte, Nyons, Crest, Donzère, Montélimar, Granne, SaintRoman, Sahune, Rémuzat ; dans les Hautes-Alpes vers Rosans, Ribiers. - Remonte dans l'Ardèche vers Viviers, Rochemaure, Cruas, mont Charray, l'Escrimet, Vallon, Baix, Celles.

G. angliea, L. - Coteaux arides des terrains siliceux. - Nul dans les chaînes vosgiennes et jurassiques; manque aussi dans les cantons du Valais, Vaud et Genève. - Còte-d'Or : massif gianitique du Morvan autour de Saulieu et d'Arnay-le-Duc, sables tertiaires des environs de Louhans, Abergement-les-Seurre. S.-rt-L. : montagne granitique de Beuvray. - Rh. : granite de Tássin, Dardilly, Craponne, l'Argentière. - Loire : massif granitiçue du Pilat. - Ain : terrains argilo-sıliceux de la Dombes et de la Bresse. - Is. : sables tertiaires des environs de la CôteSaint-André. - Ard. : terrains volcaniques du mont Mlezenc, Chaplas-du-Plaignal, le Bez. - Gard : terrains granitiques et 
schisteux des environs du Vigan, Campestre, Genollac, Concoule, Alais. - Hér. : massif granitique el schisteux du Caroux et de Fraïsse. - Nul dans la Provence, les Alpes-Mlarilimes et la région basso du Gard, de l'Hérault et du Roussillon.

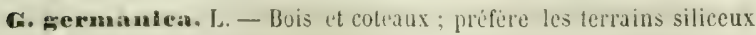
et argilo-siliceux mais non-exclusivement. - Haute-Saône et arrondissement de Belfort : zône vosgienne à Champagney, la Justice, bois de Vezelois. - Jura : granite de forêt de la Serre, argiles à cailloux siliceux de la forêt de Chaux, plateaux de Salins à Saint-Amour, là oủ existent des terrains de transport. - Valais: Zwischbergen. - Environs de Genève dans les bois sablonneux de la Bàtie, ues Frères, du Vangeron. - Cóte-d'Or : Vielverge. - S.-et-L. : Saint-Emilland, Cluny, Cuiseaux. Terrains de transport des environs de Lyon, d'Annecy, Bonneville, Chambéry, Gap, Rosans, Crest, Saint-Vallier, et dans l'Isère à Monestier-de-Clermont, Vouilland; dans l'Ardèche à Bez. - Gard : Barsac, l'Espérou. - Région montagneuse des Alpes-Maritimes. - Nul dans Vaucluse, les Bouches-du-Rhône, le Var et la partie basse du Languedoc et du Roussillon. Dans ces deux dernières provinces il occupe la région montagneuse.

f. hispaniea, L. - Coteaux stériles de la région méridionale. Dr. : Chamousse près Séderon, Nyons, mont d'Angèle, Beaufort. - H.-Alp. : Ribiers. - B.-Alp. : Sisteron, Gréoux. - Vaucl. : mont Ventoux, Saint-Amans, Caromb, Lourmarin. - B.-duRl. : environs de Marseille et d'Aix, Mauret. - Var : environs de Toulon, du Luc et de Fréjus. - Alp.-Mar. : Grasse, Vence, l'Escarène, Cimiez et Falicon près Nice, Contes, l'Aggel audessus de Menton. - Gard: le Vigan, Alzon, Campestre, l'Espérou. - Hêr. : Grammont, Grézac, environs de Lodève, le Larzac à Saint-Pierre-de-la-Fage, le Cros, le Caylar, SaintMichel-dez-Sers, Gardies-sur-l'Escandolgue. - Roussillon.

C. candicans, L. - Bords des buis des terrains siliceux. - B.-duRh. : les Baux, les Apines. - Var et Alp.-Mar. : phyllades, quartzites d'Hyères, ille de Porquerolle, environs de Toulon, granites et porphyres des Maures et de l'Estérel, Cannes. Gard: sables siliceux des environs de Nimes, de Cygnan et de 
Broussan, Manduel, Anduze. - Hér. : sables siliceux de Grammont près Montpellier, environs de Pézenas et de Lodève. Aude et Pyr.-Or. : Salses, Opol et toutes les Corbières, PortVendres, Banyuls-sur-Mler.

G. radiatn, Scop.-Lieux arides des montagnes calcaires. - Valais : Ardon, ravin de la Sionne au-dessus de Sion, la Chaux-de-Lens, Croumaclire, Savièze. - H.-Alp. : montagne de Mlaraysse, Combe-Noire, mont Séuse. - B.-Alp. : montagne de Lure.

G. Inifolia, L. - Var : phyllades siliceuses et quartziles de Carqueiranne et Colle-Noire entre Toulon et Hyères, Hle de Porquerolles.

G. horridn, D. C. - Coteaux de Couzon près Lyon. - Pyr.-0r. : montagne à l'est de Bellegarde, entre Saint-Marsal et Runet. Cette rare espèce ne se trouve ailleurs en France que dans les Hautes-Pyrénées à Ordincẻde dans la vallée de Campan et au mont Sacon dans la Barousse.

CXTisUs Iaburnum, L. - Bois des terrains calcaires. - Nul sur les terrains siliceux des Vosges, du Morvan, Beaujolais, Lyonnais, Forez, Pilat, Vivarais, Cévennes, Alpes, Bresse et Dombes.- Caractéristique des chalnes jurassiques de Côte-d'Or, Saône-et-Loire, Doubs, Jura, Revermont, Bugey, Dauphinẻ septentrional et parties de la Haute-Savoie et de la Savoie voisines du Bugey aux monts Vuache, Salève, Pringy, mont du Chat, Ifont-d'Or lyonnais. - Cet arbrisseau manque dans le bassin helvétique si ce n'est à Saint-Haurice et Chemin-Neuf dans le Valais. - Vaucl. : massif néocomien du mont Ventoux. - Var : Saint-Pons, Gemenos, Ampus ; nul ou très-rare dans le reste de la Provence. - Manque dans le Gard et l'Hérault, quoiqu'il reparaisse dans le Roussillon, vers Força-Réal, SaintPaul et Caudiès.

C. alpinus, Miller. - Bois des montagnes. - Nul dans la chalne vosgienne. - Doubs : Pontarlier et sur le versant helvétique au Suchet. - Jura : la Dôle, Faucille, les Rousses, Colombier, Reculet, la Chaux-du-Dombief, Champagnole, Mirebel près Lons-le-Saunier, Salins. - Ain : fort de l'Ecluse, bords de l'Albarine, entre Chaley et Hauteville, Hotonnes, Colliard, Co- 
lombier-du-Bugey, cluses de Nantus. - Haute-Savoie: environs d'dnnecy, vallée de Thônes, Barberine à Tête-Noire. - Valais : Salvan, la Forclaz, Chemin-Neuf, Fully. - Sav. : mont du Chat, montagnes des Bauges, de la Tarantaise et de la Maurienne. - Manque dans l'Isère. - H.-Alp. : Boscodon, Rabou, Chaudun, mont Séuse. - Drôme : les Goulets près la Chapelleen-Vercors, bois de Rochecourbe près Crest, Chartreuse de Durbon. - Var : Ampus près du Verdon. - Alp.-Mar. : forèt de la Maïris, mine de Tende, Sainte-Anne-de-Vinaï, Clans, vallon de Salèze, Saint-Etienne-le-Sauvage, Coursegoule. Pyr.-0r.: vallées de Carença et de Prats-de-Balaguer vers le Roc-del-Buc.

C. sessllifolius, L. - Coteaux secs. - Sav. : les abînes de Hyans près Chambéry. - Is. : Crémieu, Bastille de Grenoble, Sassenage, Comboire, la Motte-les-Bains, Mens. - H.-Alp. : environs de Gap et d'Embrun, Rosans, montagne de Faye près Ventavon. - B.-Alp. : la Condamine. - Drôme : Granne, Nyons, Die. Ard. - Crussol, le Pouzin, la Voulte, Privas, mont Charray, Aubenas, rocher de Sastre, Baix. - Vaucluse : Saint-Amans, Mornas, mont Ventoux, Flassan. - B.-du-Rh. : environs d'Aix, Mauret. - Var : Toulon, Aups, Draguignan, le Luc, HautReyran, Mfontrieux, Bagnols. - Alpes-Mar. : Estérel, Cannes, Nice, Saint-André, Menton. - Gard : Alais, Anduze, le Vigan, Alzon, bords du Gardon. - Hér. : Saint-Loup, Capouladou, Cambous, Viols, Montpeyroux, Sérane, le Caylar. - Assez commun dans l'Aude et les Pyrénées-Orientales.

C. nigricans, L. - Indiuué par Mutel à Brie de Saint-Chizi au pied du mont Viso oủ il n'a pas été retrouvé. M. Godron dit qu'il existe en Savoie où personne, à ma connaissance, ne l'a vu.

C. decumbens, Walp. - Pelouses des montagnes. - Doubs : Bélieu, Russey, Pontarlier, Besançon.- Jura : Salins, Poligny, Dòle, forêt de la Serre. - Nul dans la chaîne rosgienne el dans le bassin helvétique. - Cote-d'Or : chaine jurassique de Gevrey à Beaune.- S.-et-L. : coteaux calcaires de Dezize, Saint-Cernin, Berzé-le-Chàtel, Mercurey, Remigny, Saint-Sorlin, Cuiseaux.

C. triflorus, Lhér. - Bois des lerrains siliceux de la région méri- 
dionale. - Var : forêt des Maures, Garde-Freynet, bords du Reyran et du Reyranet. - Alp.-Mar. : l'Estẻrel, Auribeau, Cannes, Biot, Menton. - Hér. : cnvirons de Lodève, entre Hérépian et les Aires. - Pyr.-Or. : vallée d'Argelès-sur-Mer, forêt de la Massane, vallon de Collioure, Consolation, Banyuls-surMer, Cases-de-Pena et Corbières.

C. binorus, Lhẻr. - Collines arides. - Is. : Crémieu, balmes de Décines. - Ain : Néron près Lyon.

C. elongatus, Waldst. et Kit. - Coteaux calcaires de l'Ardèche à Châteaubourg, mont Combier. - Se trouve aussi sur l'autre rive du Rhône dans la Drôme, d'après J. Fourreau.

C. hipesutus, L. - Bois des Alpes-Har. : la Madona pres Menton, Levens, Berre, Saint-Cassien-de-Siagnes près Grasse.

C. enpitatus, Jacq. - Bois. - II.-Saone : calcaires jurassiques de Valay, Venère, Vallerois-le-Bois. - Doubs et Jura : plaines et collines de la région du vignoble, la citadelle de Besançon et Chalezeule, Villers-Farlay, Quingey, Belin et Poupet près Salins, Arbois. - Côte-d'Or : chaîne jurassique de Gevrey à Beaune. - S.-et-L. : Gergy, Saint-Bonnet-en-Bresse, Demigny, Givry, Buxy. - Bh.: Oullins. - Ain : la Pape, Saint-Ramberl, Ceyzériat, Hautecour, Bohas, Villereversure, le Revermont. Is. : Crẻmieu, Vernaz, Annoisin, Jonage, Mollard de Décines, les Dauphins-sur-Romanche. - Savoie : les abìmes de Myans près Chambéry. - Hér. : environs de Béziers. - Pyr.-0r. : montagne de Força-Réal, basses Albères, montagne de Céret.

C. supinas, L. - Pelouses des hasses montagnes. - Cote-d'Or: Vielverges. - Sav, : les abimes de Myans, Apremont, SaintMichel. - Is. : mont Rachais, Comboire, Claix, Monestier-deClermont. - H.-Alp. : mont Bayard, bois de la Selle et de SaintJean. - Var : forêı des Maures, Frîjus. - Alp.-Mar. : col de Tende, mont d'Or près de Luceram.- Gard : l'Espérou._- Pyr.Or. : montagne de Saint-Laurent-de-Cerdans, Costujes, la Sadeille au-flessus de la Manère, tours de Cabreins.

C. aIpese ras, , Thuret et Bornet. - Alp.-Nar. : vallons de Nanducbis et de la Nadone, mine de Cérèse et col de Salèse.

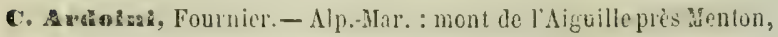
monts du Chier et du Cheiron, Bezaudun. 
C. axgenteus, L. - Coteaux secs. - Ain : Neximieu aux Peupliers, Chazey, Château-Gaillard, Loyette, Serrières, le Sault, SaintSorlin. - Sãv. : abîmes de Hyans.-Is. : Crémieu : Hyères, Bastille de Grenoble, Parisct, Comboire, Mens.-Drôme : Gervan, Loriol, Montélimar, Nyons, Barnave, Luc-en-Diois. - Ard. : Tournon, la Voulte, le Pouzin, Baix, vallée d'Ouvèze, mont Charray, Rochemaure.-H.-Alp.: Gap à la Roche, au vallon de l'Ayasse, Rosans, Ribiers.-B.-Alp. : la Baume prèss Sisteron. - Vaucl. : environs l'Orange et d'Avignon à Mont-de-Vergues. - B.-ilu-Rh. : les Alpines, environs de Marseille et d'Aix. Var : Toulon, Porquerolles, Bagnols, Saint-Quenis près Camp, le Luc. - Alp.-Nar. : Grasse, de Menton à Nice. — Gard : Nìmes, Uzès, Beaucaire, Cabrières, colline des Angles et de Villeneuve. - Hér. : Saint-Loup, Fonfrède, Maurin, Montpeyroux, Cette et la plus grande partie des départements de l'Hérault, de l'Aude et des Pyrénées-0ricntales.

ADEXOCAREUS graxahiforus, Boiss. - Coteaux arides. Var : Hyères.-B.-du-Rh. : golfe des Lèques près la Ciotat, Cassis. - Dans l'Aude et le Roussillon vers Port-Vendre, Banyuls-sur-Mer, les Basses-Corbières et Fontfroideprès Narbonne.

A. conmmutatus, Guss. - Coleaux arides. - Gard : le Vigan, l'Espérou, Anduze, Alais, Concoule. - Hér. : Ganges. - Aude : Pinède de Fontfroide. - Ard. : Joyeuse, Viviers, vallées de Vals et d'Entraigues, la Bégude, Prades.

A. eompheatus, Gay. - Cette espèce des Pyrénécs hautes et orientales se trouve, par une singulière exception, dans la Côte-d'or vis-à-vis Flammerans dans les bois d'Auxonne, puis dans la partie voisine du Jura sur les coteaux granitiques de Menotey près Dôle et aussi sur la lisière occidentale de la forêt de la Serre jusqu'à Mloisscy. M. Legrand l'indique dans la Loire au-dessous de Notre-Dame-de-Grâce dans lo vallon qui descend à Cessieux.

LEProcs termis, Forsk. - Moissons, - Var : Toulon, Hyères, Cavalaire, Maures. - Alp.-Nar. : cap Croisette à Cannes. Hér. : Roquehaute.

C. Hiz'sutås, L. - Coleaux des terrains siliceux de la région méri- 
dionale. - Var et Alp.-Mar. : gneiss, micaschisles, granites et porphyres, forêt des Maures, mont Sauvette près lęs Mayons, Hyères, le Luc, Fréjus, l'Estérel, Cannes. - Hér. : la Campagne des Hours entre Montpellier et Mauguio, bois de Lamoure, garrigues de Prègnes, Rocjuehaute, Ripaute. - S'est montré accidentellement en Savoie à Montmeillan.

L. eryptrnthos, Shuttleworth. - Var : Hyères, les Mayons.

C. reticulatus, Desv. - Champs sablonneux. - Var : iles d'Hyères, forìt des Maures, Saint-Tropez, Fręjus, Saint-Raphael. - Alp.Mar. : Cannes au cap Croisette, Grasse, la Roquette, Antibes, Menton, - Hér. : sables siliceux du bois de Grammont près Montpellier, Pézenas, Lodève. - Pyr.-Or. : Banyuls-sur-Mer.

L. angustifollus, L. - Terres cultivées. - Var, Fréjus, le Luc, la Sauvette, Callobrières. - Gard : Campagne et Broussan près Nimes, Saint-Hippolyte de Monłaigu, Treqques, Milhaud. Hér. : Pardailhan-Pontguiraud. - Pyr.-Or. : Força-Réal.

ONONIS rotundifolia, L. - Débris de rochers des montagnes, quelquefois entrainé dans les vallées basses et dans les plaines comme, par exemple, au Polygone de Grenoble. - Valais : Saxon. mont d'Orge, Saint-Leonhard, Leukerbad, Zermatt, Ganter, Simplon. - H.-Sav. : voûtes supérieures du Petil-Salève, entre Crevin et le Coin, base du Môle à Reizet près Bonneville, les Bauges. - Sav. : d'Aigueblanclıe à Moutiers, Salins, Avrieuxen-Maurienne. - Is. : col de l'Arc en descendant sur SaintPaul-de-Varces, la Motte-les-Bains, Dent de Moirans, Mens. H.-Alp. : la Grave, Briançon au fort des Têtes, Bramousse audessus de Chăteau-Queyras, environs de Gap à Charance, côte Gélive, Bayard, col de Chaudun, le Pleyne, Loubet, la Grangette, montagne d'Avançon, Puy-Maurel, Sigoyer, Durbonnas. - Dr. : le Glandaz près Die. - B.-Alp. : Barcelonnette, Condamine, Saint-Paul, Rissole. - Alp.-NIar. : Levens, le Chaudan, Touët-de-Beuil, entre Grasse et Castellane, Saint-Etienne-leSauvage. - Gard : Saint-Ambroix, Anduze, - Pyr.-Or. : vallées d'Eyne et de Llo.

O. Fruticosa, L. - Débris de rochers. - Sav. : les abimes de Myans près Chambéry, Apremont, la Maurienne - Is. : la 
Tronche pris Grenoble, Saint-Eynard, Vif, Prẻlanfrey, Monestier-de-Clermont. - H.-Alp. : environs de Gap, Romelte, SaintMens, Riotord, Pont-Sarrasin, Puy-llaurel, Rambaud, Eymeyeres, Ventavon, Tillard, col du Noyer, de Guillestre à la Maisondu-Roi, Saint-André-de-Rosans, Bruis, Ribiers au mont Rognouse. - Dr. : Montmaur près Die. - B.-Alp.: Digne, Barcclonnette, la Condamine, Tournoux, vallée de Larche. Alp. Nlar. : entre Berre et Bendigium, Utelle, Tournafort, la Maria, Saint-Etienne, Guillaumes, Entraune. - Gard : Anduze.

D. natrix, L. - Coteaux graveleux. - Cette espèce polymorphe est extrêmement commune dans la vallée du Rhône de Genève à Lyon et au-delà dans l'Isère, la Drôme et l'Ardèche, dans la vallée de la Saône à travers les départements de Saône-et-Loire et Côte-d'Or, dans les vallées de l'Arve, de l'Isère, de l'Arc, de la Drôme, de la Durance et de leurs aflluents. - Moins commune dans le Languedoc, le Roussillon, la partie basse de la Provence el les Alpes-Maritimes. - N'existe en Suisse que dans la vallée du Rhône à Martigny, Branson en Valais, puis à Bévieu, Aigle, Ollon dans le canton de Vaud, et enfin autour de Genẻve. - Rare dans le Jura à Thoirette et de là sur les bords de l'Ain jusqu'à l'embouchure de la Bienne. - Nulle dans la vallée du Rhin soit en Alsace, soit plus loin dans le Rheinpfalz. - La grandeur des fleurs et la forme des grappes présentent, dans celte espèce, de nombreuses variations.

D. arachmohlea, Lapeyr. - Débris de rochers granitiques dans la vallée de la Romanche entre les Dauphins et la Grave. B.-Alp. : Condamine.

0. ramosissima, Desf. - Lieux sablonneux. - Vaucl. : environs d'Avignon. - B.-du-Rh. : Saint-Chamas, Bouc, la Camargue. - Var et Alp.-Mar. : Hyères, Fréjus, Grasse, Cannes, Nice. Gard : Aigues-Mortes. - Hér. : Montpellier, Pérols, Maguelonne, Palavas, Cette, Agde, les Onglous, Vendres. - Littoral des Pyr.-0r.

9. viseoss, L. - Cliamps arides de la région mẻditerranéenne. B.-du-Rh. : Brignon, Tholonet, le Prẻgnon près Aix. - Var : Toulon, Baon-r!c-Quatre-Heures, Carqueiranne, Pierrefeu, Fréjus, Catal. Bassin d:ı Rhône. 
Pépières près Saint-Nazaire. - Hér. : Montpellier, Saint-Georges. - Pyr.-Or. : Cases-de-Pena, Estagel, d’Arles à Prats-de-Mollo, Prades, 'Trencada d'Ambulla, le Vernet.

Variété breviflora, D. C. - Var: Pierrefeu, le Luc, Fréjus. - Alp.-Mar. : Grasse, Antibes, ile Sainte-Marguerite, Nice, Ifenton, col de Brouis, Tende.- Hér. : Iontpellier, Saint-Loup, Pérols, garrigues du Terrail, Celleneuve, Aresquiẻs, Montarnaud, marais de Roquchaute, côtes de Bayssan près Béziers, Cartels près Lodève. - Pyr.-Or. : le Vernet, de Céret à Arles.

o. pubeseens, L. - Champs arides de la réginn méditerranéenne. B.-du-Rh. : Saint-Chamas. - Var : Toulon, Carqueiranne, le Revest, Bandols, Pierrefeu, ie Luc, les Mlaures. - Gard : carrières de Beaucaire, Villeneuve-lez-Avignon, les Angles, bois de Broussan près Nìmes. - Hér. : Mlontpellier, Gignac, Pézenas, Pas-du-Loup près Nissan.- Aude : Montfort, au bord de l'étang de Bages. - Pyr.0r. : parties basses de la Salanque, Cases-dePena.

o. cenisıl̊, L. - Pâturages des montagnes, d'oủ il descend quelquefois dans les vallées. - Sav. : la Maurienne à Saint-Jean, au Pas-du-Roc près Saint-Michel, Lans-le-Bourg, Lans-le-Villard, Bessans, mont Cenis, montagnes de la Tarantaise. - Is. : Mens. - H.-Alp. : la Grave, Briançon, bords du ruisseau de Cervières, col Malrif, mont Viso, páturages du Célar, col Agnel, environs de Gap à Saint-llens, Charance, Loubet, col de Glaise, la Grangette, col de Chaudun, le Pleyne, Bayard, Puy-Maurel, Sigoyer, Durbonnas, mont Séuse, mont Chabrières près Chorges, Rosans, mont Rognouse près Ribiers. - B.-Alp. : vallée de l'Ubaye à Malmore, la Condamine, Saint-Paul. - Drome : col de Lunel entre Crest et Saou, Barnave, montagnes des environs de Die. - Vaucl. : mont Ventoux. - Var : Sainte-Baume. Alp.-Mar. : montagnes de Grasse, Caussols, mont Cheiron, Mas et Andon, près Saint-Auban, Entraunes, Saint-Dalmas-le-Sauvage, Roubion, Briga, Tende. - Pyr.-Or. : sommités de la vallée de Nohèdes, Font-de-Comps, vallée de Conat, Cambres-d'Aze.

o. reelinata, L. - Lieux ariúes de la région méditerınéenne. Vaucl. : bois de Fargues près Aviguon. - B.-du-Rlı, : environs 
de Mlarscille, au nord de la Tour-de-Keirié près Aix, Monteiguez, Miramas, la Crau. - Var el Alp.-Mar. : Chartreuse de Montrieux, le Luc, ile Porquerolle, Saint-Tropez, Belgentier, ile SainteMarguerite, Cannes, Grasse, Antibes, Nice, Menton. - Gard : Aigues-Mortes, Sylveréal, bois de Broussan. - Hêr. : Cette, Pérols, garrigues du Terrail, Celleneuve, Aresquiès, Pézenas, Bézicrs, Cazoul-les-Béziers, Nissan, Capestang, Vendres.-Aude : Pecl-de-l'Agnel près Narbonne, Sainte-Lucie. - Pyrénées-Or. : vallée de Conat à Font-de-Comps.

0. enmpestrek, L. - Koch et Ziz. - Champs stériles dans tout le bassin.

0. antiquorum, L. - Lieux arides de Ia rẻgion méridionale. - Var : Hyères, Fréjus, - Gard : bois de Broussan prés Nimes. - Pyr.Or. : environs de Perpignan, Château-Roussillon, Cases-rlePena, Baixas, Força-Réal.

6. procurnens, Wallr. - Espèce polymorphe qui se trouve dans les lieux incultes depuis les plaines jusqu'aux sommités des montagnes.

o. striata, Gouan. - Coteaux arides. - H.-Alp. : Chaudun et les Baux près Gap. - B.-1lp. : Castellanne. la Condamine. Dròme : Crest, mont d'Angèle. - Vaucl. : mont Ventoux. Alp.-Nar. : Saint-Auban. Saint Dalmas-le-Saurage, Entraunes, Guillaumes, Saint-Martin-Lantosque, Tende. - Gard : Campestre, Alzon. - Hér. : le Larzac. - Pyrénées-0r. : Trencadad'Ambuila, Font-de-Comps, Saint-Antoine-de-Galamus.

b. altissina, Lam. - Cette espèce qui manque en France existe dans la partie lıclvétique lu bassin du Rhône près de Martigny, entre Saxon et Charrat en Valais.

D. Columure, All. - Coteaux calcaires. - Cotc-d'Or : environs de Dijon, route de Plombières et toute la châne jurassique de la Côte. - Même terrain dans Saône-ct-Loire. - Nul dans la chaine jurassique de Haute-Saône, Doubs et Jura. - Vaud et Valais : Ollon, Martigny, Fully, les Marques, Saxon, Montd'Orge, Tourbillon, Bramois, Eaint-I,conhard, Sierre, Salgesch.

- Ain: plaine d'Ambronay, Château-Gaillard, Loyettes, Clıazey, bords de l'Ain et du Rhône jusqu'à la Pape près Lyon. - Rh. : 
Couzon au Mont-d'Or. - Is. : calcaires jurassiques de Crémieu, Bouresse, Comboire, Pariset, la Bastille de Grenoble, Malcombe, Saint-Mens. - H.-Sav. : mont Brizon. - Sav. : SaIins près Moutiers, lac Saint-Marcel, Arbin, Chignin, Saint-Michel en Maurienne. - H.-Alp. : §aint-Mens et Malcombe près Gap, Briançon. - Ard. : Ucel, Mercuer. - Vaucl. : environs d'Avignon. - B.-du-Rh. : du Tholonet à Sainte-Yictoire. - Rẻgion montagneuse des $\mathrm{Alpes-Mlaritimes.-Gard} \mathrm{:} \mathrm{environs} \mathrm{de} \mathrm{Nìmes,}$ le Vigan, aqueduc du Pont-du-Gard. - Hẻr. : Lodève, Avène, Ifas-dc-Nouriès, Ia Halou, Iladières. - Pyr.-Or. : vallée de Caudiès, montagne du Cambres-d'Aze, vallées de Llo et de Vernet.

D. minutissĩna, L. - Coteaux et rochers arides de la Provence, des Alpes-Naritimes, du Roussillon et du Languedoc. - Remonte dans l'Ardèche vers la vallée de l'ouvèze et vers Baix, Vals, Payolive, les rochers calcaires de Chateaubourg; dans la Drôme vers Tain, Saint-IIay, Villeperdrix; dans les BassesAlpes à Sisteron, rochers de la Baume et de la Citadelle ; dans les IIautes-Alpes vers Ribiers, Rosans, Moydans; et dans l'Istre à la Bastille de Grenoble,

๑. mitiseima, L. - B.-du-Rh. : envir. de l'étang ge Marignane, Saint-Pierre près Martigues, étang desséché de Tourtines. Var et Alpes-Mar. : Toulon, iles d'Hyères, Antibes, ile SaintcMarguerite. - Pyr.-Or. : vallon, de Banyuls-sur-Mer, entre Arles et Prats-de-Mollo.

0. alopecuroides, L. - Plante de Corse trouvée aux environs de Toulon et de Fréjus. - Pyr.-Or. : vallée de Carença.

ANTHYLLS eytisoides, $\mathrm{L}$. - Rochers maritimes, - B.-duRh. : le Bec-de-l'Aigle près la Ciotat, Cassis derrière le Château à Beau-de-Canaille. - Alp.-Nar. : lle Sainte-Marguerite. Pyr.Or. : bords de l'Agly, Cases-de-Pena.

A. barba Jovis, L. - Rochers maritimes. - Var et Alp.-Har. : fort Lamalgue près Toulon, cap Brun, la redoute du milieu au mont Faron, iles d'Hyères et de Porcquerolles, Saint-Tropez, rocher des Lions près Saint-Raphaël et de là à la Napoule, Villefranche, Saint-Jean, Monaco, - Pyrénées-0r. : butte de l'Esparou entre Saint'Nazaire et l'embouchure du Tech. 
A. montana, L. - Rocliers des montagnes calcaires. Doubs et Jura : Pontarlier, Ornans, Bonlieu, Poupet près Salins, la Châtelaine près Arbois, Septmoncel, la Dóle, le Colombier, le Reculet. Nul dans les Vosges. - Cote-d'0r : rochers calcaires depuis Marsannay jusqu’à Chassagne. - S.-et-L. : roc. calc. de Dezize, Solutré, Vergisson. - H.-Sav. : calcaire urgonien du Salève entre te Coin et Crevin, mont Brizon, Vergy, Calvaire de Thônes, mont de Louvenaz au-dessus de Thuy, Dingy, mont Tournette. - Sav, : calc. jurass. du mont du Chat, la Chambotte, mont Nivolet, Chamoceran et les autres montagnes des Bauges, montagnes de la Tarantaise. - Ain : calc. jurass. des montagnes du Bugey autour d'Ambérieu, Saint-Rambert, côtes d'Eroges, Tenay, Rossillon, le Colombier, les monts d'Ain. Is. : calc. jurass, du mont Rachais et de Vertrieu, calcaire néocomien de la Ruchère, la Pinea, le Moucherotte, col de l'Arc. - Dr. : Barnave. - H.Alp. : calc. jurass, et néocomien de Charance, Rabou, côte Gélive, col de Glaise, Séuse, CombeNoire, Sigoyer, montagne de Crigne, Faudon, Loubet, calc. du lias des environs de Briançon, du bois Foran au-dessus de Villevieille en Queyras, les Pra-Hauts et les Pra-Bas, Rosans. - B.-Alp. : Sisteron au rocher de la Beaume, la Barge. - Ard. : Dent-d'Array. - Vaucl. : calc. néocomien du mont Ventoux. - B.-du-Rh. et Var : calcaire crétacé de Sainte-Victoire et Sainte-Baume, Ampus, Saint-Trowc. - Alp.-Mar. : calc. urgonien des montagnes de Grasse et de Caussols, Menton. - Gard : calc. jurassique Alzon, le Vigan, l'Espérou, Serre-de-Bouquet. - Hér. : dolomies jurassiques de Saint-Guilhem-le-Désert, Sérane, Lodève, Avène, le Caylar, Cros, Pas-de-l'Escalette, Viols-le-Fort. - Pyr.-Or. : Font-de-Comps, Costa-Bona, Bac del Fau près Costujes.

A. vuIneraria, L. - Espèce polymorphe qui existe dans tout le bassin depuis les plaines jusqu'aux sommités des montagnes. Les variations portent principalement sur le nombre et la forme des feuilles ainsi que sur la couleur des fleurs lesquelles sont jaunes, blanches ou rouges.

La variétẻ $A$. Dillenii, Schultes, à fleurs rouges est assez 
commune dans les montagnes du Valais au col de Fenêtre, Unterrothorn, Gornergrat, Riffel, Zwischbergen; dans l'Isère au col de l'Arc, montagne de Saint-Nizier, mont Sénèpe; dans les Hautes-Alpes à la Grave, Lautaret, Charance, Rabou, côte Gélive, col de Glaise près Gap: col Bayard, Séuse; dans les B.-Alp. autour de Larche, Barcelonnette et Sisteron; dans la Drôme à Saint-Jean près de Crest, mont d'Angèle; dans l'Ardèche à la vallée de l'Ouvèze et au rocher de Jastre; dans Vaucluse autour d'Avignon; dans les Bouches-du-Rhone autour d'Aix et de Marseille ; dans le Var près de Toulon, du Luc et d'Aups; enfin sur divers points de l'Hérault, du Gard, de l'Aude et des Pyr.-Or.

A. vulnerarioides, Boniean. - Pâturages du mont Cenis. - B.-Alp. : la Condamine.

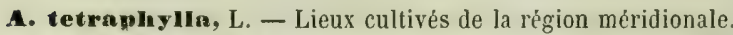

IIYMENUCA RPUS cirelnnata, Sav. - Var : Toulon au cap Brun. - Alp.-Nar. : Cannes, lles de Lérins, Nice, Villefranche. - Hérault : Saint-Jean-de-Vedas près Montpellier. - Pyr-Or. : valle̊e de l'Agly entre Cases-de-Pena et Estagel.

METYCACO matiatn, L. - Cetle espèce du Roussillon (entre Saint-Laurent-de-Salanque et Torreilles) et des environs de Narbonne doit être recherchée dans la partie méridionale de l'Hérault.

M. Inpulina, L. - Plante polymorphe, ayant des fruits tantôt glabres, tantôt hérissés de poils, commune dans les champs de tout le bassin ; souvent cultivée sous le nom de Lupuline ou de Minette.

Ir. Faleata, L. - Coteaux secs dans tout le bassin.

11. glomerata, Balb. - Coleaux calcaires du Luc (Var).

M. falcato-saliva, Reichb. - Hybride des Mr. faleata et sativa, souvent mêlé à ses deux parents.

I. sativa, L. - La luzerne est cultivée partout et devient souvent subspontanée.

MI. scutellata, All. - Moissons et lieux incultes de la région méridionale. Trouvé quelquefois dans les environs de Gap.

I. orbleularis, All. - Lieux incultes de la région méridionale 
jusque dans le sud desIIautes-Alpes, de l'Ardiche et de la Drôme. - Se rencontre parfois dans les autres parties de notre bassin à la suite de l'introduction des graines fouragères de provenance méridionale.

$\boldsymbol{M}$. ambigua, Jord. - Lieux incultes. - Environs de Lyon à Oullins, Tassin, Saint-Alban. - Ain : Massieu. - Is. : Dicines, Crémieu, Dizimieu, environs de Grenoble, Ia Tronclie, Guy-Pape, Vif. Sav. : Saint-Julien-en-Maurienne. - H.-Alp. : environs de Gap et de Ribiers. - Var.

J1. marghata, Willd. - Bords des cliemins de la Provence. A élé vu par M. Bernardin dans l'Ardèche au Grand-Pont.

M. elegan Jacq. - Pàturages des Pyrénées-0rientales entre Château-houssillon et Canet, bords des vignes de Baixas et Casesde-Pena, environs de Perpignan. A été trouvé aussi en Corse.

M. oufritutcosn, Ram. - Rochers des Pyrénées-Orientales : la Cabanasse près Mont-Cenis, commun dans la vallée d'Eyne, pentes du Canigou, vallées de Vernet-les-Bains et de Cornelladu-Conflent, environs de Céret. - Commun dans les Pyrénées centrales, - Manque dans le reste de la France.

M. Ieioearpa, Benth. - Lieux arides. - Hér. : garrigues de Ville. veyrac. - Plus commun dans l'Aude autour de Narbonne et dans les Basses-Corbières à Junquières, Fontfroide, Pech-del'Agnel de la Fenno-Horte, de Campane, Montredon, Bizanet, Fresquet, Ja Clape, au Rec, à Pech-Redon. - Pyr.-0r : Casesde-Pena, Saint-Antoine-de-Galamus, Thuir.

M. Solelroiu, Duby. - Plante de Corse trouvée près de Marseille, près de Toulon entre Ollioules et Saint-Nazaire, autour de Cannes, et enfin dans l'luérault à Valmagne.

Ir. cylindiracea, D. C. - Champs arides. - Pyr-Or. : entre Argelès et Saint-André. - Existe aussi en Corse. - Nul dans Je reste de la France.

Ir. reticulata, Benth. - Champs et vignes. - Aude : BassesCorbières à Cascatcl, Villeneuve. - Pyr.-0r. : environs d'Opol, Haut-Vernet, Perpignan, Cases-de-Pena.

M. disetformis, D. C. - B.-du-Rlı. : Montredon, cap des Goudes, la Treille. - Var : vallée de Clairet pris Toulon, la Valelte. - 
Hẻr. et Aude : Castelinau près Montpellier, Crabit, Pech-del'Agnel près Narbonne, la Campagne, Montredon. - Pyr.-Or. : Collioure, Banyuls-sur-Mer.

M. Tenoreana, D. C. - Toulon au fort d'Artigue.

M. coromata, Lam. - Lieux incultes de la région méridionale. B.-du-Rh. : vallon au sud du Prégnon près d'Aix. - Vaucl. : Montdevergues près Avignon. - Var : mont Faron, Coudon, Clairet près Toulon, Carqueiranne.-Gard : entre Nimes et et Uzès, - Aude : Pech-de-l'Agnel près Narbonne, Cap de Pla. - Pyr.-0r. : Perpignan, Château-Roussillon, Haut-Vernet.

M. proecox, D. C. - Bords des champs de la région méridionale. Var : environs de Toulon, presqu'ile Saint-Mandrier, Carqueiranne, Cannet du Luc à l'ancienne voie Aurélienne, Frẻjus ancien fort de Cavalaire. - Gard : Nimes, Campagne, Broussan, Manduel. - Hér. : environs de Montpellier, de Cette, d'Agde à Roquehaute, environs de Lodève. - Pyr.-Or. : Château-Roussillon, Collioure, Banyuls-sur-Mer.

M. polyearpa, Willd. - Espèce polymorphe dans laquelle on a distingué trois formes principales dont la distribution géographique est mal connue, ce sont :

A. tuberculata, Godr. - Fruit à tubercules obtus. - B.-duRh. : environs de Marseille. - Var et Alp-Mar. : Toulon, le Lavandou, Grasse, Vaugrenier, prís Antibes, Saint-Pons près Nice. - Hér. : Montpellier, Grabels, Béziers.

B. apiculata, Willd. - Epines courtes, non crochues. Vaucl., B.^du-Rh., Var et Alp.-Nlar. : Avignon, Marseille, Toulon, Hyères, le Luc, Fréjus, Nice. - Assez rẻpandu dans l'Hérault, le Gard, la partie méridionale de l'Ardèche et de la Dròme. Apparaît quelquefois dans le centre et le nord du bassin à la suite de l'introduction de graines de provenance méridionale. C'est ainsi (qu'on l'a observé dans la Maurienne, autour de Lyon, de Bourg, dans les plaines du Jura, Saône-et-Loire et jusque dans la Côte-d'Or autour de Dijon, Soisson, Seurre, Châteauneuf, Arnay-le-Duc.

C. denticulata, Willd. - Epines subulées, crochues au sommet. Mème dispersion que le précédent dans la Provence, les 
Alpes-Maritimes, l'Hérault et le Gard. Se montre aussi accidentellement dans le reste du bassin à la suite de l'ensemencement avec des graines du midi.

M. Inppacen, Lam. - Espèce polymorphe assez répandue dans la région méridionale.

A. tricycla, Godr. - B.-du-Rh. et Var : Cassis, Toulon, Fréjus. - Gard: Aigues-Mortes.

B. pentacycla, Godr. - Alp.-Nar., B.-du-Rh. et Var : Marignane, Toulon, ile de Porquerolles, Antibes. - Commun dans l'Hérault.

M. cillaris, Willd. - Environs de Marseille et de Toulon. - Plus commun dans les environs de Narbonnc à Rouquette, Langel, et dans le Roussillon à Baixas, Cases-de-Pena, vallée du Réart, mont Louis.

耳. macuInta, Willd. - Champs et prés dans tout le bassin.

19. minima, Lam. - Licux sablonneux et pierreux de tout le bassin.

M. Iaeininta, All. - Environs de Toulon, de Nice et de Menton, Pyr.-Or.: Baixas.

RT. maxiua, L. - Sables maritimes sur toute la côte.

M. Iittoraliø, Rhode, - Même habitat.

M. Braunii, Godr. - Variété sinistrorsa du précédent avec léruel elle est mêlée.

II. Cerarah, Willd. - Champs de la région méridionale. - Se montre accidentellement autour de Gap, de Ribiers, de Chambéry, de Saint-Julien-en-Maurienne et jusque dans la Cote-d"Or autour d'Auxonne et dans le Morvan vers Semur, la Roche-enBrenil. - Espèce polymorphe.

M. Timeroyi, Jord. - Pelouses des environs de Lyon à Saint-Alban, Mont-Chat, Villeurbanne.

M. Verloti, Perr. et Song. - Saint-Michel-en-Maurienne.

H. cincrascens, Jord. - Pelouses les environs de Lyon à Saint-Alban, Oułlins et à Saint-Jean-des-Vignes dans le Beaujolais. - Ain : Massieu et Reyrieu prìs de Trévoux. - B.-du-Rh. : Aix au chemin de la Pinette. - Var: le Luc. - Hẻr. : Saint-Pierre-de . la-Faye, Saint-Maurice. 
M. depressa, Jord.-B.-du-Rh., Var et Alp.-Miar. : Marseille, Hyères, le Revest, le Luc, Cannes. - Hér. : Cornils, Lodève.

M. tribuioldes, Lam. - Lieux secs de la région méridionale. B.-du-Rh. : Marseille, Montredon, Arles, Aix. - Rare dans le Var et les Alpes-Maritimes. - Hérault : Montpellier, Cette, Roquehaute. - Aude : environs de Narbonne. - Pyr.-Or.: Argelès, ile Sainte-Lucie.

M. murex, IVilld. - Champs de la région méridionale. - B.-duRh, et Var : Aix au quartier de Sain t-André, environs de Toulon. Gard : Aigues-Mortes. - Hẻr. : Montpellier, Celte, Agde, au pied du plateau de Roquehaute, Lodève. - Aude : environs de Narbonne.

11. trumeatulata, Gærtn. - Environs de Montpellier et de Narbonne.

M. turbbinata, Willd. - Trouvé au cap Brun près Toulon et à Prades et Baixas dans les Pyrénées-0rientales.

M. murlenta, Benth. - Champs de la région méridionale. B.-du-Rh., Var et Alp.-Mar.: Marseille, Toulon à Saint-Mandrier, Six-Fours, Fréjus, Antibes, Nice. - Hér. : environs de Montpellier.

MI. Ephaceenrpa, Bertol. - Bords des champs dans les Bouchesdu-Rhône, le Var et les Alpes-Maritimes : Bec-de-l'Aigle près la Ciotat, Toulon, Hyères, Saint-Tropez, Fréjus, île Saint-Honorat, Cannes, Antibes, Menton.

M. arborea, L. - Subspontané à Nice et à Menton.

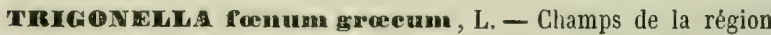
méridionale. - B.-du-Rh. et Alp.-Mar. : Martigues, Endoume, Nice. - Gard : Montfrin, Aimargues, les Angles près Villeneuve en face d'Avignon. - Hér. : Agde, Béziers, - Aude : Junquières, Lespignan. - Pyr.-Or. : environs de Perpignan.

F. glndiata, Stev. - Lieux pierreux de la région méridionale. Vaucl. : Montdevergues. - B.-du-Rh. : environs de Marseille et d'Aix. - Var : Gonfaron, le Luc, Frẻjus, Saint-Raphaël. - Alp.Mar. : Grasse, l'Escarẻne, Baus-Rous, Nice, Menton. - Gard : environs de Nîmes au bois des Espèces, Villeneuve en face d'Avignon, la Tessonne près du Vigan. - Ilér. : Montpellier, 
Pézenas, Ceyras. - Aude ; environs de Narbonne à Pech-del'Agnel, Cap-de-Pla. - Pyr.-Or. : entre Salses et la Nouvelle. Remonte dans la Drome vers Aucelon ; dans les Basses-Alpes vers Annot; dans les Hautes-Alpes vers Ribiers, Rosans.

T. monmpalinea, L. - Champs sablonneux et picreux de la région méridionale. - Remonte vers Gap, Ribiers, Aucelon, Barnave el Saint.Vallier de la Drôme, Privas, Vals, Grenoble, Feyzin ct Villeurbanne près Lyon; puis au-delà dans l'Ain à Meximieu, Ambronay, Crémieu dans l'Isère; dans la Savoie vers Moutiers et Saint-Jean-de-Maurienne; enfin dans lo Valais à Branson, Fully, Saillon, mont d'Orge, Sion, Saint-Leonhard.

T. polycerata, Benth. - Champs des Pyr.-Or. : environs de Llo et d'Osseja, Puycerda, Nyer.

T orndthopodioldes, D. C. - Prairies des environs de Montpellier. - Pyr.-Or. : vallée de l'Agly entre Espira et Estagel; de Perpignan à Cases-de-Pona.

T. Iybrida, Pourr. - Hér. : Rédémouls près Pardailhan-Pontguiraud. - Pyr.-Or. : Saint-Antoine-de-Galamus, Caramany, vallée de Nyer.

T. corniculata, L. - Champs cultivés. - Drôme : Mollans, le Buis, Vinsobres. - Vaucl. : les Alpines, Avignon, vers le pigeonnier et les Agassins. - B.-du-Rh. : paluds de Saint-Rémy, entre Saint-Rémy et Tarascon. - Gard : Nimes, Manducl, Tresques, Uzès, bords du Gardon, Pont-du-Gard, - Hér. : Pézenas, Béziers, Lunel, Boisseron, Saussines, bords du Vidourle vers Sommières.

MELTLOTU⿴囗十 mesanensis, Desf. - lieux incultes. - Vaucl. : environs d'Avignon. - Var : environs de Toulon, Hyères à l'Almanare et au Ceinturon. - Alp.-Mar. : Saint-Hospice, entre Villefranclie et Beaulieu - Hér. : montagne de Malpas, - Pyr. 0r. : entre Salses et Sainte-Marie.

M. ฮuleasa, Desf. - Champs sablonneux de la région méridionale depuis Vals et Privas dans l'Ardèche. - B.-du-Rh. : environs d'Aix. - Var : Toulon, Hyères, le Luc, Fréjus. - Alp.-Mar. : Antibes, Nice, Henton,-Gard: Nìmes, Aigues-Nortes, Sylveréal, Bellegarde, bords du Rhone à la Reyranglade. - Hér. : Rigaud 
près Agde, Roquehaute, Pézenas, Gabian, Cabrières, Lodève. Aude : environs de Narbonne.-Pyr.-Or. : Cases-de-Pena, Baixas, vallée du Réart.

M. infesta, Guss, - Espẻce de Corse naturalisée autour de Marseille, de Toulon, d'Hyères, Saint-Nazaire, Antibes. - Suivant M. Huet la plante de la Provence ne serait point le M. infesta, Guss., mais bien le MI. compacta, Salzm.

M. Italiea, Lam. - B.-du-Rh. : Aix à Puyricard. - Var: Toulon, Hyères. - Alp.-Har. : Nice au Château. — Gard : Aigues-Mortes. - Hér.: Montpellier. - Pyr.-Or. : entre Terrats et l'Hostalet le long de la Cantarane.

M. elegrans, Salzm. - Prés et champs cultivés. - B.-du-Rh. : les Iles voisines de Marseille, cap Couronne à Martigues, Becde-l'Aigle près la Ciotat. - Var: cap Brun près Toulon, lle des Imbiers et d'Hyères, Saint-Raphaël.

M. parvillora. Desf. - Champs incultes de la région méridionale. - B.-du-Rh. : Aix au clos des Capucins. - Var: Toulon, la Seyne, Porquerolles, Hyères, le Luc, Fréjus, Saint-Raphaël. - Alp.-Har. : Antibes, Ile Sainte-Harguerite, Nice, Menton. Gard: Nìmes, Manduel, Aigues-Mortes. - Hér.: Palavas, Agde, Cette, Béziers, Pézenas.-Aude: environs de Narbonne, Ia Rouquette. - Pyr.-Or. : Baixas, Château-Roussillon, Vernet, Prades. - Drôme: Crest. - Cette espèce a été trouvée quelपquefois dans les luzernières des environs de Lyon, de Seyssel et de Saint-Julien-en-Maurienne.

M. neapolitama, Ten. - Lieux arides de la région méridionale. -B.-du-Rh.: Saint-Chamas, Pilon-du-Roi, Notre-Dame des Anges, la Ciotat. - Var : les Sablettes près Toulon, Hyères, le Revest, Draguignan, Gonfaron, le Luc, Fréjus, Saint-Raphaël. - Alp.-Mar.: Grasse. - Gard : le Vigan, Campestre, l'Espérou, bords du Gardon, la Beaume. - Hér. : entre Amiane et Saint-Guilhem-le-Désert, Lodève, Cartels, Loiras, Pézenas. - Aude: Lasfons, la Fenal, Graviers de la Cesse. - Pyr.-0r. : Perpignan, Argelès-sur-Her, Collioure. - Cette espèce a été observée dans les environs de Grenoble à Comboire et au Pont-de-Claix; puis en Savoie, près de Saint-Jean-de-Ilaurienne. 
Ir. offeinnlis, Lam. - Champs dans tout le bassin. - Disséminé et peu abondant.

Ix. a1ba, Lam. - Champs. - Plus commun que le précédent surtout sur les bords des rivieres.

M. permixta, Jord. - Hyères (Var).

I. mncron'hiza, Pers. - Bords des rivieres. - Dans la partie septentrionale et moyenne du bassin. - Plus rare dans la région méridionale. On ne le signale dans la Provence que sur quelques parties des bords de la Durance, notamment vers le Pont-de-Pertuis; mais il est assez commun dans le Roussillon.

I. coerulea, Lam. - Parfois adventif ou échappẻ de cultures.

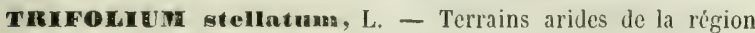
méridionale. - Se montre accidentellement autour de Lyon eb de Chambéry.

T. angusı́folium, L. -- Lieux arides de la région méridionale. - Remonte dans la Drôme vers le Buis, Montélimar, Crest, Saint-Vallier; dans l'Ardèche sur les pentes du mont Combier et vers Celles, Mercuer; dans l'Isère à Vienne, Seyssucl, Chasse, Vertrieu près Crémieu; entìn autour de Lyon et nolamment sur le talus des fortifications des Brotteaux.

T. Inearnatum, L. - Cultivé el quelquefois subspontané.

T. Molinerli, Balb. - Ce Trèlle est peut-être une variété du précédent. - Environs de Lyon, Bourg, Crémieu, Vienne, Saint-Vallier dans la Drôme; prés des bords de la Saône, de la Grosne, de l'Arroux, de la Loire, environs de Genève, d'Albertville en Savoie, Celles, Vals, Entraigues dans l'Ardèche B.-du-Rh. : Mazargues. - Var et Alp.-Mar. : Notre-Dame des Anges près Pignans, les Mayons, l'Estérel. - Hẻr. : Fraïsse, Pas-de-l'Escalette.

T. purpureum, Lois. - Bords des champs de la région méridionale. - B.-du-Rh.: bords de l'Arc à la Pioline. - Gard: Nìmes, Saint-Gilles, Manduel, Beaucaire. - Hèr. : la Planchude, bois de Grammont et de Puy-Sériẻ, bois de Lamoure, Béziers, Pézenas. - Ard. : Oubreyt, - Pyr.-Or. : Mont-Louis, vallées de Nolièdes et de Saint-Paul-de-Fenouillet. 
T. rubens, L. - Bords des bois, pelouses des terrains calcaires. Toute la chaine jurassique de Haute-Saône, Douls, Jura, Revermont, Bugey, Savoic, Dauphiné; d'autre part sur les collines jurassiques de Côte-d'Or, Saône-et-Loire, mont d'Or Iyonnais. - Bassin du Léman. - Valais : le Ziabley, l'Estroz, Leukerbad. - Ard. : mont Combier. - Dr. : Barnave, Aucelon. H. -Alp. : Durbon, Ufarnet et bois Mondet près Gap, Charance, Combe-Noire, Rosans. - Plus rare dans la région méridionale, où on ne le signale que dans les Bouches-du-Rhône à Pourrières, vallon de Vaumare, dans quelques rares localités des llaures et de l'Estérel, dans les montagnes entre Nice et Menton, ct enfin dans la région montagneuse du Gard, de l'Hérault et du Roussillon.

T. Apestre, L. - Pelouses des basses et hautes montagnes. Toute la chaine des Vosges jusqu'au Ballon de Giromagny. - Rare dans la chaine jurassique au Colombier de Gex. Assez commun sur les calcaires jurassiques de Côte-d’or à Saint-Aubin, Chassagne, Gevrey; sur ceux de Saone-et-Loire à Dezize, Rully, Buxy; sur le même terrain dans le mont d'Or lyonnais ainsi que dans le Bugey et le Revermont. - Bas-Valais. - Environs de Genève au bois de Bay près Penex.-H.-Sav. : Salève, base du Móle, col de Balme. - Sav. : montagnes des environs de Beaufort, des Bauges, de la Tarentaise et de la Maurienne. - Is. : Crémieu, mont Rachais, Pariset, mont de Lans, Mens. - B.-Alp. : Lauzannier. - H.-Alp. : Lautaret, Charance, le Pleyne, mont Séuse, mont Aurouse, col de Vars, Rosans. - Dr.: Saint-Vallier, Crest, Barnave, Aucelon. Ard. : Asperjoc. - Vaucl : environs d'Apt, la Garde, Viens. Alp.-Mar. : mont Cheiron, montagnes de Caussols et de Defens, val de Jallorgues, Saint-Martin-Lantosque. — Gard : Valbonne, Serre-de-Bouquet, Salbous, Boussargies. - Pyr.-Or. : CostaBona, Esqueros-de-Roja, Motte-de-Planès, mont Louis.

T. Ihirtum, All. - Champs incultes des terrains sablonneux. Ard.: région granit. des bords du Rhơne entre Serrières et Andance, Tournon, Celles jusqu'à Rochemaure. - Dr.: me̊me terrain entre Saint-Vallier et Tain. - Vaucl. : le Pontet près 
Avignon. - N'est pas signalé dans les Bouches-du-Rhône, dans le Var ni dans les Alpes-Maritimes. - Gard: sables siliceux, Aigues-Mortes, Sumène, Broussan, Saint-Gilles. - Hér. : sables siliceux de Grammont près Montpellier, Agde, Saint-Félix-de Lodez, Castels, Limas. - Aude: environs de Narbonne. Pyr.-0r.: Baixas, Peyrestortes, Perpignan, Banyuls-sur-Mer, Vernet, Prades, mont Louis.

T. Chcrlen', L. - Lieux secs de la région méridionale. - Vaucl. : environs d'Avignon, le Pontet, Morieres, bois du Clos. B.-du-Rh. : Marseille, Aix, la Pioline, Miramas, Martigues. - Var et Alp.-Mar. : Sablettes de Toulon, Pierrefeu, les Maures, Fréjus, Cannes. - Gard: Nimes, Manduel, bois de Campagne, de Cygnan, Aigues-Mortes. - Hêr. : environs de Montpellier, bois de Grammont, garrigues du Terrail, Saint-Loup, Maurin, Roquehaute, Agde, Pézenas, Cartels, Escandorgue.- Aude: cnvirons de Narbonne, Aussières, Fontlaurier.-Pyr.-0r. : Banyuls-sur-Mer, Prades, Molitg.

T. medium, L. - Bois de tous les terrains. - H.-Saone : zône jurassique et vosgienne. - Arrond. de Belfort. - Côte-d'Or et Saone-et-L. - Doubs et Jura : depuis les basses collines jusqu'aux sommités de la Dôle et du Colombier. - Environs de Genève au bois de la Bâtie. - Valais à Zermatt. - H.-Sav. : pied du Salève, la Roche, Brizon, Thônes, mont Châtillon, Annecy, Pringy, Pormenaz, Chamonix. - Sav. : Conflans, Pallud, Saint-Sorlin-d'Arve. - Ain: le Revermont, le Bugey, la Bresse. - Rh.: massifs du mont d'0r et aussi sur les granites de Dardilly et de Charbonnières. - Is. : Crémieu, Chamagnieu, Pariset, Bastille de Grenoble, GrandeChartreuse. - H.-Alp. : Combe-Noire de Manteyer, col de Vars. - B.-Alp. : la Condamine. - Dr. : Saint-Vallier, Crest. Ard.: Payolive. - Var: Maures. - Alp.-Mar. : Tende. Gard: Alais, Anduze, le Vigan, Chartreuse-de-Valbonne. IIir. : Ie Bouquet-d'Orb, Saint-Amand, la Sérane. - Pyr.-Or. : mont Louis, vallẻe de Nohèdes, Vernet, vallée du Tech, Pratsde-Mollo.

T. prntense, L. - Prés et champs dans tout le bassin depuis les 
plaines jusquaux montagnes. - Espèce très-variable dans sa taille, la forme de ses épis f'oraux et des fevilles, la couleur de ses fleurs.

T. pannonicum, Vill. - Forme du précẻdent à capitules gros, nombreux, à fleurs d'une couleur blanche rosée, quelquefois jaune, assez commune dans le Dauphiné dans la chaine de Belledonne, mont de Lans, Lautaret, mont Chabrières. - Au col de Balme entre la Savoie et le Valais.

T. ochroleucum, L. - Pelouses et bois sur tous les terrains. H.-Saone: Gratery, Frotey, Passavant, Clairegoutte, SaintRemy, Villersexel, Champagney, Coisevaux, Chamfey, Saulnot. - Arrond. de Belfort. - Doubs et Jura: environs de Montbéliard jusque dans la vallée de la Savoureuse, canton de Rougegemont, Ornans, Besançon, Chenaudin, Barraque-des-Violons, Salins, Dole, forêt de la Serre, Champagnole, la Faucille. Côte-d'Or: bois de la Côte. - Saône-et-L. ; Rh. et Ain.Environs de Genève, bassin du Lẻman et Valais. - H.-Sav. : Salève, Jussy, environs de la Roche, de Pringy et d'Annecy. - Sav. : Chambéry, Conflans. - Is. : Crẻmieu, la Côte-SaintAndré, Pariset, Saint-Eynard, mont Rachais. - H.-Alp. : environs de Gap, Charance, Saint-Iens, le Queyras, Rosans. B.-Alp. : la Condamine. - Dr.: Saint-Vallier, Barnave. Ard.: Cuze, Vals. - Vaucl. : environs d'Avignon à Morières, bois du Clos.- B.-du-Rh. : en Coustiero, Sainte-Victoire, entre Vauvenargues et le Bec-de-l'digle. - Var: Sainte-Baume, Notre-Dame-des-Anges près Pignans, Montrieux, Saint-Quenis près Camp. - Alp.-Nar. : Estêrel, Antibes, Nice, Menton.Gard, Hérault, Aude et Pyrénées-Orientales.

T. maritimum, Huds. - Lieux sablonneux de la région méditerranéenne. - B.-du-Rh. : Arles, Marignane. - Var: Sablettes près Toulon, Hyères, le Luc, Saint-Raphaël. - Alp.-Ilar. : Grasse, Châteauneuf, golfe Jouan. — Gard: Aigues-Mortes, Bellegarde, Saint-Gilles, Manduel, Beaucaire. - Hér.: bords des Roubines à Palavas, Roquelıaute, les Onglous. - Pyr.-Or.: Port-Vendres, Banyuls-sur-Ner, Saint-Laurent-de-Salanques, Salses. 
T. panormitanam, Presl. - Var. et Alp.-Mar. : fort Malbousquet et cap Brun près Toulon, Saint-Nazaire, Fréjus, île SainteMarguerite. - Pyr.-Or. : Collioure, Rivesaltes, berges de l'Agly jusqu’à Estagel.

T. Inpprecum, L. - Noissons de la région méridionale. B.-du-Rh. : Montredon, la Crau, Marignane, environs d'Aix au vallon de Bounouro et au-dessus de Saint-André. - Var: Toulon, Hyères, Saint-Tropez, le Luc, Fréjus. - Alp.-Mar. : Grasses, Antibes, Saorgio, Nice, Menton. - Gard : Nìmes, Man. duel, Saint-Gilles, Tresiue, Aigues-Mlorles.-Hér. : garrigues du Terrail, Mireval, Maurin, Foncaude, Saint-Loup, Roquehaute, Palavas, Béziers, Pézenas, Rabieux, Saint-Félix, - Aude : environs de Narbonne.- Pyr.-0r. : Terrats, Pouteilla, Llupia, Céret, Saint-Iaurent-de-Cerdans, Costujes.

T. supluum, Savi. - B.-du-Rh. : rives de l'Arc, vers la Pioline.

T. II gustcum, Balb.- Pelouses sablonneuses du Var et des Alp.Mar. : Toulon, illes d'Hyères et de Porquerolle, Saint-Tropez, le Luc, forế des Maures, la Sauvette, les Mayons, Frẻjus, Cannes, la Roquelte, Menton.-Pyr.-Or. : Argelès-sur-Mer, Port-Vendres, Banyuls-sur-ฏler.

T. arrense, L. - Champs sablonneux dans tout le bassin; plus abondant sur les terrains siliceux. - Présente de nombreuses variations dans la taille, les proportions du calice et de la corolle et la villosité de ses divers organes.

II. Jordan distingue les formes suivantes qu'on n'a signalées jusqu'à présent que dans les environs de Grenoble, do Chambéry et de Saint-Vallier dans la Drôme. - T. lagopinum, rubellum, arenivagum, collinum, sabuletorum, agrestinum, arvicolum. A ces formes il faut ajouter le $T$. gracile, Thuill.

T. Ingopus, Pourr. - Lieux incultes des terrains siliceux. - Rh. : granite des bords du Garon, entre Brignais et Orliẻnas, Givors. - Ard.: granite des environs de Tournon, Celles, Baix, basalte de la Roche-de-Gourdon. - Sav. : environs de Chambéry. - Var. : granites, gneiss et micaschistes des Maures au mont Sauvetle. - Se trouve communément sur les terrains granitiques des Pyrénées-Orientales, ou il remonte assez haut dans les vallées. 
T. saxatile, All. - Sables granitiques des torrents dans les hautes vallées des Alpes. - Valais : Saas, Saint-Nicolas, Almagel, glacier de Fée, pont du Furgbach.-H.-Sav. : vallée de Chamonix sur les bords de l'Arveiron et en remontant jusqu'au pied des moraines terminales du glacier des Bois, moraines du glacier de Tacconaz. - Is. : sables du Vénéon au pied du glacier du Chardon et en descendant à la Bérarde, à Saint-Christophe jusque près de Venosc en Oisans, sables de la Romanche près des Fraux, sables du Drac vers Champoléon. - H.-Alp. : sables du torrent venu du glacier d'Alefroide dans la Vallouise. Pyr.-Or. : vallie d'Urbanya, de la Preste à Pla-Guillem, environs du Boulou et de Céret.

T. Boceoni, Savi. - Terres incultes. - Le Mollard de Décines près Lyon.- Dr. : coteaux de Brandoules près Saint-Vallier.Var : île d'Hyères, Pierrefeu, les Maures, Saint-Raphaël, Gondin, Mautems, - Alp.-Mar. : Laval, Pégomas, Antibes, Bellet près Nice. - Gard : de Nimes à Saint-Gilles, Cygnan, le Vigan. Hér. : Roquehaute, la Malou. - Pyr.-Or.: Collioure, Banyulssur-Mer, pied des Albères.

T. InImaticum, Vis, - Rare à Draguignan et au Luc.

T. strintun, L. - Coteaux sablonneux dans tout le bassin; disséminé et peu abondant dans la région méridionale, excepté dans les Maures, l'Estérel et le Roussillon.

T. seabrum, L. - Coteaux sablonneux et graveleux; disséminé et peu abondant.

T. subterroneuan, L. - Pâturages des terrains siliceux. - Nul dans les chaînes rosgienne et jurassique. - Çà et là dans les pelouses sablonneuses de Cote-d'Or, Saône-et-Loire, la région granitique du Forez, du Beaujolais, du Lyonnais et du Pilat. - Terrains argilo-siliceux de la Bresse à Montluel, Thoissey, Saint-Didier, Garnerans, Pont-de-Vaux, Manziat. - Plus abondant dans le Vivarais, les Cévennes, la Provence et les AlpesMaritimes. - Pyr.-Or. : Banyuls-sur-Mer, Vernet-les-Bains. - Aude : Aussières.

T. Fraglerum, L. - Prés, bords des chemins dans tout le bassin.

T. reapinatum, L. - - Pelouses de la région méridionale. - 
Remonte jusque dans la Drôme entre Crest et Allex, les Baronnies. - Depuis quelques années on l'observe autour de Lyon au Grand-Camp et dans les environs de Bourg.

T. Clusit, Godr. et Gr. - Les auteurs de la Flore de France signalent vaguement celle forme dans la région méditerranéenne. MM. IIanry et Huet m'assurent tous deux qu'ils ne l'y ont jamais vue.

T. tomentosun, L. - Lieux herbeux de la région méridionale. - Var: Toulon, Hyères, les Maures, Saint-Tropez, Fréjus. - Alp.-Mar. : Antibes, Cannes, Nice, Contes. - Gard : Nìmes, Saint-Gilles, Manduel, Caisargues, les Angles près Avignon. -Hér. : Grammont près Iontpellier, Gibret, Saint-Jean-de-laBlaquière, Colombière, Montmau, Maguclonne, Roquehaute, Agde, Béziers, mont Saint-Clair près Cette. - Pyr.-Or. : Prades, Villefranche.

T. vesiculosum, Savi. - Plante de Corse, trouvée dans les Pyrénées-Orientales, à Port-Vendres, prairies de Paulille, vallon de Banyuls-sur-Mer.

T. sримиями, L. - Collines arides, - B.-du-Rh. : Marscille, Montredon, Arles. - Var et Alp.-Har. : Toulon, Tanneron. Hêr.: Rigaud près Agde, les Onglous, - Pyr.-Or. : base des Albères.

T. glomeratum, L. - Pelouses. - Nul dans les chaines vosgienne et jurassique, dans Côte-d'Or, Saone-et-Loire, bassin du Léman. - Sa limite septentrionale est dans l'Ain vers ChâteauGaillard; de là il descend vers Meximieu, Balan, Montluel, Reyrieu. - Rh.: Saint-Alban, Beaunant, Tassin, le Garon. - Loire: Chavanay, Malleval. - Dr. : Saint-Vallier. - Ard. : Ucel, Vals, Entraigues. - A partir de ce niveau, il devient commun dans la région méridionale.

T. suffoesatum, L. - Coleaux sablonneux de la rẻgion méridionale. - B.-du-Rh. : Marseille, Istres, Arles. - Var : Toulon, ile de Porquerolles, Bormes, les IJaures, Cavalaire, Fre̊jus. Gard: Manduel, Tresque, Broussans et Campagne pres Nimes.Hér. : Roquehante, Grammont, Villencuve-Jes-Béziers, Pízenas, mont Saint-Clair près Cette. - Aude: Aussières, Fontlaurier, Coumbo-de-Ferro. 
T. Lovigatum, Desf. - Pelouses sèches. - Rh. : Sainte-Colombe, Ampuis. - Is.: Mollard de Dérines, mont d'Annoisin près Crémieu. - B.-du-Rh. : environs de Marseille. - Var : forét des Maures, la Sauvette, Collobrières, Pierrefeu. - Hẻr.: Pardailhan-Pontguiraud, - Pyr.-0r. : pied des Albéres.

T. montanum, L. - Pelouses des montagnes et aussi des basses collines. - Toutes les chaines des Vosges, du Jura, du Bugey et Revermont ; le Valais, la Savoie, le Dauphiné et les BassesAlpes jusqu'aux Alpes-llaritimes. - D'autre part depuis Côted'Or et Saône-et-Loire jusque dans le Lyonnais. - Plus rare dans la région méridionale. - B.-du-Rh. : Cassis sur le Mole. -Var: Hyères.- Gard : Chartreuse-de-Valbonne, Serre-de-Bouquet, l'Espérou, Salbous. - Hér. : environs de Montpellier.Pyr.-0r. : mont-Louis, vallée d'Urbanya, montagne de Céret, Llaurenti, vallée de Mijanès.

T. Alpinum, L. - Pâturages des Alpes. - Nul dans les Vosges et la chaine jurassicque. - Valais: Javernaz, Fully, Saint-Bernard, Thyon, Chermignon, Zermatt, Saas, Simplon.-H.-Sav. : Brévent, jardin et moraines de la Mer-de-Glace, Buet, l'Argentière, mont Méri, montagnes de Samoëns, col de Gole̊se, påturages de Flaine, le Signal près Montrion. - Sav. : montagues des Bauges, de Beaufort, Hauteluce, mont Mirantin, Dentdu-Corbeau, montagnes des Allues, des Avanchers, de Pesey, de Macot, du Bonhomme, de la Maurienne et mont Cenis. - Is. : chaînes d'Allevard, de Belledonne, du mont de Lans, la Salette. - H.-Alp. : Lautaret, la Grave, Névache, montagnes du Queyras autour d'Abriès, de Saint-Vérand, mont Viso, mont Pelva, Ségure, col de Vars, Orcières à Jujau, mont Chaillol, Lopet, mont Chabrières.-B.-Alp. : Lauzannier, Larche, Parpaillon.- Sommités des Alpes-Maritimes.-Ard, : mont Mezenc. - A été indiqué à tort au mont Pilat, mais se trouve dans la Loire à Pierre-sur-Haute.- Pyr.-Or. : vallées d'Eyne, de Llo, de Nohèdes, de Cárença, mont Louis, Canigou, Salvanère.

T. Thalil, Vill. - Débris de rochers et pâturages des montagnes. - Nul dans les Vosges. - Chaîne jurassique au Montendre, Dôle, Faucille, Crêt-de-Chalam, Colombier, Reculet.- H.-Sav. : 
chaîne du mont Blanc, moraines de la Mer-de-Glace et de Tré-la-Téte, le Ilaupas, montagnes du Chablais, Cornette-deBise, col de Golese, patturages de Flaine, mont Charvin, Vergy, Méry. - Montagnes du Bas-Valais et aussi vers Leukerbad. - Sav. : mont Drizon, Orizan, mont Mirantin, Hauteluce, la Gitaz, le Bonhomme, le Chapieu, mont Iseran, les Allues, les Avanchers, mont Cenis. - Is. : chaine de Belledonne vers le lac Crouzel, Chamechaude, colde l'Arc, de Saint-Nizier au Moucherotte.- I.-Alp. : Lautaret, granges d'Isoire, le Queyras, Durbonas, Alp-Martin, Orcières, Pleyne-de-Chaudun, mont Queyrel, col du Noyer, mont Chabrières. - B.-Alp. : montagnes de la vallée de l'Ubaye. - Alp.-Mar. - Pyr.-Or. : vallées d'Eyne et de Llo.

T. pnllescens, Schreb. - Pelouses des montagnes et graviers des torrents des Alpes. - Valais : glacier d'Arolla, Mattmark, pied du Hornli. - H.-Sav. : chaine du mont Blanc, moraines terminales des glaciers des Bossons et de Tré-la-Tête, montagnes du Chablais, Cornette-de-Bise, Roc-d'Enfer au-dessus de SaintJean-d'Aulph, mont Clıarvin. - Sav. : col du mont Cormet, la Vanoise, les Chapieu, de Laval au col Iseran, mont Cenis à la Ramasse, - Is. : chaine de Belledonne vers le lac Crouzet, Alpe du mont de Lans. - H.-Alp. : la Grave, Lautaret, mont Chaillol, Péas, mont Pelva et Valprévaire en Queyras, col de Vars.

T. repens, L. - Prés et bords des chemins depuis les plaines jusqu'aux montagnes.

T. nigrescens, Viv. - Prés et bords des chanps de la région méridionale. - B.-du-Rh. : carrière de sable à Montredon, Mariguane. - Var : les Sablettes près Toulon, Ceinturon d'Hyc̀res, Saint-Tropez, le Luc, les Hayons, Saint-Raphaël. - Alp.-Mar. : mont du Clıeiron au-dessus de Grasse, Saint-Vallier, Antibes. - Gard: environs de Nimes, d'Aigues-Mortes, du Vigan et d'Anduze. - Hér. : Palavas, le Larzac, le Sommail.-Pyr.-Or. : vallée du Réart.

T. elegans, Savi. - Bords des bois dans les terrains sablonneux et argilo-siliceux. - H,-Saone: argiles du Keuper et du Lias 
autour de Chagey, Villersexel, Gouhenans, Lure. - Arrond. de de Belfort à Cravanche, la Miotte, étang de la Maíche. - Doubs et Jura : argiles du Keuper et du Lias autour de Montbéliard, Besançon, Villers-Farlay, Salins, Arbois, Poligny, Lons-leSaunier, terrains argilo-siliceux de la Bresse. - C.-d'Or et Saone-et-L. : région granitique du Morvan et aussi dans les bois de Perrigny près Gevrey. - Rh. : granite de Charbonnière, Tassin, Saint-Genis-Laval._Is. : alluvions sablonneuses de Bron près Lyon, Tupinières près Vienne. - H.-Sav. : environs d'Annecy. - Vaucl. : bords du Rhône près d'Avignon. - Var: mont de Pépière près Saint-Nazaire. - Alp.-Mar.: val de Pesio._Gard : bord de la Cèze à Peyremale.- Pyr.-Or.: mont Louis, Salvanère, les Albères.

T. Hybridum, L. - Plante de la Haute-Loire et de la Lozère trouvée à Miserey près Besançon et dans l'Ain à Léol près de Pont-de-Vaux. - Se trouve aussi dans les Pyrénées-Orientales dans la vallée du Tech près de la Preste, au pied du Pla-Guillem, autour de Fosse en montant à la forêt de Boucheville.

T. Michelianum, Savi. - Ce Trèfle de l'ouest de la France est inđiqué, sans localité précise, dans la Côte-d’Or par Lorey et Duret. - M. Laguesse me dit que personne ne l'a vu dans le susdit département.

T. parviftoram, Ehrh. - Pelouses. - Loire : près de Montbrison à la Blanchisserie, Bonson près du vieux château d'Essaloire; s'élève dans la vallée de Vizézi jusquu'à la Forie, Fontberland, Ecotay-l'Olme. - Pyr.-0r. : environs de Mont-Louis et bords des champs de la basse Cerdagne, métairie de l'Arbre-Sec aux Albères, environ de Maureillas. - Ce sont les seules localités connues en France.

T. angulatum, Waldst. Kit. - Bois de Grammont près Montpellier.

T. Perreymondi, Gr. - Indiqué dans le Var à Roquebrune où on ne l'a pas retrouvé. - Se trouve dans les Pyrénées-Orientales dans les vallées d'Eyne et de Prats-de-Balaguer.

T. Anforme, L. - Lieux sablonneux. - Jura : argiles tertiaires à cailloux silicenux de la forêt de Chaux. - C.-d’Or et Saône- 
et L. : Morvan granitique. - Région granitique du Beaujolars, du Lyonnais et du Forez, - Sav. : environs d'Annecy et de Chambéry, - Dr. : Barnave, - Var: sables dans les Maures et les environs de Fréjus et de Saint-Tropez. - Gard: sables lu bois de Broussan au Trou-du-Pérussas. - Hẻr. : diluviumà cailloux siliceux des bois de Grammont et de Lamoure. Pyr.-Or.: Perpignan, Olette, Fontpédrouse, entre Prats-deMollo et la Preste. - La plante de la Provence, du Languedoc et du loussillon est la forme T. micranthum, Viv.

T. agrariun, L.; aurean, Poll. - Bois et pàturages des basses montagnes. - Chaine des Vosges et du Jura jusque dans le Bugey. - II -Sav.: Salève, Brizon, environ de Thonon, Vacheresse, Pringy, Rumilly, Annecy, Veyrier.-Côte-d'Or el Saôneet-Loire. - Rh. : Rochetachon dans le Beaujolais, Saint-Cyrle-Châtoux, Fontaines, Montchat. - Loire: montagnes du Forez. - Sav. : Chambéry, Conflans, Moutiers. - Is. : Saint-Nizier, Revel, Theys, la Ferrière. - H.-Alp.: les Baux près Gap, Ribiers. - B.-Alp. : la Condamine. - Ard. : Cuze. - Gard : Trèves. - Rare dans la région méridionale excepté dans le Roussillon.

T. proeumbens, L. ; T. agrarium apud Gr. Godr. Fl. de Fr.; T. compestre, Schreb. - Pelouses sablonneuses dans tout le bassin. - Il importe de bien préciser la synonymie de cette espèce et de la suivante à cause de la confusion qui souvent a été faite entre ces deux Trèles. J'adopte sur ce point l'opinion soutenue par Fries et par M. Grenier, Flore jurassique, $1^{\text {re }}$ partie, p. 175; je réserve toutefois la question de savoir si les différences que présentent les deux plantes sufiasent pour établir une distinction spécifique.

T. minus, Rellan; $T$ procumbens, apud Gr. Godr. - Champs et prés dans tout le bassin. - Considéré par un grand nombre d'auteurs comme une forme du précédent dont il ne diffère que par l'étendard lisse ou peu strié, carẻné sur le dos et dépassant peu les ailes.

T'. patens, Schreb. - Prés humides. - Pyr.-Or. - Hẻr. : Lodève, la Salvetat, Fraïsse. - Var et Alp.-Mar. : Frêjus, Bagnols, 
Cannet, Antibes.-Ard. : Payolive, Vals, Entraigues. - Trouvé accidentellement à Louhans dans Saône-et-Loire et autour de Vizille dans l'Isère.

T. badium, Schreb. - Prairies des montagnes. - Nul dans les Vosges. - Rare dans la chaine jurassique au Noirmont au-dessus des Rousses, au Chasseral au-dessus de la source de Suze ct au Marchairuz. - Commun dans les montagnes du bas Valais et aussi dans les montagnes de Saas, Zermatt, Leukerbad. II.-Sav. : montagnes de Samoëns, Cornette-de-Bise, Haut-deVéron, pàturages de Flaine, mont Charvin, Brizon au-dessus de Solaison, chaine du mont Blane. - Sav. : montagnes de Hauteluce, mont Mirantin, Dent-du-Corbeau, Crest-Volant, mont Drizon, le Bonhomme, montagnes de Pesey, Macot, Pralognan, les Allues, les Avanchers, mont Cenis. - Is. : chaines de Belledonne, des Grandes-Rousses et du Pelvoux. - H.-Alp. : Lautaret, mont Viso, mont Pelva, Ségure, Chaillol-le-Vieil, Orcières, Sappey de la Bîtie-Neuve. - B.-Alp. : montagnes du bassin de l'Ubaye, le Lauzannier, Parpaillon. - Alp.-Mar. : - Ard. Cuze. - Pyr.-Or. : mont Louis, vallées de Nohèdes et d'Urbanya, Villefranche.

T. mpadiceum, L. - Prairies tourbeuses des montagnes.- Valais : mayens de Sion, val Sorret. - H.-Sav.: plateau des Voirons, montagnes de Samoëns, Roc d'Enfer au-dessus de Saint-Jeand'Aulph, vallée du Reposoir, mont Brizon, mont Charvin, Mégève, Nant-Borant. - Sav. : montagnes de Beaufort, Hauteluce, Crest-Volant, col de la Louze, Saint-Sorlin-d'Arves.Is. : les Fauges près du Villard de-Lans, Corençon, Saint-Pierre de-Chartreuse, forêt de Portes.-H.Alp. : Lautaret.-B.-Alp. . col de Stropi.-Rh. : Saint-Bonnet-le-Froid, Duerne, Pomeys. - Loire: Pilat, la République, Pierre-sur-Haute, les Salles, - Ard. : mont Mezenc, Gerbier-de-Jonc, Cuze. - Dr. : entre Léonce et Bouvante. - Gard : l'Espérou. Concoule. - Hẻr. : Sérane. - Pyr.-Or. : butte Saint-Pierre, Vernet-les-Bains, Cornella-du-Confluent.

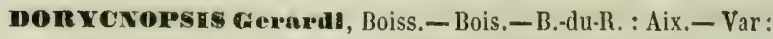
mont Fenouillet près Hyères, Cuers, Bormes, Saint-Tropez, le 
Luc, les Maures.- Pyr.-Or. : Argelès-sur-Mer, Cosperons, vallons de Collioure et de Port-Vendres, vallée de la Vall.

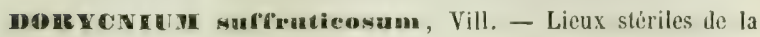
région méridionale. - Remonte dans la Drôme vers le Buis, Nyons, Crest, Die, Barnave, Erome eutre Serves et 'T'ain, Granne, Loriol, Condillac; dans l'Ardèche à Rochemaure, base du mont Combier, Roche-de-Gourdon, mont Charray, Aubenas, Ucel, Saint-Julien-du-Serre, Chassagnt, la Voulte, Châteaubourg; dans les Hautes-Alpes, vers Gap, Ventavon, Laragne, Serres, Ribiers, Rosans; dans la Savoie à Cruet, Saint-Jeande-la-Porte; enfin dans l'Isère à Pont-en-Royans, le Gua entre les Saillans et Saint-Barthélemy, Vertrieu près la Balme.

D. Collinum, Jord et Four.. - Nyons. - Tournon.

D. affine, J. F. - Avignon.

D. decumbens, Jorl. - Avignon, îles du Rhône à Valabrègues, Beaucaire, Aigues-Mortes. - Sables de la Durance vers le pont de Pertuis. - Ard. : Celles, Baix.

D. gracile, Jord. - B.-du-Rh. : Bords de l'étang de Berre, la Camargue, rives de l'Arc. - Var: les Sablettes près Toulon, les Pesquiers près Hyères. - Alp.-2Iar.: prairies du Var près Nice. - Hér. : prés maritimes de Palavas, Maguelonne, Cette, les Onglous, Pérols, Roquehaute, Vendres. - Aude : plages de Ia Clape, Etdepal.

T. Gerbaceann, Vill. - Bords des torrents, - Is.: digues du Drac prìs de Grenoble, ile de Champ près Vizille, entre Milmaze et Prébois près Mens, pont de Brion près le Monestierde-Clermont. - Sav.: Apremont, abîmes de Myans, Cruet, tlbertville. - H.-Sav. : Miolans. - Pyr.-Or: sur le littoral.

Lotes reetas, L. - Lieux humides de la région méridionale jusque dans la Drôme à Nyons et Montélimar ainsi que dans les Basses-Alpes vers les Mées.

C. Hirsutas, L. - Coteaux secs de la région méridionale. Remonte jusque dans la partic sud du Dauphiné et du Vivarais. - Je l'ai trouvé à Lyon sur les talus du chemin de ronde des Brolteaux. La villosité est très-variable dans cette espèce; elle est blanche-tomenteuse dans la forme c. sericeus, D. C. 
qu'on trouve dans l'île de Porquerolle, au cap Bregançon, à Saint-Raphaël.

L. parviflorus, Desf. - Lieux arides sablonneux. - Sablettes près Toulon, ile de Porquerolles, forêt des Maures, Fréjus, Saint-Tropez, cap Croisette près Cannes.

L. angustissimus, L. - Lieux incultes de la région méridionale. - Ard : Vals. - Var et Alp.-Mar. : Toulon, Fréjus, Saint-Tropez, Grasse, Antibes, Nice. - Gard : Broussan, Trou-du-Pérussas près le Vigan, Vézenobre, Augeac, Robiac, Avèze, l'Espérou. - Hér. : Montpellicr, la Salvetat, Fraïsse. Une forme de cette espèce se trouve aussi dans le Rhône à Charbonnières et dans l'Ain à Montribloud, Myonnay, SaintAndré-le-Corcy, Monthieu, Saint-Didier-sur-Chalaronne, SaintPaul-de-Varax.

L. hispldus, Desf.- Terres sablonneuses de la région méridionale. - B.-du-Rh. : Saint-Chamas, la Trévaresse. - Var: forêt des Maures, Saint-Tropez, le Luc. - Alp.-Mar. : montagnes de Caussols, de Défens, de Mulacé, l'Agel, golfe Jouan, Ile SainteMarguerite.-Gard: Gẻnẻrac, Poulx.

L. conimbricensis Brot. - Coteaux maritimes. - Var: SaintTropez, Fréjus, Saint-Raphä̈l. - Hér: Roquehaute où existe aussi la variété $L$. glaberrimus, D. C.

L. decumbens, Poir. - Bords des marais salés. - B.-du-Rh. : Saint-Chamas, Miramas. bords de l'étang de Marignane, les Milles sur les bords de l'Arc. - Var: Sablettes près Toulon, Ceinturon d'Hières, île de Porquerolle. — Hér. - Aude: SainteLucie.

C. Corniculatus, L. - Coteaux et pelouses depuis les plaines jus(qu'aux sommités des montagnes où il présente une forme alpestris très-remarquable. - Cetle espèce est au plus haut point polymorphe.

L. villosus, Thuill. - Distribution géographique mal connue.

L. pilosus, Jord. - Lieux arides. - H.-Sav. : Annecy. - H.-Alp. : Laragne près Serres, Ribiers. - La Provence.

L. tenuis, Kit. - Prairies humides des terrains siliceux et argilosiliceux. 
L. Delorti, Jord. - B.-elu-Rh.: Sainte-Vicloire. - Var: Rians, mont Faron, mont Coudon, le Luc. - Hér. et Aude : pic Saint-Loup, garrigues entre Nissan et la Clape, Pech-de-l'Agnel, Pastouret.

L. uliginosus, Schk, - Prés marécageux.

L. patulus, Martr. Don. - Charbonnières près Lyon.

C. Allonil, Desv. - Rochers maritimes.-B.-du-Rh. : environs de Marseille, cap des Goudes. - Var et Alp.-Mar. : fort Lamalgue près Toulon, Carqueiranne, îles d'Hyères, Bormes, SaintTropez, le Lavandou, rochers des Lions près Saint-Raphaël, Hle Sainte-Marguerite, ile Saint-Ifonorat, de Cannes à Menton. -Pyr.-Or. : vallons d'Argelès, de Collioure et de Banyuls, Trencada-l'Ambulla.

L. ornithopodioides, L. - Champs du littoral de la Provence et des Alpes-Maritimes. - Manque dans le Gard et dans l'Hérault quoiqu'il se trouve sur le littoral du Roussillon.

L. edulis, L. - Coteaux maritimcs. - B.-du-Rh. : Notre-Dame-dela-Garde, Ratonneau, Cassis. - Var et Alp.-Nar. : Toulon au cap Brun et au fort Malbousquet, Hyères, iles de Porquerolle et de Saint-Honorat, Saint-Tropez, l'Este̊rel, Agay et de lả jusqu'à Nice et Menton.

TETRAgONOLOBUS siliquosus, Roth. - Prairies humides dans tout le bassin. - Variété maritimus à feuilles glabres et charnues sur le littoral.

T. purpureus, Mlench.-Alp.-Mar. : île Sainte-Marguerite, Vence, Nice au Var. - Non loin de la frontière à Vintimiglia sur le territoire italien et, plus loin, à San Remo .

ASTIEAGAUS pentaglottis, L. - Collines arides, - B.-du-Rh. : la Serviane près Marseille. - Var : pied du mont Coudon, Carqueiranne, Pierrefeu, le Luc. - Hér. et Aude: Nissan au Pas-de-Loup, Montady près Béziers, Cazouls-les-Béziers, Clot de Nières près Lespignan, Levrettes, Junquières.

A. stella, Gouan. - Lieux incultes de la région méridionale. Vaucl. : Montdevergue, près Avignon. - B.-du-Rh. : Roquefavour, étang de Berre, entre la Mède et Châteauneuf-lesMártigues, le Pas-des-Lanciers, plateaux au N.-0. de la Tour-deKeyrié près Aix. - Var: Draguignan, Toulon. - Gard : bois 
de Broussan près Nìmes, carrières de Beaucaire, Villencuve, aux Angles. - Hér. : environs de Montpellier, Saint-Loup, Colombière, Monmau, Caunelle, Bíziers, Nissan, Capestang, Montady, Cazouls-les-Béziers, Lavagnac près Bellarga. Aude : Lespignan, bassin de Junquières. - Pyr.-Or : ChâteauRoussillon, massif du Llaurenti.

A. Sesameus, L. - Licux stériles de la région méridionale. Vaucl. : le Pontet près Avignon. - B.-du-Rh. : les Martigues, environs d'Aix aux Trois-Moulins et sur le chemin de SaintAndrẻ. - Var et Alp.-IIar. : la Garonne près Toulon, Pierrefeu, Nice.-Hér. : Colombière, Monmau, Béziers, Nissan, Cazouls-les-Béziers, Montady, Roquehaute, Agde, Saint-Jeande-Vedas. - Aude : remparts de Narbonne, Pas-du-Loup. Pyr.-Or.: Peyrestortes, Cases-de-Pena, Baixas, Estagel, SaintPaul.

A. epiglottis, L. - Lieux ariles. - Var: Toulon, Coudon, la Valette. - Hér. : Agde.

A. hannosus, L. - Lieux pierreux de la région méridionale. - B.-Alp. : Sisteron. - Vaucl. : environs d'Apt, d'Orange et d'Avignon. - B.-du-Rh.: Cuques, - Var: Toulon, le Luc, Saint-Raphaël, Gondin, Colombier. - Alp.-Mar. : île SainteMarguerite, Antibes, Nice, Menton. - Gard: environs de Nimes, Manducl, Bellegarde. - Assez répandu dans l'Hérault, l'Aude et les Pyrénées-Orientales. - Remonte dans la partie méridionale du Dauphiné vers Nyons, le Buis, Saint-André-deRosans.

A. glyeyphyllos, L. - Bois, haies, lieux humides dans tout le bassin.

A. elcer, L. - Nul dans la partie septentrionale du bassin. - Commence à paraître dans le Valais à Mlartigny, Fully, Branson, mayens de Sion, puis dans le canton de Vaud à Payerne, Mathod, Orbe, Entreroches, Chamblande, Noville, Morges, Veret, le Genet près Rolle. II se montre aussi en quelques localit’s situées en dehors de nos limites entre la chaine du Jura et les lacs de Neuchâtel et de Bienne. - H.-Sav. : au pied des Voirons, près de Lucinge, Saint-Cergues, Salève au pied de la Croisette, Viry, 
Bonneville. - Ain : environs de Ferney et de Gex. - Sav. : d'Avricux au fort de l'Esseillon, Saint-Jean-de-Maurienne, Saint-Julien, bois Champion près Moutiers. - Rh. : la Mouche et les saulaies d'Oullins près Lyon. - Is.: Feyzin et les bords du Rhone en aval de Vienne, polygone de Grenoble, Huez en Oisans, - Dr. : Saint-Vallier. - Ard. : iles du Pouzin et bords du Rhône. - H.Alp.: Villard d'Arène, Orcières, Manteyer, Durbon. - B.-Alp.: Larche. - Gard : iles et bords du Rhône à Pont-Saint-Esprit, Coudoulet

A. exscapus, L. - Valais : forêts de Sapins et do Mélèzes entre Vercorin et Travers, val d'Anniviers, bois de Finge, Zermalt, Saas, Langenthal, val Binn, Simplon. - Cette rarissime espèce se trouve aussi sur le versant italien du mont Rose, dans la vallée de Cogne.

A. aIopecuroides, L. - Pâturages des Hautes-Alpes à Boscodon près Embrun, mont Chabrières près Chorges, entre ChâteauQueyras et Villevieille près le torrent du Brasc. - B.-Alp: Bousollières près Barcelonnelte.

A. napbonensis, Gouan. - Lér. : cnvirons de Béziers à Nissan, bois Saint-Martin à Quarante, Montady, Pas-du-Loup, Capeslang à la Bastide-Neuve. - Aude : environs de Narbonne, entre Salses et la Nouvelle, Pas-du-Loup près Sigean, île Sainte-Lucie.

A. purpenreus, Lam. - Pelouses et rocailles. - Is. : Mens, SaintBaudille, Pipet, Marcieu, Savel près la Mure, pentes du mont Sénèpe, Corps, Clavans, Champ près Vizille, entrainé par le Drac jusque près de Grenoble. - H.-Alp. : Briançon, le Noyer en Champsaur, Charance, Rabou, col de Glaise, Menteyer, nont Séuse, Malcombe, Romette, Durbon, montague de Crigne, mont Rognouse, Rosans, Montmorin, Bruis, Saint-André-deRosans. - Dr. : Barnave, Die. - B.-Alp.: Lauzannier, la Condamine, Meyronnes, Tournoux, mont de Gache près Sisteron. - Vaucl. : mont Ventoux, Saint-Amans, Sablet, Séguret. B.-du-Rh.: environs de Marseille, la Bourdonnière, SaintPons, Sainte-Victoire, Saint-Germain, Pilon-du-Roi. - Var : Cuers, Bagnols, le Luc, Bezaudun, Sainte-Baume. - Alp.-Mar.: 
toutes les montagnes du littoral. - Pyr.-Or.: Canigou, Fontde-la-Conque.

A. Icontluus, Jacq. - Valais : Grand-Saint-Bernard, Bagnes, Anniviers, Zermatt, Saas, Mattmark, val Binn. - H.-Alp. : environs de Briançon et de Gap.-B.-Alp. : Sérennes, Lauzannier.

A. claux, L. - Coteaux. - Var : environs de Toulon. - Hér. et Aude : environs de Béziers à Nissan, Pierre-Plantée au bas de la Côte-Parasol, Pas-du-Loup, Levrettes, Pech-de-l'Agnel près Narbonne. - Pyr.-Or. : montagne de Céret au-dessus du MasCarol.

A. hypoglottis, L. - Pelouses des montagnes. - Sav. : de Mo dane à Lans-le-Bourg, mont Cenis. - Is. : Alpe du mont de Lans. - H.-Alp. : Lautaret, la Grave-en-Paris, Névache, mont Genèvre, mont Viso, Sappey de la Bàtie-Neuve, mont Séuse, col du Noyer en Champsaur, le Pleyne, la Grangette. -B.-Alp. : Sérennes, la Condamine, Larche, Barcelonnette. - Alp.-IFar. : montagnes de Caussols près Grasse, Estenc, col de la Maddalena, sommet de la Colmiane entre Valdiblora et Saint-Martin. Pyr.-Or. : entre Mosset et Salvanère.

A. onobrychls, L. - Lieux graveleux des montagnes. - Valais : Martigny, Fully, Branson, Charrat, Saxon, Folatères, les Marıques, entre Martigny et Sembrancher, Hérémence, Arbaz, Vercorin, Varone.-Sav. : Pas-du-Roc près Saint-Michel et plus loin en allant vers Lans-le-Bourg et au mont Cenis, de Moutiers à Bourg-Saint-Maurice. - Is. : entrainé par la Romanche et le Drac au polygone de Grenoble, Pont-de-Claix, Iles de Champagnier- II.-Alp. : entre la Grave et les Dauphins, Lautaret, Briançon, Château-Queyras, les Roux près Abriès, forêt de Boscodon près Embrun, Halcombe, Corrẻo, Puy-Montbaud et Charance près Gap. - B.-Alp. : la Condamine, Barcelonnette. - Alp.-Har. : val de Bourdous au-dessus d'Entraunes. Saint-Etienne, Isola, Sainte-Annc-de-Vinaï. - Pyr.-Or: mont Louis, prairies du Capcir.

A. vesicarius, L. - Rochers. - Sav. : Villarodin-en-Mlaurienne. - H.-Alp. : Briançon aux Fontenilles, Sainte-Ylarguerite entre la Besse̊e et Saint-Marlin, Guillestre, mont Rognouse. - Alp.- 
Mar. : mont Cheiron, Caussols, les Claps au-dessus de Grasse, Utelle.

A. austriacus, L. - Débris de rochers des Hautes-Alpes sous le fort des fêtes de Briançon, Champ-Martel à la Roche-des-Arnauds.

A. monspessulanus, L. - Pelouses des montagnes. - Vaud et Valais: Aigle, Ollon, Bex, Saxon, mont d'Orge', Saint-Leonhard, Sierre, Varone, Saas, Langenthal, Zermatt, val Binn. II.-Sav. : base du Mole ver's Ayse. - Sav : entre Cruet et Arbin, rive gauche du Doron près Noutiers, Etroit du Cieix, col du Frêne, Saint-Jean-de-Maurienne, Mndane, Lans-le-Bourg. Is. : Bastille de Grenoble, Corenc, Comboire. Claix, descend jusque vers le Polygone de Grenoble. - H.-Alp. : entre la Grave et les Dauphins, Briançon, Aiguilles en Queyras, mont Viso, bords du torrent entre Pra-Michel et les Chalmelles, SaintVéran au bas du vallon Marcel, commun autour de Gap, mont d'Avançon, Séuse, Aups.-B.-Alp. : Condamine, SaintPaul, Sisteron. - Dr.: Crest, Loriol, Condillac, Nyons, Donzère. - Ard.: Baix, Celles, l'Escrinet, Vallon, Chomérac, Rochemaure, - Vaucl. el B.-du-Rlı. : commun dans les environs d'Apt, d'Avignon, d'Orange, d'Aix et de Áarseille. Var: Baou de Quatre-Heures près Toulon, le Luc, Cabasse, Bezaudun. - Alp.-Mar. : coteaux au-dessus de Grasse, de Nice et de Menton. - Commun dans le Gard et l'Hérault autour de Nîmes, Montpellier, pic Saint-Loup, Colombière, Monnau, Fonfrède, garrigue du Terrail, Caunel, Saint-Guilhem, Béziers, - Pyr.-Or. : Mont-Louis et toute la Cerdagne, vallée d'Ille, de Cornella-du-Confluent, de Vernet el de l'Agly.

A. incanus, L. - Lieux stériles de la région méridionale. - Commence à paraître au sud de Gap à Saint-Hens, Combe-Noire, Laric, Grand-Collet près Tallard, mont Rognouse. - Dr. : le Buis. - B.-Alp. : Sisteron. - Vaucl. : Vedène, colline SainteAnne près Avignon, Viens près Apt. - B.-du-Rh. et Var: Saint-Tronc, Tour de la Keirié près Aix, Sainte-Baume, Mourières près Toulon. - Gard: environs de Nîmes, bois des Espèces, Vaqueirolle. - Hér. : environs de Montpellier, Saint- 
Loup, Saint-Mathieu-de-Tréviès. - Aude: Pech-de-l'Agnel près Narbonne, Crabit, Montredon au Mourcl. - Pyr.-Or.: Cambres-d'Aze, vallées d'Eyne, de Llo, Costa-Bona.

A. depressus, L. - Rochers des montagiıes. - Valais et Vaud: Dent de Morcles, Alpes de Lens, Château-d'Oex, - H.-Sav. : mont Brizon au-dessus de Solaison, petit Bornant, Vergy, Léchaud, au-dessus de Sézenet près Dingy, dent d'Oche.Sav. : petit Saint-Bernard à Leschaud, Lans-le-Villard, mont Cenis. - Is. : Saint-Eynard, col de l'Arc. - Dr. : ld CroixIlante à la montagne de Lussette, mont d'Angèle, Aucelon. H.-Alp. : Devez-de-Rabou, la Grangette, col de la Palelte, mont Séuse, sommet du Roc de Chabrières, Embrun. - B.-Alp.: Brame-Buou, la Condamine. - Vaucl. : mont Ventoux. Alp.-Mar.: montagne du Cheiron, entre Tende et Garlin. Pyr.-Or.: Bac-de-Bolquère, vallée de Prats-de-Balaguer.

A. tragnesutha, L. - B.-du-Rh. et Var: sables maritimes à Montredon, Saint-lyr, golfe des Liques vers les ruines de Taurentum, batterie Saint-EIme et les Sablettes près Toulon. Hér.: Agda vers l'embouchure de l'Hérault. - Aude : étangs de Salses, de la Nouvelle, île Sainte-Lucie.

A. aristatas, Lhẻr. - Lieux secs des montagnes et des collines.

Vaud et Valais: Château-d'Oex, pied des Diablerets, la Morge, Leytron, la Rosseleina, Findelen. - Ain : bords de la Valserine à Chézery, Bellegarde, Lélex. - H.-Sav. : Passy, gras du Platet, mont Méri, Reposoir. - Sav.: les Bauges, mont Drizon, Orizan à la Fégère, mont Charvin, la Maurienne, Saint-Julien, Lans-le-Bourg, Lans-le-Villard, mont Cenis. - Is. : la Balme près Crémieu, la Salette, Mens. - Dr. : mont d'Angèle, Aucelon. - H.-Alp. : Lautaret à Prime-Messe, Briançon au fort des Têtes, Granges d'Isoire, entre Château-Queyras et Villevieille, Montmorin au col de Casset, mont Bayard, mont Séuse, Chaudun, col du Noyer, Charance, Fouillouse, mont Chabrières.-B.-Alp.: Saint-Paul, Malmore, la Condamine, Barcelonnette. - Vaucl. : mont Ventoux.-Alp.-Mar. : col de Tende, des sources du Var à Puget-Théniers, vallon de Chaus, Saint-Etienne. - Pyr.-0r. : Bac-de-Bolquère, montagne de Madres, col d'Ares, 
OX YTtorers campestrik, 1). C. - Pelouses et lieux arides des hautes montagnes. - Nul dans les Vosges et dans la chaine du Jura. - Valais : Simplon, vallice de Binn, Zermatt, Evolena, Leukerbad, Torrenthorı, Guggerhübel, Zermontana, Lavarraz, Alpes de Bagnes, descend jusqu'à la Bâtia près Martigny. II.-Sav. : les deux versants de la chaine du mont Blanc, montagnes du Chablais, dents d'0che, mont des Granges, Rocd'Enfer au-dessus de Saint-Jean-d'Aulph, col de Golèse, mont Tournette, Vergy, Mégève. - Sav. : cols de la Seigne et du Bon homme, cols Joly et Iseran, Hauteluce, Chamoceran, SaintJean-de-Maurienne, mont Cenis, montagnes des Allues. - Is. : montagnes de l'Oisans, chaîne des Grandes-Rousses, Huez, rochers entre l'Erpiat et le lac Blane, entraîné par le Drac jus(qu'au Polygone de Grenoble.-H.-Alp. : la Grave, Villard-d'Arène aux glaciers du Bec, Lautaret, Galibier, col Malrif, mont Viso, Sígure, vallon des Vaches, col Agnel, Saint-Véran à IJarcel, mont Aurouse, la Grangette, col de la Palette, Chaudun.Dr. : mont Angèle. - B.-Alp. : col de Soltron, la Manche, Stropi, Mirandole, Larche, Condamine, Parpaillon. - Montagnes des Alpes-Maritimes. - Pyr.-Or. : Costa-Bona, Canigou, Font-de-Comps, vallées d'Eyne, Llaurenti.

D. Fotida , D. C. - Pelouses des montagnes. - Valais : Torrembé, Zermontana, Zanrion, Mẻribé, Zermalt. - Sav. : vallée de Tignes, montagnes de Frẻjus, mont Cenis.-B.-Alp. : les TroisEvêchẻs au-dessus de Villard-d'Arène, Galibier, col Malrif, mont Viso, col Agnel, crêtes de Ségure, col de Ruine, col Vieux, Chaillol-le-Vieil. - B-Alp. : montagnes de Barcelonnette, rocher de Saint-0urs, la Condamine dans le vallon Bérard. - Pyr.-0r. : vallée d'Eyne.

D. Ialleri, Bunge. - Pelouses des hautes montagnes. - Valais : rochers des Folatères sur Saxon, Schalbet, Rohrberg, Saxon, arêtes de Pierre-à-Voir, Riddes, Bramois, Haudères, Saas, Zermatt.-Sav. : Termignon.-H.-Alp.: col de Gondran en descendant sur Cervières, col Malrif, entre Villevieille et Molines en Queyras, Abriès, Valprévaire, Combe de Ségure, col Agnel, Saint-Véran. - B.-Alp. : rocher des Mouchillons, la Conda- 


\section{$-164-$}

mine.-Pyr.-Or. : Canigou, vallée de la Coma du Tech, Esquerdes de Roya, Pla-Guillem, vallée d'Eyne, Cambres-d'Aze, Font-de-Comps, Llaurenti.

D. Gautini, Bunge. 0 . cyanea Bieb. - Rochers et pelouses des hautes montagnes. - Valais : montagnes de Zermatt, Rothorn, Augstelberg, Hornli, Schwarzsee. - H. Sav. : les deux versants de la chaine du mont Blanc, le Buet, vers les chalets de Barberine, la Portette sur le Platet, montagnes de Samoëns et de Sixt. - Sav. : vallée des Allues, entre Laval de Tignes et le col de la Leisse, cols de la Seigne et de ia Rocheur, mont Cenis. - H.-Alp. : les Trois-Évêchés, col du Goléon, au-dessus de la Grave, Galibier, vallée de Cervières, col Malrif, Péas, Ségure, mont Pelvas, cols de la Traversette et de Ruines, vallon des Vaches, col Agnel, col de l'Echauda au-dessus du Monestier-deBriançon, col de Glaise, Chaillol-le-Vieil, la Grangette, Durbonnas, mont Obiou, Alp-Martin, Lopet, Orcières, mont Aurouse, - B.-Alp. : rocher de Saint-Ours. - Vaucl. : mont Ventoux. - Alp.-Mar. : l'Authion, Tende, val de Strop, col de Jallorgues, Saint-Etienne.

o. Inppouiea, Gaud. - Pelouses des Alpes. - Valais: Seilon des Diez, Sasseneire, Saint-Bernard, Zermalt, Tasch, Staffel, Hornli, Rothorn, Saas, Schwarzberg. - H.-Sav. : vallée de Montjoie à Contamines, Allée-Blanche en Piémont, entre l'Aiguille et le mont Joly. - Sav. : vallée des Allues, mont Cenis. - H.-Alp. : montée au col de la Traversette, chalets du Galibier.-B.-Alp. : Lauzannier, la Condamine.

o. pyrenaiea, Gr. Godr. - Pyr.-0r. : vallée d'Eyne, Cambresd'Aze, Llaurenti.

O. montana, D. C. - Pâturages des hautes montagnes. - Jura : toute la chaine du Colombier et du Reculet. - Valais : montagnes de Val-d'Hliez, mont Fully, Morgins, pied des Diablerets, Delong, la Comba, Bellalui, Zermontana, Genmi, Guggerhubel, Torrenthorn. - II.-Sav. : montagnes de Samoëns, col de Golèse, Roc-d'Enfer, monts des Granges et de Pelloua, Charvin, Vergy, Méry, Tournette, Buet, col de Balme, Croix-de-Fer, le Platet. - Sav. : le Bonhomme, montagnes de Hauteluce, de Beaufort, 
mont Trelod, Drizon, Margériaz.- Is. : col de l'Arc, la Moucherolle, Chamechaude, Grand-Som, mont Sénèpe. - H.-Alp.: col de Chaudun, la Mielette, mont Aurouse, mont Morgon, Sappey de la Bátic-Neuve, cols Malrif et Isoire, Abriès en Queyras, col Vieux, mont Viso, Saint-Véran, cote de Clausis, les Serines. - B.-Alp. : Malmore, Mirandole, Plate-Lombarde, la Condamine à Bachasse. - Dr. : le Glandaz. - Alp.-Mar. : cntre Tende et Carlin, mont Bego, le Garret au-dessus d'Entraunes, col de Pouriac. - Pyr.-Or. : clot du Cambres-d'Aze, vallée de Llo.

0. pllosa, D. C. - Graviers des torrents de montagnes. - Valais : la Bâtia près Martigny, Folatères, Charrat, Riddes, Saint-Georges de Sion, Sierre. - Sav. : Moutiers au bois Champion, Petterain, Saint-Jean-de-Iaurienne, Saint-MIichel, Termignon, l'Echaillon, mont Cenis à Ronches et à l'Eau-Blanche. - Is. : Polygone de Grenoble, Iles Champagnier, - H.-Alp.: Briançon sous le fort des Têtes, graviers de la Durance vers la Saulce.-B.-Alp. : rochers de la Blache près Condamine.-Pyr.-Or. : Canigou, vallée de Cady, Nuria, Costa-Bona.

PIIACalpina, Wulf. - Pelouses et débris de rochers des montagnes. - Valais: Javernaz, Trient, Alpes de Bagnes, Zermatt, Munstigerthal, Griess. - H.-Sav. : col de Balme, Buet, Pormenaz, montagnes de Sixt et de Samoëns, monts des Cornettes et des Granges, Signal des Agneaux et autres montagnes du Chablais. - Sav. : montagnes des Allues, de Pesey, mont Cormet au-dessus d'Aime, vallon de la Duys vers les sources de l'Arc, montagnes des Bauges. - Is. : chaines de Belledonne et de l'Oisans, la Salette. - H.-Alp. : la Grave, le Lautaret, Galibier, mont Viso, chalets de Ruines, crêtes de Ségure, rochers de Pierre-Noire, Saint-Véran, col de Vars. - B.-Alp. : Lauzannier. - Alp.-IIar. : cols de Fenêtre et de la Maddalena, Alpes de la Briga et de Beccaroussa. - Pyr.-Or. : vallées d'Eyne, d'Err, de Llo, de Carença, bois de Salvanère, le Llaurenti.

P. Crigida, L. - Pelouses alpestres. - Valais : Javernaz, Zermontana, Zanrion, montagnes autour de Leukerbad, Gemmi, Zermatt. - H.Sav. : chaîne du mont Blanc, base de l'Aiguille du Goûté, 
versant italien dans l'Allée-Blanche, mont Lachat, vallon d'Entre-les-Eaux au pied du Buet, montagnes de Samoëns et de Sixt, col d'Anterne, mont Joly, mont Méry, montagnes de Saint-Jean-d'Aulph. - Sav. : montagnes de Hauteluce, la Gitaz prics Beaufort.

P. astragalina, D. C. - Pelouses alpestres. - Valais : mont Fully, Trient, Pierre-à-Voir, Gemmi, Zermatt. - H.-Sav. : chaine du mont Blanc depuis le col de la Seigne jusqu'au col de Balme, montagnes de Samoëns et de Sirt, col de Golise, Cornette de Bise, mont des Cornettes, Roc-dEnfer, mont Vergy, Brizon, Haut-de-Véron, pâturages de Flaine, mont Charvin, Tournette, - Sav. : col du Bonhomme, montagnes de Hauteluce, Modane, Laval de Tignes, les Avanchers. - Is. : Alpe du mont de Lans. - H.-Alp. : Villard d-Aréne sous les glaciers du Bec, mont Viso, Combe de Ségure, le long du chemin du Saure et du Fournet, Saint-Véran aux Trois-Pierres, col des Hayes, Lopet en Champsaur. - B.-Alp.: Plate-Lombarde, Larche, la Condamine, Parpaillon. - Alp.-Har. : cols de Tanarello et de Jallorgues.- Pyr.-Or : vallée d'Eyne, Nuria.

P. australis, L. - Pelouses des hautes montagnes. - Valais: Bovonnaz, Javernaz, Rosselenaz, val de Bagnes, Giétroz, Grengiols, Binn, Zermatt. - H.-Sav. : vallon d'Entre-les-Eaux au pied du Buet, Roc-d'Enfer, col de Golèse, Vergy, Tournette. Sav. : le Bonhomme, Lans-le Bourg en allant vers la Chavière, mont Fréjus, Chamoceran, mont Cenis à l'Eau-Blanche. - Is. : chaîne de Belledonne à la Combe de la Lance, Ia Salette. If.-Alp. : col de Glaise, Durbonnas, mont Aurouse, col de Chaudun ả la Mielette, la Grangette, Sappey de la Bâtie-Neuve, cols Isoire et Malrif, mont Viso, Valprévaire, Ségure, gorge de la Taillante, Saint-Vẻran au plateau de Clausis. - B.-Alp. : rocher de Saint-Ours, Lauzannier, Parpaillon, vallon de Meyronnes. - Alp.-Ifar. : cols Bertrand, de Pouriac et de la Maddalena, Alpes de Tende. - Vaucl. : mont Ventoux, - Pyr.-0r : Cambres-d'Aze, mont Louis, vallée d'Eyne.

P. Gerarali, Vill. - Pelouses des Alpes. - H.-Alp.: montagnes des environs de Briançon à Prorel cl de là à la montagne des 
Tenailles, Névache, col des. Ilayes, mont Viso, col Vieux, mont d'Autare près Ancelle. - B.-Alp. : rocher de Saint-Ours, - Pyr.-Or. : Carença, plateau de Camp-Magre, Costa-Bona.

BISEG IE E. pelechas, L. - Coleaux maritimes. - Var et Alp.-Mar. : Iles du Levanit, du Lavandou el d'Hyères, Bormes, Pierrefeu, Saint-Tropez, Fréjus, Antibes, - Hér. - Pyr.-Or. : Trencada d'Ambulla, Château-Roussillon, Força-Réal, vallée de l'Agly, Cassagnes.

COLUTEA arborescens, L. - Cotcaux calcaires, - Côte-d'or : Notre-Dame d'Etang, vallon de l'Ouche, Gevrey, FontaineFroide, mont Afrique. - Saône-et-L. : Tournus. - Nul dans Haute-Saône et Jura. - Doubs : rare à Cuse dans les carrières du mont Querelles. - Plus commun dans le bassin helvétique autour de Neuchâtel, Boudry, Nyons, Morges, Genève et dans le Valais à Saint-1laurice, Fully, Sierre et Saint-Leonhard.H.-Sav. : Bonneville près du Môle. - Ain : Genay. - Is : Frontonas, Bastille de Grenoble, Vouillant, Comboire. - Sav. : Saint-Saturnin près Chamberry, bois Champion près Houtiers, Saint-Jean-de-Maurienne.-H.-Alp. : Aspres-les-Veynes, Serres, la Garde et Bayard près Gap, bords de la Luye, bois du Chabal. -B.-Alp. : bois de Tournoux, près la Condamine.-Dr. : Rioux près Saint-Vallier, Barnave. - Plus commun dans la Provence, les Alpes-Maritimes, le Gard, l'Hérault et les Pyrénées-Orientales.- Souvent cultivé.

TO BINIA pseudo Aenein, L. - Arbre de l'Amérique septentrionale. - Cultivé et rarement subspontané.

GA AEGA orficinalis, L. - Quelquefois chehppé de cultures.

GXYCYItheza glabra, L. - Cultivé dans la région méridionale. - Naturalisẻ dans les Bouches-du-Rhône vers Puyloubier, Peynier; dans le Var à Fox-Amphoux, ile de Porquerolle; dans l'Hérault.

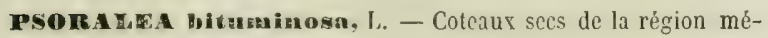
ridionale. - Remonte dans l'Ardèche, à l'Argentière, Payolive, Baix, Celles, Vals jusqu'à Tournon; dans la Drôme jusqu'à Buis, Nyons, Barnave, Saint-May, Rémusat, Saint-Vallier et Tain; dans les Basses-Alpes à Sisteron; dans les Hautes-Alpes 
vers la Saulce, Rosans; dans l'Isère jusıju'à Feyzin et Verrricu.

P. plumosa, Reichb. - Var el Alp.-Mar. : remparts de Toulon, collin's d'Hyères, cap Brégançon ; plus commun dans les AlpesMaritimes. - Naturalisé au Port-Juvénal près Montpellier.

vrCra sativa, L. - Cultivé et très-répandu en dehors des cultures.

v. cordata, Wulf. - Trouvé une seule fois par M. Cartier dans les Miaures et par M. Lannes à Saint-Tropez.

V. angustifolin, Roth. - Espice polymorphe commune dans les champs sablonneux de tout le bassin. - On n'a pas suffisamment étudié la distriłution géographique des trois formes suivantes qui ont été pourtant signalées autour de Genéve, de Thonon, de Lyon, de Grenoble, d'Albertville, de Vals, de Nimes, de Montpellier; Lodève, Béziers et dans le Var.

V. segetalis, Thuill. - V. Forsteri, Jord. - V. Bobartii, Forst.

V. Sallei, Timbal-Lagr. - Hér. : Saint-Guilhem-le-Désert.

V. cuneala, Guss. - Var et Alp.-IIar. : mont Lachen, Tanneron. Hér. : Courpouiran près Montpellier.

v. Iathyroides, L. - Coteaux arides et sablonneux. - Valais: Sion entre Valère et Tourbillon.-Environs de Genève à Peney. - Nul dans Haute-Saone, Doubs, Jura. - C.-d'Or : granite des environs de Rouvray. - Région granitique du Beau,olais, I.yonnais, Forez, Pilat. - Dr.: Saint-Vallier, Montmaur, Barnave. - Vaucl. : Orange, Gadagne. - Var et Alp.-Mar. : bois sablonneux des environs de Saint-Tropez, Fréjus, Saint-Valtier, Cannes, Biot, Nice. - Gard : terrains quartzeux des environs de Nimes dans les bois de Broussan, des Espèces, de Cygnan, Tresques.-Hér. : Valros, Pézenas, Tourbes, Capestang, Béziers. - Pyr.-Or. : Sorède, base des Albères, Trencada d'Ambulla.

v. pyrenaiea, Pourr. - Pâturages de la Miellette à Chaudun près Gap. - B.-Alp. : les Signorettes près la Condamine. Pyr.-0r. : commun dans la vallée d'Eyne et dans les prairies du Llaurenti.

v. amphiearpa, Dorth. - Lieux incultes de la région méridionale. - B.-du-Rh. : Château-Gombert près Marseille, Martigues, Carpiagne, Vaufrège, environs d'Aix au quartier de 
Bagnols et à l'ouest de Préguon. - Var: Toulon, le Luc. Alp.-Mar.: Grasse, Biot, Nice au Vinaigrier. - Gard : Ville neuve en face d'Avignon, Grand-Montagnet. - Hẻr. et Aude : cnvirons de Montpellier, les Bergines près Bézicrs, environs de Narbonne, - Pyr.-Or. : Banyuls-sur-Her, pied de la chaîne des Albères, vallée du Rẻart.

v. peregriua, L. - Champs cullirés. - Rare dans la partie septentrionale du bassin oủ il n'a été vu accidentellement que dans quelifues localités de la Côte-d'Or, notamment à Verdun et vers la ferme de Beauregard près Dijon; plus fréquent dans les plaines de la Valbonne (Ain) et autour de Lyon, Sainte-Colombe, Ampuis (Rhône). - Is. : Vienne, Balme de Claix. - Drôme : Saint-Vallier, Valence, Loriol, Crest, Barnave, la Trillière près Die. - Ard. : Vals. - H.-Alp.: Manteyer, Rambaud, SaintMens, Rosans. - Devient commun dans la Provence, les AlpesMaritimes, le Gard, l'Hérault et les Pyrénées-Orientales.

v. Iutea, L. - Champs cultivés des terrains siliceux. - H.Saône : alluvions à Chassey-les-Scey, Saint-Albin. - Jura : granite des environs de Dole, Menotey, forêt de la Serre, argiles tertiaires à cailloux siliceux entre Chaussin et Gatey. - Bois sablonneux des environs de Genève, Aïre, Peney. - Assez commun sur les terrains granitiques de Côte-d'Or, Saône-et-Loire, Rhône, Loire, Ardèche, Vaucluse, Dròme, Gard, Hérault, l'Aude et les Pyrénées-Orientales. - Terrains de transport de la Côte-SaintAndré, Crémieu dans l'Isére. - Ard.: Vals, Vallon. - B.-duRhone: Marignane, vallon des Gardes près Aix. - H.-Alp.: environs de Gap. - Var et Alp.-Mar.: le Luc, Saint-Tropez, Lavandou, Grasse, Nice, Menton.

V. Inybrida, L. - Diffère du précédent par son étendard velu et ses folioles échancrées. - Assez commun dans la Provence, les Alpes-IIaritimes, le Languedoc méridional, le Roussillon, la partie méridionale de l'Ardèche et de la Drôme. - Plus rare dans la partie moyenne et septentrionale du bassin ou il ne se montre qu'accidentellement.

v. marbonensis, L. - Bords des champs de la région méridionale. - Vaucl. : Orange. - B.-du-Rh. : Saint-Julien près Mar- 
scille, Mauret, Fontlébre, moulin Saint-Eutrope près Aix. - Var ct Alp.-Mar.: Toulon, Saint-Tropez, le Luc, Fréjus, Nice au Vinaigrier, l'Essarène, Menton. - Gard: Bellegarde, bords du Vistre, bois de Cygnan. - Hér. : Pézenas, Péret. - Assez commun dans l'Aude et le Roussillon.

Variété serratifolia, Jacq. - Var : la Sauvette. - Hẻr. : Cros sur le Larzac.

v. bithyniea, L.- Moissons de la région méridionale.-B.-du-Rh. : les Olives, Saint-Julien. - Var et Alp.-Mar. : Toulon, Hyères, le Luc, Fréjus, Grasse, Antibes, Villefranche, Menton. - Gard : Mas de Campagne prés Nîmes. - Hér.: Agde, les Onglous Roquehaute, Lézignan-la-Cèbe, Lieuran-Cabrières, Pézenas, Lunas, Lodève. - Aude et Pyr.-Or. : commun dans la plaine et dans les basses Corbières.

v. sepium, L. - Haies et pâturages dans tout le bassin depuis les plaines jusqu'aux montagnes où il présente une forme naine.

Y. tricolor, Seb. ct Mlaur. - Var: forêt des Maures à la Sauvette, Cros-de-Houton, le Lavandou.

v. pammonien, Jacq. - Moissons.- B.-du-Rh.: Fos, les Martigues. - Gard: environs de Nimes, Bagnols, Tresques, Alais, Anduze. - Hér.: Hontpellier, Lunel, Saint-Pargoire, SaintPons-de-Mlauchicns. - Pyr.-Or. : Cases-de-Pena, Baixas, Espirade-l'Agly, vallée du Réart.

v. nrgewtea, Lap. - Pyr.-Or. : bois de Salvanìre et de Boucheville, vallée de la Tet, Fonpédrouse.

v. onobryehioides, L. - Lieux arides des vallées dans les montagnes. - Valais : Hartigny, Branson, Fully, Folatères, Saillon, Sion, Sierre, Saint-Leonhard, Vex, - H.-Sav. : les Voirons. Sav. : Saint-Michel à Modane. - Is. : Mens. - Dr. : Barnave, Aucelon. - H.-Alp. : envirous de Gap, Charance, Séuse, Rabou, montagne de Grignes, à la Côte-Grimaud, mont Aurouse, Rosans. - B.-Alp. : la Condamine. - Vaucl. : mont Ventoux. Var: la Sainte-Baume, le Luc, Morières, la Roque-Broussanne, Séou-Blanc. - Alp.-Har. : Saint-Vallier, Saint-Lambert, Caussols, Vence, Coursegoules, Bougon, Tende, l'Agel. — Gard: Alzon, le Vigan, l'Espérou, Saint-Nicolas.-Hér. : sur le Larzac 
au Cros, le Caylar, Sainl-Navacelle, Madieres, Saint-Guilhem, Saint-Maurice, Lavaqueric. - Aude : environs de Narbonne. Pyr.-0r: Mont-Louis, Villefranche, Trencada d'Ambulla, vallées de Cornella du Confluent et de Vernet-les-Bains.

v. Aumetorum, L. - Bois des montagnes. - Arrond. de Belfort et 13.-Saône.-Doubs, Jura : environs de Montbéliard, de Besançon, Nans, Cubry, Salins, entre Lons-le-Saunier, Couzance et SaintAmour, remonte jusque dans les bois de sapins des environs de Pontarlier. - Ain: montagnes d'İnnimont, de Saint-Rambert, d'Arvières et de Ruffieu.-H.-Sav. : le Salève, les Voirons, Menthon. - Valais et Vaud: Aiglo, Fully, Trois-Torrents, la Chablière. - Sav. : Combe-Noire prés Tamié. - Is. : GrandeChartreuse, Saint-Pancrace, de la Terrasse à Saint-Bernard, Chalais, de Vaulnaveys à Prémol, Bouvesse près Horestel. B.-Alp. : la Condamine. - Alp-Mlar.: val Pesio, Ilonza. Pyr.-0r. : bois de Salvanère et des Fanges.

v. pisuformis, L. - Bois, - H.-Siône: calcaires jurassiques à Chariez, Noidans, Echenoz-la-Méline, Navenne. - C.-d’Or: vallon de l'Ouche, val Suzon, bois de Perrigny. - Valais : audessus de Fully, - Var : forêt des Maures, Collobrières.

V. sylvatien, L. - Bois et prairies, - Nul dans la partie septentrionale du bassin, bien qu'il existe sur le versant helvétique dans le Jura bâlois, soleurois, neuchâtelois, vaudois, dans les Alpes bernoises, dans les montagnes du canton de Vaud au-dessus de Bex et en Valais au-dessus d'Outre-Rhône et de Fully. - H..Sav. : Salève à la montée de la Croisette, Archamp, Pommier, Monelier en montant aux Treize-Arbres, mont Sion, Voirons, vallẻe de Boëge, plateau de Bornes près de la Roche, mont Saxonnet, montagnes d'Annecy-le-Vieux, de Veyrier, de la vallée du Fier, mont Charvin, mont Semnoz, vallée de Chamonix. - Sav. : Combe-Noire près 'Tamié, Moutiers, d'Lime au col du Cormet, Pralognan, Bourg-Saint-Maurice, montagnes de la Maurienne. — Is. : mont Sénèpe, prairie du Vallon au-dessus de la Chapelle en Valjoufrey, la Salette. - H.-Alp.: Lautaret, vallée du Guil près de l'Echalp dans le bois de Bonbernat et de Bouchouse, Sappey de la Bâtic-Neuve, Granon près Val-des-Près. 
v. orobus, D. C. - Cette espèce qui se trouve en dehors de nos limites dans les montagnes des Pyrénées centrales, de l'Aubrac, du Cantal, du mont Dore, du Puy-de-Dóme et de Pierre-surHaute n'existe dans notre circonscription que vers la grange du Pilat, à Planfoy aux sources du Furet, aux Citadelles; puis dans les Cévennes à Concoule, Courdouze, au mont Lozère, et enfin dans l'Hérault à Fraïsse, sur le plateau de la Salvetat.

v. eracen, L. - Prairies dans tout le bassin.

v. Geraredi, Vill. - Prés et bois. - Valais : Fully, Clıarrat, Saxon. - H.-Sav. : Conflans près Albertville. - Is.: mont Rachais, Saint-Eynard, de Quaix à la Pinéa. - H.-Alp. : Boscodon près Embrun, l'Aulanier, bois Vescal près Gap, Château-Queyras, Villevieille, Aiguilles. - B.-Alp.: la Condamine. - Ard. : l'Escrinet, la Viole. - B.-du-Rh. : la Pioline, vallon de Barret près Aix. - Var : chaîne des Maures, les Mayons, la Sauvette. - Alp.-Mar. : l'Estérel, la Roquette, Grasse, Cannes, Berre, mont Mulacé, col de Braus. - Assez commun dans l'Hérault, l'Aude et le Roussillon.

v. temuifolia, Roth. - Haies, bords des bois. - Coteaux calcaires de Saône-et-Loire. - Jura : Dole, Plumont, Champvans, Jouhe, Authume, mont Roland, Salins. - Valais : Martigny, Charrat, Branson, Fully, Saxon. - Environs de Gene̊ve à Peney, Vernier.-H.-Sav. : petit Salève au-dessus de Ilonetier, Annecy. Sav. : environs de Chambéry, de Moutiers, Saint-Michel, Modane. - Rh.: très-commun au mont Cindre, mont Toux, Couzon. - Is. : la Tronche, Pariset, Saint-Nizier, côtes de Sassenage. - H.-Alp. : Charance, col de Glaise, Pleyne de Chaudun, banc du mont Séuse. - B.-Alp.: la Condamine, Larche. - Dr. : Grest, Saint-Vallier. - Vaucl. : Saint-Amans. - Var et Alp.-Mar. : Je Luc, col de Braus, Touët-de-Beuil, Guillaumes. - Gard: le Vigan, Campestre, Lanuéjols, Coudoulet, Saint-Nicolas, Cygnan, Manduel. - Assez commun dans l'Hérault et le Roussillon.

v. varia, Host - Moissons. - Haute-Saone, Doubs, Jura. - Environs de Genève, de Chambéry et d'Annecy. - Rh.: Charbonnières, Lissieu. - Is. : Seyssinet, Saint-Nizier, Villars-de-Lans, 
la Motte-les-Bains, Saint-Didier en Devoluy. - Dr. : Crest. Var et Alpes-Maritimes. — Gard : Manduel, Broussan, Campagne. - Pyr.-Or.: basses Corbières, valléc du Réart, Fourques, Terrats.

Variété Villosa : le Luc (Var).

v. Ittoralis, Salzm. - Coteaux maritimes - B.-du-Rh. : Bec-del'Aigle. - Var: le Lazaret près Saint-Mandrier, Hyères, P'orquerolle, Fréjus.

V. atropurpurea, Desf. - Chiamps. - Var: Toulon à SainteMarguerite, ile de Portcros, de Porquerolle et du Levant, le Luc, les Maures, Fréjus. - Alpes-Mar. : la Napoule, Grasse, cap Martin près Mlenton. - Hêr.: Roquehaute, pied du mont Saint-Loup près Agde, Béziers, Paulhan, Pézenas, Saint-Chinian, Mauguio, Pérols. - Pyr.-0r. : Perpignan, basses Albères jusqu'à Céret, vallons de Collioure et de Banyuls-sur-Mer.

v. monanthos, Desf. - Champs. - Var: Six-Fours : le Luc. Alp.-Mar. : rare à Nice. - Gard: le Vigan, Alzon. - Hẻr. : la Malou, l'Auverne près Pradines. - Pyr.-0r.: Perpignan, Château-Roussilion, vallée de la Tet jusqu'à la Cassagne. S'est montré accidentellement dans Saône-et-Loire, à SaintFargeot, Tournus, Anost; dans le Rhòne à Beaunant, Chaponost, Saint-Genis-les-0llières.

v. diøperma, D. C. - Lieux incultes. - Var et Alp.-Mar. : entre Toulon et Hyères, Maures du Luc, Saint-Tropez, Fréjus, Cannes, île Sainte-Marguerite, la Roquette, Menton. - Hér.: Montpellier. - Pyr.-Or. : vallons de Collioure et de Banyulssur-Mer.

ETVUM hirsutum, L. - Moissons des terrains sablonneux dans tout le bassin.

E. tetraspermum, L. - Même habitat. - Varie à gousses glabres ou velues.

E. wubescens, D. C. - Clıamps. - B.-du-Rh. : Montredon près Marseille. - Var: Toulon, la Seyne, Hyères, Saint-Tropez. Hẻr. : Roquchaute, les Soulières près Lodève.

E. gracile, D. C. - Moissors. - Rare dans la partie septentrionale du bassin. - H.-Saône: calcaires du lias à Coulevon, 
Vesoul, Chariez, Lambrey. - Çà et là dans Doubs et Jura. Ain : plaine d'Ambronay, le Iontellier. - Rh. : Villeurbanne, la Tour-de-Salvagny, Charbonnières. - H.Alp.: Veynes, Rosans. - Dr. : Crest.-Ard. : Baix. - B.॰du-Rh.-Var et Alp.Mar. : Toulon, le Luc, Cannes. - Gard: Manduel, Capette, Sylveréal. - Assez commun dans l'Hérault, l'Aude et le Roussillon.

EnVILLA sativa, Link. - Moissons. - Doubs : Montbéliard, Arbouans. - Jura: Dole, Chaussin, Salins, vallée de la Loue. - Assez commun dans le pays bas de la Côte-d'Or et dans Saône-et-Loire. - Ain : plaine d'Ambronay, Saint-Benoît près Belley, Cormaranche près Hauteville.-H-Sav. : Voirons, SaintJulien. - Is. : Crémieu, Saint-Geoirs. - Dr. : Valence. — Plus répandu dans la région méridionale où il est souvent cultivé pour la nourriture de la volaille.

LENS eseulenta, Møench. - Cultivẻ et quelquefois subspontané. C. nigrieans, Godr. - Lieux arides. - Vaucl. : entre Montdevergues, Morières et Orange. - B.-du-Rh. : environs de Marseille et d'Aix, la Crau. - Var: environs de Toulon, fort d'Artigues, pied du mont Coudon, Ampus, le Luc.-Alp.-Mar. : Tende, la Briga. — Gard : la Foux près Alzon, l'Espérou. Pyr.-0r.: pied de Força-Réal, Cases-de-Pena.

CrCEa nrietıum, L. - Cultivé dans la rẻgion méridionale et quelquefois subspontané.

Pisum sativum, L. - Cultivẻ et subspontané.

F. arvense, L. - Cultivé et subspontané.

P. elatius, Bicb. - Lieux pierreux, - Gard: bords du Gardon entre Saint-Nicolas et la Beaume, Serre-de-Bouquet, Arî près du Vigan. - Hẻr. : rochers du Pertus au sommet de la vallée des Plans. - Pyr.-0r. : Trencada d'Ambulla, bords de l'Agly entre la Fou et Ansignan.

LATHYaUs elymenum, L. - Coteaux. - B.-du-Rh. : le Becde-l’Aigle près de la Ciotat. - Var et Alp.-Ifar. : Toulon, Hyères, Saint-Tropez, Fréjus, Cannes, Grasse, Nice, Mfenton. - Hér. : Castelnau près Montpellier: - Pyr.-0r.: vallons de Banyulssur-Mer, Collioure, bas des Albères, Perpignan, Cases-de-Pena, Baixas. 
L. Mrtieulatus, L. - Coteaux du Var et des Alpes-Nar. : Toulon,

la Seyne, Ifyères, Carqueiranne, Fréjus, Cannes, Grasse.

- Pyr.-0r. : environs de l'ermitage de Consolation, vallon de Collioure, Banyuls-sur-Mer, Cases-de-Pena, Baixas, Canet.

C. ochrus, D. C. - Coteaux. - B.-dl-Rh. : les Martigues. - Var et Alp.-Mar. : Toulon, Cannes, Antibes.-Hér. : Agde.-Pyr.-Or. : cnvirons de Mallolles, Sarrat d'en Vaquer, Perpignan, Baixas, Cases-de-Pena, vallée du Réart.

C. aphaea, L. Champs cultivés dans tout le bassin.

T. nissolia, L. - Même habitat.

C. Inisutus, L. - Moissons.

L. cleera, L. - Moissons de la région méridionale. - A été trouvé accidentellement dans la Haute-Savoie à Annemasse près de Genève, au pied des Voirons, à Saint-Julien, aux bains de la Caille et enfin dans l'Ain au fort de l'Ecluse.

C. sntivus, L. - Cultivé et quelquefois subspontané.

У. annums, L. - Moissons de la rígion miridionale. - Remonte dans la Drôme à Nyons, Valence et dans l'Isère à Revantin et Seyssuel près Vienne.

C. sylvestais, L. - Bois et buissons. - Calcaire jurassique de Haute-Saône, arrondissement de Belfort, Doubs, Jura, Coted'Or', Saônc-et-Loire; se trouve aussi sur les terrains granitiques du Morvan vers Saulieu et dans les bois sablonneux des environs de Genève. - H.-Sav. : Bonneville, Veyrier, Crẻ-du-Maure près d'Annecy, Cliamonix. - Sav.: Conflans, les Bauges. Ain : Montribloud, Polzinges, ruines du château de SaintRambert, forêt de Rothonnes près Belley, - Is.: Echirolles, Grande-Chartreuse, Saint-Geoirs. - H.-Alp. : environs de Gap à Loubet, Chaudun, Charance, côte Gélive, la Grangette, Orcières. - Ard. : Vallon, Baix, Vals. - Assez commun dans la Provence, les Alpes-Alaritimes, le Gard, l'Hérault, l'Aude et le Roussillon.

L. Intifolius, L. - Bois et haies, - Cote-d'Or au bas de Talant près de la Fontaine-des-Fées, mont Afrique. - Saône-ct-L.: Chagny, Charolles, Saint-Agnan. - Jura : entre Saint-Amour et Vauxenans, - Sur le versant helvétique au Chaumont au- 
dessus de Neuchâtel, Rolle. - Ain : la Pape, Sathonay, Reyrieu. - Rh. : Couzon, Beaunant. - Is.: Bastille de Grenoble, la Tronche, cotes de Sassenage, Vif, Mens. - Sav. : Saint-Jeande-Maurienne. - Dr. : Barnave, Saint-Vallier, Crest. - Ard. : Vals, Ucel. - H.-Alp. : Gap à Puy-Maure, mont Morgon, Rosans. - Assez commun dans la Provence, les Alpes-Ilaritimes, le Gard, l'Hérault, l'Aude et les Pyrénées-0rientales.

1. ensifolius, Badaro. - Diffère du précédent par ses folioles allongées, étroites, linéaires, acuminées. Environs d'Avignon à Gadagne, bois Jouve et Saint-Jean. - Environs de Marseille, de Brignolles et de Montpellier.

C. heterophyllus, L. - Prairies des montagnes. - Doubs : Morteau, Pontarlier vers le lac Brunet.- Jura : pentes du Colombier, entre Levier et Frasne. - C.-d'Or. : Combe-d'Arcey. - Valais : Martigny, Outre-Rhòne, Sion, Houlignon, Haudères, Leukerbad. - H.-Sav. ; vallée du Reposoir au pied du mont Méry, mont Saxonnet, val Montjoie, Bionnay. - Sav. : Tamié, les Traverses près de Beaufort, Crest-Volant, Houtiers, montée d'Aime au Cormet.- Ain : prairies du Haut-Bugey. - Is. : pré Dufour au-dessus de Saint-Ange, Chalais, la Ferrière d'Allevard, le Fréney, la Bérarde en Oisans. - H.-Alp. : Villard-d'Arène, Bramousse audessus de Château-Queyras, col de Vars. - B.-Alp. : la Condamine, fort Tournoux. - Ard. : environs d'Annonay, Asperjoc. - B.-du-Rh. : environs d'Aix, le long de l'Arc près le pont des Trois-Sautés. - Manque dans le Var. - Alp.-Mar. : SaintDalmas-le-Sauvage, la Roquette-sur-Siagne.-Gard : Campestre. - Hér. : Murviel.- Pyr.-Or. : bois des Albères au-dessus de Sorède et de Laroque, Céret, Arles.

C. cirrhosus, Seringe.-Lieux stériles.-Ard. : Thueyts, Mayres. - Hér.: Saint-Nartin d’Orb. - Pyr.-Or. : Força-Réal, Rodez, Rigarda, vallons de Prades, de Villefranche, de Cornella-duConfluent et de Vernet-les-Bains, remonte le long de la vallée de la Tet.

T. tuberosus, L. - Champs cultivés.

L. palustris, L. - Marais. - C.d'Or. : Limpré, Orgeux, Arcelot. - Bassin du Léman à Roellebot, Sionnet, Bouveret. - Valais: 
Noville, Chessel. - Sàv.: le Bourgel. - Environs de Lyon à Vassieu, Décines. - B.-du-Rh. : Arles en-Cousticro. - Gard: Saint-Gilles, Bellegarde, Franqueveau. - Pyr.-Or. : vallons de Collioure et de Banyuls-sur-Mer.

T. pratensis, L. - Prés dans tout le bassin.

L. angulatus, L. - Moissons de la régrion méridionale. - Se montre quelquefois dans les environs de Lyon à Vaugneray, Chaponost, Décines, Reyricux, Trévoux, et en remontant dans l'Ain à Bourg, Ambérieu, Ambronay ; puis dans Saône-et-Loire et enfin dans le Jura à Bletterans.

L. Bphoerieus, L. - Moissons de la région méridionale. - Rare autour de Lyon, de Genève, Dole, Cluny, Curay, Grenoble, Crémieu, Gap, Tallard, Chambéry, Nyons, Branson près Martigny dans le Valais.

צ. insconspicuus, L. - Moissons de la région méridionale. Remonte dans la Drôme vers le Buis, Barnave; dans les Hautes-Alpes vers Ribiers, Rosans, Mloydans, Saint-Andréde-Rosans; dans l'Ardèche près de Privas; el jusque vers Lyon à Sain-Fonds, Limonest.

C. strns, Vis. - Diflère du précédent par ses gousses glabres. Environs de Marscille, du Luc et de Montrieux.

C. setifolins, L. - Lieux arides - B.-Alp. : Sisteron.-II.-Alp. : Rosans, Ribiers. - Dr: Saint-Vallier, Montélimar, Bellecombe près le Buis, Nyons. - Ard.: Viviers, vallée d'Ouvèze. - Gard : Nîmes, Manduel, Marguerite, Alais, le Vigan, Anduze.-Hérault et Aude. - Pyr.-0r.: Cases-de-Pena, vallons de Villefranche et de Cornella-du-Confluent - Vaucl. : environs d'Avignon. - Var: Toulon, Hyères, le Luc. - Alp.-Nar. B.-du-Rh. : environs d'Aix, vallons de Mauret, des Gardes. Trouvé accidente!lement autour de Thonon (Haute-Savoie).

- RORUs vernus, L. - Bois des montagnes calcaires du Doubs, Jura français et helvétique, Côte-d'Or, Bugey, Revermont, Savoie, Dauphiné, Basses-Alpes jusque dans les Alpes-Maritimes. Valais : sous Ottan, la Comba, mayens de Saxon, mont d'Orge, Lavernaz. - Vaucl. : Saint-Amans, le Luberon. - Gard : Salbous, Chartreuse de Valbonne. - Pyr.0r.: vallée de Llo, en- 
virons de Mont-Louis, bois de Salvanire, de Boucheville, de la Font de Comps, et du Llaurenti. - Cette belle papilionnacẻc manque sur les terrains siliceux des Vosges, des Pyrénées, des Cévennes des Alpes granitiques et ne se montre que sur les terrains calcaires.

O. variegatus, Ten. - Remplace en Corse le précédent; se trouve aussi en Roussillon dans les vallons de Vinça et de Prades.

- Iuteus, L. - Prairies des montagnes. - Pâturages du Noirmont, de la Dôle, du Colombier et du Reculet au vallon d'Ardran. Nul dans les Vosges. - Valais: Liddes, Lavarraz, val Ferret. - H.-Sav.: Brizon, vallée du Reposoir, chalet de Colone, val d'Essert sous le Platet, Charvet près Thônes, mont Tournette. - Sav. : Arclusaz, mont Grenier, Saint-Sorlin d'Arves en Maurienne, montagnes des Allues et de Naves, d'Aime au col du Cormet, montagne du Golet. - Is. : pré Dufour au-dessus de Saint-Ange, col de la Rucliẻre, de Quaix à la Pinẻa, ChâteauBernard, Chalais aux Banettes. - H.-Alp. : Lautaret, la Fusine près Château-Queyras, Boscodon près Embrun, mont Séuse. B.-Alp. : Saint-Paul, Larche, Lauzannier. - Pyr.-0r.: Pla dels Abellans au Forat de la Chimanella dans la haute vallée de la Tet, environs des mines de Batère, vallée du Tech, montagne de la Manère, le Llaurenti.

- tuberosus, L. - Bois des terrains siliceux ou argilo-siliceux.

- Cette espèce est éminemment contrastante avec l'O. vernus; on la trouve sur les terrains de granite, grès et autres roches siliceuses; dans les chaînes calcaires elle n'apparaît que là oủ existent des terrains diluviens, glaciaires, sidérolitiques ou bien sur les argiles ct couches à silex intercalées dans les étages du lias et de l'oxfordien.

D. niger, L. - Bois. - Doubs et Jura : Grand-Combe des Bois, plateau de Chantrans au-dessus d'Ornans, Abbevillers, Bleigny près Salins, Coges près Bletterans, Loisia près Couzance. Bassin du Lẻman et Valais. - Côte-d'Or, Saône-et-Loire. Rh.: Rochecardon et tout le mont d'Or, Chaponost, Givors. - Ain : Muzin et Parves près Belley, Retord, Brénod, la Pape.H.-Sav. : Cré-du-Maure et tous les flancs du Semnoz, Magland, 
Passy, Pringy, Allinges près Thonon. - Sav.: Conllans, Moutiers. - Is. : Crémieu, Chamagnieu, Vouillant, Comboire, la Motte-les-Bains, Monestier-de-Clermont. - Dr. : Crest, Blache l'Aix et Montinaur près Die. - H.-Alp. : Charance, Sigoyer, Combe-Noire. - Ard. : mont Combier. - Gard : Chartreuse de Valbonne, Boussargues, Serre-de-Bouquet, l'Espérou. - Hér. : Lodève, Nizas, Bẻdarieux. - Pyr.-Or.: vallée de Nohẻdes, Mont-Louis, le Capcir, vallons de Villefranche et de Vernetles-Bains.

D. albus, L. O. asphodeloides, Gouan. - Prés humides de montagnes. - Is. et H.-Alp. : Monestier-de-Clermont, Mens, la Palud, Fontrenie, Puy-Maure et Lachaud près Gap, la BâtieNeuve, Rosans, Moydans.-Dr. : le Buis. - Hérault.-Pyr-Or.: Trencada d'Ambulla, montagnes de Céret et de Maureillas.

- saxatilin, Vent. - B.-du-Rh. : Martigues. - Var : environs de Toulon, la Barre près Luc. - Alpes-Maritimes. — Gard : Villeneure près Avignon. - Hẻr.: environs de Montpellier, SaintJean-de-Fos. - Aude : Clape, chemin de Ricardelle à Pech-Redon, roc de las Gueitos, Pla-Vinié.

o. camescens, L. - Bois des montagnes. - Doubs et Jura : bois de Boujailles et de la Vessoye, la Rivière près Pontarlier, Champagnole, bois de Dournon au-dessus de Salins.-H.-Alp. : ChampMartel près la Roche des Arnauds, Rosans, mont Rognouse, Saint-André-de-Rosans. - Dr. : Aucelon, la Ilotle-Chalancon. - Vaucl. : mont Ventoux, Saint-Amans. - B.-du-Rh.: pic de Bretagne, vallon des Masques près Aix. - Var: le Luc, Montrieux. - Pyr.-Or.: vallon de Villefranche, montagne SaintJacques, Trencada d'Ambulla.

sCorprures subvillosa, L. - Champs de la région méridionale.

S. vermiculata, L. - Champs des environs de Toulon, Hyères, Antibes, Montpellier, - Très-commun dans le Roussillon.

COKON⿴囗十 emerus, L. Bois des coteaux calcaires. - Collines jurassiques du Doubs, Jura, Bugey, Revermont, Valais, Vaud, Dauphiné, Savoie, mont d'Or lyonnais. - Plus rare sur les calcaires jurassiques de Cote-d'Or à Saint-Aubin et cntre Gamai et 
Nolay; de Saûne-et-Loire à Dezize, Chamilly, Cluny. - Ard. : Vals, Aubenas. - Gard : Alais, Saint-Ambroix, Anduze, Alzon, bords du Gardon à la Baume, Saint-Nicolas, Tresque.- Hérault ct Aude. - Pyr.-0r.: Trencada d'Ambulla, Vernet-les-Bains, Saint-Martin du Canigou, Font-de-Comps, Cases-de-Pena, ForçaRéal. - Vaucl.: Saint-Amans. - B.-du-Rh, et Var: collines calcaires avant le Prégnon près Aix, Salon, Lançon, pic de Bretagne, les Alpines, Sainte-Baume, Montrieux, Aups. - AlpesMaritimes.

C. shawea, L. - Cultivé el quelquefois subspontané. - Ard. : Payolive, Vals, - B.-du-Rh. et Var: Arles, le Tholonet, Teissier près Aix, Hyères. - Gard : bords du Gardon entre la Baume et Saint-Nicolas. - Hér. : environs de Montpellier, Taillade de Gignac, Saint-Loup, Capouladou, bois de la Valette, Saint-Guiraud, Ribeaute près Béziers. - Aude : Fontfroide, Clape au Rec. - Pyr.-Or. : Saint-Antoine de Galamus, Cases-de-Pena, Castelnau, Corbère, Céret, Arles.

C. valentina, L. - Rochers calcaires.-B.-du-Rh. : environs d'Aix, le Tholonet, les Pinchinats, Monteiguez. - Alp.-Mar. : Nice à Saint-André, Monaco, Menton au cap Martin et au pont SaintLouis. - Pyr.-Or. : rochers calcaires de Cases-de-Pena, ForçaRéal, calcaires de Thuir et de Castelnau.

C. montana, Scop. - Rochers calcaires. - Doubs : Côtes-duDoubs, Clos-du-Doubs, Lomont de Pont-de-Roide. - Sur le versant helvétique au mont dubert près Neuchâtel, au Chasseral et au Weissenstein. - C.-d'Or : Notre-Dame d'Étang, vallon de Messigny, val Suzon. - II.-Sav. : environs d'Annecy à Veyrier et Magériaz. - Sav. : mont du Chat, entre le col du Frêne et Apremont, mont Nivolet, bois du Nant près Avricux en Maurienne. - Is. : gorges du Manival au-dessus de Saint-Ismier, Bernin, Crolles.

C. vaginalls, Lam.-Rochers calcaires.-Doubs : Cotes du Doubs et du Dessoubre, Lomont, crêtes du mont d'Or, fort de Joux. - Jura : Dôle, Colombicr, Reculet. - Valais : Catogne, Anzeindaz, Sion, bords de la Sionne jusqu’à la Comba. - II.-Sav. : pied du Saléro entre Crevin et Archamp, Brizon vers le lac des Colombiers, 
col des Aravis, mont Tournette, Semnoz, Montmin, Arclusan, sous lo Platet au-dessus de Passy. - Sav. : mont Drizon, Charvin, Margériaz, Ilauteluce. - Is. : Bovinant, Chamechaude, Saint-Nizier, col de l'Arc, col Vert. - B.-Alp.: Condamine. - Pyr.-Or. : roches calcaires de Cases-de-Pena, Baixas, Trencada d'Ambulla, Serdinya.

C. mist ima, L. - Coteaux sces. - Nul dans Ilaute-Saône, Doulos et et bassin du Léman. - Rare dans le Jura sur les côtes de l'Ain autour de Thoirette.-Commun dans Cóte-d'Or, Saône-et-Loire.

- Valais : entre Munderessy et Dahla, Bernuna, Salgesch, Varen. - Environs de Ljon à Oullins, Beaunant, Rochecardon, chaine du mont d'Or, Néron, Miribel. - Environs de Grenoble, Gap, Saint-Vallier, Crest, Nyons jusque dans la Provence et les Alpes-Maritimes, Gard, Hérault, Aude et Pyrénées-Orientales. - Dans la Saroie il s'élève depuis les basses collines jusque dans les vallées alpines.

Variété coronata, D. C. mêlée au type dans la Provence, le Roussillon, le Languedoc et jusque dans le Vivarais.

C. juncea, L. - Coteaux, - B.-du-Rh. : environs de Marseille, vallons de Margion et de l'Auriol, Montaud, Monteiguez, les Infernets. - Var: mont Faron près Toulon, Hyères, Carqueiranne, le Luc. - Pyr.-Or. : Trencada d'Ambulla, pied de ForçaRẻal.

C. varia, L. - Coteaux dans tout le bassin.

c. scorploides, Koch. - Moissons de la région méridionale. Remonte dans la Drôme vers Nyons, Crest, Barnave, Valence; dans les Ilautes-Alpes vers Gap, Ribiers, Rosans, ; dans l'Isère vers les Balmes de Claix, Rochefort, Comboire, Saint-Martin-leVinoux, IIens; dans l'Ardèche vers le Pouzin, vallée d'Ouvèze, Celles, Mercuer, Vals. - Trouvé accidentellement autour de de Lyon.

Drontuopes ebracteatus, Brot. - Champs sablonneux du Var et des Alpes-Maritimes: Sableftes de Toulon, îles d'Hyères, Saint-Tropez, la Roquette, Cannes, Antibes, Nice, Menton. Pyr.-Or. : vallons de Collioure et de Banyuls-sur-ller, Peyrestortes, Baixas, Cases-de-Pena. 
D. perpusilus, L. - Champs et terrains incultes des terrains siliceux. - H.-Saône et arrondissement de Belfort: grès rouge, grès vosgien et schistes carbonifêres de la zône vosgienne. Jura et Ain : argiles tertiaires à cailloux quartzeux de Chaussin, Gatey, la Chênée, Pleurre, Chêne-Bernard, la Bresse et la Dombes, granite de la forêt de la Serre. - Commun sur les terrains granitiques du Morvan dans Côte-d'Or, Saône-et-Loire, chaines granitiques du Beaujolais, Lyonnais, Pilat, Vivarais. - Nul dans le bassin du Léman et dans le reste du bassin helvétique, excepté à Wyl près Bâle. - Sables de la molasse du Dauphiné et des parties voisines de la Savoie. - Diluviumà cailloux quartzeux de la Crau près Miramas. - Gard : massif granitique du Vigan, Alzon. - Hér. : Lamalou, pont de la Mouline près Saint-Amand, l'Escandorgue. - Pyr.-Or. : Perpignan, Port-Vendres, Banyuls-sur-Mer. - Nul sur les terrains calcaires.

D. compressus, L. - Champs sablonneux. - Ard. : Vals, Entraigues.-Var: Sablettes près Toulon, forêt des Maures, SaintTropez, Fréjus. - Alp.-Mar. : la Roquette, Antibes, Nice, Berre, Monaco, Menton. - Gard, Hérault, Aude et Pyrénées-Orientales.

MIPPOChePIS comosa, L. - Coteaux calcaires dans tout le bassin excepté dans la région liltorale de la Provence oủ il est remplacé par le suivant. - Manque aussi dans la partie basse du Gard et de l'Hérault; dans ces deux départements il occupe la région montagneuse. - Il en est de mème dans les PyrénćesOrientales où celte espèce se montre à la Trencada d'Ambulla et jusçu’à Fontpédrouse dans la vallée de la Tet, puis à Baixas et Notre-Dame de Pena.

H. glauea, Ten. - Lieux stériles. - Vaucl. : environs d'Avignon. -B.-du-Rh. : coteaux des bords de l'Arc, Montaud-les-Miramas, Montmayour près Arles. - Gard : environs de Nìmes au bois des Espèces, de Cygnan, Saint-Nicolas, Roquecourbe. - Assez commun dans l'Hérault et les Pyrénées-Orientales.

Y. ellata, Willd. - Lieux stériles. - Vaucl. : environs d'Orange et d'Avignon. - B.-du-Rh. : environs de Marseille, d'Arles, 
d'Aix, Cuques. - Var et Alp.-Mar. : Toulon, le Luc, Fréjus, Nice aux Baumettes. - Gard : Villeneuve-les-Avignon, bois de Broussan. - Hér. el Aude : environs de Montpellier, Murvieil, entre Juvignac et Saint-Georges, Agde, Béziers, Montady, Pézenas, environs de Narbonne. - Pyr.-Or. : entre Salses et Perpignan, Château-Koussillorı, vallée de la Tet, Prades, Olette, Fontpédrouse, Cambres-d'Azes.

Y. unisiliquosa, L. - Lieux arides de la Provence, des AlpesMaritimes, du Gard, de l'Hérault et du Roussillon.

SECURTERA coromila, D. C. - Lieux cultivés vers Nice, Monaco. - Plus commun sur la cote italienne vers Vintimiglia, San Remo. - Pyr.-0r. : Baixas, Peyrestortes et toutes les aspres de cette contrée.

HIDYSA TIM obscurum, L. - Prairies des hautes montagnes alpines, - Valais: val d'llliez, Zermontana, Pierre-à-Voir, Zermatt. - H.-Sav. : mont Blanc à la Pierre-Ronde, sous l'aiguille du Goûté, col de Balme, montagnes de Samoëns, col de Golèse, mont des Granges, mont Ardin, mont Chalune, Signal des Agneaux, Brizon, Méry. - Sav. : mont Charvin, col Joly, montagnes de Beaufort, de Hauteluce et des Bauges. - Is.: la Salelte, Chapelle en Valjouffrey. - H.-Alp. : la Grave, Villard-d'Arène à Pétarel, col Malrif, vallée du Guil, val Prévejre, Ségure, mont Vıso, col Agnel, Saint-Véran, Clausis, mont Morgon, la Bâtic-Neuve, Orcières. - B.-Alp. : col de l'Arche, mont Pela, la Condamine. - Alp.-Mar. : scurces du Var el de la Tinea, vals de Strop et de Bourdous.

F. humite, L. - Vaucl. : collines Sainte-Radegonde près Apt. B.-du-Rh.: coteaux du Barret près Aix. - Var: Toulon, Castellet, vieille route du Beausset à Cujes. - Gard: Montagnac. - Pyr.-Or. : haut Vernet pres Perpignan, Salses, Opol, pentes des Corbières.

H. capitarum, Desf. - Sables maritimes à Montredon près Marseille, étang de Berre, entre Châteauneuf et la Mede. - Var et Alp. Har. : Fréjus, Nice, vallie de Gorbio près Menton. - Hér. : Pas-du-Loup à Nissan près Béziers. - Pyr.-0r. : tour de Tautavel, Cases-de-Pena, montagne de: Saint-Antoine-de Galamus. 
OTOHIEYCIS sativa, Lam. - L'Esparcette est cultivéc partout et se montre quelquefois à l'état subspontané.

Variété montana, Gaud. - Prairies des montagnes, - Valais: Anzeindaz, Bovonnaz, Arba. - Jura : le Colombier. - H.-Sar. : montagnes du Chablais, monts de Pelloua el des Granges, Rocd'Enfer, mont Méry, Arpennaz. - Sav.: le Bonhomme, col Joly, montagnes de Hauteluce et des Bauges, mont Charvin, mont Drizon, la Rocheur, mont Cenis. - H.-Alp. : la Grave, Villard-d'Arène, Lautaret, Galibier, mont Viso, col de Glaise près Gap, Loubet, Sappey de la Bâtie-Neuve, Orcières.-B.-Alp. : prairies montagneuses du bassin de l'Ubaye. - Alp.-Mar. : col de Tende.

D. mupina, D. C. - Licux arides des hautes et basses montagnes. - Say. : mont Charvin. - Is. : balmes entre Anthon et Villette, Crémieu, Bouvesse. - H.-Alp. : Charance, la Garde, Puy-Montbard, Romette, Bayard près Gap, Bâtie-Neuve, mont Rognouse. - Dr. : Barnave. - Ard. : col de l'Escrinet, Vallon. - Vaucl. : bois de Fargues près Avignon, Saint-Amans, Üchaux. B.du-Rlı. : vallons de Saint-Pons, Eainte-Victoire, au-dessous du Bec-de-l'Aigle. - Var et Alp.-Mar. : Touris, mont Faron, Draguignan, Montrieux, le Luc, embouchure du Var, Menton.Gard: environs de Nìmes, bois des Espèces, Saint-Nicolas. Hér. : Montpellier, Bézier's, Nizas, le Cros, Pas-de-l'Escalette. - Aude: Pech-de-l'Agnel près Narbonne. - Pyr.-0r.: Trencada d'Ambulla, valléc de Saint-Laurent-de-Cerdans, Costujes.

D. saxatilis, All. - Rocher's des montagnes. - II.-Alp.: Briancon au-dessus du fort des Trois-Têtes, Eymeyère, Notre-Dame-duLaus, Rometle, Saint-Mens, Puy-Maure, Roche-des-Arnauds, Chorges, la Saulce, Tallard, mont Rognouse. - Dr. : Montélimar, Barnave, Aix. - B.-Alp. : Digne, Gréoux, la Condamine, Tournoux, Montagnetle.-Vaucl. : Bonpas près Avignon, Orange, alluvions de l'Aygues. - B.-du-Rh. : les Alpines, Saint-Remy, Montaud près Salon, Marseille, Aix, Mauret, le Prégnon. Var et Alp.-Mar. : montagnes d'Ollioules, le Luc, Touet-de-Beuil, entre Drap et l'Escarìne, Ilescend jusqu'à l'embouchure du Var. - Aude : entre Sigean et l'île Sainte-Lucie. - Pyì,-Or. : vallée de l'Agly, entre Estagel et Maury, vallon de Costujes. 
D. Caput galli, Lam. - Champs arides de la région méridionale. - Vaucl.: Orange. - B.-du-Rh. : Montaud-les-Miramas, Arles, Marseille, Aix au vallon des Gardes et an chemin de Vauvenargues. - Var et Alp.-Mar. : Toulon, Ilyères, le Luc, Fréjus, Nice, Menton. - Gard : Villeneuve-les-Avignon, Tresques, Monlagnac. - Hér.: Montpellier, Jurvieil, Nissan près Béziers, Montady, Capestang, Montagne-Percée, Cazouls-les-Béziers, Saint-Félix-de-Lodez, Pézenas, Pas-du-Loup. - Aude : environs de Narbonne. - Pyr.-Or.: Font-de-Comps, bois de Salvanère. 
156 


\section{CÉSALPINIÉES.}

CEReCIS sllquastrum, L. - Coteaux secs de la région méridionale. - Dr.: Tain, Savasse, entre Nyons et Sahune, Divajeu près Crest, Montélimar, Donzìre. - Ard. : Cruas, Rochemaure, Viviers. - B.-du-Rh. : vallon de St-Pons, Mont-Mayour, près d'Aix sur la rive droite de l'Arc, entre les Infirmeries et le pont des Trois-Sautets. - Var : Ste-Baume. - Alp. -Mar. : Mor naco, Nice. - Gard : Sauve, le long du Gardon, la Beaume, St-Privat, Pont-du-Gard, St-Hippolyte.-Hér. : Lavalette, Grabels, IIurles, St-Georges, St-Jean-de-Védas, Murviel, Béziers. - Naturalisé en plusieurs localités des Pyr.-Or. et de l'Aude notamment à Graboules, sur les bords de la Berre près du clî́teau de Gléon.

Fréquemment cultivé dans les jardins.

CERATONIA giliqua L. - Rochers maritimes. - B.du-Rh.: Château-Gombert. - Var: les Pommets près Toulon. - Alp.Mar.: Nice, Villefranche, Monaco, Roquebrune, Menton.

\section{AMYGDALÉES.}

AMTCDAMUS communis L. - L'Amandier, originaire de l'Orient, est cultivé dans la région méridionale et se montre quelquefois subspontané, ainsi que l'A, amara $\mathrm{L}$.

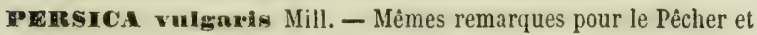
ses diverses variétés, notamment pour le P. laevis D. C.

A rencrisca vulgaris Lam. - Mêmes remarques pour l'Abricotier.

FIEUNES brigantiaca Vill, - Lieux arides des montagnes. H.-Alp. : Villard-d'Arène, Monestier-de-Briançon, val des Prés, Château-Queyras, Villevieille et Aiguilles-en-Queyras, Abriès, Mélezet près Guillestre.-B.-Alp. : vallée de l'Ubaye à St-Paul, Condamine, Larche, col de Vergous près Annot. - Alp.-Mar. : sources du Var, St-Dalmas-le-Sauvage, Estenc, col de Tanarello, la Briga.

Catal. Bassin du Rhơne. 
P. domestiea L. - Le Prunier et ses nombreuses variétés se montrent quelquefois à l'état subspontané.

P. Insititia L. - Cà et là dans les haies de la région mérid. - Rare dans les parties moyenne et septentr. du bassin. - Doubs: Besançon à Brégille, Morre, Beure, Nans. - S.-et-L. : autour d'Issy-l'Évêque. - Rh.: St-Alban, Charbonnières. - H.-Sav.: Pringy près Annecy. - Sav. : Aix, St-Innocent, Granvire, Cruet. - H.-Alp. : Embrun, Gap, Romelte, le Champsaur, Molines sous Serre-de-Chabrand.-Dr. : Lus-la-Croix-Haute. C Cà et là dans l'Ardèche. - Gard : bords du Gardon à la Beaume, près du Petit-Rhône, entre le Mas-du-Juge et le Grau-d'Orgon. Hér. : Hontpellier sur la roule de Ganges, la Salvetat. — Trèsrare dans la Provence et les Alpes-Maritimes.

MIM. Jordan et Fourreau ont démembré de ce type les espèces suivantes :

P. vapincensis, P. latifolia.-H.-Alp. : à Serres; P. sepivaga, P. dumetorum, P. agrestis, $P$. sylvestris, ces quatre derniers autour de Lyon.

P. spinosa L. - Haies dans tout le bassin. Espèce polymorphe comprenant les formes suivantes, décrites par MM. Jordan et Fourreau:

P. rusticana, E. peduncularis, $P$. oviformis, $P$. densifora.

P. frutienns Weihe. - Diffère du précédent par un fruit plus gros, des feuilles plus grandes et plus velues.-Doubs: Nans, Cuse, Beauroisin, Neublans. -C.-d’0r : Nolay. - Environs de Genève. - H.Sav. : mont Salève. - Rh. : Villeurbanne, entre Ecully, Charbonnières et Tassin. - Sav. : mont du Chat, Chignin. Is. : mont Rachais, Corenc. - H.-Alp. : Briançon, Romette, la Garde près Gap, Laye-en-Champsaur. - B.Alp.: la Condamine, Vergous près Annot.-Cả et là dans l'Ardèche. - Hêr. : Maguelonne. - Aude : environs de Narbonne. - Pyr.0r. : St-Laurent-de-Cerdans, Costujes.

Formes démembrẻes :

P. erubescens Paillot. - Chaine P. Desvauxii Bor. - Besançon, du Jura. Pont-de-Roide, Nans. 
P. rosiflora Paill. Besançon à Trois- I. cerasifora Elırlı. - Besançon Châtels. au Pont-de-Secours, Trois-

P. virescens Paill, - Chaine du Châtels. Jura.

CERASUS swium Monch, - Bois des plaines et des montagnes dans tout le bassin; peu abondant. On le regarde généralement comme la souche des divers Cerisiers nommés Merisier, Guignier, Bigarreautier.

C. vuIgrars Mill. - Cultivé et quelquefois subspontané. Le Griottier offre de nombrcuses variétés donnant toutes des fruits à chair acide.

C. mahaleb Mill. - Coteaux calcaires dans tout le bassin.

C. padus D. C. - Bois des terrains siliceux de la partie septentr. et moyenne du bassin; 's'élève très-lıaut dans les montagnes, par exemple, jusque dans les vallées qui avoisinent le mont Blanc, le mont lscran et le mont Genèvre. - Nul dans la région mérid. - En dehors de notre bassin, il commence à se montrer sur les confins de l'Hérault et du Tarn sur les terrains granitiques du versant nord de l'Espinouse à la Salvetat, puis sur les mêmes terrains à la Montagne-Noire, à la Lozère, sur les terr. granit. et volcaniques de l'Aubrac, Cantal, Hte-Loire, Puy-de-Dôme, chaînes granit. du Forez, du Morvan, des Vosges, et des Pyrẻnées.

\section{ROSACÉES.}

SPIR EA nlipendula L. - Bois et prẻs. - C.-d'Or : vallées de Messigny et de Marsannay. - Doubs: Ornans, Pontarlier. Jura : Boujailles, Champagnole, Blamont, Monnières, Salins, Lons-le-Saunier. - Vaud et Valais : Nyon, Aigle, Roche, Conthey, Chemin, Saxon, Salin, Arba, Ayent.-Envir. de Genève à Veyrier, Peney,Jussy.-H.-Sav. : envir. d'Annecy,-Ain : Belley, St-Rambert, Ambronay, Château-Gaillard, Meximieu, la Pape. - Rh.: Chaponost, Lavore près Mornant, l'Arbresle. - Sav. : envir. d'Aix. - Is. : Crémieu, Bastille-de-Grenoble, Vouillant, Monestier-de-Clermont, Mens, - H.-Alp. : col Bayard, plaine 
de Lachaud près Gap, Manteyer, Rosans, Ribeyret, col de Vars, prés du Queyras, - B.-Alp. : St-Paul, cols de la Madeleine et de Vergous. - Dr. : Aucelon. - Ard. : Vals, Entraigues. Vaucl. : Bonpas près Avignon. - B.-du-Rh. : N.-D -des-Anges, Ste-Victoire, Ste-Baume. - Régions montagneuses cu Var, des Alp.-Mar., du Gard et de l'Hérault. — Aude : les Corbières, Quillanet, Fontlaurier, Montolieu, la Mrontagne-NoirePyr.-Or. : vallée de Cornella-du-Conflent, Canet, Château-Roussillon, envir. de Perpignan. - Rare dans les Pyr.-Centr.Tarn; Lozère; Puy-de-Dôme; Cantal ; Allier; Loire.

S. ulmaria L. - Prairies humides dans tout le bassin. - Présente une forme $S$, denudata Hayn. à feuilles vertes sur les deux faces et une autre forme $S$. glanca Schultz à feuilles blanches-tomenteuses en dessous.

S. aruncus $L$. - Bois des montagnes, - Zone rosgienne de l'arr ${ }^{\mathrm{t}}$ de Belfort et de la H.Saônc. - Région des sapins du Doubs, Jura, Ain, Dauphiné, Savoie, Valais, B.-Alp. jusque dans les Alp.-Mar. autour de Clans et de St-Martin-de-Lantosque. Aude : Ste-Colombe sur l'Ebiguette. - Pyr.-Or. : le Llaurenti, Coma-du-Tech, vallon de Llapoudère, bac de Bolquère, mont Louis et dans les Pyr.-Centrales. - C'est à tort que La Tourrette a indiqué au Pilat cette espèce qui manque aussi dans le massif central de la France.

DuX As oetopetala L. - Rochers des montagnes. - Sommités du Jura helvétique et français, Chasseral, Weissenstein, Suchet, Creux-du-Van, mont d'Or, Reculet, Dôle, Colombier, Sorgiaz. Vaud et Valais : Bagnes, Saxon, Nenda, Leukerbad, Zermatt, Saas, - H.-Sav. : Salève, Môle, Brizon, Vergy, Charvin, montagnes de Flaine et de Nautau, mont Blanc au pavillon de Bellevue. - Sav. : col Joli, montagnes de Beaufort, des Allues, col de la Madeleine, Beaupré, mont Cenis, les Bauges, mont du Chat. - Is. : montagnes de la Grande-Chartreuse et de St-Nizier, - H.-Alp. : Villard d'Arène, Briançon au fort des TroisTêtes, Abriès, vallon des Vaches, St-Vẻran, Orcières, mont Chabrières, Durbonnas, la Grangette, col de Glaize et Charance près Gap. - Dr. : mont Glandasse. - B.-Alp. : vallon de Fouil- 
louse. - Alp. Mar, : Entraunes, val de Strop, cols du Ray, de l'Abisso el de Tende. - Aude : Boutairol, lac de Quérigut, le Llaurenti. - Pyr.-0r. : sommet du Cambre-d'Azes, vallée de Nohédes, sommets entre Carenca et Nuria, le Canigou. - S'étend sur les sommités des Pyr.-Centr. - Nul dans les Vosges, le Cantal, le mont Dore, le Puy-de-Dơme, la Lozire el la chaine des Cévennes.

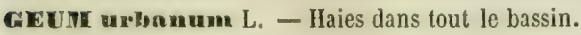

G. Internedium Ehrh. - Paraît être un hybride du prẻcédent et du suivant. - Is. : bois entre la Grande-Chartreuse et le chemin de la Courrerie. - Ain : Hauterille au-dessus de la chapelle de Mazières.

G. rvale L. - Prairies humides, bords des cours d'eaux dans les montagnes.-Zone vosgienne de l'arrt de Belfort et de la H.-Saône d'où il s'étend dans toutes les vallées des Vosges. - Région des sapins dans toute la clıâne jurassique, côtes du Doubs, du Dessoubre et de la Barbéche, plateau du Dessoubre, Lomont, la Dóle, Colombier, Reculet, Colombier du Bugey, montagnes d'Hauteville; descend jusıue près de Tenay. - Indiqué par Lorey dans le Morvan oủ il n’a pas été retrouvé. - S.-et-L. : la Coudre, Selle d'Auxy, Canada. - Vaud et Valais : depuis le fond des vallées jusque dans la région montagneuse.-H.-Sav. : Selève, Voirons, Mole, Bonnevaux, Vacheresse, Habère-Lullin, la Roche, Thorens, les Houches, Bionnassey, les Contamines. - Sav. : CrestVoland, mont d'Arith. - Rl.. : montagnes du Beaujolais. Loire : Pilat, Pierre-sur-Haute. - Ard. : le Mezenc, Bauzon. Is. : montagnes de la Grande-Chartreuse, de St-Nizier et de Villard-de-Lans, forêt de Prémol. - H.-Alp. : mont Genèvre, Gap, les Serigues, vallon de Glaise, bois des Donnes, la Grangette. - B.-Alp. : Larche, la Condamine. - Dr. : mont Glandasse. - Alp.-Mar. : sources du Var, St-Martin-d'Entraunes, St-Dalmas-le-Sauvage, St-Eticnne, Ste-Anne-de-Vinaï, alpes de Tende. - Gard : l'Espérou, Banahu. - Aude : lac de Quérigut, le Llaurenti.- Pyr.-Or. : mont Louis, forêt de Boucheville, Canigou, Costa-Bona, vallon de Collioure. - En dehors de nos limites dans les Pyr.-Centr., l'Aubrac, la Lozère, Hte-Loire, Cantal, mont Dore, Forez, Yosges. 
G. sylvatleum Pourr. - Bois de la région mérid. - B.•du-Rh. et Var: les Alpines, Ste-Baume, Maures. - Alp.-Mar. : Estérel, Berre, Tanneron. - Gard : Montdardier, Palière, Montvaillant, Toiras près Anduze, St-Paul près du Vigan, petit mas de Seine près Nìmes. - Hẻr. Murviel, Fabrègues, St-Geniès-des-MIourgues, Montarnaud, St-Martin-de-Londres, les Capouladoux, la Sérane, Ganges, St-Etienne-de-Gourgas, St-Pons, l'Espinouse. - Aude : envir. de Narbonne, Lapeyre près Quillan, Lastours. - Tarn : la Montagne-Noire. - Pyr.-Or : les Albères, vallée du Réart, envir. de Castcl et de St-IIartin du Canigou. - Manque dans les Pyrénées-Centrales.

G. pyrenaicum Willd. - Prairies des environs de mont Louis et de la vallée de Llo dans les Pyr.-Orientales._Commun dans les Pyrén.-Centrales.

G. Inclinatum Koch. - Rochers granitiques de la montagne de Sournia dans les Pyrén.-Ortentales.

C. montanum L. - Pelouses des montagnes. - Nul dans chalne vosgienne; rare dans la chaine jurassique oủ on ne l'observe que dans les crevasses du Colombier de Gex. - Commun dans toutes les Alpes du Valais et de Vaud depuis la Dent du midi et de Morcles jusqu'à la Gemmi, le Grimsel, le glacier du Rhône, les montagnes de Saas et de Zermatt. - H.-Sav. : premier piton dn Salève, Brizon, Vergy, montagnes de Samoëns et d'Abondance, mont d'Ubine, roc d'Enfer, dent d'Oche, Haut-de-Lin, col de Golèse, la Tournette, mont Charvin, toute la chalne du mont Blanc, moraines de la mer de Glace. — Sav. : Col du Bonhomme, Beaupré, Hauteluce, les Allues, col de la Madeleine, Saint-Sorlin-d'Arves, mont Cenis, les Bauges et le mont du Chat. - Is. : Massif de la Grande-Chartreuse, Bovinant, col de la Ruchère, Chamechaude, Saint-Eynard, la Pra de Belledonne, Chanrousse. - H.-Alp. : Lautaret, Briançonnais, Queyras, mont Viso, l'Obiou, Chaillol-de-Vieil, Orcières, mont Durbonnas, mont Chabrières, Séuse, Aurouse. - B. Alp. : Fouillouse, Lauzannier, Parpaillon, Annot. - Alp.-Mar. : Entraunes, l'Escarène, mont Bego, cols de Tende et de Salèse.-Aude : Boutairol, lac de l'Estagnet, le Llaurenti. - Toute la région alpine des 
Pyrénées centr. - En dehors de nos limites sur les pentes du mont Dore et du Plomb du Cantal.

G. reprams L. - Rochers des Alpes et des Pyrén.-Orient, - Vaud et Valais: Alpes de Bex et d'Aigle, Audon, Prapioz, Einzendaz, Javernaz, Granvire, les Diablerets, Morcle, Fully, Nendaz, Cleuzon, mont Velan, Saint-Bernard, Rothorn et Hornli de Zermatt, Schwarzberg de Saas, Simplon.-II.-Sav. : le Buet, col de Golèse, chaîne des Fiz et d'Anterne. - Sav. : cols de la Seigne, du Bonhomme et de la Madeleine, mont Cenis, Saut des Allues, Saint-Sorlin-d'Arves. - Is. : Piémeyan au mont de Lans, les Grandes-Rousses. - H.-Alp. : col du Golćon au-dessus de la Grave, Villard-d'Arène aux Trois-Êèchés, Galibier, col Isoard, mont Viso au chalet de Ruines, Grand-Vallon, Saint-Véran audessus de Clausis, mont Obiou, mont Aurouse, Chaillol-le-Vieil, Orcieres, col de l'Alp-Martin.-Dr. : mont Glandasse.-B.-Alp. : col Stropi, Parc-à-Sac, Parpaillon, longet de Maurin, Vallonnet à Plate-Lombarde, mont Grand-Couyer, près Annot. - Alp.Mar. : Saint-Dalmas-le-Sauvage, - Pyr.-Or, : sommet de la vallée de Carença et des montagnes qui conduisent à Nuria. Manque dans les Pyrénées centrales.

G. heteroenrpum Boiss. - Rochers calcaires de la corniche du mont Séuse près Gap.

STBEALUXA proeumbens L. - Rocailles siliceuses des montagnes. - Jura : calcaires silicifiés du Colombier, Reculet, Crètdu-Miroir. - Valais : Grand-Saint-Bernard, Thyon, Nendaz, val d'Hérẻmence, Zermatt, Simplon, la Furca, Grimsel. - H.-Sav. : toute la chaine du mont Blanc depuis le col de Balme jusqu'au Bonhomme, moraines de la mer de Glace, s'élève jusqu'au Jardin, Brévent, Aiguilles-Rouges, montagnes de Samoëns, col de Golèse, Haut-de-Lin, Signal, roc d'Enfer, Vergy de chaque côté du col de Balafrasse. - Sav, : col Joly, col du Cormet, SaintSorlin-d'Arves, mont Cenis à Pattes-Creuses et sur les bords du lac Noir. - Is. : chaines des Sept-Laux, de Belledonne et des Grandes-Rousses, versant N. E. de la Moucherolle, la Salette.II.-Alp. : la Grave, Lautaret, Galibier, val Préveyre en Queyras, mont Yiso, le Bouchas, la Tranchée, la Traversette, Saint-Véran, 
Rocheronde, Marcel, Chaillol-le-Vieil, Orcières, Alp-Martin, col de Vars. - B.-Alp. : cols de Soltron et de la Marche, PlateLombarde, Mirandole, Vallonnet, Parpaillon, Grand-Couyer près Annot. - Alp.-Mar. : sources du Var à la Stroop, lacs d'Entrecoulpes, cols de Fenêtre et de Salèse, Sainte-Anne-de-Vinaï, mont Tenibre, lacs de Raburons. - Aude et Py.-0r. : le Llaurenti, vallẻe d'Eyne, sommets entre cette vallée et le Pla-duCamp-Hagre, Canigou, Pla-Guillem. - Pyr. centrales. - Rare dans les Vosges où il n'existe que sur le versant mérid. du Hohneck.

Potentila fragariastrum Ehrh. - Commun dans les bois de la partie septentr. et moyenne du bassin; rare dans la région mérid. oủ il n’est signalé que dans les montagnes du Gard autour du Vigan, de l'Hérault à Andabre-Rosis, Castanet-le-Haut, Saint-Amand-de-Mounis, la Salvetat, Saint-Pons, - Alp.-Mar. : l'Estérel. - Pyr.-0r. : dans la zone moyenne des montagnes.

P. micrantha Ram. - Même dispersion que le prẻcédent, mais moins commun.

P. splendens Ram. - Bois. - Aude et Pyr.-Or. : le Llaurenti, bois de Salvanère et de Boucheville, Coma-de-Jau, vallée de Molitg, Canigou. - Commun dans les Pyre̊nées centrales.

P. alba L. - Bois. - Envir. de Genève à Pency, au bois de Bay, Prangins, châtaigneraies du Petit-Salève en allant à Mornex, Is. : au-dessus de Prémol. - H.-Alp. : mont Bayard, la Rochette et Romette près Gap. - Gard : l'Espérou. - Alp.-Mar. : Beuil, vallèe de Thorenc. - Pyr.-Or.: Costa-Bona, Canigou, forêt de Salvanère, montagne de Madres.

T. snxifrog Ardoino - Diffère du précédent par ses carpelles très-velus, ses feuilles à bords réfléchis, non ciliés, par sa souclie robuste et ligneuse.-Rochers des Alpes-Mar. entre SainteAgnès et Castillon au-dessus de Menton, gorges de Saorgio, Alpes de Raus et de la Briga au Chaudan, Duranus, vallée du Var depuis le confluent de la Vesubia jusqu'à celui de la Tinea, vallée de Thorenc au-dessus de Grasse. - Existe aussi dans les montagnes du Piẻmont voisines des Alpes-Mar.

*. nitida $L_{\text {. }}$ - Rochers calcaires des montagnes, - Is. : au-dessus 
de Bovinant en montant au grand Som, cols de la Ruchère et de la Cochette, Chamechaude, Haut-du-Seuil, Chalais aux Baneltes, Moucherolle. - Sav. : mont Granier. - H.-Sav. : mont Joly. Aude et Pyr.-Or. : le Llaurenti sur les escarpements de la Ventaillole et vers la Grande-Bouillouse, Salvanère.

P. caulescens L. - Rochers calcaires, - Vaud et Valais : Roche, Saint-Triphon, Lavey, Morcles, Saint-Maurice, portes du Scex, entre Inden et Varone, Leukerbad. - Jura helvétique : rochers de Fleurier, des Montets près de la Tourne, Creux-du-Van, Tetede-Rang. - Manque dans le Jura français septentrional, bien qüil soit assez commun dans les vallées du Bugey, notamment dans la vallée de l'Albarine, entre Charabotte et Nantuy, à l'entrée de la forêt de Mazières, d'Argis à Evoges, bords de la Burbanche, Rossillon, de Serrières à Ordonnaz, Parves, Muzin. H.-Sav. : base du Môle, Brizon, valléc de la Borne près Bonneville, les Aravis, ravin de la Caille, Allèves, Saint-Germain-surTalloires, Parmélan, vallée du Fier sur les rochers de SaintClair, mont d'Etalle près la Clusaz, Cornettes-de-Bise, Pormenaz, les Platets au-dessus de Passy, la Combe sur Sixt. - Sav. : entre la Balme et Yenne, Saint-Victor, Saint-Innocent, mont du Chat, Montagnole, Conflans, Villarlurin. - Is. : Bourg-d'Oisans, entre le Freney et le Pont du Dauphin. - H.-Alp. : Villard-d'Arène, Lautaret, Névache, Briançon, Cervières, gorges du Guil entre la Maison-du-Roi el Château-Queyras. - Dr. : Barnave, Pommerol. - B.-Alp.: Sérenne au rocher de Peyméan. - Vaucl. : mont Ventoux. - Alp.-Mar. : Guillaumes. - Gard : Anjeou près Montdardier, la Tessonne près du Vigan, la Foux près Alzon. - Hẻr. : Saint-Gervais, Pégayrolle-de-l'Escalette, la Sambugude, le Caylar, Madières, Saint-Haurice. - Pyr.-Or. : Font-deComps, Coba del Faits, Coba de las Possas, vallée d'Eyne, Llaurenti. - Pyr. centr. - Lozère : rochers calcaires autour de Mende, Meyrueis, Florac.

P. petiolulata Gaud. - Rochers calcaires. - Diffẻre du précédent par l'existence d'un court pétiolule à la base des trois feuilles centrales. - Valais : Clavaux, Tiny, Grone, Grosslien, Visp. H.-Sav. : Salève au-dessus du Pas-de-l'Echelle, vallon de la Catal. Bassin du Rhöne. 
Caille, roc de Chère sur les bords du lac d'Annecy. - Sav. : les Echelles, Saint-Germain, Saint-Innocent, Chambéry, d'Aigueblanche à Moutiers. - Ain : Saint-Rambert, Tenay, RossilIon. - Is. : Pariset au Désert, Sassenage, entre Saint-Etiennede-Crossey et Saint-Laurent-du-Pont. - H.-Alp. : Serres, mont Chabrières. - Dr.: Saou, Château-Bernard, Barnave.

P. nivalis Lap. - Rochers des Aipes et des Pyrénées. - Chaine de Belledonne vers le lac du Crouzet, col de l'Arc, la Moucherolle, mont Aiguille, la Salette. - Sav. : col de la Madeleine. - H.-Alp. : mont Aurouse, Pas-du-Follet en Devoluy. - Dr. : mont Glandasse. - Alp.-Mar. : sources du Var à Roche-Grande, Saint-Etienne-Jlont vers les lacs de Vens. - Pyr.-Or, : sommels de la vallée d'Eyne, pic de Finestrelle dans la vallée de Llo, Canigou, Costa-Bona, vallée de Prats-Balaguer, Llaurenti. Région alpine des Pyrénées centrales. Le $P$. alchemilloides Lap. des Pyr. centr. n'est pas signalé dans les Pyr.-0r.

P. nivea L.-Pelouses des Alpes granitiques.-Valais : Lotschberg, entre Leukerbad et Lœtschen, la Giete, Pralet d'Hérens, Zermontana, Zanrion, Schwarzsee de Zermatt.-H.-Alp. : Villardd'Arène aux Trois-Evêchẻs, Lautaret à Prime-Messe, l'Envers et le Chardoussier. - Sav. : col du mont Iseran, mont Cenis.

P. minima Hall. - Pâturages des montagnes. - Jura : montagne d'Allemogne au Creux de la Neige. - Vaud et Valais : Enzeindaz, Zermontana, Giete, Gemmi, val Lœtschen, Alliaz de Bagnes, Méribé, Seilon, Grand-Saint-Bernard au Plan-de-Jupiter et à l'Ardifagoz. - H.-Sav. : Vergy près des Petits-Lacs, montagnes de Samoëns et d'Anterne, le Buet, la Diosaz, mont Blanc au Couvercle, mont Lachat, Plan-des-Dames près du Bonhomme. - Sav. : col de la Vanoise du côté d'Entre-Deux-Eaux, Gita près Beaufort, Otherans, mont Granier. - Is. : GrandesRousses sur les bords du lac Blanc. - H.-Alp. : Galibier, col Isoard, vallon de liuine, mont Aurouse. - B.-Alp. : Vallonnet, Lauzannier. - Alp. -Mar. : les Viosennes. - Rare dans les Pyr. centr. à Vignemale et Aucupat.

P. Prigidar Vill. - Rochers des Alpes granitiques. - Valais : montagnes de Bagnes, Tzermanaire, Chenaletta près du Saint-Ber- 
nard, col de Fenctre, mont Nuoble, Maya, Orsera, Novelly, Loemmeralp, Mayens et Torrenthorn, Zermatt, Reckingen, Griess, Grimsel. - H.'Sav. : mont Blanc au Couvercle, au Jardin, aux arêtes des Charmoz, Talèfre et les Grands-Mulets, cols de Berard et de Balme. - Is. : Belledonne, Taillefer, les GrandesRousses. - H.-Alp. : pic du Bec au-dessus de Villard d'Arène, les Muandes, cols de Buffet et de Christovoul, près Névache, mont Viso, col Agnel, col Vieux, Chaillol-le-Vieil. - B.-Alp. : cols de Stropi, Mirandole. - Région alpine des Pyrén. centr.

P. grandifora L. - Prairies, pelouses des Alpes et des Pyre̊nées.

- Vaud et Valais : Grandvire, Enzeindaz, Alpes de BourgSaint-Pierre, Grand-Saint-Bernard aux rochers de la Baux, Nomaney, Outre-Rhône, Tortain, montagnes de Sion, d'Hérêmence, d'Evolena, de Zermatt et de Saas. - H.-Sav. : chaine du mont Blanc jusqu'au Couvercle et au Jardin, Brévent, montagnes de Samoüns, col de Golèse, mont Chalune, Méri, l'Etale, prìs la Clusaz. - Sav. : Hauteluce, Bonhomme, Morteret des Allues, Saint-Sorlin-d'Arves, mont Cenis, - Is.: la Pra-deBelledonne, Alpe du mont de Lans, mont Obiou, forêt d'Esparron en face du Monestier-du-Percy, mont de Roche-Sac près Mens. - H.-Alp. : la Grave, Lautaret, mont Viso, col de Vars, Chaillol-le-Vieil, Durbonnas. - B.-Alp. : Lauzannier, col de la Madeleine, Parpaillon, Longet de Maurin. - Toutes les montagnes des Alp.-Har. - Pyr.-Or. : vallée de la Manère et de Saint-Laurent-de-Cerdans. - Rare dans les Pyr. centrales à la Maladetta.

P. pedemontana Reut. - Forme du précédent, plus petite dans toutes ses parties. - Pelouses des Alpes. - Is. : chalets du Treuil au plateau du Grand-Galbert, au-dessus de Livet, col de Menée. Sav. : mont Cenis. - H.-Alp. : la Grave à Puy-Vacher, Lautaret, montagnes d'Abriès en Queyras.

P. subacaulis L. - Coteaux de la région mérid. - Vaucl. : Vaison, Flassan au bois de Fonterrasse, Bedoin, cime de Malaugii. Gard : Serre-de-Bouquet, Pouzillac. - Pyr.-Or. : vallon de Banyuls-sur-Mer, Cambres-d'Aze, Collada-de-Nuria dans la vallée d'Eyne. 
D'après SchuItz, la plante indiquée sous ce nom dans les B.du-Rh. à la Sainte-Victoire, Tête-de-Carpiagne, Roquefavour serait le $P$. Tommasiana Schultz.

P. Clementi Jord. - Coteaux secs de la Drôme : Romans, Valence, Loriol, Andancette.

P. cineren Chaix. - Rochers. - Valais, Fully, Martigny, Folatères, Conthey, Sion, Bramois, Vex, iles de Sion. - H.-Alp. : les Beaux, Rabou, mont Séuse, mont Rognouse. - B.•du-Rh.: Saint-Paul-de-Durance, vallon de la Guiramande au Montaiguez, vallon de Mangeo-Gari vers Valabre.-Alp.-Har. : Sallagriffon, près Saint-Auban.

$P$. vestita Jord. - Diffère du précédent par la teinte moins cendrée de ses feuilles. - Lieux secs des envir. de Gap au Devez-deRabou.

P. opaea L. - Coteaux secs. - Envir. de Lyon à Couzon, Beaunant, Mollard de Décines. - Ardèche. - Gard : Valat de la Dauphine, près de l'Espérou, Banahu. - Pyr.-Or. : Canigou, Pla-Guilhem, mont Louis, Cambres-d'Aze, vallée d'Eyne, Nohèdes, Salvanère, Llaurenti.

Distribution géographique mal connue; ses caractères sont d'ailleurs assez mal définis au point que souvent on l'a pris pour une forme velue du $P$. verna.

P. verna L. - Coteaux secs et bien exposés dans tout le bassin. Espèce polymorphe.

P. vivariensis Jord. - Coteaux de Châteaubourg et de Crussol (Ardéche).

P. stipularis Pourr, - Aude : les Corbiẻres, Boutairol, le Llaurenti.

P. alpestris Hall. - Pelouses des montagnes. - Chaîne des Vosges jusque sur les sommités du Ballon de Giromagny. - Toute la chaine du Jura helvétique et français depuis le Montendre jusqu'au Reculet et, plus au sud, dans les montagnes de l'Ain au Poizat, à Retord, à l'Hallériat. - Valais : Grand-Saint-Bernard, mont Cubit, mayens de la Comba, Tortain, Cleuson de Nendaz, Guggerhubel, |Zermatt. - H.-Sav. : Salève, montagnes du Chablais et du Faucigny, chaine du mont Blanc, Leschaux, Couvercle et Jardin, pavillon de Bellevue, Passy, la Tournette, Par- 
mélan. - Sav. : montagnes des Bauges, de Beaufort, de la Tarantaise et de la Maurienne jusqu'au mont Cenis. - Is. : chaines de Belledonne, des Sept-Laux, de Taillefer et de l'Oisans, mont Sẻnèpe, petite Moucherolle. - H.-Alp. : la Grave, Villardd'Arìne, Lautaret, Briançon, mont Viso, col de Glaise, col Bayard, Síuse, Alp.-Mfartin. - B.-Alp.: montagnes du bassin de l'Ubaye autour de la Condamine. - Aude : lacs de l'Estagnet et de Quẻrigut, Port de Pailhères, Llaurenti. - Pyrẻnées centr.

P. ambigů Gaud. - Valais : pâturages du Schwarzsee au dessus de Zermatt.

P. auren L. - Pelouses des montagnes. - Nul dans la chaine des Vosges. - La chaine du Jura helvétique et français, Chasseral, Suchet, mont d'Or, Montendre, Dôle, Reculet jusque dans les montagnes du Bugey.-Valais : Fully, Trouleux, Saint-Bernard, Schornen, Binnergalen, Zobourlaye, val Massa, mayens de Sion, Thyon, Zermatt. - H.-Sav. : montagnes d'Abondance et de Bonnevaux, mont Chalune, Haut-de-Lin, mont d'Ubine, Nautau, dents d'Oche, col de Golèse, Méri, Voirons, Salève, chaine du mont Blanc jusqu'au Jardin de la Mer-de-Glace, col des Aravis. - Sav. : montagnes des Bauges, mont du Chat, pâturages de la Tarantaise et de la Maurienne. - Is. : chaines des Sept-Laux, de Belledonne et des Grandes-Rousses, Chamechaude, petite Moucherolle. - H.-Alp. : Loppet, Orcières, Poligny-en-Champsaur, col de la Palette, mont Durbonnas, la Grave, montagnes du Queyras. - B.-Alp. : col de la Madeleine, Plate-Lombarde, Fouillouse, Parpaillon, Riou-German, Vallonnet. - Dr. : Aucelon. - Pyr.-Or. : vallées d'Eyne et de Carença, Font-de-Comps, Costa-Bona, Jasse de Cady. - Loire: Pilat, Pierre-sur-Haute. En dehors de nos limites, sur les sommités des monts Dômes, Dore, Cantal, Lozère. - Rare dans les Pyr. centr. à la Piquette d'Ereslids, vallées du Bastan et d'Azun.

P. pyrenaica Ram. - Pàturages des montagnes. — Aude : Boutairol, vallée de la Bruyante. - Pyr. Or.: fond de la vallée d'Eyne, Llaurenti, vallée de Mijanès, Jasse d'Outournan. - Assez commun dans les Pyrénées centr.

P. Intermedía L. - Prairies des montagnes. - Vaud : prés de 
Saint-George, le long de la route du Narchairu, à l'ouest du mont Chabert, la Comballas, l'Etivaz, Sierne-au-Cuir, Mérils, Château-d'OEx. - Valais : combe de Martigny, vallée de Champé, au-dessus de Zobourlaye. - Sav. $\therefore$ : entre Seez et Sainte-Foy, la Thuile près Tignes, entre les Allues et Pralançon, Villarlurin près Moutiers. - Is. : Grande Vaudaine au-dessus de Livet, mont de Lans, la Salette, Saint-Jean-d'Hẻrans à Toige. H.-Alp. : le Valgaudemar, Lautaret, Briançon au Chabas, Guillestre au Mélezet, Pleyne de Chaudun, Loubet près Gap, Boscodon, Durbon, col de Vars. - B.-Alp. : la Condamine, Sẻrennes.

Les cinq formes suivantes sont démembrées du P. intermedia.

P. Greneriana Jord. - Monestier- $P$. gentilis Jord. - Lautaret, la de-Briançon.

Croix-Haute.

$P$. autaretica Jord. - Lautaret. $\quad P$. medioxima Jord. - Lautaret.

P. Mathoneti Jord. - Lautaret.

P. thuringiaen Bernh. - P. parviflora Gaud.-Valais : Rossetan, les Marques, Folatères.

P. delphinensis Gren. Godr. - Rochers des montagnes du Dauphiné. - Is. : Charmanson, la Salette. - H.-Alp. : Lautaret à l'Envers, Guillestre au Mélézet.

P. multifda L. - Prairies des montagnes. - Valais : Zermontana, Zermatt, Gornergrat, Stœllenden, Findelen, Schwarzsee, Augstelberg. - Sav. : la Vanoise, mont Cenis. - H.-Alp. : Lautaret à Prime-Messe, l'Envers et le Chardoussier, Villard-d'Arène en montant aux Trois-Evêchés. - Py.-Or. : vallée de Molitg à Coma de Jau, forêt de Salvanère.

P. tormentilln L. - Lieux humides des terrains siliceux depuis les plaines jusque sur les plateaux des montagnes. Dans les chaines jurassiques, cette espèce se montre dans les lieux tourbeux à sous-sol siliceux.

Var. orophila Timb.-Aude: vallée de la Bruyante, près du lac

Quérigut.

$P$. procumbens Sibth. - Espèce intermédiaire entre $P$. tormentilla et $P$. reptans; elle a comme le premier des fleurs tetramères et comme le second des tiges couchées radicantes.-Dispersion mal connue. 
P. reptans L. - Terres incultes dans tout le bassin.

P. anserina L. - Lieux humides. - Manque dans la Provence st les Alp.-Marit.

P. rupentris I. - Rochers et débris de rochers. - Nul dans H.-Saône, Jura, Doubs, C.-d'Or, S.•ct-L. - Rh. : Beaunant, Francheville, Chaponost, Charbonnières, Fontaines. - Ain : la Pape, la Burbanche, Muzin, Parves. - Valais: les Valettes, Salvan, Combe de Martigny, Mayens de Sion, Natersberg, Blatten, entre Liddes et Bourg-Saint-Picrre, Sion. - Envir. de Genève: bois de Bay et de Prangins. - H.-Sav. : Salève, vallon des Usses, Passy, Serroz, rallée de Clıamonix, Argentière, Parmélan. - Sav. : Leınenc, Villarlurin, Moutiers, Pontamafrey près Saint-Jean-de-Maurienne. - Is. : entre Echirolles et Champagnier, Séchilienne, mont de Lans. - H.-Alp. : Lautaret, Briançon, le Queyras, Devez de Rabou, Pleyne, Loubet, Faudon près Gap. - B.-Alp. : Enchastraye, Annot. - Var : Fréjus. - Alp.Mar. : Saint-Martin Lantosque, entre Luceram et la forêt de la Maïris, montagnes de Caussols et de Roque-Bérenguier, col de Tende. - Ard. : rochers basaltiques près Thueys. - Loire : la Rive près la Valla, Saint-Jodard. - Gard : l'Espérou, le Vigan, l'Arche près Anduze. - Pyr.-Or.: le Llaurenti, bois de Boucheville et de Salvanère, Saint-Martin-du-Canigou, Vernet-lesBains, Prades, Saint-Laurent-de-Cerdans, - Pyr. centr., Puyde-Dôme, H.-Loire, Cantal.

p. rubricaulis Jord. - Forme du précédent signalée autour de Lyon, de Crẻmieu et dans le Bugey.

P. Eupina L. - Champs sablonneux et humides. - C.-d'Or : Satenay, Boncourt, Citeaux, - S.-et-L. : Louhans, Navilly, - Jura : bords de la plupart des étangs de la Bresse à Saint-Baraing, Balaiseaux, Plcurre, Chêne-Bernard, Tassenières, Fays, Champrougier. - Vaucl. : Avignon. - B.-du-Rh. : embouch. de l'Huveaune. - Gard : Beaucaire, Jonquières. - Hẻr. : Béziers aux carrières de Brégines, Nissan.

F. nrgenter L. - Coteaux secs des terr. siliceux. - Zones vos gienne et sous-vosgienne des arrond ${ }^{t s}$ de Belfort el de Montbé. liard, ainsi que dans la H.-Saône, oxfordien siliceux de Fouvent. 
- - C.-d'0r: région granitique du Morvan à Saulieu, Arnay, Semur, entre Mâlain et Baume-la-Roche. - S.-et-L. : Saisy. Valais : Saxon, Sion, Saint-Léonhard, Sierre, Zermatt.-Envir. de Genève à Peney, Gaillard, entre Veyrier et Meyrin. - Commun dans le Lyonnais, la Savoie, Dauphiné, la Loire, Vivarais, Languedoc. - Plus rare dans la Provence; Var: plateau de N.-D. des Anges près Pignans.-Alp.-Mar. : Grasse, les Mujoulx, Saint-Auban, Saint-Martin-Lantosque, Menton :

Espèce polymorphe; les formes suivantes s'y rapportent.

P. decumbens Jord.-Env. de Lyon de la Serre, Salins. - Gard :

P. confinis Jord. - Ibid. le Vigan.

P. demissa Jord. - Rh. : Beau- P. argentata Jord. - Rh. : Saintnant, Givors, Soucieu.-Env. Genis-Laval. - Is. : envir. de de Genève à Genthod.-Sav. : Grenoble,Allemont.-H.-Alp. : entre St-Innocent et Brison.H.-Alp.: Tallard,Champolẻon. Lautarel.--Loire:Montbrison. — Gard : le Vigan. - Ard. : Mirabel.

P. collina Wib. - D'après Grenier serait peut-être identique au P. demissa Jord. - Plaine et vignoble du Doubs et Jura autour de Besançon, Dolle, forêt

$P$. reclinis Jord. - Rh. : Givors.

P. cana Jord. - Is. Vienne.

P. decipiens Jord. - Rh. : Beaunant, Chaponost, Mornant, Givors.

P. inaperta Jord. - Saint-GenisLaval.

P. Inelluata Vill, - Coteaux secs. - Valais : Branson, Fully. Sav. : Conflans, entre Moutiers et Hautecour, Villarlurin. H.-Alp. : Sigoyet près Tallard, Furmeyer. - B.-Alp. : Sisteron. - Hèr. : la Sérane, Ganges. - Alp.-Mar. : mine de Tende.

P. recta L. - Pelouses des collines de la région mérid., Provence, Alp.-Mar., Pyr.-0r., Aude.-Plus rare dans l'Hérault entre Lésignan-la-Cèbe et Nizas, entre Aspiran et Paulhan. - Se retrouve dans les montagnes de la Drôme, à Lus-la-CroixHaute, au mont du Pertus, entre Barnave et Aucelon; dans les H.-Alpes, à Durbon, autour de Gap; dans la Savoie vers Albertville, à Conflans; et enfin dans le Valais aux Marques, à Martigny et Branson.

P. hirta L. - Coteaux secs de la Provence, des Alp.-Mfar., du Lan- 
guedoc et du Roussillon; remonte dans l'Ardèche vers Viviers, Ucel; dans la Drôme vers le Buis et Grignau; dans les H.-Alp. vers Ribiers, Serres et Gap.

P. Rruticosa L. - Pyr.-Or. : vallée d'Eyne.

On prẻtend avoir trouvé, en 1858 , le $P$. pensylvanica $L$. près de Saint-Christophe-en-0isans.

COMAnUn palustre L. - Marais tourbeux des plaines et des montagnes. - Granite et grès de la chaîne des Vosges. - Tourbières à sous-sol siliceux du Jura helvétique et français jusque dans les montagnes du Bugey. - Marais du Morvan granitique dans Côte-d'Or et Saûne-ct-Loire ; argiles tertiaires de Pontailler, Auxonne. - Valais : marais de Fully. - H.-Sav.: marais de Lossy au pied des Voirons, Micussy, Brizon. - Ain : les Échets, la Turchère près Bourg, Nantua, Malbronde, Meyriat, Cormaranche, Retord. - Is. : Pusignan, Charvieu, Grand-Lemps, Domène, lac Luitel au-dessus de Prémol. - Rh. : Vaugneray, Duarne, Pomeys, mont Boussivre. - Loire : Pilat, les Salles, Pierre-sur-Haute, mont Genest, Saint-Bonnet-le-Château. Ard. : le Mezenc, Chartreuse de Bonnefoi, Mezilhac. - Gard : Gourdouze près Concoule, l'Espérou. - Aude : le Donèzan. Pyr.-0r. : bois des Fanges et de Boucheville, mont Louis, Bacde-Bolquère. - En dehors de nos limites dans les terr. granit. et volcaniques du centre de la France.

Fracaitra vegen $L$. - Bois et haies dans tout le bassin.

F. collna Ehrh. - Moins commun que le précédent.

F. Ilagenbachiana Lang. - Signalé sur quelques points de la chaine jurassique, notamment entre Saint-Julien et Coligny.

F. elatior Ehrh. - Çà et là dans le bassin.

RUIUS saxntils L.-Rocailles des montagnes.-Toute la chaine des Vosges. - Région des sapins du Jura helvétique et français jusque dans le haut Bugey autour de Ruffieux, Arvières, Tenay, les cluses de Nantua et les monts d'Ain. - C.-d'Or : canton d'Is-sur-Tille, Jouvence, val de Suzon. - Valais : mayens de Saxon et de Sion, Erbioz, Leukerbad, Vercorin, Conches. H.-Sav. : Salève, Voirons, montagnes de Bonnevaux, Haut-deLin, dents d'Oche, Brizon, Parmélan, Charvin, Platets, Chamo- 
nix, N.-D. de la Gorge, entre Valorsine et Barberine. - Sav. : mont du Chat, Trévignin, Saint-Germain, bois Champion près Moutiers, Saint-Sorlin-d'Arves. - Is. : Saint-Nizier, SaintGuillaume près du Monestier-de-Clermont, mont Châtel près Mens, mont Rognon près Lalley, mont de Lans, la Ferrière d'Allevard, la Salette. - H.-Alp. : la Grave, Lautaret, Poligny en Champsaur, mont Viso au Sauze, au Bouchas et à Pra-Michel, Morgon, Boscodon près Embrun, bois Vescal près Gap, Combenoire.-B.-Alp.: Larche, Lauzannier, la Condamine.-Alp.-Har. : mont d'Or près Luceram, Colmiane entre Val-di-Blora et SaintMartin, Gars près de Briançonnet. - Ard. et H.-Loire : le Mezenc. - Aude : Salvanère. - Pyr.-Or. : forêt de Boucheville, Font-de-Comps, Canigou, Saint-Antoine-de-Galamus. - En dehors de nos limites dans les Pyrénées centrales, la Lozère, la Cantal, le mont d'Or, Puy-de-Dome, Forez, Pierre-sur-Haute.

R. Idreus L. - Bois des montagnes. - Région des sapins de la chałne des Vosges, du Jura helvétique et français jusque dans le Bugey, Valais, Savoie, Dauphiné, Basses-Alpes jusque dans les Alpes-Marit.; d'autre part dans le Morvan, Beaujolais, Lyonnais, Forez, Pilat, Vivarais, Cévennes, l'Espinouse jusque dans les Pyr.-Orient. - En dehors de nos limites, dans les Pyr. centr., l'Aubrac, Lozère, Cantal, mont Dore, Puy-de-Dôme, Forez.

R. cresilus L. - Haies. - Espèce polymorphe commune dans tout le bassin.

R. parvulus Genevier. - Bords des vignes et des champs dans les terr. calcaires. - Gard : Saint-Ambroix.

R. validulus Genev. - Haies. - Gard : Saint-Ambroix.

R. ligerinus Genev. - Bords des ruisseaux dans le centre de la France.

Dispersion mal connue.

R. delloideus Müll. - Buissons des montagnes calcaires du Dauphiné.

R. degener Müll. - Ibid.

R. debilitatus Rip. - Ibid.

R. serpens G. G. - Bois dans les envir. de Lyon à Tassin, à SaintLaurent-de-Vaux en Beaujolais. - Loire: Chalmazelle.

R. nemorosus Hayne. - Haies et bois dans la plus grande partie du bassin. Espèce polymorphe. 
1. corylifolius Sm. - Rocailles et buissons des basses montagnes dans Saone-et-Loire, Valais, Savoie, Jauphiné.-Envir. de Narbonne. Pyr.-0r.: Prades, los Masos, Estoher.

R. Igrestis Waldst. et Kit. - Buissons des plaines dans la plus grande partie du bassin, notamment dans le bassin du Léman, la Savoie, le Dauphiné.

It. dumetorum W. et N. - Haies des basses montagnes.-Particulièrement dans le Jura, Salève, Voirons, - Rh. : Chaponost, Charbonnières, Beaujeu. - Ain : Reyrieux, Trẻvoux. - Loire: Montbrison, Grand-Croix. - Ilér. : Saint-Geniès-de-Varensal, le Larzac au Caylar, Pardailhan.

B. Bellaruli W. et N. R. glandulosus Bell. - Espèce polymorphe commune dans les bois montagneux de tout le bassin, particulièrement sur les terrains siliceux.

R. calliphyllus Müll. - Bois des envir. de Saint-Étienne (Loire).

R. pendulinus Müll. - Bois. - Loire : Pierre-sur-Haute.

R. scaber W. et N. - S.-et-L. : Morvan autunois, Issy-l'Évêque.

R. hirtus W. et $\mathrm{N}$. - Haies et buissons. - Chaines des Vosges et du Jura. - H.-Sav. : Salève, Voirons, Môle, Charvin. - Is. : mont Rachais, Grande-Chartreuse. - Loire: Bois-Noir près Saint-Étienne. - Py.-0r. : vallée du Rẻart, Baixas, Saint-Estève.

R. Reuteri E. Mercier. - Envir. de Genève dans les bois de BosseyBogis, Veitay, plaıne de Saint-Georges, bois de Loëx et de la Bâtie.

R. atratus G. Genev, - Taillis. - Is. : Uriage.

R. rudis W. et N. - Bois. - Saint-Cergues au pied du Jura vaudois, envir. de Nyon au bois de Veitay. - Envir. de Genève au bois de la Bâtie. - H.-Sav. : Salève.

R. longiculp is Müll. - Bois. - Chaines du Forez, de Pierre-sur-Haute,

Montoncelle, monts Dores, monts Dômes.

R. radulı W. et N. - Haies et bois. - Envir. de Genève à Compesières,

Coppet. - Dironne au pied du Jura.

R. Schleicheri W. et N. - Loire : Pierre-sur-Haute.

R. Guntheri IV. et N. - Bois. - Jura ; Salève.

R. Menkei W. et N. - Bois. - Loire: Pierre-sur-Haute.

R. Bloxamii Lees. - Bois. - S.et-L. : bois de Poizot. - Loire : Pierresur-Haute. 
R. hispidus E. Mercier. - Au pied du Jura helvétique dans la forèt de Bonmont, bois de Bossey-Bogis près Genève.

R. piletocaulon Müll. - Buissons, - Is. : Saint-Ange au-dessus de Claix.

R. vestitus W. et $\mathrm{N}$. - Haies, broussailles principalement sur les terrains siliceux et argileux. - Chatne des Vosges. - Terrains sidérolitiques de la base du Jura suisse. - ls. : entre Vaulnaveys et Prémol, de Saint-Laurent-du-Pont à Fourvoirie. - Commun dans le centre de la France. - Loire : Pierre-sur-Haute, Saint-Georges-en-Couzan.

R. piletostachys Gr. God. - Haies. - Is. : Saint-Ange au-dessus de Claix. - Renaudiots près Autun.

א. leucanthemus Müll. - Haies. - Envir. d'Autun. - Is. : Pariset, le Sappey.

R. conspicuus Müll. - Jura suisse sur Bonmont, bois de Commugny et de Bossey-Bogis, - S.-et-L. : envir, d'Autun. — Loire : Pierresur-Haute.

R. patulus Müll. - Bois. - Is. : Vouillant au-dessus de Fontaines.

R. thyrsoideus Wimm. - Haies, bois des terrains siliceux. Chaine des Vosges, Morvan, dans S.-et-L. et C.-d'Or. - Rh. : Tassin, Saint-Genis-les-Ollières, Chaponost, l'Argentière.Loire: Saint-Martin-Lestra, Sail et Saint-Georges-en-Couzan. - Ain : Reyrieux. - Envir. de Genève à Coppet, Funnex, Genolier. - H.-Sav. : pied du Salève. - Sav. : mont du Chat. Gard : Broussan. - Espèce polymorphe.

R. albidus E. Mercier. - Bois. - Au pied du Jura de Saint-Genix à Vesancy, Gex. - H.-Sav. : Monetier au pied du Salève. - La Montagne-Noire autour de Cabarède.

R. discolor W. N. - Haies, broussailles des terrains siliceux et argileux. - Commun dans les vallées des Vosges, le Morvan, le centre de la France, Beaujolais, Lyonnais, Vivarais jusque dans l'Hérault, le Gard, l'Aude et les Pyr.-Or. : Is. : Pinet d'Uriage, entre Pariset et Saint-Nizier, Sassenage. - Bassin du Léman. H.-Sav. : pied du Salève, Arenthon, la Roche. - Pied du Jura. - H.-Alp. : envir. de Gap. - B.-du-Hh. : envir. d'Aix et de Marseille.-Var : Ie Luc.-Alp.-IIar. - Espèce polymorphe à 
laquelle se rattachent, outre le $R$. rhamnifolius et cordifolius Weihe, les deux espèces suivantes.

R. incarnatus Müll. - Broussailles. - Is. : Saint-Martin-Ic-Vinoux, Bastille de Grenoble, mont Rachais.

R. rusticanus E. Mercier. - Haies, broussailles. - Côte-d'Or; S.•et-L.; centre de la France. - H.-Sav. : Salève. - Eavir. de Genève. - Sav. : St-Innocent, le Tremblay. - Is. : Grenoble, Meylan, Seyssinet. - Envir. de Lyon.

T. tomentosus Borckh. - Broussailles. - Commun dans les vallées des Vosges. - Doubs : envir. de Montbéliard, Chapelledes-Buis près Besançon, Buillon-sur-la-Loue, entre la Main et le Val de la Loue. - Vaud : montée de St-Cergues, bois de Promenthoux. - Ain : de Belley à St-Germain, Reyrieux, la Pape. - H.-Sav. et Sav. - Rh. : Dardilly, Ecully, Charbonnières, Cogny, Sain-Fonds. - Loire: Chamazelle. - S.-et-L. : Issy-l'Evêque. - Is. : de Pariset ì St-Nizier. - I.-Alp. : Veynes, Serres, Rosans. - Gard : Nimes, St-Gilles, Bouquet, Manduel, l'Espérou. - Pyr.-Or. Céret, Arles, le Réart, la Cantarane, vallon de Castell près St-Martin-du-Canigou. - B.-du-Rh : envir. d'Aix. - Alp.-Mar. : Grasse, Clans, Nice, la Turbie, Menton, Tende. - Espèce polymorphe.

R. spectabilis E. Mercier. - Haies, bois. - Coppet près de Genève.Vaud : forêt de Bonmont.

R. collinus D. C. - Broussailles. - Au pied du Salève entre lifonetier et Mornex.-S.-et-L. : Curgy.—Ard. : Joyeuse. - Gard : le Vigan, Montdardier, l'Espérou, bois de Salbous, Auduze, Alais, St-Ambroix, Chartreuse de Valbonne. - Hér. : envir. de Montpellier, Rieucoulon, le Terral, N.-D.-de-Londres, St-Loup, Béziers. -

Vaucl. : mont Ventoux, St-Amans, plateau de Courthézon. Var: le Luc, la Verne, Pierrefeu.

R. albidus E. Mercier. - Haies. - Pays de Gex entre Gex et Vesancy,

Divonne, - Pied du Salève au-dessus de Monetier.

R. pellitus Rip. - Haies. - Loire : Montbrison, le Verdier. - Gard : Anduze.

R. cuneifolius E. Mercier. - Mornex au pied du Salève.

R. elongutus E. Merc. - Monetier au pied du Salève. 
R. undulatus E. Merc. - Versoix près Genève.

R. Riparli Genev, - Gard ả Bessèges.

IR. fruticosus L. - Haies. - Espèce polymorphe assez commune.

R. fastigiatus W. N. - Haies, bois. - Morvan dans Côte-d'Or et S.-et-L.

- Plaine et vignoble du Jura. - Centre de la France. - Hér. : la Salvetat. - Pyr.-Or.

R. plicatus W. N. - Lieux incultes. - Cote-d'Or: Morvan à Saulieu. - S.-et-L. : Issy-l'Évêque.

R. nitidus IV. N. - Buissons. - Is. : Vaulnaveys, la Ferrière d'Allovard. - Montagne-Noire dans la forêt de la Cabarède.

\section{ROSA.}

J'ai suivi la classification proposéo par MI. Déséglise, dans son Catalogue raisonné des Rosiers (Bullet. de la Soc. botan. de Belgique, $t . X V$, nos 2-3, 1876), ouvrage auquel j'ai fait, en outre, de nombreus emprunts en ce qui concerne la distribution géographique des espèces de Rosiers.

Cependant, ne partageant pas les opinions de M. Déséglise sur la question de l'Espèce, jo n'ai pu me décider à placer tous les Rosiers sur lo même rang, comme l'a fait le savant botaniste. Je crois, avec M. Crépin, qu'un très-grand nombre de formes décriteg sont des variations de quelques types principaux (Bull. Soc. bot. de Belgique, t. VIII, p. 301) qu'il importo de bien connaître et de mettre en relief. J'ai donc distingué d'abord des espèces maîtresses, suivant l'heureuse expression de M. Crépin, dont les noms seront indiqués dans ma nomenclature en caractères gras, ensuite des formes secondaires pour la désignation desquelles j'emploie les caractèros italiques.

Lorsqu'on possèdera un plus grand nombre d'observations et surtout lorsque les formes décrites auront été soumises à l'épreuve d'une culture prolongée soit dans les jardins, soit dans les stations naturelles, on pourra subdiviser encore les formes secondaires en deux autres catégories: la première comprenant celles qui sont susceptibles de se reproduire indéfiniment par le semis, à la manière des races, la seconde comprenant les variétés éphémères et non héréditaires. Mais en attendant ces observations et expériences, je dois m'en tenir à la hiérarchie provisoire des espèces maîtresses et des formes secondaires,

Je crois devoir prévenir le lecteur de no pas être trop étonné du petit nombre d'indications concernant la distribution géographique des Rosiers énumérés dans le présent Catalogue. En effet, la plupart d'entre eux n'ont été signalés que dans quelques localités de la Haute-Savoie, du Rhône, de l'Isère, des Hautes-Alpes, et, on dehors de notre bassin, du département du Cher; d'où il ne faudrait pas conclure que ces Rosicrs ne se trouvent point ailleurs, mais seulement que ce sont là les principales régions explorées par quelques rhodographes patients et habiles, tels que MMI. Puget, Boullu, Borel et Désé- 
glise. Sous le rapport géographique, commo uu point do vuo taxonomique, J'étude des Roses est encore à ses débuts.

Je remercie tous mes correspondants et, en particulier, MM. Boullu, Borel et Crépin des excellents renseignements qu'ils ont bien voulu me fournir pour l'accomplissement d'une tâche au-dessus de mes forces.

Sect. I. SYNSTYLA. Styles soudés en colonne glabre ou hérissée.

S 1. Sempervirentes. Fouilles persistantes.

ROSA sempervirens L. - Haies et buissons de la région méridionale; remonte dans l'Ardèche jusque vers Aubenas, Lhéris; dans l'Aude jusqu'au pied de la Montagne-Noire. Remonte aussi vers l'ouest de la France dans la région marit. de la CharenteInfér. et de la Vendée.

R. microphylla D. C. - Forme à pelites feuilles souvent mêlée au type. R. scandens Mill. - Forme à fleur blanche à odeur suave; fruit petit, sphérique- Collines arides de la région mérid.; moins commun que le précédent. On l'a observè dans Vaucl. autour d'Avignon; dans le Var et Alp.-Mar. autour de Toulon, le Luc, Grasse, Cannes, Antibes, Nice, Menton; dans les Pyr.-Or. vers Bagnols; dans l'Aude autour de Narbonne - Lot-et-Gar., H.Gar., Dordogne, Gironde, Charente-Infẻr.

R. prostrata D. C. - Forme à styles glabres et tige couchée observée dans les B.-du-Rh. autour d'Aix à la Gaude; dans le Var vers le Luc; dans le Gard autour du Vigan et d'Alzon; dans l'Aude vers Axat; se trouve aussi dans le Tarn à la forêt de Giroussens et au bois d'Avignon près Parisot. - Lot-et-Gar., H.-Gar.

R. ruscinonensis Déségl. et Gren. - Forme à pétioles velus-glanduleux. - Pyr.-0r. : Prades, Banyuls. - Var: Hyères..

S 2. Arvenses. Feuilles caduques.

R. bibracteatn Bast. - Tiges dressées; folioles luisantes en dessous. - Haies. - Valais à Sion. - Sav. : St-Innocent près Aix. - Rh. : Messimy, St-Christot-en-Jarret. - Is. : Crémieu. Lozère à Mende. - Tarn à Canimont, St-Urcisse, forêt de Giroussens, H.-Gar., Loire-Infêr. Maine-et-Loire.

R. arvensis Huds. R. repens $\mathrm{Scop}$. - Tiges couchées ou décombentes. - Haies. - Valais. - H.-Sav. : la Roche, Archamp, Annecy, 
Pringy. - Rh. : Charbonnières, Marcy, St-Genis-les-Ollières, Chaponost. - Commun dans les Pyrénées-0r. et Centr, le Tarn, le centre de la France.

R. érronea Rip. - Haies. - H.-Sav. à Brenthonne. - Rh. à Méginant près Tassin. - Commun dans le Cher.

R. ovata Lejeune. - Haies, bois. - H.-Sav. : Arenthon. - Cher, Deux-Sèvres.

Sect. II. STYLOSE. Styles agglutinés en colonne.

R. stylosa Desv. - Haies. - Doubs : Besançon, Nans, Cuze. S.-et-L. : Parpas, Ornée, St-Pierre près Autun. - Envir. de Genève à Vernier, Compesières, entre Genthod et Versoix, Coppet dans le canton de Vaud. - H.-Sav. : le Vuache, la Caille, Pringy. - Is. : Villette d'Anthon, Seyssins. - Rh. : Ecully, la Tète-d'Or. - Gard. - Hér. : St.-Dominique et la Gaillarde près Hontpellier, St-Jean-de-Védas, Caunelle. - Assez commun dans le Tarn, Allier, Cher, Maine-et-L., Calvados et les Pyrén.-Centr. - Le R. stylosa a les feuilles velues en dessus, les pétales blancs, tandis que le $R$. systyla Bast a les feuilles glabres en-dessus et les pétales roses.

R. systyla Bast. - Haies. - S.-et-L. : Parpas, St-Pierre près Autun. - Envir. de Genève et de Nyon. - H.-Sav.: Thonon, Lully, Arenthon, la Roche, mont de Sion, la Caille, Pringy, Chavanod et Brogny près Annecy. - Rh. : Ecully, Tassin, Charbonniëres, la Tour de Salvagny, versant nord du mont Verdun, St-Lager en Beaujolais.-Sav. : le Bourget.-Is. : Seyssins, - ¿H.-Gar., Lot-et-Gar., H.-Vienne, Indre, Loiret, Maine-et-L., Vendée, Morbihan, Loire-Infér.

R. leucochroa Desv. - Forme à fleurs blanches et à onglet jaunâtre.Autour de Lyon à Clarbonnières, Villeurbanne, Tassin. H.-Sav. : Thonon, Annecy. - Dr. : Crest. - Var : Hyères, le Luc. - Tarn, Cher, Vienne, Deux-Sèvres, Ml.-et-L., Vendée, Loire-Infér., Vosges.

R. fastigiata Bast - Forme à pétioles velus, folioles velues en-dessous sur toute la surface; se trouve mélée aux précédentes.

Les $R$. immitis Déségl. et virginea Rip. trouvés dans le dép du Cher n’ont pas été signalés dans notre bassin. 
Sect. III. GiLLICAN.E. Ramcaus ì aiguillons grêles, crochus, mêlés do soies courtes et glanduleuses; feuilles coriaces, pâles et blanchâtres en dessous.

S 1. HYвRID Styles rapprochés on colonne velue, mais non soudés.

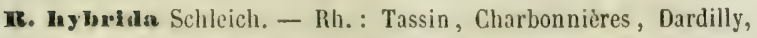
Grézicux, Corcelles, Brouilly près Saint-Lager en Beaujolais.Envir. de Genève. - Cher, Puy-de-Dôme, H.-Gar. - Plusieurs auteurs ont pensé que cette Rose est un hybride des $R$. gallica et arvonsis avec lesquelles elle est souvent mêlée.

M. Boullu a trouvé à Méginant, hameau de Tassin, une forme presque inerme qu'il nomme $R$. sublovis et en outre deux autres formes qui lui paraissent intermédiaires entre $R$. repens et $R$. gallica et qu'il appelle $R$. repente-gallica et $R$. gallico-repens. $R$. arvina Krock. - Rh. : Tassin, Charbonnières, Dardilly, Craponne. R. Polliniana Spreng. - Bois. - Rh. : Charbonnières, Dardilly, Pontd'Alaï. - Cher. - Schafihouse.

S 2. Styles libres, hérissés ou glabres.

T. austriaea Crantz. - Haies, bois des terr. siliceux. - Rh. : région granitique de Tassin, Charbonnières, Francheville, Saint-Lager en Beaujolais. - Alp.-Mar. : Pont-de-l'Ablé près de Villars-du-Var. - Cher: sur les argiles à minerai de fer superposés au calcaire d'eau douce.

$R$. arcnivaga Déségl. trouvé dans les envir. d'Orléans et $R$. Boræana trouvé dans la Loire-Infér. et près d'Angers n'ont pas encore été signalés dans notre bassin.

R. subinermis Chabert. - Haies, bois. - Rh. : Dardilly, Francheville au Gau, Charbonnie̊res.

R. geminata Rau. - Haies, bois. - Rh. : région granitique de Tassin,

Pont-d'Alaï, Charbonnières, Marcy, Ste-Consorce, Dardilly, la

Tour-de-Salvagny, St-Lager en Beaujolais. - Cher, Indre.

R. Fourrai Déségl. - Haies, bois. - Rh.: Tassin, Clıarbonnières, Ste-Consorce.

$R$. incarnata Mill. - Haies dans les terrains granitiques des envir. de Lyon, à Tassin, à Charbonnières. - Sables du bois des Catal. Bassin du Rhône. 
Frères près Genève. - Sables et argiles tertiaires de la Sologne et du Cher.

$R$. virescens Déségl. - Ce Rosier trouvẻ dans le Loiret sera peut-ếtre rencontré dans la région granitique du Rhône.

R. velutinaflora Déségl. et Ozanon. - Bois. - Rh. : Charbonnières au bois de l'Étoile, Saint-Lager sur la colline de Brouilly.

R. mirabilis Déségl. - Bois.- Charbonnières, Marcy, Tassin, Brouilly près Saint-Lager. - Cher : bois de Marmagne.

R. sylvatica Tausch. - Rh. : région granitique de Tassin, Pontd'Alaï, Francheville au Gəu, Saint-Genis-les-0llières, SainteConsorce, Charbonnières au bois de l'Étoile. - Se trouve aussi dans Maine-et-Loire, Cher, Nièvre.

R. decipiens $\mathrm{Br}$. - Bois. - Rh. : région granitique au Pont-d'Alaï, Charbonnières, Sainte-Consorce, Saint-Lager sur la montagne de Brouilly. - Le Vangeron près Genève. - Cher.

$R$. opacifolia Chabert. - Diffère du précédent par des folioles plus petites, à dentelures plus aiguës, styles glabres, fl. d'un blanc satiné. - Haies dans la région granitique du Rh. : Francheville au Gau, entre Charbonnières et Tassin.

\$ 3. Styles libres laineus.

a. gallea L. - Bois dans les terrains siliceux des Vosges, Morvan, Beaujolais, Lyonnais, envir. de Vienne. - Envir. de Genève sur les terr. sablonneux du bois de la Bâtie, des Frères, Petit-Lancy, Meyrin, Veyrier et dans le dép $p^{t}$ de l'din à Ferney. - B.-Alp. : Digne. - Schistes siliceux des Maures depuis les envir. de Toulon, jusqu'au Luc et à Fréjus. - Pyr.-Or. : Castelnau, Camélas. - N'est connu dans la chaîne jurassique qu'au mont de Brégille près Besançon. - En dehors de notre bassin on trouve cette espèco sur les terrains schisteux de Maine-et-Loire, Sarthe et Lozère; sur les granites de la Vienne; sur les sables tertiaires du Loiret, Loir-et-Cher; sur les argiles à minerai de fer du Cher; dans le Puy-de-Dôme sur les alluvions sablonn. du bois de Lezoux; elle est naturalisée autour d'anciennes cultures dans le bassin de Paris à Mlagny, Charly, Provins, Noyon.

R. protincialis Ait. - Var: le Luc, les Mayons. - Naturalisẻ en plusieurs localités de Maine-et-Loire et du Cher. 
R. assimilis Déségl. R. cordifolia Chabert (non Ilost). - Charbonnières au vallon de Ganches près du bois de l'Étoile.

R. ruralis Désígl. - Rh. : Francheville au Gau, Charbonnières au bois de l'Étoile, Tassin à Méginant. - Gher : Moulon près de Bourges, Meljun.

R. pumila L. - Doubs: Besançon au mont Brégille. - Envir. de Genève. - Rh. : Charbonnières au bois de l'Étoile, Tassin, St-Lager en Beaujolais sur la montagnede Brouilly. - Cher, Loir•et-Cher, Nièvre.

Près des gallicanes se placent le $R$. centifolia L. et ses nombreuses variétẻs cultivées dans les jardins.

Sect. IV. PIMPINELLIFOLIA. Feuilles petites, glabres; styles libros; sépales persistants.

\section{S 1. Folioles non glanduleuses en-dessous.}

R. Pimpinelufolia D. C. $-R$. spinosissima L. - Buissons et rocailles des montagnes. - Toule la chaine des Vosges jusque dans la vallée du Puix près Belfort. - Jura helvétique et français depuis les montagnes baloises jusqu'à celles du Bugey; assez commun sur les escarpements du Lomont jusque vers les sommités de la Roche près Nans, Côtes du Doubs et du Dessoubre, Chatard près Baume, Planèze et Arguel autour de Besançon; dans le Bugey on le trouve sur le Mlont, les Neyrolles, les ruines d'Arvières, la grotte d'Oncieu, Charabotte. - Valais : le Bouveret, Fins-Hauts. - Côteaux calc. de C.-d'Or, S.-et-L. - H.-Sav : Monetier au pied du Salève, le Coln au-dessus d'Archamp, Andey près Bonneville, mont Laouet, Veyrier, Crêt-du-Maure et la Puya près Annecy. - Sav. : Bessans en Maurienne, Jarrier au Châtelard près St-Jean-de-Maurienne, Trévignin, roche St-Victor au-dessus de Ifouxy. - Is. : mont Rachais, Ghalais, Comboire, St-Nizier. - H.-Alp : col Bayard, les Serigues, Côte Gélive, près Gap, pic de Chabrières, Laye en Champsaur. B.-Alp. : la Condamine. - Vaucl. : mont Ventoux. - B.-du-Rh. et Var: Ste-Victoire, St-Cyr, Ste-Baume en montant au Pilon. Alp.-Mar. : montagnes de Grasse et d'Entraunes. — Gard : le Vigan. - Hẻr. : la Sérane. - Pyr.-Or. : Font-de-Comps, mont 
Louis, vallées d'Eynes et de Llo, Llaurenti.- En dehors de nos limites dans les Pyr.-Centr., Puy-de-Dôme, Lozère, Allier.

La plupart des autcurs ont suivi Linné qui, dans le Mantissa, p. 399, avait réuni le $R$. pimpinellifolia au $R$. spinosissima ; ce serait à tort, suivant M. Déséglise. Cependant comme cette distinction n’a pas été faite par les susdits auteurs, il In'est impossible d'indiquer la distribution particulière de ces deux formes. R. \athoneti Crépin.-Ballon de St-Ilaurice dans les Vosges.-Doubs: Pont-de-Roide au Cret-des-Roches: - Is.: entre le Freney et l'Alpe du mont de Lans, St-Clıristophe-en-Oisans, mont Rachais. - H.-Alp. : mont Gondran près Briançon, la Grave. - B.-Alp :

St-Paul-de-Vars. - H.-Sav. : Salève. — Sav. : mont Nivolet.

$R$. consimilis Déségl. - H.-Sav. : Veyrier près Annecy, Mandallaz.

-Sav. : mont Nivolet, Lans-le-Bourg. - Is. : St-Eynard, la Salette. - H.-Alp. : la Grave, Villard-d'Arène. - Lozère.

R. spreı Déségl. - H.-Sav. : Veyrier. - Sav. : mont Nivolet. - Is. : mont Rachais, St-Eynard, Comboire. - H.-Alp.: la Grave, Villard-d'Arène, Devez de Rabou.-Ard. : Gerbier-des-Joncs. - Pyr.0r.: vallée d'Eyne. - Mont Dore, Cantal.

R Ozanonii Déségl. - Rameaux inermes. - Is. : St-Eynard. - H.Alp.: la Grave, Villard-d'Arène, le Puy-Vacher. - H.-Sav. : mont Brizon.

R. mitissima Gmel. - Rameaux inermes. - Ballons de Soultz, Hohnech, Hohenstaufen, Ballon de St-Maurice dans les Vosges. Envir. de Besançon. - Sav. : mont Nivolet.- Is, : Chalais aux Baneltes. - Ard. : Gerbier-des-Joncs.-Cantal, mont Dore.

$R$. reversa Waldst. Kit. - Sav. : mont Nivolet.

R. gentilis Sternb. - Ain : la Faucille au-dessus de Gex. - H.-Sav. : Salẻve. - Is. : mont Rachais. - H.-Alp. : Villard-d'Arène. Mont Chaumont, dans le canton de Neuchâtel.

$\$ 2$. Feuilles glanduleuses en-dessous.

E. myriaeantha D. C. - H.-Alp. : mont Bayard près Gap, St-Nens, - Hér, : Lamoure, St-Jean-de-Védas: Montbazin, Roquehaute, Montarnaud, Bézicrs. - Pyr.-Or.: Collioures. R. Ripartii Déségl. - Haies, bois. - H.-Alp. : mont Bayard. - LoireInfér. - Cher. 
Soct. V. SABINIdE. Tiges à soies rares et à aiguillons inégaux et distincts; foliolos doublement dontées; fruit arrondi à la base; sépales pinnatifidor, divergonts

It Sn In In Woods. - Cette espice, trouvéc par Webb et Baker dans lo Cheshire, le Warwickshire et le Yorkshire en Angleterre, a été vue aussi sur le mont Salève (H. Sav.) et à Ravellen dans le cant. de Soleure.

R. coronala Crépin. - Rochers. - Sav. : Praz-Flandet, au-dessus de Chevron.

R. sabauda Rapin. - Roch. - H.-Sav. : Salève, à la Grande-Gorge, à la Croiselte, Petit-Salève au-dessus d'Étrambières.

Sect. VI. CINNAMOME.E. Ramcaux d'un brun cannelle; aiguillons des tiges droits, inégaux, subulés non glanduloux, ceux des rameaux placés au bas des feuilles et crochus ; sépales longuement acuminés, persistants ; pédoncules munis do larges bractées.

r. cInnanomea L. - Haies. - Jura : St-Loup. - Vaud et Valais : Chailly prés Lausanne, Payerne, Lugnore, Cocherai, mont Catogne, Zermatt. - Puy-de-Dome, Creuse, Meurthe, Vosges.

R. facundissima Mœnch. - Doubs et Jura : Besançon, Salins. - H.-

S. : Pringy, Arenthon, Thonon, le Vuache. - Meurthe, Landes. R. blanda Ait, - Haies. - C.-d'or : Dijon. - Envir. de Genève au bois des Frères. - Lozère, Oise, Seine-et-0ise, Loiret.

; Sect. VII. ALPINAE. Aiguillons subulés; súpales entiers; styles libres; folioles glabres.

IR. nIpina $\mathrm{L}$. - Bois et rochers des montagnes. - Assez répandu dans la chaine des Vosges jusque dans la zone vosgienne de l'arrond. de Belfort et de la H.-Saône, dans les vallées du Puix, du Roselin, du Miélin, du Rahin, Ballons de Servance et de Giromagny. - Jura helvêtique et français; assez abondant sur les plateaux supérieurs, Clos-du-Doubs, Côtes du Doubs et du Dessoubre, Lomont, Morteau, chaînes de la Dôle, Colombier, Reculet, jusque dans les montagnes du Bugey. - Vaud et Valais : Ifontbovon, Alpes de Bex, Surchamp, Chemin, Catogne, Vex, Mayens de Sion, Vercorin, Alpes de Saxon, de St-Nicolas et de Zermatt, Oratoire-sur-Naters, vallée de Binn, glacier de Massa, 
Corbire. - H.-Sav. : Salève, Voirons, Habère-Poche, HabèreLullin, mont Laouet, col de Terramont près Vailly, montagnes de Samoëns, plateau des Gets, mont Châtillon, Parmélan, Charvin, mont Ste-Catherine près Annecy, Semnoz, Tournette, vallées de la Diosaz, de Chamonix et de Monljoie. - Sav. : Hauteluce, Crêt-du-Ré au-dessus d'Aime, col du Frêne, mont du Chat, les Bauges, St-Sorlin-d'Arves, la Ramasse et le mont Cenis. Is. : massif de la Gr.-Chartreuse, Chamechaude, St-Nizier, Villard de Lans à la Fauge, montagnes d'Allevard. - H.-Alp. : col Malrif, mont Viso au Bouchas, la Chalp, Ste-Agathe, vers PierreGrosse, Fontgillarde, envir. de Gap au Devez-de-Rabou, Pleynede-Chaudun, Séuse, Sapet de la Bâtie-Neuve, Chaillol. - B.Alp. : le Lauzannier. - Les sommités des Alp.-Har. - Loire : Pilat, Pierre-sur-Haute. - Ard. et H.-Loire : le Mezenc. Gard : l'Espérou, Concoule. - Aude et Pyr.-0r. : le haut Donézan, Llaurenti, Salvanère, Canigou, montagne de Madrès, vallées d'Eyne et de Llo, mont Louis.

En dehor's de nos limites dans les Pyr. centrales, mont Dore, Cantal, Lozère. - Espèce polymorphe à laquelle se rattachent les formes suivantes.

$R$. intercalaris Déségl. - Rocailles des montagnes. - H.-Sav. : les Voirons, vallée de Montjoie. - Is. : St-Eynard, Chamechaude, près de Vararey, Villard de Lans, Charminelle, près Voreppe, St-Christophe-en-Oisans. - Sav. : mont Nivolet. - H. Alp. : la Grave, vallon de Puy-Vacher. - Alp.-Mar. : Colmiane, près St-Martin-Lantosque. - Ard. et H.-Loire : le Mezenc. - Pyr.Or. : mont Louis. - Vosges au Hoehneck, Ballon de St-Maurice, petit Rotabac.

$R$. adjecta Déségl. - Chalne jurassique au mont Suchet. - Valais : Bovernier, Cantine de Proz en montant au St-Bernard, Zermatt. - H. Sav. : Habère-Lullin, mont de l'Offiége-sur-Brenthomme, mont-de-Coux-sur-Cervens, Bourdignin, vallée de Montjoie à N.-D. de la Gorge, Chantemerle, au-dessus de Samoëns. - Sav. : mont Nivolet. - Is. : St-Ange près le Pont-de-Claix, Villardde-Lans, St-Christophe-en-0isans. - Alp.-Ifar. : vallon de Manduebis, près St-Martin-Lantosque. - Ard. et H.-Loire : Mezenc. - Pyr.0r. : mont Louis, - Vosges au Ballon de St-Maurice. 
R. monspeliaca Gouan. - Rochers des montagnes. - Sav. : mont Cormet. - II.-Alp, : la Grave. - Gard : l'Espérou au bois des Aubrets. - Puy-de-Dome à Royal et au mont Dore, Cantal.

R. pendulina Ait.- Rochers des montagnes.- II.-Sav. : dent de Lanfond près Annecy, la Caille. - Sav. : mont St-Cassin près Chambéry, - Is. : St-Nizier. - H.-Alp. : la Grave, forêt des Fraux. - B.-Alp. : Colmars. - Pyr.-Or.-Vosges.

R. lagenuria Vill. - Rochers des montagnes, - Valais : Binn, valléc de Conches. - H.-Sav. : Salı̀ve, Habère-Poche, montagnes des cnvirons d'Annecy el de Chamonix. - Sav. : Hauteluce, Hautecombe, Lans-le-Bourg, mont Cenis. - Is. : Villard-de-Lans, col de l'Arc, St-Christophe-en-0isans. - Ain : la Faucille. - H.Alp. : Boscodon, près Embrun, Derez de Rabou. - Aude : le Donézan, près du lac de Quérigut.-Ard. et H.-Loire : le Mezenc. montagnes du Forez, Pierre-sur-Haute, mont Dore, Cantal, Lozère, Pyrénées.

R. pyrenaica Gouan. - Rocailles des montagnes. - Doubs : Pontarlier. - Pyr.0r. et centrales.

Sect. VIII. MONTAN.E. Feuilles et jeunes rameaux glaucescents; aiguillons grôles non crochus; sépales redressés après la floraison, couronnant le fruit jusqu'à la maturitó ; flours d'un roso vif.

IC. rubrifolla Vill. - Bois et buissons des montagnes. - HautesVosges au Hoehneck, Ballons de Guebriller et de Giromagny.Jura helvétique et français, Morteau, Pissoux, Chenalotte, mont d'Or, Suchet, Dóle, Faucille, Reculet, Rizoux, descend jusqu’à Poupet, près Salins; dans les montagnes du Bugey sur les monts d'Ain, Hautcville, Arvières, Colombier. - Valais et Vaud : val Ferret, Fins-Hauts, Salvan, l'Etroz, Trient, vallée de Binn, Alpes de Bex. - H.-Sav. : Salève, Voirons, Brizon, Reyvroz, montagnes de Bonnevaux, de Samoëns, plateau des Gets, mont Châtillon, Parmélan, la Clusaz, montagnes d'Annecy, Gr.-Bornant, Mégève, Chamonix. - Sav. : Mouxy près Aix, Pontamafrey en Maurienne, la Ramasse, au pied du mont Cenis. - Is. : St-Eynard, Chalais, Gde-Chartreuse, St-Ange, Villard-de-Lans, St-Barthélemy-de-Séchilienne, St-Christophe-en-0isans. - H.- 
Alp. : la Grave, envir. d'Abriès-en-Queyras, de Gap au col de Glaise, Chartreuse-de-Durbon, Ribiers au bois de St-Pierre. Dr. : montagnes de Lus-la-Croix-Haute. - B.-Alp. : la Blachière, près Maurin, col Vergous-sur-Annot. - Vaucl. : mont Ventoux. - Var : Aiguines. - Loire : Pilat, Pierre-sur-Haute, pic de Gourgon. — Gard : bois près de la Grotte de Brama-Bioou, bois de Longuefeuilles, vers Concoule. - Pyr.-Or. : bois de Salvanère, de Madrès et de Nohédes. - Pyr. centr. à Gavarnie, Héas; Lozère, Cantal, mont Dore, Puy-de-Dôme.

R. montana Chaix. - Bois et buissons des montagnes. - Valais : Fins-Hauts, Salvan, Joux-Brulée au-dessus de Branson, Sembrancher, le Clou au-dessus de Bovernier, mont d'Orge, Vercorin. - H.-Sav. : Salève au-dessus d'Archamp, la Gr.-Gorge, rochers du Coin, plateau des Voirons, mont Andey, plateau du Brizon avant la grotte, le Chenaillon au-dessus du Grand-Bornant, Semnoz, Tournette, vallée de Chamonix. - Is. : St-Christophe-en-Oisans, Villard-de Lans. - Sav. : Longefoy. - H.-Alp.: la Grave, Briançon, Abriès-en-Queyras, envir. de Gap à Charance, Rabou, Chaudun, Côte-Gélive, col de Glaise, pic de Chabrières, Aurouse, Guillestre, chartreuse de Durbon. - B.-Alp. : St-Paul, la Condamine, St-Genicz au bois de la Penne, Barcelonnette au-dessus du col de Fours. - Alp.-Mar. : Amen, près de Puget, vallon de Libaré. - Piémont à St-Remy entre le Gr. St-Bernard et Aoste.

R. salcevensis Rapin. - Rochers et bois des montagnes. - Doubs : les Saules, près Morteau, au-dessous du mont Chateleu._La Tourne dans le Jura neuchâtelois. - H.-Sav. : Salève au-dessus d'Archamp, en montant à la Croisette, au-dessus des Treize-Arbres, mont de Sion, de Thuet au Brizon, montagnes d'Abondance et de Bellevaux. - Sav. : mont Nivolet, roche St-Victor au mont du Chat. - Is. : Villard-de-Lans, St-Christophe-en-0isans. H.-AJp. : la Grave, Briançon, Devez de-Rabou, près Gap.

R. Perrieri Songeon. - Bois des montagnes. - Sav. : forêt d'Apremont, près Clıambéry, Nivolet, Margériaz.-H.-Sav. : le Vuache. - Is. et.H.-Alp. : Villard-de-Lans et Rabou.

$R$. caballicensis ruget. - Bois des montagnes. - Doubs: Pontarlier. 
Valais : Grüben, prìs Zermatt. - H.-Sav. : les Voirons, montagnes du Chablais, Bonnevaux, Reyvroz, Habère-Lullin, Habire-Poche, col de Terramont près Vailly, vallées de la Clusaz et dı Gr.-Bornant. - Sav. : St-Marcel près Mouticrs, Modane, la Ramasse au pied du mont Cenis. - H.-Alp. : Chaudun, près Gap. - Puy-de-Dome au Puy-de-Pariou, petit Puy-de-Dome, Laschamps, Randanne.-Loire : Pilat et Pierre-sur-Haute.

$R$. falcata Puget. - $R$. imponens Rip. - Bois et rocailles des montagnes. - Valais : Bovernier. - H.-Sav. : chalets de la Clappaz, près Habère-Lullin. - H.-Alp. : St-Laurent-du-Cros, près Gap. - B.-Alp. : Pas-de-Grégoire, près Tauriers, - Cant. de Fribourg à Mlontbovon, Paribaz.

$R$. glauca Vill. $-R$. Reuteri Godet.-Buissons et bois des montagnes.

Chaine des Vosges au Ballon de St-Maurice, Vagney, Gérardmer. - Jura helvétique et français autour de Pontarlier, vers le lac Brunct, au pied du mont d'Or, pied du Larmont, Bole près de la Dóle, mont Poupet, près Salins.- Valais : Bovernier, Salvan, la Forclaz, val Ferret. - Cant. de Fribourg, près Montbovon. - Cant. de Berne à Adelboden. - H.-Sav. : Salève, Voirons, chartreuse de Pommiers, Archamp, mont de Sion, Mole, de Thuct au Brizon, vallée de la Verrerie de Thorens, montagnes du Chablais à Vailly, Bonnevaux, Reyvroz, plateau des Gets, Habère-Poche, Habère-Lullin, montagnes de Samoëns, mont Châtillon, Chenaillon au-dessus du Grand-Bornant, Parmélan, vallẻes de la Clusaz et de Leschaux. - Sav. : mont Joigny et Nivolet près Chambéry, mont des Chaires, montagnes d'Hauteluce. - Is. : forêt de Portes en montant à Chamechaude, Villard de Lans, la Salette, la Ferrière-d'Allevard. - H.-Alp. : la Grave aux Lauzières, Guillestre, col Bayard, vallon de Glaise, St-Laurent-du-Cros-en-Champsaur,-B.-Alp. : St-Paul-de-Vars. -Alp.-Mar. : mont Cheiron, col de Fenestre.-Pyr.-Or., Lozère, Puy-de-Dôme à Fontanat et Lezoux.

R. complicata Gren. - Buissuns des montagnes. - Doubs : du fort de Joux à Pontarlier. - Valais et Fribourg : mont Clou sur Bovernier, la Cernaz près Montbovon. - H.-Sav. : Reyvroz, HabèrePoche, Habère-Lullin.- Puy-de-Dòme, mont Dore. - H.-Loire : Ceyssac près le Puy. 
R. intricata Gren.- Buissons des montagnes. - Doubs : Pontarlier. -

Valais : le Clou près Bovernier, Zermatt. - H.-Sav. : Habère-

Poche. - Is. : la Ferrière-d'Allevard. - Ard. : la Louvesc. -

Cantal et H.-Loire à Murat et Ceyssac.

R. fugax Gren. - Buissons des montagnes. - Doubs : Pontarlier. -

Valais et Fribourg : mont Clou sur Bovernier, Crettaz dans val.

lée d'Entremont, Cases d'Allières, près Montbovon. - H.-Sav. :

Habère-Lullin, Habère-Poche. - H.-Alp. : Villard-d'Arène. -

Pyr.-0r. : mont Louis. - Pyr, centrales.

$R$. venosa Swartz. - Buissons des montagnes. - Is. : forêt de Portes,

Villard-de-Lans. - Sav. : mont Joigny, près Chambéry. - Vosges : Saulxures, Cornimont, Liezey.

$R$. alpestris Rapin. - H.-Sav. : Salève autour des Pitons, les Voirons.

- H.-Alp. : la Grave, col Malrif. - Valais et Fribourg : Barberine, Bonnondon.

R. Schultzii Rip.-Haies. - Cette forme, indiquée dans le Cher et dans

le cant. de Fribourg à Tines, près Montbovon, sera probablement trouvée dans notre bassin.

Sect. IX. CANINAE. Aiguillons vigoureux, larges, dilatẻs à la base, crochus; folioles dépourvues de glandes sessiles entre les nervures; styles libres.

\$ 1. Nudx. Pétioles glabres, folioles simplement dentées, glabres, sans villosité sur les nervures; pédoncule et fruit glabres.

R. canina L. - R. lutetiana Lem. - Broussailles, bois depuis les plaines jusqu'aux montagnes. Espèce polymorphe au plus haut degré à laquelle se rattachent les formes suivantes.

R. glaucescens Desv. - Haies, bois. - Valais : Bovernier, val Ferret, vallée de Conches. - S.-et-L. : envir. d'Autun. - H.-Sav. : Habèrc-Poche, Pringy. - Is. : le Sappey. - Maine-et-Loire, Cher.

R. nitens Desv. - Haies, - Valais : Bovernier, Sion. - H.-Sav. :

Pringy. - Is. : le Sappey, - Vosges, Cher.

R. syntrichostyla Rip. - Haies, bois. - H.-Sav. : Habère-Poche, la Caille. - Is. : St-Christophe-en-@isans. - Rh. : la Ronzière.Cher, Vosges, Lot-et-Gar. 
R. macroacantha Rip. - Haies, bois du Cher. - A chercher dans notre bassin.

R. mucronulata Déségl. - Bois. - II.-Sav. : Ilabère-Lullin à l’Arpettaz. - Valais : Bovernier. - Cher, Alsace, Jura bâlois.

R. senticosa Achar. - Haies, bois. - H.-Sav. : Salive, au chemin de

la Croisette, Arenthon, Habère-Lullin, le Vuache, la Puya, près Annecy. - Lyon, au Gr.-Camp. - Cher, Neuchâtel (Suisse).

1. fallens Déségl. - Haies, bois. - Valais : Sembrancher, Bovernier.

II.-Sav. : grèves du Léman près Ripaille, Lully, Arenthon, Наbère-Lullin, la Roche, Pringy, la Puya près Annecy, AnnecyJe-Vicux. - Rh. : Craponne, Chaponost. - H.-Alp. : Riotors, près Gap. - Cher.

R. Tourangiana Déségl. et Rip. - Haies. - H.-Sav. : Arenthon. Envir. de Lyon. - Cher.

R. ramosissima Rau. - Haies, bois. - Doubs : Montmahoux. - S.-etL.: St.-Forgeot près Autun.- Envir. de Lyon.-Marne, Cher.

R. globularis Franchet. - Haies. - Doubs : Besançon. - S.-et-L. : la Chaume, près Autun. - Envir. de Genève. - H.Sav. : Salève, la Puya et les Ollières, près Annecy. - H.-Alp. : Gap, Matachard, Serre-Eyraud. - Rh. : Francheville. - Hẻr. : Pégayrolles-de-l'Escalette. - Puy-de-Dôme, Loir-et-Cher, Alsace.

R. montivaga Déségl. - Broussailles des montagnes. - Valais : FinsHauts, mont-Clou-sur-Bovernier, vallée de Binn. - Paribaz, Cape-au-Hoine dans cant. de Fribourg. - H.-Sav. : mont do Sion, Habère-Lullin, Habère-Poche, Bonnevaux, plateau des Gets, montagnes de Samoëns, vallée de la Verrerie de Thorens, Parmélan, Pringy, St-Germain-sur-Talloires. - Sav. : mont Nivolet. - Is. : Crémieu, mont Rachais. - H.-Alp. : envir. de Gap, col Bayard, - Puy-de-D., Cantal, Lozère.

R. spuria Puget. - Broussailles des montagnes. - Doubs : mont Brégille près Besançon, Chapelle-d'Aigremont-sur-Roulans. - H.Sav. : Pringy, Épagny, St-Martin, Annecy-le-Vieux, montagne de l'0ffiége. - Is. : forêt de Portes.- Vosges; Fribourg.

R. spherica Gren. - Haies, bois. - Assez commun dans la région des sapins du Doubs et du Jura.-Vaud et Valais : Chescières, Sion, Sembrancher, Vex.- Envir. de Genève, au bois de la Bâtie. - 
H.-Sav. : Salìve, au picd de la Gr.-Gorge et en montant aux Treize-Arbres, Habère-Lullin, Bonnevaux, plateau des Gets, montagnes de Samoëns, mont Châtillon, St-Jean-de-Sixt, la Puya, près Annecy, le Semnoz, Bluffy, Chamonix. - Sav. : mont du Chat. - Is. : le: Sappey, forêt de Portes, entre Pariset et StNizier. - H.-Alp. : Charance, Matachard, au pied du mont Au rouse. - Rh. : Charbonnières, Tassin.-Alp.-Mar. : St-MartinLantosque. - Loire : Pierre-sur-Haute, St-Rambert. - Vosges, Loiret, Cher, Puy-de-Dôme.

R. aciphylla Rau.-Rocailles. - Doubs : Besançon à Brégille.-Rh. : Beaunant, Francheville.- Montbovon dans le cant. de Fribourg. - Cher.

S 2. Bisgratex. Pétioles glabres, folioles doublement dentées, sans villosité, pédoncule ot tube du calice lisses.

R. dumalis Bechst. - Buissons des plaines et des montagnes. Commun dans les Vosges, la chaîne jurassique, C.-d'0 r, S.el-L., Rh., Valais. - H.-Sav. : Thonon, Concise, bords de la Dranse et du lac Léman, Arenthon, Reyvroz, Sałève, la Caille, Pringy, Veyrier, la Puya et.Brogny, près Annecy.-Is. : Bastille de Grenoble, mont Rachais, forêt de Portes, Sappey, Pariset.-H.-Alp.: envir. de Gap, Puy-Montbard, vallon de Glaise à Chovet, St-Laurent-de-Cros-en-Champsaur. - Alp.-Mar. : St-Martin-Lantosque, Venanson, la Maïris. - Gard. - Hér. : Montpellier, vers l'Aqueduc, la Planchude, Montauberon, Villeneuve, Lunas, Ceilhes, St-Amand-de-Mounis, St-Étienne-de-Gourgas, le Caylar, St-Maurice.-Commun dans les Pyrénées, le Tarn, le centre de la France.

R. rubescens Rip - Valais : vallée de Binn.-Cher, Allier.

$R$. rubelliflora Rip. - Lyon à Dardilly. - Is. : Chalais. - Puy-de-D. à St-Pardoux: - Cher.

R. squarrosa Rau. - Bois. - S.-cl-L. : Châl.-s..Saòne. - Doubs : Besançon au mont Brégille, Pontarlier.-H.-Sav. : Habère-Lullin, Pringy, envir. d'Annecy à Veyrier, St-Mlartin, Margériaz. Sav. : Aix, St-Innocent, Grésy, - Rh. : Francheville, Pontd'Alaï, Alix. - Ain : la Pape. - Is. : Corenc, Vernas; Vienne. - H.-Alp. : Charance, près Gap. - B.-Alp. : Barcelonnette. - 
Montbovon dans cant. de Fribourg. - Vosges, Allier, Puy-deDome, Tarn.

R. malmundariensis Lejeune. - Taies, bois. - Doubs et Jura : mont

Brégille, Salins.-S.-et-L. : Chàlon, Chagny, Tessey.-Valais :

Binn, Conches, Mayens-de-Sion. - H.-Sav. : Reyvroz, Thonon, Habère-Lullin, Pringy. - Rh. : Ecully, Tassin, Yzeron, Corcelles. - Ain : Ambronay. - Is. : Pariset, Crémieu. - Var : le Luc. - Gard : le Vigan. - Aude : Versant mérid. de la MontagneNoire à Mas-Cabardès. - Cher, Puy-de-Dóme, Allier.

R. medioxima Déségl. - Haies, buissons. - Valais : les Valettes. -

H.-Sav. : la Malvéria sur Annecy-le-Vieux, Thonon.- Is. : forêt de Portes.-Cher, Alsace.

R. Carioti Chabert. - Haies. - H.-Saone : Nans-les-IIontbozon, -

Rh. : Francheville au Gau, Tassin, Charbonnières, mont Verdun, mont Thoux. - Aude : Mas-Cabardès.-Cher à la Chapelle St-Ursin.

R. glaberrima Dumort. - Broussailles.-H.-Sav. : Pelit-Saleve près du château de Monetier.

R. oblonga Déségl. et Rip. - Iaies, bois. - H.-Sav. : Épagny. - Fribourg à Montbovon. - Cher.

r. cladoleia Rip.-Haies, bois. - Doubs : Nans-les-Rougemont, Pontarlier, Besançon, sous Rosemont. - Valais : Bovernier, Brocard.- H.-Sav. : Pringy. - Rh. : Tassin à Méginant. - Cher.

R. sylvularum Rip.- Haies, buissons.- H.-Sav. : Thonon, Pringy.-

S.-et-L. : Châlons-s.-S. - Is. : vallon de J.-J. Rousseau près Seyssinet, Villard-de-Lans, Chalais. - Fribourg à Montboron, Jura bâlois, Cher.

R. insignis Désẻgl. et Rip. - Haies. - H.-Sav. : Annecy-le-Vieux. Is. : mont Rachais, le Sappey. - Sav. : Mẻry, près Chambéry.Rh. : Francheville, Chaponost.- Allier, Cher, Loir-et-Cher.

R. Chaboissxi Gren.-Haies, buissons.-H.-Sav. : Pringy, St-Martin.

- Jura: Salins. - Is. : Vernas, près Crémieu.-Rh. : St-Genisles-0llières. - Gard : le Vigan, Alais, - Cher, Vienne, SeineInfér., Meurthe, Allier, I.-Garonne, Bâle.

R. eriostyln lip. et Déségl. - Haies, bois. - H.-Sav. : Brenthonne, Habẻre-Lullin, St-Martin, Pringy. - H.-Garonne.

R. curticola Puget.- Haies à Pringy (H.-Sav.). 
R. stenocarpa Déségl. - Haies. - H.-Sav. : Annecy-le-Vieux. - Fribourg à Montbovon.

R. villosiuscula Rip. - Haies. - Valais : Binn, Conches, la Ravoire-deMartigny. - Cher.-Fribourg.

R. stephanocarpa Déségl. et Rip. - Haies. - Is. : Villard-de-Lans, Rh. : Tassin. - Cher.

R. adscita Déségl.-Haies._Gap.-Chapelle-St-Ursin (Cher),_- Puy* de-Dome : Durtol, Royat.

R. macrocarpa Mérat. - H.-Sav. : Annecy-le-Vieux. - Is. : Chamechaude. - Cher. - Schaffouse.

R. biserrata Mérat.- Haies, buissons.-Doubs : Pontarlier.-H.-Sav.:

Salève, Habère-Lullin, Veyrier près Annecy.- Loire : St-Pauld'Uzor.- Allier, Puy-de-Dóme.- B.-du-Rh. : St-Paul-de-Roquefavour.

R. sphaeroidea Rip.- Haies, bois.-H.-Sav. : Thonon.- Cher.

$R$. brachypoda Déségl. et Rip. - Cette forme, trouvẻe dans le Cher, n'a pas encore été signalée dans notre bassin.

S 3. Hispidze. Pétioles glabres, glanduleux; feuilles glabres, simplement ou doublement dentées; pédoncule et tubo du calice plus ou moins hispides-glanduleux.

I. andegavemals Bast. - Haies, bois. - Assez commun dans la chaine jurassique, C.-d'Or, S.-et-L., Loire et le centre de la France.- Valais et Fribourg : Bovernier et Montbovon.-Env. de Genève à Compesières. - Commun dans H.-Sav. autour de Thonon; Habère-Poche, Vailly, plateau des Gets, mont Laouet, Śamoëns, Salenove, vallée de Verrerie de Thorens, vallée de Les. chaux, envir. d'Annecy, Parmélan, Chamonix. - Sav. : mont du Chat. - Rh. : Tassin, Charbonnières, Limonest, Chaponost, Vaugneray, St-Lager-en-Beaujolais.- Is. : Crémieu, Pariset, lo Sappey, forêt de Portes. - H.-Alp. : envir. de Gap, côte Gélive, col Bayard, Ange-Roubaud, la Freissinouse. - Hẻr. : Pic StLoup.-Commun dans H.-Gar., Tarn et tout le centre de la France, puis dans Cher, Loiret, Maine-et-Loire, Loire-Infér., Vosges.-Les quatre formes suivantes sont peut-être de simples variêtés du $R$. andegavensis.

R. agraria Rip - Envir. de Besançon, de Lyon à Craponne, Francheville, mont Verdun, de Gap, de Thonon, Clermont, Bourges. 
R. Lemailrei Rip.-Haies.-Annecy-le-Vieux.- Tassin près Lyon.-

Loire : Pelussin.- Freissinouse près Gap. - Cher.

R. condensata Pug.-Broussailles. - H.-Sav. : Habère-Poche, HabèreLullin.

R. purpurascens Rip. - H.-Sav. et Sav. : Hubère-Lullin, Bellevaux, mont de Veyrier, Moutiers.

R. vinealis Rip.-Haies.-Is.: Villard-de-Lans.-Doubs : Pontarlier.-

Valais: Bovernier.- H.-Sav. : Monetier au pied du Salève, Habere-Poche, Grand-Bornant.-H.-Alp. : Manse et Puy-Monbard près Gap.-Fribourg à Montbovon; Cher, Loir-et-Cher.

R. Verloti Crépin. - Is. : le Sappey.

R. Suberti Rip. - Haies. - Doubs : Pontarlier. - Valais : Bovernier.

H.-Sav. : Cruseilles, Annecy: - H.-Alp. : Gap. - Rh. : Charbonnières, Craponne.- Loire : Pelussin. - Cher. - Bâle.

R. Rousselii Rip. - Haies. - Valais : Sembrancher, Bioley. - Is. : le

Sappey, la Ferrière-d'Allevard.- Gard : Aumessas.-- Cher.

R. Kosinscyana Bess. - Haies, bois. - Besançon, au mont Rosemont;

Genève à Carouge; Parpas près Autun.-H.-Sav. : Bellevaux.-

Rh. : Francheville et Charbonnières.-Is. : le Sappey. - Alp.-

Mar. : N.-D, de Fenestre. - Puy-de-Dome, Allier, Cher, Loiret, Maine-et-Loire.

R. firma Puget.-Jura : Salins.-H.-Sav. : Bellevaux, Reyvroz.-Is.:

Villard-de-Lans.

R. aspernata Déségl. - Is. : le Sappey, Villard-de-Lans. - Niêvre.

R. verticillacantha Merat.-Valais : les Valettes.-H.-Sav. : St-Martin.

- Puy-de-Dôme, Allier, Creuse, Loire.

$R$. inconspicua Déségl. - Haies. - S.-et-L. : Chagny. - Cher, Creuse,

Loir-et-Cher, Indre.

R. Acharii Bilberg. - Valais : la Crettaz, Gabioud, Sembrancher. Rh. : Charbonnières. - Tarn.

R. Chaberti Déségl. - Haies. - Rh. : Craponne, Sainte-Consorce, Dardilly.

R. Timeroyi Chabert. - Haies. - Envir. de Lyon à Francheville, Poleymieu, la Pap̣e.-Puy-de-Dóme : Fontanat près Clermont.R. haberiana Puget.- Haies. - H.-Sav. : Habère-Lullin, Habère-Poche. - Doubs : Pontarlier. - Valais: Sembrancher.

R. transmota Grépin.-H.-Sav. : Bellevaux. - Maine-et-Loire. 
R. psilophylla Rau. - Haies. - Envir. de Genève à Compesières, Onex, Lancy.—Puy-de-Dôme : bois de Lezoux.

R. Aunieri Cariot.- Haies. - Rh. : Francheville, Tassin, Craponne.Puy-de-Dôme.

R. Laggeri Puget. - Les Cases d'Allières et la Tine près Montbovon (Fribourg).

R. Leveilløi Boullu. - Haies, - Rh. : Francheville, Sainte-Consorce.

R. scotophylla Boullu. - Haies. - Rh. : Charbonnières, Saint-Genisles-0llières.

1. Pouzini Tratt. - Broussailles de la région mérid.-B.-du-Rh.: envir. de Marseille. - Var : bois des Maures aux Escarcets, Cannet du Luc. - Commun dans l'Hér.: Vic, Valflaunès, SaintMartin-de-Londres, Montarnaud, Avène, Saint-Étienne-de-llursan, Portiragnes, Béziers, Saint-Étienne-de-Gourgas, Lunas, Saint-Martin-d'Orb, Pégayrolles-de-l'Escalette, Andabre-Rosis, Lamalou, le Larzac à Saint-Haurice. — Gard: Anduze, le Vigan. - Aude : Mas-Cabardès. - Remonte dans les H.-Alp. près de Gap à Rabou ; dans l'Is. à la Bastille de Grenoble, Corenc, Pariset, Engins, Vernas; dans le Rh. à Couzon et au mont Cindre.

$R$. leptoclada Boullu. - Forme du précédent à pédoncules presque lisses, styles velus, folioles glanduleuses sur les nervures; trouvée au Sappey près Grenoble.

R. Chavini Rapin.-Broussailles,-Valais : Bovernier au mont Clou. H.-Sav. : le Salève à la base de la Grande-Gorge, la Croisette.Is. : le Sappey, mont Saint-Martin au-dessus du Chevalon. H.-Alp. : envir. de Gap à Sauveterre, Rabou, Chaudun.

R. Wolfii de la Soic.-Broussailles, - Valais : Bovernier.

R. Martini Gren. - Gard : Auriat près Aumessas, le Vigan. - Is. : Villard-de-Lans à la Fauge.- Lozère : Bagnols les-Bains.

54. Pubescentes. Pétioles velus ou tomenteux; folioles simplement ou rarement bidentées, plus ou moins velues sur les deux faces ou simplement sur la nervure médiane; pédoncule lisse; styles velus, hérissés ou glabres.

R. dumetorum Thuill. - Haies, bois. - Ghaine jurassique où il est assez répandu. - S.-et-L. : Autun. - Envir, de Genève à Compesicres. - Valais : Saillon, Bovernier, les Valettes, Saint- 
Leonhard, vallée d'Anniviers. - II.-Sav. : Salève, Voirons, mont de Sion, la Roche, Vacheresse, Vailly, Arentlıon, Haberre-Poche, Saint-Jean-d'Aulph, Bonnevaux, Annecy-le-Yicux, Bluffy, les Espagnoux, la Puya et Argonex prìs Annecy, Chamonix. - Is. : mont Rachais, le Sappey, Chalais, Pariset, Saint-Christophe en Oisans, - II.-Alp. : Charance, Devez-de-Rabou, mont Bayard. - Rh. : Tassin, Dardilly, Charbonnières. - Ard. : Privas. Hér. : Pic Saint-Loup, Saint-Maurice sur le Larzac. - Aude : Mas-Cabardès. - Alp.-Mar. : Saint-Martin de Lantosque. B.-du-Rh., Tarn, Puy-de-Dòme, Allier, Cher, Corrèze, Maineet-L., Loiret, Loir-et-Cher.

R. oblusifolia Desv. - Haies. - Doulss : Besançon. - S.-et.L. : Parpas, Ornée près Autun. - Valais: Sembrancher, la Crettaz, Gabioud. - H.-Sav. : Thonon, Habère-Lullin, Parmélan. - Sav. : SaintInnocent. - Rh. : Pont-d'Alaï, Charbonnières, Dardilly, Chaponost, Brindas, Corcelles. - H.-Alp. : vallon de Glaise, PuyIanse près Gap, route de Veynes, - B.-du-Rh. : Aix. - Alp.Mar. : bois de Gourdon, Saint-Martin-Lantosque. - Var. : Campiès. - Gard : Anduze. - Hẻr. : Avène-les-Bains. - Loire : Chalmazelle. - H..Gar., Puy-de-Dôme, Corrèze, Cher, Loiret, Vienne, Alsace. - Scliaffhouse.

R. urbica Lem. - Haies, bois. - Région des sapins de la chaine jurassique ; descend jusque près de Salins. - Ain : montagnes d'Hauteville. - Valais : Sembrancher, Bovernier, Sion.II.-Sav. : bords de la Dranse près Thonon, Ripaille, Arenthon, Ilabère-Lullin, la Rocłı, Annecy, Veyrier, Pringy, Collonges, Alex dans la vallée du Fier, mont de Sion, Salève au-dessus d'Archamp. - Sav. : Nargériaz, Saint-Innocent, le Bourget, Beaufort, Moutiers. - S.-et-L. : Autun, Châlons. - Rh. Is. : mont Rachais, Sappey, Chlalais, Pariset, Crẻmieu.-H.-Alp. : Charance, mont Bayard, Romette. - Gard : Anduze, le Vigan. - Puy-de-Dome, Allier, Nièvre, Cher, Maine-et Loire, Loiret.

R. ramealis Puget. - Haies. - Valais : Bovernier, Mayens de Sion. II.-Sav. : Monêtier, Thonon, Saint-Jean-de-Sixt, vallée de Leschaux.

R. semiglabra Rip. - IIaies, - Valais : Bagnes à Lourtier. - H.-Sav. : Catal. Bassin du Rhône. 
Pringy. - Rh. : Oullins, Tassin, Charbonnières. - Alp.-Mar. :

Saint-Martin-de-Lantosque. - Bâle. - Cher.

R. hemilrichu Rip.-Haies. - Valais : Ravoire, Binn, Conches. - Is. :

Charvieu. - Creuse. - Bâle.

R. trichoneura Rip. - Haies. - Valais : Bovernier, le Brocard. -

H.-Sav, : Pringy, Thonon. - Rh. : Tassin. - Pyr.-Or. : mont Louis, - Cher.

R. obscura Puget. - Haies. - H.-Sav. : Salẻve, Pringy, Argonnex, la Caille, Annecy-le-Vieux, les Espagnoux près Annecy, - Is. : Vernas près Crẻmieu. - Schaffouse.

R. globata Déségl. - Broussailles. - II.-Sav, : montagne de Veyrier

près Annecy. - Sav. : Méry, Mfoutiers. - Valais et Fribourg: col de Salvan, Montbovon.

R. platyphylla Rau. - Haies, buissons. - Région dès sapins du Doubs et Jura. - Valais : Bovernier, Champé, val Ferret, Fully. II.-Sav. : bords de la Dranse près Thonon, Habère-Lullin, Habère-Poche, Vacheresse, Bonnevaux, vallée de la Verrerie de Thorens, la Caille, Parmélan. - Sav. : Moutiers. - Is. : forêt de Portes, le Sappey. - H.-Alp. : Gap à Puy-IIanse, bois de l'Aubrie au mont Bayard. - Rh.: Francheville, Tassin, Charbonnières, Corcelles. - Hẻr. : Montpellier, Pic Saint-Loup, Saint-Étienne de Mursan.-Puy-de-Dome, Allier, Yonne, Cher.

- Fribourg à Montbovon.

R. platyphylloides Déség. et Rip.- Haies, - S.-et-L. : Châlons-S.-S. Valais : Ravoire, Bovernier. - H.-Sav. : Pringy, Saint-Martin près Annecy, Brenthonne, Thonon. - Rh. : Chaponost, Charbonnières. - Puy-de-Dôme; Cher.

R. implexa Gren. - Doubs: Pontarlier. - Is. : Saint-Christophe en Oisans. - Bâle.

$R$. jactata Déségl. - IIaies, bois. - H.-Sav. : Habẻre-Lullin, HabèrePoche, Saint-Germain-sur-Talloires. - Sav. : Puy-Gros près Chambéry. - Is. : forêt de Portes.

R. sphorocarpa Puget. - Haies. - H.-Sav. : Habère-Lullin, HabèrePoche. - Sav. : Hautecombe près Aix. - Is. : la Salette.

R. coriifolia Fries. - Broussailles. - Région des sapins du Doubs et Jura. - Valais : Salvan, Bovernier, les Valettes, Champé, val 
Ferret, Mayens de Sion, Saint-Nicolas, Randa. - II.-Sav. : Salève en montant aux Treize-Arbres, près de la Croisette, audessus d'Archamp, Voirons, Chartreuse de Pommier, mont de Sion, bains de la Caille, Saint-Jean-d'Aulph, Reyvroz, HabèrePoche, Chenaillon au-dessus du Grand-Bornant, mont Châtillon, le Fernouy dans la valléc de Thónes, les Clefs, mont Charvin, la Clusaz, Mégève, Chamonix. - Sav. : montagnes d'Hauteluce, Margériaz. - Is. : le Sappey, Villard-de-Lans. - H.-Alp. : la Grave, Villard-d'Arène, Lautaret, Devez de Rabou, col Bayard, Saint-Laurent-de-Cros en Champsaur. - Rh. : Tassin, Francheville, Craponne, Chaponost. - Loire : Chalmazelle. - Gard : le Vigan. - Pyr.-Or. : valléc d'Eyne. - H.-Garonne, Cantal, Puyde-Dôme, Lozère.

5. Collines. Pétioles velus ou tomenteux; folioles plus ou moins pubescentes, simplement ou bidentées ; pédoncule hispide-glanduleux ainsi que le tube du calice.

T. collina Jacq. - Haies, bois. - Ain : Muzin près Belley. - Rh. :

Point-du-Jour, Tassin, Craponne, Charbonnières, Vaugneray.Genève à Carouge. - Sav. : Saint-Jean-de-la-Porte, bois Champion pre̊s Mloutiers. - H.-Sav. : Allonzier. - H.-Alp. : mont Bayard. - Yonne, Cher, mont Dore, Cantal.

R. corymbifera Borkh. - Haies, buissons. - H.-Sav. : Thonon. Rh.: Beaunant, Craponne, Charbonnicres, la Tour-de-Salvagny. - Var : le Luc. - Cher.

R. Deseglisei Bor. - Haies, bois. - Doubs : Besançon. - S.et-L. : Saint-Fargeot près Autun, Saint-Léger-du-Bois, Chalons.-Rh.: Tassin, Francheville, Charbonnières, Dardilly, Chaponost, Valais : Anniviers. - II.-Sav. : Thonon, Arenthon, vallée de la Verrerie de Thorens, la Roche, Parmélan, la Caille, la Puya et Argonnex près Annecy, Pringy, Salève. - Is. : Chalais, CôteSaint-André. - H.-Alp. : col Bayard. - Sav. : Houtiers, Cher. - Alsace. - Bâle.

R. hispidula Rip. - Bois. - I.-Sav. : Habère-Lullin. - Valais : Mayens de Sion.

$R$. trichoidea Rip. - Valais : Lourtiers de Bagnes. - Sav. : Salins.

$R$. imitata Déségl. - Haies, bois. - Doubs: Besançon, - Valais : 
Fins-Hauts, Valorsine.-II.-Sav. : Ilabère-Poche, Saint-Gervais.

- Rh. : Tassin, Francheville. - Is. : Allevard, Charvieu. Sav. : Macot._Ain : Priay._Fribourg à Montbovon.-Cher.

R. bellavalis Puget. - Buissons des montagnes. - Valais : Bovernier au Quercelin. - H.-Sav.: Bellevaux, col de Terramont près Vailly, Argentière, Saint-Gervais. - Sav. : forêt d'Apremont près Chambẻry. - Is. : Saint-Christophe en Oisans.- H.-Alp. : col Bayard, Saint-Laurent-du-Cros. - H.-Pyr. à Gèdre.

R. approximata Déségl. - Broussailles du plateau de Mirabelle près

Riom. - Chercher cette forme dans notre bassin.

R. fallaciosa Déségl. - Ifaies. - Rh. : Sainte-Consorce. - Maine-et-L. $R$. cinerea Rapin. - Broussailles du mont Salève.

R. bovernieriana Lag. et de la Soie,-Valais: col de la Forclaz, Bovernier. - Is. : Saint-Christophe en Oisans, - H.-Pyr. : Gèdre. R. Fricdlanderiana Besser. - IIaies, bois. - Rh. : Charbonnières, Craponne, Francheville, Tassin, Chaponost, Alix. - Cher; Maine-et-Loire.

R. macruntha Desportes. - Haies. - Rh. : Charbonnières. - Loiret; Maine-et-Loire.

I6. alba L. - Cultivé dans les jardins d'oủ il s'échappe quelquefois.

Sect. X. EGLANTERI.E. Fouilles pubescentes et glanduleuses en dossous; aiguillons des tiges droits, ceux des rameaux grands et crochus; fleurs sans bractées; zépalos divergents ; styles velus ; fleurs d'un jaune vif.

1R. Iutea Dalechamp Miller R. cglanteria L. - Souvent cultivẻ dans les jardins.

Plusieurs auteurs assurent l'avoir vu à l'état subspontané.

Sect. XI. RUBIGINOSE. folioles couvertes on dessous de glandes sessiles entro les nervures.

\$1. Tomentelle. Folioles doublement dentées, plus ou moins pubcscentes, à glandes peu abondantes et faiblement ou point odorantes; pédoncules glabres ou hispides glanduleux.

R. tomextella Lem.-Haies, bois.-Chaîne jurassiqque dans la région des vignes et des basses montagnes, mont Brégille près Besançon. - Valais : Sembrancher, Mayens de Sion. - Envir. de Genève au Petit-Lancy.-H.Sar. : Salève, Archamp, Thonon, 
Arenthon, mont de Sion, la Roche, vallée de la Verrerie de Thorens, Parmélan, Brizon, Pringy, Veyrier, Annecy. - Sav. : Conflans. - S.-et-L. : Chalons-S.-S. - Is. : Chalais, Bastille de Grenoble, entre Pariset et Saint-Nizier, la Côte-Saint-André. H.-Alp. : envir. de Gap à Rabou, Romette, route de Veynes, Manteyer. - Rh. : Tassin, Charbonnières, Dardilly, Couzon et Saint-Romain-au-Hont-d'Or. - Var: le Luc. - Ard. : Annonay. - Ilér. : Montpellier vers l'arqueduc, Saint-Barthélemy, Caunelle, Villeneuve, Grabels, Bẻziers. - Aude : Alzonne. Commun dans le centre de la France : Puy-de-Dôme, Allier, Cher, Loir-et-Cher, Indre, Maine-et-Loire. - Aisne, Meurthe.

- Lot-et-Gar. - Schaffouse, Uri, Tessin.

2i. similata Puget - Buissons. - Envir. de Genève. - Valais : les

Iles près de la Dranse, Bovernier. - H.-Sav. : Salève, Arenthon, Pringy, - Sav. : Conflans, l'Arpettaz au-dessus d'Iléry, SaintNicolas-de-la-Chapelle dans la vallée de l'Arly. - II.-Alp. : envir. de Gap. - Is. : la Salette, Villard-de-Lans. — Gard : le Vigan. - Lozère: Bagnols-les-Bains.

R. ralesiaca Lagg. et Pug. - Buissons. - Valais : Vollege, Bovernier,

Sion.

R. nebrodensis Guss.-Ain : Thoiry en montant au Reculct.-II.-Sav. :

Argentière.

Var. subintrans Gren. à styles et divisions calicinales glabres. - H.-Alp. : Gap. - Gard : le Vigan. - H.-Gar.

R. riscida Pug. - Buissons. - H.-Sav. : Cruseilles, envir. de Thonon, Chavanoz, Annecy. - Jura : Lons-le-Saunier, colline de l'Ermitage. - Alp.-Mar. : de Roccabigliere à Bertemont.

R. Blondceana Rip. - IIaies, bois. - Doubs : envir. de Besançon. Valais: la Ravoire de Martigny.-Environs de Genève.-Doubs : Besançon.-Aude : Has-Cabardès.-Puy-de-Dôme, Allier, Cher, Loiret, Loir-et-Cher.

§ 2. Glanduros $x$. Aiguillons droits, inclinés ou crochus, quel. quefois transformés on aiguillons sétacés ou glanduloux; feuilles grandes à glandes peu odorantes; pédoncules hispides glanduleux; corolle grande.

R. Irachyphylla Rau. - Ilaies et bois. - Doubs : Pontarlier. - 
Valais : Saillon, vallée d'Anniviers. - H.-Alp. : envir. de Gap. - Rh. : d'Écully à Charbonnières. - Tarn, H.-Garonne. Sclaffouse. - Loire : Champdieu, la Fouillouse, mont d'Uzor, Saint-Rambert.

R. insidiosa Rip. - IIaies dans le département du Cher; n'est pas signalé dans notre bassin non plus que $R$. dryadea Rip. et R. protea Rip., trouvés dans les mêmes localité du Cher.

$R$. consanguinea Gren. $R$. gallico-umbellata Rap. - Haies de Veyrier près Genève.

R. Godeti Gren. - Cette forme trouvée dans le Jura bâlois et neuchâtelois, ainsi que dans la Meurthe et l'Alsace, n’a pas étẻ signalée dans notre bassin.

R. Cotteti Pug. - Broussailles. - Vaud et Fribourg : vallẻe de l'Hongrin, Cases-d'Allières.

R. marginata Wallr.-IIaies. - H.Sav. : Salève au-dessus d'Archamp, Grand-Bornant, la Clusaz. - Doubs: Pontarlier, chemin de Charpillot. - Fribourg : Comballaz près Montbovon.

R. subolida Déségl. - Bois, haies. - S.-et-L. : Givry, Louhans. Rh. : Charbonnières, Sainte-Consorce, Vaugneray, Tassin, SaintLager en Beaujolais.

R. Pugcti Bar. - Haies, bois. - HI.-Sav. : bois du Barioz près Pringy, bois de Tessy sur Epangy. - Envir. de Genève à Meyrin, bois de Feuillasse.-Rh. : Tassin, Chaponost, d'Écully à Charbonnières.

- Ain : la Papa.-Is. : Décines, Veyssilieux.-Ard. : Annonay.

- II.-Garonne.

R. nemorivaga Déségl. - Bois. - Rh. : Charbonnières au bois de l'Êtoile, Chaponost, Craponne, Brignais. - H.-Gar., Cher.

Ii. Nexuosa Rau. - Haies, bois. - Rh. : Écully, Beaunant, Charbonnières, Dardilly, Couzon, Villié. - Ain : la Pape, Reyrieu, Garnerans. - Cher; Vosges. - Loire : Iontbrison, SaintJodard.

R. pscudo fexuosa Ozanon. - Haies. - Rh. : entre Tassin et Charbonnières au Chapoly, Marcy.

R. speciosa Déségl. - Haies. - Rh. : Tassin à l'Aigua, Charbonnières, Saint-Genis-les 0llières. - Cher.

R. Jundzilliana Besser. - Haies, bois. - Rh. : Pont-d'Alaï, Charbon- 
nieres, Saint-Lager en Beaujolais. - Puy-de-Dóme à Combronde. -Vosges; Loir-ct-Cher; Cher; Tarn. - Schaffouse.

S3. Psevdo rubianosie. Fouilles chargées on dessous de glandes très-odorantes; styles et pédoncules non glanduleux, glabres, (sauf dans le R. Billetii, qui les a velus).

R. sepium Thuill - Haies, bois. - Commun dans S.-ct-L., Loire, Rhône, Ardiche, Gard, Ain, Doubs, Jura, surtout dans la Bresse. - Valais : Bovernier, Sembrancher.-H.-Sav. : Salève au-dessus d'Archamp, Lully, Ripaille, Salenoves, vallée de la Verrerie de Thorens, envir. d'Annecy, Parmẻlan, les Clefs. - Sav. : env. d'Aix el de Montmélian. - Is. : Crẻmieu, la Côte-Saint-André, Saint-Eynard, Bastille de Grenoble, Tour-sans-Yenin, entre Pariset et Saint-Nizier, Uriage.- II.-Alp. : envir. de Gap, Charance, mont Bayard. - Alp.-Mar. : Antibes, Nice, Menton. Var : le Luc.-Aude : le Haut-Donézan, les Corbières. - Commun dans le centre de la France.

Une variété pubescens Rapin, dont les folioles sont pubéruIentes en dessous, ainsi que le pétiole, a été trouvé à la base du Salève, à Montmélian (Savoic), à Ebreuil, dans l'Allier, et dans le Jura bâlois.

$R$, agrestis Sav. - Diffère du précédent par des feuilles plus petites et une végétation plus grêle. Beaucoup moins commun.-S.-et-L. : envir. d'Autun et de Châlon. - Valais : Bovernier. - Sav. : Clambéry, - Rh. : Chaponost. - Is. : Bastille de Grenoble. Gard : Anduze. - Var et Alp.-NIar. : le Luc, Antibes.- Lozère, Allier, H.-Garonne.

R. mentita Déségl. - Haies, buissons. - H.-Sav. : grèves du Léman près Thonon, à Ripaille. - H.-Alp. : envir. de Gap. - Puy-deDôme à Saint-Nectaire.

R. arvalica Puget. - Buissons. - Valais : les lles, la Ravoirc. - H.Sav. : mont de Sion, Thonon, Lully, le Malvériaz et la Margériaz près Annecy, Parmélan.- Sav. : lléry près Aix, Salins. Rh. : Tassin, Craponne, Charbonnières, entre Montmélas et Blacé. - H.-Alp. : la Garde, Riotors, - Hér. : Montpellier près de l'Aqueduc.

R. virgultorum Rip. - IIaies, bois. - Valais : les Iles, la Ravoire, la 
Batiaz près Martigny. - H.-Sav. : envir. de Thonon à Ripaille, de Thuet au Brizon.-S.-et-L. : Chalons-sur-Saône.-Is. : Crě́mieu, Bastille de Grenoble, le Sappey.- II.-Alp. : Romette près Gap.-Rh. : mont Thoux, Charbonnières, Saint-Lager en Beaujolais. - Alp.-Mar. : Antibes.-Allier, Cher.

R. Billctii Puget.-Coteaux arides. - Valais : Saillon, Saint-Léonhard.

- H.-Sav. : la Puya près Annecy. - Sav. : Salins près Moutiers.

- Is. : Engins. - H.-Alp. : envir. de Gap.- H.-Gar., vers Toulouse.

$R$. Seraphini Viv.-Alp.-IIar. : St-Sauveur.

R. cheriensis Déségl._IIaies. - Valais : ban de Bovernier.-H.-Sav. :

Saint-Gervais-les-Bains. - Is, : la Salette.-Rh. : Tassin, mont

Thoux, Montmélas. - H.-Alp. : entre Gap et le col Bayard, la Garde, Rabou, Charance, Séuse. - Cher. - Fribourg : Montbovon.

R. lugdunensis Déségl. - Ilaies, lieux incultes. - Valais : Saillon, Sion, Sembrancher, Bovernier. - H.-Sav. : Arenthon, envir. d'Annecy, la Puya, Leschaux. - Rh. : le mont d'Or, Cogny, Pommiers, Tassin, de Villeurbanne au Mollard vers Pierre-Frite. Is. : Villard-de-Lans, la Salette, Crémicu au mont d'Annoisin. II.-Alp. : envir. de Gap. - Sav. : Salins près Moutiers, envir. de Chambéry, Bellecombette. - Ard. : la Louvesc.

Var. macrocarpa.-Rh.: entre Champagne et Dardilly, Couzon au mont d'Or. - Is. : St-Nizier.- Valais : Bovernier.

R. Jordani Dése̊gl. - Haies. - Envir. de Genève. - H.-Sav. : SaintGervais.-Is. : Villard de Lans, Saint-Nizier. - H.-Alp. : env. de Gap, mont Bayard, Puy-Montbard, Matachard au pied du mont Aurouse, Manteyer. - Alp. Nar. : Vence. - Yonne, Puyde-Dôme, Lozère.

R. Vaillantiana Bor, - Valais : les Iles, le Clou-sur-Bovernier, - H.Mlp. : envir. de Gap, col de Mause à Romette, Charance, chemin de Villarobert en montant à la route d'Orcières.-Sav. : Salins, 3) andugis Champion, collines de Chanaz et de Bellecombette près $\therefore$ willambery. 
5. Verfe rudigtios $x$. Feuillos couvertes on dessous de glandes odorantos; aiguillons robustes, crochus, quelquefois transformés on aiguillons sétacés et glanduleux; pédoncules hispides; stylos velus ou glabres.

18. Nubiginosa L. $-R$. apricorum Rip. - Ilaics. - Assez, commun dans les Vosges, arrond. de Belfort, II.-Saône, région du vignoble et des moyennes montagnes de la chaine jurassique. Valais : Fins-Hauts, Bovernier, Saillon. - H.-Sav. : Brogny près Annecy, Mégève, Chamonix. - Sav. - Rh. : Francheville, Craponne, Yzeron, Anse, Gleize, Corcelles,- Commun dans Is., II.-Alp., B.-Alp., Ard., envir, de Privas. - B.-du-Rh. : SainteVictoire. -Gard.-IIér. : Montpellier vers la route de Laverune et le chemin de Saint-Georges. - Aude et Pyr.-Or. : Narbonne, Saint-Intoine-de Galamus, plateau de Boucheville, Saint-Laurent-de-Cerdans, Costujes, Banyuls-sur-Mer. - Commun dans Pyr. centr., Puy-de-Dôme.

Espice polymorphe considérie par plusieurs botanistes comme un groupe d'espèces distinctes.

r. comosa Rip.- Iraies. - Forme du précédent à fruits plus gros, plus ovoïdes, couronnés presque jusqu'à la maturité par les sépales persistants. - Plusieurs des localite̊s ci-dessus mentionnées se rapportent à cette forme. - Valais : les Iles, la Ravoire, Lourtier, Chemin. - H.-Sav. : Salève au-dessus d'Archamp, Lully, Pringy. - Rh.: Tassin, Saint-Genis-les-0llières, Sainte-Consorce, Charbonnières, Vaugneray.-Sav. : Brides, cntre Chanaz et les Charmettes près Chambéry. - Is. et H.-Alp. : Crémieu, Villard-de-Lans, envir. de Gap, mont Bayard, Charance, Romette.-Ard. : Annonay.-Hér. : St-Aaurice. - Loire : mont Sémioure près Montbrison. - Puy-de-Dòme, Cher, Loiret, Vosges.

Le $R$. comosa est celui qui porte le nom de $R$, rubiginosa dans l'herbier de Linné.

r. umbellata Lcers. - Ilaies. - Fl. en corymbe fourni, fruit non couronné à la maturité par les sépales, folioles à face infér. couvertes de glandes pellucides, très odorantes. - Valais : Bovernier. - H.-Sav. : Salève au-dessus d'Archamp, Chamonix. - 
S.-et-L., Rh., Ard., H.-Alp. : Brutinel près Gap, Saint-Laurentdu-Cros. - Hér. : le Caylar, Andabre, Rosis, Lunas, SaintMaurice. - Loire : Montbrison.—Puy-de-Dôme, Tarn, H.-Gar., Cher, Loiret, Maine-et-Loire.

R. permixta Déségl_- Haies.-C.-d'Or : Meursault.-S.-et-L. : Chalons-sur-S. - Valais : les Iles, la Batiaz, Salvan. - H.-Sav. : Saleve, Cruseilles, Thonon, Pringy, Annecy. - Rh.: Tassin, Chaponost. - Is. : Saint-Eynard.-H.-Alp. : Gap à Viliarabère, Malecombe.-Ard. : Annonay.-Aude : Mas-Cabardès. - Puyde-Dôme, Allier, Cher, Loiret, H.-Vienne, Maine-et-Loire, Tarnet-Gar., H.-Gar., H.-Pyr.

R. septicola Déségl.- Haies..- S.-et-L. : Autun, Broye, Dracy-le-Fort près Chalons. - Valais : les Iles, la Ravoire, les Grangettes. II.-Sav, : envir. de Thonon et d'Annecy, la Puya, Pringy. Rh. : Tassin, Chaponost, Graponne, Charbonnières. - Is. : Crémieu, Balmes viennoises près Décines, Pariset, la Salette. Vosges, Loiret, Creuse, H.-Gar.

$R$, operta Pugel.-II.-Sav. : environ de Thonon. - Rh. : entre Francheville et Chaponost. - Cher; Alsace.

R. echinocarpa Rip.-Haies.-Valais : Bovernier. - Sav. : Bellecombette près Chambéry, Salins près Moutiers. - Rh. : Charbonnières, Saint-Lager en Beaujolais. - Is. : Crémieu. - H.-Alp. : col Bayard. - Ard. : Annonay. - Cher, Loir-et-Cher, Lot-etGar., Lozère.- Bâle.

R. sylvicola Déségl. et Rip.- Bois, buissons. - Villarobert près Gap. Cher.

R. micrentha Smith. - Haies. - Coteaux pierreux. - Valais : Bovernier. - H.-Sav, : Salève au-dessus d'Archamp. — Sav. : Salins près Moutiers. - Ain : Reyrieu. - S.-et-L. : Autun, Broye. Rh. : Izeron, Corcelles. - H.-Alp. : de la Garde à Rabou, Romette, Sauveterre, Brutinel en Champsaur. - Gard : le Vigan. - Hér. : Hontpellier vers la route de Grabels, Caunelle, Lamalou, Lunas, Avène, Ceilhes, Pégayrolles-de-l'Escalette, la Sérane. - H.-Gar., H.-Pyr., Sarthe, Maine-et-L., Vosges.

R. diminula Bor. - Broussailles. - Jura : Lons-le-Saunier. - Rh. : Izeron. - Aude : Mfas-Cabardès, Carcassonne. - Puy-de-D., Cher, H.-Vienne.-Bâle. 
R. lactifora Déségl. - IIaies. - Rh. : Tassin, Craponnc, Chaponost, Ecully, mont Cindre, Couzon.

R. Lemanii Bor. - Coteaux pierreux, bois. - Doubs : Albenans, Cuze. - H.-Sav. : Ripaille près Thonon, Lully, les Balmettes pris Annecy. - Rlı. : Tassin, Charbonnieres, Vaugneray, Corcelles. - Is. : Balmes viennoises prís Décines, Pariset, Bastille de Grenoble, la Salette. - Sav. : envir. d'Aix et de Moutiers à Salins, entre Ifontagnole et les Charmettes près Chambéry - H.-Alp. : la Garde, Freissinouse. - Dr. : Réottier. - Alp.-Mar, : bois du Farghet, Levens, - Aude : les Corbières. - Vosges, Loiret, Cher, Allier, Maine-el-L., H.-Garonne.

r. Iadanifera Timb.-Lagr. - Bois de Balma près Toulouse. - Non encore signalé dans notre bassin.

R. subdola Dísígl. - R. Kluliii Bor.-Haies. - H.-Sav. : Thonon. Loiret, Cher, Yonne, Lot-et-Gar.

Le $R$. Klukii décrit par Sprengel et tous les auteurs qui l'ont suivi n'est pas le véritable $R$. Klukii Besser, lequel a été trouvé seulement en Crimée. Je dois faire remarquer ici que j'ai placé les $R$. ladanifera et subdolu dans la section des veroe rubiginose et non dans celle des pseudo rubiginosa, comme l'a fait M. Déséglise. Je me fonde sur ce que ces deux Rosiers ont les pédoncules hispides-glanduleux. Si l'on n'admet pas cette distinction essenticlle, il n'y a plus aucun motif pour séparer les pseudo rubiginose des verce rubiginose.

$R$. rotundifolia Rchb. - Haies. - Valais : les Iles, la Ravoire, Bovernier. - Sav. : Méry près Aix. - Joire : Chalmazelle. - Rh. : Francheville, Pont-d'Alaï.-Is. · Saint-Christophe-en-0isans.II.-Alp. : la Grave, Puy-Maure près Gap. - H.-Gar., Loiret. Loir-et-Cher.

La plante de Saint-Christophe-en-0isans et de la Grave se distingue du type par ses pédoncules lisses. M. Boullu l'appelle R. ucenensis, c'est-à-dire Rose de l'Oisans. 
Sect. XII. TOMENTOSE. Aiguillons gréles, droits ou pen arqués; folioles grisâtres, pubescentos sur les deux faces, glanduleuses ou non; pédoncules hispides-glanduleux; fleur blanchâtro ou d'un rose pâlo.

\section{S 1. VERA TOMENTOSF.}

A. Folioles toutes simplement dentées.

Fe. einerssceus Dumort.- Haies, bois.- II.-Sav. : mont de Sion.

S.-et-L. : Autun. - Rh. : Tassin, Craponne, Saint-Genis-lesOllières, Marcy, Charbonnières, Limonest. - Ain : Villette. Is. : les Coltaves près la forêt de Portes.-Alsace, Cher, Sarthe, H.-Gar., H.-Vienne, Deux-Sèvres.

R. dlımosa Pugel.- Haies, bois. - H.-Sav. : Salève à Archamp, Bellevaux, Grand-Bornant, Charvin, montagnes de Samoëns, plateau des Gets, la Puya près Annecy. - Sav. : mont Margériaz. Rh. : Tassin à l'Aigua, Saint-Genis-les-0llières. - Loire : la Valla.

R. micrns Déségl. R. velutina Chabert, - Haies. - II.-Sav. : HabẻreLullin.-Ain : Villette.-Rh. : Charbonnières, Tassin:-Cher.

- Fribourg à Châtel-sur-Montsalvens.

R. marcyana Boullu.-Haies.- Rh. : Marcy, Tassin à Méginant.

R. farimulenta Crépin.- Haies. - Valais : Salvan, mont Clou sur Bovernier.-II.AIp. : Briançon. - Rh. : Saint-Genis-les-0llières. - Vosges.

B. Feuilles doublement dentées.

† Folioles à glandes éparses en dessous ou à nervures secondaires velues.

ER. Fotha Bast. - Haies. - Is. : Saint-Romain près Crémieu. Maine-et-L., Vendée, Calvados, Mayenne.

R. spiuulifolia Dematra. - Bois taillis des montagnes. - Chaîne du Jura helvétique et français, cantons de Bâle, de Neuchâtel au Chaumont el à Lignières, Pontarlier, la Faucille, Gex, Hauteville en Bugey. - H.-Sav. : Salève entre Archamp et Collonge, sous les Pitons, les Voirons, Benthomme à l'Offiége. - Sav. : mont Margíriaz, Thoiry, Aix-les-Bains. - Is. : Saint-Eynard, la Ferrière-d'Allevard.-Fribourg à Châtel-sur-Ilontsalvens, localité 
où l'espice fut découverte par Dematra, et oì M. Déséglise a trouves les formes suivantes: grandifolia, glabrescens, villosula, ambigua, glabrala, hispidella.

12. vestita Godet. - Bois : Jura neuchâtelois à Lignières. - H.Sav. :

Salève en montant aux Treize-Arbres, au-dessus d'Archamp et des Bossey, les Voirons au-dessus du chalet Baumann, Reyvroz, Allonzier. - Sav. : mont Nivolet. - Is. : Saint-Romain près Crémieu.

R. abielina Gren.- La Fresse près Pontarlier.

R. cuspidatoides Crépin.-IIaies, bois.- II.-Sav. : Pringy, Argonnex.

- Valais : Bovernier, - Doubs : mont Brégille près Besançon.

- S.-ct-L. : Parpas près Autun.-Rh. : Francheville, Charbonnières.- Cher, Calvados.-Berne, Bâle, Schaffouse.

R. genevensis Puget. - Envir. de Genève à Onex, Pinchat. - Schaffouse.

\section{t† Folioles églanduleuses en dessous.}

IR. Tomentosa Sm. - Ilaies, bois. - Commun dans les vallées des Vosges et la plaine du Rhin. - H.-Saône: Vallerois-le-Bois, Vesoul, Arcey, IIéricourt, Chagey.-La Miotte et la Justice prés Belfort. - Plaine, vignoble et moyenne montagne du Doubs, Jura, Ain, H.-Sav., Sav., Dauphiné, B.-Alp.-Envir. de Genève à Onex. - Valais : Choex entre Vourry et Port-Valais, Leukerbad. - C.-d'Or : envir. de Saulieu. - S.-et-L. : la Chicolle. H.-Sav. : Salève, Manigod, les Clefs, Ripaille près Thonon, Pringy, Arenthon, Allonzier. - Sav. : le mont du Chat et les Bauges, Saint-Cassin près Chambéry. - Is. : Crémieu, mont Rachais, Saint-Eynard, Saint-Nizier, Revel. - H.-Alp. : Chartreuse de Durbon. - Dr. : montagnes de Lus-la-Croix-Haute.B.-Alp. : montagnes de la vallée de l'Ubaye.-Gard, Pyr.-Or.Loire : Néronde, Nervieux, Saint-Just-sur-Loire, la Fouillouse, mont Sémioure, mont d'Uzor, forêt de Chorsain. - Tarn, Puyde-Dôme, Cantal, Allier, Cher, Aveyron, H.-Garonne, Maine-etLoire, Loiret, Aisne.- Fribourg à Charmey.

R. intromissa Crépin.-H.-Sav. : Salève. - Rh. : Francheville, Beaunant près des aqueducs, Craponne, Marcy.-H.-Loire : Cejssac. R. collivaga Cottet. - Haies à Montbovon près Fribourg. 
$R$ dimorpha Bess. - Haies. - Collines de la claîne jurassique notamment à Pontarlier, au mont d'Or et au mont Brégille. - S.-et-L. : Mâcon.-Rhı. : Craponne.- Puy-de-Dôme à Bussières. - Vosges à Bamont.

R. subglobosa Sm. - Haies, bois. - Basses montagnes et régions des sapins du Jura. - H.-Sav. : Salève au-dessus d'Archamp, SaintJulien, Collonges, les Voirons, Vailly, Habère-Lullin, Habère Poche, Vacheresse, la Roche, plateau des Gets, le Mole, Samoëns, Alex, le Parmélan, la Verrerie de Thorens, Veyrier près Annecy. - Sav. : montagnes de Beaufort et des Bauges. - S.-et-L. : envir. d'Autun, - Is. : Villard-de-Lans, Brié, forêt de Portes, Revel. - Rh. : Saint-Laurent-de-Vaux, Alix, Charbonnières, Saint-Genis-les-0llières, Tassin. - Alp.-Mar. : Breil, Colmiane près Saint-Martin. - Loire, Cher, Loir-et-Cher, H.-Vienne, Loiret, Vendẻe, Sarthe, Maine-et-L., Loire-Infér., Côtes-du-N., Vosges.

R. Iunoniensis Déségl. - Haies. - H.-Sav. : Tlıonon, Ripaille, Reyvroz.

$R$. confusa Puget. - H.-Sav. : Reyvroz, Habère-Lullin. - Is. : SaintRomain près Crémieu.

R. annesiensis Déségl. - Haies. - H.-Sav. : envir. d'Annecy à Sevrier, Pringy. - Sav. : mont Joigny près Chambéry.

R. Andrzeiowscii Besser. - Valais : Lourtier de Bagnes. - H.-Sav. : Salève, Bluffy, Habère-Poche, Habère-Lullin, Pringy. - S.et-L. : Chalons-sur-S. - Is. : Chalais, le Sappey, Villard-de-Lans. Rh. : Pont-d'Alaï, Écully, Chaponost. - Cher. - Puy-de-Dóme: près de Thiers.

S 2. Pomifere. Aiguilions grêles et droits; folioles tomenteuses, glanduleuses ou non; fleurs d'un rose vif; fruit gros rappelant la forme de la pomme.

R. mollis Smith. - Haies, bois, - Doubs : envir. de Pontarlier, val de Ruz, Vallorbe. - Jura vaudois et français, le Bolaz et Saint-Cergues, bois de Perrigny près Lons-le-Saunier, envir. de Salins et de Champagnole. - Envir. de Genève derrière le bois de la Bàtie, Lancy, Onex. - Valais : Zermatt, Obergestelen. II.-Sav. : Salève, Voirons, Chartreuse de Pommier, Saint-Nartin, 
bois du Barioz près Annecy, - S.-et-L. : Saint-Pierre près Autun. - Rh. : Francheville, Chasselay, Alix, Villié. - Is. : Crémieu. - Sav. : environ d'Aix et de Chambéry, Saint-Nicolasde-la-Chapelle. - Bâle. - Lozère. - H.-Loire, Puy-de-Dôme à Murol, mont Dore, Nièvre, Finistère.

R. omissa Déségl. - II.-Say. : Pringy, Tessy, Saint-Martin, Epagny, mont de Sion, Argonnex. - Is. : forêt de Portes, Villard-deLans.

R. resinosa Sternb. - Broussailles. - H.-Sav. : Chartreuse de Pommier, l'Argentière, Chamonix. - Loire : Pilat, Planfoy. - Is. : Villard-de-Lans, la Salette. - Chaine des Puy-de-Dóme et du mont Dore, Plomb du Cantal. - Il est possible que les localités indiquées pour le $R$. resinosa se rapportent au $R$. resinosoides.

$R$. resinosoides Crépin. - Broussailles. - Valais : Dixain de Conches, Bovernier. - Fribourg à la Tine près Montbovon. - H.-Sav. : Habère-Poche, mont de l'Offiẻge. - Rh.: Craponne, SaintGenis-les-Ollières, - Loire : Planfoy. - Vosges, Puy-deDôme.

R. Grenieri Déségl. - Broussailles. - Valais : Bovernier, le Clou, mont Catogne, Fins-Hauts, Salvan, Leuk, Dixain de Conches.H.-Sav. : Habère-Lullin, Reyvroz. - Is. : Saint-Chrìstophe en Oisans, Alpe du mont de Lans, vallon de Sarène près Huez.H.-Alp. : la Grave, Villard-d'Arène, Devez de Rabou, Loubet, cols de Glaise el de Bayard. - B.-Alp. : la Condamine. Pyr.-Or. : mont Louis près d'Eyne. - Lozère à Loubet.

R. pomifera Herm, - Vosges au Hohneck. - Sav. : mont Granier. - Valais : Zermatt, Dixain de Conches.-Pyr.-Or. : SaintMartin du Canigou.

R. friburgensis Lagger et Pug. - Pâturages de la Gotalaz près Fribourg.

R. recondita Puget. - Broussailles. - Valais : Dixain de Conches, Zermatt, le Borgeaux, val d'Hérémence, les Valettes, Bagnes, le Levron, la Forclaz, mont Arpile. - II.-Sav. : Reyvroz, plateau des Gets, montagnes de Samoëns et de Chamonix, l'Argentière, mont Charvin. - Is. : Villard-de-Lans, Saint-Christophe en Oisans. - Sav. : Saint-Nicolas-de-la-Chapelle, Hauteluce. - Alp.- 
Mar. : la Mairis près de Luceram, Colmiane. - Pyr.-Or. : Eynes. - Saint-Rémy et Saint-0yen dans la vallée d'Aoste en Piémont.

12. Murithii Puget. - Valais : Dixain de Conclies, Selkingen. - Canton de Fribourg aux Combes.

R. Gaudini Puget. - Valais : Dixain de Conches, Obergestelen, MunsterUlrichen, Binn.

R. gombensis Puget. - Valais : Dixain de Conches, Munster, Binn.

R. proxima Cottet. - Valais : Munster, Binn. - Trouvé dans le canton de Fribourg aux Mérils-Vert-Champs.

\section{AGRINONIÉES.}

AGrmmovia eupatoria L. - Bords des haies et des bois dans tout le bassin.

A. odlorara Mill. - Fossés, bords des chemins dans les terrains siliceux.-C.-d’Or : Epoisses, Saint-Sauveur.-S.-et-L. : SaintForgeot, Millery, Armecy, Monthelon, Roussillon près Autun. - Arr. de Belfort : le Salbert, l'Arsot. - H.-Saône: Ronchamp, Ecromagny, Ban de Champagney, Franchevelle, Saint-Germain. - Doubs : bois de Chaleseules près Besançon, marais de Saône. - Jura : Mouchard près Salins. - Vaud et Genève : Cossonay, Gingins, les Rouges, Coppet, Troinex, Compesières, Meyrin. Valais : Morgens. - H.-Sav. : Bonneville, Cluse, Saint-Mlartin, mont de Sion, plateau des Bornes près la Roche, Saint-Julien, Neydens, Molle. — Sav. : Combe-Noire près Tamié, Oncin. Ain : pays de Gex, la Bresse et la Dombes à Savigneux, les Échets. - Rh.: Couzon, Dardilly, Charbonnières. - Is. : entre SaintLaurent-du-Pont et les Échelles, la Tronche, Jiens, Monestierde-Clermont. - Dr. : environs de Die et de Luc. - Var : Maures près le Luc. - H.-Alp. : Rosans. - Gard : Aumessas près du Vigan. - Hẻr. : la Salvetat-d'Agout. - Pyr.-Or. : forêt de Boucheville, Vernet-les-Bains, montagne de Céret. - Puy-de-Dôme, Cantal, Lozère, Tarn, Aveyron, Loire, Alsace, Lorraine. 


\section{SANGUISORBÉES. (1)}

POTEIEYM sanguisorba L. - Espèce polymorphe, dissíminée dans tout le bassin, comprenant les formes suivantes dont la distribution géographique n'est pas sufflsamment étudiée.

$P$. diclyocarpum Spach. - Prís secs, bois, coteaux. - Assez commun dans G.-d'Or, S.-et-L., Jura, Doubs, Ain, Rh. - H.-Sav. : de Thuet au Brizon. - Sav. : Saint-Innocent, Trivignin. - Is. : Crémieu, Grenoble à la Bastille, Allières. - H.-Alp. : Briançon, Gap. - Dr. : envir. de Crest. - B.-Alp.: la Condamine, Sérennes. - Vaucl. - B.-du-Rh. : envir. d'Aix au Tholonet. Alp.-Mar. : sables du Var près de Nice. - Ard. : Privas. Pyr.-Or.: mont Louis, vallées de la Tet et du Tech. - Pyr. centr., Tarn; Puy-de-Dosme; Allier.

P. guestphalicum Bunningh. - Coteaux pierreux de la région mérid.

- B.-du-Rh. : Saint-Loup, Saint-Marcel. - Hér. : Madières, Navacelle. - Gard : le Vigan. - Rare et accidentel dans les parties moyenne et septentr. du bassin.

P. Delorti Jord. - Terrains incultes, prairies artificielles. - Aude: envir. de Narbonne. - Tarn; Puy-de-Dòme sur les bords de l'Allier.

P. obscurum Jord. - Påturages. - Dr.: Tain. - Centre de la France.

P. muricatum Spach. - Prés, pâturages. - Dans les parties septentr. et moyenne de notre bassin, on l'observe surtout dans les luzernières et prairies artificielles, ce qui donne à penser qu'il est introduit par des graines de provenance méridionale.

Varie à crêtes larges et saillantes P. platylophum Jord., ou à crêtes étroites, peu saillantes $P$. sterolophum Jord.

P. Magnolii Spach. - Terrains incultes de la région mérid. - Vaucl.: envir. d'Avignon, Tour-d'Espagne, Montfavet, Montdevergues. -B.-du-Rh. : envir. d'Aix à la colline des Pauvres, Saint-Narcel.

(1) Les Sanguisorbées seraient mieux placées, entre les Polygonées et les Urticées, dans la classe des Alonochlamyuées, caractérisée par une enveloppe floralo uniquo.

Catal. Bassin du Rloóne. 
- Tar: Toulon, Sainl-Tropez._Gard : Villeneuve-les-Avignon, Fournès, envir. de Nimes, bois de Cygnan, de Broussan et de Campagne. - Commun dans l'Hérault. - Aude : Durban, Villeneuve, les Corbières, la Clappe, Fontfroide, Sainte-Lucie. Pyr.-0r. : Cases-de-Pena et d'Estagel, Baixas, Salses, 0pol.

SANGUISo atBA offieimalis L. - Prés humides des plaines et des montagnes. - Commun dans les vallées des Vosges et de la chaine jurassique. - Marais de Sâone près Besançon. - Prés marécageux de la Dombes et de la Bresse, de C.d'Or, S.-et-L., Rh., Ard., Is., H.-Alp., H.-Sav., Sav. - Rare dans la rég. méridion. - B.-du-Rh. : la basse Crau. — Gard : le Caylar, Candilhac prẻs Nîmes, Concoule, l'Espérou, le Vigan. - Hér. : Lattes, Lodève. - Aude: Mijanès, lac de Quẻrigut, le Llaurenti._Commun dans les Pyrénées, mont Dore, Cantal, H.-Loire, Mezenc, montagnes du Forez, Pierre-sur-Haute.

Présente deux formes, l'une à tiges courtes, à floraison précoce S. montana Jord. qui occupe les réginns montagneuses; l'autre à tige plus longue et rameuse et à floraison tardive $S$. serotina Jord.

AxChenicha alpina L. - Pelouses des montagnes. - Rare dans les Vosges au Rotabac, au IIohneck, et au Rosberg. - Commun dans la région alpestre du Jura helvétique et français jusqu'au Colombier du Bugey. - Vaud et Valais : montagnes de Bex, Fully, Bagnes, Saint-Bernard, Thyon, Arolla, Zan, Zermatt, Comba-d'Arba, Leukerbad, Grimsel. - H.-Sav. : Salève, Voirons, montagnes du Clablais, du Faucigny jusque sur les flancs du mont Blanc à Leschaux et au Couvercle.-Sav. : mont du Chat, montagnes des Bauges, de la Tarantaise et de la Maurienne, mont Cenis. - Is. : Grande-Chartreuse, Chamechaude, Saint-Nizier, chaînes des Sept-Laux, de Belledonne et de l'Oisans. - Dr. : mont Glandasse, le Fourchat près Rosans. H.-Alp. : Lautaret, Brianconnais, Queyras, mont Viso, montagnes des environs d'Embrun et de Gap, Rabou, col Bayard, Chabrières, Séuse, Aurouse, Alp-Martin, Chaillol, Durbonnas, Chaudun, Obiou. - B.-Alp. : la Condamine, Larche. -Vaucl. : mont Ventoux._Sommités des Alpes-Marit._Loire: 
Pilat et cliaine du Forez. - Ard. : roche de Gourdon, Mezenc. Gard: Aigual, chaines de l'Espérou. - Hẻr. : l'Espinouse, le Caroux, Saint-Pons.-Aude : montagne Noire à Nore, Peyramos, vallíe de la Boulsanne, Mijanis, lac de Quérigut, Llaurenti. - Pyr.-0r. : mont Louis, vallée d'Eyne et de Vernet-les-Bains, Costa-Bona. - En dehors de nos limites dans les Pyr. centr., pic de Montalet dans le Tarn, la Lozère, le Cantal, mont Dore, Puy-de-Dôme, Pierre-sur-Iaute, Pic Pelẻ de Noirétable.

A. vulgaria L. - Prés et pâturages des montagnes; descend plus bas que le précédent. - Commun dans les Vosges et la chatne jurassique jusque dans les montagnes du Bugey, Valais, Savoie, Dauphiné, Basses-Alpes jusque dans les Alpes-Maritimes. Rare dans C.-d'Or à la Combe-d'Arcey, Baume-la-Roche, Lugny, environs de Saulieu dans la vallée du Saubiot, Hauts-d'Arneau. - Rare dans S.-et-L., à Montjeu, Broye, Auxy, - Rh. : SaintBonnet-le-Froid, Chaponost, l'Argentiẻre au Châtelard.-Loire: Pilat, Pierre-sur-Ilaute. - Gard : le Vigan, l'Espérou, Alzon. - Aude : Montolieu, les Corbières, Narbonnet, le Donézan, forèt des Fanges, col de l'Hommenet. - Pyr.-Or. : mont Louis, vallée de Nohèdes, Vernet-les-Bains, vallée du Tech, Prats-deMollo - Pyr. centr., massif de la France centrale.

A. subsericea Gr. Godr. A. lyybrida Hoffm. - Feuilles soyeuses-blanchâtres en dessous; mêlé au précédent, mais moins commun.

A. pyrenaien L. Dufour. - Rocailles des montagnes. - Nul dans les Vosges. - Jura : sommités du Colombier et du Reculet. Vaud et Valais : montagnes de Bex, Fully, Saint-Bernard, Zermatt, Saas, Simplon, Laemmeralp. - II.-Sav. : Buet, chaine du mont Blanc, montagnes de Samö̈ns et de Sixt, Roc d'Enfer, Signal, mont des Granges, mont Joly. - Sav. : Haut-de-Tours près Albertville, Hauteluce entre la Gitaz et le lac de Girottaz, col Iseran, Levanna, col de la Madeleine, mont Cenis à Ronches, Pattes-Creuses, bords du lac Noir. - Is. : chatne des Sept-Laux, Belledonne au Colon, Saint-Mury-de-Monteymont près le lac Blanc, col d'Oddie au-dessus des Adrets. - H.-Alp. : Lautaret, Galibier, mont Viso, Arcines, Rochilles, Loppet, Alp-Martin, mont Chabrières.-B.Alp. : Lauzannier, Parpaillon, Vallonnet, 
Sérennes. - Alp.-Mar. : bords du lac de Fenestre. - Aude : col de l'Hommenet, Boutairol, Pla-Bernard dans le haut Donézan, Llaurenti. - Pyr.-Or. : Font-de-Comps, Costa-Bona. - Pyr. centr.

A. pentaphyllea L. - Pelouses, débris de rochers des Alpes et des Pyrénées. - Nul dans les Vosges et dans le Jura - Valais : Fully, Bagnes, Saint-Bernard, Zermontana, Thion, Arola, Schwarzsee de Zermatt, Distelalp de Saas, Gemmi, Furca. II.-Sav. : mont Méri au Four-Métert, les Platets, la Layat, montagnes de Samoüns et de Sixt, cols d'Anterne et de Golèse, le Signal, Roc d'Enfer, Buet, Brévent, col de Balme, chaîne du mont Blanc sur les moraines des glaciers et jusqu'au Jardin de Ja Mer-de-Glace. - Sav. : Petit-Saint-Bernard, col de Forclaz au-dessus de Beaupré, montagnes d'Hauteluce entre Turnaz et Colombaz, Morteret des Allues, mont Iseran, Levanna, Saint-Sorlin d'Arves. - Is. : Grand-Charnier d'Allevard, rocher de Pendé aux Sept-Laux, Alpe du mont de Lans, la Salette. - H.-Alp. : les trois Évéchés, Galibier, mont Viso, col Agnel, Saint-Véran, Clausis, Rochilles, Chaillol-le-Vieil, Orcières, lac de Jujau, Alp.Martin, Pétarel en Valgaudemar.-B.-AIp. : Longet de Maurin. - Alp.-Ifar. : lacs d'Entrecoulpes et du Mercantourm, col de Fremamorta, Alpes de l'Enchastraye-Aude : Salvanère, Llaurenti. - Pyr.-Or. : le Canigou, à l'extrémité de la vallée de Valmanya. - Pyr. centr.

A. arvensis Scop. - Champs dans tout le bassin.

\section{POMACÉES.}

MraspruUS germaniea L. - Le Néflier est cultivê et quelquefois subspontané. - H.-Saone : bois des hospices de Gray à Vénère, Saint-Remy. - Doubs et Jura : envir. de Besançon et de Dole.-C.d'Or : Beaune, Villy-le-Iloutier, Vauchignon.-Vaud, Genève et Valais: Sauvabelin, Cery, Bursins, Aire, mont Chemin.-H.-Sav. : Annecy, Veyrier. - Sav. : Aix, Saint-Innocent, Saint-Germain, Hautecombe.-Ain : la Bresse et le Bugey. Rh. : Tassin, Chaponost, l'Argentiẻre. - Loire : Pilat. - Is. : 
Pariset, Saint-Egrìve, Poisat, Vinay, Saint-Geoirs.-Dr. : SaintVallier, Crest. - B.-Alpes : Digne. - B.-du-Rh. : rives de la Touloubre. - Alp.-Mar. : Grasso. — Gard : Ieuzet, Serre-deBouquet, l'Espérou, le Vigan.

CRATAGUS oxyaeantha L. - Espice polymorphe commune dans les haies de tout le bassin. Se présente sous les deux formes $C$. oxyacanthoides, Thuill et $C$. monogyna Jacrf. - Dans l'Ilérault, l'Aude et les Pyr.-Or. existe une troisième forme le c. ruscinonensis Gr. Blanc.

C. azsrolus L. - Bois de la région méridionale.

Cotovastede pyracantha Spach. - Ilaies de la région méridionale. Rarement planté dans les haies de la région moyenne et septentrionale.

c. vulgaris Lindl. - Rochers des montagnes. - Toules les hauteurs des Vosges jusrqu'au Ballon de Giromagny. - Jura helvétique el français, Montendre, Dổe, Colombier, Reculet jusque dans les montagnes du Bugey autour de Saint-Rambert, Tenay, Retord, Colombier, le Mont, Siint-Germain-de-Joux. - Vaud et Valais: Signal de Chexbres, Rivaz, la Sarraz, Montcherand, Martigny, Sion, Saint-Léonhard, Clavaux, Esserz. - C.-d'Or : Gevrey-Chambertin.- H.-Sav. : Salève, mont Châtillon, la Roche, Semnoz, Tournetle.-Sav. : mont du Chat, Bourdeau, Brizon, Saint-Innocent, mont Cenis. - Is. : Saint-Baudil et Vernas près Crémieu, Grande-Chartreuse, mont Rachais, col de l'Arc, mont Senèpe, mont de Lans, Mens. - II.-Alp. : la Grave, Lautaret, Briançon, mont Viso, envir. de Gap, Rabou, Séuse, col Bayard, Chaillol-le-Vieil, bois Vescal, mont Chabrières, Obiou. - Dr. : Barnave.-B.-Alp. : vallon d'Ornaye, Malmore, Sainte-Anne-dela-Condamine, Vallonnet, Grouès. - Vauel. : mont Ventoux. B.-du-Rh. : sommet de Sainte-Victoire. - Alp.-Mar. : montagnes de Caussols et de Défens, sources du Var, Colmiane, Saint-Dalmas-le-Sauvage, Tende, Utelle, Menton. - Ard. : Baix, Gerbier-des-Joncs. - Gard : l'Aigual, Hort-de-Diou, la Lozère près Concoule. - Hér. : le Caylar. - Pyr.-Or. : Trencada d'Ambulla, Saint-Antoine-de-Galamus, Corbières, montagnes de Madrès, Llaurenti. - En dehors de nos limites, dans les Pyrén. centr., 
les causses de la Lozère, les terrains volcan. du Cantal, du mont Dore, du Puy-de-Dóme el de la Haute-Loire.

C. tomentosa Lindl. - Licux pierreux des montagnes calcaires. - Nul dans les Vosges et les terrains granitiques et volcan. du centre de la France. - Jura helvétique et français jusque dans les montagnes du Bugey. - Vaud et Valais : Cheville, CheminNeuf, Tourbillon, Lavernaz, Clavaux au-dessus de Varen. H.-Sav. : base du Salève, du Môle et du Brizon. - Sav. : entre Aigneblanche et JIouticrs, bois Champion, roche Saint-Victor au-lessus de Mouxy près Aix, envir. de Chambéry, mont Cenis. - Is. : Saint-Eynard, Bovinant, Saint-Nizier, l'Enchâtre, Mens. -II.-Alp. : mont Viso à Parachalve, Saint-Véran, col de Vars, Devez de Rabou, bois de l'Aullanier près Gap, mont de Faye près Ventavon, Durbon, Guillestre, Boscodon. - B.-Alp. : Lauzannier, Annot à Ruanette. - Dr. : Barnave. - Vaucl. : mont Ventoux. - B.-du-Rh. et Var: Sainte-Victoire, pic de Bretagne, Sainte-Baume. - Hér. : le Caylar sur le Larzac. — Gard : bois de la Roquette, Cerconne, la Lozère près Concoule. - Aude et Pyr.-Or. : Boutairol dans la haute vallée de l'Aule, le Llaurenti, bois de Salvanère et de Boucheville, Font-dc-Comps. - En dehors de nos limites dans les Pyr. centr. et sur les causses de la Lozère autour de Florac et de Mende.

CYDQNi A valgaris Pers. - Le Coignassier est cultivé et quelquefois subspontané.

P' X RE'S conmunis L. - Cullivé el quelquefois subspontané.

P. amygadahformis Vill. - Terrains picrreux de la région des oliviers. - Vaucl. : environs d'Avignon à Montdevergues, Morières, Flassan. - B.-du-Rh. : le Rove, Istres, Miramas, lisière de la Crau.-Assez commun dans la partic basse du Var, Gard, IÍrault, Aude dans les Corbières, Pyrénées-Orient.

P. malus L. - Cultivé et subspontané.

P. aecrba L. - Bois depuis les plaines jusque dans les vallées des montagnes.

SOR RUS domestiea $L_{\text {. }}$ - Cultivé et subspontané.

s. виеuparia L. - Bois des montagnes. - Région des sapins dans les Vosges, le Jura helvétique et français jusque dans le Bugey, 
Savoic, Dauphiné, B.-Alp., Alp.-Naritimes. - Chaînes du Morvan. - Beaujolais, Forez, Pilat, Vivarais, Cévennes du Gard et de l'Itérault, Aulde et Pyr. centr., montagne Noire, Cantal, mont Dore, mont Dôme.

S. hybrida L. - Rochers du Jura helvétique au Sucliet, Creux-du-Van, chtre les IJauts-Genneveys et les Loges.-Iura français : rochers de Cise près Champagnole. - Loire : Pierre-sur-Ilaute aux rochers de Charnolin et de Chanchère. - Vaud et Valais : mont de Colonges sous Morcles, château de Saint-Mfaurič.-H.-Sav. : picd du Salève au-dessus d'Archamp, les Voirons vers Bonne, Chamonix, - Sav. : Saint-Innocent. - II.-Alp. : forêt de Boscodon.

s. seandlea Fries.-Rocher's el bois, - Région des sapins du Jura. —Salève. — Is. : Saint-Nizier. - Sav. : Granier, mont du Chat. - B.-Alp. : mont de Lure près Sisteron.

S. Mongeoti Soy,-Will, et Godr. - Bois et rochers. - II.-Sav. : Salève, Veyrier, Annecy, la Caille.-Jura et Ain : Reculet, Colombier du Bugey, Rossillon. - Sav. : Conflans. - Is. : GrandeChartreuse, forêt de Portes, Saint-Nizier, col de l'Arc, mont de Lans. - IH.-Alp. : la Grave, le Rif du Salp en Volgaudemar.

Diffère du précédent par ses fruits plus petits, ses feuilles plus étroites, moins profondément lobulées à la base et plus atténuées.

S. arla Crantz. - Bois des montagnes. - Chaines rosgienne et jurassique, Bugey, Savoie, Dauphiné, B.-Alpes jusque dans les Alp.-Mar., mont Yentoux et les montagnes de la Provence. Chaines du Morvan, Lyonnais, Forez, Vivarais, Cévennes jusque dans l'Espinouse, la montagne Noire, les montagnes de l'Aude et des Pyrénées-Orient. - Pyrén. centr. et montagnes du centre de la France.

S. corminalls Crantz, - Bois des collines et des montagnes. Chaînes vosgienne et jurassique, le Bugey à Saint-Rambert, Muzin, Parves. - Envir. de Genève dans les bois de la Bâthie, Veyrier, Bay. - Vaud : Roche. - H.-Sav. : Salève, plateau des Bornes près la Roche, Mandallaz, la Caille. - Sav. : Aix, SaintInnocent, Moutiers. - Is. : Chambaran, Vinay, Tullins, Chatte 
près Saint-1Iarcellin. - Dr. : Divajeu près Crest, entre Crest et Montélimar. - H.-Alp. : bois de Furmeyer et du Garon près Gap. - Vaucl. : mont Ventoux. - Var : Sainte-Baume, Mourières près Toulon, Saint-Quenis près Camp, Fréjus. - Alp.Mar. : l'Estérel, bois de Tournon-sur-Siagne. — Gard : Anduze, Palières. - Pyr.-Or. : bords du Sègre dans la vallée de Llo, entre les Bouillouses et le Aral-Pas, Canigou. - Pyrén. centr., montagne Noire, Cantal, II.-Loire, Puy-de-Dôme. - Loire : mont d'Uzor, Saint-Jodard, Fouillouse, Saint-Bonnet-lesOulles.

S. chnm cemespilus Crantz. - Bois. - Rare dans les Vosges au Rotabac, Hoehneck, Ballon de Soultz. - Sommets du Jura helvétique et français au Chasseral, Tête-de-Rang, Montendre, Noirmont, Dôle, Reculet, Rizoux. - Vaud et Valais : montagnes d'Aigle, Oltan, Ravoire, Salvan, Comba d'Arbaz, Pas-du-Loup, Mandallaz.- B.-Sav. : Brizon, dents d'Oche, Semnoz, vallée du Fier, en face du Parmélan, col de Balme, Chamonix, vallée de. la Diosaz. - Sav. : mont Cenis, la Ramasse, Saint-Sorlin-d'Arves. - Is. : col de la Ruchère, Chamechaude, le Moucherotte, col de l'Arc. - II.-Alp. : Lautaret, col des Hayes, mont Viso au Bouchas, l'Echalp, Arcines, Orcières, Valgaudemar, bois de Loubet prìs Gap. - B.-Alp. : Larche. - Alp.-Mar. : val Pesio, col de Fremamorte. - Gard : entre Brama-Bioou et Meyrueis.Aule : col de l'Ebigne dans le Donézan, Mlijanès, Llaurenti. Pyr.-0r. : Pli-dels-Abellans, bac de Bolquìre, Canigou vers la Forgc-de-Llech. - Pyrén. centr. - Cantal au col de Cabre; H.Loire au Mezenc et à la montagne de l'Ambre; mont Dore, Pierre-sur-IIaute.

S. ambigua Michalet.-Jura à la Dôle-_Pétales dressés, rosés; feuilles tomenteuses, blanchâtres en dessous.

A NIEANCHER vulgraris Mœnch. - Rochers des montagnes; plus commun sur les calcaires. - Chaines des Vosges, du Jura helvétique et français, Bugey, Revermont. - Vaud et Valais : mont Clieville, Ottan, les Marques, la Bâtiaz, Lavernaz, Clavaux, Lens, Vercorin, Aletsch.-II.-Sav. : Salève, Môle, Brizon, montagnes de Thônes et de la vallíe de l'Arve. - Sav. : Saint- 
Innocent, Brizon, Ilaulecombe, Conjux. - C.-d'Or : GevreyChambertin.-S.-et-I. : Solutri, Vergisson, Chamilly, Dezize. - Rh.: Rochecardon, Couzon, Condrieu. - Is. : Crémieu, Vienne, Bastille de Grenoble, mont Rachais, côtes de Sassenage. - Dr. : Crest. - II.-Alp. : envir, de Briançon et de Gap, mont Chabrières, le Queyras. - B.-Alp. : vallée de l'Ubaye, Sisteron. - Vaucl. : mont Ventoux, Villencuve, les Angles près Avignon. - B.-du-Rh. et Var : Saint-Loup, Roquefavour, Gignac, Toulon, le Luc, Aups, Saint-Quenis. - Alp.-Mar. : Grasse, SaintAndré pris Nice, la Turbie, Menton.- Loire : barrage de Rochetaillèe, montagnes du Forez, Pierre-sur-Haute. - Ard, : vallée d'Ouvèze, mont Combier, Privas, Aubenas. - Gard : le Vigan, borus du Gardon, Saint-Nicolas, Ganges, Anduze, Alais, Nìmes. - Hẻr. : l'Espinouse, le Larzac. - Aude : les Corbières, la Pierre-Lisse, Montolieu, la Clappe, ravin de Combemale et des Mounges, Fontlaurier, le Donézan.-Pyr.-Or. : Trencada d'Ambulla, Vernet-les-Bains, Castell, Graus "̈Olette, le Baus de l’Aze près Tech. - Pyr. : centr., causses de la Lozère, Haute-Loire, Cantal, Puy-de-Dôme, Loire.

\section{GRANATÉES.}

PUNCA granatum L.-Le grenadier est cultivé et naturalisé dans la région méridionale jusque dans le nord de Vaucluse, vers Annot dans les B.-Alpes, Saint-Paul-Trois-Châteaux, Montélimar et Tain dans la Drôme. Dans les environs d'Aubenas, on le plante dans les haies. Il s'est naturalisẻ sur les rochers de Valère et de Tourbillon a Sion en Valais.

\section{ONAGRARIÉES.}

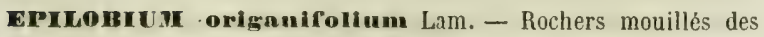
hautes montagnes. - Nul dans les Vosges. - Jura : la Dóle, Reculet au vallon d'Ardran, Colombier, Chasseron. - Valais : Bagnes, Saint-Bernard, descente du col de Balme sur Trient. H.-Sav. : montagnes du Chablais, Cornetles-de-Bise, Roc d'En- 
fer, mont des Granges, Sixt, chaine du mont Blanc, Saint-Gervais, Méri, Vergy, Charvin et Tournette.-Sav. : montagnes de Beaufort, d'Hauteluce, Crest-Voland, Crêt-du-Ré près Aime, col du Bonhomme, mont Iseran, mont Cenis, - Is. : chaînes des Sept-Laux, de Belledonne et de l'Oisans. - H.-Alp. : la Grave, Lautaret, col des Hayes, mont Viso, Chaillol-le-Vieil, Orcières, Séuse, Aurouse, Alp-Martin.-B.-Alp. : Lauzannier, Riou-German, la Condamine, Grand-Couyer sur Annot. - Alp.-Mar. : montagnes d'Entraunes, vallons de Libaré et du Boríon, col de Tende.-Ard. et H.-Loire : le Hezenc, Cuze.-Hẻr. : le Caroux. - Pyr.-Or. : pic de Costa-Bona. - Pyrénées centr., Cantal, Mezenc, mont Dore.

E. alpinum L. (non Vill.). - Rocailles liumides des montagnes, à sol siliceux. - Chaine des Vosges au Hoehneck ot au Rotabac. - Jura : Colombier, Faucille, Montendre. - Valais : Fully, Saint-Bernard, Tortain, Combire, Galen, Zermatt, Saas, - H.Sav. : Brizon, Vergy, Méri, mont d’Étale, Samoëns, 10 Layat près du Bonhomme, vallée de Saint-Gervais, chaîne du mont Blanc jusqu'au Jardin de la Mer-de-Glace, col de Balme, Buet. - Sav. : mont Iseran, Combe d'Ambin, les Allues, Crest-Voland. - Is. : chaines des Sept-Laux, de Belledonne et de l'Oisans, Grand-Som.-H.-Alp.: Lautaret, col des Hayes, mont Viso, col de Vars, Chaillol-le-Vieil, Orcières, mont de l'Ours, Alp.Martin. - B.-Alp. : Lauzannier, Fouillouse. - Alp.-Llar. : lac des Merveilles sous le mont Bego, vallée de Valmasque, Fenestre, Salsamorena, col de Jallorgue. - Loire : Pilat vers les sources du Gier, Pierre-sur-Haute-Ard. : le Mezenc.-Aude : versant mérid. de la montagne Noire, Boutairol, Llaurenti.Pyr.-Or. : vallée d'Eyne, Carença, Canigou, Font-de-Comps. Pyrén. centr., Plomb du Cantal, mont Dore.

E. paIustre L. - Tourbières et marais des montagnes; rare dans les plaines. - Chaînes des Vosges, du Jura helvétique et français. - C.-d'Or : envir. de Saulieu et d'Auxonne. - S.-et-L. : Autun, Montjeu, Roussillon. - Ain : envir. de Bourg et de Belley, Divonne. - H.-Sav. : de Nant-Borant aux Contamines, Servoz, Clıamonix.- Sav. : Saint-Innocent, Bourdeau. - Is. : 
Prémol entre Theys et Ie col du Merdaret, Corençon, Alpe du mont de Lans, Charvieu. - Dr. : Crest. - H.-Alp. : Lautaret, envir. de Gap. - Loire : Pilat, Pierre-sur-Haute, Grézieu-leFromental.-Ard. : Mezenc, Aubenas, Mauras. - Gard : chaîne de l'Aigual, la Lozère à Concoule.-Pyr.-Or. : vallées de Nohẻdes et d'Urbanya, forêts de Salvanère et de Boucheville, montagne de Madrìs. - Pyr. centr., montagne Noire et centre de la France.

E. obscurum Schreb. - Lieux liumides des plaines et des montagnes. - Chaines des Vosges et du Jura. - Ain : marais des Échets.- Rh. : Charbonnières. - Sav. : Venthon, Bourg-SaintMaurice. - Is. : Uriage. - Alp.-Mar. : Nice, Saint-Martin-Lantosque.-Loire : Pilat, la République, Pierre-sur-Haute.-Ard. et II.-Loire : le Mezenc. — Gard : Nimes, le Vigan. - Hér. : Bousquet d'Orb, Saint-Amand-de-Nounis, Pardailhan, AndabreRosis, Lamalou, Graysessac, Avène. - Lozère, H,-Loire, Cantal, Puy-de-Dôme.

E. tetragonum L. - Lieux humides dans tout le bassin.

E. Lamyii Schultz. - Forme appaurrie du précédent avec lequel elle est mêlée.

E. roseum Schreb, - Lieux frais et ombragés dans les parties sept. et moyenne du bassin. - Partie montagneuse du Gard et de l'Hérault.

E. trigonum Schrank. - Pâturages des montagnes.-Ballons de Servance, du Rotabac, du Hoelneck et de Giromagny. - Région alpestre du Jura helvétique et français au Dietisberg, Weissenstein, Chasseral, Dôle, Reculet, Faucille, Montendre. - Vaud et Valais : Saint-Bernard, Bagnes, Bovonnaz, Lavarraz, Zermatt. - H.-Sav. : Voirons, Brizon, la Diosaz, Brévent. - Is. : au dessus de Revel, forêt de Portes, Corençon.- Gard : Aulas près du Vigan.- Pierre-sur-Haute, pentes du Cantal, du mont Dore et du Puy-de-Dôme, Pyrén. centr.

E. montanum L. - Espèce polymorphe très-répandue dans les bois des basses collines et aussi des montagnes sur tous les terrains.

L. Duricti Gay. Diffère du précédent par sa souclıe rampante et stolo- 
nifère. - Ain : bois de Lavatay au-dessus de Gex, crêt de Chalam. - Loire : bois du Pic de Gourgon à Pierre-sur-Haute.

E. collinum Gmel. - Forme rameuse et rabougrie de l'E. montanum; elle habite exclusivement les sols siliceux, tandis que l'E. montanum est ubiquiste. - Commun dans les chaines granitiques des Vosges, Morvan, Beaujolais, Lyonnais, Forez, Pierre-surHaute, Pilat, Vivarais, Cévennes jusque sur l'Espinouse, l'Escandorgue et la Montagne Noire. - Terrains siliceux de la Savoie et du Dauphiné. - Aude : Boutairol, Mijanès dans le Donézan, lac de Queérigut, vallée de la Bruyante. - Pyr.-Or. : Prats-de-Mollo. - Terr. granit. des Pyrẻn. centr., de la Lozère, terr. volcan. du mont Dore et Puy-de-Dòme. Nul dans la chaine jurassique, excepté .sur les granites de la Serre vis-à-vis d'Amange.

E. Ianceolatum Seb. et Maur.—Haies, bords des bois. - Vallées des Vosges. - Nul dans la chaine jurassique, excepté sur les granites de la Serre.-Ain : envir. de Belley et de Trévoux. - Rh.: Ecully, Tassin, Charbonnières, Collonges, Couzon.-Is. : Uriage, de Vaulnaveys à Prémol, Séchilienne, la Verpillère, Diemoz. Sav. : Conflans. - B.-Alp. : Annot. - Gard : Saint-Guiral, Aumessas.-Hér. : Bousquet d’Orb, l'Escandorgue à Romiguières, Pardailhan, Lamalou, Colombières, Avènes, Saint-Martin-d'Orb, Saint-Étienne-de-Nursan.- Aude et Pyr.-Or. : entre Sigean et Salces. - Centre de la France.

E. parviflorum Schreb.- Lieux humides dans tout le bassin.

E. hirsutum L. - Lieux humides dans tout le bassin.

E. spientum Lam.- Forêts des Vosges, chaine jurassique, Bugey, Revermont, montagnes de la Savoie, Dauphiné, B.-Alpes et Alp.Marit._Manque dans le Valais. - Montagnes du Morvan, Beaujolais, Tarare et l'Argentière dans le départ. du Rh., Forez, Pierre-sur-Haute, Pilat, Vivarais. - Mezenc. - Cévennes du Gard, le long du Valat de Brama-Bioou et de la Dauphine, Espérou, Camprieux.-Aude : le Donézan.- Pyr.-Or. : mont Louis, Canigou, la Preste, Costa-Bona.

En dehors de nos limites, dans les Pyrén. centr., la Lozère, le Cantal, mont Dore, Puy-de-Dôme, Forez. 
D. rosmnxinifollum IIacncke. - Graviers et díbris de rochers.

Cette espèce se propage trìs-facilement le long des talus de chemin de fer et sur les digues des rivieres. - Rare dans le Doubs et le Jura.- H.-Saône : Chariez. - Assez commun dans le bassin du Iéman, le Valais, Savoie, Bugey, Dauphiné, environs de Lyon et bords du Rhône. - C.-d'Or : Chassagne, Rouvray, Epoisses. - S.-et-L. : Tournus, Savigny. - B.-Alp. : bassin de l'Ubaye. - Vaucl. : Flassan. - B.-du-Rh. : plateau de la colline des Pauvres, près Aix, Jouques, Roquefavour. - Var : Fréjus, Signes, Mons.- Alp.-Mar.: Grasse, le Bar, Saint-Auban, Levens, Nice, Menton.-Gard : le Vigan, Alzon, Ganges, Alais, Anduze, chartreuse de Valbonne. - Aude et Pyr.-Or. : Salvanère, Llaurenti.

T. Fleinclkeri Hoclist.-Graviers des torrents des Alpes dans Vaud, Valais, Savoie, Dauphiné, B.-Alp. et Alp.-Mar. - Pyr.-Or. et Aude : ravins du bois de Salvanère.

Enothera blemnis L. - Otiginaire de l'Amériqque du Nord, naturalisé dans les terrains sablonneux, graveleux, décombres, débris de carrières et surtout le long des cours d'eau.

(E. muricata L. - Se distingue du précédent par ses feuilles plus étroites, ses fleurs petites à pétales trois fois plus courts que le tube calicinal.-Commun sur les bords des rivières des Vosges, mais rare et accidentel dans notre bassin.

JUSST.XA grandimora Michx. - Subspontané à Vallabrègues (Gard) dans une branche du Rhône; prìs de Montpellier, sur les bords du Lez, vers les marais de Vourgaran, dans les fossés de Lattes, Gramenet, Maurin et la Madeleine.

ISNA IR DIA palustris L. - Mares et étangs, - Envir. de Belfort à Bourogne, Charmois, partie basse de la rivière Saint-Nicolas. - H.-Saone : Ferrières, Chagey, Chenebier. - Doubs et Jura. - C.-d'Or : envir. de Saulieu à Saint-Lẻger, Champeaux, Auxonne.-S.-et-L. : Saint-Jean-de-Losne. - H.-Sav. : Annemasse, Viry, Annecy-le-Vieux. - Ain : étangs de la Dombes et de la Bresse, envir. de Belley. - Sav. : Aix, Saint-Innocent. Rh. : Pierre-Bénite, Soucieu, Charbonnières, Lavore, Vaux-enVelin. - Is. : Meyzieu, Crémieu, Morestel, Eydoche, Diémoz, 
Pont-de-Beauvoisin.- Ard. : Saint-Agrève- - Var et Alp.-Mrar.: verrerie du Cannet, le long du vallon de Mourefrey, vallée d'Agay, étangs de Vaugrenier près Antibes, IIenton. - Gard : Manduel.-Pyr.-Or. : Saint-Génis, Toulouges, Vieille-Basse audelà d'Orle.

CrRCAA Iutetiana L. - Bois des collines et des basses montagnes. Dans la région méridionale se trouve seulement dans les montagnes.

C. Intermedia Ehrh. - Bois. - Arr. de Belfort dans la vallée du Puix, forêts de Lys et du Chantoisot. - H.-Saône : vallées du Rahin, de Plancher-les-Mines, Champagney.-Jura français et helvétique : crêt de Chalam, Reculet, Creux-du-Van, Chasseron, Chauffaud. - Ain : Chézery, Belleydoux, Saint-Laurent, Nantua, chartreuse d'Arvières. - C..d'Or : Nataloux près Saulieu.S.-et-L. : Roussillon, bords du ruisseau de la Cange, Armecy près Toulon.-Loire : Pilat, Pierre-sur-Haute.-Ard. : Ie Cheylard, Jaujac. - Valais : Frenière, Trient, Choex. - H.-Sav. : Brizon, Scionzier, Saint-Jean-Tholomes, le Cohennoz, Araches. - Sav. : Tresserves, Pregny, Hauteluce entre Villard et Bonnecines. - Is. : Corençon près Villard-de-Lans, Grande-Chartreuse. - Dr. : Bouvante. - H.-Alp. : Pleyne-de-Chaudun près Gap.-Manque dans la région mérid. et dans les Pyrẻnées cent. Sc rencontre dans la Montagne Noire, la Lozère, le Cantal, le mont Dore et le Puy-de-Dôme.

C. aIpina L. - Lieux ombragés et humides des terrains siliceux.Commun dans les Vosges granitiques et arénacées, notamment au Rossberg, Hoehneck, Rotabac, Champ-du-Feu.-H.-Sanne : Ballon de Giromagny, Champagney, Miélin, Fresse, vallée du Lys.-Doulss : dépôts siliceux et lieux tourbeux à Noël-Cerneux, Chenalotte, Gd.-Combe-des-Bois, Guinots, Charquemont, Bélieu, Bizot, Mémont, Russey, Saint-Julien, Frambouhans, Maîche, Damprichard.-Jura : dépôts siliceux du Creux-du-Van et de la Dòle.-Ain : de Malbronde aux monts d'Ain. - Vaud el Valais : Enzeindaz, Trois-Torrents, Veisonna, Trient, vallée de Nendaz sous Essert, Schwarenbach, Ballen de Saas, Oberwald. - H.Sav. : vallée de la Borne près Bonneville, bois du Sommier dans 
la vallé du Reposoir, Nautau, montagnes de Samoüns, Tournette, Charvin, entre le Pavillon de Bellevue et les Houches, envir. de Chamonix, vallée de la Diosaz, des Contamines à NantBorant. - Sav. : Trévignin, Hautecombe, montagnes de Beaufort et d'Hauteluce, Crest-Voland.- Is. : Allevard, Prémol près Uriage.-Alp.-Mar. : la Briga, col de Fenestre, val Pesio, SainteAnne-de-Vinai.-Gard : le long du Valat de Brama-Bioou, et en descendant de la Céreirède, Banahu. - Loire : chaîne granitique du Pilat et de Pierre-sur-Haute. - Pyr.-Or. : vallées granitiques d'Eyne, de Llo, Llaurenti.

En dehors de nos limites la Circée des Alpes se trouve dans les Pyrénées centrales, la Lozère, le Cantal, le mont Dore, le Puy-de-Dôme, la chaine de Pierre-sur-Haute.

\section{HALORAGÉES.}

MYIIOPIIYLUM verticilatum L. - Mares et ruisseaux. Moins commun dans la région méridionale.

II. epientum L.- Même dispersion.

N. sIterni forum D. C.- Plus rare que les précédents.-Manque dans la chaine jurassique. - C.-d'Or : étang des Vermouraux près Saulieu. - S.-et-L. : rivière de Millery près Autun, ètang de Bousson. - Rh.: le Bâtard près Taluyers, Lavore près Mornant.

En dehors de nos limites se trouve dans les lacs des Vosges, Puy-de-Dôme, Allier, Cantal, Corrèze.

TRAPA natans $L_{\text {, }}$ - Eaux stagnantes des terrains siliceux et argileux. - Lisière rosgienne de l'arrond. de Belfort à la Forge, Eloye.-H.-Saone: Chagey, Champey.-Doubs : alluvions entre Montbiliard et Bàle, grès des bords de l’Ognon, tuilerie de Rougemont. - Jura : étangs de la Bresse à Pleurre, Fay, Tassenière, Sellières.- Ain : environs de Bourg, Mollon.-C.d'0r : étangs à sous-sol granitique d'Arnay, la Berchère, Thoisy près Saulieu. - Is. : Mépieu, étang de la Morte près Morestel - Sav. : lac d'Aiguebellette, Pont-de-Beauvoisin. - Nul dans le Valais, les parties alpines du Dauphiné, de la Savoie, des B.-Alpes. - N'est 
signalé dans les départements méridionaux que dans les Pyr.Or. à l'Agulla de la Mar, entre Vall-Rich et l'Esparron, Salces sur les confins du départ. de l'dude.

En dehors de nos limites dans Allier, Puy-de-Dỏme, Loire.

\section{HIPPURIDÉES.}

MIPPU ItIS vuIgaris L.-Marais et étangs des parties septentr. et moyenne du bassin. Plus rare dans la région méridionale. Vaucl. : entre Saint-Véran et Réal-Pamier.-Gard : Saint-Gilles, Bellegarde.-Aude et Pyr.-Or. : étang de Salces, Canet.

\section{CALLITRICHINÉES.}

CALETTRiCrI E atagualis Scop. - Mares et fossés dans la plus grande partie du bassin, mais inégalement réparli.

C. platycarpa Kütz.-Ruisseaux, fossés.-Arr. de Belfort dans la vallée de la Savoureuse.- II.-Saûne: Pontcey. - Environs de Genève entre Lancy et Onex, Compesières, Sionet.- Valais : la Verrerie.-H.-Sav. : Douvaine, Arenthon. - Alp.-Mar. : Nice, Antibes.-Gard.—Hẻr. : la Roubine près Lattes. Plus commun dans le centre de la France,

C. verna Külz. - Mares et fossés. - Mème dispersion que le C. stagnalis.

C. hamulata Kütz. - Mares et fosse̊s. - H.-Saône : Champagney. Douls : envir. de Montbéliard. - Jura : Chaussin, Neublans, forêt de Chaux. - C.dd'Or, S.-et-L. - Envir, de Genève. - Valais : Zanrion, Saint-Bernard.-Rh. : Brindas.-Is. : Eydoche, lac du Crouzet au-dessus de Revel, plateau de Chamousset audessus de Chichiliane, lac de Brandes en 0isans.-Var : Fréjus. - Gard : le Vigan, l'Espérou, les Pises. - Hér. : Fraisse, Grammont, Prades, Roquehaute.

En dehors de nos limites, dans le centre de la France, Loire, Puy-de-Dôme, H.-Loire, Corrèze. La dispersion des diverses formes de Callitriche a été inal étudiée. 


\section{CERATOPHYLLELS.}

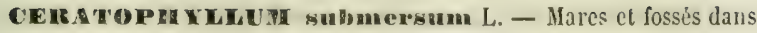
tout le bassin.

C. Jencroum L. - Même liabitat et même dispersion.

\section{I.YTHRARIEES.}

EYTHEUM sallearia L. - Lieux humides dans tout le bassin.

- Espèce polymorphe.

L. Croffere Ten.- Fossés. - Espèce assez commune en Corse, qui a ćtẻ trouvéc dans le Var et les Alp.-Mar. à Fréjus, cap Croiselte, golfe Juan, Auribeau, le Bar, Antibes, Fice, Menton. - B.-duRh. : près de Marseille, au vieux chemin de Saint-Giniex. Biarritz près Bayonne.

L. Bysopofora L. - Lieux humides des terrains sablonneux. Beaucoup plus commun dans la région mérid. - Manque dans le Valais el dans les Alpes françaises.

C. bibracteatum Salzm. - Lieux mouillés de la région mérid. B.-du-Rh. : Marignane, Istres, vallon de Sulauze.- Gard : bords de l'étang de Jonquieres, Manduel, la Capelle, Marsillargues, Aigues-Mortes. - Hér. : Agde vers les marais de Roquehaute, Maguelonne, la Madeleine.-Aude: la Rouquette près Narbonne. - Pyr.-Or.: sables des torrents des Albères à Saint-Génis et Sorède.

L. thymifolia L. - Lieux humides des terrains sablonneux de la région mérid.- Var : Toulon, Frẻjus, forêt des Maures.-Alp.Mar. : Biot près Antibes. - Gard : bois de Broussan près Nimes, Trou-du-Pérussas, Cabane d'Igonnet, ètang de Jonquières, Beauvoisin, Aigues-Mortes. - Hér. : envir. de Montpellier à Gram. mont, Lamoure, Pérols, Roquehaute à Portiragnes.-Pyr.-Or.: Sainte-Ilarie, Torreilles, parties basses du Canet, dunes de SaintCyprien et d'Argelès. - Remonte dans la partie mérid. de la Drôme à Saint-Paul-Trois-Châteaux; dans les H.-Alp. jusque près d'Embrun, vers le lac de Séguret; a été observé aussi hors Catal. Bassin du Rhône. 
de notre bassin près du Puy, dans la plaine de Clıadrac (HauteLoire).

L. geminifforam Bertol. - Terres humides autour de l'étang de Jonquières et entre Tresques et Connaud (Gard).

PEPLIS portals L. - Lieux mouillés des terrains sablonneux dans une grande partie du bassin. - Manque dans le Valais. - Rare dans la Provence et les Alp.-Mar. - Hér. : Fraisse, Pardailhan-Pontgiraud, la Salvetat,

P. erecta Req. - Lieux humides de la région mérid. - Var : le Luc, Saint-Raphaël.— Alp.-Har. : Vaugrenier près Antibes.— Gard : Trou-du-Pérussas dans le hois de Broussan, Cabane d'Igonnet entre Beauvoisin et Vauvert. - Hér. : mares de Roquehaute à Portiragnes, Grammont, Lamoure. - Aude : Montagne-Noire, envir. de Salces, la Nouvelle, ile Sainte-Lucie.

P. Timeroyi Jord.-Bords des eaux stagnantes. - Rh. : bords de l'étang de Lavore près Mornant._Ain : Montribloud — Gard : AiguesMortes.

\section{TAMARISCINÉES.}

TAMARIX galliea L. - Bords des cour's d'eaux de la rígion mérid., principalement sur le littoral. Remonte dans la partie mérid. de la Drôme vers Saint-Paul-Trois-Châteaux.

T. afrienna Desf.- Littoral méditerranéen.

MYRicaria germaniea Desv. - Graviers des torrents des

Alpes et des Pyrénées. Entrainé par l'Arve au-dessous de Genève; par le Rhone à Seyssel, Culoz et jusque à Lyon; par l'Isère à Grenoble; par la Drôme à Crest; par le Buech à Veynes; par la Durance jusque près d'Avignon et de Beaucaire; par le Var et la Roya jusque près des bordz de la mer; enfin par la Tet jusqu’à Perpignan. - Nul dans les Vosges et dans le Jura. Les pieds de M. germanica qu'on voit dans la vallée du Rhin, entre Bâle et Mannheim, viennent de graines charriées par les affluents du Rhin qui descendent des Alpes suisses. La Mlyricaire se montre aussi le long des torrents des Pyrénées. 


\section{MYRTACÉES.}

MYreTre communis L. - Haies et bois du littoral des Alp.-Mar. et des parties voisines de la Provence. - Hér. : montagne de Mireval. - Gard: Anluze, Cannes, Cérignac. - Aude : la Clappe, Corbieres, îles de Lante et de Sainte-Lucic.- Pyr.-0r. : plateau de Saint-Estève, Haut-Vernet, Banyuls-sur-Mer.

\section{CUCURBITACÉES.}

Barovia thoien L. - Haies dans tout le bassin.

ECEAYIUn elateriom Rich, - Décombres, lieux incultes de la région mérid. - Remonte dans le midi de la Drôme vers Grignan, Saint-Paul-Trois Châteaux, le Buis, Rochegude. - S'est montré accidentellement sur les bords de la Saône à Tréroux, Montmerle (Ain); dans le Jura, à la Barre près Orchamps, et aussi près de Clermont-Ferrand. Gannat et Vichy.

\section{PORTULACÉES.}

PontuUACA olerseea L. - Jardins, décombres dans tout le bassin.

HIONTIA minor Gmel. - Bords des ruisseaux dans les terrains siliceux des plaines de la région septentr. et moyenne du bassin. - Se trouve aussi dans la région mérid., mais exclusivement dans les montagnes du Vivarais et des Cévennes jusque dans l'Espinouse et la Montagne-Noire-Manque dans Vaud, Valais, bassin du Léman, Alpes de la Savoie, du Dauphiné, des B.-Alp. et des Alp.-Marit.- Commun dans les terrains siliceux du centre de la France, des Vosges et des Pyrénées. - Nul dans toute la chaine jurassiqne, excepté sur les granites de la forêt de la Serre.

I. rivularis Gmel. - Ruisseaux d'eaux vives des terrains siliceux des Vosges, du Morvan. Beaujolais, Lyonnais, Forez, Pierre-surHaute, Pilat, Visarais, Cévennes du Gard et de l'Hérault jusque 
dans l'Espinouse ct l'Escandorgue, Montagne-Noire, Pyr.-Or. Ruisseaux des terrains siliceux du Dauphininé, bois des Orciẻres au-dessus de Revel, Sept-Laux d'Allevard, Saint-Ilugon, Lavaldens.-Manque dans Vaud, Valais, Jura et Alpes-Maril.-Commun dans les Pyrén. centr., la Montagne-Noire, le centre de la France et les Vosges. Espèce exclusivement silicicole.

\section{PARONYCHIÉES.}

POrXcareon tetraphyllum L. - Lieux incultes, bords des chemins, surtout dans la région méridion.; remonte la vallée du Rhône jusqu’à Condrieu, Vienne, Lyon et Crímieu. - Nul ou très-rare dans la partie septentr.

Var. alsinefolium D. C. - Sables marit. du littoral méditerranéen.

P. peploides D. C. - Rochers marit. du littoral de la Provence et du Roussillon.

LEFLINGIA hispaniea L. - Lieux sablonneux.-Gard : bois de Broussan près Nìmes, bords du Gardon à Saint-Nicolas, Manduel, la Capelle. - Hér. : Vendre, Agde au cap Brescou, Cette aux Onglous. - Aude : Saint-Crescent, Aussières, Gaussan, Sainte-Lucie.-Pyr.-Or.

TELEPMI U M Imperati L-D Débris de rochers. - Jura : rochers de Gily près Arbois. - Valais: Saillon, Erdes, mont d'Orge, Tourbillon, Saint-Leonhard, les Plâtrières, Vercorin, Iserable. -Dr. : Nyons. - Vaucl, : Flassan, Bedoin.-B.-Alp. : Gréoulx, sur les bords du Verdon, le Châtelard, la Condamine, Jausiers, Annot, Sisteron. - H.-Alp. : Séuse, Grande-Crigne, Charance, Briançon. - B.-du-R $h$. : Vauvenargues au vallon des Masques, ravin oriental de Gardelaban, N.-D.-des-Anges, Pourrière, Arles, Sainte-Victoire à Saint-Antonin, les Alpines à SaintRemy. - Alp.-Nar. : Saint-Martin-d'Entraunes, col de Brouis, Saorgio, Briga, Tende, Levens, Utelle, vallon de Chaus, la Croix, Rimplas, Escales d'Aiglun. - Hér. : rochers de Saint-Chinian. - Aude : la Clape, Pech-de-l'Agnel, Pas-du-Loup de Sigean, la Nouvelle, Roquefort, îles de Leucate et de la Sidrière, Fitou, 
vallon du Ripau sur le Saint-Victol', col d'Estrem. - Pyr.-0r. : pont de la Fou près Saint-Paul-de-Fenouillet, Saint-Antoincde-Galamus, Cases-de-Pena, vallon de Sainte-Gatherine près Baixas, de Vingrau à Rivesaltes, bas des Albères, Céret, Prades, Trencala d'Ambulla, Vernct-les-Bains, remonte jusqu'à Nyer, Ies Graux d'Oletle et Fontpédrouse.

En dehors de nos limites, cette espece se trouve aussi dans la Lozère sur les rives du Tarn, la Malène, et dans les Pyr. centr. dans la vallée de Gavarnie, Gèdre.

PAItor renLa eymosn Lam. - Lieux incultes des terrains siliceux.- Var et Alp.-Har. : schistes des envir. d'Hyères, Maures du Luc, Fréjus, sables du golfe Juan. — Gard : schistes de Genolliac, Aulas, l'Espérou, le Ranquet, le Vigan. - Hér. : entre Madieres et Navacelle.-Lozère : schistes de Sainte-Croix, SaintEtienne-Valfrancesque, Villefort.

P. cehinata Lam. - Sables du littoral des B.-du-Rh., du Var et des Alp.-Mar. : la Ciotat, presqu'île Cepet près Toulon, Cavalaire, Saint-liaphä̈l, Cannes, Biot, Antibes.-Pyr.-0r. : Casesde-Pena.

P. argentea Lam, - Lieux incultes, - B.-du-Rh. : Saint-Louis, trouvé à Aix dans l'étendoir d'un lavage de laine et dans un jardin du cours Sainte-Anne, colline des Trois-Moulins.- Var : Sainte-Baume au Pilon. - Gard : montagne de Saint-Roman entre Jonquières et Beaucaire. - Hér. : Agde, versant ouest de la montagne de Celte, les Onglous, plage de Sérignan. - Aude et Pyr.-Or. : dunes du littoral, Leucate, la Clappe, Fontfroide, Corbières, vallons de Banyuls-sur-Her et de Collioure, SaintAntoine-de-Galamus.

P. polygonifolia D. C. - Pàturages des terrains siliceux des Alpes, des Cévennes et des Pyrénées. - Is $s_{n}$ : chaine de Belledonne à la Pra, Champrousse, Gr.-Vaudaine, Gr.-Rousses audessus d'Huez. - H.-Alpes : la Grave, Lautaret, Galibier, mont Viso à la Traversette, mont Chaillol, col de Vars. - Sav. : SaintSorlin-d'Arves.-B.-Alp. : la Condamine, Sérenne.-Alp.-Mar. : la Briga, vallée de Rabuons, vallon d'Entrecoulpes. - Ard. : Mayres. - Gard : l'Espérou, Alzon, la Baraque-de-Ribaud. - 
Hẻr. : Ie Caroux, Douch au vallon d'Eric, le Saumail, la Salvetat, Fraisse, Saint-Amand-de-Mounis. - Pyr.-Or. : massif granitique du Canigou, Font-de-Comps, mont Louis sur le chemin de Font-Romeu, garrigues de Thuir vers Castelnau.-En dehors de nos limites dans les Pyr. centr.; Lozẻre; dans les massifs granitiques et schistcux de l'Aigual, bords du Tarnoll, Massevaques près Vebron, Saint-Étienne-Valdonesse, lo Born près Mende; entraîné par l'Allier jusque sous le pont de Cournon dans le Puy-de-Dome; Tarn dans la Montagne-Noire à Ia montagne de Nore, roc de Peyramos. - Nul sur les terrains calcaires.

F. eapitata Lam. - Collines pierreuses des provinces mérid. Dr. : le Buis.-B.-du-Rh. et Var : la Pioline près Aix, Pilon de la Sainte-Baume, Lagoubran prés Toulon, Fréjus.-Gard : bords du Gardon entre Saint-Nicolas et la Baume, Blauzac. - Aude : la Clappe, Pech-de-l'Agnèle, lac de Quérigut dans le Donézan. - Pyr.-0r. : Saint-Laurent-de-Cerdans, Nohèdes, mont Louis. - Pyr. centr.

P. สerpyllfolla D. C. - Is. : en face des Fraux près le Freney, du Freney à Bourg-d'Oisans, Mizoën. - Sav. : Saint-Jean-deMaurienne.-H.-Alp. : la Roche-des-Arnauds, Manteyer, Avançon, la Saulse, Tallard, Lardier, Laragne, col de Vars, col de Casset près Montmorin.-- B.-Alp. : col de Vergous près Annot, Jauziers, la Condamine, graviers de l'Ubaye.-Alp.-Mar. : Entraunes, entre Puget-Théniers et Guillaumes, Touët-de-Beuil, Roubion, Carlin près Tende.- Vaucl. : mont Ventoux. - Pyr.Or. : Cambres d'Aze, vallées d'Eyne, de Llo et de Vernet-lesBains, Llaurenti.

P. niven D. C. - Collines pierreuses de la région mérid. - B.-duRh. : Roquefavour vers la maison du garde, Châteauneuf, Montredon. - Gard : bords du Gardon entre Saint-Nicolas et la Beaume, Blauzac, Villeneuve en face d'Avignon.- Her. : Montpellier à Lavalette, Caunclle, la Madeleine, Mireval, Fabrègues, Saint-Georges, Poussan, Pignan, Saint-Jean-de-Védas, Castries, Sussargues. - Aude : la Clappe, Villeneuve, île Sainte-Lucie, la Nouvelle, entre Saint-Laurent et Leucate. 


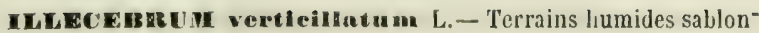
ncux ou argileux; nul sur les calcaires. - Arr. de Belfort et H.-Sàne; vallée du Puix, zone vosgienne à Champagney, Plancher-Bas, Chagey, Baslières, Fresse, vallée de Mansvillers, les Guidons près Mélisey, Servance.- Jura : granite de la forêt de la Serse, Mont-sous-Vaudrey, dipôts siliceux des forêts de Chaux, Rahon, Pleurre, Neublans. - Ain : dépôts argilo-siliceux de la Bresse et de la Dombes autour de Bourg, Pont-de-Veyle, Chatillon-les-Dombes, Marlicu, Chalamont, le Plantay, Beaumont, Saint-Germain, Montribloud. - Morvan granitique dans C.d'Or et S.-et-L. - Rh. : chaine granitique du Beaujolais depuis Chenclette jusqu'à Alix.-Is. : sables de Charelte près Crémieu, Eyloche, Saint-Elienne-de-Saint-Geoirs, Chambaran. - Gard : sables granitiques des envir. du Vigan à Alzon. - Hér. : la Salvetat, Fraisse, Andabre-Rosis, Saint-Amand-de-Mounis, Douch, l'Escandorgue à Romiguière.-Pyr.-0r. : sables des env. de Terrats, Llupra, Fosse, Boucheville entre Saint-Paul et Caramany, Llaurenti. - En dehors de nos limites dans les Pyrén. centr., la Montagne-Noire, le plateau granitique et volcanique du centre de la France.

HERNAMEA glabrat L. - Champs sablonneux dans tout le bassin.

H. hirsuta L.-Même dispersion.

Ir. elnerea D. C. - Champs sablonneux de la région mérid. Vaucl. : le Rocher près d'Avignon.-B.-du-Rh. et Var.-Gard : les plaines jusqu'à Aumessas au pied de l'Espérou.- Hér. : env. de Montpellier et de Cette-Pyr.-Or. : vallée du Réart.

II. Incana Lam. - Terrains arides surtout dans la région mérid. - Partie mérid. de la Drôme et des H.-Alp. jusque vers Die et Aucelon, ainsi qu'à Charance près Gap, mont Séuse, Roche des Arnauds, Rosans, Guillestre, roule du mont Genèvre près Briançon, Lautaret. - Is. : Huez, - Partie mérid. des B.-Alp. jusqu’a Annot. - Vaucl. : Moriẻres, Gadagne, Flassan au pied du mont Ventoux.-B.-du-Rh. et Var.-Alp.-Niar. : Levens, Touët-deBeuil, Roubion, Saint-Dalmas-le-Sauvage. - Toute la partie basse du Gard, Hérault, Aude, Pyr.-Or.; s'člève dans l'Hérault 
jusqu'au Larzac, au Caylar et à la Vacquerie. - Trouvé accidentellement autour de Lyon à Villeurbanne, Beaunant. - En dehors de notre bassin dans la Lozère autour de Mende.

H. Iatifolia Lap, - Terrains sablonneux des Pyr.-Or,; Argelès-surMer, Collioure, Banyuls-sur.Mer, vallée de Prades jusqu'd̀ Olette. - Assez commun dans les Pyr. centr. - A été trouvé dans l'Ardèche, près de Vals et d'Entraigues.

M. apinn Vill. - Lieux sablonneux des Alpes et des Pyrẻnées. Valais : Zermontana, Diez, Cotter, Sasseneire, Zermatl, Saas, Gonthorn, val de Binn, Griess, Grengiols. - Sav. : la Galise, mont Cenis. - Is. : la Pra de Belledonne.- H.-Alp. : la Grave, Lautaret, Galibier, plaine du Bourget au-dessus de Cervières, val Préveyre, col de la Croix, mont Viso à la Traversette, col de Ruines, Clausis près Saint-Véran, Orcières. - B.-Alp. : mont Pelvat.- Alp.-Mar. : cols de Fremamorte et de l'Enchastraye. - Pyr.-Or. : Cambres d'Aze, vallẻe de Llo. - Est quelquèfois charriẻ dans les plaines par les torrents, comme par exemple à Grenoble sur les alluvions du Drac. - Manque dans les Pyr. centr., le centre de la France, le Jura et les Vosges.

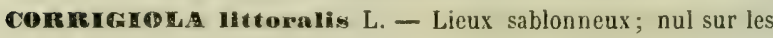
calcaires. - Zones vosgienne et sous-vosgienne de H.-Saðne et arr. de Belfort. - Doubs et Jura : terrains sablonneux de la plaine, surtout dans la Bresse et sur les granites de la forèt de la Serre.-Terrains granitiques de C.-d'Or, S.-et-L., Beaujolais, Lyonnais, Pilat, Forez, Vivarais et Cévennes. - Nul dans le Valais; rare dans le bassin du Léman, sur la mollasse gréseuse des envir. de Vevey. - Is. : sables tertiaires d'Eydoche, Champier, Mollard de Décines.-H.-Alp. : Manteyer.-Dr. : granites des envir. de Saint-Vallier. - Rare dans les B.-du-Rh. sur les cailloux quartzeux des envir. d'Arles en Coustiero.- - Var : sables siliceux des Maures d'Hyères et du Luc, Fréjus. - Dépôts caillouteux du Gard et de l'Hérault autour de Montpellier à Lamoure, Saint-Bauziile-de-Putois, Pézenas, Autignac, Béziers, Roquehaute, s'èlève dans les montagnes à la Salvetat, Fraisse, SaintAmand, Castanet, Andabre, Bousquet d'Orb, Avène, Lamalou, Pardailhan.-Terr. sablonn. des Pyr.-Or. et centrales, ainsi que 
dans tout le massif granitique et volcanique du centre de la France; vallées granitiques des Vosges.

C. relephilirolia pourr. - Terr. sablonneux de la région mérid. - Var : le Lavandou, Fréjus. - Alp.-Mar. : Agay, cap Croisette près Cannes.- Gard : terr. granit. les envir. du Vigan, SaintMartin-de-Salagosse, Alzon, Aulas. - Hér. : Fos, Faugères. Aude : sables marit. des lles de Leucate et de Sainte-Lucie, Villeneuve, col d'Estrem. - Pyr.-0r. : Perpignan, plage de Barcarès, Banyuls-sur-Mer, Boulou, Prades, Vernet-les-Bains, SaintVincent.

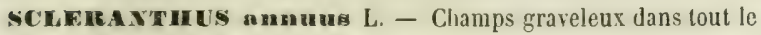
bassin. Espèce des plus polymorphes.

S. uncinalus Schur. - Terres incultes. - Gard : Aumessas, l'Espérou près Valleraugue. - Lozère, Puy-de-Dôme, mont Dore.

S. hamosus de Pouzolz. - Sables des torrents autour de Dourbie et à l'Espérou.

S. verticillatus Tausch. - Champs pierreux. - Envir. de Genève au Plan-des-Ouates, bois de Bay. - Valais : Branson. - H.-Sav. : sommet du Petit-Salève.-Partie mérid. de la Drôme vers SaintVallier, Die, le Buis. - Var : le Luc. — Gard : Grau-d'Orgon près Aigues-Mortes. - Hér. : Saint-Aunès, Roquehaute, Pẻzenas, Montagnac, l'Escandorgue. - Aude : Narbonne, Fontlaurier, vallée de la Boulsanne. - Pyr.-Or. : Sarrat d'en Vaquer près Perpignan, Château-Roussillon, Banyuls-sur-IIer.

Il y aurait lieu d'examiner si le $S$. verticillatus est le même yue le $S$. Delorti Gren., comme le veulent plusieurs botanistes. M. Lamotte met en doute leur identité.

S. marginellus Rchb.-Valais à Branson.

S. biennis Reut. - Grenier et quelrues autres botanistes soutiennent que le S. biennis n'est autre que Ie S, annuus, dont la maturité aurait été retardẻe par le froid et qui hivernerait et ne fructifierait que l'année suivante. A élé observé dans les envir. de Genève, au Salève, aux Voirons, à Conflans près Albertville et dans la Hauteluce; dans les Alpes de Morcles (Vaud), dans l'Isère au-dessus de r'rémol, Saint-Nizier, Chambaran; dans la Loire, au-dessus de Rochetaillée. 
5. perennis L. - Sables des terrains siliceux depuis les plaines jusque dans les hautes vallées. Nul sur les calcaires; ainsi dans la chaine jurassique on ne le trouve que sur l's sables granitiques de la forêt de la Serre. Dans la région mérid., on ne l'observe que dans les contrées montagneuses de l'Espinouse, l'Escandorgue, la Hontagne-Noire, la haute vallée de l'Aude.-Commun dans les terrains siliceux des Pyrénées, du centre de la France el des Vosges.

\section{CRASSULACÉES.}

TLLLEA muscosa I. - Terrains sablonneux surtout dans la région mérid. - Observé exceptionnellement dans la $\mathbf{C}$ - d'Or près de Saulieu sur les bords de l'élang de Vermouraux et dans l'Ain à Pont-de-Vaux. - Var : Hyères, le Luc, Fréjus. - Alp.Mar. : Cannes, le Biol, Antibes, Sainte-Hélène près Nice, Menton derriêre le Pigautier.-Gard : envir. de Nimes et du Vigan. - Hér. : près de Montpellier à Grammont, Doscares, SaintAunès, Roquehaute, Pézénas. - Aude : Aussières, Verdun, Ferrals. - Pyr.-0r. : Argelès-sur-Mer.

BULLA IEDA Vailantí D. C. - Mares dans les terrains sablonneux. - C.-d'Or : indiqué par Loret près des étangs de Saint-Léger où il n'a pas été retrouvé. - Var : Maures du Luc, Fréjus. - Hêr. : mares de Roquehaute à Portiragnes.

SEDU⿴囗十 Thodioln D. C. - Débris de rochers des Alpes et des Pyrénées. - Valais : près du lac Mattmark, de Zermatt à Findelen, pied de l'Albrun. - Sav. : entre Bonneval et la Levanna, sources de l'Arc. - Is. : au-dessus de Bovinant au pied du GrandSom, Charmant-Som. - H.-Alp. : mont Viso, vallons des Vaches et de Ségure, Saint-Véran à Blanchet.-B.-Alp. : col de Stropi. - Alp.-Mar. : mont Bego, col de l'Abisso, envir. du lac d'Entrecoulpes. -- Aude et Pyr.-Or. : le Llaurenti, Saint-Marsal, sommet de vallée de la Tet près d'Escaro. - Pyr. centr.

5. maximum Suter. S. Telephium L. - Rochers siliceux; nul sur les calcaires; ne sc trouve qu'en un seul lieu de la chaine jurassique sur les alluvions des environs de Thoirette, vers les con- 
fins de l'Ain et du Jura. - Commun dans les Vosges granitiyues et arénacées. - Chaine granitique qui s'étend depuis le Morvan à travers le Beaujolais, Lyonnais, Forez, Pierre-surHaute, Pilat, inontagnes vivaraises et cebennoles jusque sur l'Espinouse, l'Escandorgue et la montagne Noire. - Valais : grès talqueux de Naters à Blatten. - II.-Sav. : grès mollassique du vailon des Usses près de la Caille et d'Ayse près Bonneville, Veyrier, Talloires. - Sav. : Saint-Jean-de-Haurienne. - Is. : rochers gneissiques du Rivier d'Allemont et de la vallée de la Romanche entre Séchilienne et Gavet. - Grès nummulitique des B.-Alpes vers Annot. - Dr. : Aucelon. - Alp.-Mar. : château de la Garde près Villeneuve-Loubet, Vence, bords de la Siagne, Saint-Martin. - Aude : Escouloubre, Mijanès dans le Donézan, Montolieu sur les granites de la montagne Noire. Pyr--?r.: terr, siliceux de Saint-Laurent-de-Cerdans, vallẻe du Tech jusquu'au-delà de la Preste, mont Louis vers le moulin de la Llagone.

En dehors de notre bassin sur les rochers volcan. et gran. du Puy-de-Dóme, Tarn, Pyrénées centr.

Fries a démontré quo la planto do Suède appeléo $S$. telephium par Linné et Wahlenberg est la même que celle qui a été, plus tard, nommée S. maximum par Suter et qui, est caractérisée par les feuilles supéricures en coeur et presque amplexicaules, les feuilles inférieures sessiles. Cependant je crois que le nom linnéen doit être abandonné parce qu'il a été appliqué par un grand nombre d'auteurs pour désigner les S. purpurascens, S. fabaria Koch, ainsi que plusieurs autres formes voisines de celles-ci. Le nom de Telephium pourra être employé pour dénommer la première section du genre Sedum, ainsi que l'ont déjà fait plusieurs auteurs, Koch entre autres.

Les formes suivantes, décrites par MM. Jordan et Fourreau, ont été démembrées du $S$. maximum.

S. pachyphyllum. - Rochers granitiques d'Ámélie-les-Bains (Pyr.-0r.).

S. erubescens. - Rochers granitiques du Canigou (Pyr.-Or.).

S. cebennense. - Rochers granitiques du Vigan (Gard).

S subrotundum. - Rochers granitiques de Soucieu (Rh.).

s. apricum. - Rochers graniliques de Vienne (Is.).

S. preruplorum. - Rochers granitiques de Lyon.

S. recurvum. - Roshers granitiques de Francheville (Rh.). 
S. chloroticum. - Rochers granitiques de Saint-Gervais (H.-Sav.).

S. assurgens. - Rochers granitiques de Saint-Didier (Rh.).

s. purpursecens Koch S. telephium G. et G. Flore de Fr. (non L.). - Rochers et débris pierreux dans la plus grande partie de notre bassin, excepté dans les régions basses de la Provence, des Alp.-Mar., du Languedoc et du Roussillon; dans ces provinces on ne le voit que dans les montagnes; ainsi dans les Alp-Nar. à Saorgio et sur les bords de la Siagne; dans l'Hér. sur l'Espinouse à la Calmette, Fraisse, la Salvetat, Madeleine-de-Mounis, le Caylar; enfin dans le Roussillon à mont Louis, Fonpédrouse, bords de la Rigarda vers Gourg Colomer.

Espèce polymorphe à laquelle se rattachent les formes suivantes décrites par MM. Jordan et Fourreau.

S. rhodanense. Roch. calc. de Ser- S. buxicolum. Roc. cal de Cornod. rières (Ain).

S. subalbidum. - ibid.

S. beugesiacum. Roch. calc. de Vi- S. Navieri. - ibid.

rieu-le-Grand (Ain). • S. dumelicolum. - ibid.

S. procelsum. Roch. calc. de Hau- S. lugdunense. Caluire (Rh.).

teville (Ain).

S. pycnanthum. - ibid.

S. convexum. St-Germain-Montd'Or (Rh.).

S. saxifragum. - Cornod(Jura) S. rupivagum. Rochers calc. de

S. viridulum. - ibid.

Thoiry (Ain).

S. monticolum. - ibid.

Il me semble que c'est aussi du S. purpurascens qu'il faut rapprocher les formes suivantes, décrites par MII. Jordan et Fourreau, formes qui établissent le passage du S. maximum au S. purpurascens.

S. delphinense. Roch. calc. de St- S. serolinum. Roch. calc. de DiMartin-le-Vinoux (Is.). gne (B. -Alp.).

S. Verloli. Roch. calc. du mont S, collinum. Roch. calc. de CréRachais (Is.) mieu (Is.)

S. alpestre. Roch, calc. de Mones- S. Millieri. Roch.calc.Culoz(Ain). tier-de-Briançon (H.-Alp.).

S. fabaria Koch. - Diffère du type précédent, dont il ne serait qu'une variété suivant plusieurs auteurs, par les feuilles toujours éparses, atténuées en coin à la base et brièvement pétiolées; 
les étamines du rang inférieur sont insérées plus haut que dans lo S. purpurascens.

La dispersion géographique du S. fabaria est mal connue.

s. anacampseros $L$. - Débris de rochers des Alpes et des Pyrénées. - Nul dans les Vosges et le Jura. - Vaud et Valais: montagnes d'Aigle, Bagnes, Saint-Bernard, Alesse, Thyon, Tortain.-H.-Sav. : mont Méri, Flaine, la Diosa, val Montjoie aux Contamines et au Plan-des-Dames. - Sav. : le Mottet, Golet, Beauprẻ, les Allues, Crêt-du-Rẻ près d'Aime, lac du Cormet, Laval-de-Tignes, Saint-Sorlin-d'Arves, mont Cenis. - Is.: clıâne de Belledonne vers le lac du Crouzet, mont de Lans, mont Lalley, mont Chamoux au-dessus de la Salette. - H.-Alp. : Lautaret, Monestier-de-Briançon, mont Genèvre, Bramousse audessus de Château-Queyras, Val-Préveyre, vallon des Vaches, col de Var's, Orcières, Champoléon, mont Queyras, Pleyne de Chaudun. - B.-Alp. : Larche, Lauzannier, Parpaillon, mont Couyer sur Annol. - Alp.-Mar. : val Pesio, N.-D. de Fenestre, SainteAnne-de-Vinai, montagnes d'Entraunes. - Aude et Pyr.-Or. : Quérigut, Llaurenti, fosse du Géant au-dessus de la vallée de Prats-de-Balaguer, île du moulin de la Llagone près mont Louis.

- Pyr. centr. dans la vallée d'Aure près de Vielle et vers les bains de Penticosa sur le versant espaggnol.

s. stellatum L. - Rocailles de la région littorale. - Var : SaintTropez. - Alp.-Mar: Cannes, Antibes, Biot, Nice, Menton.Pyr.-Or. : Collioure, les Albères, N.-D, de Vie au-dessus d'Argelès. - Pyr. centr. sur le pic de Gard et à Saint-Bẻat.

S. cepaea L. - Lieux frais et ombragẻs. - Très-rare dans les régions vosgienne et jurassique; dans cette dernière on ne le voit que vers Bletterans entre Coges et Montconny. - Nul dans le Valais, - Envir. de Genève à Vernier, route de Versoix et de Coppet, Petit Sacconex, - Ain : Ferney, Thoiry. - H.-Sav. : Sillingy. - S.•et-L. : envir. de Mâcon, Cluny, Issy-l'Évêque. Commun dans les envir. de Lyon et en descendant le long du Rhône dans les départements de la. Drôme et de l'Ardèche. Is. : de Voreppe à Chalais, Vizille, de Vaulnaveys à Prẻmol, Saint-Geoirs. - Var et Alp.-Mar. : l'Esterel, Clans, Venanson, 
Auribeau, Villeneuve, Menton. - Gard : le Vigan, Valleraugue, Aujac. - Hẻr. : Andabre, Saint-Gervais, Avène, Bédarrieux, Saint-Geniès-de-Varensal, Fraisse. - Pyr. centr. et centre de la France.

S. alsinefolium All. - Diffère du S. ceprca par son inflorescence longuement paniculée, ses fleurs à pédicelles longs et grêles, ses feuilles oblongues obovales. - Alp.-Har. : rochers ombragés de la vallée de Caïros, Saint-Hartin-Lantosique, Tende. - Col de la Croix sur le versant piémontais du mont Viso en descendant dans le val Pelice.

S. rubens L. - Lieux graveleux. - H.-Saône : Noidans, Vaivre, la Motte-de-Vesoul, Chargey, St-Germain. - Jura : Dole, Chaussin, Lons-le-Saunier, Salins. - C.-d'Or: Dornoy, Auxonne, Brazey, bois de Norges. - S.-et-L. - Vaud et envir. de Genève : Lausanne, Romainmotier, Rolle, Nyon, Versoix, Vernier. H.-Sav. : Saint-Julien, Neydens, Archamp, Sillingy.-Ain : envir. de Belley, Meximieu, Thoissey, le Revermont. - 1ht. : Ecully, Charbonnières, Chaponost, l'Argentière, Beaujolais. - Sav. : Saint-Innocent.-Is. : entre Vaulnaveys et Prémol, Saint-Égrève, Saint-Étienne-de-Grossey, Châteauneuf-d'Isère, Saint-Geoirs, Diemoz. - B.-du-Rh. : Saint-Pons, - Var: le Luc, SaintRaphaël. - Alp.-Mar. : Antibes, Berre, Nice. - Gard. - Hẻr. : la Sérane, Capouladoux, Saint-Guilhem-le-Désert, Ganges, Murviel, Castelnau, Saint Ifartin-d'Orb, Avène. -Aude: la Clappe, Corbières. - Pyrénées centr., centre de la France.

S. pallidiflorum Jord. Fourr, - Rh. : Quincieux.

S. medilerraneuin J. F. - Alp.-Mar. : Cannes.

S. cospitosum D. C. - Rocailles de la région mérid. - Var: les Pesquiers, Bagnols, le Luc.-Alp.-Har. : Cannes, Biot, Antibes, remparts de Nonaco. - Gard : bois de Broussan et de Cygnan près Nìmes, bords du Gardon. - Hér. : euvir. de IIontpellier à Grammont, Juvignac, Poussan, Saussan, Murviel, Mireval, Agde, Fabrègues, Pézenas, Capouladoux, Mas-de-Londres, Saint-Martin, Pardailhan. - Aude : entre Lastours et Villardonnel. Manque dans les Pyrénées.

S. atratum L. - Rochers et pâturages. - Nul dans la chaine des 
Vosges. - Sommités du Jura helvétį̣ue et français. - Vaud et Talais: depuis les montagnes de Bex, d'Aigle et de Fully jusqu’à la Gemmi eł au Grimsel, dans la chaine mérid. depuis le Grand-Saint-Bernard jnsqu'au mont huse et au Saint-Gothard. - H..Sav. : Brizon, Vergy, montagnes du Chablais, dents d'Oche, mont Ardin, mont d'Ubine, roc d'Enfer, abbaye de Vallon, mont Ilermante, Flaine, Sixt et le Buet, toute la claine du mont Blanc depuis le col de Balme jusqu'au Bonhomme, Tournette, Charvin. - Sav. : sommités de la 'Tarentaise el de la Maurienne jusqu'au mont Cenis, montagnes des Aravis, des Bauges, d'Hauteluce et de Beaufort. - Is. : chaines des Sept-Laux, de Belledonne, des Grandes-Rousses, du mont de Lans jusifu'au Pelvoux, GrandSom, la Moucherolle. - H.Alp. : la Grave, Lautaret, Névache, mont Ginère, col Malrif, mont Viso, col de Ruines, col Agnel, Saint-Yéran à Marcel et à Longet, Aurouse, Chaillol-le-Vieil, le Noyer, mont Durbonnas, mont Queyrel près Saint-Bonnet, col de l'Alp-Martin, pic de Chabrières, col de Glaise, mont Séuse.

- Dr. : mont Glandasse. - B.-Alp. : sommet de la Tourtelle, Grand-Couyer sur Annot, Larche, Vallonnet, Bachasse, Cuguret. - Vaucl. : mont Ventoux. - Alp.-Mar.: montagnes d'Entraunes, Saint-Etienne, col de Jallorgues, Estenc, col de Tende. -Aude et Pyr.-0r.: col de Pailhères, Llaurenti, Canigou, Saint-Martin, vallée de Fillols, montagnes au-dessus de Consolation et d'Argelès, mont Louis. - Toute la chaîne des Pyr. centr. - Manque dans le massif central de la France.

s. ภnnumm L. - Rochers siliceux. - Nul dans la chaine jurassiques et dans les autres massifs calcaires. - Commun dans les vallies des Vosges granitiyues et arénacíes jusque dans la zone vosgienne de la H.-Saône et de l'arrondissement de Belfort, vallée du Rahin jusqu'à Plancher-Bas, mont de Plancher, Plancherles-Mines, Tète des Noirs-Etangs près Château-Lambert, vallée supérieure de l'Ognon et de la Savoureuse. - Vaud et Valais : gris, gneiss et granites de Dent de Morcles, Fully, Bagnes, Evolena, Saas, Zermatt. - H.-Sav. : grès nummulitique du Vergy, Méri, Voirons, dents d'Oche, Cornette-de-Bise, Colone, Tourrette, Charvin, la Vauzalle près Sixt, protogyne et schistes cris- 
tallins de la chaine du mont Blanc où il s'élève jusqu'au jardin de la Mer-de-Giace. - Sav. : sclistes cristallins des montagnes au-dessus de Conflans, d'Hauteluce et d'Aresches, les Allues, granite de la partie supér. de vallée de l'Arc en amont de Bonneval, combe de Villette près Bramans. - Loire : granite du Pilat au Pas-du-Riot, Saint-Régis-du-Coin. - Is. : gneiss et granite des chaînes des Sept-Laux, de Belledonne et de l'Oisans jusqu'au Pelvoux, schistes siliceux du Valjouffrey jusqu'au mont Chamoux près de la Salette, Chaillol-le-Vieil, Champoléon, mont de l'vurs, mont Queyrel, massifs granitiques du Briançonnais du Queyras et du mont Viso. - B.-Alp. : grès nummulitique de la vallée de l'Ubaye, Saint-Ours, lac d'Horonaye, Lauzannier. - Alp.-IIar. : massifs cristallins de Saint-Dalmas-leSauvage, col de Jallorgues, Saint-Anne-de-Vinai, col de Fenestre, Saint-Martin-Lantosque, Tende.-Aude et Pyr.-Or. : vallée de la Bruyante, Llaurenti au Bentaillole, Costa-Bona, vallée de Fulla, Escaro, fond de la vallée de Valmanya, Canigou. Pyrén. centr. - Rorhers granit. et volcaniques du mont Dore, Cantal, H.Loire notamment au Mezenc et dans l'Ardèche vers Cuze, Entraigues, roche de Gourdon._Gard ct Lozère : massifs granit. et schisteux de l'Espérou, Aigual, Hort-de-Diou, mont Lozère, Massevagnes, - Chaîne du Forez.

s. villosum L. - Prés humides des terrains siliceux et marais tourbeux des montagnes. - Commun dans les Vosges granit. et arénacées. - Très-rare dans les tourbières à sous-sol siliceux du Doubs autour de Pontarlier, Malpas et sur le versant helvétique à Porentruy; manque dans les tourbières du département du Jura. - Valais : Novelly de Nendaz, Essaz, Arolla, Zermatt, Saas, Tortain, Gruben, Simplon. - H.-Sav. : mares sur les sables sidérolitiques de la Croisette au Salève. - Sav. : molasse des bords du lac du Bourget. - Is. : sables du grès vert de la Fauge près du Villard-de-Lans, Corençon, sables molassiques de Châbons. - C.-d'Or et S.-et-L. : sables granit. du Morvan à Saulieu, Champeaux, Saint-Léger, Roche-en-Brenil, Vic-sousThil, Autun, Selle d'Auxy. - Rh. : chaines granit. du Beaujolais et du Lyonnais à Chenelette, Saint-Bonnet-le-Froid, Ise- 
ron. - Loire : chaines granil, du Forez, Pierre-sur-Haute, Pilat. - Ard. : terr. granit. et volcaniques des sources de la Loire, Mauras, Cuze. - Gard : Aigual, Hort-de-Diou. - Aude : vallée de la Boulsanne, Boutairol, Quérigut, Llaurenti. - Pyr.Or.: vallẻe de la Tet, mont Louis, Nohẻdes, Solanette près Pierrefeu, Coma du Tech. - Granite et terr. volcan. du centre de la France.

Lo S. villosum, bien que préférant les montagnes, peut aussi so montrer dans les plaines lorsqu'il y trouve le terrain qui lui convient. C'est ainsi qu'on lo voit sur les ssbles ot argiles tertiaires de la Bresse, entre Pont-de-Vaus et Bagé, dans la plaine du Forez vers Grézieu-le-Fromental. Déjà, au siècle dernier, Hermann avait remarqué sa présence sur les sables siliceux de la plaino de Haguenau, on Alsace.

S. Hirsutum All. - Rochers siliceux des montagnes. - Nanque dans les Vosges, le Jura, Bugey, Valais, Alpes françaises et génćralement dans toute la partie orientale de notre bassin. Le centre de son aire de dispersion se trouve dans les massifs granitiques et volcaniques du Puy-de-Dôme, Cantal, H.-Loire; de là il s'étend dans les chaines granitiques et schisteuses du Forez, Pierre-sur-Haute, Pilat, Allicr, montagnes de Tarare vers Panissières, puis dans les montagnes du Vivarais à la Roche-de-Gourdon, Cheylard, Vals, rochers d'Avran, Saint-Agrève, le Mezenc; sur les rochers schisteux de la Lozère; dans les Cévennes schisteuses et granitiques du Gard, autour d'Alais, Anduze, Saint$\Lambda$ mbroix, dans l'Hérault à Saint-Gervais, l’Espinouse, Lamalou, Saint-Ettienne-de-Mursan, Grayssessac; sur les deux versants de la Montagne-Noire dans l'Aude et le Tarn, et en outre dans l'Aude sur les flancs des Corbières à Lanet, Bugarach; enfin dans les Pyr.-Or. dans la vallée de la Tet à Font-Romeu, GourgNègre, Collioure au-dessus de Consolation. - Fréquent sur les rochers siliceux des Pyr. centr.

S. cruciatum Desf.-Rochers, - Cette espece, assez commune en Corse, se trouve dans les B.-Alp. à Colmars, mont Monnier. B.-du-Rh. : Sainte-Victoire à la Bouche-du-Garagay.

s. album L. - Espèce polymorphe très-abondante sur les murs et rochers et à toutes les altitudes. 
S. micranthum Bast. - Forme du précédent dont toutes les parlies sont de moitié plus petites. - H.-Sav. : vallée de Chamonix. - Is. : rochers de Bovinant au pied du Gr.-Som. - Sav. : mont Trélod, mont Drizon, la Sambuy.-II.-Alp. : Villard d'Arìne, Loubet et Chaudun près Gap, Saint-Mens, mont Séuse, mont Queyrel, mont Morgon, mont Cliabrières. - Ard. : envir. de Privas._Gard : pont de Montdardier à Avèze.-Hẻr. : roch. dolomitiques du Larzac. - Aude : Narbonne, versant mérid. de la Montagne-Noire. - Pyr.-Or. : vallées de Vernet-les Bains et de Castell, Saint-Martin-du Canigou.-En dehors de nos limites dans le Puy-de-Dôme, le Tarn.

S. anglleam Ifuds. - Débris pierreux, schisteux et granitiques.Hér. : l'Espinouse, Colombières, Saint-Bauzille près Saint-Pons, Andabre-Rosis, Fraisse, Pardailhan. - Aude et Pyr.-0r. : Montagne-Noire, vallée de la Bruyante, Llaurenti, N.-D.-de-Vie près Argelès, chaine des Albères; remonte dans la vallée du Tech jusqu'ả Prats-de-Hollo. - Toute la chaîne des Pyrẻn. centr.

S. dagyphyHum L.- Nurs et rochers depuis les plaines jusque dans les hautes vallées des montagnes.

S. brevifolium D. C. - Rochers siliceux. - Gard : Hort-de-Dion près de l'Aigual.—Hér. : Pardailhan.—Aude : versant mérid. de la Montagne-Noire autour de Mas-Cabardès, vallée de la Boulsanne, lac de Quérigut, Puilaurens près Axat.-Pyr.-Or.: Font-Romeu, moulin de la Llagone près Montlouis, Canigou, vallées de Vernetles-Bains et de Castell.-En dehors de nos limites sur les rochers siliceux des Pyr. centr.; Lozère près le pont-de-Montvert, la Borie près Vialas. - Tarn : rochers gneissiques de Moularès, Pampelonne, Mlazamet dans la Montagne-Noire.

s. alpestre Vill.- Rochers siliceux des montagnes. - Nul dans le Jura et généralement sur les terrains calcaires.-Valais: SaintBernard, Esserz, Arolla, mont Nuoble, Maya. - H.-Sav. : grẻs de la clieminée du mont Méri, grotte de Balme, Flaine, schistes el prologyne de la chaine du mont Blanc jusqu'au Jardin-de-laMer-de-Glace, les Montets près d'Argentière.-Sav. : montagnes d'Hauteluce. - Is. : chaine de Belledonne près du lac du Crouzet et de Luitel, Taillefer, massifs des Sept-Laux et de l'Oisans. 
- II.-Alp. : Villard d'Arène, Galibier, col des IIayes, Cerviùres, mont Viso, col de Vars, Chaillol-le-Vieil, mont de l'Ours près Champoléon, mont Clabrières. - B.-Alp. : Lauzannier, Horonaye. - Alp.-Mar. : mont Bego, col de l'Abisso, Saint-Martin, lac al'Entrecoulpes, col de Cérèze, Saint-Dalmas-le-Sauvage. En dehor's de notre bassin sur les rochers siliceux des Pyr. cent., mont Dore, Cantal, Lozire, Vosges dans le vallon du Frankenthat.

s. aere L. - Murs et pierrailles depuis les plaines jusque dans les vallées des montagnes.

S. sexangulare L. - Débris pierreux dans les parties septentr. et moyenne du bassin. - Rare dans la région mérid. autour de Marscille, Lantosuue, col de Tende.-Pyr.-Or. : Prats-de-Mollo cn montant à la Tour-de-Mir, derrière la Preste, Coma-du-Tech. - En dehors de nos limites dans les Pyr. centr. et le Puy-deDôme.

S. reflexam L. - Espicice polymorphe commune sur les coteaux pierreux des parties septr. et moyenne du bassin. - Plus rare dans la région mérid.-B.-du-Rh. : mont Majour près Arles. Alp.-Mar._Gard : Nìmes, l'Espérou, le Vigan.-IIér. : Ganges, Saint-Guilhem-le-Désert, Capouladoux, Fraisse, Saint-Martind'Orb. - Aude : vallée de la Boulsanne, Mijanès, Artigues, bains d'Husson, Montolieu.-- Pyr.-Or. : Oms, plateau de Saint-Marsal, haut de la vallée de Valmanya.- Commun dans les Pyr. centr. et le centre de la France.

S. rupestre L. - Forme glauque du précédent, qui se rencontre surtout dans les expositions très-chaudes, coinme par exemple dans l'Isère et les H.-Alp., à la Bastille de Grenoble, Pariset, envir. de Gap à Rabou, Chaudun, Aurouse, Champoléon, Briançon, en Savoie autour d'Aix-les-Bains; les envir. de Clermont et de Riom dans le Puy-de-Dóme, la Cabarède dans la Montagne-Noire.

Suivant Grenier, la culture dans un lieu abrite fait disparaitro la couleur glauque.

S. albescens IIaw. - Differe du précédent, avec lequel il est souvent mêlé par ses feuilles simplement glaucescentes ou même vertes, et par ses fleurs d'un jaune påle. 
s. elegrnns Lej,— Sables et rochers siliceux. - Envir. de Belfort à Charmont. - II.-Saône : alluvions de l'Ognon, Chassey-lesMontbozon, Froideterre. - Doubs : alluvions des envir. de Besançon. - Jura : autour de Dôle, Azans, Goux, Baverans, forêt granit. de la Serre, calcaire oxfordien à silex de Mesnay prẻs Arbois, diluvium de Grange-Perrey au-dessus d'Arbois. - Granite du Mforvan autour de Saulieu vers Saint-Léger-sur-Bois; du Beaujolais et du Lyonnais à la Roche-d'Ajoux, Charbonnières; du Pilat et de ses contreforts à Malleval, Saint-Julien-HolinMolette.-Ard. : Névissac.-Gard : Concoule.-Hér. : Fraisse, Saint-Geniès-de-Varensal._Aude et Tarn : les deux versants de la NIontagne-Noire. - Centre de la France, Pierre-sur-IIaute.

$S$, aureum Wirtg. - Forme glaucescente du précédent dans les sols arides, secs et bien exposés.

S. altissimum Poir.-Murs et débris pierreux de la région mérid. du bassin. Remonte dans la Dr. vers Crest, Luc-en-Diois, Châtillon; dans les H.-Alp. à Rabou, la Garde; dans l'Isère à la Bastille, Pariset, Sassenage; autour de Lyon sur les digues du Grand-Camp et à Vaux-en-Velin; dans la Savoie au mont du Chat, Chignin entre Arbin et Cruet, Saint-Jean-de-la-Porte, Saint-Jean-de-Naurienne; enfin dans l'Ain à Pierre-Châtel. Pyrẻn. centr. et Lozère.

S. anopetalum D. C. Coteaux secs et pierreux de la région mérid. et moyenne du bassin, dans les expositions chaudes; existe aussi sur le Saleve et dans le Valais ainsi que dans la partie orientale du Jura et du Bugey à Champagnole, Buffard, Salins, Mlolay, de Saint-Claude à Thoirette, Couzance, Pont-d'Ain, Nantua.—Sav. : Saint-Innocent près d'Aix. - Espéce polymorphe.

S. Verloti Jord. - Forme glaucque du prẻcédent. - Coteaux des montagnes calcaires des envir. de Grenoble, mont Rachais, SaintEynard au chemin du Sappey, Quaix; envir. de Gap à la Garde, Combe-Noire, Sappey de la Bâtie-Neuve, mont Séuse.

S. montanum Perr. et Song.-Roch. calc. du mont Rachais et de SaintEynard près Grenoble.

S. aristatum Vill. - Cette forme, indiquẻe par Villars à Sigoyer près Tallard, n’a pas élé revue dans celte localité. 
S. amplexicaule D. C. - Champs pierreux.-Mont Ventoux(Vaucl.), Saint-Guiral, l'Espérou, entre la Baraque de Michel et Camprieux (Gard), - Lozère.

SEMPER VIVUM teetorum L. - Vicux murs, toits; dans les plaines et jusque sur les rochers des montagnes. Espice extrémement polymorphe.

S. juratense Jord. Fourr. - Reculet (Jura).

L. brevirumum J. F. - Ibid.

S. monticolum J, F. - Ibid.

S. prestabile J. F.- Reculet. S, lotevirens J. F.- Ibid.

S. venustum J. F. - Iont Arbei- S. parvulum J. F. - lbid. ran (Sav.).

S. sabaudum J. F. - Mont Vergy (H.-Sav.).

S. modestum J. F.-Monestier-deBriancon.]

S. collinum J. F. - Condrieu (Rh.).

S. beugesiacum J. F. - Virieu-leGrand (Ain).

S. pyrenaicum J. F. - Vernet-lesBains (Pyro-Or.).

S. decoloratum J. F, - Culoz (Ain). S. rhodanicum J. F. - Serrièresde-Briord (Ain).

S. racemosum J. F. - Digne (B.Alp.).

S. columnare J. F. - lbid.

S. validum J. F. - Mont Rachais (Is.).

S. affine Lamotte. - Ilont Viso, Embrun.

S. robustum J. F. - Serriëres-deBriord (Ain).

S. Verloti Lamotte. - Alpe du mont de Lans.

S. Boissieri Schott, - Reculet.

S. Fauconnetti Reut.- Reculet.

S. pallidum J. F.-Monestier-deBriançon (II.-Alp.).

S. luxurians J. F. - Mont Séuse.

S. violascens J. F. - Ibid.

S. seusanum J. F.-Ibid.

S. brachyatum Lamotte. - Mont

Viso, Lautaret.

S. Boutignyanum Bill. et Gr.-Ib. - Quérigut (Aude); Pyr. centr.

S. speciosum Lamotte. - Mont Viso.

S. rigidum J. F. - La Grave.

S. saxosum J. F, - Ibid.

S. Lamottei Bor. - La Limagne d'Auvergne et Allier.

S. arvernense Lec. et Lamotte.-Rochers granit. de la Dr. entre SaintVallier et Tain, roch. granit. et volcaniques du Vivarais, Lozère, Aigual, Aire-de-Caouté, Gd-Lirou. - Hér. : Saint-Amand-deMounis au roc d'Orques,- Cantal; Puy-de-Dôme sur les rochers granit. de Champeix à Saint-Nectaire, valléc de Saint-Floret, 
roch. de Saint-Iroine près Coudes, - Loire : entre le Pertuiset et Saint-Rambert, Bö̈n, Sail-sous-Couzan, vallée de Saint-Justen-Bas, de Saint-Thurin et de Chorsain.

S. vellavum Lamotte. - Roch. granit. - Loire : Boën, Sail-sous-Couzan, bords du Lignon. - H.-Loire : roch. granit. entre le Puy et Brives.

S. lesurinum Lamoltc.- Rochers schisteux au-dessus de Saint-Étiennede-Valdonesse (Lozère),

S. cebennense Lamotte.- Rochers schisteux des envir. du Vigan, Valleraugue, la Grenouille (Gard).

S. anernensi-arachnoideıtm Lamotte. - Roç. granit. - Hér. : SaintAmand-de-Mounis au roc d’Orques. - Puy-de-Dòme, vallẻe de Champeix à Saint-Nectaire.

S. arachnoideo-arvernense Lamolte, - Puy-de-Dóme: roch. granit. de Saint-Yvoine près Coudes: - Cantal : rochers de Bonnerie.

S. calcareum Jord.- Roch. calc. des montagnes.- II.Alp. : Clarance, col de Glaise, mont Clıabrières, Brama-Buou.-Alp.-Mar., Berre, Molinet au-dessus de Menton et montagnes jusqu'au col d'Eze.

S. montanuan L. - Rochers et pelouses les montagnes. - Nul dans les Vosges, la chaine jurassique et le centre de la France.-Alp. de Vaud, Valais, Savoie, Dauphine, Alpes Basses et Maritimes. - Pyr.-0r. : sommets de la vallée de Nohédes, Canigou, Pla Guillem, Costa Bona, Llaurenti. - Toute la chaine des Pyr. centr. - Espèce polymorphe.

S. monticolum Lamotte. - Rochers du mont de Lans et du Lautaret.

S. alpestre Lamotte.- Roch. du mont Viso.

S. frigidum Lamotte. - Mont Viso.

S. piliferum Jord.-Roch. et pelouses.-Is. : mont de Lans, la Salette. - H.-Alp. : Rabou près Gap, Isautaret.

s. arachnoideum L. - Roch. et pelouses des montagnes. - Valais, Savoie, Dauphiné, Alp. Basses et Mar.-Ard, et H.-Loire : Mezenc. - Gard : sommet de l'Aigual, Hér. Saint-Amand-de-Ilounis au roc d'Orques.-Aude et Pyr.-01. : Querigut, Gorge de Boutairol, Llaurenti, la Preste, Prats-de-Hollo, Vernet-les-Bains, mont Louis. - Pyr. centr. : Puy-de-Dòme, Allier, Cantal, Creuse. Nul dans les Vosges et le Jura.

S. globiferum L. - Rochers du sommet de l'Aigual. 
S. Hirtum L. - Rochers des Alp.-Mar. : vallon de Sausse-Horène près Saint-Étienne, des chalets au lac de Vens, l'Enchastraye, Fenestre, Gardolasca, Tende.

S. Allionii. - Rochers. - B.-Alp. : Colmars. - Alp.-Mar. : col de la Madona di Fenestre.

UIIELTCUS pendulinus D. C. - Vieux murs et rochers surtout des terrains siliceux. - Commun dans la région méridionale, nul dans la partie orientale du bassin ; rare dans la partie septentr. - C.-d'Or : Semur. - S.-et L. : Cluny, Saint-Léger près Bourbon. - Rh. : rochers granit. des vallées de l'Iseron, du Garon et de la Turdine, Beaujolais. - Loire : roch. granit. Malleval, Saint-Julien, Chalmazelle. - Is. : granite des envir. de Vienne.-Dr. : granite de Saint-Vallier et environs.-Ard. : Vals, Entraigues. - A partir de ce niveau il devient de plus en plus cornmun dans la région méridionale.

En dehors de nos limiles dans les Pyr. centr., les rochers granit. schisteux et volcaniques du Puy-de-Dôme, Cantal, II.-Loire, Creuse, Corrèze, Lozère, Lot, Tarn.

J. sedoides D. C. - Rochers, - IIaute vallée de l'Aude autour du lac d'Estagnet. - Pyr.-0r. : mont Louis, Cambres-d'Aze, vallées d'Eyno et de Llo, col de Nuria, sommets de Carlite, Coma de la TCt, Canigou, vallée de Cady, roches dels Isards, sommités de Pla Guillem, Costa-Bona, Llaurenti, vallée de Mijanès.

\section{CACTÉES.}

CACrus opuntia I. - Plante originaire d'Amérique naturalisée sur les rivages de la Corse, des lles Baléares et du midi de la France à Menton, Monaco, Nice et sur divers points du Roussillon nolamment autour de Perpignan. - Valais : Sion sur les rochers de Valère et de la Majoric.

\section{FICOIDÉES.}

Mesembryanthemum nodiforum L. - Sables et rochers marit. du Var et Alp.-Mar. à Bandols, Cannes, Nice, Monaco. B.-du-Rh; : Marseille à la batterie d'Endoume, le Courtiou. 
Lo M. crystallinum est cultivé et apparait quelquefois dans les décombres et près des jardins.

\section{GROSSULARIÉES.}

mines awa crispa $L$. - Haies dans tout le bassin. - Dans le Gard, l'Hérault, l'Aude et les Pyr.-Or. il occupe la région montagneuse.

Varie à fruits glabres, pubescents ou même couverts de soies glanduleuses.

T6. nigroun L. - Le Cassis souvent cultivé se rencontre quelquefois à l'état subspontané au voisinage des habitations et dans les bois.

T. alpinam L. - Bois des montagnes. - Commun dans les vallées curitiques et porphyriques des Vosges jusque près de Belfort au Rosemont et dans la vallic du Puix; dans II.-Saơne à Champagney, vallée de Miélin, Sapois, Tête-des-Sèpes, la Prandière, Ballon de Servance; dans la zone jurassique à Rosey, Varay, Cliassey-les-IJonthozon. - Toute la chaine du Jura depuis le vignoble jusqu'aux sommités du Reculet et de la Dóle. - Montagnes de Côte-d'Or, S.-et-L., Beaujolais, Lyonnais à Saint-Laurent-de-Chamousset, le Fenoyl.-Loire : chaine du Forez, Pierresur-IIaute, Pilat. - H.-Sav. : Semnoz, Voirons, Salève, Servoz, Chamonix. - Sav. : Hautecombe, Mouxy. - Vaud et Valais: montagnes d'Aigle, de Roche, Colombey, Vionnaz, Vouvry. Ain : forêt de Valors, Retord. - Is. : mont Rachais, forêt de Portes, Pariset, Sassenage. - H.-Alp. : Charance, Devez-deRabou, mont de Chabrières, Monesticr, Montmorin.-B.-Alp. : Colmars, la Blachière près Maurin, le Lauzannier. - Vaucl. : mont Ventoux. - Région montagneuse des Alp.-Har. - Ard. : montagnes du Vivarais, mont Charray l'Escrinet. - Gard: Alzon, l'Espérou, Concoule. - Hér. : Pic-Saint-Loup, la Sérane, Avine, Pardailhan, Saint-Arnaud, la Salvetat, Saint-Pons, le Larzac. - Aude : versant mérid. de la montagne Noire vers MasCabardès, forèt des Fanges, Fontanes. - Pyr.-Or. : bois de Salvanire, le Canigou au-dessous de la Jasse-de-Cady. - En dehors de notre bassin dans les Pyr. centr. et le centre de la France. 
R. rubrum L. - Cultivê et quelquefois subspontané.

1e. petreum Wulf. - Bois et rochers des montagnes. - Escarpements des Vosges jusque dans la II.-Saône sur le Ballon de Servance, vallée du Rahin; descend jusqqu’à Nans $\left(450^{\mathrm{m}}\right)$. - Région des sapins du Jura helvétique et français, la Dôle, Faucille pris de Lavalay, Noirmont, Montendre, mont d'0r, Suchet, la Brévine, le Barboux, Dent-de-Vaulion, la Trẻlasse. - Vaud et Valais : mont Fracliz, Sion, Sierre, Trient, Combire, vallée de Chippis. - H.SSav. : Brizon à la Glacière, col de Golèse, Combe sur Sixt, Valorcine, les Contamines. - Sav. : Saint-Innocent, les Allues à Pralançon. - Rlı. et Loire : montagnes du Beaujolais, du Pilat ct de Pierre-sur-Haute.-Ard.: Bauzon, le Mezenc. - Is. : entre Revel et le lac du Crouzet, rochers de Sornin entre Engins et Autrans, bois de Naves près Sarcenaz.-H.-Alp. : Lautaret au bois de la Madeleine, Névache en allant aux Roclilles, col des Ilayes, Guillestre, Boscodon, mont Viso au Bouchas. - B.-Alp. : Lauzannier, Colmars. - Alp.-Nar. : val de Pesio, Saint-Etienne-le-Sauvage. - Aude et Pyr.-0r. : Salvanère, montagne de Céret, Saint-Laurent-de-Cerdans, bords du torrent de la Cirerole au Canigou, Vernet-les-Bains. -Pyr. centr., Puy-de-Dóme, Cantal.

\section{SAXIFRAGÉES.}

SAXIFRAGA stellaris $L,-$ Lieux humides des montagnes à sol siliceux. - Nul dans la chaine jurassique. - Toutes les hautes vallées des Vosges jusque dans l'arrondissement de Belfort et la H.-Saône vers les sources de la Savoureuse, vallěe du Rrahin dans la forêt de Saint-Antoine, Plancher-les-llines, Grande-Goutte, la Prandière, Ballons de Servance et de Giromagny. - Vaud et Valais: Alpes de la chaîne septentr. depuis les Diablerets jusqu'au Grimsel, chaine mérid. depuis le SaintBernard jusqu'au mont Rose et au Saint-Gothard. - H.-Sav. : grìs nummulitiques des massifs du Vergy, Méry, Colone, Flaine, Tournette, Charrin, Etale, chaîne du mont Blanc depuis le col de Balme jusqu'au Bonhomme; s'elève jusqu'au Jardin de la 
Mer-de-Glace. - Sav. : montagnes de Beaufort et d'Hauteluce, Crêt-du-Ré au-dessus d'Aime, vallées des Allues, de l'Isère et de l'Arc, mont Iseran, mont Cenis. - Is. : gneiss et granit des chaines du Grand-Charnier d'Allevard, des Sept-Laux, des Belledonne, de l'0isans jusqu'au pied du Pelvoux. - H.-Alp. : Laularet, Chardonnet, Ponsonnière, Névache, mont Viso, la Taillante, Chaillol-le-Vieil, Valgaudemar. - Alp.-Mar. - Ard. : Gerbier des Joncs, sources de la Ioire, le Mezenc, la Chavade. — Gard: Concoule, l'Espérou. - Aude : vallée de la Bruyante, Boutairol, Salvanère, Lac de Quérigut, Llaurenti. - Pyr.-Or. : Bac de Bolquère, vallẻes d'Eyne et de Nohèdes, Costa-Bona, Canigou, vallée de Cady. - Pyr. centr., mont Dore, Cantal, Aubrac, Lozère, Aigual, chaîne du Forez au Pic-Pelé au-dessus de Noirétable, Chalmazelle, Pierre-sur-Haute; manque au Pilat.

S. Clusii Gouan. - Rochers siliceux humides. - Ard. : Ilezenc. Gard : route de la Salle à Valleraugue, au Martinet. - Hẻr. : l'Espinouse, le Caroux. - Aude :versant mérid. de la montagne Noire à Mas-Cabardès, lac de l'Estagnet. - Pyr.-Or. : parties supér. des vallées d'Eyne et de Llo. - Rochers siliceux des Pyr. centr.; micaschistes de la Lozère entre Florac et Vebron, vallée du Gardon, Sainte-Croix, Saint-Etienne-Valfrancesque, Vialas.

- Tarn : Ambialet, le Banquet.

S. cuneifolia L. - Rochers siliceux des lieux ombragés des Alpes et des Pyrénées. - Nul dans les Vosges et la chaîne jurassique. -Valais: Chemin, Frachiz, de Trient au col de Balme, mayens de Saxon et de Sion, Mainghorn, Aletsch, Nesselalp sur Naters, val Mana, Massongex, Choex, vallées de Zermatt et le Saas. II.-Sav. : toute la chaine du mont Blauc depuis le col de Balme jusquau Bonhomme; remonte sur les moraines des glaciers; Aiguilles-Rouges, la Diosaz, Brizon, Charvin. - Sav. : montagnes de Beau fort et de Crest-Voland, vallée de l'Isère vers Tignes, entre Pesey et Hacot, vallée supér. de l'Arc. - Is. : chaînes des Sept-Laux et de Belledonne, Prémol, le Gleyzin, Theys, montagnes de l'0isans à Venosc. - H.-Alp. : Nonestier, Névache, Poligny en Champsaur, le Noyer, Chaudun. - B.-Alp. : Annot. -Alp.-Nar. : toutes les montagnes depuis Saint-Etienne, Vence, 
Levens jusqu'aux Alpes de Tende.-Gard : bois de Longefeuille à Concoule. - Pyr.-Or.: Llaurenti, vallée de Mosset près du couvent de Jau, Costa-Bona.-Pyr. centr.; Lozère au-dessus de Castelades près Villefort.

S. Inirsuea L. - Rochers humides sur le bord des eaux dans la partic supérieure de la vallée de Carença et au Llaurenti dans les Pyr.-0r. - De là s'étend dans toute la chaine des Pyrénées cen. trales.

s. cotundifolia L. - Lieux humides des montagnes. - Rẻgion des sapins du Jura helvétique et français, Bugey, Valais, Savoie, Dauphiné, Alpes Basses et Maritimes. - Nul dans les Vosges. Ard. : Mazan, Cuze, Cheylard, le Mezenc. - Gard et Lozère : l'Aigual, Durbic, de Saint-Ambroix à Villefort. - Aude : Belcaire, vallée de la Bruyante, Boutairol, Salvanère, Llaurenti. - Pyr.-0r. : Castel, Costa-Bona, la Preste, Canigou, vallées d'Eyne et du Bac de Bolquère. - Manque dans les Pyr. centr.; mais existe au mont Dore, au Cantal et dans la chaine du Forez.

S. Hireulus L. - Tourbieres du Jura helvétique et français : Pontarlier, les Rousses, la Brévine, la Trélasse, Chenalolte.-Ain : Nantua, Malbronde. - Pyr.-Or. : tourbières du bois du Llaurenti.

๘. aspera L. - Rochers siliceux des Alpes et des Pyrẻnées. - Nul sur les calcaires. - Vaud et Valais: Alpes d'Aigle et de Bex, Fully, Catogne, Bagnes, Saint-Bernard, Cleuson, les Diez, Arolla, Findelen, Schwarzgletscher, Mattmark, le Breuil, Aletsch, Simplon, Albrunpass, glacier du Rhône, Grimsel. - H.-Sav. : Buet, Flaine, chaine du mont Blanc jusqu'au Jardin, à Leschaux et au Couvercle. - Sav. : le Chapieu, Combe du mont Ambin et de Villette, mont Cenis à Ronches, mont Iseran, Saut-des-Allues. - Is. : chaines des Sept-Laux et de Belledonne au Grand-Charnier, Champrousse, Grandes-Rousses à Huez, Valjoufrey au pied du mont Chamoux. - H.-Alp. : Valgaudemar, Saint-Véran au-dessus de Cornivier, vallon d'Arcines, Mont Viso à Sélar, col de Vars. - B.-Alp. : col du Soltron, Lauzannier, Bẻrard, mont Couyer sur Annot. - Alp.-Marit. - Gard : l'Espérou, l'Aigual. 
- Pyr.-Or.: Cambre d'Azes, Canigou, vallée d'Eyne, Pla-Guillem. - Pyrén. centrales.

S. bryoides L. - Rochers siliceux des Alpes et des Pyrẻnées; s’ẻlève plus haut que le S. aspera. - Valais : Saint-Bernard, mont Ceri, Fully, Cleuson, Novelly, Thyon, Méribé, Loze, mont Nuoble, Zan, Ferpecloz, Zermatt, Schwarzgletscher, Tortain, Simplon, Schornen, Albrun, Sirwolten, Grimsel. - H.-Sav, : col de Golèse, Buct, toute la chaîne du mont Blanc; s'ẻlève sur les moraines des glaciers jusqu'à Leschaux, Couvercle, le Jardin et jusçu'aux plus hautes limites de la végétation en Europe, c'està-dire sur les rochers des Grands-Mulets. - Sav. : Morteret et Saut-des-Allues, mont Iseran, Vanoise, mont Cenis à Ronches et sur les bords du lac Noir. - Is. : chaînes des Sept-Laux, de Belledonne, des Grandes-Rousses et de l'Oisans. - H.-Alp. : Villard d'Arène sous les glaciers du Bec, Lautaret, Abriès, mont Viso à la Traversette, col de Ruines, Saint-Véran à Blanchette, vallon MIarcel, Chaillol-le-Vieil, rochers de la Brèche et du Vassivier. - B.-Alp. - Alp.-MIar. : cols de Tende et de Fenestre, lacs de Mercantourm et de Strop, sources du Var, RocheGrande. - Pyr.-Or. : Llaurenti, vallées de Llo et de Carença. - Pyrén. centr.

S. aizoides L. - Bords des ruisseaux et torrents des montagnes.Nul dans les Vosges et la longue chaine depuis le Morvan jusqu'aux Cévennes; manque aussi dans le massif central de la France. - Jura : Colombier, Reculet, Faucille. - Ain : Colombier du Bugey. - Très-commun dans les Alpes de Vaud, Valais, Savoie, Dauphiné, Alpes Basses et Maritimes. - Aude et Pyr.-Or. : Boutairol, Llaurenti, vallée d'Eyne. - Pyr. centr. - Parfois entrainé par les torrents et rivières; c'est ainsi qu'il a été trouvé près de Genève au-dessous du bois de la Bâtie el aux sables d'Aïre; on l'a vu aussi sur les bords du Rhin à Rheinfelden près Bâle.

s. granulata L. - Prés et pâturages; plus abondant sur las terrains siliceux; sa distribution est fort inégale. - Commun dans les vallées des Vosges.- Rare dans H.-Saone et arrond. de Belfort, dans la vallée du Rosemont, Leffond, Vy-les-Rupt. - Doubs et 
Jura : ne se trouve que dans les tourbières à sous-sol siliceux de Pontarlier, prés tourbeux de Pleurre, terrains siliceux de Mennières et Sampans près Dole, granite de la forêt de la Serre vers 0lltange et Brans; sur le versant helvétique à Arnex près Orbe, Verrieres, Sainte-Croix. - Nul dans le Valais.-Commun sur les terr. sablonn. des envir. de Genève à Penex, Campagne d'Ivernois, Grand-Sacconnex, Pầquis. - Extrêmement répandu sur les terr. granit. de C.-d'Or, S.-el-L., Beaujolais, Lyonnais, Forez, Pilat, Vivarais et Cévennes jusqu'à l'Espérou, Concoule, envir. d'Anduze et dans l'Hérault autour de Saint-Gervais, Douch au Caroux, Andabre-Rosis, Saint-Étienne-de-NIursan, Grayssessac, Saint-Pons, Ceilhes et Avène. - Pyr.-Or. : mont Louis, Carença, mont de Madrès, Vernet-les-Bains, Castell, Font-deComps. - Dauphiné : sur les terr. sablonn. de la Côte-SaintAndré, Crémieu, Grenoble, Saint-Vallier, Aucelon, Gap.-Provence et Alpes-Marit. : Sainte-Baume, montagnes au-dessus de Menton, Nice, Saint-Agnès, Fontan, Clans; rare dans la plaine vers le Biot et Villeneuve prẻs Cagnes.

S. bulbifera L.- Roch. du Valais à Branson, Gucuroz, Folatères, mont d'Orge, Champlan.

S. pedemontana All.-Alp.-Har.: mont Bego, col de Tende, vallée de Fontanalba, cols de l'Abisso el de Fenestre, lac d'Entrecoulpes. - Roch. entre le mont Rose et le mont Cervin sur les confins du Valais et du Piẻmont.

S. tridactylites L. - Nurs et rochers dans tout le bassin.

S. petraa L. - Débris pierreux des Alpes et des Pyrénées. - Vaud et Valais: Grandvire, Paneirossaz, Tavayanaz, Fully, Gietroz, col de Fenêtre, Oratoire de Bagnes, Cleuson, Vende, Méribé, Pasdu-Loup, Tortain, Thyon, Zermatt, Schwarzberg.- Savoie : col du mont Iseran, mont Cenis à Ronches, - H.-Alp. : mont Viso à la Traversette, col de Ruines, Saint-Véran près du pont, chapelle de Clausis, mont Aurouse, Séuse. - B.-Alp. : Longet de Maurin. - Pyr.-Or. : Llaurenti, Costa-Bona. - Pyr. centr.

S. Bellardi All. - Forme naine du précédent; je l'ai trouvée à la montagne de Jily au-dessus d'Abriès en Queyras.

S. germnioiden L. - Débris pierreux des Pyr.-Or. : vallées de 
Nohedes et d'Eyne, Canigou, Prats-de-Mollo.-Aude : Boutairol, vallée de la Bruyante, lac de Quérigut, Llaurenti, mont Alaric. - Pyr. centr.

Var. ladanifera Lap. - Pyr.-Or. : Costa-Bona, Canigou.

S. Prostu Sternb._Ard. : rochers d'Avran._Gard et Lozère : l'Espérou, Concoule, Lourette près du Vigan, mont Lozère près Villefort - Hẻr. : le Caroux, vallon d'Eric. — Aude : Axat au sud de Quillan.

s. pedatifiar Snl.-Rochers. - Sommités du Canigou (Pyr.-Or.).

S. olnseura G. G.-Rochers.-Aude : versant mérid. de la Nontagne Noire vers Mas-Cabardès. - Pyr.-Or. : fond de la vallée d'Eyne, sommets du Cambres-d'Aze et du Canigou, bords des torrents de la Comelade.-Manque dans les Pyrén. centr.

S. pexdaetylls Lap.- Rochers. - Aude : roch. granit. de la vallée de la Bruyante près Mlijanès, Boutairol, le Llaurenti.-Pyr.-Or.: sommitês des vallies de Llo et d'Eyne, sommets de la Comelade. - Manque dans les Pyr. centr.

S. nervosa Lap. - Roch. - Pyr.-Or. : vallée d'Eyne, montagne de Madrès, Llaurenti.-Pyr. centr.

S. ascendens L. - Bords des ruisseaux. - Aude : la Boutaillole près Quérigut. - Pyr.-Or. : vallées d'Eyne et de Llo, Cambresd'Aze, Canigou à la Llapoudére et au Pas-de-Cady, Costa-Bona, Coma du Tech.- Pyr. centr.

S. ajugefolia L. - Rochers siliceux. - Aude et Pyr.-Or. : Boutairol, Llaurenti au Roc-Bla nc, Cambres-d'Aze, vallées d'Eyne et de Carença, Canigou,-Pyr. centr.

S. capilata Lap. - Forme souvent mèlée aux deux précédents, dont on croit qu'il est un hybride.

s. pubescens D. C. (non Pourr.) G. G. S. mixta Lap. - Rochers calcaires. - Gard : roch. d'Enjeou près Montdardier, de la Tessone près du Vigan, Aumessas. - Hér. : ruines de Montferrand, versant nord du pic Saint-Loup, Cazillac près Ganges. - Pyr.Or. : Costa-Bona, Coma-du-Tech, Canigou, Pla Guillem, Carença, Cambres-d'Aze, vallée d'Eyne, - Lozère : roch. cal. près Mende et Florac.-Pyr. centr,

s. groenIandiea L. - Rochers, - Pyr.-Or. : pic de Costa-Bona, 
Canigou au-dessus des ćtangs de Cady, vallées d'Eyne et de Llo, au-dessus des étangs de Carlitte, Llaurenti._- Pyr. centr.- La plante indiquée sous ce nom dans les Alpes du Dauphiné, par Mutel, est une forme du S. muscoides.

s. exarata Vill. - Rochers des Alp. et des Pyrén, surtout dans les terrains siliceux. - Valais : Saint-Bernard, Zanrion, Thyon, Cleuson, Diez, Arolla, mont Nuoble, Vouasson, Zan, Hoernli, Unterrothorn, Findelen, Fée, Mattmark, Schwarzgletscher. II..Sav. : la Vauzalle près Sixt, Tournette, chaine du mont Blanc jusqu'aux Grands-Mulets, Aiguilles-Rouges. - Sav. : Morteret des Allues, mont Iseran, Saint-Sorlin-d'Arves, mont Cenis. - Is. : la Hfoucherolle. - H.-Alp. : Lautaret, mont Viso à la Traversette, chalets de Ruines, combe de Ségure, Ristolas, Pennin, la Lauze, Aurouse. - B.-Alp. : Lauzannier, Vallonnet de Heyronnes, mont Vergous et Gd-Couyer près Annot.-Alp.-Nar. : montagnes de Saint-Élienne et d'Entraunes jusqu'au col de Tende.- Vaucl. : sommet du mont Ventoux.-Pyr.-Or. : Canigou, Costa-Bona, Coma-du-Tech, Cambres d'Aze, vallées d'Eyne, de Llo, de Carol, Llaurenti.

Var. leucantha Gaud. - Valais : rochers du Trient en montant à Gueuroz, Branson, Fully.

S. inrrieata Lap. - Roch. des Pyr.-Or. à Costa-Bona, Pla Guillem, Canigou, vallées d'Eyne, de Carol et de Vall Marans. - Pyrén. centr.

ø. muscoldes Wulf.-Espèce polymorphe commune dans les Alp. et les Pyrén.; nulle dans les Vosges; rare dans le Jura sur le Reculet et les sommités entre cette montagne et le Colombier de Gex. - Vaud el Valais : toute la chaine mérid. entre le SaintBernard et le Simplon; chaine septr. depuis les Diablerets jusqu’au Grimsel.-H.-Sav. : le Môle, Brizon, Méri, Charvin, Tournetle, Platets, mont d'Étale, Anterne, le Buet, Aiguilles-Rouges, chaine du mont Blanc jusqu'aux Grands-Ilulets. - Sav. : mont du Chat, montagnes des Allues et de Tignes, Bonhomme, mont Iseran, mont Cenis.-Is. : Gde-Chartreuse, Chamechaude, SaintNizier, col de l'Arc, Gd-Veymont, la Pra de Belledonne. - H.Alpes : Loubet, col de Glaise, Séuse, Aurouse, col de l'Alp-Ilar- 
lin, Sappel, Orcières.-B.-Alp. : montagnes de la vallée de l'Ubaye, Lauzannier, Vallonnet, Bérard. - Dr. : Vassieu-en-Vercors, mont des Lussettes près la Croix-Haute.-Ard. : Mirabel. — Gard : Saint-Guiral, l'Espérou. - Aude : Boutairol, Pla-Bernard, Llaurenti. - Pyr.-Or. : vallées d'Eynes, de Llo, Nohèdes, Canigou.-Pyr. centr.

S. moschata Wulf.-Diffère du précédent par la présence sur les tiges et les feuilles de poils courts et visqueux.-Assez commun dans les Alpes du Valais, Savoie, Dauphiné, Pyrénées, dans le Jura, descend jusqu'à $3 \ddot{0} 0$ métres à la Roche de Gizia près Couzance.

S. androsacen L. - Pàturages des Alpes et des Pyrénées. - Nul dans les Vosges, le Jura et les montagnes du centre de la France. - Vaud et Valais : Boulaire, Catogne, Pierre-à-Voir, Thyon, Vende, mont Nuoble, Findelen, Augstelberg, Saas, Sirwolten, Griess, Mainghorn, Gemmi. - H.-Sav. : montagnes du Chablais, Dents d'Oche, col entre mont Ardin et Haut-de-Lin, Roc d'Enfer, Signal, montagnes de Samoëns, col de Golèse, Platets, Méri, Vergy, mont d'Etale, Buet, châine du mont Blanc jusqu'au Bonhomme. - Sav. : montagnes de la Tarantaise et de la Maurienne jusqu'au mont Cenis. - Is. : chaînes des Sept-Laux, de Belledonne, Champrousse, Oisans, Tête-de-Racha, Pelvoux, la Moucherolle. - H.-Alp. : col de l'Echauda, Lautaret, Rochilles, Jujan, col de Freissinière, mont Viso au col Vieux, envir. des lacs de la Taillante, Saint-Véran, Clausis, Aurouse, pic de Chabrières.- B.-Alp. : Lauzannier, Parpaillon, Longet de Maurin. - Alp.-Mar. : sources du Var à Rochegrande, vers les lacs de Strop au-dessus d'Entraunes. - Pyr.-0r. : vallée d'Eyne, Canigou, Costa-Bona, Pla Guillem. - Pyrénées centr.

S. Seguierl Spreng. - Valais : Zermontana, Cleuson, Thion, Vende, mont Nuoble, Galmschmeide, Riffel, Gorner, Augstelberg, Rothorn, Saas, Schornen, Krumpen, Salzgeben, Griess, Tortain, Mainghorn.

S. planifolla Lap. - Rochers des Alpes et des Pyrénées près des glaciers. - Vaud et Valais : montagnes de Bex, d'Hérens, d'Anniviers, Cleuson, Thyon, Diez, mont Nuoble, col de Fenétre, Tortain, Zermatt au Hoernli, Staffel, Unterrothorn, Schwarz- 
berg, Mainghorn et Mayen pris Leukerbad. - II.-Sav. : Verory près de l'arête de Balafrasse et ì Jalouvre, le Signal des $\Lambda$ gneaux dans le Chablais. - Sav. : Crêt-du-Ré au-dessus d'Aime, vallon de Champrossa au fond de la vallée des Allues, extrémité de la vallée du lac de Tignes, col de la Madeleine au Cheval-Noir, la Vanoise, mont Cenis à Ronches. - HI.-Alp. : Galibier, mont Viso a la Taillante, Saint-Véran aux Chalanclıes.-Pyr.-0r. : vallées d'Eyne et de Llo, Cambres-d'Aze, Costa-Bona, Canigou au fond de la vallée de Cady et au Roc des Isards. - Pyrénées centr.

s. sedoides L.- Rochers des parties supérieures de vallíe d'Eyne, du Cambres-d'Aze près des étangs de Carlite. - Nul dans les Pyrén, centr.

s. sponhemiea Gmel. - Rochers. - Jura : fort Belin prẻs Salins, Baume-les-Messieurs au fond de la vallée de Saint-Aldegrin et près des Echelles-de-Crançot, sources de la Cuisance, Gizia près Couzance.

s. hypuoldes L. - Rochers siliceux de la région mérid. et centr. - Dr. : collines granit. de Tain, Saint-Vallier, Serves.- Ard.: rochers phonolitiques et basaltiques du Mezenc, Rochemaure, mont Combier, Entraigues, Cuze, Vals, l'Escrinet, rochers gran. de Tournon. - Gard : l'Espérou, Arphy près du Vigan, Villeraugue, Aulas.-Hér. : l'Espinouse, le Saumail, l'Escandorgue, vallée de la Mare, Grayssessac. - Aude : versant mérid. de la Montagne Noire vers Mas-Cabardès. - Pyr.-Or. : vallon de Collioure, Tour de Massane, entrẻe de la vallée de Nohèdes. Manque dans les Pyrén. centr. - En dehors de notre bassin sur les micaschistes et gran. de la Lozère, rochers volcani(ques du mont Dore et Puy-de-Dome. - Loire : ruines du château de Couzan, Saint-Jodard entre Boën et Saint-Thurin, digues de Pinay, Champdieu.

Espece polymorphe dans laquelle MII. Jordan et Fourreau ont distingué les formes suivantes:

S. parvula J. F.- Serres (Dr.). S. vulcanorum J. F. - Roche-

S. helviensis J. F,-Rochemaure maure.

(Ard.).

S. basaltica J. F.-Ibid.

S. indirisa J. F. - Ibid.

S. leta J. F.- Ibid.

S. vivariensis J. F. - Ibid. 
s. aizoon Jacq. - Rochers des montagnes. - Commun dans les chainnes des Vosges, du Jura helvétique et français, le Revermont, Bugey, où il descend jusque près du fond des vallées, Valais, Sav., Dauphiné, Alp. Basses et Mar. - Ard. : Mezenc.Gard et Lozère : Arphy près du Vigan, Hort-de-Diou, Aigual, Aubrac. - Cantal. - Mont Dore. - Aude : vallée de l'Aiguette, Bains d'Ilusson, Boutairol, Mijanès, lac de l'Estagnet. - Pyr.Or. : mont Louis, Font-de-Comps, Canigou, Costa-Bona.-Pyr. centr.

Espèce polymorphe dans laquelle on a remarqué les formes suivantes :

Chondrosea valida J. F. - Boutairol, Llaurenti (Aude), Hoehneck (Vosges).

C. petroplita J. F. - Monestierde-Briançon (H.-Alp.).

C. beugesiaca J. F. - Hauteville (Ain).

C. glabrata J. F. - Saint-Véran (H.-Alp.).

C. gracilescens J. F. - Col Malrif

C. punctillata J. F. - La Grave (H.-Alp.). (H.-Alp.).

S. florulenta Moretti. - Fentes des rochers dans les Alp.-Har., autour du lac d'Entrecoulpes, mont Bego, Clapier, mont Ponset dans 'a vallée de la Gordolasea. - On l'a vu aussi dans les montagnes entre le col de Fenestre et Coni en Piémont alla Rocca dell Argentera.

S. cotyledon L. - Rowhers siliceux des Alp. et des Pyrénées. Valais : rochers d'Emousson, Nomaney, Salvan, Sparrenhorn, Sch warzgletscher, Ried de Naters, Simplon.-H.-Sav. : Bẻrard, Brévent, Buet, Chapeau de la Mer-de-Glace.-Pyr.-Or. : Collada de Nuria, vallée d'Eyne, col de las Nou Fonts, Prats-de-Balaguer, Costa-Bona, Llaurenti, vallée de Mijanès. - Pyrén. centr.-Nul sur les calcaires.

S. Iongifolia Lap. - Rochers calcaires, - Pyr.-Or. : rochers du Bordelat à l'extrémité du vallon de la Manère. - Très-commun dans les Pyrén. centr. et sur le versant espagnol.

S. lingulata Bell.-Rochers.-II.-Alp. : Ribiers.-B.-Alp. : montagnes des envir. do Digne, mont Cousson, les Dourbes près de Sisteron au nord 'de la Citadelle, mont Pierre-Impie, Larche, 
Annot, Castellane. - Vaucl, : sommel du mont Ventoux. - B.du-Rh. et Var : de Vauvenargues à Jouques, Sainte-Baume. Alp.-Nar. : depuis le col de Tende jusqu'au-dessus de Menton et jusıue vers Grasse. A été indiqué dans les montagnes de Flaine (H.-Sav.).

S. cochtearis Rchb.-Rochers des Alp.-Nar. depuis le col de Tende jusqu'au mont Mulacé au-dessus de Menton.

s. mutata L. - Pierres humides. - Valais à la Gemmi.-II.-Sav. : Pontcliy, Thuet près Bonneville.-Is. et Sav.: combe de Malafossan près Saint-Jean-d'Avellane, Ecole en Bauges. - Aude et Pyr.Or. : Llaurenti.- Pyr. centr.

s. media Gouan.- Rochers calcaires. - Pyr.-Or. : Fond-de-Comps, sommet du Cambres-d'Aze, vallée de Llo, Comarca de las Mulleres près Nuria, Costa-Bona, Canigou, rochers de la Tour de Mir à Prats-de-11ollo. - Aude : Sainte-Colombe sur l'Aiguette, lac de l'Estagnet.- Pyrẻn. centr.

S. lutco purpurea Lap.-S. arctioideo mediu Gr. Goulr.- Roc de SanFelice à l'entrée de la vallée de Llo (Pyr.-Or.).-Pyr. centr., où il est mêlé avec le $S$. media et le $S$, aretioides, ce qui l'a fait considérer comme un hybride.

Le S. medio-urctioides G. G. S. ambigua D. C. indiqué dans les Pyrẻn. centr. sera peut-être trouvé dans les Pyr.-Or., de même que le $S$. aretioides Lap.

S. diapensoides Bell. - Rochers des Alpes, - Valais : au-dessus de Lourtier, au Zeppi, Pierre-à-Voir, Bagnes, Zermatt. - Sav. : Tignes, Lans-le-Villard, mont Cenis à Ronches. - H.-Alp. : mont Viso à Ruines et à la Traversette, Guillestre en allant à Vars, fort des Salettes à Briançon. - B.-Alp. : vallon du Châtelet, Longet de Maurin. - Alp.-Mar. : mont Orno près du col de Tende, Cima dei Gelas entre mont Clapier et col de Fenêtre, plateau de Jallorgues.

S. coesia L. - Rochers des Alpes et des Pyrénées. - Vaud et Valais : montagnes au-dessus d’Aigle, mont Fully, sources de la Sionne, Kümmenalp, val Lotschen, Binn, Griess, Gemmi._Sav. : vallée de Tignes, Champrossa au-dessus des Allues, Crêt-Baudin, le Chapieu, chalets de la Ragea sous le Bonhomme. - H.-Alp. : 
Briançon, Cervières, col Isoard, col de Péas. col Agnel, col de Vars.-B.-Alp. : vallonnet de Meyronnes, lac de Praroar, Plampinet. - Pyr.-0r. : vallées de Llo, d'Err, d'Eyne, Canigou, Llaurenti. - Pyrén. centr.

S. voldensis D. C. - Mont Cenis, - H.-Alp. : col de la Croix, col Albert et col de Ruines en Queyras.

S. oppositifolia L. - Rochers des Alpes et des Pyrénées. - Nul dans les Vosges.- Rare dans le Jura, au Colombier et au vallon d'Ardran. - Vaud et Valais : toute la chaine septentr. depuis les Alpes d'Aigle jusqu'au Grimsel ; chaine mérid. depuis le SaintBernard jusqu'au Simplon.- HI.-Sav. : Brizon à Balafra, Vergy, Méri, Flaine, Parmélan, Charvin, Tournette, montagnes du Chablais à la Dent-d'Oche, Signal et Roc d'Enfer, Ayers sur Servoz, Anterne, Aiguilles-Rouges, toute la chaîne du MontBlanc depuis le col de Balme jusqu'au Bonhomme; s’élève jusqu'aux Grands-IIulets. - Sav. : montagne des Avanchers, Avrieux, la Vanoise, mont Cenis, mont Granier au col du Frêne. - Is. : Grandsom, Saint-Nizier, la Moucherolle, la Bérarde-enOisans. - H.-Alp. : Lautaret, Galibiẹr, mont Genèvre, col Afalrif, Abriès, mont Viso à la 'Traversette, col de Ruines, vallon des Vaches, Ségure, Saint-Véran, col de Glaise près Gap, mont Aurouse, pic de Chabrières, mont Fourchat près Rosans.-Dr.: Glandasse, Fondurle. - B.-Alp. : Saint-Paul, Parpaillon, Longet de Maurin, mont de Lure, Gu-Couyer près Annot. - Vaucl. : mont Ventoux.-Alp.-Nar. sur les sommités. - Aude et Pyr.Or. : lac de l'Estagnet, Llaurenti, Canigou, Costa-Bona, Cambresd'Aze, vallée d'Eyne.- Pyrẻn. centr.

S. Dimora All. - Débris des rochers schisteux et granitiques près des neiges ou sur les moraines des glaciers des Alpes. - Vaud ct Valais : Grandvire, mont des Martinets, Saint-Bernard, l'Alliaz, Cleuson de Nendaz, Galmsneide, Zermatt, Schwarzsee, Saas, monte Horo, Griess.-H.-Sav. : mont des Granges en Chablais, la Vauzalle près Sixt, col d'Anterne, Buet.- Sav. : cols du Bonhomme, de la Seigne et des Fours, fond de la vallée du lac de Tignes, mont Iseran, la Galise, la Vanoise, Saint-Sorlin-d'Arves, mont Cenis à Ronches, versant sayoyard du Galibier-Is. : 
Piẻméyan au mont de Lans, col de la Muzelle. - H.-Alp. : col Malrif, mont Viso au col Valante, Saint-Yéran au col Blanchette.

- B.-Alp. : Bérard, Crouès, Longet de Ilaurin.— Nul dans les Pyrénées.

S. retusa Gouan. - Débris de rochers des Alpes et des Pyrénées près des glaciers et des neiges. - Valais : Gornergrat au pied du mont Rose; se trouve aussi sur le versant italien du mont Rose et du Saint-Bernard. - Sav. : extrémité de la vallée du lac de Tignes, mont Cenis à Ronches et sur les bords du lac Noir. H.-Alp. : Lautaret en face de l'Hospice, pic du Bec au-dessus de Villard-d'Arène, Galibier, mont Viso au col Valante et à la Traversette.-Alp-Mar. : col de Fenêtre.-Aude et Pyr.-Or. : Llaurenti autour de l'Etang et du Roc-Blane. - Nul dans les Pyrén. centr.

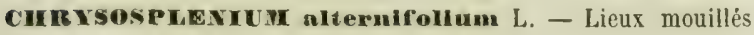
des montagnes; plus abondant dans les terrains siliceux. chaîne des Vosges, descend sur la lisière vosgienne des arrond. de Belfort, de Montbéliard et du côté de la H.-Saône; région des sapins du Doubs, Jura, Bugey, - Valais : Collombey, Massongex, Vex, Thyon, Val-Reschy, Geimen de Natersberg. - Is. : Revel, Prémol, Gde-Chartreuse, Chalais. - H.Alp. : Valgaudemar. - Dr. : forêts de sapins du Vercors. - Alp.-Mar. : vallée de la mine de Tende. - H.-Sav. : Salève, Voirons, Saint-Gervais. - Sav. : montagnes de Beaufort et de Crest-Voland. - C.d'Or et S.-et-L. : terrains granitijues du Horvan dans les env. de Saulieu, Arnay, Autun; envir. de Cluny.-Rh. : chaîne granitique du Beaujolais et du Lyonnais, Saint-Bonnet-le-Froid, Iseron, l'Argentière, coteaux du Rlı. entre Sainte-Colombe et Ampuis.- Loire : massifs granitiques du Forez, de Pierre-surHaute et du Pilat. - Ard. : le Mlezenc. - Gard : massif granitique et schisteux autour du Vigan, Valleraugue. - $\Lambda$ ude et Tarn : massif gneissique et granitique de la Montagne Noire autour de Cabarède. - Pyr.-Or.: entre la Bastide et Saint-Marsal. Lozère, H.-Loire, Cantal, Puy-de-Dome.

C. oppositifollum L. - Lieux mouillés des montagnes. - Manque dans Valais, H.-Sav., Sav., Alp.-Mar. - Existe dans toutes les 
autres régions citées à propos du C. alternifolium, et en outre dans l'Hérault à l'Espinouse, vallée de la Mare à Andabre-Rosis, Saint-Ifartin d'Orb, Lamalou, Avène, l'Escandorgue aux Rives. - Plus commun que le précédent dans la Montagne Noire. Pyr.-Or. : vallées d'Eyne, de Vernet-les-Bains, Castell.-Pyrén. centr., massif central de la France.

\section{OMBELLIFERRES.}

DAUCUS carota L. - Champs et prairies dans tout le bassin.

- Espèce polymorphe.

D. agrestis Jord. - Envir. de Lyon.

D. exiguus Herm. - Forme naine des lieux arides.

D. mauritanicus All. (non L.). - Forme luxuriante des lieux fertiles.

D. maritimus Lam. - Forme des sables marit. ayant des feuilles épais-

ses, peu velues; les rayons peu nombreux à fleurs petites. -

Sables du littoral méditerranéen.

D. serratus G. G. - Forme à fruits ayant les aiguillons des côtes secondaires confluents à la base, courts et dentiformes.

D. Bocconi Guss. - Forme à aiguillons confluents à la base et à feuil-

les triangulaires dans leur pourtour. - Sables du littoral méditerranéen.

D. maximus Desf, - Champs et vignes, - Var : Toulon, le Luc. Pyr.-Orient.

D. hispidus Desf. - Plante de Corse trouvée dans les lles du golfe de Marseille.

D. gummifer Lam. - Rochers marit. - Var: les Imbiers, ille de Porquerolle, rochers des Lions près Saint-Raphaël. - Alp.Mar. : Nice au Lazaret.

D. gingidium L. - Roch. marit. - B.-du-Rh. : Marseille à RoucasBlanc, Montredon, Cassis, Bec-de-l'Aigle. - Var : îles d'Hyères, Porquerolle. - Pyr.-Or. : Argelès, Collioure, Banyuls-sur-Mer, Canet, Perpignan.

D. sleulus Ten. - Roch. marit. - Marseille à Montredon.

D. murieatus L. Champs des Alp.-Mar. autour de Roquebrune et Menton. 
D. dentalus Bertol. - Rochers marit. des environs de Marseille.

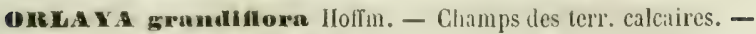
Environs de Belfort. - II.-Saône : Chariez, Noidans, Trécourt, Montigny, Chargey-les-Ports, Champagney, - Doubs et Jura : envir. de Montbéliard sur les calcaires jurassiyues et alluvions du Doubs jusqu'à Baume-les-Dames; là il disparaît, pour se montrer de nouveau près de Dole sur les alluvions du Doubs el de la Loue. - Plateaux calc. de C.-d'Or et S.-et-L. - Envir. de Genève entre Veirier et Troënex, entre Saint-Genis et Thoiry; puis plus bas dans l'Ain à Neuville-sur-Ain, Saint-Germainles-Paroisses, Ambléon, Artemare. - Vaud : Mathod, Yvonand, Goumoens, Belmont, Ecublens, Rances, Begnins, Duiller, Roche. - Valais : Branson, Liddes, mont d'Orge, Sous-la-Lex. - Is : Décines, Pariset, Claix, Mens. - Dr. : Saint-Vallier, Barnave. - H.-Alp. : Gap, Menteyer. - Var : Bagnols, le Luc, Rians. - Alp.-Mar. : envir. de Grasse, Nice et Menton. Ard.: Vals, Névissac, Mercuer, entre Privas et Chomérac, plaine du Lac, Tournon. - Assez commun dans Vaucl. B.-du-Rh., Gard, Hẻr., Aude. - Pyr.-Or. : Baixas, Cases-dePena, Haut-Vernet de Perpignan, Cornella-du-Conflent, Vernet-les-Bains, - En dehors de nos limites dans les Pyr.-Centr., Tarn, Lozère, Cantal, Puy-de-Dôme, Allier, Loire.

- platyearpos Koch. - Moissons de la région mérid. - Provence, Alpes-Har., Gard, Hérault, Aude, Pyr.-0r. - Remonte dans la Dro̊me vers Nyons, le Buis, Barnave; dans les B.-Alp. vers Ribiers.

D. maxitima Koch. - Sables marit. de la Provence et des Alp.Mar. lepuis Toulon jusqu’à Menton; Gard, Hẻr., Aude, Pyr.-Or.

TURGENA Intifolla Hofrm. - Champs cultivés des terrains calcaires. - Rare dans la partie N.-E. du bassin où il n'est connu que dans la vallée de l'ognon entre Saint-Vit et Pesme et rers Saint-Rémy sur les confins du Doubs et de H.-Saône. Valais : Sierre, Saviesse, Saint-Séverin, Saillon, Conthey. Beaucoup plus commun dans les champs calcaires de C.-d'Or, S.et-L., Loire, Rh. - Sav. : envir. de Chambéry. - Is. : autour de Grenoble. - H.-Alp. : Gap, Puy-Ilanse, Chorges, Embrun, 
Rosans.-Dr. : Barnave, Fourcinet.- Vaucl. : Orange, Bedoin, Caumont, Flassan. - B.-du-Rh. : envir, d'Aix, petit chemin du Tholonet, Bouonouro, Marseille, les Milles, Mfarignane. - Var : le Luc. - Alp.-IIar. : Gorbio, Gattières. — Ard. : Vals, plaine du Lac, de Privas à Chomérac. - Gard : envir. de Nimes et du Vigan, Saint-Hippolyte-de-Caton. - Assez commun dans l'Hér.

- Aude envir. de Narbonne, Donos, Fontjoucouse, les Corbières.

- Pyr.-0r: Salses, 0 pol, Vingrau, Baixas, Força-Réal.

CA UCALIS daucoides L. - Champs des terrains calcaires dans tout le bassin.

C. Ieptophylla L. - Champs cultivés de la région mérid. Remonte dans la Dr. vers Aucelon, Barnave, Romans; dans les H.-Alpes vers Ribiers el Gap; dans l'Ardèche jusqu'au pied de l'Escrinet; dans l'Isère à la Bastille de Grenoble, Tour-sansVenin, Vif, la Mure. - Lozère : Mende, Corsac, Florac, Meyrueis. - Trouvê accidentellement dans le Rh. à Cogny. - N'a pas été retrouvé dans la Côte-d-Or entre Semur et Rourray où Lorey prétend l'avoir vu.

TORLLiS anthriseus Gmel, - Bords des champs et des chemins dans tout le bassin. - Il est à remarquer que dans la région mérid. cette espèce est beaucoup moins commune dans les plaines que sur les montagnes, comme, par exemple, dans les Corbières, puis sur l'Espinouse à Fraisse, la Salvetal, Saint. Amand-de-Mounis, Castanet-le-Haut, le Caylar. - Dans les Alp.-Nar. elle a été vue dans les montagnes de Saint-MartinLantosque.

T. Helvetica Gmel. - Champs et bords des chemins dans tout le bassin.

T. tenuisecta Jord. - Parties mérid. de la Drôme vers Nyons, Vaison.

T. Heterophylia Guss. - Haies et bois de la région mérid. B.-du-Rh. - Vaucl. : Flassan à la Font-de-Canaud. - Var : Toulon, Morières, Fréjus. - Gard : envir. de Nìmes et du Vigan à Aulas, Aumessas. - Hér.: Fabrègues, Lavallette, Saint-Jean-de-Vẻdas, Agde, Andabre-Rosis, Camplong, Lunas, Avène, Colombières, Lodève, Saint-Chinian. - Aude : Clapeau-Rec, Fontfroide près Narbonne. - Pyr.-Or.: vallée du 
Boulou et de Céret, Baixas, Rivesaltes. - Ard. : Cuze, Vals, l'Escrinet, Craux.

T. nodosa Giertn. - Borus des champs et des chemins; plus abondant dans la région mérid. - C. d'0r: Gevrey, Marsannay, la Côte. - S.-et-L. : Cluny, Saint-Sorlin, Châne, Chalon.-Ain : la Valbonne, plaine d'Ambronay, Massieux, Trévoux. - Is. : Crémieu, Anthon, Tronche, Pariset, Claix. - Rh., la CroixRousse, Caluire, Sainte-Foy, Saint-Didier-au-Mont-d'Or, SainteFoy-l'Argentière. - Dr. : Barnave. - En dehors de notre bassin dans la Loire à l'Húpital-le-Grand, Fontanes près Montrond, Saint-Michel. - Centre de la France.

mIFora testiculatio D. C. - Moissons de la région mérid. Vaucl.: Flassan. - B.-du-Rh.: envir. d'dix au Tholonet, Rognac. - Var et Mlp.-Mar. : le Luc, Grasse, Cannes au cap Croiselle, Nice au Vinaigrier. - Hèr. : Montpellier au Mas-deComte, Lavérune, Iatles, Pérols, Viols, Valmargues, Candillargues, Saint-Guilhem-le-Désert, Beaufort près d'Olonzac.-Aude: envir. de Narbonne, Pech de l'Agnele, Montolieu, Clape à PechRedon, versant mérid. de la Montagne-Xoire, Salsigue, Cenne. - Pyr.-0r. : Salses jusqu'à l'Agly, Rivesaltes, 0pol.

B. raullans Bieb. - Moissons de la région mérid. - Vaucl. : l'Isle, Jonquières. - B.-du-Rh. : envir. d'Aix vers la gare de la Calade, Venelle. - Alp.-Nar.: Antibes, Menton. - Hér. : Fontcaude prẻs Montpellier, Lattes, Castelnau, Saint-Jean-deVédas, la Madeleine, Villeneuve, Lansargues, Cournonterral.Aude : Ifontolieu.

Corhavoredr sativum L. - Cultivé et quelquefois subspontané.

Tharsia villosa L. - Lieux stériles de la région mérid. Yaucl. : Gadagne près Avignon.-B.-du-Rh. : Ilarseille, SaintLoup, la Treille, Cassis, Fos, Aix, à l'est du vallon du Tir. Var : les Maures près du Luc, Touris, la Tourne, St-Raphaël. Alp.-Mar. : l'Estërel, Tanneron en face d'Auribeau. - Gard : Uzès, envir. du Vigan, la Tessone près d'Arré. - Hér. : la Gardiole au-dessus de Font-Cervier, N.-D. de Londres à la Jassette. -Aude : envir. de Narboune, Quillanet, Conillac, îles de l'étang Catal. Bassin du Rhơne. 
de Bages et de Leucate, versant mérid. de la Montagne-Noire près Salsigue. - Pyr.-Or, : Canet, Saint-Cyprien, Collioure, Banyuls-sur-IIer, Baixas, Saint-Antoine-de-Galamus, Caladroy, Força-Rẻal.

CASER PITY UI Iatifolí um L. - Rochers et pâturages des mon. tagnes. - Chaine des Vosges jusqu'au Ballon de Giromagny.Doubs et Jura : commun dans la chaine jurassique, montagnes et Clos-du-Doubs, Côtes dı Doubs, du Dessoubre et de la Barbèche, Lomont, Noirmont, Montendre, Dôle Reculet, Colombier. Ain : chaîne jurassicue des Monts d'Ain, Saint-Rambert, Hauteville, Colombier, montagne de Groslée. - Assez commun dans les bois montagneux de C.-d'Or. - S.-et-L. : Cluny, envir. d'Autun à Creuse d'Auxy, Sommant, Dezize, Cuizeaux.- Valais: Létroz, Trient, Crans de Lens, Leukerbad, Zermatt, entre Obergesteln et Oberwald. - Commun dans le Jura balois, bernois, neuchâtelois et vaudois, - H.-Sav. et Sav. : toutes les montagnes calcaires. - Is. : mont-Rarhais, Chalais, Grande-Chartreuse, Pariset. - H.-Alp. : Lautaret, exvirons de Gap, col Bayard, Charance, la Garde, montagne de Chabrières.-B.-Alp.: Tournoux, Malmore, Lauzannier. - Dr. : montagnes des envir. de Crest et de Barnave.-Vaucl. : mont Ventoux.-B.-du-Rh. : montagnes des environs de Marseille. - Var et Alp.-Nar. : Touris, bois de Clans, Cheiron, Entraunes, forêt de la Maïris, vallée de la Gordolasca, la Fraca.-Ard. : Cuze. - Gard : Concoule, l'Espérou, Lafoux près Alzon. - Hér. : rare à Lignères sur l'Espinouse. - Aude : montagne Noire à Cuxac, gorges de SaintGcorges, Boutairol, Mijanès, le Donezan, lac de Quérigut, Salvanère, le Llaurenti. - Pyr.-Or. : mont Louis, vallées d'Eyne et de Llo, Font de Comps, Canigou, Prats-de-Mollo, - En dehors de notre bassin dans les Pyrén. centrales, le Tarn à la montagne Noire, Lozère dans les envir. də Mende. - Loire: Pierre-sur-Haute, Gourgon, vallée de Chorsain. - Présente deux formes, l'une à feuilles glabres, L. glabrum Crantz, l'autre à feuilles velues $L$. usperum Crantz.

M. Nestlerl Soy.-Will. - Bois montagneux. - Gard : Alzon, Campestre, Camprieux, Salbous. - Hér. : le Larzac, le Caylar, la 
Vacquerie. - Aude et Pyr.-0r. : Bains d'llusson, Llaurenti. Pyr. centr. au pic de lllérie, col d'Arbas, Esquierry,- Lozère: envir. de Mende et de Florac.

4. gallieum C. Bauh. - Coteaux pierreux, - C.-d'Or: Gerrey, Beaune, Combe de Chambolle, Vougeot. - Valais : Rosseleinaz. - Ain : Serrières, Villebois, cote de Cerdon, Peyrieux.-II.-Sav。 : Sallenoves. - Sav. : Tignes, Moutiers. - Is. : Rabot, la Bastille, mont Rachais, Gières, Grande-Chartreuse, montée de Vénosc au mont de Lans, la Salette. - II.-Alp, : Villard-d'Arène, Monestier-de-Briançon, Chàteau-Queyras, de Gap à Charance, Séuse, mont de Chabrières. - B.-Alp. : la Condamine, Tournoux, Enchastraye, Annot. - Ard. : Tournon, Cruas. - Vaucl. : envir. du mont Ventoux, Flassan, Gigondas. - B.-du-Rh. : envir. de Marseille, Saint-Loup. - Var : Toulon, Bagnols, Montrieux. Alp.-Nar.: Entraunes, Villars, Margès, mont Ifulacé au-dessus de Menton. - Gard : envir. de Nimes, Serre-de-Bouquet, bords du Gardon, Navacelle près Alzon, la Tessonne près du Vigan. - Hér. : Saint-Guilhem-le Désert, Madière, Saint-Martin-deLondres, la Sérane, pic de Liausson, Lunas, Joncels, SaintÉlienne de-Gourgas, Pégayrolles de-l'Escalette. - Aude : Pechde-l'Agnèle, la Clappe, Ifontolieu, les Corbières, le Tauch, Pont de la Fou, moulin de Fontjoucouse, la Boulsanne. - Pyr.-Or. : envir. de Perpignan, Villefranche, Trencada d'A mbulla, SaintAntoine-de-Galamus, vallèe du Tech, Arles, Tour-de-Cos, la IIanère, Prats-de-Mollo. - En dehors de notre bassin dans les Pyrén. centrales; la Lozère autour de Mende, Saint-ÉtienneValdonesse, mont Vaillant près Florac, Meyrueis.

L. asprelorum Jord. - Les localités du départ. de l'Ain citées plus haut se rapportent à celte forme.

T. Siler $L_{\text {L }}$ - Rochers et bois das montages caliaires, - Nul dans les Vosges et les terrains siliceux des Alpes, des Cévennes, les Pyrénées et le centre de la France, - Abondant dans la chaîne du Jura helvétique et français : montognes du bassin du Doubs, cirque de Mauron, Côles du Doubs et du Dessoubre; descend dans le Jura jusque vers les vignobles de Salins, Arbois, Baume. - Commun sur les calcaires jurnssiques du Bugey autour de 
Saint-Rambert, Tenay, Hauteville. Virieu-le-Grand, la Burbanche, Rossillon, le Colombier, Saint-Benoît, Pierre-Châtel, le Ilont. - Vaud et Valais : montagnes d'Aigle, Trient, SaintMaurice, Comba d'Arba, Crans de Lens, Leukerbad, Zermatt.H.-Sav. et Sav. : Saleve, montagnes calcaires du Chablais, Faucigny, Bauges, Tarantaise, Maurienne. - Is. : mont Rachais, Saint-Eynard, Grande-Chartreuse, gorges d'Engins.-H.-Alp. : Lautaret, Séuse.-B.-Alp. : Horonaye, Lauzannier, Fouillouse, -Vaucl. : mont Ventoux.-B.-du-Rh. : N.-D. des Anges, SainteVictoire, Tète-de-Carpiagne. - Alp.-NIar. : toute la région montagneuse au-dessus de Levens, Saint-Vallier, Menton. - Ard. : Mauras. - Gard : rochers dolomitiques des envir. du Vigan à Montdardier, la Tessonne, Lafoux. - Hèr. : rochers dolomit. de Saint-Guilhem-le-Désert, pic de Liausson, Madières, le Larzac au Caylar, Saint-Mlaurice, la Vacquerie, Bédarieux. - Aude : Fontanes. - Pyr.-Or. : plateaux de la Font-de-Comps, forêt de Boucheville, bois de Fanges, Saint-Antoine-de-Galamus. - Rochers calc. des Pyrén. centr., de la Lozère dans les envir. de Iende, Florac, Meyrueis.

L. pruthenienm L. - Forèts et prés humides. - Doubs : Gonsans. - Jura : entre Pleurre et Rye près du moulin de Sergenon, bois de Bovard près Salins. - Envir. de Genève aux bois de Prangins, du Vangeron, de Veyrier et de la Bâtie, marais de Trélex et de Divonne; plus au sud dans l'Ain à la forêt de Rolonne près Belley, Leyment, Marlicu, Saint-André-de-Corcy, Montribloud, entre Mionnay et Tramoy, forêt de Seillon, Etang Genoux près Pont-de-Veyle. - Valais : Vervey. - H.-Sav. : Choisy, la Sémine, la Caille, Annecy, entre Cluse et Sallanches. - Sav. : env. de Chambéry et d'Aix, mont du Chat, entre Apremont et le col du Frẻne. - Is. : Voreppe, la Tour-du-Pin. S.-et-L. : Cuisery, bois près de l’étang Gallepois. - Ard. : झazan. - Pyr.-Or. : Villefranche, bois de Salvanère, forêt de Boucheville.

L. Heirsutum Lam. L. panax Gouan. - Prairies des Alpes à sol siliceux. - Vaud et Valais : Javernaz, Dent de Morcles, Fully, Isenau, Trient, Rosselcina, Alesse, Saint-Pierre, Saint-Bernard, 
Catogne, Novelli d'Hérémence, Bellalui, Torlain, Distel de Saas, Zermatt. - H.-Sav. : col de Balme, chaine du mont Blanc jusqu'au Jardin et au Courcrele, les Contamines, le Brévent, Samoins. - Sav. : Hauteluce, col Joly, la Thiupa entre Pesey et Champagny, Crêt du Ré sur Aime, les Allues, les Hottets, mont Cenis. - Is. : chaînes de Belledonne et des Sept-Laux, Colon, le Gleyzin, Brandes en Oisans. - II.-Alp. : Lautaret, Monestier de Briançon, Névache, col de Vars.

SILET trilobum Scop. - Coteaux secs des Pyr.-0r. : Costa-Bona, Canigou, Font-de-Comps, mont Louis.

Levistreude offielnale Koch. - Prairies, - Is. : la Tronche, route de Bourg-d'Oisans à Briançon. - H.-Alp. : Rosans. Gard : illes du Rhône en face de Vallabrègues, l'Espẻrou. Pyr.-Or. : mont Louis, Canigou, Capcir, Llaurenti.

ANGeLrCA sylvestris L. - Prés et bois dans la partic septentr. et moyenne du bassin depuis la plaine jusqu'aux sommités. Dans la région mérid. l'Ingélique se trouve sculement dans les montagnes; ainsi dans les $\Lambda$ lpes-Har. au mont Cheiron, le Bar, Isola, Saint-Martin, Saint-Dalmas; dans le Gard autour du Vigan, à Aumessas, l'Aigual, bords du Gardon; dans l'Ilér. sur l'Espinouse; dans l'Aude et Pyr.-Or. sur les Corbières, la Montagne Noire, le Llaurenti, Canigou, Prats-de-Mollo.

A. montana Gaud. - Diffẻre du précédent, avec lequel il est rquclqueIois mèlé, par les segments sup. des feuilles réunis et décurrents à la base; fruits moitié plus petits.

A. Tazulil Gouan.- Prairies.-Aude et Pyr.-Or. : lac de Quérigut, vallée de la Bruyante, Llaurenti, Formiguìres en Capcir, Pla dels Abellans, entre Carlitte et Coma de la Tet, bords de la Tet au-dessus des Bouillouses, mont Louis. - Pyr. centr. autour de Bagnères, Esquierry.

A. Pyrenaea Spreng. - Frairics des montagnes à sol siliceux. Espèce caractéristique des terrains granitiques, gneissiques et volcaniques, des montagnes du centre de la France, des Cévennes et de toute la chaîne des Pyrénées, Aubrac, mont Dore, Lozère, Cantal, Pilat, Pierre-sur-IIaute, montagnes du Vivarais vers les sources de la Loire, Mazan. - Aude et Pyr.-0r. : vallée 
de la Bruyante, lac de Quérigut, Boutairol, Llaurenti, Canigou, Pla Guillem, Costa-Bona, vallées d'Eyne et de Llo, mont Louis.

Commun dans les hautes Vosges depuis l'extrémité septentr. de la chainn jusqu’aux Ballons de Servance et de Giromagny. Nul dans la chaîne jurassique et dans toute la région à l'est du Rhơne.

SEgrven enrorifolin L. - Prés et bois des terrains siliceux ou tourbeux. - II.-Saône : vallée de Mansvillers, le Salbert, Plancher-Bas, Belverne, Etobon. Chagey. - Doubs et Jura : marais tourbeux de la Vèze près Besançon, Plumont, Pleurre et Sergenon près Dole, Salins. - C.-d'Or : le Morvan autour de Saulieu, Châtillon-sur-Seine. - S.-et-L. : Nontjeu, Fragny, Saisy, Bouhy, Cluny, Louhans, - Marais des envir. de Genève, bois de Veyrier, Sionet, Roellebot, Divonne. - Vaud : vallée de Joux, Iverdon, Yronand, Chailly, Vovey. - H.-Sav. : la Caille. - Rh. : Vauxen-Velin. - Is. : Charvieu, Crémieu, Bouresse. - Pyr.-Or. : Canigou, vallée d'Eyne, Llaurenti, Fontrabiouse en Capcir. Pyr. centr.

ANETHUM graveolens L. - Champs cultivés de la région mérid. - Var et Alp.-Nar. : le Luc, Drap, Puget-Théniers. Gard, - Hér. : Rieucoulon, Castelnau, Lattes, Béziers. - Pyr.Or. : Argelès, Banyuls-sur-Mer, Canet.

PeUCodanure offieinale L. - Pàturages, haies, bois de la région mérid. - B.-du-Rh. : Sommet de Sainte-Victoire. - Var et Alp.-Nar. : Bagnols, Frẻjus, Grasse, Bézaudun, Nice et Menton.-Gard : Beaucaire, bois de la Devèze près Saint-Vincent.Hér. : Montpellier à Grammont et à Lamoure.-Aude et Pyr.-Or. : pont de la Ricardo près Villeneuve, Saint-Antoine-de-Galamus. - En dehors de noz limites dans la Loire entre Sury et Précieux, entre Monthrison, Montrond et Feurs, Grézieu-le-Fromental.

P. parisiense D. C. - Bois des terrains siliceux. - Nul dans l'est de la France. - Commun dans les chaînes granitiques du Beaujolais, Lyonnais à Dardilly, Charbonnières, Tassin, Souzy. En dehors de notre bassin dans la Loire à Fouilly-les-Feurs, Néronde, Sainte-Foy, Saint Sulpice, Valeilles, bois entre la Fouillouse, Saint-Just et Saint-Bonnet-les-Oules. - Puy-deDôme, Allier, Creuse. 
P. cervaria Lap. - Coteaux secs dans tout le bassin.

F. oreoselinum Monch.- Coteaux piorreux, plus abondant dans les terrains siliceux. Commun dans la partie septentr. et moy. du bassin. Plus rare dans la région mérid. - Vaucl. : au pied du mont Ventoux vers Flassan.- Var et Alp.-Mar. : les Maures, Saint-Martin-Lantosıue, vallec de Caïros, N.-D. de Fenestre.Gard : le Vigan, bois de Salbous près Alzon. - Hér. : le Larzac au Caylar, l'Escandorgue à Romiguière, Mas-de-Mouriès, Bédarrieux.-Pyr.-Or. : forêt de Boucheville, Bellegarde, Céret, Amélie-les-Bains, Prats-de-Nollo, Fontpédrouse, Castell, Saint-Martin-du-Canigou. - Manque dans les Pyr. centr. - Commun sur les terrains siliceux du centre de la France.

P. venetum Koch.-Bois.- Valais : Ravoire-sur-Martigny, Rosey en face de Pisscvache. - Alp.-Har. : Menton, Nice, Sospel, la Giandola, Bezaudun. - Gard : Chartreuse de Valbonne, Pouzillac, bords du Rhône à Coudoulet.-Pyr.-Or. : Montlouis, vallée d'Eyne, Capcir vers Fontrabiouse, montagne de Madres entre Cornella-du-Conflent et Villefranche.

F. alsaticum L. - Coteaux pierreux. - Is, : Annoisin près Crémieu, entre Corps et la Salette. - Dr. : Romans, Crest, Loriol, Montélimar.-Ard. : Tournon. - H.-Alp. : Châteauvieux près Gap, Tallard.-B.-Alp. : Digne à Vaunavès. - Vaucl. : la Palud. - En dehors de notre bassin dans la Loire, entre Montbrison, Nontrond et Saint-Marcellin, butle de Saint-Romain-le-Puy, Merlieu.- Allier, Limagne d'Auvergne dans le Puy-de-Dome.

1. austrineum Koch. - Prés marécageux. - Envir. de Genève au marais de Sionet. - Vaud et Valais : Morcles, Fully, du lac de Tannay aux Crosses. - H.-Sav. : Brizon, vallée du Reposoir, abbaye de Vallon, Saint-Jean-d'Aulph, Bellegarde près Abondance, remonte les vallées de Mégevelte et d'Onion jusqu'à SaintGeoire.

Var. nigricans Gaud. - Vaud et Valais : Dent de Jaman, Morcles, Salvan sur Zobourlaye.

P. carvifolia Vill.- Près humides. - Chaine du Jura helvétique et français jusque dans les montagnes du Bugey autour de Retord, Arvières, Ordonnat, Tenay, Hauteville.- C.-d'Or : Roche- 
en-Brenil, Auvenet, Tournesac. - S.-ct-L. : Autun, Cluny. Is. : Gde-Chartrcuse, Saint-Nizier, Corençon. - Aude et Pyr.Or. : Belcaire, base des Albères, Saint-Génis, le Boulou, Maureillas, Céret, Arles, vallée de la Tet, Rigarda, Prades, Saint-Paulde-Fenouillet. - Pyrén. centr., Lozère autour de Mende.

p. paIustre Moench.- Prés humides de la partie septentr. et moy. du bassin. - Nul dans la région mérid.

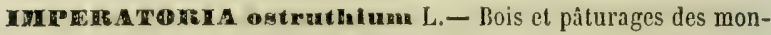
tagnes. - Rare dans les Vosges au Hoehneck et au Schneeberg; nul dans la chaine jurassique. - Valais : Saint-Bernard, Alpes de Saxon, Thyon, Arolla, Val-Reschy, Zermatt. - H.-Sav. : les Contamines sur Saint-Gervais, mont Lachat, la Diosaz, Samoëns, Sixt, mont Ardin, Brizon, Méri. - Sav. : Hauteluce entre Aime et le Crêt-du-Ré.-Is. : chaines des Sept-Laux et de Belledonne, Prémol, col d'Oddic au-dessus des Adrets, Rivier d'Allemont, Bovinant au pied du Grand-Som, Saint-Pierre-de-Chartreuse, Chamechaude, mont Chamoux à la Salette. - H.-Alp. : Lautaret, vallées de la Taillante et du col Ånnel, Saint-Véran à RocheRonde, col de Vars, Orcières, bois de Loubet près Gap.-B.-Alp.: Lauzannier, vailon du Châtelet. - Alp.-Mar. : sources du Var, bois du Boréon, vallon de Fenestre, Sainte-Inne-de-Vinai, Salsamorena. - Loire : Pilat vers le Bessac, Thélis-la-Combe, Pierre-sur-IIaute, Noirétable dans les rochers de l'Ermitage. Gard et Lozère : Concoule, l'Aigual, Pont-de-Montvert, Costelades au-dessus de Villefort. - Aude et Pyr.-Or. : Boutairol, vallèc de la Bruyante, lac de Quérigut, Llaurenti, Tartaressade, la Font-de-Comps, vallée de Molitg à Jau, Canigou, Cady, Llagone, mont Louis.- Pyrén. centr., Cantal. - Mont Dore.

Var. angustifolia Bell.-Alpes de Tende.

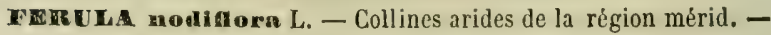
B.-du-Rh. : Marseille aux Petites-Crottes.-Var : Toulon, Hyères, Frẻjus. - Gard : bords du Gardon au Moulin de la Beaume, Anduze, Saint-Nicolas. - Hér. : la Gardiole au Trou-de-Miége, Roque-de-Fabrìgues, la Madeleine, Pic Saint-Loup, les Arcs, de Saint-Bauzile à Ganges, la Sérane à Saint-Jenn-de-Buèges. Aude : envir. de Narbonne, Clape-au-Rec, las Gueitos, Font- 
froide, Roçuefort dans les Corbières. - Pyr.-Or. : Llaurenti, Nohèdes, Font-de-Comps, le Canigou à Saint-Martin.

F. glauea L. - Var : lies des Imbiers el de Bandol. — Gard : Pierremale près $\Lambda$ nduze.

F. Ferulage L. - Var et $\Lambda$ lp.-Nar. : le Luc, Frẻjus, Grasse, Vence, Drap, col de Braus.

DPOPANAX chironium Koch. - Lieux arides de la région mérid.-B.-Alp. : Castellane. - B.-du-Rh. : pont de Mirabeau, vallon des Crides à Saint-Pons-de-Gémenos, - Var : Toulon, Iyères, lc Luc, Fréjus, couvent du Thoronet. - Hér. : montagne de la Gardiole, Saint-Jean-de-Védas au moulin Geniès, Cambous près de Viols, Pic Saint-Loup, Fabrègues, la Causse de la Selle, Béziers aux Cotes de Baissan. - Aude : versant mérid. de la IIontagne-Noire, Salsigue, mont Alaric. - Pyr.-Or, : envir. de Perthus et de Céret, plateau de Château-Roussillon, Canet, Força-Rẻal.

PASTINACA satira L. - Espèce polymorphe très-commune dans les prés et pâturages, à laquelle se rattachent les $\boldsymbol{P}$. pratensis Jord., $P$. tereliuscula Jord., $P$. propinqua Jord., $P$. opaca Bernh., dont la distribution géographique n'est pas suffisamment connue.

P. urens Req. - B.-Alp. : Annot.-Vaucl.-Iles et chaussées du Rh., Villes, Mormoiron. - Ard. : Privas. - Alp.-Mar. : Entraunes, le Bar, Sospella Giandola, Menton. - Gard : envir. du Vigan, Sauve. - Hẻr. : Saint-Martin-d'Orb, Avène, Saint-Étienne-deGourgas, Ganges.-Pyr.-0r. : vallẻe du Rẻart, Força-Rẻal.

HERACLEU Aphondylium L. - Espèce polymorphe trèscommune depuis la plaine jusqu'aux sommités des régions septentr, et moyenne du bassin : plus rare dans la région mérid. où elle est signalée dans le Var à la Sainte-Baume; dans la partie montagneuse des Alpes-IIarit.; dans le Gard à Coucoule; dans les Pyr. Or. à Mont-Louis, le Canigou, la Cerdagne. On a peu de renseignements en ce qui concerne la distribution des $H$. pratense, astivum, stenophyllum, delphinense décrits pär M. Jordan.

II. Lecókii Godr. Gr., - Différe du précédent par ses flcurs d'un jaune verditre, non rayonnantes; il remplace l'II. sphondylium dans les terrains volcaniques et granitiques du Cantal, Lozère, H.- 
Loire, partie voisine de l'Ardèche, Cévennes du Gard et de l'Hérault jusqu'à l'Espinouse, ainsi que sur les deux versants de la Montagne Noire.

Ir. Panaces L. - Bois et rocailles des montagnes calcaires. Chalne du Jura helvétique et français depuis le Chasseral et le Creux-du-Van jusqu'à la Dôle, Faucille, mont Joux, Colombier, Reculet. - H.-Sav. : Salève au pied des roches d'Archamp, Cornettes-de-Bise et Dents d'Oche dans le Chablais, les Platets au-dessus de Passy. - Is. : Grande Chartreuse, Saint-Eynard, Bourg-d'Oisans.

I. alpinum L. H. pyrenaicum Lam., - Rocailles et prairies. Jura bâlois, bernois et soleurois. - Ain : Au-dessus d'Hauteville à Planachat et au Golet de la Rochette, oủ il est très-abondant. - Aude et Pyr.-Or. : Salvanère, Laurenti, mont Louis, Borde-Givès, Formiguères en Capcir, vallées de Llo et de Vernetlcs-Bains, Castell, Saint-Vincent, Prats-de-Mollo. - Assez commun dans les Pyrén.-Centrales.

H. minimum Lam., - Rocailles des montagnes calcaires. - Is. : mont Aiguille. - H.-Alp. : Mont Aurouse. - Dr. : Mont Glandasse, entre Archianne et Glandasse. - Vaucl. : Mont Ventoux.

- Alp. Mar. : Sources du Var, Roche-Grande, Énéance sur Saint-Martin-d'Entraunes.

TORDYLXYM maximum L. - Collines incultes, moissons. Nul dans le Jura helvétique et français, bien qu'il se trouve en Alsace et dans la Lorraine. - Apparait dans l'Ain vers PierreChâtel, Meximieux, Saint-Maurice-de-Remens, Reyrieu; puis dans l'Isère à Crémieu, Décines, Vienne, Pariset, Pont-de-Claix ; dans les H.-Alp. autour de Gap; dans la Drơme à Romans, Barnave. - Très-commun dans C.-d'Or et S.-et-L.; puis autour de Lyon sur les Balmes qui s'étendent de Villeurbanne à Décines, Saint-Cyr-au-mont-d'Or, Tassin, Francherille, Beaunant, SaintGenis-les-0lliẻres. - Ard. : envir, de Privas. - Commun dans Vaurluse. - B.-du-Rl. : Narseille à Saint-Tronc, Vavenargues, Arles, Montmajour. - Var et Alp.-Mar. : La Tourne près Belgencier, Lerens, Nice à Montgros, Menton. - Commun dans le Languedoc, le Roussillon, les Pyrén.-Centr., le Tarn et le centre 
de la France jusque dans la Loire autour de Montbrison, Sailsous-Couzan, Boön, mont d'Uzor.

T. apulum L. - Dr. : Ie Buis, Nyons. - Var : Fréjus. - Aude :

Narbonne.

GAYA simplex Gaud. - Pelouses et rochers; plus commun sur les terrains siliceux des Alpes et des Pyrenées. Vaud et Valais : Enzeindaz, Grandvire, Fully, Javernaz, Saint-Bernard, Alesse, Diez, Zanrion, Thyon, mont Nuoble, Maya, Mayenhorn, Rothorn, Augstelberg, Gorner, Zermatt, Gemmi, Grimsel.-H.-Sav. : Jalouvre, Vergy, Méry, col d'Anterne, Platets, montagnes du Chablais, Cornette-de-Bise, mont des Granges, Dents d'Oche, Brévent, col de Balme, chaine du mont Blanc jusque sur les moraines de Tré-la-Tête, au Jardin et au Couvęrcle. - Sav. : Cots du Bonhominte èt de la Seigne, mont Gen is. - Is. : Chạne de Belledonne, envir. du lac Crouzet, Champrousse, Gliaînes des Sept-Laux et des Grandes-Rousses, mont de Lans, mont Chamoux à la Salette. - H.-Alp. : Lautaret, Galibier, Névache aux Rochilles, col des Hayces, mont Viso, Saint-Véran à Clausis, Pje de Chabrières. - B.-Alp. : Plate-Lombarde, Bérard. Alp. Mar. : Mont Bego, col de la Fenestre, lac d'Entrecoulpes. Pyr.-Or. Conques de Cady, Cambres d'Aze, Nurria. - Manque dans les Pyrẻn.-Centrales.

CRTTHMUM marltimum L. - Rochers et graviers maritimes. -B.-du-Rh.-Var. Toulon, ile de Porquerolle, Fréjus.-Alp.Mar. : Cannes, Nice, Menton.- Hér. : Frontignan, Cette, Balaruc, Palavas, Maguelonne. - Aude : îies de l'étang de Bages et Sainte-Lucie. - Pyr.-Or.: Argelès, Collioure, Banyuls.

ENDRESSIA Prenaica Gay. - Prairies des Pyr.-Or. à mont Louis, vallée d'Eyne et de Capcir, Llaurenti. Ce sont les seules localités de cette rarissime espèce.

MEUn athamanticum Jacc. - Prairics des montagnes à sol siliceux. - Nul sur les calcaires; ne se trouve dans la chaine jurassique que dans les prairies tourbeuses à sous-sol sablonneux depuis le Creux-du-Yan jusqu'à Mouthe et particulièrement autour de Pontarlier. - Très-abondant dans les pâturages des Vosges depuis Sarrebourg et la vallée de la Bruche jusqu’au ballon 
de Giromagny et de Servance; puis dans la zone vosgienne de la II.-Saône à Fresse, Montessaux, Champagney, Frahier, Chagey, Champey, Étobon, forêt d'Arobert, vallées de Miélin et de la Fonderie, la Prandière, Planche-des-Belles-Filles, vallées de la Combeauté et du Gehart. - Vaud et Valais : Grandvire, audessus de Bourg-Saint-Pierre vers la cantine de Proz, les Lancettes, la Pierra. - H.-Sav. : Samoëns, les Houches, monı Lachat, les Contamines. - Sav. : montagnes d'Hauteluce et de Beaufort, mont Cenis. - Is. : Prémol, col Oddie au-dessus des Adrets, Monestier-de-Clermont, col de Menée. - Dr. : Aucelon, Lalley, mont Glandasse. - II. Alp. : Lantaret, mont Viso, col de Vars. - B.-Alp. : Lauzannier, Malemort, Parpaillon au-dessus de Boussolières. - Alp.-Nar.-Loire : Pilat, Pierre-sur-Haute. Ard. : Mezenc, la Chavade, Mazan. - Gard : Espérou, Concoule. Aude et Pyr.-Or. Boutairol, Llaurenti, mont Louis, vallées d'Eyne et de Llo, Bouillouse, Canigou, envir. des lacs d'Evol et de Nohìdes. - En dehors de nos limites sur les terr. granit. des Pyr.-Centrales, sur les terr. granit. et volcaniques des monts Dore, Cantal, Aubrac, Lozère.

Ir. Mutellina Gaertn. - Pâturages et rochers des montagnes à sol siliceux. - Nul dans la chaine jurassique et, par défaut d'altitude, dans celle des Vosges. — Plus commun dans les Alpes que le précédent. - Valais: Grand-Saint-Bernard, Zermontana, Thyon, Arolla, Vouasson, Zermatt, Sirwolten, Furggen de Binn, OErnergalen, glacier du Rhône, Grimsel.-H.-Sav. : grès nummulitiques du Vergy et Léri, Mégève, mont Charvin, Cornettesde-Bise, mont des Granges et des Cornetles, col de Balme, chaine des Aiguilles-Rouges et du mont Blanc jusqu'au Jardin et au Couvercle. - Sav. : montagnes granitiques de Crest-Voland, Hauteluce et Beaufort, col Joly, Roselein, Chapieu, le Bonhomme, les Avanchers et les Allues, la Valloire, mont Cenis.-Is. : chaîne des Sept-Laux d'Allevard. - H.-Alp. : Lautaret, mont Viso à la Traversette. - Alp.-Mfarit. - Manque dans les Pyrénées; existe au mont Dore et au Plomb du Cantal.

M. adonidifolium Gay. - Prairies du mont Cenis et de Lavalde-Tignes en Savoie. 
SILACs pratengis Besser. - Prairies humides dans tout le bassin.

S. virescens Boiss. - Coteaux calc. de la Côte-d'Or entre Dijon et Beaune, Messigny, Combe des Chainaux, plateaux au nord de Sainte-Foy, Combe d'Auvenay.

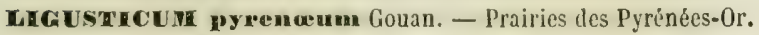
à mont Louis, vallée d'Eyne, Capcir, Trencada d'Ambulla, Vernet-les-Bains. - Pyr. centr. à Cagire, Gèdre, Pragnères, Piquette d'Endretlis, Castanèse.

L. Cerulaceum L. - Prés et pâturages des montagnes. - Manque dans les Vosges, les Alpes de la Suisse et les Pyrénées. - Jura : Reculet au vallon d'Ardran, Colombier de Gex, la Do̊le. - Sav. : mont Cenis. - H.-Alp. : col de Glaise, Chaudun, Rabou, Sẻuse, Aurouse, mont Chabrières, mont Viso. - B.-Alp. : Lauzannier. - Alp.-Nar. : sources du Var, val de Bourdous, Entraunes, Estenc, col Bertrand entre Lupega et Tende, Salsaruorena.

ATIKAMANTA eretemeis L. - Rochers calcaires des montagnes. - Nul dans les Vosges, Pilat, Forez, massif granitique, schisteux et volcanique de l'Auvergne, Velay, Cantal, Vivarais, Cévennes. - Espèce caractéristique des rochers calc. du Jura helvétique et français, bassin du Doubs, cirque de Mauron, Cotes du Dessoubre, Clos du Doubs, Lomont, Crêt des Roches, Roche du Mont près Ornans, mont d'Or, Noirmont, la Dôle, Colombier, Reculet; descend jusque près des vignobles de Salins, Arbois, Baume, Lons-le-Saunier; montagnes de Nantua, Tenay, Hauteville, Colombier du Bugey. - Vaud et Valais : chaines calcaires depuis les montagnes de Bex jusqu’à la Gemmi, dans la chaîne mérid. entre mont Nuoble et Maya. - H.-Sav. : Salève, montagnes du Chablais, Haut-de-Lin, mont des Granges, Chalune, Roc d'Enfer, mont d'Ubine. - Sav. : mont du Chat, Nivolet et autres montagnes des Bauges, Ilauteluce, montagnes calc. de la Tarantaise et de la Maurienne jusqu'au mont Cenis.-Is. : montagnes de la Grande-Chartreuse et de Saint-Nizier, col de l'Arc, la Motte-les-Bains, collines des environs de Crémieu entre Hyéres et Vernas. - Dr. : mont Glandasse, Archianne près Nonnières.-H.-Alp. : Lautaret, monl Viso, Saint-Véran, Séuse, 
Aurouse, col de Glaise, le Sapet, pic de Chabrières. - B.-Alp. : la Condamine, Sérennes, Lauzannier, Maurin, Vallonnet. Vaucl. : mont Ventoux. - Sommités des Alp.-Mar. - C.-d'Or. : calc. jurass. de Gevrey, Beaune, Couchey, Bouilland, - Gard: l'Espérou, rochers sur la route de Meyrueis. - Hér. : roch. dolomitiques du Caylar.-Pyr.-Or. : mont Louis, bords du Sègre, vallée du Llo. - Manque dans les Pyrén. centr. - En dehors de nos limites sur les calc. jurass. de la Lozère autour de Mende, Florac, Vébron.

TROCHISCANTHES nodiforus Koch. - Forèts des montagnes. - Nul dans les Vosges, la chaine jurassique, le centre de la France, les Cévennes et les Pyrénées. - Vaud et Valais : Bouveret, Port-Valais, Epinas:ey entre Saint-Maurice et Pissevache. - Sav. : bois Champion près Brides. - Is. : la Mure.II.-Alp. : bois de Rabou, de Chaudun et de l'Ermilage près Gap, mont Séuse, Embrun. - B.-Alp. : bois de Bréau près Annot. Alp.-Mar. : bois du Farghet, col de Tende, Sainte-Anne-de-Vinaï, Saint-Martin-Lantosque, Roccabigliera. - Aude : moulin de Fontjoucouse près Narbonne.

CNIDIUM apioides Spreng. - Bois des montagnes. - Is. : Vif, la Salette. - H.-Alp. : Charance, Rabou, Loubet, Combe-Noire, Séuse, mont Rognouse près Ribiers. - B.-Alp. : Digne, Annot à Ruannette. - Alp.-Har. : mont de l'Agel au-dessus de la Turbie, sommet de Rossignola.-Pyr.-Or. : Céret, Arles, Prades, Mosset, vallée du Cornella-du-Conflent.

Xataieda seabra Meissn. - Rocailles des Pyr.-Or. au fond de la vallée d'Eyne, sur les deux versants de la Collada de Nuria, Coma de la Baca près des sommités de la vallée de Carença; Pourret dit l'avoir trouvé à Salvezines près Axat (Aude); ce sont les seules localités connues de cétte espèse.

Le rarissime Dethavia tenuifolia Endl. qui existe dans les montagnes calcaires des Pyr. centr. depuis Esquierry jusqu'aux Eaux-Bonnes et à Lhéris n'a pas encore été trouvé dans les Pyr.-0r. ni ailleurs.

SESELT tortuosum L. (non Poll.). - Rochers et rocailles de la région mérid. - Dr. : Montélimar, Crest, Njons, Satnt-Paul- 
Trois-Châteaux.-B.-Alp. : Saint-Benoil près Annot.-M.-Alp.: bois de Tallard, Ribiers. - Vaucl. : Mormoiron, Mazan, envir. d'Avignon. - B.-du-Bh. : Aix, Arles, la Crau, les Milles. - Var et Alp.-lfar. : Toulon, Rians, le Bar, Cannes, île Sainte-Ifarguerite, golfe Jouan, Saint-Césaire, l'Escarène, Luceram. - Ard. : Baix, Payolive, la Voulte. - Gard : envir. de Nìmes, Beaucaire, Saint-Gilles, Villeneuve. - Hér. : commun dans la partie basse du département. - Aude: les Corbières, la Clappe et Pech-de l'Agnẻle près Narłonne. - Pyr.-Or. ; Saint-Paul-de-Fenouillet, Collioure, Port-Vendres, Banyuls-sur-Mer.

S. elntum L. - Rocailles de la région mérid. - Dr. : Crest, Nyons, Saint-Paul-Trois-Châteaux. - B.Alp.: Gréoulx, - Commun dans Vaucl, B.-du-Rh. - Var : mont Faron, Bagnols, Touris, le Luc. - Gard : Villeneuve, Beaucaire, bords du Gardon, Nimes, Uzès, Tresque, Anduze, Bagnols, Saint-Ambroix, Alais. - He̊r. : Montpellier à Montmaur, la Colombière, Fontfroide, Grabels, Saint-Clément, Montferrier, Montarnaud, Saint-Martin-de-Londres, Frontignan, Saint-Irathieu, Moulin-Genies, Prades, le Larzac à la Vacquerie. - Pyr.-Or. : Banyuls-sur-Mer, Céret, Saint-Paul-de-Fenouillet.

S. montanum L. - Coteaux calcaires; rare sur les roches siliceuses. - Calcaires des envir. de Belfort à la Miotte, la Justice, la Perche, Heroux. - H.-Saone : calc. jurass. de Chariez, Grattery, Frotey, Navenne, Arbecey, Chargey-les-Port, Fouvent. Très-commun sur les calc. jurass. de C.-d'Or, S.-et-L., Doubs, Jura, Bugey, Valromey, Revermont. - Manque sur le versant helvétique ainsi que dans la Sav. et l'Is. - H.-Alp. : Rosans, Tallard, Ribiers, Chartreuse de Durbon. - Dr. : Ie Buis, col de Lunel, Saou près Crest, Nyons. - B.-Alp. : Sisteron. - Vaucl. : mont Ventoux, Saint-Gens, Saint-Amans, Caromb. - B.-du-Rh. et Var: Sainte-Victoire, Vauvenargues, Sainte-Baume, mont Faron. - Alp.-Mar. : Saint-André près Nice, entre Tourette et Levens, au-dessus de Menton, l'Escarène, Saint-Sauveur. Commun sur les coteaux calc. du Gard et de l'Hér. - Aude : Fontanes, Rouze dans le Donèzan. - Pyr.-Or. : Cambres d'Aze, Yernet-les-Bains, Rigarda, Prats-de-Mollo, Saint-Antoine-de- 
Galamus. - Terr. calc. des Pyr. centr., du Tarn et du centre de la France.

S. glancescens Jord. - Coleaux calc. de C.-d'Or, S.-et-L. - Is. : le Manival au-dessus de Saint-Nazaire, chemin de Saint-Pierre-deChartreuse. - Ain : plaine d'Ambronay.

S. coloratum Ehrh. - Coteaux pierreux. - Nul dans le Doubs et Jura.-H.-Saône : Montigny, Arbecey, Grattery.- Vaud: Aigle, la petite Rape près de Payerne, la Bourdonnette près Lausanne, Prangins. - Valais: Saint-Maurice, Folatères au Rosey et à Tourbillon.-H.-Sav. : Crevin au pied du Salève, Collonges. - Sav. : Lans-le-Bourg. - S.-et-L. : la Chapelle-sous-Brancion, Culles. - Rh. : Couzon, Bourdelans. - Ain : la Pape, Muzin, Parves, Heximieu au sommet du Mont, Saint-Jean-de-Niost. Is. : Crémieu, Pont-Chéry, Vienne, Bastille de Grenoble, Pontde-Claix, le Touvet. - Dr. : Saint-Vallier, - H.-Alp. : Rabou près Gap. - Alp.-IIar. : envir. de Nice, col de Braus, Raus. Gard : Chartreuse de Valbonne, Pouzillac. - Pyr.-Or. : Pratsde-Mollo. - Loire : envir. de Montbrison.

S. brevicaule Jord. - Ain à Inimont.

s. earvifolium Vill. - Rochers des montagnes. - Is. et II.-Alp. : rochers des bords du Vénéon près Venosc, Noyer en Champsaur, les Andrieux en Valgaudemar, Névache, les Baux, Charance, Manteyer, Ribiers. - Sav. : envir, de Mouliers vers le lac SaintMarcel. - B.-Alp. : Saint-Paul, la Condamine. - Alp.-Mar. : Saint-Martin-Lantosque. - Manque dans les Pyrénées.

S. Libanotis Koch Libanotis montana All. - Rochers calcaires des montagnes; se trouve aussi sur les eurites et porpbyres des Vosges jusque sur le Ballon de Giromagny ainsi que sur les terrains volcaniques des monts Dômes et Dore. - Commun sur les calc. jurass, du Doubs, Jura et din vers Nantua, Maillat, Virieule-Grand, Villebois.-C. d'Or : Dijon, Messigny et toute la Cóte. - S.-et-L. : Dezize, Sampigny. - Is. : la Bastille de Grenoble, mont Rachais, Grande-Chartreuse, Chalais, gorges du Crossey, la Moucherolle. - H.-Alp. : Charance, Rabou, Combe-Noire, Séuse, Casse, Rochoux près Saint-Véran. - B.-Alp. : Longet-deMaurin. - Alp.-Mar. : les Viosennes, Lupega, Sanson. - Gard : 
Salbous, Campestre. - Aude et Pyr.-Or. : Boutairol, Llaurenti, Capcir, Cerdagne, Canigou. - Roch. calc. des Pyr. centr.

HTravonia pastinacafolla Bertol. - Espèce qui habite la Corse, Sicile, Grèce, Espagne, et qu'on a trouvẻe à Toulon.

Fon rumum officinale Gaertn. - Bords des chemins, décom. bres; souvent cultivé.

F. piperitum D. C. - Lieux incultes. - Alp.-Har. : Grasse, Antibes, Ile Sainte-Marguerite, San-Remo. - Gard : le Vigan.

RrDOLFCA segetum Moris. - Champs du Var et Alp.-Mar. : cap Brun près Toulon, cap Martin près Menton.-B.-du-Rh. : a été trouvé à Aix au Defens près de l'étendoir d'un lavage de laine.

ETHUSA eymapium L. - Champs incultes, bord des chemins, décombres, dans tout le bassin.

aNANTIE exoeata L. - Mares des Pyr.-0r. à Saint-Cyprien, Argeles, Prades.

E. pimpinelloides L. - Prés humides de la région mérid. B.-du-Rh.: le Tholonet, Arles. - Var et Alp.-Mar.: embouch. de l'Argens près Fréjus, Grasse, Cannes, Château de la Garde prìs Villeneuve, Baurous près Villefranche, cap Martin près Menton. - Gard : bois de Cyguan, de Campagne, de Candillac près Anduze. - Commun dans l'Hér. et dans l'Aude. - Pyr.Or.: Argelès, Collioure, Banyuls, Font-de-Comps, Costujes.

E. Cachenalil Gmel. - Prés marécageux. - C.-d'Or : Meursault, moulin des Etangs. - Lyon au Grand-Camp. - Is. : Décines, Meyzieu, Charvieu, Saint-Romain près Crémieu, Polygone de Grenoble, Saint-Ismier, Cosset près Claix. - Envir. de Genève aux marais de Sionet et de Gaillard. - H.-Sav. : Bossey au pied du Salève, Menthon, Saint-Jorioz. - Dr. : Montélimar. - B.duRh. : Pont-de-Pertuis, Pic de Bretagne vers la source de la Glacière, Saint-Antonin. - Var: Toulon. - Gard: Saint-Laurent, Bellegarde, Saint-Gilles. - Hèr. : Mireval, Laltes, Gramenet, Pérols, Mauguio, Saint-Hartin-de-Londres. - Aude : envir. de Narbonne.

AE. silaifolia Bieb. - Lieux mouillés. - Hér. : Mireval, Lespignan, Portiragnes près Roquehaute, Mauguio au Mas-de-Marot. - Aude: la Rouquette et Fontlaurier près Narbonne. 
E. peucedanifolla Poll. - Prés humides. - Jura : la Bresse à Pleurre, Sergenon, bois de Rye, entre Chavannes et Réconoz. Commun dans C.-d'Or, S.-et-L., Rh., partie bressanne de l'Ain. - Is. : Polygone de Grenoble, Domène. - Dr. : Saint-Vallier. B.-du-Rh. : Saint-Chamas. - Alp.-Har. : Nice au'Var, col de Tende. - Gard : Genolhac, Concoule. - Hẻr. : l'Espinouse à Fraisse et à la Salvetat, Limalou. - Pyr.-0r. : prés llu littoral, Nohèdes, Cambres d'Aze.

OE. Astuloma L. - Prés humides et marais,-Commun dans C. d'Or, S.-et-L. - Doubs : envir. de Montbéliard, bords de l’Ognon, la Ilalcombe près Besançon.- Envir. de Genève à Sionet, Choulex, Meynier, Mattegnin. - Taud : Roche, marais d'Orbe, Yrerdon, Ironand, Payerne. - Ain : Dombes et Bresse. - H.-Sav. : Menthon. - Is. : Charvieu, Estrablin, Septème. - Vaucl. : Orange, Gadagne. - B.-.Uu-Rlı. : Arles, Marignane, étang de la Palud. - Var et Alp.-Mar. : Frijus, Toulon, Grasse, Chàteauneuf, Cannes, Vaugrenier près Antibes. - Aude et Pyr.-Or. : envir. de Narbonne, étang de Salses, et du Bordigole près Torreilles.

a. globulosa L.- Prés marécageux de la région méditerr. - B.du-Rh. : borus de l'ẻtang de Marignane. - Var et Alp.-Mar. : iles d'Hyères, le Luc, Frijus, le Lavandou, Antibes, Cannes, Nice au Yar. - Hér. : pont de Galabert près Saint-Gély-du-Fesc, Agde.-Pyr.-Or. : Canet, Saint-Cyprien, Argelès, Banyuls.

E. phellandrium L. - Slarais, ruisseaux. - Assez commun dans H.-Saône, C.-d'Or, S.-et-L., Doubs, Jura, Ain. - Autour de Lyon à Yrour, étang du Loup. - Is. : ètang de Rye près Crémieu, Septeme, Pont-de-Beauvoisin.-B.-du-Rh. : Arles.-Alp.Mar. : Vaugrenier près Antibes.-Gard : envir. de Nìmes, Manduel, Aigues-Jlortes. - Hér. : marais de Marsillargues, Mas-deFavet, Lunel.- Littoral des Pyre̊n.-0r., Salses.

BLPLEVLCI rotundifolium L. - Champs et bords des chemins dans les terrains calcaires.- - H.-Saône : Chargey-les-Port. - Assez commun dans Doubs, Jura, S.-el-L.- Rh. : Collonges, Couzon, Villeurbanne, Alix. - Ain : vignes du Revermont. Envir. de Genère à Chène, Contamines, Plan-les-Ouates. Yaud: Verey, Mathod, Chardonne, Nyons. - Valais : mont 
d'Orge, Conthey, Savièse, de Saint-Léonhard à Lens.-H.-Sav. : Annecy, Veyrier, Bonneville. - Sav. : Saint-Pierre-d'Albigny. - Is. : la Molte-les-Bains, les Purtes-en-Trieres, Saint-Vizier, Beauregard, mont Rachais.-H.-Alp. : envir, de Gap.-B.-Alp.: la Condamine, Boussolières.- Yaucl. : Caumont, Védène, Jonquières.-B.-du-Rh. : les Milles près Aix.-Alp.-Mar. : Nice à Montgros, entre Castillon et Sospel, la Giandola, Levens, Ve. nanson. - Ard. : Mercuer. - Gard : le Vigan, Jeuset, SaintLaurent. - Hér. : Castelnau, Marsillargues, le Larzac au Sautdu-Lievre, Bẻdarieux. - Pyr.-0r. : entre Thuir, Llupia et Terrats, Cases-de-Pena, Rigarda, Prades, Villefranche, Prats-deMollo, le Llaurenti, vallêe de Mijanès. - Terr. calc. des Pyr. centr. et du centre de la France.

13. pxorractum Link et Hoffm.-Moissons de la région mérid., surtout dans les terrains calcaires. - Vaucl. : commun dans les arrond. d'Orange et d'Arignon. - B.-du-Rh. : envir. d'Aix et de IFarseille. - Var: Toulon, Hjères, le Luc. - Alp.-Mar. : Cannes et tout le littoral jusqu’a Nice et Menton.-Gard: Nimes, Anduze, Uzès.-Hér. : toute la partie basse du départ.- Pyr.Or. : Bairas, Cases-de-Pena, Estagel. - Remonte dans l'Ard. jusyue vers Baix; dans la Dr. vers Valence; dans les H.-Alp. Ters Gap.

B. Iongifolium L. - Pâturages. - Hautes-Yosges au Hoehneck, Rotabac et Ballons. - Jura helvétique et français au Chasseral, Creux-du-Tan, Chasseron, Suchet, la Dôle, Colombier, Reculet, Villers-le-Lac, Haut-Bugey jusqu'au Colombier. - Manque dans les Alpes vaudoises et ralaisannes. - Is. : Gde-Chartreuse, Chamechaude, la Moucherolle, Valjouffrey, mont Chamoux. - H.Alp. : Lautaret, col de l'Eclıauda, vallées d'Aiguilles et d'Abriès en Queyras, Guillestre au Mélézet.-Pyr.-Or. : Font-de-Comps, vallée de Conat. - Manque dans les Pyrén. centr. - Existe au mont Dore et au Plomb du Cantal.

B. angulosum L. - Rochers calc. de la région pyrénéenne. Aude et Pyr.-Or.: Boutairol, lac de l'Estagnet, Port de Pailhères, le Llaurenti vers Mijanès, entre la Font-de-Comps et le rocher d'Erol, Pla-de-l'Ours. - Comman dans les Pyr. centr. 
B. steliatum L. - Rochers siliceux des Alpes et des Pyrén.-Or.Nul dans les Vosges, la chaine jurassique, le centre de la France et les Pyr. centr. - Valais : Alesse, Fully, Lys de Martigny, $\mathrm{Ca-}$ togne, Alpes de Zermatt et de Saas, Simplon, Grimsel.-H.-Sav.: Brévent, vallée de la Diosaz, Buet, chaine du mont Blanc jusqu'à Leschaux, le Couvercle et Tré-la-Tête, Cornettes-de-Bise. Sav. : le Bonhomme, Chapieu, col du Cormet près Boselein, le Saut et Morleret des Allues. - Is. : cliaines des Sept-Laux, de Belledonne et de l'Oisans, notamment vers le Gleyzin, le lac du Crouzet, Champrousse, la Bẻrarde au pied du Pelvoux, 0z, PiedMottel au mont de Lans.- H.-Alp. : Villard-d'Arène, Lautaret, Rochilles de Névache, mont Viso, Fontgillarde, l'Ubac, Chaillolle-Vieil, mont Queyrel, pic de Chabrières.- Alpes-Marit. : lac des Merveilles au-dessus des mines de Tende. - Pyr.-Or. : versant espagnol de la vallée d'Eyne et du col de las Nou-Fonts.

B. ranunculoldes L. - Espèce polymorphe très-commune dans les pâturages des hautes montagnes.- Jura helvétique et français au Chasseral, Suchet, mont d'Or, Montendre, la Döle, Reculet. - Nul dans les Vosges. - Valais : col de Fenêtre, Zermatt. - H.-Sav. : Brizon, col de Balme.-Savoie : mont Cenis, la Ga lise. - Is. : Gd-Som, col de la Ruchère, le Moucherotte, col de l'Arc, mont Rognon près Lalley, la Salette.-H.-Alp.: Lautaret, mont Genèvre, Château-Queyras, mont Viso, Saint-Véran, Clausis, col de Vars. - B.-Alp. : Saint-Paul, la Condamine, Fouillouse, Lauzannier.-Dr. : mont Glandasse. - Alp.-Nar. - Var : Sainte-Baume, Pic de Bretagne. — Gard : rochers d'Anjeou près Montdardier.-Aude : Boutairol, Pla Bernard dans le haut Donézan, Llaurenti. - Pyr.-Or. : vallée d'Eyne, Prat Cabrera au Canigou, col de la Regina, vallée de Carença, Font-de-Comps.Pyr. centr.

B. caricinum D. C.-Forme naine du précédent. - Valais : Zermatt.H.-Alpes : Briançon au fort des Têtes, mont Jily près Abriès, Charance.- B.-Alp. : Sérennes, Saint-Ours, Annot.- Hẻr. : la Sérane au Roc-Blanc. - Lozère: gorges du Tarn à SainteEnimie.

IR. petroeum L.- Rochers des montagnes calcaires, - Is, : Saint- 
Nizier, la Moucherolle, mont Aiguille.- Dr. : mont Glandasse. - II.Alp. : Lautaret, Ristolas, vallon de Ségure, Aurouse, Séuse, col de Glaise, mont Maraysse en face de Montmorin, Orcières.B.-Alp. : Larche, montagnes de Colmars et d'Allos, mont GdCouyer près Annot. - Alp.-Harit. : col de Tende, le Garret, Crouès-de-l'Aze, Bouziejo. - Pyr.-Or. : Costa-Bona. - Manque dans les Pyr. centr.

B. provinciale Huet. - Versant nord du mont Faron près Toulon.

В. gramимиm Vill. - Rochers des montagnes. - Is. : la Salette au mont Chamoux.-H.-Alp. : Briançon au fort des Têtes, Chàteau-Queyras, Aurouse, col de Glaisa, mont Clıabrières. - Alp.Marit. : Saint-Auban, Saint-Dalmas-le-Sauvage, Saint-HartinLanlosque, col de Tende. - Pyr.-Or.: entre les Bains de la Preste et Peyrefeu, Villefranche, Trencada-d'Ambulla, SaintPaul-de-Fenouillet au pont de la Fou.-Commun dans la région alpine des Pyr. centr.

I. Crutlcescens L. - Coteaux. - Var: Sainte-Baume, Aups, Toulon. - Aude : Narbonne, Sigean, Font-Estramer.

B. Junceum L. - Coteaux secs. - Rh. : bords du Garon, Chaponost, Givors, - Ain : Muzin, Pierre-Chàtel. - Sav. : Mtoutiers. - Is. : Bastille de Grenoble, Comboire, Pont-de-Claix, Vif, Vienne. - Dr. : Saint-Vallier, Montélimar. - H.-Alp. : Ribiers à Antonaves. - B.-Alp. : Annot. - Vaucl, : Panisset, Morière, Orange, Carpentras, Vaucluse, Flassan. - Var : Pic-de-Bretagne, le Luc. - Alp.-Nar. : Guillaumes, Grasse, Saint-André près Nice, Monaco, Menton. - Ard. : Tournon à Saint-Joseph, Coux, vallée de l'Ouvèze. — Gard : le Vigan, Blandas, Anduze, SaintAmbroix, Alais, Serre-de-Bouquet, Pont-du.Gard.-Hér. : Montmaur, la Valette, Valène, la Sérane, Vauguières, Saint-Martinde-Londres. -Aude : Ferrols. - Pyr.-0r. et centr. - Aveyron, Cantal, Lozère, Loire à Sail-sous-Couzan. - Espèce polymorphe.

B. australe Jord. - Avignon, le Luc, Nice, Menton.

B. spinosum Gouan. - Var: l'Amour-de-Gontier près la Valette.

B. Gerardi Jacq. - Forme du $B$. junceum dont les caractères sont mal définis et qu'on a probablement confondu tantòt avec le $B$. junceum ou plus souvent avec le suivant. 
B. Jacquinianum Jord. - Coleaux secs, rochers. - Rh.: bords du Garon à Chaponost, Bully, - Is. : Décines, Meyzieu et Balmes viennoises jusqu'à Jonage. - Loire : Rive-de-Gier au Sardon, entre Chavanay et Pélussin, berges de l'ítang de Vaugirard, bords de la Loire entre Saint-Rambert el Andrézieux.

B. affine Sadler. - Diffère du précédent par le pédicelle plus court que le fruit. - Rh. : Vaugneray. - Is. : Balmes viennoises, Pusignan. - Loire : Saint-Romain-le-Puy.

B. tenuissimum L.-Champs en friche.-C.-d'Or : l'Abergement-lesSeurre. - S.-et-L. : Louhans, Navilly. - Ain : la Dombes et la Bresse, Myonnay, Montribloud, Bâgẻ. - Rh. : Saint-Jean-d'Ardières, Marcy près du bois de l'Étoile, Talancé près Villefranche. - Commun dans Vaucl. et B.-du-Rh. - Var : les Pesquiers, Salines-Neuves-d'Hyères. - Alp.-Mar. : île Sainte-Marguerite, golfe Jouan. - Gard : Nimes, Générac, Blauzac, Tresques, Aigues-Hortes. - Hér. : Montpellier à Grammont, Rieucoulon, Pérols, Maurin, Castries, Cette, Béziers. Cette espèce se plaît dans les terrains salés; c'est ainsi qu'on la voit autour des sources minérales du Puy-de-Dóme près les Martres-de-Veyre, sources du Tambour et de Sainte-Marguerite, marais de Coeur, Saint-Nectaire.-Loire : Montbrison, Précieux, Vaugirard, Grézieu-le-Fromental.

B. glaueum Rob. et Castagne. - Lieux sablonn. et salés de la région mérid. - B.-du-Rh. : Marseille, Miramas.-Var : Sablettes de Toulon, Grosse-Tour à Gien. - Alp.-Mar. : Nice à la plago de Sainte-Héléne. - Gard : Aigues-Mortes, la Fosse près SaintGilles. - Hér. : Saint-Jean-de-Védas, Cette, les Onglous, Béziers. - Aude : la Clappe, Montolieu, Sainte-Lucie.-Pyr.0r. : Canet, Argelès.

B. nristatum Bartl. - Lieux arides et pierreux. - C.-d'Or: Nolay, Gouville, Marsannay et les sommitès de la Côte. - S.-et-L. : Solutré, Navilly. - Rh. : mont Cindre, Vaux-en-Velin. - Ain : la Pape, Meximieu aux Peupliers, plaine d'Ambronay, Chenilleux près Pierre-Châtel, Voliens, Muzin, Inimont, Portes, audessus de la grotte d'Oncieu. - Is. : Décines, Jonage, Feyzin, Sérézin, Crémieu, Bastille de Grenoble, Comboire, Vif. - Dr. : 
Saint-Vallier, Barnare, Aucelon. - H.-Alp. : Gap, Tallard, Roche-des-Arnauds. - Commun dans Vaucl. et B.-du-Rh. Var et Alp.-Mar.: Toulon, le Luc, Grasse, Antibes, Nice, Caslillon, Montalban, Saint-Hnspice, la Turbie, Tende. - Gard: le Vigan, Anduze, Alais, Saint-Ambroix, l'Espeerou, Nimes, Aigues-YIortes. - Commun dans l'Hér. - Áude : Pech-de-l'Agnèle près Narbonne, Carcassonne, les Corbières. - Pyr. centr. Aveyron, Lozère, Cantal.

B. righidum L. $_{\text {- }}$ - Lieux stériles de la région mérid. - Vaucl. : bois de Sérignan, Saint-Amans, Caromb. - B.-du-Rh.: les Alpines, Rognes, l'Estaque.-Gard: envir. de Nimes, du Vigan, Saint-Ambroix et d'Anduze. - Commun dans l'Hér. - Aude : Montolieu, Pech-de-l I gnèle, la Clapne, les Corbieres.-Pyr. Or.: vallêe de l'Agly, Saint-Antoine-de-Galamus, Cases-de-Pena, Estagel, Maury, vallon de Sainte-Catherine près Baixas.

R. falcatum L. - Haies, - Trés-commun dans toute la partie septentr. et moyenne du bassin; plus rare dans la région mérid. - Vaucl. : pied du mont Venloux. - Gard : envir. du Vigan, Salbous, Brama-Bioou, Dourbie. - Hér. : la Sérane, Pic Saint-Loup, le Caylar. - Aude et Pyr.-Or. : Rouze dans le Donézan, Durban, montagne de Saint-Victor, Fontroide, Moussoulens, la Clappe, Saint-Antoine-de-Galamus, Trencada d'Ambulla, Canigou, Ternet-les-Bains, bois de Pinats et dels Manerus. Pyr. centr. et centre de la France.

B. fruticosum L. - Lieux stériles et pierreus de la région mérid. - Assez commun dans Vaucl, et B.-du-Rh. - Gard: Anduze, mont Bouquet.- Hẻr. : Frontignan, Celte, Gignac à la Taillade, Saint-Guilhem, Villeneurette, Fos, Bèdarieux. - Aute et Pỵr.Or. : Conques, Narbonne, Saint-Antoine-de-Galamus, Cases-dePena, Eslagel. - Puy-de-Dome : envir. de Chauriat et de Jirefleurs.

SICI IntIfolum L. - Fossés et marais. - Cette espèce présente une distribution trẻs-inégale; bien que commune en Alsace et jusıu'à Belfort ainsi que sur le versant helrétique vers Båle, Neuchàtel et le canton de Taud, elle manipue dans H.-Saone, Doubs, Jura ; elle est rare dans C.-d’Or à Pontailler, Auxonne, 
Seurre; puis dans S.-et-L. à Saint-Léger-du-Bois, Navilly, Verdun; elle devient très-commune en amont et en aval de Lyon jusqu'à Vienne. - Plus rare dans la région mérid. dans le Var au Luc; dans le Gard à Saint-Gilles, Bellegarde, le Vistre; dans l'Hẻr. à Lunel et enfin sur le littoral des Pyr.-Or.

BERULA angustifolla Koch. - Mares, fossés et ruisseaux dans tout le bassin.

PIMPINaduA magna L. - Prés et bois des collines et des montagnes.-Assez commun dans C.-d'Jr., S.-et-L., Beaujolais, H.-Saone, Doubs, Jura, Ain, Savoie, Dauphinè, B.-Alp. jusque dans les Alp.-Mar,-Var : Sainte-Baume, Collobrières, Pignans.

— Gard : bois de Salbous, Alzon.-Hér. : la Salvetat, Navacelle, entre Joncelle et Roqueronde, Madières. - Aude : pont de la Fou, Salvanère.-Pyr.-0r. : Céret, Vernet-les-Bains, mont Louis, Saint-Laurens-de-Cerdans. - Pyr. centr., centre de la France.

La variété à fleurs roses est assez commune dans les montagnes.

P. snxifrogr L. - Coteaux arides, bords des chemins; présente une variété laciniata à feuilles toules découpées en lobes étroits.

P. peregrina L. - Collines pierreuses de la région mérid.- Var : Toulon, Hyères, Fréjus, Saint-Tropez. - Alp.-Mar. : Nice, Roquebrune, Menton. - Hẻr. : Montpellier à Grammont, la Planchude, Lamoure, Pignans, Castries, Lodève, Pézenas.

P. tragium Vill. - Rochers calc. de la région mérid. - Dr. : Saou près Crest, Saint-Paul-Trois-Châteaux. - Ard. : rochers d'Arran, Vals, - Vaucl. : Piolenc, Montdragon, pied du mont Ventoux. - B.-du-Rh. : les Alpines, Saint-Remy, Aix, Sainte-Victoire, Luminy, Marseille. - Var : Montrieux. - Alpes-Mar. : Saint-Auban à la Clue, mont Cheiron, Bezaudun entre Levens et la Tour. - Gard : Saint-Nicolas, Chartreuse de Valbonne. Hẻr. : Saint-Guilhem, les Capouladoux aux Cambrettes, SaintJean-de-Buèges, Carlincas, - Pyr.-0r. : Prades, Trencada d'Ambulla, Villefranche à la Citadelle, mont Saint-Jacques, Força-Réal, Cases-de-Pena.

CAIUUI verticilatum Koch. - Prés marécageux et tourbeux des terrains siliceux. Cette espèce, si commune sur les terrains 
granitiques et volcaniques du centre de la France, des Pyrénées, du Tarn, manque dans les Yosges et en Allemagne (excepté vers Aix-la-Chapelle); elle manque en Suisse, dans le Jura français et helvélique et aussi dans les Alpes. - C.॰d'Or et S.-et-L. : le Morvan granitique autour de Saulieu et d'Autun; terr. granit. des départ. de Rh. et Loire, dans Beaujolais, Lyonnais, Forez, Pilat.: - Is. : molasse de l'arr. de la Tour-du-Pin. - Ain : terrains argilo-siliceux de la Dombes.-Gard: l'Espérou, Concoule, - Hér. : la Salvetat, Fraisse, Pardailhan, Cassagnols. - Aude et Pyr.-Or. : versant mérid. de la Montagne Noire, Pont-de-Conangles en Cerdagne, vallées de Vernet-les-Bains et de Cornella-du-Conflent, Arles-sur-Tech.

C. enrvi L. - Prés et pâturages des collines et des montagnes dans tout le bassin, excepté dans B.-du-Rh. et Var.- Ne se trouve dans les Alp.-Har., l'Aude et les Pyr.-Or., que dans la région montagneuse. Il en est de même dans le Gard et l'Hẻr., où cette espèce existe à l'Espérou, Concoule, le Pertus, Mauguio au Masde-Harot.-Pyr.-Centr. et centre de la France.

C. bulboesstanum Koch.- Champs.- Envir. de Belfort à Bourogne, Allanjoie, Delle, et plus loin, dans le canton de Berne, à Porrentruy, Moutiers, Délémont. - Nul dans H.-Saône, Doubs et Jura. - Commun dans les terrains calc. de C.-d'Or, S.-et-L., Rh. à Couzon, mont Cindre, et dans les parties voisines de l'Ain à Sathonay, Montanay, - Sav. : Collonges-sous-Salève, mont de Sion, Mégève. - Valais : Martigny, Fully, Sembrancher, Saas. - Is. : le Sappey, Saint-Nizier, Venosc. - Dr. : Saint-Vallier, col de Lunel près Crest.-H.-Alp. : Villard d'Arène, Briançon, Gap. - B.Alp. : la Condamine, Saint-Paul, Maurin, Annot. Vaucl. : Flassan, Bédoin.-B.-du-Rh.: Sainte-Victoire, plaine des Milles, Roquefeuille.-Var : Sainte-Baume, Ie Luc, Pignans, Fréjus. - Gard : bords du Gardon, la Beaume, Uzès, Alzon, le Vigan, - Hér. : Saint-Loup, les Capouladoux, la Sẻrane, le Larzac à la Vacquerie.-Aude : les Corbières, Tuchan, Pech-del'Agne̊le, Campane, Cap-de-Pla, la Clappe, Fontfroide. - Pyr.Or. : mont Louis, vallées de Carença, de Vernel-les-Bains et de Castell. 
Le Bunium alpinum Waldst. Kit. a été indiqué avec doute dans les B.-Alp. au vallonnet de Meyronnes, Lauzannier, vallon du Châtelet.

Egopodium podagraria L. - Haies et bois dans tout le bassin, excepté dans B.-du-Rh, et Var. - Vaucl. : íles du Rh. - Rare dans le Gard et l'Hẻr. aux bois de Salbous, Espérou, Vigan, derrière le Pic Saint-Loup, Ganges. - Pyrén. centr. et centre de la France.

AMIMI majus L.-Champs de Ia région mérid., remonte vers Vals et Mercuer dans l'Ardèche; vers Montélimar, Romans dans la Drôme; vers Gap dans H.-Alp. - Dans les parties moy. et sept. du bassin cette espèce se montre presque exclusivement dans les luzernières, ce qui porte à penser qu'elle est le plus souvent introduite par les graines de provenance mérid.

A. vignaga Lam.- Champs de la région mérid.-Dr. : Pierrelatte, Saint-Paul-Trois - Châteaux. - Vaucl, : Orange, Sarrians. B.-du-Rh. : Marseille, les Martégaux, Gadagne près du MoulinRouge, bords de la Durance, Aix vers le Moulin de la Pioline. - Alp.-Mar. : Cannes, Nice, Saint-Martin-Lantosque. - Gard : Bellegarde, Saint-Gilles, Aigues-Mortes. - Hér. : Maurin, Ville, Villeveyrac, Mèze, Agde, Roquehaute.-Aude : île Sainte-Lucie, Gleizes, Petit-Vignes près Narbonne, Villespy.-Pyr.-Or. : vallée du Réart, 0pol. - Pyr.-Occident. dans le pays basque.

srsox amomum L.- Haies et lieux humides.- C.-d'Or : Meursault, Beaune, Rouvray, Roche-en-Brenil.- S.-et-L. : Chânes, - Rh. : Saint-Fortunat, pied du mont Cindre, Anse, Alix. Ain : Crottet, Meillonas, Cuisiat, Illiat, Cruzille, Meximieu à la Côte, Rigneux-le-Franc. - Envir. de Genève, Sous-Terre, Aïre, Chatelaine, Villette, Compesières. - Is. : Vaulnaveys, Echirolles, Vif, Varces, Meylan, Seyssins, Chapareillan.-Vaucl. : îles du Rhône.-B.-du-Rh. : îles du Rhône près Arles, la Ciotat.-Var et Alp.-Mar. : Sainte-Baume, Montrieux, Fréjus. - Gard : Villeneuve, Saint-Gilles, le Vigan à Montdardier.- Hér. : Lattes, Mas-de-Mouriès, Béziers.-Pyr.-0r. : vallée du Réart, Trullas, Terrats, toute la contrée de Thuir, Salses, Saint-Antoine-deGalamus. - Pyr. centr., Puy-de-Dome, Allier. 
FALCA IRTA Heivini IIost. - Champs calcaires. - Doubs : envir.

de Montbeliard à Audincourt, Forges, Champagne, Chissey, Arc-et-Senans. - Très-rare en Suisse.-II.-Saône : Chassey-lesMontbozon, Grandvelle, Autet. - C.-d'Or: Varrois, Arcelot, Villebichat. - Dr. : Valence, Crest. - H.Alp. : Gap, la BâtieNeuve, Chorges. - Vaucl. : commun dans les arrond, d'Apt, Avignon, Orange el Carpentras jusqu'au pied du mont Ventoux à Flassan et Bédoin. - B.-du-Rh. : Aix, Fenouillères, moulin de Saint-Jérôme, Puy-Sainte-Réparade, Martigues. - Gard : Nìmes, Caveirac, Saint-Nicolas, Beaucaire. - Hér. : Montpellier au chemin de la Pompiniane, Béziers, Oupia.-Pyr.-Or. : calc. de Baixas, Cases-de-Pena, Força-Réal.- Pyr. centr. et centre de la France; Loire : Montrond entre Fontanes et Sourcieux.

PTrCHotis heterophylla Koch, - Rochers, collines pierreuses. - C.-d'Or: Dijon, Santenay, la Côte, coteaux de l'Auxois. - S.-et-L. : Dezize, Chamilly. - Jura : bords de l'Ain depuis Thoirette jusqu'à Tour-du-Meix près d'Orgelet. - Ain : Artemare, Culoz, Virieu-le-Grand, entre Bons et Rossillon, montagne de Saint-Benoit, Cascade de Glandieu. - Graviers des bords du lac Léman près de Crans, Nyons, Coppet, Promenthoux, Buchillon, Saint-Prex. - H.-Sav.: envir. de Thonon entre la Dranse et le pont de Bioge, vallée de l'Arve, - Sav. : Termignon.-Is. : Bastille de Grenoble, graviers du Drac, Bourgd'Oisans. - Dr. : Crest, Loriol, Laveyron près Saint-Vallier, Montélimar, Nyons. - H.-Alp. : Gap, Tallard, mont Chabrières. - B.-Alp. : la Condamine, Boussolières, vallon du Châtelet près Sérennes, Annot. - Vaucl. : Gigondas, Vaucluse, Malaucène, Bédoin, Flassan, Aurel. - B.-du-Rh. : coteaux de Montaiguet près Aix, envir. de Marscille. - Var : Toulon, Cabasse, Fréjus. - Alp.-Mar .: Menton, Entraunes. - Loire : Chavanay. - Ard. : Tournon, Cruas, la Voulte, l'Escrinet. - Gard : Roquecourbe, Sainte-Marguerite à Villeneuve, Alais, Saint-Ambroix, - Hér. : Avène.-Py.-Or. : Saint-Antoine-de-Galamus, vallons de Prades et de Villefranche, Serdinga, Olette, vallée du Tech, route d'Arles, Prats-de-Mollo, Canigou. - Lozère : roch. calc. des gorges du Tarn, Sainte-Enimie, Saint-Prejet, Florac, la Jonte près Meyrueis. 
P. Timbnu Jord. -- Cette espèce semée, il y a déjà longtemps, de graines venues des environs de Toulouse, à Cusset près Villeurbanne (Rhône) et sur les buttes du Mollard de Décines (Isère) continue à se propager dans ces deux localitẻs.

HEMOSCAABU⿴囗十 nodiformm Koch. - Ruisseaux, marais dans tout le bassin.

H. repens Koch. - Vaud : Rolle, Aubonne, Champion. - Valais : bords du Rhône au-delà de Noville. - Ain : envir, de Bourg. - Rh. : Chaponost à l'étang du Loup, Vaux-en-Velin, Lachassagne. Is. : Meyzieu, Saint-Laurent-du-Pont. - Gard : le long du Sumène. - Pyr.-Or. et centrales.

I. Inundatum Koch. - Fossés, marais. - Cette espèce, assez répandue dans le N.-0., l'0. et le centre de la France jusque dans le Puy-de-Dôme, l'Allier et la Loire, est rare dans notre bassin, -C.-d'Or : Seurre, Viclouge. - S.-et-L. : Millery, Monthelon, Montjeu près Autun, Surmoulin, Louhans. - Rh. : Beaunant, Chaponost à l'étang du Loup, Lavore près Mornant.-Ain : Reyrieux, Pont-de-Vaux, Bourg, Saint-Didier-sur-Chalaronne.Is. : Charette près Crémieu, Estrablin près Vienne.

TRINIA vulgaris D. C. - Coteaux calcaires. - C.-d'Or : Jouvence, Dijon, Gevrey et toute la Côte. - Commun dans S.-et-L. - Jura : la Dôle, Colombier, Reculet. - Ain : la Pape, Montluel, Meximieu, Chazay-sur-Ain, Loyettes, Ambronay, Virieule-Grand, Pierre-Châtel, forl de l'Écluse.-Rh. : Couzon, plaine de Royes. - 1s. : Bastille de Grenoble, Sassenage, Comboire, Crémieu. - Dr. : Saint-Vallier, Crest. - H.-Alp. : Gap, Tal. lard. - B.-Alp. : la Condamine, Boussolières. - Vaucl. : Avignon, Cavaillon, Vaucluse, Saint-Amans, Flassan.-B.-du-Rh. : envir. d'Aix, montagnes des Pauvres, plaine des Dédaous, Montaiguet, bois de pins des envir. de Marseille. - Var : Toulon, Hyères, le Luc, Fréjus. - Alp.-Mar. : Caussols, mont Agel audessus de la Turbie, col de Braus, Saint-Martin-Lantosque. Ard. : le Pouzin, Mercuer. - Commun dans le Gard, surtout vers Saint-Ambroix, Anduze. - Hér. : Montpellier à Lavalette, Fontfroide, Pic de Saint Loup, Montarnaud, Murviel, Castries, Valmargues, Saint-Gély-Restinclières, Frouzet, le Caylar. - 
Aude et Pyr.-0r. : Montolieu, basses Corbières, Corbières, Baixas, Cases-de-Pena, Font-de-Comps.-En dehors de notre bassin sur les montagnes calc. des Pyr. centr., de la Lozère à Mende, Florac, du Puy-de-Dôme et de l'Allier.

T. arrecta Jord. - Cette forme se trouve dans l'Ain à Viricu-le-Grand, Rossillon, Serrières.

PETIESEMNUN segetum Koch, - Champs argileux de la région mérid. - Var et Alp.-Mar. : cap Brun près Toulon, le Revesi, Nice, Menton. - Gard : Bellegarde, Saint-Gilles. - A été trouvé accidentellement dans la C.-d'Or et autour de Lyon.

P. sativum Hoffm. - Le Persil s'échappe quelquefois des cultures.

APIUM graveolens L. - Le Céleri aime les lieux salés, les décombres et environs des habitations. - Spontané dans le Jura et le Doubs autour des sources salées de Grozon et d'Arc-et-Senans; dans la Sav. vers les sources salées de Salins; dans le Puy-deDóme autour des sources salćes de Saint-Nectaire, Saint-Allyre, Royat, Médagues, Sainte-Marguerite, Gimeaux; dans la Lorraine près des salines de Dieuze, Sarrebourg; dans les mêmes conditions à Durkheim (Rheinpfalz); à Cannstat (Wurttemberg); puis dans le duché de Bade, Brandebourg, Brunswick, Westphalie, Saxe, Gallicie, Catalogne, Algérie et même dans les steppes salées de l'Asie.

Prẻs salẻs des côtes de l'Océan el de la Méditerranée dans B.-du-Rh., Var, Alp.-Ifar.; Gard à Bellegarde, Saint-Gilles, Aigues-IIortes. - Hér. : Lattes, Gramenet, Palavas, Mauguio, Avène et jusque près de Montpellier aux Prés d'Arène. - Aude et Pyr-0r. : Salses, sources salées de la fontaine Estramer et de la Font-Dame.

CiCUTA virosa L. - Marais tourbeux. - Doubs : lac d'Etalières près de la Bróvines, val de Joux, tourbières de Pontarlier et des Guinots. - S.-et-L. : Gissy, Barnaud. - IE. : Saint-Symphoriend'Ozon, Auberive près Roussillon. - Pyr.-Or. : mont Louis ef la Cerdagne, Saint-Martin près de Fosse, Perpignan. - Puy-deDôme: lacs de Chambedaze et de Bourdouze.

scs vodx peeten Veneris L. - Champs cultivés surtout dans les terrains calcaires. 
S. hispanica Boiss. - Champs incultes. - Vaucl. : Avignon, le Grand-Montagnet. - B.-du - Rh. : Montaud-les-Miramas.-Var : Toulon, Hyères. - Gard : Villeneuve-lez-Aviguon, descente de Pouls, la Beaume. - Aude et Pyr.-Or. : Narbonne, Cabestany, Saint-Nazaire. - A été trouvé près de Briançon.

S. australis L. - Champs incultes. - Dr. : le Buis. - H-Alp. : Tallard, la Saulce. - Vaucl. et B.-du-Rh.; envir. d'Avignon et de Marseille, Cuques. - Var : Toulon, le Luc. — Gard : Nimes, Campestre. - Hér. : Montpellier à la Colombière, Aiguelongue, Saint-Jean-de-Vedas, Castelnau, Villeneuve, Gigean, Celleneuve, Mireval, Pic Saint-Loup, Cette, Frontignan, Lunel, le Caylar. -Aude : Narbonne, Pech-de-l'Agnèle, Fontfroide, les Corbières, Sainte-Lucie.-Pyr.-Orientales : Port-Vendre, Paulilles, Banyulssur-Iler.

ANTwiscus vulgaris Pers.-Bords des chemins, décombres. Doubs et Jura : Besançon, Baume-les-Dames, Dóle, Baume-lesMessieurs, Arbois aux sources de la Cuisance.-C.d'Ur: Auxonne, Malain.-S.et-L。 : Cluny, Bourbon.-H.-Sav. : Salève.-Env. de Genève. - Is. : Mollard de Décines, Crẻmieu. - H.-Alpes : Rosans, Serre. - Vaucl. : Bédoin à Malaugii. - Var : le Luc, Morgès. - Ard. : Privas. - Gard : le Vigan, la Grande-Basse près Saint-Guiral. - Hér. : la Salvetat, le Caylar, le Gros, les Rives. - Aude : les Corbières, forêt des Fanges. le Donézan. - Pyr.-Or : vallée de la Tet. - Cà et là dans les Pyr. centr. et le centre de la France.

A. cerefolium Hoffm.- Cultivé el souvent subspontané.

A. sylvestria Hoffm. - Prés et bois dans tout le bassin. Présente de nombreuses variations dans la forme des feuilles.

Covorobrum denudatum Koch.- Champs et pâturages des terrains siliceux. - Nul dans les Vosges, la chaine jurassique, Savoie, Dauphiné, B.-Alp., Provence, si ce n'est à Collobrières et Tanneron; manque aussi en Suisse. - S.-et-L. : Louhans, Beuvray, Suin, Cluny, Charolles. - Rh. : Pomeys, Larajasse, Roche-d'Ajoux. - Alp.-Mar. : Estérel, Cannes, Coarazza. Loire : chaines de I'ilat, Pierre-sur-Haute- - Ard, : Entraigues, l'Escrinet, le \$ezenc. - Gard : le Vigan, Alzon, Alais, Anduze, 
l'digual.- Hèr.: sommet de la Sérane, le Caroux, Salvetat, Pardailhan, Avène, Castanet-le-Haut; descend jusquu'à Grayssessac, Saint-Étienne-de-Mursan et Saint-Martin-d Orb. - Auçe et Pyr.Or. : Tuchan, Massac, le Donézan, Salvanère, Llaurenti, mont Louis, entre Font-de-Comps et Collada de Jujols, Cambres-d'Aze, Saint-Antcine-de-Galamus. - En dehors de nos limites sur les terr. granit. et volcan. des Pyr. centr., monts Dôme, Dore, H.-Loire, Cantal, Loziere, Tarn, Allier, Creuse, H.-Vienne et l'ouest de la France.

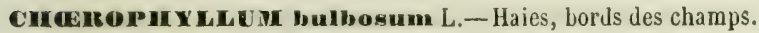

- Signalé dans les Pyr.-Or., l'Alsace et la Lorraine.

C. nureum L. - Iaies et bois. - Commun dans Doubs, Jura, Ain, Rh.-Nul dans H.-Saòne, C.-d'Or, S.-et-Loire, H.-Sav., Sav.Valais et Vaud : Chemin, la Combe, Leukerbad, bassin du Léman. - Is. : le Sappey, Gde-Chartreuse, Saint-Nizier, mont de Lans.-H.-Alp. : envir. de Gap, Rabou, Loubet, Chaudun, Villard-d'Arène. - Nul dans Vaucl., B.-du-Rh., Var.- Alp.-Nar. : Saint-Martin-Lantosque, Estenc, col de Jallorgues.-Ard. : Vals. - Gard : îles de la Barandoune au Pont-Saint-Esprit, Concoules.

- Hér. : vallée de la Mare à Andabre, Saint-Geniès, SaintGervais, la Salvetat, Fraisse. - Pyr.-Or. : mont Louis, bois de Salvanère et de Boucheville, Céret. - Pyr. centr., Tarn, Lozère, Cantal, Corrèze, Puy-de-Dôme.

C. Villarali Koch. - Prairies des hautes montagnes. Nul dans les Vosges et le Jura français, bien qu'il existe sur les confins du Jura helvétique au pied des grands rochers du mont d'Or, au Chassernn, au Passwang.- H.-Sav. : Vergy, Méri, Brizon, Parmélan, Hortaz. - Sav. : montagnes de Crest-Voland, Hauteluce et Beaufort. - Is. : chaines des Sept-Laux, de Belledonne clans la Combe de la Lance, des Gdes-Rousses, Gu-Som, col de l'Arc, la Salette.-H.-Alp. : Villard-d'Arène, Lautaret, Monestier-deBriançon, mont Viso à la Tranchee, chalets de Ruine, GrandVallon, col de Vars, bois de Rabou près Gap.-B. -Alp. : la Condamine, Sérennes. - Alp.-Mar. : vallons du Boréon, de la Malone de Fenestre et de Libaré près Venanson. - Nul dans les Pyrén.- Existe au mont Dore et au Plomb du Cantal. 
C. cicuturia Vill. - Prés et bois. - Les Vosges jusque dans la région vosgienne de la H.-Saône; chaîne jurassique jusque dans le Bugey autour de Nantua, Dortan, Retord, Arvières. - Vaud et Valais : Bex, Comba d'Arba, Ilayens de Sion, Natersberg. - II.-Sav. : Salève, Voirons, Parmelan, valléc de Saint-Gervais aux Contamines. - Sav. : montagnes des Bauges, de Beaufort et d'Hauteluce. - Is. : Gde-Chartreuse, Portes, Chalais, Sassenage. - Loire : Pilat, Pierre-sur-Haute. - Ard. : Vals, Entraigues, Accons.-B.-du-Rh. : Vauvenargues.-Alp.-Marit. : Entraunes, val Pesio. - Gard : Valleraugue, l'Aigual, Alzon, Concoule.-Hér. : la Salvetat, Saint-Pons, Pézenas, Andabre, SaintAmand.-Aude : Montfort, vallée de la Boulsanne, le Donézan. - Pyr-0r. : mont Louis, vallée d'Eyne, Carença, Vernet-lesBains, Saint-Vincent. - Pyrén. centr. ; Montagne-Noire, centre de la France.

C. umbrosum Jord. - Diffère du précédent par ses feuilles glabres et luisantes. - Loire : Pilat, Pierre-sur-Haute.

C. temulum L. - Haies, bords des bois et des chemins dans tout le bassin; moins abondant dans la région mérid., oủ il occupe presque exclusivement la portie montagneuse.

C. nodosum Lam, - Aude sur le versant mérid. de la MontagneNoire à Lastours. - Vaucl. : Flassan à la Font-de-Canau.

M YRRHIS odorata L. - Prairies des montagnes, voisinage des Itabitations, - Doubs : Gd-Combe-des-Bois, Pont-de-Roide à la Grochère. - H.-Saône : vallée du Puix, Franchevelle, mont de Vannes.- Vaud et Valais : Lavarraz, mont Seron sur l'Estivaz, Trient. - S.-et-L. : Roussillon, Auxy, Saint-Émiland.-H.-Sav.: Salève au-dessous du Gd-Piton, Voirons autour du Couvent, Brizon, Thorens, Haut-de-Lin.-Sav. : Crest-Voland, Hauteluce. - Ain : Chartreuse d'Arvières. - Is. : couvent de Gde-Chartreuse, ruines de Chartreuse de Prémol, Saint-Nizier, Monestierde-Clermont, Saint-Baudille et Pinet près Mens, Esparron près Monestier-de-Percy, Bouvesse près Morestel.-Dr. : Aucelon.- B.-Alp. : entre Colmars et le lac Signet, Lauzannier, vallon Bérard.-Alp.-Har. : montagnes de Tende, de la Briga, val de Jallorgues, Bouziejo, mont de Longeron sur Entraunes.-Aude: 
Boutairol, lac de l'Estagnet. - Pyr.-0r.: bois des Fanges et de Boucheville, montagne de Círet, Saint-Laurent-de-Cerdans. Pyrén. centr., Cantal, H.-Loire, Creuse, Loire à l'Ermitage audessus de Noirétable.

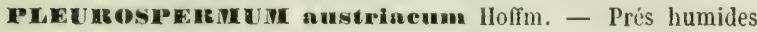
des Alpes et des Pyren.-Or. - Sav. : d'Aime au col du Cormet, les Allues. - Is. : le Valjouffrey entre Entraigues et le mont Gargas. - H.-Alp. : forêt des Fraux près la Grave, Lautaret, bois de la Madeleine près du Monestier-de-Briançon, col des Hayes, Queyras sur les bords du Guil, mont Morgon près Embrun, bois Iacour à Saint-Jean près Gap, le Valgaudemar.-B.du-Rh. : Rognes.- Pyr.-Or. : le Llaurenti.

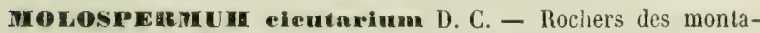
gnes. - B.-Alp. : Colmars au Grand-Couyer, Annot entre le pont de Guèdan et Entrevaux. - Alp.-Marit. : sources du Var. - Ard. : Saint-Martin-de-Londres aux Arcs, les Cambettes, Ganges, la Sérane, N.-D.-de-Londres aux lous.-Gard : I'Aigual bois de Longuefeuilles à Concoule. - Lozère : Bassi près SaintÉtienne-Valdonesse, Costelades près Villefort, envir. de Florac entre Gratagals et la baraque de Puch. - Aude et Pyr.-Or. : Boutairol, le Llaurenti, Canigou, vallées de Carença, de Pratsde-Balaguer, d'Eyne et d'Err, Bac de Bolquère, Costa-Bona. Pyr. centrales.

Le Physospermum aquilegifolium Koch a été indiqué à tort, par Mutel, au mont Viso.

ECHinorina ic spinosa L. - Sables marit. du littoral méditerranéen. - Cótes de la Crau et de la Camargue, les Sablettes de Toulon, Hyères, île de Porquerolle, Saint-Tropez, Fréjus. Saint-Raphael, littoral des Alpes-Mar. - Gard : Aigues-Mortes au Grau. - Plages de l'Hér. et de l'Aude. - Pyr.-Or. : entre Port-Vendres et Banyuls.

Sn Yav I olusastrum L, - Iaies, décombres de la région mérid. - R.-du-Rh. : Aix au vallon de Barret, Marseille. - Var et Alp.-Mar. : Toulon, Hyères, le Revest, ile de Porquerolle, le Luc, Saint-Tropez, Grasse, île Sainte-Narguerite, Nice, Monaco, Gorbio et Vintimiglia sur le littoral italien. - Gard : Nimes, Calal. Bassin du Rhône. 
Anduze, Corconne.- Hér. : Montels, Mireval, Viols, Montferrier, Castries, Dourbe, Lodève.-Aude : Carcassonne, Conques, Montolieu. - Hyr.-Or. : Perpignan, Prades, Villefranche. - Existe aussi dans les Pyr. centr. vers Saint-Béat, entre Bagnères et Lourdes, Louvie.

S. perfoliatum L. - Lieux incultes. - Var : la Verne, les Mayons. Alp.-Illar. : vallée de Thorenc près Grasse.

CONYUM maculatum L. - Décombres, bords des chemins. Autour de Belfort à Damjoutin, Bourogne; autour de Montbéliard sur les alluvions de Mandeure, Mathay, Audincourt, Charmont, Sachaux, Bavans; plus loin à Rougemont. - H.-SaOne : Velle, Echenoz, Vaivre, Frotey, Villers-Pos, vallée de l'ognon de Villersexel à Mont'̌ozon. - C.-d'Or. - Envir. de Genève à la Coulouvrinière. - H.-Sav. : la Roche. - Ain : la Bresse et la Dombes à Bourg, Manziat, Saint-Paul-de-Varaz, les Echets. Rh. : Pommiers, Arnas, Beaujeu. - Is. : Décines, Bouvesse près Morestel, Mens, mont de Lans, Villard-de-Lans, Prémol. - Dr. : Aucelon. - H.-Alp. : Gap à Rabou. — Vaucl. : le Ventouret. B.-du-Rh. : Aix, Marseille, bords de l'Huveaume à Sainte-Marguerite, Plan d'Aups. - Var et Alp.-Mar. : Hyères, Vérignon, Utelle, Tende, Roccasteron, et plus loin à Vintimiglia en Italie. — Gard: le Vigan, Alzon, Camprieux, la Bruyère. - Hér. : la Salvetat, Saint-Amand-de-Mounis, Saint-Pons, le Larzac au Cayłard et à Saint-Maurice, la Sérane au Coulet. - Pyr.-Or. et centr., le centre de la France.

CaCHuys lavigata Lam. - Rochers de la région mérid. B.-du-Rh. : Aix : pentes de la Sainte-Nictoire, Vauvenargues au vallon des Masques, vallon des Crides à Saint-Pons-de-Gémenos. - Alp.-Har. : le Cannet, la Bouverie près Roquebrune, Mauriana au-dessus de Breglia. - Gard: bords du Gardon entre Saint-Nicolas et le Mas Charlot, le long de la Grand-Combe près le Has de Seines. - Hẻr. : au-dessus du moulin Geniès près Saint-Jean-de-Védas. - Aude : Conques, Pech.-de-l'Agnèle, lles de la Sidrière, de Leucate, de Fitou et de Sainte-Lucie.

HYDrocotyLE vulgaris L. - Prés marécageux et tourbeux.

- C.-d’0r: Senlis, Magny et le Morvan autour de Saulieu. - 
H.-Saône : Saint-Germain, Francheville, la Mer. - Nul dans Doubs et Jura. - Vaud : la Sauge, Vvonand, Yverdon, marais d'Orbe, Noville, les Grangettes, bois de Bogis près Nyon, Roellebot. - Envil. de Genève à Cerex, Sionet, Troenex, Mattegnin. - Ain : tous les marais de la Bresse et de la Dombes. - Ih. : Vaux-en-Velin. - Is. : Meyzieu, Charvieu, Morestel, entre Crémicu et St-Romain, Saint-Laurent-du-Pont, Pont-de-Beauvoisin. - Vaucl, el Gard : Avignon, ètang de Pujault, Saint-Gilles. Bellegarde.-Hér. : Montpellier à la Pompignane, Mauguio à SaintMarcel, Capestang. - B.-du-Bh. : étang de Marignane. - Var : les Sablettes de Toulon. -. Pyr. : entre Elne et Argelès-sur-Mer. - Centre de la France.

ASTRANTI major L. - Prés et pâturages des montagnes calcaires. - Nul dans les Vosges. - Toute la chaine du Jura helvétique et français jusque sur les sommités des montagnes du Bugey. - Valais : Bourg-Saint-Pierre, Mayens de Sion et de Saxon.-H.-Sav. et Sav.: Salève, Voirons, toutes les montagnes calc. du Chablais, Faucigny, Bauges, Tarantaise, Maurienne. Is. et Dr. : monlagnes calc. de Grande-Chartreuse, Saint-Nizier, Vercors jusqu'au mont Glandasse. - H.-Alp. : envir. de Gap, Pleyne de Clıaudun, Séuse, Durbonnas, Orciẻres, le Queyras. - B.-Alp. : Parpaillon, Lauzannier, Bérard. - Prairies des Alp.-Mar., des Pyr.-Or. et centr., des monts Dómes, Dore, Cantal, Mezenc. C'est à tort qu'on a dit l'avoir trouvé dans les montagnes du Forez; manque aussi dans les massifs granitiques et schisteux du Pilat et Cévennes.

A minor L. Rochers et pâturages des terrains siliceux des Alpes et des Pyrénées. - Valais : val d'Illiez, Saint-Bernard, Anniviers, Zermatt, Saas, Simplon, Furca, Grimsel. - H.-Sav. : grès nummulit. de la glacière du Brizon, Vergy, Méri, massifs granit. de chaine du mont Blanc depuis le col de Balme jusquu'au col de la Seigne, chaîne des Aiguilles Rouges, vallée de la Diosaz. Sav. : montagnes granitiques de Beaufort, Hauteluce, CrestVoland, mont Iseran, la Levanna.-Is. : clsaines des Sept-Laux, Belledonne, Grandes-Rousses, Oisans jusqu'au Pelvoux, le Valjouffrey.-H.-Alp. : Villard-d'Arène, mont Viso, mont Queyrel, 
Chaillol-le-Vicil.-B.-Alp. : de Barcelonnette au pic de Séolane. - Alp.-Mar. : sommités depuis les sources du Var jusqu'aux Alpes de Tende. - Aude et Pyr.-Or. : le Llaurenti, Canigou, Coma du Tech, derrière le pic de Costa-Bona. - Terr. siliceux des Fyr. centr. - Manque dans le centre de la France et dans les chaines vosgienne et jurassique.

ERXNGIU⿴囗十⺝ a pinum L. - Pâturages des montagnes. - Jura :

Colombier du Gex, Reculet, la Dîle. - Nul dans les Vosges. Alpes vaudoises et valaisannes: Lavarraz, Nombrieux, Champéry, sur Vouvry, sur Salvan. - H.-Sav. : Méri au-dessus du Chalet de Sommier-Dessus, col de la Louze, Emousson, Mieussy, - H.-Alp. : le Miélézet et Risoul près Guillestre, l'Argentière, col de Vars, mont Viso, Orciéres. - B.-Alp. : le Lauzannier. Alp.-Mar. : montagnes de Fenestre et de Saint-Dalmas-le-Sauvage, col de la Maddalena.

C. splua siba Vill. - Débris de rochers. - Is. : base du mont Aiguille, Jarjote et mont des Lussettes près Lus-la.Croix-Haute. - Dr. : mont Glandasse. - H.-Alp. : col de Glaise et de Chaudun, Aurouse, montagne de Maraysse en face de Montmorin.Alp.-Mar. : col de Braus, Sanson, la Briga.

E Bourgati Gouan. - Débris de rochers des Pyrénées: Salvanère, sommités du Capcir, la Cerdagne, vallées d'Eyne et de Llo, plateau de la Perche, mont Louis. - Commun dans les Pyr. centr.

E. campestre L. - Lieux incultes dans tout le bassin excepté dans le Valais. - Cette espèce appartient essentiellement aux plaines et basses collines, mais ne s'élève pas dans les montagnes.

E. maritimam L. - Sables maritimes du littoral méditerranéen.

SANICUL europerea L. - Bois dans tout le bassin. - Dans le Gard, cette espèce occupe la région montagneuse des envir. du Vigan; dans l'Hérault autour de Pardailhan, Saint-Amand-deMounis, Saint-Pons, bords du Tés, Mas-de-Mouriès, Saint-Martin-d'Orb, Faugères, Bédarieux; dans l'Aude et les Pyr.-0r., elle se trouve dans les forêts de Salvanere, des Fanges et de la Salanque. 


\section{ARALIACÉES.}

Hedern Mrellx L. - Murs, rochers, troncs d'arbres dans tout le bassin.

\section{CORNÉES.}

Comenus mas L. - Ilaies, bois; plus fréruent dans les terrains calcaircs. - H.-Saône: Leffond, Vircy, Champlitte, Fouvent. Doubs : envir. de Montbeliard, de Baume-les-Dames et de Besançon, chaine du Lomont, vallée de Cuisancin. - Ain: la Pape Ambérieu, Leyment, Rigneux-le-Désert, Tenay, Belley.-Vaud et Valais: le Montet près Bex, Roche, Aigle, Vevey, Rivaz, St-Maurice au bois Noir, Folatires. - S.-et-L. : Cluny, Cuiseaux. - Rh. : Mont Cindre, St-Romain, Poleymieu, Chassclay, Arnas, Liergues. - Is. : Vienne à Levaux, Pariset, mont Rachais. Sav. : St-Jean-de-Ifaurienne, entre Salins et Villarlurin, Chambéry. - H.-Alp. : Gap, Romette, St-Jean, Serres, la Roche-desArnauds, Rosans. - Dr.:Die, Barnave. - Vaucl.: Sault. B.-du-Rh. et Var:Grans sur les rives de la Touloubre, SteBaume, Montrieux, le Luc, Fox-Amploux. - Alp.-Mar. : Vence, Grasse, le Bar. - Ard. - Gard : Alais, Anduze. - Hér. : Lavalette, St-Paul-et-Valmale, St-Loup, Montaud, Teyran, N.-D, de Londres, Gignac, le Larzac à St-Maurice et Vacquerie. - Pyr.Or : vallée du Réart, Céret, Castel, St-Marlin-du-Canigou.

C. annguinen L. - Haies dans tout le bassin.

\section{LORANTHACĖES.}

VIscen nIbum L. - Parasite sur les vieux arbres fruitiers et aussi dans les H. et B.-Alpes, sur les Pins et Sapins.

ArCaUTO IIU oxycedri Bieb. - Parasite sur le Juniperus communis et sur le J. oxycedri dans les environs de Sisteron à Château-Arnoux, Montfort, Augìs, Tèle près de Bégude la Blanche. - B.-du-Rh. : Mimet. 


\section{CAPRIFOLIACÉES.}

ADOXA mosehatellina L. - Bois humides des terrains siliceux et argileux. - Chaine vosgienne et dans les parties marneuses de la chaine jurassique.-H.-S. : Champagney, Vaivre, Chariez, Grattery, Pusy, Fouvent, St-Andoche. - C.-d'Or : Gevrey, Messigny, Noiron. - S.-et-L. : env. d'Autun, Auxy, Antully, Dracy, St-Loup, Epinac. - Ain : Bourg, St-Didier-sur-Chalaronne, Mogneneins, Retord, St-Rambert, Belley. - Vaud et Bas-Valais. - Envir. de Genève, pied du Salève. - H.-Sav. : Annecy, la Roche, Bonneville.- Sav. : Aix, Chambéry, St-Jeande-Maurienne. - Is. : Crémieu, Balmes de Fontaine, Sappey, Herbeys, St-Geoirs, Mens.-H.-Alp. : envir. de Gap à Rabou. Rh. : Ecully, Tassin, Francheville, Charbonnières, Chaponost, l'Argentière, Anse, Vaurenard.—Loire: Panissière, Rochetaillée, Pleney et Bois-Noir près Saint-Etienne, Grand-Croix, Champoly, Montbrison. - Rare dans la Provence et les Alp.-IJar. où on ne l'a signalé que près de Toulon au Revest et dans la vallée de la Mine de Tende. - Ard. : le Bez, le Mezenc, sources du Mézayon. - Gard: le Vigan et la chaîne de l'Espérou. - Aude et Tarn: Montagne Noire. - Pyr.-Or. : vallée du Réart, Ermitage de StAmans, St-Marsal.

SAMBUCUS Ebulus L. - Bords des champs et des chemins dans les terrains marneux ou argileux.

S. nigra L. - Haies et bois. Présente une variété à feuilles laciniées.

s. racemosa L. - Bois. - Très-répandu dans la région des Sapins de la chaine des Vosges et de celle du Jura jusque dans les montagnes du Bugey, Vaud, Valais, Savoie, Dauphiné, B.-Alp. et Alp.-Mar. - C.-d'Or : N. D. d'Etang, Flavignerot, Anteuil, Val Suzon, Blaizy, Saulieu. - S.-et-L. : le Morvan autunois, Cluny, Cuiseaux.-Rh., Loire et Ard. : montagnes du Beaujolais, du Lyonnais, du Pilat et du Vivarais. - Gard et Hér. : chaine de l'Espérou, l'Espinouse à Lignères. - Aude et Tarn : Montagne Noire. - Pyr.-Or. - En dehors de nos limites dans les Pyr. cent., l'Aubrac, Cantal, Lozère, monts Dômes et Dore, Forez. 
VIBURYUM TInus L. - Rochers et bois de la règ. mérid. Toulon, le Luc, Belgentier, Esterel, Ile Ste-Iarguerite, Grasse, Vallauris, vallée du Var, N.-D. de Laghet. - Gard : carrière des Angles a Villeneuve d'Arignon, bords du Gardon à la Beaume, St-Nicolas, Pont-du-Gard, le Vigan, Anduze. - Hẻr. : Mireval, Valine, Pic St-Loup, Capouladoux, Fáugère, Castries, St-Martin-de-Londres, Claret, Ganges, Pézenas. - Pyr.-Or. : St-Paul, St-Antoine-de-Galamus, Jaureillas, le Boulou, fort Bellegarde, Trencada d'Ambulla. - Souvent cultivé dans les jardins.

v. Inntana L. - Haies dans tout le bassin.

v. Opulus L. - Haies et bois.

LOXICERA Implexa Ait. - Haies et bois de la région mérid.; remonte jusque dans le sud des départ. de la Drôme et de l'Ardèche.

T. Cnprifollam L. - Cultivé dans les jardins; depuis longtemps naturalisé sur les collines de l'Alsace, de la Lorraine et aussi des Pyr.-Or. à St-Sauveur, Sarrat d'en Vaquer, envir. de Perpignan, Céret, Arles, Prades, Villefranche, Olette.

I. etrusea Santi. - Haies et bois de la région mérid. et moyenne, surtout sur les terrains calcaires. - Yar : Touris, le Luc, Montrieux. - Alp.-Nar. : Esterel, Biot, Grasse, Séranon. Commun dans le Gard, l'Hér. - Aude: Narbonne, Durban, Rennes-les-Bains, Ferrals, Mas-Cabardès. - Pyr.-Or.: bords du ruisseau de Hallolles, env. de Perpignan, vallon de Vernet-lesBains, Prats de Iollo, St-Antoine-de-Galamus. - Remonte dans l'Ard. vers Pont-de-Coux, mont Combier, vallée de l'Ourèze, Andance, Tain; dans la Dr. vers Crest, Serves, St-Yallier; dans les H.-Alp. vers Tallard, Châteauroux, Eymeyères, Ventavon au pied de la montagne de Fave, la Roche-des-Arnauds; dans l'Isère vers Vif, Claix, Vouillant, Sassenage, Bastille de Grenoble; dans la Sav. vers Jloutiers, Montmélian, Chambéry, Aix, mont du Chat, St-Germain; dans le Rh. à Couzon, St-Germainau-mont-d'Or; dans l'Ain autour de Belley; enfin dans le Valais à Conthey, Fully, Saillon, Sion. - En dehors de notre bassin sur les terr. calc. du Tarn, de l'Areyron, de la H.-Loire, du Puy. de-Dóme et de l'Allier. 
L. perielymenum L. - Haies et bois des terr, siliceux et argileux. Il est digne de remarque que dans la région mérid. on ne le voit que dans les montagnes, comme dans les Cévennes du Gard, l'Espinouse ct l'Escandorgue, la Ste-Baume, dans les vallées élevées des Alp.-Mar.

T. xylosteum L. - Haies. Même observation en ce qui concerne sa distribution dans la région mérid., avec cette différence que, relativement au terrain, il est indifférent.

I. nigra L. - Bois. - Chaîne des Vosges depuis la vallée de la Bruche jusqu'aux Ballons de Giromagny et de Servance; descend dans la H.-S. dans la vallée de Rahin et à Ruz-la-Fonderie. Région des Sapins de la chaîne jurassique dans les montagnes des arrond. de Montbéliard, Baume-les-Dames, puis au Montd'Or, à la Dole, au Colombier et jusque dans le Haut-Bugey. Vaud et Valais : Alpes de Jaman, Javernaz, Trient, Saxon, Orsera, Diez, Pas-du-Loup. - H.-Sav. : Salève, Voirons, montagnes du Chablais et du Faucigny, les Gets, Tête-Noire, Valorsine, la Diosaz, Chamonix. - Sav. : mont du Chat. - Is. : Grande-Chartreuse, St-Eynard, St-Nizier, chaîne de Belledonne entre Revel et le lac du Crouzet, envir. du Lac Luitel. H.-Alp. : Lautaret au bois de la Nadeleine, la Chalp, mont Séuse. - B.-Alp.: Tournoux, Blachiére de Maurin. - Rh. : Roche d'Ajoux, l'Argentière. - Loire : Pilat, Pierre-sur-Haute, Noirétable. - Alp.-Nar. : Clans, Tende, col de Frémamorta. Ard. : Mazan. - Gard : bois de Longues-Feuilles près Concoule, de l'Aigual près de l'Espérou. - Pyr.-0r. : mont Louis, vallées d'Eyne, de Llo et de Carença. - Pyr. centr., Puy-de-Dôme, mont Dore, Cantal, Forez.

L. pyremaica L. - Rochers. - Pyr.-Or. : Font de Comps, vallées d'Eyne et de Llo, Bac del Llo près Costujes, Tour-de-Mir, envir. de Prats de Mollo, vallée de l'Agly à St-Antoine-de -Galamus et au Pont de la Fou. - S’étend dans les Pyr. centrales.

L. nlpigena L. - Forêts des montagnes. - Nul dans les Vosges.

- Toute la chaine jurassique jusqu'au Colombier du Bugey.Vaud et Valais : Alpes de Jaman, Sembrancher, Saxon, Lens, Mayenalp, Vercorin, - H.-Sav. : Salève, Voirons, Nautau au- 
dessus de Iontriond, Morzine, plateau des Gets, montée de la Forclaz du Trient, Valorsine, Pormenaz, les Platets, les Contamines, mont Charvin, mont Clattillon, la Tournette, St-Germain, le Semnoz, Crest-Voland. - Sav. : mont du Chat, Trévignin, St-Germain, les Bréviaires, la Ramasse du Mont-Cenis. - Is. : mont Rachais, forêt de Portes, St-Nizier, bois d'Esparon près le Ifonestier-de-Percy. - II.-Alp. : Rabou, Côte Gélive, Loubet, mont Séuse, mont d'Avançon, Durbon, Montmorin au col de Casset, Briançon, Névache. - B.-Alp. : la Condamine, l'Enchastraye, Tournoux, Maurin, Larche. - Dr. : le Luc-en-Diois. Ard. : le Mezenc, Gerbier-des-Joncs. - Vaucl. : mont Ventoux. - Alp.-Har.: Morgès, Clans, Colmiane, entre St-Martin-Lantosque et Yenanson. - Pyr.-Or. : Canigou, moulin de la Llagone près Ilont Louis, bois des Angles sur la route de Capcir, vallée d'Eyne. - En dehors de nos limites dans les Pyr. centr., le mont Dore à Roche-Sanadoire et Roche-Thuillière, petit Puy-deDome. - H.-L, au bois de la Roche.

L. coerulea L. - Lieux tourbeux des montagnes, - Nul dans les Vosges. - Dans la chaine jurassique on ne le trouve que dans les tourbières de la vallée de Joux, au pied de la Dôle, forêt du Rizoux, Prémanon, Boullu. - Vaud et Valais : Alpes de Jaman, sous la Lex, Ceri, Trient, val d'Illiez, St-Bernard sous le Crêt, l'Ardifago, Catogne, Saas. - H.-Sav. : Brizon à Solaizon et à la Glacière, claalets de Colone, Nautau, Morzine, la Diosaz, Chamonix, les Contamines. - Sav. : mont du Chat, montée d'Aime au Crêt du Ré, mont Cenis, mont de Frêjus. - Is. : chaine des Sept-Laux et de Belledonne vers le lac du Crouzet, Champrousse. - H.-Alp. : Lautaret, Arcine, mont Genèvre, mont Viso au Bouchas, col de Vars, Prontic en Valgaudemar, col de Glaise.B.-Alp. : le Lauzannier, Pas de Grégoire près la Condamine. Ard. : Gerbier-des-Joncs. - Pyr.-Or. : citadelle de mont Louis, ile du moulin de la Llagone.

LINNAE borealis L. - Jolie espèce sous-ligneuse qui rampe à travers la Mousse des bois du Valais à Tête-Noire près de la frontiẻre française, sous Vende, Vercorin, Turtımann, Tufernen, Balen de Saas, Fee, Simplon. 


\section{RUBIACÉES.}

MUBIA peregrina L. - Coteaux calcaires, murs. - Commun dans la région mérid., moins dans la partie moyenne et septentrionale du bassin.

R. tinetorum L. - Cultivẻ dans la région mérid.; quelquefois subspontané.

CAMIUII Cruclata Scop. - Haies et bois dans les plaines et les montagnes. Il est à remarquer que, dans la région mérid., cette espèce occupe exclusivement les montagnes.

C. pedemontanum All. - Rochers. - Valais : Joux-Brulée audessus de Branson. - Is. : Pierre-Châtel entre Feytiny et le Collet et à Crey, la Motte d'Aveillans. - Var : coteaux du Verdon. - Gard : entre Cap-de-Coste et Arphy, Puéchagut près du Vigan.

C. vernum Scop. - Rochers des Alpes. - H.-Alp. : mont Genèvre, col de Vars. - B.-Alp. : Lauzannier, Horonaye, Tournoux, Jauziers à la Sagne, col de la Madeleine. - Alp.-Mar. : col de Tende, vallon de Fenestre, St-Martin-Lantosque, St-Étienne, Clans. - Pyr.-Or. : Mont Louis, vallée d'Eyne, Font de Comps, Canigou, Vernet-les-Bains, Cornella-du-Conflent, vallée du Tech entre Cẻret et la Preste, prairies de Pailhères. - Aude et Tarn: Montagne Noire. - En dehors de nos limites dans les Pyr. centr., l'Aubrac.

A. rotundifolium L. - Bois des montagnes. - Toute la chaine des Vosges depuis la vallée de Bruche jusqu'au Ballon de Giromagny, la chaine jurassique jusque dans le Haut-Bugey. - HteSav. : Voirons, Brizon, Habère-Lullin, Bonnevaux, les Gets, vallées de St-Gervais, de Servoz, de Chamonix et de Valorsine, Rumilly.- Vaud : districts de Payerne, Moudon, Prevon-d'Avaux. - Valais : Finshauts, Gueuroz, Chemin, Salvan, l'Etroz, Niederwald.-Sav: : mont du Chat, les Bauges. - Rh. : Chenelette. - Is. : montagnes de la Grande.Chartreuse, de St-Nizier, de Mens, de Revel et d'Uriage. - H.-Alp. : cols de Vars, le Noyer Durbon, Rabou. - Dr. : Saou, Barnave. - Alp.-Mar. : la Briga, 
Tende, St-Martin-Lantosque. - Ard. : Cuze. - Gard : bois de St-Sauveur près Camprieux.- Hér. : Avène.-Pyr.-Or. : vallée d'Eyne, forêt de Salvanère, mont de Madrès, Formiguères en Capcir, Llaurenti. - Loire: Pilat, Pierre-sur-Haute, vallée du Bonson. - En dehors de nos limites dans les Pyr. cent., la HteLoire, le Forez.

G. ellpticum Wild. - Cette espèce de Corse a êté trouvée à Hyères.

ه. boreale L. - Prés tourbeux de la région des Sapins des Vosges, Doubs, Jura. - Ain : Pradon, mont d'Ain, Dortan, Oyonnax, Ruffieu, le Vély, Arvières, de Belley à St-Germain. - C.-d'Or : Magny-sur-Tille. - Vaud et Valais: bassin du Léman, Vervey, Vouvry, Martigny, Sion, Sierre, Vercorin.- II.-Sav. : Allonzier, Douvaine, bois de Veyrier, entre Sionnet et Jussy, pied des Voirons, marais d'Arta et de Troenex, Pringy, mont Charvin. Sav. : Crêt du Ré, mont Cenis. - Is. : Grande Chartreuse. H.-Alp. : Lautaret, col de Vars, plaine de Lachaud près Gap, Charance, Chaudun, mont Bayard, mont Séuse, mont Chabriè. res, Orcières. - B.-Alp. : la Condamine, Lauzannier, SoleilleBœuf 'au-dessus de Bouzolières. - Alp.-Mfar. : mont Bego, col de Tende, Salsamorena. - Pyr.-Or. : bois de Boucheville et des Fanges, forêt de St-Martin près Fosse et Rabouillet.-Pyr. centr., Aubrac, H.-Loire, Puy-de-Dôme. Par une singulière bizarrerie, celte espèce montagnarde se trouve aussi dans les plaines de la Côte-d'Or à Orgeux, Limpré, Magny, Noiron, Châtillon, Semur.

G. verum L. - Bords des champs, prěs et pelouses dans les plaines et les montagnes.

G. decolorans et eminens Gr. Godr. - Considérés comme hybrides des $G$. verum et $G$. elatum avec lesquels ils sont mêlés.

C. purpureum L. - Champs incultes de la région mérid. - B.Alp. : Entrevaux. - B.-du-Rh. : envir. de Marseille. - Var et Alp.-Mar. : Seillans, Grasse, Thorenc, Antibes. - Pyr.-Or.: Château-Roussillon vers Canet, Sarrat d'en Vaquer.

G. mylvaticum L. - Bois. - Chaines vosgienne et jurassique. H.-S. : Mélisey, Champagney, mont de Vannes, Francheville, Linexert, Montigny, Héricourt. - C.-d'Or : Gevrey, Flavignerot, Savigny-sous-Beaune. - Rh. : l'Argentière, St-Julien-sur-Bibost. 
Ain : montagnes du Bugey, envir. de Bourg, Meximieu, Ambronay, Ferney. - Vaud et Valais : Aigle, Bouveret, Sion. - H.Sav. : Salève, St-Laurent, Annecy, Chamonix, St-Gervais. Is. : route de Bourgoin à Crémieu près de Flosaille. - B.-Alp. : la Condamine, Tournoux. - Pyr.-Or. : Prats de Mollo, la Preste, St-Laurent-de-Cerdans, Cornella-du-Conflent, Vernet-les-Bains.

G. loovigatum L. - Bois. - Is. : St-Ange, Pariset, la Morte, Monestier-de-Clermont, Mens. - H.-Alp. : Rabou, Charance, Pleyne-de Chaudun, Loubet, Durbon, Boscodon. - B.-Alp. : Tournoux, Annot. - Vaucl. : mont Ventoux. - Var:Toulon, le Luc. - Alp.-Mar. : l'Escarène, Revest, la Maïris, la Briga, Grasse. - Pyr.-Or. : vallée de Prades, Trencada d'Ambulla.

G. maritimum L. - Lieux secs de la région méditerr. - Hér. : Grammont près Montpellier, Doscare, Serre d'Oupia près d'Olon. zac, St-Chinian, St-Pons. - Pyr.-Or. : Perpignan, Prades, Olette, mont Louis, vallée de la Têt, Céret, Arles, Prats de Mollo, vallée du Tech, Cases de Pena, St-Antoine-de-Galamus, St-Paul, Caudiés, vallée de l'Agly.- Aude : Conques, Salsignes, Montolieu, Ste-Lucie, Ginole près de Quillan.

G. clatum Thuill. - Haies et bois dans tout le bassin. - Cette espèce ainsi que les suivantes sont les démembrements du $G$. mollugo L.

G. dumetorum Jord. Lyonnais. G. rigidum Vill. Dauphiné, Bugey, G. viridulum Jord. Lyonnais, Viva- Savoie, Languedoc, Vivarais. rais, Vienne. G. pallidulum Jord. Castellanne.

C. ereetum Huds. - Prẻs et bois dans tout le bassin.

G. corrudoefollum Vill. - Rochers et collines pierreuses de la région mérid. et moyenne, surtout dans les terr. calc. ou les conglomérats dans lesquels entrent des cailloux calcaires. Valais : Ottan, Valère, Comba d'Arbaz. - Ain : Gratoux et Angrières près St-Rambert-en-Bugey, Meximieu, Loyette, la Valbonne, la Pape, Vassieu. - Rh. : Ecully. - Is. : Crémieu, Vertrieu. - Sav. : mont Trélod en Bauges. - Dr. : St-Vallier, Montélimar. - H.-Alp. : la Garde, Charance, la Roche, Monestier-de-Briançon. - B.-Alp. : vallée d'Ubayc- - Commun dans Vaucl., B.-du-Rlı, et Alp.-IIar, - Var: Toulon, Hyères, 
Bormes, le Luc. - Ard. : coteaux calc. de la vallée de l'Ouvèze et des rives du Rhône. - Gard: Manduel, Nimes, Gaujac, Campestre, Montdardier, Serre-de-Bouquet. - Hér. : Montpellier à la Colombière, Bione, Fontfroide, le Crès, St-Gély, Valmargues, Restinclières, St-Bauzille, Fabrègues, Cette, Frontignan, StGuilhem, Montagnac, Pardailhan, Poussarou près St-Chinian.Aude : Sidrière de Fitou, envir. de Narbonne, Rivesaltes, Durban, vallon du Ripau. - Pyr.-0r. : vallée de l'Agly à Cases de Pena, St-Antoine-de-Galamus, Estagel, St-Paul. - En dehors de nos limites, sur les calcaires jurassiques de la Lozère et de l'Aveyron.

G. cluereum All. - Lieux incultes de la région mérid. - B.-duRh. : vallon de la Treille et de St-Pons, Ste-Victnire, chemin de la Tour de Keyrié près Aix. - Var el Alp.-Mar. : Toulon, Iyères, le Luc, l'Esterel, Grasse, entre Nice et Tende, la Giandola, Sorgio, Sospel. - Pyr.-Or. : ruines de l'abbaye de Jau au fond de la vallée de Molitg.

G. Tubldum Jord. - Coteaux incultes de la région mérid. - B.-duRh. et Var : envir, de Marseille, Toulon, Hyères, Bormes, Littoral des Alp.-Mar. et aussi près de Grasse à Andon. B.-Alp. : Digne. - H.-Alp. : Ia Garde près Gap.

A. myrianthum Jord, G. obliquum Vill.- Coteaux calcaires. - Ain : Serriêres, St-Benoit, Tenay, Nantua. - Hte-Sav. : base des dents d'Oche et de la Tournette. - Sav. : St-Innocent, Brizon près Aix, mont du Chat, Pas de la Fosse près Chambéry, St-Jeoire, Cruet, pied du Margériaz, Montmélian. - Is.: Bastille de Grenoble, Grande Chartreuse, Pariset, Pont-de-Claix, Morestel, Crémieu, Vernas. - H.-Alp. : Charance, les Serigues et Sauveterre, près de Gap, Sẻuse, Chabrières, Chartreuse de Durbon. B.-Alp. : Digne, la Condamine, St-Paul, Rochaille de Meyronnes. Vaucl. : Carpentras, Sablet. - B.-du-Rh. : Montaiguet, vallon de Mauret près Aix. - Ard. : entre Privas et le Pouzin. - Gard : le long du Valat de Brama-Bioou près Camprieux, bois de Salbous. - Hér. : plateau de Larzac au Caylar, Pegayrolles-deBuèges. - En dehors de nos limites, sur les coteaux calcaires des environs de Florac (Lozère), de Millau el St-Affrique dans l'Aveyron. 
G. Prostii Jord. - Coteaux calc. du Larzac dans l'Hér. et l'Areyron Salbous et Campestre dans le Gard et aussi dans la Lozère au tour de Mende. - If. Loret a démontré que le G. Prostii est l: forme à fleurs rouges du G. myrianthum, auquel il est très-sou. vent associé dans les localités citées de l'Hérault, de l'Aveyron de la Lozère et du Gard.

G. Iuteolum Jord. - Pelouses. - H.-Alp. : Sapet de la Bâtic Neuve, col de Vars, Brame-Buou près St-Genis-le-Désolé, mon Séuse. - B.-Alp. : la Condamine, col de Larche, Lauzannier. Vaucl. : Flassan.

G. Ieucophoeum G. G. - Pelouses, - H.-Alp. : entre Ville-Val louise et l'Echauda.

G. alpicola Jord. - Forme du précédent à panicule plus étroite, fleurs plus rapprochẻes et à pédicelles plus courts. - Is. Clavans.- H.-Alp. : la Grave, Lautaret, Monestier-de-Briançon mont Genèvre, bords de la Durance au-dessous de Briançon Guillestre, vallée du Guil. - B.-Alp. : Barcelonnette, la Conda mine, Sérennes. - Sav. : bords du lac St-IIarcel près Moutiers

G. Wrachypodum Jord. - Coteaux et bois. - H.-Alp. : Névache envir. du lac Séguret près Embrun, Guillestre, mont Chabrières Rabou, col de Glaise, ravin de Sauveterre près Gap, mont Séuse - B.-Alp. : Barcelonnette, Bachasse, la Condamine, Enchas traye.

G. Ioetum Jord. - Coteaux pierreux. - Dr. : Crest. - B.-Alp. Digne, Sisteron, Castellanne. - Vaucl. : source de Vaucluse.B.-du-Rh. : sous le pic de Bretagne.

G. collinum Jord. - Coteaux secs. - Dr. : Valence, St-Ferréo près Crest. - Ard. : Tournon, Châteaubourg. - Gard : Alais Uzès, Manduel, Tresques, route du Vigan à l'Espérou.

G. seabridum Jord. - Coteaux secs. - Is. et Dr. : Vienne, St Ferréol près Crest. - H.-Alp. : col Bayard, Puy-Montbaud, MaI teyer, Laragne. — Gard : envir. du Vigan. - Hér. : St-Geniès de-Varensal, Lunas, l'Escandorgue au Mas-de-Mouriès. - B. du-Rh. : Pas-des-Lanciers.

G. TimeroyI Jord. - Coteaux secs. - Is. et Dr. : Crémieu, Cres Ain et Rh. : la Pape, Couzon, St-Germain-au-mont-d'Or. - 
Vaucl. et B.-du-Rh. : Arignon, Pic de Bretagne. — Gard : Nimes, Manduel, Alzon, le Vigan. - Hér. : entre Grabels et la Paillade, Pic St-Loup, Lunas.

G. Implexum Jord. - Coteaux secs. - Is. : Mollard de Décines. Dr. et II.-Alp. : Valence, la Garde et Puy-Mlontbaud près Gap, Durbon. - B.-du-Rh. : Roquefavour. - Gard : Nîmes, Alais, la Tessone, Molière.

G. Intertextum Jord. - Coteaux pierreux. - Is. : la Bérarde en Oisans.-H.-Alp. : Serres, Laragne, la Garde, mont Queyrel en Champsaur. - Gard: bois de Broussan près Nimes, Serre-deBouqquet, Montdardier, Genolhac. - Hèr. : Montpellier à Lavalette, Lamoure, Fontfroide, Doscares, Grabel, Mireval, Pérols, Frontignan, Restinclières, St-IIartin-de-Londres, St-Llathieude-Londres, St-Ilathieu-de-Tréviers, Montarnaud, Joncels, la Vacquerie.

I. Loret réunit sous le nom de G. Jordani les quatre formes ci-dessus désignées et assure avoir vu entre elles des intermédiaires qui les relient l'une à l'autre.

G. Fleuroti Jord. - Coteaux de C.-d'Or et S.-et-L. autour de Saulicu aux bois de Champent et de l'Hôpital, vallon de la Coquille près d'Etalante, au-dessus de la cascade et des rocbers de Vauchignon près Nolay, entre Santenay et Chassagne et, plus loin, dans la vallée de la Dheune.

G. papillosum Lap, - Collines pierreuses. - Pyr.-Or. : vallée du Réart, Trencada d'Ambulla, vallée de Conat vers la Font-deComps, Villefranche, vallée d'Eyne, Font-Romeu, mont Louis, Llaurenti. - Pyr. centrales.

G. sylvestre Poll. - Espèce polymorphe disse̊minée depuis les collines jusqu'aux montagnes, et comprenant :

G. Bocconi D C. nitidulum Thuill. forme hispide; - G. commutatum Jord. ou lave Thuill. forme glabre;

G. montanum Vill. - Forme glabre comme la précédente à fruits plus gros et à souche cespiteuse, assez commune dans les Alpes et dans la chaîne jurassique; une variété $G$. pygmeum Iutel se trouve à Villard d'Arène sous les glaciers du Bec et au pic de Chaillol-le-Vieil, puis à la Tournette à l'est du lac d'Annecy. 
G. argenteum Vill. - Pelouses des montagnes. - Is. et H.-Alp. : Grand Som, Charmant Som, Lautaret, Rabou, Séuse, Aurousc, Chaillol-le-Vieil.

ศ. anisophyllum Vill. - Páturages et prairies des montagnes. - Sommités du Jura helvétique et français, Creux-du-Van, Suchet, mont d'Or, Montendre, Döle, Reculet. - H.-Sav. : Brizon, Vergy, Méri, Dents d'Oche, mont Laouet, la Tournette. Sav. : Haut-du-Four près Plancherine, mont Drizon, Margériaz, la Sambuy. - Is. : Bovinant, Chamechaude, St-Nizier, col de l'Arc, la Moucherolle. - H.-Alp. : mont Aurouse, mont Durbonnas.

G. tenue Vill. - Pelouses et rochers des montagnes. - Sommités du Jura helvétique et français, Creux du Van, Suchet, Montendre, Colombier, Reculet jusqu'au Colombier du Bugey. H.-Sav. : mont Brévent, la Tournette. - Sav. : Pugny, Trevignin près Aix, dent de Nivolet, mont d'Orizan au-dessus de Cléry, Pas-du-Roc près St-Michel-en-Maurienne, Arrieux, la Gitaz près Beaufort, col du Frẻne. - Is. : Grand Som, Grande Vache, le Moucherotte, col de l'Arc. - H.Alp. : col des Hayes, Orcières. - B.-Alp. : la Condamine, Sérennes.

G. Jussiœi Vill. forme naine du précédent.-H.-Sav.: Cornettes deBise, Morzine, le Buet, Barberine, Aiguilles rouges, Bẻrard.-H.Alp.: mont Viso, col de Péas, mont Aurouse. - Alp.-Mar. : Touet-deBeuil.

G. pusfllum L. - Rochers de la région mérid. - Vaucl. et B.-duRh. : mont Ventoux, Vaucluse, Cavaillon, Vacqueiras, les Alpines, Ste-Victoire. - Var : Aups, Toulon, Villecrose, la Roquebrussanne.

G. hypnoides Vill. - Forme du précédent à feuilles plus raides, plus longues, plus aiguës, non hispides. - Sav. : Villarodin en Maurienne. - B.-Alp. : la Baume sur Sisteron. - Vaucl. : mont Ventoux, source de Vaucluse.

A. pyrenalcum Gouan. - Rochers et débris pierreux. - Pyr.Or. : vallée d'Eyne, sommités de Cambres d'Aze, Canigou, Cady, la Porteille de Mantet, Costa Bona, vallée de Llo. - La région alpine des Pyr. centrales. 
G. helveticum Weigg. - Rochers et débris pierreux. - Vaud et Valais : Enzeindaz, mont Fully, Gemmi. - H.-Sav. : Brévent, Tré-la-Téte. - Is. ?: chaine de Belledonne et mont de Lans à la Tête-de-Racha. - H.-Alp. : Lautaret, Galibier, mont Viso à la Traversette, cols de Vars et du Noyer, Chaillol-le-Vieil, col do Glaise, Côte Gélive, mont Aurouse. - B.-Alp. : Larche, Lauzannier, Vallonnet, Bachasse, Bérard. - Alp.-Mar. : Tende, mont Bego, Estenc, col de Jallorgues, Bouziejo.

Var. pusillım Vill. - Mont de Lans, pic de Chaillol-le-Vieil.

G. megalospermum Vill. - Rochers et débris pierreux. - ls. : la Moucherolle, mont Aiguille. - Dr. : mont Glandasse, près de Die. - H.-Alp. : col de Glaise, Cốte Gélive, col de Chaudun. mont Aurouse. - Alp.-Hfar. : col de Tende, vallon de Hasca, de Fenestre, du Cavallé et de Rabuon. - Vaucl. : mont Ventoux. - B.-du-Rh. : Montaud-les-Miramas. - Pyr.-Or. : Collada de Nuria, vallée d'Eyne, Canigou vers la Porteille de Mantet.

๔. cometerrhizon Lap. - Rochers. - Pyr.-Or. : vallée d'Eyne, de Llo, pic de Finestreilles, Carença, Pla du Camp Magre, Costa Bona. - Pyr. centrales.

C. manntlle L. - Rochers, pâturages et bois des terrains siliceux; nul sur les calcaires. - Commun dans la chaine des Vosges depuis le Donnersberg jusqu'au Ballon de Giromagny, et aussi sur les grès vosgiens, rouges et bigarrés de la H.-Saône. - Nul dans la chaine jurassique. - Vaud et Valais : gneiss des monts Enzeindaz et Fully. - Morvan dans C.-d'Or et S.-et-L. autour de Saulieu et d'Autun. - Chaine granitique du Beaujolais, du Lyonnais, de Pilat et Pierre-sur-Haute notamment à Vaurenard, pic de Chatoux, St-Bonnet-le-Froid, Izeron, Violay au bois d'Azolles. - Granite des Pyr.-Or. entre Prats de Mollo et Pla Guilhem, vallées d'Eyne, Cambres d'Aze, col de Pailhères. Granite, gneiss et schistes de la Montagne Noire.-'Terr. volcaniques du Mezenc et de la chaine qui va du Gerbier-des-Joncs au Coyron. - En dehors de nos limites sur les terrains volcan. de l'Aubrac; sur les mémes terrains et sur les granites du Cantal, mont Dore, Forez. - Gneiss, micaschistes et granites de la Lozère et des Pyr. centr. - Cette espèce si répandue dans 
les Vosges, le massif central, les Cévennes et les Pyrénées manque complètement dans les Alpes françaises, quoique les terrains siliceux qui lui conviennent s'y trouvent en grande abondance. C'est là un fait de géographie botanique qui,s'il n'est pas fortuit, serait difficile à expliquer. Le G. saxatile n'est d'ailleurs pas la seule plante dont l'aire de dispersion géographique présente pareille anomalie.

G. palustre L. - Marais, fossés, étangs.

G. rupicola Bor. - Diffère du précédent par ses tiges décombantes ou couchées et par son fruit plus chagriné. Cà et là mélé avec le type.

G. elongatum Presl.:- Differe du G. palustre par un plus grand développement de ses rejets rampants, par les nervures de ses feuilles plus saillantes et ses pédoncules étalés.

G. debile Desv. - Marais tourbeux, prés humides. - Is. : Crémieu. -B.-du-Rh. : prẻs de Marignane, paluds de St-Remy. — Gard : Manduel, Tresques.- Hẻr. : Pérols, Mauguio, Pézenas, Mireval, Roquehaute, Agde, St-Martin-de-Londres.

G. uliginosum L. - Prés marécageux et tourbeux. - Vallées des Vosges; zone vosgienne et sous-vosgienne de H.-Saone. Tourbières de la vallée de Joux, la Trêlasse, St-Cergues. Marais de Crévin près Genève. - La Dombes. - C.-d'Or et S.-et-L. : le Morvan. - Is. : la Verpillière, Décines, Chambaran, - H.-Alp. : lac de Séguret près Embrun. - Gard : la Dourbie, Camprieux. - Hér. : Fraisse, la Salvetat. - Pyr.-Or. et centr. - Centre de la France.

G. setrceum Lam. - Collines de la région mérid. - B.-du-Rh. : envir. de Marseille, la Fare, Aix en amont du canal Zola, les Alpines. - Var et Alp. mar. : Toulon, le Luc, Grasse, St-Tropez.

C. divarieatum Lam. - Pâturages. - Is. et Rh. : Balmes viennoises, Mollard de Décines, Sérézin, mont d'Annoisin près Crémieu, St-Alban, Quincieu. - Dr. : St-Vallier, - B.-du-Rh., Var et Alp.-Mar. - Gard : bois de Cygnan près Nimes, l'Espérou, Montdardier, Molière, Aumessas, Alzon. - Hér. : Grammont, Montarnaud, Roquehaute, Bousquet de St-Martin d'Orb. G. tenuicaule Jord. - Envir. de Lyon. 
G. parisiense L. - Espèce polymorphe présentant les formes suivantes :

G. litigiosum D C. - Forme i fruit velu. - Champs sablonn. Valais : Fully. - Is. : Crémieu, St-Nlens. - B.-du-Rh. et Var : envir. d'Aix et de Marseille, Toulon, St-Raphaël. - Alp.-Mar. - Assez commun dans Vaucl., Hér. - Gard : Nimes, Uzès, Manduel, Alzon, le Vigan. - C.-d'Or : Pontailler, Magny-surTille. - S.-et-L. : Cluny, de Digoin à Bourbon. - Centre de la France, Tarn, Aveyron.

G. decipiens Jord. - Diffère du précédent par ses fruits plus gros, ses pédicelles plus longs, ses styles dressés, ses feuilles plus larges, verticilles par sept. - Lieux incultes de la région mérid. B.-du-Rh. et Var : Tarascon, Toulon, St-Raphaël. - Hér. : Cette. - Aude:St-Hippolyte. - Pyr.-Or. : port Vendres, Banyuls-sur-mer, Ermitage de N.-D. de Pena, pont de la Fou.

G. anglicum Huds.- Forme à fruits glabres.- Jura et Ain: Chaussin, Thoiry.-Bassin du Léman et Valais, - Is. : Crémieu, Dizimieu, Brezin, St-Robert. - Rh. : Caluire au Vernay. - Loire:Chavanay. - Dr. : St-Vallier. - Ard. : Vals. - Commun dans Vaucl., B.-du-Rh., Var, Alp.-Mar., Gard, Hẻr., Aude et Pyr.Or.-En dehors de nos limites, dans Loire, Allier, Puy-de-Dơme, Lozère, Tarn.

G. ruricolum Jord. - Differe du G. anglicum par ses tiges rameuses dès la base et entrelacées, ses fruits finement chagrinés, ses pédicelles plus courts. - Envir. de Lyon et de Vienne. - Tarn.

G. tenellum Jord.- Collines arides des envir. d'Antibes et d'Ampus.

C. Aparine L. - Haies, bois dans tout le bassin.

C. spurium L. - Haies, lieux incultes. Espèce polymorphe.

G. Vaillantii D. C. - Forme à fruits hispides. - Champs sablonn. Vaud et Valais. - S.-et-L. - Chaine jurassique jusque dans le Bugey. - H.-Sav. : Salève près des Treize-arbres. - ls. : Corenc, St-Eynard, Sassenage, mont de Lans. - Dr. : Die, mont Glandasse. - H.-Alp. : envir. de Gap. - Vaucl. : envir. du mont Ventoux. - B.-du-Rh. - Gard : Villeneuve. - Hér. : Pérols, Palavas, Vic, St-Georges, Grayssessac, Cetle. - Aude : envir. de Narbonne. 
G. tenerum Schleich. - Forme à tiges faibles, couchées. - Valais : Gemmi, Zermalt. - H.-Sav. : petit Salève. - Ain : Serrières. - H.-Alp. : mont Séuse. — Var : le Luc. — Gard : la Fosse près St-Gilles.

G. tricorne L. - Champs cultivés dans tout le bassin.

G. ๙acehnratum All. - Terres incultes, vignes. - B.-du-Rh., Var et Alp.-Mar. : N. D. de la Garde près Marseille, Toulon, Hyères, Fréjus, Cannes, île St-Honorat, le littoral de Nice à Menton. - Pyr.-0r. : Baixas, Cases de Pena.

A. minutulum Jord. - Iles de Porquerolle et du Levant.

G. vertiellatum Danth.- Rochers de la région mérid. - Vaucl. et Gard : Uchaux, Vaucluse, Bedoin, mont Ventoux, grand Montagnet et Villeneuve près Avignon, Blandas près du Vigan. - B.-du-Rh. : montagne des Pauvres près Aix, Ste-Victoire, Roquefavour, vallon de St-Tronc près Marseille. - Var : Mourières près Toulon, Châteaudouble. - Hér. : les Arcs près StMartin-de-Londres, mas de Mouriès sur l'Escandorgue.

G. murse All.- Lieux incultes, pied des murs. - B.-du-Rh., Var, et Alp.-Mar. : envir. d'Aix et de Marseille, Toulon, Hyères, Fréjus, littoral de Nice à Menton. - Gard : Aigues-Mortes, Sylveréal, la Fosse près St-Gilles. - Hér. : Montpellier au-dessus de Boutonnet, Gramenet, Montbazin, Cette, Palavas. - Aude : Narbonne, Mandirac, Ste-Lucie, St-Martin-de-Thoques.

VAMTEANTIA muralis L. - Rochers calcaires, vieux murs. Vaucl. : envir. d'Avignon et d'Orange. - B.-du-Rh. : Aix aux Trois-Moulins, l'Estaque. - Var et Alp.-Mar. : le Luc, îles de Porquerolle et de Ste-Marguerite, Antibes, région littorale de Nice à Menton. - Gard : Nìmes, Bellegarde, Aigues-Mortes, St-Nicolas, le Vigan, Alais, Anduze. - Hẻr. - Aude : envir. de Narbonne, Sidrière de Leucate, Ste-Lucie.- Pyr.-Or. : roch. de N.-D. de Pena.

V. Migpida L. - Alp.-Mar. : entre les Quatre-Chemins et le col de Villefranche.

ASPERUA odorata L. - Bois sur les coteaux et jusque dans les forêts des montagnes; c'est même exclusivement dans celles. ci qu'on observe cette espèce dans la région mérid. comme dans 
la chaine de l'Espérou, de l'Espinouse, des Albères, et dans les Alp.-Nar. sur les montagnes de Tende et de Pesio.

A. cymanchiea L. - Collines pierreuses dans tout le bassin. -

Espèce polymorphe.

A. rupicola Jord. - Rochers à la Grande-Chartreuse.

A arenicola Rent. - Envir. de Genève à Bernex, Sesegniens. - Sav. :

Moutiers. - Is. : Bastille de Grenoble.

A. tentifloru Jord. - Rochers calc. de St-Hippolyte (Gard).

A. tinctoria L. - Coteaux secs. - Ain : Nantua, Dortan.-Vaud : près de la grotte de Montcherand. - Gard:bois de Salbous près d'Alzon. - Pyr.-Or. : jardins St-Jacques, mont Louis. Pyr, centrales.

A. Iongiflora W. Kit. - Rocher's. - Valais : Martigny, Catogne, Chemin-Neuf, Branson, Ardon, Sion, St-Léonard, Vercorin, Stalden. - Is. : la Bérarde en Oisans. - H.-Alp. : ChâteauQueyras, Abriès, Vallouise. - B.-Alp. : vallée de l'Ubaye, StPaul, la Condamine, Lauzannier. - Sav. :Tignes, St-Michelen-Mlaurienne, Modane.- Var. : plateau de N.-D. des Anges près Pignans. - Alp.-Mar. : l'Esterel, Tende, St-Martin-Lantosque. A. Jordani Perr. Song. - Rochers. - Sav. : Tignes, St-Michel-enMaurienne, mont Cenis. - B.-Alp. : la Condamine, Rochaille de Meyronnes, Sẻrennes.

A. Hirta Ram. - Rochers calcaires. - Pyr.-0r. : Ermitage de StAntoine-de-Galamus, vallon de Prades. - Montagnes calc. des Pyr. centrales.

A. Iovigreta L. - Champs, iords des chemins. - Var et Alp.Mar. : Maures du Luc, l'Esterel, bois de Tanneron sur la Siagne. Aude : envir. de Narbonne.-Pyr.-Or. : de St-Paul à St-Antoinede-Galamus.

A. taurina L. - Coteaux pierreux. - Valais au Simplon. - Ain : montée de Culoz au Colombier. - Is. : Bourg d'Oisans, Esparon près du Monestier-de-Percy. - Dr.: les Lussettes, Aucelon au bois de la Louine. - H.-Alp. : Lautaret, Chaudun, les Baux, la Grangette, Durbon.

A. HexaphylIa All. - Alp.-Mar. : de Tende au sommet du Grammont au-dessus de Menton, Bec d'Utelle, vallon de Libaré près Catal. Bassin du Rhônte. 
Venanson, vallon de Colmiane près St-XIartin, embouchure du Var et de l'Esteron.

A. arvensis L. - Champs dans tout le bassin.

A. galioides M. B. Galium glaucum L. - Rochers calcaires. C.-d'Or : de Dijon à Plombières, Flavignerot et toute la Cóte jurassique, - S.-et-L. : Dezize, Cluny, Marmagne, Broye. Environs de Genève, à Contamines, Veyrier, Genthod, Peney, Cartigny. - Vaud : envir. d'Aigle. - Rh. et Ain : St-Clair, Vernaison, Tassin, la Pape, Tenay, Mogneneins, Meximieu sous le Mont. - Is. : Balmes viennoises jusqu'à Anthon, Crémieu, Comboire, Vouillant, Bastille de Grenoble.-H.-Alp. : St-Mens près Gap, Pleyne de Chaudun. - Vaucl. : pied du mont Ventoux, Avignon, Orange. - B.-du-Rh. : envir. d'Aix, avenue du Tholonet, la Pioline, Moulin-Fort. - Var : Toulon, le Luc, Fréjus. - Alp.-Mar. : Antibes, Auribeau. - Ard. : Payolive. - Gard : Chartreuse de Valbonne. - Hér.: Bione près Montpellier, Fontfroide, Doscares, Castelnau, Marsillargues, St-Aunès, Lattes.- Pyr.-0r. : Baixas, vallon de Ste-Catherine, Prades, Villefranche, Céret, vallée du Tech.

SHERARDIA arvensis L. - Champs des terrains calcaires dans tout le bassin.

Crucianella maritima L. - Sables maritimes. - B.-du-Rh. et Var : Marseille au cap Croisette, ruines de Taurentum, Toulon, Frẻjus, St-Raphaël. - Alp.-IIar. : Cannes, golfe Jouan. Littoral du Gard ot de l'Hér. : Aigues-Nortes, Roquehaute près Agde, Béziers. - Littoral de l'Aude et des Pyr.-0r.: Sidrière de Fitou, le Canet jusqu’à Banyuls-sur-Mer.

C. Intifolia L. - Champs cultivés. - Is. : Vienne, St-Rambert. Dr. et Vaucl. : entre Montélimar et Orange. - B.-du-Rh., Var, et Alp.-Nar.: envir. d'Aix, de Marseille, de Toulon, le Luc, Nice. - Hér. : entre Viols et les Capouladoux, St-Guilhem, St-Jean-de-Fos, Puéchabon, Aniane, St-Jean-de-Buèges. - Pyr.Or. : vallée du Rẻart, Château-Roussillon, Baixas.

C. angustifolia L. - Coteaux sablonn. - Ain ct Rh. : la Valbonne, les Neyrolles, Oullins, Francheville, Chaponost, Vernaison.Is. et Dr. : Charvieu, Vienne, Crest, Livron, Montélimar, entre 
Barnave et Montmaur. - Loire: Chavanay, Pelussin, Malleval, Bourg-Argental, Rive-de-Gier, Montbrison. - Ard. : Aubenas, Vals._Commun dans Vaucl., B.-du-Rh., Var, Alp.-Mar., Gard, Hér., Aude et Pyr.-0rientales.

\section{VALÉRIANEES.}

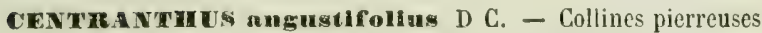
des terr. calcaires. - C.-d'Or : coteaux jurassiques de Dijon et aussi de Gevray à Santenay. - S.-et-L. : vallée de la Dheune, Nolay, Dezize, Remilly. - H. S. : calcaire jurassique de Champlitte, entre Roche et Trécourt, St-Andoche, carrières de Fouvent-le-Bas. - Doubs et Jura : calc. jur. du Saut-du-Doubs, Cotes du Doubs et du Dessoubre, Pontarlier, Salins, Poligny, St-Amour, Thoirette, St-Claude, mont d'Authume. - Ain : vallée de l'Albarine entre Tenay et Charabotte, cascade d'Evoges, la Burbanche, Inimont, Ordonnaz. - Sav. : envir. d'Aix, pentes du mont du Chat, entre Aigueblanche et Houtiers, St-Jeande-Maurienne, St-Michel. - H.-Sav. : la Tournette. - Is. : Bastille de Grenoble, St-Eynard, vallées de la Romanche et du Vénéon, Mens, Corps.-H.-Alp. : la Grave, Briancon, Cerviẻres, entre Château-Queyras et Abriès, Guillestre, Chorges, col du Noyer, les Serigues près Gap. - B.-Alp. : vallée de l'Ubaye, la Condamine, St-Paul, Larche, vallée du Verdon de Barcelonnette à Colmars. - Vaucl. : le Sault, Flassan, Fontaine de Vaucluse, Orgon, combe de Lourmarin près Apt. - B.-du-Rh. : Orgon, les Alpines. - Alp.-Mar. : Guillaumes, St-Etienne et St-Dalmasle-Saurage. - Gard : Corconne, Anduze, Alais, Ganges, St-Hippolyte, le Vigan.- Hẻr. : Pic St-Loup, Capouladoux, la Sérane, Ganges, Mas-de-Londres, St-Martin, St-Guilhem, Puéchabon, Montarnaud, Jonquières, St-Gély-du-Fesc, le Larzac au Caylar et à la Vacquerie. - Aude. - Pyr.-0r, : valle̊e de l'Agly à Cases de Pena, St-Paul, St-Antoine-de-Galamus, Villefranche jusqu'aux Graux d'Olette, vallée de Céret jusqu'à Prats-de-Mollo. - En dehors de nos limites sur les rochers calc. des Pyr. centr., de l'Aveyron et de la Lozère. 
C. Leconii Jord, - Vaucl. : Malaucine, Vaucluse, Cavaillon, mont Ventoux au bois de Fonterrasse. - B.-du-Rh.: les Alpines. B.-Alp. : Colmars, Barcelonnette. - Gard : Pont-du-Gard, Ganges, Anduze. - Aude et Pyr.-Or. : entre Vingrau et Rivesaltes.

C. ruber D. C.-Rochers calc., murs de la région mérid. - Souvent naturalisé autour des habitations dans quelques localités de la région moyenne du bassin, comme par exemple autour de Crest, Gap, Grenoble, Lyon, Aubenas, Privas.

C. calcitrapa Dufr. - Coteaux pierreux de la région mérid. et moyenne. - Is. : Crémieu, Leyrieu, Vernas, Hyères, la Balme, Vouilland. - Ain : St-Bernard, Beauregard. - Rh. : Alix au Chevrounet. - Dr. : St-Vallier, Serves, Tain, Luc-en-Diois, Divajeu, Livron, le Buis. - B.-Alp. : Digne.-Ard. : Tournon, mont Combier, Aubenas, Cuze, Vallon, Crussol. - Vaucl.: Grand-Nontagnet, Flassan, Bedoin, fort de Roquesalière prés Apt. - Commun dans B,-du-Rh., Var, Alp.-Nar., Gard, IIér., Aude et Pyr.-Or. - En dehors de nos limites dans la Lozère, le Lot, le Tarn et l'Aveyron.

VAEETRANA offieinalis L. - Bois dans tout le bassin; plus rare dans la région mérid. où il ne se trouve que dans les montagnes des Alp.-Nar. autour de St-Auban, Entraunes, St-Etienne, dans les montagnes du Vigan, d'Alzon, à l'Espérou, à l'Espinouse, à l'Escandorgue et au Caylar. Une variété sambucifolia Mick. dont les feuilles ont des segments ovales-arrondis, dentés sur les bords se trouve mêlée au type dans les envir. de Semur et de Belley.

v. Phu L. - Cultivé et quelquefois subspontané au voisinage des habitations comme, par exemple, dans le canton de Vaud à l'Etivaz, dans l'Is. au St-Eynard près de la cabane, dans les Pyr.-Or. à Cases de Pena, St-Paul, Vernet-les-Bains, Prats-deMollo, alla Roca Gallinera.

v. pyrenaien L. - Bois, lieux humides, - Pyr.-Or. : mont Louis, Fetges, vallées d'Eyne, de Llo et de Carença, forêt de Salvançre, Cosla Bona, Llaurenti, Castell près Vernet-les-Bains. - Pyr. centrales.

v. dioien L. - Lieux humides dans les parties septentr. et moyen- 
nes du bassin; rare dans la région mérid. où il se montre exclusivement dans les montagnes.

v. tuberosa L. - Rochers calcaires. - C.-d’Or : Château-Renard au-dessus de Gevrey, Curley, Chaumes d'Auvenay, entre le Mantuan et le Vergy. - Is. : Vertrieu, la Balme près Crémieu, mont Rachais, Comboire, Balmes de Fontaine. - Dr. : col de Penne. Ard. : Chomérac. - H.-Alp. : Briançon, Rabou, Charance, Côte Gélive, col de Glaise, mont Séuse. - Vaucl. : St-Amans, mont Ventoux, Caumont. - B.-du-Rh. : montagne des Pauvres, les Alpines, - Var : Toulon, Hyères, le Luc, Rians, Ste-Baume. Alp.-Har. : montagnes de Menton, Utelle, les Lattes, le Cheiron, Caussols. - Gárd : Nimes, Remoulins, Pont-du-Gard, Campestre. - Hér. : Montpellier, à la Lauze, la Paillade, Mireval, Capouladoux, St-Guilhem, la Sćrane, St-Martin-de-Londres, le Caylar.-Aude : Pech de l'Agnèle et de la Fenno-morte, Roquefort.-En dehors de nos limites, dans la Lozère sur les Causses de Sauveterre, Camp de l'Hospitalet; dans l'Aveyron autour de Millau, de Rodez et de Ste-Affrique; Pyrénées centrales.

v. globulariofolia Ram. - Rochers humides des Pyr.Or. : vallíes d'Eyne et de Conat, Font de Comps, montagnes de Madres et de Cosla Bona. - Région alpine des Pyrénées centrales.

v. tripteris L. - Rochers des montagnes; plus fréquent sur les sols siliceux, mais non exclusif. - Commun dans les Vosges euritiques et granitiques; zone vosgienne de H.-S. et des envir. de Belfort dans les vallées de Puix, de Rosemond et du Rahin, Plancher-les-Hines, Servance, Ballons de Servance et de Giromagny, vallée de Miélin, Champagney. - Rare dans la chaine jurússique sur les marnes ainsi que sur les sols tourbeux ou riches en liumus, Creux du Van, Suchet, Mont-d'Or, Pontarlier, fort de Joux, Salins, Arbois à la Châttelaine; dans l'Ain à Charabotte, Arvières, cascade de la Fouge près Poncin. - Vaud et Valais : Naie, Chemin, Trient, Clavaux, Thyon, Natersberg, Glacier du Rhone. - H.-Sav. : Salève, Voirons, Brizon, mont Laouet, Cornettes de Bise, les Contamines, Brévent, col de Balme, Vallorsine, mont Charvin, la Tournette. - Sav. : Aix, mont du Chat, St-Jean-de-Maurienne. - Is. : Grande Chartreuse, 
St-Eynard, St-Nizier, Esparon près du Monestier-de-Percy. H. et B.-Alp. : envir. de Gap, Boscodon près d'Embrun, Barcelonnette. - Vaucl. : mont Ventoux, Luberon. - Alp.-Mar. : Mine de Tende, Ste-Anne-de-Vinaï, Clans, - Loire : très-commun dans le massif du Pilat. - Ard. et H.-L. : Le Mezenc, Roche de Gourdon, Astier, Entraigues, Laprat du Cheylard. Gard : Arphy, Alzon, l'Espérou, Anduze, Concoule. - Hér. : le Larzac au Caylar, St-Amand-de-Mounis, Madières, l'Escandorgue, Pėgayrolles-de-l'Escalette. - Pyr.-Or. : Mont Louis, Motte de Planes, Cambres d'Aze, Canigou, Costa Bona, vallon de Castell. - En dehors de nos limites dans les Pyr. centrales, l'Aveyron, l'Aubrac, Cantal, Margeride, Lozère, monts Dômes, mont Dore, Forez, Pierre-sur-Haute.

v. montana L. - Débris de rochers. - L'absence du V. montana dans les Vosges contraste avec la fréquence dans cette chaine du V.tripteris. - Commun dans le Jura helvêtique et français: Cótes du Doubs et du Dessoubre, Clos-du-Doulss, Lomont, toute la châ̂ue di Montendre, mont d'Or, Suchet, Dôle, Colombier, Reculet, montagnes du Haut-Bugey. - Vaud et Valais : Javernaz, Enzeindaz, Diablerets, val d'Illiez, Comba d'Arbaz, Loze, Clavaux, pied de la Gemmi, Massagletscher. - H.-Sav. : Salève, Voirons, mont Charvin, mont Châtillon, Brizon, col des Aravis, mont des Granges, mont Laouet, montagnes de Samoëns, Bionassey, col de Balme, Vallorsine. - Sav. : mont du Chat, Nivolet et autres montagnes des Bauges, la Tarantaise et la Maurienne. - Is. : Grande Chartreuse, St-Nizier, Revel, montagnes de l'Oisans, - Dr. : Luc-en-Diois. - H.-Alp. : Lautarel, col de Vars, Chabrières, col du Noyer, la Grangette, Pleyne de Chaudun, col de Glaise, mont Séuse, St-André-de-Rosans. B.-Alp. : vallée de l'Ubaye. - Alp.-Mar. : Entraunes, col de la Maddalena, Clans, col Bertrand, bois de Caïros. - Pyr.-Or. : Mont Louis, vallée de Nohìdes, Font-de-Comps, Prats de Mollo. Pyr. centr. - Nul dans les Cẻvennes du Gard et de l'Hérault, dans le Tarn, l'Aveyron, le Cantal, le mont Dore, les monts Dômes, le Forez; dans toutes ces montagnes, comme dans les Vosges, le $V$. tripteris remplace le $\mathrm{V}$, montana. 
v. Saliumea All. - Rochers, - Vaud el Valais : les Mortais, mont Fully, Loze, Simplon, Langenthal, Tunetschhorn, Rothbinnerfurggen, Gemmi. - II.-Sav. : mont Méri à la Pointe-Percée. Is. : Chamechaude, Gresse, la Bâtie, mont Chamoux.-H.-Alp. : Villar d'Arìne aux Troix-Evêchés, Goléon, Galibier, l'Echauda, Plampinet, col de Glaise, mont Aurouse. - B.-Alp. : Bachasse, Parplaillon, Vallonnet de Heyronnes, Bérard.-- Vaucl. : sommet du mont Ventoux. - Alp.-Nar. : les Viosennes, mont Bego, col de Fenestre, mont Monnier.

v. celtiea L. - Rochers et pâturages. - Valais: St-Bernard au Tzermanaire et vers les pointes de Dronaz, mont Cervin, Distelalp, Offenthal, Simplon. - Mont Cenis au lac Blanc et sur le versant italien du mont Rose.

VALETINAMLA olitoria Poll. - Champs cultivés dans tout le bassin.

v. enrinsta Lois. - Ibid.

V. auricuia D C. - Ibid.

V. pumila D C. - Champs cultivés. - Ain et Rh. : la Pape, St-Alban, Villeurbanne, Oullins. - H.-Alp. : St-Mens au Collet, Tallard à Villevieille.-Dr. : St-Vallier, Loriol, Crest.-Ard. : Baix. - Vaucl., B.-du-Rh. : envir. d'Orange, d'Avignon, d'Apt, d'Aix, d'Arles et de Marseille. - Var et Alp.-Mar. : Toulon, le Luc, Fréjus, Grasse, Nice. - Gard : Nimes, le Vigan, Anduze. IIér. : envir. de Montpellier, de Lodève et de Béziers. - Aude : envir. de Narbonne.-Pyr.-Or. : St-Paul, Fosse, vallée de l’Agly à Ansignan.

V. echinata D C. - Champs sablonneux de la région mérid. H.-Alp. el Dr. : Gap, St-Mens, Rosans, Crest.-Vaucl. et B.-duRh. : Orange, Avignon, Morières, envir. de Marscille et d'Aix. - Var et Alp.-Mar.: Toulon, le Luc, Fréjus, Grasse, Nice, Levens, Gilette, Revest, Touet-du-Benil. - Gard : Villeneuve d'Avignon, Nìmes, le Vigan. - Hér. : envir. de IIontpellier et de Béziers. - Aude : Trèves, Villanière. - Pyr.-0r. : Céret, plaine St-Georges, vallons de Prades et de Villefranche, St-Vincent, St-Antoine-de-Galamus, vallons de Cornella du Conflent et de Vernet-les-Bains. 
v. puberula D C. - Terrains sabl, de la région mérid. - Var et Alp.-Mar. : Vidauban, le Luc, les Mayons, Grasse, l'Esterel, le Biot, Antibes. - Gard : Mas-Donzel près de la route de St-Gilles.

V. microearpa Lois. - Coteaux secs, - Ain : la Pape, les Échets.

- Is. : Seyssuel près Vienne, la Tronche, Vars, Comboire. Vaucl. et B.-du-Rh. : Fontaine de Vaucluse, Montaud-les-Miramas. - Var et Alp.-Mar. : Toulon, Antibes, Grasse. - Gard : Aigues-Mortes. - Hérault.

V. Morisomi̊ D C. - Champs cultivés. - Jura : envir. de Dóle, vallée de la Loue, Salins. - C.-d’Or : St-Remy. - Envir. de Genève; Vaud et Valais. - Is. : Seyssins, St-Nizier, Villard-deLans, Uriage, Vaulnaveys, Theys, Chasse. - Dr. : St-Vallier. H.-Alp. : col Bayard. - Alp.-Mar. : St-Martin-Lantosque, la Caille près St-Auban. - Gard : le Vigan, Alzon, Arphy, Aumessas. - Hẻr. : La Moure près Montpellier, Pardailhan.

V. truneata D C. - Champs cultivés de la région mérid. - B.•duRh. : Marseille, Marignane, l'Uminy, Martigues, la Mède. Var et Alp.-Mar.: Toulon, le Luc, Ampus, Antibes, Nice, Monaco, Menton.

V. exioenapa Desv. - Moissons, vignes. - Ain : Belley, Parves, Trévoux, Cordon. - Rh. : Lyon au Point-du-Jour. - Is. : Vernas près Cre̊mieu. - Ard. : Vals. - Vaucl. : envir. d’Orange. Var et Alp.-Mar. : Toulon, le Luc, Fréjus, Gourdon. - Commun dans Gard, Hér. et Aude. - Pyr.-Or. : vallée de Céret, Prades, Cornella-du-Conflent et Vernet-les-Bains.

v. coronata D C. - Moissons. - H.-Alp. : Gap à St-Mens, la Garde, Ange-Roubaud, Veynes, Briançon, Ribiers. - B.-Alp. et Dr.: Sisteron, Crest, Barnave, le Buis. - Ard : Vals. Vaucl. : Tour-d'Espagne, Montfavet. - B.-du-Rh. : envir. d'Aix et de Marseille.- Var et Alp.-Mar. : le Luc, Antibes, St-Vallier, Tende. - Gard : Nîmes, Aigues-llortes, Alais, Anduze, St-Ambroix, le Vigan. - Hér. - Aude : Pech-de-l'Agnèle, Aussières. - Pyr.-0r. : Força-Réal, Pezilla, Caladroy.

v. discoidea Lois. - Champs cultivés de la rógion mérid. Vaucl. et B.-du-Rh. : envir. d'Avignon, de Carpentras, d'Aix et de Marseille. - Var et Alp.-Mar. :Toulon, le Luc, Fréjus, 
Antibes, Nice, torrent de Carei, cap Martin près Menton. Gard: Nimes, Aigues-Morles, Alais, Anduze, St-Ambroix, le Vigan. - IIér. - Aude : Narbonne, mont Alaric. -- Pyr.-Or. : Céret, Banyuls-dels-Aspres, Tresserres, le Monastir, Fourques, St-Antoine de-Galamus.

V. vesiearia Mrench. - Coteaux de N.-D. de la Garde à Marseille. Pyr.-Or. : vallée du Ríart.

Fasia cornucopàa Gertn. - Cullivé el quelipucfois subspontané ì Toulon, Hyères, île de Porquerolle, Nice,

\section{DIPSACEES.}

DIPSACUS sylvestris Mill. - Bords des chemins dans tout le bassin.

D. lacinialus L. - Diffère du précédent par ses feuilles moyennes incisées-pinnatifides, ciliées de poils raides et largement connées.

D. fullowum Mill. - Cultivé pour les fabriques de drap; quelque. fois subspontané.

b. pilosas L. - Bords des chemins. - Doubs et Jura : Pontarlier, la Cluse, rẻgion du Vignoble de Salins, Arbois, Lons-le-Saunier. - C.-d'Or : la Colombière, Talant, Gevrey, Verrey, Vielverges. S.-et-L. - Valais : Martigny, Folatères, Sion. - Ain : Thoiry, Argis, Serrières, plaine d'Ambronay, Abbaye de Salignat, Genouilleux, Chazey-sur-Ain, Belley, Trévoux, Reyrieu, Néron. - H.-Sav. : Evian, Thonon, Reignier, Pringy, Faverges, Tamié, Pontchy. - Envir. de Genève, Veyrier, Vernier, St-Genis. Rh. : Pommiers, Liergues, Chırvinges, Gleizé, rives de l'Ardière et de la Turdine. - Sav. : Moutiers. - Is. : Sassenage, de Gieres à Uriage, Grande Chartreuse, Mens, Monestier-de-Clermont. - Alp.-Mar. : St-Dalmas, val Pesio.

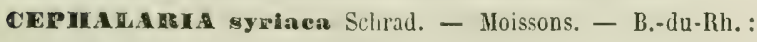
Marseille à Ste-Marthe', Château-Gombert, Roquefavour, les Milles, Berre. - Gard : Blauzac près Anduze. - Hér. : Montpellier.

C. transylvaniea Schrad, - Champs. - B.du-Rh. et Alp.-Mar. : 
Toulon, Grasse, Cannes, Antibes, Menton au cap Martin, Sospel.

C. alpina Schrad. - Prairies des montagnes. - Colombier-de-Gex, la Faucille, la Dóle, chalet des Platières, Creux-de-Pranciaux près du Reculet. - Vaud : Alpes d'Aigle et de Bex, Gryon, les Mortaix, St-Cergues, prairies du Baule. - H.-Sav. : Abbaye de Vallon, Combe-Noire près Tamié, le Reposoir. - Sav.: mont Trélod en Bauges. - Is. : Grande Clıartreuse, col de l'Arc, St-Guillaume, col de la Croix-Haute. - H.-Alp. : la Grangette, Loubet, les Baux, Crotte de Berthaud entre Rabou et Chaudun. - Alp.-Mar. : la Briga, Carlin, Tende. - Pyr.-Or. : mont Louis, Canigou. - Pyr. centr. au Pic Long.

C. Ieucanthan Schrad.- Lieux pierreux des terr. calc. de la région mérid. - Commun dans Vaucl. et B.-du-Rh. - Var et Alp.Mar. : entre Hyères et Carqueiranne, le Luc, Fréjus, l'Esterel, Grasse, Nice, Menton jusqu’à Tende. - H.-Alp. : Charance, la Garde, Collet de St-Jlens, Roche-des-Arnauds, Serres, Treschatel, N.-D. du Laus, Tallard. - Dr.: Valence, Tain, St-Vallier, Crest, Châtillon-en-Diois, Verclause, Montélimar, Donzère, Nyons. - Ard. : Châteaubourg, Crussol, la Voulte, Baix, Cruas, Rochemaure, l'Echelette d'Aubenas, Combier, Privas. - Gard : Beaucaire, Nîmes, Alais, Anduze, St-Ambroix, Je Vigan. Hér. : commun autour de Montpellier, Béziers, Lodève, St-Pons. - Aude : envir. de Narbonne, Montolieu, Durban. - Pyr.0r.: vallée de l'Agly, Cases-de-Pena, Estagel, St-Antoine-de-Galamus, vallée de la Tèt, Prades, Villefranche, Trencada d'Ambulla, vallée du Tech, Céret, Arles, Collioures, Port-Vendres. - En dehors de nos limites sur les terr. calc. de la Lozère, du Tarn, du Lot et de l'Aveyron.

KNAUT - B.-Alp. : Gréoulx. - Vaucl. et B.-du-Rh. : envir. d'Avignon, d'Orange, d'Apt, d'Aix et de Marseille, Marignane. - Var et Alp.-Mar. : cap Brun près Toulon, Plan d'Aups, Roquebrune, Fréjus, Cannes, Antibes, Menton, Estagel.- Gard : Villeneuved'Avignon, Nimes, le Vigan, Anduze. - Commun dans l'Aude et les Pyr.-Or. - On trouve à la Ste-Baume une variété integrifolia à feuilles entières. 
K. arveusis Koch. - Champs cultivés dans tout le bassin.

K. diposacifolia Ilost. - Feuilles larges et fortement nervićes; K. sylvatica Dub. forme à feuilles plus étroites et très-velues.Bois des montagnes. - Commun dans la chaine des Vosges, zone vosgienne de la II.S. et de l'arrondissement de Belfort; dans la chaine jurassique et jusque dans le Bugey el le Revermont. - S.-et-L. : bois de Canada et Paurret près Autun. Vaud et Valais. - H.-Sav. : montagnes du Chablais et du Faucigny, Voirons, Salève, la Roche, le Semnoz, St-Germain-surTalloires. - Sav. : envir. d'Aix, Mouxy, Trevignin, les Bauges, montagnes de Beaufort. - Is. : Grande Chartreuse, St-Eynard, mont Rachais. - H.-Alp. : Lautaret, envir. de Gap, mont Séuse.

- B.-Alp. : montagnes du bassin de l'Ubaye. - Alp.-Mar. - Gard: l'Espérou au Valat de la Dauphine, à Dourbie et à Rocavie. - Hẻr. : la Salvetat, Fraisse, St-Geniès-de-Varensal, Andabre-Rosis, St-Martin-d'Orb, Lunas, Hérépian. - Aude et 'Tarn : Montagne Noire sur les hauteurs de Nore et de Dresson.Pyr.-0r. : mont Louis, Salvanère, Boucheville. - En dehors de nos limites dans les Pyr. centrales, l'Aubrac, le Cantal, la Lozère, les monts Dore et Domes, le Forez.

K. puberula Jord.- Feuilles supérieures incisées-pinnatifides.

K. cuspidala Jord. - Feuilles supér. longuement attenuées en pointe.

- Is. : Fourvoirie, St-Ange, dans les bois au-dessous du col de l'Arc. - Dr. : Bas-Bouvante. - Aude et Tarn : la Montagne Noire.

K. longifolia W. Kit.-Forme à feuilles plus allongées, entières, étroitement lancéolées. - Lieux tourbeux dans les Vosges, le Jura autour de Pontarlier et de St-Laurent-en-Grandvaux. - H.-Sav. : mont Charvin, Cornettes de Bise. - Loire: Pilat, Pierre-surHaute, Ermitage de Noirétable. - Ard. : Entraigues. - Pyr.Or. : bords de la Rigarda, Glorianes.

K. Timeroyi Jord. - Collines pierreuses. - Is. : Crémieu, Morestel. - Ain : envir. de Belley, St-Benoit: - Aude et Pyr.-Or. : bois de Salvanère et de Boucheville, vallon de la Boulsane.

K. mollis Jord, - Collines des H.-Alp. : Briançon, Sapet de la Bàtie-Neuve, col Bayard, col de Glaise, Devez de Rabou, bois Mondet. - Alp.-Mar. : St-Vallier, col de Fenestre. 
K. collina Req. - Bois et champs incultes de la rỏgion mérid. Vaucl. et B.-du-Rh. : envir. d'Avignon, Orange, Apt, Aix et Marseille. - Var et Alp.-Mar. : Toulon, le Luc, Riaris, le Mas près St-Auban, Tourrette, Levens, Guillaumes, Entraunes, val de Jallorgues, St-Martin-Lantosque. - Gard: Chartreuse de Valbonne, St-Michel, la Bruyère, Tresques, Serre-de-Bouquet Vacjuières. - Pyr.-Or. : Trencada d'Ambulla et le long de la Tet jusqu'à 0lette. - Remonte dans les B.-Alp. vers Gréoulx, Sisteron, Castellanne; dans les H.-Alp. vers Laragne, la Garde, Charance.

K. subeanescens Jord. - Prairies des montagnes. - Is. : StAnge, Alpe du mont de Lans. - H.-Alp. : Lautaret, mont Séuse. - Sav. : col de la Louze.

K. carpophylax Jord. - Prairies des montagnes. - Is. : mont de Lans. - H.-Alp. : Villard d'Arène, Lautaret, rochers de la Combe du Queyras, col de Glaise. - Alp.-Mar.: mont Lachen.

SCARTOSA graminifolia L. - Rocailles calcaires. - Is. :

Gresse au pied du Grand-Veymont, la Guichardière près Mens. - Dr. : Luz-la-Croix-Haute, col des Tourrettes. - H.-Alp. : la Chalp, col de Glaise, Côte Gélive, la Grangette, Chaudun, Rabou, mont Bayard, mont Aurouse. - B.-Alp. : vallée de l'Ubaye à la Condamine, Tournoux, Rochailles de Meyronnes, Barcelonnette. - Alp.-Mar. : Alpes de Carlin, Lupega, col de Ray entre Venanson et la forêt de Clans. - Gard: Villeneuve-d'Avignon. Pyr.-Or.: St-Marsal en montant par la vallée de Valmanya.

s. stellata L. - Lieux pierreux. - B.-Alp. : Gréoulx, Sisteron. Vaucl. et Gard : Avignon, Bonpas, sur la colline près Coupine, chemin des Angles, Villeneuve. - B.-du-Rh. : Aix au chemin de St-André, envir. de Marseille eŁ d'Arles.-Var et Alp.-Mar. : Toulon, le Luc, Rians, Puget près Frẻjus, Grasse, Nice. Hér. : St-Jean-de-Védas, Murvieil, Puéchabon, Béziers.- Pyr.Or. : Collioure, ravin de Consolation.

Espèce polymorphe à laquelle se rattachent les formes suivantes:

S. brevicoma Jord. Fourr. - Béziers. - S. lobata J. F. - Toulon. - S. bipinnata J. F. - Toulon, Marseille. 
s. uerseniea L. - Champs calc. des Pyr.-Or.: Castelnau, Corbère d'Amont, Corbère d'Avail.

S. marltima L. - Bords des chemins de la région mérid.; remonte jusque dans la partie mérid. de la Drôme vers Montélimar.

S. columbaria L. - Espèce excessivement polymorphe, commune sur les collines et sur les montagnes.

S. ochroleuca L. - Variété à fleurs jaunes trouvée accidentellement à Toulon où on ne l'a pas revue depuis longtemps. - Aude et Pyr.-Or. : Montolieu, vallons de Prades, de Molitg et de Mosset. S. pratensis Jord. - Soies du calice quatre fois plus grandes que la couronne de l'involucelle, commuae dans tout le bassin.

S. affinis G. G., S. spreta Jord. - Capitules globuleux, arêtes du calice courtes, pédoncules très-longs et divariqués. - C.-d'0r : Plombières. - S.-et-L. : Dezize. - H.-Alp. : Guillestre. Vaucl. : bois du mont Ventoux. - Pyr.-Or, et Aude : bois de Salvanère et de Boucheville.

S. pulens Jord. - Capitules petits, arêtes du calice dépassant trois fois la couronne de l'involucelle, pédoncules grêles et étalés. - Ain et Rh. : Vassieu, Balan, Montluel, Belley, mont Tout. - Envir. do Genève, bassin du Léman. - Hér. : massif montagneux entre la Sérane et l'Espinouse, de Ganges à la Salvetat.

S. tenuisecta Jord. - Nîmes, Uzès.

S. brigantinca Jord. - If-Alp. : Névache, les Roux près Abriès. Sav. : Albertville.

S. sericea Jord. - Pied du mont Cenis en descendant du côté de Suze. S. suntcolens Desf. - Feuilles radic. et caul. entières, soies du calico une fois et demie plus grandes que la couronne.-Coteaux secs. - Ain : Aleximieu au Mont et aux Biolères, Chazey-sur-Ain. Is. : Balmes viennoises, Décines, Crẻmieu. - Dr. : Romans. Pyr.-0r. : plaine d'Aspres.

S. gramuntia L. - Soies du calice extrêmement courtes. - Coteaux secs. - B.Alp. : Sisteron, Laragne. - Dr. : Montélimar. Vaucl. : collines des envir. d'Orange, bois de Fargues près Avignon, Flassan. - B.-du-Rh. : Aix au vallon du Coq, envir. de Marseille, St-Loup, l'Estaque. - Gard: Nìmes, Tresques, Manduel, Comps, Alais, le Vigan. - Hér. : envir. de Montpel- 
lier, de Béziers, de St-Pons. - Pyr.-0r. : vallon de Vinç̧a, Rigarda, Perpignan, St-Antoine-de-Galamus, Mont Louis, Trencada-d'Ambulla, Collioures, Port-Vendres, Banyuls-sur-Mer.

La var. pyrenaica All., S. candicans Jord a toutes ses parties recouvertes d'un duvet blanc-tomenteux. - Alp.-MIar. : depuis Tende jusqu'à Menton, Nice, Vence, Grasse, Aiglun, St-MliartinLantosque, col de Fenestre, St-Etienne.

S. lucida Vill.- Tiges courtes à feuilles rapprochées, feuilles glabres, luisantes, soies élargies à la base et marquées d'une nervure saillante. - Pâturages des montagnes. - Région alpestre de la chaîne jurassique, la Dỏle, Faucille, Colombier, Reculet; descend jusqu'à St-Claude, Pradon, le Colombier-du-Bugey. Vaud et Valais. - H.-Sav. : montagnes de Faucigny et du Chablais Morzine, plateau des Gêts, Dents d'Oche, Cornettes de Bise, mont Chalune, les Platets, Pormenaz, col de Voza, mont Joly, mont Tournette, col des Aravis, mont Charvin. - Sav. : le Bonhomme, Bourg-St-Haurice, montagnes des Allues, la Maurienne, mont Cenis, montagnes de Beaufort. - Is. : Chame chaude, Grand Som, Charmant Som, Belledonne vers le lac du Crouzet,mont de Lans, St-Christophe-en-0isans, la Salette.H.-Alp. : Lautaret, Chaillol, Champoléon, Orsières, près de Chabrières, col de Ilanse et de Bayard. - B.-Alp. : toutes les montagnes du bassin de l'Ubaye, - Alp.-Mar. : col Bertrand. Pyr.-Or. : de Castel au Canigou.-En dehors de nos limites dans les Vosges au Hohneck, au mont Dore, au Cantal et dans les Pyr. centrales.

Une variété glabrescens Jord. se trouve à la Grande Chartreuse, au Colombier-du-Bugey et en Sav, au mont Drizon. - Diffère du S. lucida par les soies non élargies et non nerviées.

Une variété veslita Jord. tomenteuse-blanchàtre, dont les grandes corolles purpurines ont le tube pubescent, se trouve au Mont-Cenis, à Abriès en Queyras et à Colmars dans la vallée du Verdon, à la montée du mont Monnier.

Une variété alpcstris Jord. à capitules plus larges que longs se trouve dans les prairies du Collet et de la Charmette près de la Grande-Chartreuse ainsi qu'au mont Rachais. - H.-Alp. : 
Bayardon près Gap, mont Séuse. - H.-Sav. : montagnes du Chablais, mont IIermante, Dents d'Oche, mont Chalune.- Var : mont Faron, le Luc.

S. suceisa L. - Lieux humides. - Espèce polymorphe comprenant les formes suivantes:

S. platyphylla J. F. - Francheville (Rh.).

S. pubescens J. F. - Tassin (Rh.).

S. angustula J. F. - Francheville (Rh.).

S. brevis J. F. - Myonnay, les Echets(Ain).

S. syluatica J. F. - Francheville (Bh.).

S. viretorum J. F. - Sathonnay (Ain).

S. sabauda J.F. - Evian (HI.-Sav.).

S. dentata J. F. - Saint-Héand (Loire).

S. beugesiaca J, F. - Hauteville (Ain).

S. incisa J. F. - Villeurbanne (Rh.).

S. stricta J. F. - Cornod (Ain).
S. prativaga J, F, - Quincieux (Rh.).

S. procera J. F. - Mollard de Décines (Is.).

S. rhodenensis J. F. - Montluel (Ain).

S. lotevirens J. F. - Granne (Dr.).

S. tardans J. F. - Loriol (Dr.).

S. glabrata J. F. - Vernet-lesBains (Pyr.-0r.).

S. microphylla J. F. - Monestierde-Briançon (H.-Alp.).

S. parvula J. F. - Vallée d'Eyne (Pyr.-0r.).

S. subacaulis Bernardin. - Montcarra (Is.).

S. australis Wulf. - Envir. de Montluel à Niepvre, la Saulsaie (Ain). 


\section{SYNANTHÉRÉES}

\section{CORYMBIFÈRES.}

EUPATORIUM cannabinam L. - Lieux humides dans tout bassin.

ADENostrues albifroms Rchb. - Forêts humides. Commun dans la chaine des Vosges et la zone vosgienne de la H.-S. - Région des sapins de la chaine jurassique, Bugey, Savoie, Dauphiné, B.-Alp. et Alp.-Mar. - Vaud et Valais. Loire : Pilat, Bourg-Argental, Pierre-sur-Haute, Noirétable. Montagnes du Vivarais, Mezenc, Gerbier-des-Joncs, Mezilhac, Cévennes - Hêr. : l'Espinouse à Lignères, le Soulier à la Blanque.- Aude et Tarn : Montagne Noire. - Pyr.-Or. - En dehors de nos limites dans les Pyr. centr., l'Aubrac, le Tarn, la Lozère, l'Aigual, la Margeride, Cantal, mont Dore, monts Dômes, Forez.

A. Alpima Bl. et Fing.- Lieux humides des forêts dans les montanes.- Nul dans les Vosges, le centre de la France et les Cévennes.- Commun dans la rígion aipestre de la chaîne jurassique jusque dans le Haut-Bugey. - Alpes de Vaud, Valais, Savoie, Dauphiné, B.-Alp., Alp.-Mar. - Vaucl.: mont Ventoux. Pyr.-0r. : vallée d'Eyne en Capcir, bords de l'Aude entre Fontrabiouse et le col d'Arès, Costa Bona.

A. Leucophyin Rchb. - Débris rocheux. - Nul dans les Vosges, la chaine jurassique, le centre de la France, les Cévennes et les Pyr. - Valais: St-Bernard au Mont Mort, Dronaz, glacier de Proz, Arolla, Findolen, Rothorn, Gorner, Zmutt, Grimsel, le Breuil sur le versant italien du mont Rose. - H.-Sav. : chaine du Mont Blanc, moraines et Jardin de la Mer de Glace, Aiguilles-Rouges. - Sav. : St-Sorlin d'Arves, - Is. : la Pra de 
Belledonne, bords du lac Crouzct, Taillefer, la Muzelle, la Salette. - II.-Alp.: la Grave, Lautaret, Arcines, mont Viso, St-Yéran, Claaillol-le-Yieil, Champoléon à la montagne de l'ours, mont Chirat. - B.-Alp. : Lauzannier, Bérard. - Alp.-Mar. : Alpes de Nice et de Menton.

A. hybrida D. C. - Forme à feuilles glabrescentes en dessus trouvée au St-Bernard vers le Mont Mlort, vallon de Dronaz, envir. des lacs Ferret. - Is. : Taillefer en allant de Poursollet au lac Noir, Belledonne au-dessous du petit lac Domeinon.

HOMOCXVE alpina Cass. - Pàturages. - Nul dans les Vosges, le massif central et les Cévennes. - Région alpestre de la chaine jurassique jusque sur 'e Crêt de Chalam, Retord et le Colombier du Bugey. - Alpes de Vaud et Valais. - H. -Sav. : Pitons du Salève, Voirons, montagnes du Chablais et du Faucigny, Dents d'Oche, mont Chalune, chalets de Colone, plateau des Gets, Morzine, Mégève, mont Charvin, col des Aravis, Chamonix, Montanvert, col de Balme. - Sav. : montagnes de Beaufort, Hauteluce, Crest-Voland, Tarantaise et Maurienne jusqu'au mont Cenis. - Is. : massif de la Grande Chartreuse, St-Eynard, la Bàtie, Gresse, les Sept-Laux, le Gleyzin.-Dr. : mont Glandasse. - H.-Alp. : la Grave, Lautaret, montagnes du Briançonnais et du Queyras, mont Genèvre, mont Viso, Ségure, Chaillol-le-Vieil, Sapet de la Bâtie-Neuve, mont Queyrel, Obiou.-B.-Alp. : montagnes du bassin de l'Ubaye. - Alp.-Mar. : Alpes de Nice et de Menton. - Pyr.-Or. : Canigou, Carlitte, Bouillouse, Bac de Bolquère, St-Sauveur, vallon de la Preste. - Pyr. centrales.

PETAsites offieinalias lfonch. - Prés humides dans tout le bassin. Espèce polymorphe comprenant:

P. pratensis Jord. - P. riparia Jord. - P. Reuteriana Jord. Celui-ci à Bossey au pied du Salève.

P. albus Gxrtn. -- Bois humides. -- Commun dans les Vosges, la zone vosgienne de la II.-S. et de l'arrondissement de Belfort, vallées du Puix, du Rahin, Ballon de Girosnagny. - Région alpestre du Jura helvétiqque et français, Creux-du-Van, Suchet, mont d'Or, Montendre, Côtes du Doubs et du Dessoul,re, Lomont, Dôle, Reculet jusque dans le Haut-Bugey à Ruffieu, Retord, 
Arvières, en Fiez près Tenay. - Vaud et Valais : Beaulieu près Rolle, le Jorat au-dessus de Rovéréaz, Cheville, Solalex, val Champé, Vercorin.-H.-Sav. : Brizon, Charvin, la Diosaz, mont Vauthier, Fouilly, sources de l'Arveyron. - Sav. : St-Félix, Crest-Voland, St-Nicolas, Flumet. - Is. : Grande-Chartreuse, Chalais, Allevard, Esparon près du Monestier-de-Percy, Monestier de-Clermont.-Dr. : Die à la Pale près l'Abeille.- H-Alp. : Monestier-de-Briançon, Devez-de-Rabou, Charance, Loubet, la Grangette, Combe-Noire. - Var et Alp.-Mar. : Bezaudun, Alpes de Nice et de Menton. - Ard, et H.-L. : Mezenc. - En dehors de nos limites dans la chaîne du Forez à Montoncelle, Pierresur-Haute, mont Dore, Cantal.

P. niveus Baumg.-Bords des ruisseaux dans les hautes montagnes.

- Colombier de Gex, vallée de la Valserine entre Lelex et Chẻzery. - Vaud et Valais : Solalex, Pissevache, St-Bernard à Pradaz. - H.-Sav. : Vergy, le Reposoir, Méri, Sallanches, Sixt, les Fiz, les Platets, Signal des Agneaux, mont des Granges, montagnes de Samoëns. - Sav. : Abìmes de Miyans, montagnes au-dessus d'Aime, les Mottets. - Is. : Chaley, la Terrasse, StNizier, col de l'Arc, Avert près Lalley.- H.-Alp. : entre le Lautaret et la Madeleine, Briançon à la Ribière. - B.-Alp. : la Condamine, ruisseau de Bèrard. - Var et Alp.-Har. : Tende, Alpes de Fenestre. - Pyr.-0r. : Canigou, montée de Prats-de-Mollo à Pla Guillem, la Cerdagne. - Pyr. centrales.

P. frragrans Presl. - Cultivẻ et quelquefois subspontané.

TUSSHLACD farfora L. - Lieux argileux ou marneux dans tout le bassin.

Solidago Virga aurea L. - Bois dans tout le bassin.

S. minuta Vill. - Forme alpestre du précédent à capitules peu nombreux et plus gros. - Sommités de la chaîne jurassique. Valais : Thyon, Arolla, Montagny, Findelen, Rappenthal, Mainghorn. - H.-Sav. : massif du mont Blanc et des AiguillesRouges. - Is. : Grand Som, Chamechaude, St-Nizier, chaines de Belledonne, de Taillefer el de l'Oisans. - Sav. : St-Sorlind'Arves, bas du Mottet au-dessus d'Aime. - H.-Alp. : Lautaret, mont Viso, mont Morgon, Chabrières, Orcières, Chaillol-le- 
Vieil, mont Aurouse. - B.-Alp. : cime de Crouès près de la Condamine. - Vaucl. : mont Ventoux.

S. patulifolia Jord. - $\mathbf{H}_{4}-\lambda l p$. : Briançon au bois des Fontaines. B.-Alp. : Annot. - Vaucl. : mont Ventoux.

S. monticola Jord. - Bois. - Rh. et Loire : St-Bonnet-sur-Montmelas, Pilat, Pierre-sur-Haute.

s. Nthospermifolia Willd. - Bords du Rh. au Pont-St-Esprit, îles du Rh. à Coudoulet.

S. glabra Desf. - Originaire de l'Amér. septentr., naturalisé sur les bords de l'Ain, du Doubs, de la Saône, du Rhone, notamment autour de Lyon et du Pouzin, bords du Gardon d'Anduze.

LINos ats ruIgaris D C. - Coteaux secs. - Nul sur le versant français de la chaîne jurassique; rare du côté de Neufchâtel. - C.-d'Or. : Aloxe, bois de Chaigneaux, Gevrey et toute la Côte jusqu'à St-Aubin et Chassagne.-S.-et-L. : Chagny, Dezize, Chamilly, Tournus.-Ain : entre la Pape et Néron, Belley, Muzin, Pierre-Châtel, (ilandieu.-Valais : les Échelles entre Outre-Rhône et Elex, les Marques, mont d'Orge, Champlan, Tourbillon, Sierre, Briegg. - Is. : Bastille de Grenoble, Comboire, le Dauphin, Crémieu.-Sav. : Salins, St-Jean-de-Maurienne, Pas-du-Roc près St Michel.-H.-Alp. : Guillestre.- Dr. : Laveyron près St-Vallier, Crest, Loriol, Die.-Alp.-Mar. : Venanson, la Paoute près Grasse, Cannes. - B.-du-Rh. : les Alpines, Vernègues. — Var : Hyères, le Luc. - Gard : Chartreuse de Valbonne, St-Michel, Boussargues. - Hér. : Montpellier à Fontcaude, Fontfroide, bois de Valène, St-Martin-de-Londres, Prades, St-Guilhem, Hérépian, Montagnac, Cabrières près Clermont. - Aude : Cenne, Villespy. - Pyr.-0r. : Bac de Bolquère, montagne de Madrès, forêt de Salvanère.

PIMANAYON sordidum D C.- Rochers calcaires de la région mérid. - Ard. : Tournon. - Vaucl. : pied du mont Ventoux, à Flassan, Combe de Canau. - B.-du-Rh. : envir. d'Aix au chemin de Vauvenargues, vallon des Gardes, envir. de Marseille. - Var et Alp.-Mar. : mont Faron, le Luc, littoral entre Nice et Menton. - Gard : Villeneuve d'Avignon, Nîmes, Pontdı-Gard, Uzès, la Roque, le Vigan, St-Hippolyte, Anduze, St- 
Ambroix. - Hér. : Montpellier à Boutonnet, Prades, Pic St-Loup, Montbazin, Montferrand, Mireval, St-Martin-de-Londres, la Roque-Aynier, Ganges, Olargues. - Aude : remparts de Narbonne, roc de Pastouret, Pech de l'Agnèle, ravin de Combemale vers la Clape, Lastour, col de l'Épervier près Tuchan. - Pyr.Or. : fortifications de Perpignan, Trencada d'Ambulia, Roc del Grau près Vernet-les-Bains, Castell, St-Martin-du-Canigou, Molitg, Céret, St-Antoine-de-Galamus.

P. saxatile Cass. - Rochers calcaires. - B.-du-Rh. : Arles, Puy Ste-Réparade, le Bec de l'Aigle à la Ciotat. - Var et Alp.-Har. : Toulon, le Bar, Nice, Villefranche, Monaco, Menton.- Pyr.-0r. : vallée de la Tet à Prades, Villefranche, Trencada d'Ambulla, Olette, Molitg, Céret, Arles, Prats-de-Nollo, Port-Vendres, Banyuls-sur-mer, St-Antoine-de-Galamus,

P. telonense Jord. - Rochers calc., murs. - B.-du-Rh. et Var : Bec de l'Aigle, N.-D. de la Ciotat, Toulon.

CONYza ambigua D C. - Champs et lieux incultes de la région méridionale.

ERIGEHON canadensis L. - Plante originaire de l'Amérique septentr. extraordinairement répandue dans les champs et les lieux incultes de tout le bassin.

E, acris L. - Bords des champs, lieux arides.

E. serotinus Weihe. - Diffère du précédent par ses rameaux presque tous simples, les demi-fleurons plus longs que le disque, la couleur rousse des aigrettes, sa floraison plus tardive.

E. Drabuachensis Mill. - Forme montagnarde de l'E. acris dont il se distingue par ses feuilles plus longues, plus étroites, glabres. centes ou seulement ciliées, tige glabre. - Valais : Randaz. H.-Sav. : vallée de l'Arve et de ses affluents, le Giffre, le torrent de Pralong; entraîné par l'Arve jusqu'au-dessous de Genève, bords de l'Isère près d'Albertville, vallée du Drac et du Champoléon, Chorges (H.-Alp.), vallée de l'Ubaye.

E. Villa rsii Bell. - Prairies et pâturages des montagnes. - Vaud et Valais : la Hausseresse, col de Bonaudon, Alpes de Jaman et de Morcles, Chamossaire, Fully, Bagnes, St-Bernard à la Pierraz, Albrun, val Binn, Zermatt, Offenen, Saas. - H.-Sav. : 
vallie du Reposoir, Chamonix, Pormenaz, cols de Balme et du Bonhomme, Cornettes de Bise. - Sav. : montagnes au-dessus d'Aime, entre la Thuile et Laval de Tignes, entre la Fougère et la Louze, col de la Madeleine, mont Cenis, mont Trélod en Bauges. - Is. : Alpe du mont de Lans, mont Chamoux, St-Christrophe el la Bérarde en Oisans, Taillefer. - II.-AIp. : Lautaret, Briançon, Guillestre, mont Viso, col de Vars, Chabrières, Chaillol-le-Vieil, Pleyne-de-Chaudun, mont Sćuse. - B.-Alp. : Lauzannier, Tournoux, Bouzolières, Longet, Colmars.-Alp.-Nar. : col de l'Abisso, lac d'Entrecoulpes, col de Fenestre, Bonnet des Trois-Evêrues. - Nul dans les Vosges, le Jura, le centre de la France.

E. apinus L. - Pelouses des montagnes, - Nul dans les Vosges. Chaine du Jura helvétique et français depuis Ie Creux-du-Van, le Montendre, le Colombier, la Dole jusqu'au Reculet. - Vaud et Valais: Dent de Jaman, Alpes de Bex, d'Aigle et des Ormonds, Chamossaire, Javernaz, Boulaire, mont Fully, St-Bernard, mont Nuoble, Saas, Gemmi, Grimsel, Furca. - H.-Sav. : Salève aux Pitons, mont Brizon, Charvin, Tournette, Morzine, Sixt, Cornettes de Bise, Dents d'Oche, mont des Granges, Roc d'Enfer, mont Hermante, col Joly, chaine du mont Blanc depuis le col de Balme jusqu’áu Bonhomme; s'élève au Jardin de la Mer de Glace. - Sav. : montagnes de Beaufort, d'Hauteluce et de CrestVoland, bas du Mottet au-dessus d'Aime, Saut des Allues, le Chapieu, col de la Louze, du col Iseran à Tignes, mont Cenis. - Is. : Grand Som, Charmant Som, Chameciaude, la Moucherotte, col de l'Arc, petite Houcherolle, la Salette, chaînes de Belledonne, des Sept-Laux et de l'Oisans. - H.-Alp. : la Grave, Lautaret, Briançonnais, Queyras, mont Viso, col de Vars, la Bătie-Neuve, Chabrières, col du Noyer, Orcières, Durbonnas, Granon, mont Séuse, col de Glaise, mont Bayard. - B.-Alp. : Lauzannier, Malmore, Fouillouse. - Alp.-Mar. : montagnes de Nice et de Menton. - Vaucl. : mont Ventoux. - Pyr.-0r. : Cambres d'Aze, Nohẻdes, Carença, Canigou, vallẻe d’Eyne, Pla Guilhein, Costa Bona. - En dehors de nos limites dans les Pyr. centr., mont Dore. 
E. glabratus Hoppe. - Pelouses des montagnes.-Diffère du précédent par les fleurons femelles tous ligulés et par les feuilles glabres. - Chaine jurassique à la Faucille, Colombier, Reculet, Colombier-du-Bugey, Chartreuse de Portes. - H.-Sav.: Brizon, Vergy, chaine du mont Blanc, la Mer de Glace. - Is. : Chamechaude, col de la Ruchère, col Vert et col de l'Arc, Taillefer.-H.-Alp. : glaciers d'Ale-Froide, mont Viso, Chabrières, col du Noyer, Sapet de la Bâtie-Neuve. - Sav.: mont Drizon, montagnes d'Orizan.

E. uniforus L. - Pelouses des Alpes surtout dans les terrains siliceux. - Nul dans les Vosges, la chaine jurassique, le massif central et les Cévennes. - Vaud et Valais: Alpes de Bex et d'Aigle, Lavaraz, Boulaire, Javernaz, Rosseleinaz, Fully, St-Bernard, Loze, Cleuson, mont Nuoble, Rothorn de Findelen, Schwarzberg, Guggerhubel, Simplon, Tortain, Furca.- H.-Sav.: Vergy, Méri, Cornettes de Bise, Morzine, Sixt, col Joly, col de Balme, le Buet, le Brévent, chaîne du Mont Blanc au Couvercle, au Jardin et jusqu'aux Grands Mulets, dernière limite de la végétation dans les Alpes françaises. - Sav. : cols du Bonhomme et de la Seigne, la Gitaz près Beaufort, la Vanoise, col Iseran, Saut des Allues, col de la Madeleine, mont Cenis, mont Mirantin, col de la Bâthie entre Arbine et Arèches, Croix-dePierre, mont du Chat, mont Nivolet. - Is. : Champrousse, Taillefer, Tête-de-Racha au mont de Lans. - H.-Alp. : Galibier, Cervières, mont Viso, Chabrières. - Pyr.-Or. : vallée de Llo.

STENACHS ammua Nees. - Plante originaire du Canada. Vaud: Devens près Bex, Vevey, Payerne. - Is. : alluvions de l'Isère et du Drac, Polygone de Grenoble, Gières, Pont-de-Claix, Vizille. - Vaucl. : envir. d'Avignon, bords du canal de l'Hopital, entre Mondevergues et la prise d'eau.

ASTER alpinus L. - Pelouses des montagnes. - Nul dans les Vosges. - Chaine du Jura helvétique et français depuis le Creux-du-Van jusqu'au Reculet. - Alpes de Vaud et Valais. H.-Sav. : Brizon, Môle, Vergy, Méri, montagnes du Chablais et du Faucigny, Roc d'Enfer, mont des Granges, mont Ardin, 
Morzine, col de Balme, mont Lachat, mont d'Etale, la Tournette, Semmoz, mont Charvin, col du Bonhomme. - Sav. : mont Trélod, Ilauteluce, Crest-Voland, les Avanchers, mont Cenis. Is. : Belledonne, Champrousse, Grande-Chartreuse, Grand Som, Charmant Som, Chamechaude, col de l'Arc, la Moucherolle, mont Sénèpe, la Salette. - H.-Alp. : Lautaret, mont Viso, col de Vars, Chabrières, le Sapet de la Bâtie-Neuve, le Noyer, Durbonnas, Orcières, col de Glaise, col Bayard, mont Séuse. Dr. : mont Glandasse, Aucelon.-B.-Alp. : Parpaillon, Bẻrard, la Condamine, lac Praroard, Reisolle près St-Pierre. - Alp.Mar. : montagnes de Nice et de Menton. - Gard : Capellier sur la route du Vigan, St-Jean-de-Bruel. - Pyr.-Or. : vallées d'Eyne et de Llo, Cambres d'Aze, Canigou, Costa Bona, Port-de-Pallères en Llaurenti. - En dehors de nos limites dans les Pyr. centr.; les causses de la Lozère autour de Mende, la Vabre, St-Privat, Florac, Causse Méjean; les montagnes des envir. de Millau, de Séverac, de Cornus et de Tournemire dans l'Aveyron; le mont Dore.

A. Amellus L. - Coteaux secs surtout dans les terr. calc.; - les deux versants de la chaîne jurassique, Cotes du Doubs, St-Ursanne, Mandeure, de Beaucourt à Dampierre, d'Allanjoie à Bourogne, Ornans, Vauxenans près St-Amour, Marigna près Arinthod, Thoirette, pied de la Dôle et du Reculet à Trélex, le Bugey, le Revermont. - C.-d'Or : coteaux calc. depuis Larrey jusqu'à Meursault, Is-sur-Tille, Messigny. - S.-et-L. : coteaux calc. de Bussy, Culles, Cluny. - Vaud et Valais : la Cóte, Ollon, les Marques, la Morge, Sierre, Chatroz. - H.-Sav. : base des Dents d'Oche, pied du Salève, Sallenoves, Cercier, Challonges. - Envir. de Genève à Sous-Terre, bois des Frères et de la Bâtie. - Sav. : Brides-les-Bains, Salins, Aigueblanche. - Is. : Crémieu, Chamagnieu, le Touvet, Comboire, Vir. - Dr. : les Réoux près St-Vallier, St-Nazaire en Royans. - Envir. de Lyon à Couzon, Oullins, Rochecardon, Ia Pape. - Ard. : Entraigues, vallée d'Ouvèze. - B.-du-Rh. : envir. d'Arles. - Alp.-Mar. : Grasse, Venanson, Tende. - Gard : bois de Salbous près Campestre, Lannuéjols. - Pyr.-Or.: terr. calc. de la montagne de 
Madrès, des vallées de Nohèdes et d'Evol. - En dehors de nos limites sur les calc. de la Lozère, à Ste-Enimie, Causse Hejean; coleaux calc. de l'Aveyron autour de Millau, Beaulieu et Villefranche.

A. Tripolium L.- Marais salants.- B.-du-Rh. : bords des étangs de Berre et de Marignane. - Var et Alf.Mar. : Toulon, Hyères, plage de Giens, Fréjus, St-Raphaël, golfe Jouan, la Napoule, Nice au Var. - Gard : St-Gilles, Bellegarde, Aigues-Mortes. Hér. : Lattes, Gramenet, Pérols, Vic, Maurin, Villeneuve, Frontignan, Cette. - Pyr.-Or. : Port-Vendres, Banyuls-sur-Mer, Salses, la Nouvelle, lle Ste-Lucie.

A. brumalis Nees. - Plante de l'Amérique septentr. depuis longtemps naturalisée sur les les bords du Doubs, de l'Ognon, bois du Deschaux, de Tassenières, de Sergenon.-H.-Saủne: Champagney, bords de la Saône, Franchevelle, Vesoul, Bougey, Lam. brez, Contréglise, Grattery, Chariez.-C.-d'Or. : le long du Muzin entre Nuits et Quincey, Dijon. - S.-et-L. : bords de la Seille à Louhans. - Bords du Rhône autour de Lyon, du Pont-StEsprit, bords des ruisseaux à Aulas (Garl); bords de l'Isère autour de Grenoble; rives de l'Ubaye à la Condamine.

A. serotinus Wild. - Bords des eaux. - Is. : la Bâtie près St-Ismier.Dr. : Aouste près Crest. - B.-Alp. : la Condamine. - Gard : bords du Rhône près Aramon.

A. acris L. - Coteaux pierreux el bois de la région mérid.-Vaucl. : envir. d'Avignon, d'Orange et d'Apt. - B.-du-Rh. : envir. de Marseille, d'Aix à Mauret, Montaud près Salon. - Var et Alp.Mar.: Toulon, le Luc, Aups, Fox-Amphoux, Fréjus, de Nice à Menton. - Ard. : Baix, Vals, Vallon.- Gard : envir. de Nîmes, Pont-du-Gard, Chartreuse de Valiwonne, Aigues-Mortes. - Hér. : Montpellier à Grammont, Castelnau, Castries, St-Guilhem, Portiragne à Roquehaute, St-Martin-de-Londres. - Aude et Pyr.-0r : commun dans les envir. de Narbonne et les trois vallées du Roussillon. - Remonte dans la Dr. vers Nyons, le Buis, Montélimar, Crest; dans les B.-Alp. vers Sisteron; dans les H.-Alp. vers Ribiers, les bords du Buech, la Roche-Tallard.

- Espèce polymorphe. 
A. collinus J. F. - Nyons (Dr.). - A. pinetorum J. F. - St-Rémy, (B.-du-1hı.), - A. venustus J. F, - Jonquières (Gard).

A. trimervis Desf. - Terr. stériles des Pyr.-Or. vers Prades, Trencada d'Ambulla.

A. Saliznus Willd. - Espèce américaine naturalisée près de Pontde-Vaux sur les bords de la Reyssouze (Ain).

BELLIDIste Un Michelih Cass. - Páturages des montagnes. - Nul dans les Vosges, le massif central, les Cóvennes et les Pyrénées. - Sommités du Jura helvẻtique et français jusque dans le Haut-Bugey. - Vaud et Valais : Jorat, Creux-duPélesson près Payerne, cascade de Surpierre, Salvan, Barberine, St-Bernard, Pierre-å-Voir, Comba d'Arbaz, Lens, Vex, Zan, Zermatl, Leukerbad, Mainghorn, Gemmi, Grimsel. - H.-Sav. : Salive, montagnes du Chablais et du Faucigny, Abondance, Bonnevaux, chalets de Colone, Chamonix, la Roche, le Semnoz, la Tournette, Parmelan, Rumilly. - Envir. de Genève au bois de la Bâtie. - Sav. : envir. d'Aix, Mouxy, Trevignin, mont du Chat, montagnes de Beaufort, d'Aime, de St-Jean-de-IIaurienne, mont Cenis. - Is. : Grand Som, Chamechaude, St-Eynard, la Salette. - Dr. : col de Menée. - H.-Alp. : Lautaret, Monestierde-Briançon, col Isoard, mont Viso, St-Véran, Chabrières, col de Glaise, Loubet, la Grangette, mont Séuse. - B.-Alp. : Sérennes, Fouillouse, Rissolle, Seynes.- Région élevée des Alp.-Afar, d'où il descend jusqu'au Chaudan et à Ste-Agnès près Menton,

BELLIS annua L. - Terrains salés de la région littorale. B.-du-Rh. : envir. de Marseille, les Catalans, Endoume, Montredon, Martigues, Marignane. - Var et Alp.-Har. : Toulon, Hyères, ile de Porquerolles, St-Tropez, Fréjus, Cannes, Antibes, Châteauneuf, de Nice à Menton. - Gard : Aigues-IIortes, StGilles, Bellegarde, Sylvéréal. - Hér. : Palavas, Lattes, Gramenet, Cette, Balaruc, Portiragues. - Lilloral de l'Aude et des Pyr.-Or.

B. perennis L. - Prés et bords des chemins dans tout le bassin.

D. mylvestris Cyr. - Prés et pâturages de la région mérid. Vaucl. et B.-du-Rh. : envir. d'Avignon, d'Orange, d'Aix et de Catal. Bassin du Rhơne. 
Marseille, à St-Loup, Cuges. - Var et Alp.-Mar. : Toulon, Hyères, le Luc, Rians, St-Tropez, Frêjus, Cannes, de Nice à Menton. - Gard : Nìmes, St-Gilles, Tresques, Pont-du Gard, le Vigan. - Hér. - Pyr.-Or. : Port-Vendres, Banyuls-sur-Mer.

DoronYCUM plıntagineum L. - Lieux ombragés. - Gard: bords du Gardon à St-Nicolas, Pont-du-Gard, l'Espérou, envir. de Brama-Bioou. - Pyr.-Or. : au-dessus de la Borde-Girvès, vers le Pla dels Abellans, Bac de Bolquère, Llaurenti.

D. Pardaltanehes Willd. - Bois des montagnes. - Vosges. H.-S. : Nans, Uselles, Goudenans-les-Moulins. - Doubs et Jura : bois de Pont-les-Houlins près Baume-les-Dames, de Chatay à Uzelle, bois de Château près Salins. - Ain : envir. de Belley au Lit-du-Roi et au Mollard de Lavour, Châtillon-de-Michaille.C.-d'Or et S.-et-L. : Saulieu, Gevrey, Cluny, Cuiseaux. - Vaud : Lausanne, Belmont, Pandex, Rovéréaz, les Planards. - H.-Sav. : Salève près de la Fontaine de J. César en haut du Pas-del'Échelle, au-dessus d'Archamp, entre Cologny et Frontenex, Vougy près Bonneville, le Cré-du-Naure près Annecy, les Bauges. - Is. : mont Rachais, Pariset, St-Nizier, St-Barthélemyde-Séchilienne, Gavet, Peyre près Mens, Crémieu. - H.-Alp. : Boscodon, mont Séuse aux Fays. - B.-Alp. : mont des Dourbes près Digne. - Ard. et H.-L. : mont Combier, le Mézayon, Cuze, Mezenc- - Loire : Bourg-Argental, Pierre-sur-Haute, St-Georges en Couzan. - Var:Gonfaron, Cannet à St-Daumas. - Gard : bois de Salbous et de l'Espérou. - Hér. : Ganges, la Sérane, St-Pons, Olargues, Andabre-Rosis, St-Etienne-de-Mursan, Avène, Bédarieux. - Pyr.-Or. : Mont Louis, vallée d'Eyne, mont de Madrès, vallées de Castell, de Fillols et de Céret. Aude et Tarn : Montagne Noire. - En dehors de nos limites dans les Pyr. centr., le mont Dore, Aubrac, mont Dòme, Cantal, Creuse, Lozère, Forez.

D. austriacum Jacq. - Bois et bords des ruisseaux des terrains siliceux. - Nul dans les Vosges, les Alpes et la chaine jurassique. - C.-d'Or : terr. granit. du Morvan autour de Saulieu, Roche-en-Brenil près l'étang de Tournesac, prés de la Fontaine, moulin Morin, bois du Saubiot, St-Léger. - S.-et-L. : Morvan 
autunois, bois des Grandes-Queues à Roussillon, bois de Corcelle, Anost, St-Prix. - Rh. et L. : Roche d'Ajoux en Beaujolais, Pilat, Pierre-sur-IIaute, Noirétable au Pic-Pelé et à l'Ermitage. Ard. et II.-L. : Mezenc, Mazan, la Viole. - Gard : la chalne de l'Espérou, bois de Longues-Feuilies près Concoule. - Hẻr. : l'Espinouse à la Salvetat et à Salvergues. - Pyr.-Or. : valléo d'Eyne, Bac de Bolquère, Carença, Canigou, Prats-de-Mollo, la Preste, Cambres d'Aze, Llaurenti. - En dehors de nos limites sur les terrains granitiques des Pyr. centr., du Tarn, de la Creuse et du Forez; sur les gneiss, granites et rochers volcaniques de l'Aubrac, Cantal, mont Dore, monts Dómes.

ARoNicUM Doronicum Rchb. - Débris de rochers. - Valais : mont Vélan, Thyon, Esserz, Arolla, Ferpecloz, Anniviers à la Lex et à l'Arpettaz, Zermatt au Schwarzsee et à Findelen, Saas, Distelalp, Blasenhorn, Gatzenhorn, Glacier du Rhone.-H.-Sav.: chaine du mont Blanc, Jardin de la Mer de Glace, col d'Anterne, le Buet. - H.Alp. : mont Viso à la Traversette, au col Agnel, col Vieux, Ségure, St-Véran.- B.-Alp. : Lauzannier, Sérennes. - Alp.-Nar.: Tende, col de l'Abisso et de Fenestre, envir. du lac d'Entrecoulpes, Estenc. - Pyr.-0r. : vallées d'Eyne et de Llo, Canigou, Bac du Clot d'Estavel, Cambres d'Aze, Bac de Bolquère, Llaurenti.

A. scorploides Koch. - Débris de rochers. - Vaud et Valais: Alpes d'Aigle et de Bex, Boulaire, Javernaz, Chamosaire, val d'Illiez, St-Bernard, Alliaz de Bagnes, Bellalui, col de Fenétre, Donin, Méribé, Blasenhorn, Loetschen, Mainghorn, Gemmi. H.-Sav. : chaine du mont Blanc, cols de Balme et du Bonhomme, Brévent, col d'Anterne, le Buet, les Platets, col Joly, mont Charvin, Tournette, Semnoz, Dents d'Oche, Signal des Agneaux, Roc d'Enfer, Vergy, Méri, glacière du Brizon. - Sav. : les Mottets, col des Fours, la Vanoise, St-Sorlin-d'Arves. - Is. : Grand Som, Petit Som, Haut-du-Seuil, col de l'Arc, la Moucherolle, la Pra de Belledonne, Champrousse, le Gleyzin, Taillefer, la Salette. - II.Aip. : Lautaret, Galibier, mont Viso, col de Ruines, Blanchet de St-Véran, mont Morgon, Chabrières, Chaillol-le-Vieil, Alp-IIartin, mont Obiou, la Grangette, mont Au- 
rouse. - B.-Alp. : Lauzannier, Plate-Lombardo, Parpaillon, Bẻrard, Longet. - Vaucl. : mont Ventoux. - Alp.-Mar. : col de Raus, de Tende et de Fremamorte, Garret et val Strop audessus d'Entraunes. - Pyr.-Or.: Borde-Girvès près mont Louis, Pla dels Abellans, sur la rive gauche de la Têt, vallée de $\mathrm{Ca}-$ rença et de Fillols. - Pyr. centr. - Nül dans les. Vosges, la chaine jurassique et les Cévennes.

A. glaciale Rchb. - Diffère du précédent par sa tige pleine. - Valais : Blasenhorn, Griess. - Alp.-Mar. : le Garret au-dessus d'Entraunes.

Arnica montana L. - Prés et pâturages des terr. siliceux. Espèce caractéristique des granites, eurites et grès de la chaîne des Vosges depuis Wissembourg jusqu'à la vallée de Giromagny. Zone vosgienne de H.-S. à Franchevelle, St-Germain, Linexert, Bas de Mélisey, Servance, Fresse, Champagney, St-Bresson, Belfahy, Ballon de Servance, Planche des Belles Filles, ChâteauLambert. - Rare dans la chaine jurassique et seulement sur les terrains siliceux de transport, les sables sidérolithiques ou les lieux à sous-sol tourbeux superposés à des argiles glaciaires, sommet de la montagne de Boudry en allant vers le chalet du Creux-du-Van, entre le Grand et le Petit-Beauregard près du Chasseron, prés tourbeux du Vély et de Mazières près Hauteville en Bugey, Retord. - Is. : rare dans le massif de la Grande Chartreuse, sur un lambeau de grès vert au col de la Ruchère et à la Charmette, forêt de Portes, gneiss de Prémol, Champrousse, la Grande Lance, Gleyzin, la Morte, prés tourbeux du mont de Lans. - H.-Alp. : Lautaret, col Bayard sur les sables et argiles glaciaires, gneiss et granites du mont Viso, mont Loppet, Orcières, Chaillol-le-Vieil, grès nummulitiques de Chabrières, mont Obiou dans les prés de la Postelle. H.-Sav. : chaine du mont Blanc, col de Balme, Jardin de la Mer de Glace, col Joly, le Brévent, sables sidérolithiques du Semnoz et du Salève au-dessus de Cruseilles, grès nummulitiques de la glacière du Brizon, des Planets au pied du Vergy, col des Aravis, mont Charvin, la Tournette. - Vaud et Valais: Javernaz, Chamosaire, la Forclaz, Catogne, Alpes de Saxon, 
Thyon, Lens, Tortain, Nesselap, Schornen, Simplon, Binnerthal, glacier du Rh. - Sav. : les Allues, mont Cenis, CrestVoland. - B.-Alp. : Lauzannier, Parpaillon, Bérard, Longet.Alp.-Nar. : Roccabiglière. - C'est à tort que l'A. montana a été indiqué dans les B.-du-Rh. à la Ste-Victoire. - C.-d'Or et S.et-L. : terr. granit. du Morvan, Saulieu, Roche-en-Brenil, Anost; se trouve aussi dans le Morvan nivernais à Ouroux, Mlontsauche, Planchet. - Rh. et Loire : montagnes du Beaujolais et du Pilat, Planfoy, St-Bonnet-le-Château, Fierre-surHaute, Noirélable. - Ard. ct H.-L. : Mezenc, Mazan, N.-D. des Neiges. - Gard : chaine de l'Espérou, Concoule. - Hér. : le Soulier à la Blanque, Fraisse, le Saumail entre St-Pons et la Salvetat. - Aude et Tarn: granite de la Montagne Noire au roc de Peyramos, Mazamet, la Cabarède, Anglès. - Pyr.-0r. : Pla de Barres près IJont Louis, Borde-Girvès, Pla des Abellans, Canigou. - En dehors de nos limites sur les terr. siliceux des Pyr. centr., de l'Aubrac, Cantal, Lozère, mont Dore, monts Dômes, Forez, Sologne.

L'existence de l'A. montana dans les plaines sablonneuses de la Sologne est un fait de la plus haute importance et montre d'une manière frappante que les influences chimiques l'emportent sur les conditions climatériques. Voilà en effet une espèce montagnarde dont la présence a été constatée sur le territoire de treize communes de la Sologne où elle n'a pas pu étre apportée des montagnes, du moins pendant la période géologique actuelle, depuis que ce pays n'a aucune communication avec les massifs montagneux du centre de la France. La permanence de l'A. montana dans un pays relativement bas, tel que la Sologne, est d'autant plus remarquable que la culture de cette plante échoue le plus souvent dans les jardins botaniques, probablement parce qu'on ne sait pas imiter les conditions physiques d'humidité du sous-sol et les conditions chimiques de composition du terrain superficiel qni conviennent à cette espèce.

SENECIO vuigarls L. - Lieux incultes dans tout le bassin.

s. vimcosus L. - Lieux incultes, bords des chemins depuis les plaines jusqu'aux montagnes.

S. sylvatieus $L_{1}$ - Forêts des terr. siliceux. - Granite et grès des Vosges. - H.-S. : Bougnon, Chariez, Grattery, Champagney, St-Remy, Menoux. - Rare dans ta chaine jurassique sur les 
chailles siliceuses de l'oxfordien; en dehors de la chaine, sur les granites de la forét de la Serre, sur les cailloux quartzeux de la forêt de Chaux, sur les argiles de la Bresse et des Dombes. Vaud : envir. de Payerne, le Jorat, Ginel, bois des Ursins, bois de vétay près Coppet.-H.-Sav. : Orsier.-C.-d'Or, S.-et-L. et Rh. : chaines granitiques du Morvan, du Beaujolais et du Lyonnais, Saulieu, Arnay-le-Duc, Autun, St-Rigaud, St-Bonnet-de-Hontmelas, Limonest, Charbonnières, Pollionnay, St-Bonnet-le-Froid, Izeron, Vaugneray. - Loire et Ard. : route de St-Etienne à Annonay, les Salles, Pierre-sur-Haute, montagnes du Vivarais, Mayres. - Is. : Charvieu, Morestel, Eydoche, Theys. - Gard : la chaîne des Cévennes, notamment à l'Espérou et autour du Vigan. - Hér. : l'Espinouse à la Salvetat et à Fraisse. Aude et Tarn : Montagne Noire. - Pyr.-Or. : Bac de Bolquère, montagne de Madres. - Alp.-Mar. : Estenc au-dessus d'Entraunes: - Commun dans le centre de la France.

s. Ilvidus L. - Lieux incultes de la région mérid. - Var : Hyères, le Luc, les Maures, les Mayons, l'Estérel. — Gard : Aulas, lo Vigan, Grand-Combe près Alais, Palières près Anduze, - Hẻr. : Lamalou, Lunas, Grayssessac, Andabre-Rosis, Pardailhan, la Salvetat, St-Pons, Lodève, Antignac, Pézenas. - Pyr.-0r. : bois de Salvanère et des Fanges, Força-Réal, Millas, vallon de Collioure à Consolation. - Aude : envir. de Narbonne, Fontfroide, gorges de la Dure près Montolieu.

S. leucanthemifollus Poir. - Espèce de Corse trouvée à Toulon, à l'ile de Bandols.

S. crassifollus Willd. - Littoral des B.-du-Rh. et du Var : envir. de Marseille, île de Riou, Berre, Marignane, Toulon, lles de Porquerolles et des Imbiers, rochers des Lions près St-Raphaël. - Aude et Pyr.-Or. : bords de l'ètang de Salses, sur la chaussée qui conduit à Leucate, Grand-Argelès.

5. gallicus Chaix. - Champs et vignes de la rẻgion moyenne et mérid. - Ain : entre Chalamont et St-Nizier-lc-Désert, entre Tenay et les Hópitaux. - Is. : St-Romain près Cre̊mieu, Chasse, Salaize, Vienne, St-Lattier, Comboire. - Rh. : Chaponost, Givors, Violay, bois de Joux. - Dr. : Valence, St-Vallier, Tain, 


\section{$-381-$}

Die, Crest, Nyons. - Ard. : Tournon, la Voulte, le Pouzin et toute la côte du Rhône, vallẻe de l'Ouvèze, Aubenas. - H.Alp. : Gap, Puy-Naure, Ia Saulce, St-Nens, Romette, N.-D. du Laus, Tallard, Remollon, Rosans, Briançon.- Vaucl. et B.-duRh. : envir. d'Orange, de Carpentras, d'Apt, d'Avignon, d'Aix et de Marseille. - Var et Alp.-Mar. : Toulon, le Lue, Gonfaron, Fréjus, Grasse, Venanson, col de Braus, Touët-de-Beuil, Chaudan, Nice, Menton. - Gard. - Hér. : Montpellier à Grammont, Lavalette, la Pompiniane, Palavas, Frontignan, Viols-leFort, St-Guilhem, Ganges, St-Martin-de-Londres, Lamalou. - Aude : Villanière, Sidrière de Leucate. - Pyr.-0r. : envir. de Perpignan, Malloles, Château-Roussillon, Baixas, Cases-dePena, Força-Réal. - En dehors de nos limites dans la Lozère, l'Aveyron.

s. adonidifolius Lois. - Bois des terr. siliceux. - Terrains granitiques du Morvan dans C.-d'Or et arrondissement d'Autun, Saulieu, Arnay, Roche-en-Brenil; chalne granilique du Beaujolais et du Lyonnais, mont St-Rigaud, Torvéon, Charbonnières, Tassin; chalnes granitiques et gneissiques du Pilat et du Vivarais à St-Agrève, Ies Ollières, le Mezayon, Entraigues et jusqu'au Tanargues; terrains granit. et schisteux des Cévennes et de l'Espinouse aulour du Vigan, l'Espérou, Alzon, Concoule, la Salvetat, Fraisse, Grayssessac, St-Pons, St-Amand-de-Hounis. Pyr.-Or.: granite de Mont Louis, vallées de Carol, de Carença, de Llo, de Molitg, de Mosset, de Vernel-les-Bains, St-Martindu-Canigou, forêt de Salvanère, col de Pailhères.- Nul sur les calcaires; dans le département du Jura on ne l'a ru que sur les grès de mont Sous-Vaudrey, entre la Ferté et Tassenière. Son absence dans la partie vosgienne et alpine de notre bassin est d'autant plus digne de remarque que les terrains à base de silicates alumineux et alcalins sont très-répandus dans nos Alpes et dans les Vosges. - Commun sur les terr. siliceux du centre de la France, ainsi que dans l'Aveyron, à l'Aubrac, la Lozère, le Tarn, les Pyr. centrales.

s. aquatleus Huds, - Terrains argileux humides des plaines autour de Dole, la Bresse, Gex, Trévoux, Ste-Croix près Montluel, 
Résilleu près Meximieu. - Is. : Charvieu, Pont-de-Chérui, la Terrasse. - S.-et-L. : bords de la Saone, Millery, Dracy près Autun. - Cà et là dans B.-du-Rh. et Var. - Gard : Montfrin, le Caylar, la Calmette. - Aude et Pyr.-Or. : forêt de Boucheville, vallon d'Arles, ınarais de Salses, 1le Ste-Lucie, bords des étangs de Carença.

S. crraticus Bertol. - Lieux humides. - S.-et-L. : bords de la Saône, envir. d'Autun.- Jura et Ain : plaines autour de Dôle, la Bresse et la Dombes, Montribloud, Ambronay, St-Benoit, Gex. - Is. : Charvieu, Crẻmieu, Morestel.-Rh. : Dardilly. - Hẻr. : envir. de Montpellier, bords du Lez, Lattes, Villeneuve, Mauguio. Alp.-Mar. : Vaugrenier près Antibes, Tende, St-Etienne-leSauvage.

S. Jncobren L. - Très-commun dans les prés de tout le bassin.

S. nemorosus Jord. - Forme sylvestre du prẻcédent dont il diffẻre par les écailles de l'involucre lâches; de plus la plante est bisannuelle et non vivace comme le type Jacoboa.

S. fosculosus Jord. - N'a pas de fleurs ligulées, toutes sont flosculeuses; a été observé dans toute la chaine jurassique jusque dans le Bugey; dans l'Isère autour de Morestel, de Bourgoin et de St-Laurent-du-Pont; autour de Lyon à Tassin; dans la IIte-Sav. et Sav. autour du Salève et dans les montagnes du Chablais, dans les envir. d'Aix et de Chambéry; dans les Jaures vers Pignans, le Luc, Fréjus.

S. eruerefolius L. Bois, haies dans tout le bassin.

s. eaIvescens líoris et de Notaris. - Espèce de l'ile Capraja trouvée près de la Chartreuse de IIontrieux (Var).

s. Cinerarla D C. - Rochers du littoral; remonte dans l'intérieur au pied du mont Ventoux dans le vallon de la Nesque, dans la les envir. d'Apt, aux Baux près Arles, à Digne. - Assez commun d'ailleurs autour de Marseille à Montredon, Bec-de-l'Aigle, ile de Pomègue; autour de Toulon, à l'île "de Porquerolles, à Agay, sur tout le littoral de Nice à Menton; à Agde, Ste-Lucie, Mandirac, Sidrière de Leucate et de Fitou, à Banyuls-sur-Mer, au cap Biar.

s. leucophyllus D C. - Rochers. - Ard. et H.-L. : le Mezenc. 
- Pyr.-Or.: vallée d'Eyne à la Collada de Nuria, Coma de la Grave, vallées de Llo, de Prats-de-Balaguer, Cambres d'Aze, Coma du Tech, Costa-Bona, Canigou.

S. Imeinus L. - Rochers et pelouses des montagnes. - Vaud et Valais: Alpes de Bex, Enzeindaz, Fully, St-Bernard, Alesse, Bagnes, Arolla, Zermatt, Zanrion, Cleuson, Diez, mont Nuoble, Unterrothorn, Langenthal, Niederalp, Binner, Ernergalen, Furca. - H.-Sav : chaine du mont Blanc jusqu'au Jardin, Trèla-Tête, col de Balme, Buet, Morzine. - Sav. : cols du Bonhomme, des Fours et de la Seigne, Petit St-Bernard, mont Cenis. Is. : chaine des Sept-Laux, de Belledonne, des Grandes-Rousses et de l'Oisans. - H.-Alp. : la Grasse, Lautaret, mont Viso, col de Vars, St-Véran, Chaillol-le-Vieil, Orcières, Loppet, mont Queyrel, Alp.-Hartin.-B.-Alp.: Lauzannier, Longet.

S. uniflorus All. - Pâturages. - Valais : Zermalt au Gornergratt, Findelen, Grieskummen, Saas à Distelalp et dans l'Offenthal, Mattmark, Almagel, Sirwolten, Bistinen.

s. cordatus Koch. - Prés. - Vaud et Valais : Bovonnaz, Vourry, val d'Illiez, Morgins, Fee. - H.-Sav. : Haut-de-Lin, Morzine, Vacheresse, abbaye du Vallon, vallée du Reposoir près du chalet du Sommier.

S. paludosus L. Lieux humides, marais. - Plaines du Doubs et du Jura, marais de Saône, envir. de Montbéliard, de Pontarlier et de Dole, bords du Doubs, de la Seille et de la Saône.- C.-d'Or: Magny. - Ain : bords de la Saône, Trévoux, Château-Gaillard, Belley. - Vaud et Valais : bords du Rhône, Vouvry, Chessel. - Envir. de Genéve à Sionnet, Roellebot. - Sav. : bords du lac du Bourget, le Vivier, Aix, Chindrieux. - Is. : Crémieu, Amblérieu, Anthon, Meyzieu, Frontonas, Voreppe, St-Laurentdu-Pont, Domène. - Dr. : St-Vallier. - B.-du-Rh. : la Camargue, en Coustièro. - Gard : Mas de Bourri près le Caylar. Pyr.-Or.: bords des rivières de Carença, d'Eyne, de Llo, de l'Agly.

s. Fuchail Gmel. - Bois. - Commun dans les Vosges, la chaine jurassique jusque dans le Bugey, le Jorvan, Beaujolais, Lyonnais, Pilat, Pierre-sur-Haute, la Savoie, lc Dauphiné, les B.-Al- 
pes jusque dans les Alp.-Mar. - Le Mezenc, Brama-Bioou près Camprieux, bois de Longues-Feuilles prèsConcoule; les Pyr.-Or. - Cantal, H.-Loire, Lozère, Aubrac.

S. Jacquinianus Rchb. - Forme du précédent qui a les feuilles moyennes rétrécies en un large pétiole ailé et amplexicaule. - Hoins rẻpandu que le type.

s. Caeallaster Lam. - Bois et pâturages. - Cette espèce appartient surtout à la Flore du plateau central dans les monts Dore, Dômes, Cantal, Forez, sommet de Pierre-sur-Haute. - Carion prétend l'avoir trouvé à St-Rigaud dans le Beaujolais. M. Lombard l'a vue à Saulieu en Morvan (C.-d'Or).

S. Boria L. Prés humides. - Is. : Crémieu, Pont-de-Chérui, Frontonas, Vaulx-Milieu, la Verpillière, St-Quentin, Morestel, Charette, Villeneuve près St-Jean-de-Bournay, Polygone de Grenoble, Champ, la Motte-les-Bains. - Ain : Serrières - Sav. : le Bourget. - Dr. : Aouste et Saou près Crest. - H.-Alp. : envir. de Gap, Charance, les Serigues, Pleyne de Chaudun, Eymeyères, Chorges. - B.-Alp. : Beauvezer. - Vaucl. : Avignon, Mormoiron. - B.-du-Rh. : la Camargue, la Crau, les Martigues. - Alp.-Mar.: mont Cheiron, vallée de Thorenc. Gard : Aigues-Mortes, Beaucaire, Bellegarde. - Hér. : Montpellier à Lavalette, Lattes, Gramenet, Castelnau, Pérols, Mauguio, St-Narcel. - Aude : envir. de Narbonne, Clape-aut-Rec. Pyr.-Or. : vallée d'Estoher, Llech.

s. Tournefortil Lap. - Pâturages humides. - Pyr.-0r. : vallěes d'Eyne et de Llo, montagne de Madrès, Canigou, Costa Bona, Coma du Tech, Llaurenti, Port de Pallères. - Se trouve aussi dans la vallée de la Bruyante près Mijanès. - Pyr. centrales.

5. Doronicum L. Pâturages des montagnes. Nul dans les Vosges. - Chaine jurassique depuis le Montendre jusqu'au Reculet. Vaud et Valais: Alpes d'Aigle et de Bex, Bovonnaz, val d'Illiez, Trient, mont Fully, Catogne, St-Bernard, Pierre-à-Voir, mont Nuoble, Tortain, Leukerbad, Gemmi, Grimsel.- H.-Sav. : montagnes du Chablais et du Faucigny, Dents d'Oche, Hautigny, mont Ardin, Haut-de-Lin, mont d'Ubine, Nautau, Morzine, Brizon, Semnoz, Tournette, Charvin, col Joly, col de Balme, 
Bochard, Brévent, Buet, Mer-de-Glace du mont Blanc. - Sav. : les Mottets, la Gitaz, petitSt-Bernard, les Allues, les Avanchers et autres montagnes de la Tarantaise, mont Cenis et sommités de la Maurienne. - Is : Grand Som, col de la Ruchère, Chamechaude, col de l'Arc, la Moucherolle, mont de Lans, la Pra de Belledonne, la Salette. - H.-Alp. : Lautaret, Cerviẻres, mont Viso, col de Vars, mont Queyrel, Poligny en Champsaur, mont Bayard, la Grangette, Matacharre, mont Séuse. - B.-Alp. : la Condamine, Lauzannier, Fouillouse, montagnes de Castellanne. - Dr. : mont Embel. - Sommités des Alp.-Mar. - Vaucl. : mont Ventoux. - Gard : bois de la Tessonne près du Vigan et de Montdardier. - Hér. : la Sérane, Madières, les Capouladoux, Abbaye de Villemagne près Villeveyrac, le Larzac. - Pyr-Or. : mont Louis, Bac de Bolquère, vallée de Conat, Font-de-Comps, vallée de Nohèdes, Llaurenti. - En dehors de nos limites dans les Pyr.-Centr., Aubrac, Lozère, Cantal, mont Dore, Forez, Pierre-sur-Haute.

Une forme à plusieurs capitules S. Barrelieri, Gouan se trouve dans les envir. de Barcelonnette à Soleille-Bœuf, vallon du Châtelet, et dans les H.-Alp. à St-Véran.

S. Gerardi G. G. - Diffère du S. Doronicum par les écailles extérieures beaucoup plus courtes que l'involucre.-Var : Toulon, Chartreuse de Mtontrieux. - Alp.-Mar. : montagnes de Caussols ct de Défens. - Gard : Vaqueirolle près Nimes, Serre-de-Bouquet près Uzès, bords du Gardon au Pont-du-Gard, Chartreuse de Valbonne.- Pyr.-0r. : vallons de Prades et de Catllar, vallée de Nohèdes. - En dehors de nos limites à l'Aubrac.

CINERARA campestris D C., S. spathulcefolius D C. Bois et rochers. - Vosges infér. depuis Massevaux jusqu’a Barr. - C.-d'Or : val des Choues, Pothières, Riel-les-Eaux. Vaud : marais d'Orbe et đe St-Cergues, Arzier, Bière, Abbaye de Joux. - Doubs : sources du Dessoubre, Mouthe. - Is. : Lans. - B.-Alp. : Digne. - Ard. : Tain. - Aude et Tarn : MontagneNoire. - Pyr.-0r. : Fontpédrouse, vallẻe de Molitg, Mosset en montant à Jau, Llaurenti. - Pyr. centr., Aubrac.

C. aurnatiaca Hoppe. - Prés et páturages des montagnes. - 
Vaud et Fribourg: Château d’0ex, les Morlais, Praz de Crai. - Mont Cenis. - H.-Alp. : mont Viso, vallon de Ruines, vallon Agnel, St-Véran, Ceillac, chalets de Chalvet au-dessus d'Aiguilles. - B.-Alp. : Fouillouse, Lauzannier, Longet. - Alp.-UIar. : vallée de Caïrns, col de Raus, Tende, forêt de Clans.

C. lanuginosa (Tephroseris lanuginosa Jord.). - H.-Alp. : St-Vẻran à droite de Clausis, Vars, des crêtes de Ségures au lac.-B.-Alp.: envir. de Barcelonnette, Soleille-Bœuf, Bouzollières, Uvernay, Annot au col d'Allouès.

C. fuscata (Tepltros. fuscata Jord.). - Mont Viso, vallon de Ruines, rives du Guil.

C. pyrenaica G. G. - Clairières autour de Mont Louis, bases du Cambres d'Aze. - Pyr. centr.

C. Albixica L. - Prairies. - C.-d'Or : val des Choues, Combe Noire. Pyr.-0r. : le Capcir autour de Puyvalador, Pont de l'Aude, butte de Groseille dans le bois de Salvanère. - En dehors de nos limites, dans l'Aubrac, la Lozère, le Cantal et le Puy-de-Dome.

A BTEMISIA Absinthium L. - Bords des chemins, voisinage des habitations dans les plaines et les montagnes.

A. arborescens L. - Toulon, Hyères, łles d'Hyères.

A. camphorata Vill. - Débris pierreux. - Is. : Bastille de Grenoble. - H.-Alp. : Tallard, Chorges, Charance, Chaudun, N.DD. du Laus, Orcières, Rosans. - B.-Alp. : la Condamine, Rochaille de Meyronnes. - Dr. : Aucelon, Solaure près Die. - Vaucl. : Villes à Nesques. - B.-du-Rh. : envir. d'Aix, de Vauvenargues au Sambuc. - Alp.-Mar. : montagnes de Nice et de Menton. Gard : Serres de Bouquet près Uzès, St-Hippolyte. - Aude : Montolieu. - Pyr.-0r. : Céret, Trencada d'Ambulla, Villefranche, Vernet-les-Bains, Nyer, Olette. - Coteaux calc. du Puyde-Dôme et de la Lozère. - Espèce polymorphe.

Abrotanum pauciflorum Jord. F. - La Grave (H.-Alp.).

A. alpestre J. F.- Ibid.

A. pedunculare J. F. - Guillestre.

A. platylobum J. F. - Rabou près Gap.

A. ambiguum J. F. - Ibid.
A. congestum J. F. - Grenoble.

A. pulverulentum J. F. - Ibid.

A. vhodanicum J. F. - Crussol (1rd.). Loriol (Dr.). A. viridulum J. F. - Grenoble. 
A. virgatum J. F. - Meximiru anx Peupliers, berges du marais du Giron, Charnoz, Brénaz, St-Sorlin, St-Rambert, bords de la riv. d'Ain, Ambronay. - Dr. : Die, Nyons, Montjoyer.- Ard. : Cruas. - Vaucl. : mont Ventoux.

A. incanescens Jord. - Coteaux secs. - II.-Alp. : envir. de Gap, la Garde, Charance, la Roche, Collet de St-Mens, la Tourronde, Ventavon à la Montagne de Faye, Serres. - B.-Alp. : Jauziers au Pas-de-Grégoire, Sisteron. - Var : Toulon, mont de Margès près Aups.-Pyr.-Or. : Cases de Pena, Estagel, Força-Réal, Villefranche.

Les Abrotanum ponticum et Dracunculus sont cultivés dans les jardins et quelquefois subspontanés.

A. Mutellna Vill. - Rochers des hautes montagnes, - Vaud et Valais : Javernaz, Boulaire, Bagnes, St-Bernard, Zermontana, Zanrion, Pierre-à-voir, Loze, Prabé, Cleuzon, Diez, Cotter, Zan, mont Nuoble, Zermatt, Distelalp, Schwarzberg, Eginnenthal, Grimsel.- H.-Sav. : mont Méri à la Cheminée, Vergy, Brévent, Buet, Montanvert, Leschaux, Tré-la-Tête, chaîne des Aiguilles Rouges, Pierre-à-l'Echelle, montagnes de Samoëns et de Sixt, mont Jovet, mont Joly. - Sav. : col de l'Enclave, les Mottets, col Jseran, Saut des Allues, sommités de St-Martin-de-Belleville, Hauteluce, la Gitaz, mont Cenis. - Is. : chaîne de Belledonne à la Grande Lance, Grandes-Rousses, Tète de Racha au mont de Lans, St-Christophe à la Selle.- H.-Alp. : Lautaret, mont Viso, Chaillol-le-Vieil, Orcières, mont Queyrel, Alp-Martin, col de Glaise, mont Aurouse, Obiou. - B.-Alp. : Lauzannier, Bérard, Vallonnet, Longet. - Alp.-Har. : mont Bego, Alpes de Fenestre. - Pyr.-Or. : sommet du Cambres d'Aze, vallée de Llo, Canigou.

A. glacialis L. - Rochers des hautes montagnes. - Valais : StBernard a Baux, Proz et Ardifagoz, col de Fenêtre, Zermontana, Zermatt au Riffel, au Schwarzsee et à Findelen, Simplon, Binn. - H.-Sav. : Montanvert, le Bonhomme et dans l'Allée Blanche sur le versant italien du Mont Blanc. - Sav. : mont Iseran, mont Cenis à Ronches. - H.-Alp. : mont Viso à Ruines, col de Péas, col Agnel, Ségure, Guillestre. - B.-Alp.: St-Ours, crète de Bachasse, Vallonnet, Longet, mont Pelvat. - Alp.-Nar. : 
col Bertrand, mont Bego. - Pyr.-Or.: Cambres d'Aze, Pratsde-Balaguer, Pla Guilhem, vallée de Llo. - Pyr. centr.

A. pedemontana Balbis. A, lanala Willd. - Alp.-Mar. : rochers du mont Ponset du côté de Gordolasca, et au lac d'Entrecoulpes, col de Fenestre, mont Tenibre au-dessus de St-Etienne. - Il est possible qu'on trouve celte espèce sur les montagnes des B.-Alp., car elle existe à la Pra, à peu de distance de la frontière française en descendant du col de Sotron.

A. vulgaris L. - Coleaux incultes, bords des chemins dans tout le bassin. - Espèce polymorphe.

A. Imsipida Vill. - Rochers. - Bois Mondet près Gap, la Grave.

A. spicata Wulf. - Rochers des hautes montagnes. - Vaud et Valais : Javernaz, Boulaire, cols de Menouve et de Fenêtre, Zermontana, Zanrion, Orsera, Diez, Arolla, Zermatt, Leukerbad, Gemmi, Simplon, Griess. - Sav. : cols de la Seigne, du Bonhomme et du mont Iseran, Entre-les-Eaux, Saut-des-Allues, Lancebranlette, mont Cenis à Ronches, Rochemelon en Piémont. - H.-Alp. : Galibier, les Trois-Êvêchẻs, Terre-Noire sur Cervières, mont Viso à Ruines, Ségure, Clıaillol-le-Vieil, Orcières, crêtes au-dessus de Jujau. - B.-Alp. : Vallonnet, Longet, Bérard, Parpaillon. - Alp.-Mar. : col de Fenestre, sommet des Gelas à côté du Clapier. - Pyr.-Or. : mont Louis, Collada de Nuria, vallée d'Eyne, Prats-de-Ilollo. - Pyr. centrales.

A. Villarsih G. G. - Rochers des hautes montagnes. - Is. : chaine de Belledonne à la Grande-Lance, Petite Vaudaine, Taillefer, col de la Muzelle. - H.-Alp. : la Grave, Lautaret, col de l'Echauda et de l'Alp-Martin, Prontic en Valgaudemar, Chaillol-le-Vieil. - Sav. : St-Sorlin-d'Arves. - Pyr. centrales.

A. atrata Lam. - Rochers. - H.-Alp. : près d'Arcine au-dessus de Villard-d'Arène, Lautaret, Puy-Chevalier près Monestier-deBriançon. - Pyr.-Or. : plateau du Coral, Prats-de-Nollo.

A. chamomelifolia Vill. - Débris pierreux des Alpes. - Is. : la Salette. - H.-Alp. : Charance, bois Mondet, pont du Devez de Rabou, bois d'Ufarnet, Combe-Noire, col de Glaise, la Grangette, Séuse. - B.-Alp. : Larche, Maurin, Bouzollières, Sisteron.-Alp.-Mar, : val de Jallorgues, près St-Dalmas-le-Sauvage, Estenc. 
A. suavis Jord. - Rochers des envir. de Vienne et de Givors.

A. nan̊ Gaud. - Pâturages et rochers. - Valais: Findelen, Fee, Schwarzgletcher, Offenen, Langenthal de Binn. - Sav. : col de l'Enclave, vallée du Glacier en allant des Mottets au Bonhomme, l'Enclave au-dessus des Fours.

A. campestris L. - Licux pierreux et arides dans tout le bassin. Espèce très-polymorphe.

Dligosporu* pareulus Jord. F. O. brachyphyllus J. F. - Les Chè- Col de la Seigne (Sav.). res $(\mathrm{Rh}$.).

0. subsericeus J. F. - Villard O. fuscatus J. F. - I bid. d'Arène (H.-Alp.).

O. tenuifolius J. F. - Vienne.

0 . alpinus J. F. - Ibid.

0 . virescens $\mathrm{J} . \mathrm{F}, \mathrm{-}$ Ibid.

0 . argyreus J. F, - Ibid.

0. griseus J. F. - Chasse (IS.).

0. brevicaulis J. F. - Névache (H.-Alp.).

0. collinus J. F. - Nyons (Dr.).

0. pubescens J. F. - Orange.

0. laxatus J. F. - Monestier-deBriançon (H.-Alp.).

o. implexus J. F. - Vernet-lesBains (Pyr.-0r.).

O. orophilus J. F. - La Grave (H.-Alp.).

0. floribundus J. F, - Amélie-lesBains (Pyr.-Or.).

O. monticola J. F. - Ibid.

o. delphinensis J. F, - Bourg d'Oisans (Is.).

O. monspeliensis J. F. - Montpellier.

O. suberectus J. F. - Rossillon (Ain). 0. xylopodus J. F. - Ibid.

0. stenocladus J. F. - Neyron (Ain) $\quad 0$. pyramidatus J. F. - Ibid.

A. volesirea All. - Coteaux calcaires secs et chauds du Bas-Valais à Fully, Saillon, Conthey, mont d'Orge, Valère, les Plâtrières, Longeborgne, Sierre, Leuk.

A. Elutimosa Gay. - Bords des champs de la région mérid. B.-du-Rh. et Var: rives de l'Arc, Toulon, Hyères, Cannet du Luc. - Gard et Hér. : Aigues-Morles, Pérols. - Aude et Fyr.Or. : Ile Ste-Lucie, Leucate, bords de l'étang de Salses.

A. gallen L. - Sables marit. - B.-du-Rh. et Var. : littor. des envir. de Marseille, Mazargues, les Martigues, la Camargue, Toulon, la Seyne, le Ceinturon d'Hyères, St-Tropez. - Alp.Mar. : Cannes, Ile Ste-Harguerite, Antibes. - Gard : Aigues- 
Mortes, St-Gilles, Bellegarde. - Hér. : Maguelonne, Palavas, Cette. - Aude et Pyr.-Or. : Ste-Lucie, la Nouvelle, Port-Vendres.

TANACETU I vulgare L. - Souvent cultivé et parfois naturalisẻ sur les décombres et dans le voisinage des habitations. S.et-L. envir. d'Autun, Monthelon, Antully, Roussillon, Anost. Cà et là dans la II.-S., Doubs, Jura, Ain, Vaud et Valais. Commun dans les saulaies du Rhône au-dessous de Lyon.-- Is. : Vienne, Moirans, Tullins, la Terrasse, le Devoluy, le Champsaur. - Dr. : envir. de Die. - H. et B.-Alp. : envir. de Rosans et de la Condamine. - Ard. : Baix, Darbres, Mauras. - Vaucl.: envir. d'Apt, la Garde,Viens.- Alp.-Mar. : la Napoule, Cannes, Antibes. - Gard : le Vigan, Serre-de-Bouquet, Montpezat, Coudoulet. - Hér. : le Larzac à la Vacquerie, le Caylar, la Sérane au Coulet, St-Etienne-de-Gourgas, les Lavagnes au-dessus de St-Guilhem.

T. annuum L. - Champs cultivés. - Vaucl. et B.-du-Rh. : entre Château-Renaud et le pont de Rognon, Tarascon, Arles, Eyragues. - Var et Alp.-Mar. : la Garde prés Toulon, Gourdon. Gard : Beaucaire, St-Gilles, Bellegarde.-Pyr.-Or. : St-Laurentde-Cerdans, la Manère.

T. Baisamita L. - Cultivé et quelquefois subspontané.

PLAGIUs ageratifolius Lhér.- Pyr.-Or. : vallées de Valmanya et du Tech.

LEUCANTREMUM vuIgare Lam. - Prés, pâturages dans tout le bassin.

L. discoideum All. - Plagius Allionii Lhẻr. - Alp-Nar. : Castillon, Nice, St-Martin du Var, Levens et jusqu'à Tende.

I. pallens D C. - Bois de la région mérid. - Vaucl. et B.-du-Rh : Orange, Uchaux, Sablet, envir. d'Aix, bords de l'Arc, le Tholonet. - Var et Alp.-Mar. : Hyères, le Luc, Montrieux, Châteaudouble, l'Esterel et toute la région littorale jusqu'à Nice et Menton. - Gard : Alais, bains de Fonsanges près du Vigan. Hẻr. : Serre d'Oupia près Olonzac, tout le massif montagneux entre la Sérane et l'Espinouse. - Pyr.-0r. et Aude : basses Corbières, St-Laurent de Cerdans, Costujes, Bouscaillon près 
Durban, Fontlaurier, Fontfroide. - Remonte dans les II.-Alp. vers St-André-de-Rosans, Ventavon, Tallard, Sigoyer, Gap; dans la Dr. vers Sahune, Villeperdrix; dans l'Ard. vers Thueyts, Aubenas.

L. moximum D C. - Prìs et páturages. - H.-Sav. : montagnes du Chablais, les Corncttes, mont de Pelloua, mont Charvin, mont Jovet, Tré-la-Tête, la Grand Pariraz. - Is. : Bastille do Grenoble, Sassenage, St-Ange. - H.-Alp.: Lautaret, Chabrières, col du Noyer, Charance, col de Glaise, mont Séuse. B.-Alp. : la Condamine, Tournoux, Malmore. - Dr. : Rioux près St-Vallier. - Alp.-Nar. : mont Cheiron. - Gard : la Tessonne, Concoule, Anduze.-Pyr.-Or.: Canigou, Font-deComps, Prats de Mallo.

L. atrotum D C. - Prés et pâturages. - Région alpestre de la chaîne jurassiqque du Reculet, à la Dôle et à la Faucille jusque dans le Haut-Bugey. - H.-Sav. : Salève, Haut-de-Lin, chaine des Cornettes, mont Pelloua, mont Charvin, la Cluzaz, Tournette, Parmelan, chaine du mont Blanc, les Contamines, col Joly, Allonzier. - Is. : col de l'Arc, Chamechaude, Champrousse. - H.-Alp. : envir de Gap. - Alp.-Mar. : montagnes de IIenton, Nice, St-Vallier, col de Brouis, Venanson, St-MartinLantosque, St-Dalmas-le-Sauvage. - Gard : la Tessonne, l'Espérou, Anduze. - Aude : Villespy.

L. graminifollum Lam. - Coteaux secs et pierreux des terr. calcaires. - Dr. et Vaucl. : entre Montélimar et Orange. B.-du-Rh. : Ste-Victoire, entre Vauvenargues et le Bec-de-l'Aigle. - Alp.-Mar. : montagnes de Caussols et de Defens au-dessus de Grasse, Gourdon. - Gard : Campestre, Montdardier. - Hér. : Prades, à la source du Lez, la Sérane, St-Guilhem, le Caylar, Pégayrolles-de-1'Escalette, St-Geniès-de-Varensal, Caunelle, Grabels, Madières, St-Mlartin-de-Londres, Viols-le-Fort. Pyr.-Or.: vallon de Prades, Trencada-d'Ambulla, basses Albères jusqu'à Céret.

I. coronopifolium G. G. - Rochers. - Valais: Thyon, Gottier, mont Nuoble, Cotter, Mayenhorn, Tortain, Gemmi, Riffel, Augstelberg, Unterrothorn. - II.-Alp. : Combe de Ségure, val Catal. Bassin du Rhónc. 
Preveyre et la Monta en Queyras, pied de Parachalve près du Guil, col de Vars, St-Vêran à Clausis. - B.-Alp. : Horonaye, Lauzannier, Parpaillon, Longet. - Région montagn. des Alp.Mar. - Pyr. centrales.

C. palmatum Lam. - Débris pierreux. - Ard. : Entraigues, Gerbier-des-Joncs, Neyrac, Aubenas, Mayres._ Gard : St-Jeandu-Gard, l'Espérou au Valat de la Dauphine, Valleraugue, Peyremale. - Hẻr. : Lodève, Castelnau-de-Guers, St-Gervais, le Poujol, Lamalou, Avène, St-Pons, Montagnac. - Aude : MasCabardès, Lastours, la Clape, Montolieu, gorges de la Dure. Pyr.-Or. : Prats-de-Mollo. - En dehors de nos limites dans l'Aveyron, les gorges de la Dourbie et du Lot.

L. corymbosum G. G. - Bois, coteaux. - Jura et Vaud : Salins, Trélex. Envir. de Genève. - Coteaux calc. de C.-d'Or, S.-et-L. - Rh. : le mont d'0r lyonnais. - Is. : coteaux calc. du mont Rachais, St-Eynard, Comboire, Chalais, Grande-Chartreuse, Monestier-de-Clermont. - H.-Alp. : Gap, Rosans, - Dr. StVallier, Barnave, Crest. - B.-du-Rh. et Var : envir. d'Aix, Roquefavour, les Olives, l'Etoile, Ste-Baume, le Luc, Coudon, les Maures. - Alp.-Nar. : Grasse, l'Esterel, Levens, Nice, StAndré, Menton. - Ard. : coteaux calc. de vallée de l'Ouvèze et du Rhöne. - Gard. - Hèr. : calc. du Larzac, Lavalette, Mireval, Sussargues, Fabrègues, Montarnaud. - Pyr.0r. : Trencada d'Ambula.

L. Pourrettii Timb Lagr. - Aude: Cascatel près Narbonne, l'Espinassière, Conques, Montolieu.

L. alpinum Lam. - Débris pierreux des terr. siliceux des montagnes. - Vaud et Valais : Alpes de Morcles, la Tornette dans les Ormonds, Javernaz, Fully, val d'Illiez, St-Bernard, Leukerbad, Simplon, Schornen, Ernegalen, Schinnern, Albrun, Zermatt, Glacier du Rhône. - H.-Sav : grès nummulitiques du Méri, toute la chaine du mont Blanc depuis le col de Balme jusqu'au Bonhomme, s'élève jusqu'au Jardin et aux Grands-Mulets, Brévent et Aiguilles-Rouges, Morzine, Samoëns. - Sav. : col de la Seigne, les Allues, partie supérieure des vallées de l'Arc et de l'Isère, la Levanna, le mont Iseran. - Is. : chaines des Sept- 
Laux, de Belledonne à la Pra et à Champrouse, Taillefer, les Grandes-Rousses, massif du Pelvoux, col de la Muzelle. - II.Alp. : Lautaret, Galibier, Névache, mont Viso, la Taillante, col Agnel, St-Véran, col de Vars, Alp.Martin, Champoléon à la montagne de l'Ours, Orcières, mont Lopet, pic de Chaillol, mont Queyrel, pic de Chabrières, mont Aúrouse. - B.-Alp. : Bachasse, Parpaillon, Lauzannier. - Sommités des Alpes de Nice et de Menton. - Pyr.-0r. Cambre-d'Aze, vallées d'Eyne, de Llo, de Prats-de-Balaguer, col de las Nou-Fonts, Carença, Canigou, Costa-Bona, Coma-du-Tech. - Pyr. centr.

La variété minimum Vill. à feuilles tomenteuses se trouve çà et là mêlée au type.

L. tomentosum G. G. - Cette espèce a êté trouvée, dit-on, à los Basibes au Canigou.

I. porthenlum G. G. - Vieux murs, voisinage des habitations. Présente une variêté flosculosum.

CHRYSANTHEMUM segetum L. - Champs de la région mérid.; remonte jusque dans la partie mérid. de la Drôme; so montre porfois accidentellement dans la région moyenne du bassin.

C. myconla L. - Champs de la région mérid., dans la Provence, les Alp.-Mar., et dans la région littorale du Roussillon.

PINA ador A coronarla Less. - Champs, bords des chemins. Var et Alp. Mar. : Toulon sur les glacis du fort Malbousquet Ile St-Honorat, entre Agay et le cap Roux, Antibes, Nice, Roquebrune. - Pyr.-Or, : Bellegarde, Perthus, Prats-de-Mollo, Tourde-llir.

MaTricaria Chamomilla L. - Champs sablonneux. - Jura et Ain : forêt granitique de la Serre, cailloux siliceux el argiles tertiaires de la forêt de Chaux, Thoissey, Vancia, Rillieu, terr. granit. de C.-d'Or et S.-et-L.. - H.-SaÔne : Chariez, St-Rémy, Echavanne.-Région granit. du Lyonnais à Francheville, Tassin, Charbonnières. - Sables molassiques des envir. de Genève et des parties voisines de Ia Savoic. - Envir. de Grenoble, Gap, Crest. - Terr. sablonn. des envir. de Toulon à St-Mandrier, Rians, Abbaye du Thoronet, les Maures et l'Esterel. - Gard, Pyr.-Or. 
M. Inodora L. Champs cultivés dans la plus grande partie du bassin.

ANTHEMIS nobnis L. - Pelouses et bois des terr. siliceux. Jura et Ain : terr. argilo-siliceux des plaines, la Bresse, les Dom. bes. - Terr. granit. du Morvan autunois et de la G.-d'Or. Région granit. du Lyonnais.- Sables de Montaud-les Miramas, Orgon, St-Paul-de-Durance, les Maures, Béziers.

A. mixta L. Champs de la région mérid.-Var et Alp.-Mar.: Toulon, île de Porquerolles, le Luc, Fréjus, Cannes, golfe Jouan, Nice. — Gard: Aigues-Illortes, St-Gilles, Bellegarde. - Hér. : Grammont, Castelnau, Pérols, Mauguio. - Aude et Pyr.-Or. : envir. de Narbonne, de Prades, Villefranche, Olette, vallée du Réart.

A. Puseata Brot. - Champs des environs de Toulon, la Garde, le Luc, le Cannet.

A. arvemsis L. Champs dans tout le bassin. Espèce polymorphe.

La var. incrassata Bois., à pédoncules épaissis ct fistuleux est assez commune dans le Var, et les Alp.-Mar. à Toulon, Hyères, Rians, le Luc, Nice; dans le Gard à Nimes, St-Gilles, Bellegarde, Aigues-Ilortes; dans l'Hér. à Grammont, St-Jean-deVédas, Caunelle, Pẻrols, Roquehaute; dans les envir. de Narbonne, de Perpignan et de St-Antoine-de-Galamus.

A. cotula L. - Champs dans tout le bassin.

A. secundirsomea Biv.- Champs. - B.-du-Rh. et Var : Marseille, Montredon, les Goudes, Fos-les-Martigues, le Luc.

A. marltima L. - Sables marit. - B.-du-Rh. et Var : Marseille, lle Ratonneau, étangs de Berre et de Marignane, les Martigues, Fos, Stes-Mfaries, Toulon. — Littoral du Gard, de l'Hérault, de l'Aude et des Pyr.-Or.

A, montana L. - A. collina Jord. - Collines des terr. siliceux de la région mérid. - Vaucl. : Vaucluse. - Var et Alp.-Mar. : Hyères, le Luc, Pignans, les Maures, la Sauvette, Esterel, Roquebrune, bois de Gourdon, yallées de Fenestre et de Salèze, la Briga, Tende. - Gard : l'Aigual, Hort-de-Diou, Bonpérier, entre la Salle et Valleraugue. - Hér. : col Ste-Colombe entre Olonzac et St-Pons, St-Amand au Roc d'Orques, le Caroux, Pardailhan. - Pyr.-Or. : mont Louis, vallées d'Eynes et de 
Vernet-les-Bains, St-llartin-du-Canigou, vallée de l'Agly, forêt de Boucheville, Collioure, les Albères, Céret, Prats-de-Mollo.Loire : terr. de gneiss et de granite, Malleval, Lupé, Maclas, Grand-Croix, la République, St-Julien-Molin-Molette, dans la vallée de la Loire au Pertuiset, St-Victor, St-Rambert, St-Just. - Ard. : mêmes terr. dans le Vivarais.

A. Gerardiuna Jord. - Se distingue du précédent par les folioles de l'involucre pâles, scarieuses, non noirâtres sur les bords, l'involucre est ombiliqué à la base; on le trouve dans les Maures du Cannet et à St-Tropez.

A. altissıma L. - Champs de la région mérid. - Vaucl. et B.-duRh.: entre Védẻne et St-Saturnin, Orange, Mliramas, Marseille. - Var et Alp.-Mar. : Toulon, Hyères, le Luc, Fréjus, Grasse, St-Vallier, Vaugrenier près Antibes, Lantosque, Nice. — Gard: commun dans les environs de Nimes, Alais. - Hêr. : Montpellier à la Colombière, Fontcaude, St-Aunès, Doscares, Fabrègues, St-Brès, Maguelonne, St-Martin-de-Londres, Lésignan-la-Cèbe.-Aude et Pyr.-Or. : envir. de Narbonne, Sidrière de Fitou, envir. de Perpignan, la Salanque, remonte dans la valléo de la Tet jusqu'à Olette, vallées du Tech et de l'Agly. On trouve aussi cette espèce dans la Dr. à la Vacherie près Embel, Barnave, Pommerol, entre Crest et Chabrillant et jusque dans l'Is. à la Richardière près Chichiliane.

A. tinctorin L. - Coteaux secs. - Rh. et Is. : Ste-Colombe, Ampuis, Vienne, St-Maurice-d'Exiles. - Dr. : Valence, Tain, St-Vallier. - Loire: Chavanay, Malleval, Lupé, Pelussin. Ard. : Tournon, Celles, la Voulte et toute la côte du Rhone. - Vaucl. : bords du Rhone, Orange.-Alp.-Mar. : Briga, Tende, Saorgio, Roccabigliera, Ifas près St-Auban. — Gard : Iles du Rh. à Valabrègue. - Hér. : Pézenas. - Pyr.-Or. : Baixas, Cases-dePena, Trencada d'Ambulla, Sauto, vallée du Réart. - Une variété discoideum All. a les fleurs toutes tubuleuses.

A. TriumphetI All. - Bois des montagnes, - Var et Alp.-Nar. : Ampus, entre la Gilette et le Revest, Lupega, Tanarello.-Hér. : Avène-les-Bains. - Aude : Sarrat de Salabert, près Tuchan. - Pyr.-0r. : envir. de Perpignan, remonte la vallée du Tech jusqu'à Prats-de-Mollo et celle de la Tet jusqu'à Olette. 
ANACYCLUS clnvatus Pers. - Bords des bois, lieux incultes. Toulon au fort Malbouquet, Ampus. - Gard et Hér. - Aude : vallon du Ripau; Ste-Lucie, envir. de Narbonne.

A. radiatus Lois. - Bords des champs. - Vaucl. et B.-du-Rh. : Mornas, Arles, Istres, Montaud-les-Miramas. - Var et Alp.Mar. : Sablettes de Toulon, Frôjus, St-Raphaël, Cannes, ile Ste-Marguerite, Nice. - Gard et Hér. : Aigues-Mortes, Grammont, Pérols, Cette, Mauguio, Marsillargues, Mèze.-Pyr.-Or. : vallées du Tech et de la Tet.

A. valentinus $L_{\text {. }}$ - Bords des rivières des Pyr.-Or., vallée de la Têt de Perpignan à Prades, vallée du Tech, le Boulou, Céret, vallée de l'Agly, Risevaltes.

Drotis candidismima Desf. - Sables mar. - Littoral du Var et des Alp.-Mar. : St-Aigou, presqu'ile de Giens, St-Tropez, Cannes, Antibes, St-Laurent-du-Var. - Hér. : Palavas, Maguelonne, Agde, Portiragnes en face de Roquehaute. - Aude et Pyr.-Or. : Argelès, Collioure, Banyuls-sur-Mer, lle Ste-Lucie.

santourna Chamoeyparissus L. - Coteaux calcaires. B.-Alp. : Sisteron, Forcalquier, bords du Verdon. - Vaucl. et B.-du-Rh. : Avignon, Mornas, envir. de Marseille, Ste-Victoire, les Milles, la Trévaresse. - Var et Alp.-Mar. : le Luc, Flassans, St-Tropez, la Roquette, Nice, Menton. - Gard : Nimes, Beaucaire, Uzès. - Hér. : Nissan, Béziers, Servian, St-Chinian, Puysserguier, entre Mèze et Montagnac. - Aude : Conques, Durban, vallon du Ripau, Pech de l'Agnèle. - Pyr.-Or. : N.-D. de Pena, bords de l'Agly, environs de Salses, vallée du Tech, envir. d'Arles et de Montferrer.

La variété incana Lam. à feuilles et rameaux couverts d'un tomentum blanc se trouve dans le Var aux envir. de Toulon au mont Faron, Baou de Quatre-Heures, Nans, Ste-Baume, la Roquebrussanne; dans Vaucl. autour d'Orange et d'Apt, au Luberon.

La variété squarrosa D C. à rameaux peu velus, d'un vert blanchâtre, à feuilles caulinaires très-brièvement pétiolées se trouve aussi dans les envir. de Toulon au cap Brun et dans les localités de l'Hérault citées plus haut. 
La variété viridis Willd. dont les feuilles ont des dents aiguẻs et mucronées se trouve sur les bords du canal du Midi et autour de Salses et de Sigean dans les Pyr.-Or.

S. rigidula J. F. - Aude : Ville- S. provincialis J. F. - Pyr.-0r. : neuve, Durban. Vingrau.

S. Benthamiana J. F. - Aude: S. intricata J. F. - Pyr.-Or.: le Tausch.

Vingro, Costo-Lounguo.

S. pectinata Lag. - Coteaux des Pyr.-Or. à Prats-de-Mollo, Roc de las Abellas, Costujes, Montferrer, Cortsavi, Ermitage de St-Anyol.

A CIILLEA tomentosn L. - Lieux incultes. - Valais: Fully, Branson, iles de Conthey, Longeborgne, Sierre, St-Pierre, Vex, Visp, val d'Hérens, Natersberg, Briegg, Ausserbinn. - Sav. : envir. de Moutiers. - Is. et H.-Alp. : Champ près Vizille, entre Bourg d'Oisans et Venosc, le Ghampsaur à Chabottes et Pont-duFossé, Rousset près Remollon.- Dr. et B.-Alp.: Tain, Sisteron, Annot. - Vaucl. et B.-du-Rh. : Orange, Pernes, Vaucluse, les Barbiers près Apt, Bedoin, Aix vers St-Pons, envir. de Marseille. Var.: Pilon de la Ste-Baume, Bagnols, Mourières, Nans. Alp.-Mar.: Grasse, St-Vallier, Caussols, Touët-de-Beuil, Clans, Utelle, Nice. - Ard. : Chomérac, Rochemaure.-Gard : Nimes, Beaucaire, bords du Gardon près du Mas Charlot, St-Ambroix. - Pyr.-Or. : Trencada d'Ambulla, la Preste.

A. oilorata L. - Bords des chemins. - H.-Alp. : Guillestre au Champ du Pin, entre Baratier et St-André d'Embrun, plaine de Lachaud près Gap, les Baux, Chaudun, St-André-de-Rosans, Montjai, Chanousse, Lazer, Ventavon. - B.-Alp. : Sisteron. Dr. : Beaufort, Crest, Grignan. - Vaucl. et B.-du-Rh. : envir. d'Avignon, d'Orange, d'Aix et de Marseille. - Var et Alp.-Mar. : Toulon, Ste-Baume, entre Ampus et le Verdon, Mourières, Drap, Contes, Berre. - Gard : Nimes, le Vigan, Alzon. - Commun dans l'Hérault et l'Aude. - Pyr.-Or. : Prades, Trencada d'Ambulla et toute la vallée de la Tet jusqu'à Olette et Fontpédrouse, vallées de Nohèdes et de Molitg.

A. MHlefollum L. - Prés, champs dans tout le bassin. Espèce polymorphe.

Variêté setacea Koch. - Valais : Branson, Charrat, Saxon, 
mont d'Orge, Tourbillon, Visp, montée du Simplon. - B.-duRh. et Var : Ste-Victoire, Nans, N.-D. des Anges près Pignans, Fréjus. - Gard : envir. de Nimes à Uzès, Broussan, Tresques. - Pyr.-0r.: Cases de Pena.

A. alpina Parlat. - St-Bernard entre l'aqueduc et le lac.

A. compneta Lam.- Prairies. - H.-Alp. : Lautaret, le Briançonnais, le Queyras, Chaudun, Laragne. - Is. : mont de Lans. B.-Alp. : mont Monnier, Meyronnes, Larche, Tournoux, Sẻrennes. - Vaucl. et B--du-Rh. : Orange, Gigondas, Salon.

A. tamneetifolia All. - Prairies des montagnes. - Valais : Simplon au Schwischberg. - Sav. : la Gitaz entre Beaufort et le Bonhomme, vallée des Allues entre le Glacier de Gebrulaz et le chalet du Saut, mont Cenis. - H.-Alp. : Lautaret, mont Viso à Ruines, Pra-Michel, Ségure, Ristolas, Boscodon, mont Séuse. - B.-Alp. : Lauzannier. - Alp.-Mar. : vallon du Cavallé audessus de St-Martin, Ste-Anne-de-Vinaï. - Gard : Alzon. Pyr.-Or. : bois de Salvanère et de Boucheville, la Manère, Pratsde-Hollo à N.-D. du Coral.

A. dentifera D C. - Diffère du précédent par les feuilles moyennes et supér. à rachis ailế et denté. - Is. : mont de Lans, Grande Vaudaine. - Sav. : la Gitaz, mont Cenis. - H.-Alp. : Lautaret, mont Viso, col de Vars, mont Horgon, Chabrières près Chorges, Molines près St-Bonnet. - B.-Alp. : Lauzannier, Grange-Commune, mont Monnier.

A. nobılıs L. - Débris de rochers, bords des chemins. - Belfort à la Citadelle, la Miotte, la Justice. - Valais : Martigny, Branson, Fully, Saxon, Sion, St-Léonard, sur Naters, entre Visp et Stalden. - Sav. : de Feissons à Aigueblanche, Moutiers, Brides, Bourg St-Maurice, St-Jean-de-Maurienne. - Is. : Ornon, de Bourg d'Oisans à la Grave, mont de Lans. - H.-Alp. : Gap à St-Mens, Tallard, Guillestre. - Dr. : Grane, Loriol. - Vaucl. : envir. d'Avignon, mont Ventoux. - B.-du-Rh. et Var : Orgon, St-Paul-de-Durance, Colle-Noire, mont Faron, Hyères, - Alp.Mar. : Menton, Tende. - Gard : Nimes, Beaucaire, la Roque, Alais, Anduze, St-Ambroix. - Pyr.-0r. : St-Antoine-de-Galamus, Prades, vallée de la Tet jusqu'à Mont-Louis, Vernet-les-Bains, 
Casteli, Cinnigou, vallée du T'ech, Céret, Arles, Prats-de-Mollo. - En dehors de nos limites sur les causses do la Lozère à Meyrueis, Sauveterre, Causse Méjean.

A. IIgustien All.- Haies, coteaux, - Var et Alp.-Mar. : Toulon, la Crau d'Ilyères, mont de Leuse, mont Gros, entre Nice et la Turbie.-C'est à tort que Mutel indique cette espece en Oisans.

A. chrmomelifolia Pourr. - Rochers des Pyr.-Or. : Trencada d'Ambulla, Grau d'Olette, Vernet-les-Bains, vallée de Nohèdes, Font-de-Comps, vallée du Tech, Céret jusqu'à Prats-de-Hollo, vallée de Llo.

A. Agerstum L. - Terrains incultes. - H.-Alp. : entre Laragne et Ventavon. - Vaucl. : bords du Rhône et de la Durance, Orange, Courthézon, Pécoulette, Vacqueyras, Rustrel, Flassan. B.-du-Rh. et Var : Marseille, Rognac, Berre, Entressen, Aix à Fenouillères, Bompart, bords de la Jougne, Hyères, le Luc, le Cannet, Vidauban.-Alp.-Mar. : Grasse, Antibes, Nice, Menton. Gard: Nìmes, St-Gilles, Aramon, Bellegarde, Anduze. - Hér. : commun dans la plaine, plus rare dans la région montagn. à Lamalou, Pégayrolles-de-l'Escálette. - Aude et Pyr.-0r. : les Corbières.

A. Ptnimiea L. - Prés humides dans tout le bassin. - Espèce polymorphe.

A. valesiaca Sut. - Prés de Vaud et Valais à Bovonnaz, Unterwassern de Conches, Scliwibbogen. - Dans celte forme les feuilles sont poilues, à rachis largement ailé; les segments de feuilles sont cunéiformes à la base.

A. pyrenaica Sibth.- Pâturages.- Gard : les Lopies, le Prunaret près de Dourbie. - Pyr.-0r. : au fond de la vallée d'Eynes. - Cette espèce se trouve aussi à Quérigut dans l'Ariège et dans les Pyr. centr. à Castanèse, vallée de Gistain. - Se distingue de l'A. Plarmicu par sa pubescence et ses feuilles ponctuées-excavées, non luisantes.

A. Kerbarota All. - Rochers. - H.Alp. : mont Viso, depuis le rocher de Côte-Ronde jusqu'au col de la Traversette, col de Ruines. - Sav. : mont Iseran au-dessus des granges de la Lenta, sources de l'Arc au pied de la Levanna, la Galise, mont de 
Fréjus. - Alp.-Nar. : Tende, bois du Borćon au-dessus de StMartin, Ste-Anne-de-Vinaï, lac de Vens près St-Etienne, Estenc, Cette espèce se trouve en Piémont dans les Alpes de Vinadio, Valderia, Vieusonne, Ceresole, Lanzo, Giave, Fenestrelle.

A. macrophylla L. - Bois des Alpes. - Vaud et Valais : Chamosaire, Derborence, Corbassières de Bagnes, St-Bernard, Trient au bois Magnin, Anniviers, Staffel de Zermatt, Eginnenthal, Glacier du Rhoóne, Grimsel. - H.-Sav. : vallée du Reposoir, Méri, Haut-de-Lin, Vacheresse, mont de Pelloua, inont des Granges, Nautau au-dessus de Montriond, Signal des Agneaux, Morzine, plateau des Gets, Sixt à la Vauzalle et à la Combe, Chamonix au bois de Taconnaz et de Montanvert, les Contamines, Brévent, col de Balme. - Sav. : Beaufort, Hauteluce, le Bonhomme, les Allues, Lans-le-Bourg, mont Cenis. - ls. : entre Revel et le lac du Crouzet, Prémol, le Gleyzin près Allevard, Valjouffrey au pied du mont Chamoux, la Moucherolle. - Dr. : col de Menée, Luz-la-Croix-Haute. - H.-Alp. : Lautaret, mont Viso à Pra Michel, Valgaudemar au Petit-Clot, Durbon, mont Morgon, Sapet de la Bâtie-Neuve, Loubet, Pleyne-de-Chaudun. - B.-Alp. : la Condamine, le Lauzannier. - Alp.-Mar. : Tende, bois du Boréon au-dessus de St-MIartin, Ste-Anne-de-Vinaï.

A. nnnn L. - Rochers et pâturages des montagnes. - Vaud et Valais : Alpes d'Aigle et de Bex, Fully, Alesse, Zermontana, St-Bernard, Cleuson, Arolla, Diez, mont Nuoble, Zermatt, Saas, Tortain, Simplon, Glacier du Rhone, Furca, Grimsel. - H.-Sav. : Morzine, montagnes de Sixt et de Samoëns, les Gets, chatne du mont Blanc, le Tacul, Leschaux et le Couvercle. - Sav. : cols du Bonhomme, des Fours, de la Seigne et de l'Enclave, Lavalde-Tignes, mont Iseran, la Vanoise, Saut-des-Allues, mont Cenis. - Is. : chaine de Belledonne à la Grande-Lance et vers le petit Domeinon, la Citre au-dessus de Ste-Agnès, Taillefer. H.-Alp. : la Grave à Puy.Vacher, Lautaret, Galibier, Goléon, Frêjus au-dessus de Monestier-de-Briançon, col de l'Alp-Martin, mont Viso, col de Ruines, col Agnel, St-Véran, pic de Chaillolle-Vieil, Orcières, mont Lopet. - B.-Alp. : sommités des montagnes du bassin de l'Ubaye. - Alp.-Jar. : col de Fenestre, 
de Jallorgues et de la Maddalena, le Jarret. - Pyr.-Or. : le Canigou, sommités entre Costa Bona, Carença et Nuria, la Fosse du Géant, Coma de la Baca.

A. atrata L. - Rochers calcaires des Alpes. - Vaud et Valais : mont Cheville, Fully, val d'Illiez, St-Bernard, Feuilleret, Mayenalp de Leukerbad, montẻe de la Gemmi, Eginnenthal. - H.Sav. : roch. calc. du Buet, du col d'Anterne et de tout le massif entre Samoëns, Sixt, Morzine et le Valais, plateau des Gets.

A. moselacta L. - Rochers siliceux des Alpes. - Vaud et Valais : creux de Jaman, Dent de Morcles, St-Bernard, Cleuson, Thyon, Diez, Ferpecloz, Arolla, mont Nuoble, Saas, Schwarzberg, Zermatt, Tortain, Sirwolten, Alpes de Conches, Glacier-du-Rhone.

- H.-Sav. : Buet, Brévent, chaîne du Mont-Blanc, Tré-la-Tête, le Bonhomme, col de la Seigne et dans l'Allée-Blanche sur le versant piémontais du Mont Blanc.

A. hybrida Gaud. - Diffẻre du précédent par ses feuilles velues-laineuses. - Vallées de Saas et de Zermatt.

BrDENS trlpartita $L$. - Fossés dans tout le bassin; plus rare dans la région méridionale.

B. hirta Jord. - Lieux humides à Charvieu, Pont-de-Chérui, Crémieu, la Verpilière, entre Tullins et N.D. de l'0zier.

B. radlata Thuill. - Etangs desséchés de la Bresse à Pleurre, Chaussin, Chaumergy. Ce sont les seules stations connues de cette espèce assez commune en Suède.

B. cernua L. - Fossés, lieux humides; plus rare dans la région méridionale.

KERNERY blpinnata G. G. - Champs cultivés de la région mérid. - Dr. : Crest. - Var : la Garde près Toulon. - Gard : le Vigan, Anduze, Sumène, Sauve.- Hẻr. : Montpellier à Grammont, Rieucoulon, St-Georges, St-Bauzille-du-Putois, Ganges. - Pyr.-Or.

BUPHTHanMuM alletfollum $L$. - Bois et coteaux. Assez rẻpandu dans la partie mérid. de la chaine jurassique; remonte jusquau pied de la Dole, de la Faucille et du Reculet, sur le plateau des Bouchoux, des Molunes, de Belleydoux; depuis St-Claude jusqu’à Morez, Thoirette, Moirans; remonte le cours 
de l'Ain jusqu'à Champagnole; lans les envir. de Nantua s'élève au pied du Mont, Crozet, Montange, le Grand Abergement, les Neyrolles; sur le versant oriental autour de Thoiry; enfin dans les envir. de Tenay en Bugey, à la Birottière. - C.-d'Or : Marey-sur-Tille, envir. de Châtillon. - Vaud, Fribourg et Valais : Bex, Ollon, les Mortais, Château d'Oex.-H.-Sav.:Sixt à la Vauzalle et à la Combe.- Sav. : envir. de Mloutiers à Brides, Chambéry. - Is. : St-Egrève, Pont-de-Claix, Morêtel près StPierre-d'Allevard, St-Christophe en Oisans. - Dr. : Nyons. Vaucl. : Villes au vallon de la Nesque. - Pyr.-Or. : St-Martindu Canigou, Tour-de-Batère, Cortsavi.

B. grandiforum L. - Forme du précédent à fleurs grandes et à feuilles presque glabres et plus étroites. - H.-Sav. : combe de Sixt. Sav. : mont Cenis. - Is. : Bastille de Grenoble, mont Rachais, Chalais, Pariset, St-Nizier, Vif, route de Vizille à Laffrey, la Salette.-H.Alp. : la Bâtie-Neuve, Chabrières, Manteyer, la Garde, la Grangette, ChailloI, le Champsaur, la Vachette, val des Prés, Névache.-B.-Alp. : Sérennes, la Condamine, Rochaille de Meyronnes.-Dr. : Barnave.- Var et Alp.-Mar. : Bezaudun, envir. de Grasse, St-Vallier, toutes les collines de Nice et de Menton jusqu’à St-Martin-Lantosque et Tende.

AsTERISCUS maritimus Mœench. - Rochers marit. - B.-duRh. et Var: Endoume, Montredon, l'Estaque, lle Ratonneau, littoral de la presqu'ile de Giens. - Pyr.-0r. : entre Argelès et Collioure.

A. aquaticus Monch. - Lieux humides de la région mérid. depuis le sud de la Dr. à Montélimar, Dieu-le-Fit et les envir. d'Aubenas et de Vallon dans l'Ard. - Vaucl. et B.-du-Rh. : envir. d'Avignon à Caumont, Orange, Bédoin, Flassan, Mormoiron, Mourepiane près Marseille, 1̂le de Pomègue, Istres, envir. d'Arles. - Var et Alp.-Mar. : Carqueiranne, ile de Porquerolles, presqu'lle de Giens, le Luc, Fre̊jus, littoral de Nice à Menton. Gard : Nîmes, bords du Gardon à Fournès, Montbrin. - Hér. : Montpellier à la Colombière, St-Clément, Maguelonne, Restinclières, Lunel-Viel, St-Mathieu, Claret, Béziers, Agde. - Aude et Pyr.-Or. : Ste-Lucie, Prades, Villefranche à la Trencada d'Ambulla, Cases-de-Pena. 
A. spinosus G. G. - Borls des chemins de la région mérid.; remonte dans la Dr. vers Mfontélimar, Dicu-le-Fit, Crest, Allex, Cabonne, Nyons.

TNU Ma Kelenium L. - Cultivé et naturalisé autour des habitations.-H.-Saone: Fougerolles, St-Remy, Nans-les-Rougemont. -S.-ct-L. : Roussillon, St-Bonnet-cn-Bresse.- C.-d'Or: Magnysur-Tille, Talmay, Cussigny, Villebichot, - Doubs et Jura : le Mémont, le Russey, Clıarmont, Voujeaucourt, Présentevillers, Chaussin, Mont-sous-Vaudrey, Bletterans, Nans-les-Rougemont. - Is. et H.-Alp. : Froges, St-Geoirs, St-Baudille, Pipet, Lalley, Gap, Rosans. - Dr. : Barnave, - Vaucl. : le Luberon. - Var : le Luc au Prignolet. - Gard: le Boultou près de l'Espérou, Aujargues, Manduel. - Pyr.-0r. : pied des Albères, Céret, vallée de St-Laurent-de-Cerdans. - En dehors de nos limites dans le Tarn, le Puy-de-Dóme, l'Allier, la Haute-Loire.

C. Conyza D. C. - Coteaux pierreux des terr. calcaires dans tout le bassin.

I. bifron L. - Rochers et rocailles calcaires. - Is. et Dr. : Varces, Vif, Solaure près Die. - H. et B.-Alp. : : Charance, la Garde, Rabou, ravin du Busson, la Grangette, la Condamine, St-Paul, Barcelonnette, Gréoulx. - Vaucl. et B.-du-Rh. : Villes à la Nesque, envir. d'Aix au chemin de Banon et à Fontlèbre.Var et Alp.-Mlar. : Bezaudun, Grasse, le Cheiron, Vende, Venan. zon, Luceram, entre Levens et le Chaudan. - En dehors de nos limites sur les coteaux calcaires de la Limagne et de l'Allier.

X. fplroifolin L. I. squarrosa L. - Coteaux secs et pierreux. C.-d'Or et S.-et-L. : Gevrey et toute la côte, Dezize, Sampigny, Brancion.- Ain : envir. de Belley à Muzin, Parves, Pierre-Châtel, St-Benolt, Serrières, Villebois, St-Sorlin, Virieu, Tenay.Is. : entre Crémieu et la Balme, Bastille de Grenoble, Comboire, Rochefort. - Dr. et H.-Alp. : St-Vallier, Charance, Reynier près Tallard. - Vaucl, et B.-du-Rh. : Plavignal, Aix à Mauret et à la colline des Pauvres, - Var et Alp.-Mar.: Toulon, le Luc, Montrieux, Bagnols, Grasse, entre la Tour et St-Sauveur, Nice, Menton. - Gard : Nîmes, Beaucaire, Chartreuse de Val- 
bonne, le Vigan, Alais, St-Ambroix, Tresques, Bagnols, Seyne, Serre-de-Bouquet. - Hẻr. : Montpelier à Grammont, Montmaur, Castelnau, St-Briés, Mireval, Mauguio, Montferrant, Castries, St-Guilhem, St-Bauzille, Cette, les Arcs. - Aude et Pyr.-Or. : envir. de Narbonne à Fontfroide, Salsigue, Collioure, Banyuls-sur-Mer.

I. Ifrta L. Coteaux. - Rh., Ain et Is. : envir. de Lyon, la Pape, entre Brenod et Meyriat, Crémicu, Villette-d'Anthon. - Dr.: les Rioux près St-Vallier, Crest. - Var et Alp.-IIar. : Bagnols, la Roquette. - Pyr.-0r. : le Canigou, St-Martin, Cortsavi, Prades, Fosse.

I. sallelua L. Coteaux calcaires. - Envir. de Belfort à la Perche et Meroux. - H.-S. : Bucey-les-Traves, Albenans. - Vignoble et basses montagnes de la chaine jurassique.-C.-d'Or, S.-et-L. : Gevrey, Sombernon, vallée de l'Ouche, Arcelot, coteaux calcaires de Dezize, Santenay, Chassagne.- Envir. de Lyon, de Chambéry, d'Annecy, Bonneville, Clıamonix et Sixt. - Is. : Crémieu, Bastille de Grenoble, Comboire, Claix. - Dr. et Ard. : St-Vallier, la côte du Rhône, l'Escrinet, Vals, vallée de l'Ouvèze. - H. et B.-Alp. : envir. de Gap, de Chorges, de Barcelonnette et de Jauziers. - Vaucl. et B.-du-Rh. : St-Amans, Morières, Flassan, Prégnon, Montaiguet, le Tholonet.- Var et Alp.-Mar. : Ste-Baume, Montrieux, les Maures, Pignans, Auribeau, Villeneuve, St-Anne-de-Vinaï, St-Martin-Lantosque. - Commun dans le Gard et l'Hér. : envir. de Nîmes, d'Alais, Mas-de-Londres, la Sérane, Morntarnaud, St-Bauzille de Montmel, Lodève, Bédarieux. - Pyr.-Or. : Costujes, St-Laurent-de-Cerdans.

1. Vafllantil Vill. - Bois et páturages. - Envir. de Genève au bois de la Bâtie. - H.-Sav. : Bonneville, bords de la Borne, lles du Fier près Annecy, St-Germain-sur-Talloires. - Rh.: iles du Rhône en face de Vaulx-en-Velin. - Is. : Polygone de Grenoble, Comboire, la Moucherolle, Bourg-d'Oisans. - Ard. : Tournon. - H.-Alp. : envir. de Gap, Tallard, la Saulse, Chiorges, Boscodon. - Vaucl. : envir. d'Apt. - Pyr..Or. : vallée d'Eyne, Collada de Nuria, Carença.

I. semiamplexicaulis Reut. - Hybride des I. salicina et Vaillantii. - Rive gauche du Rhône au-dessus de Genève. 
I. crlthmoldes $\mathrm{L}$. - Terrains humides et salés du littoral. - B.du-Rh. : les Martigues, la Camargue, étang de Berre. - Var et Alp.-Mar. : Toulon, les Pesquiers des salines d'Hyères, Fréjus, lle Ste-Marguerite, Antibes, Nice.- Littoral du Gard et de l'Hérault. - Aude et Pyr-Or. : Ste-Lucie, la Nouvelle, étang de Salces.

T. montnun L. - Coteaux secs calcaires.-H.-S. : Bucey-les-Traves, Port-sur-Saone. - C.d'Or et S.-et-L. : Dijon et toute la côte calcaire, Nolay, Chassagne, Santenay, Dezize, vallée de la Dheune, Brancion. - H.-Sav. et Sav. : Annecy, Rumilly, St-Innocent, mont du Chat, St-Alban près Chambéry. - Ain et Rh. : Cordon, Pierre-Châtel, Belley, Château-Gaillard, Ambronay, Ambérieu, la Pape, Couzon. - Is. : Crémieu, Vienne, Champ près Vizille, Roissard, entre Bourg-d'Oisans et Venosc. - Dr. : St-Vallier, Barnave, Crest. - H.-Alp. : Gap à Puy-Montbaud, Puy-Jlaure, St-Mens, N.-D. du Laus, Rosans, le Devoluy. Vaucl. et B.-du-Rh. : envir. d'Avignon, de Carpentras, d'Orange et d'Apt jusqu'au pied du mont Ventoux à Flassan et Bédoin, Cuques. - Var et Alp.-Mar. : Toulon, le Luc, Grasse, Nice, Menton. - Ard. : St-Pierre, Privas, Mercuer. - Gard : Nîmes, le Vigan, Anduze, St-Ambroix, Beaucaire, Serre-de-Bouquet. Aude : Villespy, Saissac, Conques, mont Alaric, Tuchan, St-Victor, les Corbières.-Pyr.-Or. : St-Marsal, Prats-de-Mollo, Costujes, vallée de Conat en montant à la Font de Comps. - En dehors de nos limites dans les Pyr. centr., les coteaux calc. de la Lozère, du Tarn, de l'Aveyron el du Puy-de-Dôme.

I. Jritamniea L. - Alluvions, bords des rivières. - Doubs et Jura : depuis Montbéliard jusqu'au-dessous de Dole, alluv. de la Loue, de l'Orain et de la Seille. - C.-d'Or et S.-et-L. : Magny-sur-Tille, vallée de la Dheune et de la Saòne. - Rh. et Ain : bords de la Saône à Couzon, entre Charnoz et Giron près Meximieu. - Dr. : St-Uze près St-Vallier, Furmeyer. - Vaucl. et B.-du-Rhône:envir. d'Avignon et de Carpentras, Arles, les Alpines, - Var : Pignans, le Luc. - Gard : Nimes, Beaucaire, Manduel, Jonquières, St-Gilles, Bellegarde, Aigues-Mortes.

I. Inelenlohtes D C. - Min : St-Martin-du-Mont, Heximieu. - 
Aude et Pyr.-Or. : Conques, les Corbières entre St-Paul et Estagel, vallon de Prades, Molitg, Ria, Belloch, vallée de Finestret et Estoher, Cornella-du-Conflent au camp del Forn, la Cerdagne à Estavar. - Pyrénées centrales.

PUCTCA Ria odora Rehb. - Coteaux sablonn. - Var et Alp.Mar. : Toulon, Hyères, lle de Porquerolles, le Luc, Frẻjus, SiTropez, Cannes, ile Ste-Narguerite, Antibes, Nice, Monaco, Menton. - Pyr.-Or. : St-Antoine-de-Galamus, Collioure, PortVendres, Banyuls-sur-lier.

P. dyenteriea Gærtn. - Lieux humides dans tout le bassin.

P. vulgaris Gærtn. - Ibid. Moins commun que le précédent.

P. slcula Moris. - Marais, fossés, champs humides de la région mérid. - B.-du-Rh. et Var : Arles, Pourcieux, Hyères au Ceinturon et aux Pesquiers, Fréjus, Cannes, Grasse.-Gard: St-Gilles, Aigucs-Mortes, Bellegarde. - Hẻr.: Maurin, Pérols, Roquehaute, Béziers, Vendre. - Aude et Pyr.-Or.: envir. de Narbonne et tous les champs du littoral.

CUPUEA RYA graveolens G. G.- Champs cultivés. - C.-d'0r: Boncours-la-Ronce. - Rh. : Charbonnières, la Tour-de-Salvagny, Echallas, de Malataverne à Messimy, Talluyers. - Is. et Dr. : St-Corps près la Cốte-St-André, Romans, Laveyron près St-Vallier. - Ard. : Andance, Celles, Flaviac, Vals. - Vaucl. : envir. d'Orange, de Carpentras, d'Avignon et d'Apt. - B.-duRh. : envir. d'Aix sur les bords de l'Arc, étangs de Marignane et de St-Chamas, Miramas, Arles. - Var et Alp.-Nar. : Toulon, Ile de Porquerolle, le Luc, St-Tropez, Cannes, de Nice à Menton. Gard et Hér. - Pyr.-Or.: Prades, de Villefranche à Olette, Banyuls-sur-Mer, Prats-de-Hollo. - En dehors de nos limites dans les Pyr. centr., la Lozère dans les vallées de Ste-Croix, St-Etionne, Valfrancesque, l'Aveyron, le Tarn.

c. viscosa G. G. - Lieux incultes de la région mérid. - Vaucl. et B.-du-Rh. : envir. d'Orange, d'Avignon et d'Apt, graviers de la Durance, bords de l'Arc, Pont-des-Gardes près Aix, envir. de Marseille. - Var et Alp.-Mar. : Toulon, Hyères, le Luc, Fréjus, St-Tropez, Cannes, Nice, Menton. - Gard et Hêr. - Aude : Lastours, Villemagne, Villardonnel, Montolieu, les Corbières, 
Villeneuve, Tuclian, envir, de Narbonne, Gruissan, la Clappe, Fontfroide. - Pyr.-0r. : Château-Roussillon, bords du Tech, Boulou, Céret, Prades, Villefranche.

JAsoviA glutinosa D C. - Rocailles de la régrion mérid. B.-du-kh. et Var : envir. de Marseille et de Toulon, le Revest, Baou-de-Quatre-Heures, mont Caoumé, mont Faron, N.-D. des Anges près Pignans. - Pyr.-Or. : vallée de la Tet, Prades, Villefranche, Olette, Cornella-du-Conflent, d'Arles à Prats-de-Mollo. - Pyr. centrales.

J. tuberosa D C. - Terrains argileux humides de la région mérid. - Ard. : Joyeuse, Vallon. - Gard : envir. de Nimes, Uzès, Alais, St-Ambroix, Bouquet, le Vigan, Montdardier, Tresques. - Hér. : Montpellier à Fontfroide, Fabrègues, St-Gély, Grabelş à Valmargues, Montferrier, Montarnaud, St-Martin-de-Londres, Teyran, Pic St-Loup.-Aude : Montolieu, les Corbieres, Arques. Pyr.-Or. : Prats-de-Mollo à Can-Pobil, près du Coral.

HeLrCH XSUM arenarhum D C. - Graviers, alluvions des riv. des Pyr.-0rientales.

H. decumbens Camb. - Rochers. - Var: Hyères, St-Raphä̈l. Aude et Pyr.-Or. : ile Ste-Lucie, vallons de Port-Vendres et de Banyuls-sur-Mer.

IF. Stcechas D C. - Terrains arides et pierreux, plus commun dans les terrains calcaires. - Très-répandu dans la région mérid., remonte dans l'Ard., la Dr. et le Rh. vers Joyeuse, Aubenas, Nyons, Crest, St-Vallier et jusqu'à Lyon, dans les B. et H.-Alp. autour de Sisteron, Gap, St-André-de-Rosans; dans l'Is. vers St-Marcellin, Décines, Villette-d'Anthon, et jusqu'à Crémieu. - En dehors de nos limites sur les roch. calc. de la Lozère autour de Meyrueis, Mende; dans l'Aveyron autour de Millau, Rodez, Ste-Affrique, Villefranche; dans le Tarn sur les coteaux calcaires. - Espèce polymorphe.

II. syncladum Jord. Fourr. - Aix H. collnnum J. F. - Décines (Is.), (B.-du-Rh.). Durban (Aude).

II. marilinum J. F. - La Ciotat II. Rexiramum J. F. - Granne (B.-du-Rh.).

(Dr.). 
H. libanolis J. F. - Aude : col d'Estrem, vers Durban.

H. brachycladum J. F. - Rognac (B.-du-Rh.).

H. rigens J. F. - Ard. et Dr. : H. parvulum J. F. - St-Tropez Tournon, St-Vallier, Tain. - (Var).

H. lutescens J. F. - Ibid.

Y. sngustifolium D C. - Rochers de la région mérid. - Var et Alp.-Mar. : Sablettes de Toulon, Cannes, ile Ste-Marguerite, Antibes, Nice à Ilontboron. - Gard. - Hér. : Olargues, Lodève, Pézenas. - Aude et Pyr.-Or. : envir. de Narbonne, à la Clappe, Montoulieu, Durban, Bizault, Consolation, Céret, Prades. Lozère.

Var. serotinum Boiss, à feuilles plus longues, vertes en dessus, akènes non glanduleux. - Gard: le Vigan, Valleraugue, $\mathrm{Au}$ messas, rochers de Bonpériers. - Hér.: Gignac, vallée supér. de l'Orb, Avène, St-Martin-d'Orb, Lodève, Castanet-le-Haut, le Poujol, Lamalou, St-Pons, la Salvetat, Fraisse. - Aude : envir. de Narbonne, la Clappe à l'Espitalet, Rennes-les-Bains, les Corbières, Leucate, Ste-Lucie. - Pyr.-Or. : Trencada d'Ambulla, Prades jusqu'à Olette, vallée du Tech, Collioures, les Albères, Céret, vallée de la Têt, Perpignan, St-Feliu, Ille. - Aveyron près de Ste-Affrique. - Tarn.

H. libanotis J. F. - Prades (Pyr.-Or.). - H. leplophyllum J. F. - Valleraugue (Gard).

L'H. orientale est cultivé dans le Var à la Seyne et à Bandols.

GNAPHAMIUM luteo album L. - Collines sablonneuses de C.d'Or, S.et-L., H.-S., dans les zônes vosgiennes et sous-rosgiennes, plaines du Doubs, Jura, Ain, Vaud et Valais, Maurienne. Envir. de Lyon, de Grenoble, Crest (Dr.), Chorges et St-Andréde-Rosans (H.-Alp.), Aubenas et Joyeuse (Ard.). - Assez commun dans la Provence et le Languedoc.

C. ByIvaticum L. - Bois. - Commun dans les chaines des Vosges et du Jura jusque dans les montagnes du Bugey. - H.-S., C.-d'Or et S.-et-L. dans le Morvan, chaine du Beaujolais, Lyonnais, Pilat. - Vaud et Valais, Savoie, Dauphiné, B.-Alp. jus- 
que dans les Alp.-Mar. - Chaine vivaraise, le Mezenc, l'Espérou, l'Espinouse, la Montagne Noire, les Pyr.0r. - En dehors de nos limites dans les Pyr. centr., l'Aubrac, Lozère, Cantal, Puyde-Dome, montagnes du Forez.

C. norvegicum Gunn. - Pâturages des montagnes, - Les IlautesVosges depuis les lacs Noir et Blanc jusqu'au Ballon de Giromagny dans la H.-Saone. - Nul dans la chalne jurassique. Vaud et Valais: Clusanfe sous la Dent-du-Midi, Fully, Bagnes, St-Bernard, Simplon, Mayenwand entre le Glacier du Rh. et la Grimsel. - H.-Sav. : Vergy, Méri, Buet, Brévent, col de Balme, Leschaux, Montanvert, les Contamines, Tré-la.Tête, Dents d'Oche, Haut-de-Lin, mont Chalunc et des Granges, plateau des Gets, montagnes de Samoëns, de Sixt et de Morzine, mont Charvin, Tournette. - Sav. : mont Mirantin, Hauteluce, Arbine. - Is. : chaine des Sept-Laux et de Belledonne, lac du Crouzet, Pas de la Coche, col Oddie, la Bérarde en Oisans. - It.-Alp. : Lautaret. Névache aux Rochilles, bois du Casset près du Lauzet. - Alp.Mar. : bois du Boréon au-dessus de St-Martin. - Rh. : pic de St-Bonnet sur Montmélas. - Ard. : le Mezenc. - Pyr.-Or. : Fontpédrouse, Cambres d'Aze. - En dehors de nos limites dans les Pyr. centr., l'Aubrac, le Mont Dore, le Plomb du Cantal, les Salles dans le Forez.

G. Hoppeanum Koch._Forme plus petite, à feuilles plus élroites et plas blanches, à nervures moins marquées, capitules moins nombreux en grappe plus courte. - H.-Sav. : mont Méri à la Croixde.Fer, col Joly. - Le Ilont Tendre dans la chaine jurassique, - H.-Alp. : val Préveyre, mont Viso.

G. ulighnosum L. - Lieux humides, prés et champs inondés pendant une partie de l'année.

G. supinum L. - Rochers et pâturages. - Nul dans les Vosges. Rare dans la chaine jurassique au N. 0 . du piton du Reculet.Yaud et Valais: Boulaire, Essez, Thyon, Arolla, Zermatt, Saas, Grimsel. - II.-Sav. : Vergy, Méri, Haut-de-Lin, Cornettes de Bise, Morzine, Buet, Aiguilles Rouges, col de Balme, Mont Blanc, au Couvercle, à Leschaux et au Jardin, Tré-la-Téte, chaîne des Aravis.-Sav. : cols du Bonhomme el de la Seigne, mont Iseran, 
petit St-Bernard, col du Cornet au-dessus d'Aime, montagnes des Allues, col de la Gordaz, mont Cenis. - Is. : chaines des Sept-Laux, de Belledonne à la Pra, col de la Coche, les Grandes Rousses au-dessus d'0z, de Vaujany et d'Huez, petite Moucherolle. - H.-Alp. : Lautaret, col de l'Echauda, col Isoard, Ségure et Péas en Queyras, mont Viso, Chaillol-le-Vieil, Orcières. B.-Alp. : Lauzannier, vallonnet de Mleyronnes, Parpaillon, vallon du Chåtelet, Bérard. - Alp.-Mar. : col de Fenestre, lac d'Entrecoulpes, St-Anne-de-Vinaï, mont Tenibre au-dessus de St-Étienne, col de Jallorgues. - Pyr.-Or. : Cambres d'Aze, vallée d'Eyne, Canigou, Pla Guilhem vers le cap de la Roquette, Llaurenti. - Pyr. centrales.

ANTWNA RiA earpatiea Bl. Fing. - Rochers siliceux des Alpes. - Vaud et Valais : Boulaire, Lavarraz, St-Bernard au mont Cubit, Fully, Folatères, mont Nuoble, Guggerhubel, Distelalp, Gornern, Simplon, Gemmi. - H.-Sav. : Vergy au col de Balafrasse, base de la pointe du Midi, mont Méri sur les rochers à l'ouest de Solaison, mont d'Etale près la Clusaz, mont Charvin, Tournette, mont des Granges, Roc d'Enfer, Aiguilles Rouges, col de Balme, Jardin de la Mler de Glace, Tré-la-Tête, l'Allée Blanche sur le versant italien du Mont Blanc. - Sav. : mont Trélod, les Allues, la Galise, mont Cenis. - Is. : la Pra de Belledonne, Champrousse. - H.-Alp. : la Grave à Puy-Vacher, Lautaret, Galibier, mont Viso, col Vieux, Ségure, col de Vars, mont Queyrel et Lopet en Champsaur, mont Aurouse.-B.-Alp.: Fouillouse, IParpaillon, Bérard, Longet. - Alp.-Nar. : mont Formose près de Tende, lac d'Entrecoulpes, col de Fenestre. Pyr.-0r. : vallée d'Eyne, Canigou. - Pyr. centrales.

A. dloica Gærtn. - Pâturages des montagnes. - Toute la chalne des Vosges et celle du Jura jusque dans le Bugey. - Vaud, Valais, Savoie, Dauphiné, B.-Alp. jusque dans les Alp.-Mar., mont Ventoux, Ste-Victoire, - Le Morran, le Beaujolais, le Mont-d'Or lyonnais, le Pilat, la chalne du Vivarais, de l'Aigual et de l'Espinouse; Pyr.-Or. - En dehors de nos limites dans les Pyr. centr., l'Aubrac, la montagne d'Anglès dans le Tarn, Lozère, Cantal, Puy-de-Dôme, Forez. 
A. margaritncen L. - Naturalisé sur les bords de l'Ubaye près de Barcelonnette.

LEONTOPODYUX alpinum Cass. - Páturages des montagnes. - Nul dans les Vosges. - Sommet de la Dole. - Vaud et Valais : Boulaire, Chamosaire, Bagnes, Catogne, St-Bernard, Zermontana, col de Fenêtre, Pierre-à-Yoir, Cleuson, Barma, Seillon, Diez, Esserz, mont Nuoble, Cotter, Vende, Lœmmeralp, Zermatt, Saas, Distel, Binn, la Gemmi, - II.-Sav. : Vergy, Tournette, mont d'Etale près la Clusaz, Cornettes de Bise, mont Bostan près Samoëns. - Sav. : mont Trélod, la Sambuy, Crêt du Ré, petit St-Bernard, mont Cenis, cols de la Seigne et du Bonhomme. - Is. : la Pra de Belledonne, St-Christophe et la Bẻrarde en Oisans, la petite Moucherolle. - II.-Alp. : la Grave, Lautaret, Briançon, Malrif, mont Viso, col de Vars, St-Véran, mont Morgon, mont Séuse, Chaudun, mont Aurouse, collet d'Ancelle. - Dr. : mont Glandasse. - B.-Alp. : Vallonnet, Ho. ronaye, Longet, Bérard. - Alp.-Mar. : Capelet de Raus, Alpes de Tende, la Colmiane près St-Martin, Entraunes. - Pyr.-Or. : vallée d'Eyne et de Prats-de-Balaguer au Roc del Buc.

FLCAGo sorthulata Presl. - Champs calc. dans la plus grande partie du bassin.

F. germaniea L. - Champs des terr. siliceux. Espèce polymorphe tantôt couverte d'un duvet blanchâtre $F$. canescens Jord, tantôt recouverte d'un duvet jaunâtre ou verdâtre $F$. lutescens Jord.

F. eriocephaIn Guss, - Terrains sablonneux du Var: lles d'Hyeres et de Porquerolles, Maures du Luc et du Cannet.

F. arvensis L. - Champs des terr. siliceux; nul sur les calcaires purs.

F. minima Fries. - Champs des terrains siliceux ; nul sur les calcaires purs.

F. gnlliea L. - Champs des terrains siliceux; nul sur les calcaires purs.

Var. teniufolia Cass. - Ile de Porquerolles.

MICRoPUS erectus L. - Sables et champs pierreux. - Valais : mont d'Orge, St-Léonard, Sierre, Varone. - Vaud : envir. de Nyon, Prangins, Clarens, Genolier, Sezeniens, Coinsins. - 
H.-Sav. et Sav. : Frangy, St-Germain, Moutiers, St-Michel-enMaurienne. - Ain : fort de l'Ecluse, Ambronay. - Is. : Bastille de Grenoble, Vars, Pariset, la Mure. - H.-Alp. : Gap, entre Embrun et Briançon. - Commun dans la C.-d'Or, la Provence, Jes Alp.-Mar. et le Roussillon.

M. bombycinus Lag. - Champs pierreux. - Vaucl. autour d'Avignon, d'Orange, Bédoin.-B.-du-Rh. : la Grau, Montaud-les-Miramas.

- Pyr.-Or. : Baixas, Força-Réal, entre Camélas et Thuir.

EVAX pygmsen Pers. - Champs du littoral des B.-du-Rh., du Var et des Alp.-Mar., Toulon à la Grosse Tour, île de Porquerolle, Cannes au cap Croisette, la Napoule, littoral de Nice à Menton. — Gard : la Sylve près Sylveréal. - Hér. : pied de la montagne de Frontignan, Cette près du fort St-Pierre, Roquehaute, $\Lambda$ gde. - Aude et Pyr.-Or.: Ste-Lucie, la Nouvelle, la Clape au ravin de Rouquette, Pech André de Montredon, Paulille, Salses, Cabestany.

CARPESIUM cernumm L. - Lieux ombragés. - Vaud et Valais : Bex, Ollon, Rennaz, Cully, Fully, Salaz. - H.-Sav. : Sallenove près Frangy, Pontchy, Dessy, Scionzier, Doussard, Faverges, St-Ferréol, Evian. - Sav. : le Trembley près Aix. Is. : Balmes de Fontaine, Gières, Herbeys, Echirolles, Revel, Allevard, la Terrasse, Morestel, entre Crémieu et les Avenières. - Pyr.-Or. : vallée de Llo, Prats-de-Hollo à la Tour de Mir et au Mas-Xatard.

CATENDUMA arrensis L. - Champs dans tout le bassin.

C. bicolor Raf. - Var:talus du fort Malbousquet à Toulon. Pyr.-Or. : St-Antoine-de-Galamus.

\section{GYNAROCÉPHALES}

ECHirops sphorocephalus L. - Bords des chemins. Valais : Prafalcon, Branson, Sion, Sierre, Naters, Mœrell. Is. et Dr. : graviers du Drac près Grenoble, de l'Isère à Crolles, Mens, Crest. - H.-Alp. : la Grave, Aiguilles en Queyras, Pontde-Corbières en Champsaur, Pommerol près Rémusat, Charance, la Garde, bois Mondet. - B.-Alp. : fort Tournoux. - Ard. : 
Alissas. - Vaucl. : Flassan, Castellas, Luberon. - Alp.-MIar.: la Clue de St-Auban, St-Dalmas-le-Sauvage, Entraunes, Luceram, entre Levens et Coarazza, au-dessus de Castellar et de la Giandola. - Pyr.-0r. : entre Prades, Villefranche, Olette et mont Louis, vallées de Llo, de Cornella du Conflent et de Vernet-les-Bains. - En dehors de nos limites dans les Pyr.-Centr., la Lozère entre Mlende et Bagnols, l'Aveyron autour de Villefranche et de Capdenac.

E. Titro L. - Bords des chemins de la rígion mérid. et moyenne, - Rh., Loiro et Is. : Vienne, Condrieu, Chavanay, Corps, la Nure, Roissard, Varces, Bernin.-Ard. et Dr. : Andance, Tournon, la Voulte, Privas, mont Charray, l'Escrinet, St-Vallier, Tain, Crest, Monte̊limar. - H.-Alp. : Gap, la Garde, les Serigues, Rambaud, N.-D. du Laus, Manteyer, Sigoyer, Rosans, Guillestre, la Grave, Briançon.- B.-Alp. : la Condamine, Annot. - Vaucl. et B.-du-Rh. : envir. d'Avignon, d'Orange, de Carpentras, d'Apt, d'Aix et de Marseille.- Var et Alp.-Mar. : TouIon, le Luc, Rians, Ampus, Grasse, Nice, Menton. - Commun dans le Gard, l'Hér., l'Aude el les Pyr.-Or. - En dehors de nos limites dans la Lozère, le Tarn, l'Aveyron et le Lot.

GATACTIES tomentosa Hoench. - Bords des chemins de la région mérid.

TXRYMNUS leueographus Cass. - Lieux incultes de la région mérid. - B.-du-Rh. : Aix au vallon des Gardes, rive droite du petit chemin du Tholonet, Bompard. - Var et Alp.-Mar.: Toulon, le Luc, St-fropez, Grasse à la Paoute, Antibes, St-André près Nice. - Hér. : Montpellier à la Colombière, Montmaur, Veyrassi, St-Jean-de-Védas, Cette, Pignan, Pic St-Loup. Pyr.-Or. : plateau des Terrats et de Fourques, Banyuls-sur-Mer vers Can-Campa.

SYLIBUM Marinnum Gœrtn.-Bords des chemins de la région mérid. et moyenne; apparait accidentellement en divers lieux de la partie septentrion., mais n'y est pas véritablement indigène.

ONOPORDoN acanthium L. - Lieux incultes dans tout le bassip. 
0. Delorti Timb. Lagr. - Aude et Pyr.-Or.: entre Durban et Villeneuve, Vingrau.

D. tauricum Willd. - Lieux incultes; naturalisẻ aux SaintesMaries près Marseille, à Cette, au Port Juvénal, à Grammont et au chemin de Pérols près Montpellier. - Introduit au moyen des laines de provenance étrangère.

D. 11 yricum L. - Lieux incultes de la région mérid. - Dr. : Die, Crest, Hontélimar. - Vaucl. et B.-du-Rh. : envir. d'Aix, Toulon, la Ste-Baume, le Luc, Vidauban, Fréjus. - Gard : Nìmes, Beaucaire, St-Gilles, Aigues-Mlortes, le Vigan, Uzès. - Hér. : dans les plaines, s'êlève dans la région montagn. jusqu'à la Vacquerie. - Aude et Pyr.-0r. : envir. de Narbonne à la Clappe, Ste-Lucie, les Corbières, col d'Estrem, Villeneuve, Pont de la Fou près St-Paul, partie basse des vallées de l'Agly, de la Tet et du Tech.

D. acaule L. - Aude et Pyr.-Or.: fontaine de Merquiol près Tuchan, plateaux d'Ambulla et d'Escaro, vallée de Conat, au sud-ouest de la Font de Comps vers Bellioc.

D. aralbicum L. - Naturalisé autour de l'ancien jardin des plantes de Marseille.

CYNAIt cardunculus $\mathrm{C}$. - Cultivê et quelquefois subspontané dans le Languedoc et le Roussillon; Hẻr.: Mireval, St-Jean-deVédas, St-Aunès, Clapiers, Montarnaud, Castries, Restinclières, derrière St-Loup. - Gard : mas de Broussan près Bellegarde. - Aude et Pyr.-0r. : Villeneuve, Durban, les Corbières, Laute, la Clappe, Fontfroide, Ste-Lucie, Salses, Château-Roussillon.

NOTOBAsIS eyriaca Cass. - Terr. incultes des B.-du-Rh. et du Var aux Martigues et sur les remparts de Toulon. Probablement introduit et non indigène.

PICN@MON acarna Cass. - Lieux incultes. - Ard. et Dr. Cruas, Rochemaure, Lussas, Nyons, Montélimar. - Vaucl. et B.-duRh. : Carpentras, Valrẻas, Bédoin, envir. d'Aix et de Marseille. - Var et Alp.-Mar. : Toulon, Carqueiranne, Rians, Ampus, le Luc, le Mas, Levens, col de Braus, Nice, Menton,- Gard : Nimes, Beaucaire, Aramon, Corconne, le Vigan, Blandas. - Hér. : Lavalette, Montmaur, Montbazin, St-Gély, Villeveyrac, Baillar- 
gues, Valmargues, Cette, Balaruc, Montferrier, St-Martin-deLondres, Teyran, Valène, Olargues, Aniane, Montarnaud, Pègayrolles-de-l'Escalette. - Aude : Gruissan, le Rec, Fontfroide, Tuchan, Durban, mont Alaric, vallée de la Tet, Força-Réal, Villefranche, Font de la Barjoanne, montagne d'Ambuila, vallée du Rẻart, Monestir del Camp, St-Antoine de Galamus.

Crmsrum Innceolatum Scop. - Lieux incultes dans tout le bassin.

C. crinitum Boiss. - Lieux incultes. - Ilér. : Sérignan à Valras, Portiragne à Roquellaute, Agde. - Aude et Pyr.-Or.: Fontfroide, les Portes, lle de Ste-Lucie, Sigean, Gruissan, Vingrau, mont St-Victor près Durban, entre Durban et Villeneuve, entre St-Paul de Fenouillet et St-Antoine-de-Galamus.

C. echinatum D C. - Lieux incultes, - Aude: Ste-Lucie, Durban, Tuchan, les Corbières, Crabit, la Clappe, Vingrau, vallée du Verdouble.

c. ferox D C. - Licux incultes. - Sav. : Combe de Villette audessus de Bramans. - Is. : Grenoble sur les digues du Drac, la Mure, les Portes-en-Trièves. - Dr. : St-Vallier, Crest. - Ard. : mont Charray. - H.-Alp. : envir, de Gap à la Garde, Rabou, St-Mens, Rambaud, les Serigues, montagne de Faudon, - Commun dans la Provence, les Alp.-Nar., le Languedoc et le Roussillon.

C. odontolepin Boiss. - Coteaux pierreux. - Hér. : près de l'étang desséché de Montady, petit bois de Béziers. - Pyr.-Or.: vallon de Collioure en montant à Consolation.

C. erlophorum Scop. - Lieux incultes dans lout le bassin.

C. paluatre Scop. - Prés humides.

C. palustri monspessulanum G. G. - Pyr.-Or. : vallée d'Eyne, Coma de la Tet.

C. palustri bulbosum D C. - Signalé en Alsace et en Lorraine, n'a pas encore été remarquẻ dans notre bassin.

C. palustri acaule Hampe. - Se trouve dans le canton de Vaud à Gimelle, Coinsins.

C. palustri oleraceum Nœgeli. - Vaud et Ain : Payerne, Roche, Autagne, Allaman, Nyon, Divonne. 
C. palustri erisithales Nogeli. - Aubrac, mont Dore, Cantal, A rechercher dans notre bassin.

C. mowspessulanum All. - Bords des ruisseaux et lieux humides.-Is. : Polygone de Grenoble, mont Rachais, Pont-de-Claix, Beauregard.-Dr. : Crest, Nyons, Barnave. - H.-Alp. : envir. de Gap, les Serigues, la Garde, Charance, Montmorin, Rosans, Châteauroux près Embrun, Vallouise, Briançon. - B.-Alp. : la Condamine, Barcelonnette. - Vaucl. et B.-du-Rh. - Var et Alp.-Ifar.: Toulon, le Luc, Frẻjus, Auribeau, Nice près du Var, Menton. - Gard: Bellegarde, Tresques, St-Gilles. - Commun dans l'Hẻrault, les Corbières de l'Aude et des Pyr.-Or., Montolieu, Bigarras, bords de la riv: de la Basse, le Canigou, mont Louis, Font de Comps, St-Paul près le pont de la Fou, Prats-de-Mollo. - Pyr, centrales.

Varie à fleurs blanches.

C. oleraceum Scop. - Prés humides. - Zone sous-vosg. de la H.-S. et toute la chaine jurassique jusque vers Divonne, Gex, St-Genis, Lélex, Nantua, St-Rambert et Tenay en Bugey. Valais : val d'llliez, Sion.- C.-d'Or : Saulon, Arcelot, Orgeux, Citeaux, St-Bernard. - H.-Sav.: Abondance, mont Charvin, N.-D. de la Gorge, Hégève, Pringy. - Sav. : Hauteluce, CrestVoland. - Is. : Crémieu, entre Bourgoin et la Verpillière, Pont-de-Beauroisin. - Gard : bois de l'Aigual. - Commun dans les Pyr.-Or. - Trouvẻ accidenteliement en amont de Lyon dans les îles du Rhône.

C. oleraceo acaule Nogeli. - Prairies du Doubs, Ain et Vaud à Pont-de-Roide, Pompierre, Baume, vallées des Rousses et de Mijoux, Divonne, Gingins, Nyons. - H.-Sav. : mont Brizon. C. oleraceo vivulare Nogeli, - Prairies humides de la région des sapins de la chaine jurassique.

C. oleraceo heteroplygllum Nœgeli. - Valais : entre 0bervald et Obergestelen.

C. erisithales Scop. - Bords des bois et des prés. - Toute la chaine jurassique depuis le Montendre, la Dóle, le Vuarne jusqu'd la Faucille et au Reculet. - Valais: les Aviollaz, Morgins, la Comballaz. - Ard. et H.-L. : mont Mezenc, la Sapette. 
- Alp.-Mar. : toute la région montagn.; descend jusqu’aux bois du Farghet et de la Maïris. - Gard : le long du Valat de Brama-Bioou près de Camprieux. - Pyr.-Or.: bois de Céret, mont de la Majoral à Arles, St-Laurent-de-Cerdans, St-Martin du Canigou, forêt de Boucheville. - En dehors de nos limites dans l'Aubrac, Cantal, mont Dore, Puy-de-Dome, Pierre-surIIaute.

C. erisithali acaule Michalet. — La Faucille au-dessus de Gex, le Noirmont.

C. erisithali oleraceum Nogeli. - La Faucille, le Noirmont, la Dỏle.

C. bulbosum D C. - Prés humides dans une grande fartie du bassin.

C. bulboso oleraceum Nogeli. - Alsace. - A rechercher dans notre circonscription.

C. bulboso acanle Nœe. - Is. : Jonage. — Gard : bois de Salbous près Campestre.

C. anglleum Lob. - Prairies des sols siliceux. - Chaine des Vosges - Morvan autunois et du pays de Saulieu, Val Combe, Messigny. - Terr. sabl. de vallée du Rh. à Montélimar, Orange. - En dehors de nos limites sur les terr. granit. de Puy-deDôme, Allier, Creuse, Aveyron.

C. rivulare Link. - Prés humides. - Chaine jurassique dans les vallées de Joux et de Mijoux, les Rousses, St-Laurent-en-Grandvaux, Lélex,- Vaud et Valais : vallée de la Sarine, les Ormonds, Morgins. - H.-Sav. : vallée du Reposoir, Abondance. - Is. : Sassenage, Engins, St-Nizier, Villard-de-Lans, le Sappey. Gard : Concoule. - Aude et Pyr.-Or. : Escouloubre, base de Costa Bona, Mont Louis. - Ard. et H.-L. : Mezenc. - En dehors de nos limites dans la Lozère, l'Aubrac, H.-Loire, Cantal, Pierre-sur-Haute.

C. rivulari oleraceum Nœeli. - Prés humides de la rẻgion des sapins de la chaine jurassique.

C. rivulari acaule Nogeli. - Jura : St-Laurent-en-Grandvaux, - Faud et Valais : vallẻe de Joux, les Mosses, la Lécherette, Château d'OEx.

C. spinorissimum Scop. - Pâturages des Alpes. - Vaud et Va- 
lais : Enzeindaz, val d'llliez, Catogne, Saxon, St-Bernard, Thyon, Arolla, Staffel de Zermatt. - H.-Sav. : Vergy, Méri, Cornettes de Bise, Roc d'Enfer, mont des Granges, plateau des Gets, montagnes de Passy, de Somans, de Morzine et de Sixt, chaine du Ilont Blanc depuis le col de Balme jusqu'au Bonhomme, mont Charvin, Tournette. - Sav. : montagnes de la Tarantaise et de la Maurienne jusqu'au mont Cenis. - Is. : chaines des Sept-Laux, de Belledonne, de Taillefer et de l'Oisans, mont de Rochesac près Mens. - H.-Alp. : Lautaret, Devoluy, Queyras, mont Viso, St-Véran à Clausis, col de Vars, le Champsaur. - B.-Alp. : Lauzannier, Parpaillon, Bérard, Colmars. Alp.-Mar. : lac des Merveilles, vallées de Fenestre et d'Entraunes. - Pyr.-Or. : plateau de Régleille, cnvir. d'Ille, Força-Réal, Baixas, Cases-de-Pena, Salses.

C. spinosissimo heterophyllum Nœgeli. Carduus autureticus Vill. - Valais à Fee.-H. et B.-Alp. : Lautaret à Prime-IIesse, Lauzannier. - Mont Cenis.

C. spinosissimo oleraceum Nœg, - Vaud : Boronnaz, Frachis. - H.Sav. : mont Brizon.

C. spinosissimo acaule Nog. - Vaud : Bovonnaz.

C. glabrum D D. - Bords des ruisseaux dans les Pyr.0r. : StLaurent-de-Cerdans, Canigou, mont de Madres. - Pyr. centrales.

C. hoterophyllum All. - Prés humides des Alpes. - Valais : Saas, Zermatt, Obergestelen. - Is. : le vallon près de la Salette. Sav. : vallée de Tignes, mont Cenis. - H. et B.-Alp. : Lautaret, Larche, Lauzannier. - Alp.-Mar.

C. heterophyllo spinosissimum Noeg. - Prés de Fee en Valais. Il y a lieu de croire que cet hybride est le même que le $C$. spin osissimo heterophyllum mentionné plus haut.

C. heterophyllo acaule Nœg. - Prés des montagnes.

c. seanthifollum Arv. Touv. - B.-Alp. : Larche, Lauzannier, Parc-à-Sac.

C. heterophyllo acanthifolium Arv. Touv. - Mêmes localitẻs.

C. acaule All. - Pâturages des hautes montagnes et des basses collines. 
Une variêté caulescente $C$. Roseni Vill. se prẻsente quelquefois dans les H. et B.Alp.

C. acauli spinosissimum Nog. - Pâturages entre le Lautaret et le Galibier au-dessus des chalets de la Mandette, Cote des Colses en face du Rivet.

C. arvense Scop. - Champs dans tout le bassin.

CariduUs temuinorus Curt. - Bords des chemins de là région méridionale et moyenne.

C. corbariensis Timb. Lagr. - Aude : les Corbières, envir. de Durban vers le pont de la Ricardo, vallon du Ripau, entre Tuchan et Villeneuve, Rivesaltes.

C. corbariensi tenuiflorus Timb. Lagr. - Les Corbières.

c. pyenocephalus L. - Bords des chemins de la région méridionale et moyenne.

C. pycno-tenuiflorus Timb. Lagr. - Aude: Alzonne.

C. neieularis Bert. - Var : le Luc, Cannet du Luc.

C. Personata Jacq.. - Prés et bois. - Hautes Vosges jusqu'aux Ballons de Servance et de Giromagny dans la H.-S. - La chaine jurassique depuis le Creux-du-Van et le Mont d'Or jusqu'à la Dóle, la Faucille, le Reculet, le Crêt de Chalam et jusque dans les montagnes du Bugey. - Vaud et Valais : vallée de la Sarine, les Ormonds, montagnes de Bex et de Roche, Joux de Genêt audessus de Gryon, val d'Illiez, Bourg-St-Pierre, Leukerbad, II.-Sav. : vallée du Reposoir, Mégève, Chamonix. - Sav. : Laval de Tignes. - Is. : Sissenage, Grande Chartreuse, la Salette. H.-Alp. : Mélézet près Guillestre, mont Morgon, Molines près St-Bonnet, Molines en Champsaur. - Nlp.-JIar. : val de Pesio, Ste-Anne-de-Vinaï, Bouziejo aux sources de la Tinea.

C. personato crispus Michalet. - Ain : Crêt de Chalam en descendant sur Chẻzery.

C. personato nutans Gren. - Doubs à Pontarlier.

C. personato defloratus Gren. - Sommet du MIont d'Or, la Faucille, le Warne.

C. crispues L. - Bords des chemins, décombres.

C. crispo nutans Jord. C. acanthoides L. - Lieux incultes. - Envir. de Dịon, de Besançon, de Dóle, de Nantua, d'Autun, de Ljon, de Privas, d'Avignon. - Fyr.Or. 
C. nutans L. - Lieux incultes dans tout le bassin.

La var. C. Amansii Bor, a des feuilles très-rapprochées et hérissée de longues et fortes épines.

C. nigreseens Vill. - Bords des champs et des chemins de la région mérid. - H. et B.-Alp. : Gap à Rioutort, Puy-Maure, StMens, Tallard, la Saulce, Ventavon, Montague-de-Faye, StAndré, Sisteron, Castellane, la Condamine. - Dr. : le Buis, St-Paul-Trois-Châteaux. - Ard. : Entraigues. - Vaucl. : envir. d'Orange, de Carpentras, d'Avignon et d'Apt. - B.-du-Rh. : Cuques. - Var et Alp. Mar. : Toulon, St-Tropez, Antibes, Nice. - Gard : envir. de Nîmes et d'Alais. - Hér. : Lamoure, St-Jean-đe-Védas, Candillargues, le Terral, Lavalette, St-Drézéry, Vendargues, Grabels, St-Etienne-de-Mursan, Béziers, Montagnac. - Aude et Pyr.-Or. : envir. de Narbonne, mont StVictor, Ste-Colombe-sur-l'Aiguelte, Axat, vallée de Vernet-lesBains. - S’étend dans l'Aveyron vers Ste-Affrique.

C. Martrinii Timb. Lagr. - Forme à folioles involucrales toutes dressées-êtalées; se trouve dans l'Hér. à Andabre-Rosis et à Fraisse; dans l'Aude et les Pyr.-Or. à Monbassou et à Rivesaltes. C. australis Jord. - Var : Toulon, le Luc. St-Raphaël.

C. spinigerus Jord. C. hamulosus Ehrh. - Lieux incultes, bords des chemins de la région mérid. - B.-du-Rh. et Var : envir. de Marseille et de Toulon, Vérignon. - H. et B.-Alp. : StMens près Gap, Sisteron. - Gard : Nimes, Tresques, le Vigan, Blandas, Luc, - Hér.-Aude : la Clape, Jonquières, Conques. Pyr.-0r. : Graus-d'Olette, mont Louis, col de la Perche, route de Saillagouse. - En dehors de nos limites dans l'Aveyron à Tournemire, Villefranche, Najac.

C. vivariensis Jord. - Lieux incultes. - Ard. : St-Pierreville, Aubenas, mont Charray, Burzet, les Vans, Vals, Celles, Tournon, le Cheylard, Thueyts, Mayres. - Dr. : St-Vallier. Gard : Luc, Valcroze. - Hér. : Capouladoux, Lunas, St-Martind'Orb, le Poujol, Andabre-Rosis, St-Etienne-de-Mursan, Frouzet, St-Pons, Pardailhan, Montmaur, Mauguio, St-Aunès, - Aude et Pyr.-Or. : les Corbières, base des Albères, entre le Boulou et Céret, d'Arles à Prats-de-Nollo, Pas-del-Llop, vallée de la Tet, Prades, Villefranche, Oletle, Graus, Font-Pédrouse. 
C. Sametre Balmre Lois. - B.-Alp. : Sisteron, Digne, Castellane, - Var et Alp.-Mar. : la Ste-Baume, vallon de Cardeau près Nans, Grasse, l'Esterel, Nice à St-André, l'Escarène, Tende, StMartin-Lantosque.

C. auronicus Vill. - Débris pierreux des montagnes. - II.-Alp. : mont Aurouse entre Matachard et la Grangette, au-delả de FontAlibaud, la Cluse-en-Devoluy.

C. carlinofolius Lam. - Prés et pâturages des montagnes. - H. Alp. : lá Grave, Galibier, Monestier-de-Briançon, St.Véran à la côte Grimaud, col Agnel, mont Donne près Guillestre, Plan-dePhazy, côte Gélive près Gap, mont Aurouse, Chaillol-le-Vieil. - B.-Alp. : Lauzannier, Vallonnet, Bérard, Barcelonnetle, Seyne, Colmars. - Alp.-Mar. : vallon de Fenestre, St-MartinLantosque, val de Jallorgues. - Vaucl. : mont Ventoux, Var : N.-D.-des-Anges près Pignans, Hontrieux. - Pyr.-Or. : vallée de Llo près de la Jasse du collet de Dalt, vallée d'Eyne, Font-Romeu près mont Louis, Cases-de-Pena. - Pyr. centrales.

C. defloratus L. - Bois des terrains calcaires. - Nul dans les Vosges, les Cérennes et le massif central. - Région des sapins de la chalne jurassique, jusque dans les montagnes du Bugey. - C.-d'Or : val Suzon, val Combe, Francheville. - Vaud et Valais : Dent de Jaman, val d'Illiez, Branson, Leukerbad, Zermatt. - H.-Sav. : Salève, col des Aravis, chalets de Flaine, St-Clair, Parmelan, mont Tournette, mont Charvin, Semnoz, le Moie. - Sav. : la Sambuy, Hauteluce. - Is. : Grande-Chartreuse, Chamechaude, Casque de Nẻron, St-Nizier, col de l'Arc, descend jusqu'au polygone de Grenoble. - II. Alp. : Monestierde-Briançon, Lautaret, mont Chabriêres, col de Vars, Charance, Rabou, Pleyne-de-Chaudun, col de Glaise, mont Séuse. B.-Alp. : la Condamine, St-Paul, le Lauzannier. - Alp.-Mar. : Ste-Anne-de-Vinaï. - Pyr. centrales.

C. mediu Gouan. - Prairies des Pyr.-Or. : le Canigou, entre la Preste et Costa Bona, Peyrefeu, mont de Flotte à Arles. - Pyr. centrales.

C. carlinoides Gouan. - Débris rocheux des Pyr.-Or. : vallée d'Eyno, Costa Bona. - Pyr. centrales. 
Carduncellu» mitıssimus D C. - Pâturages, champs incultes des terr. calc. de la région mérid. - Gard : le Vigan, Campestre, Salbous, Lanuejols, Alais, Anduze, St-Ambroix. - Hér. : la Sérane, le Larzac au Cros, Sorbs, le Caylar, Serre-d'Oupia, Joncels, Pardailhan, - Aude : Axat, au-dessus de Quillan, Pierre-Lisse, Montolieu, Cenne, - Pyr.-0r. : vallée du Réartvers Passa et Monestir, Opols, Cases-de-Pena. - En dehors de nos limites à St-Béat et sur les calc. jurassiques de la Lozère, du Tarn et de l'Aveyron.

C. monspeliensium D C. - Coteaux pierreux des terr. calc. de la région mérid. - Dr. : Crest, Montélimar. - H.-Alp. : envir. de Gap à Charance, les Serigues, St-Mlens, Malecombe, col de Glaise, col Bayard, Tallard, Moydans, St-Romans. Vaucl. : envir. d'Apt aux Barbiers et à Rustrel, Mormoiron, Luberon, mont Ventoux, le Sault, Gigondas. - B.-du-Rh. : Beaurecueil, Puyloubier, Pic de Bretagne, Aix à la Lèqque, StHippolyte, Trévaresse. - Var et Alp.-Mar. : Ste-Baume, Morières près Toulon, Suscolles, au-dessus de Luceram, Utelle, bois de St-François près de Grasse. - Gard : Campestre, Sommières, Montpezat. - Hér. : Prades, St-Gély, Grabels, StMathieu, Montarnaud, St-Martin-de-Londres, Valflaunès, Claret. - Pyr.-0r. : Pont de la Fou près St-Paul, Prats-de-Mollo.

RII PONTICUM eymaroides Less. - Coteaux pierreux des Pyr.-0r. : St-Laurent-de-Cerdans, Canigou, Font-Romeu, lo Llaurenti. - Pyr. centrales.

R. heleniffoltum G. G. - Rocailles calcaires. - H.-Alp. : fort des Salettes à Briançon, Granon près Val-des-Prés, pic de Chabrières, mont Queyrel-en-Champsaur, col de Glaise, mont Aurouse. - Pyr.-Or. : bois de Salvanère.

R. meariosum Lam. - Rochers siliceux des montagnes. - Vaud et Valais : Alpes de Morcles et de Fully, Alesse, Zobourlaye, Zinal d'Anniviers, Conches, Gerenthal. - H.-Sav. : la Diosaz, Tré-la-Téte. - Sav. : vallée des Allues, le Couard au bas de la Louse. - Is. : St-Hugon près Allevard, entre la Pra de Belledonne et l'Oursière, rochers de Bâton au-dessus de Livet, la Bérarde, au pied du mont Chamoux en Valjouffrey, - B.-Alp. : 
Salces au-dessus de l'Argentière. - Alp.-Mar, : vallon de RioFreddo au-dessus de Tende, Ste-Anne-de-Vinaï.

CENTA Q dans la région mérid. - Espèce polymorplıe dans laquelle la coulcur de l'involucre, la forme des feuilles et la taille de la plante sont très-sujets à varier.

C. serotina Bor. - C.d'Or.: Montbard. - S.-et-L. : St-Forgeot près Autun. - Rh. : Tassin, Couzon, Denicé, St-Julien-sur-Montmélas.

C. slbar L. - Espèce d'Italie naturalisée à Lyon sur les talus du chemin de ronde des Brotteaux.

C. Ineea L. - Prés, bois dans tout le bassin. - Espèce polymorphe.

C. Duboisii Bor. - Environs d'Autun et de Lyon.

c. nigreserns Willd.-Prairies de la région mérid.-Gard : Concoule. - Ilér. : St-Geniès de Varensal, Castanet-le-Haut, St-Etiennede-Mursan, St Chinian à Poussarou. - Pyr.-Or. : bois de Salvanère. - Apparait quelquefois accidentellement dans la région moyenne du bassin.

C. siggra L. - Prés des terrains siliceux des montagnes. - Commun dans la chaine des Vosges. - Terr. siliceux du Morvan, monta. gnes du Beaujolais, du Lyonnais, du Pilat, du Vivarais, des Cévennes, de l'Espinouse, de l'Escandorgue, des Pyrénée:, de Ia Montagne Noire, Aubrac, Cantal, mont Dore, mont Dômes, Forez. - Espèce polymorphe.

C. nemoralis Jord. - Bois des terrains siliceux des plaines et basses collines. - C.-d’Or, S.-et-L., la Bresse, forêts de Chaux et de la Serre, la Dombes, le Lyonnais, le Vivarais. - Envir. de Genève au bois des Frères. - Is. et Dr. : envir. de Crémieu et de StVallier. - Hér. : Mas de Mouriès, Paulhan, St Pons.

C. tubulosu Chabert. - Rh. : Brignais aux Ronzières, Charbonniẻres.

C. decipiens Thuill. - S.-et-L. : bords de l'étang de Poisson près

Mâcon. - Rh. : St-Julien-sur-Montmélas.

C. nigro solstititialis G. G. - Pyr.-Or. : Trencada d'Ambulla, Cornelladu-Conflent, Vernet-les-Bains.

C. proeumberns Balb. - Alp.-Har. : Tourrette, Levens, Duranv"

Utelle.

Catal. Bassin du Ahone. 
C. Jordanlann G. G. - B.-Alp. : Annot.

C. pectinata L. - Lieux incultes de la région mérid. - Dr.: le Buis, Bellecombe, Nyons, Saou près Crest, Montélimar.-Ard. : Tournon, Vals, Entraigues, Thueyts, Cuze, remonte jusqu'au Gerbier-des-Joncs. - Gard: Nimes, Alais, Anduze, AiguesMortes, Salbous, l'Espérou. - Hẻr. : envir. de Montpellier, de Béziers et de St-Pons. - Aude : envir. de Mas-Cabardés, Cuxac, col d'Estrem, Fontlaurier, Fontfroide, la Clappe, Ste-Lucie. Pyr.-Or. : bords de la Basse, Rigarda, Trencada d'Ambulla, StMartin-du-Canigou, Graus d'Olette, Fontpédrouse, Mont Louis, Boulou, Céret, Arles, Prats-de-Mollo, Baus de l'Aze. - Vaucl. et B.-du-Rh. : Bédoin, Flassan, Piolenc, Uchaux, Gigondas, Lafayette de la Garde, les Alpines. - Alp.-Mar. : Levens. - En dehors de nos limites dans la Lozère et l'Aveyron.

C. rufescens Jord. - Ard. : mont Combier, la Voulte, Tournon, Vals, Mezenc, Gerbier-des-Joncs. - Loire : Rochetaillée, le Pertuiset, St-Rambert et St-Just-sur-Loire.

C. acutifolia Jord.-Ard.: Mayres. C. supina Jord. - Nimes, Uzès.

C. comata Jord. - Ibid. C. fuscata Jord. - Ard. : Thueyts, Neyrac.

c. uniqora L. - Prairies des hautes montagnes. - Sav. : entre

Laval de Tignes et la Galise, mont Iseran, mont Cenis, mont de Frôjus. - Is. et H.-Alp. : la Salette, Lautaret, Névache, mont Genèvre, mont Pelvoux, mont Viso, Bramousse au-dessus de Château-Queyras, Guillestre, col de Vars, mont Morgon, forêt des Andrieux en Valgaudemar, mont Chabrières, Orcières, Champoléon, Chaillol, Pleyne-de-Chaudun, col de Glaise, mont Séuse. - B. Alp. : Lauzannier, Longet, Bachasse, Colmars. Alp.-Mar. : Commun dans les montagnes. - Pyr. Or. : Carença en allant vers Nuria.

Il est digne de remarque que cette Centaurée ne s'étend pas dans les Alpes au nord de la Galise; elle manque complètement dans les montagnes de la Suisse, du Tyrol, Salzburg, Styrie, Carinthie.

D. mervona Willd. - Prairies des hautes montagnes. - Vaud et Valais : Boronnaz, Surchamp, St-Bernard, Zermatt, Saas, Sim- 
plon. - H.-Sav. : mont Méri, Parmelan, Charvin, Tournette, col des Aravis, Dents d'Oche, mont Laouet, cols de Balme el de Voza, chaine du mont Blanc, mont Joly. - Sav. : Pralognan, Champagny, Pesey, Macot, Hauteluce, Roselein, Bonhomme, la Sambuy. - Is. : chatnes de Belledonne et de l'Oisans, Monestier-de-Clermont. - II.-Alp. : montagnes du Queyras, mont Viso, val Préveyre, col de Vars, mont Durbonnas. - B.-Alp. : Longet, Lauzannier, Bérard. - Alp.-Mar. : N.-D.-de-Fenestre. - Pyr.-Or. : de Prats-de-Mollo à la Preste, vallée de Carença, Mont-Louis. - Espèce polymorphe.

ambigua Thomas, C. helvetica Gaud., C. Ferdinandi Gren. - Lieux pierreux. - Is. : entre les Etages et la Bérarde-en-0isans. Sav. : entre Laval-de-Tignes et la Galise, St-Sorlin-d'Arves. Alp.-Nar. : Colmiane, vallon de Nanduébis, la Briga.

c. pullata L. - Terrains incultes de la région mérid. - Is. et Dr. : Vienne, Valence, Montêlimar. - Gard et Hêr. : Campestre, de Grabels à Villeneuve, Jeu-de-Nail, la Iladeleine, Bione, Lavérune, St-Jean-de-Védas, Maurin, St-Brès, la Planchude, Fabrègues. - Pyr.-Or. : vallée du Réart, Pollestres, - Trouvé près de Toulon par M. Huet.

c. montana L. - Bois et prairies des montagnes. - Chatnes vosgienne et jurassique jusque dans les montagnes du Bugey. - Vaud et Valais : Alpes de Bex, val d'Illiez, Trois-Torrents, Champé, Saxon, Sanetsch, Gemmi. - H.-Sav. et Sav. : Mole, Salève, Dents d'Oche, col des Aravis, Semnoz, Tournette, Mieussy, Charvin, pavillon de Bellevue près Chamonix, mont Iseran, mont Cenis, Entre-deux-Eaux. Dent-de-Nivolet, les Bauges. - Is. : massifs de la Grande-Chartreuse, de Belledonne, de l'Oisans, de la Salette. - Dr. : mont Glandasse, col de Menée. - H.-Alp. : Lautaret, Bramousse au-dessus de ChåteauQueyras, mont Viso, col de Vars, mont Morgon, Boscodon, envir. de Gap, Pleyne-de-Chaudun. - B.-Alp. : Larche, col de la Madeleine, Malemort, Maison-Méane, Parpaillon. - Alp.-Mar.: toutes les montagnes de Grasse, Vence, Nice, Menton. C.-d'Or : sur toutes les hauteurs de la chalne jurassique. Ard. et H.-L. : Mezenc. - Gard : Serre-de-Bouquet, Barjac, 
Blauzac, Chartreuse de Valbonne, Salbous, vallon d'Aulas près du Vigan. - Hẻr.: Pic St-Loup, la Sérane, Madières, St-Raphaël près Bédarieux, St-Pons, l'Escandorgue à Pertus. - Aude et Pyr.-Or. : Montolieu, Martis, les Corbières à Massac, vallée de Nohèdes, St-Antoine-de-Galamus. - D'après M. Roux, les localités indiquées dans les B.-du-Rh. se rapportent, non au C. montana, mais au C. semidecuryens Jord. - En dehors de nos limites, existe dans les Pyrénées centr., l'Aubrac, Lozère, Cantal, mont Dore, mont Dôme, Forez.

C. Iugduneneis Jord. - Coteaux boisés. - Ain et Rh. : monts d'Ain, entre Retord et Châtillon, la Pape, Couzon. - Is. : mont d'Annoisin près Crémieu.

C. intermedia Cariot. - Forme du précédent à feuilles moins longuement décurrentes, aigrettes à poils plus courts. $-\mathrm{Rh}$. : Chaponost sur les coteaux du Garon, - Is. : plateau de Salat au-dessus de la Bastille de Grenoble, gorges d'Engins. - Ard. : Rochemaure.

C. memidecurrens Jord. - H. et B.-Alp. : St-Genis-le-Désolé près Serre, Sapet de la Bàtie-Neuve, mont Rognouse près Ribiers, Sisteron. - Vaucl. et B.-du-Rh.: St-Amans, St-Paul-de-Durance, entre Vauvenargues et Rians. - Var et Alp.-Mar. : SteBaume, Chartreuse de Montrieux, St-Etienne. - Aude : MasCabardès, la Tourrette.

C. axillaris Willd. - Pâturages des hautes montagnes. - Valais : Simplon, envir. du glacier d'Aletsch. - Is. et H.-Alp. : mont de Lans, la Bérarde-en-0isans, mont Viso, col Agnel, col de Ruines. - B.Alp. : vallon du Châtelet près Sérennes, Longet, Malemort, Horonaye. - Alp.-Har. : col de Tende, Colmiane, vallons de Nanduébis près de St-IIartin, de la Salèze et de la Madone.

C. Meusana Chaix. - Prairies des montagnes calcaires. - Ain : le Poizat au-dessus de Nantua. - H.-Alp. : mont Séuse, envir. de Gap au bois Mlondet, à Bayardon et au col de Glaise. - Vaucl. : mont Ventoux près la Font-de-Canau. - Var et Alp.-Ilar. : entre Nans et la Ste-Baume, Aiguines, Ampus, les Mayons, mont Lachen. 
C. Cyanue L. - Champs dans tout le bassin.

C. Serolow I. - Champs dans tout le bassin. - Espèce polymorphe.

C. calcrier Jord. - Forme dont les feuilles ont des segments lancéolés, entiers. - Vaucl. : Flassan.

C. alpestris Hegetsch., C. Kotschyanu Heuff. - Formes à calathides plus grosses, à cils des folioles plus longs. - Prairies. - Sommités de la chaine jurassique. - Sav. et H.-Sav. : mont Trélod, la Sambuy, mont Cenis, le Chapeau près de la Mer-de-Glace. Is : St-Christophe et la Bérarde-en-Oisans, - H.-Alp. : Lautaret, mont Viso, Bramousse-en-Queyras, Abriès, les Roux, Valpréveyre, col de Vars, col de Glaise, Pleyne-de-Chaudun.

C. silvatica Pourr. - Hybride du $C$. scabiosa et du $C$. paniculata. Aude : Castelnaudary, Durban.

C. cinerea Lam. - Espèce d'Italie trouvée au Baou-Roux, près de Villefranche et à Luceram (Alp. Mar.).

C. semżerviens L. - B.-du-Rh. et Var : les Martigues, StJulien près Marzeille, Martegaux, Baou-de-Quatre-Heures près Toulon.

C. Intybacea Lam. - Rochers calcaires de la région mérid. B.-du-Rh. et Var : île de Maïré, St-Michel-d'Eau-Douce, mont Faron, mont Coudon. - Hér. : Cruzy entre St-Chinian et Capestang. - Aude et Pyr.-Or. : Ste-Lucie, la Clape, Cases-dePena, Estagel, St-Antoine-de-Galamus, El-Hoto-de-Corbère, Calce-de-Thuir.

C. corymbona Pourr. - Rochers calc. - Aude et Pyr.-Or. : Clapeau-Rec, Las Portes, Montbassou, Prades, Villefranche.

C. maculoan Lam. - Rochers. - Dr. : Gigors près Crest. - Ard. : Vals. - Vaucl. : St-Amans, Ville, Flassan. - Mlp.-Mar. : SteBaume près du cap Roux, Esterel, Stc-Anne-de-Vinaï. - Gard : le Vigan, Montdardier, Valleraugue, bois de Broussan près Nimes, Bérias, Anduze. - Hér. : Pégayrolles-de-l'Escalette, StMaurice, le Caylar. - En dehors de nos limites dans l'Aveyron, la Lozère, H.-Loire, Puy-de-Dôme, Loire, Allier. - Espèce polymorphe.

C. tenuisecta Jord. - Feuilles profondément découpées en lanières 
linéaires, appendice à cils blancs. - Rh. et Loire : vallon du

Mornantet, Givors, Rive-de-Gier. - Vaucl. : Bedoin.

C. subalbida Jord. - Ard, : les Vans,

C. Mureti Jord. - Valais : Schuols.

C. coerulescens Willd. - Bois et lieux arides de la région mérid.

- Gard : le Vigan, Blandas, Aulas. - Pyr.-0r. : Collioure, Banyuls-sur-Mer.

C. Hanrll Jord. - Bois. - B.-du-Rh. et Var : Ste-Victoire, SteBaume, mont Sauvette et mont Freinet.

C. Ieucophren Jord. - Lieux incultes. - Is. et H.-Alp. : Grenoble sur les digues du Drac, Briançon, Guillestre, Embrun, Chorges, Tallard, Serres, Gap à la Garde, à Puy-Maure, à Rambaud, Rabou, Chaudun, St-Mens, la Saulce, Roche-des-Arnauds. B.-Alp. : la Condamine, Tournoux, Bouzolières, Castellane, Sisteron. - Vaucl. : envir. d'Orange', alluvions de l'Eygue, Flassan. - Var et Alp.-Mliar. : Châteaudouble, Grasse, Nice, Menton. - Pyr.-Or. : Prades, Graus-d'Olette, Canaveilles.

C. diffusa Lam. - Champs, - B.-Alp. : Beauvezer. - B.-du-Rh. : Aix à la plaine des Milles, Marseille. - Espèce étrangère trouvée aussi au port Juvẻnal près Montpellier.

C. paniculata L. - Terres incultes dans tout le bassin. - Espèce polymorphe.

C. valesiaca Jord. - Capitules une fois plus gros, folioles de l'involucre à cinq nervures très-saillantes, appendice brun, large. Coteaux incultes. - Valais : Branson, mont d'Orge, Sion, StLéonard, Sierre, Leuk. - Sav. : Termignon, Lans-le-Bourg.

C. paniculato calcitrapa Loret. - Hér. : Bédarieux, Lattes à Maurin.

C. polycephala Jord. - Lieux incultes. - B.-du-Rh. et Var :

Marseille, Montaud-les-Miramas, le Luc, Rians, Montrieux.

C. rigidula Jord. - Lieux incultes. - Var et Alp.-Mar. : St-Raphaël, Antibes.

C. collma L. - Champs cultivés, vignes. - Ard. : Chomérac, StPierreville, Payolive, mont Charray, Rochemaure. - Dr. : Die. - Vaucluse : envir. d'Avignon aux Agassins, le Pontet, Morières, envir. d'Orange, de Carpentras et d'Apt, Flassan. - Var et Alp.-Har. : Touris près Toulon, le Luc, Grasse, Antibes, 
Nice entre Eza et la Turbie. - Gard : Nimes, Manduel, AiguesMortes, Alais, Anduze, St-Ambroix, le Vigan. - Hêr. : envir. de Montpellier. - Aude : envir. de Narbonne à la Clape, Fontfroide, Nissan, Ste-Lucie, Sigean, Junquières, Montfort, Bigarras, Villespy, Conques, Durban, Villeneuve. - Pyr.-Or. : envir. de Perpignan à la Passion-Vieille, Sarrat-d'en-Vaquer, Orle, Baixas, Cases-de-Pena, Estagel, Maury, St-Paul, Prades. Serdinya, Olette.

C. mapifolln L. - Espèce de Corse trouvée accidentellement près de Marseille et de Toulon.

C. monchifolin L. - Coteaux calc. de l'Aude et des Pyr.-0r. : lle de Leucate, Cases-de-Pena, Baixas, Força-Réal, Salses, Opol et toute la chaîne calc. jusqu'à Tautavel.

C. aspera L. - Champs incultes, bords des chemins. - Ain et Is. : iles du Rhône en amont de Lyon, Neyron, Hiribel, Vertriou, Vienne. - Loire : bords du Rh. à Chavanay. - Dr. : Laveyron près St-Vallier, Romans, Crest, Saillans, Montélimar. - H.-Alp. : Tallard, la Saulce. Partie mérid. de l'Ardèche. - Commun dans la Provence, les Alp.-Mar, le Languedoc et le Roussillon. Espèce polymorphe.

C. pretermissa Martr. Don. - Dr. et Vaucl. : Nyons, Valréas, Carpentras. - Ard. et Aude : envir. de Privas et de Narbonne. - Var :

Sablettes de Toulon. - En dehors de nos limites dans le Tarn.

C. Mierghii Jord. - Ard. : les Vans, Joyeuse.

C. aspero calcitrapa G. G. - Gard : Tresques, Manduel, Monterin. Hér. : Montpellier à la Citadelle, Grammont, Rieucoulon, Montels, Castelnau, Lavérune, St-Aunès, Lansargues, Banquières, Vauguière, Béziers, Sérignan, St-Etienne-de-Gourgas.

C. calcitrapo aspera G. G. - Gard : Nimes, Bellegarde, Comps, Manduel, Montérin. - Hér. : envir. de Montpellier, de Béziers et de Pézenas. - Pyr.-0r. : Perpignan, Malloles, Baixas, Cases-dePena.

C. sordidn W. Kit. - Espèce de la Croatie, Istrie, Dalmatie, trouvéo près de Greoulx (B.-AIp.).

C. CaIcitrapa L. - Lieux incultes dans tout le bassin. - Espèce polymorphe. 
C. Pourretiana Timb. Lagr. et Thév. - Aude : Bouscaillon près Durban, Villeneuve.

C. myacanutha D C. - Bords des bois. - Gard : Vallescure, sur la route de Bellegarde à Beaucaire. Trouvé accidentellement ả Lyon et dans le Jura à Crissey, près Dole, entre Amans et Longwy.

đ. meihtensis L. - Lieux incultes de la région mérid. - B.-duRh. et Var : Roquefavour, Istres, Arles, Toulon, île de Porquerolle, Frêjus. - Gard : bois de Broussan près Nìmes, Roquecourbe près Marguerite. - Hér. : Lavalette, Castelnau, le Crès, Hontmaur, Cauneile, St Jean-de-Védas, Montbazin, Frontignan, Prades, Cetle, Agde. - Aude : envir. de Narbonne, Pech-del’Agnèle, la Clape, Grissan, Fontfroide, Campane, Ste-Lucie, Sidrière de Fitou, Bouscaillou près Durban, vallon du Ripau. Pyr.-Or. : Banyuls-sur-Mer, la Roca, St-Génis, le Boulou, Maureillas, Font-Estramer près Salses, Vingrau. Trouvé acciden ${ }^{-}$ tellement à Lyon, à la silouche.

C. solstittalis L. - Bords des champs de la région mérid. et moyenne du bassin. - Apparait accidentellement dans les luzernières de la région septentrionale; on le voit même élajıli depuis longtemps autour de Dijon.

C. subalbida Jord. - Vaucl. : colline d'Orange. - Ard. : les Vans, Banne.

C. nícaensis All. - Espèce d'Italie et d'Espagne trouvée accidentellement autour de Marseille, de Toulon et de Nice.

Plusieurs autres Centaurea ont été observés autour des villes susdites, ainsi 'qu'au Port Juvenal, près de UIontpellier. On en trouvera l'indication dans le Florula massiliensis advena de Grenier et dans le Florula juvenalis de M. Godron.

MrCRononChIS samanteas D C. - Lieux incultes de la région mérid. - Vaucl. et B.-du-Rh. : Lafare, le Thor, La Rose près Narseille, envir. d'Aix. - Var : Fréjus. - Gard : envir. de Nimes, Aigues-Mortes, le Vigan, Alais, Anduze, StAmbroix. - Hér. : envir, de Montpellier, de Béziers. - Aude : Pech-Redon à la Clape, Salsignes, Conques, Cenne. - Pyr.-Or. : envir. de Perpignan à St-Sauveur et Chàteau-Roussillon, entre Vingrau et Rivesaltes, Cases-de-Pena, Força-Rẻal, vallẻe du Réart. 
9. Clusin spach, - Commun dans l'Hérault,- Trouvé par M. Roux à la Pomme (B.odu-13h.) et par M. Huet à foulon.

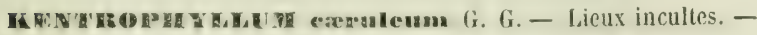
B.-du-Rh. et Var : St-Julien près Marseille, Ste-Marguerite et les Pradets près Toulon. - Pyr,-Or. : pied des Albères, le Boulou, Céret, Cases-de-Pena, Prades, Molitg.

K. Invatum D C. - Licux incultes dans la région mérid. Remonte dans l'Aril. vers l'Eserinet, Aubreyts, Chassagne, Privas; dans la Dr. vers Pierrelatle, Crest, St-Vallier; dans les II.-Alp. autour de Gap.; dans l'Is. et le Rh. vers Varces, Lyon, Crémieu; dans S.-et-I. à Sampigny, la cote jurassique et le Pays-Bas de la C.-d'Or; entin, dans le bassin du Léman, Gaillard près Genève, Nyon, Sulry, Pandex, Préverenge, Buchillon, Clarens.

Cricus benedictus L. - Lieux incultes de la rigion mérid. Vaucl. et B.-du-Rh. : envir. d'Avignon, d'Orange, de Carpentras, d'Apt; d'Aix et de Marseille. - Var et Alp.-Mar. : Toulon, Hyères, le Luc, Pierrefeu, Grasse, Cannes, Tanneron en face d'Auribeau. - Gard et Hér. - Aude et Pyr.0r. : envir. de Narbonne, Pech de l'Agnèle, Pont de la Fou près St-Paul, Ornaisons, Axat au-dessus de Quillan, butte du Moulin-à-Vent près Perpignan, Collioure, Passa, Villemolaque, le Boulou, vallée du Réart.

CrIPIN vulgris Cass. - Coteaux calc. - Valais : Branson, Nazembre, Folateres, Fully, mont d'Orge, St-Léonard, Grange, Salgesch. - Ain et Rh. : Culoz, St-Sorlin, la Pape, Caluire, Oullins. - Is. : Crẻmieu, Bastille de Grenoble, St-Eynard, Comboire. - Sav, : Montmélian. - H.-Alp. : Charance, StMens, Puy-llaure, Tallard. - Dr. : St-Vallier, Livron. Vaucl. : Avignon, Orange, Malaucène, Flassan, Mlazan, Apt à la Cucurone et au fort de Buoux. - B.-du-Rh. : envir. d'Aix et de Marseille. - Var et Alp.-Mar. : Toulon, le Luc, Rians, Grasse, Nice, Menton. - Ard. : St-Etienne, Aubenas, rochers de Jastre. - Gard : Nimes, Labeaume, Alais, Anduze, Uzès, le Vigan, Alzon. - Commun dans l'Hér. - Aude et Pyr.-Or. : garrigues des envir. de Narbonne, Pech de l'Agnèle, Pas-du-Loup de Si- 
gean, toutes les basses Corbières, Estagel, Cases-de-Pena, Baixas, Força-Réal, St-Sauveur, Perpignan, Prades, Villefranche. - En dehors de nos limites sur les calc. jurassiques de la Lozère, et de l'Aveyron.

C. brachypappa Jord. - Sion en Valais, Gap, Briançon.

SERFATUMA tiuctorin L. - Bois et prés. - Chaine des Vosges et du Jura depuis les collines jusqu'à la région des pâturages, bords du Doubs, forêt de Chaux, Etrépigney, Salins, Champagne, St-Laurent, Boujeailles, Pontarlier, la Dôle, le Colombier, le Reculet, pâturages du Bugey. - S.-et-L. : bords de la Saône et de la Seille. - Envir. de Genève au bois de la Bâtie et des Frères. - Vaud et Valais : Mathod, Prangins, Sublin, Vervey, la Grange. - Envir. de Lyon, de Gap et de Crest, et de Privas. - Is. : Rochefort près le Pont-de-Claix, Comboire, mont Rachais. - B.-du-R. : marais de la basse Crau, Berre. - Var et Alp.-Mar. : Maures du Cannet, les Mayons, Bezaudun, pont de Tournon sur la Siagne, Menton. - Gard : envir. de Nimes, d'Alais et du Vigan. - Hér. : Mauguio, St-Marcel, entre Montarnaud et la Boissière, Lodève. - Aude et Pyr.-Or. : envir. de Narbonne, Vernet-les-Bains, Céret, bois de Boucheville et des Fanges, le Capcir.

Var. montana Bor. Capitules plus gros que dans le type, presque sessiles. - Chaine du Jura à la Dole, au Colombier et au Reculet. - H.-Sav. : montagnes du Chablais, Dents d'Oche, Cornettes de Bise, mont des Granges, mont Brizon. - Is. et H.-Alp.: Chamechaude, petite Moucherolle, la Salette, env. de Gap aux cols de Glaise et de Bayard, Chaudun. - Ard. : le Mezenc. - En dehors de nos limites à Pierre-sur-Haute et au Montoncelle dans la Loire.

5. heterophyila Desf. - Prairies des montagnes. - H.-Alp. : Lachaud entre Gap et Tallard, Ventavon à Berthaud, Laric, Ose près Veynes, prairies du Quint entre Gap et la Roche-desArnauds, prairies du Peynier au-dessus de Barcelonnette de Vitrolles. - Alp.-Mar. : montagnes de Caussols, de Thorenc et du Cheiron. - Pyr.-Or.: montagnes du Caillau de Mlosset et toute la vallée jusqu'à Jau, envir. du bois de Salvanère. 
5. mudicaulis D. C. - Pàturages des montagnes calcaires. II.-Sav. : Salève au-dessus d'Archamp, mont Vuache. - Is. et H.-Alp. : mont Chamoux au-dessus de la Salette, Charance, Pleyne-de-Chaudun, mont Séuse aux Fays, montagne de Faye au-dessus de Ventavon, Annot au col de Vergons. - Vaucl. et B.-du-Rh. : mont Ventoux, sommet du vallon de Nicolas près du Pic de Bretagne. - Var et Alp.Mir. : Châteaudouble, Caussols, col des Champs entre Entraune et Colmars, mine de Tende.—Gard : St-Mlichel-dei-Sers. — Hèr. : la Sérane, Lozère sur le Causse-Méjean.

JU RINEA Bocconl Guss. - Pâturages de la région mérid. B.-du-Rh. et Var : l'Étoile, Pilon-du-Roi, Ste-Baume, sommet des Béguines, St-Cassien. — Gard : entre Campestre et le bois de Salbous. - Hér. : Sorbs. - Pyr.-0r. : entre Rabouillet et Sournia, bois de Boucheville. - En dehors de nos limites à Sauclières dans l'Aveyron.

J. pyrenaica G. G. - Différe du précédent par des calathides plus petites, à écailles toutes appliquées. - Pyr.-Or. : vallée d'Eyne près du Pla de la Baguda et sur le revers de la Collada de Nuria. - Pyr. centrales.

LEUZEA conifera D C. - Débris pierreux calc. - Rh. : Couzon au mont d'Or. - Sav. : St-Julien-en-Maurienne. - Is. : mont Rachais, Comboire, Varces, Roissard près du Monestier-de-Clermont. - H.-Alp. : Rosans, Fouillouse, Ventavon, Eymeyères, la Garde, Roche-des-Arnauds. - Dr. : Divajeu près Crest, Nyons. - Vaucl. et B.-du-Rhı. - Var : le Luc, Rians, Grasse, Nice, la Turbie, Menton, Saorgio, la Briga, St-Martin-Lantosque. Ard. : Pont d'Arc, rocher de Jastre, vallée de l'Ouvèze, la Voulte, Celles, Cruas et la côte du Rhône. - Gard : Ntmes, StGilles, Beaucaire, Villeneuve, Manduel, Tresque, le Vigan, Alais, Anduze, Uzès, Vacquières. - Hér. : Montpellier au Chàteau-d'Eau, la Colombière, Lamoure, Fontfroide, Lavalette, Grabels, Pic St-Loup, Restinclières, St-Gély, les Cambrettes, St-Martin-de-Londres, Cette, Balaruc, St-Pons, Avène, le Larzac à la Vacquerie. - Aude : envir. de Narbonne, Pech-de-l'Agnèle, la Clape, Gruissan, Sigean, Ste-Lucie, Sidrière-de-Fitou, 
Bouscaillou près Durban, vallon du Ripau, Conques, Villespy.

- Pyr.-0r. : Cases-de-Pena, Estagel, St-Antoine-de-Galamus, Trencada d'Ambulla, N.-f).-de-Vie. - En dehors de nos limites dans le Tarn et l'Aveyron.

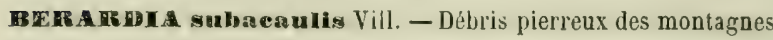
calc. - Is. : entre le mont Aiguille et le Grand-Vehmont. H.-Alp. : Val-des-Prés à la Roche-Gautier el à la Lauze, col des Hayes, col Isoard, Cucullet près Guillestre, St-Véran à Marcel, cols de Glaise et de Chaudun, la Grangette, Orcières, col du Devoluy au Noyer, mont Horgon, Pic de Chabrières, Sapet de la Bâtie-Neuve. - B.-Alp. : Bachasse, Parpaillon, Vallonnet, Bérard, lac de Praroard près Maurin, col de Fours, Colmars. Alp.-Har. : éboulis de la Grande-Roche au val de Strop audessus d'Entraunes, entre St-Dalmas-le-Sauvage et Bouziejo.

SAUSSU Re depresga Gr. - Rochers des Alpes. - H.-Sav. : mont Méri au Château. — Sav. : mont Cenis, les Aiguillesd'Arve. - Is. : les Grandes-Rousses. - H.-Alp. : le Goléon, les Trois-Évêchés, Grand Galibier, mont Viso, Orcières, lac de Jajaud. - B.-Alp. : Bachasse, Parpaillon, entre Bouzolières et Bérard, cime du vallon de Riou-German. - Alp.-Mar. : sommet du col de Jallorgues.

Var. leucantha Jord. : les Trois-Évêchés (H.-Alp.).

5. macropkylin Saut. - Rochers calc. des Pyr.-Or. : Font-deComps, inont de Madrés sur le versant du Capcir, vallée d'Eyne. - Rochers calc. des Pyrénées centrales.

9. alisealoz D C. - Rochers siliceux des Alpes. - Valais : vallée de Binn, mont Stock en Conches, entre le Simplon et Gondo sur le versant italien. - Is. : chaine de Belledonne vers le lac Crouzet. la Grande-Lance, sur les rochers au-dessus du petit Domeinon, envir. du lac de la Sitre, col Oddie au-dessus de Theys.

s. a!nima D G. - Rochers des Alpes, - Vauil et Valais : Grandvire, Branleire, Fully, St-Bernard, au-dessus du Jardin du Valais, entre la Combaz et Tschalaire, Zermontana, Hornli et Staffel de Zermatl, mont Stock-en-Conches, val Tournanche sur le versant italien. - H.-Sav. et Sav. : moraines du glacier de Tré-la-Tête, col de la Seigne, les Allues au sommct du Borgne, mont Cenis. 


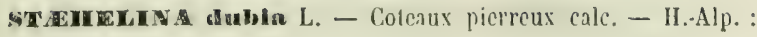
envir. de Gap à Charance, N.-D.-du-Laus, St-Mens, Lettret près Tallard. - Dr. : Nyons, entre Crest et Cabonne, - Vaucl. : Bédoin, Flassan, Sérignan, St-Dílier, Gadagne, bois de St-Jean près Avignon, envir. d'Apt à Viens et Roussillon. - B.-du-Rh. : Aix à Monteiguet. - Var et Alp.-Mar. : T'oulon, île de Porquerolles, le Luc, Iréjus, ile Ste-NIarguerite, l'Escarène, Villefranche, Menton. - Ard. : Cruas, Vallon. - Gard : envir. de Nìnes à Marguerite, la Roque, St-llichel-d'Euzet, Chlartreuse de Valbonne, Palière près Anduze, Vacquières. - Hér. : la Colom. bière, Caunelle, la Paillade, Grabels, St-Gély, entre St-Georges et Murviel, Mireval, Teyran, Cette, Capouladoux, entre StGervais et Hérépian, Rabieux près Lolève, Pézenas, Béziers, St-Étienne-de-Gourgas, Olargues. - Aude : envir. de Narbonne à la Clape, Pech de l'Agnèle, Fontfroide, Cap de Pla, Villespy. Pyr.-0r. : Cases-de-Pena, Força-Rẻal, St-Antoine-de-Galamus. - En dehors de nos limites sur les calc. du Tarn et de l'Aveyron.

CHAMAPEUCE Canabana D C. - Cette espece de Corse et de Sardaigne se trouve en France dans l'lle du Levant (Var).

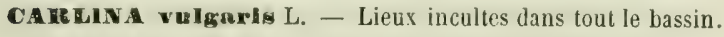

C. nebrodensis Guss, - Lieux pierreux. - H.-Sav. et Sav. : mont Chalune, Roc d'Enfer, au-dessus de la côte d'Arbroz, Feissons-sous-Briançon, entre la Fougère et Coutabella. - En dehors de nos limites au. mont Dore dans la vallée de Chaudefour, au pic de l'Aiguiller.

C. Ianata L. - Lieux incultes de la région mérid. - B.-du-Rh. : Aix près du pont des Gardes. - Var et Alp.-Nar. : Toulon, le Luc, Fréjus, Grasse, Ile Ste-Marguerite, Antibes, Nice, Beaulieu, Alenton. - Gard : Nimes, Calvisson, Bellegarde. - Hêr. : plaines des arrondissements de Montpellier et de Béziers. Aude et Pyr.-Or. : Ste-Lucie, col de l'Epervier près Tuchan, Prades, Céret, le Boulou, Espira de l'Agly, Cases-de-Pena, Clıâteau-Roussillon, envir. de Perpignan.

C. corymbosa L. - Lieux incultes de la région mérid. - Dr. : St-Paul-Trois-Châteaux, Montélimar. - Vaucl. et B.-du-Rh. : 
envir. d'Avignon, d'Orange, d'Apt et d'Arles. - Var et Alp.Mar. : Toulon, le Luc, Fréjus, Grasse, Nice, Menton. - Gard : Nimes, Uzès, Anduze, le Vigan. - Hér. : plaines des arrondissements de Montpellier, de Béziers et de St-Pons. - Aude et Pyr.-Or. : envir. de Narbonne, Gruissan, Quillan, Villeneuve, entre Durban et Montbassou, Rivesaltes, Collioure, Sarrat de las Quillas et d'en Vacquer, Malloles, Perpignan. - Tarn.

C. acaulis L. - Lieux incultes dans une grande partie du bassin. - Espèce polymorphe souvent caulescente.

C. acanthifolia All. - Pâturages des montagnes. - Is. : mont Rachais, Comboire, St-Michel-les-Portes, Marcieu, St-Christopheen-0isans. - Dr. : envir. de Die et du Luc. - H.-Alp. : Charance, la Garde, Puy-Montbaud, les Serigues, Cote Gélive, mont Chabrières, la Cluse-en-Devoluy, le Queyras. — Vaucl. - mont Ventoux, Flassan, Rustrel, Croignes, le Luberon. - B.-du-Rh. : coteaux de Beaulieu près Rognes, Mimet, Pic de Bretagne. Var et Alp.-Mar. : Ste-Baume, Morières près Toulon, Caussols, St-Vallier, Aggel, Cima d'Ours, Rasel, Vence, Nice, Menton, jusqu'au col de Tende. - Rh. : St-Julien-sur Bibost. - Loire : entre St-Chamond et le Bessat. - Ard. : mont Charray, l'Escrinet, Jaujac, Aubenas, Thueyts, Joyeuse. - Gard : le Vigan, Alzon, Montdardier, l'Espérou. - Hér. : derrière le Pic StLoup, Caylar, Mas-de-Mouriès, Romiguẻres et les Rives sur l'Escandorgue, St-Martin-d'Orb. - Aude et Pyr.-Or. : cours supérieur de l'Aude, Montagne-Noire, mont Louis et toute la Cerdagne, vallée d'Eyne, Carença, Canigou, do Costa Bona à Nuria. - En dehors de nos limites dans les Pyr. centrales, Aveyron, Lozère, Tarn, H.-Loire, Puy-de-Dóme, Pierre-surHaute.

C. cinara Pourr. - Coteaux pierreux. - Hér. : l'Espinouse, vallée de la Mare, Pégayrolles-de-l'Escalette, Pardailhan, Avène. Aude et Pyr.-Or. : cours supérieur de l'Aude, Montagne-Noire, Font-Romeu. - En dehors de nos limites au Puy-de-Come en Auvergne.

ATRACTXKus humilie L. - Rocailles calc. - Hér. : Nissan au Pas-du-Loup, Béziers sur le plateau de la Madeleine. - Aude 
et Pyr.0r. : la Clape à l'Espitalet et aux Emphenets, Thuir, Castelnau, Corbère, Bellegarde.

A. cancellata L. - Lieux pierreux des Alp.-Mar. : Cannes, Monaco, Villefranche aux Quatre-Chemins et à la Petite-Afrique, Menton au cap Martin et à St-Louis, puis plus loin sur le territoire italien à Vintimiglia.

CAPPA minor D C. - Bords des chemins. - Espèce polymorphe.

La variété $L$. pubens Bor. a des calathides deux fois plus grosses, pourvues de quelques poils aranéux qui ont souvent tait prendre cette forme pour le L. tomentosa.

L. intermedia Rchb. - Lieux incultes. - H.-Sav. : mont Salève, vallée de Thones. - H.-Alp. : vallée de l'Aullanier près Gap.

L. major Gœrtn. - Bords des chemins. - Moins commun que le L. minor.

L. tomentosa Lam. - Bords des chemins. - Chaine jurassique aux Rousses, vallée de Mijoux, St-Point, St-Cergues, le Bugey et le Revermont, Abbaye de Meyriat. - C.•d'Or : val Suzon, Plombières, Auxonne. - Vaud et Valais : Vevey, Mayens de Sion, de Visp a Stalden. - Sav. et H.-Sav. : vallée du Reposoir, Habère-Poche, Habère-Lullin, Boëge, Hauteluce, Pralognan. Is. et Dr.: le Trièves entre Roissard et le mont Aiguille, Valence, Crest. - Envir. d'Aix en Provence. - Pyr. Or.

XERANTHEMUM annuum L. - Champs calcaires. Vaucl. et Var : envir. d'Apt, Toulon, la Martre, Fréjus. - Ard. : mont Charray. - Pyr.-Or. : Villefranche, Trencada d'Ambulla, Casesde-Pena.

x. Innpertum Willd. - Coteaux incultes. - Valais : Martigny, Saillon, mont d'Orge, Sion, de St-Léonard à Lens, Sierre, Leuk. - Sav. : St-Julien, St-Michel et St-Jean-de-Mfaurienne. - Ain et Rh. : Meximieu aux Peupliers, Pont-de-Chazey, entre Cogny et St-Cyr-le-Chatoux. - Is. et Dr. : Comboire, Monestierde-Percy, la Mure, Crest. - H. et B.-Alp. : Briançon, Cervières, l'Argentiẻre, Guillestre, Gap à Charance, Puy-Maure, St-Mlens, N.-D. du Lauzet, la Baume-sur-Sisteron. - Vaucl. et B.-duRh. : Avignon, Carpentras, Orange, Apt a Roussillon, Croignes, Viens, Flassan, Marseille, Aix, Cuques, Istres. - Var et Alp. 
Mar. : Ste-Baume, Gourdon, Tence, la Briga, Utelle, Menton.Ard. : Aubenas, Joyeuse, l'Escrinet, Charray, Lasuel. - Gard: Luc, Salbous. - Hér. : Mireval, Fabrègues, St-Jean-de-Védas, Murviel, St-Gély, Valmargues, Montarnaud, Pic St-Loup, StMarlin-de-Londres, Tréviers, Capouladoux, la Boissière, Cette, Frontignan, Balaruc. - Aude et Pyr.-Or. : la Clape, Ste-Lucie, Cap de Pla, Moussan, Tuchan, Rigarda, Vinça, Prades, frencadad'Ambulla, Serdinya, Graus d'Olette. - En dehors de nos limites dans le Tarn, l'Aveyron, la Lozère et le Puy-de-Dôme.

X. eylinubeeum Sibth. et Sm. - Champs incultes. - C.-d'Or : levée du canal entre Longvic et la Colombière, le long du chemin des Romains, à l'entrée du chemin d'Aloxe près de la Douée. - B.-Alp. : mont Rognouse près Ribiers. - Ard. : Payolive. - B.-du-Rh. et Var : Marseille, ferme de la Route près Luminy, Toulon, le Luc. - Gard : le Vigan, Salbous. Hér. : Mauguio à Doscares, Fabrègues, derrière le Pic St-Loup, St-Martin-de-Londres, la Boissière, la Sérane, les Capouladoux, St-Guilhem, Lamalou, Villemagne, Lunas, Avène, Fscandorgue au Mas-de-Mouriès. - Aude et Pyr.-Or. : Saissac, St-Paul, Prades, vallon d'Estoher.- En dehors de nos limites dans le Tarn, H.-Loire, Puy-de-Dôme, Loire au mont Claret et a Chalain-leComtal.

\section{CHICORACÉES.}

CATANANCHE coeruleá L. - Coteaux calcaires bien exposés au soleil. - Is. : Bastille de Grenoble, St-Eynard, Comboire, Corps, Vienne. - Dr. : St-Vallier, Valence, Die, Barnave, Montélimar, Loriol, Donzère. - H.-Alp. : envir. de Gap, la Garde, Chorges, Rosans. - B.-Alp. : Bouzolières, Barcelonnette. Vaucl. : commun dans les arrondissements d'Orange, Carpentras, Apt, Avignon. - B.-du-Rh. : envir. d'Aix et de Marseille. - Var: Bau de Quatre-Heures, le Luc, Rians, Bagnols, FoxAmphoux, le Luc. - Alp.-IIar. : envir. de Grasse, Nice, Menton. - Ard : entre Privas et Chomérac, Pont de Coux, Charray, Aubenas, Chassagne, l'Escrinet, Baix, Mercuer, Celles, Payolive, 
la Voulte, Gruas, Rochemaure, Viviers. - Gard : Nimes, le Vigan, Manduel, Tresques, Uzès, Anduze, Alais, St-Ambroix.IIér. - Aude : commun sur les coteaux des envir. de Narbonne, sur les pentes des Corbières, et jusqu'au pied de la Mlontagne Noire, à Ilontolieu, Villespy, Conques. - Pyr.-Or.: Prades, Trencada d'Ambulla, Graus d'Olette, Sahorre, Cornella-duConflent, Vernet-les-Bains. - En dehors de notre bassin sur les calcaires jurass. des envir. de Mende, Florac, Meyrueis; calcaires de l'Aveyron autour de Millau, Ste-Affrique, Villefranche; calc. du Tarn.

CrCHoniear intybus L. - Champs dans tout le bassin.

C. divaricalım Schousb. - Coteaux incultes. - Var et Alp.Har. : Toulon au cap Brun, le Luc, Antibes, Nice.- Aude et Pyr.-Or. : Sidrière de Fitou, envir. de Durban vers la Fontaine de Ricardo, Vingrau, envir. de Perpignan, Chăteau-Roussillon, le Vernet.

TOLPIs barbata Willd. - Collines sèches des terr. siliceux de la région mérid. - Ard. : Vals, Asperjoc. - Vaucl. à la Fontaine de Vaucluse. - B.-du-Rh. : la Ciotat au Bec de l'Aigle. Var et Alp.-Nar. : Porquerolles, le Luc, St-Tropez, Cannes, Auribeau, Antibes, Nice, Menton.-Gard dans les lieux sablonn. - Hér. : terr. à cailloux siliceux de Grammont près Montrellier, Flaugergues, Lamoure, Pérols, Nurviel, St-Marcel, Montarnaud, Agde, Pézenas. - Aude : Aussiéres. - Pyr.-Or. : Rivesaltes, Cornella-du-Conflent, Vernet-les-Bains, St-Martindu-Canigou. - En dehors de nos limites sur les terr. schisteux de la vallée du Gardon et de St-Etienne-Valfrancesque; Lot sur le granite des envir. de Figeac; terr. schisteux et granit. de l'Aveyron. - Espèce polymorphe.

T. discolor Jord. F. Béziers.:- T. concolor J. F. Béziers. - T. dichron J. F. Celles-les-Bains (Ard.).

T. viraga a Bertol. - Bords des champs de la région mérid. - Var et Alp.-Nar. : Hyères, le Luc, St-Tropez, Cannes, Antibes, Auribeau, Nice, Menton. - Pyr.-Or. : Cornella-du-Conflent, Vernet-les-Bains.

WEDYPYOIS polymorpha D C. - Bords des champs de la région mérid.-Commun dans Vaucl., B.-du-Rh., Var et région Catal. Bassin du Rhône. 
litt. des Alp.-Mar. - Plaines du Gard, de l'Hér., de l'Aude et des Pyr.-0r.; remonte jusque dans la partie mérid. de la Dr. et de l'Ardèche.

II. cretica L. - Variẻté à pédoncules renflés-fistuleux, involucres à folioles arquées et muriquées. - Çà et là avec le type.

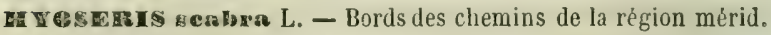
- B. -du-Rh. et Var : Marseille à Endoume, N.-D. de la Garde, phare d'Antibes, ile Ste-Marguerite, Nice, Villefranche, Monaco. - Pyr.-Or. : vallée du Réart vers Calmeilles, Prades, de Mont Louis à Fontpédrouse.

H. radisư L. - Région littorale de la Provence et des Alp.-Nar., la Ciolat, Toulon, la Seyne, Carqueiranne, Nice, Menton. Pyr.-Or. : Perpignan, St-Sauveur, Baixas, Collioure, Montesquieu au pied des Albères. - Remonte dans l'Ard. jusque vers Mercuer, Vals, l'Escrinet.

RIIAGADTOUUS atellatus D C. - Champs de la région mérid.; commun dans Vaucl., B.-du-Riı., Var, Alp.-Mar., Gard, Hér., Aude et Pyr.-Or.; remonte dans l'Ardèche jusque vers Privas.

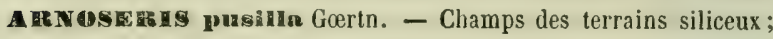
nul sur les calcaires. - Chaine des Vosges jusque dans la zone vosgienne de la H.-Saône, des envir. de Montbéliard et de Belfort; dans le restc des départements du Doubs, Jura et Ain, on ne le trouve jamais sur la chaine jurassique proprement dite, mais seulement sur les granites de la forêt de la Serre et les terr. argilo-siliceux de la Bresse. - Commun sur les chaînes granitiques qui s'étendent depuis le Morvan à travers le Beaujolais, le Lyonnais, le Pilat, le Vivarais jusque vers l'Espérou et la Lozère: plus loin sur l'Espinouse à la Salvetat, le Saumail, Fraisse, St-Amand-de-Nounis, Andabre-Rosis, Pardailhan. Granite et schistes de l'Aude et du Tarn à la Montagne Noire. - Pyr.-Or. : vallée du Réart, plateau de la Cantarana en allant aux IIostalels. - Canton de Vaud sur les sables mollassiques à Treystorrens, Chavanne, Champtauroz, Murist, la Molière, Granges-di-Vesin. - Dans l'Isère on le trouve sur les mollasses et sables de St-Nizier, St-Lattiers, St-Geoirs, Charvieu; dans la Dr. sur les granites de St-Vallier. - En dehors de nos limites 
sur les terr. siliceux des Pyr. centr., du Tarn, de l'Aveyron et de tout le massif central jusque dans les montagnes du Forez et du Bourbonnais.

APOSErers foetidn Less. - Pelouses des montagnes. - Vaud et Valais : Solalex, Salvan, Trient, Combe de la Forclaz. H.-Sav. : Sirt à la Vauzalle. - Sav. : mont Margériaz. - Is. : col de l'Arc, col Vert, la Moucherolle, la Salelte. - Dr. : mont Glandasse, col de Menée. - II.-Alp. : Sapet de la Bâtie-Neuve.Pyr.-Or. : entre Mont Louis et Formiguères, col d'Ares en allant du Capcir au Llaurenti.

LA MIPSANA communis L. - Bords des champs et des chemins. 1 X Xociraenis ginlora L. - Champs sablonneux dans tout le bassin.

1. sadierta - Champs sablonnenx.

Ex. maculata L. - Prairies et pâturages des coteaux el des montagnes.

I. uniflera Vill. - Pâturages des Alpes et des Pyrénées. - Nul dans les Vosges, la chaine jurassique, dans le massif central et les Cévennes. - Vaud et Valais : Alesse, Lavarraz, entre Naya et mont Nuoble, Schwarzsee de Zermatt, Schiwischbergen près du Simplon, Blasenhorn, Binn, Mayenwand. - Sav. : mont Cenis. Is. : mont Chamoux. - H.-Alp. : mont Viso, la Traversette, col Agnel, Fontgillarde, col de Vars, la Cluse en Devoluy. B.-Alp. : Lauzannier, Horonaye, col de la Madeleine. - Alp.Mar.: Tende. - Pyr.-0r. : mont Louis, bois des Angles, Formiguères.

SETHOLA retuenaíz L. - Bords des champs de la région méditerr. - B.-du-Rh. : Châteauneuf près les Martigues. - Var et Alp.-Mlar. : Toulon, St-Raphaël, Cannes, de Nice à Menton. Pyr.-Or. : Haut-Vernet près Perpignan, Baixas, Força-Réal.

THIRNCA hixta Roth. - Bords des chemins des terrains sablonneux et graveleux.

T. Bnisplala Roth. - Terr. sabl. et graveleux de la région mérid.Var: Toulon au fort de Malbousquet, la Martre au Logis-du-Pin. - Aude : Villeneuve. - Pyr.-Or. : Banyuls-sur-IIer.

T. tuberoå D C. - Licux pierreux de la région mérid. - B.-du- 
Rh. : envir. d'Aix au chemin du Tholonet et de Berre, Marseille. - Var et Alp.-Har. : ceinturon d'Hyères, ile de Porquerolles, le Luc, de Nice à Menton. — Gard : Nìmes, Broussan, St-Gilles, Manduel, Uzès. - Hér. - Pyr.-Or. : vallées de l'Agly et du Tech.

LEONTOBON autumnalis L. - Prairies dans tout le bassin.Dans la région mérid. il occupe spécialement les montagnes de l'Espérou, l'Escandorgue, l'Espinouse, Hont Louis et la Cerdagne, les Alp.-Nar.

L. Taraxnel Lois. - Rocailles des Alpes et des Pyrénées. - Nul dans les autres montagnes de la France. - Vaud, Valais : pied d'Argentine, Fully, St-Bernard, Barberine, Maya, Zan, Bellalui, Gemmi, Furca. - H.-Sav. : Méri, Morzine, la Balme près du Bonhomme, Tournette.- Sav. : la Vanoise, Iseran, mont Cenis. - Is. : Téte-de-Racha au mont de Lans, Chamoux, les Pélats entre mont Aiguille et Grand Vehmonl. - H.AAlp. : Galibier, col de l'Echauda, mont Prorel près Briançon, col Isoard, mont Viso, Lopet et Poligny en Champsaur, Orcières, col de l'AlpMartin, mont Aurouse. - B.-Alp. : Lauzannier, Bachasse, bérard. - Alp.-Nlar. : Jallorgues, Alpes de St-Etienne et de StDalmas. - Pyr.-Or. : forêt de Salvanère, vallée d'Evol, sur les flancs de la montagne de Nadres, Vernel-les-Bains.

L. pyrenaicus Gouan. - Prairies des montagnes à sol siliceux.Nul dans la chaine jurassique. - Commun dans la chaine des Vosges depuis le Champ-du-Feu jusqu'aux Ballons de Giromagny et de Servance dans la H.-SaOne. - Valais:St-Bernard, Catogne. - H.-Sav. : grès nummulitiques du Vergy, Méri, toute la chaîue du Hont Blanc; s’èlève jusqu'au Jardin de la Mer de Glace, Dents d'Oche, mont Chalune, Morzine, mont d'Etale près la Clusaz, le Móle, la Tournette. - Sav. : mont Cenis, mont Trélod. - Is. : la Pra de Belledonne, Champrousse, les SeptLaux, Taillefer, Alpe du mont de Lans, col de la Croix-Haute.Ii.-Aip. : la Grave, Lautaret, mont Pelvoux, mont Viso, St-Véran, Chailtol-le-Vieil, Orcières, mont Queyrel et mont Lopet en Champisaur, mont Chabrieres. - B.-Alp. : Lauzannier, Vallonnet, Mirandole. - Alp.-Mar. - Gard : l Aigual aux sources de 
l'Hérault. - Pyr.-0r. : vallèes de Nohèdes, d'Urbanya, d'Eyne et de Llo, Canigou, Coma du Tech, Costa Bona, Llaurenti. Ard, et H.-L. : mont Mezenc. - Loire : Pilat, Pierre-sur-Haute, Montoncelle. - Lozère, mont Dore, Cantal, Pyr. centr.

I. proteiformis Vill. - Prés et bords des chemins Présente une forme glabre $L$. hastilis $L$. et une forme hérissée $L$. hispidus. II existe aussi dans cette espèce de nombreuses variations dans la forme des feuilles.

L. ulpinus Vill. - Diffère du précédent par le pédoncule cannelé et renflé au sommet. - Prairies des hautes montagnes. - Valais : Thyon, Esserz, mont Nuoble, Zan, Guggerlubel, Distelalp, monte Moro, Blasenhorn. - I.-Sav. : chaîne du mont Blanc au Chapeau. - Sav. : mont Trélod. - Is. : chaines de Belledonne et des Sept-Laux, mont de Lans, mont Sénèpe.-H.-Alp. : Lautaret, mont Genèvre, mont Viso, Durbon, mont Séuse. B.-Alp. : Lauzannier, Vallonnet, Crouès, - Dr. : montagnes de Die. - Alp.-Mar. : St-Agnès au-dessus de Menton, Tende.

L. Villarsil Lois. - Coteaux pierreux de la région mérid. - Vaucl.: Orange, Carpentras, Piolenc. - B.-du-R. : collines des envir. de Marseille, Aix à Montaiguet. - Var ct Alp.Mar. : Touris près Toulon, Ste-Baume, le Luc, Grasse, Puget-Theniers, Nice, Braus, Menton. - Gard : le Vigan, Uzís, Manduel, Tresques, Villeneuve-d'Avignon. - Hér. : Montpellier à la Paillade, Lavalctte, Fontfroide, Grabels, Pic St-Loup, Montferrier, St-Gély, St-Brès, Montarnaud, Soubès. - Pyr.-Or. : Trencada d'Ambulla, Olette, Serdinya, entre Estagel et Maury. - Remonte dans les B.-Alp. vers Annot, Sisteron, Digne; dans les envir. de Gap à Charance, Malecombe, les Serigues, Puy-Montbaud, St-Mens, Eymeyères, Ventavon, Rosans, Chorges, Guillestre; dans la Drôme vers Barnave, Dieu le-Fit, Divajeu près Crest, St-PaulTrois-Châteaux, Nyons; dans l'Isère vers Mens; enfin dans l'Ard. à Cruas, Rochemaure, la Voulte, le Pouzin, Crussol.

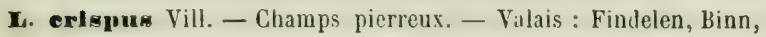
Glacier du Rhône. - Is. : Bastille de Grenoble, Pariset, Comboire, Rochefort, Vernas et Leyrieu près Crémieu. - Dr. : Nyons. - H.-Alp. : Briançon, Gap, St-Hens, Charance, Bayard, 
la Roche-des-Arnauds. - Ard. : Châteaubourg, Crussol, le Pouzin. - Vaucl. : Orange, Rouain, St-Amans. - B.-du-Rh. : Aix au vallon des Gardes, envir. de Marseille. - Var et Alp.Mar. : Toulon, le Lue, montagnes de Nice et de Menton. Gard : le Vigan, Campestre, Nimes au bois des Espèces et de StNicolıs, Manduel, Villeneuve-d’Avignon. - Hér. : Montpellier a Lavalette, Courpoiran, Bione, Castelnau, Grabels, Montarnaud, Pic St-Loup, Soubès et jusque sur le Larzac. - Aude : Peclı de l'Agnèle. - Pyr.-Or. : Prades, Estoher, Rigarda, Doma-Nova, Vernet-les-Bains. - En dehors de nos limites dans les Pyr. centr.; dans la Lozère, sur les calc. jurass. de Mende, Florac, Meyrueis; dans l'Aveyron sur les mêmes terrains autour de Millau, Rodez, Ste-Affrique et Villefranche.

HCrens sprengerians Lam. - Lieux pierreux. - B.-du-Rh. : entre Cassis et la Ciotat. - Var et Alp.-Nar. : Toulon au Cap Brun, Abbaye du Thoronet, Nice à Carabacel, Villefranche.

P. paucifor Willd. - Bords des champs de la région mérid. Vaucl, : Flassan. - B.-du-Rh. : les Alpines, St-Loup, envir. d'Aix au vallon des Gardes et de Bouonouro. - Var et Alp.Mar. : mont Coudon, le Luc, Belgentier, Grasse, Nice au Vinaigrier, Gilette, Utelle, entre Tende et Fontan. - Gard : Nimes, Margueritte, St-Nicolas, Russan. - Itér. : St-Jean-de-Védas, Puéchabon, Cette, Lunel, St-Loup, St-Guilhem, Ganges, StJean-de-Buèges, St-Martin-de-Londres. - Pyr.-Or. : Serdinya, Olette, Graus, gorges de la Tet jusqu'à Fontpédrouse.

P. pinnatifida Jord. - Nimes, Uzès.

P. atrietn Jord. - Bords des champs de la région mérid.

P. hlerneioides L. - Bords des chemins dans les plaines et les montagnes. Espèce polymorphe.

P. crepoides Saut. - Prés des montagnes. - Hontée de la Dóle, valléc de Joux près du pont, mont d’Or. - H.-Sav. : mont Méri, mont Charvin. - H.-Alp. : Lautaret. - Ard. : Cuze, Chassagne. Diffère du précédent par les feuilles supérieures longuement acuminées, les capitules et les akènes plus gros.

P. pyrenaien L. - Prairies des montagnes. - Vosges au Hohneck. - Sommités de la chaine jurassique, Dôle, Faucille, Colombier. 
- II.-Sav. : mont Méri. - II.-Alp. : la Grave, Lautaret, Briançon, Guillestre, mont Viso. - B.-Alp. : Meyronnes, Larche, Barcelonnette. - Pyr.-Or. : Mont-Louis, Cornella-du-Conflent, Vernet-les-Bains, Llaurenti. - Pyr. centr. à Cauterets.

l. Villarsii Jord. - Le Reculet au vallon d'Ardran, la Dóle, valléc de Joux. - Prés de Compesiêres près Genève. - II.-S. : vallée du Reposoir. - C'est probablement à cette forme que se rapportent les localités des H.-Alp. citées plus liaut. Cette forme diffère du typo $P$. pyrenaira par des feuilles plus étroites, des pédoncules non épaissis, resserrés au milieu.

R. corymbosa G. G. - Lieux incultes des Pyr.-Or. à ChâteauRoussilion, Haut-Vernet, Baixas, Cases-de-Pena.

Helminthia celnioldeg Gaertn. - Liẹux incultes de la rérion mérid.; se montre accidentellement dans les trèfles el luzernières dans la partie moyenne et septentr. du bassin.

Urosperanuar Ealechangail Desf. - Clıamps de la rég. mérid.; remonte dans la Drôme et dans l'Ardèche. Dans le reste du bassin se montre quelquefois accidentellement.

u. pierolales Desf. - Bords des chemins de la région mérid. Dr. : le Buis, Donzère, Montélimar. - Ard: : Viviers. - B.Alp. : Annot. - B.-du-Rh., Vaucl. - Var et Alp.-Mar. : TouIon, Carqueiranne, St-Haxime, le Luc, de Nico à Menton. Gard: Nimes, le Vigan, Uzès, Corconne, St-Ambroix, Anduze.Hér.: Montpellier à la Colombière, Grammont, Montauberon, Lavérune, Caunelle, Cellencuve, Mireval, St-Gély, St-Jean-deVédas, Mauguio, les Capouladoux. - Aude : envir. de Durban au pont de la Ricardo, et au bois de Monbassou, vallon de Ripau, Villanière. - Pyr.-0r. : Trencada d'Ambulla, ForçaRéal, Cases-de-Pena, vallée de l'Agly jusqu'à St-Paul.

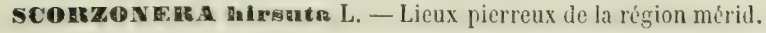
- Vaucl. : envir. d’Avignon et d'Orange, Flassan, Bédouin. B.-du-Rh. : envir. de Marseille et d'Aix, Montaiguet, Cuques, colline des Paurres, - Var : mont Coudon. - Alp.-Nar. : Nice, Sospel, col de Tende. — Gard : Nimes, Campestre, Montdardier. - Hér. : Montpellier à la Colombière, St-Martin-de-Londres, Valflaunès, Restinclière, Pic St-Loup, Capouladoux, la Sśrane, 
Viols, St-Guilhem, le Caylar, St-Maurice, la Vacquerie, Nissan, Pardaillan. - Pyr.-Or. : vallée du Tech entre Prats-de-Ilollo et la Preste, vallée de la Tet, bas Conflent, Vinça, Prades.

Renonte dans la Drôme vers Chaudebonne, Aucelon, StVallier; dans les H.-Alp., verı Rosans, Moydans; dans l'Isère vers Ciémieu; et enfin dans l'Ain vers Meximieu aux Peupliers, Loyelte, plaine d'Ambronay.

S. purpurea L. - Rocailles calcaires de la région mérid. - Hér. : le Larzac au Cros, le Caylar. - En dehors de notre bassin il s'étend dans le Larzac de l'Aveyron à Cornus et Tournemire; puis dans la Lozère au bois de Vabre près Mende.

s. nustrince Willd. - Rochers calcaires. - C.-d'Or : Gevrey, Couchey. - S.-et-L. : rochers de Givry à Buxy. - Vaud et Valais : Ollon, St-Maurice, Branson, Saxon, mont d'Orge, StLéonard, les Plâtrières, Clavaux, Sierre. - H.Sav. : Salève audessus d'Archamp et à la Grande Gorge, mont Vuache. - Sav. : St-Jean-de-Maurienne, la Saussette près Moutiers, mont Cenis. - Is. : Casque de Néron près Grenoble, Rochefort prìs du Pontde-Claix. - H.-Alp. : la Grave, Villard-d'Arène, Lautaret, Bramebuou près St-Genis, mont Donne près Guillestre, mont Chabrières, Sćuse. - Ard. : Gerbier-des-Joncs, - B.-du-Rh. et Var : Ste-Victoire près Aix, Rians, Ampus. - Pyr.-Or. : forêts de Salvanère et de Boucheville. - En dehors de nos limites existe dans l'Aveyron vers la Panouse et à Tournemire.

S. bupleurifolia De Pouz., S. crispa D C. - Bois. - Gard. : Serre de Bouquet près Uzès. - Aude : Pech de l'Agnèle près Narbonne.

S. humilis L. - Prairies des terrains siliceux. - C. -d'Or : envir. de Saulieu, St-Léger, Vignolles, Limpre, Orgeux, Arcelot, Noiron. - S.-et-L. : Morvan autunois. - H.-S. : zone vosgienne à Ecromagny, Giromagny, Plancher-Bas, grès bigarrés de Champagney, Clairegoutte, Franchevelle, Linexert, Belmont. - Terr. argilo-siliceux des plaines du Doubs, Jura, Ain, et aussi sur l'oxfordien à Chailles siliceuses. - Environs de Genève au bois de Cranves sous Monthoux, Coppet. - Région granitique du Beaujolais, Lyonnais, montagnes de la Loire et du Vivarais. Is. : argiles du bois de Chambaran. - Hẻr. : le Caroux, 
Ligneres et Cambon sur l'Espinouse, Pardailhan. - Aude et Tarn : terr. granitiques de la Montagne-Noire. - Gard : le Vigan, Alzon, l'Espérou, Concoule.-Pyr.-Or. : Mont-Louis, Borde-Girvès sur les rives de la Tet, le Canigou à Raudé. - En dehor's de notre bassin sur les terrains granitiques du centre de la France et des Pyr. centr.

S. provifiora Jacq. - Pâturages humides des bords de la mer. B.-du-Rh. : bords de l'étang de Marignane, - Gard : AiguesMortes à la Pinède. - Hér. : Maurin, Lattes, Palavas, Pẻrols, Mauguio, St-Ilarcel, Lamotte, Fréjorgnes. - Aude : la Clape, Montolieu.

S. aristata Ram. - Pyr.-Or. : vallée de la Tet, Mont-Louis, Trencada d'Ambulla, vallée de l'Agly, Cases-de-Pena.

s. Inspanea L. - Cultivé pour l'usage alimentaire; quelquefois subspontané dans les prairies. - Is. : Cordéac près Mens. H.-Alp. : Henteyer, mont Bayard, col de Glaise, la Garde. B.-Alp. : la Condamine, Bouzolierres, Longet, Melezen. - Vaucl. : Sorgues, pied du Inourre de Séve. - Var : mont Coudon. Alp.-Mar. : montagnes de Grasse, Vence, Entraunes, col de Tende. - Pyr.-Or. : Mont-Louis, Villefranche, envir. de Perpignan.

S. glistifolia Wallr. - Rochers. - Is. : mont Rachais, Comboire, base de la Moucherolle. - II.-Alp. : mont Séuse, la Grangette près Gap, Chauvet, Glaise, Ia Saulce, Romette, le Ilélezet près Guillestre. - Sav. : Apremont près Chambéry. - Vaucl. : St-Amans, Vacqueiras, mont Ventoux à Canau. - Gard : Nimes au bois des Espèces, St-Michel-dei-Sers. - Hẻr. : Mireval à la Gardiole, Fontfroide, Pic St-Loup, St-Martin-de-Londres, St-Guilhem, la Sérane, Argelliers, pic de Liausson, Montarnaud, Lodève, Pégayrolles-de-l'Escalette, Joncels, St-Maurice. - Aude : mont StVictor.

S. crispatula Boiss. - Aude et Pyr.-0r. : Cases-de-Pena, étang de Leucate.

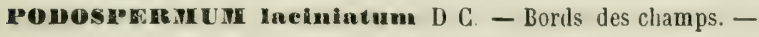
C.-d'Or et S.-et-L. : Dijon, Gevrey, Beaune, Santenay, Curgy, Dezize. - Envir. de Lyon, de Grenoble, de Gap, St-Vallier, 
Crest. - Assez commun dans la Provence, le Gard et les Pyr.Or.

P. decumbens G. G. - Diffère du précédent par ses tiges latẻrales décombantes, la centrale courte et dressée. $-\mathrm{H}_{0}-\mathrm{Alp}$. et B.Alp. : la Grave, Névache, la Garde près Gap, les Serigues, les Baux, Guillestre, Abriès, la Condamine, Bouzolières. - Is. : entre Bourg-d'Oisans et le Fréney. - Dr. : St-Vallier, Crest. B.-du-R. : envir. de Marseille, d'Aix et d'Arles, Eyguière. Var : Toulon, le Luc. — Gard : NImes, Aigues-Mortes, Alais, St-Ambroix. - Hér. - Montpellier au Plan des Quatre-Seigneurs, Mireval, Frontignan, le Crès près du Salaison. - Pyr.Or. : Perpignan, vallée de Conat, Villefranche, Trencada d'Ambulla, Nohèdes.

TRACOROGON pratengia L. - Prés dans tout le bassin.

T. orientalis L. - Hoins répandu que le précédent, dont il diffẻre par les capitules plus grands, ayant les folioles de l'involucre plus courtes que les fleurs.

T. croeifollus L. - Coteaux et montagnes calcaires. - Sav. : Arbin, Cruet, Chignin. - Is. : Bastille de Grenoble, St-Eynard, Engins. - H.-Alp. : envir. de Gap, Puy-Maure, les Serigues, St-IIens, la Garde, Rabou, Bayard, la Roche-des-Arnauds, Tallard, la Saulce, Monestier-de-Briançon. - Dr. : Barnave, Die, Crest, Montélimar. - B.-Alp. : la Condamine, Vaunaveys près Digne, Sisteron. - B.-du-Rh. : Montaiguet près Aix, montagnes autour de Marseille. - Var: mont Faron, Ampus. - Alp.-Mar. : St-Vallier, Menton à l'Agel, col de Tende. — La rẻgion montagneuse du Gard. - Hér. : Mireval, la Sérane, les Cambrettes, St-Guilhem, Liausson, St-Etienne-de-Mursan, Olargues. - Aude : Belcaire. - Pyr.-Or. : Cornella-du-Conflent, Vernet-les-Bains, Port-Vendres, Banyuls-sur-Ier. - Ard. et H.Loire : le Nezenc. - En dehors de notre bassin les coteaux calc. de la Limagne, de la Lozère autour de Mende, Florac, Meyrueis, du Tarn, de l'Aveyron et des Pyr. centrales.

T. stenophyllus Jord. - Bords des champs de la région mérid. B.-du-Rh. et Var: la Treille près Marseille, pied de Gardelaban, Hyères, le Luc. - Aude : Bouscaillou près Durban. - Pyr.-Or.: Prades, vallon d'Estoher, Céret. - Aveyron. 
'A'. porrifollus L. - Le salsifis souvent cultivẻ se montre quelquefois à l'état subspontané dans la Provence et le Languedoc.

T. ansiralle Jord. - Champs de la région mérid. - Vaucl. : envir. d'Orange el d'Avignon. - B.-du-Rh. et Var : Cassis près Marscille, le Luc, Rians. - Alp.-Mar. : Grasse, Nice, Monaco, Menton. - Assez commun dans les garrigues et pâturages du Gard, de l'Ỉér. et de l'Aude. - Pyr.-Or. : Sarrat-d'en-Vacquer ct de las Guillas près de Perpignan, vallée du Réart, St-Antoinede-Galamus. - Remonte jusque dans la partie mérid. de l'Ard., de la Drôme et des B.-Alpes. - Aveyron.

'r. mojor Jacq. - Champs de la région mérid. - B.-du-Rh. : Fos, Roujuefavour, la Vieille près Allauch. - Gard : Nìmes, Manduel, Uzìs, Beaucaire. - Hẻr. - Pyr.-Or. : Château-Roussillon, vallée du Tech, pied des Albères jusqu'au Boulou. - H.-Alp. : Ribiers à Antonaves. - Remonte dans le départ. de l'Ain vers Meximieu au Mont, pont de Chazey, Rigneux-le-Désert; dans l'Ard. vers la Viole; dans la C.-d'Or sur divers points de la Côte autour de Nuits, Beaune, Dijon.

T. dubius Vill. - Champs de la région mérid. - Vaucl. : Orange, Piolenc. - B.-du-Rh. : St-Pons-de-Gémenos, vallon de Nicolas.

- Var : Château Double. - Alp.-Mar. : Nice, Breglio, St-MIartin-d'Entraunes. - Remonte dans les B.-Alp. vers la Condamine, Faucon, St-Paul; dans les H.-Alp. au col de Vars et au IIonestier-de-Briançon, Charance, mont Séuse; dans la Savoie vers Aime. - Aveyron.

T. Hefrutus Gouan. - Champs de la région mérid. - Var et Alp.Mar. : Toulon, le Luc, Antibes, Nice. - Pyr.-Or. : vallée du Réart au Mas-Deu et jusqu’à N.-D.-du-Col.

A FEOFOCON giaber L. - Champs pierreux. - B.-du-Rh. : bords de l'étang de Caroute. - Var et Alp.-Mar. : Toulon, le Luc, Grasse, Antibes, Nice. - Hér. : Grabels à Valmargues.

CHI N DPELA juncen L. - Licux incultes dans tout le bassin

Var. latifolia M. B. - Rh. : Brignais, Condrieu. - Gard. Hér. : Montpellier, St-Martin-d'Orb. - Pyr.-0r. : vallon de la Rigarda. - Coteaux calcaires de la Limagne, de la Lozère, de la Loire. 
WHLLE ETIA apargloldeş Cass, - Pâturages des Pyr.-0r. : le Capcir à Balcère, le Llaurenti à Mijanès, el plus loin dans l'Ariège à Quérigut.

w. prenantholdes G. G. - Var : Fréjus.

TAmAXACUM vulgare Wigg. - Prés dans tout le bassin; espèce polymorphe.

T. affine Jord, - Envir. de Lyon.

T. rubrinerve Jord. - La Grande-Chartreuse, le Sappey (Is.).

T. levigatum D C. - Collines siches. - Is. : Bastille de Grenoble, Pariset, Comboire. - H.-Alp. : envir. de Gap. - Gard : Nimes, Marguerite, Laidenom. - Hér., Pyr.-0r.

T. leucospermum Jord, - Rochars calc. des envir, de Toulon, - Pyr.-

Or. : roch. calc. de Salses, Opol, Cases-de-Pena.

T. gymnanthum D C. - Licux incultes. - B.-du-Rh. - Var : Toulon, mont Faron. - Gard : Manduel au Mas-de-Vianès, le Vigan. Hér. : Montpellier, Castelnau, Lattes, St-Martin-de-Londres, Puéchabon. - Pyr.-Or. : Collioure.

T. obovatum D C. - Coteaux. - Vaucl. : Montdevergues près Avignon.- B.-du-Rh. : envir. d'Aix au Chicalonet, vallon du Coq. - Gard : Nimes, Manduel, Milhaud, Chartreuse de Valbonne. - Hér. : envir. de Montpellier. - Pyr.-Or. : envir. de Perpignan.

T. erythrospermum Andr. - B.-du-Rh. : bois de Pins à St-Julien près Marseille. - Var : le Luc. - Gard, - Pyr. centrales.

T. palustre D C. - Prés humides dans tout le bassin.

T. udum Jord. - Mélé au précédent.

T. maculatum Jord. id.

LACTUCA ramosissima G. G. - Graviers, - B.-du-Rh. Miramas, St-Julien, vallon de N.-D. des Anges. - Var : Touris, le Luc, Fréjus. - Alp.-Mar. : Nice à Ste-Hélène, Menton au cap Marlin. - Hér. : Frouzet, Villeveyrac, Ccilhes, Lamalou, StGeniés-le-Bas, Olargues. - Aude et Pyr.-Or. : vallon de Ripau près Villeneuve, Oms, vallée du Réart. - Puy-de-Dôme, H.-Loire, Lozère.

U. viminea Link. - Lieux pierreux bien exposés au soleil. - C.d’Or: route de Dijon à Plombières et de Dijon à Chagny. - 
S.-ct-L. : Vergisson, Mercurey, Sampigny, Dezize. - Valais : Martigny, Longeborgne, entre St-Léonard et Lens, les Plâtrières, Sierre, Salgesch, entre Visp et Stalden, Twrbel. - Ain : St-Sorlin, St-Rambert-en-Bugey, - Sav. : Salins entre Moutiers et Brides, les Allues, détroit du Ciex. -- Rh. : Condrieu, Ampuis, Chavanay, Pelussin, Malleval. - Dr. : Barnave, Châtillon-enDiois, Menée, Roussieux. - H.-Alp. : Rosans. - Vaucl. : Orange, Vaucluse, Malaucène, Bédoin, Apt. - B.-du-Rh. Var : mont Faron, le Luc. - Alp.-Mar. : Grasse, Nice, l'Escarène, Sospel, la Briga, Tende, St-Martin-Lantosque. - Ard. : commun dans les parties chaudes des vallées de l'Ardèche, de l'Eyrieux, du Doux et de leurs affuents. - Gard : NImes, Beaucaire, Uzès, lo Vigan. - Aude : Villespy. - Pyr.-Or. : vallées de la Tet, du Tech et de l'Agly; remonte jusqu'à Mont Louis et Prats-de-mollo.

L. chondrilloflora Bor. - Débris rocheux. - C.-d'Or et S.-et-L. : Dijon et toute la Cote jusqu'à Santenay, Dezize. - Is. : Bourg d'Oisans, Venosc. - H.-Alp. : la Garde près Gap, N.-D. du Laus, Espinasse, Remollon, Roche-des-Arnauds, Embrun, mont Dauphin, Guillestre. - B.-Alp. : Annot. - Dr. : entre St-Vallier et Tain, Crest. - Ard.: Tournon, Andance, entre Privas et Chomérac, l'Escrinet, mont Charray, Prades, Vals. - B.-duRh. - Alp.-Nlar. : montagnes au-dessus de Menton, - Gard : le Vigan, Campestre, Arphy. - Hér. - Pyr. centr., Tarn, Loire à Pertuiset, Grandjean, Veauche.

L. saligna L. - Champs incultes. - Doubs et Jura : alluvions du Doubs autour de Dole, Chaussin, Menotey, Salins. - C.-d'Or et S.-et-L. : Nolai et une partie de la Cote, vallée de la Dheune. Bassin du Léman, Genève, Nyon. - Valais: Fully, Saxon. Ain : la Bresse, le Bugey et le Revermont. - H.-Sav. : Frangy, Vẻsenaz. - Kh. : Oullins, St-Alban, Villeurbanne. - Is. : Crémieu, la Tronche. - H.-Alp. : N.-D. du Laus, St-Jean près Gap, Roche-des-Arnaud, Ribiers, Rosans. - Ard. : Privas. - Dr. : St-Vallier, Crest, Barnave. - Vaucl. - B.-du-Rh. : Montaiguet près Aix, vallée de Chicalon. - Var : Carqueiranne, St-Tropez. - Gard et Hêr. - Pyr.-Or. : vallée de Cornella-duConflent et de Vernet-les-Bains, Olette, Graus d'Olette. 
L. menrlola L. - Lieux incultes, décombres dans tout le bassin.

L. dubia Jord. - IIêlé au précédent dans les environs de Genève, de Grenoble, Gap.

T. Virosa L. - Lieux incultes. - H.-S. : Chariez. - Nul sur le versant français de la chalne jurassique, bien qu'il existe sur le versant helvêtique dans les cantons de Neuchâtel, Vaud et Genève. - C.-d'Or : envir. de Dijon, - S.-et-L. : Cluny, petite Verrerie. - Rh. : Oullins, Villeurbanne. - Ain : Belley vers le lac de Bar. - Sav. : Aiguebelle. - Is. : Crémieu, Rochefort, Pont de Claix. - Dr. : Crest. - Ard. : le Cheylard. - Gard, Hér., Pyr.-Or.

L. Ravida Jord. - Lieux incultes. - Envir. de Genève, de Lyon entre St-Clair et la Pape, envir. de Grenoble au Polygone, aux carrières de Fontaine et aux Côtes de Sassenage, entre Claix et St-Ange. - Sav. : Aime, Moutiers, St-Mlichel-en-Maurienne. Dr. : Crest. - Envir, de Gap à la Garde. - Hér., Gard, Pyr.Or.

L. antiva L. - Cultivê et quelquefois subspontané sur les décombres.

L. Chaixi Vill. - Bois des montagnes. - Envir. de Gap au Devez de Rabou, les Baux, Chaudun. - Alp.-Mar.: Alpes de Tende, de St-Étienne et de St-Dalmas.

On a trouvé dans les H.-Alpes une variété à feuilles laciniées.

L. muralls Fresen. - Bois et bords des chemins.

C. perennis L. - Rochers calcaires. - Calc. jurass. de H.-S. à Chargey-le-Port, Grattery. - Doubs et Jura : calc. jurass. des envir. de IIontbéliard et de Besançon, Côtes du Dessoubre et de la Voyèze, rochers de Clémont, de Bief et de Pont-de-Roide, vignoble jurassien depuis Salins jusqu'à Lons-le-Saunier, Brans près Montmirey. - C.-d'Or et S.-et-L. : toute la chaine jurassique de Givry à Santenay puis dans la vallée de la Dheune, St-Sernin, Nolai, Dezize. - Vaud et Valais : montagnes calcaires d'Aigle, les Diablerets, vallon d'Outhannaz au pied des Mortais, St-Maurice, Branson, les Marques, Sion, St-Martin, entre Visp et Stâlden. - Ain : calc. jurass. du fort de l'Écluse. Culoz, Virieu-le-Grand, Arvières, Tenay, St-Rambert. - H.- 
Sav. : calc, du Vuache, Molle, vallée du Reposoir, Mieussy, calvaire de Thônes. - Sav. : calc. des envir. de Moutiers, mont Cenis. - Is. et II.-Alp. : calc. jurassique de Bastille de Grenoble, la Salette, Gap, Chorges, Briançon. - Coteaux calc. de l'Ardéche, de la Drome et de Vaucl. - B.-du-Rh. et Var:montagnes calc. des envir. d'Arles au mont Majour, d'Aix et de Marseille, de Rians, du Luc. - Alp.-Nar. : Grasse, St-Yallier, Nice, Menton, gorges de Saorgio, St-Martin-Lantosque. - Gard : Villeneuve près d'Avignon, bords du Gardon, Chartreuse de Valbonne, le Vigan. - Montagnes calc. de l'Hér. - Aude : calc. des envir. de Narbonne, Durban, Lastours, Montolieu. - Pyr.Or. : rochers calc. de Trencada d'Ambulla, route de Mont Louis, vallon de Port-Vendres, Cases de Pena, Pont de la Fou et toutes les Corbières. - En dehors de notre bassin sur les coteaux calc. des Pyr. centr., Lozère, Lot, H.-Loire, et exceptionnellement sur les rochers granitiques et porphyriques des environs de Boën, Sail-sous-Couzan jusqu'à St-Thurin et Noirétable dans la Loire; l'analyse chimique de ce substratum serait intéressante à connaitre.

T. teneruma Pourr. - Rochers et vieux murs du Languedoc et du Roussillon, - Hér. : Cette, St-Chinian à Poussarou, Madeleine-de-llounis, - Aude : entre Durban et Villeneuve, col de l'Épervier près Tuchan, ravin de Combemale près la Clape. Pyr.-0r. : vallons de Collioure, de Port-Vendres et de Banyulssur-ller, vallée du Tech, Céret, Arles, route de Prats-de-Mollo, vallée de la 'Tet, Vinça, Prades, Villefranche, Olette jusqu'ả Mont Louis, Cornella du Conflent, Vernet-les-Bains, vallée de. l'Agly, Cases de Pena, St-Antoine-de-Galamus, St-Paul. - Pyr centr. dans la vallée d'Argelès.

PRENANTH purpuren L. - Bois des montagnes, - Toute la chaîne des Vosges jusíue dans la H.-Saône à Belfahy, Champagney, Ronchamp, vallée du Rahin. - Toute la région des sapins de la chaine jurassique jusque dans le Haut-Bugey. Le Morvan dans C.-d'Or et S.-et-L.; les montagnes du Beaujolais, du Lyonnais, du Pilat, du Vivarais et des Cévennes jusque dans la chaine de l'Espérou à Salbous et Concoule. - L'Espinouse à 
Ligniêres, St-Gervais, Andabre-Rosis, St-Amand-de-Mounis. Is., II.-.llp. et Dr. : montagnes de la Chartreuse, de St-Nizier, de Mens, de Montmorin, de Gap et de Barnave. - La région montagn. de Vaud, Valais, de la Savoie, des B.-Alp. et des Alp.-Nar. - B.-du-Rh. : montagne de Cordes près Arles. Pyr.-Or. : Mont Louis, Canigou, Bac de Bolquère, Prats-deHollo. - En dehors de nos limites dans les Pyr. centr, la Lozère, les monts Dore, Domes, Cantal, Forez, H.-Loire notamment au Hezenc sur les confins de l'Ardèche, les montagnes du Tarn, de l'Aveyron à l'Aubrac.

sovChys tenerrlmus L. - Bords des chemins de la région mérid. - Vaucl. : Avignon, Vaucluse. - B.-du-Rh. et Var: Endoume près Harseille, le Revest, Hyères, St-Naxime, St-Raphaël. - Alp.-Har. : entre Roubion et St-Sauveur. - Gard : envir. de Nîmes. - Hér. : Montpellier autour du Pérou et de l'Esplanade, la Piscine, Nissan. - Aude : envir. de Narbonne.Pyr.-Or. : vallons de Port-Vendres, de Banyuls-sur-Mer, Collioure, vallée du Réart.

S. olernceus L. - Lieux cultivés dans tout le bassin.

S. asper Vill. - Champs dans tout le bassin.

S. glaucescens Jord. - Lieux pierreux de la région littorale. B.-du-Rh. : Montredon, les Catalans, île de Pomègue. - Var : Carqueiranne, cap Brun près Toulon, lle Porquerolle, presqu'lle Taillat près St-Tropez.

S. arvenmis L. - Moissons dans tout lo bassin.

S. maritimus L. - Bords de la mer. - B.-du-Rh. et Var : envir. de IIarseille et de Toulon, la Seyne, cap Brun, Carqueiranne, ile de Porquerolle, Hyères, St-Tropez, Fréjus. - Littoral de l'Hérault. — Gard : Jonquières, Bellegarde, Beaucaire, StGilles, Aigues-Mortes. - Aude et Pyr.-Or. : Sidrière de Fitou, Ste-Lucie, Port-Vendres, Collioure, Banyuls-sur-Her, St-Paul.

S. decorus Castagne.- Forme luxuriante du précédent. - B.-du-Rh. : Miramas, St-Chamas, Salon.

s. aquatilis Pourr. - Aude et Pyr.-Or. : Narbonne vers le moulin de Gua, Pont de la Fou près St-Paul-de-Fenouillet.

5. palustrìs L. - Prés marécageux. - C.-d'Or : Saulon, Arcelot. - 
Is. : la Verpillière. - B.-du-Rh. : envir. d'Arles. - Pyr.-0r. : bords de la riv. de la Basse près Perpignan, bords de l'Agly près Rivesaltes, prés de la Cerdagne, du Capeir et du Llaurenti.

s. H'Iumieri L. - Bois des terr. siliceux. - Chaine des Vosges depuis le Hohneck et le Rotabac jusqu'aux Ballons de Giromagny et de Servance dans la H.-Saone, vallée du Rahin à la Grande Goutte. - Nul dans la chaine jurassique. - Morvan autunois, mont Beuvrai, Roussillon. - Vaud et Valais : vallée du petit Ilongrin, ravins de Kubli, mont Jorogne, bois au-dessus de Charnex, les Ormonds près le plan des Isles, Dents de Jaman, Bovonnaz, Massongex, Zweisimmen. - H.-Sav. : vallée du Reposoir, Nautau. - Sav. : Galopaz au-dessus de Puisgros, mont Joigny, au-dessus de Leussé près du col de la Louse, Pralognan, Crêt du Ré. - H.-Sav. : Revel au-dessus de la cascade de l'Oursière. - Rh. : montagnes du Beaujolais, la Roche-d'Ajoux, entre Ampuis et Ste-Colombe. - Loire : Pilat au Saut-du-Gier, la Madeleine, les Salles, Pierre-sur-Haute, vallée de Chorsain, - Aude et Pyr.-0r'. : vallée de la Bruyante près Mijanès, MontLouis, Bac de Bolquère, vallées d'Eyne, de Llo et de Carença, Costa Bona, Llaurenti, Canigou. - En dehors de nos limites dans les Pyr. centr., Lozère, Aubrac, Cantal, mont Dore, Tarn, Forez.

S. nIpinus L. - Forêts humides. - Chaine des Vosges au Hohneck, Ballon de Soultz et dans Ia H.-Saône au Ballon de Servance, vallée du Rahin et du Fray. - Région alpestre de la chalne jurassique sur le vereant helvétiçue et français, Mont d'Or au bois de Longeville, la Dôle. - Vaud et Valais : Charavex, les Planards, Dents de Jaman, Trient, Grand St-Bernard, Emanex, Savonay, Saxon, Glacier du Rhône, Grimsel. - H.-Sav. : mont Brizon près de la Glacière, mont Ardin, Cornettes-de-Bise, Morzine, Sixt, la Diosaz, Chamonix à la Pendant, mont des Faux. Sav. : Crêt du Ré, mont Cenis, Bonhomme, mont du Chat. Is. : chaine de Belledonne au lac du Crouzet et à Prémol, StHugon près Allevard, entre St-Ange et le col de l'Arc, la Moucherolle, mont Chamoux. - H.-Alp. : Lautaret, le Champsaur. -Alp.--Har. : vallée du Boréon au-dessus de St-Martin, Ste-Anne. 
de-Vinaï. - Pyr.0r.: mont Louis, Bac de Bolquère, vallée d'Eyne, Canigou, Costa Bona, Llaurenti. - En dehors de nos limites dans les Pyr, centr., mont Dore, Cantal, Forez, Pierresur-Haute à Coleigne.

PICRIDIUM vulgare Desf. - Champs cultivés de la région mérid. - Vaucl. : dans tout le département jusqu'au pied du mont Ventoux, - B.-du-Rh. : commun dans les envir. d'Aix, d'Arles et de Marseille. - Var: Toulon, Hyères, le Luc, StTropez, Fréjus. - Alp.-Mar. — Gard : Nìmes, le Vigan, Alais, St-Ambroix, Anduze, Uzès, Beaucaire. - Hér. : envir. de Montpellier, de Béziers et de St-Pons. - Aude : envir. de Narbonne, de Durban, jusqu'au pied de la Montagne-Noire vers Villespy, Montolieu, Mas-Cabardès. - Pyr.-Or. : Collioure, Port-Vendres, Banyuls, Força-Réal, Cases-de-Pena, Villefranche, Cornella-duConflent, Nyer, et jusqu'à Mont-Louis. - Remonte dans la vallée du Rhône du côté de la Drôme à Montélimar, Nyons, Serves, Tain, St-Vallier; du côté de l'Ardèche jusqu’à Vals, Coux, Tournon ; puis au-delà dans la Loire et le Rhône jusqu'à Malleval, Chavanay, Condrieu, Ampuis.

zACINTHA verueosa Gærtn. - Lieux incultes de la région mérid.-B.-du-Rh. : Aix au vallon des Masques. - Var : Maures du Luc, St-Tropez, cap des Issembres, Fréjus. - Alp.-Mar. : Cannes, Antibes, Nice. - Gard : le Vigan près du château de Tessan, Aubard, route de St-Gilles et de Génerac, Manduel à Campuget. - Hẻr. : Ganges, les Capouladoux.

PTEROTHECA nemausensis Cass. - Champs et bords des chemins, surtout dans la région mérid. - Depuis quelques années, l'extension géographique de cette espèce s'est considérablement accrue; le $P$. nemausensis est actuellement très-commun dans la plaine qui s'étend à l'est de Lyon; on le suit jusque vers Ambérieu en Bugey, d'où il s’étendra probablement encore plus loin.

CREPIS vesiearia L. - Environs de Marseille et de Toulon.

C. taraxacifolis Thuill. - Champs et prés. Une variété à feuilles cntières existe dans les champs de la rẻgion mérid.

C. recognita Hall, - Forme du précédent à tiges plus courtes et étalées. 
Lieux secs de la région mérid. - B.-du-Rh. : envir. de Marscille, envir. d'Aix au vallon du St-Esprit. - Vaucl. : Montdevergues près Avignon. - Var : Toulon, le Luc. - Gard : Villencuve, Nimes, Aigues-Mortes, le Vigan, Beaucaire, Uzès, St-Gilles. - Aude, Lastours, Narbonne. - Remonte dans la Drüme vers Crest. - Se montre parfois accidentellement dans les luzernières.

C. Inursifolia L. - Espece d'Italic trouvée par M. Roux autour de Marseille, et par M. Huet dans les environs de Toulon.

C. erucafolia G. G. - Trouvé autrefois vers le Lazaret de Marseille et dernièrement vers la gare de Toulon.

C. setoza Hall. - Champs incultes, bords des chemins dans les parties moyenne et mérid. du bassin; plus rare dans la partie septentr. - Très-abondant entre Lyon, Villeurbanne et Vauxen-Velin. - Ain : la Pape, Tréroux, Reyrieux, Garnerans, StLaurent-les-Mâcon. - S-et-L. : Tournus. - Doubs et Jura : envir. de Montbéliard, de Dôle, Pesmes, Salins. - Envir. de Genève et de Neuchâtel. - Is. : Vienne, Tencin. - Dr. : Crest. - Ard. : les bords du Rhône, Tournon. - B.-du-Rh. : Marseille à Larose, chemin de Château-Gombert, vallon de Valbarelle. - Vaucl. - Var : le Luc, Pierrefeu, SaintRaphaël. - Alp.-Ilar. : Bollena, Roccabigliera, St-Sauveur. Gard : Pont-Rouge, près le canal de Beaucaire à Aigues-Mortes. - Hér. : Vendres près de l'êtang.

C. Ieontohomoides All. - Rochers du littoral, - B.ddu-Rh. : Bec de l'Aigle près de la Ciotat, Châteauneuf-les-Martigues, ile de Pomègue. - Var : Baou-Rouge près de la Seyne, ile de Porquerolles.

C. Sufremiana Lloyd. - Lieux graveleux ou sablonn. - B.-duRh. : Entressen-en-Crau, Fos, Carpiagne, sommet de Ste-Victoire. - Gard : Nimes, bords du Gardon près du Pont-du-Gard.

C. Foetida L. - Lieux incultes dans tout le bassin.

C. alvida Vill. - Rochers calc. - Is. : col de l'Arc. - H.-Alp., Lautaret, Névache, St-André près Briançon, mont Genèvre, Charance, col de Glaise, mont Aurouse, Jansac, Rosans. - B.Alp. : St-Paul, Sérennes, St-Ours, Bouzolières, la Condamine, 
Larche. - Dr. : Solaure près Die. - Vaucl, : mont Ventoux. - B.-du-Rh. et Var : Ste-Victoire, Ste-Baume, mont Coudon, Nans, Montrieux. - Gard : le Vigan, Alzon, Campestre, StAmbroix, Anduze. - Hẻr. : Pégayrolles-de-l'Escalette, Lauroux à Labeil, Ceilhes, le Caylar, la Sérane, pic de Liausson. - Alp.Mar. : Menton, Braus, la Briga, Tende, St-Dalmas-le-Sauvage, Bouyon. - En dehors de nos limites sur les calc. jurass. de la Lozère à Corsac, Florac, Meyrueis; calc. jurass. de l'Aveyron autour de Sauclières et de Villefranche.

C. Hulbora Cass. - Lieux incultes de la région mérid. - B.-duRh. - Var : Toulon, le Luc, Almanarre, St-Tropez à la Pinède, presqu'lle Taillat, St-Raphae̊l. - Alp.-Nar. - Gard : Bellegarde, St-Gilles, Aigues-Hortes, bois de Cygnan près Nimes, Montels, Aulas près du Vigan. - Hér. : Montpellier à la Colombière, St-Jean-de-Védas, Cournousec, Castries, Restinclières, Ganges, Cette. - Pyr.-Or. : Rivesaltes, Banyuls-sur-Mer.

C. aurea Cass. - Pâturages des montagnes. - Nul dans les Vosges, le massif de la France centr. et dans la chaine des Cévennes. Jıra helvétique et français, Chasseral, Weissenstein, Chasseron, Montendre, Creux-du-Van, Noirmont, la Dôle. - Vaud et Valais : Alpes de Bex et d'Aigle, de Saxon, de Thyon, Combad'Arbaz, Zermatt, Saas. - H.-Sav. : Salève, Môle, Brizon, Vergy, Méri, montagnes de Colonne, mont Chalune, chaine des Cornettes, toute la chaine du Mont Blanc, depuis le col de Balme jusqu'au Bonhomme, s'élève jusqu'au Jardin de la Mer de Glace, Sixt, Morzine, Brẻvent, Semnoz, Tournette. - Sav. : mont Charvin, les Bauges, mont du Chat, toutes les montagnes de la Tarantaise et de la Maurienne jusqu'au mont Cenis. - II.-Alp. : Lautaret, Galibier, mont Gondran, les Rochilles, Cervières, mont Viso. - B.-Alp. : montagnes de la vallée de l'Ubaye. Vaucl. : mont Luberon près Buoux. - Alp.-Jfar. : la Briga, mont Bego, Ste-Anne-de-Vinaï. - Pyr.-Or. : Mont-Louis. Pyr.centr.

C. ehrysnntha Frœl. Rocailles. - Valais : Zermatt au pied du Hornli, val de Bagnes. - Sav. : col du mont Iseran.

Le Crepis alpestris Tausch, assez répandu dans les Grisons, manque en Valais. 
c. premorsn Tausch. - Bois des terr, calc. - Cette espice, assez commune sur les collines calc. de l' $\mathrm{A}$ lsace el de la Lorraine, est fort rare dans notre bassin; on l'a signalée dans le Valais à Lamuraz-de-Thion; en Sav. au col du Frêno; dans l'Ain à la Grange-du-Cimetière, au-dessus d'Arvières; dans les II.-Alp. à la Grave; dans les environs de Châtillon, Tarsul (C.-d'Or).

C. benuis L. - Prairies dans une grande partie du bassin.

c. ulcoensiss Balb. - Prairies de la région mérid. - B.-du-Rh. et Var : près du chàteau de Vauvenargues, Pic de Bretagne, SteBaume, Montrieux.-Alp.-Mar. : Grasse, Antibes, Nice, Castillon etCastellar, au dessus de Menton.-Gard: Tresques, Montdardier, Aumessas, Lanuejols. - Hér. : Montarnaud, Lunas. - Remonte dans les B.-Alp. vers Barcelonnette, la Condamine, Bouzolières; dans les H.-Alp. vers Gap et Chorges; dans l'Is, vers Pariset, Comboire, mont Rachais, Crémieu ; dans le Rh. vers Tassin, le mont fout. - Se montre parfois accidentellement dans la partie septentr. du bassin.

C. virens Vill - Champs cultivés. - Espèce polymorphe répandue dans tout le bassin, et présentant des feuilles tantot pennatifides ou pennatiséquées, $\tan t o t$ entières.

C. agrestis W. Kit. - Mélé au précédent, dont il diffère par des capitules plus gros, hérissés de longs poils noirs glanduleux. Beaucoup moins commun.

C. tectorum L. - Lieux sablonneux. - Côte-d'Or. - Valais : Evionnaz, Martigny, Grone.-Gard : Banahu près de l'Espérou, Mas Charlot aux bords du Gardon; - les trois bassins des Pyr.Or.

C. pulehra L. - Bords des champs des terr. calc. - C.-d'Or : chemin de Chenove, Fontaine des Fées au-dessous de Talant et toute la Côte. - S.-et-L. : vallẻe de la Dheune, Nolai, Dezize. Jura : Champvans près Dôle, bords du Doubs à Choisey, Salins; parait manquer sur le versant helvêtique. - Is. : mont Rachais, la Tronche, Venosc, Huez-en-Oisans. - H.-Alp. : Monestier-deBriançon, la Vallouise, Charance, Rabou, Collet de St-Mens, la Garde, la Roche, les Baux. - Sav. : Aime. - B.Alp. : la Condamine, St-1'aul, Sisteron. - Vaucl. : bords du Rhône. - B.- 
du-Rh. et Var:mont Cordoa près Arles, envir. d'Aix, Marignane, Rognac, Toulon, le Luc. - Alp..Har, : Caussols, Antibes, Nice, col de Braus, Sospel. — Gard, Hér. et Aude. - Pyr.-0r. : base des Albères, Collioures, Port-Vendres. - En dehors de nos limites sur les terr. calcaires de l'Aveyron, de la Lozère, de la Limagne et de l'Alsace.

C. pygmaea L. - Rocailles des Alpes et des Pyrénées. - Vaucl. ct Valais : Enzeindaz, Bovonnaz, Lavarraz, Dent du Midi, Leukerbad à la Gemmi. - H.-Sav. : chaîne des Fiz, Platets, col d'Anterne, Sixt, la Porte-des-Aravis. - Is. : mont de Lans. - H.Alp. : la Grave, Galibier, les Hayes, col de Vars, mont Viso, mont Aurouse, mont Séuse, mont Obiou. - B.-Alp. Vallonnetde-Ileyronnes, Parpaillon, Bérard. - Vaucl. : mont Ventoux.Alp.-Mar. : mont Monnier, d'Entraunes à Villars et à Jallorgues, val de Strop, Bouziejo au-dessus de St-Dalmas-le-Sauvage. Pyr.-Or. : vallée d'Eyne, Collada de Nuria, Coma de la Baca. Pyr. centr. au Narboré.

C. Iampaanoldes Frœel, - Pâturages des Pyr.-Or. : Canigou, cap de la Roquette, sommités de la chaine entre Costa-Bona et Carença, Mont-Louis. - Col de Pailhères.

C. succisafolia Tausch. - Prẻs et pâturages des montagnes. Nul dans les Vosges et les Cívennes. - Commun dans le Jura helvêtique et français, depuis le Vogelberg, le Wasserfall, le Passwang et le Weissensteir jusqu'à la Dôle, le Colombier, le Reculet, le Colombier du Bugey. - H.-Sav, : montagnes du Chablais, Morzine, mont Brizon, le Vergy. - Loire : Pilat, Planfoy, Chalmazelle, Gourgon, Courreau. - Ardèche : Mezenc. - Pyr..0r. : Coma du Tech, Font-de-Comps, la Bentaillole au Llaurenti. - En dehors de nos limites sur les hauts plateaux du Puy-de-Dôme, mont Dore, Cantal, Aubrac, Forez.

C. blattarieides Vill. - Prés des montagnes. - Rare dans les Vosges. - Commun dans le Jura helvétique et français, Paswang, Chasseral, Weissenstein, Suchet, mont d'Or, Montendre, Dole, Faucille, Reculet jusqu'au Colombier du Bugey. - Vaud et Valais : Dent de Jaman, Alpes de Bex, Lavarraz, Bovonnaz, val d'llliez, Leukerbad. - H.-Sav. : le Môle, Brizon, 
Petit Bornand, Parmelan, Semnoz, St-Germain, Tournette, Charvin, Haut-de-Lin, Morzine, Mégive, le Tour, entre Chamonix et le col de Balme. - Sav. : le Bonhomme, entre Beaubois et Roselein, la Sambuy, Bozel, mont Cenis. - Is. : Chamechaude, Bovinant, la Moucherolle, la Morte, mont Chamoux. - II.-Alp. : la Grave, Lautaret, col de Vars, Sapet de la BáticNeuve, Durban, Séuse, Devez-de-Rabou, la Grangelte, le Pleyne, Orcières. - B.-Alp. : Bérard, Horonaye, Lauzannier, Colmars. - Var : mont Bessillon près Cotignac. - Alp.-Mar. : mont Frontero, Ste-Anne-de-Vinaï, Entraunes. - Pyr.-0r. : mont Louis, Cambres-d'Aze, Bac de Bolquẻre, Llaurenti. - Gard : Gourdouse sur la Lozère, Concoule. - Pyr. cent.

C. grandinora Tausch. - Prairies des montagnes. - Nul dans les Vosges, la chaine jurassique. C'est à tort que Boreau l'a indiqué au Pilat. - Vaud et Valais : Bovonnaz, Fins-Hauts, Zermatt, Almagel, Glacier du Rhone, Grimsel, Gerenthal, Blasenhorn. - H.-Sav. : montagnes du Clablais, mont Chalune, Nautau au-dessus de Montriond, Sixt, Pavillon de Bellevue sur St-Gervais, la Tournette. - Sav. : montagnes 'de. Hauteluce, mont Cenis. - Is. : la Morte, mont Chamoux. - H.-Alp. : Lautarel, mont Gondran, mont Viso, col de Vars, col de Glaise, pic de Chabrières, Orcières, Valgaudemar. - B.-Alp. : Lauzannier, Horonaye, col de la Madeleine. - Alp.-Mar. : mont Bego, N.-D.-de-Fenestre, vallée du Boréon, Ste-Anne-de-Vinaï. - Ard. et H.-L. : mont Mezenc, Mazan. - En dehors de nos limites dans les Pyr. centr., mont Dore, Cantal, Lozère.

C. montann Monn. - Prairies des montagnes. - Nul dans les Vosges et les Cérennes. - Sommet de la Dôle et du Reculet. Vaud et Valais: Jaman, Enzeindaz, Bovonnaz, Lavarraz, Surchamp, Mayens de Sion, Cran-sur-Lens, Mayenwand, près du Glacier du Rhone. - H.-Sav. : mont Brizon, Méri, montagnes de Samoëns et de Morzine, mont de Pelloua, Tournette, Charvin. - Sav. : Crêt du Ré au-dessus d'Aime, Hauteluce, mont Cenis.

- Is. : mont de Lans. - H.-Alp. : Lautaret, Cervières, mont Gondran, col de Vars, Chaudun, col de Glaise, mont Sẻuse, la Grangette, col du Noyer. - Dr. : mont Glandasse. - B.-Alp. 
et Alp.-IIar. : Malemort, St-Anne-de-la-Condamine, col de la Maddalena. - Pyr.-Or. : vallées d'Eyne et de Mijanès, Llaurenti.

C. paIudoen Monch. - Prés maréc. des terr. siliceux. - Chalne des Vosges. - H.-S. : zone vosgienne à Mansvillers, mont de Vannes, Chauves-Roches, vallées de lliélin, du Rahin, du Fray, Belfahy, la Prandière, Ballon de Servance, Franchevelle, Linexert, Champagney.-C.-d'Or. : envir. de Saulieu, bois du Sautde-Gouloux. - Jura: terr. argilo-siliceux de la Bresse à la forêt de Chaux, Pleurre, Sergenaux, bois de Rye. - Ain: marais de Cormaranche et deDivonne, Mazières, envir. de Belley. Vaud et Valais : Jaman, Frachiz, Bovonnaz, Alesse. - H.-Sav. : Salève, Voirons, envir. de Thonon vers les Allinges, Haut-de-Lin, Vacheresse, mont Charvin. - Sav, : col du Frêne. - Is. : Côtes de Sassenage, Bovinant, Pre̊mol.- H.-Alp.: Lautaret, mont Genèvre, col de Vars, mont Aurouse. - Rh. : le Garon, Vaugneray aux Jumeaux, St-Bonnet-le-Froid, Izeron, Vaurenard, St-Juliensur-Montmélas, Boucivre. - Loire : Pilat, le Bessat, St-Christoen-Jarret, Planfoy, les Salles, Pierre-sur-Haute. - Ard. : Nayres. - B.-du-Rh. : Arles. - Alp.-Nar. : Alpes de Tende, St-Mfartin-Lantosca. - Gard : le Vigan, Trèves, Alzon, Genolhac, Concoule. - Pyr.-0r. : de la Preste à Pla Guilhem, forêt de Salvanère. - Pyr. centr., centre de la France, Aubrac.

Ix. ataticefollum All., Tolpis staticafolia Schultz bip., Chlorocrepis staticcefolia Gris. - Lieux pierreux, rocailles, graviers.Les deux versants de la chaine orientale du Jura helvétique et français, de Thoiry au fort de l'Écluse et dans la vallée de la Valserine de Lelex à Chézery et jusqu’à Bellegarde; vallée de l'Ain de Coysia à Thoirette. - Envir. de Genève, bords du Rhône, Anglefort, Pierre-Châtel, Balan et jusque sur les balmes qui s'étendent en amont de Lyon sur la rive droite à lâ Pape et sur la rive gauche à Jonage. - Vaud et Valais : bassin du Léman, pied des Diablerets, la Crottaz, St-Maurice, Fully, Orsières, Barrière du Rhône, Comba d'Arba, Leukerbad, Zermatt, Findelen. - Commun dans la Savoie, le Dauphiné et les BassesAlpes le long: des cours d'eau, l'Arve jusqu'aux sources de 


\section{$-463$}

l'Arveiron, l'Isire, l'Arc, l'Arly, lo Drac, la Romanche jusqu'à la Grave et au Lautaret, la Durance et ses affluents, la Guisane, le Guil, l'Ubaye et l'Ubayette, la Bléone, l'Asse et le Verdon.Environs de Gap, de Chorges. - Vaucl. : Malaucene, Lafare, Sablet, Cavaillon. - Alp.-Mar. : St-Vallier, montagnes de Nice et de Menton. - Pyr.-Or. : vallon de Hosset, gorges d'Estoher.

Les botanistes sont loin d'étre d'accord sur la place qu'il convient d'assigner à l'H. staticaf lium; les uns, avec Grisebach, ont placé cette plante dans un genre nommé Chlorocrepis; d'autres, à l'imitation de Schultz, l'ont rangée parmi les Tolpis, lesquels ont pour caractère d'avoir les akènes surmontés d'une aigrette à poils uni-sériés.

Cependant si on considère que, par d'autres caractères beaucoup plus importants, et notamment par ceux des organes de végétation, l'H. statavicafolium ressemble beaucoup plus aux Hieracium et aux Crepis qu'aux Tolpis, on sera amené à lo maintenir parmi les premiers, ainsi que je l'ai fait après Villars et un grand nombre de botanistes.

D'autre part, tout en reconnaissant que l'H. stalicxfolium tient le milieu entre les Crepis et les Hisracium, j'estime que la création du genre Chlorocrepis, si légitime qu'elle soit, est un premier pas dans une voie dangereuse : car les motifs qu'on peut alléguer en faveur de cette scission conduiraient de proche en proche, et avec non moins de raison, à subdiviser en une multitude de genres nouveaux les Crepis et les Hieracium.

Or si on examine combien sont insignifiants et variables les caractères invoqués par Koch, Fries et divers autres auteurs pour établir des sections parmi les Crepis et les Hieracium, on sera peu disposé à transformer ces sections en coupes génériques.

Le genre Hieracium est un Protée excessivement polymorphe et presque insaisissable. S'il est vrai qu'on puisse constater l'existence de quelques types principaux, il est certain aussi qu'entro eux se placent une multitude de formes intermédiaires qui échappent à toute tentative de classification. La difficultó d'un arrangement systématique des nombreuses espèces qui composent ce genre paraitra plus insurmontable encore aux botanistes qui admettent la possibilité de la formation de nombreuses races d'Epervières douées de la faculté de se reproduire par semis, et aussi à ceux qui, avec Schultz, avec MM. ArvetTouvet et Timbal-Lagrave, assurent qu'il existe une multitude d'Hicracium hybrides, peut-être susceptibles de se perpétuer indéfiniment par génération. Que sera-ce donc si les hybrides eux-memes peuvent engendrer à leur tour d'autres hybrides d'une fécondité plus ou moins durable! 
En attendant que l'expérimentation nous ait éclairés sur la valeur des hypothèses relatives à la variabilité spontanée et à l'hybridité, j'ai cru devoir énumérer impartialement toutes les formes qui sont arrivées à ma connaissance soit par la lecture des ouvrages, soit au moyen des documents fournis' par mes collaborateurs et surtout par M. Arvet-Touvet, auteur de la Monographie des Hieracium du Dauphiné. J'ai reçu de ce botaniste, aussi savant que modeste, des renseignements très-détaillés qui m'ont été d'un précieux secours. Je suis heureux de lui exprimer ici la reconnaissance de la Société botanique de Lyon, et la mienne en particulier, pour la généreuse et importante contribution qu'il a bien voulu apporter à notre cuvre de statistique.

IIIERACUII Phosella L. - Pelouses, bords des champs et des chemins dans tout le bassin et à toutes les hauteurs. Espèce polymorpho qui tantôt a les calathides couvertes de poils noirs glanduleux, nigrescens Fries; tantôt a les calathides hérissẻes de longs poils non glanduleux, pilosissimum Fries. Dans la forme virescens Fries, les feuilles sont presque vertes en dessous.

II. Saussurboides Arv. T. - Pâturages de la montagne de Serres sous Taillefer (Isère).

II. velutinum Heg. et Heer; II. Camerarii Callay. - Pâturages du mont Viso, la Traversette, vallon de Ségure, col Fromage. - Mont Cenis.

I. bimorum Arv. T. - Is. : prairies de Brandes au-dessus d'Huez, col de Clavans, bords du torrent de Sarène.

H. caricinum Arv. T. - Is. : prairies de la Morte.

II. Faurei Arv. T., H. pilosello-glaciale? - H.-Alp. : prairies du Lautaret, de Riftort et de Paris au-dessus de la Grave, col d'Arcines, le Désert sous le mont Olan, Malrif. - Valais : La Furca.

II. subrubens Arv. T., H. glaciali-Pilosella? - Mêmes localités que le préce̊dent, et, en outre, au col de Vars.

H. hypoleucum Arv. T., H. velutino-glaciale? - Massif du mont Viso, vallon de Ségure.

II. Schultesii F. Schultz, II. Pilosello-Auricula - Jura : forêt de la Serre. - Dans les Vosges an Hohneck.

bifurcum M. B., II. Pilosello-praaltum? F. Schultz. - On prętend l'avoir trouvé à Chamonix près de la source de l'Arveiron. 
II. fallacinum F. Schulz, II. Pilosello-fallax? - Cetle Eperviere, trouvie d'abord dans l'Alsace et la Lorraine, a été vue enstaite autour de Neuchâtel et de Lausanne.

I. Auricula L. - Prairies dans tout le bassin. - Dans l'llér., les Alp.-Nar. et les Pyr.-Or., on ne le voit pas dans les plaines, mais seulement dans la région montagn. Espèce polymorphe qui, d'apress Schultz, formerait facilement des hybrides avec les autres Epervières.

20. forentinam All. - Graviers le long des rivieres. - Valais : bois de Finge près de Sicrre, Visp, Zermatt, Briegg. - H.-Sav. et Sav. : bords de la Ménoge et de l'Arve jusque vers Sallanches et Passy, bords de l'Arly vers Ugine, bords du Doron de Beaufort et de l'Isère autour de Moutiers. - Is. : Bourg d'Oisans, Venose, St-Christophe-en-Oisans, Polygone de Grenoble, oủ il est amené par la Romanche. - H.-Alp. : la Grave, la Vallouise, le Queyras, le Champsaur, le Valgaudemar, envir. de Gap à Lachaud, St-IIens, Eymeyères, la Saulce, Sigoyer, Manteyer, Montmaur. - B.-Alp. : bords de l'Ubaye, la Condamine, StPaul, Barcelonnette. - Alp.-Mar. : Tende, val de Pesio, StEtienne.

11. pronItam Vill. - Prés marécageux. - H.-Saône : Champagney, Nans. - Doubs et Jura : envir. de Montbéliard, de Pontarlier, de Salins, de Dole et de Lons-le-Saunier. - Ain : GrandAbergement, Bellegarde, Confort, Culoz, Cormaranche, Dortan, Cerdon, Muzin, Belley.-Envir. de Genève, bois de la Bàtie, Peney, marais de Troenex. - Vaud et Valais : Bex, Aproz, Zermatt. H.-Sav. et Sav. : Arenthon, col de la Forclaz, de Voza, Plampraz au pied du Brévent, les Contamines, Chardonnet, N.-D.-de-laGorge, Annecy aux Balmettes, Crest-Yoland, Nivolet, mont Cenis. - Is. : mont Rachais, le Sappey, St-Ange, St-Nizier, Prémol. H.-Alp. : Briançon, Chorges, Sigoyer, col de Glaise. - B.-Alp. : la Condamine à Ste-Anne, Bouzollières, Longet. - Alp.-Mar. : montagnes de Nice et de Menton. - Var : Toulon, le Luc. - Hẻr. : Montpellier à Grammont, Mauguio à Doscares. - Pyr.-Or. : Canigou, Carença, loutpédrouse.

11. eуmosam L. - Prairies des montagues. - Valais : Marligny, 
Valorsine, Folatères. - Ain : de Tenay à Hostiaz. - Sav. : la Maurienne, St-Sorlin-d'Arves. - Is. : St-Eynard, Chamechaude, Charmantsom, col de la Ruchère, plateau de Chamousset près le mont Aiguille, la Salette. - H.-Alp. : Lautaret, Malrif, ChâteauQueyras, vallée du Guil, Molines, Poligny-en-Champsaur, Sapet de la Bâtie-Neuve, Chabrières, Charance, Bayard, col de Glaise, la Grangette. - Dr. : Aucelon. - B.-Alp. : Bouzolières près de Barcelonnette. - Var : Colle-Noire près Toulon, Maures du Cannet et des Mayons.

H. hylbridum Chaix. - Pâturages des montagnes. - H.-Alp. : Devez-de-Rabou, bois Mondet, col de Glaise, Chabrières, Manteyer.

II. anchusoides Arv. T.-Is. : St-Eynard, Champrousse, les Voudaines. - H.-Alp. : mont Viso près du chalet de Ruines, col de Vars. - Se trouve aussi dans les Pyrénées et en Espagne.

I. gIaciale Lach. - Pâturages des Alpes et des Pyrénées. - Vaud et Valais : Anzendaz, mont Fully, St-Bernard, Saas au Distelalp, Rothorn et Schwarzsee de Zermatt, Glacier du Rhone, Grimsel.H.-Sav.: Vergy, Méri, montagnes du Chablais, Cornettes-de-Bise, Morzine, Sixt, Aiguille à Bochard, Montanvert, moraines de la Ner de Glace, le Chapeau, Aiguille-du-Tour, col de Balme, Brévent, col Joly, les Contamines. - Sav. : cols du Bonhoinme et de la Seigne, les Mottets, bords du lac du Cormet au-dessus d'Aime, Hauleluce, Dent du Corbeau, Laval-de-Tignes, la Levanna, St-Sorlin-d'Arves, mont Cenis. - Is. : les Sept-Lauxd'Allevard, mont de Lans, Brandes et la Bérarde-en-0isans. H.-Alp. : Lautaret, Guillestre, mont Viso, col de Vars, St-Véran, Clausis. - B.-Alp. : Vallonnet, Bachasse, Plate-Lombarde, Longet, col de la Madeleine. - Alp.-Mar. : mont Monnier, col de l'Enchastraye. - Pyr.-Or. : Cambres-d'Aze, vallée d'Eyne au Pla de lá Bayuda, Carença.

II. Smithii Arv. T. - II. glaciali-Atricula? - H.-Alp. : la Grave aux prés de Riftort et de Paris, Lautaret. - On l'a trouvé dans le Tyrol.

II. Laggeri Schultz. - Forme plus grande que le H. glaciale et à capitules plus nombreux. - Is. : prairies de Serres sous Taille- 
fer et de Brandes-en-0isans. - H. et B.-Alp. : Lautaret, mont Pelvoux, mont Tabor, mont Viso, valiée de Maurin à Longet.

I. pratense Tausch, - Prés. - C.-d'Or : Saulieu, Semur, Savignyles-Beaune. - Valais : mont d'Orge, Sion, les Marques, StLéonard. - Is. : le Sappey, Vienne.

Ir. snbinum Seb. et Maur. - H. multiflorum Schleich. - Prairies des montagnes. - Vaud et Valais : mont de Naye et des Verraux, le Crais, Alesse, mont Fully, Folatères, val Ferret, Zermatt. - H.-Sav. : entre col de Golesse et col de Couz, mont Pelloua. - Is. : Proveysieu, Brandes et Auris-en-Oisans, la Salette. - H.-Alp. : Lautaret, mont Viso, Pleyne-de-Chaudun, col de Glaise, Lopet, Orcières. - Sav. : les Allues, les Mottets, le Bonhomme, mont Cenis. - Alp.-Nar. : mont Mulacé, Tende. — Gard : envir. de Nimes, bords du Gardon à St-Nicolas, Pontdu-Gard, Aigues-Mortes.

II. nurnntiseum L. - Prairies des montagnes. - Vosges au Hohneck, ballon de Soultz. - Chaine jurassique au mont d'Or, Montendre et Reculet. - Vaud et Valais : Enzeindaz, Morgins, St-Bernard, la Chaux, Thyon, la Furca. - H.-Sav. : Brizon, Vergy, Méri, mont de Pelloua et des Granges, Cornettes de Bise, Morzine, cols d'Anterne et de Balme, Plampraz au pied du Brévent, la Flégère, les Viaux, col Joly, les Contamines. - Sav.: col du Bonhomme, Grand-Bornand, Crest-Voland, Hauteluce, Dent du Corbeau, les Allues, mont Cenis. - Is. : col d'Oddie, Theys, Allevard, Brandes et Auris, la Salette, forêt d'Esparon près le Monestier-de-Percy, la Croix-Haute. - H.-Alp. : le Goléon, mont Viso, col Agnel, la Grangette. - Alp.-Mar. : cols de Fremamorta et de la Maddelena. - En dehors de notre bassin au Cantal et au mont Dore.

14. pumllum Lap. - Rochers des Pyr.-Or. et de l'Aude, Canigou, Cambres-d'Aze, vallée d'Eyne à la Collada de Nuria, Carença, Costa Bona, montagnes du cours supérieur de l'Aude.

If. glaueum All. - Rochers. - Valais : Ermitage de St-Maurice, la Bâtiaz, entre Saas et Fee. - H.-Sav. : Salève au pied de la Grande Gorge, pied du Môle, grotte du mont Brizon, Bonnevaux, pont de Bioge près Thonon, St-Germain, la Tournette. - Jura 
helvétique et français, la Tourne du côté de Noiraiguc, Creuxdu-Van, Pont-de-Roide, Beaume-les-Messicurs, mont d'Ain, Charabotte et Retord en Bugey. - Is. : Villard-de-Lans, le Freney, Venosc, la Bérarde. - H.-Alp. : la Grave, Laularet, Briançon, mont Genèvre, Cervières, col Isoard, Vallouise, Côte Gélive près Gap, mont Séuse. - Nul dans les Vosges, le centre de la France, les Cévennes et les Pyrénées. - Espèce polymorphe, dont une forme, le H. brigantiacum, Jord., se trouve dans les H. et B.-Alp. à Monestier-de-Briançon et au Grand éboulement près du lac Praroard.

H. leucophaum G. G. - Rochers calc. - Is. : gorges d'Engins, col de l'Arc, Villard-de-Lans, Lans, St-Nizier.

1r. polituna G. G. an H. politum Fries? - Débris de rochers. Is. et H.-Alp. : St-Nizier, la Salette, la Bérarde-en-Oisans, Briançon, Abriès en Queyras.

II. saxetanum Fries. - Is. : la petite Moucherolle au Grand-Arc, le Fréney, la Bérarde.

HY. chondriloides Vill. - H. glencopsis G. G. - Débris de rochers. - Is. : montagne de Serres-sous-Taillefer, massifs des Grandes-Rousses et du Pelvoux, gorges du Diable à St-Christophe-en-0isans, la Bérarde. - H.-Alp. : la Grave snus les glaciers, Villard-d'Arène, mont Genèvre, pic de Chabrières, Rabou, col de Glaise, Séuse. - B.-Alp. : Longet. - Adoptant l'opinion de M. Arvet-Touvet, j'ai réuni le II. glaucopsis G. G. au chondrilloides Vill.

耳. bupleuroides Gmel. - Rochers. - Vaud et Valais : Enzeindaz, mont Fully, St-Bernard, val de Binn. - Ain : rochers au-dessus de la cascade de Charabotte. - H.-Alp. : entre Abriès et les Roux.

I. faleatum Arv. T. - Débris pierreux en montant au Galibier (H.-Alp.).

HT. Ieucophocum G. G. - Rochers calc. - Is. : gorges d'Engins, col de l'Arc, Villard-de-Lans, Lans, St-Nizier.

II. ehlorsefollum Arv. T. - II.-Alp. : vallée du Guil au pied des prairies de la Traversette et en allant au col de Valante.

IX. ealyeinum Arv. T. - Massif du Pelvoux, Valbonnais, le Désert au pied du mont Olan. 
I. scorzonerefollum Vill., II. glabratum Iloppe. - Prairies. -

Toutes les cimes de la clialne jurassique depuis le Weissenstein, Creux-du-Van, Suchet, Mont-d'Or, Montendre jusqu'à la Dôle et au Reculet. - Vaud et Valais: Alpes de Bex et d'Aigle, Fully, Gemmi, Zermatt et toute la clıaine mérid. jusqu'au St-Bernard. II.-Sav. : Salève à la Grange-Gorge, Brizon, Abbaye de Vallon, Vacheresse, mont Chalune, la Tournette. - Sav. : montagnes autour de Tignes, mont Cenis. - Is. et Dr. : col de l'Arc, les Fauges près de Villard-de-Lans, la petite Moucherolle et tout le massif calc. du Vercors jusqu'au mont Glandasse près Dic, la Salette, Poursollet au pied de Taillefer et toutes les montagnes de l'0isans. - H.-Alp. : la Grave, Villard-d'Arène, Lautaret, massif du Pelvoux, col de l'Échauda, mont Viso, la Chalp, StVéran, col de Vars, St-Sauveur près Embrun, pic de Chabrières. - B.-Alp. : Malemort, Lauzannier, Longet, Colmars. Alp.-Mar. : mont Frontero, val de Pesio, mont Bego.

Espèce polymorphe; la forme décrite par Villars a des feuilles velues; celle qui a été nommée glabratum par Hoppe a les feuilles presque glabres, étroites, la tige courte, les capitules plus petits.

II. clegrns Arv. T. - Is, et Dr. : prairies des montagnes de Villars-deLans et du Vercors.

Ir. villosum L. - Rochers des montagnes. - Nul dans les Vosges.

- Jura helvétique el français, Chasseral, Sonnenberg, Creux-duVan, Weissenstein, Mont d'Or, Suchet, Montendre, Dóle, Reculet. - Vaud et Valais : Bovonnaz, Anzendaz, mont Rosslen sur Sex, Fully, Méribé, St-Bernard, Zermatt, Gemmi. - H.-Sav. : Pitons du Salève, le Môle, Brizon, Flaine, Signal des Agneaux, Haut-deLin, mont Hermante, mont Méribel, Dents d'0che, Cornettes de Bise, mont des Granges, mont Laouet, col de Balme, la Flégère, le Brévent, Ia Tournette, Semnoz, Parmelan, Charvin, col Joli. - Sav. : col du Bonhomme, la Sauce, les Mottets, montagnes au-dessus d'Aime, Fort de l'Esseillon, mont Cenis, Crest-Voland, IIauteluce, mont du Chat, Dent de Nivolet, le Trélod et autres montagnes des Banges. - Is.: Grand Som, Haut-du-Seuil, St-Nizier, col de l'Arc, col Vert, la Moucherolle, mont Sénèpe, 
Grandes-Rousses, la Salette. - H.-Alp. : Lautaret, Péas, Abriès, mont Viso, pic de Chabrières, mont Morgon, col du Noyer, Poligny-en-Champsaur, Orcières, Sapet de la Bîtie-Neuve, Durbonnas, Chaillol, Alp-IIartin, la Grangette, Pleyne-de-Chaudun, mont Séuse, col de Glaise. - Dr. : mont Glandasse. - B.-Alp. : Horonaye, Lauzannier, Longet. - Alp.-Mar. : montagnes de Nice et de Menton. - Pyr.-Or. : Costa Bona, Cornella-du-Conflent, Vernet-les-Bains. - Espèce excessirement polymorphe; on a distingué notamment les formes pilosum Schl., semi.gltobrum Billot, nudum Gr., elongatum Froel.. grossidens Fries, et les suivantes dont la distribution géographique mérite quelques détails.

H. dentatum Hoppe, forme du villosum à fl. grandes, à feuilles sinuéesdentées non amplexicaules. - Rochers des montagnes. - Is. et Dr. : au-dessus de Ste-Ange, col Vert, col de l'Arc, Villardde-Lans et toute la chaine calc. du Vercors jusqu'au mont Glandasse près Die. - Ain et H.-Sav. : le Reculet, le Salève. H.-Alp. : envir, de Gap.

H. dasytrichum Arv. T., H. glandulifero-villosum? - H. et B.-Alp. : prairies du Lautaret, de Riftort et de Paris, vallée de Maurin en face du col de la Noire. - Chaine du Mlont Blanc.

II. nigritellum Arv. T. - Sav. : le Châtelard près St-Jean-de-llaurienne. H. et B.-Alp. : prairies du Lautaret, de Riftort et de Paris, col de Vars, mont Viso, val Agnel, val Longet au-dessus de Morin.

II. valdepilosum Vill. - Prairies. - Is. et II.-Aip. : mont Sénèpe, la Salette, l'Oisans, Villard d'Arène, Arcines, Lautaret, col de Vars. - Mont Cenis.

H. speciosum Hornn. - Suivant MI. Arvet-Touvet, c'est à tort que cette espèce a été indiquée dans les montagnes du Dauphiné.

M. ugtulatum Arv. T. - H.-Alp. : entre le col Malrif et celui des Thurres.

I. armerioldes Arv. T., H. Murithianum Favre (Bull. Soc. murith. 1876). - Sav. : mont Châtelard près St-Jean-de-Maurienne, mont Cenis. - II.-Alp. : Lautaret au vallon des Roches-Noires et autour de l'Hospice, col d'Arcine.

II. trichocladum Arv. T. - H.-Alp. : entre le col Alalrif et celui des Thurres. 
II. leucochloum Arr. T. - Yéme localití; est peut-être une variété du H. pilifertm.

I. pillferum Honpe. - Pelouses des terr. siliceus des Alpes; nul dans la chaine jurassiqque. - Taud et Valais : Anzendaz, mont Fully, colferret, St-Bernard à la Baux et à la Pierra, Thyon, Bagnes, mont Nuoble, Zan, Schwarzsee de Zermatt, Augstelberg, Offenthal.-II.-Sar.: Vergy, Méri, Tournette, mont des Granges, Morzine, Sixt, col de Balme, Jardin de la Jer de Glace, - Sar.: le Buahomme, les Jottets, la Yanoise, vallée des Allues vers le glacier de Gébrulaz, Sources de l'Arc. - Is. : chaines des SeplLaux, de Belledonne, de Taillefer et des Grandes-Rousses. II.-Alp. : massif du Pelroux, Lautaret, Galibier, Nérache aux Rochilles, cols Jalrif et de Tars, mont Queyrel et mont Lopet, Orcières à Jujau, mont Aurouse. - B.-Alp. : Lauzannier, Vallonnet de Meyronnes.

I. glonduliferum Hoppe. - Pelouses des terr. siliceux des Alpes; nul dans la chaine jurassique. - Vaud et Talais : Alpes de Morcles, Anzendaz, mont Fully, St-Bernard, mont Catogne, vallée de Saas à Distelalp et Jattmark, mont Joro, Simplon, Grimsel, Furca.- H.-SaF. : toute la chaine du Mont Blanc depuis le col de Balme jusqu'au Bonhomme; s'élite sur les moraines des glaciers, Jardin de la Mer de Glace, Couvercle, versant italien italien du Mont Blanc, sur les moraines des glaciers de l'allée Blanche, - Sav. : les Mottets, la Sauce, la Gitaz, les Allues, Jarrier, sources de l'Arc et de l'Isère, mont Cenis, mont Mirantin. - Is. : chaines des Sept-Laux, de Belledonne, de Taillefer, des Grandes-Rousses, du Pelroux, Pied-Jlottet au mont de Lans, la Salette. - H.-Alp. : la Grave, Lautaret, Galibier, col des Hayes, ral Prévayre et Ségure en Queyras, mont Viso, mont Queyrrel et Lopet en Champsaur, col de l'Alp-עartin, Chaillol-le-Yieil, Valgaudemar. - B.-Alp. : Fouillouse, Lauzannier, col de la Iadeleine, Bachasse. - Alp.-IIar. : sommités des Alpes de Nice et de Menton. - La rariette calvescens Fries a les feuilles glabres, on la troure sourent mêlée au type.

I. subnirale G. G. - Paturages des Alpes. - H.-Sar. et Sar. : le Maurais-Pas au-dessus de la Jler de Glace, Saut des Allues, Catal. Bassin du Rhône. 
mont Cenis. - II.-Alp. : cols de Paga, d'Isoard, de la Groix et de Ruines, rochers de Ségure du côté de la Taillante. - B.-Alp. : VaHlonnet de Meyronnes, Longet, la Portiolette.

I. aaxatile Vill. H. Lawsonii Vill. - Rochers calc. des Alpes et des Pyrénées.- Valais : entre la Bâtiaz et les Marques.-H.-Sav. et Sav. : Dents d'0che, mont Laouet, mont Cenis, cascade de Coux. .- - Is. : entre St-Laurent-du-Pont et la Grande Chartreuse, Bovinant. - Dr. : Barnave. - H.-Alp. : Briançon, mont Genèvre, Cervières, la Chalp, mont Queyrel, Orciẻres, Sigattier, Ėmbrun, pic-de Chabrières, col de Glaise, Charance, la Grangette, Matachard, mont Séuse - - B.-Alp.: Fours à la Baume, ravinș du Bourgel. - Alp.-Mar. : au-dessus de St-Sauveur. - Gard : Montdardier, Pradines, Campestre, Brama-Bịoou. - Hér. : la Sérane, le Larzac au Caylar, St-Naurice, la Vacquerie. - Aude : Axat audessus de Quillan. - Pyr.-Or. : Jont, Louis, Bac de Bolquère.

. La forme que Villars avait distinguée sous le nom de H. Law. - sonii ne differe du type que par des capitules plus nombreux et plus grandș.

Ir. intertexiun Arv. T. - H.-Alp. : Lautaret, col de Vars, col de la Croix.

I. ollvaceum G. G. - Pyr.-Or. : vallon de Consolation près Collioure, Trencada d'Ambulla.

H. oleotirens Arv. T. - Pyr.-Or: : méme localitê...

H. neocerinthe Fries. - Bois des Pyr.-0l. : Bac de Bolquêre, $\therefore$ fortifications de Iont Louis, Prats de Sollo au pied de la Toursq belinide-Mir.

H. compositum Lap. - Pàturages des Pyr.-Or. : Prats de Mollo, la. Preste, bords du Tech.

Ir. 'olatum Lap: - Rocailles des Pyr.-0r. : vallée d'Eyne.

H. cerinthoides Fries, II, longifolium Șchleich. - Rochers. Vaud et Valais : Alpes de Bex, Surchamp, mont Fully, BourgSt-Pierre, St-Bernard, Binn.- H..Say, et Sav. : mont Chalune, Mer de:Glace, à Clıamonix, Lans-le-Bourg. - H.-Alp. : col de Vars. - Pyr.-Or. : Bac de Bolquère, Coma du Tech, Llaurenti. II. cordifolium Froel. - Pyr.0r : Prats de Mollo.

I. vogesiacum Mougeot. - Rochers et pâturages des Vosges au 
Ilohneck et au Rotabac et de la chaine jurassique à la Dole, au Colombier et au Reculet; en Suisse au Wasserfall, Dornacherberg. - Alpes vaudoises et savoyardes à Bovonnaz el à Chamonix. - Gard : Brama-Biou près de Camprieux. - En dehors de nos limites au Mont Dore et au Cantal.

II. sonchoides Arv. T., H. jurano-vogesiacum ? - Mont Dore d'Alvergno."

H. amplexienule L. - Rochers calcaires. - Nul dans les Vosges. - Jura helvétique et français, sommités entre Montbéliard et le Saut du Doubs, Cótes du Dessoubre, Clos du Doubs, Lomont, la Grand Combe, fort de Joux, Morcy, St-Claude, le Colombier, la Faucille, Thoirette, fort de l'Ecluse, la Valserine, la Bienne, l'Albarine, Colombier du Bugey. - Vaud et Valais: Aigle, St-Maurice, Rossetan, mayens de Saxon, Sion, Leukerbad, Stalden. - П.-Sav. : montagnes d'Abondance, mont Méribelle, Dents d'Oche, la Diosaz, Aiguille à Borhard, Brévent, GrandBornand, rochers de St-Clair entre Annecy et Thones, Sevrier, Cuvat, mont Charvin, Salève. - Sav. : mont du Chat, St-Saturnin près Chambéry, Moutiers, St-Jean-de-Iaurienne, fort de l'Esscillon, mont Cenis. - Is, et Dr. : Grande Chartreuse, StNizier, le Fréney, Crémieü, Barnave, Nyons. - H. -Alp. : Lautaret, Monestier-de-Briançon, St-Véran, col Marcel, Guillestre, Chorges, Durbon, Chaillol-le-Vieil, Loubet, Chaudun, col de Glaise; mont Aurouse. - B.-Alp. : St-Paul, la Condamine, Sérennes. - Alp.-Mar. : montagnes de Grasse, Nice et Menton. - Vaucl. : mont Ventoux, St-Amans. - B.-du-Rh. et Var:- SteVictoire, Pic de Bretagne, Ste-Baume, Ampus. - Ard. : rochers basaltiques de St-Julien, Entraigues, Mayres. — Gard et Hér. : le Vigan, Campestre, Pic St-Loup, le Larzac au Caylar et à St-Nlaurice, Madières, St-Amand-de-Mounis, St-Chinian, Avène. - Aude et Pyr.-0r.: Mas-Cabardès, les Corbières, Axat, Villefranche, Trencada d'Ambulla, St-Vincent, Vernet-les-Bains, murs de Mont Louis, Prats de Mollo à la Roca Galinera. - En dehors de nos limites sur les rochers calc. de l'Aveyron autour de Millau et de' Ste-Affrique, roch, calc. de la Lozère à Mende, Florac, ainsi que dans le Cantal, la II.Loire et les Pyr. cen- 
trales. Par une exception singulière, il se trouve aussi sur les rochers granitiques des bords de la Loire vers le Pertuiset et Cornillon. Il serait intéressant de connaitre la composition chimique de ces rochers. Il importe de remarquer que le $I$. amplexicaule, qui manifeste une préférence très-marquée pour les rochers calcaires, crolt aussi sur les rochers basaltiques du Vivarais, dans la composition desquels entre le silicate de chaux, l'un des éléments constitutifs des feldspaths labradoriques.

H. rupicola H. Jord., H. leplocladum Gris. - Roch. calc. de Sisteron (B.-Alp.).

II. pulmonarioides Vill. - Diffère de l'amplexicaule par les feuilles caulinaires lancéolées-sessiles et non amplexicaules. - Roch. calc. - Jura et Ain : St-Claude, Nantua, vallée de l'Albarine, Tenay, Chaley, Charabotte, Chartreuse de Portes, Ordonnaz, Rossillon, Chavornay. - Valais : St-Ilaurice, Sembrancher, Bovernier. - H..S. et Sav. : Salève, Sommier, vallée de Thônes, bains de la Caille, Chambéry, Albertville, Tamié, Moutiers. Is. et Dr. : Bastille de Grenoble, mont Rachais, Grande-Chartreuse, la Terrasse, Sassenage, St-Nizier, montagnes de Die. II. et B.-Alp. : la Grave, mont Chabrières, Chaudun, Séuse, Aurouse, Sẻrennes, Fouillouse. - Alp.-Marit. : Tende. Vaucl. : mont Ventoux. - Pyr.-Or. : Mont-Louis, - Avec Villars et plusieurs autres auteurs, j'incline à considérer l' $I$. pulmonarioides comme une forme de l'amplexicaule.

II. pseudo-cerinthe Koch. - Rochers des montagnes. - Vaud : Jaman, Bovonnaz, les Diablerets. - H.-Sav. : Pitons du Salève, Vergy, rochers du pont St-Clair dans la vallée de Thônes, Cornettes de Bise, Signal des Agneaux, Nautau, montagnes de Samoëns et de Sist, Jardin de la Mer de Glace. - Is. et H.-Alp. : le Sappey, la petite Moucherolle, la Grave, Lautaret, mont Genévre, pic de Chabrières, Charance, Loubet, col de Glaise, mont Séuse. B.-Alp. : Digne, la Condamine, Sérennes. - Pyr.-Or. : Pratsde-Mollo ả la Tour-de-Mir.

Ir. urtieaceum Arv. T. - Is. : Engins, St-Nizier, Villard-de-Lans, Corençon, la Rampe-des-Commères après le Bourg•d'Oisans. II. rhombifolium Arv. T. - Cuves de Sassenage, Engins, St-Nizier. 
II. ligusticum Fries. - Rochers calc. - Ain : Fort de l'Ecluse, Tenay, Argis, Ordonnaz, Inimont. - II.-S. ct Sav. : Salève au Coin et au Grand-Piton, Montmélian. - Valais : Bagnes. - Aude : mont St-Victor près Villeneuve, Tuchan. - D'après M. ArvetTouvet, la plante de ces localite’s n'est pas le II. ligusticum Fries, mais bien le $I$. rhombifolium.

H. nlpinum L. - Rochers siliceux des Alpes; nul dans la chalne jurassique. - Vaud et Valais : Alpes de Jaman, d'Enzeindaz et de Morcles, Fully, Alesse, Thyon, Gerenthal, Griess, Musithal dans Eginenthal, Glacier du Rhone, Schwarzsee de Zermatt, vallée de Saas à Mattmark et à Distelalp, Simplon, St-Bernard. II.-Sav. : col de Balme, Montanvert, Prarion de St-Gervais, Brévent, Jardin de la Mer de Glace, Vergy au pied du col de Balafrasse. - Sav. : cols du Bonhomme et de la Seigne, Croixde-Pierre et Grand-Pariraz près IIauteluce, mont Mirantin, Dent du Corbeau, Crest-Voland. - Is. : chalnes des Sept-Laux, de Belledonne autour des lacs du Crouzet et de la Sitre, Taillefer près du lac de Poursollet, la Croix-IIaute, massif du Pelvoux. - H.-Alp. : le Valgaudemar, Nérache aux Rochilles, mont Viso, St-Véran, Clausis. - Pyr.-Or. : vallẻe d'Eyne, Cambres-d'Aze, Prats-de-Balaguer. - On prétend l'avoir trouvé dans les Vosges.

Ir. heterodon Arv. T. - Rochers calc. du Dauphiné dans toute la chaine qui s'êtend de Lans à St-Nizier, au col de l'Arc et au Grand-Vehmont. - Envir. de Briançon.

II. Jacquini Vill. - Rochers calcaires. - Nul dans les Vosges. - Jura helvêtique et français jusque dans les montagnes du Bugey, à Rossillon, Lhuis, St-Rambert, Belley, Châtillon-deMichaille, Montanges. - H.-Saône : calc. jurass. de Nans. C.-d'Or : toute la Côte jurassique de Dijon à Chagny, — Vaud et Valais : grotte de Montcherand, les Clées, St-Tryphon, Roche, Villeneuve, St-Maurice, St-Léonard, Gemmi. - H.-Sav. et Sav.: Stc-Harie en face des Houches, mont des Granges, Dents d'Oche, IIaut-de-Lin, Pont-St-Clair entre Annecy et Thônes, GrandBornand, Verrerie de Thorens, mont Charvin, petit Salève, Pasde-l'Echelle, Saleve-au-Coin, mont d'Etale, mont du Chat, mont Nivolet, St-Jean-de-Naurienne, Bourg-St-Haurice. — Is. : Cré- 
micu, Grande-Chartreuse, la Terrasse, Pariset au Désert, StNizier, Villard-de-Lans, petite Moucherolle, Poursollet au pied de Taillefer, la Pra de Belledonne. - H.-Alp. : Rabou, mont Sẻuse, col du Noyer. - B.-du-Rh. et Var : Ste-Victoire, pic de Bretagne, Ste-Baume, Pilon-du-Roi. - Aude : Lapeyre audessus de Quillan, les Corbières. - Pyr.-Or. : Mont-Louis, Molle-de-Planès, Bac de Bolquère.

IK. Ianatum Vill. - Rochers. - Valais : ravin de la Morge près Conthey, Saxon, Saillon, entre Bovernier et Sembrancher, Chemin-Neuf, mont d'Orge, entre Varone et Inden, Findelen, Visp, Briegg, Schalberg, Binn. - H.-Sav. et Sav. : Salève, Mole, Chilly et Veyrier près Annecy, St-Jean-de-Haurienne, St-Michel, Lans-le-Bourg, Champagny, Tignes, mont Iseran. Ain : Villebois, Serrières, St-Rambert, Portes, d'Argis à Evoges, Tenay, Inimont, St-Benoît, Pierre-Chàtel, Mfontanges. Is. : entre la Balme et Yenne, mont Rachais, mont de Lans à PiedMottet, mont Chamoux. - H.-Alp. : la Grave, Lautaret, Cervières, mont Genèvre, Malrif, mont Viso, Ségure, Guillestre, Ceillac, mont Chabrières, col du Noyer, mont Queyrel en Champsaur, Pleyne-de-Chaudun, la Garde, mont Aurouse. B.-Alp. : Longet, la Condamine, Sérennes, Bouzolières. Alp.-Mar. : montagnes de Nice et de Menton. - Pyr.-Or. : vallées de Llo, d'Eyne, de Prats-de-Balaguer et de Carença, - Espèce polymorphe.

II. Lannes a trouvé sur les rochers de St-Cornille, près de la Condamine, une forme à feuilles présentant sur la face supérieure des taches coulcur lie de vin; c'est le H. anochroüm Jord. et Borel encore inédit.

I. andryaloides Vill. - Rochers calc. - H.-Sav. et Sav. : petit Salève, rochers du Fier près de Seyssel, Chilly près Annecy, mont Gargan près Moutiers. - Is. et Dr. : le Chevalon, StEynard, Sassenage, Engins, Villard-de-Lans et le Vercors, Varces, Vif, Venosc, Barnave. - H. et B.-Alp. : Briançon au Fort des Têtes, mont Viso, col Agnel, Rabou près Gap, Sisteron à la Baume, Bouzolières. - Alp.-Mar. : la Roquette, Bouyon, col de Braus. - B.-du-Rh. et Var : Ste-Baume, Bau-de-Bretagne, N.-D.-des-Anges, - Espèce polymorphe. 
II. eriopsilon Jord. - Hochers calc. - II. et B.-Alp. : Charance, col de Glaise, Château-Queyras, la Condamine. - Dr. : mont Glandasse.

II. pichum Pers. - Forme à feuilles glabres sur la face supér. et tachées

de brun. - Valais : Binn. - Sav. : Leysse près Chambéry.

II. Liollardi Vill. - Rochers. - Is. : Sassenage, Villard-de-Lans. -

H. et B.-Alp. : entre lo Galibier et le Lauzet, Vallouiso vers le col de l'Echauda, col de Glaise, Bouzolières, Itoronaye.

Suivant M. Arvet-Touvet, l'II. Lioltardi Vill. est une variété dressée et simplement dentée de l'H. andryaloides. L'Epervière décrite par Grenier et par Fries sous le nom de II. Liotlardi est celle qui sera désignée plus bas sous le nom de II. psendo-lanatum Arv.-T.

Tr. Kochisuum Jord. - Rochers calc. - Is. et Dr. : Mcylan, la Terrasse, St-Bernard, le Touvet, Pariset, col de l'Arc, montagnes de Die. - Sav, : cascade de Coux, Pas-de-la-Coche.

I. Iansleum Arv. T. - Rochers, - Molasse des envir. de Villardde-Lans, notamment à la Roche-Pointue et Bréduire sur les rochers de la cascade, chemin de Corençon, en face du moulin, bords de la Bourne, Tourtre et Vernaison, près des Goulets.

Une forme plus verte et plus allongée se trouve mêlée au type.

II. Reboudianum Arv. T. - Rochers des environs de Villard-de-Lans et des Grands-Goulets.

H. Ravaudii Arv. T., H. andryaloidi-amplexicaule? - Rochers du chemin de la Tancanière près Villard-de-Lans, des bords du Nan à Cognin, près St-Marcellin (Is.). - Var. virescens : rochers avant d'arriver à Chấteau-Queyras (H.-Alp.).

IT. verbaseifollum Vill., II. pelititum Fries. - Débris de rochers. - Is., H. et B.-Alp. : envir. d'Uriage, mont Genèvre, Larche, le Lauzannier.

I. thapsoides Arv. T. - Bois de Pins au mont Genèvre près de Briançon, col de la Croix et col Malrif en Queyras.

I. melandrifolium Arv. T. - Prairies du Lautaret.

I. floceosum Arv. T. - Lautaret, col Malrif, mont Viso.

H. Iychnloides Arv. T. - Bois de Pins près Briançon, col Malrif, entre Abries et les Roux. 
I. mollitum Arv. T. - Prairies du bord du Guil, entre li Chalp et la Traversette, envir. du Chalet de Ruines.

I. ehloropsis G. G. - Débris de rochers. - Is. et II.-Alp. : Taillefer et toutes les montagnes de l'Oisans, la Salette, massif du Pelvoux, Valgaudemar, Villard d'Arène, Lautaret, au-dessus du Lauzet, Névache, Malrif, vallée du Guil.

Var. mespilifolium Arv. T. - Prairies de la Traversette.

I. Pamphin Arv. T. - Lautaret, le Valbonnais au pied du mont Chamoux.

I. rupestre All. - Rochers, - Vaud : d'Aigle au Sepey, Enzeindaz, mont Parey.- Ain et Is. : Inimont, Marcieu, Monteynard. - H. et B.-Alp. : Cervières, entre Malrif et Abriès, Guillestre, Embrun, Gap, mont Séuse, Castellanne, Sérennes, Bouzolières. - Alp.-Miar. : St-Auban.

I. pseudo-Ianatum Arv. T., H. Liottardi G. G., Fries (non Vill). - H. et B.-Alp. : Villard d'Arène, Lautaret, vallée du Guil au-dessous de Valante, pic de Chabrières, mont Séuse, val Longet au-dessus de Maurin.

II. lanatellum Arv. T. - Is. : Grande Chartreuse, Chalais, mont Jusson au-dessus de Voreppe. - H.-Alp. : Lautaret, mont Genèvre, Malrif, col de la Croix, vallon du Guil en face du chalet de Ruines, col Fromage au-dessus de Ceillac, St-Véran, Pic de Chabrières.

II. Farinulentum Jord, - Rochers calc. - Ain : Rossillon, Inimont, Tenay, Argis, Portes. - Is. : Côtes de Sassenage, gorges d'Engins, Pariset, St-Nizier, Grandes-Rousses, Clavans. - H.Alp. : la Grave, mont Genèvre, le Queyras, massif du Pelvoux. - Sav.: St-Jean-de-Maurienrie.

I. leiopogon Gren. - Rocailles calc. — Is. et H.-Alp. : le Désert au fond du Valjouffrey, Devez de Rabou, col de Glaise.

I. cephalodes Arv. T. - H.-Alp. : vallon de Guil en allant au col de Valante.

H. oligocephalum Arv. T. - Is. et H.-Alp. : mont Chamoux, le Valjouffrey, vallon du Guil.

II. murorum L. - Bords des chemins, débris de rochers, graviers dans tout le bassin depuis les plaines jusqu'aux montagnes. - Espèce polymorphe au plus haut degré. 
II. pracox C. II. Schultz. - Rérgion des Sapins de la chaine jurassique.' - Prangins près Genève. - II.-Sav. et Sav. : Sale̊ve, Montmélian, - Var: les Mayons.

II. medium Jord. - Collines de la chalne jurassidue. - Is. et II.-Alp. : pont d'Eybens, Varces, Prémol, Lautaret, Rabou près Gap. - Consiléré par plusicurs auteurs comme variété du précédent. II. petiolare Jord. - Clıaine jurassigue. - Rh. : Francheville, Dardilly, Chaponost. - Loire. - Aude : Montolieu.

II. adscitum Jord. - Nont Pilat. - Auvergne.

II. glancimum Jord. - Rh. : Dardilly, Chaponost, Vaugneray. - Dauphines. - S.et-L. - Centre de la France; Tarn.

II. palulipes Jord. - Rh. : Chaponost. - Centre de !a France. - Tarn. II. comatulum Jord. - Curgy près Autun. - Rh. : Chaponost.

II. subdolum Jord. - Rh. : Dardilly, - Centre de la France.

H. rubescens Jord, - Rh. : Tassin.

II. adscitum Jord. - Mont Pilat. - S.-et-I. : Ornée, Hontjeu. - Auvergne.

II. fragile Jord. - Rh. : Couzon, Charbonnières, Tassin, Francheville,

Vaugneray, - Is, et H.-Alp. : Crémicu, moulin de Ste-Ange, pied du Grand-Galbert, Villard-d'Arène. - Gard : le Vigan, Concoule. - Plaine de l'Hérault. - Aude et Tarn: la Montagne Noire. - Pyr.-Or. : Font-Romeu, la Cerdagne, Saillagouse, Bourg-Madame.

II. Jaubertianum Timb et Loret. - Haies et bois. - Hér. : Prades, Mireval, Montarnaud, Bédaricux, Madières, la Vacquerie, Pézenas.

II. robustum Martr. Don. - La Montagne Noire.

II. parile Jord. - Rh. : St-Romain-au-Mont-d'Or.

II. adjunchum Jord. - Rh. : Écully.

II. divergens Jord. - Rh. : Chaponost. - Centre de la France.

II. retrodentstum Jord. - Rh. : Écully, Francheville.-Aude et Tarn:

la Montagne-Noire. - Centre de la France.

II. montolcarense Timb. Lagr. - Aude : Coume de Guitard près Montolieu, Ste-Colombe-sur-l'Aiguette.

II. mucronulatum Timb. Lagr. - Aude: Coume de Guitarl, mamelon de l'Ermitage de St-Roch. 
I. sessilifoliun Timb. Lagr. - Aude: mont St-Victor près Durban.

II. similatum Jord. - Rh. : Narcy. - S.ect-L. - Tarn et centre de la France.

H. inclinans Jord, - Rh. : L'Arbresle.

H. ovalifolium Jord. - Terr. granit, du Morran autour de Saulicu, du Lyonnais à Ecully, Charbonnières, L'Arbresle, Liergucs. Terr, granit. du Tarn el du centre de la France.

H. brevipes Jord. - Rh. : Charbonnières. - Aude et Tarn : IIontagne Noire. - Centre de la France.

H. fallens Jord. - Rh. : St-Cyr. - S.-et-L. : Montmin près Autun. Tarn et centre de la France.

H. bounophilum Jord. - Bh : St-Cyr. - S.et-L. : Brisecou près Autun. - Aude et Tarn: Montagne Noire et centre de la France.

H. rarinœum Jorl. - Rh. : Couzon. - Montagne Noire et centre de la France.

H. collivagum Jord, - Is. : Vernas près Crémieu.

H. furcillatum Jord. - Rh. : Vaugneray, - Tarn et centre de la France.

H. conjugatum Jord. Rh. : Vaugneray, - Tarn et centre de la France. H. prasinifolium Jord. - S.-el-Loire : Montjeu, la Porolle. - Rh.:

Charbonnières. - Tarn et centre de la lrance.

II. fissifolium Jord. - Rh. : Francheville.-Tarn et centre de la France. II. oigocludon Jord. - Rh. : Francheville. - Tarn et centre de ha France. II. pinetorum Jord. - Rh. : Vaugneray. - Tarn et centre de la France. II. recensitum Jord. - Rlı. : Vaugneray. - Tarn et centre de la France. II. scabripes Jord.-Rh. : mont Verdun. - Tarn et centre de la France. H. sparsum Jord, - Rh. : St-Germain, - Tarn et centre de la France. - Montagne Noire.

If. gentile Jord. - Rì. : St-Genis-Laval. - Tarn et centre de la France. II. pilosulum Jord. - Mont Pilat. - Aude : Montolieu. — Centre de la France.

II. Pourretianum Timb. Lagr. - Pyr.-0r. : St-Antoine-de-Galamus. H. cinerascens Jord. - S.et-L. et C.-d'Or. - Rh. et Is. : Charbonnières, Dardilly, L'Arbresle, forêt de Chambaran.-Gard : Salbous, Pont-du-Gard, Arphy. - Aude et Pyr-Or. : Pech de l'Agnèle, la Clape, Gruissan, Fontfroide, Fontlaurier, les Corbières, mont St-Victor près Durban, col d'Estren, Tuchan, Iontagne Noire, St-Antoine-de-Galamus. 
11. pilosissimum Pourr., non Fries. - Pyr.0r. : St-Antoine-de-Galamus, Pont de la Fou.

II. viridicoltum Jord. - Rh. : Vaugneray. - Centre de la France.

II. syngenes Jord. - Mont Pilat.

II. cardiophyllum Jord. - Envir, de Genève.

II. virens Jord. - Rh. : Francheville.

II. exolericum Jord. - Rh. : Francheville. - S.-et-L. : Ornée. Centre de la France.

II. sylvivagum Jord. - Envir. de Lyon. - La Montagne Noire.

II. abieticolum Jord. - Mont Pilat.

II. serratifolium Jord. - Envir. de Lyon.

II. sylvularum Jord. - Envir. de Lyon. - S.-et-L. : Pauvret, Mont-

jeu près Autun. - Centre de la France.

H. oblongum Jord. - Chaine jurassique. - S.-et-I. : Montjeu. -

Rh. : Charbonnières, Alix. - H.-Alp.: Rabou pris Gap, la

Grangette. - La Mlontagne Noire.

II. nemorense Jord. - Chaîne jurassique de l'Is. : St-Eynard, Grande

Chartreuse, Pariset, col de l'Arc, entre Corps et la Salette. -

H.-Alp. et Dr. : Devez de Rabou, Bas-Bouvante. - Sav, el H.-

Sav. : montagnes du Chrablais et du Faucigny, le Saléve, les

Bauges, Maurienne et Tarantaise.

II. tenuipes Jord. - Envir. de Lyon.

II. aspreticolum Jord. - Envir. de Lyon. - Centre de la France.

II. petrogenes Jord. - Mont Pilat. - Centre de la France. - Aude :

Iontolieu.

I. pallidulum Jord. - Envir. de Lyon. - Mont Pilat. - Ard. : StAgrève.

II. albulum Jord. - Ard..: Thueyts.

II, albogilvum Jord. - Gard : St-Nicolas.

H. campylotrychum Jord, Gard : St-Nicolas.

H. laciniosum Jord. - Rh. : Dardilly. - Montagne Noire. - Is. : St-Nizier.

H. consociatum Jord. - Nontagne Noire.

H. Inchsum Koch, H. cersium G. G. an Fries? - Région alpestre de la chalne jurassique, Mont d'Or, Suchet, Dôle, Reculet. H.-Sav, et Sav. : Salève aux Treize-Arbres et à la Croisette, le 
IIôle, mont Cenis. - Is. : chaîne de Belledonne vers le lac Crouzet, St-Nizier, mont Chamoux. - Pyr.-Or. : valléc du Réart autour de Llinas, Calmelles, Iontoriol, N.-D. du Col, Font de Comps.

K. bifdum Kit. - H.-Sav. : gorges de la Diosaz. - Plaines et basses montagnes de l'Hérault. -- Pyr.-0r. : Font-de-Comps.

H. cirritum Arv. T. - Cetle espèce comprend trois formes : $1^{\circ}$ celle à péricline noir, hérissé de poils glanduleux; Is. et II.Alp . : Grande Chartreuse, de St.Ange au col de l'Arc, Lautaret, Clıorges, Rabou près Gap; a été rapporté par II. Verlot à l'H. atratum Fries; $2^{\circ}$ forme à péricline arrondi et ventru à la maturité, hérissé de poils roussâtres, décrite dans le Catal. de II. Verlot sous le nom d'H. saxifragum Fries, Is., H.-Alp. et Sav. : les Sept-Laux, Taillefer, l'Oursière, sous les glaciers de la Grave, les Alpes de la Maurienne; $3^{\circ}$ la forme grêle, cendrécglaucescente à péricline non poilu (II. elisum Arv. T.); II.-Alp.: la Chalp en Queyras, Malrif, montée du col des Thurres.

Ir. porrectum Fries. - Rocailles à l'entrée du vallon d'Ardran au Reculet.

I. papyra ceum Gren. - Forêts de Sapins de la chaine jurassique, le Suchet, le mont d'Or jusqu'au Reculet.

H. Inevleaule Jord. - Bois des plaines et des montagnes, principalement sur les terrains siliceux. - Doubs et Jura : chailles siliceuses de l'exfordien de Chalezeule, près Besançon, forêt granit. de la Serre. - Chaine granit. du Beaujolais à Chenelette, Monsols, Alix. - Is. et H.-Alp. : argiles de la forèt de Chambaran, mont Séuse.

H. virgulntum Arv. T. - Vallée du Guil en Queyras.

1. vuIgatum Fries, H. sylvaticum Lam. - Bois des plaines et des montagnes. - Chaîne des Vosges jusqu'au Ballon de Giromagny et au mont de Vannes dans la H.-Saône, chaine jurassique jusque dans le Bugey, Savoje, Dauphiné, Alpes Basses et Marit. jusque vers Tende. - Le Morvan, le Beaujolais, Lyonnais, Forez, Pilat, Vivarais, Cévennes jusque dans les environs du Vigan, d'Alzon, de Concoule, l'Espérou, l'Escandorgue et l'Espinouse. - Aude et Pyr:-Or, : la Montagne-Noire, Canigou, 
forêt de Salvanère. - Pyr. centr.; Tarn, Lozère, Aveyron et massif central. - Espèce très-polymorphe.

II. acuminatum Jord- - Châne jurassique. - Les Echets en Dombes.

- Rh. : Chasselay, Quincieux, St-Germain. - Manteyer près Gap.

II. fuscescens Jord. - Les Echets en Dombes au bois des Volières.

II. sciaphyllum Jord. - Rh. : Charbonnières.

H. adelphicum Jord. - Rh. : Narcy.

II. tortifolium Jord. - Mont Pilat et centre de la France.

II. pracedens Jord.- Nêron. (Ain),

H. brevidentatum Jord. - Mon I Pilat.

H. cheriense Jord. - Montjeu près Autun.

II. finilimum Jord. - Bh. : Alix. - Centre de la France.

II. argillaceum Jord. - Terr. argileux de la Dombes et de la Bresse. -

Rh. : Vaugneray aux Jumeaux. - Is, et H.-Alp. : St-Christopheen-0ișans, Lautaret. - Centre de la France.

II. crubescens Jord. - S.-et-L.

H. festinum Jord. - S.-et-L.

II. chlorophyllum Jord. - Ain : Ordonnat.

II. perscissum Jord. - Mont Pilat, Pierre-sur-Haute.

II. improperum Jord. - Envir. de Lyon.

H. inumbratum Jord. - Is. : Pont-de-Chérui.

II. aviicolum Jord. - Envir. de Lyon.

H. medioximum Jord. - Envir, de Lyon. - Mont Pilat.

II. celsicaule Jord. - Mont Pilat.

II. approximatum Jord. - Rh. : Tassin, Charbonnières, Dardilly, Alix.

- Aude: : Montagne-Noire.

H. inquinatım Jord. - Ain : Néron.

H. tinctum Jord. - Rl.. : Izeron.-S.-et-L. : Ornęe.

$\boldsymbol{H}$. divisum Jord. - Rh. : Oullins, Francheville, Givors, Sain-Bel.

H. laceratum Jord. - Rh. : Dardilly,

H. asperwatum Jord. - Rh. : Dardilly, - Pierre-sur-Haute, - Montjeu près Autun.

II. nemorale Jord. - Mont Pilat.

H. laciniosum Jord. - Rh. : Dardilly. - Is. : St-Nizier. - MontagneNoire. 
II. pallidifolizm Jord. - Envir. de Lyon. - Pierre-sur-Haute. S.-et-L. : Montjeu.

II. pilatense Jord. - Mont Pilat.

II. partitum Jord. - Mont Pilat.

H. commixtum Jord. - Hont Pilat, Pierre-sur-Haute. - Rh. : St-Bonnet-le-Froid. - Is. : Theys, St-Nizier. - Ard. : le Mezenc. Aude et Tarn : Montagne-Noire.

H. dissidens Jord, - Mont Pilat.

II. fictum Jord. - Rl. : St-Bonnet-le-Froid.

II. incisifolium Jord. - Rh. : Vaugneray. - Mont Pilat.

II. confine Jord. - Rh. : l'Arbresle.

H. intersitum Jold. - Rh. : Dardilly.

II. cruentum Jord. - Rh. : Francheville, Charbonnières, Dardilly, Alix, Soucieu.

II. pinicolum Jord, - Rh. : la Merlanche près Givors.

H. macellum Jord. - Rh. : la Merlanche près Givors, - S.-ct-L.

II. particeps Jord. - Vienne.

H. stellgerum Frœl. - Rochers des montagnes de l'Hér. : Pic St-Loup, Capouladoux, St-Guilhem-le-Désert, St-Mlartin-deLondres aux Arcs.

H. ropunculoiden Arv. T. - Is. : bois des Follets entre $\mathrm{Oz}$ et Vaujany en Oisans, montée du Valbonnais au mont Chamoux, le Désert en Valjouffrey, massif du Pelvoux.

II. buglossoides Arv. T. - Pyr.-Or. : rochers de Molitg.

I1. vestitum (x. G. - Pyr.-Or. : Mont Louis. - C'est à tort, suivant M. Arvet-Touvet, que cette espèce a été indiquée au Lautaret.

H. Insiophyllum Koch. - H.-Alp. : N.-D. de Laus, Savines, Embrun.

H. palldum Biv. - Is, et II.-Alp. : le Valbonnais au-dlessous du Mont Chamoux, le Désert en Valjouffrey, Lautaret, St-Véran et col de la Croix en Queyras.

Var. persicifolium Fries. - Champrousse près Uriage (Is.).

Var. intricatum Arv. T. - Le Lautaret (H.-Alp.).

H. pallidiforme Arv. T. - Valais. - Is. et H.-Alp. : Champrousse, Taillefer, le Valbonnais, le Valgaudemar et tout le massif du Pelvoux, col de la Croix en Queyras. 
II. ceralodon Arv, 'T. - Iybride ou variété du précédent. - Is. et II.-Alp. : montagne de Scrres au-dessus du Villard-St-Clıristophe, massif du Pelvoux, Lautaret. - Varie à dents des feuilles longues ou courtes.

11. melanospermum Arv. T. - Is. el II.-Alp. : Taillefer, montagnes de l'Oisans, massif du Pelvoux, mont Tabor, Lautaret. - Pyr.0r. : Mont Louis.

H. brunellaforme Arv. T. - Le Désert en Valjoufirey, pentes du mont Olan.

II. Guillonianum Arv. T. - Pyr.-0r. : Mont-Louis, rochers apres la Cabanasse.

I. aconpioidew Arv. T. - Panicule de forme scorpioide, écailles de l'involucre obtuses. - Is. et H.-Alp. : vallon entre la Salette et le Valbonnais, pentes du mont Olan, Navette en Valgaudemar, pentes au-dessous du lac de Pétarel, Lautaret du côté du Lauzet.

I. eoerulewm Arv. T., non Scop. - Is, et H.Alp. : vallon sous le mont Chamoux, cols de Vars et de la Croix, vallée du Guil sur les pentes de la Traversette et de Valante, hauteurs du. vallon de Ségure.

II. viride Arv. T. - II.-Alp. : entre le col Malrif et celui des Thurres, mont Viso à la bifurcation des sentiers de la Traversetle et de Valante, col de la Croix.

II. maellentum Fries. - Envir. de Gap.

H. subalpinum Arv. T., II. prenanthoideo-murorum? - Is, et H.-Alp. : montagnes de Villard-de-Lans, col de l'Are, Champrousse, montagnes de l'Oisans, du Pelvoux, du mont Viso, du Valbonnais, Lautaret. - Pyr.-Orientales.

II. subsecundum Arv. T. - Mont Viso, au pied des prairies de la Traversette, bords du Guil.

II. psendoviride Arv. T. - H.-Alp. : col Malrif.

I. Themaipleeum Arv. T. - Is. : mont Sénèpe près la Mure, massif du Pelvoux, vallon de la Salelle, les Clots au fond du Valgaudemar. - Cette forme a été décrite par M. Arvet-Touvet sous le nom d'H. grandifolium (Monogr. p. 39), remplaces par celui d'H. hemiplecum, parce que l'épithète de grandifolium a été díjà appliquée par Schulız à une Eperviere d'Afrique. 
1. doronieifoliam Arv. T. - Is, et II.-Alp. : chalne calc. de StNizier à Die, col Vert, mont Sénèpe, montagnes de l'Oisans, Villard d'Arène, bords' du torrent qui descend des Trois-hivêchès.

II. isatifolium Arv. T. - Is. et H.-Alp. : le Désert en Valjouffrey, sous le mont Olan, chaîne de St-Nizier à Die, les Fauges du Villardde-Lans, Alpes de l'Oisans, Lautaret, vallée du Guil depuis la Chalp jusquaux prairies de la Traversette.

IX. juranum Fries. - Bois et pâturages. - Sommités de la chaine jurassigue depuis le Creux-du-Van, le Suchet et le Mont d'Or jusqu'au Montendre, à la Dôle et à la Faucille: - H.-Sav. : Salève, Voirons, Brizon, Móle. - Sav. : Arclusaz au-dessus de St-Pierre-d'Albigny, base du mont Granier ver's Entremont-leVieux, Dent du Corbeau, entre la Fraidaz ct Balastat sur Arbine, Colombaz près Hauteluce, mont Drizon à la Bouchasse, St-Pierred'Albigny, bois entre Plan-Bérard et la Fougère près Fessonssous Briançon. - Is., Dr. et H.-Alp. : mont Sénèpe près la Mure, Villard-de-Lans et la chaine calc. qui s'étend dans le Vercors et jusqu'à Die, Mélézet sur Guillestre, col de Vars, vallée du Guil, mont Séuse. - B.-Alp. : Larche, le Lauzannier, - Alp.-Mar. : val de Pesio, descente de N.-D. de Fenestre. - La plupart des localités attribuées à l' $H$. elatum Fries par quelques auteurs doivent être rapportées à l'II. juranum ou au pscudo-juranum. Du reste, suivant M. Arvet-Touvet, l' $I$. clatum Fries n'cxiste pas en France.

II. pseudo-juranum Arv. T. - Is. : montagnes de Villard-de-Lans. H. et B.-Alp. : Lautaret, Abriès en Queyras, col de la Croix, vallée du Guil, le Lauzannier, Parc-à-Sac près Larche.

1r. elchoriaceum Arv. T. - Mont Viso au pied des prairies de la Traverselte.

1. ochroleucum Schleich., Kocli, H: picroides G. G. et Verlot, non Vill., nec Koch, H. cydoniäfolium Fries, non Vill., suivant la synonymie adoptée par M. Arv. T. - Is. et II.-Alp. : l'Oisans, le Valbonnais, Combe du Loup au fond du Valgaudemar, Lautaret, mont Viso au pied des prairies de la Traversette et autour du chalet de Rulnes. 
I. pieroides Vill., non G. G. nec Verlot, II. pallidiforum Jord. -

B.-Alp. : Colmars, seule localité française oủ, suivant M. ArvetT., il ait été rencontré jusqu'à ce jour. II. lactuccefolium Arv. T., II. amplexicanli-prenanthoides? - Is. et H.Alp. : Villard-de-Lans au bord de la forêt des Clots et au bois du Seigneur, entre Molines el Château-Queyras. - B.-Alp. :entre St-Paul-de.Vars et le col de Mirandole.

Var. II. viscosum Arv. T., H. prenanthoidi-amplexicaule? - Abriès en Queyras, la Condamine, mont Ventoux.

I. cydoniaefolium Vill. - Bois des montagnes. - Vaud et II.-Sav. : Alpes de Morcles, mont Jorogne, mont Cenis et Brévent. - Is., Dr. et H.-Alp. : Revel, Prémol, la Moucherolle, Corençon et toute la chaine de St-Nizier et du Vercors jusqu'd. Die, le Valjouffrey, Lautaret, Névache aux Rochilles, col de Vars, pic de Chabrières, mont Séuse. - B.-Alp. : Malemort, Lauzannier, Parpaillon. - Alp.-Har. : val de Pesio, col de Fremamorta, bois de la Fraca. - Vaucl. : mont Ventoux, au Grand Vallon. - En dehors de nos limites au Mlont Dore, Cantal, Forez, Pierre-sur-Haute.

1X. prenanthoides Vill. - Bois et prairies. - Sommités des Vosges. - Chaine jurassique au Chasseral. - Vaud et Valais: Surchamp, Alesse, mont Jorogne. - H.-Sav. et Sav. : Pitons da Salève, Abbaye de Vallon, Cornettes de Bise, Haut-de-Lin, Morzine, Dents d'Oche, Ste-Marie près des Houches, col des Aravis, mont d'Etale près la Clusaz, le Parmelan, la Tournette, Tamié, la Turnaz près Hauteluce; entre le col de la Louze et de la Fougère, montagnes de la Maurienne, combe du mont Ambin, mont Cenis. - Is. et H.-Alp., vallée de la Romanche entre Bourgd'Oisans et le Freney, Clavans, la Grave, mont Viso, col de Vars, St-Véran, Morgon, Boscodon, Orcières, la Bâtie-Neuve, Prontic en Valgaudemar, N.-D. du Laus, Pleyne de Chaudun, Devez de Rabou, les Baux, mont Séuse. - B.Alp.: Parpaillon. Alp.-Mar. : val de Pesio, Alpes de Beuil et dẹ St-Etienne. Var: Ampus. - Pyr.-Or. : Canigou, Cornella-du-Conflent, Font de Comps, vallée du Tech, Coma du Tech, Costa Bona.

II. perfoliatum Froel.-Montagnes de l'Oisans et de Villard-de-Lans(Is.). Catal. Bassin du Rhóne. 
H. Ianceolatum Vill., H. spicatum Bor. non All., H. strictum Fries, H. pseudo-prenanthes Serres. - Alpes de l'Oisans, le Freney. Venosc, St-Christophe, le Pelvoux, la -Vallouise, le Lautaret, montagnes des environs de Villard-de-Lans et de Gap. - Mont Dore d'Auvergne.

H. bifrons Arv. T. - Is, et H.-Alp. : Uriage, Séchilienne, St-Barthélemy, vallée du Vénéon, mont Sénèpe, Ville-Vallouise. St-Jean-de-Maurienne en Sav.

I. symphytaceum Arv. T. - Is. : bois d'Uriage et de Prémol, Söchilienne, vallẻe du Vénéon, mont Sénépe.

H. heterospermum Arv. T., H. symphytaceo-boreale? - Is. : Prémol, mont Sénèpe.

I. Iycopifolium Froel. - Bords des chemins, bois. - Vaud et Berne : Rolle, Bienne. - H.-Sav. et Ain : Salève du côté de Cruseilles, d'Arbent à Viry. - Rh. : près des aqueducs de Chaponost. - Gard : Anduze, Chartreuse de Valbonne.

I. trldentatum Fries, $H$. levigatum Schultz, H. rigidum Koch. Bois des terr. siliceux. - Chaine des Vosges. - Le Morvan dans C.-d'Or et S.-et-L., envir. de Rouvrai et d'Autun, mont Beuvrai. - Terr. sabl. du canton de Vaud et de H.-Sav, à Rolle et Cruseilles. - Is. : chaine des Sept-Laux, de Belledonne, des Grandes-Rousses et autres montagnes de l'Oisans. - Ain : la Dombes à Montribloud. - Loire : Pilat, Pierre-sur-Haute, rochers de l'Olme, bois de Courreau.

En dehors de nos limites se trouve encore sur les terrains volcan., les gneiss et granites de l’Allier, du Puy-de-Dôme, du Tarn:- Espèce polymorphe:

H. firmatum Jord. - Gneiss, granite dù Tárn!

H. firmum Jord. - Montribloud et les Echets dans la Dombes. - Mont Pilat.

H. viretorim Jord. - Mont Pilat.

H. conspicuum Jord, - Pilat.

H. auristylon Jord. - Pilat.

H. stenocladum Jord. - Izeron (Rh..).

H. pseudo-gothicum Arv. T. - Le Morvan.- Is. : les Fauges de Villarsde-Lans, Corençon. - L'H. gothioum Fries se trouve-t-il en France? 
I. insuetum Jord. - Tassin à l'Aiğua (Rh.).— La République (Loire).

II. cuspidatum Jord. - Tassin:

II. picturiense Sauzé et Maill. - La Montagne Noire.

1. anbaudum L., II. borcale Fries. - Espòce polymorphe répandue dans la plus grande partie de notre bassin. - C'est à tort qu'on a prétendu séparer spécifiquement l'Il. boreale de l'H. sabaudum, en alléguant que le premier a des feuilles lancéolées, plus étroites. L'II. sabaudum doit, à notre avis, étre considéré comme une unité spécifique dans laquelle on peut distinguer un grand nombre de formes très-voisines les unes des autres; telles sont les suivantes:

II. gallicum Jord. - Le Morvan autunois, les Renaudiots, Roussillon, St-Léger-sous-Beuvrai. - Rh. : Charbonnières, Tassin. - Ain : la Bresse et les Dombes, monts d'Ain. - Is. : digues do la Vence près St-Robert. - Gard: le Vigan, Campagne. - Tarn et centre de la France.

H. grandidentatum Jord. - Terr. granit, du Lyonnais à Écully, Charbonnières, Tassin. - Tarn et centre de la France.

H. Carioni Bor - Leș Renaudiots près Autun.

H. pyrenaicum Jord. - Aude et Tarn : la Montagne Noire.

II. provinciale Jord. - Var: Chartreuse de la Verne, les Mayons,

Garde-Freinet, Gonfaron.

H. depauperatum Jord. - - Is. et H.-Alp. : St-Ghristophe-en-Oisans,

Briançon. - Sav. et Piémont: Lans-le-Bourg, Suze.

II. dissilum Jord. - Is, et H.-Alp. : Uriage, St-Christophe, la Grave.

HI. vallicolum Jord, - Is. : Bourg d'Oisans, Livet, mont de Lans.

II. dumosum Jord. - Chaîne jurassique. - Forêt de Viry près Genève.

H.-Sav. : Allinges. - Rh. : Francheville, Dardilly, St-Cyr-auMont.d’Or. - Is. : Crémieu, Bourgoin, le Sappey, Allemont. Gard: Aumessas, le Vigan, Alzon. - Hér..: St-Martin-d'Orb, Andabre-Rosis, St-Geniès-de-Varensal. - Tarn et centre de la France.

II. obliquum Jord. - C.-d'Or et S.-et-L. : envir. de Saulieu, Forêt-Sacrée près Autun. - Rh. : Ivour, Vaugneray. - Dr. et Ard. : Crest, Thueys:-Hér:: Andabre-Rosis, la Salvetat.

H. aspericaule Jord. - Forêt-Sacrée près Autun. - Rh. : Tassin. 
H. subhirsutum Jord. - S.-et-L. et Rh. : Autun, le Beaujolais, Izeron, Vaugneray. - L'Auvergne, le Tarn.

II. curvidens Jord. - Rh. : Francheville, Dardilly, la Tour-de-Salvagny.

- H.-Sav. : Salève au-dessus de Cruseilles. - Ain : Trévoux,

Reyrieu. - Gard : Mandagout.

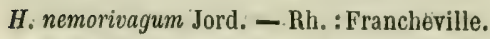

H. tardiflorens Jord - Rh. : Francheville.

H. salignum Jord. - Rh. : Tassin.

H. dispalatum Jord. - Rh. : Tassin.

H. virgultorum Jord. - Chaîne jurassique. - S.-et-L. et Rh. : Morvan autunois, Vaugneray, Charbonnières, Beaunant. - Is. : Revel. - Gard : St-Sauveur.

H. chloroticum Jord. - Rh. : Charbonnières.

H. eminens Jord. - Rh. : Charbonnières.

H. procelsum Jord. - Rh. : Charbonnières. - Ain : Nêron.

H. nemogeton Jord. - Rh. : Marcy.

H. hypselocaulon Jord:- Rh. : Francheville.

H. aviorum Jord. - Envir. de Lyon.

H. occitanicum Jord. - Gard et Hér. : Anduze; la Sérane.

H. aspretorum Jord. - Rh. : Vaugneray.

H. editicaule Jord. - Rh.' : Francheville.

H. proelongum Jord. - Rh. : Dardilly.

H. hirsutuhum Jord. - Rh. : L'Arbresle, St-Bonnet.

H. rigidulum Jord. - Rh. : 'Tarare: - Is. : Livet.

H. rigens Jord. - Chaîne jurass. - Rh. : Francheville, Charbonniêres,

Soucieu, entre Chaponost et Brignais. - Is. : Bastille de Greno-

ble, St-Egrève, Balmes de Fontaine, Revel, Venosc, St-Christophe-en-Oisans. - Gard : le Vigan, St-Vincent, Manduel.

H. editum Jord. - Rh. : L'Arbresle, St-Bonnet.

H. recticaule Jord. - Ain : Néron.

H. salticolum Jord. - Rh. : Limonest; Oullins.

II. macrodontum Jord. - Rh. : Tassin.

H. vivariense Jord. - Ard. : Thueyts.

H. concinnum Jord. - Chaine jurassique. - Les Renaudiots près Autun. - H.-Sav. : les Allinges. - Rh. : Francheville, Beaunant. - Is. : Revel, Allevard, Vaulnaveys, Venosc. - Gard : Chartreuse de Valbonne. 
II. anceps Jord. - Rh. : Francheville.

II. suberectum Jord. - Montagnes du Lyonnais et de Tarare.

II. streptophyllum Jord. - Rh. : Tassin.

II. vagum Jord. - Chaine jurassique. - Envir. de Genève, Vangeron.

- H.-Sav.: le Cháble. - Rh.: Chassagny, Givors, le Beaujolais. - Is. : entre le Freney et mont de Lans, - Ard. : Toulon, Mayres. - Gard : Aulas.

II. rigidicaule Jord. - Envir, de Lyon.

H. depilatum Jord. - Is. : Crẻmieu.

II. Roffavieri Jord. - Rh. : St-Gyr-au-Hont-d'Or.

II. beugesiacum Jord. - Ain : Ordonnat.

II. ilicelorum Jord. - Gard : Jonquières.

II. serum Jord. - Rh. : Francheville. - Brisecou près Autun.

H. pervagum Jord. - Rh. : Marcy, - Montjeu près Autun.

II. umbelliforme Jord. - Rh. : Charbonnières.

H. amphibolum Jord. - Rh. : Givors.

H. Boreanum Jord. - Vallée du Garon.

H. orophilum Arv. T. - Lautaret.

H. Didieri Arv. T. - St-Jean-de-Maurienne.

H. hypericifolium Arv. T. - Is. : Gières, St-Martin-d'Hères.

II. subvirens Arv. T. - H.-Alp. : les Navettes, les Clots en Valgaudemar.

H. conicum Arv. T. - Lautaret.

II. valesiacum Fries. - Vaud et Valais. - H.-Sav. : Veyrier près Annecy.

H. hiresutum Bernh. - Pyr.-Or. : Prats de Mollo.

Y. umbellatum L. - Bois. - Chaine vosgienne et jurassique. Zone vosgienne et sous-vosgienne de H.-Saône. - Le Morvan dans C.-d'Or, S.-et-L. et toute la chaîne des montagnes du Beaujolais, du Lyonnais, du Pilat, du Vivarais, des Cérennes du Gard et de l'Espinouse à Fraisse dans l'Hér, - Sav. et H.-Sav. Is. et Dr. : terr. sabl. de la Côte-St-André, St-Geoirs, Echirolles, Laval, St-Robert, Crest. - Var et Alp.-Mar.: Chartreuse de la Verne, Alpes de Tende. - Aude et Pyr.-Or. : envir. de Quillan, Vernet-les-Bains, Cornella-du-Conflent, Villefranche, Trencada d'Ambulla. Espèce polymorphe. 
H. monticola Jord., H. restivum G. G. non Fries. - Prairies.- H.-Sav. et Sav. : mont Brizon, du col de Tamié à Praz-Flandet près Chevron. - Is. et H.-Alp. : Alpe du mont de Lans, Lautaret à Prime-Messe.

H. robustum Fries, II. laureolum Arv. T.— Sav. : St-Jean-de-Haurienne. H. salicifolium Arv. T. - H.-Alp. : mont Rognouse près Ribiers.

H. slbidum Vill. - Prairies, débris de rochers. - Ilautes Vosges depuis le Hohneck jusqu'aux lacs Noir et Blanc. - Nul dans la chaine jurassique. - Valais : entre Saas et Mattmark, Blasenhorn, le Mayenwand en montant à la Grimsel. - H.-Sav. et Sav. : col de Balme, les Aiguilles Rouges, Plampraz, la Flégère, le Brévent, les Posettes, la Tournette, col de Fenêtre entre Hauteluce et Nant-Borant, bas du Mottet et bords du lac du Cormet au-dessus-d'Aime, cols de la Roue et de la Seigne. - Is. et H.Alp. : le Collet d'Allevard, Haut-du-Pont, la Cochette, la Bérarde, col d'Arcines au-dessus de Casset, le Désert sous le mont Olan. - Alp.-Mar. : Alpes de Tende, vallée de la Gordolasca, vallon du Cavallé près du col de Cérèsé.

ANDRYA LA sinuata $L$. - Terres incultes de la région mérid. et moyenne. Très-commun dans la Provence, les Alp.-Mar., le Roussillon, le Languedoc; il s'étend d'une part dans la Drôme et l'Is. vers Livron, Hontélimar, Crest jusqu'à Vienne, d'autre part, à travers le Vivarais, jusque dans la région granit. du Lyonnais où il existe abondamment. Devient très-rare en remontant au nord. - Nul dans les Vosges, la H.-Saône; C.-d’0r, Doubs, Jura, Ain, Vaud et Vaud et Valais, Savoie et dans une grande partie du Dauphiné.

A. undulata Presl. - Ile du Levant.

A. ragusima L. - Terr. sablonn. des Pyr.-Or. : envir. de Perpignan, vallée du Réart, entre Vingrau et Rivesaltes, bords de l’êtang de Villanova, Céret, Cases de Pena, vallée de l'Agly, les Corbièrés.

SCOLYMUS maculntus L. - Bords des chemins. - Vaucl. et B.-du-Rh.: envir. d'Avignon à Caumont et Gadagne, les Martigues. - Var : Castignaux près Toulon. - Gard et Hẻr. : Caderousse, Mornas, Agde, Villeneuve, Béziers, cap de Loupian, Mèze, Villeveyrac, Portiragnes, Cette, Vias, Olargues. 
s. Inispanieus L. - Bords des chemins de la région mérid., audelà de laruelle il se propage rapidement depuis quelques annẻes. C'est ainsi qu'il est devenu très-commun près de Lyon, autour du fort de Villeurlanne, et plus au nord sur les bords de la Saône, à Collonges.

S. grandinorus Desf. - Bords des chemins de la région littor. du Roussillon vers Collioure et Banyuls-sur-Jler.

\section{AMBROSIACEES.}

XANTHXU1 strunarlum L.-Décombres, roisinage des habitations, bords des cours d'eau et des chemins dans notre bassin; du reste assez fugace, comme le sont beaucoup de plantes ruderales dont les stations sont sourent détruites par les changegents opérés dans les voies de communication.

X. macrocarpum D C. - Champs, bords des routes; plus rare que le précédent. - Signalé autour de Genève oủ on ne l'a pas revu depuis plusieurs années, autour de Lyon à Sain-Fonds et à Feyzin, dans les environs de Gap à Manteyer. - Beaucoup plus commun dans les plaines du Gard, de l'Hérault, de l'Aude cl des Pyrénées-Orientales. Moins rẻpandu dans la Provence, si ce n'est sur les bords du Rhône vers Avignon, Arles, Tarascon, ainsi yue sur les bords de l'Arc, vers Fenouillères. On le trouve aussi çà et là sur d'autres points de la vallée du Rhone, notamment, vers Baix, le Pouzin, Tournon.

X. italicum Moretti. - Diffère du précédent par son fruit plus hérissé, terminé par deux becs non convergents supérieurement.- Cette espèce, très-roisine de la précédente, a été trouvée dans les Alpes-Maritimes à Menton et à St-André; dans le Var à Cannet, Entraigues, 0n prétend l'avoir vu aussi à la Cité, près de Lyon, sur des décombres extraits d'un jardin.

X. splnosum L. - Décombres, lieux incultes, bords des chemins.

- Assez commun dans la région méridionale d'oủ il se propage rapidement vers la partie moyenne du bassin, le long du cours du Rhône et dans le voisinage des chemins de fer. Depuis quelques années le $X$, spinosum est devenu fort commun autour de 
Lyon, particulièrement sur la rive gauche du Rhône. Au surplus il est digne de remarque que, même dans la région méridionale, il n’a pas toujours été aussi répandu qu'il l'est actuellement : la date de son apparition autour de Montpellier serait entre l'époque de Gouan et celle de Magnol, de 1700 à 1763, c'est-àdire postérieurement à l'année pendant laquelle le cẻlèbre botaniste Magnol publia la première édition de son Botanicum monspeliense, ouvrage dans lequel il n'est fait aucune mention du $X$. spinosum. Au commencement du xvir siẻcle, la Lampourde épineuse n'existait pas en Italie, en Grèce, non plus qu'en Algérie. Il est probable qu'elle aura été apportée de l'Amérique méridionale par les Portugais.

On peut prévoir que le $X$. spinosum est destiné à se propager de plus en plus dans la partie moyenne de notre tassin où il est déjà largement naturalisé. Peut-être même s'étendra-t-il de proche en proche dans la partie septentrionale, ou actuellement il ne se montre que d'une manière accidentelle et adventive.

AMBRoSIA tenuifolla Spreng. - Espèce originaire de l'Amérique méridionale naturalisée à l'extrémité d'un faubourg de Cette dans l'Hérault; elle ne parait pas s'être propagée hors de cette station restreinte, et pourrait bien disparaître un jour par suite de l'accroissement rontinu du nombre de constructions nouvelles sur le terrain qu'elle occupe.

A. artemisisefolia L. - Espèce originaire de l'Amérique septentrionale qu'on a signalée en plusieurs localités de l'Angleterre et de l'Allemagne. M. Chanrion l'a trouvée il y a deux ans, entre Durette et Lantignié, dans le département du Rhône. L'introduction de cette espèce exotique dans notre région beaujolaise est de date trop récente pour qu'il soit permis de considérer l'A. artemisicfolia comme définitivement acquis à la Flore du bassin du Rhône. 


\section{LOBÉLIACÉES}

LOBE⿱IA A urens L. - Landes incultes. - Gard : Bourdezach, seule localité du bassin du Rhône. - Cette espèce existe dans le Tarn, l'Aveyron, la région pyrénéenne, l'ouest et le centre de la France.

LA UTENTA Michelinna D C. - Lieux humides. - Var : îles d'Hyères, le Luc, le Cannct, Fréjus, St-Raphaël.

\section{CAMPANULACÉES}

JA10NW montnna $\mathrm{L}_{\text {. }}$ - Landes, bruyeres, lieux incultes des terrains siliceux des plaines et des montagnes (l'épithète de montana ne convient point à cette espèce et pourrait êtro remplacée par celle de vulgaris). - Commun dans la chalne des Vosges et dans les zônes vosgiennes et sous-vosgiennes des arrondissements de Belfort el de Montbéliard. - Argiles et sables des plaines du Jura autour de Chaussin, Chaumergy, Sellières, Mont-sous-Vaudrey, forèts de Chaux et de la Serre, alluvions sablonneuses de l'Ognon. - Commun dans toute la longue chaine qui, depuis le Morvan granitique, s'êtend à travers les montagues charollaises, mâconnaises, beaujolaises, lyonnaises, jusqu'au mont Pilat et, plus loin, à travers les montagnes vivaraises jusque dans les Cévennes du Gard el de l'Hérault, et ensuite à la Montagne Noire, ainsi que dans toute la chaine des Pyrénées. - Se montre sur quelques points du Valais et du canton de Vaud; devient plus commun sur les sables mollassiques des environs de Genève, autour du Salève, entre Etrembières et Mornex, au bois de Bay près Peney, à Aĩre; sur la mollasse de Hle-Sav. et Sav, ; sur le même terrain dans les plaiCatal. Bassin du Rhône. 
nes du Dauphiné, non loin de Grenoble sur les gneiss à Revel, Vaulnaveys, sables du Mollard de Décines, piès Lyon, St-llaurice en Valgaudemar; collines de la Drôme à Saou près Crest, St-Vallier; terr. sablonn. de Vaucl., B.-Alp., Gard, Hér., Aude et Pyr.-0r.; B.-du-R. : plaines à cailloux quartzeux de la Crau. - Var et Alp.-Nar. depuis les plaines du Lue, d'Auribeau et d'Antibes jusqu'ả la région montagneuse.

3. perennis Lam. - Plante vivace stolonifère, silicicole comme la précédente. - Commune dans les Hautes-Vosges. - Chaine beaujolaise au pic de St-Bonnet-sur-Montmelas et de Sévelette; chaine du Pilat, du Vivarais, des Cévennes, du Gard et de l'Hér. à l'Espérou, Concoule. - Aude, Ariège et Pyr.-Or. : Montagne Noire, pays de Sault, lac de Laurenti, Jasse do la Bentaillole, port de Paillères, Quérigut, Mijanès, bois de Boucheville, MontLouis.

En dehor's de nos limites, sur les montagnes du Forez, Puyde-Dôme, mont Dore, Cantal, Aubrac, Espinouse, Lozère, Pyrínées centrales.

Une variété naine, J. pygmaea, est quelquefois mêlée au type. J. Cariomiana Bor.-Forme du précédent à tiges étalées, puis arquéesascendantes, à racines émettant de nombreux rejets terminés par des rosettes de feuilles. - C.-d'Or et S.-et-L. : Morvan granitique autour de Saulieu, Semur, Autun, le Beuvray, mont de Pauvray près Curgy. - Chaine beaujolaise et lyonnaise à Chenelcte, St-Bonnet-sur-Nontmelas, Vaugneray, St-Bonnet, Izeron, Charbonnières, Chaponost. - Loire : Pélussin, Maclas, St-Martin-Accoallieu. - En dehors de nos limites dans le Morvan nivernais et probablement ailleurs.

\$. Luminis Pers. - Rochers. - Pyr.-Or. et Ariège : sommet du Cambres d'Aze, vallée d'Eyne au col de Nuria, vallée de Llo, plateau des Bouillouses, Carlitte, Carença, Costa Bona, Canigou, Prats de Mollo, Nohèdes, Carlitte, Port de Paillères, vallon de Boutadiol, Pic del Ginèvré.

PHyThuma paucinorum L. - Rochers et pâturages des Alpes et des Pyrénées. - Valais : Zermatt au Furgalp et au Niltaghorn, Saas au Schwarzberg et à l'Offenthal, Cabane de 
Vincent au pied du Mont Rose. - Sav. : mont Iseran, Bec de Dix-Heures, sur le versant italien près de Courmayeur, mont de Fréjus, mont Cenis à Ronches. - IR. et B.-Alp. : Galibier, Terre-Nière sur Cervières, mont Viso au col de Ruines, col Agnel, Clausis, St-Véran, col de la Croix, vallons de Ségure et de Péas, Valpréveyre, col Malrif, Lauzanier, Vallonnct, Grand-Couyer sur Annot, mont Monnier, - Alp.-Har. : Entraunes, et les sommités au-dessus de St-Etienne, St-Nartin, Tende. - Pyr.-Or. : vallée d'Eyne, Nuria, Madrès en Capcir, Canigou. - Pyrẻnées centrales.

L2 variété globularifolium Hoppe se trouve dans les montagnes d'Abriès en Queyras, au mont Cenis, au Simplon, aux pieds du Nont-Rose et du Cervin.

Ep. hemisphnericuan L. - Rorhers et páturages des montagnes à sol siliceux. - Vaud et Valais : Jaman, Javernaz, les Martinets, la Tour près Anzendaz, Fully, les Ecoulayes-sur-Saxon, Ferpécloz, Zermontana, Zan, Thyon, St-Bernard, Evolena, Zermatt, Saas, Simplon, Guggerhübel, Conches, Glacier du Rhône, Ilayenwand, Grimsel. - II.-Sav. et Sav. : toute la chaine du mont-Blanc depuis le col de Balme jusqu'à ceux du Bonhomme et de la Seigne, Montanvert, moraines et Jardin de la Mer de Glace, Leschaux, les Grands Mulets, chaines du Brévent et des Aiguilles-Rouges, la Levanna, le mont Iseran. - Is. : chaines du Pelvoux, des Grandes-Rousses, de Taillefer, de Belledonne et des Sept-Laux, mont Chamoux près la Salette.-II. et B.-Alpes: Lautaret, Bufféra près Névache, vallon d'Arcines près le Monétier, Alp.-Nlartin, Champoléon, Orcières, Chaillol, Valgaudemar, Pic de Chabrières près Chorges, mont Viso, Lauzanier. Alp.-Mar. : sommités des vallées de la Vesubja, Tinea, Roja. Gard et Lozère: L'Espérou et l'Aigual. - Ard. et H.-Loire : Mont Mezenc, Gerbier des Joncs, sources du Mézayon. - Pyr.Or. et Ariège : montagnes d'Evol, vallée d'Eyne, Canigou, ciryue des Aiguettes et d'Artounant, lac d'Estagnet, la Bentaillole.En dehors de nos limites au Puy-de-Dôme, mont Dore, Cantal, Pyrénées centrales.

La variété humile Schl. a les feuilles supér. denticulées, les 
bractées égales au capitule. - Valais : Zermatt au Riffelhorn, Saas au Toelliboden, Schwarzkerg, Offenthal, monte Moro. Sav. : le Bonhomme.

P. Iongibracteatum, P. Charmelii Vill., P. Scheuchzeri All. Rochers des montagnes alpines et subalpines. - Valais: StBernard, Zermatt, Saas en montant à Fée, puis en allant vers Mattmark, Simplon, le Breuil sur le versant piémontais du mont Rose. - Is. : Sassenage près du pont Charvet, entre Bourgd'Oisans et le Freney, col de l'Arc. - Sav. et H.-Alp. : mont Cenis, Briançon, mont Viso, bords du Guil, Château-Queyras, mont Dauphin, Charance, mont Séuse, mont Aurouse, le Noyer, mont Morgon. - B.-Alp. : St-Paul à la Reissolle, la Condamine, Bachasse, col de Fours, vallon Bérard, Colmars, mont Couyer sur Annot. - Var et Alp.-Niar. : La Sainte-Baume, sources du Var, Alpes de Tende, d'Entragues, de Vinaï et de Vaudier. Hẻr. : Pic St-Loup, St-Guilhem-le-Désert.

Dans cette espèce, les dimensions des bractées sont très-variables; les feuilles tantôt entières, tantôt dentées.

PP. orbiculare L. - Bois et prairies des collines et des montagnes.

- Espèce très-variable quant à la forme des feuilles, des bractées et du capitule: d’où les variétés appelées lanceolatum, ellipticum, cordifolium, brevifolium, comosum.

P. scorzonerifolium Vill. - Prairies des Alpes. - Vaud et Valais : Alpes de Bex, de Saxon et de Sion, Simplon. - Sav. : Turnaz dans la Hauteluce, Laval-de-Tignes, mont Cenis. - Is. ct H.-Alp. : mont de Lans, mont Chamoux près de la Salette, la Grave, Lautaret, Galibier, col de l'Echauda, Buftẻra-sur-Névache, l'Argentière, mont Viso, Combe de Ségure, col de Vars et des Hayes. - B.-Alp. : Lauzanier, la Madeleine, Sérennes.

La forme décrite par Villars a l'inflorescence en épi; celle qui a été nommée P. Michelii par Allioni, a des capitules presque globuleux.

P. betonleifolium Vill. - Prairies des Alpes. - Vaud et Valais : Alpes de Jaman, Panerossaz, Lavarraz, Alesse, Saxon, l'Etroz, Cleuson, Arolla, Nayens de Sion, Guggrerhübel, Natersberg, Taesch, Saas, Simplon, Champé, St-Bernard à la Pierraz, 
Gemmi. - II.-Sav. et Sav. : Sixt, Chamonix, le Reposoir, mont C.larvin, Vergy, mont de Pelloua, la Tournette, Laval-de-Tignes, Crest-Voland, Hauteluce. - Is, et H -Alp. : Belledonne vers le lac du Crouzet, Taillefer, mont Pelvoux, la Grave, Lautaret, Bufféra-sur-Névache, vallée du Guil au pied du mont Viso, col Bayard, Prontic en Valgaudemar. - B.-Alp. : Lauzanier, col de la Madelcine, Longet de Maurin, Soleil-Buou, Parpaillon, mont Monnier. - Alp.-Mar. : Tende, Colmiane, vallée du Borẻon près St-Martin, Salsamorena, Séranon, mont Lachen.Pyr.-Or. : vallon de Castell, St-Martin du Canigou, d'après Companyo (1). - Pyr. centr.

P. mpleatum L. - Bois et prés des collines et des montagnes. Dans cette espèce, les fleurs sont tantôt d'un blanc jaunâtre, tantôt bleues comme, par exemple, dans les environs de Grenoble et de Gap. La variêté nigrum Sm., persicifolium D C., que quelques auteurs ont élevée au rang d'espèce, a les feuilles moins cordiformes et plus étroites, le capitule presque ovoïde, les fleurs d'un bleu-violet foncé. Nulle sur les calcaires, elle est assez répandue sur les terrains siliceux de la clıâne des Vosges jusqu'au ballon de Giromagny et à Champagney dans la HauteSaône; sur les sables des bords de l'Ognon, dans les bois entre

(1) Companyo, auteur d'une Histoire naturello des Pyrénées-Orientales, a mis une sorte d'amour propre patriotique à enrichir son Catalogue de plusieurs espèces qui, examen fait, ne se trouvent point dans les PyrénéesOrientales, et a accepté, sans les contrîler, une multiturle d'indications fausses de localités citées par Lapeyrouse et par Pourret. - M. Timbal-Lagraveayant ou l'obligeance de m'écrire pour me mettre en garde contro les assertions fantaisistes du naturaliste roussillonnais, j'ai voulu, quoique le témoignage du sayant botaniste toulousain n'eût pas besoin de confirmation, m'assurer par moi-même de l'inexactitude de certains faits avancós par Companyo, et jo suis revenu de mon voyage avec le profond regret d'avoir répété des assertions fausses dans les fascicules précédents du Catalogue de la Flore du bassin du Rhône.

Le lecteur est donc prévenu que, jusqu'à plus ample information, il devra tenir pour suspectes la plupart des indications concernant la végétation des Yyrénées-Orientales. Il serait fort à désirer que quelque botaniste entreprît sur cet intéressant pays un travail analogue à celui que MM. Timbal-Lagrave et Jeanbernat ont publié, en 1879 , sur lo massif du Laurenti. 
Geneuille, Cussey et Moncley, dans les environs de Dôle, puis dans la vallée de Joux; dans l'Autunois à Canada et sur tout le plateau d'Antully; dans le Gard à l'Espérou, près de Concoule; dans les Pyr.-Or., près de Mont-ILnuis. - En dehors de nos limites, dans le Puy-de-Dơme, le Cantal, la Lozère, Margeride, l'Aubrac.

B. urstelfolium Clairv., P. Halleri All. - Prairies des Alpes et des Pyrénées. - Valais : mont Fully, Leukerbad, val de Loetschen, glacier du Rhône, Hayenwand. - Sav. : Laval de Tignes, Bas du Mottel au-dessus d'Aime, Entre-deux-Eaux, mont de Fréjus, mont Cenis. - Is. et H -Alp. : Clavans, forêt des Fraux dans la vallee de la Romanche, la Groix-Haute, Lautaret à PratBrunet, Bufféra, la Vachette, les Rousses près Abriès, val Préveyre, vallon de Ruines, col Agnel, col de Vars, Boscodon. B.-Alp. : St-Paul, Tournoux, col d'Allos, Lauzanier, vallon de Grange-Commune, Enchastraye, Parpaillon, Vallonnet, Bérard, Colmars. - Alp.-Har. : Estenc au-dessus d'Entraunes, sources du Var, Salsamorena, vallée du Boréon, col de Tende. - Ard. : Mezenc, la Pra du Cheylard. - Pyr.-Or. : Canigou, Costa-Bona. - En dehors de nos limites à Sacon, près de Mauléon (Pyr.Basses), pentes du Puy-Nary (Cantai), bois au-dessus du Born (Lozère).

SPECULA TE IA vugarla, S. Speculum Alph. D C. - Moissons dans les plaines et les montagnes.

5. Inybrida Alph. D C. - Champs cultivés, moissons de la région mérid., surtout dans les terrains calcaires; apparait quelquefois accidentellement dans les champs de la partie moyenne et septentrionale du bassin.

s. faleata Alph. D C. - Bords des champs de la région mérid. B.-du-Rh. : envir. de Marseille à St-Loup, St-Tronc, la Ciotat au Bec-de-l'Aigle. - Var et Alp.-Mar. : Fort-Rouge près TouIon, Ile du Levant, le Luc, Grasse, Roqucbrune, l'Esterel, la région littorale de Cannes à Jlenton. - Aude et Pyr.-Or. : envir. de Narbonne, les Corbières, Argelès, Collioure.

s. pentagonia Alph. D C. - Espèce du nord de la Grèce et de l'ile de Crète trouvée dans un champ de blé à Gignac (B.-duRh.) et au Port-Juvénal près de Montpellier. 
CAMPANULA grandinora Lam. C. Medium L. - Bois de la région mérid. et moyenne; manque dans la partie septentr. du bassin, ou du moins, ne s'y montre que subspontané dans le voisinage des jardins, - Commence à paraître autour de Lyon à Couzon, au mont Cindre et au-dessus de Limonest; puis dans l'Ain, près de Trévoux, entre Rossillon et Virieu. - Is. : Crémieu, Bastille de Grenoble, la Tronche, base du St-Eynard. Sav. : Apremont, St-Jean-de-la-Porte. - Dr. et Ard. : Crest, Divajeu, St-Vallier, Baix et les coteaux des bords du Rhône. H.-Alp. : Charance, la Garde, Aspres-les-Veyno. - Vaucl. : envir. d'Orange, Sablet, Gigondas, - B.-du-Rh. : envir. d'Aix à Montaiguet, Prignon, le Tholonet, envir. de Marseiłle, vallon de la Treille jusqu'au pied de Garlaban. - Var et Alp.-Mar. : Mont Faron, Montrieux, Rians, Mourières, le Luc, Fréjus, Grasse, Nice, Menton. - Gard : Roquebrune près le Pont-SaintEsprit, envir. d'Aujac à la Chartreuse de Valbonne, Grand'Combe près d'Alais, bords de la Cize entre St-Ambroix et Bessège.

C. speciosn Pourr. - Rochers calcaires du Languedoc et de la région pyrénéenne. - Gard : rochers d'Anjeou près Montdardier et de Brama-Bioou. - Hér. : la Sérane, les Capouladoux, Liausson, St-Guilhem-le-Désert, N.-D. de Londres au Pous, le Larzac prìs du Cros, Pégayrolles de l'Escalette, Joncels, St-Ilichel d'Alajou, Cervières, Roqueredonde. - Aude, Ariège et Pyr.-Or. : envir. de Narbonne à la Clappe, Pech-Redon, les Corbières, Axat, mont St-Victor près Durban, couloir des Courtalets près Tuchan, lac du Laurenti et de Rabassoles, St-Paul-de-Fenouillet, Pont de la Fou, St-Antoine de Galamus, Font de Comps, Villefranche, Prats de Mollo. - En deliors de nos limites dans les Pyrén. centr. l'Aveyron et la Lozère.

C. unna Lam. C. Allionii Vill, - Rocailles calcaires des Alpes francaises; manque dans les Alpes de la Suisse. - Ses stations les plus septentrionales sont au mont de Frijus au-dessus du tunnel des Alpes, et au mont Cenis à la montagne de Ronches sur le territoire italien. - Is. et Dr. : les Pélats entre le mont Aiguille et le Grand Veymont, Gresse, la Bâtie, mont des Lussettes et 
col des Aiguilles près de Lus-la-Croix-Haute, mont Glandasse. - H. et B.-Alp. : Galibier, cols Isoard, des Hayes, Malrif et de Ruines, mont Viso à la Traversette, vallon des Brun, montée de St-Véran au col Ceillac, col de Vars, col de Glaise, mont Aurouse, Plampinet, mont Séuse, Poligny et col du Noyer en Champsaur, mont Morgon, pic de Chabriures, Prapioz, Bérari, Parpaillon, Maurin, Lauzanier, Bousollières, mont Grand-Couyer près Annot. - Vaucl. : mont Ventoux. - Alp.-Mar. : col de la Maddalena, Bouziéjo au-dessus de St-Etienne, Alpes d'Entraunes et de Tende.

C. barbata L. - Prairies des Alpes; manque dans les Vosges, le Jura, les Pyrénées et le massif central de la France. - Commun dans Ies Alpes de Vaud, Valais, Savoie, Dauphiné, Basses-Alpes jusque dans les Alpes-Haritimes.

C. petraea L. - Alp.-Mar. : Escales d'Aiglun, Sallagriffon, vallée de l'Esteron, Cheiron.

C. gromerata L. - Bois et prairies des coteaux et des montagnes calcaires. - Espèce polymorphe qui présente de nombreuses variations quant à la forme des feuilles et des capitules; les feuilles sont tantôt glabres, tantôt pubescentes.

c. cervicarla $L$. - Bois des collines et des basses montagnes surtout dans les terrains arénacés et argileux; manque dans la chalne jurassique. - Genève et Vaud : bois de la Bâtie, de Crevin et de Veyrier, Nyon, les Montets près Bex. - H.-Sav. et Ain : Bossey, Héry près d'Ugine, Mogneneins, les Echets. Chaine granitique du Beaujolais, Lyonnais, Pilat.-B.-du-Rh. : la Crau et la Camargue.-Commun sur le gneiss de l'Alsace.

La variété cervicarioides Mut., qui a les feuilles inférieures atténuées en pétiole plus long que le limbe, se trouve quelquefois mêlẻe au type.

C. Eplenta L. Prairies des Alpes et des Pyrénées. - Valais : Fully, les Marques, la Bâtiaz près de Martigny, Orsières, Bagnes, Hérémence, Longeborgne, Vercorin, Anniviers, entre Visp et Stalden, Zermatt, Simplon. - Sav. : Ste-Foy en Tarantaise, Lans-le-Villard. - Is. et H.-Alp. : St-Christophe-en-Oisans, mont Chamoux près la Salette, la Grave, Laularet, Granon près 
Val-des-Pres, Plampinet, Fort des Salettes à Briançon, vallee du Guil à Villevieille, Abriès, clıalet de Ruines, col de Glaise, Charance, Chabrières près Chorges. - B.-Alp. : Larche, Sérenne, Iloronaye, au-dessus de Bousollières, Fouillouse, Colmars, mont Grand-Couyer pròs Annot. - Alp.-Alar. : StEtienne-IIont, St-Dalmas-le-Sauvage, St-Martin, Ste-Anne-deVinaï, col de Tende. - Vaucl. : St-Martian.

Une variêté ả épis interrompus se trouve dans les B.-Alp., à la Condamine, entre Sérennes et Fouillouse.

c. thyrsolden L. - Prairies des montagnes; manque dans la chaîne des Pyrénées. - Jura helvétique et français : chaines du Chasseron, Montendre, Noirmont, la Dole, Colombier, Reculet, Sorgiaz. - Vaud et Valais : Dent de Jaman, Anzendaz, Bovonnaz, la Tour, Nombrieux, Dent de Morcles, Fully, val d'llliez, Catogne, St-Bernard à l'Ardifagoz, coldes Fours, Levron, Thyon, Diez, mont Nuoble, Laemmeralp. - Hte-Sav. et Say. : Dents d'Oche, mont Chalune, Hautigny près Bonnevaux, Iole, Semnoz, Parmelan, Brizon, Tournette, mont Trélod en Bauges, col Joli, Sixt, col de Voza, mont Lachat, les Platets de Passy, col d'Anterne, Hauteluce, la Gita, la Thiopa près de Champagny, les Allues, les Aranchers, Golet, Beaupré, col de la Madeleine.Is. et Dl. : Grand Som, Chamechaude, le Moucherotte, la Moucherolle, col de l'Arc, mont St-Michel près Lamure, la Salette, forêt d'Esparon près le Ionestier-de-Percy, mont Glandasse près Die. - H.-Alp. : la Grave, Villard d'Arine, Lautaret, mont Viso, Cote Gélive près Gap. - Alp.-Mar. : Berthemont près Roccabigliera.

C. Intifolla L. - Bois des montagnes. - Ballons des Vosges, Rotabac, Lauchen, Hohneck jusque dans la Haute-Saone au Ballon de Servance et au Plain-des-Voleurs au-dessus de Miélin. Jura helvétique et français: Weissenstein, Chasseral, Creux-duVan, mont d'Or, la Dôle, le Colombier, la Faucille, Côtes du Doubs et du Dessoubre à Consolation, le Bélieu, Meyriat près de Nantua. - Vaud et Valais : envir. de Bex à lirenières, les Plans, pied des Diablerets, le Brocart, Saxon, Combe de Martigny. Hte-Sav. : vallée du Reposoir. - Is. et H-Alp. : massif de la 
Grande-Chartreuse, St-Ange, Combe Noire de Manteyer, Pleyne de Clıaudun et Loubet près Gap, le Hêlézet près Guillestre. Alp.-Mar. : les Viosennes, val de Pesio, Ste-Anne-de-Vinaï, StEtienne-IIont.

C. urticifolin Schm., C. Trachelinm L. - Bois des plaines et des montagnes dans tout le bassin. Espèce polymorphe.

Une variété à calice hérissé, dasycarpa G. G., se trouve dans les envir. de Gap, dans la vallée de l'Ubaye, ainsi que dans les vallées de Chamonix et de Sixt.

C. rapunculoldea L. - Bois et bords des champs dans les plaines et les montagnes.

C. bononiensis L. - Bois des vallées montagneuses. - Valais : Fully, Conthey, Longeborgne, St-Léonard, Sembrancher. Sav., Is., II.-Alp. : Cruet près Montmélian, du Bourg-d'Oisans à Huez, envir. de Gap, mont Rognouse près Ribiers. - B.-Alp., B.-du-Rh., Alp.-Mar. : Entrevaux, la Crau, la Camargue? (d'après Castagne et Derbès), Villeneuve-d'Entraunes, la Croix, St-Dlartin-Lantosque, St-Sauveur, entre Tende et le col Levens, mont Rasel au-dessus de Nenton, St-Vallier, Caussols.

C. parvillora, $C$. Erinus L. - Hurs et rocailles de la région mérid. remonte dans les B.-Alp. vers Gréoux, Sisteron; dans la Drômo vers IIontélimar, Crest, Valence, Tain et St-Vallier; dans l'Is. vers St-MIarcellin et Vienne; dans l'Ardèche, la Loire et le Rhône à Privas, Entraigues, Chomérac, Tournon, Serrières, Malleval, Chavanay, Condrieu, Ampuis, Ste-Colombe.

C. clatine L. - Cette espèce qui est particulière aux Alpes piêmontaises dans le val Vegezzo, dans les montagnes de Giaveno, Viu, Lancei, dans les Alpes vaudoises du versant italien du mont Viso, remonte, d'aprìs M. Lannes, juscque près de la frontière française, non loin du col de la Croix, ainsi qu'à la montée du mont Cenis.

Des recherches ultérieures la feront peut-être découvrir sur le territoire français.

Mlutel l'a indiqué, d'après Ménard, au mont Genèvre; mais aucun Lotaniste n'a encore pu l'y trouver.

C. macrorrhiza Gay. - Commun sur les rochers des Alp.-Mar., 
depuis Tende et St-Martin-Lantosque, jusigüa Menton, Monaco et Nice; se retrouve à St-Vallier, le Bar, Caussols et Gourdon près Grasse. - Var : la Ste-Baume.

C. pyramidalis L. - Cette Campanule, propre à la Carniole, à la Vẻnétie, à l'Istrie, à la Dalmatie, est souvent cultivée; on l'a vue subspontanéc sur les rochers de St-Germain frès Aix-lesBains (Sav.). Je ne sais si elle y existe encore.

C. rhombohalls L. - Prairies des montagnes. - Jura helvétique et français : Chasscron, Suchet, Hont d'Or, Noirmont, Montenure, Dole, Reculet, Colombier du Bugey, Poisat. - Alpes de Vaud, Valais, Chablais, Faucigny, Voirons, Salève, les Bauges, mont du Chat, la Maurienne jusqu'au mont Cenis, montagnes de Beaufort, de Crest-Voland, de Hauteluce. - Is., Dr. et I.Alp. : massifs de la Grande-Chartreuse et des Quatre-IIontagnes, chaines de Belledonne, de Taillefer, mont de Lans, la Salette, le Vercors, montagnes d'Omblèze et de Die, la Grave, Lautaret, le Briançonnais, le Queyras, mont Viso, montagnes d'Embrun et des envir. de Gap, Chabrières, Orcières, Durbon. - B.-Alp. et Alp.-Har. : Larche, Lauzanier, val de Pesio, Ste-Anne-de-Vinaï, Vaucl. : St-Lambert. - Pyr.-Or. et centrales.

C. Inuceolata Lap. - Rochers des Pyrénées : Mont Louis, Pla des Abellans, Carença, Capcir, Laurenti au lüc de Rabassolès, vallon de Boutadiol, Pic de Canrusc, Port de Paillères. - Pyrénées centrales.

C. Inifolla Lam. - Prairies et pâturages des montagnes; beaucoup plus commun sur les terrains siliceux que dans les chaines calcaires. - Chaine des Vosges, manque dans la chaine jurassique. - Vaud et Valais : Bovonnaz, St-Bernard, Bagnes, Anniviers au Senlienna, Zermatt. - Hte-Sav. et Sav. : Brizon, Vergy, Méri, chaine du mont Blanc jusqu'au jardin de la Mer de Glace et au Couvercle, Charvin, Hauteluce, Beaufort, mont Cenis. - Is. et H.-Alp. : chaine de Belledonne vers le lac du Crouzet et à la Pra, Champrousse, Lautaret, mont Gondran, les Hayes, mont Viso, col de Vars, Guillestre, col de Glaise, pic de Chabrières. - Dr. et II.-Alp. : mont Embel, montagnes de la vallée de l'Ubayo. - B.-Alp. : Maurin à la Blachière, St-Paul, 
Larche. - Alp.-Mar. : Entraunes, Guillaumes, St-Martin-Lantosque, col de Tende. - Pyr.-Or. et Ariège : Mont Louis, vallée d'Eyne, la Preste, Quérigut, les Aiguettes, l'Estagnet, le Laurenti. - Rh. et Loire : Liergues, Pic de St-Cyr-le-Chatoux, mont Pilat, le Bessat. - Ard. et H.-Loire : le Mezenc, Mazan, Freyssenet, Ste-Agrève. — Gard : l'Espérou et l'Aigual.

En dehors de nos limites, dans les massifs de Pierre-surHaute, mont Dore, Cantal, Lozère, Aubrac, Pyr. centrales. Espèce polymorphe.

C. nutans, C. Scheuchweri Vill. - Tige naine, uniflore; fleur grande et penchée. - Vaud et Valais : Bovonnaz, Panerossaz, mont Fully, Gemmi, Furca, Glacier du Rhône, Simplon, Saas à Mattmark, Anniviers, St-Bernard. - Hte-Sav, et Sav. : Buet, chaine des Aiguilles-Rouges, col de Balme, Jardin de la Merde-Glace, col de Golèse, Vergy, col du Bonhomme, Hauteluce, mont Mirantin. - Htes-Alp. : Galibier, Monestier-de-Briançon, Alp.-Martin, Claaillol, Orcières, Champoléon, Péas, mont Aurouse. - B.-Alp. el Alp.-Har. : St-Paul, Bousollières, GrandCouyer-sur-Annot, Entraunes, vallon du Cavallé, au-dessus de St-Martin, Colmiane, Tende. - Pyr.-0r. et Ariège : Canigou, mont Louis, Cambres d'Aze, vallées d'Eyne et de Llo, lac de Rabassollès, cirque des Aiguettes, vallon de Boutadiol. - Pyrén. centrales.

La variêté valdensis All. diffère du type, avec lequel elle est quelquefois mêlée, par ses tiges et feuilles velues.

C. precatoria Timb. Lagr. - Pelouses des montagnes de la chaine pyrénẻenne. - Ariège : lacs du Laurenti et du Quérigut, cirque des Aiguettes et d'Artounant, Port de Paillères, vallon de Barbouillẻre, roc d'Escrouts, Bosc-Négré, Pic de Fontargente. Cette espèce avait été confondue par Lapeyrouse avec le C. rhomboidalis.

C. ficarioides Timb. Lagr. - Pelouses des montagnes de la chaine pyrénéenne. - Áriège: lacs du Laurenti et de Valbonne, Pic de Tarbézou, vallons de Barbouillère et de Boutadiol, jasse de Bentaillolle. - Présente une variété hirsuta.

C. rotundifolln L. - Rochers, bois et páturages depuis les plaines jusqu'aux montagnes. - Espèce extrêmement polymorphe. 
C. pennina Reut. - Valais entre Bourg-Saint-Pierre et la Cantine de Proz.

C. ruscinonensis Timb, Lagr. - Pyr.0r. : Collioure à Consolation, Tour de la Massane.

C. stenocodonta Boiss. Reut. - II.-Alp. : montagnes d'Aiguilles en Queyras, col de Ia Croix, Chalet de Ruines. - Alp.-Mar. : val de Pesio, col de Tende.

C. Bocconiana Vill. - H.-Alp. : montée du mont Genèvre.

C. velutina D. C. - Pyr.-Or. : mont Louis.

C. caespitona Scop. (non Vill. nec All.). C'est probablement par suite d'une erreur de synonymie que cette espèce des Alpes Noriques, Dinariques et Juliennes a été indiquée dans les montagnes alpines, subalpines ainsi que dans la chalne du Jura où elle n'existe pas. Les botanistes qui ont commis cette erreur, n'ont pas pris garde que les Campanules appelées caespilosa par Villars et par Allioni sont des formes du C. pusilla Haenke, espèce polymorphe, qui est en effet très répandue sur les rochers calcaires des montagnes de l'est de la France et de la région py rẻnẻenne. (Voir la note insérẻe dans le Bull. Soc. bot. France, t. XXI, p. CXIII, session de Lyon).

C. puslila Iraenke. - Rochers calcaires. - Toute la chaîne jurassique jusque dans les montagnes du Bugey. - Alpes calcaires de Vaud et Valais. - H.-Sav. et Sav. : montagnes du Chablais, du Faucigny, Charvin, Reposoir, Grand et Petit Bornand, montagnes de Hégève, de Crest-Voland, Hauteluce, la Gitaz, les Bauges, la Tarantaise et la Maurienne jusqu'au mont Cenis. - Is ct H.-Alp. : massifs de la Grande-Chartreuse et des Quatre-Montagnes, la Grave, Lautaret, Briançonnais, Queyras, Rabou, Chaudun et col de Glaise prìs Gap, Aurouse, Sćuse, Chabrières, Chaillol. - B.-Alp. et Alp.-Mar. : depuis les montagnes de la Condamine jusqu'au Lauzanier, Alpes de St-Martin, de StEtienne et de Tende. - Vaucl. : envir. d'Apt. - Pyr.-0r. et Ariège: Cambres d'Aze, Prats de Balaguer, lac du Laurenti, vallon de Boutadiol, cirque d'Artounan, Escale de Valbonne, lac de l'Estagnet, Roc de Lorry. - Nul dans les Vosges, les Cévennes et le massif central. 
Espice polymorphe comprenant $C$. pulchella Jord., C. tenella Jord., Malhonetiana Jord, subramulosa Jord, gracilis Jord.

C. excisa Schl. - Lieux pierreux du Valais: vallée de Saas à Balen, Fée, Mattmark, Distelap, Simplon, Binn, Munstigerthal.

C. rapuncula L. - Bords des bois et des chemins; inégalement réparti.

C. patula L. - Haies, lieux ombragés. - Rare dans les Vosges et en Alsace, - Jura : Coges près Bletterans. - Envir. de Genève à Vernier, Meyrin, entre Bossey et Divonne. - Vaud et Valais : le Jorat au-dessus de Lausanne, Tour de la Molière, Vevey, Allaman, Aigle, Massongex, Folatères, Martigny, Maladeire, Entremont. - H.-Sav. : Pommier, St-Gervais. - Commun dans la région granitique de C.-d'Or, S.-et-L., Beaujolais, Lyonnais, - Terrains sablonneux de l'Is. à Uriage, Vaulnaveys, St-Nizier, Corençon, mont Rachais, Poligny en Champsaur, Valgaudemar. - Alp.-Mar. : St-Martin-Lantosque, entre St-Sauveur et St. Etienne, Clans, Bollena, mont Rasel au-dessus de Menton. Ard. et Gard : le Cheylard, l'Espẻrou, Dourbie. - Hér. : StAmand-de-Mounis, la Salvetat, Fraisse. - Pyr.-Or. et Ariège : St-Martin, Fosse, bois de Boucheville et de Salvanère, vallée de l'Aude aux bains d'Usson, Mijanès, cirque des Aiguettes.

c. persicifolla L. - Bois et prairies des plaines et des montagnes. - Commun dans les parties septentr. et moyenne du bassin. Plus rare dans la partie mérid. oủ il occupe exclusivement la région montagneuse.

Une variété lasiocalyx à calice hérissé est parfois mêlée au type ou même le remplace.

C. cenisia L. - Rocailles des Alpes et des Pyrénées - Vaud et VaIais : Lavarraz, Grandvire, les Martinets, les Diablerets, mont Fully, Barberine, Valsorey, Proz de Bagnes, Diez, Mayenhorn de Leukerbad, Simplon au Raut et Kaltwassergletscher, UnterRothorn, Zmult, Schwarzberg près Mattmark, Distel d'Eginna, Griess. - H.-Sav. et Sav. : le Buet, Barberine, les Aravis, le Bonhomme, la Vanoise, mont Tabor, mont Cenis à Ronches. Is. et II.-Alp. : Grandes-Rousses au-dessus du lac Blanc, Villard-d'Arìne, Galibier, entre Vallouise et Argentière, col de 
l'Alp.-Marlin, Orcières, col Malrif, crêtes de Ségure, Cervières à Terre-Nière. - B.-Alp. : Bérard, près la Condamine.

WAUMENBERGIA hederacen Rchb. - Prairies tourbeuses des terrains siliceux; nul sur les calcaires. - Commun sur le granit et le grès vosgien du versant lorrain de la chaine des Vosges; par une bizarrerie de dispersion gíographique, il man. que sur le versant alsacien où cependant existent les mêmes terrains. Nul dans la chaîne jurassique, - Morvan granitique dans C.-d'Or et S.-et-L. autour de Saulieu, St-Léger, la Roche en Brenil, Semur, envir. d'Autun, Petite Verrière, Grande Verrière, Roussillon, Bourbon. - Rlı. : chaine granitique du Beaujolais à Propières, St-Ri:aud, la Carelle près Beaujeu, Chenelette. - Aude et Tarn : toute la Montagne Noire, les Martis, Mliraval. - Gard et Hér. : l'Espérou, l'Espinousse à Salvergues, le Caroux, Fraisse, St-Amand-de-IIounis, Andabre-Rosis, envir. du Pont-de-Gignac, au-dessus de St-Pons. - En dehors de nos limites sur les terrains siliceux des Pyrénées centrales, do l'Aveyron, du Tarn, de la Lozère, du Cantal, du Puy-de-Dome, Loire, Allier, Yonne, Maine-et-Loire, et tout l'ouest de la France jusque dans la Bretagne et la Normandie.

\section{VACCINIÉES}

VACCINIUM myrt11um L. - Bois des montagnes; beaucoup plus commun sur les sols siliceux que sur les calcaires oủ il ne se montre que lorsque ceux-ci sont recouverts de terrains do transport ou lorsqu'ils contiennent des bancs de grès, des couches de silex, ainsi qu'il arrive assez souvent dans les élages oxfordien, néocomien et nummulitique. On le voit quelquefois descendre jusque dans les plaines sablonneuses, comme, par exemple, dans celles de Haguenau et de la Bruche en Alsace.

v. uliginosum L. - Marais et tourbières des montagnes à sol siliceux; ne se montre dans les chalnes calcaires que là oủ des tourbières sont établies sur des argiles glaciaires. - Hautes Vosges depuis le Schneeberg et le Champ du Feu jusque dans la IIaute-Saone aux ballons de Giromagny et de Servance, Bel- 
fahy, vallée du Rahin, les Arrachies. - Jura helvêtique et français : Sonnenberg, Chasseral, Ponts, Sagne, Brévine, Creuxdu-Van, tourbières de Maîche, Bélieu, Russey-Guinots, Mémont, Bonnétage-St-Julien, Pontarlier, Bonlieu, Joux, les Rousses, la Trélasse, Colliard près Nantua, et le Vély près Hauteville en Bugey. - Vaud et Valais : Dorchau, Gourze, Grand-St-Bernard. - IIte-Sav. et Sav. : Brizon, col de Balme, Valorcine, mont Sambuy, Hauteluce, Crest-Voland. - Is. : chaines des SeptLaux et de Belledonne au dessus de Jasse-Vieille, la Pra, Cham. prousse, lac Luitel, Grand Som, l'Obiou. - H. et B.-Alp. : La Grave, Lautaret, IIonestier-de-Briançon, mont Viso, Chaillol, Orcières, Durbonnas, Valgaudemar, St-Véran, col de Vars, Lauzanier, Parpaillon. - Alp.-Har. : St-Dalmas-le-Sauvage, cols de Salèze et de l'Abisso. - Pyr.-Or. et Ariège : massif du Canigou et du Laurenti. - En dehors de nos limites dans les Pyrénées centrales, la Lozère, Cantal, mont Dore, Puy-deDóme, Pierre-sur-Haute.

v. rubrum, $V$. Vitis Idaea L. - Pâturages et bruyères des montagnes à sol siliceux; dans les chaines calcaires il ne se joontre que sur les sols tourbeux ou lorsque à la surface existent des couches siliceuses. - Commun dans les Iiautes-Vosges jusque dans la H.-Saòne sur les Ballons de Servance et de Giromagny, Belfahy, Champagney, Tète des Noirs-Etangs. - Jura helvélique et français : lieux tourbeux au Paswang, Weissenstein, Sonnenberg, Chasseral, Creux-du-Van, Chasseron, mont d'Or, Suchet, Montendre, St-Julien, le Reculet, montagnes de Nantua. - Vaud et Valais : la Bretonnière et Bois-Girard près de Payerne, Cugy, Bottens, Rovéréaz, Ecublens, val Massa, Mayens de Sion, Vercorin, Simpłon. - H.-Sav. et Sav. : Salève, derrière le Piton du milieu, Voirons, Servoz, Chamonix, la Sambuy, Crest-Voland, Hauteluce, les Bauges. - Is. et H.-Alp. : chaines des SeptLaux, de Belledonne, Chamechaude, Bovinant, St-Nizier, le Noyer en Champsaur, Valgaudemar, Valpréveyre en Queyras.B.-Alp. et Alp. Mar. : Tournoux, Alpes de St-Dalmas-le-Sauvage, col de la Maddalena. - Rh. et Loire : montagnes de Tarare, le Boucivre, Pilat. - Gard et Lozère : entre Brama-Bioou 
ct Meyrueis. - En delrors de nos limites, ì la Margeride, Cantal, mont Dore, P'uy-de-Dome, Pierre-sur-Llaute. - Descend en $\mathrm{Al}$ sace jusque dans la plaine de Ilaguenau à 150 mètres.

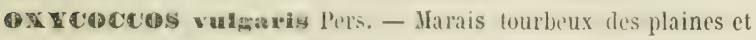
des montagnes. - Vosges granitipues et arénacies jusque dans la Haute-Saone au ballon de Giromagny, Servance, la Bravouse, vallée du Rahin, Fresse, Ecromagny, Annegray.- Jura helvétique et français : Chasseral, Eplatures, tourbiěres d'Eloyes, d'Auxelles, Pontarlier, la Brévine, les Ponts, la Trélasse, les lRousses, vallée de Joux, le Vély près Hauteville en Bugey. Vaud et Valais : Gourze, Jongny, bords du lac de Champé. Is. : lac Luitel au-dessus de Prémol. - C.-d'Or et S.-et-L. : Morvan granitiçuc autour de Saulieu, Anost, Planche, Gien, Montsauche. - Pyr.-0r. et Ariège : Canigou, Madrẻs, Laurenti dans les mouillères de Soucarat, la Maouré de Riplaou. - En dehors de nos limites dans les parties de l'Yonne et de la Nièvre qui confinent au Morvan, Cher, Indre, Creuse, Pierre sur-Haute, Puy-de-Dơme, Lozère, bassin parisien.

\section{ERICINÉES}

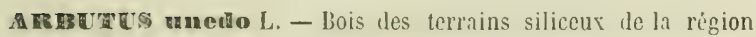
mérid.; nul sur les calcaires. - B.-du-Rh. : cailloux quartzeux de la Ciotat, St-Loup, N.-Dame-des-Anges, - Var et Alp.-Mar. : granit, grès, schistes des Maures depuis Hyères, Pierrefeu, le Luc, l'Esterel. - Vaucl. : Orange, Gadagne, bois de Sẻrignan. - Dr. : Nyons, Montélimar. - Gard : le Vigan, St-Ambroix, Alais, Nìmes. - Ilér. : Murviel, la Gardiole, St-Guilhem-leDẻsert, Montarnaud, Viols, Valène, St-Martin d'Orb, Montpeyroux, Arboras, Soumont, Loiras. - Aude et Pyr.-0r. : Fontfroide, basses Corbières, St- $\Lambda$ ntoine de Galamus, Céret, Llauro, Oins, Montoriol.

A Jura, des Alpes et des Pyrénées. - Jura lıelvélique et français au Chasseral, Creux d'Allemogne. - Vaud et Valais : Jaman, Chapuise, les Martinets, les Plans à Ormonds-dessus, Anzendaz, Catal. Bassin du Rhône. 
Boulaire, Catogne de Martigny, St-Bernard, Gičtroz, Comba d'Arbaz, Miayens de Sion, Tortain, Leukerbad, la Gemmi, Simplon. - Hte-Sav, et Sav. : Glacière du Brizon, Vergy, mont de Chalune et de Pelloua, Sixt, Brévent, Aiguilles-Rouges, col de Balme, chaîne du mont Blanc, Taconnaz, Bionnassey, mont Lachat, les Contamines, Hauteluce. - Is. : Chamechaude, Charmant Som, col de l'Arc, la Moucherolle. - H. et B.-AIp. : Lautaret, Vergons sur Annot. - Pyr.-Or. et Ariège : Canigou, Coma du Tech, Carlitte, Madrès, Laurenti au vallon de Barbouillère, Roc Blanc, Pic de la Camisette, Valbonne. - Pyr. centrales.

A. ofideinans Wimm. - Rochers des montagnes. - Jura helvétique et français: Weissenstein, Suchet, mont d'Or, Noirmont, Hontendre, Dôle, Colombier, Reculet, entre Chẻzery et Lélex, Sorgiaz, mont de Nantua, Colombier du Bugey. - Vaud et Valais : Anzendaz, mont Fully, Morgins, Rosselenaz, Chemin, Liduls, Tolrembé, Thyon, Montorge, Comba d'Arbaz, Ayent, Vercorin, Simplon, Bellap, val de Binn. - Hte-Sav. el Sav. : Salève, montagnes du Chablais, du Faucigny, de la Tarantaise et de la Maurienne, les Bauges, mont du Cliat. - Is. : mont Rachais, Chalais, Grande-Chartreuse, de Pariset à St-Nizier, la Salette. - H. et B.-Alp. : la Grave, Lautaret, la Vachette, Plampinet, envir. de Briançon et de Gap, Tournoux, Horonaye. Alp.-MIar. : montagnes d'Entraunes, mont Cheiron au col de Vagay. - Ard. : le Nezenc, Gerbier des Joncs. — Gard : Trèves, Lanuejols, Anduze, l'Espérou. - Aude, Pyr.-0r. et Ariège: les Corbières, Canigou, Costa Bona, Carença, Font de Comps, Madrès, mont Louis, Laurenti. - En dehors de nos limites sur les Causses jurassiques de la Lozire, de l'Aveyron, Pyr. centr., grès rosgien de l'Alsace.

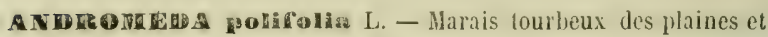
des montagnes. - IIautes-Vosges, jusque dans la Haute-Saône au ballon de Giromagny, Ecromagny, Annegray, Franchevelle à la Pile, St-Germain. - Jura septentr. : tourbières des Ponts, la Sagne, la Brèvine, l'ontarlier, Miouthe, Bèlieu, Nö̈l Cerneux, Chenalotte, Bizot, Narbief, Mémont, Russey, Guinots, Bonné- 
tage, St-Julien, Chaux-du-Dombief, Vaux, Boujailles, Noiraigue, Ste-Croix, Val de Joux, les Rousses, la Trélasse. - Vaud : Gourze, Jongny, les Mosses dans les Ormonds. - IIte-Sav. : Mieussy dans Ies marais de Somans. - En dehors de nos limites a l'Aubrac sur les bords du lac de Saliens, Cantal, mont Dore, Pierre-sur-Haute.

C. ceux depuis les plaines jusqu'aux montagnes. Se montre quelquefois dans les chaines calcaires sur les couches d'argile, sur les amas de silex, ou sur les dépôts siderolithiques. La Bruyère commune est indifférente aux conditions climatériques : on l'observe dans tous les pays de l'Europe et à des altitudes différentes et jusque dans les hautes vallies des Alpes. En outre elle croit sur les terrains siliceux de toutes les formations géologiques, quelle que soit leur structure physique: sur les argiles aussi bien que sur les graviers et les sables; dans les terrains les plus secs ainsi que dans les tourbières constamment mouillées. Suivant les staiions dans lesquelles elle vit, elle semblerait tantot xérophile, tantôt hygrophile ; ici elle paraîtrait être psammophile, ailleurs pélophile. En réalité elle s'accommode de tous les sols, pourvu qu'ils contiennent une forte proportion de silicates alcalino-terreux.

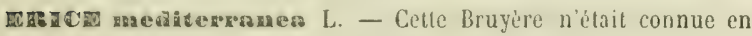
France que dans les landes de Cissac (Gironde); ensuite elle a été trouvée dans les Pyrénées-Orientales sur la route d'Argelès à Laroque d'Albère.

E. muntakora L. - Collines arides de la région mérid, - B.-duRlı. : envir. de Marseille à Si-Marcel, Mazargues, Montredon à Bandol, St-Loup, - Alp.-Mar. : vallon de Hagnan et Bellet près Nice, St-Hospice près Villefranche. - Hér. : Montferrier à Fontfroide, la Tour de Piquet, Murviel, Montarnaud, St-Guilhem-leDésert, les Capouladoux aux Cambrelles, Gignac à la Taillade, Mourèze, Villeneuvette, Aumclas, Castelnau-de-Guers.-Aude: rochers entre la Nouvelle et Leucate.

E. leciplens St-Amans, L. ruguns G. G. (an L. ?) $\rightarrow$ Bois et collines des terrains siliceux. - Var: les Sablettes près Toulon, ile de 
Bandols. - Pyr.-Or.: Amélic-les-Bains. - Is. : bois de Chambaran au S.-0. de Roybon. - En dehors de nos limites cette Bruyère existe dans l'ouest de la France, puis dans la Vienne, Maine-et-Loire, Sarthe, Loir-et-Cher, Indre-et-Loire, Indre, Cher, bassin sous-pyrénćen.

Les botanistes ne sont pas d'accord sur la synonymie: les uns, avec Reuter et Grenier, identifient l'Erica vagans L. avec l'E. decipiens St-Am.; d'autres avec Chaubard et M. Debeaux, soutiennent que le véritable $E$. vagans L. est une plante d'Orient qui n'existe pas en France.

I2. tetralax L. - Cette Bruyìre qui habite les terrains sablonneux de l'ouest et du centre de la France ne se trouve dans le bassin mediterranéen que dans les vallées appartenant au massif du Laurenti au col des Ares, rochers de la Soucarade, bois de France, Grand Pla, Roc de l'Ermite, jasse de Campeilles, Roc de Campeil, pic de Canrusc, pointe de Lesquerde.

E. cinearea L. - Collines arides et clairières des bois dans les terrains siliceux. - Is. et Dr.: Tour de Monfalcon près Roybon, Nyons. - Ard. : St-Pierreville, l'Argentière, vallée du Nezayon. - Gard: dans la partie montagneuse des arrondissements d'Alais et du Vigan. - Hér. : Montarnaud, Argelliers, Villeneuvette, Octon, Soumont, Loiras, Usclas, Madières, St-Christol, Neffiès, Lodève, Béziers, l'Espinouse, St-Martin d'Orb, St-Chinian. - Aude, Pyr.-Or. et Ariège : Villespy, Fontíroide, Fontlaurier, Aussières, Carcanières, Corbère et les Aspres de la vallée du Réart, Salvanẻre, col des Ares, Quẻrigut, Pic de Lieurous.

En dehors de nos limites, dans le bassin sous-pyrénéen, Aveyron, Tarn, et le centre de la France jusque dans la région granitique de Loire, Allier, Yonne, Nièvre, S.-et-Loire; puis dans le bassin parisien et dans l'ouest.

E. arborea L. - Terrains incultes, bois des terrains siliceux de la région mérid. - B.-du-Rh. : conglomérat à cailloux quarıeux du Bee de l'sigle et de N.-D. des Anges. - Vaucl. : Gadagne, Strignan. - Var et Alp.-IIar. : granite, schistes, porphyres grès des Naures et de l'Esterel, ille de Porquerolle, le Luc, Cannes, sables mollassiques de Menton. - Gard : Nîmes, Nanduel, 
la Beaume, le Vigan, St-Ambroix, St-Jean-du-Gard, Anduze, entre Valleraugue et Ganges. - Hér. : Murviel, Montarnaud, Lodeve, pentes du plateau de Fignols, entre Olargues et St-Pons, Lamalou, Bédarieux, St-Aiartin d'Orb, Octon, Jérifon, Ambeyran, le Caroux. - Aude et Pyr.-Or. : Fontlaurier, Fontfroide, Albères, Argelès, Collioure, Banyuls, Céret jusqu'à Prats-deMollo, vallées du Réart et de la lèt.

D. peoparıa L. - Bois et garrigues de la région mérid, - Dr. : Valence, Montẻlimar, Grignan, Nyons. - Vaucl. : Sẻrignan, Uchaux, Rustrel, Gignac, Roussillon, Flassan, Gadagne. - Var el Alp.-Mar.: depuis Toulon, les Maures et l'Esterel jusqu'à Cannes, Antibes, Nice et Menton. - Ard. : Bourg St-Andéol, Jovyac. - Gard et Hér. : commun dans la partie basse des arrondissements de Nimes, d'Uzès, du Vigan, de Montpellier, de Béziers ct de Lodève. - Aude et Pyr.-0r.: Montolicu, partie basse des Corbières, les Albères, vallée du Réart, Céret et route de Pratsde-Nollo, Villefranche et route de Hont-Louis.

canea L. - Bois. - Vaud et Valais : coteaux au-dessus de Bex et d'Ollon, forêt d'Epinacey, Solalex, Alpes de Chateau d'Oex, vallée de Gérignoz, col du Pillon, Bois-Noir près St-Ilaurice, Lalex, bois de Finge, vallée de la Lizerne au-dessous du lac de Derborence, vallée de la Norge près du Pont-Neuf, Brieg près de Schalberg et le long du Riedwald, pied du Glieshorn. H.-Sav. et Sav.: rochers de la base d'Andey près de Bonneville, Dessy près Pontchy; suivant Fauconnet on l'aurait trouvé vers le chalet des Avenières au-dessus de Cruseilles, Rumilly, St-Laurent, entre Bramans en Iaurienne et la Combe de Villette, entre Avrieur et le fort de l'Esseillon. - Alp.-Mlar.: Val de Pesio, col de Tende. - La Bruyère incarnate, de mèrae que la précédente, est moins exclusivement silicicole que les autres Bruyères; elle prospère très-bien sur les roches calcaires recouvertes d'humus.

LOISEACUA proeunbeng Desr. - Rochers siliceux des Alpes et des Pyrénées. - Vaud et Valais: Anzendaz, Javernaz, Lavarraz, Isenau, mont Fully, Catogne de Sembrancher, StBernard, Zermontana, sommités des vallées d'Hérémence, d’Evo. 
lena et de Zermatt, Leukerbad, Simplon, Conches. - H.-Sav. et Sav. : le Buet, Brévent, Sixt, claine du mont Blanc à Leschaux, le Couvercle, Entre-la-Porte, Montanvert, mont Lachat, col de Balme, Méri, les Contamines, Laval-de-Tignes, mont Cenis. - Is. et II.-Alp. : chaînes granitiques du Grand-Charnier, des Sept-Laux, de Belledonne vers le lac du Crouzet et à Champrousse, Villard d'Arène sous les glaciers du Bec, col Nalrif, col de Vars. - B.-Alp. et Alp.-Nar.: Tournoux, Pizzo d'Ormea dans les Viosennes. - Pyr.-0r. et Ariège: Cambres d’Aze, vallée d'Eyne, Carol, Canigou, lac du Laurenti, Pic del Ginevré et de Tarbézou, col de Lègue, Barbouillère. - Pyr.-centrales.

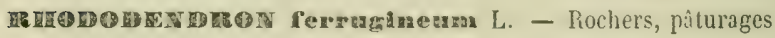
et bois des montagnes du Jura, des Alpes et des Pyrénées sur les sols siliceux; nul dans les Vosges et le centre de la France. Jura helvêtique et français: Chasseral, Creux-du-Van, Noirmont, Montendre, Dôle, Reculet, la Faucille du côté de Gex. Vaud et Valais : la plus grande partie de la chaine mérid. depuis le St-Bernard jusqu'au Simplon, Conches, Glacier du Rhône, Grimsel, Mainghorn. - H.-Sav. et Sav. : les Voirons sur les sables mollassiques du coté de Boëge, le Môle, Brizon, Vergy, Méri, toute la chaine du mont Blanc jusqu'au Couvercle et à Entro la-Porte, la plupart des sommités à sol siliceux de la Tarantaise et de la Maurienne jusqu'au mont-Cenis. - Is. et H.-Alp. : chaines granitiques des Sept-Laux, de Belledonne de l'Oisans jusqu'au Pelvoux, Lautaret, le Briançonnais, mont Viso, Sapet de la Bâtie-Neuve, Champoléon, Valgaudemar, le Champsaur; se montre sur quelques points des massifs de la Grande-Chartreuse, à Bovinant, et entre Villard-de-Lans et la Moucherolle, là où existent des couches de grès verts, au Pic de Chabrières sur les grès nummulitiques. - B.-Alp. et Alp.Mar.: sommités des Alpes de Tournoux, Fouillouse, Lauzanier, Grange-Conmune, Entraunes, St-11artin-Lintosifue, St-Etienne et St-Dalmas, Tende.-Pyr.-Or. et Ariège : mont-Louis, vallées d'Eyne et de Llo, Canigou, Costabona, massif du Laurenti. Pyrénées centrales.

Te. Hersutum L. - Roclsers calcaires des Alpes de Vaud et Valais : 
Tours d'Ai, Ansex au-dessus d'Aigle, Bovonnaz; Lavarraz, Anzendaz, Gemmi près de Schwarrenbach, Simplon, val d'Anniviers. - C'est à tort que Villars l'avait indiquué dans le Valgaudemar. - Haller prétend l'avoir reçu du lleculet, mais personne ne l'y a jamais trouvé. Lamon et Godet assurent l'avoir vu dans le Jura bernois au Chasseral.

D'après l'examen des stations connues dans les Alpes helvétiques, bavaroises el autrichiennes, le Rhododendron hirsutum est une espece calcicole.

\section{PIROLACÉES}

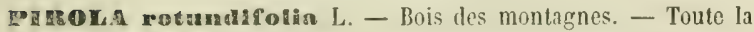
partie orientale du bassin depuis les Vosges et le Jura jusqu'aux Alpes-lfaritimes. - C.-d'Or, S.-et-L. : Marcy-sur-Thil, Bligny, Marsannay, Couchey, Baulme-la-Roche, Val-des-Choues, Meursault, Beaune, Nuits, Cuiseaux. - Rh.: chaine beaujolaise à Chenelette, entre Brouilly et Jarnioux. - Ard. et Gard : la Sapette, St-Sauveur près Camprieux.

En dehors de nos limites dans les Pyrénées centrales, l'Aubrac, Cantal, mont Dore, monts Dômes, Forez, Yonne, bassin parisien. P. media Swartz. - Lieux ombragés, buissons. - H.-Sav. : Salève au-dessus d'Archamp. - Is.: St-Nizier, - En dehors de nos limites en Alsace entre Guebwiller et Soulzmatt, Schaefferthal.

Cette forme est intermédiaire entre le $P$. rotundifolia dont elle se distingue par ses étamines toutes courbées vers le style, et le $P$. minor dont elle differe par son style plus long que l'ovaire.

IP. manor L. - Bois des montagnes surtout sur les terrains siliceux. - Commun dans les Vosges granitiques et arénacées jusque dans la H.-Saône aux Ballons de Servance et de Giromagny, Ruzla-Fonderie, vallée de Miélin, Belfaliy, Fresse, Champagney, Mélisey. - Beaucoup plus rare dans la région des Sapins de la chaîne jurassique. - Morvan autunois à Antully, forêt de Planoise, Fragny, St-Sernin-du Bois, Roussillon, St-Prix, mont Beuvrai. - Chaines du Beaujolais et du Lyonnais à Aujoux, St-Bonnet-le-Froid, Meys. - Massif du Pilat. - Gard et Hẻr. : Cam- 
prieux, la Salvetat. - Pyr.-Or, et Ariége : Ml-de-Madrès, cirque des Aiguettes, bords du lac du Laurenti. - Vaud et Valais : entre Genollier et Coinsins, Panerossaz, Zermalt, Simplon. - H.Sav. : Saleve près des Pitons, les Voirons, chaîne du mont Blanc, forêl des Bossons près Chamonix. - Is. : Grande-Chartreuse, St-Ange, St-Nizier, Prémol, Mens, la Salette. - H.-Alp. : Nêrache au Queyrelin, et à la Combe des Thures, Rabou et Chaudun près Gap. - Alp.-1Iar.: Luceram, Alpes de l'Authion, de Fenestre et de l'Isola.

En deliors de nos limites dans les Pyrénées centrales, l'Aubrac, les montagnes de Lacaune (Tarn), Cantal, mont Dore, Creuse, Allier au Mayet-de-Montagne, Loirc à Pierre-sur-IIaute, mont Sémioure et Noirétable, bassin parisien.

P. chlorantha Swartz. - Bois des montagnes. - Vaud et Valais: Rovérẻaz, Sauvabelin, Vennes, les Montets près Bex, bois de Chene près Coinsins, environs du lac de Derborence en allant vers le Chemin-Neuf, bois Magnin pres de Trient, Vende, Arolla, Finge, Saas, Zermatt, Simplon. - H.-Sav. et Sav.: chaine du mont Blanc, entre les glaciers deș Bossons et de Taconnaz, col de Voza, Semnoz, Colonies d'Annecy, mont Charvin, bois de Barioz près de la Caille, ljois Champion près Moutiers, Bramans.

- Is., H. et B.-Alp. : Pinel d'Uriage, St-Nizier, Villard-de-Lans, Guillestre, forêt de Boscodon prìs Embrun, la Vachette près de Briançon, mont Genèvre, Gảp à Rambaud, la Condamine. Alp.-Nar. : Entraunes à la Combe de Bourdoux. - Gard: BramaBioou près Camprieux. - En dehors de nos limites, dans la Loire au mont Sémioure, dans la Haute-Loire à Costaros, dans l'Aveyron à Vinnac près Millau.

P. secunda L. - Bois des montagnes. - Vosges, chaine du Jura jusque dans le Bugey. - Vaud et Valais, Savoic, Dauphiné, B.-Alp. et Alp.-Mar. - Vaucl. : mont Ventoux. - Ard. et Gard: la Sapette, Brama-Bioou et St-Saureur près Camprieux. - En dehors de nos limites au mont Dore, Cantal, Haute-Loire, Loire.

T. uniforer L. - Bois des montagnes. - Vaud et Valais : Coinsins, Jorat près du Chalel-à-Goybet, l'Alliaz, Solalex, bois de Chexbres, 
les Plans, Creux de Champ, vallon de Gérignoz, Frenières, Catogne de Sembrancher, la Comba, Chemin-Neuf, Saxon, sous Vende, Vercorin, Leukerbad, Zermatt, simplon. - II.-Sav. et Sav. : vallées de Servoz, de Chamonix et de N.-D. de la Gorge, l'Allée-Blanche sur le versant italien au mont Blanc, Pesey, mont Jovet en descendant sur Macot, Bramans, Lans-le-Bourg, I.ans-le-Villard. - Is. et Dr. : St-Nizier, Bois-Grand près Mens, Lus-la-Croix-Haute, forêt de la Penny. - II. et B.-Alp.: la Vachette près Briançon, mont Genèvre, col Isoard, Ceillac, Guillestre a Combe Chauve, la Chal p Ste-Agathe à Roart, Boscodon, Venterol, Chartreuse de Durbon, le Noyer, la Condamine, Tournoux. - Alp.-Mar. : St-Dalmas, Clans, col de Braus, bois du Farghet au-dessus de l'Escarène. — Gard : St-Sauveur près Camprieux. - Pyr.-0r. et Ariège: mont Louis, vallée de Llo, d'Olette à la Font de Comps, mont de Madres, massif du Laurenti, cirque d'Artounant et des Aiguettes, foret du Paillariel et de Riplaou. - En dehors de nos limites dans les Pyrénées centrales; II.-Loire dans les bois de Bauzit, Taulhac, Chacornac, Costaros; Lozère à Langogne; Loire au mont Sćmioure près Montbrison; les hautes Vosges dans la vallée des Granges, le Khliertoft au Pont-des-Fées.

Le Pirola umbellata L. n'existe pas dans notre bassin. - Il se trouve en Alsace dans la forêt de llaguenau.

\section{MONOTROPIÉES}

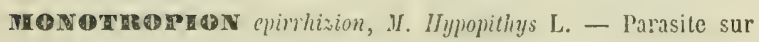
les racines des Pins, Sapins, Chênes, Hêtres, etc. 


\section{COROILHLORES}

\section{LENTIBULARIÉES}

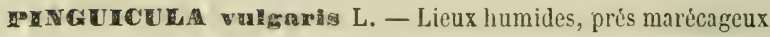
surtout dans les vallées montagneuses. - Chaînes vosgienne et jurassique jusque dans le Bugey du cotẻ oriental, et sur le versant occidental jusque vers les marais d'Artaz, de Divonne et de Troënex. - Vaud et Valais : montagnes de Bex, de Champéry, Trient, Arola, Binnerthal, Grimsel. - H.-Sav. et Sav. : pied du Salive au-dessus de Collonges et d'Archamp, montagnes du Chablais, du Faucigny, de Beaufort, de la Tarantaise et de la Maurienne. - Is, et H.-Alp.: le Sappey, Revel, Laffrey, Lautaret, Ia Vachette, mont Gondran, St-Véran, vallon de Ségure, les Baux et col Bayard près Gap, Poligny en Champsaur, la Condamine, Sérennes, - Dr. : mont Glandasse. - Alp. Mar.: vallées du Var, de la Vesubia et de la Tínea. - Ard. et Gard. : mont Mlezenc, la Tessonne, la Foux près d'Alzon. - Hér. : St-Amand au Roc d'Oryues, Madieres au-dessus de Navacelle. - Pyr.-0r. et Ariège: Canigou, vallées de Carença, d'Eyne, de Llo, massif du Laurenti. - En dehors de nos limites dans les Pyrénées centrales, l'Aubrac, Cantal, mont Dore, mont Dòmes, et à travers le centre de la France jusque dans le bassin parisien.

P. grandiftoru Lam. - Forme du précédent à fleurs plus grandes, à corolle ventrue, aussi large que longue, à éperon égalant presque la corolle. - Jura helvétique et francais : Chasseral, Creuxdu-Van, Chasseron, Dôle, Colombier, Reculet, bois de la Vattay à la Faucille, Crêt de Chalam, Credo, Colliard, Poisat près Nantua, Isernore, le Vély près IIauteville. - Valais: Alpes de Saas, Fée, Simplon, val Massa, Zermatt, val Ferret, St-Bernard. - H.Sav. et Sav. : montagnes du Chablais, Dents d'Oche, Signal des 
Agneaux, mont Laouct près Reyvroz, mont Cenis. - Is., Dr., I. et B.-Alp.: : Seyssins, descente du Pas-de-la-Clé sur Montaud, Monestier-de-Clermont, mont Glandasse, la Vachette près Briançon, Sérennes. - Pyr.-Or. ct Ariège: Canigou, bords du lac de Nohẻdes, Prats-de-Mollo, lac du Laurenti, val de Barbouillère. - En dehors de nos limites dans les Pyrénées centrales, Aubrac, Cantal, mont Dore.

Une forme longifolia Ram., qui a les feuilles allongées, atténuées en pétiole, se trouve dans les Pyrénées et la Lozère.

Le $P$. grandiflora se relie au $P$. vulgaris par de nombreux intermédiaires dont le principal est:

P. leptoceratia Rihb. - Observes sur les hauteurs de la chalne du Jura ; dans l'Is. au col du Frêne près d'Entremont-le-Vieux; dans les B.-Alp. à St-Anne près de la Condamine.

P. alsolna L. - Rochers humides des montagnes, - Chaine jurassique à la Dôle, Montoisê au-rlessus de Crozet, le Reculet, montagne d'Allemogne. - Vaud et Valais : Alpes de Montreux, Anzendaz, Solalex, Lavarraz, mont Fully, Alesse, Vende, P'ierreà-Voir, Arolla, Arba, Lens, Leuk, Zermatt, Simplon. - H.-Sav. et Sav. : Ie Mole, Brizon, Vergy, Méri, mont de Sion, Salêve audessus d'Archamp, Parmclan, plateau des Gets, Hautigny, Signal des Agneaux, col de Balme, les Mottets, col de la Seigne, HauteIuce, mont Cenis. - Is. : Grande-Chartreuse à Bovinant, Chamechaude, St-Nizier au-dessus des Trois-Pucelles, bords du lac de la Morte, la Pra de Belledonne, Champrousse, Haut-du-Pont et St-Hugon près Allevard. - II. el B.-Alp. : Lautaret, Gondran, Valpréveyre en Queyras, Péas, Ségure, Lauzanier, Ste-Anne. Alp.-Hlar. : val de Pesio, Carlin, mont Bego, col de Fremamorta.

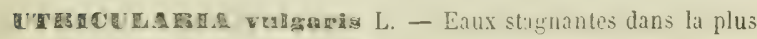
grande partie du bassin. Espèce polymorphe.

J. intermedia Ifayn. - Lacs et étangs des Vosges; envir. de Belfort dans le ruisseau de la tourbière d'Eloje. - Jarais des Verrières et des Ponts.

U. neglecta Lehm. - Mares, - Jura : Mou-de-Pleurre près Chaussin. Alp. Mfar. : Vaugrenier près Antibes, Nice aux Grenouillères du Var. 
ข. minor L. Eaux stagnantes des terrains siliceux. - H.-Saône et Belfort: Evette, Chagey, l'Arsot, entre Eloye et Roppe. - Tourbières de la clraine jurassique: Andelot, St-Laurent, St-Pierre, les Riousses, Menotey dans la forêt de la Serre, Divonne, Belley. - Vaud et Valais: Gourze, Jongny, Yronand, Duiller, Guercet, Verrerie. - H.-Sav. : Aïse près Bonneville, marais de Lossy, Bossey, Epagny, - Rh. et Is. : Vaux-cn-Velin, Décines, Pontde-Beauvoisin, Rives, Varces, - C.-d'Or: Horvan granitique à Saulieu, Roche-en-Brenil. - B.-du-Rh., Var et Alp.-Mar. : marais de Raphèle près Arles, Castignaux près Toulon, Nice au Var. - Assez répandu sur les terrains siliceux du centre et de l'ouest de la France.

\section{PHIMULACÉES}

HiotTon a galustris L.--Mares et fossés, -Jura, S. et L., Ain : la Bresse à Rye, la Chassagne, Neublans, Parcey, Champvans, Damparis, bois de Varennes à St-Bonnet-en-Bresse, bois de StDidier près St-Martin-en-Bresse, Louhans, Navilly, Verdun, Ecuelles, Bourg, Manziat, Loyes, Marboz, Pont-de-Vaux, Curciat. - Rh. et Is.: Yvour, Vaux-en-Velin, Décines, Tigneux et Hyères près Crémicu, Morestel. - Ċ.d'Or: Citeaux, Broin, Gilly, Boncourt, Argilly. - Vaud : d'drenches à Missy et à Salavaux, Cudrefin, Yvonand, Yverdun. - Gard : marais du Cayla.

Hay sant français de la chậne jurassique septentrionale, bien qu'il existe dans le Jura lorrain, balois, soleurois, bernois, neuchatelois et vaudois, ainsi que dans tout le bassin du Léman, et en remontant le Rhơne dans le Bas-Valais jusqu'à Sion. - A partir de Genève, il devient commun dans le Jura méridional à travers le Bugey et ensuite dans quelques parties de la Savoie, du Dauphiné et des B.-Alp. jusque dans les Alpes-Mar. - Très-abondant dans les environs de Lyon. - Vaucl. : mont Ventoux, St-Amand. - Gard et Hér.: Alais et StJean-du-Gard, Anduze, Chartreuse de Valbonne, Lodève, St- 
Pons. - Lozère, Aveyron, Tarn, ouest de la France. - Manque dans C.-d'Or, S.-et-Loire, B.-du-Rh., Var, la chaine des Pyrénées et une grande partie du centre de la France. Comme on le roit, celte espèce a une dispersion géographique fort irréguliere.

D2. officionsaim Jacil. - Pris, hois, depuis les plaines jusquaux montagnes; beaucoup plus répandu que le précédent. - Dans les départements méridionaux il se montre surtout dans la rée gion montagneuse et plus rarement dans les plaines.

Le $P$. suavelens Bertol. est une forme à feuilles blanchatres et tomenteuses en dessous, qui quelquefois est mêlée au type ì feuilles concolores sur les deux faces, et devient même plus commune que celui.ci dans les parties les plus chaudes des parties montagneuses de notre bassin.

$P$. variabilis Goup., $P$. officinali-grandiflora, hybride souvent mélé à ses deux parents et dans lequel la couleur des fleurs est trèsvariable. Il est actuellement démontré que cet hybride peut être obtenu expérimentalement par la fẻcondation arlificielle. Godron a aussi produit, par le mème moyen, et en intervertissant le rôle des générateurs, le $P$. grandifloro-officinalis.

P. elation Jaç. - Bois et prés des collines et des montagnes. Chaine jurassique jusque dans le Bugey; remonte au nord sur les collines calcaires de la Lorraine et de l'Alsace. - Vaud, Valais, Savoie, Dauphiné, B.-Alpes. - C.-d'Or, S.-et-Loire. Vaucl.: mont Ventoux. - Gard: bois de Salbous pres Campestre, Chartreuse de Valbonne, remonte jusqu'à Brama-Bioou. Hér.: St-Amand-de-Mounis, Fraisse, la Salvetat. - Ariége : cirque des Aiguettes, Barbouillère, Grand-Pla, Pic des Fronteils, Jasse de Ja Bentaillole. Companyo l'indique dans les Pyr.Or. à la Preste et au Canigou et à Pla Guillem. Les indications fournies par ce naturaliste ne peuvent être admises sans une nouvelle vérification.

Le $P$. clatior produit avec les $P$. grandiflora et officinalis des hybrides nommés $P$. grandifloro-elatior, clatiori-grandiflora, elatiori-officinalis. Ce dernier, décrit par Muret, a été rarement observé jusqu'à présent et d'ailleurs n'a pu ètre obtenu au moyen de la fécondation artificielle. 
Le $P$. intricata G. G. est-il aussi un hybride du $P$. officinalis et de l'elatior, ou est-il seulement une forme alpine du $P$. elatior dont les feuilles seraient insensiblement atténuées en un long pétiole ? En attendant que cette qquestion soit élucidée, je me borne à signaler sa présence dans I'Is., H. et B.-Alpes au col de l'Arc, la Moucherolle, Grand-Veymont, mont Genèvre, col de Vars, Lauzanier, Horonaye, Fouillouse, Crouès. - Alp.-Mar. Pyr.-Or. et Ariége : Canigou, vallée d'Eyne, mont Louis, lac du Laurenti, vallon de Boutadiol, Valbonne, Barbouillère.

17. Prarinosa L. - Prés marécageux clu Jura, des Alpes et des Pyrénées. - Claaine jurassịue à Délémont, Diesse, Pontarlier, Morteau, Houthe, St-Laurent, Chapelle-des-Bois, Morey, Val de Joux, Montendre, les Rousses, St-Georges, Longirod, Baumeles-Dames, le Marchairu. - Vaud et Valais : Anzendaz, Javernaz, mont Fully, St-Bernard, montagnes de Sion, Thyon, Leukerbad, Zermatt, Natersberg, Simplon, Albrun. - H.-Sav, et Sav.: montagnes d'Abondance, Roc d'Enfer, mont de Pelloua, plateau des Gets, Roselein, la Gita, Crest-Voland. - Is, et II.Alp.: Alpe du mont de Lans, St-Jean-d'Ilérans près Mens, bords des lacs de Laffrey et de Lamure, Lautaret, Valpréveyre en Queyras, vallée du Guil au pied du mont Viso, cols Bayard et de Glaize, Charance, N. D. du Laus, Chaillol, Orcières.-B. Alp. et Alp. Nar.: sommités de la vallée de l'Ubaye, Alpes diEntraunes, Estenc, col de Jallorgues, St-Dalmas-lc-Sauvige, col de la Maddalena, Tende. - Pyr. centrales.

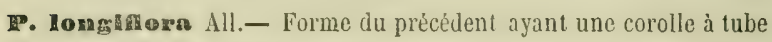
très-long.-Valais, vallẻe de St-Nicolas, Alpe de Taesch, pied du passage de l'Alphübel, Findelen, vallée de Saas au-dessus d'Almagel et de Fée, Schwarzberg, val de Zwischbergen, Simplon à Gondo vers le Steinersand, Sallinischalp, vallée de Binn, Gistiberg dans le Muntigerthal. - H.-Alp. : col de Vars, St-Véran à la Grangeasse el à la Pierre-de-l'Ours, vallon Agnel. M. Lannes l'a aussi trouvé sur le versant piémontais en descendant du col de St-Véran à la Chianale.

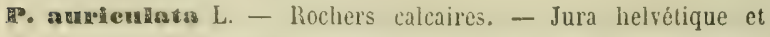
français : Passivang, Fallienstein, Gcisftuh, IIauenstein, Weis- 
senstein, cluses de la Birse et de la Sorne, Baume-les-Dames aux rochers de Chatard, vallée du Dessoubre, roches de Houtiers et de Court. - Vaud et Valais: Jaman, Javernaz, Anzendaz, mont Fully, Prabé, val d'llliez, Alpes de la Sionne, de Lens, Simplon, Zwischbergen, - II.-Sav.: vallée du Fier au Pont-StClair, Parmelan, Charvin, Semnoz, Brizon, Vergy, Jalouvre, vallé de la Borne, vallon des Usses près de la Caille, la Tournotle, col des Aravis, Roc d'Enfer. - Sav.: mont du Chat et de l'Epine, Trérignin, rochers d'lenne, mont Trélod en Bauges, montagnes d'Aime.- Is. ct Dr.: Grande-Chartreuse à Bovinant, Chamechaude, col Vert, col de l'Arc, la Houcherolle, St-Nizier, St-Paul près du Monestier-de-Clermont, mont Glandasse.

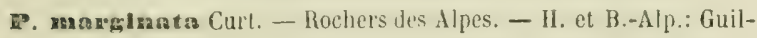
lestre, Chateau-Qucyras, Villevieille, Valpréveyre, col Agnel, col de Ruines, vallon des Vaches, St-Véran, col Isoard, de Valdes-Prés à Plampinet, le Pouzin prìs d'Embrun, Naurin, Larche, Vallonnet, la Condamine, St-Paul à la Reissolle, Castellanne, mont Grand-Couyer sur Annot. - Alp.-Mar.: Entraunes et les vallées montagneuses au-dessus de Thorenc, mont Cheiron, Brouis, Alpes de Nice et de Menton.

GP. vireosa Vill. - Rochers siliceux des Alpes et des Pyrénées. Vaud et Valais : Dent de Morcles, mont Ottan, mont Fully, StBernard, Vernayaz, Trient, Thyon, mont Nuoble, Evolena, Ferpecloz, Zermatt, Guggerluubel, Fée, Aletsch, Grimsel, val Massa, Natersberg, Schalberg, Simplon, Albrun, Viescherthal, Munstigerthal. - H.-Sav. et Sav.: le Buet, les Montets près de Chamonix, chaine du mont Blanc au Chapeau, au Jardin, au Couvercle et sur les moraines de la Mer-de-Glace et de Tré-la-Tête, l'Allée Blanche sur le versant italien, le Chapieu, la Sauce, Saut des Allues. - Is.: chaînes granitiques des Sept-Laux, de Belledonne à la Pra et à Champrousse, col d'Oddie, Taillefer, St-Christophe-en-0isans, massif du Pelvoux, mont Chamoux.II.-Alp.: La Grave, Lautaret, Monestier-de-Briançon, Pra-Brunet, Chaillol-le-Vieil, Guillestre à la Chapelue, mont Viso à la Traversette et au col de Ruines. - Alp.-Har.: les Viosennes, Alpes de Tende et de Fenestre, la Clue de St-Auban. - Pyr.- 
Or.: Canigou, Costa-Bona, Cambres-d'Azes, vallée d'Eyne. Ces dernières indications de localités pyrénéennes sont à vérifier.

P. pedemontana Thomas. - Rochers des Alpes de la Savoie à Ia Levanna, mont Iseran, Laval-de-Tignes, col du petit mont Cenis, Savines en Piémont.

F. Iatifolia Lap. - P. hirsula Vill., P. graveolens. Hegetschw. Rochers des Alpes et des Pyrénées. - Sav. les Allues, sources de l'Arc, mont Thabor, mont Cenis. - Is. et H.-Alp.: second tunnel entre Bourg-d'Oisans et le Fréney, la Grave à Puy-Vacher, Pic du Bec au-dessus de Villarù-d'Arène, Lautaret, vallon d'Arcines, Alp.-Martin, mont Genèvre, mont Gondran, Névache, Orcières. - B.-Alp. et Alp.-Mlar.: Grand-Couyer sur Annot, col de Tende, vallée de la Gordolasca, vallon de Nanduébis, lac d'Entrecoulpe, la Clue au-dessus de St-Auban. - P Pyr.-0r.: Cambres-d'Aze, Costa-Bona, Canigou.

TP. integrelfolla L. - Pâturages des hautes vallées pyrénéennes: Costa-Bona, Cambres-d'Azes, vallée d'Eyne, Madrès, Carlitte, lac du Laurenti, Jasse de la Bentaillole, Pic de Tarbézou, vallons de l'Estagnet et de Boutadiol. - Pyr.-Centrales.

- Allontana Lois. - Rochers des Alp.-Mar. dans les gorges de Saorgio, vallée de Caïros, Alpes de Fenestre.

GREARA Iuten, G. Vitaliana Duby. - Rochers des Alpes et des Pyrénées. - Valais : sommités de la vallée de Zermatt, Gorner, Rothorn, Riffelhorn, Saas, Torrent et Guggerhübel, Roswald, Simplon, Binn. - Sav.: cols de la Vanoise et du Palet, mont de Fréjus, mont Cenis. - Is. et H.-Alp.: glacier du mont de Lans, Lautaret, Galibier, mont Genèrre, St-Pierre près Briancon, cols Malrif, Isoard, des Hayes et de la Croix en Queyras, cols de Ruines et de la Traversette, montagnes de St-Véran, mont Aurouse, Charance, Cùte Gúlive, col de Glaise et Chaudun près Gap, pic de Chabrières, Boscodon.-B.-Alp. et Alp.-Nar.: la Condamine, Sérennes, Lauzanier, Annot au cols d'Allous et de St-llichel, Alpes de St-Etienne et d'Entraunes, sommet de l'Abisso. - Vaucl.: mont Ventoux. - Pyr.-Or. et Ariége : Canigou, la Gallinassa, les Set-Homens, Cambres d'Aze, Collada de Nuria, Valbonne sous le Roc de la Musicque, Port de Paillères sur le versant oriental, - Pyrẻn.-centrales. 
ANDHESACES Felveticum Gaud. - Rocher's calcaires des Alpes. - Vaud et Valais : Dent de Jaman, Clapuise, Anzendaz, les Diablerets, Surchamp, Javernaz, Cheville, Dent-du-Midi, Conthey, Prabẻ, Loze, Pierre-Rouge, Bellalui, Gemmi, Mayenhorn, Zermalt. - H.-Sav. et Sav.: Jalouvre, Vergy, Méri, Roc d'Enfer, Signal des Agncaux, Cornetles-de-Bise, Chaîne du mont Blanc où il s'élève jusqu'au col du Géant et aux Grands-Ilulets, le Buet, les Aravis, mont Drizon, la Tournette- Is, et H.-Alp.: Villard-Eymond, Villard-d'Arène, mont Viso à la Traversette, mont Aurouse, Obiou.

A. Imburicatum Lam. - Rochers des Alpes et des Pyrinces. Valais : Catogne, Cleuson, Zmutt et Findelen près Zermatt, vallée de Saas au Trift et au-dessus de Balen, Alpes de Naters, sur Lutschen. - Is. et II.-Alp. : chaine de Belledonne près du lac Crouzet, les Sept-Laux, Brandes en Oisans, Lautaret, mont Viso à la Traversette et à Ruines. - Alp.-Mrar. : sommet de l'Abisso, Ste-Anne-de-Vinaï. - Pyr.-Or.: Canigou, Cambres d'Aze, vallées d'Eyne et de Llo. - Pyrén. centrales.

A. glaciale Hoppe, A. alpina Lam. - Rochers siliceux des sommités les plus élevées des Alpes. - Valais : St-Bernard à la Baux, Montmort, Chenaletta, col de Fenêtre, Zermontana, Cleuson, col de Severeu, Thyon, Orsera, Arolla, Hérémence au Cret, Ferpécloz, Vouasson, Pierre-Rouge, Mayenhorn, Mainghorn, Alpes autour de Zermatt au Gorner et au Schwarzsee, col StThéodule, envir. de la cabane de Vincent au pied du mont Rose, Alpes de Saas et du Simplon, Griess. - H.-Sav. et Sav.: cols du Géant, du Bonhomme, des Fours, Iseran, la Galize, col de la Madeleine au Cheval-Noir. - Is.: Grandes-Rousses vers le lac Blanc, Pyramide des Sept-Laux, mont de Lans au glacier du Chamois.

J'ai trouvé au pied du mont Cervin, sur les moraines du Furgengletscher, une forme à pédoncules allongés que j’appelle variélé peduniulata. On sait que dans le type les pédoncules sont extrêmement courts et restent inclus dans les rosettes des feuilles.

A. pabegcens D C. - Rochers des Alpes et des Pyrénées. Catal. Bassin du Rhône. 
Vaud et Valais: Javernaz, Lavarraz, Dent de Morcles, Alpes de Vouvry, Bellalui, Nufenen, Saas, arêtes sur Donin, Mainghorn, Gemmi. - H.-Sav. et Sav.: Dents d’Oche, Signal des Agneaux, mont de Pelloua, Roc d'Enfer, Vergy, Méri, mont Blanc à Leschaux et jusqu'aux Grands-Mulets, Tournette, Drizon, Trélod, col des Fours, mont Cenis. - Is. et H.-Alp.: le Gleyzin d'Allevard, Taillefer, Petite Moucherolle, mont Aiguille, Tréminis, l'Obiou, Villard d'Arène aux Trois-Évêchés, Galibier, mont Genèvre, mont Viso à la Traverselte, la Grangette près Gap, mont Aurouse, Chaillol. - B.-Alp.: Fouillouse, Bérard, Parpaillon.Ariège : Laurenti au Roc Blanc, Boutadiol. - Pyrén. centrales. Dans cette Androsace, la longueur des pédoncules est très-variable : la forme cylindrica D C. a les pédoncules beaucoup plus longs que les feuilles; celles-ci persistent sur la tige et forment de petites colonnes. Dans la forme ciliata D C., les feuilles sont glabres sur les faces et ciliées seulement sur les bords, mais non persistantes en forme de colonne.

A. villossan L. - Rochers calcaires des hauts påturages du Jura, des Alpes et des Pyrénées. — La Dôle, le Vuache et le mont dı Chat. - Is. et Dr. : le Moucherotte près St-Nizier, Gresse, la Bâtie, col Vert, col de l'Arc, Grand Veymont aux Bachats, mont d'Angèle, Brette près la Motte-Chalancon. - H. et B.-Alp.: les Aiguilles près Plampinet, mont Viso à la Traversette, mont Vergons sur Annot. - Vaucl.: mont Ventoux. - Alp..Mar. : cols de Tende et de Fremamorta. - Aude et Pyr. Or. : le Milobre, Cambres d'Aze, vallées d'Eyne et de Llo, Carença, Font-deComps. - Pyrẻn. centrales.

A. villosissimum, A. Chamaejasme Jacq. - Diffère du p̧récédent par l'abondance des poils qui hérissent le calice, la tige et les pédoncules, par ses feuilles obovées et linguement ciliées. Le nom bizarre et insignifiant donné par Jacquin sera avantageusement remplacé par celui de villnsissimaum qui présente le double avantage d'exprimer très-clairement le caractère distinctif et de rappeler en mème temps l'étroite affinité de cette forme avec l'A. villosa. - Rochers calcaires de Vaud et Valais : Dent de Jaman, Anzendaz, Boulaire, Panerossaz, les Diablerets, la 
Gemmi, Sanetsch, Prabé, Comba d'Arba, Bellalui, enire Roswald et Binn, Schoenhorn, Glieshorn.-L'A. villosissimum remplace l'A. villosa dans les Aipes calcaires non-seulement de Vaud et Valais, mais aussi des cantons de Berne et des Grisons. - On le trouve aussi dans les montagnes calcaires de Salzburg, Tyrol, Styrie, Autriche, Transylvanic, Bohême, Bavière.

A. Iacteam L. - Rochers calcaires des liautes pelouses du Jura et des Alpes.-Jura helvétique et français: Háuenstein, Passwang, Weissenstein, Chasseral, Creux-du-Van, Chasseron, Suchet, mont d’Or, diguille de Baume, Tête-de-Rang, Pertuis, Cluses de la Birse et de la Sorne, Côtes du Doubs, col des Roches, RocheBlanche près St-Claude. - Sc trouve aussi dans les Alpes calcaires du canton de Berne à la Gemmi, au Stockhorn ct au Sigriswilgrat. - Is. et Dr.: la Moucherolle, mont Glandasse. H. et B.-Alp. : Lautaret, Charance, mont d'Avençon, Longet de Maurin.

A. earaean L. - Rochers siliccux des montagnes. - Nul dans la chaîne jurassique. - Vaud et Valais : Alpes granitiques de Morcles, Fully, St-Bernard au mont Cubit, Arpettaz d'Orsières, Catogne, Thyon, Prabé, Colter, Sassencire, Ferpécloz, Guggerhübcl, Unterrothorn, Findelen, Scliwarzee de Zermatt, Mattmark, Simplon.-Sav.: le Bonhomme, la Levanna, mont Cenis. - Is.: chaines des Sept-Laux et de Belledonne à la Pra, Champrousse, Taillefer, mont de Lans, mont Chamoux, la Houcherolle. - H. et B.-Alp. : Lautaret, N.-D. des Neiges et Gondran près Briançon, Alp-Martin, mont Viso à la Traverselte, envir. de Gap à la Grangette, col de Glaise, Chaudun, Orcières, Chaillol, Pic de Chabrières, la Condamine, Sérennes, Maurin, Lauzanier, Parpaillon. - Alp.-LIFar.: sommitts des Alpes d'Entraunes, StMartin-Lantosque, St-Etienne, St-Dalmas, Tende. - Pyr.-Or. et Ariége : Cambres-d'Aze, vallée d'Eyne, Canigou, Laurenli, Valtonne, Barbouillere. - En dehors de nos limites dans les Pyrén. centrales, Cantal, mont Dore, Vosges au sommet du Ballon de Soultz. - Espèce polymorphe à laquelle se rattachent les formes suivantes :

A. roscum Jord. Fourr. - Ballon de Soultz dans les Vosges, mont Dore, Plomb du Cantal, Pyr. Or. 
4. puberulum J. F. - Mont Viso, mont Cenis.

A. brigantiacum J. F. - Briançon, Lautaret, mont Viso (II. -Alp.); col d'Allous (B.-Alp.); Entraunes (Alp.-Nar.).

A. Reverchonianum J, F. - Mlont Viso au col Agnel, St-Vérah, Briançon à Granon.

A. Obtusaifoninana All, - Pelouses rocheuses des Alpes. - Vaud et Valais: Dent de Jaman, Javernaz, Panerossaz, Anzendaz, les Mortais, les Martinets, la Tour près d'Argentine, Sanetsch, Gemmi, Mainghorn, St-Bernard, Zanrion, Thyon, Loze, Vende, Sasseneire, Ferpécloz, Cotter, mont Nuoble, Zermatt, Saas, Simplon, Naters, Binn, Glacier du Rhône, Grimsel. - H.-Sav. et Say.: mont Méri, Crêt du Ré au-dessus d'Aime, les Allues, Petit St-Bernard, col Joly, mont Cenis, versant italien du mont Blanc dans l'Allée-Blanche. - Is. et H.-Alp. : chaine des SeptLaux et de Belledonne, Champrousse, Taillefer, Montagnes de l'Oisans, Pelvoux, mont St-Hichel près la Mure, la Grave, Lautaret, Lauzet, mont de Prorel, N. D. des Neiges près Briançon, mont Genèvre, cols des Hayes et Isoard, montagnes du Queyras jusıu'au mont Viso. - B.-Alp.: Bẻrard, Parpaillen, Fouillouse, Longet. - Apl.-Mar.: St-Anne-de-Vinaï, cols de la Motta, Alpes d'Utelle.

A. geptentrionale L. - Pelouses des Alpes. - Valais : Randaz, Findelen près Zermatt, Distelap de Saas, Mattmark, - Sav. : Combe de Villette entre Bramans et le mont Ambin.- H.-Alp.: la Grave, Villard d'Arène, Lautaret, Névache, Citadelle de Briançon, la Salle, Gaudissart, mont Genivre, la Monta, mont Viso, col Agnel. - Est-il vrai que celte Androsace se trouve dans les bois de l'Espérou, comme le prétend de Pouzolz ?

A. divarieatasm, A. Chaixii G. G. - Bois. - Is. et Dr. : pied de la Petite Moucherolle, forêt près de Grimone et entre Lus et Glandage. - II.-Alp.: Loubet et Rabou près Gap, Manteyer, mont Séuse, Briançon au Fort des Trois-Tètes. - B.-Alp. et Alp.-Mar.: Annot, col St-Michel, Sisteron, Guillaume, Bec d'Utelle, mont Lachen au-dessus de Séranon, mont Cheiron. Vaucl.: mont Ventoux. - D'après M. Loret cette espèce est la mème que l'A. lactiflorum Pall. de la Sibérie. Nais ce nom de 
lactiforum ne me parait pas convenir à une pla te tont la fleur est habitucllement rose ; c'est porrquoi je l'appelle A. divaricalum.

A. maxinuma L. - Champs cultivés des terrains calcaires. C-d'Or : Dijon, Pouilly, Norges, Messigny, Fixin, Broclıon. Valais: pied de Montorge, Grone, Grimisuat, les Plâtrières, Sierre, entre Conthey et Plan-Conthey, Aven, Brigerberg. Sav.: St-Jean-de-Maurienne. - Is., H.-Alp. et Dr.: entre Bourg d'Oisans et le Freney, Villard d'Arène, Monestier-de-Briançon, Ja Vachelte, entre Briançon et Cervières, Malrif, Guillestre, Embrun, Tallard, envir. de Gap et de Die. - B.-Alp. : la Condamine, Annot, Castellane. - Var et Alp.-Mar.: Signes près la Ste-Baume, la Malle et Caussols au-dessus de Grasse, Villeneuve d'Entraunes, Guillaume, Touët-de-Beuil, Valdiblora, N.-D. d'Utelle. - Vaucl.: entre Montdevergues et Bonpas, Apt, Lacoste, la Garde. - B.-du-Rh.: envir. d'Aix et de Marseille. Gard : Camprieux et Campestre près d'Alzon. - Hêr. : le Larzac au Caylar el à la Vacquerie, Cros, St-Michel-d'Alajou. Pyr-0r.: Trencada d'Ambulla, Belloch, mont Louis, Collioure, Banyuls. - En dehors de nos limites dans l'Aveyron sur le plateau du Larzac et des Arnals; dans la Lozère près de Flöac; dans le Puy-de-Dôme et l'Allier sur les coteaux calcaires de la Limagne et des envir. de Vichy; Yonne, Maine-et-Loire, Vienne, Indre-et-Loire, Loir-el-Cher, Loir, Champagne et Lorraine.

Cromaños europaeus L. - Bois. - Jura helvetique et français: Soleure, la Neureville, Jougne, bois du Pertuis-duSoc, l'Iter au-dessus de Cressier, les Gras près Morteau, Cótes du Doubs sous les Plancheltes, St-Claude à la montẻe de Septmoncel, Mijoux, vallẻe de Flumen, Molinge, bois de la Billaude près Champagnole, la Tour-de-Meix, Châtillon-de-Hichaille, entre Confort et Chézery, Dortan, Oyonnax, Serrières-sur-Ain. - Vaud et Valais : Château d'Oex, Vevey, entre le Bouveret et les Evoueltes, la Praisse près de Roche, Port-Valais, Bois-Noir près St-Maurice, versant italien du Simplon. - II.-Sav. : pentes du Salève, entre Thonon el Evian, Reignier, Pralong, Cluses, Thônes, mont Magériaz près Annecy, vallon des Usses près 
de la Caille. - Is. et H.-Alp.: le Touvet, St-Vincent-de-Ilercuze, Ribicrs. - Ard.: St-Laurent-les-Bains.

C. rofpandus Sibth. Sm., C. hederifolius Ait. - Bois de la région mẻrid. - B.-du-Rhone et Var : Roucas-Blanc près Marseille, envir. de Draguignan au bois des Seives, - Gard et Hér.: moulin de la Beaume sur Gardon, Anduze, les Capouladoux aux Cambrettes. - Pyr.-Or. : vallée de l'Agly à St-Antoine-de-Galamus.

C. nexpolitsanas Ten. - Espèce d'Italie et de Corse trouvée près de Marseille et dans lo Gard à Nozières,

SOLANGLA alpina L. - Pelouses des montagnes, immédiatement après la fonte des neiges. - Chainnes jurassiques : Suchet, Montendre, Crêt-de-Chalam, la Dôle, Colombier, Beculet. - Vaud et Valais: Alpes de Bex, d'Aigle, de Sion, Aletsch, Leukerbad, Champéry, St-Bernard, Bagnes, Zermatt, Saas, Grimsel. - II.-Sav.: Môle, Brizon, Vergy, Méri, Dents d'Oche, mont Ardin, plateau des Gets, Nautau, Ror d'Enfer, Sixt, chaine du mont Blanc depuis le col de Balme jusqu'au Bonlomme, Parmelan, Semnoz, Charvin, Tournette, la Clusaz, Mésève, la Gita près Beaufort, col de la Seigne, Crest-Voland, la plupart des sommités de la Tarantaise jusqu'au mont Iseran et de la Maurienne jusqu'au mont Cenis. - Is. et H.-Alp. : chaines de Belledonne, de l'Oisans, du Pelvoux, Grand-Som, St-Eynard, la Moucherolle, Lautaret, mont Genèvre, le Queyras, val Préveyre, Ségure, mont Viso, le Gapençais à Charance, Chaudun, Durbonnas, mont Aurouse, Chaillol, Orcières. - B.-Alp. : la Condamine à Ste-Anne, Parpaillon, Maurin, Lauzanier. Alp.-Mar.: montagnes d'Entraunes, de St-Martin, St-Etienne, St-Dalmas, Tende. - Pyr.-Or. et Ariége : vallẻe d'Eyne, Canigou, Coma du Tech, Costa Bona, val de Paillères au Roc de Lorry, lacs du Laurenti, vallons de Barbouillère et de Valbonne, col de Lègues. - Pyr. centrales, Cantal, mont Dore.

5. uusilla Baumg. - Pelouses des sommités des Alpes du Valais dans les vallées de St-Nicolas et de Saas, près du lac Merjelen, Budmen, Schornen, Ennergalen, puis à la Furca, au glacier du Rhône et à la Grimsel: - En dehors de nos limites dans les Alpes des Grisons, de Glaris et au Faulhorn. 
COATES nogea, C. Mathioli L. - Bois et grotles humides, Sav.: grotte vers te premier pont sur l'lsère entre tignes et Laval, mont Cenis dans le bois situé à gauche à l'entrée de la valice de Savalain, ou cette jolie Primulacie fut, dit-on, semie par Molineri. - En dehors de nos limites, dans plusieurs vallées du Piémont, de la Lombardie et de quelques autres provinces italiennes; on la trouve, en outre, dans la Hongrie, Croatie, Transylvanie, Autriche, Bavière, Russie septentrionale.

GL. IX maritima L. - Var, Aute et Pyr.-Or.: littoral d'llyires, de l'ille Ste-Lucie, étang de Salces, St-Laurent-la-Salanque.

- Existe aussi sur le littoral de l'Ocẻan, et en Auvergne dans les marais arrosés par les eaux salées de Martres, Tambour, StNectaire, marais de Cour et de Gimeaux.

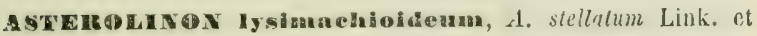
IIofim.-Lieux sablonneux de la région mérid.; remonte dans la Dròme vers Romans, St-Vallier; dans l’Ardéche ver's Andance. - Ouest de la France depuis Bayonne jusqu'à Nantes.

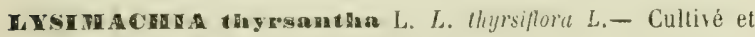
quelquefois subspontané autour des jardins.

L. gIauea Moench, L. Ephemerum L. - Pyr.-Or.: vallées de Prades, de Vernet-les-Bains, de Cornella-du-Conflent, Graus d'Olette, Serdinya, Thuès, Céret.- En dehors de nos limites, Pyrénées centrales dans la vallée de Larboust près de St-Aventin et de Trébons.

L. vulgaris L. - Lieux humides dans toute la France.

C. nummularia L. - Prés loumides et le long des haies.

L. nemoralis L. - Bois des montagnes. - Les Tosges, la chaine jurassique. - Valais: Gueuroz, Salvan, pied de la Dent-duMidi, Champéry.- H.-Sav. et Sav.: Salève, Voiron, Môle, Semnoz, St-Laurent, Crest-Voland. - Is.: Crémieu, Uriage, Prémol, Grande-Chartreuse, Pont-de-Beauvoisin. - C.-d'Or, S.-et-Loire, Rh.: Morvan, chaînes beaujolaise et lyonnaise. - Loire, Ard., Gard, Hér.: massif du Pilat, châ̂ne vivaraise, Cévennes du Gard et de l'Hér. à l'Espérou, l'Espinouse, la Salvetat, St-Pons, Andabre-Rosis, St-Amand, Avène. - Aude et Tarn : massif de la Jontagne noire. - Pyr. Or. el Ariége : vallées de Vernet-les- 
Bains et de Cornella-du-Conflent, Céret, d'Arles à Prats-deMollo, Laurenti a Valbonne, bois de Fach, Pla del Bose, forêt de Paillariel, Prat Lastou. -En dehors de nos limites dans les Pyrén. centrales, l'Aubrac, les montagnes du centre et de l'ouest de la France.

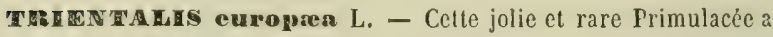
été découverte par II. le chanoine Chevalier au Grarid-Bois de Crest-Yoland (Sav.) - Non loin de la Furca, elle existe dans le canton d'Uri entre Hospenthal et Réalp et au-delà de Zumdorff en descendant la Reuss. On la trouve encore dans les montagnes du Tyrol, Autriche, Transylvanie, Silésie, Moravie, Iongrie, Gallicie, Bohême, en diverses narties de la Bavière, au Bayerischerwald, Fichtelgebirge, Lindelberg, Hesselberg, Spessart, Rhoen; dans les Ardennes belges; en Hollande; enfin en Angleterre, Ecosse, Suède, Norvège et Russie.

CORES monspeliensis L. - Coteaux calcaires ct bien exposís de la région mérid.; remonte dans la Drôme et les Basses-Alpes vers Nyons, Montjoyer, Livron, Montélimar, Crest, Sisteron, Gréoulx, Château-Arnoux, Entrevaux; dans l'Ardèche jusqu'à Cruas, Baix, la Gorce.

CENTENCUES minimas L. - Lieux humides, sablonneux ou argileux; nul sur les terrains calcaires. - Lisière vosgienne et alluvions de l'arrondissement de Montbéliard. - Jura et Ain : commun dans la Bresse et dans la Dombes. - C. • d'0r et S.-ct-Loire : Morvan, la Roche en Brenil à l'étang de Tournesac, Monthelon, St-Sernin-du-Bois, la Bresse à Verdun, Chevigny, Vielverge, Boncourt, Longway et la Bresse louhanaise. - Rh., Is. et Dr.: Chaponost, Beaunant, Viennc, Horestel, Romans. Genève el Vaud : plaine de St-Georges, environs du bois de la Bâtie, Vernier, Bernex, Colovrex, Nyon, Payerne, le Jorat. Sav.: entre Confans et Venthon, Arenthon. - Ard.: Gard et IIér.: Vals, le Vigan, Andabre-Rosis. - Var : Chartreuse de la Verne. - Aude et Pyr.-Or.: Cuxac, Villemagne, Ferrals, Villespy, Verdun, pied des Albères, Prats-de-Mollo.

Cette plante est probablement plus répandue qu'il ne semble d'après les indications précédentes; car, à cause de son exiguité, clle échappe facilement à l'observation. 
ANACAuLIS arvensis $\mathrm{I}_{\text {. }}$ - Cette espece, commune dans les champs cultivés de toute la France, comprend deux races qui se maintiennent distinctes par la culture: l'une A. phenicea Lam. à corolle rouge souvent ciliolẻe-glanduleuse, l'autre $A$, ccortlea à corolle bleue ordinairement non ciliolée. - Plusieurs auteurs les considèrent comme deux espèces distinctes.

A. tenelia L. - Marais des terrains argileux; nul dans Ia chaine jurassique. - H.-Saone : Auxelles, - S.-et-L. et C.-d'Or: Norvan autunois, envir. de Saulieu, la Roche en Brenil, Saut-deGouloux. - Rh.: chaîne du Beaujolais à Chenelette, Pin-Bouchain près Tarare. - Vaud: marais des Gonelles, Blonay, Chailly et Chaulins près Vevey, lac de Bret. - Ain et Is.: SteCroix près Montluel, Charvicu, Tigneu, Vaux-Milieu, Fronto. nas, marais de Fulie à la Verpillère. — Gard : Aigues-Hortes, Anduze, Genolhac, bois de la Calmette, étang de Puyau.- Irér.: Montpellier à la Pompiniane, Palavas, St-Brès, Fonfroide, Castries, Marsillargues, Avène, St-NIartin-d'Orb, la Salvelat, Fraisse. - Aude, Tarn et Pyr.-Or.: la Montagne-Noire, Fontlaurier, Pont de la Fou près St-Paul-de-Fenouillet. - B.-du-Rh.: vallon des Ouides près Marseille, bords de l'étang de Marignane, envir. d'Istres. - Var. et Alp.-Mar.: les Sablettes près Toulon, le Revest, St-Tropez, Fréjus, l'Esterel, Nice au Var et à St-André, cntre St-Agnès et l'Aiguille au-dessus de Menton. - En dehors de nos limites dans les Pyrénées centrales, Aveyron, Tarn, Cantal, Puy-de-Dôme, Allier, Loire et jusque dans le nord de la France.

SAWOEUS palustris, $S$. Valerandi L. - Marais, prés lumides ou quelquefois arrosés par des eaux salées.- C.-d’or : Orgeux, Genlis, Argilly, Villebichot. - Doubs et Jura : Froidefontaine, Pontarlier, Jouhe près Dole, Aumur, $\Lambda$ bergement-la-Ronce. Genève el Vaud: marais de Sionnex, Roellebot, Meinier, Aigle, les Devens près Bex. - Ain, Rh. et Is.: Culoz, lac Bertrand, Belley, Vaux-en-Velin; Villeurbanne, Décines, Meyzieu, la Tour-du-Pin, Grenoble, - Sav.: Seyssel, Moutiers, - H. -Alp. : près des sources minérales du Plan-de-Phazy non loin de Mont Dauphin. - Commun dans la Provence, les Alp-Ilar., le Lan- 
guedoc, le Roussillon, la partie mérid. de l'Ardeche et de la Drôme.

\section{EBÉNACÉES}

mros ap Y R@ Iotus L. - B.-du-Rh.: la Camargue, lles dlu Rhơne. - Cultivé dans le Midi et quelqucfois subspontané.

\section{STYRACÉES}

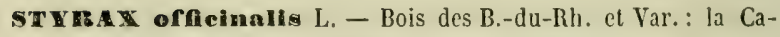
margue, Hyères, mont Coudon, Touris, Montricux.

\section{OLÉACE்ES}

FraxyNuS excelsior $L$. - Bois humides, bords des fossés dans tout le bassin. - La variété australis Gay, qui a les feuilles plus étroites et oblongues-lancéolées, se trouve çà et là dans la région méditerranéenne.

F. oxypliylla Bieb. - Forme du précédent à folioles lancéolées, longuement acuminées, profondément dentées. - Bords des rivières de la région mérid. - Vaucl., B.-du-Rh. et Var. : Bords du Rhone, de l'Arc et du Gapeau. - Gard : bords du Gardon au Pont-du-Gard et à la Bcaume, bords du Contre-Canal à Bellegarde. - Pyr. Or.: gorges du Rẻart. - Espèce polymorphe.

La var. obtusa G. G. a les samares oblongues, arrondies au sommet; la variété rostrata Guss. a les samares lancéoléesaigueës; la variété biloba G. G. a les samares fortement écỉancrées et bilobées au sommet.

F. purvifolia Lam. - Forme à fuullles ovales trouvée près de Montpellier sur les bords du Lez, et autour d'Aix en Provence.

On a encore observé d'autres formes de Frênes, comme, par exemple, le $F$. monophylla Desf., dans lequel la foliole terminale est seule développéc, les paires latérales de folioles restant rudimentaires ou mème manquant totalement; le $F$. leptocarpa D C. qui a des samares étroitement linéaires et très-petites dans toutes leurs parties. 
T. thyresantina, $F$. Ormus L. - Vallons ombragés, - Alp. mar.: le Bar au bord du Loup, Nice, Monaco._-Gard et Lozere : entre Brama-Bioou et Meyrueis. - Pyr.-Or. : Força-Réal, Cases-dePena, Baixas. - Culliré et quelquefois subspontané.

ST RiXG a vagaris. - Cultivé el quelquefois subspontané dans les haies. - La coulcur des fleurs est variable: tantôt blanche, tantôt rougeattre, plus souvent offrant les diverses nuances du blanc violacé.

OLE eurogaen L. - L'Olivier, qui est cultivé dans tout le midi de la France et qui caractérise une région botanique s'étendant jusque dans les parties mérid. de lả Drôme, des BassesAlpes et de l'Ardèche, se montre souvent à l'état sauvage sur les rochers et les garrigues incultes.

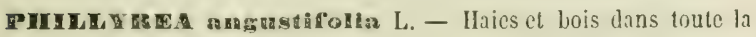
région de l'Olivier; comme ce dernier arbre, il remonte dans la partie mérid. de la Dr., des B.-Alpes et de l'Ardèche. - Il ミe présente sous deux formes principales, l'une, plus commune, à feuilles étroites, l'autre à feuilles plus larges, P. latifolia lyaby. Quelques botanistes admettent encore, sans motif à mon avis, une forme intermédiaire, $P$. media $\mathrm{L}$.

LCUSTre UM vagare $L$. - Haies dans tout le bassin.

\section{JASMINÉES}

Jastrex remonte dans la Drôme, les H. et B.-Alpes, l'Ardèche et l'Isère à Nyons, Charance près Gap, Ribiers, Rosans, Sigoyer près Tallard, Sisteron aux rochers de la Baume, Jastre, Aubenas, la Voulte, Crussol, Châteaubourg, Gervan, St-Vallier, Vienne, Bastille de Grenoble. - Observé accidentellement dans quelques haies autour de Lyon et de Mâcon, et dans ce cas échappé des jardins oủ il est cultivé. - En dehors de nos limites sur les terrains calcaires du Tarn, de la Lozère, des envir. de Clermont, Riom, Pont-du-Château ; puis dans le Cher, Loir-et-Cher et l'ouest de la France.

Le Jasmimum officinale $\mathbf{L}$, est cultivê et se montre quelquefois à l'état subspontané. 


\section{APOCYNÉES}

VINCA minor L. - Haies et bois dans tout le bassin.

v. major L. - Uaies et buissons de la région mérid.; remonte dans l'Ardèche, la Drôme, les H. et B.-Alpes, l'Isère à Coux, Privas, Châteaubourg, Montélimar, Crest, St-Vallier, Romette près Gap, Tallard, la Roche-des-Arnauds, Sassenage, St-Egrère, Corenc. - Rh., Ain, Sav. et H.-Sav.: Rochecardon, Sl-Alban, Souzy, la Pape, St-Germain-Ies-Paroisses, Moutiers, Veyrier près Annecy. - Genève et Vaud : entre Aïre et Châtelaine, Chailly près Vevey, Ollon, St-Tryphon,

Comme la Pervenche à grandes fleurs est souvent cultivée dans les jardins, il y a lieu de croire qu'elle est échappée des jardins dans toutes les localités où elle a été observée en dehors de la région méridionale.

Veutiflora Bertol., V. media G. G.-Forme du précédent à divisions du calice glabres et plus courtes, à lobes de la corolle plus acuminés, à feuilles non ciliées sur les bords; par ces caractères elle semble intermédiaire entre les $V$. major et minor. Du reste on pourrait considérer ces deux dernières Pervenches comme des races de la même espèce. - B.-du-Rh., Var et Alp.-Mar.: entre Fos et St-Mitie, Toulon, Hyères, Rians, de Nice à Menton. - Hér.: Montpellier au Cimetière et à Montels, Castelnau, St-Guilhem, les Capouladoux, Frouzet, Ganges, StFélix-de-Lodez, Cuyras, Rabieux-St-Félix, Poussaroux près StChinian. - Aude et Pyr.-Or.: envir. de Narbonne, Pastouret, Font-Froide, Ste-Lucie, Port-Vendres, Argelès, Collioure, Banyuls, Prades, Vernet-les-Bains.

$\boldsymbol{V}$. difformis Pourr. - Aude et Pyr.-Or.: Bouscaillou près Durban, St-Antoine-de-Galamus.

FET ION Iauriforme Lam., $N$. Oleander L. - Var, et Alp.Mar.: Toulon, Hyìres, le Revest, Dardennes, Bormes, le Muy, Bagnols, Fréjus, St-Raphaël au fond de la Vallongue, Maures de Vallauris, vallon de Lattes entre Menton et Vintimiglia et près de cette dernière ville, sur le territoire italien, dans la vallée de la Nervia. - Le Laurier-Rose est souvent cultivé. 


\section{ASCLEPIADEES}

CYNA NCHOV aeutums L. - Terrains sablonneux de he région méditerranéenne. - B.-du-Rh. el Var.: Arles, le Luc. - Gard - et Hér.: Aigues-Mortes, Pérols, Vic, Mireval, Mauguio au GrandTravers, - Aude et Pyr.-Or.: Ste-Lucie, Banyuls-sur-Mer.

Le C. monspeliacum à grandes feuilles n'est qu'un état particulier de cette especce et ne mérite mème pas le nom de variété.

ALExTOXICON offeinale, Vincetoxicum officinale Nonch.

- Lieux pierreux et incultes dans tout le bassin. - Espèce très-polymorphe dans laquelle la forme des feuilles, du calice et de la corolle présente de nombreuses rariations. - L'utilité du genre Alexitoxicon (Vincetoxicum des auteurs) est elle-même fort contestable. On pourrait en effet, sans inconvénient, fairc rentrer dans le genre Cynanchon toutes les formes et espèces ciaprès énumérées et conserver le nom d'Alexiloxicon comme titre de section. Les formes suivantes se rattachent à l'Alexiloxicon officinale:

A. albidum Jord. Fourr. - Quincieu (R.h.).

A. dumeticolum J. F. - Neyron (Ain).

A. bcugcsiacum J. F. - T'enay (Ain).

A. ochroleucum J. F. - Tassin (Rh.).

A. petroptilum J. F. - Digne (B,-AIp.).

A. alpicolum J. F. - la Grave (H.-Alp.).

A. luteolum J. F. - Bourg. d'Oisans (Is.)

A. Shutllewortianum Thuret el Bornet. - Montagnes du Var.

A. pubcrulum Timb. Lagr. - Tuchan, Durban (Aude).

Les formes $A$. laxum el contigum G. G. sont souvent mêlées au type dans les parlies méridionale et moyenne de notre bassin.

L'A. nigrum Mœench, à corolle d'un pourpre noirâtre, a élé trouvé dans le Gard autour de Nimes, Uzès, Anduzc, le Vigan, Aigues-Nlortes; dans l'Hérault sur divers points des arrondissements de Montpellier et de Béziers; dans l'Aude et les Pyr.-01: autour de Quillan, vallée de l'Agly, Molitg, Collioure, Banyuls; 
dans Vaucluse autour d'Arignon à Gadagne, entre Gigognan et le Mourre de Sève. A l'A. nigrum se rattache la forme A, melanthum J. F. trouvée près de Béziers (Hér.).

Ascture CAs Cornutiana Decaisne. - Cette plante, originaire de la Virginie et non de la Syrie comme le croyait Linné (A. syriaca L.), est souvent cultivée dans les jardins. Grâce à la facilité avec laquelle ses graines à aigrettes sont transportées par les vents, elle s'est abondamment naturalisée sur plusieurs points de notre bassin, notamment dans les iles du Rhône à Vallabrègues, Beaucaire, Coudoulet, Baix, le Pouzin, le Péagede-Roussillon, Serves, Tournon, St-Vallier, St-Maurice-d'Exil, Cliavanay, Condrieu, Irigny, Feyzin, et dans la vallée de la Saone à St-Georges de Reneins près Villefranche, Pont-deVaux. - On l'a vue aussi autour de Belley.

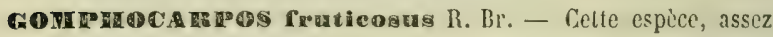
commune dans les îles de Corse, de Sardaigne et de Sicile, a élé trouvée sur la rive gauche du Tech près de Céret (Pyr.-0r.), el aussi dans les environs de Monaco:

\section{GENTIANÉES}

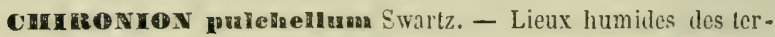
rains argileux, dans laquelle la forme et les dimensions des feuilles varient beaucoup. - La forme latifolium Smith, à feuilles courtes, larges et obtuses, se trouve sur Ic littoral méditerrancen.

C. corryahosama Dulac. C. Centaurium D C. - Pelouses humides et prairies dans tout le bassin.

C. Iinarifolianan D C., Erylhrea linarifolia Gris. - Gravicrs de la Durance près d'Avignon, envir. d'Orange.- Hér. et Aude : Palavas près IIontpellier, la Corrège près de Narbonne.

C. Spiestum Willd. - Licux humides de la rígion méliterranéenne. - Vaucl. el B.-du-Rh.: graviers de la Durance, envir. d'Aix le long des ruisseaux du petit chemin du Tholonet et à l'entrée du vallon de Valcros, borls des étangs du littoral. Var et Alp.-Mar.: Toulon, Hyères, Gonfaron, le I.uc, Frẻjus, 
Tournon-sur-Siagne, tle Ste-Narguerite, golfe Jouan, Nice, Menton.-Gard : Bellegarde, Aigues-Jiortes, St-Gilles. - Ilér.: Palaras, la Madeleine, Mireval, bords de la Mosson, Mauguio, Villeveyrac. - Aude et Pyr.-Or.: bords de l'Agly à Cases de Pena. - Littoral de l'Océan.

C. maritiman Willd. - Prairies humides du littoral de la Móditerranée et de l'Océan. — Gard : Aigues-Mortes et aussi, dans l'intérieur, au bois de Cygnan près Nîmes. - Hér.: plage de Pẻrols et de Palavas, bois de Lamoure près Montpellier, St-Aunès, Castrics. - Aude et Pyr.-Or.: prairies du littoral à SteLucie, Leucate, le Canet. - Var. et Alp.-Nar. : région littorale.

CHCENA Aurormis Delarbre. - Bords des étangs, pelouses humides des sols siliceux. - Jura et Ain : la Bresse dans les cantons de Chaussin, Chaumergy, Montbarrey, Bletterans, lisière de la forèt de la Serre, la Dombes vers Marlieu, St-Germain, Pont-de-Veyle, Chalamont, étang de la Chambrière près Bourg, Pont-de-Vaux. - C.-d'Or et S.-et L.: Morvan, Roche-enBrenil à l'étang de Tournezac, étang de Champeau, Thoisy, Autun, Fragny, Marmagne, Monthelon, Tavernay, - Rh.: Alix, Arnas, Clarbonnieres. - Gard : Brama-Bioou près Campricux. - Aude et Pyr.-Or.: Villespy, Ferrals, la Nouvelle, bords de l'étang de Salses. - B.-du-Rh. et Var.: le Tholonet, Sablettes prìs Toulon, le Luc, St-Raphaël.

c. pusina Gris. - Prés humides, bords des étanğs dans les terrains siliceux.- Jura : la Bresse autour de Pleurre, Gatey, Tasseniẻres, Fays, Chaumergy, Neublans, Commenailles. - S.-etL.: Monthelon, Tavernay, Ternans. - Vaucl. : envir, d'Apt à Gignac, Roussillon, Rustrel. - Gard et Hér. : entre Beauvoisin et Vauvert, mare de Grammont près Montpellier, mare de Roquehaute près de Vias. - Aud. et Pyr.-Or. : Ferrals, plages d'Argelès et de Banyuls-sur-iler.

En dehors de nos limites dans l'Aveyron, le centre et l'ouest de la France.

CHLoreor perfolistum L. - Colcaux incultes, licux humilcs dans tout le bassin. 
C. serolinum Koch. - Differe du précédent par ses fleurs d'un jaune plus pâle, à divisions du calice plus larges et plus longues. Environs de Lyon, d'Annecy, de Grenoble et de Montélimar. B.-du-Rh. et Var.: Fos-les-Martigues, Berre, le Luc. - Gard et Hér.: Uzès, Manduel, Maguelonne.

C. imperfoliatuna L. - Sables du littoral de la Méditerranée ct de lOcéan. - B.du-Rh. et Var; Berre, Fos, la Camargue, Toulon, Hyères, île de Porquerolle. - Gard et Hêr. : AiguesIIortes, Palavas, Maguelone, Sérignan à Valras.- Aude et Pyr.Or.: Ste-Lucie, Leucate, Quillanet, Canohès, Banyuls-surMer.

GENTIAv Iutea L. - Prairies cies montagnes. - Disséminé d'une manière irrégulière dans la chaîne des Vosges depuis Aubure jusque dans la H.-Saône aux Ballons de Giromagny et de Servance, la Jumenterie. - Plus abondant et plus uniformément répandu sur les hauts pâturages de la chəine jurassique jusque dans le Revermont et le Haut-Bugey. - Très-commun dans les montagnes calcaires, moins abondant sur les terrains siliceux de Vaud, Valais, Savoie, Dauphiné, B.-Alpes et Alp.Ifar. jusque dans les montagnes de Thorenc et de St-Auban. C.-d'Or: Irés-abondant au nord et à l'ouest de Dijon, depuis l'altitude de $290 \mathrm{~m}$. jusqu'à celle de $584 \mathrm{~m}$. au mont Afrique, mais exclusivenent sur les calcaires, comme par exemple sur les couches jurassiques de l'Auxois à Villy et Marcellois, sur lis calcaires tertiaires d'eau douce du Châtillonnais, sur ceux de Montbard, du Val Suzon et de Chanceaux près des sources de la Seine. - Rh., Loire el Ard. : montagnes granitiques et porphyriques de Tarare, Pilat au Pré Lager, St-Sauveur à Taillard, le Mezenc volcanique, Cuze, Gourdon, - Gard et Lozère : chaine de l'Espérou et de Concoule, montagnes du Soulier, de l'Espinouse et de Saumail au Saut-de-Vésoles, à Salvergues et à Lignères. - Aude et Tarn : la Montagne Noire. - Pyr.-Or. et Ariége : mont Louis, vallée d'Eyne, de Llo, Bolquẻre, Canigou, Costa Bona, le Laurenti, cirque des Aiguettes, Roc d'Escrouts. - En dehors de nos limites dans les Pyrénées centrales, Tarn, Aveyron à l'Aubrac et au Levezou, Creuse, Cantal, mont 
Dore, monts Domes, Yonne sur les calcaires depuis Vincelles jusqqu’à Cruzy, Loire au Montoncelle et à Pierre-sur-Haute.

On sait que la Grande Gentiane croit ordinairement dans la chaino jurassiqque, dans les Alpes et dans les Pyrénées, à partir do 900 jusqqu'à 1,800 mètres : par consécquent, son existence à une altitude de 300 à 580 métres, sur les collines calcaires de l'Yonne et de la Cóte-d'Or, est un fait de géographie botanique digne de remarque.

Quoique la Grande Gentianc puisse venir sur les terrains siliceux, comme on le voit dans les Vosges, le Schwarzwald, les montagnes du fiorez et du centre de la France, cependant l'observation montre qu'elle est beaucoup plus commune sur les terrains calcaires, ainsi que de Candolle l'avait déjà constaté. J'ajoute que les roches volcaniques et porphyriques, lesquelles contiennent des éléments calcaires, lui conviennent presque aussi bien que les sols calcaires eux-mêmes.

La distribution géographique de cette espèce en Europe est fort singulière : on la voit s'étendre depuis la chaine orientale des Karpathes dans la Hongrie et la Transylvanie, c'est-ì-dire depuis les Alpes de Bersava, du Marmaros et de Rodna, à travers la Croatie, la Carniole, la Dalmatie, l'Istrie, le littoral autrichien, la Vénétie, la Lombardie, le Tyrol méridional, moyen et occidental, le Vorarlberg et le Piémont, jusque dans les Alpes helvétiques et françaises, ainsi que dans la chaine du Jura et des Vosges. Celles-ci, le Schwarzwald et quelques rares localités du Jura Souabe paraissent être sa limite septentrionale. Au sud elle s'étend dans les montagnes septentrionales du Portugal, de l'Espagne, dans les iles de Corse et de Sardaigne, et enfin dans l'Apennin italien. - Elle manque dans les Alpes de la Styrie, Carinthie, Salzburg, Haute-Autriche, Bavière, Moravie, dans toute l'Allemagne moyenne et septentrionale, dans la Russie, dans les iles britanniques et les Etats scandinaves. De son absence dans ces derniers pays, on peut conclure que la théorie qui place le berceau des plantes alpines dans le nord de l'Europe ne lui est pas applicable, et que la Grande Gentiane doit ètre considérée comme étant autochthone dans la longue zone étendue de l'est à l'ouest, à travers l'Europe centrale, depuis les Karpathes de la Transylvanie jusqu'aux montagnes de la Galice espagnole.

G. brevifissa, G. Burseri Lap. - Prairies des sommités des Alpes et des Pyrénées. - Sav.: la Grand-Pariraz près Hauteluce. II. et B.-Alp.: mont Viso vers les chalets de la Traversette et au col de Ruines, Combe de Ségure, col de Vars, Lauzanier, Fouillouse, Grange-Commune, Parpaillon. - Alp.-Mar. : cols de la Calal: Bassin du Rhöne. 
Nadủalena et de Fremamorti, Ia Fraca, Clans, vallon du Boréon à Peirastreccia. - Pyr.-0r. et Ariẻge : Canigou, Costa Bona, Cambres-d'Aze, vallées d'Eyne et de Lto, Laurenti au Soula de Joucarets, cirque des Aiguettes, forèt de l'Ermite, vallon de Barbouillère.

G. punctnta L. - Rochers siliceux des Alpes et des Pyrínces. Vaud et Valais : Morcles, Münstigerthal, Glacier du Rhône, Mayenwand, Grimsel. - H.-Sav. et Sav. : Dents d'Oche, Hautde-Lin, montagnes de Morzine, de Samoëns et de Sixt, le Brévent et les Aiguilles-Rouges, chaîne du mont Blanc jusqu'au Jardin et au Couvercle, Brizon, Vergy, Méri, Flaine, Tournette, col Joly, Hauteluce, col de la Madeleine, la Levanna près des sources de l'Arc, mont Cenis, mont Iseran, Laval, le Golet, Beaupré, la Vanoise, les Allues, montagnes de Presle au-dessus de la Rochette. - Is.: St-IIugon, chaines des Sept-Laux et de Belledonne, la Pra, Champrousse, Poursollet au pied de Taillefer, sommet du Grand Som, la Salette. - H.-Alp. : la Grave, Lautaret, mont Genèvre, mont Viso, col Agnel, Pennin, mont Queyrel, Lopet en Champsaur, Orcières, Durbonnas, mont Aurouse.

G. Iuteo-punctata G. G., G. liybrida Vill. non Schleich. - Probablement hybride des $G$. lutea et punctata. La corolle est ponctuée, divisée jusqu'à la moitié en cinq segments aigus. - Is. et H.Alp.: Lancey, Uriage, Allevard, Valbelle au-dessus de Guillestre, Combe-Noire de Nanteyer, mont Séuse? - B.-Alp.: Halemort, Enchastraye, Lauzanier, col de Fours? - Il y a lieu de croire que la plante de Guillestre, des envir. de Gap et des B.-Alpes n'est pas le véritable $G$. luteo-punctata, mais une forme ponctuée du G. lutea. M. Borel n'a jamais vu au mont Séuse ni le luteo-purpurea, ni même le G. punctata.

G. purpurea L. - Prairies des Alpes. - Vaud et Valais : Dent de Jaman, Panerossaz, Boulaire, mont Fully, Pas de Cheville, StBernard à la Pierraz et à la Baux, Catngne, Tlıyon, Anniviers à l'Arpettaz, Evolena, Reschy, Tufernen de Zermatt, Fée, Mallmark, Simplon, Alstaffel dans l'Egginenthal, Münstigerthal, Glacier du Rhône, Mayenwand, Furca. - H.-Sav. : chaine du 
mont Blanc depuis le col de Balme jusuqu'au Bonhomme, s'elève au jardin de la Mer-de-Glace et au Couvercle, Brévent, Pormenaz, Môle, Brizon, Vergy, plateau des Gets, Nautau, Roc d'Enfer, Cornettes de Bise, Tournette, Charvin, les Contamines, col Joly. - Sav. : l'Aiguille près Hauteluce, plateau du Cornet enIre Chapieu et Roselein, Bas du Mottet au-dessus d'Aime, la Thiopa près Champagny, Saut-des-Allues, Dent-du-Corbeau, mont Mirantin, Haut-de-Seytenex, la Sambuy.

Une variété à lleurs jaunes a été observée en Valais sous $\mathrm{Za}$ cheur d'Iserable; je l'ai trouvée aussi au mont Fully.

G. Iuleo purpurea, G. hybrida Schl. non Vill., G. Thomasii Gillab. Probablement hybride des G. lutea ct purpurea. - Pâturages alpestres. - Vaud et Valais : Boronnaz, Chamosalles, Barma d'Esserz, Cleuson de Nendaz, Glacier du Rhône. - H.-Sav. : Môle, Glacière du Brizon, Vergy en descendant du cólẻ du Reposoir, chaine du mont Blanc, cols du Vieux-Emousson et du Genevrier. - Sur le versant italien du mont Blans, dans l'AlléeBlanche.

G. purpureo-punctata, G. Charpentieri Thom. - IJjbride des G. purpurea et punctata. - Vaud: Alpes de Lavarraz et de Bovonnaz. - H.-Sav. : mont Méri.

G. eruelata L. - Pelouses des collines et des montagnes calcaires de la partie septentr. et moyenne du bassin; plus rare dans la région mérid. et seulement dans les montagnes du Gard, de l'Hérault et des Alpes-Maritimes.

G. nsclepiadea L. - Prairies humides des montagnes. - Taud et Valais : les Plans, Bovonnaz, Javernaz, les Pléiades, marais de Villars près de l'Alliaz, mont Jorogne, le Jabloz dans la vallée de l'Etivaz, pâturages de Gros-Mont dans les Alpes de Gruyère (Fribourg), val d'Illiez, Fée près Saas, Simplon. - II.-Sav. et Sav. : mont Méri, Nautau, Haut-de-Lin, Combe-sur-Sixt, Chamonix, Barberine, Anterne, Semnoz, Tamié, Dent de Nivolet, les Allues, mont Cenis. - Is, et H.-Alp. : St-Hugon, combe du Veyton, vallon du Gleyzin, la Ferrière d'Allevard, Lautaret, l'Echauda, Orcières, col de Vars, - B.-Alp. et Alp.-Mar. : Lauzanier, Horonaye, Tende, bois de la Fraca, Ste-Anne-de-Vinaï. 
- En dehors de nos limites dans le Jura helvétique au Passwang, au Vogelsberg et au Weissenstein.

c. mneroearpophors, G. Pneumonanthe L. - Prairies marécageuses ou tourbeuses. - Jura helvétique et français: Val de Travers, Ste-Croix, Yverdon, pied du mont d'Or, St-Laurent, Champagnole, Clairvaux, tourbières du Bélieu, marais de Saône près Besancon, Pleurre, Chaussin. - Vaud et Valais : Crassier, Bonmont, Trélex, St-Georges, Estavayer, Saxon, Chandolin de Savièse, Lens. - H.-Saône : Franchevelle, Linexert, Bois-Derrière, Grands-Bois près Mélisey, Champagney, Palante, Bouhans, la Villedieu, monts Revaux entre Ecromagny et la Lanterne. - C.-d'Or et S.-et-L.: Orgeux, Arcelot, Satenay, Boncourt, Limpré, Antully, Cuiseaux. - Ain : Divonne, Pouzafol près Lagnieu, Boz et Chevroux près Bourg, étang Genoud, près Pont-de-Veyle: - Rh. et Is. : l'Argentière, St-Martin-enHaut, Vaux-en-Velin, Décines, Meyzieu, Jareyriat, Pont-deChérui, St-Quentin, la Verpillère, Bouvesse, Hyères près Crémieu. - Dr. et H.-Alp. : Nontmiral, près Romans, Saou près Crest, Gap à la Palud, col Bayard, la Roche-des-Arnauds. - Ard. : le Mezenc, Vernoux. - Gard et Hér. : prairies tourbeuses du Lengat près de l'Espérou, la Salvetat. - Pyr.-Or. et Ariège : le Capcir, Salvanère, le Laurenti, la Bentaillole, col des Ares. - B.-du-Rh. et Alp.-Mar. : en Coustiero, près d'Arles, Fos, Thorenc au-dessus de Grasse. - En dehors de nos limites, dans la Loire, à Pierre-sur-IIaule, les Salles, mont Sémioure. Une forme naine et pauciflore, G. humilior, Pneumonanthe montana J. F., se trouve au Bessat (L.oire); je l'ai vue aussi au Jezenc, ensuite entre Puy Valador et le col des Ares (Py.-Or.), enfin dans le Puy-de-Dỏme, entre Chateldon et Lachaud. Il est probable que cette forme naine est plus commune qu'on ne croit dans les marais des montagnes.

C. aenulis L. -- Espèce polymorphe, très commune sur les pelouses et rocailles du Jura, des Alpes et des Pyrénées, comprenant plusieurs formes bien distinctes les unes des autres et constituant des races parfaitement caractérisées.

G. Iatifolia G. G. G. Kochiana Perr. Song., G. excisa Koch. - Forme à 
feuilles largement oblongues, corolle grande, d'un bleu leinte de pourpre, divisions calicinales rétrieies à la base, réunies par une membrane, à sinus tronqués. - Jura helvétique et français: Aiguilles de Baume, Chasseron, la Tourne, Charbonnières, Creux-du-Van, Luisans, Bélicu, Barboux, Grand-Combe des Bois, Russey, Bonnélage, Vallanvron. - Valais: la Forclaz, StBernard, Havoire, Chemin, Mayens de Sion, Thyon, mont Nuoble, Distelap de Saas, Simplon, Blatten, Bellalp, Ernergalen, Mainghorn, Gemmi. - II.-Sav. : Cornettes de Bise, col de Golèse, plateau des Gets, clıâne du mont Blanc jusqu'au Couvercle, col Joly, Tournette, Méri, Charvin. - Sav. : montagnes de Hauteluce, entre Turnaz el Colombaz, la Gita et la Sauce près Beaufort, Cornet près Roselein, les Allues, les Avanchers, montagnes de IIautecour, col de la Madeleine, mont Mirantin, col de la Bâthie, mont Drizon sur Tamié, Galopaz, montagne de St-Hugon, mont Cenis. - Ain : Arvières, Retord, IIauteville. - Is. et H.-Alp. : chaine de Belledonne, la Pra, Prémol, mont Senèpe, la Grave, Lautaret, mont Genèvre, mont Viso au col de Ruines, St-Véran, col de Vars, col Bayard, Avançon, Durbon. - B.-Alp. ct Alp.-Mar. : la Condamine à Ste-Anne, Lauzanier, Parpaillon, Bousollières, vallées du Var, de la Vesubia et de la Tinea.

G. alpina Vill. - Forme à feuilles courtes d'un vert pâle, fleurs petites, plante naine. - Pelouses des terrains siliceux des Alpes et des Pyrénẻes. - Vaud et Valais: Alpes de Morcles, de Fully, de Salran, de Lens, Orsera, Pierre-à-Yoir, Métailler. - H.-Sav. : Cornettes-de Bise, montagnes de Samoëns, le Buet, Pormenaz, chaine du mont Blanc, depuis le col de Balme jusqu'au col de la Seigne, s'élève au Jardin, au Tacul et au Couvercle. - Sáv., Is. et H.-Alp.: montagnes de St-Hugon, les Sept-Laux, Belledonne à la Grande-Lance, col Oddie, Champrousse, Taillefer, Grand-Galbert, Piéméyan au mont de Lans, la Grave sous les glaciers, Villard d'Arène, Chaillol, Champoléon, mont Aurouse. - Pyr.-0r. el Ariẻge : Cambres d'Aze, massif du Laurenti, vallon de Boutadiol, col de Lègue, lac Noir, Sarrat-d'en-Binada. G. angustifolia Vill. - Forme à feuilles luisantes à la face supér., 
oblongues, longuement atténuées vers la base, fleur d'un bleu d'azur sans teinte purpurine. - Rochers calcaires des Alpes et des Pyrénées, - Sav. et Is. : Saint-Cassin près Chambéry, cascade de Coux, Montagnole au Pas de la Fosse, St-Badolph au Pas de la Coche, Apremont, mont Granier, col de la Ruchère, Bovinant, mont Rachais, Chalais, Pariset, Comboire, St-Nizier, col Vert. - H.-Alp. et Dr. : Charance et Rabou, cols de Glaise, du Noyer, Poligny en Champsaur, mont Séuse, mont Aurouse, montagnes entre le col de Lus et Glandage, entre Lus et le Grand-Ferrand. - Pyr.-0r. : Cambres d'Aze, vallée d'Eyne, lac du Laurenti, jasse de la Bentaillole, lac de Rabassolès, vallon de Couneq.

G. coriacea, G. Clusii Perr. et Song, - Forme à feuilles coriaces, lan. céolées, à pointe cartilagineuse très aiguë, divisions calicinales appliquées sur le tube de la corolle, élargies à la base et à bords denticulés; fleur d'un bleu foncé à gorge non tachée de vert, ce qui la distingue nettement des précédentes. - Rochers calcaires. - Jura helvétique et français. - Vaud et Valais : rochers de Naye, la Tour, Anzendaz, les Diablerets, Fully. H.-Sav. : le Môle, Glacière du Briznn, Haut-de-Véron, Roc d'Enfer, Nautau, mont d'Ubine, Hautigny près Bonnevaux, Dents d'Oche, Samoëns et Sixt, la Tournette, col Joly. - Sav. : montagnes de IIauteluce aux Lanches de la Savonne, la Sauce, la Gita, mont Arclusan au-dessus de Nontmin, Chamoseran, mont Drizon, sur Tamié, mont Margériaz près des Déserts.

G. pyrenaiea L. - Pelouses humides des Pyrénées. - Pyr.-Or. el Ariège: mont Louis, col de la Perche, Cambres d'Aze, vallées d'Eyne et de Llo, Canigou, Carença, Coma du Tech, Costa Bona, Salvanère, Bentaillole en Laurenti, lacs Blcu et Noir, lac du Laurenti, mouillères de Barbouillère, Boutadiol, la Restanque, las Cucques, Soucarrat. - Pyr. centrales. Cette espèce remplace dans les Pyrénées le G. bavarica.

G. bavariea L. - Pelouses humides des Alpes. - Vaud et Valais : Dent de Jaman, Anzendaz, la Tour, Bovonnaz, Panerossaz, Javernaz, St-Bernard, col de Fenêtre, Tortain, Severeu, Thyon, Zermontana, mont Nuoble, Chermignon, Mayenlıorn, Gemmi, 
Alpes de Zermatt, Iloernli, Gornergrat, vallée de Saas à Mattmark, Distelilp, monte Moro, Mittarhorn, Sihwnhorn, Wildhorn, Simplon. - H.-Sav. et Sav.: Cornettes de Bise, col de Golèse, roc d'Enfer, mont Ardin, col de Balme, Vergy, Méri, Brizon, montagnes de Sales, Charvin, Tournette, vallon d'Entre-les-Eaux, col de la Seigne, les Mottets, mont Cornet près Roselein, Hauteluce, Crest-Voland, les Avanchers, col de la Madeleine, mont Iseran, mont Cenis, l'Allée-Blanche sur le versant italien du mont Blanc. - H. et B.-Alp. : Lautaret, Galibier, col de l'Echauda, col des Hayes, Buffera, Laval près Névache, mont Viso, vallée de Ségure et de la Taillante, mont Aurouse, Chaillol-le-Vieil, Lauzanier, Vallonnet de lleyronnes, col d'Allos, sources du Var.

Dans la variété imbricata Schl., les liges sont courtes, rapprochées et comme imbriquées; existe dans les Alpes du Valais.

G. Rostani Reut. - Differe du G. bavarica par ses feuilles ovales-lancéolées, atténuées aux deux extrémités et non obovales-spàtulées, par les dents du calice égalant la corolle. - H.-Alp.: mont Genèrre, Gondran, haut de la vallée du Guil, Refuge du col Agnel, Valpréveyre, cols de la Croix, d'Abriès et de Vars.

C. verna L. - Pâturages du Jura, des Alpes et des Pyrénées. Jura helvétique et français : Wasserfall, Vogelberg, Weissenstein, Moron, mont Terrible, Chasseral, Chaumont, Creux-duVan, mont d'Or, Suchet, Noirmont, Montendre, Morteau, Bonne̊lage, Maîche, Bréseux, Lomont, de St-Ursanne à St-Braix, Dúie, Colombier, Reculet, Sorgiaz, Poisat, mont d'Ain, Colombier du Bugey, montagnes de Hauteville, Molard de Don. Vaud et Valais : Anzendaz, Panerossaz, Diablerets, mont Fully, Salin, Vex, St-Léonard-sur-Drône, Arbaz, Mainghorn, Gemmi, Alpes de Zermatt et de Saas, Natersberg, Sirwolten, St-Bernard, pied du mont Rose jusqu’à la Cabane de Vincent (3158m.). II.-Sav. : Dents d'Oche, montagnes de Sixt, Roc d'Enfer, mont Ardin, Brizon, Salève, Voirons, plateau des Bormes près de la Roche, Charvin, Tournetle, Servoz, Passy, chaine du mont Blanc depuis le col de Balme jusqu'au Bonhomme; s'élève jusчju'aux Grands-IIulets ( $3{ }^{\prime} 70^{\circ}$.), col Joly. - Sav. : Hauteluce, 
Crest-Voland, mont Cornet près Roselein, montagnes de la Tarantaise jusqu'au mont Iseran, de la Maurienne jusqu'au mont Cenis et à la Levanna, mont Drizon, les Bauges au mont Nivolet et au Margériaz. - Is. ct H.-Alp. : Grande-Chartreuse, Sappey, St-Eynard, col Vert, la Moucherolle, mont de Lans, Taillefer, Crand-Galbert, Champrousse, Laularet, Monetier, la Vachelte, mont Genèvre, le Queyras, mont Viso, St-Véran, montagnes du Gapençais, du Clıampsaur et de l'Embrunais. - B.Alp. et Alp.-Mar. : la Condamine, Malemort, Lauzanier, Maurin, mont Cheiron, sommités des vallées du Var, de la Vésubia, de la Tinea et de la Roya, - Pyr.-Or. et Ariège : Mont-Louis, vallée d'Eyne, Evol, Nohèdes, Font de Comps, Canigou, lac du Laurenti, Rabassolès, Valbonne, Paillères, Barbouillère, Boutadiol, la Bentaillole, les Aiguettes, Artounant. - En dehors de nos limites au Cantal et au mont Dore, Pyrénées centrales.

Dans la variété angulosa M. B., les angles du calice sont plus saillants et ailés; une autre variété naine, G. pumila Vill. se trouve quelquefois mêlée au type.

Le G. brachyphylla Vill. a des feuilles courtes, arrondies au sommet et obtuses. - Vaud et Valais: Anzendaz, Taveyanaz, Cape-au-Hoine, Alpes de Morcles et de Fully, St-Bernard, col de Fenêtre, Zermontana, Thyon, Loze, Sasseneire, Mainghorn, dlpes de Zermatt et de Saas, Grimsel, Simplon. - H.-Sav. et Sav. : vallée de Bérard, col de Balme, Aiguille du Tour, Bionassey près de St-Gervais, col Joly, entre la Gitaz et le Grand-Pariraz, le Cornet près Roselein, Saut des Allues, col du Cormet, mont Iseran, mont Cenis à Ronches, col de la Seigne et des Fours, l'Allée-Blanche sur le versant italien. - Is. et H.-Alp. : chaîne des Sept-Laux et de Belledonne, lac Domenon, Piéméyan au mont de Lans, mont Pelvoux, la Moucherolle, Galibier, mont Genèvre, St-Véran à Clausis, col de Vars et de Ruines, col Vieux, Chaillol, Pic de Chabrières, mont Aurouse. - B.-Alp. : Lauzanier, Bachasse, Longet, Vallonnet.

G. germaniea Willd. - Prairies et pâturages des collines et des montagnes, surtout dans les terrains calcaires, $-\mathrm{H}$.-Saone : Grattery, Leffond, Chargey-les-Port, Fouvent, Larret, Athe- 
sans. - Doubs, Jura et Ain : assez répandu dans les arrondissements de .Iontbéliard et de Besanron, Salins, Arbois, Poligny, Voiteur, Lons-le-Saunier, St-Amour, montagnes du Bugey et du Revermont, descend dans la plaine d'Ambronay. - Genève ct II.-Sav. : bois de la Bâtic, des Frères, Peney, le Vangeron, Salève, Semnoz, - Vaud et Valais : Fenalet, Pisserache. - Is. el H.-Alp. : St-Pierre-de-Chartreuse, Entremont, Chalais, StPaul-les-Monestier, Morestel, Pont-en-Royans. - Alp.-Mar. : mont Sciaccarré et col Bertrand, - C.-d'Or, S.-et-L. : collines calcaires des env. de Dijon el de l'Auxois, Cuiscaux, Givry, Garenne de Chamilly. - Ard. : mont Mezenc. - En dehors de nos limites, sur les coteaux calcaires du centre, de l'ouest et du nord de la France.

G. obtusifolia Willd. - Forme du précédent à feuilles obtuses, plus longuement pétiolées, à fleurs plus petites, d'un blanc jaunâtre, teinté de violet, quelquefois même complètement jaunes. $(G$. flava Lois.) - Valais : assez commune dans les vallées de Zermatt et de Saas, Haenstegen, Niederalp, Schornen, Kaltwasser, Furca. - Ain : de Belley aux Paroisses, St-Haurice-deRémen, St-Martin-du-Frène. - La variẻté flava à Chazey, entre Lhuis et Serrières (Ain); à Meyzieu près du Moulin Cheyssin (Is.); à Vaux-cn-Velin et Villeurbanne (Rh.).

Le G. campestris est une race dérivée du G. germanica. En eflet, dans celui-ci le calice et la corolle sont divisés en cinq lobes égaux; tandis que dans le $G$. campestris les divisions du calice et de la corolle sont au nombre de quatre, celles du calice inégales et plus profondes. Le G. germanica est donc le type régulier, le G. campestris une forme déviée et anomale, ce que semble témoigner l'inégalité des lobes calicinaux. En vain alléguerait-on pour les.séparer en detix espẻces distinctes que l'une est pentamère, l'autre tétramère : nous savons que ce n'est pas là un caractère spécifique, et que, par exemple, il existe plusieurs espèces d'Alsinées qui tantôt sont pentamères, tantôt tétramères.

G. campestris L. - Prairies des montagnes. - Commun dans la chaine du Jura helvétique et français jusque dans le Bugey. - Vaud, 
Valais, Savoie, Dauphiné, B.-Alpes et Alp.-Mar. - Massif du Pilat, chaîne vivaraise, Mezenc, Gerbier-des-Joncs, Cévennes du Gard à l'Espérou, St-Guiral, Concoule. - Pyr.-Or. et Ariège : Canigou, Prats-ile-Nollo, col de la Perche et des Ares, lac du Laurenti, Valbonne, roc de 1 lascaras, val de Paillères. En dehors de nos limites dans les Pyr. centr., l'Aubrac, montagnes du Tarn, Lozère, Cantal, monts Dómes, Pierre-sur-Haute, Creuse, Haute-Vienne, Vosges, - Varie à fleurs blanches ou jaunes.

G. TenelIa Rottb. - Gazons humides des Alpes et des Pyréntes. Vaud et Valais : Grandvire, mont Fully, St-Bernard, val Ferret, Torrembé, Thyon, Diez, Arolla, Arpelta d'Anniviers, Gemmi, Alpes de Zermatt au Riffel et au Schwarzsee, vallée de Saas à Almagel, Mattmark, Scliwarzberg, Simplon, Sirwolten.-H.-Sav. et Sav. : col de Balme, Brévent, cols de la Seigne et du Bonhomme, mont Iseran, entre Laval et Tignes, les Allues, col de la Madeleine, mont Cenis à Ronches. - Is, et H.-Alp. : Piẻméyan au mont de Lans, Lautaret, Galibier, mont Genèvre, col des Hayes, Péas, Ségure-en-Queyras, mont Viso, St-Véran à Clausis, col Agnel, col de Vars, Orcières. - B.-Alp. et Alp.-Mar. : Mirandol, Vallonnet de Meyronnes, Fouillouse, Longet, Bérard, Maurin, sources du Var, les Viosennes. - Pyr.-0r. : vallée d'Eyne. - Pyr. centrales.

G. nivallas L. - Gazons humides du Jura, des Alpes et des Pyrênées. - Ne se trouve dans la chaine jurassique qu'au sommet du Hontendre et du Chàtel, dans les près de Biére et de St-Livre. - Vaud et Valais : Anzendaz, mont Fully, St-Bernard, Valsorey, Barberine, Arpetta d'Anniviers, Bombire, Cleuson de Nendaz, Diez, Vende, Zan, Alpes de Zermatt et de Saas, Simplon, Mainghorn, Gemmi, glacier du Rhone. - H.-Sav. : chaine du mont Blanc depuis le col de Balme jusqu'au Bonhomme, s'élève jusque sous l'Aiguille du Gouté et au Jardin, moraines de la Mer-de-Glace au Mauvais-Pas, Buet, Pormenaz, Entre-les-Eaux, montagnes de Sixt et de Morzine, Cornetles de Bise, plateau de Flaine, Méri, glacière du Brizon, Tournette, les Aravis, Clıarvin. - Sav. : mont Iseran, la Vanoise, lac Bleu 
près de Brides, les Allues, Bas-du--Ioltet au pied du Crêt-du-Ré au-dessus d'Aime, Petit-Saint-Bernard, mont Cenis。 - Is. et H. - Alp.: chaine de Belledone à la Pra, Pí́meyan au mont de Lans, Villard de-Lans entre les Fauges et la Moucherolle, la Salette, Yalsenestre, Lautarat, Galibier, la Ponsonnière, col des Rochilles sur Névache, plateau des Thurres, Valpréveyre en Queyras, col de Ruines et de Vars, valion de Ségure, mont Viso à la Traversette, Valgaudenar, Durbonnas, Faudon près Gap. - Dr. et B.-Aip.: mont Glandasse, Grange-Commune, Mirandol, Longet, vallon du Châtelet, Fouillouse, bois de Tournoux. - Alp.-Nar.: les Viosennes, mont Bego, col de Fenestre, le Garret au-dessus d'Entraunes, suurces du Var. - Pyr.-Or. et Ariège: Cambres d'Aze, Canigou, lac du Laurenti, Port de Paillères, Valbonne, Barbouillère, lacs Bleu et Noir, Pic del Ginèvré, Soucarrat. - Pyr. centrales.

G. utriculosa I. - Cetle Gentiane qui existe dans les prairies humides de l'Alsace, ainsi que vers Schaffouse, Bâle, Eglisau, est indiquée dans le bassin du Rhône, en Valais à Vervay, Senliena, Hochbalm de Zermatt, Alpe infër. de Saxon, Mayens de Sion, sur Lens et Cran, Findelen, glacier de Zmutt. - Je soupsonne que ces indications se rapportent à un Gentiana verna à ailes du calice très saillantes, forme appelée angulosa ou alata. Je n’ai jamais vu le véritable G. utriculosa en Valais.

G. elliata L. - Pelouses des terrains calcaires. - Claine du Jura helvétique et français jusque dans le Bugey et le Revermont. Chaînes calcaires de Vaud, Valais, Savoie, Dauphiné, B.-Alpes jusque dans les Alpes-Mlaritimes. - Collines calcaires de Côted'Or, dans l'Auxois et le Châtillonnais, Saône-et-Loire, Mont d'Or lyonnais. - Gard et Hér. : env. du Vigan et d'Alzon, l'Espérou, Saint-Amand-de-Iounis au Roc d'Orifues, l'Escandorgues, Ceilhes, - Versant nord du mont Ventoux. - Pyr.-Or. et Ariège: Prats de Mallo, lac du Laurenti, Valbonne sous le Roc de la líusique. - En dehors de nos limites sur les terrains calcaires des Pyrénées centrales, de la Lozère, Aveyron, Tarn, Cantal, Yonne, Alsace et Lorraine.

SWEETTA perennis L. - Marais tourbeux des montagnes. 
- Jura lıclvétique et français : Lignères, Pouillerel, Brévinc, Morteau, Bílieu, Noël-Cerneux, Chenalotte, St-Julien, Pontarlier, Mouthe, Vaux, Chapelle-des-Bois, Ste-Croix, le Boulu, Prémanon, Noirmont, la Dôle, vallée de Joux, des Rousses, des Dappes, Trélasse, Malbroude, Brenod et Cormaranche près IIauteville en Bugey. - Vaud : bains d'Alliaz, marais de Gangane sur les Pléiades. - H.-Sav. el Sav. : Somans, les Gets, St-Martin-de-Belleville, les Allues, mont Cenis. - Is., H. et, B.-Alp. : chaines des Sept-Laux et de Belledonne, col Oddie audessus des Adrets, Brandes au pied des Grandes-Rousses, Lau. taret, Arcines, mont Viso, St-Véran, col de Vars, Mélezet, Orcières, Chaillol, Lauzanier, Parpaillon, Fouillouse, col d'Allos. - Alp.-Mar. : col de la Maddalena, Alpes de Tende et de la Briga. - Ard. : la Champ-Raphaël. - Pyr.-Or. et Ariège: mont Louis, Font-Romeu, entre les lacs d'Evol et de Nohèdes, lacs du Laurenti, la Bentaillole, Valbonne, Barbouillère, Soucarrat, Boutadiol. - C.-d'Or : val des Choues dans le Châtillonnais. - En dehors de nos limites dans les Pyrén. centrales, l'Aubrac, la Guiolle dans l'Aveyron, mont Dore à la Croix-Morand.

WH EN NTH trifoliatum, Menyanthes trifoliata L. - Marais, prés tourbeux des plaines et des montagnes des parties sept. et moyenne du bassin; manque dans la Provence et les Alp.Mar. - Gard : l'Espérou à la Baraque de Michel, Concoule sur la Lozère. - Hér. : l’Espinouse à Cambon, Lignères, la Salvetat, le Saumail à Cassagnoles. - Pyr.-Or. : Balcera en Capcir, les Bouillouses, col des Ares, mouillères de Noubals, Al-Remus d'Artigues, Soucarrat, Pis, las Cucquès, la Galinière.

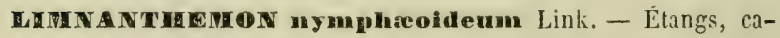
naux, rivières. - Commun dans la Bresse et la Dombes, bords cie la Seille, du Doubs, de l'Ognon et de la Saône dans II.-Saône, C.-d'Or et S.-et-L. - Rh. : bords de la Saône et du Rhône à St-Georges-de-Reneins, Anse, Yvour, Oullins, la Mulatière, fossés du Parc de la Tête-d'Or à Lyon oủ il a été introduit. Is., Sav. et Dr. : Décines, Voiron, Gières, lac du Bourget, StVallier au marais de Villeneuve. - B.-du-Rh. et Var : la Crau, 
la Camargue, l'Agoutier près Toulon, la Garde. — Gard et Hẻr. : Bellegarde, St-Gilles, Aigues-Mortes, le Vistre près Nimes, la Robine et Fossés de Laltes, Maurin, canal du Midi à Béziers, le Canalet près Roquehazte. - Centre et ouest de la France, Alsace.

\section{POLEAIONIACÉES}

BOLENGNYON earuleum L. - Jura helvétique et français : abondant dans le val de Travers, sur les bords de la Reuze près de Fleurier, cntre le Villers et les Pargots, pâturages des Fourgs, Jorteau, pied du fort de Joux, la Bre̊vine, Birseck, - Vaud : Panex sur Ollon, Salins sur Aigle, l'Etivaz, les Ormonds. - En dehors de nos limites dans les Pyr. centrales au mont Cagire et au Pic de Gard; dans la H.-Loire entre l'Aubépin et la Grangette, Troisson près de Bessamorel, à Taulhac-le-Château; dans le Cantal près de Moissac; dans le Puy-de-Dôme, au Cheire de Come. - Il est fort douteux que cette plante soit indigène dans Ies localités indiquées, et tout porte à croire qu'elle est échappéc des jardins d’agrément où elle était cultivée.

\section{CONVOLVULACEES}

Convonverus sepineola, C. sepium Ilaies dans tout le bassin.

C. soldanellum L. - Sables maritimes. - B.-du-Rh. et Var : envir. de Marseille au Prado, côte vis-à-vis de l'île Mä̈ré, île Plano, Sablettes près Cannes, golfe Jouan. - Gard et Hér. : Aigues-Mortes, Pinède de Ste-Marie, littoral de llauguio, Frontignan, Celte, Agde. - Aude et Pyr.-0r. : la Corrége, Leucate, littoral d'Argelès à Banyuls.

C. arvensis $L,-$ Champs dans tout le bassin.

C. tomentosus Choisy. - Champs et bords des chemins entre Toulon et liyères, Cannes.

C. althreoideus L. - Murs, bords des chemins et des champs. B.-du-Rh., Var et Alp.-Nar.: île Verte, env. de Cassis, de Marscille et d'Aix, Toulon, île de Porquerolle, le Luc, St-Raphaül, 
St-Aigou, Cannes, golfe Jouan et le littoral de Nice à Jienton.

- Hér. : montagne de Cette, route de Ganges au Mas de Comte.

- Aude et Pyr.-0r.: Ste-Lucie, Leucate, bords de l'étang de Salses, Fitou, Elne, Estagel, Cases de Pena, Força-Réal, Trencada d'Ambulla, Collioure, Port-Vendres, Banyuls.

Une variété argyreus D C., qui a les tiges et les feuilles argentées-soyeuses, est quelquefois mêlée au type.

C. Ianuginosus Desr., C. linearis D C. - Rocher's de l'Aude ct Pyr--Or.: ravin de Rouquette près Hontolieu, la Clappe, EIne, route de Cases-de-Pena à Estagel.

Comme pour l'espèce précédente, il existe une variété à feuilles argentées-soyeuses, argenteus. Celle-ci a été trouvée sur les rochers au-dessus de la gare de la Ciotat, la Cadière, montée de Cujes, le Camp (B.-du-Rh.); Leucate, la Clappe, redoute de Montoulieu (Aude).

C. cantabrieug L. - Collines pierreuses calcaires de la région méridionale d'où il s'élève quelquefois jusque dans la région montagneuse, comme, par exemple, à Alzon (Gard) et sur le plateau du Larzac (Hér.). - Remonte dans la Drôme vers Nyons, Crest, Die, St-Vallier; dans les B. et H.-Alp. vers Sisteron, Digne, Ribiers, Gap; dans l'Is. à la Bastille de Grenoble, Feyzin, Mollard de Décines, Crémieu; dans l'Ain à la Pape et sur les coteaux du Rhône jusque vers le conlluent de l'Ain; dans Ardèche et Loire sur les côtes du Rhòne jusqu'à Joyeuse, Aubenas, Baix, Tournon, Malleral, Chavanay. - Commun sur les coteaux calcaires de la Cote-d'Or depuis Beaune jusqu'à Santenay, puis à Auxey et Montrachet. - En dehors de nos limites sur les coteaux calcaires de l'Yonne, de la Limagne d'Auvergne, du Cantal, de la Lozère, du Lol, de l'Aveyron, de Tarn, Tarn-et-Garonne, Haute-Garonne, les Charentes.

C. Lineatus L. - Lieux pierreux, bords des chemins. - Vaucl., B.-du-Rh. et Var: env. d'Avignon, de Jonquières, d'Apt, d'Aix, de Marseille, de Toulon et de St-Tropez. - Gard: Villeneure en face d'Avignon, St-Pons, Fournès, Laudun, Tresques. - Hẻr. : env. de Montpellier, Pérols, Castelnau, Prades, Juvignac, Villcneuve à la lifadeleine, Pézenas, Béziers. - Aude et Pyr.-Or. : 
env. de Narbonne, Ste-Lucie, Sidrière de Fitou et de Leucate, Collioures, Port-Vendres, Céret, Arles, Trencada d'Ambulla, St-Antoine-de-Galamus

Naturalisé dans le Puymde-Dôme à Puy-Long et dans les plaines de Cournon.

C. trieolor L. - Naturalisé autour de Toulon au Forl-Rouge et aux Pradets. - Cullivé dans les jardins.

La forme nommée par Bertoloni C. pseudotricolor, a élé trouvée accidentellement dans les moissons autour de Nice, entre Eza et la Turbie, à Castillon au-dessus de Menton.

C. slculus L. - Rochers et rocailles. - Var et Alp.-Mar. : Clairet près Toulon, Hyères, presuu'île de Giens, entre Monaco et Eza, Menton au Pont Saint-Louis.

Le C. hirsutus Stev., espece de Grèce, Turquie et Tauride, a été trouvé accidentellement près de Narseille et entre Toulon et Hyères.

CRESGA mierophylin, $C$, cretica L. - Lieux humides de la région méditerranéenne.-B.-du-Rh. et Var:env. d'Arles, Marignane, Toulon, Hyères, les Pesquiers, - Alp.-Mar. : Cannes, îte Ste-Marguerite, golfe Jouan, Antibes, Nice au Var. Gard et Hér. : Bellegarde, Aigues-Mortes, Pẻrols, Mireval, les Aresquiers, Maugnio, Villeneuve, Frẻjorgues, Portiragnes.

CUsCUTA densifora Soy. Will. - Parasite sur le Linum usita. tissimum.

La distribution géographique des Cuscutes ne présente aucun intérêt, parce qu'elle est liée à celle des plantes qui leur servent de support.

C. major D C., C. europeca L. - Parasite sur l'Ortie, le Clanvre et le Joublon.

C. epithymiphyta, C. Epithymum L. - Parasite sur les Thymos vulgaris et serpyllus, sur le Satureia montana, sur les Luzernes, Trèfles, Genêts et autres Papilionacées.

La var. C. Trifolii Babgt. et Gibs. a des fleurs plus grandes, en glomérules plus denses. - Plusieurs botanistes assurent avoir vu le $C$. Trifolii devenir $G$. epithymum lorsqu'il est exposé à une longue sẻcheresse. S'il en est ainsi, le $C$. Trifolii ne serait 
même pas une variété, mais un état particulier produit par l'exubérance de végétation du support.

Le C. Muelleri Strail differe du C. Trifolii par les styles plus courts que le tube de la corolle; celle-ci toujours fermée et plus courte que le calice, portant des écailles dressées à bords laciniés.

C. Godroniana Desmoul., C. alba Godr. non Presl. - Parasite sur les Bruyères, le Serpolet, le Genista scorpia, les Galion, et plusieurs autres plantes de la région méridionale.

C. corymbosa Choisy, C. suareolens Seringe. - Parasite sur la Luzerne.

C. momogyna Vahl, - Parasite sur la Vigne, quelquefois mais plus rarement sur l'Osyris, le Genista sagittalis et le Daucos salivus (D. Carota).

\section{RAMONDACÉES}

RAMONDA wyremaica Rich. - Rochers calcaires. - Pyr.-0r. : vallée du Tech au Mas de la Guardia, d'Arles à Cortsavi, Pratsde-Mollo vers la Roca Galinera, vallée de Nohèdes. - Pyr. cent. à st-Sauveur, vallée de Lus jusqu'à Gavarnie, lac d'0o, Esquierry, Sarrancolin, cirque d'Arbison, Gedre.

\section{BORRAGINÉES}

CEMRNTHON asperum Roth, Cerinthe major Lam. - Champs pierreux de la région mérid. - Var et Alp.-Mar. : Toulon, le Luc, Fréjus, la Rocuette, Grasse, Mouans, Cannes, Antibes, Villefranche. - Gard : Sommières, Sylvéréal, Aigues-Mortes. - Hér. : Lésignan, Saussines, Agde, Pézenas. - Aude et Pyr.Or. : ile Ste-Lucie, le Riveral, la Salanque.

C. minus L., C. glubrum Scop. - Prairies du Jura, des Alpes et des Pyrénées. - Jura helvétique et français : bords de la Reuze en face de Fleurier, route de Boveresse, pâturages de la Chaux, les Bulles au mont Dard au sud de la Sagne, le Crouzet, Siche-des- 
Embernats pris St-Cergues, Cerneux-Pépuignot près Morteau, Cotes du Doubs près Vallanvron. - Vaud et Valais : Dent de Jaman, Bovonnaz, Solalex, Morgins, Trient, Bagnes, Méribé, les Diez, Anniviers, Simplon.-II.-Sav. el Sav. : Dents d'Oche, Cornettes de Bise, Roc d'Enfer, vallée du Reposoir en montant au Méri, vallée de la Borne, Clıarvin, Tournelte, mont de Fréjus, Combe de Villette, mont Cenis. - Is. et H.-Alp. : Bovinant de la Grande-Chartreuse, Alpe du mont de Lans, la Salette, la Grave, Lautaret, Briançon, la Vachelte, mont Genèvre, Abries en Queyras, mont Viso. - B.-Alp. : la Condamine, St-Paul, Lauzanier, Bousolières, Annot. - Alp.-Ilar. : St Etienne et StDalmas-le-Sauvage, Entraunes, Mine et col de Tende, le Mas, Thorenc, mont Cheiron, Caussols. - Pyr. centrales. - Espèce polymorplie.

C. alpinum liit. - Forme du précédent à corolle plus grande, dont les lents sont ovales-obtuses, non conniventes, et un peu courbécs en dehors. - Trouvée par M. Lannes à Longet de Maurin et au mont Viso. - 0 n assure l'avoir vue sur les rochers de Naves au-dessus d'Autrans (Is.), ainsi que dans le val Pesio et au col de Tende. - En dehor's de nos limites, dans les Pyr. centrales au Pic de Lhéris et dans la vallée de Barétous au-dessus d'Arette. C. auriculatum Ten. - Forme qui diffère du C. minus par ses tleurz de couleur pourpre deux fois plus petites; les divisions de la corolle sont aiguës et dressées. - H. et B.-Alp. : vallon de Glaise près Gap, mont Séuse. - B.-Alp. et Alp.-Nar. : Colmars, vallée de Thorenc, val de P'esio, col de Tende.

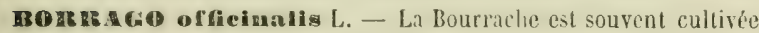
lans les jardins el quelquefois subspontanée dans les champs et les vignes.

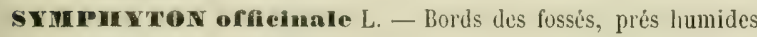
dans une grande partie du bassin.

S. tuberosum L. - Bords des fossés ct des ruissenux ombragés de la région mẻrid; remonte vers Crest, Gap et jusque dans les env. de Lyon à Francheville, Brignais, Givors, et près de Grenoble à Seyssins, Veurey. - L'ouest, le centre et le sud-ouest de la France.

Catal. Bassin du Rhône. 
s. buIbosum Schimp. - Alp.-Mar. : Nice à Saint-Roch, Menton. A NCUUSA officinalis L. - Champs, bords des chemins autour de Marseille, Briançon, Chaillol, Perpignan. - En dehors de nos limites dans l'Alsace, Charente-Inf., Vendée, Loire-Infér.

A. undulata L. - Bords des champs de la région mérid. - L'Estaque près Marseille, remparts de Toulon, Cannes, Nice.

A. iraliea Retz. - Champs, vignes, bords des chemins surtout dans les régions mérid. et moyenne; rare dans Jura, Doubs, H.-S., Genève, Vaud et Valais où il se montre adventif dans les luzernières. Cependant il semble s'étendre de plus en plus dans les susdites régions à la faveur des chemins de fer; c'est ainsi qu'il s'est abondamment naturalisé le long de la ligne de Lyon à Genève, - Espèce polymorphe dans laquelle la forme des feuilles présente plusieurs variations.

A. gempervirens $\mathrm{L}$. - Le long des murs et des haies. - Gard : le Vigan, à Arphy, Galary près d'Aulas. - L'ouest de la France.

A. Barelieriana Bellardi. - Alp.-Mar. : la Briga, Tende; plus abondant sur le versant italien à Limone, Robilant, Vaudier.

NoNNIA alba D C. - Terrains sablonneux. - B.-du-Rh. : Tarascon, bords de l'Arc à Berre. - Gard: Beaucaire au Château de St-Roman, bords du Rhône à Vallabrègues, bords du Gardon entre St-Nicolas et la Beaume. - Hér. : Castelnau, bords du Lez près de la première écluse, Pézenas, Agde, Béziers, Villeveyrac. Aude et Pyr.-Or. : env. de Narbonne à la Clape, bois de Célciran, la Fenal, Raounel, Vicledaigue, vallée de l'Agly à Casesde-Pena, Baixas, Força-Réal.

ALI N N Iucea D C. - Sables maritimes. - Iles d'Hyères. Port-Venilres, Banyuls-sur-Mer. - On a prétendu que cette especce existe autour de Marseille, cependant M. Roux m'a dit ne l'y avoir jamais vue.

A. tinetoria Tausch. - Terrains sablonn. des B.-du-Rh., Vaucl. - Var: le Revest près Toulon. - Gard : bois de Broussan près Nimes, bords du Gardon près de St-Nicolas, Tresques, Villeneuve en face d'Avignon. - Hér. : Hontpellier à la Pompignane, Fabrègues, Sussargues, Viols-le-Fort, Pézenas, Bédarieux. - Aude et Pyr.-0r, : Carcassonnc, Leucate, env. de 


\section{$-561-$}

Perpignan, Collioure, Port-Vendres. - Dr. et Ard. : Donzère, Andancette, Romans, Lasuel. - Sa station la plus septentrionale est près de Lyon aux Sables de Montchat et au Molard de l)écines.

ovos.ra echioideun $\mathrm{L}_{\text {. }}$ - - Sables et graviers, - Sav. : StJean el St-Michel en Maurienne. - Is., Ain et Rh.: Vienne, Lérieu près Crémieu, Mleximieu, la Pape, Vernaison, Yvour. - II.-Alp. et Dr. : Guillestre, la Saulse et Charance près Gap, Montmort près Veynes, Serres, St-Andró-de-Rosans, Moydans, mont Queyrel et Noyer en Champsaur, le Guard près Nyons, Crest. - B.-Alp. et Vaucl. : col d'Allos, Sérennes, Meyronnes, Maurin, Jélezen, la Condamine, Digne, Avignon, Orange, Sérignan. - Alp.-Mar. : Grasse, St-Dalmas-le-Sauvage, Entraunes, Tende. - Ard. : Lussas, Baix. - Gard et Hér. : assez rẻpandu dans les arrond. de Nîmes, d'Alais, d'Uzès et du Vigan, la Sérane, Madières, le Larzac au Cros et au Caylar, la Vacquerie, Causse-de-la-Selle. - Pyr.-0r. : St-André, St-Laurent-deCerdans, Costujes, Prats-de-Hollo. - En dehors de nos limites dans la Lozère, l'Aveyron, la Charente-Inf.

L'O. arenarium W. Kit. a la corolle plus petite, les anthères exsertes, le stigmate entier et n'est peut-être qu'une variation individuelle, non permanente par hẻrédité.

L'O. stellulalım W. Kit. a de petits poils disposés en étoile à la base des poils principaux. - Valais : rochers des Marques, la Bâtiaz, Fully, Saillon, Montorge, St-Léonard, Brigerbad.

LITHOSP IRION fruticosum L. - Lieux incultes de la région mérid. - Vaucl, et B.-du-Rh. : St-Didier près Carpentras, Gargas, Baumes, env. d'Aix, collines du Montaiguet et de la Keirié, les Pennes, Rorquefavour, entre. Cassis et la Ciotat, I'Estaque, la Ste-Baume. - Gard et Hér. : env. de Nîmes, d'Uzès, de Tresques et d'Anduze, Lavalette près Montpellier, Grabels, la Colombière, St-Guilhem, Mlontarnaud, Murviel, St-Mathieu* de-Tréviers, Valflaunès, Claret, Pézenas, Nissan. - Aude et Pyr.Or. : env. de Narbonne au Pech-de-l'Agnèle, Moux, Bages, Pradines, Pas-du-Loup, mont Alaric, Cases-de-Pena, Força-Réal, Prades, Villefranche, Olette. 
L. oleifolium Lap. - Rochers des Pyr.-0r. sur les bords de la rivière de St-Anyol, et au Camp de Bassagudes près Costujes.

L. пurpureo creruleum L. - Ilaies, broussailles, bois dans tout le bassin. Plus commun dans les terrains calcaires.

L. offinale L. - Haies, bois et bords des chemins.

L. a rvense L. - Champs, vignes.

L. permixlum Jold. - Forme à carpelles bleuâtres, ligèrement tuberculeux, fleurs bleues. - Ain, Rh. et Loire : env. de Trévoux et de Belley, Vaugneray, Francheville, Montbrison. - H. et B.Alp. : Briançon, bois Mondet et Rabou près Gap, la Grangette, Avançon, Ribiers, la Condamine, Maurin, Larche, Allos. Vaucl. et Alp.-Ylar. : mont Ventoux, Caussols, mont Cheiron, Entraunes.

L. apulum Vahl. - Lieux arides de la région mérid. - Vaucl. et B.-du-Rh. : env. d'Avignon à Montfavet, Horières, envir. d'Apt et d'Aix au vallon les Gardes et à la colline des Pauvres, Mazargue près Marseille, Istres. - Var et Alp.-Mar.: les Sabletles près Toulon, Fréjus, St-Raplıä̈l, Antibes, Villeîranche, Menton. - Gard : env. de Nimes au bois de Broussan, St-Gilles, Bellegarde, Aigues-Mortes. - Hér. : Montpellier au-dessus de la Gaillarde, St-Jean-de-Vẻdas au Terral, Lattes à Maurin, StGeniès-des-Mourgues, Caunelle, Fabriggues, Vauguières, Pignan, Sussargues, Pérols, Frouzet, Nefliès, Pézenas, Béziers. - Aude et Pyr.-0r.: env. de Narbonne à Fresquet, Fontlaurier, Aussiẻres, Laraguoux, vallée de l'Agly à Força-Réal, Baixas.

GCHION italieume L. - Terrains incultes, bords des champs de la région mérid.; remonte dans la Drôme jusqu'à Montélimar. - Cotes de l'ouest de la France.

E. pyranidale Lap. - Pyr.-Or. : Perpignan, St-Esteve, le Soler, Argelès, rives de la Massane, Collioure.

E. abereanum Naulin et Debeaux. - Chaine des Albères prẻs de Collioure.

L'E. Rauuolfanum Delile, espèce d'Egypte, a été trouvée à Marscilie près des lavoirs à laine.

E. vulgnre L. - Lieux incultes, bords des chemins dans toute la France. 
La variété tuberculatum Link et IIofim., qui a les fruits plus fortement tuberculeux, les fleurs plus grandes est assez commune dans la région mérid.

La var. parviftorum, E. Wierzbickianum Hab., qui a des fleurs petites, dépassant peu le calice et des étamines incluses est çà et là mêlée au type et à la forme tuberculalum.

E. maridinum Willd. - Sables marilimes près de Toulon, lles d'IIyeres, Si-Tropez.

E. creticum L. - Bords des chemins, - B,-du-Rh., Var et Alp.Mar. : Tarascon, Bormes, Lavandou, Fréjus, St-Raphaël, l'Esterel, $\Lambda$ gay. - Gard et Hér. : Beaucaire, Celte.

E. plantaglneum L. - Lieux incultes. - Vaucl, B.-du-Rh., Var et Alp.-Mar, : env. d'Apt, d'Aix, de Salon, de Marseille, île de Porquerolle, le Luc, Cannes, Antibes, Nice. - Gard : Garon, Broussan, Cygnan, Campagne près de Nîmes. - Hér.: Castelnau, Roquehaute près de Vias, Agde, Béziers, Nissan, Olargues. - Aude et Pyr.-0r. : env. de Narbonne, Ste-Lucie, env, de Perpignan, Château-Roussillon, le Boulou, Collioure, Banyuls. - Remonte dans l'Ardèche dans la vallée de l'Ouvèze près du mont Charray.

Calyeinum Viv. - Bords des chemins. - B.-du-Rlı, Var et Alp.-Mar. : Marseille, lle Ratoneau, Endoume, St-Julien, la Ciotat, Toulon, Fréjus, île Ste-MIarguerite, Nice, Villefranche, Monaco, Menton.

E. arenarium Guss. - Espèce de Corse, d'Italie, Sicile, Grèce, trouvée à Agde et près de Cette, sur les bords de l'étang de Thau.

PULVONA IT silvestris. - Bois dans tout le bassin.

Espèce extrêmement polymorphe dans laquelle on observe de nombreuses variations dans la couleur des fleurs, le lieu d'insertion des étamines, la Iongueur du style, la villosité ou la glabresience du tube de la corolle au-dessous de l'anneau normal de poils que présentent à la gorge toutes les Pulmonaires, la forme et la maculature des feuilles, la longueur des pétioles. Aussi la classification des Pulmonaires est-elle un sujet perpétuel de controverse entre les botanistes, d'autant plus que les 
caractères au moyen desquels on les subdivise en plusieurs races (especces pour un grand nombre d'auteurs) ne présentent pas une constance absolue, et que ces races ou prétendues espèces sont reliées les unes aux autres par de nombreux intermédiaires. Malgré la variabilité des caractères, et tout en admettant l'existence d'une seule espèce de Pulmonaire à laquelle je donne le nom banal de P. silvestris, j'estime cependant que, dans la pratique, il y a avantage à distinguer plusieurs formes secondaires assez nettement distinctes les unes des autres, et qui d'ailleurs se perpétuent héréditairement, soit dans les stations naturelles, soit mème par semis dans les jardins botaniques. Ces formes sont les suivantes :

$P$. officinalis L. - Forme à souche grêle, oblique, caractère par lequel elle se distingue de toutes les suivantes qui ont une souche épaisse, plus ou moins rameuse. - Bois de la chaine des Vosges, Doubs, Jura, Salève, Annecy, Pringy; rare dans le Bugey. Parait manquer dans le reste du bassin.

P. tuberosa Schrank, P. angustifolia L. - Forme qui se distingue des trois suivantes par la présence de quelques poils glanduleux sur la tige, et par le tube de la corolle ordinairement poilu au-dessous de l'anneau normal de poils qui hérisse la gorge. C'est la forme la plus commune dans notre bassin; toutefois, dans les départements méridionaux, elle occupe exclusivement la région montagneuse.

P. azurca Besser. - Forme à fleur d'un bleu d'azur, à feuilles étroites, les radicales linéaires-lancéolées, les caulinaires étroites, ordinairement non tachées. - Pâturages des montagnes. - ls., H.-Alp, et Sav. : mont Sénèpe, la Grave, Lautaret, N.-D. des Neiges près Briançon, mont Cenis. - Ariège : le Laurenti. En dehors de nos limites au Cantal, mont Dore, Puy-de-Dôme, Forez, Pierre-sur-Haute.

P. affinis Jord., P. saccharata Mill. - Forme à feuilles largement ovales, atténuées en long pétiole et marquées de taches plus larges que dans les autres formes. - Bois des montagnes de la vallée de la Brévenne à Sain-Bel, Bessenay, St-Germain près l'Arbresle. - Env. de Grenoble, de Gap, de St-Vallier (Dr.), Ca- 
gnes (Alp.-Mar.). - Aude et Ariège: vallée de l'Aude, des bains d'Escouloubre à Fontanes, Pont du Marchand, Bentaillole, Estagnet, Valbonne, Boutadiol.

Au mont Dore et au Cantal, dans la partie supér. de la région des Sapins, M. Lamotte a observé une forme qu'il appelle alpestris et qui parait intermćdiaire entre l'a urea et l'affuis. Elle differe de cette dernière Pulmonaire par ses feuilles caulinaires plus larges, embrassantes et non tachées.

P. mollis Wolf. - Forme dans laquelte la villosité des feuilles et de la tige est douce au toucher; se rapproche du P. tuberosa par la présence de poils dans le tube de la corolle et par les nombreux poils glanduleux qui hérissent la tige. - Existe au Canigou et au Laurenti d'après de Candolle. MII. Timbal et Jeanbernat n'en parlent pas.

MYosots palustris With. - Marais, prés humides dans les parties septent. et moyenne du bassin; dans la partie mérid. il n'existe que dans les montagnes. - Espèce polymorphe.

M. lingulata Lehm. - Diffère du précédent par la racine non rampante, par la tige arrondie à la base, le style trés court, la fleur petite, d'un bleu pâle. - Mêlé au précédent qu'il remplace même dans les sols siliceux, argileux, tourbeux.

II. pushlla Lois. - Bois et sables humides. - B.-du-Rh. et Var : Montredon près Marseille, bords de l'étang de Marignane, les Pesquiers, Ceinturon d'Hyères, St-Tropez, Fréjus, St-Raphaël. - Corse.

II. stricta Link, - Champs sablonneux, - Commun en Alsace et près de Belfort à Damjoutin. - Dans le Jura on ne l'a vu que sur les rochers de Beaume près de Lons-le-Saunier. - Rh. et Loire : Lyon à Montchat, Villeurbanne, Beaunant, Chaponost, Vaugneray, St-Bonnet-sur-Montmelas, Lamure, Fleurie, Chénas, Pilat, Chalmazelle, - Is. et Dr. : Beauroir près St-Ilarcel. lin, Romans, St-Vallier, Crest. - H.-Alp. : env. de Gap, Rabou, col de Glaise, mont Séuse. - Alp.-Mar.: St-Vallier. - Gard : env. de Nimes et du Vigan. - Pyr.-0r. et Ariège : la Basse, la Salanque, Mijanès, Artigues, St-Félix, le Pla, Usson, le Puch.

及. verskeolor Pers. - Champs sablonneux et graveleux. - Commun 
dans les vallées des Vosges, la H.-Saone, les plaines sablonneuses de l'Alsace, de Belfort et de Montbéliard, la Bresse du Jura ct de l'Ain, les terrains granitiques du Lyonnais, du Forez et du Vivarais, les sables de la mollasse du Dauphiné, des env. de la Roguette et de Menton (Alp.-Mar.). - Gard : le Vigan, Aulas, St-Jean-du-Bruẹl. - Champs sablonn, de l Hérault et des Pyr.-Or. - Espèce polymorplıe.

M. Intea Balbis, M. Balbisima Jord. - Dans cette forme, le calice est peu ouvert après la floraison, les fleurs petites, toutes jaunes. Terrains siliceux de S.-et-L.et Rh., autour de Cluny, Romanèche, Pruzilly, Chẻnas, Fleurie, Beaujeu, St-Bonnet-le-Froid, Chasselay, Lamure, Vaugneray. - Loire : Doizieu, le Planil au pied des bois du Pilat, Ruthiange, St-Genest-1Falifaux, Ecotay, Ies Salles, Chalmazelle. — Gard : Arphy, Dourbie, Puéchagut, le Vigan, Aumessas. - Hér. : vallée de la llare à Andabre, StGeniès-dc-Varensal, Castanet-lc-Hauı, St-Pons, Fraisse. - En dehors de nos limites dans le Tarn, Tarn-et Garonne.

Entre les $M$. versicolor et lutea, existe une forme intermédiaire, M. fallacina Jord., dont les fleurs présentent la sírie de couleurs du M. versicolor, et ont les corolles petites à tube non saillant du M. lutea. Ces deux formes sont du reste mêlées au type dans les localités citées plus haut.

M. hispida Schlecht. - Licux incultes dans tout le bassin.

7Y. Intermedia Link. - Champs, bords des chemins.

PI. silvatien Hofim. - Lieux humides des bois dans les montagnes des Vosges, chaîne jurassique, Vaud, Valais, Savoie, Dauphiné, B.-Alp., Alp.-Mar. - Chaîne montagneuse du Morvan, Beaujolais, Forez, Lyonnais, Pilat, Vivarais, Cévennes du Gard et l'Hérault, centre et ouest de la France. - Espèce polymorphe. I. alpestris Schm. - Forme du précédent a fleurs plus grandes, en grappes plus serrées, à pédicelle floral plus court et à calice légèrement ouvert. Ne vit pas comme le $M$. silvulica dans les bois, mais bien dans les pâturages des hautes montagnes. Vosges depuis le Ballon de Soultz, le Hohneck, les sommités de la Vallée de Munster. - Chaine jurassique: Chasseron, mont d'Or, Montendre, la Dôle, Colombier, Crêt de Chalam, Reculet, 
Sorgiaz, Retord. - Sommités des Alpes de Vaud, Valais, Savoie, Dauphiné, B.-A!p, et Alp.-Mar. - Vaucl. : mont Ventoux. - Pyr.-Or.: Canigou, vallées d'Eyne, de Nohèdes, Laurenti. - Pyr. cent., Cantal, mont Dore, monts Dómes.

M. pyrenalea Pourr. - Pâturages des montagnes, - Pyr.-Or. et Ariège: Canigou, Costa Bona, Carença, Cambres d'Aze, vallées d'Eyne et de Llo, élangs de Nohides, vallons du Laurenti et de Barbouillère, Valbonne, Pic de la Camisette. - Sav. : mont Iscran, mont Cenis. - Pyr. centrales.

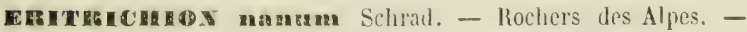
Valais : sommmités des Alpes de Zermatt, Saas, s'élève au pied du mont Rose jusque vers la Cabane de Vincent (3158 m.), Simplon. - H.-Sav. et Sav. : Buet, Brévent, Jalouvre, Méri, Aiguilles d'Arves, col de la Madelcine, mont Cenis sur les bords du lac Noir. - Is. el II.-Alp. : chaîne de Belledonne à la Grande-Lance, Taillefer, Grandes-Rousses au-dessus de Brandes, Villard d'Arène, Lautaret sur le Haut-Ricliard, le Combeynot, la Bérarde, glacier Carré (375\%”.), au pied du Pic de la Ileige, mont riso, glaciers de l'Asty, col Agnel, St-Véran à Blanchette, Orciores, Chaillol-le-Vieil, col de Valestresse.Alp.-Mar. : Alpes de St-Etienne, bords du lac de Mercantourm, Alpes de Fenestre, sommet du mont Bego.

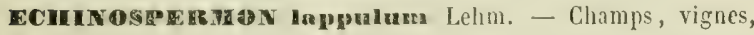
bords des chemins. - Jura et Ain : env. de Dole, les Arsures près Salins, Vauxenans près St-Amour, Thoirette, le Revermont et le Bugey. - Versant neuchatelois et vaudois : val de Ruz, Boudry, Nyon, Rolle. - Talais: St-Maurice, les Marques, Sion, St-Léonard, Sierre, val St-Nicolas. - H.-Sav, et Sav. : pied du Salève, Pas-de-l'Ec!telle, Pont d'Annemasse, vallée de l'Arve, Chavanod, Marcilly, Pringy, Bramans et St-Michel en Maurienne, Brides-les-Bains. - Env, de Lyon à la Pape, StGenis-Laval, Oullins, bords de l'Izeron el du Garon, Brouilly, Quincié et Chiroubles en Beaujolais, - C.-d'Or, S.-et-L. : Dijon, route de Plombiẻres el toute la Cóte, Dezize, la Molhe-StJean. - Is. et II.-Alp. : Cotes de Sassenage, vallée de la Romanche, remonte jusqu'au Lautarct, vallée de la Guisane, le 
Mlonestier, Briançon, la Vachette, Vallouise, valléc du Guil en Queyras, Gap, Tallard. - B.-Alp. la Condamine, St-Paul, Serennes, Castellane. - Vaucl., B.•du-Rh. - Gard ct Hèr. : le Vigan, Tresques, Montpellier à Grammont, Castelnau, Montferrier à Fontfroide, Maguclone, Mèze, Montagnac. - Aude., Pyr.-0r. et Ariège : env. de Narbonne, de Perpignan, vallée de l'Aude, Usson, Rouze, Mijanès.

E. deflexum Lehm. - Forme du précédent à pédicelles fructifíres réfléchis, plus longs que le calice. - Valais : Bagnes, bois de St-Barthélemy, d'Hérémence, Leziferujo, Chapelle de la Garde sur Evolena, entre Ayer et Zinal, entre Kalpetran et St-Nicolas, Zermatt, Findelen, vallée de Saas, Lax. - H.-Alp.; Boscodon près Embrun, Combe-Noire de Manteyer.

CY ToG cossoy elneinifounan L. - Bords des champs et des chemins de la région des Oliviers. - Vaucl., B.-du-Rh. et Var : env. d'Avignon, d'Orange, Sérignan, Flassan, Graveson, les Alpines, Arles, Aix, Marseille, Toulon, Fox-Amphoux, Château-Double, le Luc, Frẻjus. - Alp.-Mar. : Grasse, St-Vallier, Lucéram. - Gard et Hẻr. : env. de Nîmes, d'Uzès, d'Anduze, Tresques, Pont-du-Gard, Montpellier à la Paillade, la Colombière, Villeneuve, Restinclières, St-Loup, Mireval, Frontignan, Pézenas, Béziers. - Commun dans l'Aude et les Pyr.-Or. Remonte dans la partic mérid. de Ia Drôme vers St-Paul-TroisChâteaux, Montélimar; dans les B.-Alp. vers Sisteron.

C. pictum Ait. - Champs et bords des chemins de la région mérid.; remonte dans l'Ardèche, la Drôme, les H. et B.-Alp., l'Isère et le Rhône à Privas, Grignan, Crest, Chamaloc, Valence, Laragne, Vienne, Marcieu, Givors et les bords du Garon. - Cette espèce s'étend assez loin dans le bassin de la Garonne, dans le centre et l'ouest de la France, à travers la Loire, l'Yonne, Nièvre, Indre, Loir-et-Cher, Loir, Vienne, Maine-et-Loire, Sarthe, Charente-Infér., Ille-et-Vilaine, Vendée.

C. offeimale L. - Lieux incultes, bords des chemins dans les parties septentr. et moyenne du bassin; plus rare dans la partie mérid. oủ il lne se montre que dans les régions montagneuses.

C. montanum Lam. — Bois des montagnes. - Chaîne des Vosges. 
- Jura helvétique et français : Weissenstein, mont Terrible, Chasseral, Creux-du-Van, Suchet, env. de Montbéliard, col des Roches, Côtes du Doubs et du Dessoubre, Lomont, Crèt de Vaubierge, château de la Roche, Champey au-dessus de Salins, sources de la Loue, forêt d'Arrières en Bugey. - Vaud et H.Sav, : les Frachis au-dessus de Bex, forêt de la Cheneau audessus d'Aigle, Saleve au-dessus d'Archazip et de Pommier, Annemasse, vallon de la Caille, Bonneville, Reignier, Rumilly, Veyrier près Annecy, Thones, Charvin, vallèe de la Borne, Tamié, mont Charvin. - C.-d'Or: Velars, Combe de Flavignerot, Trouhaut, Nolay vers la cascade, Vauchignon, Lugny. Is. et Dr. : vers l'ancienne cabane de St-Eynard, forêt d'Esparon près le IIonestier-de-Percy, col de Rousset près Die. B.-Alp., Var et Alp.-Har.: la Condamine, les Mayons, val de Pesio. - Vaucl. : Flassan. - Gard : Corconne entre St-Bauzelly et Ganges. - Hér. : Pic St-Loup, la Sérane, les Capouladoux, St-Guilhem, St-Bauzille-de-Putois, St-Iartin-de-Londres aux Arcs. - Ariège et Pyr,-0r. : Roc de Mascaras, bois de France, foréts de l'Ermite et de Riplaou, bois de Fach, de Boucheville et de Salvanere.

C. Dioscoridianum Vill. - Lieux incultes, - C.-d'Or : Gouville à l'entrée des bois de Savigny, Nuits à Chambolle et à la Serrée, Tarsul. - Is., Dr. ei H-.Alp. : Bastille de Grenoble, Sassenage, Comboire, Clelles, Claps-du-Luc, entre le Dauphin et la Grave, Briançon, Puy-St-André, Puy-St-Pierre, Vars, Sigoyer, Veynes, Roche-des-Arnauds, le Noyer en Champsaur. B.-Alp. : la Condamine, Mélezen, Toumoux, Hochaille de Meyronnes, Annot. - Alp.-Mar. : entre Duranus et Levens, Venanson, Tende. - Vaucl. : Flassan, bois du mont Ventoux. Aude et Pyr.-0r.: env. de Tuchan sur le plateau de Tauch, Pont de la Fou près St-Paul-de-Fenouillet.

Le $C$. clandestinum Desf., de l'Espagne et de l'Italie mérid. a été trouvé accidentellement au Port-Juvénal près Montpellier.

Dr PMALODEs Innifolium Hœnch, - Cultivé et quelquefois subspontané. - Pied du mont Ventoux, Carpentras.

D. vernum Mønch. - Cultivé et quelquefois subspontané. 
ASPEITCO procumbens L. - Décombres, hords des routes. - Très-inégalement réparti; assez abondant dans la région mérid., dans les montagnes des Alp.-Mar., B. et H.-Alp, Dauphiné, Savoie, Vaud et Valais. - Ain : sous le rocher de PierreChâtel à l'entrće des Grottes. - C. d'Or: autour de Dijon et le long de la Côte.

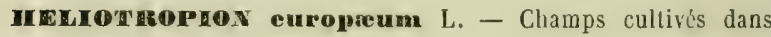
tout le bassin.

1. supluuzn L. - Terres sablonn. sur le bord des étangs, des marais et des rivières de la région mérid. - B.-du-Rh. : vallée de Vauvenargues, Ste-Victoire, env. de Harseille. — Gard: bords de l'étang de Jonquières, la Tour d'Anglas près du Caylar. IIér. : Villeneuve à la Madeleine, Mauguio, Agde, Roquehaute, Palaras, Cette. - Bords des rivières des Pyrénées-0r.

Mr. cupasgavicum L. - Espèce américaine naturalisée à Porlde-Bouc, les Martigues, Cette, Agde, Pérols, Palavas, Ste Lucie, la Nouvelle, Leucate.

Le Rochelia stellulata Rchb. d'Espagne a été vu au Port-Juvénal près Montpellier et aux Catalans près Marseille.

Le Solenanthus lanatus Alph. DG. d'Algérie a été aussi trouvé au Port-Juvénal.

\section{SOLANÉES}

LYCrUn barbarum L. - Le nom donné à cette espèce semble indiquer que Linné la croyait originaire de l'Afrique septentrionale. - Grisebach supposait qu'elle nous est venue de la Podolie et de la Gallicie. Comme elle est plantée dans les haies, elle n'offre aucun intérêt sous le rapport de sa distribution géographique; la variẻté sinense Lam. présente un cálice à cinq dents inégales, non bilabié; les feuilles sont plus larges que dans le type.

L. europrenm L., L. mediterraneum Dun. - Planté dans les haies de la région méditerranéenne.

L. afrum I. - Haies des champs et des vignes autour de Perpignan, 
SOLAXUTI nimeum L. - Lieux cultivés, bords des chemins dans tout le bassin.

La couleur des fruits, habituellement noire, est quelquefois jaune dans le $S$. chlorocarpum Spenn. ou rouge dans le S. miniatum II. Koch.

S. villosim Lam. - Forme du S. nigrum à corolles plus grandes, à tiges et feuilles couvertes de poils, - Lieux cultivés, décombres; plus commune dans la région mẻridionale.

s. tuberosuma L. - La Pomme de terre (ou micux Parmentiere) devient quelquefois subspontanée sur les décombres, autour des labitations. Il en est de même des Solanum sndomeum L., S. lycopersicum L., S. melongenum L.

S. duleamarum L. - Bois, haies, dans toute la France.

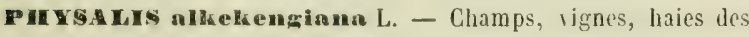
terrains calcaires. - II.-Saône: Andelarre, Vouhenans, Coulevon, Purgerot, Gouhenans, Gray, Chassey-les-Montbozon, Nans. - Doubs et Jura : env. de Montbéliard, de Dole et de Chaussin. - Ain : Villereversure, Belley, St-Rambert, la Birottière près Tenay, Trévoux, Reyrieux. - Genève, Vaud el Valais: bords de l'Árve près de Pinchat, chemin des GrandsPhilosophes, Vandouvres, Nyon, Pandex, Hontreux, Villeneuve, Roche, Aigle, Bex, Orbe, Rances, Beaulmes, Mathod, Fully, Martigny, Conthey, Sion, Sierre. - C.-d'Or et S.-et-L. : Dijon, Plombières, Larrey, Chaux, Beaune, Santenai, Dezize, Remigny. - Rh. : Limas, Pommiers, Vaux-en-Velin, Ecully. - H.-Sav. : env. d'Annecy, Magériaz, env de Bonneville et de Rumilly, Tamié. - Is., Dr. et II-Alp. : Balmes de Fontaine, Pont-deClaix, la Terrasse, Sinard, Veynes, Ribiers, Rosans, Barnave. - Vaucl. el B.-du-Rh. : env. d'Avignon, d'Aix, d'Apt, iles du Rhône. - Var et Alp.-Mar. : le Luc, Bar, St-Auban, Aiglun, Vallauris, Grasse, Ste-Agnès au-dessus de Menton, Tourette, Levens, Touët-de-Beuil. - Gard et Hér. : Bouquet, Lussan, Tresques, Alzon, Brissac, Ceilhes. - Aude et Py, -Or.

IELL DO DAccifera Lam. Atropa Belladona L. - Bois des montagnes. - Chaine des Vosges, H.-Saone, env. de Belfort, de Monbéliard, de Besançon et de Dole, forêts de Serre et de 
Rochefort, le Revermont, forêt d'Arvières en Bugey, Mazières, Tenay, Jujurieux, Cerdon, Parves. - Vaud et Valais : les Plans, Chemin-Neuf, bois de Finge. - H.-Sav. : Petit Salève, couvent de Pommier, Voirons, vallée du Reposoir, St-André dans le val de Fier, Tamié. - Is., Dr. et H.-Alp.: GrandeChartreuse, Chalais, la Terrasse, St-Guillaume, Chichilianne, Durbon, Bouvante, Granne, Rabou et Loubet près Gap, CombeNoire de Manteyer, Sigottier, Boscodon, Guillestre, entre la Ilaison-du-Roi et Château-Queyras. - B.-Alp., B.-du-Rh. et Var : Barcelonnette, Sisteron, les Alpines, Ste-Baume, ile de Porquerolle. - Alp.-Mar. : forêt de la Maïris, entre la Tour et Si-Sauveur, bois de la Fraca, forết de Clans, mont d'Andon audessus de Grasse. - C.-d'Or et S.-et-L. : Arc-sur-Tille, Arcelot, Val Combe, Sombernon, Messigny, la Côte, Montbard, Montjeu, St-Didier, Cluny, - Rh. : Pic de St-Cyr-le-Chatoux, - Vaucl. : bois du mont Ventoux aux Charbonniers, montagnes de la Garde et de Lure. - Gard et Hér, : bois de Salbous et de Bouquet, Pic St-Loup, Capouladoux, la Sérane, Pégayrolles-del'Escalette, St-Bauzille-de-Ilontmel, entre Madières et Navacelle, St-Amand-de-Hounis, la Vacquerie, Bédarieux.

DATU EA tramonia L. - Décombres, voisinage des habitations. Originaire de l'Amérique septentr. Une forme à corolle violacée, $D$. tatula, s'échappe quelquefois des jardins où on la cultive. Il en est de même du Tabac, Nicotia tabaca L. - Je dis, à dessein, Nicotia el non Nicotiana pour désigner la plante dédiée à Nicot. (Voyez mes remarques sur la Nomenclature bolanique.)

Le $D$. Metelia L., originaire de l'Asie, s'est naturalisé dans le vallon de Banyuls-sur-Mer (Pyr.-Or.), et aussi, d'après Robert, autour de Toulon.

Iryoscramos niger L. - Décombres, bords des chemins.

H. albus L. - Hurs, décombres, bords des chemins de la région mérid.

H. major Nill. - Forme du précédent a gorge d'un pourpre noirâtre et à fleur plus grande; mêlée au type. 


\section{VERBASCEES}

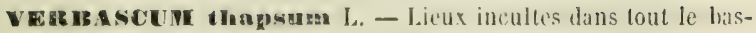
sin. - Espéce polymorphe dans laquelle la grandeur de la corolle, la villosité des filets staminaux, la décurrence des feuilles présentent de nombreuses variations.

Dans la variété montanum Schrad., la décurrence, au lieu d'aller d'une feuille à l'autre, n'a lieu que dans la moitié de l'intervalle par deux ailes cunéiformes.

v. thapaiforme Schrad. - Licux incultes dans tout le bassin.

Dans la variété crassifolium Hoffm. et Link, les filets staminaux sont entièrement glabres.

V. phlomoideum L. - Lieux incultes, bords des rivières dans tout le bassin. - Forme du précédent à décurrence moins prononcée.

Dans la variété australe Schrad., la décurrence a lieu par deux ailes cunéiformes jusqu'au milieu seulement de l'intervalle entre les deux feuilles conséculives. - Assez commune dans la rígion méridionale.

v. slnaram L. - Lieux incultes, hords des chemins de la région méridionale; remonte dans la vallée du Rhône vers Crest, $\mathrm{Va}$ lence, Tain et s’étend dans le bassin garumnien ainsi que dar.s l'ouest de la France.

V. maiale D C., $V$. Boerhanii L. - Lieux incultes de la région mérid. - Vaucl., B.-du-Rh., Var et Alp.-NIar.: Montdevergues près d'Avignon, Orange, Piolenc, Montaud-les-Miramas, Pic de Bretagne, Toulon, Fréjus, Nice, Menton. - Gard: envir. de Nìmes, Manduel, St-Gilles, Uzès, le Vigan, Alzon, Anduze, Villeneuve en face d'Avignon. - Hér.: Montpellier, Hireval, Rieucoulon, Grammont, St-Jean-de-Védas, Doscares, Sussargues, Montarnaud, St-Guilhem, les Capouladoux, St-Elienne-de-Goulgas, Bousquet d'Orb, Olargues. - Aude et Pyr.-Or.: envir. de Narbonne au Pech de l'ignèle et à la Clape, cap de $\mathrm{Pl}_{\mathrm{i}}$, Baixas, Cases-de-Pena, Perpignan, lc Boulou, Prades, Olclle, Collioure, Port-Vendres. - Remonte dans II. et Ba-Alpes ver's la Roche-des-Arnauds, Charance près Gap, la Baume-sur-Sisteron, Annot. 
v. pulverulentana Vill, V. flocesum W. Kil. - Lieux incultes, bords des chemins. L'abondance plus ou moins grande du tomentum ne saurait être considéríe comme un caractère pouvant servir à séparer spécifiquement les $V$. pulverulentum et floccosum.

v. Iyelanitasas L. - Licux incultes depuis les plaines jusqu'aux montagnes. - Espèce polymorphe dans lacpuelle la couleur des fleurs est très-variable.

V. nigman L. - Lieux incultes depuis les collines jusyue dans les montagnes. - Manque dans la région des Oliviers et occupe exclusivement la partie montagneuse des départements méridionaux.

v. dentatum Lap., V. Chaixii. Vill. - Bords des chemins. Sav.: Arbin, Cruet, Lémenc sur Chambéry. - Is., H.-Alp. et Dr.: Parisel à la Tour-sans-Venin, Sassenage, la Bastille de Grenoble, Vienne, Embrun, Charance et les Baux près Gap, StVallier. - Ard.: Entraigues. - Vaucl., B -du-Rh., Var et Alp. Mar.: Flassan à Canaud, Pic de Bretagne, Sainte-Baume, Rians, les Mayons, Grasse, Aggel et Grammont au-dessus de Menton. - Gard: le Vigan, Corconne, Anduze, Candillac, Serre-deBouquet, Broussan. - Hér.: Grabels, Valẻne, St-Loup, Capouladoux, Joncels, Avène. - Pyr.-0r.: Vallée du Tech, Céret, Arles, Prats-de-Mollo, Prades, Villefranche, Olette.

v. blattarium L. - Bords des chemins, lieux incultes.

V. virgatum With., V. bluttarioidenm Lam. - Jura : Alluvions du Douls et de la Loue au-dessous de Dole. - Ain et Rh.: bords le la Saône à Trévoux, la Dombes aux Echets, Quincieu, Villié, Arnas. - C.-d'Or el S.-et-L.: Beaune vers la source de la Bouzoise, Pontailler'sur'Saòne, Louhans, St-Didier, Issy-l'Evế,ue.Ard.: Coux.

Les Verbascum s'hybrident facilement entre eux et donnent les produits suivants que je me borne à énumérer, attendu que leurdistribution géographique est capricieuse et mal connue:
V. nigro-thapsum Fries.
V. nigro-thapsiforme Fries.
V. thapso-nigrum Schiede.
V. thapsiformi-blatlarium G. G.
V. thapsiformi-nigrum Schiede.
V. phlomo-blattarium G. G. 
r. sinualo-phlomoideum G. G.

$V$. thapso-sinuatum G. G.

$V$. sinuato-thapsum Loret.

$V$. sinuato-pulverulentum Noulet.

V. nigro-lychitum Schicde.

$V$. nigro-pulverulentum Smill.

V. lychnilo-blattarium Koch.
V. sinuato-blattarium G. G.

$V$. blaltario-sinuatum Loret.

r. thupso-floccosum G. G.

V. thapso-lychnitum H. Koch.

V. Ihapsiformi-lychnitum Schiede.

$V$. lloccoso-lhapsiforme Wirtgen.

V. lychnito-floccosum Ziz.

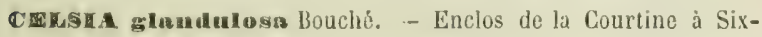
Fours près Toulon.

c. oxdentalis L. - Introduit à la Colombiere près Montpellier.

\section{SCROPHULARIÉES}

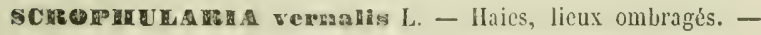
Vaud et Valais: les Devens près Bex, pied du Mont-Chemin. Sav., Is, et H.-Alp.: St-Michel en Haurienne, Séchilienne en Oisans, Séchier-de-St-Jacques, Lubac en Valgaudemar, mont IIorgon, Devès-de-Rabou. - C.-d'Or : parc d'Agey. - Rh.: cimetière et murs du presbytère de Duerne. - B.-Alp. ct Alp.Ilar. : Annot au col d'Allous, Venanson, vallée de la Gordolasca, cntre Tende et Carlin. - Gard: l'Espérou. - Ariège : cirque des Aiguettes et d'Artounant, Couillade-du-Prat-Lastou, Soucarrat. - En dehors de nos limites autour du Puy, de Blois, de Tours, d'Angoulême et en Alsace.

S. peregrina L. - Haies, vignes, lieux incultes de la rígion mérid. - B.-du-Rh., Var et Alp.-Mar. : Marseille près la GrotteRoland, St-Pons, Toulon, le Luc, St-Tropez, île Ste-Mlarguerite, Chàteaudouble, çà et là entre Nice et Menton. - Gard et Hẻr. : Franqueveau et Montpellier aux Arceaux, Aigueslongues, Masde-Cros, la Paille, Castelnau, la Pompiniane, Lavaletle, Agde et Cette. - Pyr.-0r.: St-Laurent-de-Cerdans, Port-Vendres, Banyuls. - S'étend dans le bassin sous-pyrénéen et l'ouest de la France.

s. alpestris Gay. - Prairies des montagnes à sol siliceux. Hẻr. : Ja Salvetat, Fraisse, St-Amand-de-Hounis. - Aude, Pyr.Or. et Ariège : Montagnc-Noire, Mas-Cabardès, Canigou, PratsCatal. Dassin du Rhône. 
de-Mollo, Mont-Louis, Formiguères-en-Capcir, Mijanès, forêt de l'Ermite, cirque des Aigueltes, roc d'Escrouts. - En dehors de nos limites dans les Pyrén. centr., l'Aubrac, les monlagnes granitiques du Tarn.

5. nodosa L. - Lieux humides des plaines et des montagnes; manque dans la région des Oliviers et occupe exclusivement la partie montagneuse des départements méridionaux.

5. ađuatlea L., S. Balbisii Horn. - Bords des fossés et des ruisseaux dans tout le département.

S. alata Gilib., S. Ehrharti C.-A. Steven. - Chaine des Vosges. H†e-Saône: Chemilly, Franchevelle. - Doubs, Jura ct Ain : Pontarlicr, St-Point, Ste-Marie, Champvans et Moirans près Dole, Chézery sur les bords de la Valserine, Thoiry. - Envir. de Genève à Satigny, Coppet. - Itte-Sav. et Sav. : Maxilly, Pringy, Albigny près Annecy, Chevron. - Is. : Proveyzicu, St-Laurent-du-Pont, Pariset, Laffrey, Marcieu, Chichiliane, Bourg-l'Oisans. - C.-d'Or et S.-et-L : Mlessigny, Val-Combe, Marcellois, Montbard, Tournus, la Chapelle-sous-Brancion.

5. Ineida. L. - Lienx pierreux de la Provence et des Alpes-Naritimes: env. de Harseille à l'Estaque, vallon du Puits-de-Paul, Toulon, le Luc, Grasse, de Nice à Menton.

S. eanina L. - Lieux pierreux el sablonn. des plaines et des basses montagnes. - Espèce polymorphe. - Une variété pinnatifula a été observée dans l'Aude à Nlontfort, dans les Pyr.-Or. autour de Perpignan, à la Tour-de-Hassane et à Banyuls.

Au S. canina se rattachent encore les formes suivantes:

S. rutamuraria Pourr. - Pyr.-Or.: Pont-de-la-Fou près St-Paul-deFenouillet.

S. Pourretiana Timb. Lagr. - Aude: Nontfort, Ste-Colombe.

S. stipulacea Timb. - Aude: Quillan, Ginoles.

S. humifusa Timb. et Gautier. - Aude et Pyr.-Or. : sables marit. prés de Narbonne, Leucate, plage de Canet, bords des étangs de St-Nazaire et de Cagareil.

S. luteola Timb. - Pyr.-Or.: Tour-de-Massane près Argelès.

S. juralensis Schl., S. Iloppii Koch. - Forme montagnarde du S. canina à fleurs plus grandes, ayant la lèvre supérieure de la 
corolle une fois plus longue que le tube, à pédicelles plus longs, à feuilles bi-pennatiséquées. - Débris de rochers calcaires. Nul dans les Vosges, le massif granitique du centre de la lirance, les Cévennes. - Commun dans le Jura lielvétique et français: Weissenstein, Iassenmal, Creux-du-Van, nont d'Or, Suchet, côtes du Doubs et du Dessoubre, la Dôle, Colombier, la Faucille, Reculet, Sorgiaz, Fort-de-l'Ecluse, envir. de St-Claude et de Baume-les-Nessieurs, Colombier du Bugey, vallée entre Tenay et Rossillon, Virieu-le-Grand. - Is. et II.-Alp. : polygone de Grenoble où il est amené par les eaux du 1)rac, St-Nizier, col de l'Arc, la Noucherolle, St-Didier-enDevoluy, la Grave aux Lauzières, envir. de Gap au col de Glaise, Cote-Gélive, mont Aurouse. - B.-Alp. et Alp.-Mar. : Annot au mont Couyer, Lauzanier, Meyronnes, mont Monnier, val de Pesio, col de Tende. - C.-d'Or et S.-ct-L. : toute la Côte depuis Gevrey jusquà Santenay, Dezize à Ia montagne des Trois-Croix.

- Il est douteux qu'il se trouve dans les Pyrénées et en Aurergne, où l'indiruent les auteurs de la Flore de France.

S. manerosissinan Lois. - Espèce de Corse, Sirdaigue, Espagrie, qu'on a trouvée autour de Toulon, Fréjus, St-Raplıäl, Cagnes, Nice à la pointe de Carras.

AN'Tre le bassin.

A. manjus L. - Vieux murs, rochers de la région mérid.; se montre subspontané sur les murailles des jardins dans les parties moyenne et septentr. du bassin. - Espèce polymorphe.

A. Huetianzm, - Aude : a Belcaire.

A. tortuosum Bose. - Forme de l'A. majus à lieurs et feuilles glabres.

Vicux murs. - Var et Alp.-Mar. : ruines romaines de Fríjus, Grasse, vallée de la Vesubia. - Pyr.Or. : les bains sous les fortifications de Perpignan.

A. latifolium DC. - Forme à feuilles largement ovales-lancéolées, à lleurs jaunes. - Vieux murs, débris de rochers. - H.-Sav., Is. et Dr.: Annecy, Bastille de Grenoble, Ruchefort près du Pontde-Claix, Vif, entre Corps el la Salette, vallon de Rioufrold et les Corrẻardes près Lus-la-Croix-Ilaute, St-Vallicr. - Ard. : le Pou- 
zin. - II. et B.-Alp. : Rabou, Chaudun et Charance près Gap, Nont-Dauphin, Plan-de-Phazy, Briançon, la Condamine, Sisteron. - Vaucl. : pied du mont Ventoux à la Canaud, Malaucène, Sérignan. - B.-du-Bh., Var ct Alp.-Mar. : Ile de Märé, Auriol, N.-D.-des-Anges, les Alpines, Toulon, Rians, le Luc, de Nice à Menton. - Aude, Pyr-Or. et Ariège: les Corbières, Perpignan, Villefranche, Mont-Louis, Prades, Céret, Graux-d'Olette, Cases-de-Pena, St-Paul, St-Antoine-de-Galamus, vallée de l'Aude, bains el château d'Usson, Rouze, Mijanès, vallon de Canals.

A. Banerviperas Lap. - Murs de Perpignan. - Pyr. centrales.

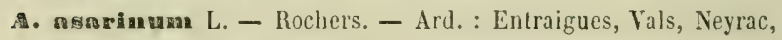
Iontpezat, Mayres. - Hèr. : St-Gervais-sur-Mare, St-Genièsde-Varensal, St-Guilhem-le-Désert, St-Pons, le Caroux au vallon d'Eric, Lamalou, Babeau près Chinian, Ganges. Gard: Alais, Anduze, le Vigan, vallon d'Aulas, l'Espérou, Concoule, Bourdezac. - Aude : Montagne-Noire, Mas-Cabardès, Lastours, Ferrals, les Ilhes. - Pyr.-0r. : Céret, Arles, Pratsde-Hollo, Foręa-Réal, Trencada-d’Ambulla, Amélie-les-Bains, Cases-de-Pena, St-Antoine-de-Galamus, Balcera en Capcir, entre Argelés et la Pava. - En dehors de nos limites dans les Pyr. centr., Tarn, Aveyron, Lozère, II.-Loire.

A. รiculsam Ucria. - Remparts de Perpignan.

L'A. calycinum Lam., d'Esp̧agne et de l'tialic mérid., a été trouvé accidentellement près de Marseille et au Port-Juvénal près de Hontpellier.

ANA nul sur les calcaires. - C.-d'Or, S.-et-L. : le Morvan granitique autour de Saulieu, Villargoix, la Selle, Cussy, envir. d'Autun, Marinagne, Hontcenis, le Creusnt. - Commun dans la chaine granitique du Beaujolais, Forez, Lyonnais, Pilat, Vivarais, Cévennes du Gard et de l'ilérault, Montagne-Noire, Pyr.-Or. Manque dans la chaîne des Vosges, H.-Saône, Doubs, Jura, Ain, Vaud et Valais et toute la Suisse, excepté dans une localitẻ des envir. de Genève entre Peney et le bois de Bay. - Rare sur les sables de transport et la Molasse du Dauphiné et de la Savoie près du Pont-de-Beauvoisin, Verna et St-Romain près 
Crẻmicu, Mollard-de-Décines, Valence, Crest, granile de St-Vallier. - Rare dans la Provence et les $\Lambda$ lp.-Mar. au vallon des Masques près Aix, le Revest près Toulon, Belgentier, route de Grasse à St-Vallicr, ile Ste-Mlarguerite. - En dehors de nos limites sur les terrains siliceux des Pyr. centr. où il n'est pas trés-répandu; plus abondant dans les montagnes de l'Aveyron, Lot, Lozère, Cantal, H.-Loire, Puy-de-Dome, dllier, Loire et tout le massif de la France centrale. - Manque dans l'oucst et le nord de la France.

Par les détails qui précèdent, on voit que cette espèce a pour foyer principal le milieu du plateau central de notre pays, doù elle s'irradie, au sud à travers les montagnes siliceuses du Lot, de l'Aveyron et du Tarn, puis dans les Pyrénées où elle se rarétie ; à l'ouest dans la Corrèzc, la Creuse, H.-Vienne et Vienne; à l'est jusque dans la longue chaine qui, depuis la Montagne-Noire et les Cévennes, s'étend à travers le Vivarais, le Pilat, le Forez, Lyonnais, Beaujolais, Charollais, jusqu'au Morvan.

Outre la localité des envir. de Genève citée plus haut, l'A. bellidifolium ne se trouve en Europe que dans un petit nombre de stations, dont la plus septentrionale est près de Trèves, sur les bords de la Moselle; les autres en Piémont, Lombardie, Corse, Espagne et Portugal.

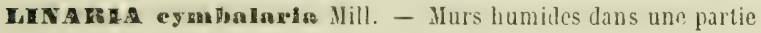
du kassin. Souvent cultivé dans les jardins d'oủ il s'èchappe facilement.

L. apuria Mill. - Champs cultivés, surtout dans les couches mar. ncuses des terrains calcaires.

L. elntine Desf. - Champs cultivés des terrains argileux el siliceux.

L. Eraeen Chav. - Licux incultes. - B.-du-Rlı., Var et Alp.-Mar.: envir. de Marseille à St-Julien, vallon du Rouet, Aubagne, les Maïres, Cassis au Château, les Sablettes près Toulon, Salins d'Hyères, ile de Porquerolle, Brẻgançon, Maures du Cannet et de Tanneron-sur-Siagne. - Gard et Hér.: Aigues-Mlortes à Milhaud, Montpellier à Grammont, Lamoure, Ricucoulon, la Madeleine, Lavẻrune, Saussan, Lausargues, Mauguio, Pérols, Maguelone, Roquehaute près Vias, Celte. - Aude et Pyr.-Or. : la Clape près Narbonne, bords de l'étang de Salses, mares 
entre Argeles ct Collioure, Port-Vendres, Banyuls. - Celte espèce a été trouvece aussi sur le littoral de l'Océan à Belle-Ile et à Fouras.

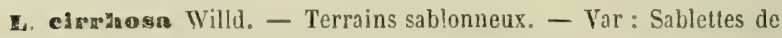
Toulon, ille de Porquerolle, le Cannet. - Gard el Hér. : AiruesMortes, Mauguio à Lamotte.

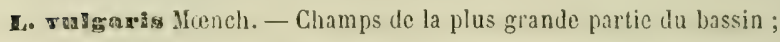
manque dans quelques régions, notamment dans le Gapençais.

L. Itrlisen Trev., L. genistifolia D C. - Prairies des Alpes. - Valais: St-Pierre, entre Sembrancher et Bovernier, vallée de la Visp et de Saas. - Sav.: partie supér. de la valléc de l'Isère. Is. et H.-Alp.: mont Sénèpe, St-Clıristophe-cn-Oisans, la Bésrarde, la Chapelle-en-Valjouffrey, Lautaret à Prime-Hesse, Monestier de Briançon, les Eduits et le Poet près Briançon, vallie de Cervières, la Vallouise, vallée du Guil entre Abriès et Risto!as, la Chalp, bois de Pra-Roussin, montagne de Combes en Champsaur, St-Haurice-en-Valgaulemar. - B.-Alp et Alp.Mar.: St-Paul, partie supér. des vallées de la Vesubia, de la Tinea et de la Roja. - Pyr.-Or.: Perpignan, Cases-de-Pena, Estagel, Collioure, Anse de Paulille, Banyuls.

4. Fenceroiann Mill. - Bois, côtcaux, champs surtout dans la région mérid. - Var et A!p.-Mar.: Toulon, le Luc, St-Tropez, la Roquette, Cannes, Antibes, Nice, Menton. - Gard et Ilér.: commun dans les arrond. de Nîmes, Alais, Uzès, le Vigran, envir. de Montpellier à Grammont, Lamoure, Montpeyroux, Lodève, St-Pons, l'Espinouse, Madières, Andábre-Rosis, Pardailhan, Ceilhes, Graissessac. - Aule et Pyr.-Or. : Fontfroide, Aussières, Fontlauricr, pied des Albères, Banyuls. - Apparait souvent dans les environs de Lyon, de Crémieu, d'Heyrieux, de Bourgoin, de Chambéry et dans la Dombes, notaminent entre les Echets et Tramoye, puis dans la Côte-d'Or vers Auxonne, Seurre, Marsannay, Saulieu. - En dehors de nos limites, dans le bassin sous-pyrénéen, l'ouest, et accidentellement sur plusieurs points du centre de la France.

4. arvensis Desf. - Champs cultivés. - din, Rh. et Loire: Chátillon-en-Dombes, Pierre-Châtel, Chiroubles, Chaponost, envir. 
de Lyon, à St-Alban et Ste-Foy, Rive-de-Gier, St-Julien-MolinMolette. - Is. et Dr.: Charvicu, St-Geoirs, St-Vallier. - Haut.Alp.: Moncstier-de-Briancon, la Vachette. - Var et Alp.-Mar.: Toulon, St-Tropez, les Maures, la Roquelte près Grasse. - Ard. el Gard: St-Agrève, arrond. de Nimes, d'Uzès, d'Alais, du Vigan. - IIér.: Montpellier à Grammont, St-Georges, Pignan, Sl-Aunès, Pézenas, St-Martin-d'Orb, Lunas, Avène, Pardailhan. - Aude, Pyr.-0r. et Ariège: les Corbières à Villeneuve, St-Antoine-de-Galamus, Mijanès, Quérigut, Carcanières, le Puclı, Rouze.

L. simplex D C. - Forme du précìdent à corolle jaune teintée de stries violettes, à éperon droit. - Commune dans la Provence, les $A l p .-$ Nar., le Gard, l'llérault, l'Aude et Pyr.-Or.; reinonte dans la Dróme et l'Ardèche, vers Nyons, Grest, Montmaur, SaintVallier, entre Rochemaure et Tournon; dans les B.-Alp. vers la Condamine, Pas-de-Grégoire, Annot; dans Ies H.-Alp. et l'Is. vers les Baux près Gap, Remollon, Ribiers, Briançon, St-Lattier, Moidieu, Vizille, Champagnier, Charvieux; dans la Loire et le Rhône, vers Pelussin, Chavanay, St-Hiclıel, Givors, vallée du Garon, Lyon à St-Clair et Villeurbanne; dans la H.-Savoie à Rumilly, Challonges, Bassy, Chilly.

L. micrantha Spreng. - Dans cette forme les feuilles des tiges florales sont ovales-lancéolées. - Hér., Aude et Pyr.-0r.: vignes des environs de Frontignan, Mireval, Narḅonne, Durban, lle SteLucie, Opol, Salses, Perpignan.

L. sparten Hoffm. et Link. - Celte espèce des Landes entre Bayonne et Bordeaux a été indiquée par Companyo dans le Conflent (Pyr.-0r.); je ne l'y ai point vue et n'ai pas connaissance qu'on l'y ait observée. La même remarque est applicable à la mention par le même botaniste du L. thymifolia.

L. chnlepensis Mill, - Champs de la région mérił. - Var et Alp.Mar.: Toulon, le Luc, Ramatuclle près St-Tropez, Grasse, Antibes, Nice, Ste-Agnès, Menton. - Gard et Hér.: Visscq, Montdardier, Campestre, Alzon, l'Espérou sur la Tessone, Murviel, la Sérane. - En deliors de nos limites à Mont-Vaillant près Florac (Lozère). 
I. strarata D C. - Cliamps incultes dans uno grande partie du bassin. - Quoiqque assez commune dans l'Alsace, la Lorraine et Ia H.-Saône, cette Linaire est rare et fugace dans la Dombes et le Jura autour de Besançon, de Salins, d'Arbois, entre Morey ct les Rousses et à Busy ; elle estencore plus rare sur le versant helvétique.-Très-abondante dans la C.-d'Or., S.-et-L., Rh., Loire, Ard., Gard, Hér. et jusque dans l'Aude, les Pyr.-Or. et l'Ariège. - Rare en Savoie, excepté sur quelques points de la Maurienne; plus rare encore autour de Genève et dans Vaud et Valais.

Dans son voisinage on trouve quelquefois un liybride $L$. $v u l$ gari striata dont la fleur est jaune et plus grande.

L. đxugaylla Mill. - Cette Linaire de l'Algérie, Espagne. Italie mẻrid., a êté vue à Toulon, Cannes, Grasse, Nice ct au PortJuvénal près Montpellier. - Cultivée dans les jardins.

C. aIpina D G. - Rocailles des montagnes. - Nul dans les Vosges, le centre de la France et les Cévennes. - Vaud et Valais: Bovonnaz, Anzendaz, les Diablerets, mont Fully, St-Berrard, Catogne, mont Nuoble, Mayenhorn, Mainghorn et moraines des gIaciers de Leukerbad, Gemmi, Schwarzberg, Zermatt, vallée de Saas, s’élève sur les fancs du mont Rose jusque ver's la Cabane de Vincent, Bellalui, Tortain, Simplon, Sirwolten, Eginenthal, Albrun, Furca. - H.-Sav. et Sav.: Vergy, Méri, vallée de la Borne, chaine du mont Blanc depuis le col de Balme jusqu’au Bonhomme, Jardin de la Mler-de-Glace, Chırvin, sommités des montagnes de la Tarantaise jusipu'au mont Iseran, et de la Maurienne jusqu'au mont Ambin ct au Mont-Cenis. - Is. et H.-Alp.: chaine de Belledonne, des Grandes Rousses, Mont de Lans, la Salette, l'Obiou, la Grave, Lautaret, mont Gondran, Vallouise, mont Viso, col Agnel, Valpréveyre, Pic de Chabrières, Chaillol, Champoléon, Orcières, Côte $\backsim$ Gélive et col de Glaise près Gap.B.-Alp.: St-Paul au Pas de la Louve, Crouès, Bachasse, Vallonnet, Lauzanicr, Parpaillon, Bẻrard, Grand Couyer sur Annot.Dr. et Vaucl.: mont Glandasse, mont Vertoux. - Alp.-IIar.: entre St-Auban et Castellanne, Entraunes, col de Jallorgues, Alpes de Tende. - Pyr.-Or. et Ariège: plateaux supérieurs des 
vallics de Nohides, Evol, Eyue, Cambres d'Azc, Canigou, Laurenti. - Descend que!quefois dans les plaines de l'Arve, du Fier, du lilıone et du Drac jusque près de Genève, d'Annecy, de Lyon et de Grenoble.

L. petraca Jord. - Forme du précédent à grappes fructifères plus allongies et plus lâches; la corolle a des lobes plus étroits, plus allongés, d’un violet plus foncé, à éperon droit ct non comprimé; les feuilles plus longues et plus étroites sont en verticilles plus écartés; les tiges sont dressées ot non étalées-diffuses.

Celte forme habite les rochers calcaircs du Jura français et helvetique au Weissenstein, Chasscral, Creux-du-Van, Colombier, Dòle, Faucille, Reculet, Creux-de-Pranciaux;' Tenay en Bugey. - II.-Sav.: rochers calcaires de la Tournette, cascade d'Arpennaz, Dents d'Oche et probablement en beaucoup d'autres localités où elle a été désignée sous le nom de $L$. alpina.

E. swpina Desf.. - Lieux sablonn. et picrreux des plaines, res collines et surtout des montagnes. - C.-d'Or: Nuits, Meursault, Nolay, Vauchignon, sables de l'Ouche entre le Parc et Longvic. - Ain el Sav.: Culoz, plaine d'Ambérieu, combe d'Ambin. Is, Dr. et H.-Alp.: gorges d'Engins, St-Nizier, Pusignan, Janeyriat, entre Pont-de-Chérui et Loyettes, Crémicu, graviers de la Drôme à Jansac, Fondeurle près Die, Saou près Crest, valléc de la Bourne près de la Balme, envir. de Gap au col de Glaise et de Chaudun, mont Aurouse, Poligny et le Noyer en Champsaur, Embrun, Briançon, Ribiers. - B.-Alp. et Alp.-Niar.: la Condamine, Tournoux, Serennes, Annot, sommités des Alp.-Har. d'oủ il descend jusqu'à L'Escarène, Tourrette, Caussols, l'Esterel. - Var et B.-du-Rh.: mont Faron, Ste-Victoire, envir. d'Aix au Tholonet, vallon de Fontgamate, le Luc. - Vaucl. : ille de la Bartelasse près d'Avignon, mont Ventoux, - Ard.: coteaux du Rhòne depuis le Pouzin jusqu'à Tournon. - Gard : bords du Gardon à Corconne, le Vigan, Alzon, St-Ambroix, Anduze, Hér : Montferrand, Montarnaud, St-Loup, les Arcs, St-Guilhem, la Sérane, les Capouladoux, St-IJartin-d'Orb, Ie Caylar, Villecelle, la Vacquerie, Lodève. - Aude, Pyr.-0r, et Ariége: Mon- 
tolieu, envir. de Narbonne, St-Antoine-de-Galamus, Pont de la Fou, Mont-Louis, la Cerdagne, le Capcir, vallée de l'Aude, Usson, Rouze, Mijanès, Roc de Mlascaras. - Espèce polymorphe. La varièté pyrenaica $\mathrm{D} \mathrm{C}$., plus robaste ct plus élevée que le type, a la lèvre inférieure de la corolle de couleur plus foncée, le palais barbu el taché de deux lignes rousses. - La Moucherolle, col de l'Are, envir. d Avignon, Pyr,-Or. el centrales.

L. maner Desf. - Champs, bords des chemins lans tout le bassin. La variété praetermissu Delastre, qui a la capsule, la tige et les feuilles glabres, a été trouvėe dans la C.-d'Or, le Gard entre Sauve et Quissac, sur les graviers du Vidourle.

4. rubrifolia D C. - Vieux murs, rochers de la région mérid. - Vaucl.: Flassan, Bédoin, Vaucluse. - B.-du-Rh. et Var: envir. de Marseille et d'Aix, vallon des Gardes, Barret, Toulon, le Luc, Draguignan, St-Raplıäl. - Alp.-Mar.: St-Vallier, Levens, vallon du Renard près de l'Escarène, Tende, Braus. Gard et Her.: entre St-Bauzely et Ganges, Pignan, Gigean, Assas, Montarnaud, Moulin de Figuières, St-Guilhem, les Capouladoux, les Cambrettes, St-Etienne-de-IIursan, St-Geniès-de-Varensal, Bédarieux, St-Pons. - Pyr.-Or.: Font de Comps.

I. or mérid. - B.-du-Rh., Var el Alp.-Mar.: montagnes des env. de Marseille, Ste-Victoire, Ste-Baume, entre Ascros et Tondon dans la vallée du Var. - Gard : le Vigan, Campestre, Anduze, Uzès, Pont-du-Gard, St-Nicolas. - Hér.: pic St-Loup, la Sérane, les Capouladoux, St-Guilhem, Ganges, Andabre, St-Geniès-de-Varensal, St-Amand, Lunas, Mas-de-Nouriès, Madières, le Caylar, la Vacquerie, Olargues. - Aude et Pyr.-Or.: envir. de Narbonne, Cases-de-Pena, St-Antoine-de-Galamus, Pont de la Fou, Font de Comps. - H.-Alp. et Is.: St-André de Rosans, Pont en Royans, ruines de Beauvoir près de St-Marcellin, Varces, Vif, Pariset à Beauregard, la Tronche, porte St-Laurent ả Grenoble. En dehors de nos limites sur les rochers calcaires des Pyr. cent., Tarn, Aveyron, Lozère; transporté par l'Ariège et la Garonne jusqu'au-dessous de Toulouse.

L. villosa $\mathbf{D}$ C. - Forme du précédent à tiges, pédoncules et calices 
couverts de longs poils laineux. - Garrigues calcaires des Cor. bières à Albières, Caudiès, St-Paul.

L.e L. reflexa Desf, de l'Algérie et Italie mérid, a été rencontié á Roquebrune près Menton.

Les L. scariosa et virgala Desf, onl été trouvés au Porl-Juvénal près de Ilontpellier.

Le L. Innigera Desf. a été vu au lazaret de Harseille et au Port-Juvinal.

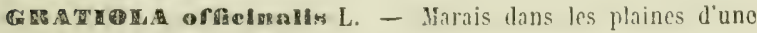
grande partie du bassin.

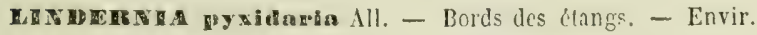
de Belfort à Bourogne, Vézelois, Sermamagny. - C.d'Or et S.-ct-L. : Citeaux, Boncourt, Branges, Issy-l'Evẹue, Bresse loulanaise autour de St-Bonnet. - Bresse du Jura et de l'Ain à St-Baraing, Balaiscaux, le Deschaux, Villers-hobert, Montsous-Vaudrey, Tassenières, Fays, Commenailles, Chaumergy, Champrougier, Neublans, étangs de St-Seine, de Samerey et de St-Symphorien, entre Dole et St-Jean-de-Losne, envir. de Trévoux, la Dombes à Villars, Marlieux, le Plantay, Lapeyrouse, St-Laurent près llâcon. - Is. : envir. de Bourgoin. - En dehors de nos limites dans l'Alsace, Lorraine, bords de la Loire pris de Montbrison, bords de l'Allier pris Moulins, rives de la Mlayenne cl de la Sèvre, étangs de la Nièvre.

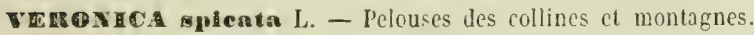
- Doulis, Jura et Ain : Audincourt, Arbouans, Orgelet, Clairvaux, Arinthod, Moirans, envir. de St-Claude, de Thoirette à Champagnole, le Bugey autour de Nantua, Hauteville, Arrieres, Belley.-C.-d'Or: Gerrey, bois de Saulon, Broindon, Auxonne. - Genève, Vaud et Valais: Genthod, bois de la Bàtie, bassin du Léman, Martigny, Fully, Saxon, Sion, le Simplon. - H.-Sav, et Sav. : Semnoz, Tamié, mont Trélod. - Is., II.-Alp., Dr. : Décines, Meyzicu, Jonage, Crémicu, Bourgoin, envir, da Grenoble, mont Rachais, Pariset, Lautaret, Monestier-de-Briançon, envir. de Gap à Charance, Rabou, col Bayard, Côte-Gélive, col de Glaise, Manteyer, Séuse, Durbon, Orciêres, mont Glandasse, Andancette. - B.-Alp. et Alp.-Harit.: la Condamine, Horo- 
nayc, St-Martin Lantosque, St-Dalmas-de-Tende. - Nontagnes du Vivarais, du Gard, de l'Espérou et au bois de Jalès près Bérias. - On trouve dans les montagnes une variété naine.

V. tearionshlla, $V$. Tencrium L. - Coteaux pierreux sur les collines et les montagnes. - Espère polymorphe dans laqueile les feuillcs sont tantôt lancéolécs, $r$. lanccolata, tantòt plus larges el ovales, $\boldsymbol{V}$. latifolia, d'autres fois blanchâtres-pubescentes, $V$. cancscens.

V. prostrata L. - Forme couchée du précédent dans laqquelle les divisions du calice et la capsule sont glabres. La floraison est plus précoce que celle du V. tencrioply?lla, que sourent il remplace, particulièrement sur les coteaux calcaires de la partie septentr. de notre bassin.

W. chaningunga L. - Bords des chan?ps, des chemins et des bois. Dans la var. pilosa Benth., il existe, outre les deux lignes de poils, une pubescence sur toute la tige.

v. urtieñfolia L. - Bois des montagnes calcaires du Jura, des Alpes et des Pyrẻnées orientales. - Nul dans les Vosges, le centre de la France, les Cévennes, la Margeride, la MontagneNoire, l'Aubrac. - Commun dans les bois du Jura helvétique et français jusque dans le Bugey. - Montagnes calcaires de Vaud, Bas-Valais, Savoie, Dauphiné, B.-Alp. et Alp.-Mar.

v. beceabunga L. - Bords des foseís et des marais dans tout le bassin.

ซ. anngahis L. - Mêmes lieux que le précédent.

V. anagalloidea Guss. - Forme du précédent, dont les feuilles et les divisions dı calice sont plus étroites, la capsule ovale presque deux fois plus haute que large. - Bords des eaux. B.-du·Rh. : Marignane. - Gard et Hẻr. : Manduel, Montpellier, Castelnau, St-Aunès, Villeneuve, Lamalou, Pézenas, Roquchaute près Vias. - Aude et Pyr.-Or. : envir, de Narbonne et de Perpignan. - Is. et Dr. : Echirolles, Montélimar. - Rare dans les parties moyenne et septentr. du bassin.

v. seutellata L. - Lieux marécageux des plaines et des montagnes.

La variété pubescens Koch, $V$. parmularia Poit, et Turp. est pubescente-glanduleuse dans toutes ses parties, 
V. Eentama L. - Bois loumides dés colcaux el des monlagnes sur les sols siliccux el argilcux. - Vosges granitiques et arénacées jusque dans la H.-Saône à Cỉauves-Roches, Etobon, Clragey, Frahier, Champagncy, vallée de Miélin, St-Remy, Nenoux, Chassey les-Niontbozon, Cubry, Clairegoutte. - Doubs, Jura et Ain: zone sous-vosgienne des envir. de Montbéliard, Roelies, Vandoncourt, Dasle, Belchamp, Elupes, Sochaux, Charmont, argiles et cailloux siliceux des forêts de Chaux, de liahon, de Neublans, forèt de Scillon près Bourg, Coillard, St-Rambert, Meximieu, - Vaud et Valais : la Rippe, St-Cergues, Bonmont, Longirod, Prevon-d'Avaux, bois de Vincy, Alpes de Jaman, Ste-Croix, les Plans, Massongex, Dent-du-Midi, Sembrancher, Chemin, Pierre-à-Voir. - H.-Sav.: Pitons du Salève audessus de Pommier, les Voirons, Chamonix. - Is. et Dr. : Balmes-de-Fontaine, Prémol, Grande-Chartreuse, Diémoz, Crand Combe près Heyrieux, Crest. - C.-d'Or et S.-et-L. : Citeaux, Pontailler, Boncourt, Villebichot, Autun, Roussillon, bois de la Cange, de Canada, de la Coiffe-au-Diable, Loulans. - Rh. et Loire: le Beaujolais, Rochecardon, Tassin, Pontd'Alaï, vallon de Fontaines, Pilat, Pierre-sur-Haute. - Hér. : le Soulier au bois de la Planque.

w. aplayiar L. - Rocailles du Jura, des Alpes et des Pyrénées. Nul dans les Vosges, le massif contral et les Cévennes. - Jura helvétique et français : Dent-de-Vaulion, Montendre, Noirmont, Dóle, Faucille, Reculet, Colombier du Bugey. - Vaud et Valais: Bovonnaz, Panẻrossaz, Creux-de-Jéman, mont Fully, Trient, St-Bernard, Torrembé, Pierre-à-Voir, Loze, Délong, Zan, Rothorn, Alpes de Saas, Mayenhorn, Nainghorn, Gemmi, Simplon, Grimsel. - II.-Sav.: Dents d'Oche, Signal-des-Agneaux, Hautigny près Bonnevaux, col de Golèse, Vergy, Brizon, Buet, Brévent, col de Balme, Jardin de la Mer-de-Glace, pavillon de Bellevue, Semnoz, Charvin, Mégève, col Joly. - Sav.: Hauteluce, la Sauce, la Vanoise, mont Iseran, mont Cenis, AlléeBlanche sur le versant italien du mont Blanc. - Is., H.-Alp. et Dr.: Chameclsaude, Grand-Som, col Vert, col de l'Arc, PetiteMoucherolle, Belledonne, la Salelte, Lautaret, Monestier-de- 
Briançon, Cervières, col des Hayes, mont Viso, St-Véran, col de Vars, Chaillol, Orcicres, pic de Chabrieres, Obiou, Chaudun, mont Aurouse, mont Séuse, mont Glandasse. - B.-Alp. et Alp.Mar. : Lazanier, Vallonnet de Meyronnes, Farpaillon, Bẻrard, mont Monnicr, Grand-Couyer, Entraunes, mont Bego, Alpes de Tende. - Vaucl, : mont Ventoux, - Pjr.-Or. et Ariège : Cambres-d'Aze, valtées d'Eyne et de Carenca, Madrẻs; Font-deComps, lac du Laurenti, vallon de Boutadiol. - Pyrénées centrales.

v. orformans L. - Bois des coteaux et des montagnes, - Rare dans la région des 0liviers. - Espèce polymorphe dans laquelle les feuilles présentent plusieurs variations quant à leur dimension et à la forme.

V. Allioniana Vill. - Forme alpine du précédent, dont il diffëre par la corolle plus grande, d'un bleu foncé, la capsule plus haute, les feuilles luisantes et plus épaisses. Les $V$. officinalis et Allioniana ont entre eux, sous les rapports morphologique et gẻographique, les mêmes relations que les Myosotis silvatica et alpestris. - Nanque dans les Vosges, le Jura, le massif central, les Cévennes, la Suisse, les Alpes de la Bavière et des Etats autrichiens. Cette espèce est propre aux A!pes françaises et piémontaises. - Sav. : mont de Fréjus, mont Cenis. - Is. et HI.-Alp. : mont de Lans, la Bẻrarde en-0isans, Villard-d'Arène, Lautaret, Galibier, Briançon au Chabas, nont Genèvre, GonUran, Cervières, Valpréveyre en Queyras, Ruines, St-Véran, col (le Vars, mont Morgon, pic de Cliabrières, Poligny et le Noyelen-Champsaur, col de Glaise, monts Aurouse et Séuse. B.-Alp. et Alp.-Mar.: Lauzanier, Mirandole, Vallonnet de Meyronnes, Parpaillon, Bérard, col d'Allous près Annot, Entraunes, Alpes de St.Etienne, de St-Dalmas-le-Sauvage et de Tende.

v. mumanularia Gouan. - Pyr.-0r. et Ariège: Cambres-d'Aze, vallée d'Eyne, cols de Nuria et de la Fosse-du Gẻant, vallée de Prats-le-Bałaguer, Canigou, Laurenti en montant à la Porteillede-Baxouillade, Pas du Roc-Blanc. - Pyrénées centrales.

ע. Prudenaloga L. - Débris de rochers des montagnes. - Jura 
liclvétique et français: le Noirmont, les Rousses, la Dóle, Faucille, Colombier, lieculet, Sorgiaz, descend jusqu’à Morez et à Mijoux, - Vaud et Valais: Morcles, Diablerets, mont Fully, Fins-Hauts, Surchamp, Leukerbad, Alpes de Zermall, au-dessus de Blatten, de Naters, Comba-d'Arbaz. - II.-Sav, et Sav. : Saleve aux Pilons, Möle, Vergy, le Reposoir, Brizon, mont d'Armonnaz près Reyvroz, mont Ilermante, entre Servoz et Chamonix, la Flégère, IIauteluce, Crest-Voland, Crêt-du-Ré. - Is., Dr. et H.-Alp. : Grande-Chartreuse, IIaut-du-Seuil, St-Nizier, col Vert, col de l'Arc, la Moucherolle, Corençon et la Salette, montagnes de Lus-la-Croix-Haute, mont Glandasse, Lautaret, Névache, Val-des-Prés à Granon, pic de Chabrières, Chaudun, Chaillol, Durbonnas, Charance, col de Glaise, nont Aurouse, Séuse. - B.-Alp. et Alp.-Mlar. : Lauzanier, Malemort, Bachasse, Longet, Grand-Couyer sur Annot, Entraunes, Estenc, sommilés les rallées de la Vesubia, Tinea, Hoja. - Gard : sommet de l'Aigual (d'après de Pouzols). - Pyr.-Or. et Ariège: Canigou, mont Louis, vallon de Boutadiol, lac de l'Estagnet, port de Paillères, lac de Quérigul.

$V$. saxatilis Jacr. - Forme du précédent ayant la corolle a'un beau bleu à gorge purpurine; les poils des pédoncules et du calice ne sont pas glanduleux. Celte race se maintient très-bien par semis; sans être tout à fait exclusive, elle est plus commune sur les terrains siliceux que sur les calcaircs, contrairement à ce qui arrive pour le $V$. fruticulosa. - Jura helvélique et français: Chasseral, sommet du Crêt-du-Miroir au-dessus du fort de l'Écluse, Credo, Colombier du Bugey. - Vaud et Valais: montagnes de Gryon, Bovonnaz, Diablerets, Surchamp, sur la Crottaz, mont Fully, Fins-Hauts, val de Trient, Catogne, GrandSt-Bernard, Novelli de Nendaz, Sassencire, Alpes de Leukerbad, de Zermatt et de Saas, Schwarzberg, Simplon, Richenen de Niederwald, Schornen, Eiria, Bellap, Grimsel. - H.-Sav. : Cornettes de Bise, col de Golèse, montagnes de Sixt et de Samoëns, col de Balme, chalne du mont Blanc, Leschaux, Clıamonix, moraines de la Mler-de-Glace, le Buet, Brévent, chaine des Aiguilles-Rouges, Vergy, Brizon, Méri, Mégève, col Joly, col des 
Aravis, l'Allée-Blanche sur le versant italien du mont Blanc. Sav. : la Sauce, col de Fenêtre, Cornet près Roselein, CrestVoland, mont Jovel sur Montagny, crêt du lié, mont Cenis. Is. et H.-Alp. : chaîne de Belledonne à la Pla, Champrousse, Grand-Galbert, Taillefer, Brandes en Oisans, Alpes du mont de Lans, Lautaret, Névache, mont Viso, col de la Traversette, pic de Chabriẻres, Champoléon, mont Queyrel, Valgaudemar, mont Aurouse. - B.-Alp. et Alp.-Mar. : Lauzanier, Malemort, Longet, Maurin, cols de Fenestre et de Tende. - Pyr.-Or. et Ariège: vallée de Nohẻdes, lac du Laurenti, vallons de l'Estagnet et de Boutadiol, Valbonne, pic de Mounégou. - En dehors de nos limites dans les Pyrénées centrales, massif granitique du Ilohneck jusqu'au Rotabac dans la chaine des Vosges.

V. Genudifolis, I. bellidioides L. - Pelouses des Alpes et des Pyrénées. - Vaud et Valais: Creux de Jéman, Panerossaz, mont Fully, St-Bernard, Cleuson, mont Nuoble, Diez, Chermignon, Torrenthorn, Gemmi, Alpes de Zermatt et de Saas, Simplon, Einergalen, Nesselalp, Bellalp, Glacier du Rhône, Furca Grimsel. - H.-Sav. et Sav.: col de Balme, chaine du mont Blane sur les deux versants, Jardin de la Mer-de-Glace, Buet, Brévent, chaîne des Aiguilles-Rouges, col de Golèse, Méri, Charviri, col des Fours et de la Seigne, Hauteluce, la Thiopa au-dessus de Champagny, Cret du Ré, mont Iseran, IIont-Cenis. Is. et II.-Alp.: chaine de Belledonne, Champrousse, Bovinant au pied du Grand Som, col de l'Arc, mont Sénèpe, la Salette, la Grave, Pelvoux, Lautaret, col de la Ponsonnière, Valgaudemar, mont Viso, cols de Ruines, Agnel et Vieux, la Taillante. - B.-Alp. et Alp.-Mar.: Lauzanier, Vallonnet de Meyronnes, mont Bego, Alpes de Tende, col de l'Abisso. - Pyr.-Or. et Arì̀ge: Canigou, Cambres d'Aze, vallée l'Eyne, vallon de l'Estagnet en montant au col de Lègue, Pic de Valbonne. - Pyrénées centrales.

v. alpian L. - Rochers et pelouses da Jura, des Alpes et des Pyrénées. - Jura français : la Dole, le Colombier, Reculet, Sorgiaz. - Vaud et Valais: Anzendaz, Bovonnaz, Lavarraz, StBernard, Zanrion, Diez, mont Nuoble, Alpes de Zermatt, 
Hoernli, Zmutt, Gorner, Alpes de Saas, pied du mont Rose vers la Cabane de Vincent, Gemmi, Grimsel, Glacier du thône. H.-Sav. et Sav.: Cornettes de Bise, Dents d'Oche, Iautigny, col de Golèse, montagnes de Sixt, Buet, Brẻvent, col de Balme, chaine du mont Blanc, Jardin de la Mer-de-Glace, Tacul, Couvercle, Entre-la-Porte, Mégève, col des Aravis, Charvin, Méri, Vergy, Jalouvre, Sernnoz, Crêt du Ré, Hauteluce, Tournette, col du Bonhomme, mont Jovet, mont Iseran, Mont-Cenis. Is. et H.-Alp.: chaines des Sept Laux, de Belledonne, de Taillefer, des Grandes-Rousses, du Pelvoux, Grand-Som, Haut-duSeuil, Roche-à-Sac près Mens, petite Moucherolle, Lautaret, mont Pẻas en Queyras, mont Viso, St-Véranà Blanchet, Champoléon, Valgaudemar, Chaillol, Orcières, mont Queyrel, Pic de Chabrières, mont Aurouse.. - B.Alp. el Alp.-Ylar. : Lauzanier, Vallonnet, Parpaillon, Fouillouse, Bérard, Grand Couyer sur Annot, sommilés des vallées du Var, Vesubia, Tinea, Roja. Pyr.-0r. et Ariège: Canigou, vallées d'Eyne et de Llo, sommet de Comelade près Prats-de-Mollo, lac du Laurenti, vallon de l'Estagnet, de Boutadiol, de Valbonne et de Paillères. - En dehors de nos limites dans les Pyrénées centrales, Mont Dore, Plomb du Cantal, Puy-Mary et Bataillouze.

V. lilacina Townsend. - Forme du précédent à longs stolons feuillés, les feuilles des rosettes et de la tige régulièrement dentées; corolle de couleur lilas pâle teintée de rouge avec la gorge blanche. - Valais : Bellap et sommet du Riederhorn. - Pyr.-Or. et Ariège : Cambres d'Aze et Port de Pailleres.

v. serpyllifolia L. - Prés et lieux humides depuis les plaines jusqu'aux montagnes; rare dans la région des Oliviers.

La forme tenella All. est une plante naine à tiges étalées sur la terre. Suivant Boreau, elle se maintient par le semis.

v. Ponaeana Gouan. - Pàturages des Pyrénées. - Pyr.-0r. et Ariege: Mont-Louis, vallée d'Eyne, mont de Madrìs, vallons de Valbonne, de l'Estaguet, de Boutadiol, de Barbouillère, Port de Paillères au Roc de Lorry. - Pyr. centrales.

v. peregrina L. - D'après Companyo, dont le témoignage est suspect, cette Véroniqgue se trouve dans les bois de Boucheville Calal. Bassin du Rhône. 
et des Fanges, ainsi qu'au Riveral de St-Rélix et de Millas. Elle est naturalisée depuis longtemps dans les allées du jardin botanique de Montpellier.

v. arvensis L. - Lieux cultivés, bords des chemins.

v. verna L. - Lieux arides des terrains siliceux. - Nul dans les chaines calcaires du Jura helvétique et français. - Abondant sur le versant alsacien des vallées granitiques et arénacées de la chalne des Vosges. - Genève et Valais : bois de Peney, Gueuroz, Branson, Creuset, Montorge, Tourbillon, Valère, Longeborgne, St-Léonard. - H.-Sav. et Sav.: vallée de Chamonix, Conflans, Combe de Villetle. - Ain : Montluel, Pont-de-Vaux. - Is., Dr. et H.-Alp.: Bourgoin, Vienne, Laveyron près StVallier, Honesiier-de-Briançon, Briançon, St-Véran, mont Viso, dans les prairies de la Traversette, Clâteauroux près d'Embrun, Poligny en Champsaur, Rabou et les Baux près Gap. - C.-d'Or, S. et-L.: le Jorvan granitique à Saulieu, Premeaux, Orgeux, Dijon, Nuits, Solutré, Issy-l'Evếque. - Rh. et Loire: Beaunant, Dardilly, Pollionnay, bords du Garon, l’Argentière, les Salles, Chalmazelle, Pic Pelé de Noirétable, St-Jodard, IIontbrison. - Gard et Hér.: le Vigan, Cap de Coste à l'Espérou, la Salvetat.

v. acinifolia L. - Champs sablonn. et argileux. - H.-Saone, Doubs, Jura et Ain: Quincey, Chassey-les-Hontbozon, entre Montbéliard et Etupes, Brognard, Charmont, la Bresse et la Dombes, - Envir. de Genève à Peney, Meyrin, Compesières, Nyon. - C.-d'Or et S.-et-L.: la Bresse, Cluny, bords de la Loire. - Rh., Loire et Is.: région granitique du Lyonnais, Forez, envir. de Vienne. - Gard et Hér.: St-Jean-du-Bruel, bois de Campagne, Trou-du-Pérussas, Montpellier à Grammont, Lamoure, Mlézouls, Lamalou, Lodève, Pézenas. - Var et Alp.-Nar.: Hyères, St-Tropez, Maures du Cannet, la Roquette, Antibes, Nice. - Ariège : St-Félix, le Mas, Quérigut, Carcanières, entre Rouze et $\Lambda$ rtigues.

v. triphylı L. - Chramps sablonneux, - Envir. de Montbéliard, de llole, de Genève, Bas-Valais, Annecy, Aix-les-Bains, Crémieu, cnvir. de Lyon, St-Vallier, Valence, Briançon, la Conda- 
mine. - Le Morvan, la Bresse, Cluny. - Région granitique du Lyonnais et du Forez. - Gard et Lozère : entre BramaBioou et Meyrueis.

ד. praceox All. - Champs sablonneux. - Cette Véronique, commune en Alsace et dans la plaine rhénane, manque dans les départem. de H.-Saone, Doubs, Jura. - C.-d'Or, Dijon, Nuits, Gevrey. - Ain et lih.: Trévoux, Pont-de-Vaux, Tassin près Lyon. - II.-Sav. et Sav.: bords du Fier près Annecy, Moutiers et Aime en Tarentaise, envir. de Genève, bassin du Léman, Bas-Valais. - Dr., H. et B.-Alp.: St-Vallier, Briançon, Gap à Charance et Rabou, Serennes, St-0urs, Sisteron. - Vaucl.: Flassan, Bédoin. - Var.: île de Porquerolle. - Gard et Hér.: le Vigan, Alzon, Campestre, le Caylar.

* perwier Poir., V. Buxbanmii Ten. - Champs de I.-S., Doubs et Jura : Vesoul, Quincey, Frotey, Champagney, St-Hippolyte, Besançon, Lons-le-Saunier. - Envir. de Genève. - Valais : St-Maurice, Sion. - S.-et-L, C.-d'Or. - Ain, Sav., H.-Sav., Rh., Is,: envir. d'Autun, de Dijon, Gevrey, St-Rambert et Tenay-en-Bugey, Annecy, Aix, Chambẻry, Villeurbanne et Vacix prìs Lyon, Vienne, Grenoble, Uriage, Seyssins, Voiron. II.-Alp., Yar. et Alp.-Mar.: Briançon, Toulon, ille de Porquerolle, le Luc, Nice, Menton. - Gard et Hér. : Aigues-llortes, Montpellier, Gigean, Balaruc, Pézenas.

W. sorestis L. Lieux cultivés dans tout le bassin.

V. didymn Ten., $V$. polita Fries. - Lieux cultivés.

v. Hederifolla L. - Lieux cultivés.

ซ. cymbaInxia Bodard. - B -du-Rh.: Marseille à N.-D.-de-laGarde, la Ciotat au Bec-de-l'Aigle, ile Verte. - Var. et Alp.Mar.: Toulon, la région littor. des Alpes-Maritimes. — Gard et Hér.: Nimes, Montpellier, Castelnau, Mireval, Frontignan, Lavalette. - Littoral des Pyr.-0r.

V. longipes Jord. - Décombres à Toulon.

CHIOSELA autatien L. - Lieux humides, bords des élangs. - Envir. de Montbéliard, Chagey, Courcelles, la Chapelle. sous-Chaux, Roppe, Eloye. - S.-et-L., Jura, Ain : dans la Bresse et la Dombes. - Bassin du Liman à Versoix , les Pier- 
rettes près Lausanne; Valais dans les marais de Fully. Bords du Rhône au Pont-St-Esprit (Garu). - St-Martin-d'Entraunes (Alp.-Mar.)

Exivos alpinus L. - Rochers calcaires. - Nul dans les Vosges et dans le massif central. - Jura helvétique et français : Hauenstein, Passwang, Weissenstein, cluses de la Birse et de la Sorne, Dent-de.Vaulion, Lomont, Morey, St-Claude, Mijoux, St-Laurent, les Molunes, Joux, les Rousses, les Dappes, Noirmont, Montendre, la Dôle, Colombier, Faucille, Reculet, montagnes de Nantua, Tenay, Hauteville, Colombier du Bugey. Vaud et Valais : Anzendaz, Dent-de-Jaman, mont Fully, Pissevache, val d'Illiez, Derborence, Chemin-Neuf, Comba d'Arbaz, Lens, Gemmi. - H.-Say, et Say.: Salève au Pas-de-l'Echelle, vallée de la Borne, montagnes de Thônes, IIagériaz et les colonies d'Annecy, Charvin, les Bauges. - Is. et H.-Alp. : GrandSom, Chamechaude, Sassenage, col Vert, col de l'Arc, Lautaret, vallée du Guil en Queyras, Obiou, mont Aurouse, Chaudun, col de la Palette, col du Noyer en Champsaur. - Alp.-Mar.: la région montagneuse d'où il descend jusque près de Menton ct de St-André près Nice, bois de Gourdon près Grasse. - Gard : envir. du Vigan à Lafoux près d'Alzon, l'Espérou. - Hér.: la Sérane, Madières, Pígayrolies-de-l'Escalette, le Pertus, Caylar, la Vacquerie. - Pyr.-Or., Arięge et Aude: Font-de-Comps, Cẻret, Port-le-Paillères, Usson, vallée de l'Aude. - En dehors de nos limites sur les rochers calcaires des Pyr. cent., de l'Aveyron, Tarn, Lozère.

DIGITALs purpurea L. - Bois, taillis, lieux incultes des terrains siliceux; nul sur les calcaires. - Chaîne granitique et arénacée des Vosges jusqu'à la lisière vosgienne des envir. de Belfort au Salbert et à l'Arsot, et jusqu'à la zone vosgienne de la H.-Saône, à Etobon, Chagey, Champagney, Ronchamp, Fresse, Ecromagny, vallée de Mansviller, Mont-de-Vannes, Belfahy, Revers-aux-Chiens, Ruz de la Fonderie, Ballon de Giromagny; grès bigarrẻ et alluvions de Linexert, St-Germain, Franchevelle. - Lisiẻre vosgienne de l'arrond. de Nontbéliard à Fresches, Dampierre, Elupes.- Gneiss et granites de la longue 
chaine qui s'étend depuis le Morvan à travers les montagnes charollaises, màconnaises, beaujolaises, lyonnaises, foréziennes jusqu'au Pilat; puis sur les mêmes terrains avec schistes siliceux du Vivarais, des Cérennes, de l'Espinouse et de la Montagne Noire. - Dans le massif du Laurenti à la Baouzeille du Tarbézou, dans la vallée de la Lauze.

En dehors de nos limites, la Digitale pourprée existe sur les terrains siliceux des Pyrénées centrales, Aubrac, Lozère, Margeride et toutes les montagnes du massif central; s'étend dans l'ouest depuis le Nontronais, l'Angoumois, lo Poitou' l'Anjou et le Limousin jusque dans la Bretagne et la Normandie. Dans le N. E. on la voit sur les versants alsaciens et lorrains des Vosges, sur les grès verts de la Champagne, les schistes des Ardennes. - Elle existe en Corse, Sardaigne, Espagne, Portugal, Belgique, Hollande, provinces rhénanes, Wurtemberg, Fichtelgebige, Thuringerwald, Harz, Grande-Bretagne, Danemark, Suède et Norvége.

Manque dans la chaine jurassique, les Alpes françaises, helvétiques, italiennes, dans la Bavière mérid,, le Tyrol, Salzburg, Styrie, Carinthie, Hongrie, Transilvanie, Carniole, Croatie, Slavonie, la péninsule turco-hellénique, la Russie, l'Italie et, en un mot, dans toute la partie sud-est de l'Europe.

D. parvimora All, D. lutea L. - Bois, taillis, lieux pierreux des coteaux et des montagnes, surtout, mais non exclusivement, sur les terrains calcaires. - Chaine des Vosges et du Jura jusque dans le Bugey. - Vaud, Valais, Savoie, Dauphiné, B.-Alp., Alp.-Mar., Vaucl. au mont Ventoux et au Luberon, Var et B.-du-Rh. à la Ste-Baume, Pic de Bretagne, les Mayons. C.-d'Or et S.-et-L.: toute la Côte jurassique, l'Auxois, régions calcaires de l'Autunois, du Mâconnais, Beaujolais, mont d'Or lyonnais, Vivarais. - Gard : le Vigan, Serre-de-Bouquet, Chartreuse de Valbonne. - Hér.: calc. jurass, deș vallées de l'Hérault, de l'0rb et de leurs affluents. - Aude, Pyr.-Or. et Ariége : Corbières, vallées d'Eyne, de Llo, de Vernet-les-Bains, vallée de l'Aude, Fach, Rouze, Soucarrat, vallons de Councq et de Canals.

D. purpuren-lutea Mey., D. purpurascens Roth. - IIybride des deux précédents. - C.-d'Or, S.-et-L.: Semur, Canada d'Auxy, Azé près Jlacon, Pruzilly, Issy-l'Evêque. - Loire : eutre Chavanay 
et Pélussin, entre Terrenoire et Rochetaillée, de la Terrasse au col de Pavézin, St-Paul-en-Jarret. - Gard et Hér.: Anduze, Trèves, entre Hérépian et St-Gervais-sur-Ilare.

D. grandiflora $\mathbf{A}$ ll. - Bois, taillis sur tous les terrains, - Chaîne des Vosges jusque dans la H. Saone à Champagney, Mansvillers, monts de Vannes et de Ternuay, Ballon de Giromagny. - Commun dans la chaine jurassique jusque dans le Bugey, Vaud et Valais, Savoie, Dauphiné, B.-Alp., Alp.-Mar. - S.-et-L., Rh., Loire: Cluny, Charolles, le Beaujolais, Theizé, Cogny, Soucieu, Courzieu, St-Bonnet-le-Froid, Fenoyl, Chayanay, Pélussin, Soulages, Valfleuri, Terrenoire, Rochetaillée, Pierre-sur-Haute. Ard.: l'Escrinet.

D. Iuteo-grandiflora Bavoux, D. media Roth. - Doubs et Jura : Chapelle-des-Buis près Besançon, Salins. - Is. : entre Pariset et St-Nizier. - H.-Sav.: mont Brizon.

aUPHaAs A offeinaliğ L. - Prés et pâturages des plaines et des montagnes. Espèce très-polymorphe dans laquelle on distingue les formes suivantes qu'on peut répartir en deux groupes : $1^{\circ}$ le groupe officinalis L. ; 20 le groupe nemorosa Pers.

\section{Groupe officinalis.}

Fleurs grandes. Corolles à tube saillant hors du calice.

E. campestris Jo!d. - Differe du type officinalis par une pubescence plus courte et plus glanduleuse et par la capsule plus longue que la feuille axillante. Assez commune dans tout le bassin. Cependant, comme la plupart des observateurs ne se sont pas appliqués à la distinguer, il n'est pas possible de donner un aperçu complet de sa distribution géographique.

E. uliginosa Ducon. - Variété peu importante qui diffère du type par les bractées moins acuminées.

E. montana. Jord. - Peut être considérée comme étant la forme montagnarde de l'E. campestris dont elle differe par sa grappe plus manifestement interrompue à la base, par ses feuilles plus larges et obtuse̊ment dentées. - Cette forme a été observée dans les chaines du Pilat, du Forez, des monts Dômes, monts Dore, Cantal, Lozère, Tarn, Montagne-Noire, Aveyron, dans 
I'Hérault à l'Espinouse autour de la Salvetat, Andabre-Rosis, Fédou. - Elle est indiquée aussi dans les $\Lambda$ lpes du Dauphiné et de la Savoie; est-ce bien la même forme que celle du centre de la France?

E. alpin Lam. - Forme très-distincte des précédentes par ses feuilles et son calice glabres, par sa corolle d'un violet pourpre, ses capsules fortement échancrées, munies d'un long mucron. On pourrait la considérer comme la forme grandiflora de l'E. salisburgensis; ou, vice versa, on pourrait dire que l'E. salisburgensis est la forme parviflora de l'E, alpina.-Valais : Simplon. H. et B.-Alp. : mont Genèvre, mont Viso, col Agnel, Ruines, col de Vars, Orcières, Malmore, Fouillouse, Lauzanier, Bachasse, Parpaillon, Longet. - Vaucl.: mont Ventoux. Alp.-Mar. : col de Tende, mont Lachen. - Pyr.-Or. et Ariẻge : mont Louis, Carença, vallée de Llo, Collada de Nuria, vallons du Laurenti, de Boutadiol et de Barbouillère, Ascou, Mijanès.

Groupe nemorosa.

Fleurs petites, Corolles à tube inclus dans le calice.

E. hirlella Jord. - Tiges, feuilles et calice couverts d'une pubescence grisâtre, mêlẻe de poils glanduleux. Valais: Simplon. - H. Sav. et Sav. : montagnes du Chablais, Cornettes de Bise, Signal des Agneaux, Roc d'Enfer, montagnes de Sixt, col de Golèse, vallée du Reposoir vers le chalet de Sommier, montagnes d'Aime. - Is. et H.-Alp. : Chamechaude, Villard-de-Lans, Taillefer, la Grave, Lautaret, col de Vars, mont Viso, Devez de Rabou, Ventavon. - Alp.-Mar.: Estenc, sources du Var. Ariège: bassin supérieur de l'Aude, Rouze, vallon de Riplaou, Canáls, Councq, Barbouillère, Quérigut.

E. puberula Jord. - Diffère du précédent par sa pubescence non mêlée de poils glanduleux. - H. et B.-Alp. : la Vallouise, mont Genc̀vre, val Préveyre, Ségure et la Monta en Queyras, Annot au col de Vergons. - Alp.-Har.: Entraunes.

E. rigidula Jord., E. gracilis Fries. - Feuilles et calice à poils très. courts appliqués, non glanduleux; corolle d'un pourpre lilas. - Loire: chaine de Pilat et de Pierre-sur-Haute. - Hér. : la 
Salvetal, Fraisse, St-Amand-de-Mounis, Avène, Pardailhan. S.-et-L. : le Morvan, Roussillon. - Ard. : Thueyts. - Ariège : vallée de l'Aude à Quérigut, Mijanès, vallon de Canals.

E. mainlis Jord. - Diffère du précédent par le calice plus poilu, à dents plus acuminées, par sa capsule non émarginée. - Envir. de Besançon, sur le plateau de Trois-Châtels. - S.-et-L. : Montjeu près Autun, Roussillon en Morvan. - Gard et Hér. : envir. du Vigan, Aumessas, le Caylar. - Environs de Lyon sur les collines des bords du Rhone. - Is. et H.-Alp. : Pariset près Grenoble, chalet du Gleyzin près d'Allevard, Pic du Bec audessus de Villard d'Arène.

E. cuspidata, E. ericelorum Jord. - Se distingue par ses tiges noiràtres couvertes d'une pubescence fine et non glanduleuse, sa grappe dense, ses feuilles supérieures à dents cuspidées - Celte forme est assez commune dans les prairies et pâturages des collines et des montagnes de la chaîne jurassique, C.-d'Or et S.-et-L. ; dans la Côte de Dijon à Beaune, Marcellois, le Morvan autunois. - Chaine du Beaujolais, Forez, Lyonnais, les montagnes du Dauphiné, les Cévennes du Gard et de l'Hérault à l'Espérou, l'Escandorgue et l'Éspinouse, la Montagne Noire. H.-Sav, et Sav. : Petit Salève, le Coin, Avenières au-dessus de Cruseilles, le Vuache, montagnes d'Abondance, de Bonneraux, de Thônes, le Magériaz et le Roc de Chere près du lac d'Annecy, Chamonix, Servoz, montagnes de Hauteluce et des envir. d'Albertville. - Ariège : Rouze, Jasse de la Bentaillole, Pic de Canrusc.

E. nitidula Reut. - Tige grosse, brune à pubescence non glanduleuse; capsules larges, fortement émarginées arec un mucron aussi long que l'échancrure. $\rightarrow$ Doubs à Pontarlier; Vaud entre St-Cergues et la Dôle; Valais au Simplon. - Ariègo: Mijanès au vallon de Canals, Barbouillère, Jasse d'Espagne.

E. cuspidatissima, E. salisburgensis Funk, - Tige ordinairement rameuse dès la base, à pubescence fine, non glanduleuse; feuilles glabres d'un vert clair; dents des feuilles florales trois fois aussi longues que larges et toutes cuspidées. - Pâturages de la chaine du Jura helvêtique et français: Chasseral, Creux-du- 
Van, Chasseron, Mont d'Or, Noirmont, la Dôle, Colombier, Faucille, Reculet, Colombier du Bugey, montagnes autour de Hauteville. - Vaud et Valais : Alpes de Bex, Fully, Bois-Noir, Trient, Zermatt, Saas, Simplon. - II.-Sav. et Sav. : Salève, la Roche, Cuvat, Dents d'0che, Abondance, Bonnevaux, Abbaye de Vallon, vallée du Fier, Parmelan, Charvin, vallée de Chamonix, chaine du mont Blanc, mont Iseran, mont Cenis. Is. et II.-AIp. : grotte des Échelles, la Ruchère, la Bérarde, Villard d'Arène, Valpréveyre en Queyras, Boscodon, Champo-. léon, Valgaudemar, Orcières, Chaudun, Séuse, Aurouse. B.-Alp. : la Condamine, Parpaillon, Bachasse, Annot. Alp.-Mar. : Entraunes, St-Martin-Lantosque, Ste-Anne-de-Vinaï. - Vaucl. : mont Ventoux. - Hér. : le Caylar et l'Escandorgue. - Ariège et Pyr.-Or. : Quérigut, Carcanières, Pic de Peyre-Planque, le Capcir et probablement les hautes vallées des Pyr.-0rientales.

E. cupraea Jord. - Diffère du précédent par sa tige noirâtre, à rameaux ćtalés, à feuilles d'un vert sombre et comme cuivré. Grenier et quelques auteurs l'identifient avec l'E. salisburgensis. Il me parait qu'il y a avantage à maintenir la distinction, surtout si, comme je le fais, on considère toutes ces Euphraises comme des races appartenant à une seule espèce l' $E$. officinalis.

- L'E. cupraea ne s'élève pas aussi haut dans les montagnes 'que l'E. salisburgensis et se montre de préférence sur les collines peu élevées ou quelquefois dans les parties infẻrieures des vallées montagneuses; il est, d'ailleurs, assez commun dans une grande partie de notre bassin.

E. minima Jacq. - Tige naine; pubescence non glanduleuse ; corolle à lèvre supér. lilas, à lèvre infér. jaune. - Chaine jurassique: Montendre, la Dole, Noirmont, Colombier, Reculet, pied oriental du Vuarne. - Valais : mont Fully, Catogne, St-Bernard, Esserz, Diez, Arolla, Alpes de Zermatt et de Saas, Simplon, Grimsel. - H.-Sav. et Sav. : Mốle, glacière du Brizon, Vergy, Méri, montagnes de Samoëns et de Sixt, Dents d'Oche, mont Hermante, Nautau, Hautigny, Semnoz, Charvin, Brévent, Aiguilles-Rouges, chalne du mont Blanc, les Allues, Hauteluce, 
Crest-Voland, les Motte!s. - Is. et H.-Alp. : chatnes des SeptLaux, de Belledonne, Prémol, Colon, la Moucherolle, l'obiou, Villard-d'Arène, Alp-Martin, Lautaret, Cervières, mont Viso, Orcières, Chaillol, pic de Chabrières. - B.-Alp. et Alp-Ilar.: Lauzanier, Plate-Lombarde, Fouillouse, col de la Madeleine, Alpes de St-Étienne, col de Fenestre, lac d'Entrecoulpes, col de Tende. - Pyrénées, chaines des monts Dore, Dômes, Cantal.

Odontritrs rubra Pers. - Hoissons dans tout le bassin depuis les plaines jusqu'aux montagnes. Espèce polymorphe.

0. serotina Rchb. - Diffère du précédent par les feuilles plus étroites, moins profondément dentées, les feuilles florales moins longues; floraison plus tardive. Même habitat.

Dans la var. divergens Jord., les rameaux sont plus étalés.

0. viscos $R$ Rchb. - Lieux arides. - Vaucl. et B.-du-Rh. : envir. d'Avignon, de Carpentras, de Flassan, de Bedoin, d'Aix et de Mar'seille, bords de l'Arc, Montaiguez, chaine de l'Etoile, vallon de St-Pons. - Alp.-Har.: Ste-Anne-de-Vinaï. — Gard : Villeneuve-les-Avignon, Campestre. - Aude et Pyr.-Or. : envir. de Narbonne, Port-Vendres, Paulille, Banyuls-sur-Ner, St-Laurentde-Cerdans, Trencada-d'Ambulla, Vernet-les-Bains. - On prétend l'avoir trouvé entre Modane et Bramans en Maurienne et dans les H. et B.-Alp. à Ribiers et à Annot.

o. alpestris Jord. Fourr. - Forme du précédent observée dans la Dr. et les H.-Alp. vers le Buis, entre Crest et Beaufort, la Saulse, mont Genèvre, Briançon. - Valais : de Sierre à Varen, bois de Finge.

D. Iutea Rchb. - Coteaux arides dans tout le bassin.

0. lanceolata Rchb. - Forme montagnarde qui diffère du type par les bractées plus longues, par les poils glanduleux qui hẻrissent le calice, et enfin par les feuilles plus larges. Remplace l'o. lutea dans les moissons de la partie supér. des vallées de l'lsère, de l'Arc, de la Romanche, de la Durance, de l'Ubaye, du Verdon, du Var, de la Tinea, de la Vesubia, de la Roja et autres rivières de la Savoie, de l'Isère, des H. et B.-Alp., Alp.-Mar.; montagnes des envir. de Gap, d'Annot et de Grasse.

BA RTSCHMA alpina L. - Cette espèce est une des plus gẻné- 
ratement répandues sur les sommités du Jura helvétique et français, des Alpes de Vaud, Valais, Savoie, Dauphiné, B.-Alp. et Alp.-Mar., Pyr.-Or., Ariège. - En dehors de nos limites dans les Pyr.-Centr., le Plomb du Cantal, les Vosges au Hohneck.

B. spleata Ram. - Bords des bois, licux arides. - Pyr.-Or.: montagne de Céret, St-Laurent-de-Cerdans, Rigarda, dans les ravins du bois de St-Sauvcur. - Pyr.-Centrales.

I. trixngo L., Trixago apula Stev. - Lieux arides, bois de la rẻgion mérid.-B.-du-lih.: Cuques, Marseille, Rassuen, Arles. Var. et Alp.-Mar.: Toulon, Hyères, le Luc, Cannes, Ile SteMarguerite, la Roquette, Antibes, Villefranche au cap Ferrat, Beaulieu. - Gard et Hér.: bois de St-Nicolas près de la Grange, Montpellier au Mas de Bosc près de Baillargues, Cette, plage de Sérignan. - Aude et Pyr.-Ur.: envir. de Narbonne, Fontfroide, Quillanet, Port-Vendres, Banyuls-sur-Mer. - Littoral de l'Océan.

B. viscona L., Eufragia riscosa Benth. - Sables humides de la région méditerran.-B.-du-Rh., Var. et Alp.-Mar.: Entressen, Ia Camargue, Ceinturon d'Hyères, Cannes, ille Ste-Marguerite, la Roquette, Antibes, Nice au Var, Menton au cap Martin. Hér.: Cette. - Bords de l'Océan.

B. Intifolia Sibth., E. litifolia Gris. - I.ieux sahlonneux de la région mérid.- B.-du-Rh.: envir. d'Aix, bords de l'Arc, vallon de la Guiramande, St-Tronc près Marseille, bois de Mazargues, Ste-Victoire, - Var. et Alp.-Mar.: Morières près Toulon, le Luc, les Mayons, l'Esterel, Grasse à St-Jacques, Cannes, Antibes, Nice au Var, Berre, la Fraca. - Gard et Hér.: toute la partie basse des arrond. de Nîmes, d'Uzès, Palavas, Mauguio au Grand-Travers, plaine de Juvignac à Caunelle, Cette à Villeroi, St-Pons.-Aude: Carcassonne._Littoral de l'Océan et une partie du sud est de la France.

RHINANTIOS majus Ehrh. - Prairies des plaines et des montagnes. Le calice est tantot glabre, $R$. glabrum, tantôt velu, R. hirsutum.

R. minus Ehrh, - Prairies des montagnes. - Chalnes des Vosges 
ct du Jura, Taud, Valais, Savoie, Dauphiné, B.-Alp. - Morvan, montagnes du Forez et du Vivarais. - Gard : envir. du Vigan, Concoule. - Pyr.-Or., Ariège, Pyr. centrales, montagnes du centre de la France. - Espèce polymorphe.

Une variété angustifolium est quelquefois mêlée au type.

$R$. alpinum Baumg., $R$, angustifolium Gmel. - Cette forme remarquable par ses bractẻes acuminées et fimbriẻes, ainsi que par la lèvre supér. de la corolle fortement courbée-ascendante, se trouve dans l'Ain et le canton de Vaud au-dessus de Dironne, Gimel, St-Georges, Vallorbes, liontreux, Leysin. - Valais : Findelen près Zermatt, vallẻe de Saas. - H.-Sav. et Sav.: Salève, Mont-Cenis.

PEDrCuLAris vertlcillata L. - Prairies humides des Alpes et des Pyrénées. - Vaud et Válais: Alpes d'Aigle et de Bex, Jaman, Bovonnaz, Anzendaz, Panérossaz, Cheville, les Diablerets, St-Bernard, Pierre-à-Voir, Catogne, Zermontana, Thyon, Vende, mont Nuoble, Pas-du-Loup, Alpes de Zermatt et de Saas, Simplon, Gemmi, Hainghorn, Oberwald, Glacier du Rhone. H.-Sav. et Sav.: Môle, Vergy, Méri, Brizon, Charvin, Tournette, nontagnes de Samoëns, Cornettes de Bise, Dents d'0che, mont d'Ubine, mont Ardin, Hautigny, Signal des Agneaux, mont Pétetod, $\Lambda$ iguilles-Rouges, chaîne du mont Blanc, le Bonhomme, col de la Seigne, la Gita, les Mottets, Roselein, mont Joly, mon. tagnes d'Aime, de Tignes, des Allues, Crest-Yoland, Hauteluce, montagnes de la Maurienne jusqu'au Mont-Cenis. - Is, et H.Alp.: chaines des Sept-Laux, de Belledonne, mont de Lans, Pelvoux, la Salette, l'Obiou, la Grave, Lautaret, Briançonnais, mont Genèvre, Queyras jusqu'au mont Viso, St-Véran, col de Vars, mont Aurouse. - B.-Alp. et Alp.-Jlar.: Serennes, Malemort, Longet, Horonaye, Entraunes, Jallorgues près St-Dalmasle-Saurage, col de la Mladdalena. - Pyr.-Or.: Mont-Louis, col de la Perche, Nohièdes. - Pyr. centr., Plomb du Cantal au PuyMary.

P. follosn L. - Prairies et pâturages des montagnes. - Chaine des Vosges. - Jura helvétique et français: Chasseral, Reculet au vallon d'Ardran, creux du Noirmont au-dessus des Rousses. 
- Vaud el Valais: Jaman, Bovonnaz, Croumaclire, Chamosaire, Cheville, Proz, l'drpettaz, val Ferret. - H. Sav. et Sav.: Dents d'Oche, Cornettes de Bise, Signal des Igneaux, Hautigny, mont d'Cbine, Roc-d'Enfer, mont Pétetol, montagrie de Samoëns et de Sixt, col de Golese, vallee du Reposoir, Tournette, les Ayers sur Servoz, St-Germain, Jontmin, la Sambuy, Laval-de-Tignes, montagnes d'Aime, mont Trélod en Bauges, Mont-Cenis. - Is. et H.-Alp.: colde la Ruchère, Borinant, Chapelle-en-Taljouffrey, Lautaret, Vallouise, mont Genèrre, mont Gondran, mont Viso, Vars, Séuse, col de Glaise, la Grangette, Durbonnas. - B.-Alp. et Alp.-Mar.: Lauzanier, Malemort, Parpaillon, Ste-Anne, vallon de Nauduébis prẻs St-Martin-Lantosque. - Pyr.-Or. et Ariège : Munt-Louis, Bac de Bolquẻre, vallée d'Eyne et de Tohèdes, Canigou, Pla Guillem. Prats-de-Jollo, Coma du Tech, Salvanère, Balcera en Capcir, Laurenti, vallon de Paillères à Sou carrat, la Waouré, Councq. - Pyr. centrales, Plomb du Cantal, Puy Mary, monts Domes, montagnes du Forez, Pierre-surHaute.

P. recutitn L. - Prairies humides des Alpes. - Valais, St-Bernard à la Baux, la Laivraz, les Herbagères du col de Balme, col de Fenétre, la Lex, Anniviers, Glacier du Rhơne, Grimsel, Simplon, St-Gothard. - Sar.: Bas du Mottet entre Aime et le Crêt du Ré, lac de la Giroltaz, mont Mirantin à l'Haut-de-Tours.

P. rosea Wulf. - Rochers des Alpes. - Sav.: Iaval-de-Tignes, mont Iseran, Entre-deux-Eaux, l'Ecot, Jlont-Cenis à Ronches et au lac Blanc. - H. et B.-Alp.: col de Buffert au-dessus du Monestier-de-Briancon, col des Désertes au-lessus de Yérache, Granon près Briancon, Terre-Xière près Cerrières, mont Pelras, val Préreyre et val Ségure en Queyras, haute vallée du Guil, vallons de Ruine et de la Taillante, crêtes de Ségure, col Agnel, col de Péas, St-Yéran, Croùè près la Condamine. - Il est digne de remarque que cette Pédiculaire manque dans les Alpes de la Suisse; elle existe dans les Alpes du Tyrol, Styrie, Carinthie, Autriche, Lombardie.

P. polustris L. - Prairies marécageuses el tourbeuses de la plaine et des basses montagnes. 
P. sllvatiea L. - Prairics liumides; beaucoup plus commun que le précédent dont il se distingue par ses tiges ètalées sur lo sol et par son calice à cinq lobes inégaux.

P. comosa L. - Prairies des montagnes, - H.-Sav., Sav., Is. et Dr.: col de Balme, mont Lachat, Pavillon de Bellevue, mont de Fréjus, Mont-Cenis, les Bauges, mont Nivolet, Orizan sur Cléry, mont St-Michel près la Mure, la Salette, la Croix-Haute, les Lussettes. - H. et B.-Alp.: la Grave, Lautaret, Monestier-deBriançon, Névacle, la Vachette, mont Gondran, col du Bourget au-dessus de Cervières, Guillestre, cols de Vars et de Glaise, la Grangette, mont Séuse, Durbonnas, mont Rognouse, Serennes, col de Vergons près Annot. - Alp.-Har.: Ste-Anne-de-Vinaï, col de Fenestre, Alpes de Tende, col de Raus. - Vaucl.: mont Ventoux. - Pyr.-0r. et Ariège: Canigou à la Jasse de Cady, Coma du Tecl, vallée d'Eyne et d'Evol, Laurenti en montant à la Porteille de Baxouillade, lac Noir, vallons de Barbouillère et de Valbonne, Port de Paillères. - En dehors de nos limites dans les Pyr. centr., Plomb du Cantal, mont Dore, monts Dômes, H.-Loire, Lozère. Celte Pédiculaire manque dans les Alpes de la Suisse. - On la trouve dans les Alpes de Lombardie, Vénétie, Tyrol, Hongrie, Transilvanie, Gallicie.

P. Incarnata Jacq. - Prairies des Alpes. - Valais : Chamosaire, Fully, St-Bernard à la Baux, Zermatt, Simplon, Grimsel. H.-Sav, et Sav.: Cornettes de Bise, mont Charvin, Bonhomme, Saut-des-Allues, mont Cenis. - Is. et H.-Alp.: la Pra de Belledonne, St-Mury-de-IIonteymont près du lac Blanc, Taillefer, Alpe du mont de Lans, la Salette, Lautaret, Buffera, mont Genèvre, Valpréveyre et Ségure en Queyras, mont Viso à la Traversette, St-Véran à Blanchet, col de Vars, Orcières. - B.-Alp. et Alp.-Mar.: Lauzanier, Horonaye, Bérard, Longet, Alpes d'Entraunes, col de Salèse, lac d'Entrecoulpes, cols de Tende et de Raus, l'Authion, Saorgio. - Manque dans le reste de la France.

P. pyrenaica Gay. - Prairies et pâturages des Pyrénćes. - Pyr. Or. et Ariège : vallée d'Eyne, Cambres d'Aze, lac de Nohẻdes, col de las Nou-Fonts, Prats-de Mollo pris de la Tour de Mir, 
Canigou sur le plateau de Cady, vallée de Galba en Capcir, lacs du Laurenti, de l'Estagnet et de Valbonne, Boutadiol, Barbouillere, Cirque des Aiguettes et d'Artounant, Pic del Ginèvré, la Bentaillole. - Pyr. centrales.

P. cenisia Gaud. (apple lie à tort $P$. gyroflex

- Prairies des Alpes. - Sav.: cols du Bonhomme et des Fours, Tignes, Cirapieu, les Allues, mont Cenis.-H. et B.-Alp.: Lautaret, Villard-d'Arène aux Trois-Evêchés, Galibier, col du Chardonnet, col de Névache, Pic de Chabrières, Malemort, Parpaillon, Fouillouse. - Alp.-Mar.: Salsamorena.

IP. Eyroflexn Vill., P. fasciculata Bell. - Prairies des Alpes. Valais : St-Bernard å l'Ardifagoz, bas des lacs du col de Fenètre. - Sav.: col de la Seigne, le Chapieu, mont Cenis à Ronches, mont Granier. - Is. et Dr.: col de la Ruchère, col Vert, col de l'Arc, plateau de Chamousset derrière le mont Aiguille, la Croix-Haute. - H. et B.-Alpes. : Lautaret, mont Genévri', St-Véran, vallon Agnel, Boscodon, Pic de Chabrières, col du Noyer, Orcières, Durbonnas, la Grangette, mont Aurouse, Toulnoux, Horonaye, Parpaillon, Malemort, mont Grand-Couyer sur Annot, les Dourbes près Digne. - Alp.-Mar.: Entraunes, lac de Rabuons près de St-Etienne, vallon de Fenestre, StcAnne-de-Vinaï, col de Tende.

P. ntrorubens Schl. - Prairies humides du Grand St-Bernard à la Baux, val Ferret, mont Cenis.

P. rostrata L. - Pelouses des terrains siliceux des Alpes et des Pyrénées. - Valais : St-Bernard, Zermontana, Cleuson, Thyon, Vouasson, Diez, Arolla, Maya, Sasseneire, Alpes d'Anniviers, de Zermatt et de Saas, Simplon, Tortain, Sirwolten, Albrun, Bodmeralpen, Grimsel. - II.-Sav, et Sav.: col de Balme, chaine du mont Blanc au Montanvert et sur les moraines des glaciers, Griaz, Taconnaz, chaines des Aiguilles-Rouges, mont fovet, mont Iseran, mont de Fréjus, col de la Seigne, Allée-Blanche sur le versant italien, mont Cenis. - Is, et II.-Alp. : chaine de Belledonne vers les lacs Doménon, Taillefer, l'Alp. de Villardd'Arène, Cervières à Terre-Nière, Buffera sur Névache, col de l'éas, Ccillac à la Collelle-Verte, Valprẻveyre et Ségure en 
Qucyas, mont Viso, Champoléon. Chaillol. - B.-Alp. et Alp.Mar.: Lauzanier, Vallonnet, Longet, sommités des vallées du Var, de la Vesubia, Tinea et Roja. - Pyr.-Or. et Ariège: Cambres d'Aze, vallées d'Eyne, Pla Guillem, val de Paillères à Soucarrat, mouillère de Riplaou. - Pyr.-centrales.

La forme P. Letourneuxiana Personat, qui a le calice très-velu, les pédoncules égaux au calice, a été trouvée đans la chaine du mont Blanc, sur les rochers de l'Aiguilles des Charmoz, et au Jardin de la Mer-de-Glace.

P. tuberosa L. - Prairies des Alpes et des Pyrínées. - Vaud et Valais: Anzendaz, Boulaire, Panerossaz, les Diablerets, val d'Illiez, Catogne, Chermignon, Cotter, Loze, Thyon, Tortain, Findelen, Simplon, monte Moro, Glacier du Rhône, Grimsel. H.-Sav. : Salève à la Croisette, Cornettes de Bise, montagnes de Six, Platets au-dessus de Passy, chaîne du mont Blanc, pavillon de Bellevue, mont Lachat, col Joly, Charvin, Bas du Mottet audessus d'Aime, Plan-des-Dames et la Sauce près du Bonlomme, Hauteluce, mont Cenis. - Is. et H.-Alp.: chaînes des Sept-Laux et de Belledonne, col Odie, Champrousse, Taillefer, Alpe du mont de Lans, la Salette, mont Sénèpe, Lautaret, Galibier, Prorel près Briançon, haute vallée du Guil au pied du mont Viso, vallon de la Taillante, Lopet, Orcières, Chaillol, Champoléon - B.-Alp. et Alp.-Mar.: Tournoux, le Garret au-dessus d'Entraunes, Sainte-Anne-de-Vinaï. - Il est douteux que cette Pédiculaire existe dans les Pyr.-Or. et l'Ariège où elle est indiquée par Lapeyrouse et par Companyo. - Espéce polymorphe.

P. Barrelieriana Rchb. - Forme glabre, à lobe du calice entiers. Valais : Chemin, Gemmi, Bellalp, Pierre-à-Voir. - H.-Sav. : Dents d'Oche, mont d'Ubine, mont Ardin, Signal des Agneaux, Roc d'Enfer, Hautigny, mont Pétetod, col de Golèse, montagnes de Samoëns, Buet, col de Balme, pavillon de Bellevue, Aiguilles de Varens, vallon d'Entre-les-Eaux. — Sav.: col de la Madeleine, Hauteluce, la Sambuy, les Bauges, mont Trélod, mont Drizon, l'Arclusaz au-dessus de Saint-Pierre d'Albigny, mont Granier.

MELANIPYN cristatum L. - Collines boisées des parties septentr. et moyennes du bassir. Dans la région mérid. il 
occupe exclusivement les montagnes, celles de Grasse, de l'Espérou, de Lodéve, des Pyr.-Orientales.

7r. arvense L. - Champs cultivés des terrains calcaires. La couleur des fleurs et des bractées varie.

M. nemorosum L. - Bois. - Manque dans les Vosges et le Jura septentr. - Ain: de Culoz au Colombier du Bugey. - Sav. et II.-Sav. : montagnes du Chablais, du Faucigny, de la Tarantaise et de la Maurienne. - Is. : montagnes de la GrandeChartreuse, de Chalais, de St-Nizier, Prémol. - II., B.-Alp. et Alp.-Mar. : montagnes des envir. de Gap, Tournoux, vallées du Var, de la Vesubia et de la Tinea. - Gard: Alzon, bois de Salbous près de Campestre. - Hér. : St-Pons, le Caylar.

Tr. pratense L. - Bois et taillis des plaines et des montagnes.

R. allvateun L. - Forêts de sapins des montagnes des Vosges, chaine jurassique, Alpes de Vaud, Valais, Savoie, Dauphiné, B.-Alp. et Alp.-Mar. - Gard : Alzon et l'Aigual. - Pyr.-Or. et Ariège : le Capcir et le Laurenti.

'TozzHA alnina L. - Bois et taillis des montagnes. - Nul dans les Vosges. - Jura helvétique et français: Passwang, Weissenstein, Chasseral, Creux-du-Van, Clıasseron, Noirmont, Montendre, la Dôle, Faucille, Colombier, Lélex. - Vaud et Valais : Jaman, les Plans, Ormonds-Dessus, Solalex, Bovonnaz, Dent du Midi, Champéry, l'Eau-sur-Vouvry, l'Etroz, Simplon, Glacier du Rhone. - H.-Sav. et Sav. : Dents d'Oche, col de Golèse, montagnes de Sixt et de Samoëns, Roc d'Enfer, plateau des Gets, Cornettes de Bise, mont des Granges, rochers de Flaine et de Véron, mont Ardin, Haut-de-Lin, bois de Sommier au pied du mont Méri, la Tournetle, Charvin, Galopaz au-dessus de Puisgros, col de la Mladelcine, glacier des Cerces. - Is. : CharmantSom, Grande-Chartreuse à Vallombrey et au Collet, GrandeVache, Gigneux au-dessus de la Placette, St-Eynard, entre St-Ange et le col de l'Arc, cascarle de l'Oursière au-dessus de Revel, Haut-du-Pont près Allevard. - H.-Alp: vallée du Guil au bois de l'Echalp près de l'Envers, bois de Pilat, la forêl de Pennin près d'Aiguilles, Durbon. - Alp.-MIar. : col de l'Abisso, vallon de Nanduébis près St-Martin-Lantosque, col de Fremamorta, Ste-Anne-de-Vinaï. - Pyr. centrales, Cantal. 


\section{OROBANCHÉES}

PHELTEA crerulea C. A. Mey. - Parasite sur les racines de l'Achillios millefoliatus. - Doubs et Jura : Grand-Combe-desBois, Consolation, bords du Doubs à Parcey et à C.haussin, Salins, Baume près Lons-le-Saunier, Dole. - Vaud et Valais: Rolle, Branson, Folatères, entre Sembrancher et Vence, la Morge, Tourbillon, Mont-d'Orge, Platlaz, Géronde. - H-Sav. : les Voirons, Vacheresse, Hatere-Lullin, Petit-Brogny, Annecy-le-Vieux. - Is., Dr. et II.-Alp.: le Sappey, pic du Loup au-dessus de Vif, Clavans, Mizoën, Abriès, Fontgillarde, col Agnel, Crest, Aucelon. - B.-Alp. : vallon du Bourget près Bousollières. - Vaucl. et B.-du-Rh. : mont Ventoux, Apt, le Luberon, Cucuron, graviers de la Durance, la Ste-Victoire, - Var et Alp.-Jlar.: Toulon, St-Raphaël, Cannes, St-Martin-Lantosıue. - C.-d’Or et S.-et-L. : Meursault, Beaune, Bèze, Saulieu, le Morvan, Curgy, Cluny. - Pyr.-Or. : Trencada d'Ambulla, Céret.

$\boldsymbol{P}$. casia Reut. - Parasite sur les racines de l'stremisia gallica el du Camphorosma monspeliacum. - B.-du-Rh. et Var: Ratoneau, Pomègue, Montredon piès Marseille, envir. d'Aix, mont Sauvette près les Mayons. - Gard el Pyrr.-Or.: Aigues-Mlortes, Molitg, Banyuls-sur-Mer.

P. arenaria Walp. - Parasite sur les racines de l'Artemisia campestris. - Canton de Genève à Sezegniens. - Valais et H.-Sav. : Martigny, Sierre, Chamonix, Courmayeur sur le versant italien, St-Andrẻ dans le val du Fier. - Rh. et Ain : entre Villefranche et St-Georges, le Vernay près l'Ile-Barbe, la Pape, Néron. Is. : Beauregard au-dessus de Pariset, Huez en Oisans. - Alp.Mar. : entre Duranus et Figaret, col de Braus, Tende. - Gard : bois de Broussan près Nìmes, la Pinède-des-Saintes près AiguesMortes. - Hér. : Palavas, Cette, Roquehaute, Lamotte. - Aude ct Pyr.-0r.: Leucate, graviers de la Tet vers St-Estève.

P. olbiensis Coss. - Parasite sur les racines de l'II. stoechadense. - Ile de Porquerolle près Hyères.

P. Iavandulacea F. Schultz. - Parasite sur les racines des 
Psoralea biluminosa, Thapsia villosa. - B.-du-Rh. et Var: Ste-Victoire, le Luc, St-I'ropez, St-Vallier, Nice à St-Roch et à Brancolar, Baus-Rous prìs de Villefranche. - Pyr.-0r.: Caladroy.

R. ramosa C. $\boldsymbol{A}$. Mey. - Parasite sur le Chanre, le Tabac et quelques autres plantes dans tout le bassin.

R. Muteliann Reut. - Forme du précédent olservie sur diverses Composées et Papilionacées de la rẻgion mérid. - B.-du-Rh. et Var: envir. d'Aix, de Narseille, Toulon, Hyères, le Luc, St-Tropez, Antibes, la Roquette, Nice, Mlenton. - Gard et Hér.: envir. de Nimes, lu Vigan, de Iontpellier, Lavérune, Grabels, Mauguio, Castelnau, Cette, Castries, Lunel, Béziers, Mèze, Pardailhan, Pézenas. - Pyr.-0r.: Argelìs, Collioure, PortVendres, Banyuls-sur-Mer.

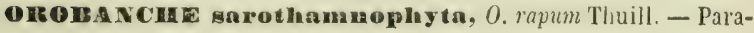
site sur les Sarothamnos vulgaris et purgans dans tout le bassin.

D. crinita Viv. - Parasite sur les racines du Lotos cytisoideus. Var. : presqu'île de Gien, île de Porquerolle.

D. eruenta Bertol. - Parasite sur les racines des Papilionacées, particulièrement sur les Genêts.

D. variegata Wallr. - Parasite sur les racines des Genêts, Coronilles et Antbyllides. - B.•du-Rh., Var et Alp.-Mar. : Ste-Victoire, la Ciotat, Fréjus, St-Martin-Lantosque, entre Tende et Lupega, entre Fontan et Tende, entre l'Escarène et Sospel. Gard: envir. du Vigan. - Pyr.-0r.: vallée du Rẻart et de Caladroy, les Albères.

- speciosa DC. - Parasite sur les racines de la Fève dans la Provence, les Alp.-Mar., le Roussillon et le département du Gard.

- galionepiphyta, 0 . Galii Vauçh. - Parasite sur les Galion dans tout le bassin.

D. thymonepiphyta, O. Epilhymum DC. - Parasite sur les Thymos, Saturein, Teucrion.

-. senbioshareng, $O$. Scabiose Koch. - Parasite sur Scabiosa columbarin, Carduns defloratus et australis. - Jura helvétique el français: Combe de Lavaux, St-Sulpice, Noirmont, Dôle, 
Faucille, au dessus de Thoiry, Colombier, St-Hippolyte, St-Rambert en Bugey. - Vaud et Valais: les Mortais, Leukerbad. H.-Sav. : Brizon au-dessus de Solaison, Salève à la GrandeGorge. - HI.-Alp. : la Grave, Villard d'Arène, Lautaret, Abriès en Queyras, mont Séuse. - B.-du-Rh.: St-Julien près Marseille.

0. fuliginosa Reut. - Parasite sur le Cineraria maritima. - Iles de Porquerolle, Ste-IIarguerite et Ste-Lucie.

0. columbariharens, 0 . Columbarice Vauch. - Parasite sur Scabiosa columbaria, Minthe artensis. - Var et Alp.-Mar.: Hyères, Fréjus, Cannes. - Pyr.-Or. : Doma Nova dans le vallon de Riu Fagès.

- teucrionepiphyta, 0 . Tetcrii Hol. et Schultz. - Parasite sur les Teurrion, Thymos. - B.-du-Rh. et Alp.-Mar. : pic de Bretagne, Tende. - Gard et Hér. : le Vigan, la Sẻrane, Avène-lesBains. - Pyr.-Or. : St-Laurent-de-Cerdans, Costujes, Carença, Prats-de-Balaguer, - Is. et H.-Alp. : la Bastille de Grenoble, digues du Drac, Comboire, Chorges, les Serigues près Gap. - B.-Alp.: Serennes, Maurin, la Condamine. - H.-Sav. : Annecy-le-Vieux, Pringy, le Salève. - Ain: Nantua, Maillat, Belley, la Pape. - Commun sur les coteaux calcaires de la C.-d'Or et de S.-et-L.

o. rhy trosepiphyta, $O$. Ritro G. G. - Parasite sur l'Echinops paucifora (Ritro L). - H.-Alp. : Cervières, Guillestre, Charance, Rabou, les Roustans près Gap, Roclie-des-Arnauds, Ventavon. Vaucl. et Var: Flassan, Bédoin, Plan d'Aups près de la SteBaume. - Pyr.-Or. : envir, de Perpignan, Malloles, vallée du Réart.

D. rubens Wallr. - Parasite sur les racines des Medicagn. -- Envir. de Lyon, de Grenoble et de Crest. - Gard et Hêr.: Cernach, Vallabrègues, Béziers. - Py.-Or.: la Salanque, le Riveral, Vernet, Vinça, Baixas, Cases-de-Pena, Prats de Ilollo.

- Mllerhaerens, 0 . Laserpitii-Sileris Rapin. - Parasite sur le Laserpitium siler. - Jura helvétique et français: Creux-duVan, Vuarne, Dôle, Colombier, Faucille, Colombier du Bugey, montagnes d'Ambéricu. - H.-Sav. : Salève à la Grande-Gorge. - Is. : mont Rachais, St-Eynard, Chalais. 
D. major L. - Parasite sur les racines du Centururon scabiosifo!ium. - Envir. de Besancon, de Grenoble, Aix, Marseille, Toulon, Fréjus; dans le Gard à Tresques, Chartreuse de Valbonne; dans l'Ilêr. à Cette; dans les Pyr.-Or. à Céret, Vernet-les-Bains, vallée de l'Agly.

9. cervarhnerens, 0 . Cercariac Suard. - Parasite sur ies racines du Peucedanon cervarium. - Doubs et Jura : mont Brégille près Besançon, monts Poupet et Belin près Salins, Perrigny près Lons-le-Saunier, Colombier de Gex, St-Ramberi en Bugey. - Bois des Frères près Genève. - H.-Sav. : Petit-Salève près Monetier. - Is.: mont Rachais. - Rh.: Couzon et StRomain au Mont-d'Or. - C.-d'Or. : Poinçon, Velais. - Gard : envir. de Nîmes.

D. picridepiphyta, 0 . picridis Tauch. - Parasite sur les racines du Picris hieracioidea. - Jura: Crissey près Dole. - C.-d'Or: St-Remy, Pont de Passy, Montigny, Hontfort. - Envir. de Grenoble vers le Petit-Séminaire, Comboire, mont Rachais, Couzon près Lyon, le Luc (Var).

- artemisiepiphyia, $O$. artemisiue Vauch. - Parasite sur les racines de l'Artemisia campestris. - Vaud et Valais : Coppet, Martigny, entre Sembrancher et Vence, Folatères, Sion, Vex. II.-Sav. : entre Rumilly el Frangy. - II.-Alp. : Lautaret, vallée de Cervières. - B.-du-Rh. et Var: Nazargue, envir. d'Aix, Brignoles. - Gard et Hér. : Aigues-Mortes, Tresques, Clapiers, Jacou. - Pyr.-Or. : envir. de Perpignan, entre Olette et MontLouis.

D. saIvihnerens, O. Salviae F. Schultz. - Parasile sur les racines du Salvia glutinosa. - Envir. de Gap. - Alp.-Mar.: St-Ilartin Lantosque, Tende.

- pubescens d'Urv. - Parasite sur les racines du Crepis bulbosa. - B.-du-Rh.: envir. de Marseille à Montredon, Bonneveine, Mazargue, vallon de Margiou. - Var et Alp.-Mar. : Hyères, le Luc, l'Escarène.

D. Hederihaerens, O. Hederae Vauch. - Parasite sur les racines du Lierve dans tout le bassin.

L'o. lawina Ch. Bonaparte, trouvé sur le Laurus nobilis ne 
Jiffère pas du précédent. Du reste, il est probable que le nombre des Orobanches serait rểduit à un petit nombre d'espèces si on connaissait bien les modifications que peut produire la nature de la plante qui sert de support.

- minor Sutton. - Parasile sur les racince des Trèlfes, des Pois, de plusieurs Ombellifères, Carduacées, Chicoracées et Labiécs dans tout le bassin. Espèce assez variable à laquelle s'applique très-bien l'observation qui vient d'être faite relativement à l'influence du support.

L'0. Crithmi Bertol., parasite sur le Crithmum maritimum ne diffère pas assez de l'O. minor pour en faire une espèce distincle.

o. amethyøtea Thuill. - Parasite sur les racines des Eryngion, Chrysanthemon, surtout dans la région méridionale; cependant on l'observe aussi autour de Gap, de Vienne, de Vals, de Grenoble et de Lyon.

D. cernua Laff. - Parasite sur les racines des Artemisia gallica, $A$. campestris, Galion erectum, Lachuca viminea. - Gard: AiguesMortes, Rochefort, Bouquet, bords de l'étang de Pujaut. - Hér. et Aude: Cette, Frontignan, Pérols, Palavas, Vic, Frontignan, Mauguio, Sidric̀re de Leucate. - Vaucl., B.-du-Rh. ct Var: Avignon, cap Croisette près Marseille, Martigues, St-Tropez.

TATHEA senuamarla L. - Parasite sur les racines des arbres dans tout le bassin, mais disséminé et peu abondant.

CLANDESTINA rectinora Lam. - Parasite sur les racines des arbres. - Gard et Hér. : Salbous près Campestre, Ganges, Fraisse, St-Pons, Navacel!e. - Aude et Pyr.-Or. : Belcaire, Mar. sal, Villespy, Mijanès, bois du Pla d'en-Bosch près Frontells en descendant du Laurenti, foret du Paillariel. - Plus commun dans l'ouest de la France.

En terminant, faisons la remarque que les Orobanches, comme toutes les plantes parasites, sont peu intéressantes au point de vue de la géographie botanique; e'est pourquoi j'ai cru inutile de donner de longs détails à leur sujet. Au surplus, ainsi qu'il a été dit plus haut, la distinction des espèces de cette famille restera fort incertaine, tant qu'on ne saura pas, d'une manière expérimentale, quelle est l'étendue des variations que peut produire, dans chaque espèce, Ia nature du support. 


\section{LABIEEES.}

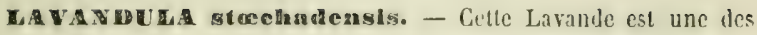
plantes les plus communes des coteaux incultes de la région mérid, elle est caractéristique des terrains siliceux. - Vaucl. et B.-du-Rh.: terr. sablonn. des envir. d'Avignon à Gadagne, de Marseille à Ifazargues, de la Ciotat vers le cap de l'Aigle. Var et Alp.-Mar.: toute la zone des grès bigarrés, des schistes micacés, gneiss, granites, porplıyres, depuis Toulon jusqque dans la forêt des Maures, puis au-delà de St-Raphaël dans l'Esterel, les envir. de Cannes. - Gard: garrigues à cailloux siliceux des envir. de Nìmes, Tresques, la Capelle, remonte jusque dans les régions des grès et granites d'Anduze, St-Jeandu-Gard. - Hér.: garrigues à cailloux siliceux de Grammont près Montpellier, Lamoure, Murviel, Murles, St-Aunès, Castries, Montarnaud. - Aude et Pyr.-Or.: coteaux pierreux de l'arr. de Narbonne, les Corbières, Cases-de-Pena, Força-Réal, Baixas, St-Paul, Sl-Antoine-de-Galamus, Salses, Opol, Prades, de MontLouis a Fontpédrouse, Ternet-les-Bains, Collioure, Banyuls-surMer, picd des Albères, Céret, Arles.

L. spieata L. - Gariigues des montagnes, Cetle espèce, comprise dans le sens le plus large et en y rattachant les formes ci-après énumérécs, occupe de vastes espaces non-seulement dans la région méridionale jusque dans l'Ardèche, la Drôme et les B.Alpes, mais encore dans les H.-Alpes et l'Isère où elle remonte très-haut dans les vallẻes montagneuses. - La Lavande a élé naturalisée dans le Jura et le Doubs autour de Salins, au for St-André, aux rochers de St-Joseph, de St-Thiébaud, puis près de Besançon, à Brégille et à la montagne de Rosemont. - Espèce polymorphe.

L. flagrans Jord. - Bh. et Ain: Couzon, Muzin près Belley. - Is., II.-Alp. el B.-Alp.: vallée de la Romanche depuis Bourg-d'Oisans et le Fréney jusqu'à la Grave et à Villard d'Arène.

L. dilphinensis Jord.- Is,, Dr., H. et B.-Alp.: Vif au Pic du Loup, envir . de Gap, à la Garde, Rabou, Puy-Maure, Montjay, la Bâtie-Neure, 
1a Roche-des-Arnauds, St-Mlens, Manteyer, do Jausiers à StFlary. - Dr.: Nyons.

L. latifolia Vill. - Dr. et H.-Alp.: Nyons, le Buis, Loriol, la Saulse, Laragne, Ribiers, St-Genis。 - Ard.: Baix, St-Vincent-de-Barrès, les Nonnières. - Vaucl. et B.-du-Rh.: envir. d'Apt, de Flassan, mont Ventoux, Luberon, env. d'Aix et de Marseille. Var et Alp.-Mar.: Toulon, Carqueiranne, Rians, le Luc, Antibes, Nice, Menton. - Commun dans les garrigues du Gard et de l'Hérault. - Aude et Pyr.-Or.: Villespy, Montolicu, pentes des Corbières, Durban, N.-D. de Pena, St-Antoine-de-Galamus. Espèce polymorphe.

L. erigens J. F. Béziers. - L. intervupta J. F. Béziers. - L. inclinans J. F. Aix (B.-du-Rh.).

La connaissance des Menthes du bassin du Rhone est fort arriérée, parce que la plupart des botanistes de notre région ont négligé l'étude de ce genre difficile. Comme, malgré mes efforts je ne suis pas parvenu à réunir un nombre sufisant d'informations, je me vois forcé d'ajourner à une autre époque le tableau de la distribution géographique des espèces et $d \epsilon s$ nombreuses races de Menthes, ainsi que de leurs hylurides. Présentement je me borne à l'indication sommaire des principaux types distribués suivant la classification de M. Malinvaud.

D'après ce savant botaniste, les Menthes peuvent être divisées en deux groupes : Eumentha et Mfnthoidea. Le premier est subdivisé, d'après la forme de l'inflorescence, en trois sections: Spicatx, Capitatx et Verticillatx. L1 signification de ces noms est trop connue pour qu'il soit nécessaire d'en donner une définition. - Il est certain que de tous les caractères, celui de l'inflorescence est le plus facile à constater et le moins sujet aux rariations. Comme base d'une classification des Menthes le mode d'inflorescence est, sans contredit, de beaucoup préférable à la forme des stolons épigés ou hypogés, à la villosité ou glabréité de l'intérieur du tube de la corolle, et, à plus forte raison, à la présence ou absence de verrues sur les achaines, à la longueur des pétioles et pédoncules, à la forme des feuilles, au plus ou moins de villosité des pédicelles, à la longueur des étamines tantôt incluses, tantòt exsertes, et erfin à la forme du calice, de la corolle et des feuilles. Toutefois ces caractères secondaires méritent d'être pris en considération pour distinguer entre elles les espèces de Menthes et leurs nombreuses races. 


\section{A. Eumentha.}

\section{\% 1. - Spicatx}

MINTIIE rotundifolin L. - Bords des fossés et des cours d'eaux dans tout le bassin. Espèce polymorphe.

Feuilles glabrescentes sur les deux faces, var. glabrescens Timb.

Feuilles profondément dentées, sinuées ou laciniées sur les bords, var. crispa nonn. auct.

Feuilles larges, fortement rugueuses, en épis serrés et épais, var. rugosa Hoffm.

Epis grêles, corolle très-petite, var. parviflora Wirtg.

Epis allongés, interrompus à la base, var. mucrostachya Ten.

M. Allvestris L. - Bords des ruisscaux ct rivières. - Espèce polymorphe dont les formes les plus remarquables sont les suivantes:

M. viridis $\mathrm{L}_{\text {.., }}$ forme à feuilles vertes et glabres.

M. candicans Crantz, à feuilles grandes, blanches-argentées en dessous par un duvet soyeux.

II. mollissima Borkh. à tige fortement tomenteuse-laineuse, à feuilles longues, oblongues-lancéolées, blanches cendrées en dessous.

II. nemorosa Willd, à feuilles largement ovales-elliptiques, pubescentes-cendrées en dessous.

Entre les 1 . rotundifolia et silvestris existent un grand nombre de formes intermédiaires que plusieurs botanistes considérent comme des hybrides. J'ajoute que l'hybridité de ces formes intermédiaires, bien que probable, n'est point démontrée expérimentalement, attendu que la fécondation artificielle des Menthes est une opération que l'exiguité des fleurs rend extrêmement difficile. Il est donc impossible, dans l'état présent de la science, d'assigner à chacun des parents le róle qui lui revient ct, par conséquent, on ne saurait établir actuellement une nomenclature régulière des formes hybrides, conformément aux principes de Schiede. Aussi, comme le remarque fort judicieu- 
sement M. Malinvaud, si compétent en celte matière, est-il permis de donner des noms simples aux Menthes hybrides, ou qu'on croit telles, tant qu'on n'aura pas des notions précises sur leur origine.

Outre les formes hybrides, $\boldsymbol{M}$. velutina Lej, M. gratissima Wirtg., produites par le croisement des $M$. rolundifolia et silvestris, il en est d'autres, les nepetoidea Lej., pubescens et hirta Willd., Ayassei et Deseglisei Malvd., urlicifolia Ten., suavis Guss. qui résultent probablement de la fécondation du M. uquatica par le M. silvestris, ou vice versa; et enfin les Maximilianea F. Sch., M. Schultziana Bout. et rolundifolio lirsula Timb. engendrés par le croisement des $H$. aquatica et rotundifolia.

$$
\text { 2. - Capitatæ. }
$$

PINTHE aquatiea L. - Marais, fossés dans tout le bassin. Espèce polymorphe dans laquelle la villosité est très-variable, d'où les expressions de $\boldsymbol{M}$. hirsuta, M. glabrescens.

$$
\text { \%. - Verticillate. }
$$

I. arvensis L. - Champs dans tout le bassin. - Espèce polymorphe dans laquelle les pédicelles sont tantôt glabres, tantôt velus.

La forme agrestis Sole a les feuilles largement crénelées. dentées.

La forme parietarifolia Beck a les feuilles faiblement dentées, rétrécies en coin à la base, le calice à dents courtes et ólargies. Il est probable que, comme le croit M. Malinvaud, les formes décrites sous les noms de M. Mulleriana, Wohlwerthiana, stachyoidea Host, mollis, scordiastrum, micrantha, Schultz, sont des hybrides des $M$. arvensis et rotundifolia.

Le . . saliva $\mathrm{L}$. el les diverses formes qui s'y rattachent, comme M. verlicillata Riv., M. ballotifolia 0piz, M. subspicata Weihe, M. origanifolia Host, M. pulchella Host, M. viridula Host, M. crenata Beck, M. palustris Moench, M. austriaca Jacq., sont probablement des hybrides des $M_{0}$ aquatica et arvensis.

Le croisement des $\boldsymbol{M}$, arvensis et viridis produit une séric de formes hybrides désignées sous le nom général de $\boldsymbol{M}$. gentilis 
Lej, et qui se distinguent des autres Nenthes à inflorescence verticillée par la glabréité de la base du calice ct de la face interne de la corolle. Parmi ces formes, les unes ont le calice sem.

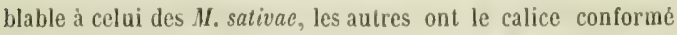
comme celui des $M$. arvenses, ce qui a conduit M. Malinvaud à les diviser en deux sections appelées, la première arvensastrum, la seconde sativastrum. Les formes les plus remarquables de 1\%. gentilis sont M. cardiaca, gracilis, pratensis, rubra, variegata, des auteurs anglais, Agardhiana de Fries, Pauliana et Wirtgeniana de F. Scluultz, resinosa d'Opiz, grata de Host, elegans et clliptica de Lejeune, et enfin le cuntalica d'Héribaud.

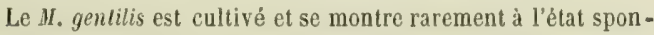
tané; cependant on l'a observé autour de Genève et en Savoie, à la Gravière près de Ilurat (Cantal), et enfin M. Fray l'a vu à Anse (Rhòne) et à Coligny (Ain).

Le $M$. rubra Sm., souvent cultivé et quelquefois spontané, est un hybride des $M$, viridis et sativa.

Le I. piperita IIuds., également cultivé et quelquefois subspontané, résulte du croisement des $M$. viridis et aquatica. Quant aux $M$. citrata Ehrh. et odorata Sole, ils sont probablement issus d'un croisement en sens inverse des mêmes générateurs.

Lc II. Noulctiana Timb. paralt être un viridi-rotundifotia; le M. amaurophylla Schultz un rotundifolio-viridis.

Enfin, les M. dentata Roth et Skofitsiana Kerner semblent étre des silvestri-arcenses. (Puur plus amples détails, voir l'intéressant article publié par M. Malinvaud, Bull. Soc. bot. Fr., 1. $27 .-1880$.

\section{B. Menthoidea.}

MINTIrE pulegia L. - Licux humides, bords des mares et des ćtangs. - Plusieurs auteurs, considérant que le Pouliot a le calice fermé après la floraison par un anneau de poils el porte à la base une gibbosité unilatérale, en ont fait, avec Miller, un genre particulier appelé Pulegium. S'il est vrai que le Pulcgium vulgare Mill. ne forme jamais d'hybrides avec les véritables 
Ienthes du groupe Eumentha, la séparation scrait assez légitime et, pour ma part, je n'y contredis point, non plus qu'à la crẻation du genre Preslia caractérisé par son calice à quatre dents concaves et aristées, par ses achaines oblongs.

I. cervina L. - Preslia cervina Fresen. - Lieux humides. Bords du Rhône près d'Avignon, Arles. - Lieux humides des parties basses du Gard, de l'Hér, de l'Aude et des Pyr.-0r.

LYCOPUS enropaeus L. - Lieux humides dans tout le bassin. Grecañon vuIgare L. - Licux incultes dans tout le bassin. 0. megastachyum Link. - Forme du précédent à épis prismatiques composés de fleurs nombreuses.

Thymos vulgaris L. - Coteaux secs de la région mérid.; remonte dans les Alpes Basses et Hautes, la Drôme vers Sisteron, Digne, Annot, Ribiers, Gap, Serres, Laragne, Tallard, Nyons, St-Paul-Trois-Châteaux, Grignan, Crest, Die; dans l'Ard. vers Rochemaure, Cruas, Baix, Privas, Celles, la Voulte.

T. serpylius L. - Lieux arides dans tout le bassin depuis les plaines jusqu'aux montagnes. Espèce polymorphe dans laquelle les dimensions des feuilles sont très-variables; d'oủ les variétés ovatus, angustifolius, confertus.

T. chrmaedryus Fries. - Forme du précédent à rameaux hérissés de poils disposés par rangées; les verticilles inféricurs des fleurs sont plus écartés. Dans la variẻtẻ lanuginosus les feuilles sont grandes et très-velues. Dans la variẻtẻ pannonicus Fries, toute la plante est hérisse̊e de poils blanchâtres, les feuilles sont atténuées aux deux extrémités, les fleurs forment un long épi.

Grysopos officinalis $I_{\text {. }}$ - - Rochers de la région mérid. et moyenne. - Vaucl, et B.-du-Rh.: envir. d'Avignon à Bonpas, Védènes, Orange, Piolenc, la Crau à Cannebiero près Graus, Salon, envir. d'Aix à Puyricard, Meyreuil. - Var et Alp.-Mar.: Toulon, Grasse, Caussols, le Bar, Tende, Levens, l'Agel. H.-Alp., B.-Alp., Is. et Dr.: envir. de Gap à Rabou, N.-D. du Laus, Tallard, Rosans, vallée du Guil en Queyras, Sisteron, Varces, Pariset, Bastille de Crenoble, Corps, Die, Rochegude, St-Vallier. - Gard : St-Ambroix, Anduze, Aramon. - Ain : Muzin, Virieu-le-Grand, Ambronay. - Sav.: entre Moutiers et 
Salins, St-Mlichcl. - Valais : Fully, Folateres, Branson, Stalden. - Naturalisé dans le Jura et le Doubs aux rochers de Poupet, ruines du château de Vaugrenans, Pontarlier.

Les formes suivantes sont démembrées de celte espèce.

II. bengesiacus J.-F. - Ain : Virieu-le-Grand, Muzin, Serrières, Ville bois.

II. decumbens J.F. Névache (H.-Alp.), de St-Flory à Bouzollières (B.-Alp.), Guillaumes (Alp.Mar.), Echirolles (Is.)

II. recticaulis J.-F. - Manteyer près Gap, St-Benoit, Annot (B.-Alp.).

II. aristatus Godr. - Pyr.-Or.: Mont-Louis, St-Thomas, Cassagne.

II. montunus J.-F. - Briançon.

II. cinerascens J.-F. - Védènes, Bonpas près Avignon, la Crau. Aramon (Gard).

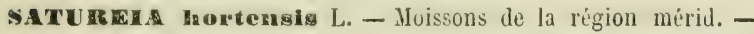
Dr., II. et B.-Alp.: St-Nazaire-en-Royans, Valence, Barnave, Crest, St-Paul-Trois-Châteaux, Gap, Montmaur, Ventavon, Rosans, Montjay, Sisteron, Annot à St-Benoît, Vaulvanès, Vaucl., B.-du-Rh. et Var:mont Ventoux, envir. d'Orange, de Carpentras, d'Avignon et d'Apt, bords de la Durance, Meyrargue à St-Canadel, la Ste-Baume, le Luc, Frèjus. - Gard : Bagnols, St-Gervais, St-Michel, Sumène, St-Ambroix, le Vigan. - Hẻr.: sources du Lez, St-Jean-de-Védas, Montferrier, pic St-Loup, St-Etienne-de-Gourgas, Soubès, Si-Bauzille-de-Putois, Ganges, Madières. - Ard.: entre Privas et Chomérac. - Souvent cultivê et quelquefois subspontané.

S. montana L. - Rochers calc. de la région mérid. - Dr. et H.-Alp.; Montélimar, Chanousse, Serres, Roche-des-Arnauds, Rabou et Charance près Gap, Guillestre, Chăteau-Queyras, Embrun. - B.-Alp. et Vaucl.: envir. de Sisteron, Digne, Annot, Serennes, la Condamine, mont Ventoux, Luberon, envir. d'Apt à Viens, Croignes, envir. d'Avignon sur les bords du Rhône, le Thor sur le monticule de Thouzon. - Ard.: envir. de Vallon, Pont-d'Arc. - Collines calcaires des B.-du-Rh., Var et Alp.Mar., du Gard, de l'Hér. et Pyr.-0r. - Naturalisé sur les coteaux de Muzin près Belley. - Espèce polymorphe.

S. brevis J.-F. - Digne. 
S. petraea J.-F. - St-Rémy (B.-du-Rh.).

S. provincialis J.-F. - Collobrières (Var).

S. flexuosa J.-F. - Laragne (H.-Alp.).

S. rigidula J.-F. - Aix (B.-du-Rh.).

Micronenia graeen Benth. - Espèce de Grèce, de Corse, d'Algérie et d'Espagne qu'on prétend avoir trouvée près de Toulon.

M. Juliama Benth. - On dit l'avoir vu sur les coteaux des envir. d'Avignon, ainsi que dans la vallée de l'Agly (Pyr.-0r.) sur les coteaux de Cases-de-Pena, Estagel, Vingrau, Salses, Opol. Ces indications me paraissent fort suspectes.

M. piperella Benth. - Rochers des Alp.-Nar. depuis Tende jusqu'au Brès et à l'Agel au-dessus de Henton, St-Sauveur.

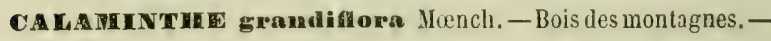
Rh. et Loire : chaine du Beaujolais et du Pitat, Pierre-surHaute. - H.-Sav. et Sav.: Servoz, Mégève, entre Héry et Flumet, St-Gervais, les Contamines, mont Charvin, Manigod, Serraval, Crest-Voland, le Cohennoz, Hauteluce, Beaufort. - ls., H.-Alp. et Dr.: Chalais, Grande-Chartreuse, Portes, mont Rachais, Prémol, la Morte, St-Nizier, mont Sénèpe, la Salette, la Grave, Lautaret, Briançon, Guillestre, mont Séuse, bois de Rabou et de Chaudun, Charance, Durbon, Chaillol, la Condamine, Barnave, Jansac, forêt de la Penny près Lus-la-CroixHaute. - B.-Alp. et Alp.-Mar.: la Condamine aux Tardées, Bérard, Annot au col d'Allous, Caille, mont Lachen, Entraunes. - Vaucl.: mont Ventoux. - Ard.: Mezenc, Gerbier-desJoncs, lac d'Issarlès, Baizon, Mayres. - Gard et Hér.: chaines de l'Aigual, de l'Espérou au Valat de la Dauphine, la Céreirède, bois de Peire-Besses près Alzon, St-Jean-de-Buèges à Combescure. - Ariège et Pyr.-0r. : Laurenti, aux cirques des Aiguettes et d'Artounant, Jasse-de-Councr, Salvanère. - En dehors de nos limites dans les Pyr. centr., la Lozère, l'Aubrac, la II.-Loire, Cantal, Forez.

C. officinalis Monch, - Bois et coteaux dans tout le bassin. Es. pèce poymorphe.

C. ascendens Jord., C. menthifolia G. G. an Host 9 Diffère du précédent 
par ses fleurs plus petites, moins saillantes, ayant les dents supérieures du calice dressíes et non courbées en dehors, par ses feuilles plus larges, faiblement dentécs, par sa souche moins radicante. - Environs de Geneve, Collonge, Thonon, Veyrier près Annecy, Lyon, Montélimar, St Guilliem le Désert et StMartin de Londres dans la vallée de l'Hérault, la Sérane, Lamalou, vallée de l'Lude d'Usson à Fontanes, Rouze, Mijanès.

C. nepela Savi. - Bords des champs et des chemins de la région mérid.; remonte la vallée du Rlione jusqu'à Lyon. - Assez commun dans l'ouest de la France.

C. nepetoidea Jord. - Lieux pierreux, - H. Sav. : pied du Salève, Archamp, Veyrier, Talloires. - Is. et II.-Alp. : envir. de Grenoble, Rochefort près Varces, St-Ange, entre Corps et la Salctte, Pabou et la Garde près Gap, mont Séuse, la Bâtie-Neure, Serres, Champoléon, Guillestre, la Grave. - B.-Alp. el Alp.-Mar.: Barcelonnette, la Condamine, Serennes, Annot, St-Paul, Sisteron, Digne, Castellanne. - Vaucl. : Flassan, Bedoin, mont Ventoux. - Très- commun dans les montagnes des Alp.-Mar. où il remplace le $C$. nepeta.

C. alplna Lam. - Pâturages du Jura, des Alpes et des Pyrínées. - Jura helvétique et français : Weissenstein, Chasseral, Creuxdu Van, Montendre, Noirmont, la Dôle, Colombier, Reculet, Colombier du Bugey. - Vaud et Valais : Anzendaz, Diablerets, Saivan, St Bernard, Mayens de Sion, Leukerbad. - H.-Sav. et Sav.: Salève aux Pitons, Nôle, vallée de la Borne, Dents-d'Oche, mont d'Armonnaz près Reyvroz, Bellegarde près Abondance, Prarion, Chamonix, Crest-Voland, Beaufort, École en Bauges, Combe de-Villette près Bramans, mont Iseran. - Is., II.-Alp. et Dr.: Grande Chartreuse, St-Nizier, col de l'Arc, la Salette, la Grave, Lautaret, mont Genèvre, St Vêran, mont Chabrière, Is Grangette, col du Noyer et Lopet en Champsaur, col de Glaise, mont Aurouse, Aucelon. - B.-Alp. et Alp.-Nlar.: la Condamine, Serennes, lạ Blachière, Soleille-Boeuf, vallées du Var, de la Vésubia et de la Tinea. - Ariège, Pyr.-0r. et centrales.

Manque dans les Vosges et dans les montagnes du centre de la France. 
C. aclua Clairv. - Champs dans tout le bassin.

Chroponyon vulgare L. - Haies, lieux incultes dans tout le bassin.

MELISSA officinalis L. - Cultivé dans les jardins et quelquefois subspontané.

TOSMATLNUS offehnalis $\mathcal{L}$. - Coteaux rocheux de la région mérid.; remonte jusque dans les B.-Alp. et le sud de la Dr. et de l'Ard.: à St Paul-Trois-Châteaux, Granne, Nyons, Sisteron et sur les coteaux de la rive droite du Phône jusque près de Tournon. - Espèce polymorphe.

$R$. rigidus J.-F. - Dr. et Ard.: de Tain à Romans et jusqu'à Genissieu, Tournon. - Var à Toulon.

R. Alexuosus J.-F. - Dr.: Nyons, $-R$. tenuifolius J.-F. Aix (B.-du-Rh.).

SALVIA offeinalis L. - Souvent cultivé dans les jardins et quelquefois subspontané dans la région mérid. et moyenne de notre bassin.

S. vertieilata L. - Bords des routes, - Vaud : Charpigny près St-Tryphon, le Furet, le Sepey, Leysin. - H.-Sav. : Bernier et Reyvroz près Evian, Chenevoz, Abondance, Vacheresse. C.-d'Or: Dijon. - Lyon à Sans-Souci où il a été semé. - Alp.Mar.: l'Ariane près Nice, Menton, Sospel, vallée de la Roja de Fontan à Tende. - Gard: le Vigan, Mandagout. - Hér.: Pégayrolles-de-l'Éscalette. - Pyr.-0r.

s. selarea L. - Coteaux secs, lc long des murs. - C.-d'Or et S.-et-

L.: Gevrey, Meursault, Santenay, Dezize. - Valais: Fully, Conthey, Savièse, Montorge, Tourbillon. - Envir. de Lyon à Villeurbanne, Montchat, Chevinay. - Sav. Is. et Dr.: BourgSt-Maurice, St-Martín-le-Vinoux, St-Lattier, Romans, StVallier, Moydans, Die, Vaulnaveys près Crest. - H. et B.-Alp. : Guillestre, Ribiers, Ste-Ilarie près Rosans, Digne, Castellanne, Valbelle près Sisteron. - Vaucl.: Flassan, Bẻdoin, Croignes, Viton. - B.-du-Rh. Var et Alp.-Mar. : Montaiguet et vallon de Brunet près d'Aix, Toulon, le Duc, Draguignan, Grasse, Bar, Nice, la Turbie, de Menton à Tende. - Gard et Her. : arrond. de Nimes, d'Uziss, Alais, envir. de Iontpellier au chemin de 
Grabels, entre le pont de Lavalette ct Nontferrier, Ganges, Claret, Gabian, Octon. - Ariège: pentes du Roc de Cira audessus de Rouze. - Cetle Sauge s'échappe souvent des jardins oil on la cultive.

s. nethiopls L. - Bords des chemins. - Sav. : St-Jean-deMaurienne. - H.-Alp. et Dr. : Briançon, Guillestre, Aiguilles cn Queyras, Ribiers, Charance, la Gárde, Rabou, Roclıe-desArnauds, Rosans, Romans, Barnave, Nyons. - B.-Alp. et Vaucl. : Jausiers, Gadagne, Serignan, Apt, Joucas, Gargas, Roussillon. B.-du-Rh. et Var: Ste Victoire, Vauvenargues, Hargès près d'Aups. - Gard et Hér. : Campestre, Trèves, Pradines près Lanuejols, le Caylar. - En dehors de nos limites dans l'Aveyron, la Lozère, le Puy-de-Dôme.

5. gluthosn L. - Bois. - Nul dans les Vosges. - Rare dans le Doubs el le Jura à Charquemont, Thoirelte; devient plus commun dans l'Ain à partir de Lélex et de Chézery; puis sur les pentes du Colombier du Bugey, St-llambert en Bugey, la vallée de l'Albarine, envir. de Belley. - Assez commun dans Vaud, Talais, H.-Say., Sav., Dauphiné, Alp.-Mar. - Gard: le Vigan, Aumessas, l'Espérou, Bessèges, Anduze, St-Ambroix. - Ard.: Entraigues, Vals, Privas. - Pyr.-Or.

\$. protensis L. - Prés dans tout le bassin.

5. vezonaen L. - Bords des champs et des chemins de la région mérid.; remonte jusque dans le sud de la Dr., des H.-N/p. el de l'Ard. - On l'a même trouvé dans les envir. de Lyon et de Dijon. Une forme de cette espèce à coroile dépassant à peine le calice, à style inclus, est le $S$. horminoides de Pourret, distinct du S. clandestina I.

s. clandestina L. S. horminoides G. G. non Pourr. - Bords des cliamps et des chemins dans la Provence, les Alpes-Haritimes, le Languedoc et le Roussillon; remonte dans les B.-Alpes vers Sisteron, Annot; dans les H.-Alpes vers Ribiers, Laragne.

NEREC Iarceolata Lam. - Pierrailles, Jords des routes, gravier's des torrents dans les montagnes. - Sav. : vallée de l'Arc cntre la Chambre et Lans-le Bourg. - Is, et II.-Alp. : Champagnicr, Séchiliennc, vallées de la Romanche et du Catal. Bassin du Rhöne. 
Vénéon, la Grave, de Corps à la Salette, Villard de-Lans, Monestier-de-Briançon, envir. de Briançon, vallée du Guil, Rabou, col de Glaise, Chaudun, Séuse, mont Aurouse, Rosans, Moydans. - Dr. et B.-Alp. : les Corrẻardes près Lus, Die, Montélimar. B.-Alp. et Vaucl. : la Condamine, St-Paul, Larche, Annot, Castellanne, mont Ventoux et Luberon. - B.-du-Rh. Var et Alp.-Mar. : pic de Bretagne, Stc-Baume, Gémenos, Escragnoles, vallées du Var, de Vesubia, Tinea et Roja jusqu'au col de Tende. - Hér. : rochers de la Sérane à Pégayrolles-de Buiges.

N. pariflora, N. nepelella L. - Forme du précédent à petites fleurs; calice plutôt duveteux que laineux. - Vallée de l'Arc à Saintde-Maurienne, Termignon, Lans-le-Bourg.

N. entaria L. - Décombres, bords des chemins dans une grande partic du bassin.

N. Iatifolia DC. - Bords des chemins et des prairies. - Aude ct Pyr.-0r. : Belcaire, Mont-Louis, la Cabanasse, la Cerdagne, Madrès, Formiguères en Capcir.

N. nuda L. - Lieux pierreux des montagnes. - Vaud : ChableRouge près de Roche, entre Frénière et Gryon, Búvieux, entre les Gauges et le Furet. - Is. et H.-Alp. : de Corps à la Salette, envir. de Gap à Loubet, la Grangette, Séuse, Chaillol, Champoléon, Prontic en Valgaudemard, mont Genèvre à Clavière, mont Rognouse. - Vaucl. et Alp.-Mar. : la Garde, val de Pesio, cols de Fenestre et de Tende.

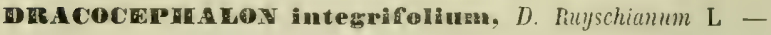
Rochers et pâturages des Alpes et des Pyrénées. - Vaud ct Valais: Nombrieux, Panerossaz, Prapioz, Boulaire, Surchamp, Lavarraz, Comba sur Vouvry, Salanfe, Dorénaz, Grand-Lui près de la Líchère d’Orsières, les Crépans dans la vallée de Bagnes, val St-Nicolas au Jungenalp. - II.-Sav, et Sav. : St-Jeand'Aulph, Nautau, Aiguille-à-Buchard au Pas de l'Ours, montagnes d'Aime aux Mottets, Pralognan, mont Cenis. - Is. et H.-Alp.: mont St-Nichel près Lamure, la Bẻrarde, Lautaret, le Mélezet près Guillestre, mont Bayard et Grangetle de Rabou près Gap. - B.-Alp. et Alp.-Nar.: Bousollières, Faucon à SolcilleBuou, col de la Madeleine, l'Enchastraye. 


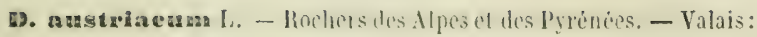
rochers de Dorénaz au-dessus d'Outre-ihone, entre le Rosey et le Ziabley, Iserable, entre Riddes et Nendaz. - II. et B.-Alp. : le Noyer au l'ré-de-l'Aigle où il n'a pas élé retrouvé, montagne des Dourbes pres Digne. - l'yr.-Or. : lont de Comps au pied du rocher de la Coba del Fatj. Companyo dit que la station a été ravagée et détruite par la rapacité de quelques botanistes centuriateurs.

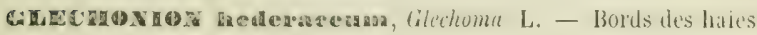
et des bois dans toute la France.

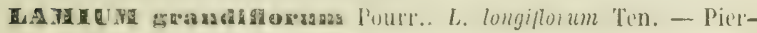
railles des montagnes, - II. et B.-Alp. : pic de Chabrières près Chorges, Pommerol, Scrennes, Fouillouse, Colmars, mont Grand-Couyer près Annot, Digne, Faillefeu, Coulebrousse près Seyne. - Vaucl., Var et Alp,-Mar. : mont Ventoux, St-Amans, Baumes, Rợuesalières, St-Pilon à la Ste-Baume, mont Faron. mont Lachen, St-Auban, montagnes de Nice et de Menton. Hér., Aude et Pyr-0r.: montagnes de St-Pons, les Corbières à Pierre-Pertuse, couloir des Courtalets près Tuchan, Pont de la Fou près St-Paul-de-Fenouillet.

L. amaplexisaule L. - Cliamps incultes dans tout le bassin. Dans cette espèce, comme chez plusieurs autres Labiẻes, la corolle reste parfois incluse dans le calice. Cet état particulier a reçu le nom de $L$. clandestimum.

E. Ineigam Willd, L. hybridum Vill. - Champs et bords des chemins; plus rare que le précédent.

L. purpureum I. - Champs et bords des haies dans tout le bassin.

L. maculatum L. - Lo long des haies et des murs dans tout le bassin.

L. nlburan L. - Nême habitat.

L. Hexuagum Ten. - Le long des haies dans les envir. de Montpellier, Perpignan, Prats-de-Mollo. - Ard. : Entraigues.

E. galeobololman Crantz. - Licux ombragis dans tout le bassin.

E. ENE TES trinlobatus Lam. L. Cardiaca L. - Dícombres, envir. des habitations surtout dans les parties septener. et moyennes 
du bassin; plus rare cians les provinces mérid. où il se montre dans quelques parties des rígions montagneuses.

C. marrablfolias, L. marruliastrum L. - Dícombres, boris des chemins. - Jura : Beauregard près de Chaussin, la Borde près Peseux, St-Scine près Dole. - Ain: Vésines, Heximieu, plainc d'Ambronay. - C.-d'Or et S.-et-L : Seurre, St-Jean-de-Losne, Quétigny, Domois, Buffière, Navilly, St-Bonnet-en-Bresse au moulin de Taprey, Saunière prés Verdun, Surmoulins près Autun. - Env. de Genève à Mont-Fleuri près Meyrín.

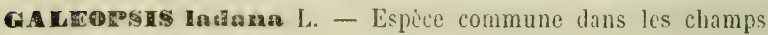
cultivés et qui se présente sous de nombreuses formes. Les fleurs ordinairement purpurines, sont quelquefois blanches; la teinte, le plus souvent verte, est, en certains cas, blanchâtre; les feuilles sont larges (G. latifolia), ou étroites ( $G$. angustifolia Ehrh.) Cetle derniẻre forme est extrêmement commune dans les terrains calcaires des plaines, et présente dans les montagnes une variété naine. De Candolle, Gussone et Godron pensent que le G. ladana de Linné n'est pas le G. angustifolia, mais bien le $G$. intermedia Vill. qui, dans les régions septentrionales de l'Europe, est assez commun, comme, du reste, il l'est dans nos Alpes.

G. intermedia Vill. - Forme à glomérules de fleurs écartés les uns des autres, à bracties plus courtes que les calices, à feuilles ovales-lancéolées; assez abondante dans les champs des partics montagneuses du Valais, Jura, Savoic, Dauphiné, B.-Alp. et Alp.-Mar., ainsi que dans les montagnes du Gard, de l'Hérault, des Pyr.-Or. et de l'Ariège.

G. arvatica Jord. - Forme à tige courte, à rameaux nombreux, étalés; feuilles d'un vert cendré; calice plus petit et plus lérissé. - Mêlé çà et là avec le $G$. anyustifolia.

A. ochroleuen Lam. - Champs des terrains siliceux.

G. pyrenaica Bartl. - Forme dlu précident, à feuilles ovales, obtuses, arrondies à la base. - Rocailles des terrains siliceux des Pyr.Or. à Collioure, Port-Vendrcs, Cosperons, Jes Albères, Amélieles-Bains, Villefranche, Nolitg, le Vernet, Olette et jusqu'à IIont-Louis, - Une forme naine se trouve au Cambres d'Aze, aux cols de Nuria et de las Nou-Fonts. 
G. nodosa Mocnch, G. Tetrahit L. - Haies, bois dans tout le bassin. Espece polymorphe dans laquelle on a particulierement remarqué les formes suivantes:

G. preciox Jord, - Valais: Bagnes. - HI.-Sav. : Saleve, Voirons, Arache, Pernant, Abondance. - Ariege: les Aiguettes, Boutadiol, Rouze.

G. pallida Jord. - Dispersion mal connue.

G. Reichenbachiana Reut. - II.-Sav. et Sav. : Salève, Dents d'Oche, mont Méribelle, Cihamonix au Saut-des-Pèlerins, à Taconnaz et au Bouchet, Ies Contamines, Chapieu. - Ain: la Faucille, Chalets d'Ardran. - Is. : Bovinant, la Moucherolle, Revel à Jasse-Vieille.

G. bifida Boenn. - Clamps et régions montagneuses du Jura et de l'Ain. - Ariège : les Aigueltes, Bentaillole.

G. glaucescens Reut. - Alp.-Mar. : Aiglun, Guillaumes.

f. sulfurea Jord. - Sav. el Is. : envir, de Chambéry, Allevard, St-Laurent-du-Pont, Domènc, Theys, Morestel, les Avenières, St-Jeand'Avellane. - Pyr.-Or.: les Albères.

G. Verlotiana Jord. - Is. : St-Nizier, le Sappey, Vaulnaveys, Revel.

G. alpicola Jord. - Villard d'Arène (H.-Alp.). - G. Gaiognei Jord. Briançon, la Condamine.

G. loucantha Jord. - Crémieu (Is.).

SaAchys germanicas L. - Champs et bords des chemins.

s. Itakeus Nill. - Espice italienne trouvée par M. Roux dans les B.-du-Rh. près de la Treille au vallon des Bellons, et entre Cassis et la Ciotat au vallon de Peiro-Redouno.

S. Ieraclens All. - Coteaux rocailleux. - Var et Alp.-Mar.: le Luc, Fréjus, Grasse, Antibes, montagnes au-dessus de Nice et de Menton, nolamment à la Turbie. - Pyr.-Or.: Prats-deMollo, Font-de-Comps, Costujes. - En dehors de nos limites dans le Puy-de-Dôme, l'Allier, Aveyron, Tarn, Cher et les Charentes.

5. nlpiaus L. - Bois. - Jura helvétique et français, montagnes et collines calcaires de II.-Saone, C.-d'Or, S.-ct-L., mont d'Or lyonnais, Vaud, Valais, Savoie, Dauphiné, B.-Alp. et Alp.-Nar. - Aude, Pyr.-0r. et Ariege. - En dehors de nos limites dans les Pyr. centr., l'dveyron, le massif central. 
S. silvatieus L. - Bois et borils des haies dans tout le bassin.

S. poIustrils L. - Fossés, prés humides dans tout le bassin.

Le S. palustri-silvatica Schiede, S. ambigua Sm., probablement hybride des deux précédents, se trouve quelquefois mêlé ả eux.

5. arvensis L. - Champs dans une grande partie du bassin.

s. hintus L. - Champs de la région mérid. - Var et Alp.-Mar. : glacis du fort Malbousquet à Toulon, iles d'Hyères, Cannes, île Ste-Marguerite, Nice, Villefranche, Monəco, Menton. - Hér.: entre Grammont et Mézouls.

S. anuuns. - Champs dans tout le bassin.

S. maritimas. - Champs sablonneux des bords de la mer. B.-du-Rh. : Montredon, Mazargues. - Var et Alp.-Mar. : TouIon, Hyères, St-Tropez, Fréjus, Cannes, golfe Jouan, Nice. Hér.: Palavas, Pérols, Cette, Frontignan, Maguelone. - Aude et Pyr.-Or.: le Corrège, Leucate, Cannet, Argelès, Collioure, Port-Vendres, Banyuls.

S. rectus L. - Champs pierreux dans tout le bassin.

S. delphinensis Jord. - Forme du précédent à fleurs d'un blanc opalin.

- Is. et Dr. : la Motte-les-Bains, Prunières près la Mure, Claps du Luc, Luc-en-Diois.

Ba g calcaires. - Is. et II.-Alp. : Grande-Chartreuse à Bovinant et au col de la Ruchère, la Salette, Lautaret. - Pyrénées centrales.

B. Hirsuta L. - Pâturages des montagnes. - Vaud et Valais: Alpes de Château-d'Oex, de Vouvry, Val d'Illiez, St-Bernard, les Combes, Bella Comba, Grand-Lui, Clavanx, Leukerbad. H.-Sav.: Cornettes de Bise, Signal des Agneaux, plateau des Gets, montagnes de Samoëns, Châtillon, Calvaire de Thônes, Parmelan, vallée de la Borne, Charvin, Tournctte, mont Méri, Pormenaz, Brérent, Buet, col de Balme, mont Lachat, mont Joly. - Sav. : le Bonhomme, Hauteluce, mont Iseran, Golet, Pralognan, montagnes de Tignes, mont Cenis, mont Trélod en Bauges. - Is. et Dr.: Grande-Chartreuse, Grande-Lance, ClampRousse, Gleyzin, Sept-Laux, la Moucherolle, Mont de Lans, mont Sénèpe, la Bérarde, la Salette, l'Obiou, mont Glandasse. - H. et B.-Alp.: la Grave, Lautaret, mont Viso, col de Vars, 
mont Sćuse, cols de Glaise et de Chaudun, Durbonnas, Lopet, montagne de Chabrières, Lauzanier, Parpaillon, mont Monnier, Colmars. - Alp.-Mar. : parties supér. des vallées du Var, de la Vesubia, Tinea, Roja; descend à une faible altitude dans le vallon de St-Michel pris d'Eza. - En dehors de nos limites dans les Pyrénćes centrales. - Les deux formes suivantes ont élé démembrées de cette espèce :

B. alpestris J. F. - La Saletto (Is.). - B. sabauda J. F. Mont Brizon (II.-Sav.).

F. officinalis L. - Prós et bois dans tost le bassin. - Espèce polymorphe à laquelle se rattachent les espèces suivantes, décrites par MM. Jordan et Fourreau :

B. occilana. - Béziers (Hêr.). B. pratensis. - Chaponost (Rh.).

B. parvula. - Vallée d'Eyne (Pyr.- B. nemorosa. - Tassin (Rh.).

Orient.).

B. stricticanlis.-Hauteville(Ain).

B. subcarnea. - Pierrefeu (Var). B. silvicole. - Anse (Rh.).

B. brachystachya. - Evian (Sav.). B. hylebia. - Sathonay (Ain).

B. pilostachya. - Ascou, Bouta- B. polyclada. - Neyron (Ain).

diol, les Aiguettes, Artounant, B. validula. - Neyron (Ain).

lac Bleu (Ariège). B. drymophila. - Quincieux (Rh.)

B. recurvata. - Soucarrat, Ben- B. recurta. - Caluire (Rh.).

taillole, Pla del Bosc (Ariège). B. rigida. - Pierre-sur-Haute B. angustifolia. - St-Pons (IIér.). (Loire).

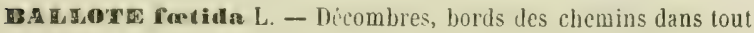
le bassin.

B. spinosa Link. - Rochicrs ombragés. - B.-Alp. : Beauvezer, Entrevaux. - Alp.-Mar.: le Bar, St-Arnoux, Villars, Duranus, Levens, Nice, Eza, entre Ste-Agnès et Gorbio, entre Castillon et Castellar, Saorgio, Breglio, Sospel, Vintimiglia et plus loin sur le littoral d'Italie.

E⿱艹 - Dr. et Vaucl.: Niyons, Montderergues près Avignon, Carpentras, Gigondas. - B.adu-Rh. el Var : collines des envir. d'Aix et de Marseille, Barbentane, Graveson, mont Caoumet près Toulon. - Gard et Ilér.: garrigues calcaires des envir. de Nîmes, 
Anduze, Montlardier, Aramon, Beancaire, Villeneure, Montpellier, Bézicrs, Cette. - Aude et Pyr.-Or. : la Clape, Pech de l'Agnèle, Fontfroide, les Corbières, Tuchan, ile Ste-Lucie, Salses, Opol, Força-Réal, Baixas, Cases-de-Pena, Estagel, St-Antoine-de-Galamus, Pont de la Fou.

F. ventosa, $P$. Ierbit-venti L. - Bords des chainps et des chemins de la région mérid, - II.-Alp. : Serres, Laragne, Ribiers, Ventavon. - Dr. : Bourdeaux, Saillans, Crest, Njons, Montélimar. - Ard. : Aubenas, Jastre. - Vaucl., B.du-Rh. et Var: Monidevergues près Avignon, Gadagne, Orange, Courthízon, Flassan, Viens, envir. d'Aix à St-Donat et au Défens, la Crau, St-Pons, vallon de Fabregoule près Septèmes, Stc-Baume, St-Zacharic, Toulon. - Gard : Nìmes, Serre-de-Bouquet, Manduel, Bouillargues, St-Gilles, Alais, Anduze, St-Ambroix, Montdardirr. Hér. : cotcaux des arrond. de Montpeltier, Béziers, St-Pons. Aurle et Pyr.-Or. : Lastour's, mas Ste-Puelle, cnvir. de Narbonne, Pech Redon, la Clape, Crabit, Quatourze, Montfort, île Ste-Lucie, Sigean, le Boulou, Château-Roussillon près Perpignan.

R. Praticosa L. - Espèce du sud de l'Italie et de l'Espagne trouvée dans l'ile du Levant et dans les reboisements du mont Faron près Toulon.

SIDERTTES romana L. - Champs secs de la région mirit. Dr.: Valence, Montélimar, Fierrelatte, Suzc-la-Rousse. B.-Alp. : Gróoulx, Castellanne. - Vaucl. et B.-du-Rh. : envir. d'Avignon, de Carpentras, d'Orange, d'Apt, d'Aix et de Marseille. - Var et Alp.-Mar. : Toulon, ile de Porquerolle, le Luc, St-Tropez, Fréjus, Rians, St-Vallier près Grasse, Nice, Menton. - Commun dans la région des Oliviers du Gard et de l'Hérault. - Aude et Pyr.-Or.: envir. de Narbonne, Pech de l'Agnèle, la Clape, le Peyron, Fontlaurier, Baixas, Cases-lle-Pena, Thuir, Perpignan, Villefranche, Collioure, Port-Vendres, Banyuls.

S. montana L. - H. et B.-Alp., Alp.-Mar.: Barrème, Annot, Villeneuve d'Entraunes, Guillaume. - B.-du-Rh.: Valentine près Marseille.

S. Hirsuta L. - Lieux secs et pierreux de la région mérid. Dr. et Vaucl.: St-Paul-Trois-Châteaux, le Buis, Mormoiron, 


\section{- $631-$}

Mazan, Roussillon et Viens près d'Apt, Gigondas, Sérignan, Jontfuret. - II. el B.-Alp.: Ribiers, Château-Arnoux, Digne. - B.-du-Rh. : cnvir. d'Aix, de Marseille el de Salon, les Alpines. - Var et Alp.-Mar. : mont Coudon, Montrieux, le Luc, Roquebrune, Antibes, Villefranche, Levens, Uitelle, col de Braus. - Gard et Hér. : Franqueville près St-Gilles, AiguesVives, Beziers, Nissan, Vendres. - Aude et Pyr.-0r. : envir. de Narbonne, Fontfroide, Quillan, Pierrelin, Sigean, Perpignan, Collioure, Port-Vendres, Banyuls.

Une forme nommée ittloralis par M. Timbal-Lagrave, se trouve sur le littoral de l'Aude.

5. seoraloiden L. - Lieux pierreux de la région mérid. - B.Alp., Vaucl, et B.-du-Rh.: Castellanne, Gréoulx, vallées du Verdon et de la Durance, Cucuron, Apt, env. de Marseille.Gard: Nimes, Uzès, Manduel, Beaucaire au bois de Broussan. - Hẻr.: Montpellier à la Colombière et à Veyrassi, St-Gély, Grabels, la plaine depuis Prades jusqu'au Pic St-Loup, SteCroix, Fontanès, Montferrant, la Sérane. - Aude el Pyr-Or.: envir. de Narbonne, la Clape, Pech-de-l'Agnèle, Fontfroide, Gruissan, Ste-Lucie, vallée de l'Agly, envir. de rerpignan et de Prades. - Une variété fortement velue-laineuse, S. tomentosa Pourr., existe à Quillan (Aude) e à Franquevau près St-Gilles (Gard).

La forme provincialis Jord. Fourr. est signalée à St-PaulTrois-Châteaux (Ur.), Orange (Vaucl.), St-Rémy, les Alpines, Barbentane, Graveson, Mazargues (B.-du-Rh.).

La forme nemausensis J. F. dans les envir. de Nimes, Jonquières, Broussan (Gard).

La forme ruscinonensis Timb. Lagr, dans les Pyr,-0r, au mont Taillefer près de Port-Vendres.

5. hyssopoplaylla L. - Coteaux rocailleux calcaires, graviers des terrains de transport. - Jura et Ain : Poupet près Salins, St-Claude et vallées environnantes, bords de la Bienne et de de l'Ain jusqu'à Thoirette, bords de la Valserine, crêt de Chalam, la Dole, Faucille, Colombier, Reculet, Tenay, St-Ram. bert-en-Bugey, le Hont près de Nantua, Ambronay. - Hte- 
Sav.: Annecy, fles du Fier, Thônes, le Parmelan, Tournette, Jalouvre, Reposoir, pied des $₹$ is, Pormenaz. - Is.: GrandeChartreuse, Chamechaude, St-Xizicr, col de l'Arc, mont Aiguille. Jonage et îles du Rhône en amont de Lyon. - Dr.: St-Vallier, Glandasse, Lus-la-Croix-Haute. - H.-Alp.: cols de Glaise et de Chaudun, Durbon, col du Noyer, mont Aurouse. - Vaucl.: envir. d'Apt. - Pyr.-Or.: vallée d'Eyne, Cambres-d'Aze, Madrès, Font-de-Comps. - Pyr. centrales. - Espèce polymorphe à laquelle se rattachent les formes suivantes :

S. pyrenaica Pourr. - Pyr.-Or.: Eyne, Font-de-Comps. - Ariège:

lac du Laurenti, vallons de Boutadiol, de Barbouillère et de

Valbonne, lac de Rabassolès, Port de Paillères du côté oriental.

S. remotiflora J. F. - Jonage (Is.).

S. longicaulis J. F. - Jonage, îles du Rhône (Is.), Chazay (Ain).

S. stricta J. F. - Jonage, îles du Rhơne (Is.), Chazay (Ain).

S. orophila J. F. - Le Reculet.

S. jurana J. F. - Reculet et montagnes d'Inimont, d'Ordonnaz et de Nantua (Ain).

S. integrifolia J. F. - Tenay (Ain).

MAREUBIUN vuggare L. - Bords des champs dans tout le bassin.

M. npulum Ten. - Pyr.-Or.: Pont-de-la-Fou près St-Paul-de-Fenouillet.

Mellttis meligsophylla $L$. - Bois dans tout le bassin.

SCUTELLA TE alpima L. - Collines et montagnes calcaires.

- C.-d'Or: Dijon, Gouville, mont Afrique, Pommard, Neursault, Beaune, Nuits. - Vaud et Valais: Alpes de Bex, Morcles, Fully, Hausseresse, Orsières, Liddes, Sembrancher, gorges de Durnant, Pierre à Voir, Mauvoisin. - H.-Sav.: Dentsd'Oche, les Platets, vallée du Reposoir, Héry au pied du mont Charvin. - Sav.: petit St-Bernard, Ciserette près Champagny, Combe-d'Ambin, St-Jean-de-Ilaurienne, Mont-Cenis. - Is.: la Grande-Chartreuse, Revel, Bour ${ }^{\gamma}-d^{\prime}$ 'Oisans, Brandes, Mont de Lans du côté de Venose, la Salette. - Dr. et H.-Alp.: Toussières et les Lucettes près Lus-la-Croix-Haute, Sappet de la Bàtie-Neuve, côte Gélive, col de Glaise, Séuse, Lautaret, Ia 
Grave, mont Genèvre, col Isoard, Abriès-cn-Queyras, St-Véran. - B.-Alp. : la Condamine, Lauzanier, Larche, mont GrandCouyer-sur-Annot. - Alp.-Mar.: Entraunes, St-Dalmas-le-Sauvage, mont Lachen au-dessus de Séranon, cols de Tende et de Fenestre. - Pyr.-0r.: Cambres-d'Aze, vallée d'Eynes, Nohèdes. - Roch. calc. des Pyr. centrales.

g. Inastifolin L. - Prés marécageux. - C.-d'Or, S.-ct-Loire et Ain : l'Abergement-les-Seurre, Chalon-sur-S., St-Bonnet-enBresse, Ecuelles, St-Laurent-les-Mâcon, Asnières, Vésines, bois de Seillon, la Dombes, Villars, les Echets, - Rh.: bords de la Saône et du lih. à Anse, Collonges, Oullins, Pierre-Bénite.

s. galerieulata L. - lieux bumides, bords des étangs dans une partie du bassin.

8. minor L. - Prés marécagcux des terrains siliceux et argileux. - Envir. de Belfort à l'Arsot, Salbert, la Vaivre. - H.-Saône : Champagney, Mansvillers, mont de Vannes, Menoux, Franchevelle, Mélisey. - Côte-d'Or, S.-et-L., Jura, Ain : l'Autunois, le Morvan, la Bresse, la Dombes, forèts de Chaux, de la Serre, de Rahon. - Is.: Charette, Revel, Chambaran, Eydoche, Flachère. - Gard et Iİr.: Gourdouze près Concoule, Fraisse, la salvetat, St-Vincent à Cassagnoles.

BRUNECIA hyssopongylla C. Bauh. - Coleaux secs, bords des chemins,-Dr.:Valence, Montélimar, Barnave, Grest, Nyons. - II. et B.-Alp.: la Garde et Charance près Gap, Manteyer, Sigoyer, Tallard, la Bàtie-Neuve, la Freissinouse, Roche-desArnauds, Digne, Sisteron, Annot. - Vaucl. et B.-du-R.: envir. d'Avignon entre Morières el Gadagne, bois de Fargues, envir. d'Orange, St-Amans, pied du mont Ventoux, env. d'Apt à Roussillon, Viens, Roquesalière, envir. d'Aix, bords de l'Arc, Montaiguet, envir. de Marseille. - Var et Alp.-Nar.: Toulon, Carqueiranne, Iyyères, le Lur, Fréjus, Cannes, Nice, Itenton. - Ard.: Joyeuse, Aubenas, entre Privas el Chomérac. - Gard el Hér.: Nîmes, Sauve, Montpezat, Blauzac, Alais, Anduze, StAmbroix, le Vigan, Tresques, commun dans les arrond. de Montpellier, de Béziers et de St-Pons. - Aude et Pyr.-0r.: envir. de Narbonne, Levrettes, Jonquières, la Clape à PechRedon, les Corbières, Bugarach, vallée du Tech, Costujes. 
E. vulgareis L. - Prés et bois dans tout le bassin. Les feuilles sont tantôt entières, tantôt pennatifides. - Espèce polymorphe.

B. allic Pallas. - Forme du précédent à fleurs blanches, plus grandes, à feuilles quelquefois entières, le plus souvent pennatifides. Plus rare que le type et paraît préférer les terrains calcaires et les expositions chúudes. M. Timbal-Lagrave a observé à Mijanès (Ariège) une Brunelle qu'il croît être hybride des $B$. alba et vulgaris et qu'il appelle B. albo-zulgaris. Si ce n'est pas un hybride, c'est au moins une forme intermédiare.

B. grandiflora Jacq. - Coteaux secs dans tout le bassin. - Varie à fleurs purpurines ou blanches, à feuilles entières (integrifolia), pennatifides (pennatifida) ou hastées (hastifolia). Cette dernière variété a été observẻe dans le Roussillon, l’Ariège, le Languedoc, le Tarn.

A ERGA (Ajuga) reptans L. - Prés, bois dans tout le bassin. Espèce polymorphe.

Une forme $A$. alpina Vill, dépourvue de stolons, se trouve sur les hautes montagnes du Valais, de la Savoie, du Dauphiné et des Pyrénées, notamment au St-Bernard et au col de Fenêtre - Cornettes de Bise, Brévent, mont Charvin, Tignes, Morteretdes-Allues. - Sepl-Laux-d'Allevard, forêt des Fraux près la Grave, - Massif du Laurenti.

A. pyramidalis L. - Pàturages des montagnes. Vaud et Valais : Anzendaz, Outre-Rhône, Fully, Sembrancher, St-Bernard, val Ferret, Bellalp, Natersberg, Zermatt. - H.-Sav. et Sav: Buet, col de Balme, flancs du mont Blanc, Vergy, Méri, la Sauce près Beaufurt, col de la Seigne. - Is.: chaîne de Belledonne près du lac du Crouzet, col Oddie, Champrousse, Grand-Charnicr d'Allevard, Taillefer, Lovitel près Venosc. - H.-Alp. : la Grave, Villard-d'Arène, St-Etienne-en-Devoluy, mont Séuse, mont Aurouse, Durbonnas, Chaillol, Péas. - B.-Alp. el Alp.Mar.: Lauzanier, Parpaillon, Fouillouse, Alpes de St-Etienne, lac d'Entrecoulpes, cols de Fenestre et de Salèse, Val di Blora, Alpes de Tende. - Pyr.-Or.: et Ariège : Canigou, Mont-Louis, lac de Quérigut, jasse d'Espagne, col de Lègue, lac du Laurenti, crête de Salsafrage, Soucarrat. - En dehors de nos limites au mont Dore, Plomb-du-Cantal, Aubrac, Pyr. centrales. 
ג. genevengis L. - Prairies et clamps dans tout le bassin.

A. chamaepitya Sclireb. - Jachèces et champs cultirés dans tout le bassin.

A. Iva Schreb. - Coleaux pierreux de la région des Oliviers dans la Provence, les Alpes-IIaritimes, le Languedoc et le Roussillon.

Une forme à fleurs jaunes, appelíe A. pseudo-iva par Robert et Castagıe, est ruelquefois mêlée au type à fleurs purpurines ou le remplace.

TEUChiov frutieans L. - Cotiaux pierreux des Pyr.-Or. à Banjuls-sur-Ner et au cap Cerbère.

'E. tripartiturs, $T$ pseudochamnepitys L. - Terrains pierreux du littoral. - B.-du-Rh. et Var : envis. de Marseille à St-Henry, les Aygalades, l'Estaque, Mourrepiane, Fréjus.

s. botrydium, T. Jiotrys L. - Champs cultivís et jachéres dans tout le bassin.

T. scordium L. - Bords des étangs, prés lumides.

T. scordioideum Schreb. - Forme du précédent à feuilles cordiformes, crénelées sur tout leur pourtour; quelquefois mêlée au type.

T. seorodonium L. - Bois et haies dans tout le bassin.

T. maสgniense L. - Ile du Levant près Hyères.

T. Chranacedryuma $L$, - Bords des chanaps, des haies et des bois.

T. Itucidum L. - Bois. - B.-Alp. et Alp.-Nar.: la Condamine, Meyronnes, la Reissolle, Barcelonnette, Castellanne, Annot, Seyne, Digne, Colmars, Entrevaux, St-Étienne, St-MartinLantosque, Entraunes, Séranon, mont Cheiron, mont Lachen, les Lattes, St-Sauveur, Utelle, Duranus.

T. Inxum L. - Rochers et pierrailles de la région mérid. Vaucl. et B.-du-Rh.: envir. d Avignon, d'Aix au Ttıolonet et au Prégnon, Montredon, Ste-Marguerite, iles de Pomègue et de Maïré, St-Pons, St-Loup. - Var et Alp.-Mar.: Toulon au mont Faron, le Luc, ile Ste-Marguerite, Grasse, Nice, St Hicheld'Eza, Menton au Pont-St-Louis, grolte do Marenco. - Gard et Hér.: bords du Gardon à St-Laurent-le-Minier, Anduze, St-Jean-de-Yédas au Moulin Geniès, pic St-Loup, St-Martin-deLondres aux dres, Ganges, la Sérane, les Capouladoux, St- 
Guilliem-le-Désert. - Aude et Pyr.-Or. : Tuchan, St-Victor, la Clape au Rec, Vingrau, Trencada-d'Ambulla, Cases-de-Pena, Pont-de-la-Fou prés St-Paul, St-Antoine-de-Galamus. - Espèce polymorphe à layuelle se rattachent les deux formes suivantes :

T. virescens J. F. - Montpellier. - T. pulverulentum J. F. - La Ciotat (B.-du-R.).

T. marum L. - Rocailles. - Var et Alp.-Nar. : lles du Levant, de Portcros et de Porquerolle, la Cascade près Grasse.

T. pyreuaicuna L. - Rochers calcaires. - Pyl'-0r. et Ariège : mas de la Guardia près Arles, Costujes, St-Antoine-de-Galamus, pic de Bugarach, Salvanère, roc de la Pinouse au sommet du vallon de Canals. - Is. : entre le Ionestier-de-Clermont et Gresse, la petite Moucherolle. - Pyr. centrales.

T. montanum L. - Coteaux calcaires dans tout le bassin.

T. aureum Schreb. - Dr., H. et B.-Alp.: Nyons, Saou, les Baux près Gap, Tallard, Ventavon, Roche-des-Arnauds, Laragne, Ribiers, Serres, Rosans, St-André-de-Rosans, Sisteron. - Ard.: Chassagne. - Vaucl.: Orange, Jaalaucène, Gigondas, Vaucluse, Cavaillon, - B.-du Rh. et Var : Ste-Victoire, Tête-de-Carpiagne, Ste-Baume au St-Pilon.-Gard : Nimes, Manduel, Montdardier, Alais, St-Ambroix, Anduze. - Hér.: St-Guilhem, les Cambrettes, la Sérane, Ganges, Madières, l'Espinouse. - Aude et Pyr.-Or.: les Corbières, vallée de l'Aude d'Aunat à Quillan, Salses, Cases-de-Pena, St-Antoine-de-Galamus, Pont-de-la-Fou, Trencada-d'Ambulla, Prats-le-Mollo. - Espèce polymorphe à laquelle se rattachent les formes suivantes:

T. saxicolum J.-F. - Vaucluse. - T. massiliense J.-F. Arles à Montmajour.

T. polium L. - Rocher's et rocailles de la région mérid. - Remonte dans les H. et B.-Alp. vers Sisteron, Digne, Laragne, Serre, Fouillouse, Ventavon, Veyne, Rosans; dans la Drôme vers Nyons, Dieu-le-Fit, Loriol, Crest, Valence, St-Vallier, Serves; dans l'Isère vers Vaugris et Vienne; dans l'Ardèche et la Loire vers Rochemaure, Aubenas, Tournon, Serrières, St-Pierre-deBouf. 
Espece polymorplıc comprenant les formes suivantes distingućes par Jordan et liourreau :

P. supinum. - Commun dans la règion mérid.

P. album. - Commun dans la régrion mérid.

P. microphyllum. - Pampelonne près Rochemaure (Ard.).

P. vivariense. - Crussol (Ard.).

P. rhodunicum. - Vienne, entre

St-Vallier et Tain (Dr.) Tournon (Ard.).

P. graniticum. - Serves (Dr.).

P. flexuosum Vaugris (Is.), Granne (Dr.), Cruas (dru.).
P. vestitum. - Nyons (Dr.).

I. supravirens. - Celles (Ard.).

P. drumense. - Loriol (Dr.).

I. collinum. - Nyons (Dr.).

P. brevicaule. - Celles (Ard.).

$P$. debile, - Vaison (Vaucl.).

P. leptocludum. - Vaugris (Is.).

P. drucntinum. - Avignon, bords de la Durance.

l. arausicum. - Orange(Vaucl.).

$P$. canescens, - Vaucluse.

$P$. lagopinum. - Tresques (Gard).

\section{AGANTHACÉES}

ACANTMOS moLis L. - Fentes des rochers, - Var et Alp.Mar.: Toulon, Hyères, le Cannet, Rians, le Bar, Grasse, Biot, Nice à Magnan et à St-Philippe. - Gard et Hér.: pied des anciens remparı de Nimes, chemin d'Alais, bords du Gardon à Anduze, parc d'Uzès, Pech-Bebios près Béziers, Vendres, Lespignan. - Pyr.-0r. : citadelle de Perpignan, lle Ste-Lucie. Dans plusieurs des localités ci-dessus mentionnẻes l'Acanthe a été planté et, par conséquent, n'y est pas indigène.

\section{VERBÉNACÉES}

VERENA officinalis L. - Bords des clumins, clumps dans tout le bassin.

VTr agnus, $V$. agnus-castus L. - Bords des torrents, lieux incultes. - B.-du-Rh. : la Ciotat. - Var et Alp.-IIar.: Toulon, La Scyne, Hyères, Antibes, Châteauneuf, Foux-de-Mouans, Nice, Menton, cap Martin. - Ilér. : bords du Libron, Cers. - 
Aude et Pyr.-Or.: Nontoulieu, la Clape, Collioure, Port-Vendres, Banyuls-sur- Her.

\section{PLANTAGINEES}

PL NTAC majoñ L. - Prairies, bords des chemins dans tout le bassin.

P. intermedia Gilib. - Forme du précédent à hampe velue, couchée, arquée-ascendante, à feuilles hẻrissíes de poils blanchâtres, sinuées-dentées, à pédoncules étalès-arqués. Se montre de préférence dans les terrains sablonneux lıumides. La variété minima DC. est une forme naine à épis courts et ovoïdes qu'on observe quelquefois dans les terrains sablonneux très-secs.

P. nltisgaimara Lois., P. Cormuti Gouan. - Terrains salès du littoral méditerranéen de l'Ilérault et du Gard.

F. media L. - Bords des chemins, prés dans tout le bassin.

P. brutin Ten. - Forme naine du précédent à bractées et lobes de la corolle aigus. - H.-Alp.: prairies de la Grave, Villard-d'Arène, Lautaret.

P. coronopoura L. - Lieux secs, bords des chemins de la région mérid. - Kremonte quelquefois la vallée du Rhône jusqu'à Lyon. - Espèce polymorphe présentant tantôt des feuilles larges ( $P$. latifolia DC.), tantôt des feuilles charnues, étroites (P. maritima Godr.), quelquefois des feuilles entières ( $P$. integrifolia Godr.)

P. carasifolia Forsk. - Plage méditerranéenne de la Provence, des Alpes-llar., du Languedoc et du Roussillon.

P. maroina L. - Lieux salés des côtes de la Méditerranéc, et aussi dans l'intérieur des terres. - B.-du-Rh. et Var: borls de l'ćtang de Berre, Fos, la Crau, la Camargue, Montrieux, Brignoles, jardin de l'hospice de la Ste-Baume. - Gard: Saure, Servier, boris du Vidourle, à Quissac. - En dehors de nos limites sur les côtes de l'Ocẻan el dans les marais salés du Puyde-Dòme à St-Nectaire, Ste-Narguerite, eaux du Tambour, Médagues, marais de Coeur, de Marmillat, de Sarliève.

2. serpentimn Vill. - l'icrrailles, bords d's chemins. - Com- 
mun dans la région mérid.; plus rare dans le centre et le nord llu bassin. - Doubs et Jura : Chapelle-des-Buis près Besançon, 'larcenay, Ornans, Salius. - Valais, II.-Sav.: le Simplon, Archamp, St-Julien, Allonzier, Cuvat, Modane, - Ain : Peyrieu, Virignin, Culoz, lles du lihône en face de la Pape. - Is. et Dr. : bords du lhoone à Feyzin, Genas, Vif, St-dnge, polygone de Grenoble, Vaunaveys près Crest, Granne, Montélimar, Lusla-Croix-Haute. - H. et B.-Alp.: Lautaret, Plan-de-Phazy, Poligny en Champsaur, Chaudun, mont Séuse, Charance, Glaise, Chorges, Durbonnas, Jausiers, la Condamine, Lauzanier, Annot. - Ard. : Charray, Flaviac, Vernoux. - Commun dans la Provence, les Alp.-Mar., le Languedoc et le Roussillon.

r. apina L. - Pelouses des montagnes. - Chaine jurassique : la Dole, le Reculet. - Alpes de Vaud, Valais, H.-Savoie où il s'élève jusı̧u'au Jardin de la Mer-de-Glace, Savoie, Dauphiné, B.-Alpes et Alp.-Maritimes, - Pyr.-Or. et Ariège. - En dehors de nos limites dans les Pyrénées centrales, mont Dore, Cantal. La variété incana Ram. se trouve dans la chaine des Pyrénées.

P. subulatu I. - Rochers maritimes. - B.-du-Rh., Var et Alp.Mar.: envir. de Marseille, lles Pomègue, Ratoneau et Riou, la Crau, Montaud, envir. de Toulon et de Cannes. - Pyr.-Or.: Collioure, Port-Vendres, Banyuls-sur-Mler.

P. carinata Schrad. - Rochers siliceux. - Chaines granitiques du Rhône, de la Loire et de l'Ardèche. - Is., Dr. et Ard. : Vienne, St-Vallier, Tain, Crest, Montélimar, Vernoux, Celles, 'Tournon. - H.-Alp. : Valgaudemar au-dessus de Vallon-CIot. - B.-du-Rh. et Var: bords de la roule de dliramas à Eyguières, et de Miramas à Istres, la Sauvette daus les Maures. - Gard: l'Espérou, l'Aigual, le Vigan, Alais, St-Ambroix, Anduze, Concoule. - Hér.: St Chinian, St-Pons, la Salvelat, Fraisse, le Caroux, Pardailhan, vallée de la Hare, St-llartin-d'Orb, Avèneles-Bains. - Aude et Tarn : la Montagne-Noire. - Pyr.-Or. et Ariège : Argelès, Port-Vendres, Prades, Vernet, Olette, Carol, lac Noir de Rabassolès, pic de Fontnègre, port de Paillères. En dehors de nos limites dans les montagnes du Forez, la vallée de la Loire, le massif central et jusque dans l'ouest de la France. - Nul sur les calcaires. 
P. lagopoda L. - Champs pierreux de la région mérid. - Váucl. et B.-du-Rh.: Montdevergues près Avignon, envir. d'Orange, de Carpentras, d'Aix et de Marseille. - Var et Alp.-Mar.: Toulon, Hyères, le Luc, Cannes, Nice, Menton. - Gard : Villeneuve en face d'Avignon, St-Gilles, Nìmes. - Hér. : Montpellier, la Colombière, Hireval, Vic, St-Aunès, Vendargues, Cette, Pézenas, Bẻziers, St-Chinian. - Aude et Pyr.-Or.: littoral de la Clape, Ste-Lucie, Leucate, Canet, Argelès, Collioure, PortVendres, Banyuls-sur-Mler.

P. Ianceolata L. - Bords des chemins, champs et prís, dans tout le bassin. Espèce polymorphe dans laquelle la forme et la villosité des feuilles, la taille, la longueur et la forme des épis présentent de grandes variations.

P. Timbali Jord. a les feuilles linéaires-lancéulées.

$P$. lanuginosa Koch a les feuilles couvertes de poils laineux.

P. capitata Ten. a un épi globuleux et des feuilles laineuses à la base.

P. argentea Chaix. - Prairies des montagnes. - H.-Alp.: envir. de Gap, Charance, Rabou, col de Glaise, Bayardon, mont Séuse, mont de Faye près Ventavon. - Vaucl. et B.-du-Bh.: StAmans, Ste-Victoire, les Baux. - Var et Alp.-Mar.: N.-D. des Anges près Pignans, mont Cheiron, St-Martin-d'Entraunes. Gard et Hér.: bois de Salbous, Campestre, rochers de la Sérane, St-Guilhem-le-Désert. - En dehors de nos limites dans l'Aveyron près de Tournemire et au Devèze-de-la-Panouse.

P. albieans L. - Bords des chemins. - B.-du-Rh. et Var.: envir. d'Aix sur le chemin de Berre, Moquefavour, envir. de Marseille, Toulon au Fort Ste-Catherine, Hyères. - Hér.: Béziers, Pézenas, Nissan, Montpellier derrière la Citadelle, Lunel. Aude et Pyr.-Or.: envir. de Narbonne, la Clape, Pech de l'Agnèle, Ste-Lucie, les Corbières, Sigean. Vingrau, Perpignan, Baixas, Cases-de-Pena.

P. holostea Lam., P. Bellardi All. - Lieux sablonn. de la région méditerranéenne. - B.-du-Ph. et Var: envir. de Marseille, les Martigues, Toulon, Hyẻres, St-Tropez, Fréjus, Cannes, Nice, Jenton. - Gard et Hér.: envir. de Nimes, Pont-du-Gard, Sylvéréal, Roquehaute près Vias. - Aude et lyr.-Or.: région lit- 
torale, St-Crescent, Aussieres, Ste-Lucie, Collioure, Port-Vendres.

P. Puscencenss Jord. - Pâturages des Alpes, - H. et B.-Alp.: mont Viso en montart à la Traverselte, col Agnel, Lauzanier, Horonaye, Malemort. - Alp.-Mar.: cols de la Maddalena et de Tende, l'Authion, la Maïris, Berre, l'Escarène.

P. montana Lam. - Pâturages des montagnes. - Jura helvétique et français. - Hte-Sav. et Sav.: montagnes du Chablais, Tournette, Semnoz, Hauteluce, Crest-Voland, montagnes de la $\mathrm{Ta}-$ rantaise et de la Maurienne. - Is. et H.-Alp.: Grande-Chartreuse, St-Eynari, Chalais aux Banettes, col Vert, col de l'Are, la Moucherolle, Charance, col de Glaise, Bayard, Chaudun, mont Séuse, Durbonnas, Poligny en Champsaur.

P. monosperma Pourr. - Pelouses de la région alpine des Pyrénées. - Pyr.-0r. et Ariège : Canigou, Mont-Louis, St-Pierre, vallée d’Eyne, Laurenti en montant à la Porteille de Baxouillade, pic del Ginévré. - Pyrénées centrales.

P. pryllia L. - Bords des champs et des chemins de la région mérid.; remonte dans la Drôme vers Nyons; dans l'Isère entre Corps et la Salette.

P. arenaria $\mathbb{W}$. Kit. - Lieux sablonneux de la région mérid. et moyenne du bassin. Apparait quelquefois dans les champs de Trèfle et de Luzerne de la région septentr. où il est probablement apporté du midi avec les graines de plantes fourıagères.

P. суnops L. - Lieux incultes de la région mérid. et moyenne; moins commun dans la partie septentr. du bassin.

HTTO IE ELA Ineustris L. - Lacs et élangs. - Envir. de Genève à l'embouchure de la Versoix, pointe de Bellerive, près du Vangeron, Promenthoux près Nyons. - Arrond. de Belfort, Doubs : entre Roppe et Eloye, étang de la Forge, Cravanche, Chaux, la Chapelle. - Jura et Ain: la Bresse à Sergenaux, Fays, Champrougier, forèt de la Serre, la Bresse de l'Ain et la Dombes à Villars, St-Andrẻ-de-Corcy, St-Jean-Thurigneux. C.-d'Or, et S.-et-L.: le Morvan à Saulieu, étangs de Champrin, Beauvais, Létaumé, Vic-sous-Tille, la Bresse louhanaise. - Rh.: Lavore près de Chassagny. - Is.: Bouvesse, étang de Bressieu, marais de Janeyriat. 


\section{PLUMBAGINÉES}

A RMERA maritima Willd. - Cette jolie espéce des côtes de l'Océan est souvent cultivée dans les jardins d'où elle s'échappe quelquefois.

A. rusclnonensis Gir. - Rochers du littotal des Pyr.-Or. a Collioure, Port-Vendres, Banyuls-sur-Mer.

A. Juneea Gir. - Sochers. - Gard et Hér. : Montdardier à Blanılas, le Vigan, St-Guilhem, Viuls-le-Forl, la Sérane, les Capouladoux, St-Martin-de Londres, Madières, le Larzac au Caylar et à la Vacquerie, Lodève, Liausson, Carlincas.

A. majellensia Boiss. - Rocher's de's Pyr.-Or. au pic de Taillefer au-dessus de Consolation.

A. pIantaginer Willd. Colcaux inculles des parlies mérid. et

moyenne du bassin. Espèce polymorphe comprenant les formes suivantes :

A. sabulosa Jord. - C'est la forme la plus répandue; elle a des fleurs blanches en petits capitules.

A. buplearcidea G. G. - Coteaux de la région mérid. - Forme à involucre pâle dont les folioles ne dépassent pas les fleurs, à calice divisẻ en lobes terminés par une longue arête. - Vaucl. et B.-du-Rh. : Orange, Piolenc, Cavaillon, Flassan, mont Ventoux, Luberon, collines de Carpiagne, Ste-Victoire, chaîne de l'Étoile.

-- Var : Toulon a cap Garonne, N.-D.-des-Anges près Pignans, mont Sauvette, Montrieux, Fréjus. - Gard et Hẻr.: Uzès à Serviers, Valflaunès, Lamalou, St-Chinian, Riols.

A. praecox Jord. - Fleuis grandes, blanchâtres, à floraison précoce; feuilles larges et courtes. - H. ot B.-Alp. : le Lauzet, Névache, mont Genèvre, St-Étienne en Deroluy, entre St-Firmin et la Chapelle en Valgaudemar, la Roche-des-Arnauds dans la prairie de Quint, mont Hognouse près Ribiers, Annot.

A. flicaulis Boiss. - Var à la Tourne près Belgentier (Huel, 1863).

A. alpina Willd. - Rochers et pâturages des Alpes et des Pyr. - H.-Sav. et Sav.: Cornetles de Bise, Vergy, Tournette, la Vanoise. la Thiopa au dessus de Champagny, Crêt-du-Rí au-llessus d'dime, 
Ic Bonlsomme, mont Cenis, l'Allée Blanche sur le versant italicn du mont Blane. - Is. Dr. et H.-Alp.: Belledonne, Champrousse, Taillefer, Grandes-Rousses, St-Pierre-de Chartreuse, la Moucherolle, sommet de Toulau près Embel, Lautaret, Galibier, vailee du Guil, mont Viso, col Vieux, mont Aurouse, Chaillol, mont Queyrel. - B.-Alp.: Lauzanier, Vallonnet, Parpaillon, Bachasse. - Nip.-Mar. : lac de Vens, le Jarret, cols de Jallorgues et de Fencstre, Ste-Anne-de-Vinaï, mont Bego, Raus, l'duthion. - Pyr.-Or. : Canigou, Pla Guillem, Cambresd'Aze, vallée d'Eyne. - Pyrénées centrales.

STATCE simuta L. - lles du Levant (Var), Lazaret de Nice (Alp.-Mar.).

*. Limonin L. - Commun sur les rivages de l'0céan; la forme serotina Rchb. du littoral méditéranéen a des rameaux plus grẻles, plus ílalés, flexueux; elle est assez répandue sur les plages maritimes du Roussillon, de l'Aửe, de l'Hérault et du Gard. - Envir. de llarseille, Sablettes de Toulon, Hyères, ile de Porquerolle, St-Tropez. On ne l'a pas revue à l'embouchure du Var, à Antibes et à l'île Ste-Marguerite où elle existait autrefois. - Espece polymorphe.

6. Iychinanifolia Gil. - Espece des côtes de l'Océan observie sur le littoral de la Mćditerrannée près de Narbonne à la Clape et à Ste-Lucie, puis à Leucate.

๙. Oleifolia Wild., S. Dodartima Gir. - Cette Statice du littoral de l’océan a śté vue dans l'Hérault sur la plage des Onglous près de Cetle, à Valréas prés Sérignan.

s. confusn G. G. - B. du, Rh. : la Camargue aux Ste-Haries, Martigues à l'étang de Caroute. - Aude: Ste-Lucie, bords du canal de la Nouvelle.

\$. densiaora Gir., S. Girardiana Guss. - B.-du-Rh. : Arles, les Martigues, Fos. - Gard et Hér.: Aigues-Mortes, Bellegarde, St-Gilles, Pẻrols, Palavas, Maguelonne, Cette, les Onglous, Mireval, Valras, Portiragne à Roquehaute. - Aude et Pyr.Or. : Ste-Lucie, la Nouvelle, St-Nazaire, le Canet.

6. duriuscula Gir. - B, du Rh. et Var : Fos, Istres, les Martigues, Toulon. - Hér.: Cette, Maguelonne. - Aude et Pyr.-0r.: Ste-Lucie, la Nouvelle, Leucate. 
S. Legrandiana Timb. et Gautier. - Plages de Vendres et de Leucate.

S. minutr L. - B..du-Rh. el Var : Ile Pomègue, Ratoneau et Riou, plage de Montredon, lle de Porquerolle, St-Raphaël.

s. virgata Willd. - B.-du-Rh. et Var: Marseille, les Martigues, Arles, Hyères aux Pesquiers. - Gard et Hér. : Aigues-Mortes, Roquehaute, les Onglous, Cette, Haguelonne, Palavas. - Aude et Pyr.-Or. : la Clape, Ste-Lucie, Port-Vendres.

s. pubescens DC. Var et Alp.-IIar.: Antibes, Fréjus, Cannes, îles de Lérins.

S. belldifolia Gouan. - B.-du-Rh.: les Martigues. - Gard et Hér.: Bellegarde, Aigues-Mortes, Pérols, Palavas, Maguelonne, Mireval, Frontignan, Cette, Sẻrignan à Valras. - Aude : (iruis. san, la Clape, Ste-Lucie, Leucate.

s. cohioidea L. - Bords des étangs, terrains sablonneux des plages maritimes et même de l'intérieur des terres. - B.-du-Rh. Var et Alp.-Har. : Montredon, iles Pomègue et Ratoneaı, Montaud près Salon, Roquefavour, Toulon, le Lucc, Frêjus, Cannes, de Ifenton à Vintimiglia. - Gard et Hér. : Aigues-Hortes, Sylvéréal, Caunelle, Sussargues, Madières, St-Guilhem-leDésert. - Aude et Pyr.-Or.: Creizel, la Clape, Ste-Lucie, Leucate.

S. diffusa Pourr. - Plages de Ste-Lucie et de St-Pierre (Aude).

Lrmonion monopetaium Boiss. - Plages de la Clape, SteLucie, la Nouvelle (Aude).

PLUMBaco europaea L. - Bords des chemins de la région méditerranéenne; remonte dans les B.-Alpes vers Cháteau. Arnoux; dans la Drome vers Clansayes, Montélimar; dans l'Ardèche vers Payolive.

\section{GLOBULARIE்ES}

GLOBULARIA vugaris L. - Coteaux incultes surtout des terrains calcaires.

La forme G. Wilkommiana Nyman a les divisions calicinales deux fois plus longues que le tube, le capitule plus petit. 


\section{$-640-$}

G. nudicantis L. - Rochers et rocailles des montagnes calcaires.

Vaud et Valais: Lavarraz, Solalex, Chemin-Neuf, mont Fully, Comba d'Arba. - II.-Sav.: Salève, le Mole, Glacière du Brizon, Parmelan, Tournette, mont Péletod, Dents-d'Oche, montagnes de Samoens, Tré-la-Téte, mont Joly. - Is. : Grande-Chartreuse, col de la Ruclière, forct de Portes, le Collet près du CharmantSom, Ies Bancttes de Chalais, St-Ange, St-Nizier, entre Corps et la Salette. - Ir. : Barnave, Vassieux en Vercors. - Alp.Mar.: Alpes de Clans et de Tende. - Pyr.-Or. et Ariège: Font de Comps, Cambres d'Aze, vallée d'Eynes, Prats-de-Mollo, St- $\Lambda$ ntoine-de-Galamus, lac de Rabassolès, vallon de Councq, Rouze, Usson, forét de Salvanère.

G. condufolia L. - Rochers des montagnes calcaires. - Jura helvétique et français: Hauenstein, Wallenburg, Passwang, Chasseral, Tete-de-Rang, la Tourne, Creux-du-Van, Suchet, Montendre, Noirmont, la Dole, Colombier, Reculet, les Rousses, Iloret, Bonlieu, St-Claude, Montanges, Poisat près Nantua, Colombier du Bugey. - Valais : St-Maurice, Martigny, Saillon, Vétroz, Tourbillon, Comba d'Arba, Leukerbad. - H.-Sav. et Sav. : Salève, Brizon, Vergy, Méri, Parmelan, Charvin, monlagnes de Thônes el de Veyrier près Annecy, Dents-d'Oche, montagnes calc. de la Tarantaise et de la Maurienne. - Is: Bastille de Grcnoble, Grande-Chartreuse, St-Nizier, Villard-deLans, Pont-en-lioyans, la Salette. - Dr. et II-Alp.: Pas de Lauzens et Saou près Crest, Villard-d'Arène, la Vachette, St-Véran, Charance, cols de Glaise et Bayard, mont Chabrière, Séuse. - B.-Alp. et Vaucl. : montagnes de Gache et de la Baume près Sisteron, Annot, la Condamine, Serennes, Bouzollières, mont Ventoux. - Alp.-Mar. : montagues calc. de Grasse, des vallées du Var, Vesubia, Tinea, Roja.

G nı Lam. - Forme du précédent, à feuilles étroites et à pédoncules courts, observée prés de Sisteron aux rochers de la Baume (B.-Alpes), au mont Ventoux et à St-Amans (Vaucl.), à la Ste-Vicloire (B.-du-Rh.), dans les Pyr. Or. et l'Ariège, à Saint-Antoine-de-Galamus, Cambres d'Aze, vallẻe d'Eyne, Prats-de-Mollo, lacs du Laurenti et de Rabassolès, Valbonne, 
$-646-$

Port-de-Paillères, vallon de Canals, de Boutadiol et de Barbouillère, Rouze, Pic de Soulanès, Roc-Rouge au-dessus de $\mathbf{M i -}$ janès. - Pyrên. centrales.

C. alypa L. - Coteaux pierreux de la région mérid. - Commun dans les B.-du Rh., Var et Alpes Mar. - Gard et Hér.: Chartreuse de Valbonne, bords du Gardon à la Baume, montagne de Cette, Nissan, St.-Vartin-de-Londres, St-Guilhem, la Sérane à St-Jean-de-Bueges, Mas-de-Vareithe, Capouladoux, St.-Étiennede-Gourgas. - Aude et Pyr.-Or.: environs de Narbonne et de Perpignan, Cases-de-Pena, St-Antoine-de-Galamus, Trencada d'Ambulla. 


\section{MONOCHLAMYDÉES}

\section{PHYTOLACÉES.}

PIITTOLACCA decandra L. - Espèce américaine naturalisée dans les bois de la Chartreuse de Valbonne et à la Pinẻded'Aigues-Mortes (Gard); au pied des Albères, à St-Génis-desFontaines et à St-André (Pyr.-Or.); autour de Fréjus et de l'Abbaye de Ste-Marie du Thoronet (Var); vers le Bar, Nice, Mlonaco, IIenton (Alp.-Mar.); autour d'Aix (B.-du-Rh.) et d'Avignon (Vaucl.).

\section{AMARANTACEES.}

ARA RANTON deflexum L. - Bords des chemins, déconbres, lieux incultes. - Commun dans la Provence, les Alp.-Harit., le Languedoc et le Roussillon; remonte dans les B.-Alp. vers Annot; dans la Drôme vers St-Vallier. On l'a aussi observé à Lyon, à Trévoux (Ain) et à St-Julien (H.-Sav.), à Genève dans le quartier de Carouge.

A. blitonlum L. - Bords des chemins, décombres, dans tout le bassin.

A. milvestre Desf. - Lieux cultivés dans les parties mérid. el moyenne du bassin; plus rare dans la partie septentrionale.

A patulum Bertol. - Licur cultivés, voisinage lles habitations. B.-du-Rh. : Aix. - Région littorale des Alp.-Mar, - Gard et Hér.: Manduel, Lattes et Grammont. - Aude et Pyr.-Or.: envir. de Narbonne, pied des Albères. - Is.: naturalisè à Grenoble par des graines échappies du jardin botanique, Pont-de-Chérui.

A. retronexum I. - Dérombres, bords les chemins dans les 
parties mérid. el moyenne du bassin. D'année en année, on voit cette espèce gagner du terrain dans la partie septentrionale. Déjà elle est largement naturalisée sur plusieurs points de la Côte-d'Or et il y a lieu de croire que, grâce aux chemins de fer, elle étendra de plus en plus son aire de dispersion.

A. album L. - Espèce de l'Amérique septr. naturalisée près des habitations et le long des chemins de la région mérid.; remonte dans la Drôme vers Crest; je l'ai vue à Lyon aux Brotteaux.

POLYCNEMUV arvense $L$. - Bords des chemins el des champs de tout le bassin.

Présente deux formes, l'une $P$. minus qui a les bractées égales au périgone; l'autre $P$. majus dont les bractées dépassent le périgone.

\section{SALSOLACÉES.}

ATIRPLE Inortensis L, - Cultivé et qnelquefois subspontané.

A. microthecus Hoq. T. - Forme du précédent à feuilles vertes en dessus, glauques en dessous, ovales, lancéolées. - H. et B.Alp.: sous les forts de Briançon, Château-Queyras, la Condamine. - B.-du-Rh. et Hér.: Aix, au-dessous du cimetière, plages d'Agde et de Cette.

A. roseus L. - Licux incultes, - Vaucl,, Gard et B.-du-Rhône.: envir. d'Avignon, Gadagne, Villeneuve, envir. de Marseille, St-Geniez, les Martigues. - Var et Alp.-Mar.: Toulon, Salins d'Hyères, entre Nice et le Var, Vintimiglia sur le territoire italien. - Hér.: Mireval, Béziers, Pézenas, St-Nazaire, St-Martin-d'Orb. - Aude et Pyr.-Or.: envir. de Narbonne, Ste-Lucie, Salses, Canet, St-Nazaire.

A. crnssifolius C. A. Mey. - Terrains salés. - B.-du-Rh. et Var : Montredon, étang de Berre, Marignane, Toulon, Salins d'Hyères. - Gard et Hêr.: Aigues.IIortes, Cetle, Balaruc. Aude et Pyr.-Or.: toute la région littorale.

A - Iaciniatus L. - Décombres, terrains salés. - B.-du-Rh. el Alp.Mar.: envir. de Marseille, les Martigues, Nice, - Gard et Hêr : 
Bellegarde, Aigues-Mortes, Palavas, Portiragnes, Cette. - Littoral de l'Aude et des Pyr.-Or.

A. hallmu* L. - Partout planté dans les haies de la région mérid.; souvent subspontanê.

A. Hnstatus L. - Bords des champs et des chemins dans une grande partie du bassin. - Espèce polymorphe dans laquelle les graines, le périgone et les feuilles prisentent de nombreuses variations

La variété salimus Wallr. ou oppositifolius DC. qui a des graines petites, des feuilles épaisses, blanchâtres, farineuses, ordinairement opposées, croit dans la région littorale de la Provence, des Alp.-Mar., du Languedoc et du Roussillon.

A. patulus L. - Bords des chemins et des champs dans tout le bassin; plus commun que le précédent. - Espèce polymorphe.

DIBIONE portulacifolia Moq. 'T. - Littoral méditerranéen de la Provence, des Alp.-Nar., du Languedoc et du Roussillon.

SPINACIA glabra Mill, et S. oleracea L. - Cullivés et quelquefois subspontanés.

BETA vulgaria L. - Cultivé ct iquelquefois subspontané.

B. marltima L. - Bords de la mer et des étangs.

CHENOPODHON ambrosioldeum L. - Cultivé dans les jardins et subspontané autour de Toulon, Nice; - autour de Montpellier à Castelnau, Lavérune, Mauguio, Candillargues, Castries, Andabre Rosis, St-Gervais sur-Mare, Hérépian, Limas (Hér.); - Alais, Chartreuse de Valbonne, Vallabris près Uzès (Gard); Perpignan, Vernet, Céret (Pyr.-0r.).

C. botrydium L. - Terrains graveleux et sablonneux de la Provence, des Alp.-Mar., du Languedoc et Roussillon. - Remonte dans les B.-Alpes et la Dr. vers Annot, Nyons, Valence; dans l'Ard. à Flaviac; dans la Loire et le Rh., le long du Gier, Lyon; dans l'Is. et la Sav. à Vienne, Venoš, St-Christophe-en Oisans, de Moutiers à Bourg-St-Maurice et Ste-Foy; dans Vaud et Valais, à Payerne, de Branson à Saxon, Marligny, Sion, Bra mois, Longeborgne, de Visp à Stalden, vallée de St-Nicolas et de Saas. - En dehors de nos limites dans les Pyr. centrales, la Lozère, le Tarn et l'Aveyron. 
C. polyspermum. - Lieux cultivés, décombres dans le bassin. - Espèce polymorphe.

C. Foctidum Lam., C. Vulvaria L. - Décombres, bords des chemins.

C. fieifolium Sm. - Champs humides des environs de Lyon, Grenoble, Romans, Bourg-d'Oisans, Nîmes, Beaucaire, Perpignan, le Luc, lle de Porquerolle, Rians, le Bar.

C. album L. - Lieux cultivès, décombres. - Espèce polymorphe.

C. opulifollum Schrad. - Lieux cultivés, décombres dans la Provence, Alp.-Itar., Languedoc, Roussillon, Briançon, Grenoble.

C. hybridum L. - Lieux cultivés, roisinage des habitations.

c. urbieum L. - Voisinage des habitations, bord des chemins.

c. murale L. - Nême habitat.

C. glaueum L. - Lieux lıumides, - H.-Saône : Recologne, Ronchamp, Champagney, - Doubs : envir. de Montbẻliard. C.-d'Or, S.-et-L. et Rh.: Dijon, Genlis, bords de la Saone, Anse, Collonges près Lyon.

C. rubrum L. - Lieux humides, bords des étangs et des rivières dans la Provence, Alp.-Har., Languedoc, Roussillon. Lorsque cette espèce vit dans les terrains arrosés par des eaux salées, elle acquiert des feuilles èpaisses el charnues (C. crassifolium (Hornm).

C. Inantatum, C. Bonus Henricus L. - Voisinage des habitations dans les plaines et jusque dans les montagnes autour des chalets et des bergeries.

BLITON vikgatum $L$. et B. eapitarum L. - Cultivés dans les jardins et quelquefois naturalisés dans leur voisinage.

EROUEIEVA multifida Moq. T. - Naturalisé sur les glacis des fortifications de Toulon ainsi qu'au port Juvénal près de Hontpellier.

BoCHIA prostrata Schrad. - Bords des caux. - B.-du-kh.: Tarascon, Arles, - Hér., Aude et Pyr.-0r.: bords du canal à Béziers, Nissan au Pas-du-Loup, cap de Pla près Narbonne, Leucate, Rivesaltes, Perpignan, Elne, Olette, vallon de Cerbère, Collioure, drgelès. 
K. arenaria Roth. - Cliamps sablunneux. - Is. et Dr.: Chasse, les Balmes près Romans, St-Paul-Trois-Châteaux. - Vaucl.: Carpentras, Bédoin, Moulin-de-la-Grotte, Mormoiron. - Gard.: les Angles au Trou-du-Renard et à la descente du MourreBlanc à l'étang de Pujault, Tresques.

Ex. Hirauta Nolte. - Bords des etangs, terrains salés du littoral. - B.-du-Rh. et Var : étangs de Berre et de Marignane, Toulon. Gard et Hér.: littoral d'Aigues-Mortes, de Mauguio à Agde. Littoral de l'Aude et des Pyr,-Or.

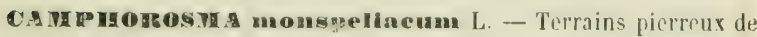
la région méditerranéenne. - Vaucl., B.-du-Rh, Var, Alp.Mar.: envir. d'Avignon, d'Orange, de Carpentras, d'Aix, de Narseille, Toulon, le Luc, St-Tropez, Brégançon, Antibes, entre Eza et St-Hospice. - Gard et Hẻr.: Villeneure en face d'Avignon, Monfrin, Nìmes, envir. de Montpellier, de Cette et de Biziers. - Aude et Pyr.-Or.: envir. de Narbonne, la Clape, Pech de l'Agnèle, Ste-Lucie, Vingrau, Perpignan, Argelès, Port-Vendres, Collioure.

COIRTERTION HyssopophyHum L. - Terrains sablonneux de la région mérid. - Vaucl. et B.-du-Rh.: graviers de la Durance et de l'Aygues, Arles. - Garl et Hêr.: Villeneuve. les Angles, Tresques, Pont-du-Gard, Beaucaire, Aigues-Mortes, Pérols, Palavas, Cette, Agde, Portiragnes. - Remonte sur les bords du Rhône à St-Vallier, Laveyron, Feyzin près Lyon.

Garicorvia herbacen L. - Bords des étangs el des mares d'eau salée. - B.-du-Rlı., Var et Alp.-Mar.: étang de Berre, la Camargue, Toulon, Ile de Porquerolle, salins d'Hyères, ile Ste-Margucrite, golfe Jouan. - Littoral du Languedoc et du Roussillon depuis Aigues-llortes jusqu'à Banyuls. - Espèce polymorphe dans laquelle M. Duval-Jouve a distingué deux formes: l'une qu'il appelle $S$. patula à rameaux étalés, à entrenouds courts, à écusson à face concave, dont la graine a des poils roulés en crosse; plante annuelle; - la seconde, appelée par Dumortier S. stricta, S. Emerici Duv,-Jouve, a des rameaux dressẻs, des entre-nœuds longs, l'écusson légèrement convexe, les graines ont des poils droits; la plante est bisannuelle. 
\$. Fruticoma L. - Bords des étangs salés. - B.-du-Rh., Var et Alp.-Mar.: étang de Marignane, St-Tropez, golfe Jouan. — Gard, Hér., Aude et Pyr.-Or.: littoral depuis Aigues-Mortes jusqu’ả Banyuls-sur-Mer.

La forme sarmenlosa Duv.-Jouve a des tiges peu ligneuses, couchées, sarmenteuses, étalées, des épis courts, des graines à poils crochus.

La forme macrostachya Moric. a des épis gros et longs, à périgone des fleurs saillants, la floraison est précoce. Se trouve sur le littoral languedocien dans les mèmes localités que la forme précédente et que le type. On l'a aussi vue sur le littoral de la Provence à Toulon et aux Pesquiers d'Hyères.

SUAEDA fruticosa Forsk. - Terrains salés du littoral de la Provence et Alp.-Mar., du Languedoc et du Roussillon: de Martigues à Marseille, Toulon, salins d'Hyères, Antibes. - Bellegarde, St-Gilles, Aigues-Mortes, Cette, Pérols, Palavas, Agde, Ste-Lucie, la Nouvelle, Leucate, Salses.

s. maritima Dumort. - Terrains salés du littoral de la Provence, Alp.-Mar., Languedoc, Roussillon : étangs de Berre, Marignane, Montredon, Toulon, Hyères, entre l'embouchure du Var et Nice. - Bellegarde, St-Gilles, Aigues-Mortes, Pérols, Cette, Palavas, Agde, Ste-Lucie, la Nouvelle, Leucate, Salses.

s. splendens G. G. - Terrains salés du littoral dans les mèmes localités que le précédent.

SALSOLA decumbens Lam., S. Kuli L. - Terrains salés et décombres. - Vaucl., B.-du-Rh., Var et Alp.-Nar.: bords de la Durance en amont d'Avignon, bords de l'Arc près Aix, Martigues, Berre, Marignane, Sablettes de Toulou, lle de Porquerolle, St-Tropez, de Cannes à Menton. - Commun sur le littoral du Languedoc et du Roussillon. - Remonte dans la vallée du Rbone jusqu'à Vienne, Givors, Feyzin, Lyon, où il s'est natura. lisé sur des décombres du quartier de la Mouche.

S. tenuifolia Moq.-T., S. Tragus L. Forme du précédent à feuilles longues et étroites. - Terrains sablonneux. - B.-du-Rh. et Var: Arles et Aix sur les bords du Rhone et de l'Arc, bords de l'Argens près du Cannet. — Gard: Manduel, Beaucaire, Aramon. 
s. Iougifolia Lam., S. Soda I. - Terrains salés du littoral. B.-du-Rh., Var et Alp.-Mar.: étangs de Berre, de Marignane et de Caroute près des Ifartigues, Sablettes de Toulon, Hyères, St-Tropez, golfe Jousn, lle Ste-Marguerite. - Gard, IIer., Aude et Pyr.-Or.: Aigues-Mortes, Bellegarde et tout le littoral des envir. de Celte, d'Agde jusqua'à Ste-Lucie et Salses.

\section{POLYGONÉES}

0XXIA ATHON digynum, Oxyria digyn Campil. - Rochers et rocailles des terrains siliceux des Alpes et des Pyrénées. Vaud et Valais: Taveyannaz, Javernaz, St-Bernard, Corbassière, Cleuson, Esserz, mont Nuoble, Zmult, Rothorn, Aletsch, Sirwolten, Grimsel. - H.-Sav, et Sav.: Buet, Montanvert, les Bossons, Tré-la-Tête, le Miole, Vergy, Méri, Charvin, Roc d'Enfer, Hauteluce, la Sauce près du Bonhomme, mont Iseran, l'Ecot près Bonneval, mont Cenis. - Is. : chaines des SeptLaux et de Belledonne, la Bérarde, l'Obiou, la Grave, Villard d'Arène, col des Hayes, mont Viso, St-Véran à Clausis, Champoléon, Orcières, Chaillol. - B.-Alp. et Alp.-Mar.: Lauzuinier, Parpaillon, Vallonnet, Bérard, Grand-Couyer sur Annot, partie supér. de la vallée du Var, lacs de Vens et du Mercantourm, vallon de Strop près d'Entraunes, col de Fenestre, mont Bego, le Clapier. - Pyr.-0r. et Ariège: Canigou, Cambres d'Aze, vallée d'Eyne, cols de Nuria et de las Nou-Fonts, Laurenti, Barbouillère, Roc Blanc, Valbonne, lac de l'Estagnet. - Pyrénées centrales.

IU UnX paImstris Sm. - Espèce polymorphe qui se présente sous deux formes principales: l'une, que j'appelle $R$. palustris laxiflorus, à verticilles floraux espacés et qu'on trouve assez communément dans la région bressanne, C.-d'Or, S.-et-L., Jura et Ain; l'autre, que j'appelle R. palustris densiflorus, à épi dense et dont les fleurs ont les dents du périgone au moins aussi longues que la valve. Le nom de Rumex maritimus donné à cette forme par Linné ne lui convient pas, car elle se montre le plus souvent dans les marais de l'intérieur des terres. L'erreur 
de Linné à ce sujet vient probablement de ce que les échantillons qu'il avait reçus avaient été récoltés dalı des marais situés au voisinage de la mer. - Dans notre bassin du Rhone, le Rumex pulustris laxiflorus se trouve sur le bord des étangs et des mares de la C.-d'Or à Saulon, Citeaux, Boncourt; dans S.-et-L., Jura et Ain sur quelques points te la région bressanne à Navilly, Ecuelles, Verdun, St-Baraing, Pleurre, Fuys, Champrougier, Neublans, Bâgé, Trévoux, les Echets; dans l'Isère à Janeyriat, Charvieu, à l'étang de Mépieu, Montceau près Bourgoin, les Abrets. - En dehors de nos limiles dans une grande partie du centre, du nord et de l'ouest de la France.

16. pulcher L. - Lieux pierreux, bords des chemins. - Une variété hirsutus a les feuilles et la tige couvertes de poils.

rt. obtusfroliun L., R. Friesiamus G. G. - Bords des chemins avec le précédent.

F. conglonersetas Murr. - Bords des mares et lles fossés.

mo. sanguimeus L., $R$. nemorosus Schrad, - Lieux liunides, bois frais. - Les tiges et nervures des feuilles sont de couleur tantôt rouge, $\tan t o t$ verte.

nt. crispus L. - Bords les chemins et des champs.

I. macrophyıus, $R$. hydrolapalhum Huds. - Fosses et marais. - Manque dans la Provence, les Alp.-Alar. et le Roussillon.

K6. acutus L., $R$. pratensis M. K. - Prairies, - C.-d'Or et S.-et-L.: Citeaux, Collonges, Autun sur les bords de l'Arroux, Cluny, Charolles. - Lh. et Is : Francheville, Pierre-Bénite, Crémieu.

- En dehors de nos limites dans l'Alsace et la Iorraine.

18. Hortensis Lam., R. Putientia L. - Cultivé et quelquefois subspontané de inême que le $R$. domesticus Hartm.

F6. aquatieus L., R. hippolapathum Fries. - Bords des rivières. Bords du Doubs autour de Pontarlier; descend à Morteau et audessōus du Saut-du-Doubs.

IR. Alpinu* L. - Voisinages des chalets et bergeries des Alpes, Pyrénées, Vosges, Mezenc. - mont Dore, Cantal.

R. bucephalophorus L. - Champs sablonueux de la région mérid. - Espèce polymorphe.

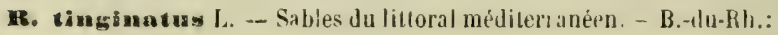


Arles. - Gard el Hér: sigucs-Nortcs, linèle de Saintes, entre Celle et Agde: Porliragnes pris Rorquehaute. - Littoral de l'sude.

2.. serututus L. - Iiurs, rocailles calcaires ou dibris de roches volcaniques. Rare sur les lochers de granile, gneiss, grès. Lorsqu'il vit sur un substratum siliceux, les feuilles sont vertes et n'ont pas la teinte glaugue-blanchàtre quelles présentent lorsgue la plante croit sur les rochers calcaires.

Kb. arifollus All. - Buis el prairies des montagnes. - Tosges, chaine jurassique, Saroie, Dauphiné, B.-Alp. et Alp.-Har., Pyr.-Or. - En deliors de nos limites dans les Pyr. centr., mont Dore, Cantal, Forez.

R. amplexicuulis Lap. - Forme du précédent à gaines persistantes, allongées et longuement ciliées sur les bords. - Ariege: Laurenti au Soula de Joticarets et au cirque des Aiguetles, vallon de Barbouillere, lac de l'Estagnet, roc d'Eecrouts, vallon de la Mlaouré, Soucarrat. - I'yr. centr.

F. nectosus L. - Prés et bois humides. - Cultivé dans les jarlins.

Et. Engroideas Desf., R. intermedius DC. - Licux incultes de la région mérid.; remonte dans la Drôme à Grignan.

ri. acetoselins L. - Champs des terrains siliceux; nul sur les calcaires. Il est digne de remarque que le chaulage à haute dose le fait disparaitre

POL L dans tout le bassin.

P. vivigaruma L. - Pàturages humides des montagnes. - En dehors de nos limites dans les Pyr. centr., mont Dore.

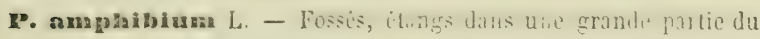
bassin. - Lorsque l'eau se retire, la tige de celte espéce se dresse, les feuilles se hérissent de poils, les gaines des feuilles deviennent cilices.

F. Iapathiưoliuma L. - Lieux lumides, mares, étangs. Espèce polymorplie dans laquelle les épis floraux, les feuilles, les gaines, les nouds des tiges, les fruits prisentent de nombreuses variations dans leurs dimensions, leur forme et leur couleur.

Catal. Eassin du Thöne. 
p. persienrium-L. - Lieux humides, fossís dans tout le bassin.

- Espèce polymorphe.

F. serrulatum Lag. - Fossés du litloral du Tar el des Alp.-Nlar.: Toulon, IIyères, golfe Jouan, Nice au Var. - Aude : envir. de Narbonne.

Thite Schrank, P. dubium Stein. - Lieux humides, foszés.

P. minum Iluds, - Licux humides des terrains siliceux.

R. Eydaopiperinuma L. - Bords des eaux, fossés.

Les Polygonon aquatiques forment entre eux des bybrides dont la nomenclature manque de certitude, attendu qu'il est impossible de savoir au juste quel rỏle a joué chacun des deux parents. Leur distribution géographique étant d'aillcurs liće à celle des générateurs, je m’abstiendrai, pour ces deux motifs, de les mentionner.

F. maritimum L. - Sables du lilloral de la Provence, AlpesMaritimes, Languedoc et Roussillon. - Espèce polymorphe.

T. Littorale Link. - Forme du précédent à gaìnes plus courtes que les cntre-nouds, à six nervures. - Sables maritimes à Celte.

P. Intermedium Robert., P. Robertianum Lois. - Sables marit. - Var: Toulon, Hyères, ile de Porquerolle, St-Tropez.- Hér. et Aude: Cette, Palavas, la Nouvelle.

Wagellaxe Spreng. - Sables. - Var: Toulon, le Luc, entre St- Tropez et Camarat. - Gard et Hér. : Aigues Mortes, Agce près des marais de Rigaud.

P. aviculare L. - Bords des chemins, lieux incultes dans toute la France. Espèce polymorphe dans laquelle les tiges, les feuilles et les fruits sont très-variables. Il est probable que les nombreuses variations de cette plante sont en rapport avec l'état physique du sol, suivant que celui-ci est sablonneux ou argileux, perméable ou compacte, sec ou humide.

P. arenarium W. Kit, - Sables marit, des envir. de Toulon et d'Antibes. - Bords de l'étang de Jonquières (Gard).

P. virgatum Lois, P. Bellardianum All. - Champs de la région mérid. - B.-du-Rh. : les Milles, Roquefavour, bords de l'Arc et de l'Hluveaume. - Var: Sableltes de Toulon, le Luc. - Gard: 
Bellegarde, St-Gilles. - Hér. : Colte, Latles, Larérune, Sesquier près Mèze, Valmagne, Villeveyrac, la Sérane, St-Martin-deLondres. - Remonte dans les B.-Alpes à Beauvezer, Annot; dans les H.-Alp. à Manteyer.

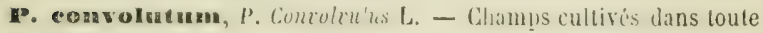
la France.

E. Alumeticola L. - Champs cultivés dlans tout le bassin.

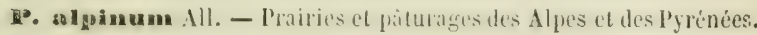
Valais: Steinhaus au Simplon, Gondo sur le rersant italien, Obergesteln, pied mérid. du Griess, le Berbelet près du pont de Geschinen, entre Münster et Reckingen aux Rossaeckern. Is. et II.-Alp. : descente du Grand-Galbert sur Oulles, mont Viso, prés de la Traversette, de Ségure et de Valpréveyre, StVéran à Clausis, mont Genèvre. - B.-Alp. el Alp.-Mar. : col de la Madeleine, Salsamorena et Bouziejo au-dessus de St-Dalmasle-Sauvage, vallons de Nanducbis et de Fenestre, les Viosennes. - Pyr-0r. el Ariège: Canigou, Prats-de-Nollo, Cambres d'Aze, vallées d'Eyne et de Nohèdes, lac du Laurenti, vallon de Boutadiol, Barbouillire.

P. Fagopyrum, tatarieum et orientale L. - Cultivés et quelquefois subspontanés.

\section{DAPHNOIDEES}

DAPpre Interiflora. D. YeNereum L. - Bois ct pâturages des montagnes d'où il descend quelquefois dans les vallées. - Dans la Provence on ne le trouve qu'à la Ste-Baume; dans le Gard à Camprieux, le long du Brama-Bioou. - Nanque dans l'Hérault. - En dehors de nos limites dans les Pyrénées, l'Aubrac, le Lévezou, montagnes du Tarn, mont Dore, inonts Dômes, Cantal, Forez, les Vosges.

DP. Iaureola L. - Bois des montagnes et coteaux des terrains calcaires et rolcaniques. - Nul dans les Vosges, sur les terrains de granite, gneiss du massif central et des Cévennes.

\$D. alpina L. - Rocliers des montagnes calcaires. - Nul dans les Yosges, le massif central et les Pyrẻnées. - Jura helrétique et 
français: Weissenstein, mches de Fleuricr, Creux-du-Van, Cluses de Moutiers, Cótes du Doubs, cirque de Mauron, la Rochc-Pesante, Tête-de-Calvin près du Saut-du-Doubs, Dessoubre à St-Julien, Pont-de-Roide, Crêt-des-Roches, Crêt-Châtard, Lomont de Roulans, Belin et Poupet près Salins, la Châtelaine près d'Arbois, St-Georges. - Vaud et Valais : les Plans, Solalex, les Diablerets, envir. du lac de Derborence, Catogne, Roc-Percé de Scmbrancher, le Fary, Leukerhad, Grand-Sex, entre Visp et Stalden. - II.-Sav. : Saleve, Veyrier, Crevin, le Coin, Archamp, Montmin, pont St-Clair sur le Fier, montagne de Veyrier près Annecy, Thonon entre la Dranse et le pont de Bioge. - Sav. : mont du Chat, St-Martin-la-Porte, entre Aigueblanche et Moutiers, mont Gargan, Brides, St-Jean-de-Maurienne. - Is.: St-Eynard, Sassenage, Comboire, St-Nizier, Villard-de-Lans, borls du lac de Lovitel près Vénose en Oisans. - H.-Alp. et Dr. : la Grave, Lautaret, la Vachelte, col du Noyer, col de Glaise, Louhet ct Rabou près Gap, Lus-la-Croix-Haute, Jansac. B.-Alp. : la Condamine, Serennes, Annot. - Alp.-Mar. : partio supér. de la vallée du Var, Estenc au-dessus d'Entraunes, la Clue de St-Auban, Thorenc, entre Tende et Carlin, Feriou audessus de Coarazza, mont Mulacé sur Menion. - Vaucl., B.-du-Rh. et Var: mont Ventoux à la Font de Canau, pic de Bretagne, Ste-Victoire, Stc-Baume. - Gard et Hér.: Alzon, Campestre, St-Guilhem-le-Désert, bois de Montmédys près do Ganges, la Vacquerie, St-Privat, le Caylar, St-Etienne-deGourgas. - C.-d'Or: rochers calcaires de Gevrey, Couchey, Chambolie, Nuits, Bouilland. - En dehor's de nos limites sur les causses de la Lozère et de l'Aveyron.

D. striata Tratt. - Rochers du Lautaret, col des Rochilles-surNévache (H.-Alp.).

E. exasagrginasta, D. Cincomm I. - Rocailles et pelouses des montagnes. - Chaine jurassique au Crêt du Trembiaz, Montendre, Noirmont, Seiche-des-Embornels, pré de Bière au-dessus du Brassu, rochers de Cise près Champagnole, Maurnn, Chapelledes-Bois, le Mont près Nantua, Poisat. - Is. : pied du St-Eynard sur Corenc, chalet près Mens, balmes du Rhơne entre Anthon 
ct Villette où il a été amené des montagnes par les caux du Bhône. - II.-Alp.: Briançon, Gondran, Névache au dessus de Ilampinet, col Isoard du coté de Cervieres, Guillestre, pic de Chabrières près Cliorges, Chaillol, col du Noyer, mont Aurouse, St-André-de-Rosans. - B.-Alp. : la Condamine, Bousollières, Tournoux. - Dr. : Lus-la-Croix-IIaute. - Nlp.-Mar.: montagnes de Caussols et de Defens au-dessus de Grasse, St-Etienne, col de Fenestre, mont Lachen. - C.-d'Or : le Châtillonnais à Essarois, Voulaines, Leuglay. - Gard: Sallous, le Vigan, Bourdezach. - Dans cette espèce les feuilles sont échancrées au sommet, et quelquefois portent un mucron dans l'échancrure, comme dans la variété $D$. Verlotima $G$. G.

D. orthophylia, D.Gniditu L, - Bois et rocailles de la région mérid. - Vaucl.: Avignon, Uclaux, Piolenc, Sérignan. B.-du-Rh., Var et Alp.-Mar.: envir, d'Aix, de St-Remy, de Marseille, de Toulon, île de Porquerolle, entre Nice et Menton. Toute la partie méridionale du Gard, de l'hérault, Aude et littoral des Pyr.-Or.; remonte dans la rallée de l'Agly vers St-Antoine de-Galamus.

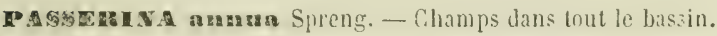

PP. daymelea DC. - Coteaux pierreux de la région mérid. B.-Alp.: Annot, Castellanne. - B.-du-Rh, et Var: le Pilon-duRoi, Morıtrieux, Morières. - Gard el llér. : Nìmes au chemin d'Uzìs, N. D. de Londres, derrière St-Loup, Gignac, St-Chinian, Argelliers, Montarnaud, Nissan, Liausson, Aniane, Bédarieux, Montagnac, la Vacquerie, Pardaillan. - Aude et Pyr.-Or.: Caunes, envir. de Narbonne, la Clape, Aussières, Pradines, Levretles, les Corbières, Villefranche, Trencada d'Ambulla, Vernet-les Bains, Font de Comps, entre Fontanes et Usson dans la vallée de l'Aude, au-dessous des ruines du Château d'Usson. - Pyr. centrales.

Po. Inetoria Pourr. - Bois de la Chartreuse de Vallonne au quartier des Cabreries (Gard).

P. eandieans Lam. P. Tarlon-Raira L. Rochers. - B.-du-Rh, Var et Alp.-Mar. : Montredon, ille de Maïré, les Martigues, ile de Porquerolle et du Levant, Sablettes de Toulon, fle Ste-Narguerite. 
P. Birguta L. - Licux picrreux de la région mérid. - B.-du-Rlı, Var el Alp.-Mrar.: Montredon, île de Maïré, Toulon, Hyères à Brégançon, St-Tropez, St-Raplıël, îles Ste-IIarguerite et StHonorat, Antibes. - Aude et Pyr.-Or. : Ste-Lucie, la Nouvelle, Collioure, Port-Vendres, Paulille, Banuyls-sur-Ner.

\section{LAURINÉES}

LAU U⿺ nobilis L. - Cultivé dans la régrion mérid, et quelquefois subspontané.

\section{SANTALACÉES}

THLSION nlpinum L. - Pâturages des montagnes. - Chatnes vosgienne et jurassique jusque dans les montagnes du Bugey. - Valais : Alpes de Saxon et de Sion. - H.-Sav, et Sav. : Salève, les Voirons, Brizon, Jalouvre, Tournette, Grand-Bornart, Clıarvin, mont Pétetod, Dents d'Oche, chaînes du mont Blanc et des Aiguilles-Rouges, Brévent, mont Joly, les Allues, mont Cenis. - Is. et Dr. : col Oddie au-dessus des Adrets, Poursollet au pied de Taillefer, St-Nizier, Villard-de-Lans, mont Chamoux, mont Glandasse, Lus-la-Croix-Haute. - H. Alp.: la Grave, Lautaret, Névache, Valpréveyre en Queyras, St-Véran, Chaillol, Morgon, la Grangette, Rabou près Gap, mont Séuse. - B.-Alp. et Alp.-Mar.: Lauzanier, col de la Madeleine, Alpes d'Entraunes, de Ste-Anne-de-Vinaï, de Fenestre et de Tende. - Vaucl. : mont Ventoux. - Gard et Hêr. : Brama-Bioou près Camprieux, Concoule sur la Lozère, St-Guiral, St-Sauveur, la Salvetat. Pyr.-Or. et Ariège: Canigou, Pla Guillem, vallée de Llo, lacs du Laurenti et de l'Estagnet, roc de Nascaras, vallon de Barbouillère. - Loire: Pilat. - C.-d Or: Combe-Noire dans lo Châtillonnais, - En dehors de nos limites, dans les Pyr. centrales, l'Aubrac, Cantal, mont Dore, monts Dômes, Haute-Loire, Forez dans les chaînes de Noirétable et de Pierre-sur-Haute.

'T. intermedium Schrad. - Pâturages des montagnes des envir. d'Annecy, de Gap, col Bayard, Devez de Rabou, mont Sćuse, 
Chorges. - B.-Aip. et Alp.-Mar. : Sisteron, vallon de Fouillouse, mont Mulacé, bords de la Roja près de Tende. - Gard: Montdardier, Aulas, bois de St-Sauveur et du Prunaret,

T. pratense Ehrh. - Pâturages de la chaine jurassique jusque dans le Bugey. - II.-Saone: vallée de la Savoureuse, Clampagney, Nans. - C.-d'Or: F'ontainc-llerle à F'anges, Valcourbe. Valais: le Trient. - I1.-Sav. : Salève, Dents d'0che, - Is.: Grande-Chartrcuse, col de la Ruchère, mont Rachais, Pariset, St-Nizier. - II. et B.-Alp.: Lautaiet, la Vachette près Briançon, mont Morgon, Séuse, Fouillouse. - Aude et Tarn: versant méridional de la Montagne-Noire. - Pyr.-0r. et Ariège: Mas Christine près Argelès, val de Quérigut à la Jasse d'Espagne el au Pla Bernard, Soucarrat, val de Paillères. - Loire: Pilat, Pierre-sur-Haute. - Pyr. centr., Cantal, mont Dore, monts Dòmes, Vosges.

T. pyrensicum Pourr. - Pyr.-0r. el Ariege: mont de Madrès, lac du Laurenti, cirque des Aiguettes, escale de Boutadiol, roc des Braguẻs de Rabassolès.

T. Lumifusum DC. - Pàturages des terrains calcaires, - C.-d'Or et S.-et-L. : Vauclignon près Nolai, Cluny, Paris-l'Hopital. Jura : Dóle, Champrans, mont Roland, Authume, Chatenois, Amange, calcaire autour de la forêt de la Serre. - Envir. de Genève au bois de Bay, Peney, bois du Lignon au-dessus d'Aïre. - Is. : Jonage. - Ard. : Mazan. - Ariège, Aude et Pyr.-Or. : Mijanès, Rouze, vallée de l'Aude, Collioure, Argelès.

T. diverientum Jan. - Bois, coteaux secs. - C.-d'Or et S.-etLoire: Dijon, Gevrey, Bcaune et toute la Côte, Dezize, Sampigny, Cluny, Vergisson, Solutré. - Ain : Thoirette, Pont-d'Ain, coteaux entre Montluel et la Pape, Trévoux, Belley, Rossillon. Rh. : St-Alban, mont Cindre, Couzon, Beaunant, - Is. et Dr. : Crémicu, Comboire, Vif, Rochefort, St-Vallier, Crest. - H. et B.-Alp.: Puy-Maure et la Garde près Gap, Roche-des-Arnauds, Sisteron, la Condamine, Serennes, Meyronnes. - Var et Alp.IIar. : Toulon, le Luc, entre Nice et IIenton. - ArJ.: mont Charray. - Commun dans le garrigues de Vaucl, B.-du-Rh., Gard, Hér., Aude, Pyr.-Or. et Ariège. 
OS Lran alba L. - Ilaies et tcrrains incultes de la région mérid.

- Remonte dans l'Ardẻche à Viviers, Rochemaure, Baix, Cruas, la Voulte el jusque vers les confins du département de la Loire à Limony; dans la Drôme ct les B.-Alp. à Nyons, Montélimar, Donzère, Romeyer, Crest, Tain, St-Vallier, Sisteron; dans I'Isère à Rochefort, Comboire, la Bastille de Grenoble; dans l'Ain à Mluzin, Parves, le Lit-au-Roi, Pierre Châtel, Glandieu St-Benoit; dans la Savoic et H.-Sav. entre Chambéry et Montmélian, bords du lac du Bourget, entre Annecy et Mandallaz.

\section{ÉLÉAGNÉES}

graviers des rivières el des torrents. - Très-commun dans Vaud, Valais et le long du Rlıone; puis au-dessous de Genève jusqu'à Lyon et de là dans les iles du Rhône et de la Durance près d'Avignon, de Beaucaire et de Vallabrègues. - Vallées de l'Arve, de l'Iscire, de l'Arc, du Drac, de la Romanclie, de la Drôme, de la Durance et de la plupart des aflluents de ces rivières.

EDAGNOS angastiforas L. - Cultiví et quelquefois subspontané.

\section{CYTINÉES}

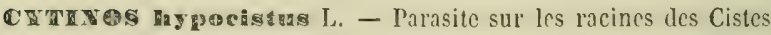
lans la région mérìd. - La forme luteus se montre de préfírence sur les Cistos monspeliensis et salvifolius des terrains siliceux; la forme ruber sur lo Cistos albidus des terrains cal. caires. Cette dernière est si différente de la précélente que plusieurs botanistes n'lésitent pas à la considérer comme une espèce distincte. Cependant j'incline à croire cque la différence, très-notable entre les deux Cylinos jaune et rouge, lient à la diversité du support. La même observation est applicable aux Viscnm album et V. luteum, aux Orobanches, aux Cuscutes et peut-être à une multitude d'espèces épiplıjtes phanérogames et cryptogames. 


\section{ARISTOLOCHIÉES}

A5. T20 อ enroprean $I_{\text {. }}$ - Bois des montagnes. - Chaine du Jura helvétique et français jusque dans le Bugey. - Vaud et Valais : envir. de Bex, de Afonthey, Val d'Illiez. - II.-Sav.: pied du Salève, Arenthon, Thonon, la Roche, les Balmettes d'Annecy, le Semnoz, le Reposoir, St-Gervais. - Is : Morestel, forêt de St-Serverin, Montalieu, entre Bouvesse et Charette, St-Laurent-du-Pont, Grande-Chartreuse, Sassenage, Livet, Mens. H.-Alp.: Villard d'Arène, St-Maurice en Valgaudemar. - Alp.Mar.: val de Pesio, valtée de Castarin près Tende, mines de 'Tende. - C.dor et S.-et. L. : Vauchignon près Nolay, Meursault, Nuits, Gevrey, Dijon, Dezize, Cluny, Salornay. - Loire: Pilat à Thélis-la-Combe, St-Galmier.

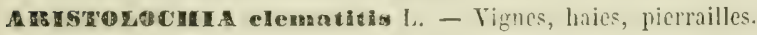
- Jura : envir. de Dole. - Environ de Genc̀ve à Martinet d'Avuzy. - Commun dans les envir. de Lyon sur les digues du Rhone et dans les vignes du Mont-d'Or. - Is, Dr., H. et B.-Alp.: Pont-de-Chérui, St-Maurice-d'Exil, St-Vallier, Châteauneuf, Livron, Crest, Gap, Sisteron. - Sav.: ervir. de Chambeiry. - Commun dans la Provence, Ics Alp.-Mar., l'Aude, Pyr.-Or., ainsi que dans C.-d Or et S.-ct-L.

A. pistoloehis L. - Rochers, garrigues de la région mérid. Vaucl. et B.-du-Rh.: envir. d'Avignon, d'Orange, de Carpentras, d'Apt, d’Aix à St-Marc, de Marseille à Montredon, St-Loup, lle de Pomègue. - Var et Alp.-Nar.: Toulon à la Seyne, Hyères, le Luc, Rians, Grasse, l'Esterel, Utelle, Gilletta, Levens, Nice. Gard: Nîmes, Manduel, Jonquières, le Vigan, Alais, Anduze, Uzès, St-Ambroix. - Hèr.: Montpellier, Si-Jean-de-Védas, Fabregues, la Madeleine, Lattes, Murviel, Agde, Lunas, Madières, St-Martin-dc-Londres, Capouladoux, Pardailhan, le Caylar. - Aude et Pyr.-Or.: Pech de l'A Łnnèle près Narbonne, Levrettes, Crabit, Ste-Lucie, Cascs-de-Pena, Baixas, Pont de la Fou près St-Paul-de-Fenouillet, Prades, Villefranclie, vallon de Cerbère. - Remonte dans la Drôme vers Nyons, Crest, Beau- 
fort, Aucelon, Die, Montélimar; dans les B. et H.-Alp. vers Sisteron, Ribiers, Roche-des-Arnauds, Neffes près Gap; dans l'Ardèche à Alissas, Coux.

A. rotundn L. - Haies et champs de la région mérid. - Vaucl. et B.-du-Rh.: Orange, Avignon, Apt, Aix aux Mloulins-Forts, envir. de Marseille. - Var et Alp.-Nar.: Hyères, Revest, le Luc, Rians, St-Tropez, Torretta, Nice. - Gard : Nìmes, St-Gilles, le Vigan, Anduze, Alais, St-Ambroix. - Aude et Pyr.-Or.: envir. de Narbonne, les Corbières, Baixas, Cases-lle-l'ena, Argeiès, Collioure, Banyuls. - Remonte dans l'Ardeche vers Rochemaure et Baix; dans les H.-Alp. el' la Drôme vers Gap, Nyons, Crest, Bourdeaux, la Motte-Chalancon, Moydans.

A. pallida W. Kit. - Var et Mlp.-Mar.: mont Sauvette près les Mayons, Ampus, Ste-Baume, Thorenc.

A. Iongre L. - Bords des champs et des haies de la région mérid. - Vaucl. et B,-du-Rh. : envir. d'Apt, Peyrolles. - Var et Alp.-Nar. : Belgentier à la Tourne, Nice, entre le vieux Castellar, le Mulacé et le Grammont au-dessus de IIenton. - Hér.: Montpellier, St-Aunès, Villeneuve, Pérols, Lattes, Lunel, Marsillargue, Agde, Clermont, Béziers, Viols, St-Nlartin-de-Londres. - Pyr.-Or. : bords de l'Agly, Perpignan, Villefranche, vallon de Cerbère, anse de Peyrefite près Banyuls.

\section{EMPÉTRËES}

EMPETRON nigrum L. - Rochers et rocailles humides des montagnes. - Tourbières de la chaine jurassique, Creux-duVan, les Rousses, vallée de Joux, le Boulu, Prémanon, Reculet, montagne d'Allemogne au-dessus de Thoiry. - Vaud et Valais: Lavarraz, Fully, St-Bernard, Pierre-à-Voir, Giétroz, Zermatt, Saas. - H.-Sav. et Sav.: Glacière du Brizon, Vergy, Buet, Brévent, Montanvert, Leschaux, Couvercle, les Contamines, Bionassey, mont Joly, cols du Bonhomme et de la Seigne, mont Iseran, les Allues, mont Cenis. - Is.: la Pra de Belledonne, Champrousse, Grand-Charnier, Sept-Laux, Taillcfer, Chamechaude, mont Chamoux. - H.-Alp.: la Grave, Lautaret, le Champsaur, 
le Valgaudemar, le Briançonnais et le Queyras. - Alp.-Nar.: col de Fremamorta, Alpes de T'ende et de la Briga. - Pyr.-Or.: Canigou, Pla Guillem, Cambres d'Aze. - En dehors de nos limites dans les Pyr. centrales, mont Dore, Cantal, Forez à Pierre-sur-ilaute, les Vosges.

\section{EUPHORBIACEES}

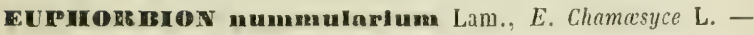
Licux cultivés de la région mérid.; remonte vers Nyons, Montélimar, Tain, St-Vallier, Romans et jusqu'à Lyon.

E. auriculatum Lam., E. Peplis L. - Sables maritimes du littoral méditerranéen.

E. helioseopium L. - Lieux cultivís dans tout le bassin.

E. platyphyllum L. - Bords des chemins et des champs humides.

S. strictum L. - Forme grêle du précédent à tiges courtes, fleurs petites, capsules moins grosses à sillons plus profonds et à tubercules plus saillants. - Mème habitat.

E. nkenoenrpun Guss. - Espèce d'Italie el d'Espagne qu'on avait vue à Marseille aux Catalans et à la montée de la Gineste, mais qui a disparu de ces localités.

E. pubescens Desf. - Bords des fossés el des champs humides de la région méditerranćenne. - B-du-Rh.: bords de l'Huveaune, IIarignane. - Var et Alp.-Mar.: Toulon, Fréjus, Grasse, Cannes, golfe Jouan, Antibes, Pégonnas, Nice, Menton. - Gard : Aigues-Mortes, St-Gilles, Bellegarde. - Hér.: Mauguio, Lattes, Celte, Palavas, Pérols, Mireval, Maguelonne. - Aude et Pyr.-Or.: envir. de Narbonne, Craboules, la Robine, Perpignan, Argelès.

Une variètẻ de cette espèce, qu'on pourrait appeler glabrescons, présente une pubescence très-courte.

E. pilosum L. - Forme du précédent à feuilles plus velues et obtuses et à graines lisses, qui se trouve dans plusieurs départements du centre et de l'ouest de la France, mais qui est assez rare dans notre bassin. J'en ai des échantillons venant des environs de Toulon au Revest, de Veynes près Gap, enfin des Alpes-IJari- 
times entre St-Martin-du-Var et Chaudon, - De Pouzols l'indique au pont de Malaygue près Blauzac (Gard). - A propos de cette Euphorbe, il n'est pas inutile de rappcler aux botanistes qu'ils doivent se tenir en garde contre une erreur de synonymic qu’on n'a pas toujours su éviter et que l'E. pilosum de Linné n'est pas l'E. pilosum de Brotero et de Bertoloni. Ce dernier est le même que l'E. pubescens Desf. à graines hérissées de petites crêtes, et dont les feuilles, moins velues que celles de l' $E$. pilosum, sont en outre lancéolies-aiguës.

E. paIustre L. - Fossis, bords des marais. - C. d'Ol' et S.et-L.: plaines marécageuses de la vallée de la Saône et des envir. de Charolles, de Louhans, de Verdun, Navilly, Ecuelles, St-Bonneten-Bresse. - Jura et Ain: la Bresse à Chaunergy, Rye, la Cliassagne, Bletterans, Tivaux, Aumur, l'Abergement-laRonce, bords de la Reyssouse et de la Saône, Belley. - Vaud: la Sauge, Cudrefin, Yronand, Yverdon, Orbe, Villeneuve, Roche. - Sav, et Is.: le Bourget, Chasvieu, Pont-de-Chérui. Gard et Hér.: Bellegarde, St-Gilles, Beaucaire, Aigues-Mortes, Mauguio à Lamotte et à St-Marcel, Sérignan. - B.-du-Rh. et Var: Arles en Coustiero, Vidauban près du bord d'Argens. Rh.: Vaux-en-Velin.

E. Ibernicum L. - Bois humides. - Alp.-Har. : Gourdon, St-Auban, Villars du Var, Berre, bois du Farghet, mont Mulacé au-dessus de Nenton. - Iér.: Fraisse à Lignières. - Pyr.-Or. et Ariège: col de Baladon, Canigou, Villefranche, Mont-Louis, forêts de Salvanère, de la Limouse, du Paillariel et du Riplaou, Pla del Bosc, Prat Laston, Valbonne, cirque des Aiguettes.

Aulee L. - Bois dans tout le bassin.

E. Capinosuan de Pouzols. - Pâturoges. - Gard: Alzon, Campestre, Blandas au bois de Salbous. - Aude : mont Alaric. En dehors de nos limites dans la Lozère autour de Mende et de Florac, Causse Méjean, Mont-Vaillant.

E. Verrucosum Lam. - Bois et prés des terrains calcaires des parties septentr. et moyenne du bassin. Mloins rêpandu dans la région mérid., où il est remplacé par le suivant.

E. Aniconum DC. - Coteaux calcaires. - II. ct B..Alp.: Ri- 
biers au mont llognouse, Larche. - Dr: : plaine de Vaumeil vis-à-vis le l'oët. - Vaud.: Avignon à Vélènes, colline SteAnne, Carpentras, montegnes entre la Fare et Vacqueiras. B.-du-Rh., Var et Alp.-Mar.: Bédoule de Cassis, montée de Nans à la Ste-Baume, mont Coudon près Toulon, les Mayons ả Mavallon, Grasse, Braus, Menton. - Gard: Campestre, le Vigan, Anduze, St-Ambroix, Alais, - Itr. : envir, de Montpellier à Bionne, Caunclle, Lavalete, Cournonsec, Sussargues, Mlireval, St-Loup, la Sérare, les Capouladoux, Montarnaud, St-Guilhem, St-Martin dc-Londres, le Larzac à St-Haurice, Castelnau-de-Guers près Pizenas. - Aule : envir. de Narbonne, Pech de l'Agnèle, Sie-Lucic, Leucate.

E. solnosuma L. - Fentes des rochers de la region mérid. B.-Alp.: Gréoulx, Digne, Annot. - Yaucl, et B.-du-12h : Mirabeau, envir. d'Aix à Montaiguct, entrie du vallon du Mayol, Roquefavour. - Var et Alp.-Nar.: T'oulon, Draguignan, Cannetdu-Luc, Fréjus, St-Raphaëi, Cannes, Grassc, Nice, Menton.

E. Dineardelism Lam, E. Gerardianum Jacq. - Champs pierreux des parties mérid. et moyenne du bassin; moins commun dans la partie septentrionale.

Une forme naine, E. saxrtile Lois., se trouve dans les tocailles du sommet du mont Venloux el du mont Alaric.

5. meusifolina Lam., E. Pilyusa L. (1). - Sables du littoral mediterancen. - B.du-Rh. et Var: cnvir. de Marscille, iles Ratonneau et Pomigue, cap Croisctte, Toulon, Hyères, ile de

(1) C'est à tort que, depuis Linné, la plupart des auteurs écrivent pithyusa le nom de l'Euphorbe qu'on avait cru, sans motif plausible, être l'espèce de Tithymalos quo Dioscoride et Galien appelaient pituousa (pityusa dans la transcription cn caractères romains). Le raclical de ce mot étant pitus (pitys, Yin), il est évident qu'il fautécrire, avec Pline et tous les anciens botanistes, pityusa.

Au sujet de I'E. hiberni'um, citó plus haut, je rappelle que c'est par distraction que Linné a écrit Euphorbia hiberna (d'hiver). Il est clair que le nom d'Euphorbe d'hiver no saurait être donné à une plante qui fleurit aux mois do juin et juillet; il est certain d'ailleurs que Linné avait bien l'intention do dire Euphorbe d'Jrlande, car il ajouto habitat in Hibernia. On doit done diro Euphorbion hibernicum. 
Porqucrolle, St-Tropez, Fréjus, St-Raphaël. - Hér., Aude et Pyr.-Or.: bords du canal de Bourdigue à Celte, Ste-Lucie, la Nouvelle, St-Pierre, Leucate, Salses, Collioure, Port-Vendres.

E. maritimum Lam., E. Paralins L. - Sables maritimes du littoral des B.-du-Rh., Alp.-Mar., Languedoc et Roussillon.

E. dendroikena L. - Rochers, coteaux pierreux du littoral méditerranécn. - Var et Alp.-Mar.: Toulon au Fort Ste-Marguerite, presqu'île de Gien, îles de Portcros et du Levant, Bormes, île Ste-Margucrite, assez commun de Nice à Menton.

E. nieneense All. -- Lieux arides de la région mérid. - Vaucl. et B.-du-Rh.: Sablet, Apt, Flassan, Montaiguet près Aix, vallon de l'Evêque près Marseille, vallons autour du Pilon-du-Roi, Roçuefavour. - Var et Alp.-Mar.: Hyères, le Luc, Fréjus, Cannes, Grasse, Bouyon, Touët-de-Beuil, mont Férion, Nice, la Turbie, Roquebrune, Menton, Castillon. - Gard : Nimes, StAmbroix, Alais, Anduze, le Vigan, Montdardier, Alzon. Hér.: assez commun dans les arrond. de Hontpellier el de Béziers. - Aude et Pyr.-0r.: garrigues des arrond. de Carcassonne et de Narbonne, notamment à Aussières, la Clape, les Corbières, Durban, Villeneuve, vallée de l'Agly à St-Antoinede-Galamus et St-Paul-de-Fenouillet, Rennes-les-Bains, entre Vingrau et Rivesaltes, parties basses des vallées de la Tet et du Tech oủ il remonte vers Prades, Graux d'Olette, Argelès, Banyuls-sur-Mer. - Dr.: Nyons, le Poëı près du Buis. - H.-Alp.: entre Ventavon et Lazer, Ribiers, Châteauroux-sur-Chabre.

E. esulum L. - Bords des cours d'eau. - Jura : alluvions du Doubs et de la Loue au-dessous de Dole. - C.-d'Or et S.-et-L.: envir. de Dijon, vallon de Suzon, Chenove, Nuits, de Digoin à Bourbon. - Ain et Rh.: bords de la Saône et du Rhơne à Trévoux, Inse, St-Germain, Pierre-Bénite, Vernaison. - Is, et Dr.: Vienne, St-Vallier, Montélimar, Grignan. - Gard, Vaucl. et B.-du-Rh.: bords du Rhône depuis Aramon jusıu’aux Graux d'Orgon, Avignon, Tarascon, Arles, bords du Gardon au Pontdu-Gard, Montfrin. - Hér.: bords du Lez, de la Mosson et de l'Hérault. - Espèce polymorphe dans laquelle la forme des feuilles présente de nombreuses variations qui ont fourni à M. Jordan les distinctions ci-après désignées : 
E. pseudocyparissium, E. salicctor um, E. araricum, E. riparium.

C. temuifolasm Lam. - Licux frais. - Vaucl, B.-du-Rh. et Var: Carpentras, St-Rémy, le Tholonet près Aix, les Martigues, Arles en Coustiero el à la Pissaroto, Ampus.

E. terrecisum L. - Sables maritimes. - Var et Alp.-Mar.: Hyères, Bormes, Fréjus, St-1łaphaël, Cannes, Nice, Vintimiglia. - Gard et Ifér.: Grau-d'Urgon en face des Saintes-Maries, Pinède-des-Saintes au poste des Quatre-Mlaries, embouchures de l'Aude et de l'Orb. - Aude et Pyr.-Or.: Sle Lucie, Collioure, Port-Vendres, Paulille, Banyuls.

E. seratum L. - Champs cultivés de la région mérid.; remonte dans la Dr, les B. et H.-Alpes vers Hontélimar, Loriol, Grignan, St-Vallier, Sistcron, Embrun, Gap, libiers, Tallard; dans l'Ardèche de Viviers à Chiteaubourg.

E. aleppieam L. - Champs des rives de l'Arc depuis son embouchure dans l'étang de Berre jusque vers la Fare (B.•du-Bh.). - Toulon.

2. сурпrissifozme L. - Licux incultes depuis les plaines jusqu'aux montagnes.

2. exiguum L. - Champs cultivés dans tout le bassin. - Espèce polymorphe dans laquelle les feuilles sont tantôt tronquẻesmucronulées (E. retusum DC.), tantòt dentées (E. rubrum Cav.), d'autres fois échancrées et presque trilobées ( $E$. tricuspidatum Lap.'.

E. suleatum Delens, - Champs cultivẻs. - B.-du-Rh.: envir. d'Aix aux vallons du Cascaveou et de la Guiramande, la Mède près des Martigues, Châteauneuf, Marignane, Roquefavour, plan d'Aups, - Hér.: Roquehaute près Portiragnes.

E. Faleatum L. - Champs cultivés des parties mérid. et moyenne du bassin; plus rare dans la partie septentr. - Vaud et Valais: bassin du Lèman, Ayen, les Plâtrières. - Doubs, Jura et Ain : alluvions du Doubs et de la Loue, Thoiry, Royssiat, Ambronay, St-Rambert, bords de la Burbanche, Belley, la Pape. - Envir. de Lyon. - Is. : Corenc, Claix, Pariset, Brie. - S.-et-L., C.-d'Or : Cluny, Cortembert, Cormatin, Quincey, Cussigny, Longric. - H.-Saône: Boursières. 
E. tameinense All. - Champs cultivís. - H. et B.-Alp.: envir. de Gap a Charance et Rabou, Embrun, Guillestre, Briançon, Ribiers, la Condamine, Annot, Sisteron, Senez, Castellanne. B.-du-IRh, et Var : les Alpines, envir. d'Aix au vallon des Gardes et au Tholonet, envir. de Harscille au vallon de Forbin et à la Treille, Draguignan, le Beausset, Grasse, Fréjus, Tende, Fontan, Saorgio, la Giandola, Breil, Brouis, entre Castillon et Sospel. - Ard.: Thueyts.

E. пpeproran L. - Champs cultivés dans une grande partie du l)assin.

E. peploideum Gouan, - Forme du précédent à graines plus petites, portant deux fossettes sur dkux des faces adjacentes au raphé, tige plus petite. - Champs cultivés de la rẻgion méridionale.

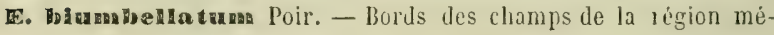
rid. - Var et Alp.-Har.: la Seyne, Toulon, Hyères, St-Tropcz, Fréjus, l'Esterel. - Gard: Villeneuve en face d'Avignon, digues-Nortes. - Pyr. Or.: Argeles, Collioure, Port-Vendres, Banyuls, Ceret.

2. segerale L. - Champs cullivés de la région mérid.; remonte dans la Drôme, les H.-Alpes et l'Isère vers le Buis, Suze-laFousse, Gap, Briançon, Comboire. - Espèce polymorphe. Dans une rariéie, appelée $E$. longibracteatum par de Candolle, les tractées s'allongent et deviennent semblables aux fouilles.

E. densifoliums, E. Pinea L. - Sables et rochers maritimes. Var: Ilyères, Frẻjus. - Gard et ryr. Or.: Fourques, PortVende:, Banyuls.

E. morotanareuma L. - Espice du littoral de l'Ocían indiquéc par Lecog et Lamotte autour de St-Jean-clu-Gard el d'Anduze (Gard).

E. Batrateuna Jacq, - Bois dans tout le bassin.

E. qurpaream Lam., E. chanacias L. - Coteaux arides de la région mérid. - Remonte dans la Dròme et les B.-Alpes vers Nyons, le Buis, St-Paul-Trois-Châteaux, Siıu, Grignan, Rémusal, Valence, Digne; dans l'Ardeclıe vers Rochemaure, Trivas et Crussol.

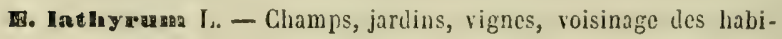
tations. 


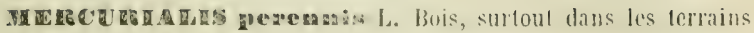
calcaires.

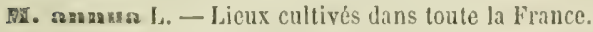

12. Huctina Hanry. - Forme remarquable, entierement glabre, et dans laquelle les pointes vertes de la capsule sont peu nombreuses. - Var: le Luc, Bormes. - Hér.: Montpellier à la Colombiere, Villeneuve, Cetic, Fabrigues, St-Jean-de-Vedas, l'Lscalette.

ND. ambiga La - Lieux cultivés de la région méridionale.

75. comaendosa L. - Licux incultes, hords des chemins de la région mérid. - B.-du-kh.: les Martigues, envir. de Marseille. Hér.: Montpellier, Béziers, Agde. - Aude et Pyr.-Or.: Carcassonne, Lastours, Conques, la Clape près Narbonne, Ste-Lucie, Bouscaillou près Durban, Rivesaltes, Perpignan, Céret, Banyulssur-Mer.

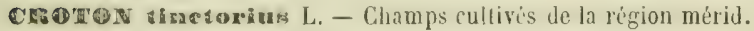
- Vauci.: Carpentras, Avignon, Sérignan, Mormoiron, envir. d'Apt, les Barbiers, le Luberon. - B.-du-13h.: Martigues, Miramas, envir. d'Aix et de Marseille. - Var : Toulon, Hyères, le Luc, Cuers, Rians, Fréjus. - Alp.-Mar.: Grasse, Antibes dans la plaine de la Brague, Nice, Villefranche, Eza, Menton. - Gard et Hér.: envir. de Nimes, d'Alais, d'Anduze, de Montpellier, de Béziers et de Cette. - Aude et Pyr.-Or.: envir. de Narbonne et de Perpignan.

BUXg's sempenvinens L. - Coteaux arides, bois dans tout le bassin, surtout sur les terrains calcaires, quelquefois sur les basaltes décomposés, les gneiss et micaschistes calcifères; rare sur le granit.

\section{MORÉES}

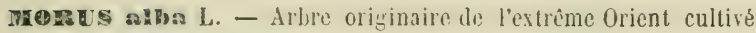
pour la nourriture des vers à soie.

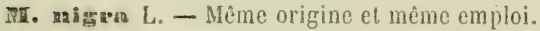

Ficus ennoea L. - Cultiré dans le midi de la France et quelquefois subspontané. 


\section{CELTIDÉES}

CaTTS australis L. - Cultivé et quelquefois subspontané dans la région mérid. - Remonte dans l'Ardèche, la Loire et le Rhơne à Rochemaure, Coux, Vals, Andance, Nalleval, Chavanay, Condrieu, Ampuis; dans la Drôme et l'Isère vers Tain, St-Vallier, Vienne.

\section{ULMACÉES}

ULMUS campestris Smith. - Haies et bois des plaines et des coteaux.

U. montana Sm. - Bois des montagnes, bords des routes. - Cá et là dans le Doubs et le Jura, Vaud et Valais. - Hte-SaOne : Champagney, vallées de Mansvillers, de Miélin et du Rahin, Tète-des-Sèpes, Plain-des-Voleurs, Chagey, Etobon. - Ain : Charabotte entre Tenay et Hauteville, Ruffieu, Grand-Abergement, Champagne. - H.Sav.: Salève, le Môle. - Is, et H.Alp.: entre St-Laurent-du-Pont et la Chartreuse, Prémol, Chichiliane, St-Christophe, la Salette, la Grave. - B.-du-Rh.: bords de l'Huveaume et du Jarret, Aix à l'entrée de la Pinette sur le chemin de Vauvenargues. - Gard : l'Espérou à Dourbie, Lanuéjols.

U. efrusa Willd, - Planté le long des chemins.

\section{URTICEES}

URTiC urens L. - Lieux cultivés, décombres dans toule la France.

U. membramacen Poir. - B.-du-Rh., Var et Alp.-Nar. : Arles, la Camargue, Toulon, Hyères, Fréjus, lle Ste-Marguerite, Nice, Menton. - Gard et Pyr.-Or.: Sylvéréal, Perpignan.

d. dloiea L. - Décombres, voisinage des habitations. - Une forme velue, $U$. hispida, se montre quelquefois dans les vallées montagneuses. 
ข. nunufera L. - Dicombres, voisinage des habitations dans la région mérid. - Remonte dans la Drôme à St-Paul-Trois-Château, Grignan. - On l'a trouvé à Lyon sur des décombres, mais il n’a pas reparu.

PATKETARA officinalis L. - bécombres, vicux murs. Se prísente sous deux formes, l'une, la plus commune $P$. diffusa M. K. a des tiges étalées, diffuses et ordinairement rameuses; l'autre $P$. erecta M. $K$. a la tige dressée, habituellement simple. EP, Iusitaniea L. - Murs et rochers. - B.-du-Rh. et Var : envir. de Marscille, vallon de Possoteu à la Treille, Cassis, la Ciotat, vallon du Duc à Kognac, Toulon, la Valette. - Pyr.-Or.: ermitage de Baixas, Cases-de-Pena, Banyuls.

THELGONGN Iongiatipulatum, T. cynocrambe L. - Rocisers ct rocailles. - B.-du-Rlı., Var et Alp.-Nar.: la Ciotat au Becde-l'Aigle, Toulon, le Luc, Cannes, Grasse, de Nice à Menton. - Hér.: Mireval au Trou-de-Mliège, bords de la Mosson à StJean-de-Védas, Frontignan, Cournonterral, Cournonsec. Pyr.-Or.: vallon de St-Martin près Maureillas, Banyuls.

\section{CANNABINÉES.}

CANNALIS sariva L. - Cultivé et quelquefois subspontané sur les décombres.

HUMULUS Impulus L. - Haies, buissons dans tout le bassin.

\section{JUGLANDÉES.}

JUGravs regin L. - Cultivé pour l'alimentation et la production de l'huile.

\section{CUPULIFERES.}

FAGUS allvatiea. - Bois.

CASTANE valgaris $L$. - Bois des terrains siliceux; ne prospère pas sur les terrains calcaires.

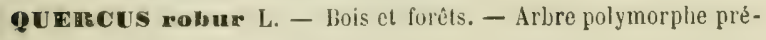
sentant les formes suivantes: 
Q. brevipedunculata, Q. sessilifiora Sm. - Race à pédoncules courts ou presque nuls. Présente une variété pubescens Willd. dans laquelle les feuilles sont tomenteuses surtout en dessous, la tige moins élevée.

Q. longipedunculala, Q. pedunculata Ehrh. - Race à pédoncules beaucoup plus longs que les pétioles. - Dans la variété apennina Lam. les feuilles sont tomenteuses en dessous, la tige moins élevée. Celle-ci a été observée dans les Albères (Pyr,-Or, ), au Cannet-du-Luc (Var’, à Charbonnières et Givors (Rhône), Belley (Ain).

Dans la variété fastigiata Lam. les branches sont ascendantes et offrent l'aspect pyramidal quion remarque dans le Populus fastigiala Poir.; se trouve dans les Pyr.-Or. à Saint-Laurentde-Cerdans et à Costujes.

Q. tomentosa, Q. To $\approx a$ Bosc. - Race à feuilles couvertes en dessus de poils roussâtres, étalés, et en dessous d'un épais duvet tomenteux qui recouvre aussi le pétiole. - Se trouve à la Tessonne et à Avèze (Gard), à Rigarda et à Finestret (Pyr.-0r.).

Q. recurvisquamosa, $\varrho$. Cerris L. - Dans ce Chêne les écailles de la cupule sont recourbées en dehors; les feuilles ont des lobes aigus. - Doubs et Jura : Quingey, Villars-St-Georges, St-Vit, Antorpe, forêt de Chaux. - Alp.-Mar. : Grasse à la Fons-des-Gavots.

G. dentata, Fontanesii Guss. - Race du précédent qui a aussi les écailles de la cupule recourbées en dehors, mais dont les feuilles sont tomenteuses en dessous, ovales et non oblongues, pourvues de lobes dentés. - Var et Alp.-Mar.: Montauroux, à deux kilomètres de Pont-de-Tournon-sur-Siagne, Font-desGavots près Grasse, Tourette, Callian.

Q. subera L. - Terrains siliceux des Maures, de l'Esterel et de l'lle de Porquerolle. - Terrains siliceux des Albères, Collioure, le Boulou, Céret, Arles, vallée du Rẻart (Pyr.-Or.).

Q. Hex L. - Commun dans la région méridionale. - Arbre polymorphe dans leiquel les feuilles et les fruits présentent de nombreuses variations. - Remonte dans la vallée du Rhone jusqu’au niveau de Vienne. 
Q. Auzendiann G. G. - Race du précédent remaruuable par la glabrescence de ses feuilles, toujours dentées, épineuses. - B.-duRh. et Var. : St-Loup et St-Tronc près Marseille, envir. d'Aix à la Repentance, quartier de la Félicité et du Seuil, le Ceinturon d'Hyères, lo Luc et le Cannel. - Gard.: les Angles près Villeneuve.

Q. coceifera L. - Commun dans les garrigues calcaires de la région mérid. - Remonte dans la Drome et l'Ardèche, vers Nyons, Donzère, Viviers.

Cortyus avellana L. - Haies et bois dans toute la France.

CARPINUS betulus L. - Bois et taillis. - Cultivé sous le nom de Charmille.

OSTRX A cripinifolla Scop. - Bois. - Var et Alp.-Mar.: Fréjus, Auribeau à N.-D. de Vaucluse, Grasse, le Bar, St-Arnoux, Aiglun, Vaugrenier près d'Antibes, Nice, Sospel, Castillon, Henton.

\section{SALICINÉES.}

SALIX pentandra L. - L Marais et tourbières des montagnes du Jura et des Alpes. - En dehors de nos limites dans les massifs de Pierre-sur-Haute, du Puy-de-Dôme, de la Creuse, du Cantal et de l'Aubrac. - Plusieur's botanistes," sur la foi de Lapeyrouse, l'indiquent vaguement dans les Pyrénées, oủ son existence est douteuse; il manque dans les Vosges.

s. rraglis L. $\rightarrow$ Lieux humides.

S. alba $L .-$ Bords des ruisseaux et des rivières.

Le S. vitellina est cultivé pour servir de lien.

s. babyloniea L. - Cultivé dans les jardins.

S. ineana Schrank. - Borls des ruisseaux et rivières des montagnes d'où il descend souvent dans les plaines. C'est ainsi qu'il est naturalisẻ sur les bords du Doubs, de la Loue, de l'Ain, du Rhône, de l'Isére, de la Durance, du Gardon, de la Mlosson, de la Lironde et de plusieurs aflluents de ces cours d'eau.

S. purpurea L. - Bord des eaux.

s. rabra Huds. - Bords des eans, - Rives du Doubs prés Rochefort. - Vaud : Payerne, le long de la Broie, Orny près d'Orbe, 
Goumoens. - S.-et-L.: bords de la Seille à Louhans et de la Grosne à Cluny, - Bords du Rhơne à Lyon, St-Vallier, Loriol, Beaucaire, Vallabrègues. - Bords do la Drôme à Crest et du Gardon près de Comps.

ง. Wimmerlana G. G. - Rives du Doubs près de Hontbéliard.

s. daphnoiden Vill. - Bords des rivières. - Genève, Vaud et Valais : confluent de l'Arve et du Rhône, Veyrier, rives de la Sarine, près de Château-d'0ex, de la Tourneresse à l'Etivaz, le Bas-Valais jusqu'à Sion. - H.-S.: Gaillard, Arenthon, la Roclıe, vallée de Boëge, vallée de la Borne, Grand-Bornand, Thones Annecy, Pringy. - Sav.: Tignes, bords de l'Isère près d'Albertville, bords de l'Arc près de Bessans. - Is.: Polygone de Grenoble, lles de Champagnier, Bourg-d'Oisans, vallée du Bréda au Fond-de-France. - H. et B.-Alp.: Villard d'Arène, le Monestier, Névache, bords de la Durance près de Briançon, Guillestre, le Champsaur, le Valgaudemar et le Devoluy, env. de Gap au col Bayard, les Serigues, Ia Condamine, Larche, Maurin. - Depuis longtemps ce Saule est naturalisé en amont de Lyon, entre la Tête-d'Or et Vaux-en-Velin, ainsi que dans les lles voisines el à Villette-d'Anthon.

S. vimimalis L. - Bords des ruisseaux et des rivières.

S. Smithians Willd, S. viminali-cinerea Wimm. - Vallée de Joux (Vaud); route du fort de l'Écluse (Ain), coteaux entre la Condamine et Jausiers (B.-Alp.).

s. oleifolia Vill. - Bois des montagnes. - Is. et H.-Alp.: Urtières près de Tullins, combe de Lancey, St-Ange, mont St-Martin, env. de Gap à Loubet, les Baux, montagne de Crigne près de la Saulse. On n'a pas pu retrouver ce Saule dans les localités indiquées par Villars. Serait-ce, comme le croyaient Chaix et Villars, un hybride des $S$. viminalis et caprea, ou comme l'a soupçonné Grenier des S. cinerea et incana. Il est fort problable que, sous les noms de S. oleifolia, S. Seringiana on a décrit plusieurs hybrides des S. cinerea, incana, et caprea, et que c'est à l'un d'eux, particulièrement au S. incano-cinerea qu'il faut rapporter l'individu que nous observons depuis plusieurs années sur la route de Tenay à IIauteville (Ain), celui que Reuter 
a vu pris du fort de l'Écluse, et enfin l'arbre signale par $\mathbf{M}$. J. Verlot près de la forêt de Portes en montant à Chamechaude (Is.). - Ce même hybride a depuis longtemps été aperçu dans la plaine rhénane, autour de Bảle et de lhheinfelden.

S. cinerea L. - Bords des caux depuis les plaines juspue dans les vallées montagneuses.

Faute de documents précis, je m'absticndrai de parler des hybrides que forme le $S$. cinerea avec les $S$. caprea pt purpurea.

s. Erandifolise Ser. - Bois des montagnes calcaires. - Chaino jurassique au mont d'Or, Suchet, côtes de Noiraigue, la Dole, Noirmont, Faucille, Reculet, Rizoux, de Tenay à Hauteville en Bugey. - II.-Sav, et Sav.: Salève, les Voirons, Dent de Lanfond pris Anriecy, les Avanchers, Hautcluce, Queige, mont Cenis. Is. et H.-Alp.: Grande-Chartreuse, forêt de Portes, Saint-Nizier, l'Oisans, le Valjouffrey, Villard-d'Arène, Lautarel au bois de la Madeleine.

s. enprea $L_{\text {. }}$ - Bois des coteaux et des montagnes.

๙. aurita L. - Lieux humides des plaines et des montagnes. Plus fréquent sur les terrains argileux et siliceux que sur les sols calcaires, à moins que ceux.ci ne soient fortement marneux ou recouverts d'une argile glaciaire, comme on le voit dans les tourbières établies dans les chaines calcaires. - Rare dans les départements méridionaux, dans les montagnes de l'Espérou, St-Guiral, le Vigan, Alzon (Gard), ainsi que dans celles d'Andabre-Rosis, St-Amand, Fraisse (Hér.).

S. aurilo-repens Wimm. S. ambigua Ehrh. - Lieux tourbeux et marécageux, surtout dans les montagnes. Jura helvétique et français : Tourbières des Ponts, de la Brévine, de la Chaux-de-Fonds, de la Trélasse, de Pontarlier, de la vallée de Joux et des Rousses. - Existe en plaine dans les marais de Saône près de Besançon et sur le plateau peu élevé des Échets (Ain).

s. repens L. - Prés tourbeux des plaines et plus souvent des montagnes à sol siliceux ou argileux. - Chaines vosgienne et jurassique jusque dans les montagnes de Nantua et de Hauteville en Bugey, à Colliard, Malbroude, Cormaranche, le Vély. Environs de Genève et Haute-Say.: marais de Veyrier et de 
Roellebot, de Lossy au pied des Voirons, de Chesse près Thonon et des Mouilles près Bellevaux. - Is. et H.Alp.: bords du lac de St.-Julien-de-Raz, Quirieu, bords du lac de Saves près Arrandon, Guillestre, mont Bayard près Gap, Manteyer. - C.-d'Or, le Morvan, Saulieu, Alligny, St-Julien. - Gard: Barague de Ifichel à l'Espírou, le Lengas près de Concoule sur la Lozère. - Pyr.-0r. et Ariẻge : Canigou, Laurenti dans les mouillères de Valbonne, Boutadiol, le Pis, le Pla dè l'Agré, la Cesseilla. - Loire et Ard. : Pilat au Pré-Lager, le Mezenc. - En dehors de nos limites, dans les montagnes de Pierre-sur-Haute, (Loire), mont Dore, Cantal, Lozère, Margeride, Aubrac, Pyrẻn. centrales, ouest de la France. - Espèce polymorphe dans laquelle on voit de nombreuses variations dans la forme et la couleur des feuilles et des capsules : d'où les variétés angustifolia, lanceolata, fusca, argentea, leiocarpa.

5. Hastata L. - Pâturages et bois humides des Alpes et des Pyrénées. - Nul dans les Vosges, le Jura et le centro de la France, - Vaud el Valais : Lavarraz, Anzendaz, Thyon, Orsera, Lens, Diez, Leukerbad, Zmutt, Münstigerthal. - H.-Sav, ct Sav : Glacière du Brizon, Méri, les Gets, montagnes de Samoëns, Laval-de-Tignes, mont Iseran, les Allues, les Avanchers, mont Cenis, - Is. et H.-Alp.: Alpe du mont de Lans, Grandes-Rousses, Villard-d'Arène, Lautaret, col du Chardonnet du côté de Névache, Gondran, Abriès en Queyras, haute vallée du Guil au pied du mont Viso, St-Véran à Clausis, St-Chafrey près d'Embrun. - B.-Alp. : Lauzanier, Fouillouse. - Ariége : lac de Quérigut, cirque des Aiguettes, Mijanès au Roc de Campeil.

S. nigroleans Smith. - Lieux humides des montagnes, d'où il descend le long des torrents et des rivières jusque dans les plaines. - Jura helvétique et français: Chasseral, Tête-de-Rang, mont d'Or, vallée de Mijoux, Noirmont, Nontendre, bords du lac de Joux, les Rousses. - Valais : Zermatt, Leukerbad, plaine du Rhòne. - H.-Sav., Sav. et Genève: Salève au Pas-de-l'Échelle, Scientrier, Bonneville, bords de l'Arve jusque près du confluent en aval de Genève, graviers de la Dranse près de Thonon, graviers de l'Isère et de l'Arly près d'Albertville, mont Cenis. 
- Is. et I.-Nlp : la Mateysine, le Valjouffrey, graviers du Drac, Polygone de Grenoble, Villard d'Arène, Lautaret, le Monestier, bords de la Durance près de Briançon, Guillestre, Boscodon près d'Embrun, Séuse. - B.-Alp. et Alp.-Alar.: la Condamine, la Blachière, Malemort, St-Étienne, Salèse, Ste-Anne-de-Vinaï.

S. Helveties Vill. S. Lapponum L. - Jarais des haules montagnes. - Vaud et Valais: Lavarraz, Catogne de Martigny, l'ierre-à-Voir, Zermontana, Clcuzon, Novelli, Thyon, Orsera, Combire, Diez, Mayenhorn, Binnergalen, Mattmark, Mlünstigerthal, glacier du Rhone. - H.-Sav. et Sav. : chaine du mont Blanc au Montanvert et à Entre-la-Porte, col de Balme, mont Cenis. - H.-Alp. : mont Viso à Ruines et à la Traversetle, Combe de Sigure. - Le Saule indiqué au mont Dore sous le nom de S. Lapponum est-il le même que celui des Alpes?

5. eaesia Vill. - Lieux tourbeux des Alpes. - Vaud: entre Solalex et Anzendaz. - H.-Sav. et Sav. : la Tournette, les Allues, Tignes, mont Cenis. - H. et B.-Alp.: Lautaret, mont Viso, col de Vars, Lauzanier, Mfalemort, la Blachière.

S. glauea L. - Prés humides des Alpes. - Vaud et Valais : Lavarraz, mont Fully, St-Bernard, Munsterthal. - H.-Sav. : bords de la Her-de-Glace, versant italien du mont Blanc dans l'AlléeBlanche, bas du Hottet au-dessus d'Aime, Fornet entre Lavalde-Tignes et le mont Iseran, mont Cenis. - Is. et H.-Alp. : col des Baisses entre la Salctte et le Valjouffrey, col de Sereine audessus de Clavans, la Grave, Lautaret, Monestier-de-Briançon, mont Genèvre, Orcières sous le pic de Mourefrey, haute vallée du Guil au pied du mont Viso, col de Ruines, col de Vars. B.-Alp. : Fouillouse. - - Le Saule indiqué sous le nom de $S$. glauca dans le massif du Laurenti est, suivant M. TimbalLagrave, une forme à feuilles soyeuses du S. pyrenaica Gouan.

s. arbuseula L. - Pàturnges humides des Aipes et des Pyrénées. - Vaud et Valais: Lavarraz, Nufenen, Majenhorn, Gemmi, Ilünstigerthal, Grimsel. - II.-Sav, et Sav. : Cornetles de Bise, mont Pétetod, les Avanchers, les Allucs, la Galise, lac de la Girottaz, la Sauce, col de la Seigne, Allée-Blanche, mont Cenis. H. et B.-Alp. : Lautaret, Monestier de Briançon, Gondran, mont 
Viso, Lauzanier, Parpaillon, sources du Var. - Pyr.-0r. et Ariège: vallées d'Eyne et de Carol, Valbonne sous le Roc de la Musique, Pla Bernard. - Pyrénées centrales.

S. myrsinitis L. - Prairies humides des Alpes et des Pyrénées. Vaud et Valais: Lavarraz, Bagnes, Arolla, Zermontana, Giétroz, Comba d'Arba, Zan, Mayenhorn, Feuilleret, Chermignon, Saas, Iainghorn. - Il.-Sav. et Sav. : mont Blanc à Entre-la-Porte, I'Écot entre Bonneval en Maurienne et la Levanna, mont Cenis. Is. et H.-Alp. : Alpe du mont de Lans, Lautaret, Névache, mont Viso, col Agnel, col Vieux. - B.-Alp. et Alp.-Mar. : Fouillouse, les Viosennes. - Pyr.-Or. et Ariège: montagne de Jadrès, lac du Laurenti en montant aux Jassettes, Valbonne au Roc de la Musique, Barbouillère sous le pic de la Camisette. - Pyrénées centrales.

s. pyremaiea Gouan. - Roches ct rocailles de la chaine des Pyrénées. - Pyr.-Or. et Ariège : Canigou, vallées d'Eyne et de Llo, mont de Madrès, val de Paillères à Soucarrat, vallon de Boutadiol, lac du Laurenti, Escale de Valbonne. - Pyrẻnées centrales.

S. reticuiata L. - Rochers et pâturages de la chaine jurassique, des Alpes et des Pyrénées. - Jura helvêtique et frarçais : versant nord du Chasseral, montẻe de la Dôle du côté des Rousses, le Reculet. - Vaud et Valais: Anzendaz, val d'Illiez, Pierre-àVoir, Orsera, Arolla, Zan, Giélroz, Sasseneire, Nainghorn, Gemmi, Zermatt, Simplon, Gerenthal. - H.-Sav. et Sav. : Dents d'Oche, Roc d'Enfer, Brizon, Jalouvre, Vergy, Méri, le WIole, col Joly, Montanvert, Entre-la-Porte près du Jardin de la Mer-de-Glace, Tré-la-Tête, col de Forclaz, mont Jovet, bas du Mottet au-dessus d'Aime, les Allues, les Mottets au pied du col de la Seigne, mont Cenis. - Is. et H.-Alp. : chaine de Belledonne vers le lac du Crouzet, Mont de Lans à Piéméyan, Brandes, Valjouffrey, mont Chamoux, Galibier, mont Viso, col Vieux, St-Yéran à Clausis, Plampinet, l'Aiguille-Rouge, Péas et Valpréveyre en Queyras, pic de Chabrières près Chorges. - B.-Alp. et Alp.-Nlar.: Vallonnei, Fouillouse, Crouès, Entraunes, cols de Jallorgues et de l'Abisso. - Pyr.-0r. et Ariège: vallẻe 
d'Err, lac du Laurenti, vallon de Boutadiol, Roc Blanc au-dessus de Barbouillère. - Pyrénées centrales.

s. retusa L. - Rochers et pelouses de la chatne jurassique, des Alpes ct des Pyrénées. - Jura helvétique et français: Chasseral, Tête-de-Rang, Creux-du-Van, Chasseron, Suchet, Noirmont, Colombier, Reculet. - Vaud et Valais: Solalex, Lavarraz, Alesse, mont Fully, Arpellaz, Pierre-à-Voir, Comba d'Arba, Cleuson, Arolla, Thyyon, Bellalui, Giétroz, Mayenhorn, Torrenthorı, Zermatt, Findelen, Saas. - II.-Sav, et Sav. : montagnes du Chablais, du Faucigny, de Beaufort, de la Tarantaise jusqu'au Petit-St-Bernard et au mont Iseran, montagnes de la Maurienne jusqu'au col de la Seigne et au mont Cenis; s'élève sur les flancs du mont Blanc jusqu'au Jardin de la Mer-de-Glace. Is. et H.-Alp.: chaines des Sept-Laux, de Belledonne, de la Grande-Chartreuse, de St-Nizier, la Moucherolle, l'Oisans, la Salette, le Lautaret, le Briançonnais ct le Queyras jusqu'au mont Viso, pic de Chabrières près Chorges, Chaillol, Durbonnas. B.-Alp. et Alp.-Mar. : Lauzanier, Vallonnet, Fouillouse, Parpaillon, Jallorgues, Alpes de l'Abisso et de Tende. - Fyr.-Or. et Ariège: Cambres d'Aze, vallée de Lìo, Laurenti, Valbonne, Barbouillère, pic del Ginèvré. - Pyr. centrales.

S. serpyllifolia Scop. - Forme à petites feuilles qu'on trouve dans le Valais à Alesse, mont Fully, Findelen, Saas, Fée, Arolla, Torrenthorn, Mayenhorn. - Sav. et H.-Sav. : mont Cenis et le Cramont en Italie, col de la Seigne, la Galise, Tré-la-Tète, col d'Anterne. - Is. et H.-Alp.: Piéméyan au mont de Lans, les Trois-Érêchés, Galibier, mont Aurouse.

S. herbacea L. - Rocliers et pâturages des Alpes et des Pyrénées. - Vaud et Valais : Panerossaz, St-Bernard, Zermontana, Thyon, Arolla, Galmscheide, Zermatt, Schalberg, Gemmi, Grimsel. II. Sav. et Sav. : Vergy, Méri, Jalouvre, Signal-des-Agneaux, Cornettes-de-Bise, chaine du mont Blanc, Jardin, Entre-la-Porte, Tré-la-Têle, mont Jovet, Saut des Allues, la Forrlaz, mont Cenis. - Is. et H.-Alp.: chraines des Sept-Laux, de Belledonne près des lacs Doménon, des Grandes-Rousses, Haute-Téte-de-Rachıa au Jont de Lans, la Bérarde, les Trois-Évêchés, Galibier, Lau- 
taret, Ruffère, mont Viso, Chaillol, pic de Chabrières près Chorges, mont Aurouse, Durbonnas. - B.-Alp. et Alp.-Mar.: Lauzanier, Vallonnet, Estenc, la Stroop, le Garret au-dessus d'Entraunes, Salsamorena, col de Fremamorta, lac d'Entrecoulpes, Alpes de Tende. - Pyr.-Or. el Ariège: Canigou, envir. de l'élang de Las-Nous, Laurenti, au sommet du pic del Ginèvré et du Sarrat d'en Binada, Pla Bernard. - Pyr. centrales, mont Dore.

POPUEUS tremala L. - Bois humides.

P. alba L. - Bois, bords des rivières, - Souvent planté.

1. canescens Sm. - Planté et parfois subspontané.

P. virginians Desf. - Planté.

R. nigra L. - Bois humides, bord des caux.

P. pyramidalis Ros. - Planté le long des chemins ct des cours d'eau. - Suivant M. Decaisne, ce Peuplicr est une variétẻ du précédent, due à la culture.

\section{PLATANEES}

PLATavos orientalis L. - Planté dans les promenades publiques.

P. occidentalis L. - Planté.

\section{BETULACÉES}

BeTULA alba L. - Sols humides des terrains siliceux des plaines et des montagnes. - Dans les chaines calcaires le Bouleau ne se voit que sur les tourbières à sous-sol siliceux. - Nul dans la région des Oliviers. - Dans les départements méridionaux il n'existe que que dans les montagnes. - Espèce polymorphe. La variété pendula Roth a les rameaux longuement pendants; c'est la forme des plaines. - La variété laciniata G. G., à feuilles laciniées, est quelquefois mêlée au type.

B. pubescens Ehrh. - Forme rabougrie du B. alba, à feuilles et pétioles pubescents et souvent glutineux pendant leur jeunesse. En vicillissant, cet arbre se rapproche du Boulcau blanc. 
La variété carpalica W. Kit. a des rameaux visqueux pendant leur jeunesse.

B. intermedin Thomas. - Comme ce Bouleau vit souvent en société arec les $B$. alba et nana, on a supposé qu'il pourrait être hybride de ceux-ci. Il est plus probable que, comme le croyaient Gaudin el Thomas, ce Bouleau est simplement un état intermédiaire entre les $B$, alba et nuna. - On l'a observé dans les tourbières de la chârue jurassique aux marais de Chasseral, des Ponts et de la Brévine.

B. nana L. - Forme naine du Bouleau blanc, trouvée dans les tourbieres des vallées de Joux et des Rousses, du Brassu, des Ponts, de la Brévine et de Mouthe.

ACNes viridis DC. - Bois et prairies des montagnes. - Nul dans les Vosges et la chaine jurassique. - Vaud et Valais : Jorat, bois de Payerne, mont Vully, Bovonnaz, Solalex, Zermatt, Bérisal, Grimsel. - H.-Sav. et Sav.: Salève du coté de Cruseilles, les Voirons du côté de Boëge, le Môle, Brizon, Vergy, Méri, Parmelan, Charvin, unont de Sion, chalets de Colone, mont Cenis. - Is. et II.-Alp.: Chamechaude, Revel, Prémol, entre la Ferrière et les Sept-Laux, Lautaret, le Briançonnais, vallêe du Guil, col de Ruines, Champsaur. - B.-Alp. et Alp.-Mar.: la Condamine, Serennes, vallées du Var, de la Vésubia, Tinea, Roja.

A. glutinosa Gaertn. - Bords des eaux dans toute la France.

A. Incrana DC. - Bords des caux, beaucoup moins répandu que le précédent.

\section{ABIĖTINÉES}

PINUS silrestris L. - Bois des terrains siliceux des plaines et surtout des montignes où il est fréquemment planté. - Ce Pin re prospère pas dans les terrains calcaires. L'ignorance de celte antipathie a été cause de nombreux mécomptes soil pour les particuliers, soit pour l'administration forestière. Le Pin d'Autriche est celui qui convient aux terrains calcaires.

P. uneinata Ram. - Forêts des montagnes. - Vaud et Valais : Anzendaz, Arbignon, Alpes de Lens et de St-Haurice-de-Lac. 
- Ain : montagne d'Allemogne au Reculet. - Is, et H.-Alp.: lac Luitel entre Prémol et Champrousse, Chamechaude, Bovinant, St-Nizier, plateau de Chamousset près du mont Aiguille, N.-D. du Laus près Gap, vallée de Cervières près Briançon. B.-Alp. et Alp.-Har.: la Condamine, Serennes, Alpes de Fenestre, forêt de Molières et de Fremamorta. - Vaucl.: mont Ventoux. - Pyr.-Or. et Ariège: Canigou, vallée d'Evol, HontLouis, Font-Romcu, le Capcir, le Laurenti à Bosc-Nẻgré, Sarrat d'en Binada. - Pyr. centrales.

Une forme naine, S. pumila (Pumilio Hacnke), au tronc couché, tortueux et rameux, sc trouve dans les tourbières de la chaine jurassique au Chasseral, au Suchet, dans les vallées de la Brévine, des Rousses, et vers le lac St-Point.

P. Iarieio Poir. - Bois. - Gard et Hér.: vallée de Bessège à Bourdezach, St-Guilhem-le-Désert, entre Bédarieux et Carlincas, Montarnaud. - Pyr.-Or.: Fillols, montagne de Sahorre, les Albères.

P. pyrenaicn Lap. - Forme intermédiaire entre le précédent et le suivant, qui se trouve dans les Pyrénées centrales et ne paralt pas avoir été introduite dans le bassin du lhône.

P. halepensis Mill. - Très répandu dans la région des Oliviers.

P. pinea L. - Littoral de la Provence et des Alpes Maritimes. La Camargue, Pesquiers d'Hyères, l'Esterel, Cannes ; a été planté dans l'intérieur autour du Luc, du Cannet et de Vidauban. - Gard: Pinèdes de Ste-Marie et d'Aigues-Hortes. Pyr.-Or.: St-Genis au pied des Albères.

P. maritima Lam. - Terrains siliceux de la région mérid. B.-du-Rh., Var et Alp.-Nar.: bois de Mazargues près Marseille, Hyères, île de Porquerolle, le Luc, St-Tropez, l'Esterel, Cannes. - Gard: Aigues-Mortes aux Quatre-Maries, Manduel. - Pyr.Or.: Argelès, Collioure. - Depuis quelques années, le Pin maritime a été abondamment planté dans les Landes sablonneuses de Gascogne et sur les terrains siliceux de diverses parties du centre et de l'ouest de la France. On l'a mème introduit sur les sables de la Sologne oủ il a très-bien réussi. Cependant il ne résiste pas aux hivers rigoureux : celui de $1879-80$ a fait périr un grand nombre de sujets. 
P. cembra L. - Bois des liautes montagnes, - Vaud el Valais: Anzendaz, entre les Balmes et la Forclettaz, Fully, Rosselena, Catogne, Valorcine, Thyon, Arolla, Leukerbad, Zermatt, Saas, Reschy, Graechen. - II.-Sav. et Sav.: Sixt, Chamonix, St-Gervais, Crest-Voland, Beaufort, Saut-des-Allues, Lans-le Bourg. Is. : Champrousse, Poursollet au pied de Taillefer, mont Chamoux. - II. et B.-Alp.: le Briançonnais, le Queyras, St-Véran à Clausis, col de Vars, le Noyer en Champsaur, mont d'Ancelle, mont Morgon, la Grangette près Gap, les Tardées près la Condamine. - Alp.-Mlar.: forêt des Mollières, vallon de Jallorgues, col de Salèze, vallon d'Entrecoulpes, mont Bego, mont Vanca au-dessus de Fontan.

P. pectinata Lam., L. Picect L. - Planté dans les montagnes de tout le bassin jusqu'à 700 mètres.

P. exceloa Lam., P. Alics L. - Planté dans les montagnes à partir de 600 mètres.

P. Iarix L. - C'est l'essence qui résiste le mieux aux froids rigoureux des hautes régions des Alpes. On l'a aussi planté dans les plaines.

\section{CUPRESSINEEES}

JUNCPRUS communis L. - Bois, coteaux depuis les plaines jusqu'aux montagnes.

J. nana Willd. - Forme alpine du précédent qu'il remplace sur les hautes montagnes et dont il se distingue par sa tige et ses rameaux couchés.-Ain : la Dôle, Colombier, Reculet, Sorgiaz, Crêt de Chalan. - Valais: St-Bernard, Thyon, Grimsel, Furca. H.-Sav, et Sav.: Brizon, Vergy, mont de Laouet, Semnoz, montagne de Veyrier près Annecy, les Contamines, Beaufort, du Pont de la Balme aux Bréviaires dans la vallée de l'Isère. - Is., II. et B.-Alp.: Grand-Som, Chamechaude, Champrousse, Mont de Lans, Lautaret, montagnes de Chatteau-Queyras et de Guillestre, col de Vars, Sapet de la Bâtie-Neuve, Devez de Rabou près Gap, Allos. - Vaucl. et Alp.-Mar.: mont Ventoux, parties supér. des vallées du Var, de la Vesubia, Tinea et Roja. - Pyr.-Or. et Ariège: Canigou, pic del Ginevré et de Tarbézou, Port-de- 
Pailleres, lac de Rabassoles, rocs de l'Estagnet et de Salsafrage. Ard. et II.-Loire: Gerbier-des-Joncs. - Pyrén. centrales.

Le Sabina Villarsii Jord. est une forme à gros fruits (S. macrocarpa) du J. nana.

J. ozycedrus L. - Coleaux de la région mérid.; remonte dans la Drôme vers Solaure, Saou, Montélimar, Propiac, Tain, St-Vallier ; dans l'Ardèche à Rochemaure, Vals, Tournon.

J. phonicea $L$. - Rocher's de la région mérid.; remonte dans la Drôme à Luc-en-Diois, Montmorin, Donzère, Nyons, Montmaur; dans les H.-AIpes et l'Isère à Rabou, St-Clẻment-d'Embrun, Comboire, montagne de Níron près Grenoble.

J. lycia L. - Forme du précédent ayant une taille plus élevẻe, des rameaux dressés, des fruits plus gros. - Var: presqu'ile de Giens, les Pesquiers d'Hyères, île de Porquerolle.

J. macrocarpa Sibth. - Forme à gros fruits trouvée dans les Alp.-Hlar. à Margès près Vérignon, Fox-Amphoux, te Biol, Menton.

J. sabina L. - Coteaux des montagnes. - Nul dans la chaine jurassique. - Vaud et Valais: château d'Oex, mont Fully, Pissevache, Martigny, Orsières, Scmbrancher, Tortain, de Visp à St-Nicolas, Findelen près Zermatt, bois de Finge, Conches. - H.-Sav. el Sav.: montagne de Veyrier à Rampon, mont Fucille sur Vailly, la Gita, les Gets, Vallorsine, St-Jean-de-Maurienne. - H. et B.-Alp. : entre la Maison-du-Roi et ChâteauQueyras, vallée de Cervières, la Condamine. - Pyr.-Or. el centrales.

TAXUs bacenta L. - Bois ct coteaux calcaires. - Doubs, Jura et Ain: Cotes du Doubs, du Dessoubre et de la Loue, Lomont, montée de la Dóle et du Reculet, Torcieux, le Valromey, Ruffieu, Monts.d'Ain. - Vaud et Valais: Alpes de Bex et de Châteaud'Oex, bois de Sauvabelin, Aproz, Ayen, val Reschy, Naters. H.-Sav.: Salève, les Voirons, Brizon, Pontchy, Rumilly près Bonneville, Menthon, Talloires, Veyrier. - Is. : Grande-Chartreuse, St-Nizier, Autrans, Infernet près Voreppe. - Dr., H. el B.-Alp.: Aucelon, pic de Toulans, le Noyer, les Baux, Montmort, Annot. - Var et Alp.-Har.: Ste-Baume, mont Courmes au- 
dessus de Grasse, Collongues dans la vallée de l'Esteron, vallon de Libaré près St-Martin-Lantosque, forêt de la Maïris. - Gard et Hér.: Chartreuse de Valbonne, rochers du Caroux.

\section{GNÉTACEES}

E. rigida comprenant les variétés E. hebrodensis Tin. (E. Villarsinna G. G.) et E. helvelica C. A. Meyer. - Forme à pédoncules très-courts ou même nuls, à rameaux durs, non flexibles, tantot dressés dans la var. nebrodensis, tantot plus ou moins décombants dans la var. helvelica. La tige et les rameaux, au lieu d'avoir une couleur glauque comme dans l'E. distachya, ont une couleur d'un vert clair ou sombre. - Les deux variélés existent à la Baume près Sisteron et à la Brèche-deVergons près Annot (B.-Alp.), ainsi qu'à Orange (Vaucl.).

La variété helvetica, d'ahord observée dans le Valais sur les rochers de Folatères, Saillon, Châteauneuf, Montorge, Sion à Tourbillon, a été vue ensuito à Embrun, Laragne, Châteauneufde-Chabres, Ribiers (H.-Alp.'; sur les pentes du pic de Bretagne, près Gémenos (B.-du-Rh.); Villencuve-les-Avignon au Grand-Montagnet (Gard).

La variété nebrodensis se distingue de la précédente par ses rameaux toujours dressés d'un vert plus foncé, son style droit et plus court. - A été trouvée à Valernes (H.-Alp.); vallée de l'Yeuse près Mérindol, mont St-Jacques près Cavaillon (Vaucl.); les Alpines à Eyguières, St-Remi, mont Peuzin, montagne de Cordes près Arles (B.-du-Rh.); ruines du château de Gigors près Crest; Châteâu de Pierre-Pertuse dans les Corbières (Aude).

E. maritima, E. distachya L. - Forme du précédent particulière aux sables et coteaux du littoral méditerranéen et océanique. B.-ilu-Rh.: îles de Pomégue et de Ratoneau, route de Narseille aux Martigues, bords de l'ètang de Berre. - Gard : Pinèded’Aigues-Mortes, bords de l'ẻtang de Pujault. - Hẻr.: Pérols, Palavas, Frontignan, Cette, Fabrègues, les Aresquiers, St-Jeande-Védas au moulin Geniès, Agde. - Aude et Pyr.-Or.: la Catal. Bassin du Rhöne. 
Clape, Ste-Lucie, Leucate, Salses, Banyuls-sur-Mer. - En dehors de nos limites sur les sables maritimes des côtes de la Vendée, de la Loire-Infér., du Morbihan et du Finistere.

Contrairement à l'opinion de tous les auteurs qui, depuis C.-A. Meyer, Tineo jusqu'à M. Bonnet, ont considéré les trois Ephedra ci-dessus énumérés comme des espèces distinctes, je réunis sous le nom d'E. rigida les $E$. nebrodensis et helvetica. En outre, je regarde l' $E$. distachya comme une forme maritime de l'E. rigida dont il ne diffère que par la flexibilité et la couleur glauque de ses tiges et de ses rameaux, ainsi que par la longueur des pédoncules, attributs qui me paraissent influencés par l'aura mavitima. Du reste, il serait facile de vérifier si ma supposition est fondée en cultivant pendant longtemps, sur des rochers calcaires arides et éloignés de la mer, quelques pieds d'E. distaclya récoltés sur les sables maritimes. Dans le cas où ceux-ci perdraient leur teinte glauque, deviendraient rigides et présenteraient des pédoncules courts, il n'y aurait plus aucun motif d'hésitation à accepter le rapprochement que je propose et à considérer définitivement l'E. distachya comme la forme maritima de l'E. rigida, lequel, lui-même, présento au moins deux variétés, trop voisines l'une de l'autre pour qu'il y ait lieu d'établir entre elles une distinction spécifique. 


\section{MONOCOTYLEDONEES}

\section{ALISMAGEEES}

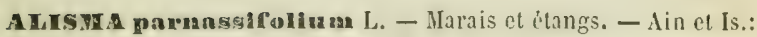
environs des forêts de Seillon et de la Chambrière, marais de Charvieu, Arandon près Morestel, les Aveniêres. - Sav.: l'Echaillon près de St-Jean-de-Maurienne.

A. plantagineum L. - Fossís et marais dans tout le bassin. Espèce polymorphe dans laquelle la forme des feuilles présente de nombreuses variations suivant que celles-ci sont inondées ou exondées. Dans le dernier cas, les feuilles sont habituellement larges, ovales, en cour ou arrondies à la base (A. latifolium); mais si de fréquents changements arrivent dans le niveau de l'eau, elles sont lancéolées (A. lanccolatum Rchb.). Lorsque les feuilles restent constamment inondẻes, elles sont linéaires (A. graminifolium Ehrh.).

Les modifications produites sur les organes de végétation des plantes aquatiques doivent être souvent rappelées aux botanistes afin de les préserver de la tentation qu'ils pourraient avoir d'établir des distinctions spécifiques basées uniquement sur le caractère si variable de la forme et des dimensions des tiges et des feuilles.

A. crcuatum Michalet. - Forme du précédent à tige et rameaux recourbés. - Jura et $\Lambda$ in : bords du Doubs et de l'Orain à Chaussin, Longwy, Peseux, Champdivers, Molay, Gevry, Villey-enBresse, St-Seine et Laillon, le Plantay près Marlieu. - Environs d'Avignon, d'Hyères et de St-Tropez et probablement ailleurs.

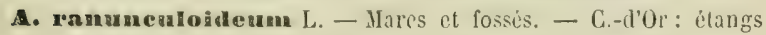
de Larrey, près de Châtillon. - Rh., Ain, Is. et Sav. : Vaux-enVelin, les Echets, lac Bertrand, Ceyzéricu, Lavours, Décines, Meyzieu, Crémieu, Morestel, Romagnieu, Crolle, St-MauriceCalal. Bassin du Rhóne. 
d'Exil, marais de llyans près Chambéry. - Assez commun dans les parties basses de la Provence, des Alpes-Mfaritimes et du Languedoc.

Dans la var. repens Cav., les tiges sont couchées, sauf la centrale, et s'enracinent aux nœuds.

A. natans $\mathrm{I}_{\star}$ - Mares et fossés. - G.-d’Or: le Morvan autour de Saulieu dans les étangs Fortier, Morin, Larmier. - La Bresse, la Dombes, Lyon à la Mouche et aux Brotteaux. - Is. : Décines et Meyzieu.

Dans celte espèce, comme dans un grand nombre de plantes aquatiques, les feuilles immergées sont linéaires, les feuilles flottantes sont ovales.

DATI SON⿴囗十 stellatum Pers. - Etangs et fossés. - C.-d'Or: étangs de St-Seine-en-Bâche, de Seurre et de Bagnot:- Doubs, Jura, S.-et-L., Ain : étangs de St-Seine, Laillon, l'Abergement, St-Symphorien, la Bresse et la Dombes où il est commun. B.-du-Rh. : la Crau, la Camargue, Montmajour. - Gard et Hẻr.: Jonquières, Bellegarde, Agde, Roquehaute. - Pyr.-Or.: bords de l'étang de Salses, Argelès.

SAgirera aquatica Pline, Sayittaria sagittifolia L. - Fossés et ruisseaux dans tout le bassin.

Lorsque les feuilles sont constamment submergées, elles sont linéaires et réduites au pétiole (S. vallisnerifolia Cosson et Germain).

EบTON⿴囗十 umbellatus L. - Rivières et étangs. - Arrond. de Belfort et HI.-Saône : Bourogne, Pontcey, Chemilly, Vesoul, Trécourt, Genevrières, la Lanterne, bords de la Saone ct de l'Ognon. - Jura, C.-d'Or, S.-et-Loire, Ain: bords de l'Ognon, du Doubs, de la Loue, de l'Ouche et du canal de Bourgogne, Chalon, Autun, Louhans, bords de la Reyssouze, bois de Bouvant près Bourg, étangs de la Bresse et de la Dombes, Reyrieux. - Rh. ct Is.: bords de la Saône à Anse, Fontaines, bords du Rhône à Yvour, Oullins, Feyzin, St-Symphorien-l'Ozon, la Verpillière, Virieu. - Dr.: Loriol, Montélimar. - Vaucl. et B.-du-Rh.: envir. d'Avignon et d'Arles, - Var et Alp.-Mar. — Gard: StGilles, Franquevau, Bellegarde, Jonquières, Aigues-Mortes. - 
Hér. : Maurin, St-Marcel, Fre̊jorgues, Lunel, Vendres. - Pyr.Or.; Agulla de la Mar, entre l'étang de Villeneuve, Vall-Rich el l'Esparrou d'après Companyo.

\section{COLCHICACEES}

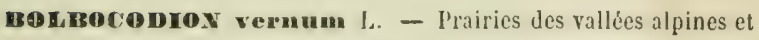
pyrénéennes, - Valais: Branson, la Barma, Folatères, collines des environs de Sion, Miéville, Montorge, Aproz, Mayens de Sion, Plâtrières de Sierre, Eischoll, Visperterminen. - Sav. : de Lans-lc-Bourg au Mont-Cenis. - II.-Alp. : Lautaret, Monestierde-Briançon, Val des Prés, mont Genèvre, col des Hayes, col Agnel, l'Echalp, St-Véran à la Grangeasse, pic de Chabrières, Charance, col de Glaise, mont Séuse. - B-Alp. : Malemort, Horonaye, Crouès, Annot au mont Vergons. - Dr.: Aucelon, entre le Pas de Jansac et le Pas de la Posterle près le Luc-enDiois. - Alp.-Mar. : montagne de Trottes au-dessus d'Entraunes. - Pyr. centrales.

Le Merendera filifolia Camb. d'Espagne el des îles Baléares a été trouvé dans les B.-du-Rh., à Carro, non loin de la Couronne et aux Martigues.

L'indication donnée par Companyo, du Merendera bolbocodia Ram, dans la vallée de Carol et au col de Jau dans les Pyr.-Or. est probablement erronée. Il n'est pas venu à notre connaissance que celte espèce ait été trouvée en dehors des Pyr. centrales.

COLCHECO autumale $L$. - Prairies dans toute la France. Espice polymorphequi fleurit quelquefois au printemps lorsque son développement a été retardé par une immersion prolongée des prairies.

C. longifolium Castagne, C. arenarium $\mathrm{W}$. Kit. -- Forme dans laquelle les bulbes et les capsules ont des dimensions moindres, les étamines toutes insérćes au mème niveau, les feuilles lancéoléeslinéaires. - B.-du-Rh. et Var: Aix au vallon de Cascaveou au N. 0 . de la Kéirié, bords de la Crau, vallon de Luminy près Marseille, le Luc, St-Tropez, entre Gassin el Cavalière, Cannes, 
I'Estérel, Antibes, Nice ả Mont-Gros et à St-Roch, Menton au cap Martin. - Gard el Hér. : Manduel, Villeneuve en face d'Avignon, bois de Fargues, çà et là dans Ies arrond. de Montpellicr et de Béziers.

C. provinciale Loret. - Diffère du précédent par son périgone deux fois plus long, à divisions linéaires, non veinẻ. - Var: les Maures, les Mayons.

C. alpinum DC. - Resscmble aux deux précédents par le mode d'insertion des étamines, la petitesse du bulbe et la forme des feuilles; mais il en diffère par sa capsule ovoïde mûrissant plus tôt et entourẻe de deux feuilles. - Prairies des Alpes. - Valais : Bourg St-Pierre, Champé, Val Ferret, Mayens de Saxon et de Sion, Findelen, Saas, Berbelegg prés Munster, Schwischbergen, Simplon. - H.-Sav. et Sav. : Nant Borant, Chamonix, Lans-leBourg en montant au mont Cenis. - Is. et H.-Alp. : Alpe du Mont-de-Lans, Brandes et St-Christophe en Oisans, Lautaret, Plampinet, Val-des-Prés, mont Genèvre, mont Viso, col de Glaise, Côte Gélive près Gap. - B.-Alp. et Alp.-Mar. : Bachasse près de la Condamine, col de Vergons sur Annot, sources du Var, St-Etienne-le-Sauvage, val de Jallorgues, mont Cheiron au-dessus de Grasse, Berre, Tende.

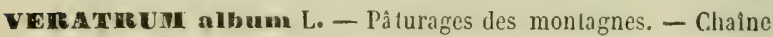
des Vosges jusque dans la II.-Saône aux Ballons de Servance et de Giromagny. - Claine jurassique, Vaud, Valais, Savoie, Dauphiné, B.-Alpes et Alp.-Mar. - Chaîne du Mezenc, Mazan, Cuze et Mezilhac dans l'Ard., l'Espérou. - Pyr.-Or. el Ariége dans le massif du Llaurenti.

En dehors de nos limites dans les Pyr. centrales, Aubrac, Lévezou, Cantal, mont Dore, chaine du Puy-de-Dỏme, la Crcuse.

La variété Lobelianum Bernh. à fleurs vertes se trouve quelquefois mêlée au type à fleurs blanchâtres.

NATER Aude, Pyr.-Or, et Ariège: Martis, Coma de Vall Marans, col de Jau, Salvanère, versant oriental de Paillères, val de l'Estagnet, cabane de Fournet et de la sire. - En dehors de nos limites dans le Cantal et l'ouest de la France. 


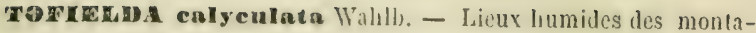
gnes. - Clıalne jurassique, Vaud et Valais, Savoie, Dauphine̊, B.- $\Lambda \mid p$, et $\Lambda \mid$ p.-Har. - Pyr.-Or. et Ariege: Mont-Louis, le Llaurenti.

T. borealis Walhlb. - Lieux humides, - Valais: sous le Kallwassergletscher. - H.-Alp. au col Malrif, mont Viso aux sources du Guil vers Pierre-Noire.

\section{LILIACÉES}

TULTPA CIushama DC. - Bois des champset des rignes de la région mérid. - Vaucl, et B.-clu-Rh. : envir. d'Avignon, Aixà la Torse, Montaiguet, Peiblanc, Roquevaire, Var et Alp.-Mar. : Toulon, le Luc, Fréjus, Cannes, Grasse, Nice, Menton. - Gard et Hér.: Nìmes à la Tour Magne et sur le chemin de Sauves, Bézierssur la rive droite de l'Orb, Pézenas. - Subspontané á St-Pierre-d'Albigny (Sav.) et à St-Genis-Laval près Lyon.

T. Lortetiana Jord. - Champs des B.-du-Rh. et du Var: Aix, Marseille, Cassis, Grasse, Cannes, Antibes.

T. acutifora Pourr. - T. Oculus solis St-Am. - Cluamps et vignes de la région mér. - B.-Alp. et Vaucl. : Sisteron, Castellane, Apt. - B.-du-Rh., Var et Alp.-Mar.: Aix, Toulon, la Seyne, le Luc, Draguignan, Grasse, Cannes, Nice à Cimiez. - Gard et Hér. : Manduel, Nìmes, Montpellier au Pré d'Arêne et à Rondelet. - En dehors de nos limites dans le bassin de la Garonne.

T. praeeox Ten. - Champs cultivés de la région mérid. - B.-duRh., Var et Alp.-Ilar. : envir. d'Aix au vallon de Brunet, Buonouro, le Luc, Draguignan, Mougins, Grasse, Cannes, Antibes, Nice, Menton. - B.-Alp. et Dr. : Sisteron, Aouste près Crest, le Faury. - IIér.: Ilontpellier au prẻ d'Arène, Flaugergues, le Caylar, Fraisse, - Naturalisẻ autour de St-Jean-de Maurienne, au plan de l'Aiguille de Vienne, à Marcy près d'Anse (Rh.) et à Ste-Foy-sur-Lyon.

T. Didieriana Jord. - Cliamps, - Talais : Condemine de Sion.Sav.: la Fortune prìs Aime, Macot, le Clappet près St-Jean-deMaurienne. 
Une forme appelée par M. Jordan $T$. platystigma, ayant la fleur d'un pourpre pâle ellilacé, odorante, a êté trouvée près de Guillestre (H.- $\Lambda \mid$ p.)

T. allvestris L. - Champs, vignes et prés. - Envir. de Belfort. - H.-Saône, Doubs et Jura : Vesoul, Chariez, Montbéliard, Besançon, Salins, Arbois, Poligny, Dole, Baume, Lons-le-Saunier. - Vaud et Valais: Nyon, Coppet, Orbe, Beaulmes, Eclépin, Morges, Myolan près Vendouvre, Sion, vallée de Loctschen, Natersberg, Simplon. - Envir. de Gene̊ve et H.-Sav.: entre Veyrier et Sierne, Neydens prẻs St-Julien. - Ain : Ambléon et St.-Germain près Belley, Trévoux. - C.-d’Or et S.-et-Loire: Châtillon, Neuilly, Léry, Varois, Cluny, Mâcon, Romanèche. Rh.-ct-Loire: Limas, St-Tryx, Dracé, Fleurie, Charnay, StGenis-Laval, Chavanne près St-Chamond, Grand-Croix, - Sav.: Moutiers, Aime, St-Jean-de-IIaurienne, - H. et B.-Alp. : Embrun, Gap, Ribiers, Sisteron, Castellane, Faucon, Bousolięres, Entrevaux. - Vaucl., Var et B.-du-Rh.; envir. d'Apt, d'Aix et de Toulon au mont Faron. - Ard. et Gard: Roche de Gourdon, Aramon, St-Guiral, Anduze. - Hẻr.: Nontpellier au Pré d'Arêne, le Caylar, Fraisse. - En dehors de nos limiles dans les Pyr.-centr., l'Aveyron, la Lozère, la H.-Loire, Charente-Infér., Loire-Infér. - Espèce polymorphe à laquelle se rattache le T.alpestris Jord. Fourr. trouvé dans les H.-Alpes au mont Genèvre, à St-Véran, Fontgillarde.

T. gallea Lois. - Var: mont Faron, Hyères, le Luc, Fréjus. Aude: Massac dans les Corbières.

T. Celsiamn DC. - Bords des champs de la région mérid. et mojenne. Ain : sommet du Colombier du Bugey, - Sav. : Galopaz au-dessus de Puisgros, Orizan sur Cléry. - Is. et H.-Alp. : mont Rachais, Claix, Gresse, St-Nizier, la Bâtie, Clıamprousse, Charance, col de Glaize, Séuse, Aurouse, Ventavon, Ribiers, Montmorin, Bruis, Rosans. - B.-Alp. et Vaucl. : mont Vergons sur Annot, Castellane, Sisteron, mont Ventoux. - B.-du-Rh.: envir. du mont Ventoux. - B.-du-Rh. : environs d'Aix, Montaiguet aux vallons du Coq et de Fontgamate, Ste-Victoire, St-Loup, Roquevaire. - Var el Alp.-Mar.: Toulon, Le Iuc, Fréjus, L'Esterel, 
mont Cheiron près Grasse, Clans, col de Tende, Sitorgio, la Giandola. - Gard et IIér. : l'Espérnu, borls du Garion, la Beaume, pont du Gard au chemin d'Uzès, Douch sur le Caroux, le Caylar, Fraisse. - Aude et Pyr.-Or. : envir. de Narbonne au Pech de l'Agnèle, Sidrière de Fitou, Roquefort et Fraisse dans les Corbières, Collioure, Argeles. - En dehors de nos limites dans l'Areyron, les Deux-Sèvres, Maine-ct-Loire.

T. Gesmerlama L. -- Souvent cultivé et quelipuefois subspontané, comme, par cxemple, au Champ Lélé à la Forlune près Aime (Sav.), et dans lesenvir. de Grasse (Alp.-1lar:).

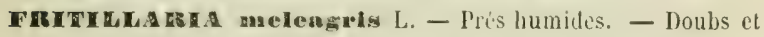
Jura: Pontarlier, Morteau à Biaufond, Grand'Combe des Bois, Blancheroche, Poset près Pont-de-Roide, Nozcroy, Bletterans, vallée de Syam. - Ain: Nantua à Long-Favre, le Mont, la Tour, la Cluse, Brẻnod, St-Rambert au Chenavarey, Champdor, Hauteville, Chaley, Mogneneins, entre Pont-de-Vaux et Thoissey. Rh. : Belleville, Anse, lles Royes, Tassin. - S.-cl-L. : Louhans, St-Bonnet-en-Bresse, Charnay, Verdun, Ecuelles près Mâcon. Is.: Vézeronce et le Bouchage près Morestel, Crémieu, Vienne. Sav.: envir. de Chambéry. - Gard: Campestre, St-Guiral, l'Espérou.

F. pyremniea L. - Rochers, bois des montagnes, - Hér.: StPons, les Rives, St-llichel-des-Sers, le Caylar. - Pyr.-0r.: Mont-Louis, Bac de Bolquère, les Bouillouses, vallée d'Eyne. Pyr. centrales, Aveyron, Tarn.

F. deIphinensis Gren. - Prairies des montagnes. - Dr., H. et B.-Alp.: les Lussettes près la Croix-Haute, Laularet, Rabou, bois Mondet et Charance près Gap, col de Glaise, bois de Chaudun, mont Síuse, Châteauroux près Embrun, la Traversette, de Fontgillarde au col Agnel, St-Véran, LLazanier, Certamussat, Larche, mont Vergons sur Annot. - Alp.-Nlar. : bois du Farghet, Alpes de Breil, l'Authion, Tende.

F. Involuenata All. - Piturages des montagnes. - B.-Alp.: mont Vergons sur Annot, col St-Hlichel, les Dourbes près Digne, Sisteron, Castellane. - B.-du-Rh. et Var : Ste-Victoire, Vérignon. - Alp.-Mar. : St-Etienne-Mlont à Saune-Morène, St-Mar- 
tin d'Entraunes, Utelle, Clans, Tende, Breil, Molinet, bois du Farghet et mont Mulacé au-dessus de Menton.

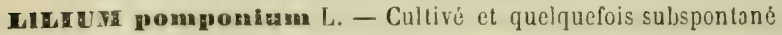
dans les B.-Alp. à Annot, Castellane; dans les Alp.-Mar.à Guillaume, St-Martin d'Entraunes, Grasse à St-Christoplic-desBois, St-Vallier, le Mas, Aiglun, Malaucène, Saorgio, l’Aggel, Grammont au-dessus de Menton.

C. pyrenalcuan Gouan. - Bois et bruyeres, Aude, Ariège et Pyr.Or.: Montagne Noire, cirques d'Artounant et des Aiguettes, col de Ligue, lac de Quérigut, Roi d'Escrouts, Mont-Louis, Bac de Bolquère, Balcera en Capcir, Carlite, Carença, Canigou. Pyr.-centr., Aveyron, Tarn.

¿. martagonum $L_{\text {. }}$ - - Bois des collines et des montagnes des parties septentrionale el moyenne du bassin; plus rare dans la partie mérid, où il occupe exclusivement les régions montagneuses, le mont Ventoux, montagne de St-Amans et de la Garde, la Ste-Baume, montagnes de Montrieux, de Grasse et des Alpes-Maritimes.

L. exoceun Chaix. - Lieux pierreux des montagnes. - Chaine jurassique française el helvétique: Mouthe à la Croix de la Bonde, revers nord du mont de Buttes, Neuveville, de Voens à Lignières, pente mérid. de la Roche de Château-Vieux près St Blaise. - Valais: Chemin-Neuf, Comba d'Arbaz, Simplon, Swischbergen, entre Casermetta et Gondo. - H.-Sav. et Sav. : Taninges, St-Sigismond, Micussy, Chamonix au Chauderon et au Mont, Conflans, Brides, Aigueblanche, Champagny, mont Nivolet au-dessus de Chambéry. - Is. et H.-Alp. : St-Eynard. la Terrasse, Gavet, Séchilicnne, la Grave, Briançon, la Vallouise, la Monta et l'Echalp en Queyras, lac Séguret près Embrun, Charance, Rabou, Chaudun et les Baux près Gap, mont Séuse. - B.-Alp. et Alp.-Mar. : la Condamine, Serennes, St-ÉtienneMont, Fntraunes et sommités des vallées du Var, de la Vesubia, Tinea, Roja.

4. canaldum L. - Cultivê et quelquefois subspontané sur les rocliers de Comboire près Seyssins (Is.); vignes de St-Antoinede-Galamus (Pyr.-Or.) ; Hyères, Toulon, le Luc, Mlouans, Grasse et Nice (Var et Alp.-Mar.). 


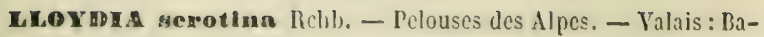
gnes, Giétroz, Evolena, Iiérémence, Nendaz, Zanrion, ZermonLana, Zermatt, Mattmark, Leukerbad, Simplon. - Sav. : col Iseran, Entre deux Eaux, St-Sorlin-d Arves, mont Cenis. Is, et H.-Alp. : la Pra de Belledonne, Champrousse, Allemont, Brandes en Oisans, Chantelouve, la Salette, Lautaret, vallon d'Arcine, mont Genervre au Pic Clienaillet, la Taillante, mont Queyrel en Champsaur, pie de Clıaillol. - B.-Alp. et Alp.-Har.: Longet de Maurin, lac d'Entrecoulpes, col de Fenestre, vallées de la Gordolasca et de Rabuons, Alpes de Tende, lac des Merveilles.

UROPETACON serotinum Gawl. - Rochers et rocailles calcaires. - Dr. et B.-du-Rh. : Nyons, Aucclon au Devez, entre Barbentane et Boulbon. - Gard et Ilêr. : envir. de Nîmes, Pontdu-Gard, de la Beaume à Collias, St-Chinian. - Aude et Pyr.Or. : envir. de Narbonne, Pech de l'Agnéle, cap de Pla, Quillanet, Cases-de-Pena, St-Antoine-de-Galamus, Força-Réal, Trencada d'Ambulla, dunes de Canet.

SCILLA maritima L. - Sables du littoral. - Var ct Alp.-Mar.: Sablettes de Toulon, Hyères, Monaco, Menton aux BaussiRossi.

s. autumnalis L. - Pelouses des terrains sablonneux et pierreux.

- La distribution géngraphique de cetle espece est fort irrégulière: quoique très-conmune sur les collines calcaires de l'Alsace, elle manque dans le N.-E. de la France, en Suisse, en Allemagne, en Bavière et dans les États autrichiens, exceptẻ sur le littoral, en Istrie et en Dalmatie. Elle existe en Belgique, en Angleterre et dans tous les pays de l'Europe méridionale.

s. Iygeinthoidea $I_{\text {. }}$ - - Clıamps pierreux. - B.-du-Rh., Var et Alp.-Mar. : envir. d'Aix, aux vallons de Valcros et de Buonouro, la Valette, Hyères, le Luc, Fréjus, Grasse, Cannes, Antibes, Levens, Nice, Menton.

S. nmoena L. - Lieux sablonneux des envir. de Toulon au Baou de Quatre-Heures — Sur la côte ligurienne à San-Remo.

S. itallea L. - Rochers, - H. et B.-Alp.: Antonaves près Ribiers, Sisteron à la Citadelle. - Var et Alp.-Mar.: Fréjus, Grasse, 
Foux de Mouans, le Bar, Gourdon, Nice, la Turbie, Gor'bio, Stc-Agnès, Castellar, Menton.

S. vernn Huds. - Pelouses de la région pyrénéenne. - driège et Pyr.-Or.: col des Ares, Quérigut, col d'Aspills, bois de France, le Grand-Pla, Jasse de Campeilles, mont de Madrès en Capcir, Mont-Louis. - Pyr. centrales.

S. squamosa Lam., S. liliolyacinthus L. - Bois des montagnes.

- Hér. : St-Amand de Mounis, Fraisse à Lignières. — Ariège et Pyr.-0r. : val de Paillères à Soucarrat, eirque des Aiguettes el d'Arlounant, pic de Canruse à la Cabane de la Hire, Balcera en Capcir, Prats de Mollo. - En dehors de nos limites dans les Pyr. centr., le Tarn, Aveyron, Cantal, mont Dore, monts Dômes, Creuse, Allier, Forez, l'ouest de la France.

s. Bifolia L. - Coteaux dans tout le bassin, excepté dans lés parties basses de la rẻgion méridionale dont il occupe exclusivement la région montagneuse.

DrNITHOGATON narbonenge L. - Bords des champs et des vignes de la région méridionale.

D. suifuream Schult. - Bois et bords des champs des terrains calcaires.

๑. nutans L. - Champs et prés. - Neuchatel, Vaud, Genève: château de Colombier, à l'entrée du bois de Reuse, Nyon, Montreux, Cursier, Ouchy, Rolle, Crans, - H.-Sav. et Sav. : Neydens près St-Julien, la Roche, St-Innocent près d'Aix. C.-d'Or et S.-et-Loire: envir. de Saulieu, Crottet près Mâcon, entre Romanèche ei Grèches. - Rh. et Is. : Ste-Foy-sur-Lyon, Villeurbanne, Charette et Amblérieu, Sassenage, Balmes de Fontaine. - Dr. et H.-Alp.: St-Vallier, Crest, Gap. - B.-duRh. Var et Alp.-Har. : envir. d'Aix, Fenouillet près Hyères, entre St-Dalmas et Fontan. - Gard : Nimes, Marguerite.

Ф. prollferum Jord. - Champs et vignes de la région mérid. Envir. d'Aix et de Marseille, région littorale des Alp.-Mar. Parties basses du Gard, de l'Hér. et des Pyr.-Or.

D'après M. Loret, les 0 . divergens Bor., 0 , proliferum Jord. et 0 . pater-familias Godr. sont des états divers de la même espéce suivant l'état physique du sol dans lequel elle crolt. 
D. umbellatuma L. - Clamps, tignes et prés dans tout le bassin.

- Espèce jolymorphe à laquelle se rapportent les formes dislinguées par MaI. Jordan et Fourreau, sous les noms de:

0. rigidulum, alpestre, propinquum à St-Véran (II.-Alp.o).

O. monticolum à Monestier-de-Briançon (II.-Alp.).

O. parviflorum, lardans, rusticum envir. de Lyon.

O. coespilicium à Cornod (Jura).

O. anguslifolium Bor. à Verdus de Privas (Ard.).

D. Centafoliura Guss. - Rorailles des coteaux et des montagnes. - H. et B.-Alp.: Lautaret, mont Genèrre, col de Glaise et Bayard, mont Séuse, Malemort, Soleil-Buou, Annot aux cols de Vergons el d'Allous, Grand-Couyer. - Vaucl. et B.-du-Rh.: mont Ventoux, vallons de Fabregoule près Septime, du Cascavẻou et de la Tour de Keirié près Aix. - Var: Seou-Blanc près Toulon, Plan du Pont près Hyères, Montrieux. - Ard. : Privas. - Hér. : Montpellier à la Colombière, Valmargues, St-Jean-de. Védas, St-Loup, Andabre-Rosis, Béziers, Magalas, le Cros, le Caylar, l'Espinouse à Lignières.

D. nrabicum L. - Coleaux du Var et des Alp.-Mar. : Toulon, Ile de Porquerolles, Cannes, Nice entre Ste-Hélène et Carras.

GAGIA tenopetnia Fries. - Champs pierreux. - Envir. de Genève, du chemin de Prégny au Grand Sacconex, entre Laconnex et Soral. - H. et B.-Alp.: mont Rognouse près Ribiers, Annot, St-André. - Vaucl.: mont Ventoux. - Alp.-Mlar.: Guillaumes, mont de l'Aiguille au-dessus de Menton. - Hér. : le Larzac au plateau de la Prade, le Cros.

C. Iutea Schult. - Prés et bords des bois dans les montagnes. Chaine des Vosges, Jura helvétique et français: Val de Travers et de St-Imier, bords du ruisseau de Neuveville à Lignières, Combe de Biaufond, Cul-des Prés, Belfort, Côtes du Doubs à Mauron, Grand-Combe, val de Fuans, Côtes du Dessoubre à Consolation, Pouillerel, Nozeroy, Andelot, pied de la Bôle, de la Dole, du Colombirr et du Reculet, Crêt de Chalam, Lompnes près Hauteville en Bugey, Charabotte, St-Rambert, Portes. Vaud et Valais: Fenalet, St-Tryphon, Roche, Château d'Oex, Gucuroz, montée du Brocard sous le Chemin-Neuf, Nayens de 
Sion, entre Brieg et Brigerberg. - Envir. de Geneve à Collonges, Etrambières, Veyrier, Soral, - H.-Sav. et Sav. : Brizon, Annecy, Alex, Domancy, pied du mont Jovet, Hermillon près St-Jean-de-Nlaurienne, mont Cenis. - Is. : Amblérieu près Crémieu, St-Julien-de-Raz, Jusson près Voreppe, GrandeChartreuse, Allevard. - H. et B.-Alp. : Mélezet de Guillestre, Manteyer, mont Rognouse près Ribiers, cols de Vergons et d'Allous près Annot. - Var et Alp.-Mlar.: Touris, Gonfaron, mont Cheiron, Caussols près Grasse. - Gard : l'Espérou, l'Aigual, St-Guiral - En dehors de nos limites, dans les Pyr. centr., la Lozère, Hte-Loire, Puy-de-Dôme, Vosges.

C. minima Schult. - Espèce des Alpes d'Autriche, Bohême, Moravie, Gallicie, Hongrie, Croatie, Styrie, Lombardie, Grisons et St-Gall ru'on a trouvée dans le canton de Vaud, à Solalex, les Frachis, col de Jaman. - Elle a une tige très-petite, portant une seule feuille radicale filiforme, une seule feuille florale spathiforme, des pédicelles glabres.

C. fistulosa Duby. - Prairies des Alpes el des Pyrénées. - Vaud et Valais : Alpes d'Ollon et de Bex, Anzendaz, les Mortais, Bagnes, St-Bernard, Cleuson, Thyon, Loze, la Zour, Gemmi, Galen, Guggerlü̈bel, Zermatt, Distelalp de Saas, Simplon. H.-Sav. et Sav. : Brizon, Vergy, Méri, cascade de la Sallanche à la montagne de St-Roch, Roc-d'Enfer, Nautau, mont Darbon près Vacheresse, Dents-d'Oche, Sixt, les Platets, la Tournette, Charamillon, col de Balme et col Joli, la Sauce, Cormet près Roselein, les Allues, mont Granier près Chambéry. - Is., Dr. et H.-Alp. : Bovinant, Grand-Som, la Pra de Belladonne, Piémeyan au mont de Lans, Lus-la-Croix-Haute, la Grave, Lautaret, Monestier-de-Briançon, l'Echauda, Grand-Vallon près du mont-Viso, la Taillante, col de Glaise, mont Sćuse. - B.-Alp.: Parpaillon, Malemort, Horonaye, cols de Vergons et d'Allous. - Alp.-Mar.: Entraunes, cols de Jallorgues et de Fenestre, Alpes de Tende, Mangiabou au-dessus de Sospel. - Pyr.-Or.: Canigou, vallée d'Eyne. - Pyr. centrales.

G. axvensis Schult. - Champs cultivés dans tout le bassin.

G. saxatuLs Koch. - Rochers siliceux. - Valais: granite de 
Branson, quartzites des envir. do Sion. - Is.: granite à l'enIrée du rallon de Levau, près Vienne, - Gard : cailloux quartzeux du bois de Campagne près Nîmes, gneiss des environs du Vigan. - Hér. : gneiss des envir. de St-Pons.

En dehors de nos limites, sur les quartzites et schistes siluriens de la Loire-infér. et de Haine-et-Loire, sur les granites el gneiss des Deux-Sivres et de l'Allier, sur les cailloux siliceux des envir. de Nemours (Seine-et-Marne); sur le basalte des envir. d'Issoire (Puy-de-Dome).

Espèce polymorphe dans laquelle on observe des modifications dans la forme de la capsule et des divisions du périanthe, dans la pubescence ou la glabrescence de la tige, dans le nombre des fleurs, variations d'après lesquelles on a prétendu élever au rang d'espèces les formes désignées par les auteurs sous les noms de G. bohemica, andegavensis, Fourracana. Ayant eu occasion d'examiner un grand nombre d'échantillons recueillis dans plusieurs localités, j'ai reconnu le peu de constance des caractères inroqués pour établir 'des distinctions spécifiques.

ALDT mativam L. - Cullivé et rarement subspontané autour des jardins.

A. scorodoprasum L. - Champs cullives. - Envir. de Genève et H.-Sav. : Compesières, Confignon, St-Julien. - H. et B.-Alp.: Manteyer, Aurouse, St-Léger en Champsaur, la Condamine, entre Jauziers et St-Flavy. - B.-du-Rh. el Alp.-Mar. : Aix près des bords de l'Arc, Montaiguet, St-Vallier. - Gard: AiguesMortes, Concoule, Manduel. - Ariège: Llaurenti, pic de Canrusc.

A. vineale L. - Vignes et champs dans tout le bassin.

A. porrum $e t$ ampeloprosum L. - Cullivẻs et rarement subspontanés autour des jardins.

A. Eolyanthum Roem. Schult. - Champs et vignes de la région mérid. - S'est montré accidentellement dans les envir. de Gap et de Grenoble.

A. rotundum L. - Champs ef vignes, - C.-d'Or: Semur, Montbard, St-Rẻmy. - Is., H. et B.-Alp. : Comboire, Huez, St-Paulles-Monestier, Briançon, Puy-Maure près Gap, Annot, col de la Madeleine. - B.-du-Rh., Var et Alp.-Mar.: çà et là dans les envir. d'Aix et de Marseille, Coudon près Toulon, Hyères, Cannes, Entraunes. - Gard et Hér. : Castillon près Rémoulins, 
St-Gilles, St-Martin-de-Londres, Pic St-Loup, Frouzet - Aude et Pyr.-0r. : Sidrière de Fitou, St-Paul-de-Fenouillet, St-Antoinede-Galamus, Vernet-les-Bains.

A. neatiforuan Lois. - Rocailles de la région mérid, - B.-duMh., Var et Alp.-Mar. : envir. de Marseille, le Grand-IIontredon aux Goudes et au cap Croisette, ile Pomègue, Toulon, Hyères, les Imbiers, île de Porquerolle, Fréjus, Cannes, îles Ste-Harguerite et St-Honorat, Antibes, Nice, Menton, remonte jusqu’ả l'Escarène et à Tende.

A. Sphatecephalum L. - Champs et lieux incultes des plaines et des montagnes, surtout sur les terrains calcaires. - Espèce polymorphe dans laquelle la longueur et la direction des pédicelles, la forme et la rouleur du périgone ordinairement purpurine, quelquefois blanche, la forme de l'ovaire, la présence ou l'absence de bulbilles autour du bulbe ont donné lieu à l'établissement de variétés appelées $A$. approximatum G.G., tenuiflorum Delastre, descendens L. Cette dernière existe en Provence autour de Fos, de Harseille, Toulon, Hyères, île de Porquerolle.

A. ascalonicum, cepreun ct fistulosam L. - Cultivés pour les usages culinaires.

A. sehoenopranum L. - Lieux humides. - Jura helvétique et français : rives de l'Orb sous le Brassus, val de Joux, tourbières de Belle-Fontaine et de la Chapelle, bords du lac Ste-Marie, Chapelle-des-Bois, Montbéliard, St-Laurent, pentes de la Dole. - C.-d'Or : de Gouville à Gevrey, Marsannay, Château-Renard. - Envir. de Genève à la pointe de Bellerive, Aïre, embouchure de la Versoix, Nyon, Chancy. - Iles de l'Ain sous Mollon, iles du Rhône en amont de Lyon. - Ariège : Llaurenti, Barbouillère, l'Estagnet, Valbonne.

La forme alpinum Koch, sibiricum Vill., foliosum Clarion a des tiges plus élevées et plus feuillues. - Vaud et Valais: Lavarraz, Bovonnaz, Zan, Grimsel, Eginna, Simplon. - II.-Sav. et Sav.: Bise, Roc-d'Enfer, Signal des Agneaux, Sixt, Platets, mont Blanc, Aiguilles-Rouges, valléc de Bérard, mont Joly, la Layat, Vergy, Méri, Hauteluce, la Sambuy, montagnes des Allues et de Belleville près Noutiers, mont Cenis. - Is., Dr, et 
II.-Alp.: la Pra de Belledonne, Brandes, la Moucherolle, la Salette, mont Glandasse, la Grave, Lautaret, Monestier-deBriançon, Péas en Queyras, mont Viso, col de Vars et Bayard, la Grangette, Cliaillol, Orcières, le Valgaudemar. - B.-Alp. et Alp.-Mar. : la Condamine, Lauzanier, Bachasse, col de la Made. leine, Entraunes, parties supér. des vallées du Var, de la Ves bia, Tinea, Roja.

A. chamacmoly L. - Sables des bords de la mor. - B.-du-Rh. ct Var: Montredon, plage de Fos, Salins d'Ilyères, cap des Issembres, St-Tropez, cap Lardier. - Pyr.-Or. : Argelès, Collioure. - IIér. : s'étend dans l'intérieur des terres sur les bords de la Mosson, entre le pont de Villeneuve et le moulin Geniès, au bois de Baillargues et dans les envir. de Béziers.

A. subhirsutura L. - Lieux secs le la Provence et des Alp.-Mar. : envir. d'Aix au Prégnon et Tholonct, Mourières près Toulon, Cannes, Grasse, Nice, Monaco, Menton.

A. triquetram L. - Bords des champs humides, - Var et Alp.Mar. : Toulon, le Lavandou, Chartreuse de la Verne, Bormes, les Mayons, St-Tropez à l'Aumède, Fréjus, Nice, Menton. - Pyr.Or. : Collioures, Banyuls-sur-Mer.

A. roseum L. - Champs, prés et vignes de la région mérid. Espèce polymorphe comprenant A, corbariense Timb. Lagr. trouvée dans les Corbieres et à.St-Antoine-de-Galamus (Pyr.-Or.); A. subalbidum J. F, trouvé à St-Tropez (Var).

A. nonpolitanum Cyrill. - Champs de la région mérid. Var et Alp.-Mar. : Toulon, IIyères, Cannes, Grasse, Nice, Monaco, Ilenton. - Aude et Pyr.-Or.: Narbonne, Ste-Lucie, Banyulssur-Mer.

A.- nimcuan L. Chomps de la région mérid. - B.-du-R., Var et Alp.Mar.: les Figons près Aix, Toulon, Cannes Grasse, Nice, Menton. - Ilér.: Candillargues.

A. moly L. - Cette espèce, dont l'existence en France avait été niée, a été trouvée par M. Gautier au mont Alaric prẻs Moux dans l'dude.

A. Iongipetiolntum, A. ursinum L. - Bois et taillis humides; dans la région méridionale on ne le voit que dans les montagnes. 
A. reticulntum, A. viclorialis L. Pelouses et rocailles des montagnes. - Hautes-Vosges jusque dans la H.-S. aux Ballons de Servance et de Giromagny. - Jura helvẻtique et français: Chasseron, Chasseral, Creux-du-Van, Mont-d'Or, la Dole, Reculet au vallon dArdran, vallon de la Brévine près RondeFontaine. - Vaud et Valais: le Grand Jabloz, Alpes d'Etivaz, la Hausseresse, les Verraux, Lavarraz, Barberine, Vallorsine, Catogne de Sembrancher, Glacier du Rhône. - H.-Sav. et Sav. : Dents d'Oche, Roc d'enfer, Nautau, la Flégère, Brévent, Vergy, Méri, Samoëns, Mieussy, vallée du Fier à Thuy, mont Dron près Thônes, la Tournelte, sommet de Joigny près Chambéry, mont Cenis. - Is. et H.-Alp. : Champronsse, col Oddie, Charmant Som, Grande Sure, la Salelte, Lautaret à l'Envers, la Madeleine. - Alp.-IIar.: mont Frontero. - Ard. et H.-L. : mont Mezenc. - Gard et Hér. : Prunaret près de Dourbie, Valat de la Dauphine près de l'Espérou, Fraisse. - Pyr.-Or. ct Ariège: Canigou, Mont-Louis, vallée d’Eyne, Madrès, lac de Rabassolès, montée du pic de Tarbézou, vallon de l'Estagnef. - En dehors de nos limites dans les Pyr.-Centr., Lozère, Aubrac, Margeride, Tarn, Cantal, mont Dore et Dômes, Forez, Pierre-sur-Haute de Coleigne à Porché, bois de la Madeleine, Montoncelle.

๑. oleraceum L. - Champs, vignes depuis les plaines jus¡u'aux montagnes. - Espèce polymorple comprenant notamment $A$ alpicolum J. F., forme montagnarde trouvẻe au mont Viso; $A$. complanatum Bor. ayant les étamines incluses comme dans l'A. oleraceum, et des feuilles semblables à celles du carinatum L.

A. carinntum L. - Champs et vignes. - Jura et Ain: Salins, Arbois, Lons-le-Saulnier, Bellegarde, Nantua, Parves, la Pape et lles du Rhône, Sathonay. - Vaud, Valais, envir. de Genève, d'Annecy, de Lyon à Yvour et dans les îles du Rhône, envir. de Grenoble à la Bastille, St-Ange, Allevard. - C.'dor: entre Dijon et Chagny, Saulieu, Rochc-en Brenil. - B.-duRh. : la Crau, les Alpines, entre Fonvielle et St-Remy. - Gard.

Espèce polymorphe qui s'élève assez haut dans les vallées 
montagneuses, à Villard d'Arèno et Roche-des-Arnauds (HI.Alp.); à la Condamine, Maurin, col de la Madeleine (B.-Alp.); enfin au mont Cenis,

La forme A. pulchellum Don, qui n'a pas de bulbilles dans l'ombelle, a été signalée dans le canton de Vaud, dans l'Ain et l'Isère à Aleximicu, Ambronay, Meyzieu, mont d'Annoisin près Crémicu; dans les Alp.-Mar. entre Levenș et la Tour, col de Brouis, Castillon, Ste-Agnès, col d'Eza.

La forme A. flaxifolium Jord. a les feuilles étalées et parfois contournées.

A. Aavum L. - Clampes pàturages de la région mérid. et moyenne. - Dr., II. et B.-Alp.: Die, Barnave, montagne de Bluye près le Buis, Rosans, Ribiers, la Maison-Ilu-Roi entre Guillestre et Château-Queyras, Mantejer à Combe Noire, Sisteron. - Vaucl. : pied du mont Ventoux à la Font de Canat, envir. d'Avignon à Caumont, Jaron près Apt. - Ard.: Rochemaure, Jastre. Gard et Iír.: Campestre près Alzon, Lanuéjols, le Larzac au Caylar, l'Escandorgue à Rorqueronde, Fougères. - En dehors de nos limites dans la Lozère et le Puy-de-Dòme, dans le Loir à Malesherbes et dans S.-et-MI. à Fontaincbleau.

A. pandeulatuma L. - Champs et vignes. - Bh., Is. et H.-Alp.: Gleyzé, la Tronche, Corenc, Meylan, Briançon, Molines, St-Véran. - B.-Alp. : la Condamine, de Meyronnes à Larche. Vaucl., B.-du-Rh., Var et Alp.-IIar. : Flassan, Aix le long de la Torse, Miramas, Toulon, le Luc, Fréjus, Antibes, Touel-de-Beuil. - Gard et Hér. : Nìmes, Manduel, Bessèges, St-Ambroix, Anduze, le Viran, Ceilhes, St-Maurice. - Aude et Pyr.-Or.: Sidrière de Filou, Perpignan.

La -variété pallens $L$., qui a des fleurs d'un blanc terne, a êté observéc à Ribiers, Annot, Toulon, Fréjus, golfe Jouan, Menton, dans les parties basses du Langucloc et du Roussillon.

A. strietum Schrad. - Prairies des montagnes, - Is. et H.-Alp. : la Salette au mont Chamoux, la Grave, Lautaret, vallée du Guil, mont Viso, liontgillarde.

A. nosehehana L. - liveailles et lieux arides de la réggion mérid.

- Dr.: Montjoyer, entre Montélimar et Orange. - II.-Alp. et Catal. Bassin du Rhône. 
Vaucl.: mont Rognouse près Ribier's, Flassan au pied du mont Ventoux, Montdevergues et bois de Fargues près Avignon, Carpentras. - B.-du-Rh. et Var: envir. d'Aix aux vallons du Cascavéou, de Lévèze, de Fongamate, N.-D. ile la Garde à Marseille, vallon de la Nerthe, Pas-des-Lanciers, mont Faron, Morière, Ampus, Cannet du Luc. - Gard et llér.: Villeneuve en face d'Avignon, Campestre, Pouzillac, Ilontpellier à la Lauze, Fontcaude, St-Jean-de-Védas, Maurin, Montferrier, Castries, Claret, St-Martin-de-Lundres, Colombière, St-Clinian, Capouladoux et jusqu'au Larzac. - Aude et Pyr.--0r. : envir. de Narbonne et de Perpignan.

A. nareissiflorum Vill. - Rochers et rocailles calcaires. Is., Dr. el II.-Alp. : St-Nizier, la Moucherolle, Grand Veymont, mont Aiguille, mont Glandasse, Séuse, Côte Gélive et col de Glaíse près Gap, Aurouse, le Noyer et Poligny en Champsaur, mont Morgon. - B.-Alp. et Alp.-NIar. : Vallonnet de Meyronnes, St-Ours, Roche-Grande aux sources du Var, val de Stropi audessus d'Entraunes, cols de la Maddalena et de Tende. - Vaucl.: mont Ventoux près de la source Fontfiliole.

A. fallax Dom, - Rochers des montagnes, - Jura helvétique et français: Tête de Rang, Chasseron, Creux du Van, Noirmont, Foncines, Ornans, Cótes de la Loue, Champagnole, Arbois, Salins, Haute-Pierre, Colombier, Reculet, Colombier du Bugey, Hauteville. - Vaud et Valais: Alpes de Château d'Oex, de St.Tryphon et d'Aigle, Jaman, Sion, Zermatt, Saas, Simplon. II.-Sav. et Sav. : montagnes du Chablais, Brizon, Grand-Bornant, 1a Tournette, mont Drizon et Trélod en Bauges, Nivolet, Arclusaz, Haut du Col, mont Cenis, Allée Blanche sur le versant italien du mont Blanc. - Is. et H.-Nlp.: St-Eynard, Belledonne vers le lac du Crouzet, Champrousse, Pariset, St-Nizier, St-Véran, Côte Gelive, cols de Glaise et de Chaudun, mont Séuse. - Alp.Mar.: Ste-Anne-de-Vinaï, Alpes de Guillaumes, montagnes de Grasse el de St-Auban. - Vaucl: Avignon, Carpentras. Ard.: Gourdon, Mayres, Aubenas. - Gard: le Vigan, Alzon, St-Guiral à Sumẻnes, St-Jean-du-Gard, Alais, Anduze, St-Ambroix. - Hẻr.: Grayssessac, St-Amand-de-Mounis, Pardailhan 
à Rodomouls. - Pyr.-Or. et Ariègo: vallée d'Eyne, Llaurenti au Soula de Joucarets, Boutadiol, Roc de l'Estagnet, ruines du château d'Usson. - L'A. fallax descend quelquefois dans les plaines, comme par exemple, dans la vallée de l'Ain à l'ouest d'Ambronay, dans la vallée du Rlıone en amont de Lyon. Espèce polymorplie dans laquelle les nervures des feuilles sont plus ou moins saillantes, les étamines tantot exsertes, tantot incluses.

A. acutangulum Schrad. - Forme du précédent, à étamines incluses et à feuilies plus fortement nerviées, qui habite les prés marécageux des bords du lhoone, de la Saône, de l'din et de l'Isère.

A. Sâeulum Ucria. - Var et Mlp.-Mar.: le Malpey près Frejjus, Agay, riviere de l'Esterel.

A. Pragrans Vent, Nothoscordon fiagrans Kunth. - Coteaux des des envir. d'Hyères, île de Porquerolles.

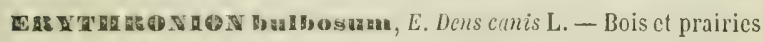
des colcaux et des montagnes. - Envir. de Genève, H.-Sav. et Ain: colline de Pinchat au bois de la Bâtic, bois des Frères, d'Onex et de Bernex au Nant de l'Agnon, Chancy, le Vuache, Vulbens, Vougy, Scionzier, St-Geoire, Ambéricu, la Croix du Reposoir près Belley, Ambléon, Evoges, T'enay, Chaley, mont d'Ain, bois d'Aromas et de Montdidier, Arintlıod. - Is., Dr. et II.-Alp. : la Balme et IIjères près Crémieu, Morestel, l'EchailIon près Vif, Vassieu en Vercors, le Dauphin près la Grave. Alp.-Nuar.: Grasse, l'Escaréne, Menton. - Gard et IIér.: Lánućjols, l'llort-de-Diou près de l'Espérou, l'Espinouse, le Caroux, la Salvetat, St-Pons. - Fyr.-0r. et Ariège: Canigou, Prats de Mollo, vallées d'Eyne et de Llo, Mont-Louis, col des Ares, Quérigut à la Jasse d'Espagne, Jasses de Campeille et de Bentaillole, lioc de l'Ermile. - Aude et Tarn : la Nontagne Noire.

- En dehors de nos limites dans les Pyr.-Centr., l'Aveyron, le Lot, la Lozère, Puy-de-Dôme, Creuse.

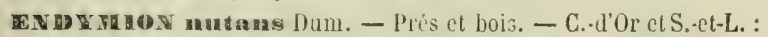
le Morvan, Saulieu dans les prẻs de Beauvais et de Turlin, Roussillon au pré do la Goutte, Petite-Verrière. - Valais: 
Martigny, Montorge, Sion à Talère. - Rh.: entre Ecully et Charbonniêres. Cette Liliacẻe est très irrégulièrement distribuée en France: outre les localités citées, on la trouve dans l'Ouest, le bassin parisien, la Champagne ct la Lozère:

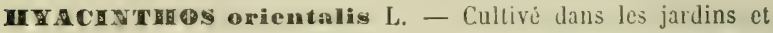
quelquefois subspontané dans la Provence et les Alp.-Mar. autour de Toulon, Fox-Amphoux, le Luc, le Bar, Mougins, Nice, Menton.

L'H. provincialis Jord., plus grêle dans toutes ses parties que que le type, a été observé au Luc, à Cannes et à Nice.

L'H. albulus Jord, ayant une grappe de fleurs blanchåtres moins lâche et des feuilles uressẻes, a élé cueilli à Grasse (Alp.-Mar.).

H. ant etaystinus L. - Suivant Companyo, celte espice des Pyrénées centrales se trouverait à St-Martin du Canigou, autour des forêts de Boucheville et des Fanges. Celte indication a besoin de contrôle.

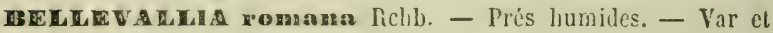
Alp.-Mar.: Sablettes de Toulon, la Garde, Hyères, St-Cassien près Cannes, Antibes, Levens, Menton. - Hér. : Cers et Portiragnes, Lattes. - Aude et Pyr.-0r.: Ste-Lucie, Salses, Argelès, St-Nazaire, Mas Larglade près Perpignan, - Tarn.

D. Ixifoliata Kunth. - Clıamps aux Pradets près Toulon.

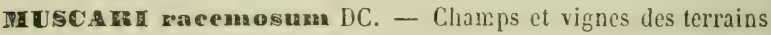
calcaires dans une grande partic du bassin. - Espèce polymorphe.

II. neglectum Guss. - Forme robuste du précídent, à grappes plus grosses, à feuilies plus largement canaliculées, à valves de la capsule peu ou point échancrées.

7. botryoideum DC. - Champs, prés et vignes. - Doubs, Jura et Ain : envir. de Montbéliard, Champagne d'Arbouans, Besançon, Dole, rives de l'Albarine près de St-Denis, Vaux. - Vaud et Valais: envir. de Payerne et de Lausanne, Lutry, Cully, Rolle, Sembrancher, Vollẻge. - II.-Sav., Sav., Is. ct II.-Alp. : Neydens, envir. de Chambéry et de Grenoble, mont Genèvre, Guillestre. - Vaucl., Var et Alp.-Jlar. - Ard. : Gourdon, le 
Mézayon. - Gard et Hêr. : Alzon, le Vigan, Anduze, Campestre, Nimes, derrière le Pic St-Loup, la Sérane. - Aude: Ferrals, Narbonne.

79. coneromasa Mill. - Di tous lis Muscari cést le plus commun dans les champs et vignes des terrains calcaires de notre bassin.

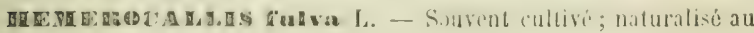
coleau Jourans près de Montbéliard (Doubs); dans Yaud et Valais entre Béricux et F̈renière, Ilauterille, Ridles, Martigny, les Fosses; dans les B.-du-Bh. et Varà Pouričres, Ste-Victoire, les Mayons pris le Luc.

H. flava L. - Sourent cultivé; naturalisé, comme le précédent, au coteau Jouvans; dans Vaud et Valisis a St-Tryphon, entre Honthey et Mnssongex, St-Georges, Montorge, sous le Sex de Valère.

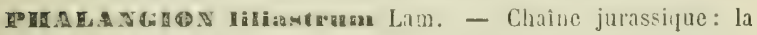
Dole, Colombier, Reculet au vallon d'Ardran. - Vaud et Valais: de Glion à Clamosalles, Dorchau, Ies Mérils, Outhannaz, rocher de la Dent près Château d'Oex, en Praz, Chainpé, Chemin-Neuf, Vex, Mayens de Sion, Gemmi, Brieg, Simplon, Zermalt, Mehlbaum sur Imfeli, Ausserbinn. - U.-Sav, et Sar.: Dents d'Oche, Roc d'Enfer, montagnes de Sixt, col de Balme, Chamonix aux Chauderons et Entre-les-Champs, la Layat, Vergy du côté du Reposoir, Glacière du Brizon, Mieussy, la Tournette du cóté de Serraval, les Avanchers, le Golet, mont Nivolet, mont Cenis. - Is. et II.-Alp. : les Banettes sur Voreppe, Charmant Som, Grand-Som, col de la Ruchère, Chamechaude, Champrousse, col Oddie au-dessus des Adrets, Moneslier-de-Clermont, la Silette, Lautaret, mont Genèvre à Clavières, col des IIayes, mont Viso, St-Véran à la Grangeasse, mont Bayard, Charance, col de Glaise, Chaudun. - B.-Alp. et Alp.-Mar.: Parpaillon, Serennes, Lauzanier, sources du Var, Estenc. - Vaucl. : St-Lambert, la Garde. - Ard. et H.-Loire: Mezenc. - Pyr. centrales.

PP. Niliacenus, Anthericon liliago L. - Cotcaux incultes, surtout des terrains calcaires.

P. ramosum Lam. - Coteaux incultes dans tout le bassin.

P. planifolium Pers., Simethis bicolor Kunth. - Lieux sablon- 
neux de la rẻgion mérid. - Var et Alp.-Mar.: Coudon, Iyères, Léoubes, Carqueiranne, Brẻgançon, entre Agay et Théoules, cap Croiselte près Cannes, golfe Jouan, Antibes. — Gard et Hér. : Grand'Combe près Alais, St-Pons. - Aude et Tarn : la Montagne-Noire. - En dehors de nos limites dans le Tarn, l'Aveyron, l'Ouest, la Vienne, l'Indre, Cher, Loir-et-Cher, Loiret.

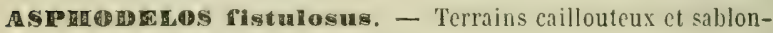
neux. - B.-du-Rh.: Narseille à N.-D. de la Garde, la Viste, la Crau. - Var et Alp.-IFar. : Toulon, Fréjus à l'embouchure de l'Argens, St-Raphaël, ile Ste-Marguerite, Nice, Villefranche, Monaco. - Gard et Hér. : les Saintes-Maries, Pinède d'AiguesMortes, Béziers, Cette. - Aude et Pyr.-Or. : Narbonne, Perpignan, Collioure.

A. miezocrnpus Viv. - Sables marit. - B.-du-Rh., Var et Alp.Mar.: iles Ratoneau et de Bandol, Fréjus, Agay, Auribeau, lle Ste-Marguerite. - Aude et Pyr.-Or.: de Narbonne à Collioure, Port-Vendres, Banyuls, vallon de Cerbère.

Les espèces suivantes appartiennent toutes à un groupe appelé tantôt $A$. ramosus, tantot $A$. albus. Laissant indécise la question fort controversable de savoir si ce sont de véritables espèces ou des variétés d'un type $A$. ramosus L., nous énumérons ci-après celles de ces formes qui ont été le mieux étudiées.

A. delphinensis G.G. - Prairies des hautes montagnes. - Sav. : Orizan sur Cléry, entre Bozel et le mont Jovet. - Is., Dr. et II.-Alp. : Alpe du mont de Lans, vallon au-dessus de la Chapelle en Valjouffrey, la Salette, Lus-la-Croix-Haute, Lautaret, les Bayards, col de Glaise, Séuse, Chabrières. - B.-Alp. et Alp.Mar.: Horonaye, Lauzanier, Soleil-Buou, Annot, Entraunes, col de Pouriac, vallons de Jallorgues près St-Dalmas, de Fenestre et de Salèse près St-Martin, la Trinité. - Vaucl. et B.-duRh.: mont Ventoux, Flassan à la Combe-de-Canau, envir. d'Apt et de Montredon, St-Loup, Ste-Victoire, Arbois, les Alpines, Graveson, Arles. - Aude et Pyr.-Or.: la Montagne-Noire, Canigou, Nohèdes.

A. cerasifer Gay, A. Villarsii Verlot, - Bois des montagnes calcaires. - Sav., Is. et H.-Alp. : Aime en Tarantaise, Comboire, 
mont Rachais, Chalais, Chamechaude, la Pinéa, Corenc, Charance près Gap. - Ard. : mont Charray. — Garrigues du Gard, de l'ilér., de l'Sude et des Pyr.-Or.

Les formes suivantes ont êté dẻcrites par MII. Jordan et Fourreau.

A. olbiensis, - IIyères, Porlcros A. grandiforus. - Toulon.

\section{(Var).}

A. Stachadensis. - Ile Porteros.

A. monticola. - Mont Séuso (H.Alp.).

1. fuscatus, - Mas-Cabardès (Aude).
A. procerus. - Roquefavour (B.du-kh.).

A. australis. - La Ciotat (B,-du. Ish.).

A. cminens. - Tour de la llassane (Pyr,-0r.).

A. collinus, - Clramagnieu (Is.).

A terrains calcaires de la région mérid. : remonte sur les coteaux calc. de la Drome et de l'Ardeche, à Châteauneuf, Nyons, Montélimar, Viviers, Rochemaure, Cruas, Baix, Alissas, Privas, Celles; des B. et H.-Alp. et de l'Isère à Sisteron, Clıarance, Tallard, la Saulce, Serres, Rosans, St-Ismier près Grenoble; dans la Savoie ct le Rhône au col du Frêne près Chambéry, envir. d'Aix-les-Bains, coteaux de Neyron et de Couzon près Lyon.

En dehors de nos limites sur les collines calcaires de la Lozere, Tarn, Aveyron, Gers, Pyr. centrales.

\section{SMILACÉES}

PARs emadrifolia L. - Bois frais des coteaux et des montagnes.

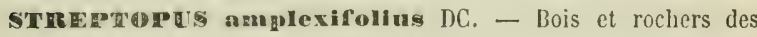
hautes montagnes. - Hautes-Vosges jusque dans la H.-Saône au ballon de Giromagny. - Jura helvétique et français: Chaseral, Vallanvron à la Chaux-d'Abel, Pouillerel entre le Locle ct les Planchettes, Châteluz, Combe de Lavaux à la Cornée et aux prés Rolliers, Combe, Biosse, la Dôle du côlé de la Rippe, Faucille à la Vattay. - Yaud et Valais: la Verda, Château- 
Chamois, Alpes de Château, Lavarraz, Boronnaz, l'Etroz, la Crotta, Champé, Mayens de Borernier, Simplon. - II.-Sav. et Sav. : Roc d'Enfer, Filloz, Blailière, Pointe des Agnesux, plateau des Gets, Glacière du Brizon, Clıamonix au Montanvert, les Contamines, lac de la Girotlaz, Crest-Voland, Monthion, col de la Louze entre Plan-Bérard et Grand-Naison, Tamié, mont du Vuache, mont Cenis. - Is. et H.-Alp. : entre Revel et le lac du Crouzct, Villard-de-Lans au bois des Touches, Charmant Som, la Charmette, Grande-Sure, Grande-Vache, lo Valgaudemar, bois Foran et de Château à Villevieille en Queyras. - Alp.-Mar.: St-Dalmas-le-Sauvage, Ste-Anne-de-Yinaï, bois du Boréon, col et mine de Tende. - Gard : l'Aigual, Valat de la Dauphine, bois de Longues-Feuilles près Concoule. - Pyr.Or. ct Ariège: Canigou, le Vernet, Bac de Bolquère, Llaurenti au Soula de Joucarets, pic de Canrusc, roc des Braguès de Boutadiol. - En dehors de nos limites rlans les Pyr.-centr., l'Aubrac, Lozère, Cantal, mont Dore, Picrre-sur-Haute.

COUVA ELAREA Holygonata L. - Bois dans tout le bassin, excepté dans les parties basses de la région méricl, oủ il occupe exclusivement les montagnes.

C. multidera $L_{\text {. }}$ - Dois des montagnes; moins répandu que le précédent dont il diffẻre par sa tige arrondie et ses étamines à filets poilus.

C. verticillata All. - Bois des montagnes.

C. maialis L. - Bois des coteaux et des montagnes.

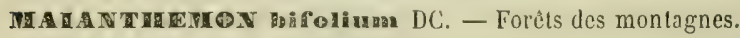

ASTRAGOS temuifolius Lam. - Lieux incultes. - Sav. : abimes de Nyans et mont Nivolet sur Chambéry, bois Champion près Moutiers. - Is., Dr. et H.-Alp. : mont Rachais, Comboire, Varces, Roche-Colombe près Clest, Rosans, Manteyer, Ribiers, Guillestre, Tallard, Séuse, Charance près Gap. - Vaucl. : base du mont Ventoux vers la Font de Canau, St-dmans, Carpentras. — Gard et Hér. : Salbous près Campestre, Alais, St-Ambroix, Chartreuse de Valbonne, le Larzac au Caylar. - Pyr.-Or. Lozère, Aveyron.

A. offecinalis L. - Cultivẻ et quelquefois subspontané. - Espęce polymorphe. 
A. seaber Brign. - Sables marit. - B.-du-Rh. : la Camargue et la Crau. - Gard ct Ilér.: Aigues-llortes, Palavas, Pérols, Maguclonne, Lattes à Maurin et à Gramenet.

A. aentifolias $\mathrm{L}_{\text {. }}$ - Haies, bnis et lieux pierreux de la rigion mérid. - Remonte dans la Drome à Nyons, St-Paul-Trois. Châteaux, Crest, Donzère, Montélimar ; dans les H. ct B.-Alp. : à Ribiers, Annot, Sisteron; dans l'Ardèche à Rochemaure, Viviers, Cruas, Baix, le Pouzin, Ruoms, Pont de Coux, la Voulte.

- En dehors de nos limiles dans le Tarn, l'Aveyron, le Lot et diverses autres parties du bassin de la Garonne.

nusces nealeatus $L_{\text {. }}$ - Ilaics et bois dans tout le bassin.

Et. Inypoglossua L. - Hyẻres autour du Château (Var).

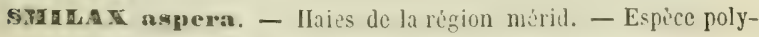
morphe dont les feuilles sont quelquefois très-larges et inermes. S. mauritanica Desf.. - Remonte dans la partie mérid. de la Dròme, à Nyons, Donzère, Chàteauneuf, Montélimar.

\section{DIOSCORIDEES}

my ATMOS (Tamus) connmunis L. (1) - Bois et buissons dans tout le bassin.

(1) Le nom de cet arbrisseau grimpant est un exemple des altérations qu'on a fait subir à certains mots grecs. Déjà les copistes qui nous ont transmis l'Histuire naturelle de Pline avaient commis une première faute d'ortho. graphe en écrivant Tamnus au lieu de Thamnus (0xpy05). Depuis Gesner, les botanistes ont écrit Tamus, probablement parce qu'ils ont cru que le Tamnus dont parle Pline dans le livre XXI, 50 de son Histoire naturelle est la mème plante que l'Uúa taminia dont les propriétés thérapeutiques sont indiquées dans les livres XXIII, 13, 14 et XXVI, 86 du mẻme ouvrage. Or, suivant Pline, l'Uva taminia est le Labrusca appelé par. les naturalistes grecs Ampclos agria (Vigne sauvage). Il est d'ailleurs généralement admis, et avec raison à mon avis, que le Tamus communis de Gesner et de Linné est l'Ampelos melaena (Vigne noire ou Bryone noire) assez bien décrit dans le livre IV, 182 de la Matière médicale de Dioscoride. Faute de description, il est impossible de savoir si notre Tamus est la même plante que Je Tamnus de Pline. Quoiqu'il en soit à cet égard, il y a lieu de croire que le naturaliste romain, dont l'ignorance en botanique est bien connue, aura pris pour un nom de plante le terme générique thamnos que les Grecs employaient pour désigner un arbrisseau quelconque. 


\section{IRIDÉES}

Cro COS rexnus $\Lambda 11$, - Pelouses des moningues, - Nul dans les Vosges. - Jura lielvétique et français: Chasseron, Clıasseral, Suchet, mont d'Or, Lomont, mont Terrible, Cotes du Doubs et du Dessoubre, Clos du Doubs, Franches-IIontagnes, Boujailles, Hautes-Joux, Chaux-de-Fonds, Pontarlier, Nozeroy, Nouthe, Champagnole, la Dôle, Crèt de Chalam, Reculet, Colombier du Bugey, Molard de Don, Ruffieu, monts d'Ain. - Vaud et Valais: Payerne, Yvonand, Moudon, St-Cierge, Peney, Villar's-Mendraz, entre la Malarliere et la Bourdonnette près Lausanne, la MaisonRouge près Burtigny, Choex, Massongex, la Combe, St-Pierre, Vex, Arvillard, Mayens de Sion, Thyon, Naters, Simplon. H.-Sav. et Sav.: Salève, Voirons, col des Aravis, plateau des Gets, Crest-Voland, montagnes de la Tarantaise et de la Mlaurienne. - Is. et H.-Alp. : Grande-Chartreuse, Chalais, mont Rachais, St-Nizicr, la Grave, le Brianconnais, le Queyras et le Gapençais. - Dr. et B.-Alp : montagnes di Vercors, du Diois, des vallées do l'Ubaye et du Verdon. - Alp.-Mar. : Entraunes, forêt de la Maïris, Tende. - Loire, Ard. et H.-Loire : le Pilat, St-Agrève, les Coirons, le Mezenc, l'Échelette, sources du Mézayon. - Gard: l'Espérou à Dourbie, Cerviliêre, Causse-Noire près de Lanuejols. - En dehors de nos limites dans les Pyrénées, Lozère, Aubrac, Cantal, H.-Loire, Puy-de-Dôme, Forez.

C. versicolor Gawl. - Licux arides des montagnes calcaires, surtout dans la région mẻrid. - Is. et H.-Alp. : Comboire près Seyssins, Embrun à Caleyères, Ribiers, Charance, col de Glaise. - B.-Alp., Var et Alp.-Mar. : montagne de Courchon sur St-André, mont Faron, Morières, Bagnols, Rians, le Luc, Draguignan, parties infér. des vallées du Var, de la Vésubia et de la Tinea. - Vaucl, et B.-du-Rh.: mont Ventoux, Flassan, le Luberon, Tour de Keirié près Aix, Vernus entre le Regage et Peypin.

C. medius Balbis. - Bois des Alp.-Mar. entre Gorbio et Roquebrune, mont Mulacé au-dessus de Menton, Tende. 
C. nudifozrus Sm. - I'rairies et pâturages. - Ilér. : St-Pons, Douch, Fraisse, la Salvetat. - Aude, Pyr.-Or. et Ariège : montagne Noire, les Corbières, le Llaurenti au Soula de Joucarets, Montmija, jasses de la Bentaillole, des Aiguettes, de Boutadiol et d'Artounant. - En dehors de nos limites dans les Pyr. centr., T'arn, Aveyron, Ger's, Landes.

C. sativus All. - Subsiontané autour de Brieg, de Naters (Valais), près d'Aime en Tarantaise où il a élé autrefois cultivế.

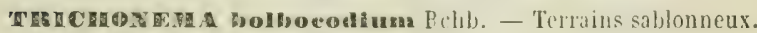

- Gard et Hér.: Graus d'Orgon, Aigues-llortes, Portiragnes, entre Palavas et Maguelonne, Pont de Villeneure. - B.-du-Rh., Var el Alp.-Ifar. : bords de l'étang de Berre, Toulon, le Luc, St-Tropez, Fréjus, Antibes, Nice, Menton. - Espèce polymorphe qui existe dans les Landes, la Gironde.

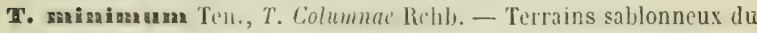
littoral méditerranéen. - Gard et Hér.: Aigues-liortes, Graus d'Orgon, Montpellier à la Gaillarde, la Planchude, Bione, les Aresquiers, Portiragnes, entre Villeneuve et St-Jean-de-Védas. - Pyr.-Or.: Salses, Leucate, Collioure, Port-Vendres. - Var et Alp.-Ilar.: coleau de Pipière à St-Nazaire, Giens, Hyères, Cannes, Antibes, Menton, au Casino de Carnolès. - En dehors de nos limites sur les coteaux maritimes du littoral ocẻanique, depuis la Charente infér. jusqu'à la Nanche.

Espèce polymorphe à laqquelle se rapportent les formes suivantes:

Trichonema ramiflorun Ten., subalbidum et flaveolum Jord., trouvés toutes les trois à St-Tropez.

IxIS pumina Yill., I. chamaciris Bertol, - Coteaux pierreux de la région mérid. - Vaucl., B.-du-Rh. et Var: Orange, Carpentras, Sérignan, de Flassan au bois de Honterrasse, env. d'Avignon à Montdevergues, d'Aix à Keirié et à Montaiguet, env. de Marseille, le Revest, Fréjus. - Gard et Hér.: envir. de Nimes au bois des Espèces, Calvas, Salbous, Villeneuve en face d'Avignon, plaines des arrond. de ilontpellier et de Béziers. - Aude et Pyr.-0r. : Trèbes, ile des Ouilloux, Sidrière de Fitou et de Leucate, Pech de l'Agnèle, envir. de Perpignan. 
I. Inquescus Lam., I. olbiensis Hénon. - Rochers et rocailles de la région mérid. - Var ct Alp.-Mar.: Hyères, le Revest, le Luc, les Maures, Eza. - Gard et Ilẻr. : le Mas de Seine près Nimes, la Beaume, Serre de Bouquet, Anduze, Valbonne, Liausson près de Clermont, Pégayrolles de l'Escalette. - Espèce polymorphe.

Ir. germaniea L, - Rochers et murailles. - C.-d'Or et S.-et-L. - Vaud et Valais : château d'Orbe, Belmont, St-Saphorin, StTryphon, Martigny, Fully, les Marques, Folatères, Montorge, Tourbillon, les Plâtrières, Naters. - H.-Sav. : rochers du Vuaclie prés de Cliaumont, Vulbens, Magériaz près Annecy. - Is: Grenoble à la Porte-de-France, Sasscnage, Seyssinet, la Terrasse, - D. : Divajeu près Crest, St-Paul-Trois-Châteaux, - Vaucl. et B:-du-Rh. : envir. d'Avignon, d'Apt, d'Aix et de Marseille. - Var et Alp.-Nar.: Toulon, Bagnols, le Muy, Rians, le Luc, Nice, Menton. - Gard et Hér. : Anduze, Alais, le Vigan, Montpellier. - Aude et Pyr.-Or.: les Corbières, St-Antoine-deGalamus, Opol, Castelnau, Thuir.

耳. parlida Lam. - Naturalisé autour d'Anglefort et de Culoz (Ain) oủ il a êté cultivé.

I. Morentina L. - Vieux murs, - B.-du-Rh., Var et Alp.-Mar. : Marseille à St-Jẻrôme, Toulon au cap Brun, Ilyères, Grasse, Nice, Monaco. - Hẻr. : les Onglous entre Agde et Cette.

I. Iutea Sam., I. pseudacorus L. - Fossés, marais et élangs dans tout le bassin.

I. Fotidigsinna L. - Bois et taillis. - Doubs et Jura:St.Vit, Busy, Liesle, Mouchard, Salins, Arbois, St-Amour, Planoise, Montferrand. - C.-d'Or et S.-et-L. : Antilly, Verrey-sous-Salmaise, IIontbard, Flavigny, vallée de Thénissey, Semur, Villars-Fontaine, Cluny. - Rh., Is, et II.-Alp. : Couzon près Lyon; Cessieu, entre St-Rambert et Anneyron, la Cote-St-André, la Grave. -B.-du-Rh., Var et Alp.-Mar.: Arles, la Camargue, Toulon, le Luc, le Canret, bords de la Brague près Antibes. - Gard et Hér.: Mas charlot aux bords du Vigan, la Foux près du Pontdu-Gard, envir. de Montpellier et de Béziers. - Aude: bois de Céleiran près Narbonne, bords du ruisseau de Guitard près Montolieu. 
E. maritima Lam., I. spuriu L. - Prairies du littoral. - B.-duRh. et Var: Berre, Arles, Fos-les-Martigues, Toulon au Fort Melbousquet, Ilyères. — Gard el Hér. : St-Gilles, Aigues-Mortes, Sylvéréal, Barjac, Mauruio à Saint-Marcel, Agde à l'embouchure de l'Hisrault et au cap Brescou, Vias, Nissan. - Aude et Pyr-Or, : Elne, St-Laurent de la Salanque, Perpignan.

耳. gromalnea L. - Prairies du littoral de l'Aude et des Pyr.-Or.: Sidricre de Lcucate, envir. de Perpignan.

耳. slbinciea L. - Bords du lac de Joux entro l'Abbaye et les Bioux.

I. xiphis Ehrh. - Prairies du littoral, - B.-du-Rh. : chemin de Pourières à Puyloubier, - Hér.: Portiragnes_ en face de Roquehaute.

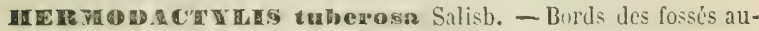
tour de Toulon, Hyères, le Luc, Fréjus, Grasse à St-Jacques, Nice au vallon de Magnan.

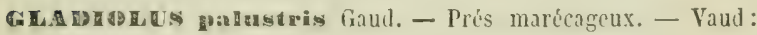
Vouvry, Roche. - H.-Sav, et Ain: bois de Cranves au pied des Voirons, marais de Pouilly près St-Genis, Chalex près du fort de l'Ecluse, bois de Peissy, Noville, Nantua au pied du mont à Colliard, Oyonnax.

C. BIny reas koch. - Cóteaux incultes. - Var: le Baou de QuatreHeures près Toulon, Hyères, Le Luc. - Hér.: St-Guilhem-leDésert, Viols. - Aude et Pyr.-Or.: moulin de Ripau près de Baixas, Port-Vendres.

G. conmanais L. - Champs et prés de la région mérid. - Espęce polymorphe comprenant un grand nombre de formes, au nombre desquelles se trouve le G. Lannianus Jord. trouvé par MI. Lannes à St-Tropez.

G. gegetalls Gawl. - Clsamps de la région méril. Remonte dans la Drôme et les II.-AIpes vers Crest et Gap; dans l'Is. à Corenc, Seyssins, Pariset; dans l'Ard. et la Loire à Roclsemaure, Vesscaux, Aubenas, Baix, St-Lager, Clomérac, Privas, St-Chamond; à Lyon derrière la bulte du Grand-Camp; dans la II.-Sav. à Neydens près St-Julien, Archamp. 


\section{AMARYLLIDÉES}

Aa a THes nivale L. - Prés et bois. - Belfort, Delle.- Vaud et Valais : Etabloz près Payerne, Aubonne, Treycovagnes, Mor. ges, Lausanne, Glérolles, Corseaux, Chernex, Rossinière à la Frasse, Bex, Fenalet, Monthey, Vourry. - Ain, Is., Sav. el H.Alp.: Trélex, Vouillant, Pragondran en Ilaurienne, CombeNoire de Manteyer. — Gard et Hér. : bois de Salbous près Campestre, St-ımand-de-IIounis, la Salvetat, le Jarzac à St-Pierrede la Fage, St-Michel, le Cros, le Pertus près Lodève. - Aude, Pyr.-0r. et Ariège: Montagne-Noire, Canigou, Bac de Bolquere ct Borde-Girvès près Mont-Louis, Mijanès, Artigues, Quérigut, Jasse Bédeillère, Carcanières. - En dehors de nos limites dans les Pyr. centr., Aveyron, Tarn, Lot, H.-Loire, Puy-de-Dòme, Allier.

UEDCOION verunun L. - Prés et bois, - Envir, de Belfort à Pérouse, Eloye dans la Vaivre. - H.-Saone: bois de Pusy, Nans, Rougemont, Chassey-les-IIontbozon, Champagney, Plancher-les-Mines. - Assez commun dans la chaine jurassique jusque dans le Bugey autour de Nantua, de Ruffieu, Belley, Tenay, St-Rambert, Drom en Revermont. - C.-d'Or et S.-et-L. : vallées de Marsannay, de Gevrey, de Ilessigny el de toute la Côte, Lugny, Charolles. - Vaud: pied du Jura près de Gingins et de Bonmont, le Jorat, Lausanne, la Tine, château d'0ex, chalets d'Allières, St-Loup. - H.-Sav.: Petit-Salève près du Pas de l'Echclle, château d'Etrembières, roûtes supérieures, vallée des Usses à la Caille, la Roche, plateau des Gets, Pontclıy, Brizon, Gyez, Cran près Annecy. - Is. : Chalais, Balmes de Fontaine, Pariset, Montcarrat à la Louvatiére, la Balme, Amblérieu, Vienne à Lévau, bois d'Esparon près du Monestier-de-Percy. - Dr. et H.-Alp. : Embel, Toulau, col Bayard, bois de St-Laurent-duCros et de Poligny en Champsaur. - Alp.-Mar. : Tende, St-Dalmas de Tende. - Rh. et Loire: Vaurenard, Ruthiange entre la Chambaz et la Chamboite, Pierre-sur-Haute.

L. aestivum L. - Prés humides de la ríg. mérid. - B.-du-Rh. 
et Var: Arles, Castignaux pris Toulon, ile de Porquerolles. Gard et Hér.: Candilhac, Milhaud, Manduel, Montpellier au Pré d'Arène, Lattes, Gramenet, St-Brès, St-Narcel, Lansargues, St-Jean-de-Védas, Fabrègues, Portiragnes, St-Martin-de-Londres. - Pyr.-Or. : Salses, Perpignan, Argeles.

4. Ifemale DC. L. nicaeense Ardoino. - Rochers entre Nice et Menton, Montalban, Villefranche, Eza, la Turbie, au-dessous de Roquebrune, Pont-St-Louis.

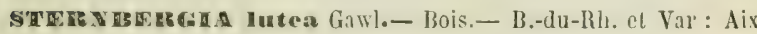
près de la maison Alexis, Six-Fours, Cogolin. - Gard : bois de Salbous près Campestre, Manduel.

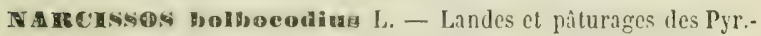
Or. : Port-Vendres, Prades, Villefranche. - Pyr. centr. jusque dans les Landes et la Gironde.

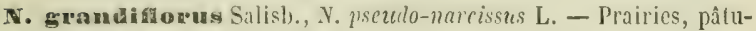
rages et bois des collines et des montagnes. - Chaîne des Vosges jusque dans la H.-Saône au bois de Frotey, Rougemont, Nans, Fallon, Chavanne, Luze, Couthenans, val d'Ajol. - Jura lielvélique et français: Sonnenberg, Chasseral, Pouillerel, JouxUu-Plane, Tête-de-Rang, Noirmont, Moron, Franches-Montagnes, coteaux jurassiques de l'arrond. de IIontbéliard et de Besançon, Salins, la Dôle, Reculet, Poisat, Brenod, Retord, Colombier du Bugey, Ambronay, St-Germain, Portes, forêt de Seillon. - Vaud et Valais: Payerne, Rougemont, Lausanne, Bex, descend sur les rives du Léman jusque vers Collonges, Belleville, val d'Illiez, Massongex, Sion, Viesch. - H.-Sav. et Sav. : Salève à la Pierre-aux-Fées, Thonon, Crans près Annecy, Serraval, Albens, mont de l'Épine. - Is. et Dr. : mont Rachais, Chalais, GrandeChartreuse, Corbelin, Vienne, St-Martin-en-Royans, Haut et Bas-Bouvante, Chironne près Die, - C.-d'Or et S.-et-L. : vallèe de Alessigny au-dessus de Fontaine de Jouvence, Combe de Gevrey, Nuits, Semur, Autun. - Rh. et Loire: mont Cindre, Violay, le Pilat. - Chaîne des Cévennes, du Gard et de l'Ilérault, l'Espérou, la Sérane, Lodève, les Plans, St-Gervais, Rosis, Ceilhes, le Larzac, St-Pons, le Saumail. - Aude, Pyr.-Or. et Ariège: Montagne-Noire, Canigou, Nohèdes, Mont-Louis, Mija- 
nès, Port de Paillères. - En dehors de nos limites, dans les Pyr, centr., Axeyron, Aubrac, Tarn, Lozère, Cantal, II.-Loire, mont Dore, Puy-de-Dôme, Creuse, Pierre-sur-Haute ct Noirétable (Loire).

Espèce polymorphe dans laquelle la grandeur et la couleur des fleurs présentent de nombreuses variations qui ont donné lieu à la distinction de $N$. major Curt., $N$. bicolor Lap., $N$. abscissus Roem. et Schult, N. Bernardi Hénon, - Ce dernier est une forme à couronne courte, à hampe anguleuse, que Grenier a considérée, sans motifs plausibles, comme une hybride des N. grandiflorus et poeticus. - Doubs et Ain : prairies de Ia Vrine près Pontarlier, le Poisat, la Bertroude, Combe de Lachaux, Retord, Colombier du Bugey.

N. incomparabilis Mill. - Prís et bois, - Belfort. - Ain: Pierre-Ghâtel, Poisat, côte de Cerdon, Bourg. - H.-Sav. et Sav. : St-Félix, Albertville, Gilly. - Rh. : St-Romain-au-llont-d'Or, Fleurie. - Vaucl., B.-du-Rh., Var et Alp.-Mar: Avignon, Tarascon, Aix, St-Giniez près Marseille, Toulon, le Luc, Grasse, Menton. - Gard et IIér. : Baraque de Jíchel près de l'Espérou, Doscare. - Aude : Carcassonne.

Il est probable que dans plusieurs des susdites localités le N. incomparabilis est échappé des jardins dans lesquels il a été cultivé.

N. poetieus L. - Prairics surtout dans les montagnes. - Jura helvétique et français: Creux du Van, la Tourne, Châteluz, Franches-llontagnes, Valanvron, val de Travers, la Brévine, Pontarlier, Levier, Boujailles, Russey, Guinots, Charquemont, Fuans, Consolation, Lomont, Reclere, Damrant, Pierrefontaine, Salins, Arbois, Prẻmanon, les Molunes, les Bouchoux, Noirmont, la Dóle, Mont d'Ain de Nantua, Poisat, Brenod, Colombier du Bugey, Evoges, Mazières près IIauteville, le Revermont. Vaud et Valais : Jorat, Frenières, val d'llliez, Sion. - II.-Sav. : Crevin, Collonges, Vangeron, les Bauges, Orizan sur Cléry, Puisgros, la Thuile. - Is. et Dr.: col de la Ruchère, Sappey, St-Nizier, Vaulnaveys, Crest. - II. et B.-Nlp. : la Grave, Ie Queyras, le Gapençais, la Condamine, Lauzanier, Serennes. - 
Montagnes de Vaucl. - B.du-Rh., Var ę Alp.-Nar. - C.-d’Or et S.-ct-L. : Nuits au bois de Laresse, Lugny, Léry, Baigneux, Valcombe, Cluny. - Rh. et Loire: Panissières, Violay, Pilat, St-Genest-Malifaux, Bard, Pierre-sur-IIaute. - Ard. : St-Ciergedu-Cheylard, Croix de Milet, forét de Mazan. - Gard et Hér. : assez répandu dans les prairies montagneuses des arrond. d'Alais et du Vigan, Lattes, St-Brès, Mauguio à St-Marcel, St-Bauzillede-Montmel, Sussargues, Castries, - Pyr. Or. el Ariège : Canigou, Mont-Louis, Evol, Nohèdes, Capcir, Mijanès, Noubals, Artigues, le Pla, St-Félix. - En dehors de nos limites, dans les Pyr. centr., l'Aveyron, Jes montagnes du plateau central.

Espèce polymorphe, quelquefois billore, dans laquelle la couleur du périgone et la forme des feuilles présentent plusieurs variations. Une forme appelée $N$. radiiflorus par Salisbury a une tige ancipitée et des feuillez étroites.

Le $N$. biflorus Curt. a des fleurs géminées dont les divisions périgonales sont d'un blanc jaunátre, la couronne d'un jaune foncé devenant blanchâtre sur le bord, la tige est comprimée, ancipitée. - Genève, Vaud, Valáis, H.-Sav, et Say.: Troinex, Evordes, Collonges, Compesières, Sierne, Nyon, sous le Sex de Valère, Marthod près Albertville. - Hẻr. : Hauguio, Gramenet. - Alp.-Jlar.: Grasse, Castellar, Menton.

v. juncifolias Req. - Garrigues de la rigion mérid. - Vaucl. et B.-du-Rh. : mont Ventoux, Caromb, envir. d'Apt à Plavignal, Mauragues, la Keirié et le Montaiguet près Aix. - Gard: Valbonne, Serre de Bouruet, Nimes au bois des Espèces, Campestre, Alais, Anduze. - Hér. : Iontpellier à Lavalette, Murviel, Villeneuve au Mas de Maigrel, N.-D, de Londres, St-Martin de Tréviers, St-Loup, Capouladoux, Castries, Restinclières, StGeniès-le-Bas, le Larzac à la Vacquerie et à St-llichel-des-Sers, Pardailhan. - Aude et Pyr.-Or. : Narbonne, les Corbières à Roquefort et liraisse, Trébies, St-Antoine-de-Galamus, entre Baixas et N.-D. de Pena, Villefranche, Prats de Mollo, Céret, les Albères, Collioure à Consolation et à Taillefer, Port-Vendres, Banyuls, vallon de Cerbère. - En dehors de nos limites, dans les Pyr. centr., le Lot et l'Aveyron. 
N. junquilıs L. - Cultivé et quelquefois subspontané.

N. ochroleneus Lois. - Champs autour de Toulon el de Grasse.

N. odorus L. - Prairies près de Toulon, de Grasse, d'Auribeau et de Menton.

N. ehrysanthus DC. - Prairies des envir. de Toulon, Grasse, le Bar. - San Remo sur la côte ligurienne. - Une forme à fleurs pâles N. subalbidus Lois. a été vue au Luc (Var).

N. aureus Lois. - Prairies du Var et des Alp.-Mar. : íle de Bandol, le Luc, Grasse, le Bar, Magagnosc, Nice, Menton.

N. niveas Lois. - Bords des champs du Var et des Alp.-Mar. : île de Bandol, Grasse, Nice, St-Hospice, Menton.

N. Iubius Gouan. - Rocher's et garrigues de la région mérid. Vaucl., B.-du-Rh. et Var: Avignon, Caromb, Sérignan, la Keirié et Montaiguet près Aix, envir. de Marseille et de Toulon au Baou de Quatre-Heures. - Gard et Hér. : la Foux au Pont-duGard, la Beaume, Lédenon, Villeneuve en face d'Avignon, les Angles, Montpellier à Bione, les Aresquiers, entre Fontfroide et Montferrier, le Trou-de-Miège, St-Jean-de-Védas, Frontignan, la Gardiole, St-Loup, Neflics, Castelnau-de-Guers, Béziers, les Capouladoux, les Cambrettes, St-Guilhem-le-Désert.

N. polyanthos Lois. - Bords des champs du Var et des Alp.-Mar. : Toulon, le Luc, Cannes.

N. tazettus L. - Prés de la région mérid. - B.-du-Rh., Var et Alp.-Mar. : envir. de Marseille, de Berre et de Toulon, presqu'île de Giens, illes de Porquerolle, de Bandol et de St-Honorat, le Luc, Frẻjus, Cannes, Grasse, Antibes, Nice, Menton. - Gard et Hér. : partie basse des arrond. de Nimes, d'Uzès, de Montpellier et de Béziers. - Aude et Pyr.-Or. : envir. de Narbonne, de Carcassonne, de Perpignan, de Port-Vendres et de Banyulssur-Mer.

Espèce polymorphe, sujette à varier quant à la couleur des fleurs, ainsi que dans les dimensions de la hampe: d'où les variêtés patulus Lois., orientalis Lois., intermedius Red., et la série suivante des IIermione décrits par MMI. Jordan et Fourreau.

N. micranthus, - Toulon. N. pratensis, - Montpellier. hololeucus. - Cannes, Grasse. monspcliensis. - Ibid. 
N. mediterraneus. - Antibes, Ba- N. modestus - Ibid.

nyuls.

discolor. - Antibes.

neglectus. - Ibid.

antipolensis. - Ibid.

liltoralis. - Ibid.

lobatus, - Ibid. discretus. - Ibid.

conlortus, - Ibid.

Ganymenoideus, - Ibid.

debilis. - Ibid.

chloroticus. - Ibid.

xantheus. - Ibid.

PAXCaATON maritimum L. - Sables maritimes. - B.-du-

Rh. : Montredon, Cap Croisette, fles de MaÏrẻ et de Riou, Fos, la Couronne, la Crau, la Camargue. - Var et Alp.-Mar. : TouIon, Brégançon, St-IIaxime, Fréjus, Cannes, ile Ste-IIarguerite, la Napoule, Vintimiglia sur la côte italienne. - Littoral du Gard et de l'Hér. - Aude et Pyr.-Or. : Montolieu de la Clape, Canet, St-Nazaire, St-Cyprien, Argelès, P'ort-Vendres, Banyulssur-Mer.

\section{ORCHIDÉES}

CAICEOLUS alternifolius, Cypripedilon calceolus L. - Bois des montagnes. - Jura helvétique et français : Chasseral, Creux du Van, Côte de Noiraigues, Val de Ruz, Fertuis, derrière Trémont, pied du Cliaumont à Sous-le-Mont, Poliez-le-Grand, audessus de Bierre et de Trélex, Lélex au-dessus des chalets Girod, entre Lochieu et Irvières. - Vaud et Valais: Roche-Cornet près St-Cierge, Boulens, Bercher, Roveréaz, Gourze, bois de Genet au-dessus de Fenalet, Château d'Oex, Vex, Val Reschy, Sembrancher, Vercorin, Mayens d'Hẻrẻmence. - H.-Sav. et Sav. : entre Collonges et Pommier, les Voirons, Brizon à Solaison, Beaumont, bois du Barioz près Pringy, Bluffy sur Talloires Serraval, Montmin près Faverges, les Bauges, Apremont près Chambe̊ry, bois Champion près Moutiers. - Is., Dr. et H.-Alp. : St-Ange à la prairie Dufour, Echevis près St-Jean-en-Royans, St-Aignan en Vercors, St-Dizier près de la Motte-Chalançon, Combe-Noire de Manteyer, Rabou à Ufernette, Rambaud près Gap, Boscodon près Embrun. - B.-Alp. et Alp.-Mar. : entre Serennes et Fouillouse, Tournoux, forêt de Clans, - C.-d'Or: bois des Thermes, dans la vallée de Voulaines, Val des Choues. 
SPIRANTIEOS aestivale Rich. - Prés humides. - Belfort à l'Arsot et au Salbert. - Jura et Ain: Vriange, forêt de la Serre, étang Genoud près Pont-de-Veyle, Dironne, le Plantay, lac de Bar près Belley, lac Bertrand, Château-Gaillard, Chazay. - Ge. nève et H.-Sav. : marais de Veyrier, de Bossey, entre Annemasse et Jussy, de Lossy, de Collonges, de Chessé près Thonon, la Roche, marais de Torex sous le mont de Sion. - Vaud et Valais: Fétigny près Payerne, Yverdon, Rances, St-Sulpice, Vevey, Morges, Coinsins, Gingins, Roche, St-Mlaurice. - G. d'Or et S.eetL.: Vielverge, Citeaux, Seurre, Nolay, Semur, - Rh. et Is. : Yvour, l’Argentière à la Mure, Décines, Meyzicu, Vaux-Milieu, Pont-de-Chérui, Morestel, Fontagnieu près Varces, Polygone de Grenoble, St-Jean-d'Hérans. - Vaucl., B.-du-Rh., Var et Alp.Mar. : Avignon, Bédoin, Piolenc, Marseille, Marignane, Hyères, le Luc, le Cannet, Grasse, Maures de Tanneron, golfe Jouan, Nice au Var. - Gard et Hẻr. : Alais, Anduze, le Vigan, Tresques, Aigues-Hortes, Palavas, St-Guilhem, Bédarieux, Lodève, la Salvetat.

s. autumnale Rich. - Prés. - Doubs, Jura et Ain : envir. de Montbéliard, de Besançon, Morteau, Salins, Arbois, Ferney, Mionnay, Ambronay, Meximieu, Jujurieu, :Leyriat, Châtillonsur-Chalaronne. - Vaud et Valais: Trélex, Vevey, Jorat, Agiez, Rance, Payerne, Branson. - Envir. de Genève à Veyrier, Aïre, Compesières, - H.-Sav. et Sav. : Feigères, Thoiry, - C.-d'Or: Citeaux, Seurre, Boncourt, Brazey. - Rh., Loire et Is. : Chaponost, Dardiliy, Lentilly, Chasselay, mont Verdun, Lidrgentière, Rive-de-Gier, Grand'Croix, Frontonas, Crémieu, Horestel, Polygone de Grenoble. - Dr. : Laveyron près St-Vallier, Loriol, - B.-du-Rh., Var et Alp.-Ilar. : envir. d'Aix, de Marseille, le Luc, Grasse, Nice, Mfenton. - Gard et Hẻr. : envir. de Nimes, d'Uzès, de Montpellier et de Béziers.

GDODHER a repens R. Br. - Bois des montagnes. - Jura helvétique et français : Délémont, Passwang, Weissenstein, Cluses de Moutiers, Franches-Montagnes, St-Imier, val de Ruz, Chasseral, Mont-d'0r, Ncuchâtel, Boudry, Valangin au-dessous de Mouthe, Colombier, Crêt de Chalam, Mijoux, la Faucille au- 
dessus de Vattay, Colombier du Bugey, Hauteville. - Vaud et Talais: Payerne, Moulon, Orbe, Chitel, Chateau d'Oex, Solalex, Lavernaz, Mayens de Sion, Champé, entre Bovernier el Sembrancher, pied du Simplon. - H.-Sav, et Sav.: Crevin, Archamp, bois du Vangeron, Brizon, Genollier, la Roche, Pringy, Chalets de Colone, Chamonix sous le Bois-Rond, entre Aillon ct Chatclard en Banges, hois Champion pris Moutiers, Tignes, de Modane à Bramans et à Lans-le-Bourg. - Is, et H.-Alp.: Grande-Chartreuse, col de la Ruchère, Perquilin, col de la Rochette, St-Nizier, gorges de Lans, Villette près Mens, Lèches près Luc-en-Diois, la Grave, Noyer en Clıampsaur, Durbon. B.-Alp. et Alp.-Mar. : Tournoux, Thorenc, forêts de Clans et de la Maïris. - Gard: St-Sauveur, Camprieux, Lanuéjols.

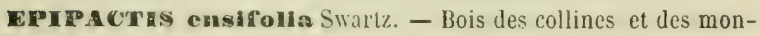
tagnes calcaires.

E. Inncifolin DC. - Même habitat.

E. Tubra All. - Bois des collines et des montagnes.

v. Iatifolin All. - Même habilat, mais plus commun que les trois précédents. - Espèce polymorphe ayant tantôl des fleurs verdâtres (E. viridiftora BI. Finge), tantôt des fleurs d'un pourpre foncé ( $\boldsymbol{E}$. atrorubens Hofim.). La largeur des feuilles est trèsvariable.

E. nicroplyylla Swartz. - Forme à feuilles plus courtes que les entrenœuds, à nervures lisses. - Vaud et H.-Sav.: Trélex au pied du Jura, vallée du Reposoir. - Rh. : mont Cindre, St-GenisLaval. - B.-du-Rh. : Montaud-les-Miramas. - \$lp.-Mar. : Nice, à Brancolar près Cimiez, Gairaut, vallon de Laghet, bois du Farghet. - Hẻr.: Montpellier à Châteaubon, Baillargues, Mauguio, la Sérane, St-Pons, St-Étienne-de-Mursan, Grayssessac, St-Geniès de Varensal.

E. palustrels Crantz. - Prés marécageux dans une grande partie du bassin.

CISTEE Avata Rob. Br. - Bois et prés des plaines et des montagnes.

L. cordata Rob. Br. - Bois des montagnes. - Hautes Vosges jusque dans la H.-Saone au Ballon de Giromagny. - Jura helvé- 
lique el français : Chasseral, Creux-du-Van, Franches-Montagnes, Moutiers, Pouillerel, Rizoux, Noirmont, Montendre, la Dolle, Faucille, Colombier, Reculet, Crêt de Chalam. - Vaud et Valais: Solalex, Mayens de Sion. - H.-Sav. et Sav. : les Voirons près du Couvent, Brizon, vallée du Reposoir au pied du Mẻri, Flumet, les Contamines, Clamonix, montée du Montanvert, Hauteluce, Roselein, N.-D. de Bellecombe, Crest-Voland, le Laitelet près Conflans. - Is. et II.-Alp. : entre Prémol et Champrousse, Chamechaude, Grande-Cliartreuse, Durbon. - Alp.Nar. : col de Fenestre, forêt de Clans. - Loire: Pilat aux sources du Furens, Grand-Bois entre Tarentaise et Prẻ Lager. - En dehors de nos limites, dans les Pyr. centr., Cantal, mont Dore, Forez à Noirétable et à Pierre-sur-Haute.

NEotma orobanchoiden, $N$. nidus avis Rich. - Parasite sur les racines des arbres dans les forêts des montagnes.

Emononov abortivum Swartz. - Coleaux pierreux, clairières des bois des terrains calcaires. - C.-d'Or et S.-et-L. : bas de Gouville, Marsannay, Concœur, Meursault, Vauchignon près Nolay, Cluny, Brancion, Cuiseaux. - Doubs el Jura : bois de Brégille près Besançon, Gilly près Arbois. - Vaud et Valais : coteaux du Léman, la Russille, Valeyres, St-Maurice, Folatères, les Marques, Clamoson, Ardon, Savièse, Lens, Sion, Sembrancher. - Genève, H.-Sav, et Sav. : bois des Frères, de Vernier et de Crevin, Collonges-sous-Monthoux, bois de Bon près Bonneville, la Roche, Mieussy, Allonzier, les Usses près la Caille, Thonon, Pringy, Colonies d'Annecy, Abimes de Myans près Chambe̊ry. - Ain et Rh. : envir, de Belley à Muzin et St-Germain, entre Béon et Talissieu, Néron, la Pape, Couzon, St-Romain, mont Cindre, mont Toux, Curis. - Is., Dr., H. et B.-Alp. : Crémieu, la Tronche, Pariset, Aucelon, Cresl, Nyons, Embrun, Montmort près Veynes, Malecombe, la Garde et Jarjayes près Gap, Annol. - Vaucl. et B.-du-Rh. : envir. d'Avignon et d'Apt, mont Ventoux, Luberon, envir. d'Aix et de Marseille. - Var et Alp.-Mar. : Toulon, Hyères, Montrieux, Rians, le Luc, StTropez, les Maures, Fréjus, Cannes, Nice, Menton. - Gard et Hér. : coteaux calcaires des arrond. de Nìmes, Uzès, Montpellier, 
Béziers. - Aude el Pyr.-Or.: cotraux calcaires des envir. de Narbonne, Fontfroide, Aussières, Villefranche, Céret, Collioure.

EProberox aphallum Suartz. - Bois des montagnes, - Jura helvétique et français : Chasseron, Cluses, de la Birse près Délẻmont, entre les Ponts et la Chaux-du-dlilieu, au-dessus de Nutrux, forêt d'Etroits près Ste-Croix, Gras près Morteau, la Planée, la laucille du coté de Mlijoux, montée de la Dôle du côté des Rousses, - Vaud: mont Aubert, Alpes de château d'Oex, d'Aigle et de Bex, bois de Saufaulaz, de Bovonnaz et de Lavernaz. - II.-Sav. et Sav. : Saxonnet au-dessus de Brizon, Chartrcuse du Reposoir, Maglan, Colone, la Frasse, au-dessus des Granges près IIauteluce. - Is, et H.-Alp. : Grande-Chartreuse, Chalais, bois de la Traverso à Corençon, Durbon, Bertaud et Lreux de l'Ours près Chraudun. - Alp.-IIar.: St-Martin d'Entraunes au bois de Sucisse, St-Dalmas-le-Sauvage, Entraigues. - Ard. : la Sapelte. - En dehors de nos limites dans les Vosges aux vallons du Frankenthal et de la Schlucht, Soulzbach, Ballon de Guebwiller.

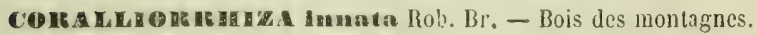
Jura helvêtique et français : Passwang, Weissenstein, Wasserfall, Délémont, Franches-montagnes, Chasseral, Pouillerel, Chaux-de-Fonds, Brévine, Creux-du-Van, Dent de Vaulion, Montendre, Russey et Guinots, Maiche, bois du Rizoux, de la Faucille, de la Dóle, d'Arvières et de Retord en Bugey. - Vaud et Valais : Solalex, Mayens de Sion, Simplon près de la galerie du Kaltwasser. - H.-Sav. et Sav.: Grand Piton du Saleve, trouvé autrefois aux Voirons où depuis longtemps on ne l'a pas vu, Brizon, Prarion de St-Gervais, montée du Pavillon de Bellevue, N.-D. de la Gorge, St-Laurent près Rumilly, mont Charvin du côté de Mranigod, Colone, au-dessous du lac de la Girotta près Hauteluce. - Is., Dr. et H.-Alp. : la Houcherolle, St-Nizier, Prémol, Revel, forêt de Portes, la Morte, forèt de la Penny pris Luz-la-Croix-Haute, bois de l'Infernet, Villard d'Arène, la Grave, Guillestre, Boscodon près Embrun, Poligny et le Noyer en Cbampsaur, Rabou et Loubet près Gap. - Alp.Mar. : forêt de Clans, Alpes de Tende, — Gard: vis-à-vis de 
St-Sauveur, Camprieux. - En dehors de nos limites, dans les Pyr. centr, l'Aubrac.

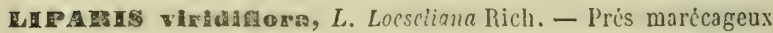
et tourbeux. - Jura : entre Sergenon et le bois de Rye, Mou do Pleurre près Chaussin. - Vaud, Valais et H.-S.: tourbières de Jongny, Maladeire, Sion, marais de Bossey et de Lossy au pied des Voirons, St-Laurent près la Roche, Brizon, Serraval, les Contamines. - Ain et Is. : Ste-Croix près Montluel, lac de Bar près Belley, Meyzieu près du Houlin de Cheyssin, le Grand. Lemps.

Le Malaxis paludosa Swartz, qui existe en Alsace, en Lorraine, sur quelques points du centre et de l'ouest de la France, n'a pas été trouvé dans le bassin du Rhône.

SEMAPLAS cordigerra L. - Bois sablonneux de la région mérid. - Var et Alp. Mar. : coteau de Pipière près St-Nazaire, lles du Levant, Lavandou, le Luc, l'Esterel, Cannes, ille Ste-Marguerite, la Roquette, Berre, Contes, Nenton. - Ard. : Aubenas, Vals. - Aude et Pyr. Or. : Montagne Noire, Saint-Antoine de Galamus, entre Oms et St-Harsal. - En dehors de nos limites dans les Pyr.-Centr., les envir. de Toulouse, l'ouest de la France.

5. longipetals Poll. - Bois. - Var et Alp.-llar.: les Pradets près Toulon, ille de Porquerolle, Vidauban, le Luc, Grasse, Nice, Menton. - Gard : bois de Cygnan près Nimes, Alais, Anduze, Barjac. - Pyr. Or. : St-Antoine de Galamus. - En dehors de nos limites dans le bassin de la Garonne.

s. Ilngua L. - Bois sablonneux de la région méditerranéenne. Var et Alp. Mar. : Salines Neuves d'Hyères, le Luc, Nice,! Monaco, Menton. - Gard et Hér. : le Vigan, St-Ambroix, Anduze, Alzon, Corconne, Montpellier à Lamoure, entre Mauguio et la Planchude, Portiragnes, St-Pons, St-Genis-de-Varensal, Castanet-le-Haut, St-Chinian, Pardailhan, la Salvetat, Fraisse. Pyr. 0r. : Collioures, Port-Vendres.

s. megleeta de Notaris. - Var : Toulon, le Luc, le Lavandou, la Garde-Freinet, St-Tropez.

S. oceultata Gay. - Coteaux incultes du Var à la Seyne, Hyères, Carqueiranne, Te Pradet, Lavandou, le Luc, St-Raphaël. 
ACER as arathagoplnozum R. Br. - Pelouses et coteaux calcaires dans tout le bassin.

A denaiforum Boiss. - Bords des bois. - B. du Rh. et Var : Marseille au fond du vallon de St-Tronc, Mazargues, vallons de l'Évêque et du Rouet, Hyères, lle de Porquerolle, le Luc, les Mayons, Chartreuse de la Verne, Frẹus. - Pyr. Or. : Perpignan, Argeles, Collioure.

A. Iongibracteatuan Rehb. - Bords des bois. - B. du Rh., Var et Alp. Mar. : Arles, Toulon, Hyères, la Valette, Gonfaron, Frẻjus, Nice, Menton. - IIér. : bois de la Gardiole près Mireval.

A. Heirelnum Lindl. - Coteaux et pelouses dans une grande partie du bassin:

A. pyramidale Rchb. - Coteaux inc:ıltes, pre̊s et bois.

Orchis papilionaceus L. - Pelouses. - Ain : la Pape, StMaurice de Gourdans. - Var et Alp.Mar.: le Luc, l'Esterel, Châteauneuf, Biot, Berre, Contes, Nice. - En dehors de nos limites autour de Toulouse et en Corse.

0. morio L. - Prés et bois dans tout le bassin. - Espéce polymorphe.

D. pletus Lois. - Forme du précédent à fleurs pIus petites, à éperon plus long et à feuilles mucronées. - Bois de Pins de la région mérid. - Vaucl., B. du Rh. et Var: le Luberon, env. d'Aix, de Marseille et de Toulon, Iyères, ille de Porquerolle, le Luc, St-Tropez, Fręjus. - Hẻr. : envir. de Montpellier, de Béziers et de Lodève. - Aude et Pyr. Or. : environs de Narbonne, de St-Cyprien et de Port-Vendres.

L'O. Champagneuxianus (Barnéoud), trouvẻ à Hyères et aux Cannebiers de St-Tropez, présente une échancrure au sommet de l'ovaire, et a ordinairement trois tubercules.

b. ustulatus L. - Prés et pelouses des coteaux et des montagnes.

b. coreosmus, 0 . coriophorus L. - Prés, bois et buissons. Doubs et Ain : envir. de Montbéliard, de Nantua, de Seyssel et de Meximieu. - Vaud et Valais : pied du Jura à Trélex, bassin du Léman, Orbe, Nyon, Payerne, Outre-Rhône, Collonges, Fully, Folatères, entre Clarat et Saxon. - Envir. de Genève et H.-Sav. : marais de Veyrier et de Troinex, Cranves, Lossy, la 
Roche, Arenthon, Albens, Passy. - Is, et H.-Alp. : Décines, Pontde-Beauvoisin, St-Mlartin d'Hicres, Lachaud prís Gaip, Manteyer, Roche-des-Arnauds. - B.-Alp. et Alp.-Mar. : Annot, Nice, Ienton. - Vaucl. et B.-du-Rh. : envir. d'Avignon, d'Orange et de Marseille. - C.-d'Or et S.-et-L. : Magny, Jouvence, mont Afrique, Liernais, Messigny, Chambolle, Charolles, Bourbon, Louhans. - Rh. : Clıarbonnières, Vaugneray, Mornant, l'Argentière. - Ard. : Ste-Harguerite-de-Vals, Cros du Roure près Privas. - Gard : Villeneuve en face d'Avignon, Montpezat, Chartreuse-de-Valbonne, le Vigan, Alais. - Hér. : St-Genièsde-Varensal, la Salvetat, Fraisse, l'Escandorgue, Pardailhan. - Pyr.-Or. : Vernet-les-Bains, Castell, Mont-Louis, - Espèce polymorphe dans laquelle les fleurs, habituellement à odeur de punaise, sont quelquefois inodores, ou à odeur agréable comme c'est le cas dans la forme 0 . fragrans Poll.

o. fragrans Poll. - Pâlurages secs. - Rh. et Ain : Vaux-en-Velin, la Pape, Balan, Meximieux-aux-Peupliers, Loyettes. - B.-du-Rh. et Var: envir. de Marseille, Sablettes de Toulon, Hyères, le Luc, Garde-Freinet. - Ard. et Gard : bords ilu Mízayon, AiguesMortes, bords de l'étang de Pujault. - Hér. : Montpellier à Grammont, Mauguio, Pérols, Maguelonne, Cette, Portiragnes, Pic St-Loup, Capouladoux, la Sérane, Montarnaud.

D. tridentatus Scop. - Bois et pâturages. - C.-d'Or : Is-sur-Tille, Santenay. - Ain, Rhône et Loire: la Pape, Caluire, entre Beaunant et St-Genis-Laval, Panissières. - Is. : Charvieux, Pariset à Beauregard. - Vaucl., B.-du-Rh., Var et Alp.-Mar. : bois de Sérignan, Ste-Victoire, Aups, les Mayons, Caussols audessus de Grasse, le Bar, Ferrion, Nice au mont Calvo, Menton. - Gard : bois de Cygnan, sur la route de Nìmes à St-Gilles. Espicce polymorplıe: la forme IIanryci Hénon se trouve au Luc, au Cannet, à Garde-Freinet.

D. slmius Lam. - Prés, pâturages et bois. - Doubs et Jura : Mont Brégille près Besançon, entre Menotey et Chevigny. - C.-d'Or, et S.-et-L. : Nuits, Dijon. - Envir. de Genève, Vaud et II.-Sav. : bois des Frères et de Peney, pied du Salève, collines du Léman, Nyon, Lossy, Cranves, la Roche, Annecy, Menthon. - Assez 
commun dans le Bugey, Mont d'Or lyonnais, envir. de Grenohle, do St-Vallier et de Crest. - II. et B.-Alp.: Guillestre, Gap, Annot. - Vaucl. et Alp.-Mar. : bois de Fargues près Avignon, Chateau-Double, Berre, vallon de L.ouda, la Tour, Utelle, Menton. - Gard: le Vigan, Chartreuse de Valbonne, Tresques, Boussargues, Pauzillıac. - Hér.: Ganges, Viols, Grabels à Font-d'Aurelle.

D. galestus Lam. - Bois et prairies dans une grande partie du bassin.

- Fuseus Jacq. - Bois et pelouses des terrains calcaires. - Envir. de Genève, collines du Léman, Trélex au pied du Jura vaudois, Martigny et Sion en Valais. - II.-Sav. : Salève, Annecy, Pringy, Allonzier, la Roche, Serraval, Thonon. - C.-d'Or et S.et-L. - Ain et Rh. : le Revermont, Belley, Parves, Pont-d'Ain, Meximieu, Trévoux, Ecully, Oullins, Francheville, mont Cindre, Couzon, Fontaines, Bully. - Is., Dr., H. et B.-Alp. : Bastille de Grenoble, Comboire, Pariset, Claix, St-Vallier, Die, Crest, la Garde près Gap; Manteyer, Annot. - Vaucl. et Alp.-Mar.: Avignon, Carpentras, Orange et Apt, Nice, Drap, Fontan, la Briga, Eza, cap Martin. - Gard: le Vigan, Alzon, Nìmes, Chartreuse de Valbonne, Tresques. - Ilér.: Montpellier à Château-d'Eau, Montferricr, Lodève. - Aude et Pyr.-Or. : Narbonne, Prades, Mont-Louis.

Espèce polymorphe qui, suivant M. Timbal-Lagrave, forme plusieurs hybrides avec les 0 . galeatus et simius.

o. globosus L. - Prairics et pâturages des montagnes. - Hautes Vosges jusque dans la Hautc-saône au Ballon de Giromagny. - Jura helvélique el français: Passwang, Weissenstein, Clıasseral, Pouillerel, Téte-de-Rang, Tourne, Chasseron, Châteluz, Mont d'Or, Larmont, Boujailles, Pontarlier, Poupet, Arinthod, Montendre, Dôle, Reculet, Mont-d'Ain, Poisat, Retord, Colombier du Bugey, Mazières, Golet de la Rochette. - Vaud et Valais : Bovonnaz, les Jeurs, Sublin, val d'Illiez, Loetschen, Ardon, Imfeld. - II.-Sav. et Sav, : Dents d'Oche, mont Pétetod, plateau des Gets, Vergy, le Mole, Ies Voirons, Salève, Parmelan, col des Aravis, Vergy, Semnoz, Tournette, Bluffy, Thorens, la Sambuy, 
mont du Chat, mont Cenis, - Is. et Dr. : col de la Ruchère, Charmant-Som, St-Ange, Ionesticr-de-Clermont, le Queyras, Glandasse, col de Menée, envir. de Gap. - B.-Alp. et Alp.Mar.: Lauzanier, Longet, Malemort, Parpaillon, Entraunes et toutes les sommités des vallées du Var, Vesubia, Tinea, Roja. - En dehors de nos limites, dans le Cantal et la Hte-Loire. Il est douteux qu'il se trouve dans les Pyrénées oủ il a été indiqué par Lapeyrouse et par les bolanistes qui ont copié cet auteur.

D. masealar L. - Prés et bois des plaines el des montagnes.

0. pallens L. - Prés et bois des montagnes. - Is. et H.-Alp. : St-Eynard, mont Rachais, St-Ange, St-Nizier, Monestier-deClermont, Rabou près Gap. - Sav. : Château de Melph près Moutiers, mont Cenis. - Var et Alp.-Mar. : Ste-Baume, Pignans, bois du Farghet, Mangiabou au dessus de Sospel, l'Authion, cols de Tende et de Tanarello.

D. provincialis Balb. - Forme du précédent à fleurs en épi plus allongé, à éperon plus long et à feuilles plus étroites et plus allongées. - Bois et prés. - Sav., Dr. et Is. : envir. de Chambẻry, Voreppe, mont de St-0urs pris Voiron, Bastille de Grenoble, Pariset, Crest. - B.-Alp., Var et Alp.-Ifar. : Annot, Sto-Baume, Fréjus, la Garde-Freinel, les Mayons, le Cannet, St-Tropez, Auribeau, l'Esterel, ile Ste-Marguerite, Berre, Contes, Nice, Menton. - Gard et Hér. : bords du Gardon au Moulin de la Beaume, Anduze, Lamalou, St-Geniès-de-Varensal, Bẻdarieux, Fos, St-Pons. - Pyr.-0r. : Collioure, Port-Vendres.

- palustris Jacq. - Prés marécageux dans tout le bassin. Espèce polymorphe.

o. laxiforus Lam. - Forme du précédent à bıactées plus courtes, à lobe moyen du labelle plus court que les latéraux.

o. alatus Fleury. - Trouvé par II. Gillot à Givry et Mouthier-enBresse (S.-et-L.). C'est sans motif plausible que Reuter a considéré cette espice comme une hybride des 0 . morio et laxiflorus.

- saceatus champagneux. - Collines schisteuses des environs d'Ilyères, Pierrefeu.

D. snubachus L. - Prairies des montagnes, - Chaîne du Jura 


\section{- 733 -}

mérid. : descente de la Dole à la Vasserode, Colombier, Poisat, Retord, Colombier du Buscy, Rumieu, l'ortes, Molard-de-Don. - Vaud et Valais: Alpes de Chateau-d'Oex, Branson, Folatères, Gucuroz, Cliemin sur Zobourlaye, Trient, Natersberg, Niederwald, Simplon. - H.-Sav. ct Sav. : Pitons du Salève, Reposoir, Roc d'Enfer, Dents d'Oche, Nautau, plateau des Gets, col des Aravis, Hauteluce, la Sambuy, mont Granier près Chambéry, mont Cenis. - Is. et H.-Alp.: St-Eynard, Charmant-Som, Prémol, mont Sénipe, col de Mence, Lautaret, col de Glaise, Devez de Rabou, Séuse. - B.-Alp. et Alp.-Mar. : Lauzanier, Horonaye, Parpaillon, Entraunes et toutes les sommités des vallées du Var, Vésubia, Tinea, Roja. - S.-et-L. et Rh.: Pruzilly, Chênas, Rochetachon, St-Bonnet-sur-Montmélas. - Loire : Pilat au Planit, vallée du Janon, le Bessat, St-Genest-Malifaux. - Ard. et Gard: Cuze, le Béage, l'Éspérou à Banahu, l’Aigual, Anduze. - Her. : Lauroux aux Sièges. - Aude et Ariège: la MontagneNoire, val de Paillères à la Mouillère de Soucarrat. — En dehors de nos limites, dans les Pyr. centr., Cantal, Aubrac, mont Dore, Puy-de-Dôme, Forez dans les chaines de Noirétable et de Pierresur-Haute. - Espece polymorphe dans laquelle les fleurs sont jaunes, rouges ou blanches.

D. Intifolius L. - Prés humides dans tout le bassin. - Espèce polymorphe.

- incarnatus L. - Forme du précédent à bractées et à feuilles plus longues, celles-ci non maculées.

D. maculatua L. - Prés et bois humides.

D. bifolius L. - Prés et bois des coteaux et montagnes calcaires. - Espèce polymorphe dans laquelle le nombre des feuilles varie de 2 à 3 ; les fleurs, le plus souvent odorantes, sont quelquefois inodores et de grandeur variable.

D. chloranthus Cust. - Forme du précédent à éperon plus renflé, à fleurs inodores, à loges des anthères divergentes. Plus rare que le type.

D. conongeras L. - Bois et prairies dans les parties septentr. et moyenne du bassin, ainsi que dans les montagnes des Alp.Mar., Cévennes du Gard et de l’Hêr., montagnes des Pyr.-Or. 
et de l'Ariège. - Espèce polymorphe dans laquelle la forme des épis floraux et des feuilles présente de nombreuses variations. Comme dans plusieurs autres Orchis, les fleurs sont tantôt odorantes, tantôt inodores.

o. odoratissimus L. - Forme du précédent à fleurs plus petites en épi grêle, à éperon plus court. - Jura helvétique et français : Paswang, Weissenstein, Cluses de Moutier, Creux du Van, Dôle, Faucille, Reculet, Mont de Nantua, Colombier du Bugey, Portes, Groslée. - Vaud et Valais : Gingins, Trélex, Burtigny, Vevey, Alpes de Château d'0ex et de Bex, Gueuroz, l'Etroz, Sublin, Drône, Arbaz, Vex, Sembrancher. - H.-Say. et Sav.: Salève au bas de la Grande Gorge sur Archamp, Vergy, Brizon, Pringy et Marcellaz près Annecy, St-Jorioz, Doussard, bois Champion près Houtiers, mont du Chat, les Bauges. - Is. et H.-Alp. : Grande-Chartreuse, Corenc, Claix, St-Nizier, le Queyras à Villevieille et Molines, mont Bayard près Gap. - Alp.Mar. : mont Tenibre au-dessus de St-Étienne, Clans, la Gordolasca. - C.-d'Or: vallée de Messigny et de Savigny, sources de la Seine. - Loire et Gard : Pilat, bois de l'Espérou et de l'Aigual. - Cette forme se montrequelquefois dans les plaines autour de Lyon, à Meyzieu, Reyrieu, sur les bords de l'Ain vers Château. Gaillard.

D. viridis Crantz. - Prés et pâturages des montagnes. - Les Hautes-Vosges jusque dans l'arrond. de Belfort et la H.-Saone à la Miotte, la Justice, Champigny, Chảlonvillars, Ternuay, Nans, Cravanche, Offemont. - Jura helvétique et français : Paswang, Monterrible, Chasseral, Franches-Montagnes, Larmont, Creuxdu-Van, Chasseron, Suchet, Boujailles, Noirmont, Dole, Reculet, le Bugey. - Vaud et Valais : Bovonnaz, Branson, Combe de Martigny et d'Arbaz, Mayens de Sion, Zermatt au Schwarzsee, Grimsel, Simplon. - H.-Sav. et Sav. : Salève, Voirons, Hole, Dents d'Oche, Abondance, col de Balme, Chamonix, sur les flancs du mont Blanc, col des Aravis, mont Cenis. - Is. et H.-Alp. : col de la Ruchère, Corenc, Champrousse, Sénèpe, Lautaret, le Briançonnais et le Queyras, Durbon, mont Bayard, Chaudun, Orcieres, - B.-Alp. et Alp.-Har., Parpaillon, la Ma- 
deleine, Entraunes, St-Etienne, St-Martin-Lantosca, Venanson, la Briga, Tende, la Maïris. - C.-d’Or et S:-et-L. ; envir. de Saulicu a Liernais, la Guette, Noiron-les-Citeaux, Combe-Noire, Val des Choues, Antully, Auxy, St-Émiland. - Rh., Iooire et Ard. : montagnes entre Lentilly ct Izeron, le Pilat, Gourdon, le Mezene. - Gard: Baraque de Michel près de l'Espérou. Aude, Pyr.-0r. et Ariége : Lapeyre près Quillan, Prats de Mollo, Coral, val de Pailleres, la Restanque, foret de la Limouse. - En dehors de nos limiles, dans les Pyr.-Centr., Aubrac, Mont-d'Or, Cantal, Puy-de-Dôme, Vosges.

D. albidus Scop. - Piturages des montagnes. - Hautes-Vosges jusque dans la I1.-Saone aux Ballons de Servance et de Giroma. gny, Planche-des-Belles-Filles, Belfahy. - Jura helvétique et français : Weissenstein, Chasseral, Tête-de-Rang, Chasseron, Châteluz, Suchet, Montendre, Mont-d'Or, Poupet, Boujailles, Dóle, Colombier-du-Bugey. - Vaud el Valais : Bovonnaz, Trient, Chemin, Mayens de Sion, Catogne de Martigny, Thion, Leukerbad, Simplon. - II.-Sav. et Sav.: Pitons du Salève, Voirons, Brizon, Vergy, Méri, Dents d'Oche, col des Aravis, mont Blanc au Couvercle, col du Cornet entre Chapieu et Roselein, mont Cenis. - Is. et H.-Alp. : Sept-Laux, Champrousse, St-Nizier, Col de l'Arc, mont Chamoux, Névache, Granon près Briançon, Cervières, Ristolas el Ségure en Queyras, la Chalp Ste-Agathe, Chaudun, Aurouse, Durbon. - B.-Alp. et Alp.-Har.: montagnes de l'Ubaye, St-Etienne, vallées de Caïros et de la Gordolasca, la Mairis, cols de Salèse et de Tende. - Loire et Ard. : Pilat, Longes, Mezenc. - Gard : Baraque de Michel à l'Espérou. - Pyr-Or. et Ariègè : Canigou, Pla Guilhem, Nohèdes, val de Paillères, vallon de la Mouré, Pla del Bosc, Paillariel, l'Estagnet, Riplaou. - En dehors de nos limites, dans les Pyr.-Centr., Aubrac, Cantal, mont Dore, Forez dans les chaines du Montoncelle et de Pierre-sur-Haute, Vosges.

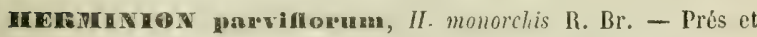
pâturages des montagnes. - Jura helvétique et français: Wasserfall, Monterrible, Chasseral, Creux-du-Van, Ferrière, Pont, Locle, Pontarlier, Cirque de Mauron, Grand'Combe des Bois, 
Barboux, Noël-Cerneux, Sussey, Charquemont, Malche, Consolation, Lomont, Roche, Blamont, Vandoncourt, Cotes du Dessoubre et de la Loue, entre les Rousses et Morey, Cise près Champagnole, Andelot près Salins, Lélex, le Vély près Hauteville en Bugey, Meyriat. - Envir. de Genève à Veyrier, bois des Frères et de la Bátie. - H.-Sav. et Sav. : les Voirons, Brizon, Pringy, St-Jorioz, Chamonix au-dessus du hameau des Nants, CrestVoland, Montmélian. - Is. : les Fauges de Villard-de-Lans, Corençon, St-Nizier, Pariset, la Terrasse. - H. et Bi-Alp. : Briançon au marais de Chamandrin, Val-des-Prés, col Bayard, Montmaur et la Palud près Gap, Manteyer, Durbon, Boussolières, Barcelonnette. - Alp.-Mar. : Chapelle de la Trinité près StMartin-Lantosque, Tende. - C.- d’or : Cussy-la-Colonne, Semur. - Pyrénées, Alsace, Lorraine, bassin de Paris.

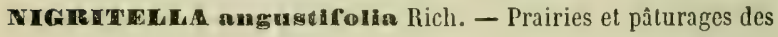
montagnes. - Nul dans les Vosges. - Jura helvétique et français : Paswang, Weissenstein, Chasseral, Tête-de-Rang, la Tourne, Pouillerel, Creux-du-Van, Châteluz, Chasseron, Dent de Vaulion, Mont d'Or, Suchet, Noirmont, Montendre, Dole, Colombier, Reculet, Poisat, Petord, Colombier-du-Bugey, Golet de la Rochette et le Vély près Hauteville. - Vaud et Valais: Anzendaz, Fully, Comba d'Arbaz, Pierre-à-Voir, Thyon, Zan, Zermatt, Aletsch, Simplon, Conches, Leukerbad. - H. Sav, et Sav. : Dents d'Oche, Flaine, Salève, les Voirons, le Môle, plateau des Gets, Sixt, col des Aravis, col de Balme, Brevent, Pavillon de Bellevue, col Joly, Charvin, Semnoz, Nivolet, les Bauges et la plupart des montagnes de la Tarantaise jusqu'à Tignes et au mont Iseran et de la Maurienne jusqu'au mont Cenis. - Is., Dr. et H.-Alp. : Grand Som, Chamechaude, Taillefer, Champrousse, St-Nizier, col de l'Arc, la Salette, Durbon, col de Menée, Lus-la-Croix-Haute, Glandasse, Saou, le Briançonnais, le Queyras et le Gapençais. - B.-Alp. et Alp.-Har.: Lauzanier, Malemort, Longet, Soleil-Buou, Entraunes et toutes les hautes montagnes des vallées du Var, Vesubia, Tinea, Roja. - Ard. et H.-L. : Mezenc. - Pyr.-Or. et Ariège: Canigou, Mont-Louis, Llaurenti, Boutadiol, Soucarrat, Port de Paillères. - En de- 
hors de nos limites dans les Pyr. centr., la H.-Loire au Mégal. - Espèce polymorphe.

N. suaveolens Koch. - Forme du précédent plus développée dans toutes ses parties; les divisions du tablier, l'éperon, et l'épi tloral sont plus allongés. Quelques botanistes ont prétendu sans preuves que c'est un hybride du $N$. angustifolia et de l'O. conopeus. - la Dole; la Moucherolle, Grande-Chartreuse; St-Bernard à la Pradaz entre la Baux et l'Ardifagoz; Vergy, Méri, Brizon, Petit Bornand, mont Blanc at Lèchaud, entre Mlégève et Hauteluce.

OPHIES amenifern lluds, - Pelouses, clairières des bois surtout dans les terrains calcaires. - Espèce polymorphe variant quant à la grandeur et à la couleur des fleurs: d'oủ les distinctions de 0. parviflora, grandiflora, ziridiflora, virescens, atrata, squalida.

D. Bertolonisana Moretti. -- B.-du-Rh., Var et Alp.-Mar. : Entressen; Toulon, Hyères, Grasse, Gourdon, la Roquette, St-Vallier, Antibes, Contes, Nice, Menton.

D. tenthredinifera Willd. - Espice de Corse trouvée près de Celte dans les terrains salés de Villeroi.

- Fueifera kchb. - Bois, pâturages dans une grande partie de notre bassin.

o. npifera Huds. - Même habitat.

D. Lombylialora Lin. - Espéce de Corse trouvée dans les Alp.Mar. dans les prairies des bords de la Brague près Antibes, vallée de Gorbio près Ifenton. - Sur la côte italienne à Castel d'Appio près de Vintimiglia.

- scolopax Cav. - Pâturages de la région mérid. - Var et Alp.Mar. : Toulon, lc Luc, le Cannet, Rians, Montrieux, Cannes, Ile Ste-Marguerite, Val di Blora, Contes, Berre, Nice. - Hér. : Montpellier au Mas de Rey, Lavérune, Caunelle, Prades, Murviel, Castries, Béziers, Ribaute. - Aude et Pyr. Or.: bords du ruisseau de Guitard près Montolieu, St-Antoine-de-Galamus, St-Paul, Caudiès. - En dehors de nos limites dans le Tarn et l'Aveyron.

0. muscifera Iluds. - Pelouses des terrains calcaires dans les 
parties septentr. et moyenne du bassin; moins répandu dans la région mérid., où il est signalé près de Toulon; - Fenestre, St-Martin, Tende, la Giandola, Breil (Alp.-Mar.); bois de l'Espérou et de Salbous (Gard).

D. hirsuta, O. speculum Link. - Espẹce dont le labelle est hérissé de longs poils roux, et qui a été trouvée près de Menton derrière Garavan (Alp.-Mar.).

D. Pusea Link. - Pelouses de la région mérid. - Vaucl., B.-duBh., Var et Alp.-Mar. : le Luberon, Aix à Montaiguet, ruines de Meyreuil, St-André, vallon des Gardes, envir. de Marseille et de Toulon, Hyères, Grasse, Vallauris, Drap, Contes, Nice, Menton. - Gard: Broussan près Nìmes, Pinède d'Aigues-Mortes, - En dehors de nos limites dans les Pyr. centr., Tarn et Aveyron.

D. Iutea Cav. - Pelouses, clairières des bois. - B.-du-Rh., Var et Alp.-Mlar.: St-Mitre près d'Istres, St-Cyr, Toulon, Hyères, Grasse, St-Hospice, Eza, Menton. - Gard et Hér. : Pinède d'Aigues-Mortes, Montpellier à la Colombière, Fontfroide, Fontcaude, Caunelle, Lavalette, Doscares, Pignan, Béziers. - En dehors de nos limites dans le bassin de la Garonne jusque dans les Landes, l'Ouest.

o. aIpina L. - Pâturages des Alpes. - Vaud et Valais : Panerossaz, Diez, Cotter, Zermatt au Hoernli, à Findelen et à Zmutt, Schwarzberg de Saas, Sirwolten, Binn, Simplon sous le Kaltwassergletscher et sur le Schienhorn. - H.-Sav. et Sav. : Brévent, col de Balme, Vergy, Méri au-dessus de Sommier-Dessus, la Gitaz, mont Drizon à la Sambuy, Laval de Tignes, col du mont Iseran, les Mlottets, la Gita près Beaufort, les Allues au Saut, mont Cenis. - H.-Alp. : col Malrif, de Fontgillarde au col Agnel, St-Véran à la Haufe-Planche de Clausis.

\section{HYDROCHARIDELS}

II X DTOCHA ERIS condifolla, $I I$. morsus ranae $L$. - Fossés et étangs. - H.-Saône, Doubs et Ain : Scey-sur-Saone, bords de l'Ognon, la Dombes, la Bresse, Thoissey, St-Didier, Pont-de- 
Veyle, Crottet, St-Jean-de-Lone, bords de la Seille. - Vaud : Yronand. - Rh. et Is. : Yvour, Villeurbanne, Vaux-en-Velin, Décines, Meyzieu, Morestel. - B.-du-Rh. et Var: Arles, entre la Garde el le Pradet près Toulon, Frểjus. - Gard et Hêr. : Nimes, Manducl, Bellegarde, St-Gilles, Beaucaire, Villeneuve à la Madeleine, Narsillargues, Mauguio à St-Marcel.

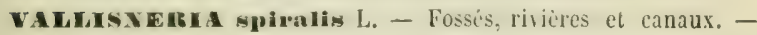
Vaucl. et B.-du-Rh.: Orange, Arles, la Camargue. - Gard, Hér. et Aude : bords du kiıone à Vallabrègue, canaux à Bellegarde, St-Gilles, Franqueveau, le Lez, canal de Lunel, Portiragnes, Lattes, Narbonne. - Depuis longtemps introduit à Lyon dans les fossés des fortifications, dans la Saône oủ il remonte jusqu'à Mâcon, canal de Pont-de-Vaux, canal de Bourgogne à St-Remy, Buffon, Rougemont, Montbard, Venarey.

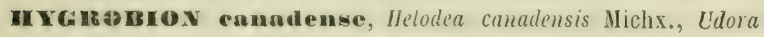
canadensis Nutt. - Espéce aquatique originaire de l'Amérique septentr. naturalisée autour de Lyon, de Grenoble, dans le canal de Bourgogne à St-Rémy, Buffon, Telars, entre Dijon et Plombières, dans le canal du Rhône au Rhin près de Montbéliard (Contejean), dans la Bresse louhanaise et le canal du Centre (Gillot).

\section{JUNCAGINÉES}

TREGLOCHN palustre L. - Lieux marécageux depuis les rivages de la mer à Aigues-Mortes, Mauguio et Pérols jusque dans les hautes vallées des Alpes.

T. Bnrrellerinnum Lois. - Marécages des bords de la Méditerranée et de l'Océan. - B.-du-R. el Var : Arles, Marignane, Castigneaux prè Toulon, Salins d'Hyères. - Gard et Hér.: Sylcéréal, Aigues-Mortes, Pérols, Vic, Maguelone. - Aude et Pyr.-0r. : Ste-Lucir, Leucate, Salses, St-Laurent.

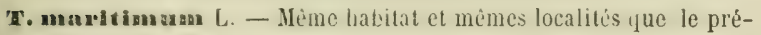
cédent. Existe en outre dans les marais salés de l'intérieur, dans la Lorraine, en Auvergne à St-Nectaire,

SCHFUCHzER palestris L. - Marais tourbeux des montagnes. - Hautes-Vosges jusque dans la H.-Saône à Frcsse, étang 
Bonjean. - Chaine jurassique dans les marais des Ponts, de Verrières, Planée, Brévine, Chataigne, Cachot, Vraconne, les Rousses. - Vaud et H.-Sav. : les Mosses, Chamonix au Bouchet. - C.-d'Or : étang Larmier et Fortier près Saulieu, l'Abergement-les-Seurre. - Is. : lac du Grand-Lemps, lac Luitel dans la forêt de Prémol. - Pyr.-Or.: lacs du plateau de Carlite, Pla de Bonas IIoras près de la Grande Bouillouse, suivant Companyo. - En dehors de nos limites dans les Pyr.-Centr., Aubrac, mont Dore, Pierre-sur-Haute à la Sagne de la Morte.

\section{POTAMEES}

POTAMOGTTON matans L. - Fossés, mares et étangs dans les plaines de tout le bassin.

P. Muitans Rotb. - Cours d'eau et étangs. - Envir. de Belfort à l'étang de la Forge. - Jura: Orain à Tassenières et Villers-Robert, la Dorme à Chaumergy, la Chassagne, Rye, le contrecanal à l'Abbaye-Damparis, Dammartin près St-Amour, l'Orain et le Doubs à Chaussin, Longwy. - Vaud et Valais, Ain, Rhône, C.-d'Or et S.-et-L. - Ard, et Gard: Vals, Banahu près de l'Espérou, Concoule.

P. polygonifoliug Pourr. - Etangs à fonds tourbeux. - I.Saône: Chaux, Sermamagny, Champagney, Lantenot, Mélisey. - C.-d'Or et S.-et-L. : envir. de Saulieu et d'Autun. - Loire : le Bessat. - Hér. : l'Espinouse, le Saumail, Cambon, Fraisse, la Salvetat.

P. rufeseens Schrad. - Eaux stagnantes. - Arrond. de Belfort el H.-Saône : plaine du Champ-de-Mars, Boursières, Buc, étang d'Arfin. - Doubs, Jura, Vaud et Valais : marais de Saone près Besançon, envir. de Dole, St-Cergues à la mare de la Givrine, l'Orbe près du Brassus, les Ormonds, lac inf. du mont Fully. H. -Sav. et Sav. : Lac Léman et du Bourget. - Is. et I.-Nlp. : Taillefer, lac du Volant à Brandes en Oisans, lac de Cervières près Briançon. - C.-d'Or et S.-et-L. : envir. de Saulieu à Nataloux et St-Léger, Brandon, Antully, Auxy. - Pyr.-0r. et Ariège : lac d'Aude en Capcir, lac de Quérigut. 
P. oramineus L., P. heterophyllus IDC. - Mares et etangs. Doubs et Jura, Vaud et Genive : marais de la Brévine, lacs de Joux et des Rousses, bords du Doubs entre Chaussin et Longwy, étang de St-Scine près Dole, marais de Thiclle et du Landeron, St-Sulpice, Morges, Nyon, bords du lac Léman entre Genthoix et Versoix, pointe de Bellerive, - C.ed'Or, S.eet-L. : envir. de Saulicu à Censerey, Citeaux, Brandon, Bresse chalonaise et louhanaise, étangs do Pontoux, de Mouthier et de Dissey.

Cette espèce, de mème qุu'un grand nombre d'autres plantes aquatiques, varie suivant qu'elle est submergée ou émergée: dans le premier cas les feuilles sont lancéolées-linéaires, dans le second cas, elles sont ovales.

P. plontagineus Ducros. - Mares et fossés. - Ain, envir. de Genève, Vaud: Divonne, Veyrier, Duilliers, Coinsins, Valeyres, Bossey, Roellebot. - Envir. de Lyon, de Grenoble, de Gap, Nice au Var, golfe Jouan.

P. Iucens L. - Ilares, ètangs el rivières. Espece polymorphe dans laquelle la forme des feuilles présente plusieurs variations.

$P$. decipiens Nolte. - Forme du précédent à feuilles presque sessiles, arrondies à la base. - Le Rhône près de Genève.

P. praelongus Wrulf. - Doubs et Vaud : lac d'Etalières près de la Brévine, lac de Breltaye.

Perfoliatus L. - Rivières, fossés dans unc grande partie du bassin.

F. crispus L. - Étangs, mares et rivières.

P. compressus L. - Fossís el étangs. - II.-Saône et envir. de Belfort à Hersuay, Eloye, Roppe, Bourogne. - Doubs et Jura : grand et petit lac d'Etalières près de la Brévine, lac des Rousses. - C.-d’Or. : Saulieu, Auxonne. - Is. : Décines, Meyzieu, Charvieu. - Var.

F. acutifolius Link, - Mares et fossés. - H.-Saône : Chagey, Buc, Mersuay. - Jura et Ain : mares des bords du Doubs et de la Loue au-dessous de Dole, la Bresse. - Is. : Charvieu.

P. obtusifolius M. K. - Hares et étangs, - Arrond. de Belfort, H.-Saône et Doubs : Eloye, Bourogne, Clıarmois, Sermamagny, 
Buc, Montferney, Chariez, Chassey-les-Nontbozon, Verne, lac d'Êtallières près de la Brevine. - C.-d'Or : Saulieu.

P. pusillus L. - Mares, étangs et fossés. - II.-S , Doubs et Jura : Boursières, Mersuny, Chagey, Couthenans, Montferney, Chasseyles-Montbozon, mares des bords du Doubs et de la Loue. S.-et-L. et Ain : Ecuelles, Thoissey, - Genève, Vaud, Valais, H.-Sav, et Ain : canal de Prangins, Divonne, marais au pied des Voirons, Bonneville, Vernayaz, Alpe de Fully, - - Vaucl. et B.-du-Rh. : envir. d'Avignon et d'Arles. - Ard., Gard et Hér. : la Bastide, le Vistre, Pont de Car près de N’mes, le Lez près de Sauret, Castelnau, Villeneuve à la Madeleine, Portiragnes.

P. trlchoideus Cham. et Schl. - Mares de la Bresse jurassienne à Balaiseaux, Pleurre. - Envir. de Saulieu (C.-d'Or).

P. pectinatus L. - Étangs et rivières dans les plaines et les montagnes.

P. marinus L. - Lacs des montagnes. - Les Rousses (Jura); - mont Cenis; - Villard d'Arène, Puits St-Guillaume près Embrun, Brunissard près du col des Hayes (H.-Alp.). - B.-Alp. et Alp.-IIar. : lac Ligny sur Colmars, col de la Maddalena.

P. densus L. - Fossés, marais et étangs. - Espèce polymorphe.

ZANYCIELCIA palustris L. - Nares, fossés et rivières. Espèce polymorphe variant suivant qu'elle est inondée ou exondée.

La forme tenuis Reut., qui a les carpelles et le style plus petits, se trouve sur les bords du Léman entre Genthod et Versoix.

Dans la forme pedicellata Fries, les carpelles sont plus longuement pédicellés et portent sur le dos une aile membraneuse dure, crénelée-dentée.

A CTumena filiformis Petit. - B.-du-Rh. : étangs de la Camargue et de Valcarès. - Gard el Hér. : Aigues-Mortes, élangs de Pérols, de Fréjorgues et des Onglous près Cette. 


\section{NAIADELS}

NAYAs mojor Roth. - Mares, étangs et riviires. - II.-Sañe, Doubs, Jura, Ain, C.-d'Or et S.-et-L. : la Saone, l'Ognon, le Doubs, la Loue et la Seille, étangs de la Bresse. - C Cà et là dans le Rhône, de Lyon à Arles. - La Drôme près Crest. - L'Hẻrault près Agde. - Is. : marais du Grand-Lemps et de St-Jeande-Bournay.

x, minor All. - Hares, ćtangs et rivières dans les mêmes régions que le précédent.

\section{ZOSTERREES}

Posmbonion oceanieum Delile. - Bas-fonds de la Méditerranéc sur le littoral de la Provence, des Alp.-Har. et du Languedoc.

CTIODOCE nodosa Ascherson. - Fond de la mer près de Toulon, Cannes, Antibes. - Maguelonne, entre Palavas et Carnon.

KUPRA moritima L. - Étangs, fossés, marais salés du littoral méditerranéen. Espèce polymorphe.

R. rostellata Koch. - Forme du précédent à pédoncules courts, non roulés en spirale, à fruits moins ovoïdes que dans le type et presque semi-lunaires. - Golfe Jouan (Alp.-Mar.), entre l'embouchure du Salaison et le Mas de Marot (Hér.).

Une forme brachypoda Gay, à podogynes courts, a été observée à Castignaux pı’ès Toulon.

ZOSTER marimus L. - Livagés de la Méditerranée el des élangs voisins. - Élang de Berre, Castignaux. - Aigues-Mortes, le Caylar. - Littoral de l'Iér., de l'Aude et des Pyr,-0r.

z. กณnus Roth.- Mêlé au précédent.

\section{LEMNACÉES}

LEMNA trisulen, minor, giblon, polyrohiza et nrrhiza L:

- Eaux stagnantes dans tout le bassin. 


\section{AROIDÉES}

A rov dracuncuinm L. - Var : Toulon, Fenouillet.

A. mreculatum L. - Haies et bois des plaines et des montagnes.

A. Itnlleum Mill. - Haies, lieux ombragés de la région mérid. Remonte dans l'Ard. vers Pont-d'Arc, Privas. - C.-d'Or : entre Montbard et St-Rémy.

A. nrisarum L. - Lieux ombragés de la Provence et des Alp.Mar. : Arles à la montagne de Cordes près Montmajour, la Ciotat au Bec-de-l'Aigle, Toulon, Hyères, lle Porquerolles, Fréjus, les Mayons, Cannes, Antibes, Nice, Menton. - Pyr.-Or. : Collioure, Paulille, Banyuls sur-Mler.

ACOIB as aromatieus, A. calamus L. - Envir. de Belfort entre Valdieu et Allanjoie, canal et rivière de St-Nicolas, étang de la Forge, la Douce, de Bavilliers à Bermont. - H.-Saône : Chagey, Frahier, Champey, Champagney, Mersuay, Linexert, Franchevelle, Bois-Derrière. - Doubs : Grand'Combe des Bois, Guinots, Bonnẻlage, St-Julien, Chenalotte, Montandon, Doubs à Vaufrey, Dessoubre à Rosureux, Fesches, Étupes, Brognard, Exincourt, Sochaux, Montbéliard, Courcelles, Luze, Couthenans, canal depuis Allanjoie jusyu'au Doubs, Morteau, les Gras, Pontarlier, Besançon. - Vaud et Valais : les Grangettes, Longirod, Morat, le Rhone sous Saillon, Vouvry, - Ain, Rh. et Is. : rives du Rhône sous Pougny, I.yon à la Tête-d'Or, Pont-de-Beauroisin, Grant-Lemps. - Planté dans la mare de Graminont près Montpellier. En dehors de nos limites dans l'dlsace, Lorraine, ouest.

L'Acore est originaire de l'Asie mineure et fut introduit par J. Bauhin en Alsace, en Lorraine et autour de Montbéliard.

\section{TYPHACÉES}

T Y PIIE Intifolia L. - Étangs, marais et fossés. - Espẻce polymorphe. Une varieté appelée Shuttlewortiana Koch et Sonder, a le stigmate plus court que le type. 
T. angustrolln L. - Héme habitat. - Expece polymorphe.

La variété lugdunensis Clıbert a les chatons presque contigus sur un axe glabre; se trouve dans les lles du Rhone en amont de Lyon.

T. minima Ifoppu. - Bords des cours d'eau. - Borls du Rlóne depuis l'embouchure de l'Arre jusque vers Avignon et Arles; bords de l'Arve vers Arenthon, Bonneville, Marignier; de I'Isère à Grenoble, Bourg dOisans; rives de la Dróme et de la Durance. - Espèce polymorphe. - La forme gracilis Jord., observée autour de Lyon et dans le Beaujolais, diffère du type par une plus grande longueur des feuilles, lesquelles dépassent la tige florale, et par sa floraison plus tardive.

SFA HEA TOX monosum Huds. - Fosses, itangs et marais dans les plaines de tout le bassin.

S simplex Huds. - Mares et fossi's des plaines et des montagnes; moins rêpandu que le précèdent.

5. nntans $L_{4}$, - Lacs et marais des montagnes. - Vaud et Valais : tourbières de Jongny et de Gourze, bois de Chêne près Genolier, bois de Bogis près Nyons, étang du Drezon près Soral, marais de Visp, Simplon. - H.-Sav. et Sav. : marais de Viry et de Lossy, Aresche, Chamonix au Bouchet, col Joly. - Is.: la Pra de Belledonne, lac Achard près Champrousse, Taillefer, Grandes-Rousses, - C.-d'Or : Saulieu, Roche-en-Brenil.

\$. minimum Fries. - Marais des plaines et des montagnes. Doubs et Jura : tourbières des Guinots, la Rivière, le Loquiat, le Landron, les Ponts, la Brévine, lac d'Étalières, Champvans près Dole. - C.-d'Or : Sauliea à Chambrun, mares de Pontaquin. - H.-Sav, et Sav. : marais de Viry, de Lossy et d'Allonzier, col du Cormet, Prarion de St-Gervais, les Glaisins près Annecy-le-Vieux. - Ain et Is. : marais de Charignin près Belley, envir. de Bourg, Vescours, Genas, Charvieu. - H.-Alp.: lac sur Névache au vallon des Thures, Orcières. - Pyr.-Or. et Ariége : Mont-I_ouis, lac de Carlite, Valbonne au Laquet du Roc de la Musique, las Cucquès. 


\section{JONCEEES}

JUNCUS conglomerntus $L_{\text {. }}$. - Fossés, lieux marécageux.

y. effusus $L_{\text {. }}$ - Meme habitat.

J. diffusms Hoppe. - Lieux humides; plus rare que les précédents.

- glaneus Ehrh. - Lieux humides des plaines et des montagnes. Espèce polymorphe.

अ. paniculatus Hoppe. - Forme du précédent à tige plus grèle, à fleurs d'un fauve pâle en panicule plus lâche et plus écartées ; c'est la forme la plus commune sur le littoral méditerranéen du Languedoc et du Roussillon.

ॠ. nreticus Willd. - Lieux humides des hautes vallées alpines. Valais : vallée de Saas entre Allmagel et le lac de Mattmark et de l'autre côté du lac au pied du Schwarzberg, Findelen près Zermatt. - Sur le versant italien du mont Rose et du mont Blanc au Breuil et dans l'Allẻe Blanche. - Sav. : bas du Mottet au-dessus d'Aime. - H.-Alp. : Lautaret, col de l'Echauda, col des Hayes à la Combe de l'Ourserette, Cervières, bords du Guil sous Ruines, la Taillante sur les bords du lac Foréon, col Agnel, St-Véran à Clausis et Roche-Ronde.

Ð. furonmis L. - Mares des hautes montagnes à sol siliceux. Hautes Vosges jusque dans la H. Saône aux Ballons de Servance et de Giromagny, Champagney, Fougerolles, Vauvillers, St-Germain. - Vaud et Valais: St-Bernard, Thyon, Almagel, Simplon. - H.-Sav. et Sav.: Brizon près des chalets de Solaison, chalets de Bise, Montanvert, col de Voza, Prarion de St-Gervais, col du Cornet au-dessus de Roselein, bords des lacs du mont Cenis. - Is. et H.--tlp: Belledonne sur les bords du lac du Crouzet, Prémol, Poursollet sous Taillefer, Sept-Laux, Alpe du mont de Lans, Lautaret, col des Rochilles sur Névache, Monestier-de-Briançon. - B.-Alp. et Alp.-Mar.: Lauzanier, bois du Boréon, lac d'Entrecoulpes, la Trinité près St-Ilartin, lac des Merveilles près Tende. - Loire, Ard. et Gard : Pilat, Mezenc, Gerbier-des-Joncs, Espérou, Lozère, Concoule. Ariège: lacs de Rabassolès et de l'Estagnet, Laquet de Valbonne. 
- En dehors de nos limites dans les Pyr. centr., Aubrac, Lozère, Cantal, mont Dore, Hte-Loire, Forez à Pierre-sur. Haute.

J. neutus L. - Rivages do la Miditerrance de l'ecean ; s'etend quelquefois dans l'intérieur lo long des cours d'eaux.

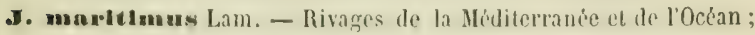
espèce polymorphe dans laquelle la forme de la panicule est tris-variable.

J. atrotus Lam., J. Jacquinianus L. - Lieux humides des Alpes, Vaud et Valais: Alpes de Morcles et de Fully, Salanfe, St-Bernard, Zermontana, Cleuson, Novelli de Nendaz, Diez, Arolla, Anniviers, Riffel, Saas, Belalp, Simplon, Nestlergrad, Eginnenthal, Glacier du Rhône. - H.-Sav. et Sav. : mont Blanc, Mer de Glace, Jardin, Chapeau, Couvercle, Aiguille à Bochard, Tré-la-Tête, Laval-de-Tignes, mont Iseran, Levanna à la Combe des Eivettes, les Allues, Valloires sous le Galibier, col de la Jouze, mont Cenis à Ronches et au lac Noir. - H.-Alp. : Péas en Qucyras, Ségure, Ruines, vers les sources du Guil, la Traversette, la Taillante, col Vieux, de Fontgillarde au col Agnel, St-Véran à Clausis et à Blanchette. - B.-Alp. et Alp.-Mar. : Longet de Maurin, val de Rabuons, mont Ténibre sur St-Etienne, Ste-Anne de Vinaĩ, lline de Tende.

J. Iriglumis L. - Lieux marécageux des Alpes. - Valais: Fully, Nendaz, Hérémence, Evolena, Anniviers, Reschy, Zermalı, Saas, Leukerbad, Belalp, Simplon, Glacier du Rhône. - H.-Sav. et Sav. : Chamonix au Chapeau et au Montanvert, Prarion de StGervais, col du Cormet, les Allues, les Ilottets, mont Cenis. Is, et H.-Alp. : Gleyzin, le Merdaret au-dessus de Theys, GrandesRousses, St-Christophe en Oisans, Lautaret, mont Genèvre, Val Préveyre, Péas, Ruines, sources du Guil, la Traversette, la Taillante, St-Véran à Clausis, Orcières. - B.-Alp. et Alp.-Mar. : lac de Ligny sur Colmars, mont Garret au-dessus d'Entraunes, Salsamorena au-dessus de St-Etienne, N.-D. de Fenestre, mont Bego, col de Raus. - Pyr.-0r.: Canigou, val d'Eyne, Carlite. - Pyr. centrales.

J. trifidus L. - Rocher's des Alpes et des Pyrénées. - Vaud el Valais: Alpes de Taveyanaz, Morcles, Fully, St-Bernard, Zer- 
montana, Thyon, mont Nuoble, Ferpecloz, Zmult, Mont-NIort, Saas, Sirwolten, Eginnenthal, Simplon. - S. et II.-Sav. : Buet, Brévent, mont Blanc jusqu'au Jardin et au Courercle, moraines de la Mer-de-Glace et de Tré-la-Tête, la Layat, le Bonhomme, la Sauce, mont Cormet, Combe des Eivettes au pied de la Levanna, mont Cenis et petit mont Cenis. - Is, et H.-Alp : Belledonne près du lac du Crouzet et à la Pra, Combe de la Lance, Sept-Laux, Gleyzin, Taillefer, Brandes en Oisans, la Bérarde, Lautaret, Alp.-Martin, Pelvoux, Chaillol, Orcières, mont Viso à la Traversette, la Taillante. - B.-Alp. et Alp.-Har. : la Reyssole, Lauzanier, Longet, Grand Couyer sur Annot, sources du Var et sommités des vallées de la Vesubia, Tinea, Roja. - Pyr.Or. et Ariège: Canigou, vallées d'Eyne et de Llo, Carlite, vallon de Boutadiol, Pic del Ginèvré, Pla de Monpudoux. - Pyr. centrales.

J. pygmaeus Thuill. - Bords des mares et des étangs. - C.-d'Or: Saulieu à St-Didier, Maison Baude. - Rh. et Ain : Lavore près Mornant, Scillon près Bourg, Marlieu, Chalamont, le Plantay, Beaumont, St-Germain. - B.-du-Rh. et Var : la Crau, la Camargue, Montmajour près Arles, Sablettes de Toulon, le Luc, les Maures, St-Raphaël. — Gard et Hér. : Nîmes, Manduel, Broussan, Jonquiẻres, Montpellier à Grammont, Cette, Portiragnes à Roquehaute. - Le centre et l'ouest de la France.

J. copitatus Weig. - Lieux sablonneux, humides. - G.-d'Or et S.-et-L.: Rouvray, Saulieu, Auxonne, Ornée près Autun, Issyl'Évêque. - Ain et Rh. : Chevroux près de Pont-de-Vaux, Brignais, bords du Garon vers le moulin de Barail, élangs de Lavore.

- Var et Alp.-Mar. : Hyères, les Maures, Cannes, golfe Jouan, IIenton. - Hér.: Montpellier à Grammont el à Lamoure, Roquehaute. - En dehors de nos limites dans Lozère, Tarn, Aveyron, Hte-Loire, Puy-de-Dôme, Allier, Forez, le centre et l'ouest de la France.

J. supinus Hoench. - Ruisseaux et mares des terrains siliceux et argileux. - H.-Saône: Ecromagny, Mansvillers, Ruz-la-Fonderie, Champagney, Grands-Bois, Linexert, Franchevelle, St-Remy, Senoncourt, Grattery. - Envir. de Belfort le long 
de la Savoureuse. - C.-d'Or et S.el-L.: Morvan autour de Saulieu et d'Autun. - Jura et Ain: forêts Je Chaux et de la Serre, le Vernay, la Bresse et la Dombes, envir. de Bourg, Pontde-Vaux, Hontribloud, les Echets. - Rh. et Loire: St-Bonnetle-Froid, Yzeron, les Jumeaux de Vaugneray, l'Argentière a Arjol, Pilat, Montbrison, Pierre-sur-Haute. - Is. : Cliambaran, Chabons, Eydoche, Flachere. - Gard: Peyremale, le Vigan, l'Espérou, Alzon, Concoule. - Ilér. : l'Espinouse, Fédou, le Saumail, Fraisse. - Aude et Tarn : la Montagne Noire. - En dehors de nos limites sur les terrains siliceux du Tarn, Aveyron, Puy-de-Dóme, Sologne, Limousin, Ouest.

Espèce polymorphe dans laquelle les tiges sont tantôt couchées et radicantes (J. uliginosus Roth), tantot allongées et flottantes (J. fuitans Lam.), quelquefois présentant des fascicules de de feuilles arec les capsules ou à leur place (J. viviparus). De meme que dans un grand nombre de plantes hygrophiles, les feuilles sont capillaires quand elles restent submergées (J. confervaceus).

J. Iamproearous Ehrh. - Lirux humides dans lout le bassin; espèce polymorphe.

J. Ingenarius Gay. - Lieux humides de la région mérid. - B du-Rh. et Var: bords de la Durance et de l'Arc, Toulon, la Seyne à Balaguier. - Gard et Hér.: Manduel, Pujault, Monfrain, Mauguio, Montpellier au Pré d'Arène, la Paillade, Béziers, Pézenas, Capestang, Lodève, Vias, Roujan, Lunel. - Aude et Py.-0r,: Narbonne, Ste-Lucie, Leucate.

J. Striatus Schoush), - Fossẻs de la région mérid. - B.-du-Rh., Var et Alp.-Mar. : Raphèle, Caoumé près Toulon, Nice au Var. - Hér. : Montpellier à Lamoure, Mauguio à St-Marcel, Pérols, Roquehaute, Courpoiran, St-Martin-de-Londres. - Aude et Pyr.-0r. : Narbonne, Ste-Lucie, Leucate.

J. neutiflorus Ehrh, - Lieux humides des marais.

J. anceps Laharpe. - Espice intermediaire entre le J. lamprocarpus auquel elle ressemble par les fruits et le J. acutiforus dont elle a le port. - Envir de Crémieu, Grenoble, Gap, Toulon, golfe Jouan. - Gard et Hêr. : St-Gilles, Bellegarde, Aigues- 
Mortes, étang de Pujault, Mireval, Pérols, Palavas, Courpoiran, St-Marcel.

J. alpinus Vili. - Lieux marécageux des montagnes. - Doubs et Jura : Bélieu, Russey, Guinots, St-Julien, Pontarlier, la Dôle, Colombier, Faucille, Reculet. - Commun dans les montagnes de Vaud, Valais, Savoie, Dauphiné, B.-Alp. et Alp.-Mar. - Ard., H.-L. et Gard: le Mezenc, l'Espérou, la Grandès, la Lozère à Concoule. - Pyr.-0r. : Canigou, Pla Guilhem, vallée d'Eyne, Mont-Louis, Carlite, Llaurenti. - En dehors de nos limites dans les Pyr. centr., Aubrac, Lozère, Cantal, Mont Dore, H.-Loire, Forez à Pierre-sur-Haute. Le Jonc des Alpes est souvent entraîné jusque dans les plaines. C'est ainsi qu'on l'a vu autour de Genève, de Lyon, de Grenoble, dans la plaine du Rhin en Alsace.

J. obrusiloras Ehrh. - Lieux marécageux dans tout le bassin.

J. cquarrosus L. - Lieux marécageux et tourbeux des montagnes à sol siliceux, - Nul dans la chaine jurassique. - Hautes Vosges jusque dans la $\mathbf{H}$. Saone aux Ballons de Giromagny et de Servance, mont de Ternuay, Château-Lambert, sources de la Lanterne, vallée du Rahin aux Arachies, Fresse, Annegray, Ecromagny, descend sur les terrains de transport et les grès bigarrés à Franchevelle, Linexert, St-Germain, Tourbière de la Pile; s’êtend sur la lisière vosgienne dans les envir. de Belfort. - C.-d'Or et S.-et-Loire autour de Saulieu, St-Léger, Roche-en-Brenil, Semur, Morvan autunois, Montjeu, S.-Georges. - Chaînes beaujolaise et lyonnaise, Chansailles, St-Bonnet-leFroid, les Jumeáux de Vaugneray, l'Argentière à l'Arjol. - Pilat, montagnes de l'Ardèche à St-Agrève, le Mezenc, Gerbierdes-Joncs. - Chaîne de l'Espẻrou à Concoule, l'Espinouse, Cévennes de l'Hérault à Saut de Vésolles, Mons, Fraisse, la Salvetat. - Montagne-Noire. - Mont-Louis, lacs du Llaurenti et de Rabassolès, mouillères de Riplaou, de Cesseilla de la Restanque et d'Al Touch dans l'Ariège. - En dehors de nos limites sur les terrains siliceux de Tarn, Aubrac, Cantal, mont Dore, Puy-de-Dome, Forez, contreforts du Morvan dans l'Yonne et la Nièvre, Creuse, Cher, Loir-et-Cher, Loire, Maine-et-Loire, l'ouest. 
Deux faits principaux caractérisent la distribution géographique du J. squarrosus: c'est d'abord son alsence sur les terrains calcaires pour lesquels il a une répulsion manileste ; c'est ensuite sa localisation toute particulière. En eftet du massif central de la France où il est à son maximum do fréquence, il s'étend d'un cûté dans l'Ouest, d'un autre cóté vers le sud de la France jusqu'à la Montagne-Noire et au Llaurenti, enfin du cóté de l'Est dans les Cévennes, le Pilat, Forez, Morvan jusqu'aux Vosges et au Schwarzwald, sans pénétrer dans les montagnes jurassiques, alpines et subalpines de la France, de la Suisse et du Piémont, excepté sur les grès verts entre Villard-de-Lans et Corençon (Isère).

Il est probable que les indications données par quelques botanistes relativement à la présence du J. squarrosus au pied du Gothard, ainsi que dans la Hongrie, Transilvanie, Croatie, sont erronées, comme l'ont déjà soupçonné Fuss et Neilreich,

J. temuis Willd. - Ce Jonc qui n'était connu jusyu'à ce jour en France que dans quelqques localités de la Loire-Infér. entre la route de Vannes et Orvault, à la Dénerie, à Port-Durand sur l'Erdre, à la Caillerie près les Cléons, et enfin dans le Finistère autour de Brest, a été trouvé par M. Vendrely dans la HauteSaone sur l'ancienne route de Luxeuil à St-Valbert, dans le bois du Banney, ainsi que dans un sentier longeant au sud le bois de Chatigny, puis dans Saone-et-Loire à Mouthier-en-Bresse d'oủ il a été envoyé à M. le $\mathbf{D}^{\mathrm{r}}$ Gillot par M. l'instituteur Bi. geard; enfin sur les bords de l'étang de Rye (Jura).

J. multiforus Desf. - Terrains humides et salés du littoral méditerranéen. - B.-du-Rh. et Var : bords de l'étang de Berre, Marignane, Toulon, Hyères, Castignaux. - Gard et Hér. : StGilles, Aigues-Ilortes, Frontignan, Vic, Marseillan aux Onglous, Lattes à Maurin, Pẻrols, Portiragnes, - Aude et Pyr.-Or. : SteLucie, Leucate.

J. compressus Jaca. - Lieux humides dans tout le bassin. Espèce polymorphe.

J. Gerardianus Lois. - Forme du précédent à fleurs plus petites, à styles plus longs, particulière aux terrains salés du littoral ou de l'intérieur. - B.-du-Rh., Var et Alp.-Mar. : envir. de Marseille et de Toulon, la Seyne, golfe Jouan, Nice, Monaco. Gard, Hér.,. Pyr.0r. : St-Gilles, Bellegarde, Aigues-Mortes, Perols, Cette, Agde, Salses, St-Laurent de la Salanque. 
Celte forme existe aussi au centre de la France dans les marais salés de llarmillat, Coeur, St-Nectaire (Puy-de-Dome).

La forme J.tricephalus Gay a été observée à la Tourne près Belgentier (Var).

J. tenagelus $I_{\text {. }}$ - Lieux humides des terrains siliceux.

ॠ. Hufonius L. - Lieux humides, - Espèce polymorphe. Dans la forme fasciculatus Berlol. les fleurs sont rapprochées en fascicules, les rameaux plus courts et plus épais; - dans la forme ranarius Perr. Song. les divisions externes du périanthe égalent presque la capsule; les internes sont plus courtes que la capsule, celle-ci est oblongue-ovoïde.

cuzua pilosa IVilld, - Bois dans les parties septentr. et moyenne du bassin; plus rare dans la région mérid. - Gard : le Vigan, Alzon, l'Espérou. - Pyr.-Or. et Ariẻge: Mont-Louis, Prats-de-Balaguer, col des Ares, bois de France et de Lesquerde, forêts de Salvanère et de l'Ermite.

C. erecta, L. Forsteriana DC. - Forme du précédent à pédoncules toujours dressís, à divisions du périgone plus longues, à feuilles plus étroites. - Même dispersion que le type, mais moins abondant.

耳. Rlavescens Gaud. - Bois de Sapins du Jura helvétique et français, des montagnes calcaires de Vaud, Valais, Savoie, Dauphiné, Basses-Alpes. - Pyr.-0r. : Canigou, Carença. - Pyr. centr. Nul dans le reste de la France.

T. maxima DC. - Bois des terrains siliceux des plaines et des montagnes.

L. Glabrata Desv, L. Desvauxiana Kunth. - Bois des montagnes. Ard, et H.-L. : Mezenc. - Ariège et Pyr.-Or. : vallon de l'Estagnet en montant au col de Lègue, Prats-de-Alollo, Evol, Valmanya. - En dehors de nos limites dans les Pyr. centr., Cantal, mont Dore, Pierre-sur-Haute.

T. opadicea DC. - Rochers et rocailles humides des Alpes, des Pyrénées el des Vosges. - Vaud et Valais: Anzendaz, Fully, Champé, St-Bernard, mont Nuoble, Guggerhubel, Laemmeralp, Zermatt, Saas, Simplon, Glacier du Rhone, llayenwand, Grimsel.-H.-Sav. etSav.: Vergy, Méri, Roc d'Enfer, Colone, Brévent, 
Aiguilles-Rouges, Sixt, col d'Anterne, mont Btanc jusqu'au Jardin et au Couvercle, Aontanvert el Tré-la-Tète, col des Fours et du Bonlromme, la Sauce, Reselein, St-Sorlin d'Aives, la Levanna, mont Cenis. - Is. ct II.-N I p. : Belledonne vers le lac du Cronzet, col Oddic, Sept-Isax, Taillefer, la Salette, Lautaret, col des IIayes, sources du Guil, mont Viso, la Taillante, St-Véran, Chaillol-le-Vieil, Vallonpierre en Valgaudemar. - B.Alp. ct Alp.-Mar. : Bérard, Roche-Grande aux sources du Var, col de Jallorgues, lac du Mercantourm, col de Fenestre, Salsamorena, cols de l'Abisso et de Tende, mont Bego, Mine de Tende. Pyr.-0r. et Ariège: Canigou, cirque des Aiguettes et d'Artounant, Roc de l'Estagnet, Jasse de Bentaillole, Pic del Ginèvré.

- En dehors de nos limites dans les Pyr. centr. et les Vosges.

E. alboida DC. - Bois des montagnes à sol siliceux, - - liaules Vosges jusque dans la zone vosgienne et sous-vosgienne des envir. de Belfort, de Montbéliard et de la II. Saône au Ballon de Giromagny, vallẻe du Géhard et de Miélin, Château-Lambert, Champagney, Beulotte-St-Laurent, mont de Vannes, Ecromagny, sur l'oxfordien siliceux et le minerai de fer à Grattery, Chariez, Fouvent, Larret, Dampierre, Noidans-le-Ferroux. - Doubs et Jura: oxfordien siliceux au Saut-du-Doubs, Lomont de Villars et de P'ierre-Fontaine, Roche d'Or, Chalezeule et la Vèze près Bcsançon, Quingey, Villersfarlay, terrain de transport à cailloux siliceux de la forêt de Chaux, grès et granite de la forêt de la Scrre. - Très-rare dans le Bugey à Colmaranche, Hauteville ct Parves. - C.-d'Or : granil des envir. de Saulieu, Collonges, Viévigne. - Vaud: Jorat au-dessus de Lausanne, Payerne, Avenche, Bex. - Alp.-Mar.: Thorenc, St-Martin, Lantosque, Alpes de Hollières, vallée de la Gordolasca, col de l'Abisso, Alpes de Tende. - Ard. : le Mézayon, la Viole. - Aude, Pyr.. Or. et Ariege: Durban, les Bouillouses, Mont-Louis, Jasse de la Bentaillole, Escale de Valbonne, lacs de l'Estagnet, de Quẻrigu et de Llaurenti, cirques des Aiguelles et d'Artounant, lloc de Mascaras, Val de Paillères à Souccarat.

4. nivea DC. - Bois des montagnes dans tout le bassin du Rhỏne.

- Pyrénées, centre de la France, Vosges.

Catal. Dassin du Rhone. 
L. Inten DC. - Pelouses et rocailles des Alpes et des Pyrénées. Valais: Fully, Catogne, St-Bernard, Thyon, Guggerhubel, Aletsch, Simplon, Zermatt, Saas, Glacier du Rhône, Grimsel. II.-Sav. ct Sav. : cols de Balme et de Voza, mont Blane jusqu'au Jardin et au Couvercle, moraines de la Mer-de-Glace et de Trẻla-Tête, Brévent, la Layat, la Sauce, Hauteluce, les Bréviaires, les Allues, mont Jovet, mont Cenis à Paltes-Creuses. - Is. et II.-Alp. : Belledonne à la Pra, Champrousse, Sept-Laux, St-Hugon, Taillefer, la Salette, Arcines, mont Genèvre à Chenailler et à Gondran, Conranvert en Valgaudemar, Péas en Queyras, la Traversette, la Taillante, col Agnel, St-Yéran à Clausis, col de Vars. - B.-Alp. et Alp.-Mar. : Lauzanier, Longet, Fouillouse à Plate-Lombarde, mont Grand-Couyer, sources du Var à Estenc, mont Garet au-dessus d'Entraunes, lac d'Entrecoulpe, vallons de Nanduebis et du Clapier, col de Tanarelio. - Pyr.Or. et Ariège: Canigou, Cambres d'Aze, vallées d'Eyne et de Llo, Capcir, Valbonne au Roc de la Musique, Pas du Roc Blanc. - Pyr. centrales.

E. campestris DC. - Pelouses ct clairières des bois dans tout le bassin.

M. multifforn Lej. - Bois et pâturages des collines et des montagnes. - Espece polymorphe ayant quelquefois les divisions du périgone blanchâtres et scarieuses (L. pallescens Kioch); les épis très-courtement pédicellés et rapprochés en capitules ( $L$. congesta Lej.); les fleurs noirâtres disposées en épis courts ( $L$. nigricans Desv.). Cette dernière forme est assez commune dans les hauts pâturages des Vosges, de la chaine jurassique, Valais, Savoie, Dauphiné jusqu'aux Alpes-Naritimes; d’un autre côtê au Pilat, dans les Pyr.-0r. et l'Ariège; - puis, en dehors de nos limites dans la Lozère, Aubrac, Cantal, mont Dore, Pierresur-Haule.

L. spicata DC. - Púlurages des liautes montagnes. - Claaine jurassique au Noirmont, Montendre, Dòle, Colombicr, Reculet. - Vaud et Valais : Anzendaz, Bovonnaz, Diablerets, Fully, StBernard, Pierre-à-Voir, entre Orsera et Novelli, Thyon, Mayenhorn, Gemmi, Saas, Belalp, Grimsel; s'élève jusqu'au pied du 
mont hose à la Cabane de Vincent. - II.-Sav. el Sav. : Brevent, col de Balme, nont Blane jusyu'au Jardin, au Couvercle et aux Grands-Mulets, Montanvert, Mont Charvin, la Sauce, petit StBernard, Cormet, Praman sur Pesey, Hauteluce, petit mont Cenis, mont Cenis. - Is. el H.-Alp. : Belledonne, Champrousse, Sept-Laux, Tele-de-llachat, Taillefer, Grand Som, Charmant Som, la Salelte, St-Christoplie et la berarde en Oisans, mont Pelvoux, la Grave, Lautarel, Chabas près Briançon, sources du Guil au pied du mont Viso. - B.-Alp. et Alp.-Mar. : Grand Couyer sur Annot, Entraunes, mont Tenibre au-dessus de St-Etienne, Ste-Anne-de-Vinaï, lac d'Entrecoulpe, cols de Salẻse et de Tende. - Ard. et Gard : le Mezenc, l'Aigual, l'Espérou. Pyr.-0r. et Ariège: Canigou, Mont-Louis, Carlite, Pas du Roc Blanc, Pic des Frontcils, Pla Bernard, Pic del Ginèvé. - En dehors de nos limites dans les Pyr. centr., Cantal, mont Dore.

E. Lиeăiformis DC. - Prairies des Alpes et des Pyrénées. - Is. et II.-Alp.: Belledonne à la Pra, Champrousse, Taillefer, Brandes en Oisans, Alpe du mont de Lans, la Bérarde, la Salette au mont Chamoux, Lautaret, Lauzet, Monestier-de-Briançon, mont Genèvre, Gondran, Chaillol-le-Vieil, Jurbonnas, col Bayard et de Glaise, cote Gélive, Rabou, Grangette, mont Aurouse. - Sav. : de Coutabella à Retord près du col de la Louze, col de la Madeleine, mont-Cenis à Pattes Creuses, - B.-Alp. et Alp.-Nar.: Lauzanier, Alpes de St-Dalmas, col de Tanarello. - Pyr.-Or. et Ariège: Canigou, Cambres d'Aze, vallon de Boutadiol, lacs de Valbonne et de Rabassolès, cirques d'Artounant el des Aiguettes, Val de Paillères à Soucarrat. - Pyr. centrales.

\section{CYPIRACEES}

CWurags longus L. - Fossés, lieux humiles. - C.-d'Or : Premeaux, Argilly, Saulon, Arcelot. - Jura et Ain : bords de la Seille au-dessus de Bletterans, Belley, Muzin, Viricu-le-Grand, Tréroux. - Rh. : Lyon à la IIouche el à Gorge-de-Loup, Yvour. - Vaud et H.-Sar. : Abbaye de Sales, Veyrier près Annecy. Is. et Dr. : Pont-de-Chérui, Charvieu, Renage, Loriol. - Ard. : 
sources de St-Romme près Aubenas. - Assez commun dans la Provence, lo Languedoc ed le Roussillon.

C. badius Desf. - Forme du précédent à épillets plus nomlreux, en fascicules plus denses et moins dresses. - Var ot $\mathrm{Alp}$.-Mar. : Toulon, la Seyne, ile de Porquerolle, St-Tropez, Nice, Menton. - ILér. : Lavalette, St-Georges, Villeneuve, Bézicrs, Vias, Lodève, Lamalou, Vendres, Andabre-Rosis, - Aude et Pyr.-0r. : Ste-Lucie, Salses, Grau d'Argelès, Banyuls-sur-Mfer.

C. ollwaris Targ.-Tozz. - Pâturages des bords de la mer. Marseille à la Belle-de-Hai, Toulon, Cannes, Nice, Menton. Pyr.-Or. : Mas Piras et St-Victor près Perpignan, la Salanque.

E. aureus Ten, - Lieux sablonn. du littoral des envir. de Toulon et de Menton.

C. Puseus L. - Lieux sablonneux humides.

C. sehnoenoldeas Gris. - Sables maritimes du Littoral. - B.-du-Kh., Var et Alp.-Mar. : Montredon, Nazargues, Toulon, Hyères, lic de Porquerolle, Fréjus, St-Raphaël, Cannes, Nice, Menton. Plages du Languedoc et du Roussillon.

C. gerotiaras Rottb., C. Montianus L. - Licux humides, fossís. Ain et Rh. : envir. de Belley, Pont-de-Cordon, entre Thil et Niribel, la Houche, Irigny. - Is. : Villette d'Anthon, sables de l'Isère près de Grenoble, îles du Drac au-dessous de Fontaine. - Vaucl. et B.-du-Rh. : Réal-Panier près Avignon, Arles, la Camargue. - Var el Alp.-Mar. : Petit-Argens et ancien lit d’Argens près Fréjus, Nice au Var. - Gard el Hér. : Vallabrègues, Sylveréal, bords du canal du Midi à Béziers et à Portiragnes. - En dehors de nos limites dans les Landes et la Gironde.

C. Anregcens L. - Lieux sablonneux humides.

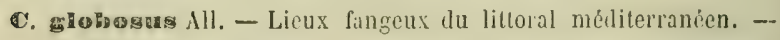
Nice au Var, Menton, Vintimiglia: - Envir. de Perpignan à Château-Roussillon, bords du Tech à Amélic-les-Bains.

C. dikatrelayogs All. - Fossés du littoral méditerranéen. - Nice au Var. - Pyr.-0r. : étang de Salses du côté de la FontEstramer.

SCमำ 
- Channe du Jura helvétique et français près des lacs de Joux, de Chalain et de Doucier. - Min, Vaud cl Valais : existo on plaine dans Ies marais de Divonne, d'Orbe, do Rolle, de Vervay. - H.-Sav., Is., II. et B.-Alp. : Nant-Borant, Prémol, Lancey, Laularet, envir. de Gap, Lauzanier. - C.-d'Or' : Voulaines, Marcy-sur-'Tille.

\$. m/grateans L. - Plés marécagcux et tourbeux. - Doubs, Jura et Ain : marais de Saône près Besançon, de Vaucy près Arbois, de Divonne, Lélex, Belley, Sle-Croix prés Montluel. - G.-d'Or : envir. de Saulieu. - Vaud et Valais : Troenex, Praz-Diez, Thyon. - Is. et Dr. : Meyzicu, Puzignan, Bourgoin, Polygone de Grenoble, Corene, Vif, Roissard, Villeneuve près St-Vallier, Saou, Caborne près Crest. - H,-Alp. : Lachaud et Manteyer près Gap. - Commun dans la Provence, Alp.-Mar., Languedoc, Roussillon et la partie mérid. de l'Ardèche.

CLADTN mariseum R. Br. - Marais et lacs.

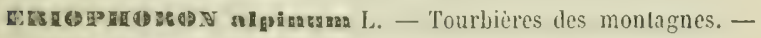
Jura helvétique et français, Pontins, Echelette, Eplatures, Ponts, Pouillercl, Tếle-de-lRang, Brévine, Verrières, Pontarlier, Bélieu, Mémont, Bonnétage, St-Julien au marais des Mouilles, NoëlCerneux, Chenalotte, Boujailles, Chapelte-des-Bois, Andelot, Villencuve, vallée de Joux, marais de la Pile près de la Trélasse, Forêt d'Échallon au lac Genin, Colliard, Retord, Cormaranche, lo Vély près Hauteville en Bugey. - Vaud et Valais : les Pleiades, les Mosses, la Verda, mont Fully, chalets de Catogne, Champé, Zermalt on montant au Riffel. - H.-Sav. et H.-Alp. : Valorcine, col de Balme, les Contamines, col de Vars. - Alp.-Mar. : Alpes de Ste-Anne-de-Vinaï, de Carlin et de Tende, - En dehors de nos limites dans le mont Dore, Cantal.

E. copicatom IIost, E. Schcuchicrianum II oppe. - Tourbieres des Alpes. - Vaud et Valais : Bovonnaz, Lavarraz, Fully, St-Bernard, Cleuson, Thion, Esserz, Zan, Zermatt au Schwarzsee, Saas à Mattmark, Eginna, Grimsel. - H.-Sav., Sav., Is. et II.-Alp. : cols de Balme, d'Anterne et Joly, Planets au pied du Vergy, Jalouvre, Crest-Voland, Bas du Mottet, col du Cormet, Bellevaux, mont Cenis au lac Noir, la Pra de Belledonne, 
Combe de la Lance, Sept-Laux, Taillefer, Galibier, col de Vars, pied du mont Viso, Ruines, la Taillante vers les lacs, Ségure. B.-Alp. et Alp.-Mlar. : Valonnet, Horonaye, Longet, lac de Ligny sur Colmars, sources du Var à la Strop, plateau de Jallorgues près Si-Dalmas, Salsamorena, Ste-Anne-de-Vinaï, lac de Frc- mamorta, col de Tende. - Pyr.-0r. : Canigou, vallée d'Eyne. - Pyr.-Centrales.

F. vagianatara L. - Tourbières des montagnes, - Hautes-Vosges jusque dans la H.-Saône au Ballon de Giromagny, vallée du Rahin, Ecromagny, tourbière de la Pile près St-Germain. Jura helvétique et français : Sonnenberg, Echelette, Ponts, Bré. vine, Pontarlier, Bélieu, Boujailles, Chapelle des Bois, Andelot, Villeneuve, les Rousses, Coillard, Retord, le Vély près Hauteville en Bugey. — Vaud et Valais: marais de la Pile près St-Cergues, Gourze, les Mosses, la Sicrne-au-Cuir près Château d'Oex, Gryon, Thion, Orsera, Zermalt. - H.-Sav. et Sav. : derrière le Crand-Piton du Salève, Bellevaux au marais des Mouilles, col de Balme, la Flégère, Combe sur Sixt, plateau des Gets, Crest-Voland, bords du lac de la Girottaz. - Is. : Prémol au lac Luitel. - B.-Alp. et Alp.-Mar. : Lauzanier, lac des Merveilles. - C.-d'Or et S.-et-I. : Saulieu à St-Didier et au bois de la Vente à l'ltalien, élangs de Montjeu et des Cloix près Autun, Antully, Roussillon. - Loire : du Pic de l'Oeillon à la Croix de Montvieux, entre Tarantaise et la Rẻpublique. - Gard : marais du Lengas à la Grandès-Haute près de St-Guiral, la Lozère près Concoule. - Pyr.-Or. et Ariège : Canigou, les Bouillouses, Madrès, lac du Laurenti, Ascou, val de Paillères à Soucarrat. En dehors de nos limites dans les Pyr.-Centr., Lozère, Cantal, mont Dore, Forez, Creuse, Sarthe, Maine-el-Loire.

E. folystachyum L. - Lieux marécageux. - Espèce polymorphe se subdivisant en trois races ci-après désignées et différant les unes des autres par la présence ou l'absence des stolons, la longueur, la rudesse ou la glabrescence des pédoncules, les di. mensions des feuilles, la forme des achaines tantôt acuminés, tantôt mutiques.

E. gracile Koch. - Prés marécageux. - Jura lıelvétique et français : 
Wasserfalt, Pleurre, Fays, Sellieres, Champvans près Dole. Vaud : Châtel St-Denis, mont Jorogne, les MIosses, - II.-Sav. et Sav. : marais de Lossy au pied des Voirons, mont Cenis. C.-d'Or et S.-ct-I. : envir. de Saulieu, Millery près Autun, Ilontgillard pris Issy-l'Évéque. - Ain el Is. : Ste-Croix près Montluel, Mcyzieu, Chatva près Pusignan. - Gard et Loz. : l'Espérou, la Lozère près Concoule. - Ariége : P'ic del Ginevré, Sarrat d'en Binada, Pla Bernard. - En dehor's de nos limites dans Aubrac, Lozère, lacs du mont Dore, Puy-de-Dome, HauteLoire, Creuse, Vienne, Itte-Vienne, Maine et Loire, Ouest et bassin de Paris.

E. angustifolium Roth. - Prisente de nombrenses variations dans la taille, la longueur des pédoncules, le nombre des capitules, la forme des feuilles.

E. latifolinm IIoppe differe de la forme précédente par ses capitules plus nombreux, par ses achaines arrondis et mutiques, ses feuilles plus larges et plus courtes, sa souche non stolonifére.

SCrato silvadieas L. - Fossés, prés el bois liumides.

s. Michellanus L. - Mares et étangs, - C.-d'Or et S.-et-L. : étang d'Arnay, mares de Boncour, Citeaux, bords de la Saone à Màcon, Cluny, St-Didier, Issy-l'Évêque. - Jura, Ain et Rh. : la Bresso et la Dombes, St-Laurent-de-l'Ain, envir. de Bourg à Viriat et Corgenon, Trévoux, Quincieux, Collonges, PierreBênite. - Is. : Montceau près Bourgoin. - En dehors de nos limites dans l'Allier, la Nièvre, le Cher, les Landes.

5. maritianus L. - Mares et ćtangs, - II.-Saône : Chariez, la Mer. - Jura et Ain : la Bresse et la Dombes. - C.-d'Or : Laignes, Flagey, Boncourt, Genlis, Vielverge. - Vaud et Valais: rives du Léman à Concise, Grandson, Yrerdon, Yronand, Estavayer, Roche, Maladeire, Montorge. - Rh. et Is. : Bourdelans près d'Anse, Pierre-Bénite, Polygone de Grenoble, Gières. Assez répandu dans la Provence, les Alp.-Mar., le Languedoc et le Roussillon. - Espèce polymorphe à inflorescence variable. Dans la forme digymus les épillets sont ramassés en capilule compacte, les stigmates au nombre de deux.

s. compressus Pers. - Prés humides des plaines el surtout des 
montagnes. - Assez commun dans la chalne jurassiquo jusque dans le Bugey. - H.-Saone, C.-d'Or, S.-et-L. : Chariez, Chassey-les-Hontbozon, Semur, Saulieu, Blaisy, Gevrey, Nolay, Saisy, Sully, - Valais : Mayens et iles de Sion, St-Barthêlemi d'Hérẻmence, Vercorin. - H.-Sav. et Sạv. : Salève, Voirons, Collonges, Brizon, montagnes du Chablais, du Faucigny, de la Tarantaise ct de la Mlaurienne jusqu'au mont Cenis. - Is., Dr. et II.-Alp. : Champ près Vizille, St-Nizier, les Fauges de Villard-de-Lans, le Merdaret au-dessus de Theys, Montmaur près Die, Lautaret, mont Genèvre, le Queyras et le Gapençais. B.-Alp, Alp.-IIar, et Var' : vallée de •l'Ubaye, St-Dalmas-leSauvage, vallée du Boréon, la Maïris, col de Tende, Nice au Var, la Ste-Baume, Gonfaron. - Envir. de Lyon à Vaux-enVelin et sur les bords du Rhône en remontant vers la Pape. Ard. : Vals, Gerbier-des-Joncs. - Gard et Hér. : Uzès, Alais, Bouquet, l'Espérou, Avène, Lunas, St-Martin-d'0rb, Lauroux et Labeil au-dessus de Lodève.

s. Holoseracenas L. - Licux humides de la région mérill. Plus rare dans la région moyenne à Gap, Crest, St-Vallier (Dr.); - Pont-de-Claix, Seyssins, Meyzieu (Is.); - le Teil, Aillan, Celles, Vals (Ard.); - envir. de Lyon à Craponne, Yvour, Vaux-en-Velin, St-Georges-de-Reneins près Villefranche; - bords de la Saône à I'révoux; - envir. de Genève entre Genthọd ct Versoix. - Ĺspèce polymorphe à inflorescence variable.

5. Iacusta-ls L. - Fossés, marais, étangs, rivières. - Espèce polymorphe.

La forme digynus (S. Tabernaemontanus Gmel.) est plus grêle dans toutes ses parties, a une teinte glauque et n'a que deux stigmates au lieu de trois; mêlée au type qu'elle remplace quelquefois.

5. eanolnatus Sm., S. Duvalianus Hoppe, - Bords des eaux, - Rh. et Is. : envir. de Lyon à la Tête-d’Or, Vaux-en-Velin et Feyzin.

s. Hittoralls Schrad., S. triqueter L. - Marais, bords des eaux de la région mérid. - B.-du-Rh. et Var: St-Mitre, Ccinturon d’Hỳres, Fréjus. — Gard et Hér. : St-Gilles, Bellegarde, Aigues- 


\section{$-761-$}

Mortcs, Mauguio, Mireval, Pérols, Villencure, Vendres, - Aude et Pyr.-Or. : Ste-Lucic, Salses. - Espèce polymorphe.

5. Pollichianus G.G. - Forme dans laquelle une des faces de la tige est faiblement canaliculée. C.-dl'Or et S.-et-L. : Saulieu, Limpré, envir. de Louhans et de Macon. - Ain el Is. : Trévoux, Massignieu, Nattages, Peyrieu, Polygone de Grenoble, Domène.

- Envir. de Lyon à la Téte-d'Or, la Mouche, Pierre-Bénite, Irigny, Vaux-en-Velin. - Ard. : T'ournon.

s. Wougens Vahl., S. Rothianus Hoppe. - Ce Scirpe, assez commun dans les marais du litloral de l'Océan, se trouve à Salses et Grau d'Argelès (Pyr.-0r.), à St-Louis, près de l'embouchure du Rhône, à Nice près du Var; on l'a même observé autour de Lyon dans les iles du Rione sous la Pape, au Grand-Camp el à la Nouche, puis près de Màcon à Feillans.

S. maz exonatus L. - Marais, étangs. - H.-Saûne : étangs du hois de Citers et du Grand-St-Maurice, des Monts-Revaux, de BoisDerriere, de la Maugenotte et des Grands-Bois. - Douhs, Jura et Ain : Bourg, la Bresse à Champrougier, Tassenières, Chaumergy, Blelterans, Scillon et la Chambrière près Bourg, la Dombes à Montribloud, Villeneuve, la Peyrouse, Villars, St-Paul-de-Varax. - Valais : Villeneuve, Noville. - S.-ct-L. : mare de Brouailles près Louhans, Mouthiers en Bresse, étangs de la Verre. - Is. : Pont-de-Chérui, Charvieu, Charva, Crémieu, St-Martin-de-Yaulserre près le Pont-de-Beauvoisin.

5. suplau L. - Bords des eaux, - C.-d'Or et S.et-L. : Citeaux, Nuits, Boncour, Gergy, Sully, Gerland, Verlun-sur-Doubs, étangs de Moux-Guillot et de Pontoux. - Genève, Vaud et Valais: bords du lac Léman entre Genthod et Versoix, embouchure du Boiron près Nyon, Grangettes près de Villeneuve, St-Sulpice, les Pierrettes, loords du Rhône. - Jura et Ain : člangs de St-Seine, St-Baraing, Cervat, Commenailles, Bletterans, la Dombes à Montribloud, Châtillon, Baneins, St-Germain, le Plantay, Villars, Versailleux, Chalamont. - Is. : Charvieu, Montceau, St-Martin-de-Vaulserre près le Pont-de-Beauvoisin. - Gard : étang de Jonquières.

\$. setreeus L. - Terrains siliceux humides. - Lisiere rosgienne 
des arrond, de Belfort et de Montbéliard, - H.-Sav. : HabèreLullin, Roc de Chère sur les bords du lac d'Annecy. - Ain: lo Bresse, forêt cé Seillon, la Dombes à Marlieu, Pont-de-Vaux, Reyrieux, Trévoux. - Rh., Loire et Ard. : chainne granitique du Lyonnais, du Pilat, du Forez et du Vivarais, puis danz les Cévennes du Gard el de l'Hérault ainsi que dans les Pyr.-Or. - Is. : les Terres-Froides, Mens, Echirolles, Prémol. - Rare dans la Provence et les Alpes-llaritimes.

5. finformis Savi, S. Snvianus Seb. Mlaur. - Lieux humides de la région mérid. - Bords des étangs de la Camargue, de la Crau, de Berre, Sablettes de Toulon, Hyères, île de Porquerolle, les Maures, les Mayons, Cannes, la Roquette, Antibes, Nice, Menton. - Gard et Hêr.: Aigues-Hortes, Chartreuse de Valbonne, Ganges, Palavas, Pérols, Mauguio, Montarnaud, Lamalou, Lunas, St-Etienne-de-Gourgas. - Aude et Pyr.-Or. : envir. de Narbonne, bords de la Tet, Salses, N.-D. de Coral, Massane.

S. Guitans L. - Ifares et étangs. - C.-d'Or et S.-et-L. : étang Fortier près Saulieu, étangs de Brnuailles et de St-Bonnet près Louhans. - Ain: St-Laurent-les-Htâcon, étang Genoud près Pont-de-Veyle. - Centre et ouest de la France.

S. pancifioneus Ligthf. - Licux marécageux des plaines et des montagnes. - Tourbières de la chaîne jurassique à Pontarlier, Boujailles, Champagnole, Chapelle-des-Bois, les Rousses. Vaud et Valais: Trélex, Diez, Rothorn, Zermatt au Hoernli, à Findelen et au Schwarzsee, Saas, Simplon, Glacier du Rhône. - H.-Sav. el Sav. : marais de Lossy aux pieds des Voirons, pied du Salève au-dessus de Collonges et d'Archamp, col de Balme, N.-D. de la Gorge, mont Cenis, - Ain, Rh. et Is. : Lyon à la Tête-d'Or, Vaux-en-Velin, Anse, Polygone de Grenoble, St-Nizier, les Fauges de Villard-de-Lans, - H. et B.-Alp.: Lautarct, Galibier, Guillestre, vallée du Guil, Manteyer, Séuse, Sigoyer, Noyer en Champsaur, Vallonnet de Meyronne, Annot. - Alp.Mar. : Estenc au-dessus d'Entraunes, col de Salèse et de Fenestre. - Gard et Hér. : l’Espérou, Monjardin près Lanuéjols, Mauguio.

S. eaespitosus L. - Licux marécageux et tourbeux des plaines 
el des monfagnes. - Tourbières des Vosges jusque dans la II.-Saône au Ballon de Servance, - Tourbières de la chaine jurassique, les Ponts, Brévine, Chaux-du-Milieu, Vraconne, Noiraiguc, Mortcau, Pontarlier, Mouthe, Boujailles, Val de Joux, les Rousses, Colllard. - Vaud et Valais: la Sicrne-au-Cuir près de Chateau d'Oex, les Mosses, Alpes de Morcles et de Fully, Emousson, lac do Champé, Gottier, Zermalt au Riffelhorn et Augstelberg, Simplon, Grimsel. - II.-Sav. et Sav.: col de Balme, Chamonix, la Layat pres Nant-Borant, les Allues, mont Cenis. - Envir. de Lyon dans les iles du Rhône près de la Pape et à Oullins. - Is. et H.-Alp. : Belledonne, Prémol, col du Merdaret, Sent-Laux, Taillefer, Lautaret, l'Echauda, mont Genèvre, le Champsaur, Orcières, le Valgaudemar, le Queyras. B.-Alp. et Alp.-Mar.: Lauzanier, Parpaillon, Longet, sources du Var, Estenc au-dessus d'Entraunes, col de Salèse, forêt de Clans, col de Tende. - C.-d'Or' et S.-et-L. : envir. de Saulieu au bois des IIauts-d'Arneaux, Roussillon. - Gard: l'Espérou, la Lozère à Concoule, Gourdouzc. - Pyr.-Or. ct Ariége: Nohèdes, Nadrès, Matemale en Capcir, lac de l'Estagnet, Baouzcille de Tarbézou. - En dehors de nos limites dans les Pyr. centr., Lozère, Aubrac, Tarn, mont Dore, Forez à Noirétable et Pierresur-Haute, centre et ouest de la France.

5. alplinus Schl. - Lieux marécagcux des Alpes. - Valais: Zermatt à Findelen et au Stock de Zmutt, Simplon sous le Sirwoltensee. - H.-Sav. et Sav. : col de Balme et Chapeau près de la Mer-de-Glace, les Allues, mont Cenis sur le plateau et, d'après MIM. Gillot et Déséglise, sur le versant sud de la montagne do Thure au-dessus da col de la Ramasse. - H.-Alp. : au-dessus du col Malrif, mont Pelvas, la Traversette, Pierre-Noire aux sources du Guil, au-dessous de Fontgillarde, lac du Pontet près Villard d'Arène.

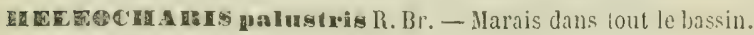
- Espèce polymorphe.

E. uniglumis Koch. - Forme du précédent dans laquelle l'écaille inférieure embrasse complètement la base de l'épi. Plus rare que le type. 
II. mullicaulis Dietr. - Forme à soucho fibreuse, non-rampante, ayant, le plus souvent, trois stigmates, quelquefois deux seulement, les achaines triquètres, plus rarement comprimés. C.-d'Or et S.-et-L. : Roche-en-Brenil, Autun, Broye, entre Maltat et Viry. - Ain, Rlı. et Is. : étang Genoud près Pont-deVeyle, Vaux-en-Velin, Meyzieu, Polygone de Grenoble, Comboire, lac de Jarrie. - Var et Alp.-Nar. : Fréjus, l'Esterel, Cannes, Nice au Var. - Hér: Fraisse.

19. ovrta R. Br. - Étangs. - H.-Sav. el Doubs: Plancher-Bas, Chenebier, Frahier, Clagey, la Mer, Lure, Franchevelle, Lincxert, bois de Citers, la Maugenotte, élangs des Monts-Revaux près St-Germain, Senoncourt, Mont, Grand-Villard. - Jura ci Ain : forêt de Chaux et de la Serre, la Bresse et la Dombes autour de St-Paul-de-Varax, Villars, Villeneave, Montribloud. C.-d'Or' et S.-et-L. : envir, de Saulieu, Citeaux, Nuits, Saulon, Boncourt, Autun, Millery, la Bresse louhanaise. - Is. : Charette près Morestel, St-Martin-de-Vaulserre près le Pont-deBeauvoisin, Grenoble.

耳T. Relezandis R. Br. - Marais. - Mêmes localités que le précédent dans la partie septentr. du bassin. - Genève, Vaud, Valais ct H.-Sav. : bords du lac Léman, Guercet, Plan Conthey, borls de l'Arve à Arenthon, Crest-Voland, - Rh., Is, et Dr. : envir. de Lyon, Crémieu, bords de la rivière d’Ain, Eybens, Laffrey, Sept Laux, Saou près Crest. - Gard : le Vigan, Concoule.

1If. Inxa Vahl. - Prés humides. - Nice aux Grenouillères du Var. L'Argentière (Ard.).

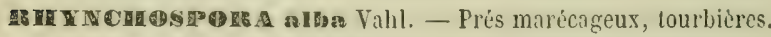
- H.Saone et arrond. de Belfort: les Ballons, les Guidons, sources de la Lanterne, entre Frahier et Planchicr-Bas, Fresse, Champagney, Mourière, vallée du Rahin, Grands-Bois, Bas do Mélisey, Franchevelle, Lautenot, tourbière de la lile près StGermain. - Doubs et Jura : lisière vosgienne de l'arrond. de Montbéliard aux Guinots, Clamesol, Pontarlier, Mouthe, Boujailles, Villeneuve, Marigny, Sone, les Rousses. - Ain : envir. de Bourg, Coillard, Culoz, les Echets. - Vaud et Valais: Vervey, Villeneuve, tourbières de Jongny et de Gourze, lac de Châtel- 
Denis, les Mareottes sur Salvan. - II.-Sav. et Is. : marais de Lossy, de Chessé pris Thonon, Sillingy, Epagny, Chindrieux, Chambaran. - C.-d'Or et S.-ct-L. : lo Morran, Saulieu, BoisMorin, étang lortier, Auxonne, envir. d'Autun. - Loire: Pilat, Chalmazelle. - Gard et IIér, : Gourdouze près Conconle, la Salvetat, Fraisse, Lignieres. - Aude : Montagne-Noire, Martis.

0. Paser lloem. Schult. - Marais tourbeux de H.-Saone entre Annegray et Ecromagny, sources de la Lanterne, lranchevelle, étang de la Maugenotte près Bois-Jjerrière, tourbière de la Pile pris Sl-Germain. - En dehors de nos limites dans les lourbières du grès vosgien entre Kaiserslautern et Bitche, centre et ouest de la France, Landes, Pyr. centrales.

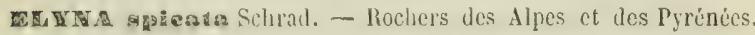
- Vaul et Valais: Panerossaz, Anzendaz, Alesse, Fully, SiBernard, Zermontana, Diez, Cotler, Zermalt, Saas, Cabane de Vincent au pied du mont llose, Simplon, Gemmi, Grimsel. II.-Sav. et Sav. : col de Balme, Joly et des Fours, Charvin, la Sauce, les Noltets, entre la Ciclaz et la Gittaz près Beaufort, la Grande-Motte près du lac de Tignes, entro Tignes et Laval, mont Iscran, les Allues, mont Cenis. - Is. et H.-Alp. : Grande-Vaudaine, Taillefer, Piémeyan au mont de Lans, la Houcherolle, l'Obiou, la Grave, Lautarel, Galibicr, Guillestre, mont Viso, col Vieux, la Grangette, Aurouse, Chaillol. - B.-Alp. et Alp.Mar. : Fouillouse, Vallonnet, Parpaillon, Bachasse, sources du Var à la Strop, Roche-Grande, Alpes le Carlin et de Tende. Pyr-0r. et Ariège: Canigou, Cambres d'Aze, vallée d'Eyne, Valbonne à la base du Roc de la Musique, Pic de la Camisette, Pas du Roc-Blanc. - Pyr. centrales.

ESORE Pyrénées. - Valais: Glacier du Rhône, Furca. - Sav.: vallée de la Rocheur, bords du lac de Tignes, Plagne de Pesey, mont Cenis en montant à Ronches, la Savine cntre Bramans et Chaumont. - En deliors de nos limites, dans les Pyrénẻes au sommet des arêtes du Camp-Long, à la montagne de Vignec-d'Aure à côté du lac d'où sort le Bodet, base Ǵu Gabiédou, sources d'Asne entre Gavarnic et le Port de Boucharo. - Canton de Berne au 
Stockhorn et au Faulhorn. - Norvège et Suede septentrionales, Laponic, Écosse.

CAex aloicostrongyla, C. dioica L. - Ilarais tourbcux. II.-Saône el C.-d'Or : Ferrelte, Roche-en-Brenil, Saulieu. Doubs, Jura et din : Chenalolte, Narbief, Guinots, Bonnétage, St-Julien, Ste-Croix, Pontarlier, Bélieu, la Trélasse, Levier, Salins, Divonne, Hauteville en Bugey. - Vaud, H.-Savoie et Sav. : Nyon, Glarens, les Mossettes sur Grion, marais de Bossey sous lo Salève, Lossy au pied des Voirons, Chamonix au Chauderon, Combe-de-Villette, mont Cenis. - Is. et B.-Alp. : Meyzieu au moulin de Cheyssin, la Condamine.

C. Aoleotrigona, C. Davalliana Sm. - Prís marécageux. Assez commun dans les tourbières de la lisière vosgienne des arrond. de Belfort et de IIontbéliard, chaine jurassique, Vaud, Valais, Savoie, Dauphiné, B.-Alp. jusque dans les Alp.-IIar. C.-d'Or, S.-et-L. et Rh. : le Morvan, Prémeaux près Nuits, SteFoix près Dijon, l'Abergement-les-Seurre, vallée de la Seille, St-Genis-Laval. - Pyr.-Or. et Ariège : la Cerdagne, le Capcir et le Llaurenti.

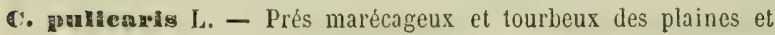
montagnes.

C. macrostyln Lap. - Pelouses des Pyr.-0r. et de l'Ariége: le Capcir et le Llaurenti, Pic de Tarbézou, Port de Paillères. Pyr. centrales.

C. microgroekin Wahlb. - Marais des Alpes valaisanes et savoyardes : Alliaz-de-Bagnes, Tortain de Nendaz, Diez, Anniviers, revers mérid. du Griess, bord du lac de Tignes, les Allues, entre Lans-le-Bourg et Bessans, mont Cenis près du lac.

C. מauciflon Ligthf. - Prés tourbeux des montagnes, - Hautes Vosges jusque dans la Hte-Saone au Ballon de Giromagny, vallée du Rahin aux Arrachies et à la Grande-Goutte. - Doubs et Jura : Pontarlier, Bélieu, Boujailles, les Rousses, la Pile pre̊s de la Trélasse, les Rousses, Chapelle-des-Bois. - Vaud et Valais : montagnes au-dessus de Vervey, les Mosses, Grimsel. - H.-Sav, et Sav, : col de Balme, Prarion de St-Gervais, ma= 
rais du Grand-Bois à Crest-Voland, mont Cenis. - Is. et B.Alp. : Prémol au lac Luitel, entre Lans el Villard-de-Lans, Longet de Maurin. - En dehors de nos limites dans l'Aubrac, mont Dore, Forez à Pierre-sur-Haute, Vosges.

C. Hyzenaien Wah!b. - Polouses des muntagnes des Pyr-Or. et de l'Ariège: Canigou, Cambres-d'Aze, vallée d'Eyne, Carlite, la Nous dans la vallée de Carol, vallon de l'Estagnet, lac Bleu et Noir, Pla de Monpudoux, Roc de Salsafrage. - Pyr. centrales.

C. rupestrin All. - Rochers des Alpes et des Pyrénces. - Valais : mont Fully, Alesse, Sanetsch, Pointe sur les Folatères, Gemmi, Grimsel, Riffelberg, Simplon. - Hte-Sav. et Sav. : col de Balme, Brivent, entre Tignes et Laval, mont Cenis. - Is. : la Prá de Belledonne, Taillefer, montagnes des Salles à St-Christophe en Oisans, la Salette. - II. et B.-Alp. : la Grave, Pic du Bec, Loularet, Galibier, Col Isoard et des Hayes, mont Viso, la Portiole, Vallonnet, mont Aurouse. - Vaucl. et Alp.-Nar. : mont Ventoux, mont Frontero. - Pyr. centrales.

C. chordorhiza Ehrh. - Tourbieres des montagnes de Pontarlier, la Brévine, Mouthe, le Bélieu, la Chenalotte, les Ponts, Ste-Croix. - En dehors de nos limites à l'Aubrac et au mont Dore.

C. Poetida Vill. - Lieux humides des Alpes et des Pyrénées. Vaud et Valais: Anzendaz, lac Lioson, Panerossaz, col entre la Capc au Moine et la Tournette, mont Fully, Alesse, Bagnes, Catogne, St-Bernard, Ferret, Zanrion, Vouasson, Thyon, Arolla, la Lex, Guggerbübel, Tortain, Belalp, Simplon, Zermatt, Saas à Mattmark et à l'Offenthal, vallée d'Eginna au Kif, Glacier du Rhône, Grimsel. - H.-Sav. et Sav. : col de Balme, Brévent, chaine du mont Blanc jusqu'au Jardin et au Couvercle, la Layat, cols dú Bonhomme, des Fours et de la Seigne, Vergy, Méri, Jalouvre, Roc d’Enfer, col de Golèse, la Sauce, mont Bellaclsat, mont Iseran, Levanna et Cenis. - Is. et H.Alp. : la Pra de Belledonne, Combe de la Lance, Sept-Laux, la Bérarde, Lautaret, Galibier, Pelvoux, col de l'Alp-Nartin et des IIayes, la Traversette, Ruines, la Taillante, St-Véran, Chaillolle-Vieil, Orcières. - B.-Alp. et Alp.-Mar. : Vallonnet, Lau- 
zanier, Estenc, lac de Rabuons au mont Ténibre, St-Étienne, Stc-Anne-de-Vinaï, col de Fenestre, lac dEntrecoulpe. - Rare dans les Pyr. centr. aux Ports de la Picade, de la Fraiche, de Venasque et à Esquicrry.

C. Enderostyla Gay, - Licux humides des Alpes de Vaud et Valais: Lavarraz, Panerossaz, Sl-Bernard, Thyon, Lamon, Simplon, Grimsel.

C. juncifolla All.-Licux humides. - Valais: Zermalt au Schwarzsee, Hoernli, Riffel, Augstelberg, Saas au Schwarzberg. Sav. : Cabane du col Iscran, mont Cenis entre la Cenise et la route et, d'après MM. Gillot et Déséglise, sur le ver'sant mérid. de la montagne de Thure au-dessus du col de la Ramasse.

c. diviฐa Huds. - Lieux humides de la région mérid. - Remonte à la Saulse près Tallard (H.-Alp.); Lyon à Vaise; plaine du Forez. - Espèce polymorphe.

C. setifolia Godr. - Forme grèle du précédent, ayant des utricules à bec plus long, qu'on trouve assez communément dans la région des Oliviers et jusque dans les parties mérid. de la Drôme et de l'Ardèche.

C. Aistielna Huds. - Prés marécageux dans tout lo bassin.

C. Sethabebrama Schrank. - Lieux sablonneux. - Doubs, Ain et Rh. : prairie de l'Allan à Montbéliard, bords de la Saône à Thoissey, St-Didier, Montmerlc, Reyrieux, Villeurbanne, StAlban, Chaponost, Dardilly, - Is. et Dr.: Vienne, Laveyron près St-Vallier, Crest. - Vaucl. et Var : Avignon, le Luc. Gard et Hẻr. : Nìmes, Anduze, Sylveréal, Uzès, Montpellier à Lavalette, Pic St-Loup, St-Jean-de-Védas.

C. brazoidea L. - Fossés, marais ct bois humides des terrains siliceux. - Hte-Saône, Doubs, Jura et Ain : grès bigarrés et alluvions des arrond. de Lure et de Montbéliard, la Bresse et la Dombes. - C. -d'Or et S.-et-L. : envir. de Saulieu, d'Auxonne, Citeaux, Collonges, Louhans, Autun.

C. Tappoina L. - Lieux marćcageux, fossés dans tout le bassin.

C. Bameleada L. - Bois, pelouses, borls des chemins. - Espice polymorphe dans laquelle l'épi est tantôt continu, à écailles brunes ( $C$. contigua Hoppe), tantôt interrompu à la base, à 
icailles vertes, l'inféricur muni d'une longue bracléc filiforme (C. virens Lam.), d'autres fois composé d'épillets très-écartés les uns des autres ( $C$. divulsa Good.).

C. panieulata L. Marais des plaines et des montagnes.

C. paradoxr Willd. - Prís tourbeux. - Doubs et Jura : Mandeure, Audincourt, marais de Saone près Besançon, Salins, Pleurre, Rye, Bonlieu. - C.-d'Or : cnvir. de Chátillon. - Vaud: Payerne, St-Cierge, Cugy, Froideville, Vidy. - Ain et Rh. : Divonne, les Echets, Ste-Croix près Nonlluel, étang du Loup ả Chaponost, Yrour. - Is. et Dr. : marais de St-Romain près de Pont-de-Chéruy, Eydoche, lac de Raz près Voreppe, Villeneuve près St-Vallier. - I1.-Sav. : marais de St-Félix.

c. ceretiusenin Good. - Marais et près tourbeux. - H.-Saône, Doubs ct Jura : étang de Bellerue près Giromagny, Clsenalotte, Guinots, St-Julien, marais de Saòne, les Ponts, Pontarliel, Bélicu, Boujailles, Chapelle-des-Bois, les Rousses, la Trélasse. - II-Sav. et Ain : marais de Lossy au pied des Voirons el de Trège près Annecy, de Ste-Croix près Montluel. - C.-d'Or el S.-et-L. : envir. de Saulieu et d'Autun, Broye, Roussillon, Issy-l'Érêque, bords du canal du centre près Montchanin, Mouthiers-en-Bresse. - Is. : Meyzieu, Grand-Lemps, Grande-Chartreuse.

C. Ineleomstis Ehrll. - Tourbicres de Pontarlier, la Brévine, Bélieu, les Roussez, lac do Joux, Stc-Croix, les Ponts.

C. elonmata L. - Bords des ruisseaux et des mares surtout dans lés terrains siliceux. - Jura et Ain : forêt de la Serre, la Bresse, Ferney. - II.-Saône : Chiampey, Mersuay, Cubrial. C.-d'Or et S.-el-L. : Saulon, Limpri, Montjcu près Autun, Branges pris Louhans. - Vaud : Jongny, Gourze, Sauvabelin, le Jorat, Jois de Bogis près Niyon.

C. ovnlis Good., C. leporina L. - Lieux lrumides des plaines et des montagnes. - Espèce polymorphe présentant de nombreuses variations dans la coulcur, les dimensions et la forme de l'épi.

C. angmoximata Hoppe, C. lagopina Wahlb. - Lieux humides des Alpes. - Valais: Entremont, St-Bemard, Toursson, Arolla, bistelalp el Ofientlal de Saas, Tustain, Simplon, Grimsel, GlaCatal. Bassir du lihône. 
cier du Rhone, Furca. - H.-Sav, et Sav. : Aiguilles-Rouges, Tignes, mont Iseran, mont Cenis.

Lagger a trouvé au Grimscl une forme qui parait liybride entre les $C$. lagopina et $C$. foctida et que Wimmer a nomméc C. Laggeriana. M. Favrat l'a aussi trouvée au Simplon sur le plateau de Hohlicht.

C. stellulata Good. - Prés marécageux et tourbeux des filaines et des montagnes.

C. caneseens L. - Prés el loois humides des monlagnes. - Hautes Vosges jusque dans la H.-Saône au Ballon de Ciromagny, Frahier, Plancher-Bas, Champey, Chagey, puis plus bas sur le grès bigarré et les alluvions. - Doubs, Jura et Ain : Brévine, Ponts, Pontarlier, Mouthe, Andelot, Val de Joux, Rousses, Trélasse, Lélex. - Vaud et Valais : les Mosses, Châtel-St-Denis, Morgins, Erbignon, Thyon, Saas, Belalp, Simplon, Glacier du Rhône. - H.-Sav. ct Sav. : Roc d'Enfer, mont Clalune, Chamonix au Bouchet, col de Voza et Joly, Rosclein, les Allues, entre le Laitelet et l'Haut-du-Pré au dessus de. Conflans, mont Cenis, - Is. : Belledonne vers le lac du Crouzet, lac Luitel, chalets de Gleyzin en montant aux Sept-Laux. - Alp.-Mar. : Ste-Anne-de-Vinaĩ, entre Lupega, et Tanarello. - C. d’Or et S.-et-L. : envir. de Saulieu et d'Autun. - Rh, et Loire : l'drgentiẻre, bords de la Gimont entre Aveyze et Grézicux, Pilat au Pré Lager et au Bessat. - Gard et Ariège : chaîne de l'Espérou, Pic de Mounégou, vallon de la Maouré, Pla de Monpudoux, lacs de Rabassolès et de l'Estagnet. - Espéce polymorphe.

C. vitilis Fries. - Forme du précédent ayant le bec de l'utricule fendu au sommet, et croissant non dans les prés liumides, mais sur les rocliers des Alpes. - Valais : Alesse, Fully, Catogne de Martigny, Guraz de Bovernier, St-Bernard, Simplon, Grimsel. - LI.-Sav. et Sav. : col de Balme, Montanvert, Roc d'Enfer, entre le Laitclet th l'Hant-le-Pré, - Is. : Belledonne au lac du Crouzct et à la Combe de la Lance, col Oddie, lac Robert, Gleyzin.

C. remata L. - Bris et taillis humides des phaines et des montagnes. 
C. Winkiana Schk. - Pelouses, bois de ła région mérid. - B.-du13h., Var et Alp.-Mar. : Bonneveine, la Ciotat au Bec de l'Aigle, 'Toulon, liyères, le Luc, Fréjus, Cannes, la Roquelte, Vallauris, Biot, Monaco, Menton. - Gard : bois de Broussan, de Cygnan et de Campagne près Nimes, Pont-du-Gard à St-Nicolas, Aumessas. - Hér. : Montpellier à Grammont, Lamoure, Doscares, Hireval, St-Bris, Murviel, bois de Valène, Capouladoux, Bézicrs, Cetle, Agde, Pézenas, St-Martin d'Orb, St-Elienne-de-Mursan, Avène. - Aude el Pyr.-0r. : envir. de Narbonne, Perpignan, Argelis, Collioure, Port-Yendres, Banyuls.

C. eurrevin All. - Rochers et pelouses des Alpes et des Pyrénées. - Vaud el Valais: Pointe des Martinets, Alpes de Bex, liully, Bagnes, Zermontana, Ricumatien, Gottier, Vouasson, mont Nuoble, Gemmi, Zermalt, Saas, Grimsel. - II.-Sav, et Sav. : col de Balme, Brévent, Aiguilles-Rouges, chaîne du mont Blanc jusqu'au Jardin et au Couvercle, le Bonlomme, lac Combal daus l'Allée Blanche, Grand Pariraz près IIauteluce, mont Ise. ran, mont Cenis a Ronches et au lac Blanc. - Is. ct II.-Alp. : Bellelonne à la Pra et vers le lac Domenon, Grand Veymont, la Salclle, Pelroux, la Grave, Lautaret, Galibier, Chaillol, mont Viso, col Vieux, St-Féran, Aurouse. - B.-Alp. et Alp.-Mar. : Vallonnet de Meyronnes, mont Grand-Couyer sur Annot, Estenc à lioche-Grande. - Pyr. centrales.

C. eypirodera L. - Iares ct ítangs. - Belfort derrière la Caserne. II.-Saône : étangs des Mlonts-lievaux, entre Franchevelle et StGermain, Grand St-Maurice, élang du Milieu et Grand étang, entre la Chaux el la Chapelle. - Jura et Ain : la plupart des étangs de la Bresse, la Dombes. - C.-d'Or et S.-et L. : Saulon, Citeaux, Collonges, l'A bergement-les-Seurre, la Bresse louhanaise, Gergy près Chalons-sur-S. - Is. : bords de l'étang de Mépieu.

C. bicolor All. - Lieux humides des Alpes. - Valais: Torrembé, Zermontana, Tortain, Thion, Diez, Arolla, col de Fenêtre, Zermatt au IJoernli et à Zmult, Oberstaffel cle St-Nicolas, Saas à Mattmark, Simplon. - Sav. : Laval de Tignes, les Mollets, les Allues, mont Cenis. - II.-Alp. : Villard d'Arène, Galibicr, col 
de l'Échauda et des Hayes, la Traversette, St-Véran, mont Aurouse. - B.-Alp. et Alp.-Mar. : Vallonnet de Mleyronnes, lac de Ligny sur Colmars, sources du Var à la Strop.

C. eaespitasn Gijod., C. Goodenoughiana Gay. - Prés narécageux des plaines et des montagnes. - Espèce polymorplıe.

C. strietn Good. - Marais el fossés dans toul le bassin.

C. aeuta Fries. - Marais, bords des cours d'eaux. - Commun dans les parties septentr. et moyenne du bassin; plus rare dans la région mérid. - Espèce polymorphe dans laquelle la couleur et les diinensions des écailles prísentent de nombreuses variations. Dans la forme prolixa Fries les écailles dépassent longuement les fruits et sont cuspidées, les utricules sont fortement nerviés. - Dans la forme Tourangiana Bor. les écailles ont une couleur d'un brun jaunâtre, dépassent longuement les fruits; ceux-ci ont des nervures peu saillantes.

C. grtanaca Scop. - Lieux humides des plaines et des montagnes. Espèce polymorplie présentant des variations dans la longueur des pédoncules et la longueur des écailles.

C. peraluia Iluds., C. maxima Scop. - Licux liumides; commun dans. les arrond. de Belfort et de Montbéliard, la H.-Saone, Chalezeule près Besançon, forêts de la Serre et de Chaux, Belley et St-Rambert en Bugey, - Envir. de Genève et H. Sav. : StGeorges, Archamp, Crevin, Feigères. - S.-et-L. et Rh. : bois des Renaudiols près Autun, Canada d'Auxy, Anost, mont Beuvrai, Cluny, Rochecardon, entre Ste-Colombe et Ampuis. - Is. et Dr. : Solaise, Grande-Chartreuse, Sassenage, Balme de Fontaine, Pont-de-Beauvoisin, Romans, - Envir. d'Aix, de Marseille, de Toulon, d'Hyères, l'Esterel, bords du Loup et de la Siagne, Nice, Menton. - Gard : Anduze, Alais, Chartreuse de Valbonne, Tresques. - Hér. : Lavérune, Lamalou, Lunas, Pézenas, Bédarieux.

C. mienocarpa Salzm. - Plante de Corse qu'on dit avoir trouvée à Toulon et à Grasse.

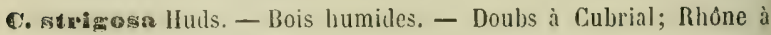
Dardilly, cntre Thizy et St-Jean-]a-liussière, entre Vaux et Avenas. 
C. niba Scop. - Iraies, bois et paturages des terrains calcaires. Jura helvétique et français : Hauenstein, Wasserfall, Noutiers, Moron, Chaumont, Champagnole, la Dole, Colombier, Côtes du Doubs et du Dessoubre, Pont-de-Roide, Mandeure, Mrbois, Salins, Lons-le-Saulnier, Lélex, Oyonnax, Dortan, Nantua, Culoz, StGermain près Belley. - Vaud et Valais : Bex, Sion, St-Léonard, Vex. - II.-Sav, el Sav. : pied du Saleve, les Voirons, Crêt du Maure près Annecy, Poisy, mont du Chal. - Is. et II.- Ilp. : mont d'Annoisin près Crémieu, Jonc, Villette d'Anthon, Grenoble, Comboire, St-Ange, Vif, Gap, Seyne, Embrun, Briançon. - C.-d'Or et S.-et-L. : vallon de Ste-Foix, au-dessus de la Fontaine de Jouvence, Val-Combe, entrée de la Vau de Gevrey, Marcy-sur-Tille, Buxy, entre Rully et Chamilly. - Gard : bois de Salbous près d'Alzon, l'Espẻrou. - Aude ct Pyr.-Or. : collines calc. des Corbières.

C. capullaris L. - Lieux lıumides des Aipes et des Pyrénées, Vaud et Valais : Lavarraz, Bovonnaz, pied des Diablerets, Fully, Catogne de Sembrancher, St-Bernard, Giétroz, Sanetsch, Gemmi, Arolla, Diez, bois de Cythere, Alpes de Zermatt et de Saas, Simplon, Belalp, Grimscl. - II.-Sav. et Sav. : Abondance, col de Balme, Prarion de St-Gervais, N.-D, de la Gorge, cols du Bonhonme, des Fours et Joly, les Mottets, la Gitaz, les Allues, les Bréviaires, montée de Iaval de Tignes au mont Iseran, mont Cenis, - Is. et H.-Alp. : Champrousse, Grand Veymont, Lautaret, col de l'Échauda, Briançon au Chabas, col des Hayes, Péas el Abriès en Queyras, la Traversette, la Taillante, de Fontgillarde au col Agnel, St-Véran, Orcières. - H.-Alp. : Parpaillou, Lauzanier, Serennes, Soleil-Buou, lac d'Allos. - Pyr. centrales.

C. pallescens L. - Bois et prairies humides des coleaux et des nontagnes surtout dans les parties septentr. et mojenne du bassin; - plus rare dans les montagnes du Gard et de l'Hẻr. vers le Vigan, Alzon, l'Espérou, l'Espinouse à Fraisse, StGeniès-de-Varensal, Pardaillan. - Pyr.-Or. à Prats de Ilollo. Canigou, Mont-Louis.

C. olbiengis Jord. - Bois de la région mérid. - Bois des Maures 
près Iyètes, Plan-du-Pont, los Mayons, Cannes, Menton. Ilér. : Argelliers. - Pyr.-Or. : Collioure à Consolation, vallon de Cerbère en montant à la Tour de Quer-lioig.

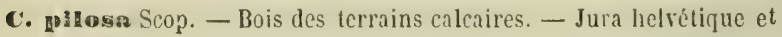
français : le Chaumont, Lassarraz, Bière, Cossonay, Salins, Poligny, Arbois, Lons-le-Saunier, Panessières, bois de Baume, de Montrond et de Moidons jusqu'à Pont d'Héry, commun dans Ics envir. de Belley à Thuy, St-Germain, St-Benoil, Glandieu, Parves et Muzin. - Genève et Vaud : bois des Frères et de la Joux, Onex, Bernex, Vernand, Pompaples, Entreroche. H.-Sav, et Sav. : bains de la Caille, la Puya el le Crêt du Maure près Annecy, bois de Jacob près Chambéry.

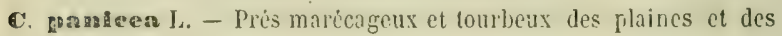
montagnes.

C. natha Host. - Pâturages secs. - Genive, Vaud ct Talais : bords du Léman, du Rhône el de l’Arve, Branson, Saillon, Sion, les Plâtrières, Sembrancher. - Ain : Confort, Meximieu au pâturage de Giron, Balan, la Pape. - Rh. : Vaux-en-Velin, St-GenisLaval, Millery, mont Cindre. - Is, el Dr. : Polygone de Grenoble, Corenc, Laveyron, St-Vallier. - H.-Sáv. el Sav. : bords de l'Arve du Fier et de l'Isère. - H.-Alp. : envir. de Gap. Vaucl. et B.-du-Rh. : envir. d'Avignon et de Marseille. - Ard. et Gard : Mayres, Uzès, Alais, Anduze, St-Ambroix, le Vigan. - Hér. : Lavaletle, Restinclières, Caunelle, la Paillade, StMichel-des-Sers, Madięres.

C. ugtulata Wahlb. - Lieux marécageux des Alpes. - Valais : Torrembé dans lo val de Bagnes, Dicz. - H.-Alp. : bords du Guil au pied du mont Viso, la Taillante autour du lac Foréon. - Ariège : vallon de Barbouillère, lac de l'Estagnet, Port de Paillères.

C. åtrota L. - Rochers el pâturages des Alpes et des Pyrénées. Vaud et Valais : Anzendaz, Pannerossaz, Fully, St-Bernard, Bagnes, Zermontana, Tortain, Zanrion, Sanctsch, Méribé, mont Nuoble, Zan, Vouasson, bois de Cythère, Gemmi, Alpes de Saas, Simplon. - H.-S. et Sav. : Vergy, Méri, Roc d'Enfer, Entredeux-Pertuis près Abondance, col de Balme et d'Anterne, Bré- 
vent, Aiguilles-liouges, la Flégèere, Tré-la-Téte, le Bontromme, les llottets, la Sauce, lac de la Giroltaz, Bas du Mottet all-dessus d'Aime, les Allues, Laval de Tignes, 'lignes, col de la Madeleine, mont Cenis. - Is. : la Pra de Belledonne, Champrousse, Sept Laux, Taillefer, mont Clamoux. - II.-NIp. : Lautaret, Galibier, col des Rochilles sul Névache, mont Viso, Orcières, le Champsaur et le Valgaulemar. - B.-Alp. et Alp.-Mar. : Lauzanier, Mirandol, Alpes de St-Étienne, val de Jallorgues, les Viosennes. - Pyr.-0r. et Ariüge : Canigou, Cambres d'Aze, Prats de Balaguer, Capcir, vallon de la Maouré, Riplaou, val de Paillères. - Pyr. centrales. - Espéce polymorphe.

C. nigra All. - Forme grêle du précédeut à épis tous ovales, dressés, portés par des pédoncules plus courts, à utricules obovés. llemes localités qne te C. atrata, mais plus commun. Valais : Fully, Zanrion, Zermontana, Thyon, Diez, Donin, Gemmi, Guggerhubel, Alpes te Zermatt, de Saas et du Simplon, Tortain, Eginnentlial, Glacier du litıone; s'élève sur les flanes du mont Rose jusçu'à la Cabane de Vincent. - II.-Sav. el Sav. : montagnes du Faucigny, de la Tarentaise et de la Maurienne, notamment sur les flancs du mont Blane où il s'élève jusqu'au Grands-Mulets, au Petit St-Bernard, à la Vanoise, au mont Iseran, sur les sommités des Allues et du mont Cenis. - Is, et II.-Alp. : Belledonne vers le lac Doménon, Sept-Laux, Piéméyan au mont de Lans, Lautaret, Galibier, mont Viso, col Agnel.-B.-Alp. et Alp.-Mar. : Longet, Vallonnet, mont Grand Couyer, mont Garret près Entraunes, mont Bego. - Pyr.-0r. et Ariege : Costa Bona, Pla Guilhem, Cambres d'Aze, lac du Llaurenti, Barbouillère, Campeilles, Roc de la Musique à Valbonne, lac de Quérigut. - Pyr. centrales.

C. aterrima lloppe. - Forme robuste à taille élevée, à tige rude, à épis oblongs-cylindriques. Moins commun que les C. nigra el atrata. - Valais : Zermontana, Orsera, Vousson, Zan, Gottier, Leukerbad, Staffel de Zermatt, Simplon, Mayenwand. - H.-Sav., Sav. et Is. : la Layat près Nant Borant, glacier de Tré-la-Tête, la Pra de Belledonne, les Sept-Laux.

C. Limosa L. - Marais tourbeux des plaines et surtout des mon- 
tagnes. - Hautcs-Vosges jusqque dans la II -Saơne au Ballon de Giromagny, mont de Ternuay, Lantenot. - Chaine jurassique à Pouillerel, Ponts, Brévine, Pontarlier, Bélicu, Chapelle-desBois, Mouthe, les Rousses, la Trélasse, en plaine dans les marais de Pleurre près Dôle. - Vaud et Valais : les Mosses, Sicrne-auCuir près Cuâteau d'Oex, Saas à Mattmark, Grimsel. - Il.-Sav. et Sav. : Lossy au pied des Voirons, Cranves, Chamonix au Bouchet, mont Alirantin, mont Cenis. - Is. et HI.-Alp. : Prémol, Grand-Lemps, Quirieu, col Bayard près Gap. - C.-d'Or : Saulieu, St-Léger, Limpré. - Gard : Gourdouze près Concoule. En dehors de nos limites dans la Lozère, Aubrac, Jlargeride, mont Dore, Creuse, Mnrvan nivernais, Forez.

Une forme irrigua Sm. a les épillets plus courts et les feuilles planes.

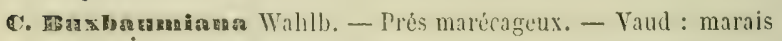
d'Orbo. - Rh. : l'Argentière. - H.-Alp : mont Bayard près Gap. - En dehors de nos limites en Alsace.

C. Inisgada Willd. - Marais de la région mérid, - Var et Alp.Mar. : Sablettes de Toulon, Hyères, le Luc, Fréjus, Cannes, golfe Jouan, Nice, Vintimiglia. - Ilér. : Mireval, Vic, Mauguio, à St-Marcel et au Mras-le-Larot, Pérols, Lavalette, Lansargues.

C. precox Jaci. - Pelouses sèches, - Espèce polymorphe à taille variable, ayant quelquefois un ẻpi gynobasique.

C. polyrrhiza Waltr. - Forme cespiteuse, non stolonifere, à feuilles égalant ou dépassant les tiges, qui vit, non sur les coteaux secs comme le type C. precex, mais dans les bois humides des terrains siliceux des parties septentr. et moyenne de notre bassin.

C. Comentosa L. - Bois et prairies humides des terrains argileux ct marneux.

C. polubifera L. - Bois humides des terrains silicenx. - HutesVosges jusque dans la H.-Saône à Champagney, Écromagny, Franchevelle, Linexcrt, Henoux, Amance.. - Lisière vosgienne des arrond. de Belfort et de Montbéliard, Chalezeule près Bcsançon, Salins, Arbois. - Vaud : Tourbière de Vraconnaz près Ste-Croix, molasse de Payerne. - H.-Sav, et Sar. : Ics Voirons, Vergy aux Planets, les Villards sur Thônes aux Cabassières, 
Haut-du-Pró au-lessus do Conflans, mont Mirantin au Laitelet. - Is. et II.-Alp. : Chambaran, Flachere, Eyúoche, Prémol, la Garde ct Clarance pris Gap. - C.-d'Or et S.-et-L. : coteaux granitipues du Morvan, Saulieu, Boncourt, St-Bernard, Val des Choues, collines granitigues de S.-CL-L. - Rh. et Loire : région granitique du Lyonnais, Charbonnières, Dardilly, Larajasse, l'Argentière, Pilat et Pierre-sur-Haute, - Ard., Gard et Hér. : chaîne du Vivarais, Gerbier-des-Jones, Nìmes, le Vigan, Alzon, Concoule, Ic Srumail. - Pyr.-Or. et Ariège : Cérel, StLaurent-de-Cerdans, Cornella-du-Conflent, lacs du Laurenti, cirques d'Artounant el des Aiguettes, vallon de Boutadiol.

C. menabranneese Ioppe, C. ericetorum Poll. - Pelouses séches des terrains siliceux. - Vaul et Valais : Dent de Jaman, les Verraux, mont d'Alesse, Alpes de Morcles et de Fully, Bagnes, St-Bernard, Lens, Sanetsch, Evolena, Cotter, Zermatt, Taesch, Alpes de Mühlibach, Simplon. - Sav. : mont Iseran, mont Cenis à Ronches, à Pattes-Creuses et au lac Blanc. - H.-Alp. : Villard d'Arène, Lautaret, Briançon au Poüt.

C. montana I. - Bois et pelouses des terrains calcaires. - Chaîne jurassique jusque dans le Bugey. - Collines calc. de Vaud, BasValais, Savoie, Dauphiné, B.-Alpes. - Calcaires jurassiques de Côte-d'Or, Mont d'Or Iyonnais. - Gard et Hêr. : bois de Salbous, Dourbie, l'Espérou, entre Madière et Navacelle, - Aude et Pyro-Or. dans les Corbières.

C. baeilarla Jord. - Lieux humides du Var et des Alp.-Mar. : cap Croisette près Cannes, Grasse à St-Jean, la Roquelte, Menton.

C. mynobasis Vill. - Bois des collines et des montagnes calcaires.

- Calc. jurassicque de H.-Saone au Sabot de Frotey, Nans, Goudenans. - Toute la chaine jurassique jusque dans le Bugey et le Revermont. - C.-d'Or jurassique et Mont-d'Or Jyonnais. - Montagnes calc. de Vaud, Bas-Valais, Savoie, Dauphiné, B.-Alpes, Provence et Alp.-Mal', - Calc. jurassiquc des Corbières. - Nul dans les Vosges et les montagnes à sol siliceux du centre de la France.

C. wedipostyla Dural-Joure. - Coteaux secs du Var el de l'Hér. : Maures du Luc et du Cannet, Montpellier à Lamoure, Montarnaud, Argelliers, Mas-de-Londres à Gabriac. 
C. brevicollas DC. - Páturnges, bois taillis, - Ain : montagne de Parres au-deseus de Coron, Tenay sous les rochers d'Hostiaz. - Aude: entre Durban et Tuchan dans les Corbières. - En dehors de nos limites au puy de Wolf près Decazeville (Areyron). - Banat au mont Domugled près des Thermes d'Hercule, Château Versec dans le Comté Temes, mont Siméon près Csiklova, mont Skofayna près Mogdan, mont Tilfamare près 0ra. vicza. - Transilvanie dans le Creux de Thorda, - Serbie à Ravanica dans le cerele Cupria.

C. Eumails Leyss. - Coteaux secs des terrains calcaires ou des terrains de transport contenant des cailloux calcaires.

C. digitata L. - Bois des partics septentr. et moyenne du bassin surtout dans les terrains siliceux. - Espèce polymorphe.

C. ornilhopoda Willt. - Forme du précedent, particuliere aux terrains calcaires, ayant les épis rapprochés, les pédoncules inclus dans les gaines des bractées, les utricules dépassant les écaillles. Nul dans les Vosges granitiques et arénacées oủ le $C$. digitata est très-commun. - Assez commun dans le Jura helvétique et français jusque dans le Bugey, - Calcaire jurassique de C.-d'Or, S.-et-L., Mont-d'Or lyonnais. - Montagnes calcaires de Vaud, Valais, Savoie, Daupliné, B.-Alp.; plus rare dans les Alp.-Mar. — Gard à l'Aigual et à l'Espérou. - Aude, Pyr.-0r. et Ariège : collines calc. des Corbières, Escale de Valbonne, Boutadiol, Counq, Canals.

C. mueronata All. - Pelouses et rochers des montagnes. - Sav., Is. et H.-Alp.: mont Cenis, col de l'Arc, petite Houcherolle, Lautaret, col de Glaise, mont Bayard, Séuse, Aurouse, Poligny en Champsaur. - B.-Alp. et Alp.-Alar. : Serennes au rocher du Pin, les Viosennes, Lupega.

C. Frigida All. - Licux humides des hatites montagnes à sol siliceux. - Vaud et Valais : Lavarraz, Alesse, Fully, St-Bernard, Méribé, Giêtroz, Zanrion, Prasgras, Loveigne, Blattenalp et Loemeralp, Alpes de Zermatt et de Saas, Simplon, Grimsel. H.-Sav, et Sav. : chaine du mont Blanc jusqu'au col du Bonhomme, les Mottets, col du Cormet, Bas du Mottet, mont Cenis. - Is. et H.-Alp. : Belledonne, Combe de la Lance, Sept-Laux, 
Poursollet au pied de 'Taillefer, la Berarde, Lautarct, Galibier, Champolén, Orcières, Valgaudemar, col Mairif, Péas en Queyras, vallée du Guil, col de Vars. - B.-Alp. el Alp.-Alar. : Lauzanier, Longet, sommités des vallées du Var, Vesubia, 'Tinea, loya, - Gard: Ilort-de-Diou près de l'Aigual, Valat de la Dauphine près de l'Espérou. - Pyr.-Or. et driège: Canigou, l'la Guilhem, cirques des Aiguettes, Sarrat d'en Binada, lloc de Campeilles, vallon de Boutadiol, Roc de l'Estagnet. - En dehors de nos limites dans les Pyr. Centrales, dans les Vosges au IIokneck. - Nul dans la chaine jurassique.

C. Inpidula Gaud. - Rocailles et pelouses des $A l$ pes. - Valais : Bagnes, Zermatt au Riffel et à Augstelberg. - H.-Alp. : la Grave, Villard d'Arène, Lautaret.

c. Cerruglnea Scop. - Patturages humides des montagnes surtout dans les terrains calcaires. - Chaîne jurassique à la Dôle, Reculet, Brassus. - Vaud et Valais : Alpes de Bex, Boronnaz, Diez, Lens, Leukerbad, Gietroz, Saas, Zermatt. - II.-Sav. et Sav. : Salève, Vergy, Méri, Glacière du Brizon, Colone, Roc d'Enfer, col d'Anterne, Chamonix, Parmelan, Semnoz, Tournette, le Mole, Grest-Voland, les Moltets, mont Cenis. - Is. ct II.-Alp. : col de la Ruchère, forêl des Fraux, Lautaret, bois de Rabou et Loubet près Gap, la Bâtie-Neuve, Durbonnas, - B.-Alp. et Alp.Mar. : la Condamine aux Tardées, Entraunes, col de Fenestre, vallon de Libarẻ près Venanson, - Vaucl. : mont Ventoux.

C. semperviresis Vill. - Pâlurages des montagnes calcaires. Jura helvêtique et français : Weissenstein, Chasseral, Chasseron, Creux du Van, Mont-d'Or, Suchet, Montendre, Colombier, Dôle, Reculet, Colombier łu Bugey, Monts d'Ain. - Vaud et Valais : Anzendaz, Loze, Sanetsch, Sasseneire, Diez, Comba d'Arbaz, mont Nuoble, Gemmi, Mayenwand, Saas, Zermatt, Simplon. - H.-Sav. et Sav. : Pitons et Grande Gorge du Salève, Brizon, Vergy, Méri, mont de Pelloua, Dents d'Oche, Brévent, Jardin de la Mer-de-Glace, col Joly, Somans, Tournette, mont Cenis. - Is, et H.-Alp. : Grande-Chartreuse, Clsamechaude, St-Nizier, col de l'Arc, la Moucherolle, la Saletle, l'Obiou, la Grave, le Briançonnais, Chabrières, Charance, Bayard el Chau- 
dun près Gap, Séuse, Aurouse, Chaillol-le.Vicil. - B.-Alp. cl Alp.-Mar. : Lauzanicr, Croues, col 'd'Allous, Entraunes, Alpes de Jallorgues, de Salèse, Je Fenestre et de Tende. - Vaucl. : mont Ventoux. - Pyr,-Or. et Ariège: Carença, valléce d'Eyne, lac du Llaurenti, Boutadiol, Pic de Canrusc. - Pyr. Centrales.

C. frma Host. - Rocailles des Alpes. - Vaud et Valais : Anzendaz, Panerossaz, Fully, St-Bernard, Menouve, Simplon, Grimsel. H.-Sav. : Balafra au-dessus du Brizon, Vergy, Roc d'Enfer. -

- Is. et H.-Alp. : Belledonne, Champrousse, mont Viso, la Taillante. - B.-Alp. : Ste-Anne, Lauzanier, Malemort, Croués.

C. tenuis Host. - Rochers des montagnes calcaires. - Jura helvétique et français : Paswang, Hauenstein, Weissenstein, Tête deRang, Creux-du-Van, Mont-d'Or, Chasseron, Cótes du Doubs et du Dessoubre, St-Julien, rochers de Fleurey et de Saint-Hippolyte, la Dole, Colombier, Reculet, St-Claude à la Roche-Blanche, Petites-Chiettes, Nantua entre Malbroude et les Neyrolles. Vaud, Valais ct H.-Sav. : St-Cergues, Giétroz, Pitons du Salève, Colone, Brizon, Veyrier, près Annecy. - Sav. et Is. : les Échelles, mont du Chat, Bovinant, St-Nizier, Petite-Moucherolle, les Fauges de Villard-de-Lans, Prémol, Revel. - B.-Alp. : Parpaillon, Lauzanier. - Rochers calcaires des Pyrénées el de la Lozère.

C. Sllvatica Huds. - Bois des montagnes.

C. depanperata Good. - Bois. - Doubs : Arbouans près llontbéliard, Montbart, bois de Chailluz près Besançon. - Rh. et Is : entre Royes et Fontaines, parc de Vizille. - S.-et-L : Brancion, Praye près Chissey. - Gard et Hér. : Salbous près d'Alzon, Corconnes, St-Mlartin-de-Londres aux Arcs, Pic St-Loup, le Larzac à St-Naurice. - Centre et ouest de la France.

C. hordeifonmis Wahlb. - Fossés et marais. - S.-ct-L. : Charolles. - H.-Alp. et Dr. : entre Gap et Charance, St-Julien en Champsaur, Buissard, Lus-1a-Croix-Haute. - Var : bois des Haures près du Luc. - En dehors de nos limites dans Lozère, Puy-de-Dôme, Loire, envir. de Paris, la Lorraine.

C. Aava L. - Prés marécageux. - Espèce polymorplıe variant quant à la grosseur des fruits, la couleur des écailles, l'étal 
lisse ou rugueux des liges. Le fruit est quelquefois sans achaines.

C. divaricata, C. OEderiana Ehrh. - Forme ì fruits plus petits, non réfléchis, à bec droit. - Souvent mêlé au type.

La variété le pidocarpa 'Tausch, à fruits petits et à bec recourbé, à écailles brunes, est intermédiaire entre le $C$. flava et la forme diraricata.

C. Mniriana Cosson et Germ. - Lieux humides de l'Hérault à Ganges, Lodève, vallon de Lergue à Pégayrolles-de-l'Escalette, entre Madière et Navacelle. - Alp.-Mar. : vallon de Chaux près Touët de Beuil, Nice au Vallon Obscur. - En dehors de nos limiles dans la Marne, le bassin de Paris, l'Orne, la Vienne, Charente inférícure.

C. Hornsehaehiana Hoppe. - Pris maricageux et tourbeux. Manque dans la région mérid., excepté aulour du Vigan el de St-Gilles (Gard). - De méme que dans les C. flava et distans, les fruils du G. Ilornschnchiañ sont quelquefois dépourvus d'achaines, ce qui a porté quelques botanistes à soupconner l'hybridité des individus stériles, et comme ceux-ci ont ordinairement des fruits d'un fauve pâle ou jaunâtres, on leur a donné les noms de C. fulva, C. xanthocarpa.

C. Aisınne L. - Prés marécageux, fossés dans lout le bassin. Espèce polymorplie présentant, entre autres, une forme $\left\langle C^{\prime}, b i\right.$ ncrvis Sm.) à écailles d'un brun foncé, à utricules rougeâtres n'ayant que deux nervures.

C. extensa Good. - Sables maritimes, prés du lilloral de la Provence, des Alp.-Mar., du Languedoc et du Roussillon. - Côtes de l’océan.

C. punetata Gaud. - Lieux humides de la région mérid. - Var ct Alp.-Mar.: Toulon, Hyires, ile de Porquerolle, le Luc, Cannes, Antibes, Nice au Var, Contes. - Hér.: St-IIartin d'Orb, Pardailhan, de St-Pons à la Salvetat. - Aude: Martis. - Littoral de l'ocían.

C. Inevigata Sm. - Lieux couverts des terrains tourbeux. - C.d'Or et S.-et-L. : envir. de Saulieu à l'étang des Vermouraux, bois de Saubiot, Menessaire, Marcy-sur-Tille, Roussillon, bois 
des Grandes-Queues, St-Prix, mont Beurrai. - Var: environs d'Hyères et du Luc. - Hér. : Fraisse et la Salvetat. - Aude: Mas-Cabardès, Martis.

C. Iomganractenta, C. pseudocyperus L. - Licux marécageux des terrains siliceux el argileux. - Doubs, Jura et Ain : envir. de Montbúliard et de Baume, plaine bressanne, Pleurre, forêt de la Serre, envir. de Bourg, St-Didier-sur-Chalaronne, Virieu-leGrand. - C.-d'Or et S.-et-L. : Saulieu à Champmorin, Quincey, Saulon, Brogny, Ecuelles, Verdun, Millery près Autun, Bran. don, Perrecy, Charolles. - Rh. et Is. : Vaux-en-Velin, Meyzieu, Hières près Crémicu, les Avenières, Grand-Lemps, Gières. Dr.: étang de Suze près Grignan. - B.-du-Rh. et Var: mares de Raphèle près Arles, la Foux près la Garde. - Gard : envir. de Nimes, de Manduel et de Bellegarde. - Hér. : Mireval, Mauguio à St-IIarcel. - Pyr.-Or.: Salses, Canet, St-Cyprien, Argelès.

C. ampullreer Good. - Prés marécageux.

C. vesicarin L. - Narais, fossẻs.

C. Baratiosa Guod. - Bords des eaux, marais. - Espèce polymorphe. Dans la forme Kochiana Gaud. l'écaille est longuement cuspidée.

C. riparia Curl. - Bords des eaux.

C. nutang Hosl. - Lieux marécageux. - C.-d'Or et S.-ct-L.: Scurre, Auxonne, Chalon. - Rls, et Ain : Lyon au Grand-Camp, Pierre-Bénite, Anse, Quincieu, Reyrieux, Trévoux à l'île Beyne, Thoissey. - Is. : Meyzieu, Bourgoin. - En dehors de nos limiles dans la Loire-Inférieure.

C. Anifozmais L. - Narais et tourbières. - II.-Saône: étang Billaux près Lantenot. - Doubs et Jura : Bélieu, Chenalotte, Guinots, St-Julien, Val de Joux, Rousses, Trélasse, Brévine, St-Laurent-en-Grandvaux. - C.-d'Or et S.-et-L. : Saulieu à l'étang Fortier, Collonges, Montjeu près Autun. - Vaud: Noville, Jonguy, Gourze, Ie Sentier, Seedorf près Payerne, marais de Roellebot. - Ain et Is. : les Échets, Heyzien, la Boucherette près Genas, Charvieu, Grand-Lemps.

C. Hheta L. - Lieux humides dans tout le bassin. 


\section{GRAMINEES}

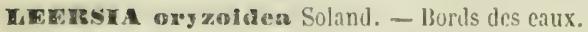

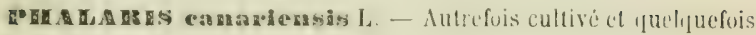
subspontané.

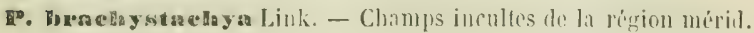
- Vaucl. el B.-du.1h. : envir. d Avignon, de Marseille aux Catalans et à St-Charles, Roquevaire. - Var et Mp.-Mar. : St-Nazaire, le Luc, Fréjus, Cannes, Grasse, Antibes, Nice, Nenton. - Ilér.: Montpellier au-dessus de Figuérolles, Baillargues, Maguclone, Méze, Manguio à St-Marcel, Cette, Agie, Candillargues, - Aude et Pyr.-Or.: envir. de Narbonne et de Perpignals.

R. minoz Retz. - Champs sablonneux de la région mérid. - B.du Rh. : envir. d'Aix sur les bords de l’Arc, la Trinité, Marseille sur les hords de Jarret, St-Loup, Martigues. - Var et Alp.Mar. : Toulon, Hyères, le Luc, Nice, Menton. - Hér.: Montpellier au Polygone, Mireval, les Aresquiers, Celte, Laltes, Frontignan, Agde. - Aude et Pyr.-0r. : envir. de Narbonne et de Perpignan, Canet, St-Nazaire, Argelès. - Litloral de l'Océan depuis les Landes j’ısqu'à la Bretagne.

Les $P$. crypsoidea d Urv. et truncala Guss. ont été vus autrefois près de Toulon et de Marscille.

T2. pandoxa L. - Champs cullivés de la région méril. - B.-duRh.: bords de l'étang de Marignane, envir. de Marseille. Var et Alp.-Mar. : Toulon, Hyères, Frẻjus, Cannes, Grasse, Antibes, Nice. - Hẻr. : Montpellier à Rondelet, Montels, Frontignan, les Aresquiers, Mauguio, Lattes, Roquchaute près Vias, Nissan. - Aude et Pyr.-Or. : envir. de Narbonne, Ste-Lucie, la Nouvelle, Salses, Perpignan. - Le Lot, la Gironde et la Vendie.

E* caruleseens Desf. - Lieux humides le la rẻgion mérid. B.-du Rh. : envir. de Marseille aux Calalans, bords de Jarret, Belle-de-Mai. - Var et Alp--Ifar.: Toulon, IIyères, le Luc, Frẻjus, Cannes, Grasse, Antibes, Vaugrenier, San-Remo sur la côte italienne. - Hér.: Bézicrs, Cette, Montpellier à Grammont et au Jas de Comte. 
P. nodosa L. - Bords des chemins et des champs de la région mérid. - B.-du-Rh. : envir. de Marseille aux Catalans, bords de Jarret et de l'Iluveaume, Belle-de-Hai. - Var et Aip.-Mar.: talus du chemin de fer entre Toulon et la Garde, Iyères, ile de Porquerolles, le Luc, Cannes, Grasse, Antibes. - Hér.: Montpellier vers le Pré d'Arène, lá Paillade, Châteaubon, Mireval, St-Aunès, Mauguio, Villencuve, Maguelone, St-Jean-de-Védas.

FP. aruatuacea $\mathrm{L}$. - Bords des rivières dans tout le bassin.

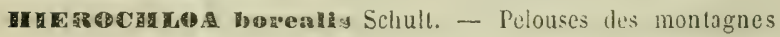
des B.-Alp. au Plan de Barcelonnetle, I’as-de-Grégoire près Jausiers, Larche.

X. W bois des plaines et des montagnes. - Espèce polymorplie présentant quelquefois des épis velus à clıaumes rudes $(X$. villosum Dum.).

X. aristatum Boiss., X. Puclianum Lecoq el Lam. - Forme à épi lâthe, à fleurs stériles, velues, beaucoup plus longues que les fertiles, l'inférieure longuement aristée; racine annuelle, plus rarement bisannuclle.

Ciest à tort, suivant moi, qu'on a roulu élablir une distinclion entre lo $X$. Puelianum el le $X$. aristatum. Celui-ci est un ètat luxuriant, à panicule plus ample, à épillets plıs grands, à chaumes plus robustes et plus èlerés.

CHA Champs et vignes des terrains siliceux. - Nul dans la partic septentr. du bassin. - Commun dans la région granitique du Lyonnais, sur les sables de transport de Décines, St-Aiarcellin (Is.), St-Vallier, Crest et Nyons (Dr.). - Champs sablonneux de la Provence, du Languedoc et du Roussillon.

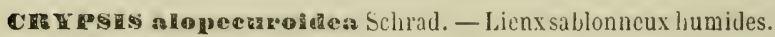
C.-d’Or et S.-et-L..: Citeaux, étang de St-Seine en Bâche, St-

(1) Chamagrosits signifiant Agrostis nain, il y aurait pléonasme à dire, avec Borkhausen, Chamagrostis minima. C'cst pourquoi, tout en conservant le genre Chanagrostis créé par Borkhausen, j'ai adopté l'épithète verna employée par Palisot de Beauvois. 
Léger, Boncour pris Nuils, Cluny, Malay, Chagny, Montchanin, bords du canal du Centre. - Jura : alluvions du Doubs pris Chaussin, étang de l'Abergement prés Neublans, - Ain et Rh.: bords de la Saone à Thoissey, Pont-de-Vaux, Trévoux, Collonges, lle de Royes. - L'Ouest, depuis la Gironde jusqu'ì la Bretagne.

C. seladenoiden Lam. - Licux humides. - Vaucl. : cnvil. d'Avignon vers les mares de Petile-IIotesse et de la Berthelasse. Var et Alp.-Mar.: Toulon, Gonfaron, Fréjus, Nice au Var. Gard et Hér. : envir. de Nìmes, Grammont près Montpellier, Plades, Villeneuve, I.attes, Balaruc, Cette, Agde. - Littoral de l'Aude et des Pyr.0r. - L'Ouest depuis la Gironde jusqu'à la Brelagne.

c. meuleata Ait. - Lieux humides. - Var et Alp.-Jar. : Toulon, Hyères, Früjus, Grasse, golfe Jouan, Nice aux Grenouillères. Gard ct Ilér.: Aigues-Nortes, Lattes à Maurin, Nauguio à StMarcel, Pérols, Maguelone, Cette, Balaruc. — Litloral de l'Aude et des Pyr.-Orientales. - L'Onest depuis la Gironde jusqu'à la Bretagne.

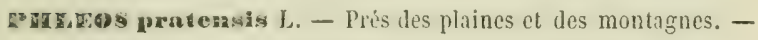
Espèce polymorphe présentant une variété nodosus L. à colle renlté en bulbe, très-commune dans la région mérid. et aussi dans quelques localités de la partie moyenne du bassin, comme par exemple, dans les envir. de Lyon oủ MI. Jordan a observé trois formes principales, l'une précoce ( $P$. procox) ayant un épi ovale et court, l'autre tardive ( $P$. serotimus) ayant un épi plus grêle et plus allongé. - Entre ces deux formes et le type $P$. pratensis se place une forme interméchiaire ( $P$. intermedius) ayant des feuilles plus larges, une taille plus élevée et des épis plus longs que les $I$ '. proecox et serotimus, lesquels vivent sur les terrains sablonneux, secs et arides, tandis que le $P$. intermedius et surtout le $\Gamma$. pratensis type croissent, l'un dans les pâturages fertiles, l'autre dans les prés humides ou artificiellement irrigués.

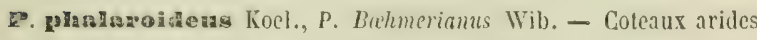
dans une grande partie du bassin.

Catal. Bassin du Rhônc. 
F. 8sper Jacr. - Bords des chemins el des champs secs; plus rare dans la région méridionale.

TP. alpônas L. - Pâturages des montagnes, - Chaine jurassique, Alpes de Vaud, Valais, Savoie, Dauphiné, B.-Alp. et Mlp.-IIar. - Pyr.-Or. et Ariège. - C.-d'Or et S.-et-L. - En dehors de nos limites dans les Pyr.-Centrales, Cantal, mont Dore. - Nul dans les Vosges et les Cévennes. - La présence du $P$. alpinus dans les montagnes peu élevées de C.-d'Or et S.-et-L. est un fait de Géographie botanique digne de remarque.

Une forme naine, $P$. commulatus Gaud, à épi ovale ou presque globuleux, à ligule très-courte, se trouve dans le Valais au Proz, Arpettaz d'Anniviers, Alpes d'Hérens et d'Hérémence, Zermatt, Saas, Simplon, Leukerbad, Glacier du Rhône; dans la H.-Sav. et Sav, au Roc d'Enfer, Sixt, Flumet, Petit-Mont-Cenis.

RP. Hix.satas Suter, P. Michelienus All. - Prairies des montagnes. - Jura helvétique et français : Chasseron, Suchet, Chasseral, Crcux-du-Van, Aiguillon, la Dóle. — Vaud et Valais : Bovonnaz, Lourtier de Bagnes, Mayens de Sion, Binn, - H.-Sav. ct Sav. : Brizon, le Reposoir, Samoëns, Aiguilles-Rouges, col du Cornct près le Chapieu, Tignes, mont Granicr, mont de Fréjus, mont Cenis. - Is. et H.-Alp. : col de la Ruchère, Clıamechaude, col Vert, col des Hayes, le Noyer et Chaudun en Champsaur. B.-Alp. et Alp.-Mar. : Lauzanier, Bérard, Bachasse, Crouès, Alpes de Saint-Étienne, de Lantosque ct de Tende.

FP. arenarias $\mathrm{J}$. - Licux sablonneux des parties moyenne ct mírid. du bassin; manque dans la partie septentrionale.

Tr. tenuis Schrad. - Champs de la Provence et des Alp.-Nar. : envir. d'Aix, de Marseille, Toulon, Hyc̀res, la Roquebrussanne, Fréjus, Cannes, Antibes.

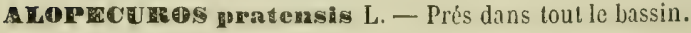

A. agrestis $L$. - Champs et vignes dans tout le bassin.

A. genieulatus L. - Marais, lieux humides.

A. fulvus Sm. - Forme glauque à arête égale aux glumes, qu'on trouve dans quelques marais des terrains siliceux et argileux. - II.-Saone, Doubs et Jura : les Guidons près Mélisey, la Mer, Champagney, Cubry, Franchevelle, vallée de l'Ognon, de Saône 
๑ Or. et Ariège : Canigou, Carença, vallée d'Eyne, Collada de Nuria, vallée de Llo, col de Lègue, Jasse de la Bentaillole, Pic de Baxouillade. - Pyr.-Centrales.

Cette Graminée a étẻ trouvée dans les Alpes du Valais au mont Catogne. Elle existe dans les Alpes granitiques des Grisons, puis plus à l'est, dans les Alpes de la Bavière, Tyrol, Salzburg, Styrie, Carinthie, Iongrie et Transilvanie.

- pellemontana Boiss. el Reut. - Rochers des B.-Alp. et Alp.Mar. : mont Viso à la Traverselte, Estenc à Roche-Grande, mont Tenibre au-dessus de St-Elienne, lacs du col de Salèse, du Mercantourm et d'Entrecoulpes, cols de Fenestre et de Fremamorta, vallée du Clapier entre le sommet de l'Abisso et le col de Tende. SCH耳 de la région mérid. - Remonte dans la Drôme, les H.-Alp. et l'Isere à Barnave, Aucelon, Claps du-Luc, Gap, Veynes, Rosans et Vienne. - S'étend dans le bassin de la Garonne et l'ouest de la France jusque près de Nantes.

CENCHE⿱ bords des chemins dans les partics mérid. et moyenne du bassin jusque vers Grenohle, St-Jean-de-IIaurienne et Lyon, puis dans Vaud et Valais vers Bex, Fully, Charrat, Montorge, Tourbillon et les Plâtrières.

SET Th glauea P. de Beaur. - Champs cultivés dans tout le bassin.

5. viridis $\mathrm{P}$. de B. - Forme à glumelles non ridées en travers, la supéricure plus courte, à soies vertes ou purpurincs. - Hème dispersion.

S. verticillata P. de B. - Lieux cultivés.

Le $S$. ambigua Guss., quelquefois mçlẻ aux deux précédents, a l'épi interrompu à la base comme le $S$. verticillata, mais les pointes des soies sont dirigées en haut et non accrochantes, ce qui a donné lieu de croire qu'il est peut-être lıybride.

S. itallere P. de B. - Cultivé dans le midi do la France et quelquefois subspontané.

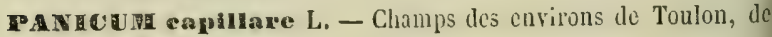
Nice à St-Roch et au Var. 
Lo P. dilatatum Poir., originaire du Brẻsil, est depuis longtemps naturalisé au Luc, au Cannot et à Entraigues près Vidauban (Var).

P. repena - Lieux sablonn. des envir. de Toulon, Ifyères aux Pesquiers.

miliaceum L. - Cultivé et souvent subspontané.

polystaehyum, P. Crus-Galli L. - Lieux humides dans tont le bassin.

P. sangaranale L. - Lieux cultivés. - Espéce polymorphe; dans la Torme ciliare le bord des glumelles est hérissé de cils blanchâtres.

- gianerum Gaud. - Lieux sablonncux. - II.-Saơne, Doubs, Jura, C.-d'Or et S.-et-L. : Clıampagncy, Chasscy-les-Scey, Bonnal, la Bresse, Loulans, Autun, Nuits. - Envir. de Genère, Nyon, Lyon, Virieu-le-Grand, Faverges, Grenolble. - Gard et Hér. : Nimes, Alais, Uzis, Fraisse, la Salvetat. - Pyr.-Orientales.

- vaginatum Sw, - Plante ćtrangìre naturalisće dans l'Hér. autour de Bédarieux, sur les bords de l'0rb, à Lattes, ainsi que dans une grande partie de la vallée de la Garonne et de quel. ques-uns de ses aflluents, puis vers Biarritz et Bayonne.

Yrovon repens, $C$. dactylon Pers. - Lieux incultes, bords des chemins.

Paitena versicolor Fabre. - Sables du littoral méditerranéen.- Var et Alp.-Mar. : Ceinturon d'Hyẻres, Frẻjus, Cap Croisette près Cannes. - Hêr. : Maguelone, Palavas, les Onglous, Sérignan.

NDEOPOGON polystaeñyus, $A$, Ischuemum $\mathrm{L}$. - Bords des champs et des chemins.

provineialis Lam. - Indiqué par Garidel à l'Estaque-Séon, au pied de la Sainte-Victoire, et par Robert à Garduèle près Rians; n'a pas élé retrouvé dans les susdites localités.

distnehyus L. - Licux pierrcux de la région mérid. - Var el Alp.-IIar.: Toulon, Fréjus, Cannes, Cabasse, Antibes, Nice, Nenton. - Pyr.-Or.: Salses, Collioure, Port-Vendres, Banyulssur-lier.

maerostachyum, A. Altionianus DC. - Lieux pierreux de la 
région littorale. - Alp-NIar. : entre Nice et Villefrancle, entre Nonaco et Mala, Menton au Pont St-Louis. - Pyr.-Or. : Collioure, Port-Vendres, Banyuls.

A. verticilstus, A. gryllus L. - Lieux secs. - Vaud : collines du Montet et du Devens près Bex. - Petit-Mollard de la Pierre. Frite à Décines près Lyon. - Carpentras (Vaucl.). - B.-du-Rlı.: envir. d'Aix, vis-à-vis des Moulins-Forts et sur la rive droite de l'Arc, les Infirmerics, Château de St-Pons, Roquefavour. Var et Alp.-Nar.: Toulon, Fréjus, Le Biot, Nice, Menton. Gard : St-Nicolas, Dions, Aigues-Mortes, Anduze, Alais:- Hér.: Montpellier à Lamoure, Fontfroide, St-Marcel, Grabels, Valmargues, St-Brès, St-Mlartin-de-Londres, Prades à la source du Lez, Montarnaud, la Sérane, les Cambrettes, Pézenas.

A. Hintus L. - Lieux pierreux de la région mérid. - B.-du-Rh. : l'Estaque, Cassis. - Var et Alp.-Nar. : Toulon, Hyères, Fréjus, Cannes, Grasse, Antibes, Nice, Nenton. - Gard : Fort-lesBains, - Pyr.-Or. : Salses, Cases-de-Pena, St-Antoine-de-Galamus, Prades, Villefranche, Vernct-les-Bains, Olette, Fontpédrouse, Paulille, Banyuls.

A. hubeseens Vis, - Lieux arides, - B.-du-Rh. el Alp.-Mar. : la Ciotat, Toulon, Hyères, Bormes, Cannes, Antibes, Nice, Henton. - Aude et Pyr.-Or.: Narbonne, Port-Yendres, Céret, Arles, Villefranche.

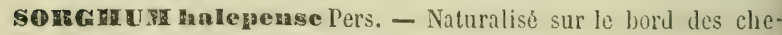
mins el des champs de la région méricl.

5. vulgare Pers. - Cultivé dans le midi de la France.

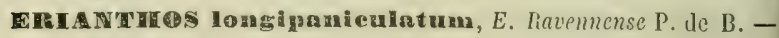
Bords de la mer et des rivières de la région mérid. - Vaucl. : graviers de la Durance, Avignon. - B.-du-Rì. : Fos, Istres sur les bords du canal de Grignan, Arles sur les bords du Rhône, Aix à Saint-Canadet. - Var: Toulon, Hyères, St-Aigou, Fréjus. - Gard et Hér. : Aigues-Hortes, Mauguio à Lamotte, Cclte, Vias, les Onglous, Roquehaute. - Pyr.-0r. : étang de Salses.

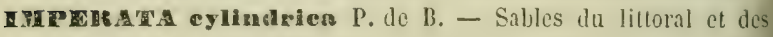
rivières, - B.-du-Rh. : Fos, la Camargue, Hontredon. - Var et Alp.-Har.: Sablettes de Toulon, Hyères, la Napoule, golfe 
Jouan, Antibes, Nice, Menton, pointo du Cap Murtola. - Gard : Aigues-IIortes, Grau-du-Roi, bords du Gardon au Pont-lu-Gard et à la Beaume, lles du lihơne à Vallabrigues. - Hér. : plages de Mauguio et de Perols, Celte aux Salines et aux Onglous, Sérignan à Valras. - Aude et Pyr.-Or. : littoral entre Narjonne et Perpignan.

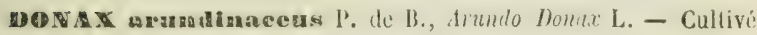
dàns la rén. mérid.

ש. Enazedtandeas Rocm. Schult. - Licux frais. - Var et Mlp.Mar. : Fréjus, St-Raphaül, autrefois au golfo Jouan et à Antibes. - Aude et Pyr.-Or. : Stc-Lucic, Salses à ta Font-Estramer.

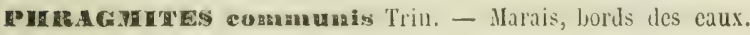
- Espèce polymorplie.

12. giganteus Gay. - Forme robuste à taille plus élevée, à épillets plus gros et plus larges, ayant des lleurs à glumes mucronées, tridentées. - Pyr.-Or. : Salses à la Font-Estramer. - Hér. : Lattes, Maurin et Vic.

P. ruscinonensis Mabille. - Plaine du Roussillon, marais de Salses en face de la Font-Estramer.

P. splendens Timb. Lagr. - Salses (Pyr.-Or.).

P. marilimus Mabille. - Salses, étang de Craboules (Pyr.-Or.).

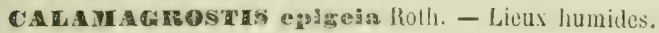

C. Iittoren LC, - Borls des rivières.

C. Ianceolata Rotg. - Prés et bois humides. - Jura : bois de Champvans et de l'Abbaye pris Dole, marais de Pleurre; sur le versant helvétique à la Brévine au bord du Bied. - Vaud et Geneve : Bains de l'Alliaz, Roche, marais de Rocllebot. C.-d'Or et S.-el-L. : Auxonne, bois de Bruailles près Louhans, Mouthiers en Bresse. - Rh., Ain et Is. : Lyon à la Mouche, iles du Rhone, les Echets, Genas.

C. villosa Vill, C. IIalleriana DC. - Prés humides, - Vaud et Valais: Alpes de Château d'Oex et de Bex, Simplon. - H.-Alp.: Briançon, la Vallouise. - Alp.-Ilar. : N.-D. de Fenestre.

C. tenella Host. - locailles humicles des montagnes à sol siliceur. - Doubs : tourbières de Pontarlier. - Vaud et Valais : Alpes de Gérignoz, de l'Etivaz et de Morcles, mont Bovinette près 
Champé, Fully, Findelen près Zermatt, Simplon. - II.-Sav. : Glacière du Brizon, Vergy au pied du Léchaud, Aiguilles. Rouges, Brévent, Montanvert, Jardin de la Mer-de-Glace, Samoëns. - Is. et II.-Alp. : Belledonne vers le lac du Crouzet, Sept-Laux, Taillefer, la Bérarde près du glacier du Chardon, Lautaret, mont Viso, - B.-Alp. et Alp.-IIFar. : Annot à Vergons, Alpes d'Entraunes el de Lantosque.

C. varia Schrad. - Forèts des montagnes. - Itautes Vosges jusque dans la II.-Saône au Ballon de Giromagny, chaine jurassique jusque dans les montagnes du Bugey, Alpes de Vaud et Valais. - H.-Sav, et Sav. : Salève, les Voirons, Parmelan, montagnes du Faucigny, da Chablais et des Bauges, Rumilly sur les bords du Chéran. - Is. et H.-Alp. : massifs de la Grande-Chartreuse, de St-Nizier et de la Salette, los Fraux près de la Grave, la Grangette près Gap, mont Séuse, Scyne. - B.-Alp. et A!p.-Mar. : montagnes de la vallée de l'Ubaye, de Digne, val de Pesio, StMartin, Tende. - Aude et Ariège : la Montagne Noire, lac de Quérigut, Jasse de Campeilles, forêt du Paillariel.

C. arranuhinacen Roth. - Forêts des montagnes. - H.-Sav. : mont Pétetod, Reyvroz. - Ain et Is. : Valromey, Grande-Chartreuse. - Loire et Ard. : Pilat, Pierre-sur-Haute, Gerbier-desJoncs. - Gard et Aude: Aumessas, les Loupies, la Montagno Noire. - Pyr.-Or. et Ariège : Canigou, Carença, vallće d'Eyne, Jont-Louis, Bac de Bolquère, vallon de l'Estagnet, Barbouillères, Roc de Salsafrage. - En dehors de nos limiles dans les Pyr.-Centr., Lozère, Tarn, Aubrac, Cantal, mont Dore, Puy-deDôme, Forez.

PPSAMMrites arenarluma Rocm. Schult. - Sables du littoral méditerranéen. - B.-du-Rh. : la Camargue, Fos, Montredon, Mazargue. - Var et Alp.-Mar. : Toulon, Hyères, ile de Porquerolle, Fréjus, St-Raphaël, Cannes, Antibes, Nice. - Littoral de l'Hér., du Gard, de l'Aude et des Pyr.-Or. : Grau-du-Roi, Vias, les Onglous, Cette, Ste-Lucie, Salses, Argelès, Collioure, Banyuls. - Espèce polymorphe.

P. australe Mabille, - Sables maritimes des envir. de Narbonne, la Franqui près Leucate, plage de Canct. 


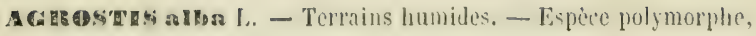
lo plus sozvent stolonifere, quelquelois dépourvue de stolons, très-variable quant à la taille, à la forme de la panicule llorale et des feuilles. De là les variétés distinguées par les auteurs sous les noms de A. stolonifera, gigantea, maritima, vulgaris.

A. vulgaris With. - Considéré par Linné comme une varićté de l'd. alba. En effet, il ne difrère de celui-ci que par la forme ćlale de sa panicule dont les fleurs ont la glumelle inféricure un peu plus longue et par la brièveté des ligules. Du reste il est bon de noter que l'A. vulgaris vit sur les terrains sablonneux secs, tandis que l'A. alba croit dans les lieux humides.

A. verticilata Vill. - Lieux humides, bords des ruisseaux de la région mérid. - Remonte dans les B.-Alp., H.-Alp., Drôme, vers Gréoux, Gap, Tallard, Crest.

L'A. olivelorum G. G., qu'on trouve çà ct là dans la région des Oliviers est une forme intermédiaire entre l'A, alva dont il a la ligule allongée et les glumelles inégales, et l'A. vulgaris auquel il ressemble par sa panicule étaléc.

A. canina L. - Bois des sols siliceux. Comme dans un grand nombre d'autres Graminécs, l'arète de la glumelle inférieure manque quelquefois : d’où la variété mutica.

A. mobra L. - Trouvé en Savoie au col de Fenêtre près Ilauteluce.

A. Ipina Scop. - Pîlurages et rochers des Alpes et des Pyrénées.

- Vaud et Valais: Lavarraz, Anzendaz, Fully, Lens, St-Bernard, Catogne, Thyon, Findelen, Augstelberg, Mattmark, Simplon, Gemmi, Grimsel. - II.-Sav, et Sav. : Dents d'Oche, Roc d'Enfer, Sixt, le Buot, col de Balme, chaîne du mont Blanc au :Iontanvert, à Leschaud et au Jardin, moraines de la Mer-deGlace, Bellevue, la Layat et le Bonlıomme, Allée-Blanche, col Joly, mont d'Etale près la Clusaz, Vergy, Méri, Tournetle, la Sauce, mont Cenis. - Is. et II.-Alp. : la Pra de Belledonne, Champrousse, Poursollet au pied de Taillefer, mont de Lans à Piémeyan, la Moucherolle, mont Chamoux, l'Obiou, Lautaret, Péas ct Abriés en Queyras, mont Viso, col Agnel, Cliaillol-le- 
Vieil, Champoléon, Orcières, mont Aurouse. - B.-Alp. ct Alp.Mar. : Parpaillon, Vallonnet, Estenc, Salsamorena au-dessus de St-Dalmas, la Trinité près St-Ifartin, mont Bego, mont Frontero. - Vaucl. : mont Ventoux. - Pyr.-Centrales.

A. filiformis Gaud., Schleicheri Jord, - Rocher's des montagnes calcaires. - Jura helvétique et français : Hasenmatt, Creux-duVan, pied des escarpements de Mijoux, Colombier, Faucille, Reculet au vallon d'Ardran. - - H.-Sav. et Sav, : montagnes de Sixt et de Samoëns, Anterne, Naulau, Maglan, Brizon, Tournette, la Cyclaz près du lac de la Girotaz, mont Granier près Chambéry, mont Cenis. - Is. ct H.-Alp. : Chamechaude, StNizier, Gorges d'Engins, les Fauges de Villard-de-Lans, col de l'Are, Villard d'Arène, mont Séuse. - Dr. : St-Paul-TroisChâteaux.

A. Rupestriø All. - Pelouses et rochers siliceux des Alpes et des Pyrénées. - Valais : mont Fully, Thyon, Augstelberg, Simplon, Glacier du lhoóne, Grimsel, s'élêve au pied du mont hose jusqqu’à la Cabane do Vincent. - II.-Sav. et Sav. : Roc d'Enfer, Vergy, Méri, Glaciẻre du Brizon, Tournetle, col de Golèse, chaine du mont Blanc au Montanvert, au Chapeau, au Jardin et jusqu'aux Grands-IIulets, Buct, Liguilles-Rouges, Flaine, mont d'Etale près de la Clusaz, mont Iscran, Combe de Villette, mont Cen is au lac Blanc. - Is. et H.-Alp, : Belledonne, Combe de la Lance, Champrousse, Sept-Laux, Gleysin, Brandes en Oisans, mont Pelvoux, Lautaret, Galibier, mont Gondran, cols Isoard et Agnel, Chaillol-le-Vieil, Orcières à Jujau, Pic de Chabrières. - B.-Alp. et Alp.-Har. : Lauzanier, col de Jallorgues el mont Jarret au-dessus d'Entraunes, lac d'Entrecoulpes, la Trinité près de St-Martin, col de Tende. - Pyr.-Or. el Ariège: Canigou, Cambres d'Azc, Madrès, lacs du Llaurenti et de Rabassolès, Bentaillole, Boutadiol, Barbouillère, Roc d'Escrouts, Port de Paillères. - En dehors de nios limites dans les Pyr.-Centrales, Cantal, mont Dore.

A. elegans Thore. - Bois de Pins à Toulon, Hyeres, Maures du Luc et du Cannct, Vidauban, Fréjus. - Landes du sud-ouest de la France. 
A. ponduda DC. - Lirux frais lu Var et Alp,-Mar. a Tonlou, Hyeres, lirejus, les Maures, Cannes.

A. ventosa, A. Spica-Venti L. - Moissons des parties septentr. et moyenne du lassin; rare dans la région mérid. - Espéce polymorphe ayant des lleurs jaunes, violelles ou rouges (1. purpurea Gaud.!

A. intencupera L. - Forme à panicule étroite, contractée, à anthères ovales orljiculaires. - Envir. de Geneve, bords du Léman, Bas-Valais, Annecy, la Bresse, envir. de Lyon, de Grenoble, Villard-de-Lans, Pont-en-Royans, Valence, St-Vallier, Avignon, Alais, St-Ambroix et Lanuejols dans le Gard, Cette ct Ganges dans l'Hérault, St-Agrève dans l'Ardèche.

A. pungens Schreb. - sables maritimes. - B.-du-kh. : Cap Croisetle, illes de Maîré et de Riou. - Var et Alp.-Mar. : Toulon, Ilyères, Frejjus, Cannes, Ile Ste-Margucrite, Nice a Carras, Vinlimiglia. - Gard et Hér. : Aigues-Mortes, Grau-du-Roi, Pérols, Palavas, les Onglous, embouclume de l'Orb et de l'Aude.

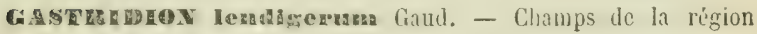
mérid. - Remonte dans la Dr. et les H.-Alp. à Crest, Aubessagne en Champsaur; dans l'Is. à Yienne, Charvieu, Seyssins; dans I'Arul. à Vals et Celles; dans le Rh. à Ste-Colombe, Charbonnières, Messimy, Chessy; dans l'Ain et les envir. de Genève à Massieu, Pont-de-Vaux, Ferney, Bernex, Compesières, bois de la Bâtic près de Peney; dans S.-et-L. à Loulıans, Charolles, Rigny-sur-Arroux. - S'étend dans les vallées de la Garonne et de la Loire, l'Ouest.

G. seabsuan Presl. - Champs ariles du Var et Alp.-Mar. : Toulon, le Lue, St-Tropez à Camarat, Fréjus, île Ste-Margucrite, Antibes.

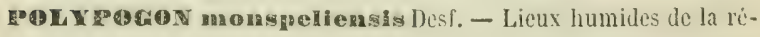
gion mérid. Remonte jusque dans le sud de l'Ardèche. - S'est naturalisé à Lyon le long des fossés du fort de Villeurbanne et du chemin de ronde.

Do. anaridimas Willd, - Litloral méditerrancen. - Espèce polymorplie.

P. Hutoralis Sn. - Sables maritimes, lieux salés. - Hér. : Ma- 
guelone, la Madeleine, Palavas, Montpellier au Pré d'Arène. - Aude et Pyr.-Or. : envir. de Narbonne, St-Nazaire, sables de Barcarès.

Suivant M. Duval-Jouve celte Graminée serait un hybride du l. monspeliensis et de l'Agroslis alba.

E,AGCROS ovatas L. - Liltoral de la Provence, Alp.-IIar., Languedoc et Roussillon.

STRP tortils Desf. - Lieux arides de la région mérid. - B.-duRh., Var : et Alp.-Mar. : Marseille, la Crau, la Garde près Toulon, Rians, Nice, Villefranche. - Pyr.-Or. : Perpignan, Argelès, Collioure, Paulille, Banyuls, vallon de Cerbère.

S. juncea L. - Lieux stériles de la région ınérid. - Vaucl. cl B.-du-Rh. : Montderergues près Avignon, Carpentras, Orange, Piolenc, Vaucluse, les Alpines, envir. d'Aix au Tholonet, vallon du Cascaveou, Cuques. - Var et Alp.-Har.: Toulon, Hyères, Rians, le Luc, l'Esterel, Grasse, Nice, Menton. - Gard : garrigues sur la route d'Uzès, la Beaume, St-Nicolas, Combe de IIangeloup, Ilas Charlot, Villeneuve en face d'Avignon. - Hér. : Lavalette, St-Jean-de-Védas, Grabels, bords du Lirou, Prades, Restincličres, Montarnaud,. Soubès, Montagnac. - Aude et Pyr.-Or. : Lastours, Cap de Pla, Pech de l'Agnèle, borls du Ripau, Cases-de-Pena, Foręa Réal, Trencada d'Ambulla.

s. capllinta L. - Lieux arides des terrains calcaires. - Vaud et Valais : Montreux, 0llon, Aigle, Fully, les Marques, la Bâliaz de Martigny, Sion à Valère et Tourbillon, les Platrieres, Sierre, Leuk, Stalden, Simplon entre Grund et Mégi, Wassermatten sous Ganter. - Ain ct Sav. : sous le fort de Picrre-Châtel, Brides au vallon des Ramicrs, St-Jean-de-Maurienne, Pas-du-Roc près de St-Michel. - Is. el H.-Alp. : Rochefort près du Pont-de-Claix, entre Bourg d'Oisans et la Garde, Briançon aux Trois-Têtes, vallée du Guil, St-Julien en Champsaur. - Dr. et II.-Alp. : Nyons, Donzère, la Condamine, Sisteron. - Vaucl. et B.-duRh. : envir. d'Avignon au Pontet, à Fargues et Cassagne, Carpentras, Bédoin, Aix à la colline dei Dédaou, Cuques, la Crau, les Milles. - Ard. et Hér. : Viviers, Montpellier à la Colombière, Lattes à Maurin, St-Jean-de-Vélas, Mauguio, Vauguiẻres, Cas- 
tries, Cette. - Pyr.-0r. : Salses, 0pol, Cases-de I'ena, Forçarical.

S. pennata L. - Rochers et lieux ariles. - C.-d'Or: Corcelles, Velars, Semur, bords de l'Armançon, Roche-en-Brenil, Marsannay-la-Cote, Gevrey. - Jura : Fort Belin, Salins, Boujailles, rochers de Baume-les-Messieurs, vallée de la Loue. - Ain : Forts de l'Écluse et de P'ierre-Châtel, St-Benoît, Muzin prìs Belley, St-Germain, Portes, St-Rambert en Bugey, Ambronay, Satlionay, la Pape. - Vand et Valais : Château d'Oex, St-Mlaurice, Fully, la Batia de Martigny, les Marques, Sembrancher, IIontorge, St-LČnart, Ies Plátrieres, Zermalt à Offenen, de Visp à Stalden, montée du Simplon dans la plaine de la Ganter. - II.-Sav, et Sav. : Sälèce près de la Grande Gorge, PetitSalive au-dessus d'Etrembières, le Vuache, base du Mole entre Aïre ct Contamines, Coppier, mont du Clat, St-Germain, Brizon, St-Innocent sur les bords du Bourget, Brides, envir. du lac St-Harcel, Pas-du-Roc pris St-Hichel en Maurienne, Bessans, mont Cenis. - Is. : Vernas près Crémieu, entre Hières et $0 p$ Ievoz, Bastille de Grenoble, Claix, la Salette. - Dr., H. et B.-Alp. : Andancelte, Chanousse, la Grave, Charance, Rabou, mont Séuse, la Condamine, Boussolières, Annot, col de la Madeleine. - Alp.-Mar. : Entraunes, Grasse, St-Vallier, Utelle, entre Clans et Venanson, Ste-Anne-de-Vinaï, Tende. - Vaucl. et B.-(lu-Rh., : envir. d'Avignon, entre Gadagne et Jonquerette, Orange, Sérignan, Vaucluse, mont Ventoux, Apt, Cucuronne, les Alpines, Carpiagne, vallon de Vède, Pichauris. - Ard. : la Côte du Rhône de Rochemaure au Pouzin, Ruoms, Pontd'Arc, Aubenas aux rochers de Jastre el au rocher Barra StJulien. - Gard et IIér. : garrigues sur la route de Nîmes à Uzìs, Campestre, région montagncuse des calcaires jurassiques de l'Hér. d'où il descend à Grabels et à Caunelle. - Aude et Pyr.-0r.: rochers calcaires de la vallée de l'Agly et de la Tet, Cases-de-Pena, Trencada d'Ambulla, les Corbières, Bouscaillon près Durban. - En dehors de nos limites sur les rochers calcaires de la Lozère, du Tarn et de l'Aveyron.

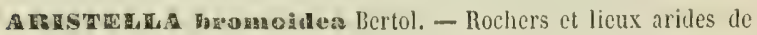


la région mérill. - B.-du-Rh., Var et Alp.-Ilar. : vallon de St-Pons à Gémenos, de Passo-Temps à la Treille, Toulon, le Luc, Fréjus, île Ste-Marguerite, Grasse, Antibes, Nice, Jonaco. - Gard : envir. de Nimes, bois de Campagne, de Broussan, de Barjac, de Candilhac, Serre de Bouquet, Concone. - IIér. : Nontpellier à Grammont, la Colombière, Castelnau, St-Aunès, St-Jean-dc-Védas, Murviel, Lavalette, le Crès, St-Aunès, Pic St-Loup, les Arcs, Lamalou, Lunas, Madières, St-Chinian, Puéchabon, St-Guilhem-le-Désert, St-Jean-de-Buèges. - Aude et Pyr.-0r. : Fontfroide, Salses, Perpignan, Cases-de-Pena, Costujes, vallée de la Mtassane.

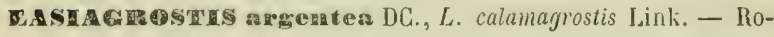
chers calcaires et pentes rocailleuses des montagnes. - Jura helvétique et français jusque dans le Bugey, Vaud et Valais, Savoie, Dauphiné, B.-Alp. jusque dans les Alp.-IIar., le mont Ventoux et les montagnes calcaires de la Provence. - Ard., Gard et Ilér. : coteaux calc. des rives du Rhône, l'Éspérou, Lanuéjols, Valflaunès prés du Pic St-Loup, Navacelle, St-Martinde-Londres aux Arcs. - Pyrénées. - Nul dans les Vosges et les montagnes granitiques et gneissiques du centre de la France.

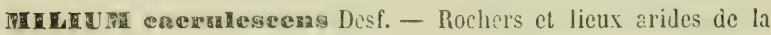
région mérid. - B.-du-Rh. : iles de Pomègue et Ratoneau, l'Estaque, vallon de Toulouse, la Ciolat. - Var el Alp.-Mlar.: Toulon, Hyères, lie de Porquerolle, le Luc, Fréjus, Cannes, StArnoux, Grasse, Antibes, Nice, IJenton. - Aude et Pyr.-Or. : envir. de Narbonne, St-Antoine-de-Galamus, Villefranche, Collioure, Banyuls.

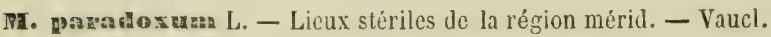
et B.-du-Rh. : bois de Sérignan, vallon de Loule à St-Paul-deGémenos, versant nord du mont Faron près Toulon. - Gard : Mas Charlot près Nimcs, Serre-de-Bouquet, Alzon, Anduze, Aumessas, St-IIippolyte. - IIér. : Mireval, Fabrègues, Murviel, Teyran, Pic St-Loup, Avène, Hérépian, St-Chinian, St-Pons, Pégayrolles-de-l'Escalette, Bédarieux, St-Guilhem, Madières, Granges, St-Martin-de-Londres, le Larzac à la Vacquerie, StMaurice, Navacelle, Béziers. - Aude et Pyr.-Or. : envir. de Narbonne, vallée de la Massane à Lavail, Collioure, Banyuls. 
M. multifiogrum Cav, - Licux arides de la région mérid.

תr. ef fousenan L. - Bois des collines et des montagnes dans les parties septentr. ot moyenne du bassin; plus rare dans les mon-

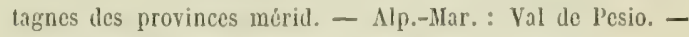
Gard : mont Cavalier près Nìmes, bois de Banahu.

M. seabrean G. Rich. - Bois. - Vaucl. et Var: plateau de StAmans, la Sainte-baume. - Oucst de la France.

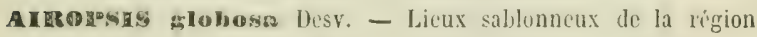
mérid. - Var et Alp.-Mar. : Sablettes de Toulon, le Luc, Fréjus, golfe Jouan, Grasse. - Hér. : Nontpellier à Grammont, bois de Lamoure, Roquehaute, Montarnaud. - Pyr.-Or. : plage du Barcarès, étang de St-Nazaire. - Littoral de l'Ouest.

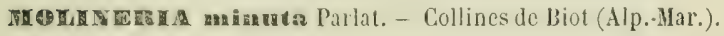

AHE A canesechs L. - Licux sablonneux, - C. - d'Or et S.-ct-L. : terrains granitiques de Saulicu, Scmur, le Morran autunois, sables de transport d'Auxonne, de la Loire et de l'Arroux. Rh., Is., Dr. et Ard. : terrains granitiques du Lyonnais, sables de transport du Mollard de Décines, Romans, Pont-en-Royans, granite de Śt-Vallier à Laveyron, Tournon, Vals. - Vaucl. et Var : Avignon, Orange, Carpentras, Apt, Sabletles de Toulon.

- Gard et Hér. : l'Espérou, Dourbie, Broussan, Peyremale, Montarnaud, Viols, St-Guilhem, Madières, la Sérane. - Littoral du Roussillon. - Nul dans la chaine jurassique et généralement sur les terrains calcaires.

A. ardiculata Desf. - Sables maritimes. - Var et Alp.-Har. : les Sabletles de Toulon, îles d'IIycires, Cavalaire, Fréjus, St-Raphä̈l, Cannes, golfe Jouan, - IIér. : littoral d'Agde, entre Argelès et Collioure.

A. fasciculuta Boiss. Reut. - Forme à épillets plus petits, à faisceau de poils plus courts. - Var : Toulon, illes d'IIyères, Ie Luc, Fréjus. Gard et Hér. : bois de Broussan, Mas de la Vache, bords du Gardon à St-Nicolas, Castelnau au Sablas, Grammont, Lamoure, St-Aunès, Lamalou, Hérépian, Colombière, St-Martin d'Orb,

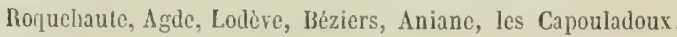
- Aude et Pyr--Or. : Narbonne, Perpignan, Collioure, PortVendres, Banyuls. 
A. earyoplayina L. - Terrains sablonneux; nul sur les calcaires. - Espèce polymorplic.

A. brachystachya, A. Tenoriana Guss. - Forme à ipillels ä-S fois plus courts que leurs pédicelles, à glumelles sans faisceaux de poils à la base, tantôt aristẻes, tantôt muliques, ofirant même quclquefois dans le méme épillet des fleurs à arêtes et d'autres sans - arêtes. - Var et Alp.-Har. : Toulon, fles d'Hyères, Carqueiranne, le Luc, Bormes, Roquebrune, St-Raphaël, IEsterel.

A. aggregata Timeroy. - Forme venant par loufles abondantes, ì ipillets ovales en panicule plus fournie, commune dans les terrains granitiques du Norvan, de Saulieu et d'Autun, Lyonnais, Beaujolais, Forez, Cévennes jusque dans l'Espinouse, l'Wscandorgne et la Monlagne-Noire. - Bresse du Jura et de S.-et-I. à Chaussin, Pleure, Gatey, Loulans.

A. divaricata Lois. - Forme à épillets espacés, portés sur des pédicelles divariqués. - Mèlée à la forme précédente, mais plus rare.

A. eleganas Gaud. - Terres sablonneuses. - Rh. et $\mathrm{Ain}$ : Chaponost au Garon, Givors, bois de Rothonne près Belley, la Pape. - Is. et Dr. : Mollard de Décines, Romans, St-Vallier. - Var : Toulon, le Luc, les Maures, Frijus : - Vaucl., Gard et Hér. : sables des bords du Rhône, Montpellier à Lamoure, Grammont, Doscares, Castrics, St-Martin-de-Londres et d'Orb, Lamalou, Lodève.

A. Lorovacialis Jord. - Licux sablonneux, - Var et Alp.-Nar. : St-Nazaire, Toulon, Brégançon prìs Hyères, les Maures, les Mayons, Frẻjus, Cannes, golfe Jouan.

A. Cupanaraga Guss. - Lieux secs et arides de la région mérid. Vaucl. et B.-du-Rh. : Orange, Sérignan, Puiseux, Mazargue, St-Tronc, la Crau. - Var et Alp.-IIar. : St-Nazaire, Hyères, le Luc, Fríjus, Antibes, Nice, Menton. - Gard et Ilér. : AiguesMortes et les garrigues de Palavas, Montpellier, Agle. - Aude : Narbonne, Careassonne, la Tourctte.

B. precer L. - Terres sablonneuses des parties septentr. ct moyenne du bassin; rare dans la partie méridionale.

A. escegovidosa L. - Prés humides, bords des eaux des plaines et 
des montagnes. - Espice polymorplic comprenant une variête alpina Gaud., plus petite, à épillets bruns, une variété lilloralis Reuter vivant sur les sables voisins des bords de la mer, une troisième variétê, A. media Gouan, à feuilles enroulécs:sétacécs, à rameaux nus à la base, à arête plus longue que la glumells, quelquefois nulle. Celte derniere forme vit dans les lieux incultes de la rigion des Oliviers; plus rare dans les parties moyenne et septentr. du bassin, autour de Gap, de Crémicu et de Dijon notamment à Larrey.

A. Alexuosa L. - Bois et rochers des collines et montagnes à sol siliceux. - Nul sur les ralcaires. - Espèce polymorphe dans laquelle les dimensions des feuilles et des épillets sont très-variables. La forme $A$. Legei Bor. a des épillets plus gros; la forme montana L. les a, au contraire, plus petits et colorés en violet noirâtre.

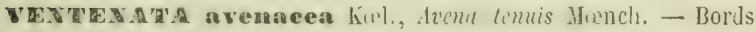
des champs et des bois, - S.-et-L. : Cluny, Issy-l'Evêque. Bh., Ain ct Dr. : Francheville, Tassin, Chaponost, Pollionnay, Bourg, Laveyron et Beausemblant près St-Vallier, - I. et B.-Alp. : Laragne, Ribiers-le-Poët, Sisteron. - Gard: l'Espérou, Camprieux, Lanuéjols, Alzon.

AVEDS satisn L., A. orientalios L. et A. strigosn Schrel. - Cultirés et quelquefois subspontanés.

A. barbata Brot. - Lieux stériles, bords des champs et des chemins de la région mérid.

A. Carua L. - Moissons de la région mérid. et moyenne; se montre quelquefois accidentellement dans la partic septentr. du bassin.

A. sterilis L. - Champs cultivés de la région mérid. d'où il remonte jusque vers Lyon. - Espèce polymorphe.

A. setncer Vill. - Rochers des montagnes calcaires, - Is., Dr. et H.-Alp.: Chamechaude, col de l'Arc, la Moucherolle, mont Aiguille, l'Obiou, col de Menée, mont Glandasse, Séuse, Aurouse, Durbonnas, Noyer en Champsaur. - B.-Alp. et Alp.Mar.: entre Serennes el Maurin, mont Frontero, Alpes de Tende, de la Briga et de l'Isola. - Vaucl. : mont Ventoux. Catal. Bassin du Rhône. 
A. Anfolia Lag. - Champs de la plaine du Roussillon à Elne, Château-Roussillon, Thuir.

A. mempervirens Vill. - Pìturages et rochers calcaires des montagnes. - H. et B.-Alp.: Lautaret, pic de Chabrières, col de Glaise, Séuse, Aurouse, col du Noyer, Rosans sur le Fourchat, Annot. - Sav. et Alp.-Mar. : mont Cenis, col du Ray cntre Venanson et la forêt de Clans, Alpes de Frontero, Carlin, la Briga, Tende.

A. montana Vill. - Prés et páturages des montagnes. - Is. et Sav. : Grande Chartreuse, Chamechaude, la Iloucherolle, Belledonne, Combe de la Lance, mont Chamoux, mont de Frijjus, mont Cenis. - Dr. et H.-Alp. : Lus-la-Croix-Haute, Durbonnas, Obiou, la Grave, Lautaret, Galibier, col de l'Echauda, Chaillolle-Vicil, Chabrières, mont Viso aux sources du Guil, col de Ruines et de Glaise, Chaudun, Séuse, Aurouse. - B.-Alp. et Alp.-Nar.: Meyronne, St-Paul, Bachasse, Lauzanier, Grand Couyer sur Annot, Entraunes, col de Pouriac au-dessus de StÉtienne. - Vaucl. : mont Ventoux. - Pyr.-0r. et Ariège : Canigou, Carença, vallée d'Fyne, Mont-Louis, lac du Llaurenti, pic de Joucarets, vallon de Boutadiol, pic del Ginèvré. En dehors de nos limites dans les Pyr. centr., Aveyron, Cantal, H.-Loire.

A. Hostiana Boiss. - Prairies et pâturages. - Is., Sav, et H.-Alp. : la Salette au mont Chamoux, mont Conis, mont Viso vers les chalets de Ruines, mont Séuse, Aurouse. - B.-Alp. et Alp.Mar. : vallon d'Horonayc, Larche, Serennes au Rocher du PIn, Bonnet de Trois-Evêques, Alpes de Tende.

A. veresicolor Vill. - Rochers et pâturages des Alpes el des Pyrénẻes. - Vaud et Valais : Anzendaz, mont Fully, Catogne, Surchamp, Bagnes, Diez, Arolla, mont Nuoble, Zermatt, Findelen, Simplon, Glacier du Rhône, Grinsel. - H.-Sav, et Sav. : Dents d'Oche, montagnes de Sixt et de Samoëns, le Buet, le Brévent, chaine du mont Blanc au Ifontanvert, au Jardin, au Couvercle el à Tré-la-Tête, la Layat près du Bonhomme, Héri, Charvin, mont d'Etale prìs la Clusaz, la Sauce, Croix-de-Pierre près Hautcluce, entre la Cyclaz et le grand Pariraz, mont Hi- 
rantin, mont Iseran, mont Cenis. - Is. et II. $-\mathrm{Al}$ p. : Belledonne près du lac du Crouzct, Sept-Laux, Brandes en Oisans, Lautaret, sources du Guil, col Vieux, Poligny en Clampsaur, Chaillol, col de Glaise, mont Bayard, Pleyne-de-Chaudun. B.-Alp. et Alp.-Har. : Horonaye, Roche-Grande près Estenc, Alpes de Tende. - Pyr.-0r. : Canigou, Pla Guilhem, Font-deComps. - En dehor's de nos limites dans les Pyr. centr., Cantal, mont Dore, Puy-de-Dôme.

A. pubeseens L. - Prairies des coleaux et des montagnes. Espece polymorphe. La forme amelhystina $\mathrm{LC}$. ou sesquitertia $\mathrm{L}$. qui a des épillets plus gros et plus violacés est quelquefois mêlece au type.

A. prentonsis L. - Prés secs et bois des plaines et des montagnes. - Espèce polymorphe.

1. bromoidea Gouan, A. australis Parl. - Forme du précédent à épillets plus étroits, contenant $5-8$ fleurs en panicule allongéc. Lieux stériles de la région mérid. ; remonte dans les $\mathbf{H}$.-Alp. et la Dr. vers Gap, Nyons, Loriol, Crest, Tain, Ponsas; dans l'Is. à Claix, Pariset, Sassenage, Grenoble.

A. elntior L. Arrhenaterum elatius M.K. - Prairies et bois. - Une forme bulbosa, qui a la racine renflée en tubercules superposés, se rencontre quelquefois dans les moissons.

1. (Trisetum) subspieata Suter. - Pelouses des hauts pâturages des Alpes el des Pyrénées. - Valais : Catogne de Sembrancher, col de Fenêtre, sommet de la Clienaletta au St-Bernard, Nendaz, Ilérémence, Evolena, Anniviers, Zermatt au Gorner et à Zmutt, Cabane de Vincent au pied du mont Rose, Saas, Leukerbad, Simplon, Conches. - H.-Sav. et Sav. : col de Golèse, mont Blanc aux Grands-Mulets, Ubine de Pelloua, col du mont Iseran, mont Cenis à Ronches. - H.-Alp. : Villard d'Are̊ne sous les Glaciers du Bec, l'Echauda, cols Malrif et Agnel.

A. gericen, Triselum Guudinianum Buiss. - Coteaux du Bas-Valais entre Fully et Saillon, Lens, Montorge, St-Léonard, les Plâtrières. - Sur le versant italien à Suse et, dans la vallée d'Aoste, à Villefranche, Nus, Aimaville.

A. pranicea Lam. - Bafterie d'Endoume prís Marscille. - Narbonne. 
A. Aavescens L. - Prairies et pâturages des plaines el des montagnes. - Espèce polymorphe. La forme alpestris DC. a des fleurs nuancées de violet.

A. distichophylla Vill. - Rocailles des Alpes. - Vaud ct Valais: Anzendaz, Diablerets, Fully, Bagnes, Zermontana, Zermatt au Schwarzsee, Saas, Gemmi, Simplon, Grimsel. - H.-Sav. et Sav. : montagnes de Samoëns et de Sixt, Allée Blanche sur le versant italien du mont Blanc, Dents d'0che, les Cornettes, Sâles sur Servoz, mont Jovet près du Bonhomme, les Aravis, Lanches de la Chavonne près Hauteluce, Tignes, Pas-lu-Roc près St-Mlichel en Maurienne, Valloire, Fort de l'Esseillon, mont Cenis à Ronches. - Is. et H.-Alp. : mont de Lans, Piéméyan, la Salette, la Grave, Lautaret, col Isoard et des Hayes, pic de Chabrières, Chaillol, Chaudun, mont Aurouse. - B.-Alp. et Alp.-Nar. : Serennes, la Condamine, Bérard, Grand Couyer, Entraunes. - Vaucl. : mont Ventoux.

GAUDIN A fragilis Pal. de Beauv. - Prairies, bords des champs et des chemins; plus commun dans la région mérid.

HOLCOS Ianatus L. - Bords des champs. - Espèce polymorphe. La forme aestivalis Jord. Fourr. se trouve à Quincieux (Rhône).

Ir. mollis L. - Champs des terrains siliceux et argileux des plaines et des montagnes.

Howera cristata Pers. - Coteaux secs des plaines et des montagnes. - Espèce polymorphe.

1. grandiflora Bert. - Forme à épillets plus grands, à glumes égalant les fleurs. - B.-du Rlı, : la Crau d'Arles. - Alp.-Nar.: Grasse, forêt de Clans.

K. Urevifolia Reut. - Forme alpestre de petite taille, à feuilles courtes, à épillets ténus en épis courts. - Sav. et H.-Alp. : mont Cenis, cols Isoard, des Hayes et de lâ Croix, Abriès, vallon de Péas en Queyras.

L. setreer Pers. - Pelouses siches des plaines et des montagnes. - Espéce polymorphe à épillets et chaumes tantôt glabres (K. valesiaca Gaud.), tantôt pubescents (K. pubescens Parlat.).

La forme alpicola G.G. a des chaumes plus longs, plus tomen. 
tcux au sommet, d'une coulcur cendrée. - Sav. et II.Alp. : mont Cenis, Villard d'Arène, Lautaret, Abriès en Queyras, Pic de Chabricres, mont Aurouse.

Ix. villosa Pers, - Sables humides du littoral méditerranéen.

R. phleoidea Pers. - Licux inculles de la région mérid. et moyenuc.

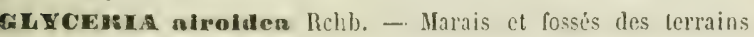
siliceux et argilcux des parties septentr. et moyenne du bassin, surtout dans la région bressanne depuis la zone orientale du départ. de la C.-d'Or. jusqu'à Lyon.

G. Auitams R. Br. - Fossés et marais. - Espece polymorplie ayant quelquefois une grappe penchée, des feuilles à gaines se déchirant en réseau fibrilleux ( $G$. plicata Fries) que quelques botanistes élèvent au rang d'espèce.

c. Iollacen Godr. - Prés liumides des terrains sablonneux. II.-Saone: Menoux, Boursières, Cubrial, Franchevelle. - Is., II. et B.-Alp. : Voreppe, Gap, Enchastraye. - B.-du-Rh, et Gard : los, les Martigucs, Jonquières.

F, aquatica Wahlb. - Bords des marais et des rivières.

Tr. fentuelformis Heynh. - Marais du litloral miditerranéen. B.-du-Rh. et Var : prẻs salés de Berre et de Marignane, bords de Jarret, Toulon. - Hér., Aude et Pyr.-Or. : Vic, Maguelone, Cette, Ste-Iucie, marais de Thuir et de Canohès.

Cr. convoluta Fries. - Licux humides du littoral méditerranéen. B.-du-Rh, et Var: Berre, Marignane, Fos, Toulon. - Gard, Hér., Aude et Pyr.-Or. : Aigues-Mortes, Palavas, Cette, Narbonne, Ste-Lucie, la Nouvelle, Salses.

- distans Wahlb. - Bords des eaux de la région littorale. Existe aussi dans l'intérieur près des sources salées du Plan de Phazy non loin de mont Dauphin, le Freney, Villard d'Arène (H.-Alp.). - Grozon près Arbois et IIontmorot près Lons-leSaunier (Jura), - Vals (Ard.) - En dehors de nos limites dans les lieux arrosés par les sources salées de la Lorraine et de la Limagne d'Auvergne.

crogsios maxginatus Pal, de Beauv, - Terres arides, - B.du-Rh. : la Crau, Marseille à Ste-Marguerite et à St-Pierre. - 
Aude et Pyr.-Or. : remparts de Narbonne, Perpignan à Canct et à Cabestany, Cases-de-Pena, Prats de Mollo.

SCLEROCHMOA dura $P$. de B. - Lieux sablonneux, bords des chemins. - Valais : Fully, Saxon, Sion. - Lnvir. de Grenoble, Gap, Ribiers, Avignon, Flassaa, Montaud-les-Miramas, Toulon, Nice. - Nìnes, Montpellier, Mireval, Vic, Latles, Palavas. En dehors de nos limites dans la vallée de la Garonne, l'Auvergne, l'Alsace et la Lorraine.

POA annua L. - Lieux cultivés, bords des clemins. - Espèce polymorphe.

$P$. supina Sclırad. - Forme alpine ayant une souche vivace, des épillets plus grands, nuancés de violet, fleurissant à lá fonte des neiges. - Valais: Saas à Nattmark, Simplon. - II.-Sav.: Vergy autour des lacs du côté du Reposoir, Chamonix au Chauderon. - Is. et H.-Alp. : Chamechaude près de l'Abreuvoir, Belledonne à la Combe de la Lance et près des lacs Domenon, Sept-Laux près du lac Carré, plateau de Clıamousset près des abreuvoirs de Chichiliane, Lautaret, mont Viso, mont Pelvas.

R. minon* Gaud. - Rocailles des Alpes. - Vaud et Yalais : Bovonnaz, Fully, St-Bernard, Zermontana, Zermatt, Simplon, Grimsel, s'élève au pied du mont Rose, vers la Cabane de Vincent. II.-Sav. et Sav. : Vergy, Roc d'Enfer, Nautau, Pétetod, montagnes de Samoëns et de Sixt, col de Golèse, Buet, Brêvent, mont Blanc au Chapeau, au Jardin, à Tré-la-Tèle et dans l'Allẻe Blanche vers le lac Combal, le Bonhomme, entre Turnaz et Colombaz près Hautelucc, Bas-du-Nottet au-dessus d'Aime, Tignes, mont Cenis. - Is. et H.-Alp. : li Moucherolle, AlpIIartin, la Grave, mont Pelvas, mont Viso, Champoléon, Orcières. - B.-Alp. : Vallonnet, Lauzanier, Malemort.

P. concimna Gaud. - Coleaux bien exposés du Bas-Talais à Branson, Montorge, Tourbillon, les Plâtriêres, St-Léonard.

P. Inxa Haenke. - Rochers siliceux et rocailles des Alpes et des Pyrénées. - Vaud et Valais : Anzendaz, St-Bernard, Zermontana, Mattmark, pied du mont Ruse à la Cabane de Vincent $\left(3,138^{\mathrm{m}}\right)$, col St-Théodule $\left(3,350^{\mathrm{m}}\right)$, Simplon, Grimsel. 11.-Sav. et Sav. : chaine du mont Blanc au Montanvert, au 
Couvercle, au Jardin $\left(2,756^{\mathrm{m}}\right)$, et aux Grands-Nulets $\left(3,470^{\mathrm{m}}\right)$, Aiguilles-Rouges, Alléc-Blanclıe, mont Mirantin, la Vanoise. Is. et II. $-\mathbf{A}$ p. : Belledonne près des lacs Domenon, Poursollet au pied de Taillefer, Sept-Laux, Piéméyan au mont de Lans, la Grave, Chaillol-le-Vieil, Orcières, mont Viso a la Traverselte. - B.-Alp. et Alp.-Mar. : Mélezen de St-Paul, N.-D. de Fenestre. - Pyr.-Or. et Ariège: vallée d'Eyne, Carlitle, Llaurenti en montant à la Porteille de Baxouillade, vallon de Quérigut à la Jasse d'Espagne. - Pyr.-Centrales.

P. caesia Sm. - Rocailles des Alpes. - Vaud et Valais : les Planards, Morcles, St-Bernard, Zermontana, Lens, Gemmi, Distelalp de Saas. - H.-Sav. et Sav. : Brévent, mont Blanc au Couvercle, aux Grands-Mulets et à Tré-la-Tête, mont Charvin, mont Cenis au Iac Blanc. - Is. et H.-Alp. : la Pra de Belledonne, Grand Galbert, glaciers de Villard d'Arène, mont Pelvoux, Iautaret, mont Viso, Orcières, mont Aurouse.

P. memoralis L. - Bois et lieux ombragés dans tout le bassin. Espice polymorplıe dans laquelle les dimensions des épillets sont variables. - La forme alpina est une plante glauque, raide, à panicule étroite.

T. serothua Ehrlı. - Bords des caux, - Envir. de Belfort sur les bords de la Savoureuse. - Vaud : Morges, Payerne à la GrossePierre, Villeneuve. - S.-et-L. : Millery près d'Autun, bords de l'Arroux, - Bords du Doubs et des ètangs de la Bresse. Envir. de Lyon à Pierre-Bénite, Vernaison. - B,-Alp. et Var : Annot, Fréjus. - Pyr.-0r. : la Massane près Argelès.

P. alpina L. - Pàturages des montagnes. - Chaines des Vosges, du Jura helvétique et français, Vaud, Valais, Dauphiné, B.-Alp. et $\Lambda$ p.-Mar., mont Ventoux. - C.-d'Or et S.-et-L. : sommités de la Côte depuis Marcy-sur-Tille, à Clıâteau-Renard près Gevrey, Nolay ont jusqu'au delà de Beaune, entre Mercurey el Chamilly. - Gard : Espinassous près Lanuẻjols. - Pyr.-Or. ct Ariège : Canigou, Costa Bona, Carenca, Eyne, Llo, Llaurenti, En dehors de nos limites dans les Pyr.-Centr., Aubrac, Cantal, mont Dore, Vosges.

Espèce polymorphe qui s'éléve jusiqu'aux dernières limites de 
la vẻgétation aux Grands Nulets du mont Blane $\left(3,170^{\mathrm{m}}\right)$, å la Cabane de Vincent au pied du mont Rose $\left(3,158^{-\infty}\right)$ et au col St-Thécdule $\left(3,30^{\mathrm{m}}\right)$, et se montre aussi sur les montagnes peu élevées de la Côte-d'Or et de Saône-et-Loire.; on la voil quelquefois descendre assez bas dans les vallées alpines.

La variété brevifolia DG., a une panicule petite et des feuilles courtes el raides. De même que les $P$. annua et bulbosa, lo $P$. alpina est souvent vivipare.

更. bulbosa $\mathrm{I}_{\text {. }}$ - Lieux incultes, bords des chemins.

T. compressa L - II urs, rocailles, licux secs et sablonneux.

P. distichophylia Gaud. - Rocailles, schistes des montagnes. - Vaud et Valais : Lavarraz, Javernaz, les Plans, Fully, Sanetsch, Catogne, St-Bernard, Findelberg, Gemmi. - H.-Sav. et Sav. : Vergy, le Reposoir, Brévent, Aiguilles-Rouges, Sixt, col de Golese, la Balme près du Bonhomme, les Moltets, Chapieu, Bas du Mottet au-dessus d'Aime, mont Iseran, mont Cenis, lac Combal dans l'Allée Blanche. - Is, : Belledonne à la Combe de la Lance, Chamechaude, Grande-Chartreuse, St-Nizier, la Moucherolle, la Salette. - H.-Alp. : Lautaret, Galibier, col de l'Échauda, Mairif en Queyras, de Fontgillarde au col Agnel, Chaillol, Orcières, mont Aurouse. - B.-Alp. et Alpr-Mar. : Malemort, Jausiers, Annot, mont IIonnier, col de Fenestre, Ste-Annc-de-Vinaï. - Vaucl..: mont Ventoux. - Pyr.-0r. et Ariège : Carença, Prats-de-Balaguer au col de Las nous-Fonts, Llaurenti en montant à la Porteille de la Baxouillade, Pic des Clots de l'Eigue. - Pyr.-Centr.

2p. pratensis L. - Prairies et pàturages. - Espèce polymorphe présentant des variations dans les dimensions des feuilles, d'où les variétés dites latifolia et angustifolia. Dans la variété anceps Gaud. les feuilles sont larges et courtes, les tiges comprimées.

E. Erivinlis L. - Bords des haies et des bois, prés liumides.

Tr. silleatiea Vill., P. sudeticn Haenke. - Buis et loruyères des montagnes surtout sur les sols siliceux. - Forêts des Vosges graniliques el arénacies jusque dans la H.-Saône aux Ballons de Giromagny et de Servance, Planche des Belles-Filles, vallée de 
Miélin. - Beaucoup plus rare dans la chaine jurassique et seuIement dans les licux tourbeux ou recouverts d'une épaisse couche d'humus, comme, par exemple, au Lomont, au Creuxdu-Yan, autour de Pontarlier, à Poupet, Arzier, Poisat. Vaud ot Valais au-dessus de Bex, Fully, Champé, Bagnes, simplon. - II.-Sav, et Sav. : Vergy, vallée du lieposoir, mont Cenis, - Is. et II.-Alp. : forêt do P'ortes, l'rémol, la Morle près Lavaldens, Pleyne de Chaudun près Gap, Sibeyrans et Durbonnas, mont Queyrel et les Combes on Champsaur, Ristolas. Alp.-Mar. : les Viosennes. - C.-d'Or et S.-ct-L. : Ie Morran granitique, Autun, Antully, Cluny, Charolles. - Loire, Ard, et liér. : chaîne granitique el gneissique du Pilat, Vivarais, Ie Mezenc rolcanique, l'Espérou jusqu'à Fraisse. - En dehors de nos limites dans tes Pyr.-Centr., Aubrac, Tarn au Pic de Montalet, Cantal, mont Dore, Puy-de-Dóme, Forez, Vosges. - Espice poIymorphe.

1'. hyjbrida Gaud. - Forme du précédent ayant les feuilles non courbées au sommet et une souche plus longuement rampante. Plus fréquent que le type dans la chaine jurassique au Weissenstein, Chasseron, Chasseral, Creux-du-Van, Aiguillon, entre le Vuarne et la DoIe, Mont-d'Or, Faucille, montagne de St-Jean, Reculet au vallon d'Ardran, forêt d'Arvières en Bug̉ey. - Vaud: Alpes de Jaman el de Bex, Château-Chamois, Alpes de Châteaud'Ocx. - If.-Sav. et Is. : col de Balme, Brizon, le Reposoir, le Semnoz, Grande-Chartreuse.

EIR AGRostrs megalostaehya Link. - Terrains sablonneux ct graveleux. - Espèco polymorphe.

L. brachystachyn, E. poacformis Link. - Forme à épillets plus petits, non fasciculés, à glumelle infér. obtuse. - Lieux sablonn. de la région mérid. - Remonte dans la vallée du Rhône à Lyon, Genève, Nyon, Lausanne et le Bas-Valais.

E. pilosa P. de B. - Terrains sablonneux et coillouteux des parties mérid. et moyenne du bassin.

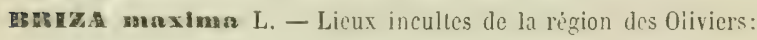
remonte dans la partie mérid. de la Drôme et de l'Ardèche.

Fi. medka L. - P'rés, pâturages des pliaines et des montagnes. 
B. minor L. - Clıamps sablonn. de la région mírid. Remonte dans l'Ardèche à St-Maurice-de-Baix; s'étend dans le bassin de la Garonne et dans l'Oucst.

FiELC cllata L. - Rochers, murs, côteaux arides et bien exposés. - Espèce dont le polymorphisme n'a pas élé suffisamsamment reconnu par les auteurs.

La forme Magnoliana G. G., à longue panicule, est la plus commune dans la région des Oliviers; Ia forme nebrodensis G. G., à panicule courte, est la plus répandue dans la partie septentr. et moyenne de notre bassin.

C'est à tort, suivant moi, que Godron dans la Flore de France, décrit, comme espèce distincte sous le nom de $H$. ciliata $\mathrm{L}$. une plante qui se trouverait seulement en Alsace, dans le Palatinat, en Saxe et en Suède. Cette prétendue espèce est un état du $N$. nebrodensis dont les graines sont un peu plus chagrinées.

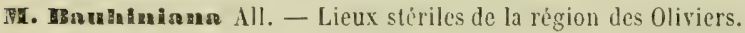

Ji. minutse L. - Lieux stériles de la région mirid. - Espice po. lymorphe sc présentant sous deux formes principales, l'une à feuilles très-étroites, enroulées-sélacées, l'autre à feuilles plus larges et planes, $M$. latifolia Cosson, $M$, major Sibth. Sm.

MF. nuta $\mathrm{L}$. - Bois des plaines et des montagnes dans les parties septentr. et moyenne du bassin. Rare dans la région mérid. et seulement dans les montagnes de St-Martin-Lantosque, vallíe de Caïros, l'Escarène (Alp.-IIar.), Salbous près Campestre (Gard).

II. unifora Retz. - Formc ayant des épillets à deux fleurs dont une seule 'est fertile, disposés en panicule peu inclinée. - Bois des plaines et des montagnes.

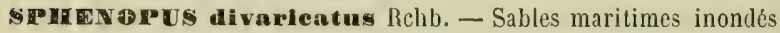
pendant l'hiver, - B.-du-Rh., Var et Alp.-Mar. : Arles, Marignane, Toulon, Salines-Neuves d'Hyères, Cannes, Nice, Eza. Gard et Hér. : Aigues-IIortes, Palavas, les Onglous, Maguelone, Celle, embouchure du Lez, Mauguio, Lattes, Vic. - Aude et Pyr.-0r. : Narbonne, Leucate, Salses, Canet, St-Nazaire, St-Cyprien.

SCHETRPOA maritimn Parlat. - Sables maritimes. - 
B.-du-Rh., Var et Alp.-Mar. : Mazargues et Montredon près Marscille, Toulon, Hyères, St-Raphaël, Cannes, golfe Jouan, Nice. - Gard et Heer. : Aigues-Nlortes, Palavas, les Onglous, Celle. - Lilloral de l'Aude et des Pyr.-Or.

ธ. คubseสsills, S. hemipoa Parl. - Sables marit. - B.-du-Rh. et Var : Mlazargues, la Seyne aux sabletles, Si-kaphaël. - Gard ct Ilér. : Aigues-IIortes, Palavas, Cette, Agde, les Onglous, Portiragnes.

s. relgh Gris, - Lieux sablonneux et caillouteux, bords des chemins dans les parties mérid. et moyenne du bassin.

\$. Ioliacea G. G. - Sables maritimes du littoral méditerrancen.

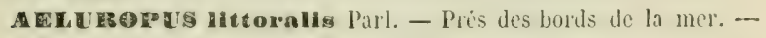
B.-du-R. ct Var: Marseille aux Catalans, Marignane, Arles, Sablettes près de la Seyne, Hyères. - Gard et IIẻr. : Aigues-Ilortes, Maguelonne, la Molte, Portiragnes, Vic, Frontignan, Balaruc. - Aude et Pyr.-0r. : ile Ste-Lucie, Salses, St-Nazaire.

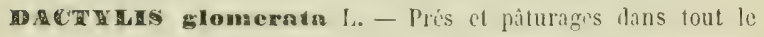
bassin. - Espèce polymorplie. Le D. hispanica Roth est la forme des lieux secs et arides; il a une panicule compacte, à glumelle inférieure obtuse ou quelquefois échancrée avec un mucron dans l'échancrure; les feuilles radicales sont persistantes.

D耳pL CUVE Gerotina Link. - Coteaux arides. - Ain : Muzin près Belley, entre Tenay et Chaley. - Sav., Is. et Dr. : rochers de l'Echaillon près St-Jean-de-Maurienne, Vienne, Bochefort près du Pont-de-Claix, Varces, St-Vallicr, Gervan, Nyons. Vauç. ct B.-du-Rh. : Avignon, Salnn, Miramas, Istres. - Var et Alp.-Mar. : Vidauban, St-Daumas au Cannet, les Ifayons, Antibes, Grasse, Utelle. - Ard. et Gard : Vallon, Vals, Tournon, le Vigan, Aulas, Anduze. - Hérr. : Moulin-Geniès, Jes Capouladoux, St-Mllartin el Mas-de-Londres, Causse de la Selle, Pézenas. - Aude et Pyr.-Or. : Narbonne, Donos, Perpignan, Villefranche, Vernet-les-Bains.

HOtr EA caerulea Moench. - Prés et bois humides des plaines et des montagnes. La taille de cette Graminée est trèsvariable.

DANThoria decumbens DC. - Prés et bois, - Plus fréIuent sur les terrains siliceux. 
D. ERPAncionis DC. - Pris liumides, - H. et B.-Alp.: mont Bayard et Corrie de la Roche près Gap, Sisteron, Castellanne. B.-du-Rh. et Var: bords du canal de Craponne au-dessus de la Roque-d'Antheron, forêt des Maures. - Collines des Alp.Mar.

CYvosures chistatua L. - Prairies des plaines el des montagnes.

C. echanatas L. - Lieux cultivés de la région mérid. jusque dans la partie mérid, de la Drôme et de l'Ardèche. Par une bizarreric de distribution géographique, cette Graminče, si commune dans le midi de la France, se montre aussi dans quelques vallées des montagnes. C'est ainsi qu'on la rencontre dans les champs de la partie supéricure du haut Valais entre Obergestelen et Oberwald; dans la vallée de la Dranse à Bovernier, Sembrancher et Jiddes; dans la haute vallée de l'Arve autour de Chamonix; en Savoie, dans la haute vallée de l'Isère à Tignes, dans la vallée du Doron entre Brides et Pralognan, autour d'Albertville, au Cohennoz, à Serraval, Crest-Voland, MontDenis et St-Jean-de-Maurienne; dans l'Isére en montant de Revel à Belledonne et de Vaulnaveys à Prémol, enfin à StNizier.

En dehors de nos limites, le C.echinatus s'étend dans le bassin de la Garonne, et dans l'Ouest jusque dans le Morbihan, le Finistère et la Hanche.

C. polybracteatus Poir. - Bois de la région mérid. - B. duRh., Var et AIp.-Mar. : rochers de la Tête-de-Carpiagne, valJons de Toulouse, de Passo-Temps, de St-Clair, Roquevaire, Cassis, sous le Baou-de-Canailln, mont Faron, la Verne, les Maures, les Mayons, l'Esterel, Cannes.

C. aureas L., Lamarckia aurea Moench. - Lieux pierreux du littoral méditerranéen. - Var et Alp.-Nar. : Hyères, Bormes, Frẻjus, Villefranche, Monaco. - Pyr.-Or. : Collioure, PortVendres, Banyuls, vallon de Cerbère.

VU1⿴囗十 myrohea,, $V$. pscudomyuros Soy,-Will. - Licux sablonneux et caillouteux, bords des champs.

v. seiuroilea Gmel. - Même habitat. 
v. cllata Link, - Lieux incultes de la région mérid, et moyenne jusqu’à Lyon et Genève.

V. serneea Parl. - Lieux arides à Fréjus et à Cannes.

\$. genieulata Link. - Dicombres auloul de Irarsille, Hyires, Menton, Argelès, Collioure, Port-Vendres, Banyuls.

v. Ligustiea Link, - Burds des chemins. - Envir. de Marseille, Toulon, Hyères, le Luc, Fréjus, Cannes, Nice, Menton. - Hér. ct Pyr.-Or. : Vias, Grammont, sables marit. de la Franqui près Leucate.

v. bromoldea Rehb. - Lieux slériles de la région mérid. Remonte dans la vallée du Rhone jusqu'à Lyon.

v. Juberula Guss., V. Micheliana Rehb. - Lieux sablonn. du littoral méditerranéen. - B.-du-Rh., Var et Alp.-Har. : Marseille à Mazargues et Montredon, la Seyne aux Sablettes, Hyères, le Luc, Fréjus, golfe Jouan, Menton. - Gard et Hér. : la Sylve près Sylvírẻal, Aigues-Mortes, Belle-Cote près de Bouillargues, Lamoure, Sérignan à Valras, les Onglous. - Littoral de l'Aude et Pyr.-Or.

FESTUC tenuifolia Sibth. - Pâturages secs des plaines et des montagnes. - Espèce polymorphe se présentant sous deux formes principales, l'une plus commune, F. ovina L., a la glumelle inférieure brièvement aristée, et doit être nommée $F$. tenuifolia, aristata; l'autre, $F$. tenuifolia de Sibthorp, a la glumelle inférieure non aristée, les feuilles plus ténues et plus longues, et mérite l'appellation de $F$. tenuifolia, mutica. En conséquence, il n'y a pas lieu de maintenir le nom insignifiant de Fétuque des brebis, ni la distinction spécifique admise par la plupart des auteurs entre ces deux formes si voisines. Au surplus il existe encore une troisième forme qu'on trouve çà et là dans les Alpes de Vaud, Valais, Savoic et Dauphiné, et qui ne diffère de la première que par sa taille plus petite et sa panicule florale plus courte; c'est celle que Suter et Gaudin avaient appelée $F$, alpina.

F. Colleriana All. - Rocliers et pelouses des Alpes et tles Pyrínées. - Vaud et Talais: Anzendaz, Fully, St-Bernard, Zermontana, Thyon, Arolla, mont Nuoble, Gemmi, Augstelberg, Mall- 
mark, Simplon, s'élève au pied du mont Rose jusqu'à la Cabane de Vincent. - H.-Sav. et Sav. : Méri, Samoëns, col de Golèse, Buet, Brévent, Aiguilles-Rouges, col de Balme, mont Blanc au Jardin, au Couvercle et aux Grands-IIulets, Val Montjoie, Charvin, Petit-St-Bernard, Hauteluce entre la Cyclaz et la Pariraz. - Is. et H.-Alp. : Belledonne vers les lacs Domenon, Brandes-en-Oisans, la Salette, la Iloucherolle, Obiou, Pelvoux, la Grave, Lautaret, mont Viso, Chaillol-le-Vieil, Clampoléon, mont Aurouse. - B.-Alp., Alp.-Mar. et Vaucl. : montagne d'Embrua, col de Fremamorta, mont Ventoux. - Pyr.-Or. et Ariège: Canigou, Cambres-d'Aze, Jasse de la Bentaillole, Pic del Ginévré et de la Trune, Pas du Roc-Blanc.

F. pyreuaica Reuter. - Rochers et pâturages des Pyrénées. Ariegge: Llaurenti aux Jassettes, lac de Valbonne, vallon de la Maouré. - Pyr. centrales.

F. Iuriuscula L. - Coteaux arides, bords des chemins depuis les plaines jusqu'aux montagnes. - Espèce polymorphe ayant des feuilles vertes ou glauçues de dimensions variables, des épillets glabres ou velus. - Une forme alpestris a le chaume court, la panicule serrẻe.

F. Indigesta Boiss. - Pâturages des sommités des Pyrénées. Pyr.-Or. : Canigou, Pla Guilhem, Carença, Prats-de-Balaguer, col de Nuria sur le versant français et espagnol.

F. violneen Gaud. - Prairies humides des Alpes. - Vaud et Talais : Anzendaz, Grand-St-Bernard, Sembrancher, Val d'Entremont, Zermatt, Saas, Gemmi, Simplon, Nufenen. - H.-Sav. et Sav.: Dents d'0zhe, Nautau, col de Golèse, Samoëns, Vergy, Méri, Tournette, Clıarvin, Brévent, Chamonix, au Montanvert et à la Flégère, mont Iseran, mont Cenis. - Is. et H.-Alp. : Chamechaude, la Moucherolle, Grandes-Rousses, Lautaret, col des Bayes, mont Viso, St-Véran, Morgon, Aurouse, Séuse, Chaudun. - B.-Alp. et Alp.-Mar. : Grand-Couyer-sur-Annot, Roche-Grande près des sources du Var.

F. rubra L. - Prés, bords des bois dans tout le bassin.

F. Heterop 耳nylla Lam. - Bois et pàturages des plaines, des collines et des montagnes. - Espèce polymorphe dans laquelle la 
couleur des fleurs et les dimensions des feuilles sont variables. La forme nigrescens Lam., à fleurs d'un violet foncé, est assez répanduc dans les monlagnes du Jura, des Alpes, des Pyrénées, Margeride, Lozère, Cantal, mont Dore, Forez.

F. pumila Chaix. - Rocailles et patturages des montagnes. - Jura helvélique et français : Chasseral, Creux-du-Van, Clıasseron; Suchet, Colombier, Reculet, la Faucillo, Retord. - Vaud et Valais: Anzendaz, Fully, St-Bernard, col de Fenêtre, Pierre-àVoir, Galmscheide, Gemmi, Riffel de Zermatt, Simplon. - H.Sav. et Sav. : Roc d'Enfer, Nautau, mont Péletod, Sixt, Brizon, Vergy, Brévent, col de Balme à la Croix-de-Fer, Chamonix au Montanvert, Antcrne, Sâles, Platets, col des Aravis, la Tournette, les Mottets, col de Fenêtre, mont Trélod, mont Cenis. Is, et II.-Alp. : Grand Som, Chamechaude, col de l'Arc, la Pra de Belledonne, mont de Lans, mont Chamoux, la Grave, Lautaret, Galibier, Alp.-Martin, cols des Hayes, Isoard, de Ruines et Agnel, Pías en Queyras. - B.-Alp. et Alp.-IIar. : Bérard, mont Grand-Couyer, Entraunes, Alpes de Fenestre et de Carlin, les Viosennes, mont Frontero.

Une variété aurata Gaud., qui a la panicule étalée, d'un jaune doré, des ligules très courtes, a étẻ trouvée en Valais dans les Alpes de Saillon, d'Héremence et d'Anniviers, de la Gemmi, et du Simplon.

F. varla Haenke. - Rochers des hautes montagnes, - Valais : StBeruard, Bagnes, Saas, Simplon. - Sav., Is. et H.-Alp. : mont Cenis à Ronches, Belledonne près des lacs Domenon, Prémol, Grandes-Rousses vers le lac Blanc, Lautaret, mont Viso. B.-Alp. et Alp.-Mar. : Serennes à Champ-Rond, Crête de CostoLoupet, mont Ilonnier, mont Frontero, Viosennes, col de Tende. - Pyr.-0r. ct Ariège : Canigou, Pla Guillem, Prals-deMollo, Cambres-d'Aze, col de Nuria, vallée d'Eyne, Font-Romeu près Mont-Louis, Salvanère, Llaurenti au Soula de Joucarets, Escale de Valbonne, Port de Paillères.

La variété flavescens Bell., qui a des feuilles capillaires molles, des épillets oblongs, pauciflores, jaunâtres, se trouve dans les H.-Alp. à la Grave, Val-des-Prés au vallon de Dormillouse, 
à Abries, Villevieille et Molines en Queyras; dans les B.-Alp. au col d'Allos et aux sources du Var.

La variété eskia liam., qui a des feuilles jonciformes, raides et épaisses, existe dans les Pyr.-0r. et l'Ariège au Canigou, Prats-de-Mollo, vallée d'Eyne, lo massif du Llaurenti. - Pyr. centrales.

T. paloøa lialler F. - Prairies des hautes montagnes à sol siliceux. - Valais : St-Bernard, Zermatt au Riffel, mont Cervin, Saas, Simplon. - H.-Alp. et Alp.-Har. : mont Viso à la Traversette, Salsamorena aux sources de la Tinea, col de l'Abisso. - Ard., H.-L. et Gard: Mezenc, Baraque de Nichel à l'Espérou, Banahu.

- Pyr.-0r. et Ariège: Canigou, Cambres-d’Aze, massif du Llaurenti. - En dehors de nos limites dans les Pyr. centr., Margeride, Aubrac, Lozère, Cantal, mont Dore.

F. nutans Host, $F$. Scheuchseriana Gasul. - Pàturages du Jura et des Alpes. - Colombier, Reculet au vallon d'Ardran. - Valais et Vaud : Bovonnaz, val d'llliez. - HI.-Sav. et Sav. : Anterne, Sixt, col de Golèse, Méri, vallée du Reposoir, Hauteluce entre Turnaz et Colombaz, mont Cenis. - H. et B.-Alp. : Névache, Lauzanier.

F. sllvatiea Vill. - Bois des montagnes. - Forêts des Vosges jusque dans la H.-Saone, aux Ballons de Giromagny et de Servance, vallée de Miélin, du Rahin et du Fray. - Jura helvétique et français jusque dans les montagnes du Bugey. - Vaud et II.-Sav. : Saufeulaz sur Bex, Salève, Voirons. - Is. et H.-Alp. : Grande-Chartreuse, Chalais, mont Rachais, Prémol, St-Ange, Pleyne-de-Chaudun près Gap. - Le Morvan, Roussillon près Autun au-dessus du ruisseau de la Canche. - Loire et Ard.: Pilat, Mezenc, Bauzon. - En dehors de nos limites dans les Pyr. cent., Cantal, mont Dore, Forez, Vosges.

F. spadicea L. - Rochers et paturages des hautes montagnes. Sav. : les Allues, montagnes au-dessus d'Aime, entre Retord et le col de la Louse, petit mont Cenis, mont Cenis. - Is. et II.Alp. : col Oddie, Alpe du mont de Lans, mont Chamoux, Lautaret, mont Gondran, cols de Ruines, Agnel et de Vars, Orcières, Chaudun, col de Glaise, mont Séuse. - B.-Alp. et Nlp.-Mar. : 
Crouès, Malemort, Entraunes, Bonnet des Trois-Evếques, col de Salèse, vallon du Cavallé, Alpes de Tende. - Ard, et H.-L.: le Mezent, Gerbier-des-Joncs. - Gard et Hér. : Clıartreuse de Valbonne, Concoule, l'Espérou, les Cambrettes, la Sérane, le Caylar. - Pyr.-Or. el Ariègo: Canigou, Costa Bona, Pla Guillem, vallée d'Eyne, Jasse de la Bentaillole, cirques d'Artounant et des Aigueltes, val de Paillères à Soucarrat. - En dehors de nos limites dans les Pyr. centr., Aveyron, Cantal, mont Dore, Pierre-sur-Haute. - Espèce polymorphe. La forme Durandiana Claus. est signalée à l'Ermitage de Consolation au-dessus de Collioures, à Port-Vendres et à Banyuls dans les Pyr.-0r.

T. anudinacea Schreb. - Bords des eaux dans tout le bassin. Espèce polymorphe.

La variêté interrupla Desf., à panicule étroite, interrompue à la base, se trouve dans l'Hẻr. et l'Aude à Palavas, Cette, Murviel, St-Martin-de-Londres, Lodève, Sérignan, Narbonne.

T. protenuis lluds. - Prairies et pâturages. - Espèce polymorple ıpui a quelquefois une pariicule réduite à des rameaux solitaires portant des épillets presque sessiles ( $F$. pseudololiacea Fries).

Figanten Vill., Bromos giganteus L. - Bois des montagnes.

IBro.nOS nutans, B. tectorum L. - Lieux incultes dans tout le bassin.

B. seaberrimus, $B$, sterilis L. - Nême habitat.

B. maximus Desf. - Forme luxuriante du précédent, à taille beaucoup plus élevée, à chaumes un peu pubescents au sommet, à ligule plus allongée, à glumes moins inégales; la panicule est tantôt penchẻe, tantôt dressée. - Lieux stériles de la région mérid. d'où il remonte accidentellement dans la vallée du Rhône et de ses aflluents à Crest, St-Vallier, Comboire près Grenoble, Veyrier près Annecy. S'étend dans le bassin de la Garonne et dans l'Ouest.

B. madritenais L. - Lieux stériles de la région mérid. et moyenne jusqu'à Lyon, Grenoble, Chambéry et Annecy.

FB. xubeng $\mathrm{L}$. - Lieux stériles de la région mérid. - Remonte dans la Drôme à Nyons et Crest.

W. asper L. - Bois des collines et des montagnes.

Catal. Bassin du Rhonc. 
B. erectus IIuds. - Prairies, bords des champs et des bois. - Varie à panicule contractée ou étalée.

8. seeallinus L. - Moissons. - La grosseur des épillets varie : d'où les formes appelées macrostachya, microstachya.

B. arvensis L. - Lieux cultivés. - Espèce polymorphe, - La forme patulus qu’on observe dans les lieux stériles de la région mérid. a la panicule penchée et les rameaux courts.

R. commutatus Schrad. - Clıamps, bor'̉s des chemins. - Espèce polymorphe.

B. racemosus L. - Forme à panicule étroite et contractée, à épillets renflés et brillants, à glumes ovales, à glumelle infér. plus régulièrement courbée en arc. - Mêlẻ au précédent dans la partie septentr. du bassin. Beaucoup plus commun que le $B$. commulatus dans le nord de l'Europe.

B. moHis L. - Bords des champs et des chemins. - Espèce polymorphe ayant des épillets tantôt glabrescents ou glabres (var. glabrescens), tantot velus ainsi que la tige (var. mollissimus). Cette dernière forme qui, en outre, a les rameaux plus contractés et plus courts se trouve dans les sables du littoral de la Provence, du Languedoc et du Roussillon.

La forme intermedius Parlat., qui a des épillets linéairesoblongs, des chaumes grêles et très-glabres, existe dans la région des Oliviers de la Provence et du Languedoc.

r. squnrrosus L. - Lieux incultes de la région mérid., d'oủ il remonte à Crest, St-Vallier (Dr.), Mercuer (Ard.), Gap et StMens (HI.-Alp.), Grenoble, Comboire, Vif, Genas (Is.), Villeurbanne près Lyon, vallée de l'Arc en Maurienne, de l'Arve jusqu’à Chamonix, du Rhône valaisan à Martigny, Sembrancher, Sion, Sierre, Visp et Brieg, et enfin dans C.-d'Or et S.-et-L. à Meursault, Santenay, Rully.

B. maerostachyus Desf. - Lieux stériles de la région mérid. d'où il remonte vers Nyons et Die dans la Dróme.

HOMDEUMI Vugare, hexnstlehtam et distichum L. Cultivés et quelquefois subspontanés.

耳. murinum L. - Bords des chemins.

15. secalinum Schreb. - Prés et bords des champs. 
19. moritimum With. - Sables du litloral méditerranéen et même de l'intérieur des terres. - Vaucl., B.-du-Rh., Var et Alp.-Mar. : Avignon, Orange, bords de l'étang de Marignane, Toulon, le Luc, le Muy, Frijus, ile Ste-Marguerite, golfe Jouan, Nice. - Littoral du Languedoc et du Roussilion.

8f. bulbesum L. - Lazaret de Toulon, la Sauvelte près des Mlayons.

Q 1 IIOs erinitus Schreb. - Lieux herbeux de la région mérid. - La Crau, Arles, Fréjus, St-Raphaël, Grasse, Nice, Villefranche. - Gard et Hêr. : Nimes, St-Jean-de-Védas, Murviel, St-Georges, Portiragnes, Roquehaute, Agde, Pẻzenas. - Aude ct Pyr.-Or. : Narbonne, Salses, Opol.

E. silvaticus IIuds., E. europaeus L. - Bois des collines et des montagnes.

SECAY cereale L. - Cultivé el yuelqufois subspontané.

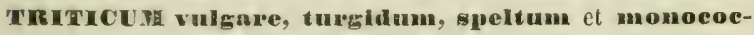
eum L. - Cultivés.

T. villosum Pal. de B. - Naturalisé pris de Marscille à St-Marcel sur la montagne de N.-D. de Nazareth, vallon de Forbin, la Penne, remparts de Toulon, la Sauvette dans les Maures, Nice. - Montpellier à Lamoure, Béziers, Pézenas.

GCILors ovata L. - Licux arides, bords des chemins de la région mérid. Remonte dans la Dr., les II. et B.-Alp. à Crest, Montélimar, Valence, St-Vallier, Laveyron, Gap, Embrun, Barcelonnette; dans l'Ard. à Viviers, Rochemaure, Aubenas et Tournon.

Espèce polymorphe daus laquelle M.I. Jordan et Fourreau ont distingué les formes suivantes :

E. virescens. - Toulon, Perpi- E. erigens. - Mas-Carbardès guan.

E. procera. - Montpellier.

(Aude), Perpignan.

A. erratica. - Valréas (Vaucl.).

E. microstachya. - Montjoyer

E. vagans, - Montpellier.

¿E. pubiglumis. - Digne.

E. nigricans, - Perpignan.

de. triuneinlis L. - Lieux stériles de la région mérid. Remonte 
dans la vallée du Rhône dans les mêmes localités que l'E. ovata.

E. Triartstata Willd. - Même dispersion que le précédent.

Les trois Egilops ci-dessus désignés forment avec le Triticum vulyare les hybrides suivants :

E. vulgari-ovata Godr., E. triticoidea Requien. - Vaucl, et B.-duRh. : Avignon, Orange; les Milles, les Martigues, Berre. Gard et Hér. : Nìmes, Montpellier à la Colombière et ả Figuérolles, Baillargues, Lansargues, Villeneuve, Roquehaute. Pyr.-Or.: de Rivesaltes à Vingrau.

E. vulgari-triaristata Godr. - Montpellier, Agde.

E. vulgari-triuncialis Loret. - Hér. : Bédarieux, Mas-de-Londres.

AGRORYQ⿻上丨 junceun P. de B. - Champs du littoral méditerranéen. - B.-du-Rh., Var et Alp.-Mar. : Fos, Montredon, Nazargues, Toulon, Hyères, ile de Porquerolle, Frẻjus, Cannes, Antibes, Nice, Menton. - Gard et Hẻr. : Aigues-Mortes, Palaras, les Onglous, Cette, Agde. - Pyr.-Or. : Argelès, Collioure, Banyuls-sur-Mler.

A. acutum Roem. Schult. - Sables maritimes du littoral. Bords de l'étang de Marignane, Toulon, Cannes. - AiguesMortes, St-Gilles. - Maguelone, Palavas, Pérols, entre Cette et Agde. - Canet, Collioure, Port-Vendres, Banyuls.

A. elongatun Host, A. scirpeum Presl. - Marais saumâtres du littoral. - Étang de Berre, les Martigues. - Aigues-Mortes, Peccais. - Maguelone, Palavas, Pérols, les Onglous, Valras, Mauguio, Frontignan, Agde. - Ste-Lucie, Salses, Canet, le Vernet près Perpignan.

A. pungens Roem. Schult. - Sables maritimes. - Nlazargues, la Madrague. - Toulon, Cannes, Antibes, Nice. - Littoral du Languedoc et du Roussillon.

A. pyenanthum Godr. - Sables maritimes du littoral méditerranéen.

A. enmpgestre G. G. - Champs, bords des chemins do la région mérid. et moyenne jusqu’à Gap, Grenoble et Lyon.

A. glaucum Rocm. Schult. - Lieux arides des montagnes. - Valais : les Marques, Conthey, Sion, Simplon. - H. et B.-Alp. : 
Laularet, St-Jean près Gap, Matachard, Causson près Digne, Castellanne.

A. mraclle de Pouzolz. - Gard : Manduel, Aigues-IIortes.

A. repens P. de B. - Lieux cultivés dans tout le bassin.

A. caninum Roem. Schult. - Bois, haies, lieux ombragés.

A. Ecouxisnum Gren. et Dus. - Prés salés de Berre (B.-elu-Rb.), marécages de la plage de Portiragnes (Hér.).

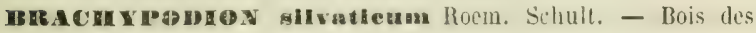
plaines el des montagnes.

B. Dinnatum $P$. de B. - Le long des haies, des loois et des chemins. - Espèce polymorphe. - Dans la forme australe, très répandue dans la région mérid., les feuilles sont étroites, raides, à la fin enroulées, la glumelle infér. plus longue.

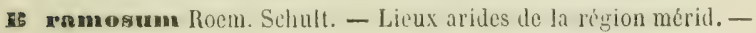
Remonte dans la Drômo à Crest, le Buis, Nyons, Donzère, Montélimar ; dans l'Ardèche à Viviers, Rochemaure.

B. distnehyum P. de B. - Licux pierreux, bords des champs de Ia région mérid. - Remonte dans l'Ardéche, la Drôme, et s'étend dans le bassin de la Garonne.

LDIIXI perenne L. - Prés, bords des champs et des chemins. - Espece polymorphe présentant une forme grèle, à épillets pauciflores, L. temue L., et une autre forme à épillels larges, multiflores et étalẻs, L. crislatum Pers.

X. Inncum A. Braun. - Prairies, lieux herbeux humides. - Cultivé sous le nom de Ray-Grass d'Italie.

7. multiforam Lam. - Lieux cultivés de la région mérid. et moyenne; plus rare dans la partie septentr. dans quelques localités de la C.-d’Or.

L. rigidum Gaud. - Lieux cultivés de la région mérid. - Remonte à Crest, St-André-de-Rosans, Grenoble, Lyon, Dole, Autun, Cluny, Genève et les bords du Léman.

C. temulentum L. - Yoissons, cliamps cultivís des plaines et des montagnes. - Espèce polymorplie ayant une arête tantôt longue, taniot courte, quelquefois nulle: d'oủ les variétés macrochaetum, leptochactum, muticum.

L. arvense Schrad., L. linicola Sond, - Champs de Lin. - Forme à 
chaumes et épis grêles, à épillets obovés et à glume courte, assez commune dans les champs de Lin des envir. de Besançon, Cuse. - Sav. à Crest-Voland.

VARDUROS unilnteralis Boiss., $N$. tenellus Rchb.-Lieux arides, coteaux pierreux de la région mérid. - Remonte dans la Dr., les H.-Alp. et l'Is. à Crest, Montélimar, Gap, Ventavon, Lazer, Vienne, Vaulnaveys, Comboire, Grenoble; dans l'Ain et le Rh. à Virieu-le-Grand, Belley, la Valbonne, la Pape, Oullins, St-Alban; près de Genève entre Versoix et Genthod, au Salève le long du Pas de l'Échelle, dans les carrières de Mornex; dans la C.-d'Or au-dessus des carrières des Chartreux, Fontaine, Ste-Anne, Couchey, entre Beaune et Savigny. S'étend dans l'Ariège, l'Aveyron, et quelques localités du bassin de la Garonne.

N. alterniflorus, $L$. Lachenalianus Godr. - Licux sablonneux arides, coteaux pierreux des terrains siliceux. - Espèce polymorphe présentant, comme la précédente, une forme aristée et une forme mutique.

N. subulatus, N. Saľmannianus Boiss. - Coteaux des B.-du-Rh. aux vallons de Toulouse et de Gémenos, Bec-de-l'Aigle à la Ciotat.

LERTUER eylindricus Trin. - Sables de la région mérid. - Vaucl., B.-du-Rh., Var et Alp.-Nar. : Avignon, bords de la Durance, les Martigues, Marseille, Toulon, Fréjus, golfe Jouan, Antibes, Nice. - Gard et Hér. : íle de la Bartelasse, Montpellicr à Grammont, Fréjorgues, Maguelone, Montbazin. - Aude et Pyr.-Or. : Narbonne, plage de Barcarès, de la Salanque el du Canet.

L. Ineurvatus Trin. - Sables du littoral méditerranécn. - B.du-Rh., Var et Alp.-Mar. : Marseille, Aix près du chàteau de la Calade, Sablettes de la Scyne, Sa!ines-Neuves d'Hyères, ile Ste-Marguerite, golfe Jouan, Antibes, Nice. — Gard et Ilér. : Aigues-Mortes, Bellegarde, Cette, Maguelone. - Aude et Pyr.Or. : Narbonne, Collioure, Port-Vendres, Banyuls.

L. Aliformis Trin. - Sables maritimes, - B.-du-Rh., Var et Alp.-Mar. : Toulon, Hyères, Fréjus, golfe Jouan, Antibes, Nice. 
- Ilér. ct Pyr.-Or.: Roquehaute, Cancl, Chảteau-Roussillon, Argeles.

FogICLas aristntus (nardoilens) Trin. - Licux sablonneux. - Hh. cl Is.: Lyon à Nontchal, Mollard do Décines, Crémieu, Clıarviou, Jarrie, entre Eybens el St-Martin. - Dr. : Romans au Nas-de-Tricot. - H. ct B.-Alp., Vaucl.: Laragne, Ribiers, Sisteron, bords de la Durance, Avignon au bois de Fargues, Jorière au chemin de Jonquerelles, Orange, Sérignan, - B.du-B. et Var: la Ciotat, Toulon, Ic Luc, St-Tropez, Fréjus, Cannes, Biol, Nice, Menton. - Gard: Manduel, Caissargues, Anduze, le Vigan, Fort-les-Bains. - Hér. : Montpellier à Grammont, Juvignac, Fabrègues, St-Jean-de-Ve̊das, Murviel, Pignan, St-IIartin-de-Londres, St-Étienne-de-Mlursan, St-dlarlind'Orb, Lamalou, Colombière, Ceilhes, Avène, St-Chinian. Aude et Pyr.-Or. : Aussières, St-Nazaire, Canet, Perpignan sur Ies graviers de la Tet.

SA It wos strleta $L$. - Piturages des terrains siliceur des montagnes ef quelquefois des plaines. - Haules-Vosges jusque dans la II.-Saône aux Ballons de Servance et de Giromagny, Planche des Belles-Filles, mont de Vannes, vallée de Mansvillers, grès bigarri et dilavium de Franchevelle, Fougerolles, Linexert, Esboz. - Nul sur de grandes étendues de la chaîne jurassique; ne s'y montre que dans les lieux tourbeux au Paswang, Lomont, Chasseral, la Tourne, Pouillerel, Aiguillon, mont d'Or, Boujailles, Reculet, Colombier du Bugey. En plaine on le voit dans la forêt granitique de la Serre et sur les sols argileux de la Bresse. - Vaud et Valais: prés tourbeux de Bovonnaz, Alpes granitiques de Bagnes, St-Bernard, Nendaz, Hérémence, Evolena, Zermatt, Simplon, Conches, Grimsel. - H.-Sav. et Sav. : Sables sidérolithiques du Salève et des Voirons, vallée de Chamonix, de Montjoie, Hauteluce, Beaufort, Crest-Voland. - Is. et H.-Alp. : chaines granitiques de Belledonne, des SeptLaux, de Taillefer et de l'Oisans, prairies tourbeuses du mont Aiguille, de la Salette, du Lautaret, col Bayard, Chaillol-leVieil, Lus-la-Croix-Haute, col de Vars. - Pâturages alpestres des B.-Alp. et Alp.-Mar. - Terrains granit. du Morvan autour 
de Saulieu, Arnay, Roche-en-Brenil, Aulun; chalne granitique et gneissique du Beaujolais, Lyonnais, Pilal, Yirarais, Iczenc jusqu'à l'digual, la Lozère, l'Espinouse à Fraisse, la Salvetat, le Saumail. - Pyr.-Or. et Ariège : gneiss et granite du Canigou, Pla Guillem, Madrès, Salvanère, Llaurenti. - En dehors de nos limiles sur les terrains siliceux des Pyr. centr., Tarn, Aveyron, Lozère, Allier, Yonne, tout le centre, l'Ouest, le bassin de Paris, l'Alsace et la Lorraine.

La distribution géographiqque du $N$. stricta à travers l'Europe fournit un puissant argument en faveur de la doctrine de l'influence chimique du sol sur la dispersion naturelle des plantes. Bien que cette Graminée se plaise de préférence dans les pâturages des montagnes à sol siliceux ou à sol calcaire recouvert de dépóts tourbeux, cependant elle peut vivre dans les plaines lorsqu'elle y trouve les conditions telluriques qui lui conviennent; c'est ainsi qu'elle croit non-seulement dans la plaine sablonneuse de Haguenau, en Alsace, où elle a pu descendre des Vosges, mais encore sur les sables et argiles tertiaires de la Sologne, loin des massifs montagneux du centre de la France et, plus loin encore au nord, à Rambouillet, Fontainebleau, Senlis, Compiègne et dans la vallée de Bray, tantòt sur les sables, tantòt sur les argiles, c'est-à-dire sur des terrains dissemblables sous le rapport des propriétés physiques, mais analogues par leur teneur en silicates alumino-terreux et par le manque d'éléments calcaires. Voyez (page 513) les mémes remarques à propos de la Bruyère vulgaire. 


\title{
ACOTYLÉDONEEES VASCULAIRES
}

\author{
FOUGERERS.
}

Botrioror Imnne $S w$. - Pâturages secs des montagnes, excepté dans les B.-du-Rh., le Var et l'Hérault. - Espèce polymorphe dans laquelle la forme des segments des frondes présente des variations.

D. Intifolium A. Braun. - Forme a fronde triangulaire, large dans lo sens horizontal, à segments crénelés-lobẻs ou même quelquefois profondément incisés-lobés. - Pâturages des montagnes. - H.-Sav. : Chamonix au bois Bouchet. - Loire : Pilat près de la ferme de Botte, Marlhes. - Vosges.

OPIrOGLOssox vulgare L. - Prís et pâturages lumides.

n. lusitunicum I. - Forme à taille plus petite, dans laquelle le limbe de la fronde stérile est lancéolé ou lancéolé-linéaire. La floraison est beaucoup plus précoce : dans le midi, elle fructifie de fin décembre à fin janvier; dans l'Ouest, du 15 février au 15 mars, tandis que la Langue de Serpent vulgaire fructifie de mai à juin. En outre, celle-ci crolt dans les prairies humides, au licu que l'O. lusitanicum se montre sur les pelouses sèches bien exposées au soleil. - B.-du-Rh., Var et Alp.-Mar. : en Coustiero, la Pissaroto, Fos, Hyères, Vidauban, Fréjus, Antibes. - Is. : Crémieu. - Ouest et sud-ouest de la France.

osmuvid regalis L. - Bois marécageux. - H.-Saône : vallon de Mourière près Ronchamp, mont de Vannes sur le versant occidental, Montessaux, Fougerolles. - Jura : Bletterans, forêt de la Serre. - C.-d'Or et S.-et-L. : Saulieu au bois de la Fiote, bois de la Vente-à-l'Italien, bois des Renaudiots près Autun, Antully, la Gravetière près Broye, Branges près Louhans. - 
Ain : Croltct, ctang Genoul, Pont-de-Vaux, Corgenon, St-Denis, Marboz. - Is. : forêt de Chambaran près Roybon, étang de Bressicux, St-Albin-de-Vaulserre el Vérel près du Pont-dcBeauvoisin, les Avenières. - Var et Alp.-Mar. : la Verne, la Napoule, l'Esterel. - Gard et Hér. : Valleraugue, Genolhac, Portes, St-Gervais à Mlècles, le Caroux, Lamalou, Pardailhan. - Pyr.-Or. : Carbassera au-dessus de Lavail près Collioure, Rigarda, Glorianes.

CETEIE ACI offelunIis Willd. - Murs et rochers.

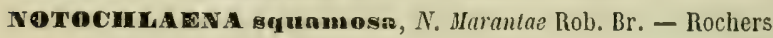
siliceux. - Dr. et Ard. : granite de St-Vallier à Champis et de Ponsas, Tournon sur la rive droite du Doux, Thueyts, les Lobelles près des Vans, Vals, Mayres. - Var et Alp.-Mar. : Indres près Fréjus, Daumas au Cannet, la Garde-Freinet au N.-0. du fort Freinet, l'Esterel, Biot près Antibes. - Gard : Anduze, le Vigan. - En dehors de nos limites dans l'Aveyron à Firmy, Najac, Ferragut gorge de la Sérène, St-Parthem.

POLYPODYOY vulgare L. - Bois, rochers, vieux murs. Espèce polymorphe dans laquelle les segments des frondes sont entiers, ou dentés (serratım), quelquefois mème les segments inférieurs profondẻment pennatilobés (cambricum).

P. villosum Dulac, $\boldsymbol{P}$. phegopteris L. - Bois des montagnes, surtout dans les terrains siliceux. - Hautes Vosges jusque dans la Haute-Saône aux Ballons de Servance et de Giromagny, Clıampagney, mont de Vannes. - Vallée du Puix près de Belfort. Disséminé dans la chaine jurassique où il ne se montre que sur les sols tourbeux et riches en humus; existe en plaine sur les cailloux quartzeux de la forêt de Chaux. - Vaud et Valais : Esserts de Bois-Girard près Payerne, Combremont-le-Petit, lc Jorat, Alpes de Château d'OEx et de Vevey, Ferrière, val d'Illiez, Bagnes, Saas, Simplon. - H.-Sav. et Sav. : Brizon, bois de Sommier dans la vallée du Reposoir, envir. de Chamonix au Bouchet, Montanvert, cascade du Dard, les Pélerins, vallée de Beaufort, Tignes. - Is, et H.-Alp. : chalnes de Belledonne, des Sept-Laux, de Taillefer et de l'Oisans, Prontic en Valgaudemar, la Chapelle, Chasseron, le Briançonnais. - Alp.-Mar. : Ste- 
Anne-de-Vinaï. - C.-d'Or et S.-et-L. : Morvan granitique, SauJieu au bas des Chemins-Blancs, Roche-en-Brenil, Premeaux, les Renaudiots et Brisccou près Autun. - Rh. et Loire: le Beaujolais à St-Rigaud, Pilat. - Ard,, Gard et Ilér. : montagnes volcaniques du Gerbier-des-Joncs, gneiss et schistes du Vigan et de l'Espinouse à Fraisse. - Pyr.-0r. et Ariège : Pratsde-IInllo, Mont-Louis, Noliedes, Llaurenti au Soula de Joucarets, forêt du Paillariel, pic de Canrusc. - En dehors de nos limites dans les Pyr. centr., l'Aubrac, Cantal, mont Dore, Puy-de-Dome, le Forez à Pierre-sur-Haute, Morvan nivernais, la Creuse et la Haute-Vienne.

P. Mneticum L. - Rochers. - Hautes Vosges jusque dans la II.Saône aux Ballons de Servance et de Giromagny. - Région alpestre de la chaîne jurassiuge à la Dòle, au Reculet et au Crêt de Chalam. - Valais et H.-Sav. : Val Ferret, Brévent, Aiguilles. Rouges, montée des cascades du Dard, des Pélerins et des Grands-Nifulets, montée des chalets de l'ognant à gauche du glacier d'Argentière, vallon de la Diozaz, bois de la Griaz près de N.-D. de la Gorge, Allée-Blanche près du lac Combal, Voirons, Brizon, Méri. - Is. et II.-Alp. : Belledonne vers le lac du Crouzet, Prẻmol, Grande-Chartreuse, Charance, Chaudun, Rabou.

P. triangulare Dulac, $P$. dryopteris $\mathbf{L}$. - Rochers, bois humides des montagnes à sol siliceux. - Hautes Vosges jusque dans la II.-Saône aux Ballons de Servance et de Giromagny, ChâteauLambert, Miélin, mont de Vannes, Ecromagny, Mélisey. Vaud et Valais; Morcles, Ferrière, Bagnes, Saas, Simplon. H.-Sav. et Sav, : sables sidérolithiques du Salève au-dessus d'Archamp et des Voirons, bois de Sommier dans la vallée du Reposoir, Chamonix au Bouchet, à la Mer-de-Glace, aux cascades du Dard et des Pélerins, les Contamines, vallẻe de Beaufort, Combe de Villette près Bramans. - Is. et H.-Alp. : chaines de Belledonne, d'Allevard, de Taillefer, la Grave, vallon de Pe̊as, Orcières, pic de Chabrières, la Grangette. - B.-Alp. et Alp.Mar. : les Sanières près Jauzicrs, col de Fenestre, Clans, Venanson, Lantosque, Utelle, la Giandola, - C.-d'Or et S.-et-L.. 
le Morvan à Nataloux près Saulieu, Montjeu, Roussillon, valléc de la Cange. - Rh. et L. : le haut Beaujolais et Lyonnais à Aujoux, St-Rigaud, Duerne, Aveize, Haute-Riroire, Yzeron, Pilat. - Ard. et Gard : Mazan, le Vigan, l'Espérou. - Aude, Pyr.-Or. el Aric̉ge: la Montagne-Noire, Nohèdes, Graux de Thuès, Llaurenti au Pla del Bosc, Baouzeille du Tarbézou, Soucarrat, Mijanès au ravin de Canals, val de Paillères. - En dehors de nos limites dans les Pyr. centr., Tarn, Aubrac, Cantal, mont Dore, Puy-de-Dóme, Forez à Pierre-sur-Haute, Morvan nivernais, Creuse, H.-Vienne. - Espèce polymorphe.

La forme calcareum Sm., particulière aux terrains calcaires, a une fronde plus étroite et plus ferme, des pétioles finement poilus-glanduleux. - Chaine jurassique jusque dans les vallées du Bugey où elle est très-commune, montagnes calcaires de Vaud, Valais, Savoie, Dauphiné et jusque dans les Alpes-Maritimes.

GRAMIITS Ieptophyila Sw, - Rochers humides de la région mérid. - Var et Alp.-Mar. : Hyères, Cavalaire, le Luc, la Garde-Freinct, vallée de l'Esteron, Maures de Tanneron, Cannes, Vallauris, Biot, Menton. - Gard et Hér. : le Vigan, vallon d'Aulas, Pardailhan. - Pyr.-Or. : Collioure, Port-Vendres, Banyuls sur-Mer. - En dehors de nos limites dans l'Aveyron, la Lozère, le Finistère. On prétend l'avoir vu au Dar près de Conflans (Sav.).

WoODSTA hyperboren $\mathrm{R}$. Br. - Rochers siliceux des Alpes. Valais: Mayens de Lourtier, Zermatt, Saas à Fee, Grundberg, Schwarzberg, Naters, pied mérid. du Simplon. - H.-Sav. et Sav. : Chamonix à la Grande, entre les ponts de Ste-Marie et du Coupeau, la Flégère, sommet des Aiguilles-Rouges, les Altues. - Is. et H.-Alp. : Belledonne au-dessus du lac Crnuzet, Bourg-d'Oisans près de la Cascade, Brandes, Villard-Eymond, lac Lovitel, Auris sur la rive gauche de la Sarène, Clapier de St-Christophe, la Bérarde près du glacier de Chardon, les Fraux, la Grave sous les glaciers, Villard d'Arène sur la rive gauche de la Romanche et au vallon de l'Arp, Chasseran en Valgaudemar, Molines en Champsaur. - B.-Alp. : Lauzanier près du lac. 
Une forme à lobes profondément incisés, $W$. ilvensis $\mathrm{R}$. Br., est assez commune dans la vallée de Chamonix, sur la rive droite de l'Arve jusqu'aux gorges de la Diosaz, puis sur l'autre versant des Aiguilles-Rouges en montant au col de Bérard et de Salenton, au Buet, et vers la cascade de Bérard.

ASPIDION Ionehitum Sw. - Bois des montagnes. - Jura helvétique et français: Chasseral, Creux-du-Van, Chasseron, Rizoux, la Dôle, Colombier, Reculet, Faucille, Ornans, Salins, Retord et Arvières en Bugey, Abergement-de-Varey. - Vaud et Valais: Anzendaz, Fully, Salvan, Trient, Catogne, Mayens de Sion, Arbaz, Mainghorn, Simplon. - H.-Sav. el Sav. : Pitons du Salève, montagnes de Samoüns et de Colone, mont Châtillon, Parmelan, lloc de Chère sur les bords du lac d'Annecy, chaine du mont Blanc, entre Argentière et la Tour, Pierre-à-Bérard, montée du Pavillon de Bellevue, de Montanvert et des Cascades, Crest-Voland, Bonneval en Maurienne, sources de l'Arc. - Is. et H.-Alp. : chaines de Belledonne et de l'Oisans, Grande Clıartreuse, col de l'Arc, la Salette, Lautaret, mont Viso, Chaillol, Orcières, Séuse, la Grangette, Aurouse. - B.-Alp. et Alp.-Mar. : Lauzanier, Parpaillon, sommités des vallées du Var, Vesubia, Tinea, Roja. - Loire et Gard : le Pilat, le Vigan. - Pyr.-Or. ct Ariège: Canigou, vallẻe d’Eyne, Madrès, cirque des Aiguetles, Boutadiol, Rabassolès, pic de Tarbézou, Counci, Port de Pailleres. - Pyr. centrales.

A. aculeatun Doell. - Bois dans tout le bassin. - Espèce polymorphe variant quant à la forme des segments des frondes.

POLXSICHON convolutum Dulac, $P$. thelypteris L. - Licux marécageux et tourbeux. - Envir. de Belfort au Salbert, marais de la Vaivre, Gouttes de l'Arsot. - Doubs, Jura et Ain : Conthenans, Dole, Doucier, Divonne, la Bresse, Cressieu, Contrevoz et Prémeyzel près Belley, envir. de Bourg, cntre Pont-de-Vaux et Vescours, Ste-Croix près Montluel. - Vaud: Seedorf près Payerne, entre Valeyres et Montchoisi, les Pierrettes, Epenex, Jongny, Roche. H.-Sav. et Sav. : Bossey, Roellebot, Lossy, Troenex, base d'Andey près Bonneville, Thonon, Annecy, Menthon, lac St-Marcel près Moutiers, la Bâtie près Tours. - Is. : Mey- 
zieu, la Boucherette près Genas, Eydoche, marais de Tignieux, des Avenières, de la Tour-du-Pin, du Pont-de-Beauvoisin, Grand-Lemps, Paladru, Chambaran. - C.-d'Or: Prémeaux aux sources de la Bouzoise, Val des Choues, marais de Combe-Noire, Beaune, Marcy-sur-Tille. - B.-du-Rh. : étang de Meyrane, Raphèle. - Hér. : étang de Capestang. - Ariège : mouillères du Llaurenti, Val de Paillères à Soucarrat.

P. gInndulosum Dulac, $P$. oreopteris $\mathrm{L} .-\mathrm{B}$ ois des montagnes à sol siliceux. - Hautes-Vosges jusque dans la H.-Saone au Ballon de Giromagny, vallée du Rahin, Plancher-Bas, Ronchamp. - Envir. de Belfort à la Vaivre et sur la lisière de l'Arsot. Rare dans la chaine jurassique à la Dôle, au Reculet, au Crêt de Chalam, dans les bois de Lavatay près de la Faucille, à une faible altitude dans les forêts à sol siliceux de Chaux et de la Serre (Jura), de Châtillon en Bresse, Brisecou près Autun (S.et-L.). - C.-d'Or : Saulieu dans les bois de Verneau près de l'etang Larmier. - Fribourg, Vaud et Valais: Alpes de Gruyère et de Château d'Oex, montagne de Perche près des OrmondsDessus, Colatel sur Bex, Sembrancher. - H.-Sav. : grès macigno et nummulitique des Voirons près du Couvent, Meillerie, vailée du Reposoir, Chamonix en montant au Biolet, aux cascades du Fouilly, du Dard et des Pélerins, au glacier des Bossons, Ste-Harie, gorges de la Diosaz. - Is. et Dr. : GrandeChartreuse, Proveyzieu en allant à la Charmette, Revel à l'Oursière, Allevard au mont Ourrard, Barnave au Sarrelong. Alp.-Mar. : bois de Molières et de l'Isola, vallée de Pesio. Ard. et Gard : Entraigues, montagnes du Vigan. - Pyr.-0r. et Ariège : Mont-Louis, Madrès, Cirque des Aiguettes et d'Artounant, forèts de Riplaou, des Braguès et de Paillariel, Roc de l'Estagnet. - En delors de nos limites dans les Pyr.-Centr., Aveyron, Puy-de-Dome, Allier, Niêvre, Creuse, H.-Vienne, Maine-et-Loire.

P. obtusum Dulac. $P$. filix-mas Roth. - Bois des plaines et des montagnes. - Espèce polymorphe dans laquelle les lobes sont plus ou moins obtus.

P. cxistatum Roth. - Bois des montagnes, - C.-d'0r: Saulieu, 
Roclıe-en-Brenil. - II.-Sav, : Chatelard près Servoz, - Ain et Is. : Hauteville, Grande-Chartreuse. - Loire : Pilat.

T. mplnulosum DC. - Bois humides des collines et des montagnes. - Espèce polymorphe ayant les lobes des segments plus ou moins distincts, les dents plus ou moins aiguës.

Dans la forme dilatatum Willd., les frondes sont pius larges, les segments, sauf les supéricurs, sont distincts. Assez commune dans les Vosges, la chaîne jurassique, les Alpes de Vaud, Valais, Savoie, Dauphiné, Alp.-Mar. - Montagnes du Morvan granitique, du Lyonnais à l'Argentière, du Pilat. - Forez, Allier, Cantal, mont Dore, Lozère, Aveyron.

La forme tanacetifolium DG., diffère peu de la prẻcédente avec laqquelle elle se trouve dans les mêmes localités. Elle a des frondes plus grandes, deux fois penníes, des segments bordés de dents moins aiguës.

P. rigidum DC. - Rocailles et fentes des rochers calcaires des montagnes. - Chalne jurassique au Suchet, à la Dôle, au Reculet dans les crevasses de la montagne d'Allemogne, - II.-Sav. et Sav. : Grand Piton du Salève, Brizon, Méri, vallée du Reposoir, rochers de Bise, Bellevaux, Haut-dc-Lin, base des Fys, entre le col et les chalets d'Anterne, au-dessus du Chapiu, Beaufort, Crêt du Ré au-dessus d'Aime. - Is. ct H.-Alp. : Grande-Chartreuse, Dent de Crolles, col de l'Arc, les Fauges de Villaru-de-Lans, Brandes, Chabrières, Orcières, la Grangette, Glaise. - Dr. et Alp.-Mar. : mont Glandasse, montagnes de Caussols, Défens, Lachen, val Pesio, col Bertrand, mont Aiguille sur Henton.

CXSTOPTEIts frogilis Bernh. - Rochers humides des collines et des montagnes. - Espèce polymorphe offrant une grande variabilite̊ dans la forme des lobes des segments.

Dans la forme C. alpina Link. le rachis est très-êtroit, les lobules profondément dentés-pennatifides. Çà et là dans les montagnes du Valais, Savoie, Dauphiné, Pyrénées.

Dans la forme C. vegia Presl, les lobes sont obtus, arrondis et peu découpés.

Dans la forme C. montana Link, les frondes sont triangu- 
laires, trois fois pennées, la souche longuement traçante. Chaine du Jura helvétique et français : Weissenstein, Côtes-duDoubs, Combe de Vallanvron, Creux-du-Van, Creux de la Neige au-dessus d'Allemogne, la Faucille du côté de Mijoux. Vaud: Alpes calcaires de Chàteau d'Oex à Château-Chamois, la Pierreuse et la Montagnette, Dent de Naye, Bovonnaz, Frachis. - H.-Sav. : Brizon au-dessus du Creux-à-Musset, la Glacière, Vergy au-dessus du lac Bénit, Méri, le Reposoir, le Grand-Bornand, la Clusaz. - Is. : entre Bovinant et Entremont, Charmant Som. - Ariège : lac du Llaurenti, Barbouillère, Valbonne, Boutadiol. - Pyr.-Centrales. La forme montana a une préférence marquée pour les rochers calcaires.

ASPLENON Ambriatum Dulac, A. filix-foemina Bernh. Bois humides des collines et des montagnes. - Espèce polymorphe variant quant à la taille, la forme et la dimension des lobules.

A. Hallerianum DC. - Rochers humides, - Calcaires des environs de Belfort à la Justice et à la Miotte. - Doubs, Jura et Ain : rochers d'Arguel et de Gonsans, Salins, Thoirette, StClaude, vallées de l'Ain, de la Bienne, très-commun dans le Bugey à Rossillon, Arvières, Charabotle, Hauteville, Ruffieu, Cerdon, Nantua, le Revermont. - Valais : Salvan, N.-D. du Sex, Tourbillon, Montorge, Leukerbad. - H.-Sav. et Sav. : Salève, les Voirons, Brizon, Crêt du Maure près Annecy, vallée du Fier au Pont St-Clair, vallée de l'Arve entre Cluse et Sallanches, base des Fys, envir. de Moutiers. - Is., H.-Alp. et Dr. : Crémieu, Grande-Chartreuse, Sassenage, Pariset, Bourg d'Oisans, la Grave, Barnave, Crest, Nyons, St-Vallicr. - B.-Alp. et Alp.-Mar. : la Condamine, Châtelard, Digne, Sisteron, Barrême, montagnes de la vallée du Var, Aiglun, Seranon. - Vaucl. et Var:mont Ventoux, Luberon, Ste-Baume. - C.-d'Or et S.-et-L. : vallon de \lessigny derrière Nuits, Youillant, la Côte chalonnaise. - Rh. et Loire: rochers granitiques des vallées de l'Yzeron et du Garon, la Terrasse, Malleval, Bessey, Ruthiange. - Ard. : rochers basaltiques de Jaujac, Thueyts, Mayres, Vals, Entraigucs. - Gard et Hér. : le Vigan, Valleraugue, l'Espérou, l'Espi- 
nouse, partic supérieure de la vallée de la Marc. - Pyr.-0r. et Ariège : Graux d'Oletto et de Thuès, St-Antoine-de-Galamus, vallée de l'Aude, Usson, Rouze, Mijanès au ravin de Canals, lac du Llaurenti vers l'Escale de Boutadiol, - Espece polymorphe.

Une variété remarquable, appelée par \I. Legrand foreziacum a des folioles grandes, à segments élargis, peu profondément divisés; a été observẻo dans les montagnes granitiques de la Loire.

Lid. IIallerianum, comme on l'a vu par l'énumération précédente, est beaucoup plus commun sur les rochers calcaires, cependant il se montre parfois sur les rochers siliceux du Lyonnais, du Forez, des Cévennes et du centre de la France, et aussi sur les roches volcaniques du Vivarais, circonstance qui ne permet pas de le considérer comme une Fougère calcicole exclusive, mais seulement préférente.

A. Ianceolazun Huds, - Rochers humides de la rérgion mérid. - Var : ile de Porteros. - Ard., Gard el Hér. : Vals, montagnes du Vigan et d'Andabre-Rosis. - Ouest.

La variété obovatum Viv., à lobes obovés-suborbiculaires se trouve ả Hyères (Var), Graissessac (Hér.)

A. smbrotumatum, A. irichomanes L. - Vieux murs, rochers dans tout le bassin.

La variêté glandulosum Lois., A. Petrarchae DC. a des frondes courtes, glanduleuses, à rachis pubescent. - Fontaine de Vaucluse. - Mont Faron près Toulon, Lavalette, le Luc, Antibes, Menton.

A. Viride Huds. - Rochers calcaires humides. - Chrîne jurassique, Vaud, Valais, Savoie, Dauphiné, B.-Alpes jusque dans les Alp.Mar. - Pyr.-Or. et Ariège : rochers calc. de la vallée de la Tet, lac du Llaurenti, Boutadiol, Barbouillère, Valbonne. Pyr.-Centr. - Nul dans la chaine qui s'étend du Morvan aux Cévennes, dans le centre, l'ouest et le nord de la France. kare en Lorraine et en Alsace.

A. ma roinam L. - Rochers de l'île du J.cvant (Var), - Littoral de l'Ouest.

Catal. Bassin du Rhone. 
A. seprearutomale L. - Rochcr's siliceux des collines et des montagnes. - Nul sur les calcaires.

La présence đe cetle Fougère sur les blocs erratiques siliceux épars dans les chaines calcaires ou sur les terrains de transport est un des exemples les plus décisifs en faveur de la doctrine de l'influence chimique du substratum sur la dispersion naturelle des plantes.

A. gernanicum Weiss. - Probablement hybride des A. septentrionale et subrotundum en société desquels il vit. A été observé sur les roches granitiques de la forêt de la Serre (Jura), dans les envir. de Chamonix au Bouchet de Servoz, Bocher, Ste-1Iarie, base de la montagne de fer, sur Coupeau, gorges de la Diosaz, Ayers sur Servoz, sous le Platet, flanc mérid. de Pormenaz, mont Lachat, les Gaillants, Chauderons. - Valais : entre Vernayaz et Salvan, Saas au-dessus de Sammaten. - Sav. : Beaufort, Arèche, Conflans. - Rochers granitiques du Lyonnais, Pilat, Forez, Oisans, Vivarais, Cévennes.

Milde a trouvé un Asplenon qn'il croit être hybride des A. septentrionale et viride. Si cet hybride existe en réalité, il doit être fort rare, attendu que ses deux parents présumés coexistent rarement ensemble : le premier vivant sur les rochers siliceux, le second sur les rochers calcaires.

A. muzrale, A. Rutc-Hururia L. - Rochers et murs. - Une variété microphyllum a les lobes plus petits et plus nombreux.

A. nigrotasn L., 1. adiantum nigrum L. - Lieux ombragés et humides. - Comme le précédent, présente unc variété microphyllum (A. serpentini Koch), assez commune dans la région mérid.

SCOIG 5. sRgittatuan DC., S. hemionotis Sw. - Murs et rochers ombragés. - B.-du-Rh. : Marseille-Veire, Mlontredon, Mazargue, ile de Maïré. - Alp.-Mar. : Antibes, Mala entre Mlonaco et Eza.

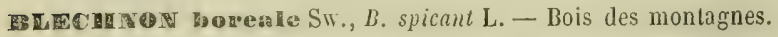
- Hautes-Vosges jusque dans la HI.-Saone aux Ballons de Servance et de Giromagny, Champey, Ronchamp, mont de Vannes, Miélin, Revers-au-Cliens, Planche-des-Belles-Filles. - Jura lielvétique ct françuis: Chasseron, Chàteluz, Vallanvron, Creux- 
du-Van, Pouillerel, Raineux, Cotes du Dessoubre, Boujailles, Fressey, Noirmont, la Dole, Crêt de Chalam. - Vaud et Valais : la Hausseresse, Isenau, la Porche, Bretlaye, les Pléiades, Jaman, Trient, Tète-Noirc, entre Fins-Hauts et Salvan. - II.-Sav. el Sav. : Salève du coté mérid., les Voirons, Glacière du Brizon au-dessus de Solaison, IIaut-de-Lin, Hoc de Chere sur les bords du lac d'Annecy, Tournette, chaine du mont Blanc, Val Nontjoie, montagnes de Beaufort et de Crest-Voland. - Is. : Grande-Chartrcuse à la Charmette et à Vallombrey, Combe de Lancey, montée des Sept-Laux, picd de la Moucherolie, en plaine à la forêt de Chambaran près Roybon, - Alp.-Mar. : entre la Napoule et Agay. - C.-d'Or et S.-et-L. : envir. de Saulieu au bois Morin, aux étangs Forticr et Champeaux, Roche-en-Brenil, le Morvan autunois. - Chaines beaujolaise et lyonnaise à St-Rigaud, Ajoux, Pierrefiland, Pic de Chatonx, Larajasse, l'Argentière au Châtelard, St-André-la-Côte. Chaines du Pilat, du Vivarais et des Cévennes au Mezenc, Cuze, l'Espérou, St-Jean-du-Gard, Portes, l'Espinouse, le Saumail, St-Pons, Lamalou, vallée de la Mare. - Aude, Pyr.-0r. et Ariège : la Montagne Noire, Canigou, Valnanya, forêts du Paillariel, du Pla del Agré, Artounant. - En dehors de nos limites dans les Pyr.-Centr., Aubrac, montagnes granitiques du Tarn et de tout le centre de la France, la Vendée, Deux-Sèvres, Char.inferer.

Le $B$. borealc est beaucoup plus rẻpandu sur les terrains siliceux que sur les terrains calcaires et ne se montre dans la chaine jurassique, en particulier, que sur les terrains de transport ou lorsque les roches sont recouvertes d'une couche épaise d'humus. Son existence à une faible altitude sur les cailloux quartzeux des forèts de Chaux (Jura), de Louhans (S.-et-L.) et de Chambaran (Is.) mérite d'être remarquée, et montre une fois de plus que certaines espèces montagnardes peuvent vivre dans les régions basses lorsqu'elles trouvent dans le substratum les conditions chimiques favorables à leur existence.

[PTERS aquHina $\mathrm{L}$. - Bois, lieux incultes eles terrains siliccux des plaines et des montagnes. - Dans les chaines calcaires la Grande Fougère ne se montre que sur les amas de silex des couches oxfordiennes et crétacies, ou encore sur les dé pôts sidérolithiques, les cailloux et argiles de transport. 
P. erenter L. - Rochers humides des Alp.-Nar. : Nico au Vallon Obscur, vallon do Donaréou près d'Aspremont, la Giandola, Tende.

ADIANTON eapinare, A. capillus Vencris $\mathrm{L},-$ Rochers, grottes et murs humides de la région mérid. ct, plus rarement, de la région moyenne où il remonte dans l'Ard. à Ruoms, Ucel, Thueyts; dans la Dr. et Is. à Remollon, Réoltier, Romans, Bourg-du-Péage, Avignonnet, Séchilienne, Toiron, St-Martinle-Vinoux à la grotte de l’Ermitage, Créinieu à Béthenas; à Lyon à la grotte des Étroits; dans l'Ain à Bons, Artemart à la source du Groin, Lhuis, cascades de Cerrérieu et de Glandieu, Pierre-Châtel, le Credo près du Fort-de-l'Écluse; dans H.-Sar. et Sav, à Brizon près St-Innocent, Rumilly au-dessous du Château des Balmes dans le ravin de Chenu; dans Valais et Neuchatel à la Ravoire de Martigny, Crévola, Boudry. - En dehors de nos limites dans la vallée d'Aoste en Piẻmont, Pyr.-Centr., Aveyron, Lozère à Meyrueis, Lot à Figeac, Tarn, Loir-et-Cher, Indre-et-Loire, Vienne, Char.-Infér., Deux-Sèvres.

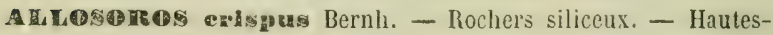
Vosges jusque dans la II.-Saone à la Tète-des-Noirs, montagne de Fresse, Étangs près Château-Lambert. - Vaud et Valais : Creux de Jéman, mont Fully, Salvan, Forclaz, vallẻe de Saas cntre Almagel et Nattmark, Simplon sous Ilobschen, la Tavernettaz, sous la Coupure. - H.-Sav. et Sav. : Buet, Brévent, la Flégère, Chamonix aux Plans, Grandes-Places, le Bouchet, Montanvert, Caseades du Dard et des Pélerins, l'Allée-Blanche, la Layat, Crest-Voland, Beaubois près Beaufort, entre le col des Fours et l'Enclave, St-IIartin-de-Belleville. - Is, et H.-Alp. : Belledonne vers le lac du Crouzet, Sept-Laux, Gleyzin, StChristophe aux Etages el à la Bẻrarde, Taillefer, Lautaret à Casse-Rousse, Chaillol, Champoléon, le Valgaudemar. - Alp.Mar. : val de Rabuons, Ste-Anne-de-Vinaï, N.-D.-de-Fenestre, cols de l'Abisso et de Tende. - Ard. et H.-L. : Mezenc, Gerbier-des-Joncs, Mirabel. - Ariège : Valbonne au Roc de la Musique, Roc Blanc, Pic de la Camisette, Boutadiol. - En dehors de nos limites dans les Pyr.Centr., Lozère, Cantal, Pierresur-Haute. 


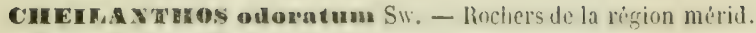

- Var et Mlp.-Mar.: Toulon, Iyères, Belgentier, St-Daumas au Cannet, Grasse, St-Císaire au quartier St-Ferréol, port de Mala entre Monaco et Eza, Roquebrune. - Gard et Hêr. : la Meiranne près St-Ambroix, lo Vigan, Lodève. - Pyr.-Or. : Collioure, Port-Vendres, Vernet-les-Bains, Thuès. - En dehors de nos limites entre Aosto el Aimaville en Piémont, Lozère à la jonction du Gürdon et de la rivière de St-Étienne-Valfrancesque, Bagneres de Luchon à Casarilh.

\section{ĖQUISÉTACÉES.}

EQUSETUM arvense $L$, - Lieux humides.

E. elourneum Roth, E. telmateia Ehrh. - Lieux humides.

E. silvaticum L. - Bois humides des montagnes, - II.-Vosges jusque dans la H.-Saone 'au Ballon de Giromagny, vallées du Rahin et de Miélin. - Lieux tourbeux de la chaine jurassique : Franches-llontagnes, Creux-du-Van, les Ponts, Brévine, Chasseral, Mouthe, Levier, Boujailles, Châtelaine, Suchet, la Dôle, Colombier, la Faucille à Lavatay, Crêt de Chalam, Nantua, Retord, le Vély près Hauteville. - Vaud, Genève et Valais: Payerne, petit Sacconex, bois Magnin près du Trient, Derborence, Vercorin, Mayens de Sion, Zermatt, Bérisal en montant au Simplon. - H.-Sav. el Sav. : Salève, Voirons, vallée de Boëge, Iabère-Lullin, Bellevaux, la Roche, vallées du Fier et de la Borne, mont Charvin, Crest-Voland, Chamonix. - Is. : les Fauges du Villard-de-Lans, Corençon, Prémol, St-Hugon. C.-d'Or et S.-et-L. : Saulieu, Arnay, St-Léger, Renaudiots près Autun. - Rh. et Loire : St-Bonnet-le-Froid, Yzeron, Aveize, Loire entre Givors et Ste-Colombe, Pilat à la République et au Pré Lager. - Gard et Hér. : l'Espérou, l'Espinouse à Cambon. - En dehors de nos limites dans les Pyr.-Centr., Aveyron, Cantal, mont Dore, Forez à Pierre-sur-Haute.

E. palustre L, - Marais.

E. Limosum L. - Fossés, étangs.

c. hiemale L. - Lieux sablonneux el graveleux humides, 
E. xamosum Schl. - Lieux sablonneux et graveleux, surtout sur le bord des rivières. - Rives du Léman, du Rhơne autour de Lyon et dans le Valais, de l'Arve, de l'isère près de Grenoble, de l'Ain à Thoirette, de la Durance et de l'Arc, du Gard, de l'Hérault, de la Tet, du Tech et de plusieurs affluents de ces rivières.

E. variegatum Schl. - Sables des bords des rivières dans les memes localités que le précédent. - Remonte jusqu'au pied des glaciers, notamment dans la vallée de Saas vers le glacier de Fee, dans la vallée de Chamonix vers la source de l'Arve et au pied du glacier du Tour.

C. umbrosum Mey. - Lieux humides. - Valais à Balen de Saas, Zermatt, val Ferret.

\section{RHIZOCARPÉES.}

MARSTLA quadurifollata L. - Mares et étangs. - C.-d'Or : mares des forêts de St-Nicolas, Broin, Citeaux, St-Seine-enBâche, Seurre. - S.-et-L., Jura et Ain : Autun, Savigny en Revermont, la Bresse et la Dombes. - Vaud : Villeneuve. Sav. et Is. : lac du Bourget, St-llaurice-d'Exil, les Avenières, étang de Salevaz près Morestel, Charvieu, Janeyriat, Décines. - Rh. : Oullins, Vaux-en-Yelin, fossés du parc de la Téte-d’or.

M. pubescens Ten. - Hẻr. : mares de Roquehaute près Portiragnes.

PILULARIA aessilis, $P$. globulifera L. (1). - Marais, étangs. - C.-d'Or et S.-et-L, : Rourray, Saulieu à l'étang Fortier, Montjeu près Autun. - Jura et Ain : la Bresse à Commerıalle, entre Champrougier et Fays, Marlieu, le Plantay, St-Germainsur-Renom, Reyrieux aux Bruyères, St-André-de-Corcy, Montribloud, les Échets. - Sav. : lac du Bourget.

(1) L'épithète globulifera formant pléonasme avec le nom générique $P_{i}$ lularia, il me parait préférable de la remplacer par celle de sessilis qui indique le caractère principal par lequel l'espèce diffère de la variété minuto Durieu, qu'on pourrait appeler, par opposition, $P$, pedicellata. 
P. pedicellatu, $P$. minula Durieu. - Forme цui differe du type par la présence d'un pédicclle fructifére recourbé en bas et par l'exiguité de toute la plante. - Hér. : mares de lloquehaute près Portiragnes. - Le centre, l'ouest et le nord de la France.

SARviva natunm floffm. - - Na'a pas été retrouvé dans les localités indiquées par Villars aux Avenières, au Pont-de-Beauvoisin et à Paladru. - B.-du-Bh. : Arles. - Pyr.-Or. : étang de Carença, marais de Cagarell à Canet. - Bordeaux le long des allées Boutaut.

\section{ISOETEEES.}

ISOECES lacustre L. - Lacs des montagnes. - Pyr.-Or. : lac d'Aude en Capcir, lac de Paradelles à l'extrémité de la Calme, mares de la Grande-Bouillouse. - En dehors de nos limites à l'Aubrac dans les lacs de St-Andéol et de Bort; au mont Dore dans les lacs de Guéry, de Chauvet et de Montsineire; dans les Vosges dans les lacs de Gérardmer, Longemer, Retournemer.

I. setreeam Delile. - Ltangs. - Var : St-Daumas au Cannet. Hér. : mare de Grammont, Roquehaute près Vias. - Pyr.-Or. : marais de Salses, Font-Estramer.

T. reticulatum, I. Hystrix Durieu. - Lieux stériles. - Alp.Mar. : Cannes, la Roquette.

1. serobieulatum, 1. Duriaei Bory. - Lieux stériles. - Var et Alp.-Mlar.: Sablettes près de la Seyne, Cannes, la Roquette, golfe Jouan, Biot. - Hér. : Roquehaute près Vias, Lamoure près IIontpellier.

\section{LYCOPODIACÉES.}

EXCOPDION selaginum, $L$. selngo L. - Bois et rochers des montagnes surtout dans les terrains siliceux. - Hautes Vosges jusque dans la Haute-Saône au Ballon de Giromagny, Tête des Noirs-Etangs et des Sèpes, Ternuay. - Rare dans la chaine jurassique et seulement sur les sols tourbeux au Chasseron, Chasseral, Montendre, la Dôle, Reculet, Tourbieres de la vallẻe 
de Joux et des Rousses, le Russey, forêt d'Arvières. - Valais : Fully, Folateres, Pissevache, Bagnes, Thyon, Zan, Mayens de Sion, Zermall, Simplon. - H.-Sav. et Sav. : Salève, les Voirons, Brizon, montagnes de Samoëns et de Bellevaux, Haut-deLin, Roc d'Enfer, Colone, plateau des Gets, Brévent, Pierre-àBérard, Ste-IIarie-au-Fouilly, mont Lachat, pied de l'Aiguille des Charmoz, Crest-Voland, Hauteluce, Beaufort, les Allues, Bonneval en Haurienne à l'Ecot, mont Cenis. - Is. et H.-Alp. : chaînes de Belledonne, des Sept-Laux, de Taillefer' et de l'Oisans, la Ifoucherolle, la Grave, Lautaret, Névache, mont Viso, StVéran, Chaillol, Valgaudemar, envir. de Gap. - B.-Alp. et Alp.-Mlar. : le Lauzanier, St-Etienne-Mont, vallon de Cavallé, mont Bego, col et mine de Tende. - C.-d'Or et S.-et-L. : le Morvan granitique autour de Saulieu vers l'étang Morin, entre Anost et Planchey, Roussillon, St-Prix, source de la Canche. Loire et Ard. : Pilat, Mezenc, Gerbier-des-Joncs. - Pyr.-Or. et Ariège : Canigou, Valmanya, cirques d'Artounant et des Aiguettes, Valbonne, Barbouillère, forêt du Riplaou. - En dehors de nos limites dans les Pyr. centr., Aubrac, Cantal, mont Dore, Forez à Pierre-sur-Haute, Morvan nivernais, Finistère, Côtes du Nord.

C. Inundatum L. - Prés tourbeux des plaines et surtout des montagnes à sol siliceux. - Hautes Vosges jusque dans la H.-Saône au Ballon de Giromagny, sources de la Lanterne, tourbières de la Pile. - Doubs, Jura et Ain : tourbières de Pontarlier, Bonlieu, Chapelle-des-Bois, les Guinots, forêt de la Serre, Coillard près Nantua, Chevroux près Bourg. - Vaud et Valais : Ste-Croix, Sierne-au-Cuir près d'Oex, Gourze, marais des Mosses. - H.-Sav. : marais de Lossy au pied des Voirons, Chamonix au Bouchet, Valorsine, en face de Pierre-à-Bérard, Bellecombe. - Is. : Terres-Froides, Eydoche, Chambaran, lac Luitel au-dessus de Prẻmol. - C.-d'Or et S.-et-L. : Saulieu aux étangs Morin et Fortier, Roche en Brenil, St-Georges près d'Autun, le Pavillon près Roussillon, Branges près Louhans. - Rh. et Loire: Cliazay-d'Azergues, Pilat dans les prés de Tarantaise. - Aude, Pyr.-Or, et Ariẻge : Montagne Noire, Carlitte, mouil- 
lères de Paillariel, de la Cesseilla, del Seillenc, de Boutadiol, cirque d'Artounant. - En detors de nos limites dans les Pyr. centr., Aubrac, Tarn, Puy-de-Dome, Allier, Forez à Pierre-surHaute et aux Salles, Creuse, ouest et nord de la lirance.

C. anzucinana L. - Buis des montagnes. - Rare dans les Vosges et la chainc jurassique : Combe de Vallanvron, Chàteluz, Creuxdu-Yan, Levier, la Dole, vallée des Dappes, bois de la Faucille, escarpements sur Mijoux. - Vaud et Valais: Ste-Croix, Alpes de Château d'0ex, les Pléiades, Solalex, Tête-Noire, Bagnes, Vercorin, Sion, Leukerbad, Saas, Simplon. - H.-Sav. et Sav. : les Voirons, Brizon, Haut-de-Lin, Roc d'Enfer, Chamonix, col de Voza, N.-D. de la Gorge, Crest-Voland, Beaufort, mont Cenis. - Is. : Grande-Ćhartreuse, Revel, Prémol, Allevard. Loire : Pilat au Bessat, sources du Furens, Pierre-sur-Haute, Pic Pelé près Noirétable. - Dans les Vosges au Champ-đu-Feu, Ballon de Soultz, Hohneck. - Il est douteux que ce Lycopode existe dans les Pyrénées oủ l'indiquent Lapeyrouse et tous les auteurs qui ont reproduit les assertions de l'auteur de l'Iistoire abrégée des plantes des Pyrénées.

C. algutnam L. - Rochers et bruyères dés montagnes à sol siliceux.

- Nul dans la chaîne jurassique, quoiqu'il existe dans les Vosges au Hohneck, au Ballon de Soultz, au Champ du Feu et au Lauchen-Weyher. - Valais : mont Fully, val d'Illiez, St-Bernard, Thyon, Simplon, Mayenwand. - II.-Sav. et Sav. : les Voirons sur les grès nummulitiques des envir. du Couvent, Brizon, plateau des Gets, Buet, Brévent, flanc mérid. de Pormenaz, Valorsine aux Mélèzes, col de Balme, moraines de la Merde-Glace, Bouchet de Chamonix, col de Voza, Pavillon de Bellevue, Prarion de St-Gervais, mont Lachat, les Contamines, mont Joly, Ia Layat, Haut-du-Pré et Nant-Bruyant au-dessus de Confans, mont Cenis à l'Eau-Blanche. - Is. et H.-Alp. : Belledonne vers le lac du Crouzet, col du Merdaret, Grand Charnier, Sept-Laux, Haut-du-Pont, Taillefer à Poursollet, Lautaret, Champoléon, Orcières. - Ard. et H.-L. : Mezenc, mont Mégal. - Ariége: lac du Llaurenti, de Quérigut, lac Bleu, Roc de l'Estagnet. - En dehors de nos limites dans les Catal. Bassin du Rhônc. 
Pyr. centr., H.-Loire, Puy-de-Dòme au bois de la Chartreuse près Pontgibaud.

T. complanatum L. variété cyparissoideum, $L$, chamaecyparissus A. Braun. - Bruyères des montagnes à sol siliceux. - HautesVosges jusque dans la H. Saône au Bailon de Giromagny. Alp.-lfar. : col del Sabbion, montagnes au-dessus de San Remo en Italie. - En dehors de nos limites dans les Vosges, la Loire au-dessus des rochers de l'olme près de Chalmazelle, l'Aveyron dans les envir. de Lacalm à Montyrac, la Corrèze.

A l'exemple de plusieurs auteur's, je considère ce Lycopode comme une variété à rameaux dressés, moins larges et moins comprimés, du $L$. complanalum du nord de l'Europe et de l'Amérique, lequel a des rameaux secondaires divergents en éventail, d'où le nom de $L$. complanalum, var. flabellatum qui lui a été donné par Dœll dans sa Flore du duché de Bade (Flora des Herzogthum Badens).

L. clavatum L. - Bruyères des montagnes à sol siliceux. Hautes Vosges jusque dans les envir. de Belfort à l'Arsot et au Salbert et jusque dans la H. Saone aux Ballons de Servance et de Giromagny, Fresse, Champagney, le Chérimont, Conthenans, Belverne. - Rare dans la chaine jurassique au Clos du Doubs, au Lomont, dans les tourbières de Bonlieu, sur l'oxfordien siliceux des bois de Chailluz el d'Aglan près Besançon. - Jura et Ain : forêt granitique de la Serre, cailloux siliceux de la forêt de Chaux et des bois des envir. de Bourg à Marboz, bois des Volières près des Echets, de Meillonaz dans le Revermont. - Vaud et Valais : tourbière de Jongny, Sierne-au-Cuir près Château d'OEx, St-Livres, Tête-Noire, Massongex, Bagnes, Leukerbad, Simplon, Grimsel. - H.-Sav, et Sav. : couches sidérolithiques de Cruseilles et de la Grande Gorge du Salève, grès nummulitique des Voirons, Bellevaux, Vallon, Chamonix au Bouchet, Prarion de St-Gervais, dépôts sidérolithiques du Crêtdu-Haure près Annecy, Crest-Voland, Beaufort, Hauteluce. Is. : Granges de Frédières au-dessus de Revel, sables du grès vert des Fauges de Villard-de-Lans et de St-Nizier, - H.-Alp.: le Noyer en Champsaur, mont Viso. - Alp.-Mar. : val de Pesio. 
- C.-d'Or et S.-et-L. : Saulieu à l'ëtang Fortier, le Gué aux Geais, bois de la Croix, Hoche en Brenil, St-Georges et Montjru près Autun, le Pavillon, les Grandes Queues. - Loire et Ard. : Pilat, Mezenc. - Hêr. : Salvergue sur l'Espinouse. - Pyr.-Or. et Ariège: Font de Comps, Nohèdes, Evol, Madrès, forêt du Pla del Agré. - En dehors de nos limites dans les Pyr. centr., Tarn, Aubrac, la Margeride, Cantal, II.-Loire, Puy-de-Dome, lorez à Pierre-sur-Haute, Morvan nivernais, Creuse, II.-Vienne, Sologne, l'ouest et le nord de la France.

Le $L$. clavatum est une plante montagnarde qui, de méme que l'Arnica montana, le Scirpus caespilosus, le Bleclenon boreale, le Calluna vulgaris et le Nardos stricla, peut vivre sur les collines basses et même dans les plaines lorsqu'elle trouve dans le sol les conditions chimiques qui lui conviennent.

SELACTNEC spinuloma $A$. Braun. - Paturages et bruyere: liumides des montagnes. - Jura helvétique et français : Chasseral, Tête-de-Rang, Chasseron, Suchet, Aiguillon, Creux-duTan, Mont-d'Or, la Dòle, Colombier, Faucille, Reculet, Colombies du Bugey, Retord. - Vaud et Valais: Bovonnaz, Fully, Folalères, Morgins, Bagnes, St-Bernard, Mayens de Sion, Zermalt, Simplon. - H.-Sav. et Sav. : Grande Gorge et Pitons du Salève, les Voirons, Vergy, Roc d'Enfer, montagnes d'Abondance, Glacière du Brizon, Colone, base du Buel, Mẻlèzes de Valorsine, Chamonix à Hortaz et au Bouchet, Nant Borant, Charvin, la Vanoise, Combe de Villette, mont Cenis. - Is. et H.-Alp. : Grande-Chartreuse, Chamechaude, Belledonne, Combe de la Lance, Clsamprousse, col Oddie, Sept-Laux, Alpe du mont de Lans, mont Chamoux, mont Aiguille, Pelvoux, Lautaret, Abriès et Ségure en Queyras, mont Viso, mont Bayard, Orcières. B.-Alp. et Alp.-Mar. : Parpaillon, Vallonnet, Enchastraye, Bírard, Lauzanier, Entraunes, N.-D. de Fenestre, Alpes de la Briga, - Loire: Pilat. - Pyr.-0r. et Ariège : Canigou, Pratsde-Balaguer, Mont-Louis, Madrès, base du Roc de la Musique à Valbonne, vallon de Boutadiol, lac du Llaurenti. - En dehors de nos limites dans les Pyr. centr., Cantal, mont Dore, Pierresur-Haute. 
s. Dêlredica Spreng. - Pàturages humides des Alpes, - Vaud et Valais : Bex, Collonges, Gueuroz, Branson, Folatères, Monthey, Pissevache, Salvan, Martigny, Sembrancher, Mayens de Sion, vallées de St-Nicolas et de Saas, Simplon à Hittenbach et ả la Tavernettaz. - H.-Sav. : pied du Brizon, Chamonix au Bouchet, en allant à Hortaz et à la source de l'Arveiron, au Cougnon, Nont-Vautier, Servoz, Nant-Borant, Bionassey, les Contamines. Alp.-Mar. : St-Martin-Lantosque, Venanson, Tende. - Is. et H.-Alp. : Villars prétend l'avoir trouvé au-dessus d'Uriage et de Revel, mais actuellement on ne l'y trouve plus. II. Borel assure l'avoir trouvé à Bobbi au mont Viso. - J'ai vu dans l'herbier de Chaberı des échantillons de $S$, helvetica récoltés à Meyzicu près Lyon, dans un pâturage voisin du Moulin de Platacul. J'ai cherché vainement, à plusieurs reprises, la Sélaginelle lielvéti [ue dans cette localité et, en adınetlant la sincéritẻ de Chabert, il y a lieu de croire que la plante aura disparu par le fait de la mise en culture du pâturage oủ elle se trouvait.

9. Dentienata hoch. - Coteaux humides de la région mérid. Var et Alp.-Mar.: Toulon, Hyères, île de Porq̨uerolle, le Luc, St-'Tropez au Jasse d'Aubert, St-Raphaël, Cannes, Antibes, Nice au Vallon Obscur, Henton dans le vallon des Châtaigniers. - Hér. : bords de l'Hér. aux pieds des Capouladoux. - Pyr.Or. : vallées du Réart et de l'Agly. 


\section{AVERTISSEMENT}

La table qui termine le Catalogue des plantes vasculaires de la Flore du bassin du Thône n'a pas seulement pour but de faciliter la recherche des noms de plantes cités dans l'ouvrage, elle a encore une utilité particulière qu'il importe de signaler à l'attention c'es botanistes.

Lin effet, pendant le temps écoulé entre la publication du cinquièmo et celle du sixième fascicule, l'auteur s'est appliqué à un travail de révision de la Nomenclature botanique, et après un minuticux examen de tous les éléments dont elle se compose, il a formulé les principes fondamentaux de la dénomination des espèces végétales. Ẻnsuite, il a appliqué cos règles dans la rédaction des sixième et septième parties et d'une seconde édition de la première partie du Catalogue, ainsi que dans plusieurs articles publiés par les membres de la Société botanique de Lyon.

Le perfectionnement du langage scientifique ayant une importance incontestable, il est permis d'espérer que quelques-unes des modifications proposécs obtiendront l'assentiment des naturalistes qui, He s'endormant pas dans une paresseuse quiétude, acceptent tout progrès de quelque part qu'il leur vienne.

Mais comme la conception et la bonne exécution d'un plan de rétorme nécessitent de longues recherches de Iinguistique que la plupart des botanistes, entièrement absorbés par l'étude si intéressante des formes végétales, n'ont ni le goùt ni le loisir d'entreprendre, nous avons cru qu'il serait avantageux au succès de notre entreprise de dresser à leur usage une liste facile à consulter sans perte de temps. Eu égard ì la vaste étendue du bassin du Rbòne, à la variété de ses climats et de ses terrains, cette liste comprend la plus grande partie des plantes de la Flore française et pourrait ètre aisément complétée par l'adjonction des noms d'espèces qui manquent à notre région rhodanienne.

Afin de faire comprendre immédiatement au lecteur en quoi consistent les modifications principales que nous avons apportées à la Nomenclature linnéenne, nous donnons ci-après l'énoncé des deux lois suivantes dont nous proposons l'alloption :

1. Les noms de genre conservent $l a$ forme qu'ils ont dans la langue, grecque ou latine, à laquelle ils appartiennent;

20 Les noms spécifiques sont des adjectifs à désinence latine, exprimant, autant qu'il est possible, un des caractères différentiels, et s'accordant grammaticalement avec le nom gézérique (1).

Le lecteur est prévenu que, par dérogation provisoire à l'article 2 et de peur de bouleverser trop profondément et d'un seul coup les vieux usages, on a conservé, tout en les adjectivant impitoyablement, quelques épithètes insignifiantes, comme, par exemple, celles qui rappellent un nom d'homme.

(1) Pour plus ample explication, on consultera la Reforme de la Nomenclature botanique, 1880, et les Nowvelles remarques sur la Nomenclature botanique, 1881. - Paris, J.-B. Baillière.

Catal. Bassin du Rhónc. 
Nous arons pensé qu'il était superflu d'ajouter un troisième article dans lequel il aurait été dit qu'on doit se conformer, dans la construction des noms, aux règles orthographiques et grammaticales, car celles-ci simposent d'elles-mèmes et ne sauraient ètre discutées.

Afin de ne pas surcharger la table, nous arons volontairement omis de signaler les modifications faciles à reconnaitre comme, par exemple, celles qui portent sur les désinences. Lorsqu'un nom a subi un changement notable, nous avons indiqué (entre parenthèse) l'ancienne dénomination. L.es noms des espècos secondaires, des races et des variétés ont été imprimés en caractères italiques, - Par suite des tâtonnements presque inévitables en pareille matière, il y a quelquefois discordance entre le texte des deux derniers fascicules et la table, mais dans ce cas, c'est à celle-ci qu'il faut définitirement se référer.

Nous nous sommes abstenu de faire suivre les termes nouveaux du nom de leur auteur, et à ce sujet nous devons une explication catégorique.

Suivant nous, la réforme de la Nomenclature doit conserver un caractère impersonnel et ne pas servir de prétexte à la satisfaction trop facile de l'amour-propre des novateurs qui espéreraient "se couvrir de gloire " en changeant ruelques noms de plantes. Cette condition nous semble d'ailleurs nécessaire au succès de la réforme.

Sans doute, le naturaliste qui, le premier, a distingué et décrit une espèce a droit au titre d'inventeur; mais celui dont le rỏle a consisté seulement à la nommer, ou à changer une expression, soit inexacte, soit vicieuse, ne peut s'attribuer que le mérite vulgaire du grammairieı connaissant la valeur des mots et les règles de leur construction. C'est pourquoi nous estimons que les dénominations nouvelles ne doivent être suivies d'aucun nom d'auteur, ou simplement, si l'on tient à indiquer leur origine, des deux lettres initiales du participe emendatum (réformé); cette dernière indication est même inutile si, comme nous l'avons fait et comme il sera nécessaire de faire pendant quelque temps, on rappelle l'ancien nom.

A ce propos, et sans nous illusionner au point de croire qu'un jour viendra où les betanistes se mettront complètement d'accord sur les questions de Nomenclature, nous demandons s'il ne serait pas avantageux, au moins lorsqu'il s'agit d'espèces non litigieuses, d'abandonner enfin le bagage encombrant de la synonymie que nous trainons sans cesse avec nous et do le reléguer dans le domaine de l'histoire où est sa véritable place.

A plus forte raison, ne pourrait-on pas omettre l'inutile répétition des signes L., DG., Vill., Lam., Willd, etc., à la suite des appellations restées univoques et au sujet desquelles il n'existe aucune ambiguité?

Nous concluons donc en priant les botanistes qui adopteront la glossologie employée dans le Catalogue des plantes vasculaires du bassin du Rhóne, de se borner à en informer, une fois pour toutes, leurs lecteurs, sans mention spéciale à l'occasion de chaque espèce. Il est hien entendu, d'ailleurs, que leur liberté d'action reste entièrement réservée pour les cas particuliers où ils voudraient apporter des améliorations que nous n'uvons pas su trouver ou que nous n'avons pas osé réaliser présentement. 


\section{TABLE DES NOMS DE PLANTES}

CITES D.INS I.E

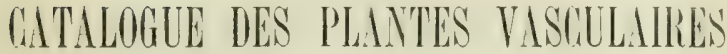

\section{DU BASSIN DU RHONE}

AbIgA (Ajuga) roptans L., alpina Vill., pyramidalis L., genovonsis L., chamaepitya Schreb., iva Schreb., iviformis (pserdo-Iva Rob.

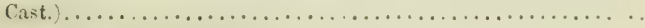

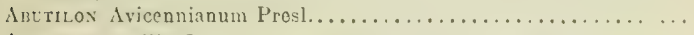

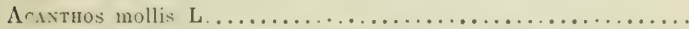
Acer platanophyllum (Pseudo Platanus L.), opulifulium Vill., mons pessulanum L., Martinianum Jord., campestre L., platanoideum L. Aceris anthropophorum R. Br., denxillorum Ijoiss., longibractratum Rchb., hircinum Lindl, pyramidale $R$ chb.................. Acurluos tomentosus L., odoratus L., millefoliatus L., setaceus Koch, alpinus Parlat., compactus Lam., tanacetifolius All., dentifer D C., nobilis L., ligusticus All., chamacmolophyllus Pourr., viscosus Lam. (ageratum L.), ptarmicus L., valesiacus Sut., pyrenaicus Sibth.; cuncifolius Lam. (Herba-Rota L.), macrophyllus L., nanus L., atratus L., moschatus L., hybridus Gaud .............. Aconiton tuberosum (Anthora L.), lycoctonum L., napellum L., pani-

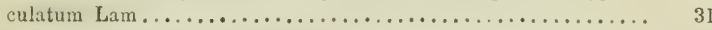

Acoros calamoideus $L$. ou aromaticus .................... 活'

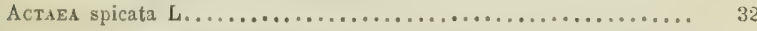

Adenocarpos grandiflorus Boiss., commutatus Guss., complicatus

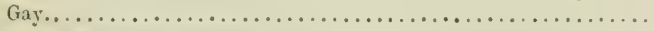
Adexostylis albifrons Rchb., alpina Bl. Fing., leucophylla Rchb., hybrida DC ........................... $31 \mathrm{~h}$

Adianton capillare (Capillus Veneris L.) .................. . . . . .

Adonis flammea L., autumnalis L., aestivalis L., vernalis L., pyre-

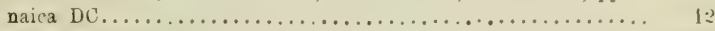

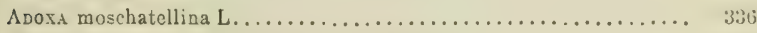

Egopodion angelicifolium Lam. (Podagraria L.)............ . 32' 
AGILops ovata I., virescens J. F., procera J. F., erratica J. F., vagans J. F., erigens J. F., pubiglumis J. F., microstachya J. F., triticiformis Requien, et autres hybrides, triaristata Willd, triuncialis L.....................................

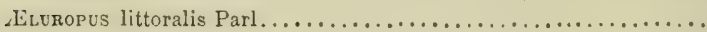

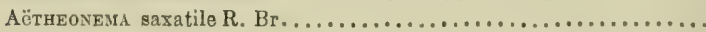

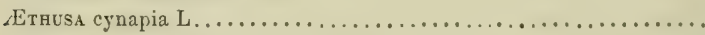

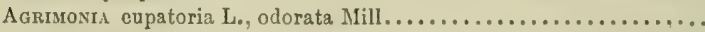

Agroprron junceum P. de B., elongatum Host (scirpeum Presl.), acutum Rocm. Schult., pungens Roem. Schult., pycnanthum G, G., campestre G. G., glaucum Roem. Schult., gracile de Pouz, repens P. de B., caninum Roem. Schult., Rouxianum G. G...........

AGrostis alba L., vulgaris L., verticillata Vill, olivetorum G. G., canina, L., rubra L., alpina Scop., filiformis (Schleicheriana Jord.), rupestris All., elegans Thore, pallida DC., ventosa (spica-venti

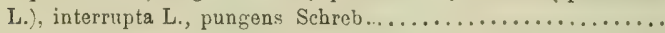

Aira canescens L, articulata Desf., fasciculata Boiss. R., caryophylla L., aggregata Timeroy., divaricata Lois,, brachystachya (Tenoriana Guss.), elegans Gaud,, provincialis Jord, Cupaniana Guss., praecox L., caespitosa L., media Gouan, flexuosa L...........

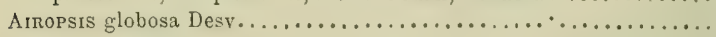

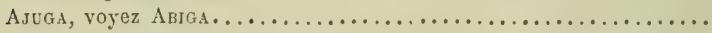

Alchrmlld alpina L., vulgaris L., subsericea G. G., pyrenaica Léon Duf., pentaphylla $L_{\text {, }}$, arvensis $L \ldots \ldots \ldots \ldots \ldots \ldots \ldots \ldots$.

Aldrotanda vesiculosa L. (B. du Rh, et Vaucl, : marais de Raphèlo et bords du Rhône..............................

Alexitoxicon officinale (Vincetoxicum officinale Moench), albidum Jord. F., dumeticolum, beugesiacum, ochroleucum, petrophilum, alpicolum, luteolum, Shuttleroortianum Thuret et Bornet, puberulum Timb.-Lagr., laxum G. G., contigurem G. G., nigrum

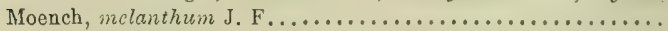

Alisma parnassophyllum L., plantaginum L., arcuatum Michalet, ranunculifiorum $L_{\text {. }}$ natans $\mathrm{L} \ldots \ldots \ldots \ldots \ldots \ldots \ldots \ldots \ldots$.

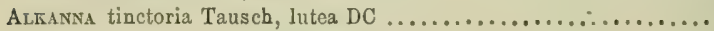

Allius sativum L., scorodoprasum L., vineale L., porrum L., ampe-

loprasum L., polyanthum Roem. Schult, rotundum L., scutiflorum Lois., sphaerocephalum L,, ascalonicum L., ceprum L., schoonoprasum L., foliosum Clarion, chamaemoly L., subhirsutum L., triquetrum L., roseum L., neapolitanum Cyr., nigrum L., moly, longipetiolatum (ursinum L.), reticulatum (Victorialis L.), oleraceum L., alpicolum J. F., complanatum Bor., carinatum L., consimile Jord., fexifolium Jord., pulchellum Don, flavum L., panlculatum L., strictum Schrad., moschatum L., narcissanthum Vill., fallax Don, acutangulum Schrad., siculum Ucria, fragrans Venten. Altosonos crispus Bernh ............................ Alvus glutinosa Gaertn, incana DC, viridis DC............... 701 836 Alopecuros pratensis L., agrestis L., geniculatus L., fulvus Sm., bul- 
bosus L., utriculatus Pors, capitatus Lam. (Gorardianus Vill.).. Alsine tenuifolia Crantz, laxa Jord., viscosa Schreb., hybrida Jord., fastigiata Rchb. (Jacquiniana Koch), mucronata L., petraea Jord., brevifolia Jord., sotacea M. Koch, verna Bartl, recurva Wahlb, Villarsiana M. Koch, striata Gren., glandulosa (Bauhinorum Gay), Cherleriana Fenzl, stricta Wablb., lanceolata M. Koch......... Althata cannabina $L_{\text {, }}$ officinalis $L_{\text {. }}$, narbonensis Pourr., hirsuta $L_{\text {. . }}$

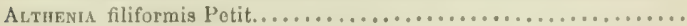
ALYSSox incanum L., maritimum Lam., porusianum Gay, halimifolium L., macrocarpum DC., Candollianum J. F., saxigenum J. F., spinosum L., pyrenaicum Lap., calycinum L., vagun Jord,, sabulosum Jord., ruderale Jord,, arvaticum Jord., campostro L., montanum L., beugesiacum J. F., psammerm J. F., brevifolium J. F, rhodanense J. F., arophilum J. F., brigantiacum J. F., cuneifolium Ten., flexicaule Jord., alpestre L., saxatile J. F.... Amaranton deflexum L., blitonium L., silvestro Desf., patulum Bert.,

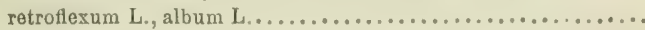

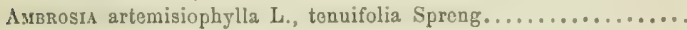

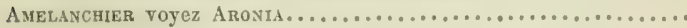
Assi majus L., dilatatum (visnaga L.) $\ldots \ldots \ldots \ldots \ldots \ldots \ldots \ldots \ldots$

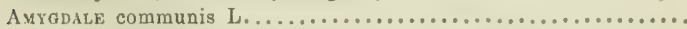
Axacrclos clavatus Pers, radiatus Lois, valentinus L............ ANAGAllis arvensis L.; phoenicea Lam., cerulea Lam., tenella L...

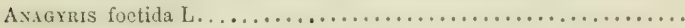

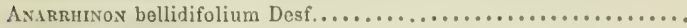
Ancuusa italica Retz, officinalis L., undulata L., sempervirens L.,

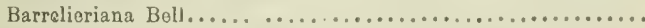

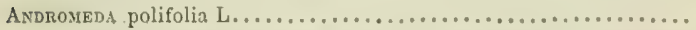

ANDROPOGON polystachyus (Ischaemum L.), distacbyus L., macrosta. chyus (Allionianus DC.), verticillatus (Gryllus L.), hirtus L.,

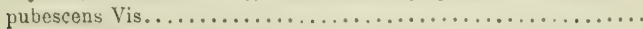

Anprosaces belveticum Gaud,, imbricatum Lam., glaciale Hoppe, pedunculatum, pubescens DC, villosum L., villosissimum (Chamaejasme Jacq.), lacteum L., carnoum L., roseum J. F., puberulum J. F., brigantiacum J. F., Reverchonianum J. F., obtusifolium All., septentrionale L., divaricatum (Chaixianum G. G.),

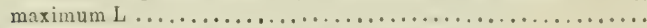

Andryala sinuata $L$., undulata Prosl., ragusina L..............

Aкемоsе fragifera Wulf. (baldensis L.), nemorosa L., ranuđculiflora L., narcissantha L., coronaria L., rosea Hanry, cyanea Risso, variegata ou Rissoana Jord., Ventriana Hanry, Mouansiana Hanry, punicea Boiss., hortensis L., pavonina Lam., versicolor Jord., lepida Jord, palmata L........................

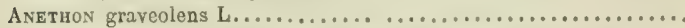

Avgelica silvestris L., montana Gaud., Razuliana Gouan, pyrenaea

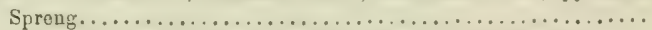
ANTenvaria carpatica Bl. Finge, dioica Gaertn., margaritacea L .... 
Anthesrs nobilis L., mixta L., fuscata Brot., arvensis L., cotula L., secundiramea Biv., maritima L., montana L., Gerardiana Jord., altissima L., tinctoria L., Triumphetiana All..............

Anthriscos vulgaris Pers., chrerephyllus (Cerefolium Hoffm.), silvestris Hoffm.

ANтHYLlis cytisoidea L., argentea Lam. (A. Barba-Jovis L.), montana L., vulneraria L., mbriflora, cenisia (vulnerariformis Bonjean), tetraphylla L................................

AntrRrhinon otontium L., majus L., Fuetianum, latifolium DC., sempervirens Lap., asarinum $L_{\text {, }}$ siculum Ueria.............

Anthoxanthum voyez Xanthonanthos , ..................

Aphyllanthos monspeliense $\mathrm{L} \ldots \ldots \ldots \ldots \ldots \ldots \ldots \ldots \ldots \ldots \ldots$

Apros graveolens $4 \ldots \ldots \ldots \ldots \ldots \ldots \ldots \ldots \ldots \ldots \ldots \ldots$

Aposeris foetida Less. .............................

Aquifolium vulgare (Ilex Aquifolium L.)....................

Aquilegia vulgaris L., atrata Koch, viscosa Gouan, nemoralis Jord., collina Jord, ruscinonensis Timb., alpina L., pyrenaica DC...

ArABIS brassiciformis Wallr., saxatilis All, verna $R . B r_{*}$, auriculata Lam., stricta Huds, serpyllifolia Vill., hirsuta L., sagittata DC., ciliata Koch, procera Jord., pubigera, accedens, collisparsa, idanensis, propera, hirtella, propinqua, gracilescens, subnitens, vesula Jord., Allioniana DC, muralis Bert., rosella Jord., saxigena Jord., alpestris Schl., cenisia Reut., Gerardiana Bess., perfoliata Lam., cebennensis DC., Thaliana L., arenosa Scop., alpina L., caerulea Jacq., bellidifolia Jacq., pumila Wulf., turrita L .....

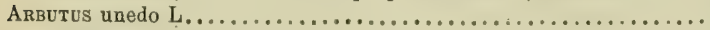

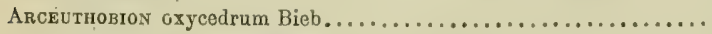
Arctostaphylis alpina Spreng., officinalis Wimm............. Arensria montana L., biflora L., ciliata L., serpyllophylla L., cinerea DC., hispida L., modesta Duf., grandiflora All., tetraquetra L., massiliensis Fenzl .............................

Aristella bromoidea Bertol. . . . . . . . . . . . . . . . . . . . Aristolochid clematitis L., pistolochia L., rotunda L., pallida WV. Kit., longa $L . . \ldots \ldots \ldots \ldots \ldots \ldots \ldots \ldots \ldots \ldots \ldots \ldots \ldots \ldots \ldots$

Arveria maritima Willd, ruscinonensis Gir., majellensis Boiss., plantaginea Willd, sabulosa Jord., bupleuroidea G. G., praecox Jord., flicaulis Boiss, alpina Willd...................

Arnica montana L...............................

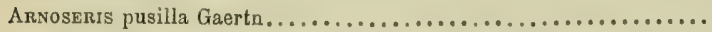
Aron dracunculum L., maculatum L., italicum Mill., arisarum L.... Aronia rotundifolia Pers. (Amelanchier vulgaris Moench)........... Aronicus doronicum Rchb., scorpioideum Koch, glaciale Rchb...... Arrhenateron elatius M. Koch; voyez Avena elatior.............. Artemisia absinthia L., arborescens L., camphorata Vill., pauciflora J. F., alpestris, peduncularis, platyloba, ambigua, congesta, incanescens Jord., Mutellina Vill., glacialis L., pedemontana Balb., vulgaris L., insipida Vill., spicata Wulf, Villarsiana G. G., atrata 
Lam., chamaomelophylla Vill, suavis Jord, nana Gaud., campes. tris L., parvule J., H., subsericen, alpine, urgyrea, brevicaulis, laxata, orophila, monticola, delphinensis, suberecta, stenoclada, brachyphylla, fuscata, temifolia, virescens, grisea, collina, pubescens, implexa, Horibunda, monspeliensis, xylopoda, littorea, pyramidata J. F, valesiaca All., glutinosa Gay, gallica L......

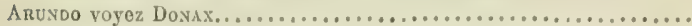

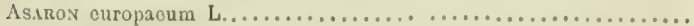

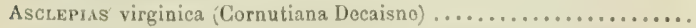

Aspardgos tonuifolius Lam., oflicinalis L., scaber Brign., acutifolius L.,

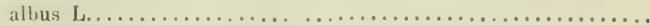

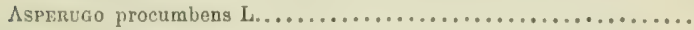

AspenvLd odorata L., cynanchica L., rupicola Jord., arenicola Rout., tenuiflora Jord., longiflora W. Kit., Jordaniana P'orr. Song., tinctoria L., hirta Ram., laevigata L., taurina L., hexaphylla All.,

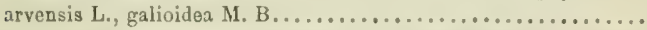

Asphodelos fistulosus L., microcarpus Viv., delphinensis G. G., cerasifer Gay, olbiensis J. F., stoechadensis, monticola, fuscatus, collinus, grandiflorus, procerus, australis, eminens J. F....

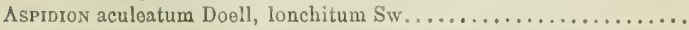

ASplevon fiubriatum Dulae (Filix-focmina Bernh.), Hallerianum D C., lanccolatum Hud.., obovatum Viv., subrotundum (Trichomanos L.), glandulosum Lois. (Petrarchae DC.), viride Huds., marinum L., septentrionale L., alternifolium Wulf. (gormanicum Weis.), murale (Ruta-Muraria L.), nigrum (Adiantum nigrum L.), microphyllum Aster alpinus L., amellus L., maritimus ('Tripolium L.), brumalis, Nees, serotinus Willd., acris L., collinus J. F., pineticola J. F., venustus J. F., trinervis Desf, salignus Willd..............

Asterrscos maritimus Mfoench, aquaticus Moench, spinosus G. G....

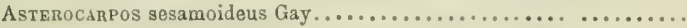

Asterolinon lysimachioideum (stellatum Link et Hoffm.)......... Astragalos pentaglottus L., stellatus Gouan, sesameus L., epiglottus L., hamosus L., glycyphyllus L., cicerinus L., exscapus L., alopecuroideus L., narbonensis Gouan, purpureus Lam., leontinus Jacq., glaux L., hypoglottus L., onobrychus L., vesicarius L., austriacus L., monspessulanus L., incanus L., depressus L., spi-

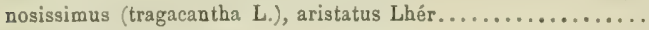

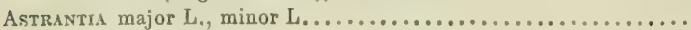

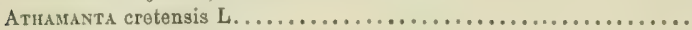

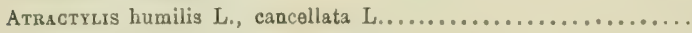

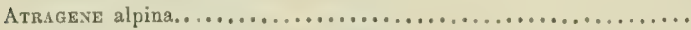
Atriplex hortensis L., microthecus Moq. T., roseus L., crassifolius C. A. Mey., laciniatus L., halimus L., hastatus L, patulus L...... 386 791 663 $5 \div 0$

Avena sativa $\mathrm{L}$, oriontalis L., strigosa Schreb., barbata Brot., fatua L., sterilis L., setacea Vill., filifolia Lag., sempervirens Vill, montana Vill., Hostiana Boiss., versicolor Vill, pubescens L., pratensis L., bromoidea Gouan, elatior L. (Arrhenateron), subspicata Suter, 
sericea (Trisetum Gaudinianum Boiss.), panicea Lam., flavescens

L., distichophylla Vill.,..........................

Ballote foetida Lam., spinosa Link.....................

BARBAREA vulgaris $\mathrm{R}$. Br., longisiliqua Jord., brevistyla Jord., arcuata

Rchb., stricta Andrz., intermedia Bor., praecox R. Br........

BArtschis alpina L., spicata Ram., trixago L., viscosa L., latifolia

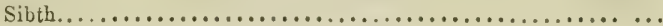

Belladona baccifera Lam. (Atropa Belladona L.).................

Bellevallia romana Richb., trifoliata Kunth.................

Bellidiastrum Michelianum Cass.........................

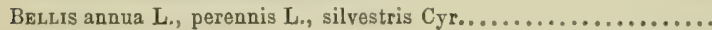

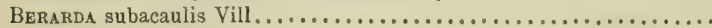

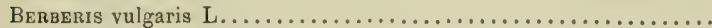

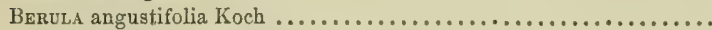

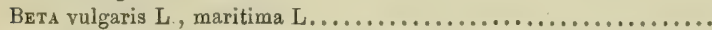

Betonice flava (Alopecuros L.), hirsuta L., alpestris J. F., sabauda

J. F., officinalis L., occitana J. F., parvula, subcarnea, brachys tachya, pilostachya, recurvata, angustifolia, pratensis, nemorosa stricticaulis, silvicola, hylebia, polyclada, validula, drymophila, recurva, rigida J. F...............................

Betula alba L., pubescens Ehrh., carpatica IV. Kit., intermedia

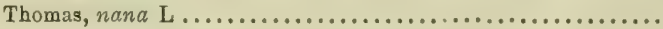

Bidens tripartitus L., hirtus Jord., radiatus Thuill., cernuus L., bipin-

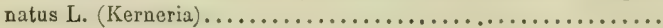

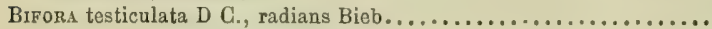

Biscutella laevigata L., intricata Jord, collina, lima, mediterranea, polyclada, picroidea Jord., mollis Lois., coronopophylla Vill., cichoriophylla Lois .................................

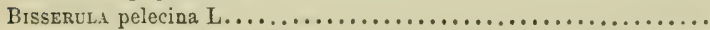

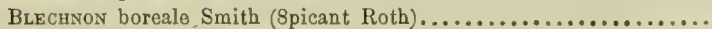

Blrton virgatum L., capitatum L... ....................

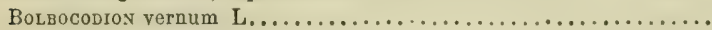

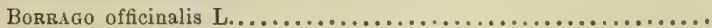

Botrxpron lunare (Botrychium Lunaria Sw.), rutifolium A. Br....

Brachypodron silvaticum Roem. Schult., pinnatum P. de B., ramosum

R. Schult., distachyum P. de B.......................

Brassica oleracea L., Robertiana Gay, napa L., Richeriana Vill, nigra

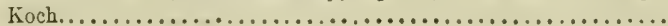

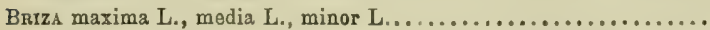

Bromos nutans (tectorum L.), scaberrimus (sterilis L.), maximus

Desf, , madritensis L., rubens L., asper L., erectus Huds., secalinus L., arvensis L., commutatus Schrad., racemosus Parl., mollis

L., squarrosus L., macrostachyus Desf ..................

Brunella hyssopophylla C. Bauh., vulgaris L., alba Pall., grandiflora Jacq ..........................................

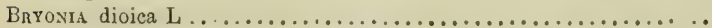

Bufronia macrosperma Gay, tenuifolia $L \ldots \ldots \ldots \ldots \ldots \ldots . . . . . .$.

801

629

600

571

708

375

375

434

$32 b$

322

649

628

682

401

299

54

167

834

650

691

559

825

821

809

633

261

90 


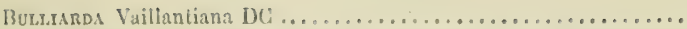

Bunias crucago L, arecnsis Jord, urachyptera Jord, macroptera

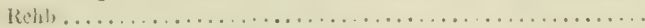

Buptrtulyon salicifolium L., grandiform L...............

BupLeuron rotundifolium L., protractum Link et Hoffm., longifolium $L_{*}$, angulosum $\mathrm{L}_{\text {., }}$ stollatum L., ranunculillorum L., cariciıum DC, potracum L., provinciale Huet, graminoum Vill., fruticosecns L., junceum L., australe Jord, spinosun Gouan, Gerardianzm Jacq., Jacquinianum Jord, affine Sadler, tenuissimum L., glaucum Rob. Cast., aristatum Bartl., rigidum L., falcatum L.,

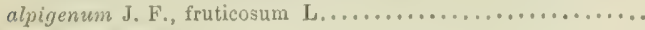

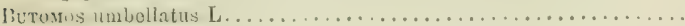
Buxes sempervirens $L \ldots \ldots \ldots \ldots \ldots \ldots \ldots \ldots \ldots \ldots \ldots \ldots \ldots \ldots \ldots$

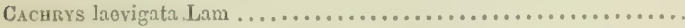

Cactos opuntius $\mathrm{L}$. ou Opuntia vulgaris Haw .................

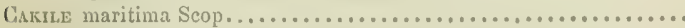

C.sLAmgrostis epigoia Roth, littoron DC., lanceolata Roth, villosa Vill., tenclla Host, varia Schrad., arundinacca Roth...........

Caldsintue grandiflora Moench, officinalis Moench, ascendens Jord., nepcta Savi, nepetiformis Jord, alpina Lam., acina Clairv......

CAlceorus alternifolius (Cypripolilon Calecolus L.).............

Calendula arvensis L., bicolor Raf, . . . . . . . . . . . . . . . .

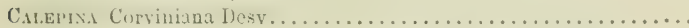

Callimax stagnalis Scop,, platycarpa Kïutz, verna Kütz, hamulata liü $\ldots \ldots \ldots \ldots \ldots \ldots \ldots \ldots \ldots \ldots \ldots \ldots \ldots \ldots \ldots \ldots \ldots \ldots$

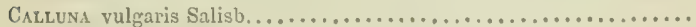

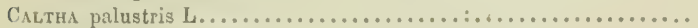

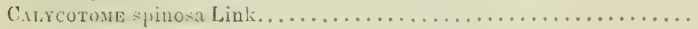

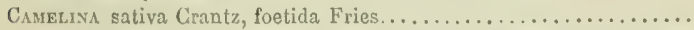

Campanuld grandifora Lam. (Medium L.), spociosa Pourr., nana (Allioniana Vill.), barbata L., petraea L., glomerata L., cervicaria L., cervicariformis (cervicarioidea Mutel), spicata L., thyrsoidea L., latifolia L., urticifolia Sehm. (Trachelium L.), rapunculiformis L., bononiensis L., parviflora (Erinus L.), elatine L., macrorrhiza Gay, pyramidalis L., rhomboidalis L., lanceolata Lap., linifolia Lam., nutans (Schcuchzeriana Vill.), valdensis All., precatoria Timb., ficariformis Timb., rotundifolia L., pennina Reut., ruscinonensis Timb., sterocodonta Boiss. Reut., Bocconiana Vill., velutina DC., caespitosa Scop., pusilla Haonke, excisa Schl., rapuncula L., patula L., persicifolia L., conisia L..............

CAmprorosus monspoliacum $\mathrm{L} \ldots \ldots \ldots \ldots \ldots \ldots \ldots \ldots \ldots \ldots \ldots$

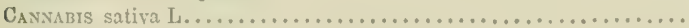

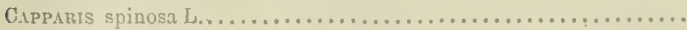

CApsella triangularis (Bursa pastoris Mlocnch), agrestis Jork,, virgata, praecox, ruderalis, sabulosa, rubella Reut............

Cardamine asarophylla L., thalictroidea All. (Ilumeriana Vill.), pratensis L., praticola Jord, herbivaga J., udicola J., Matthioliana Catal. Bassin du Rhonc. 
Mor., amara L., impatiens L., hirsuta L., silvatica Link, parviflora L., alpina TVilld., resedifolia $L . . \ldots \ldots \ldots \ldots \ldots \ldots \ldots \ldots \ldots$ Carduxcel.tus mitissimus DC., monspeliensis DG..............

Cardeves tenuiflorus Curt., corbariensis Timb., corbariensi-tenuiflorus Timb., p.ycnocephalus L., pycno-tenuiforus Timb., acicularis Bert., personatus Jacł., personato-crispus Michal., personato. nutans Gren., personato-defloratus Gron., crispus L., crisponutans Jord., nutans L., nigrescens Vill., MIartrinianus Timb., australis Jord., spinigerus Jord., vivariensis Jord., balmensis (Sanctac Baimae Lois.), aurosicus Vill., carlinifolius Lam,, defloratus L., mcdius Gouan, paniculatus Lam. (carlinoides Gouan)..

Carex dioicostrongyla (dioica L.), dioicotrigona (Davalliana Sm.), pulicaris L., microglochin Wahlb., pauciflora Lightf, pyrenaica Wahl, rupestris All., chordorrhiza Ehrh., foctida Vill, microstyla Gay, divisa Huds, setifolia Godr., disticha Huds., Schroberiana Schr., brizoidea L., vulpina L., muricata L., divulsa Good., juncifolia All., paniculata L., paradoxa Willd., teretiuscula Good., heleonastis Ehrh, elongata L., ovalis Good., approximata Hoppe (lagopina Wablb.), stellulata Good., canescens L., vitilis Fries, remota L., Linkiana Schk., curvula All., cypiroidea L., bicolor All., caespitesa Good., stricta L., acuta Fries, glauca Scop., pendula Huds. (maxima Scop.), microcarpa Salzm., strigosa Huds., ulba Scop., capillaris L., pallescens L., olbiensis Jord., pilosa Scop., panicea L., nitida Host, ustulata Wahlb., atrata L., nigra All., aterrima Hoppe, limosa L., Buxbaumiana Wablb., hispida Willd., praecox Jacq., polyrrhiza Wallr., tomentosa L., pilulifera L., membranacea Hoppe, montana L., basilaris Jord., gynobasis Vill., œdipostyla Duv.-Jouve, brovicollis DG., humilis Leyss., rligitata L., ornithopoda Willd., mucronata All., frigida All., hispidula Gaud., ferruginea Scop., sempervirens Vill., firma Host, tenuis Host, silvatica Huds., depauperata Good, hordeiformis Wahlb., Hava L., divaricata (EEderiana Ehrh), Hornschuchiana Hoppe, Mairiana Coss. Germ., distans L., extensa Good., punctata Gaud., laevigata Sm., longibracteata (pseudo Cyperus L.), ampul. lacea Good., vesicaria L., paludosa Good., Kochiana Gaud, riparia Curt., nutans Host, flliformis L., hirta L........... CARLiNa vulgaris L., nelorodensis Guss., lanata L., corymbosa L., acaulis L., acanthophylla All., cinara Pourr...............

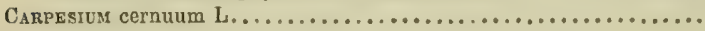

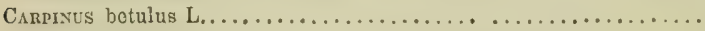

CARon verticillatum Koch, carvi L., bolbocastanum Koch.........

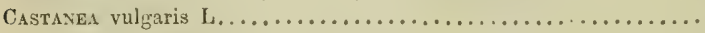

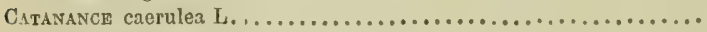

CAuCalis daucoidea L., leptophylla L....................

Celsia glandulosa Boutché, oriontalis $4 \ldots \ldots \ldots \ldots \ldots \ldots \ldots$

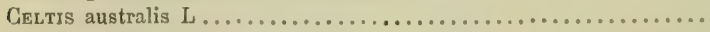

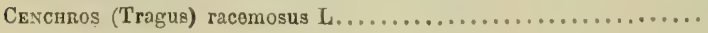


Centaurton amarum $I_{4}$, serotinum Bor., album L., jaceum L., Duboisianum Bor., nigrescens Willd., nigrum L., nemorale Jord, tubulosum Chabert, decipicns 'Thuill., nigro-solstitiale G. G., procumbons Balb., Jordanianum G. G., pectinatum L., rufescens Jord., acutifolizen J., comatum J., supinum J., fuscaum J., uniflorum L., nervosum Willd, ambiguum Thom., pullatum L., montanum L., lugdunense Jord., intermedium Cariot, somidocurrens Jord., axillaro WVilld, Seusanum Chais, cyanoum L., scabiosifolium L., (Scabiosa L.) calcareum Jord., alpestre Hog., silvaticum Pourr., cincroum Lam., semporvirens L., intybacoum Lam., corymbosum Pourr., maculosum Lam., tenuisectum Jord., subalbidum Jord., Muretianum Jord., cacrulescens Willd., Hanryanum Jord., diffusum Lam., lencophacum Jord., paniculatum L., valesiacum Jord., paniculato-calcitrapum Lorot, polycophalum Jord., rigidulum Jord., collinum L., napifolium L, sonchophyl . Ium L., asperum L., practermissum Martr.-Don., Mierghiarum Jord., aspero-calcitrapum G. G., calcitrapo-asperun G. G., sordidum IV. Kit., calcitrapum L., Pourretianum Timb. et Thev., myacanthum DC., molitenso L., solstitiale L., subalbidum Jord.,

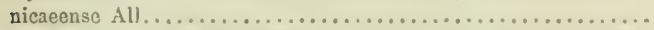

Centranthos angustifulium DG., Lecoqianzm Jurd, rubrum DC.,

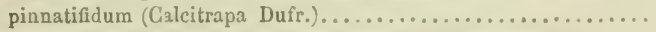

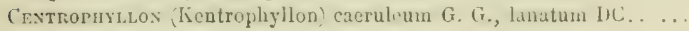

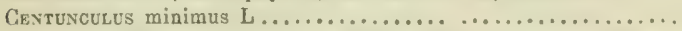

Cephalodes (Ciphalaria) syriacun Schrad., transsilvanicum Schral. alpinum Schrat., leucanthum Schrad ..................

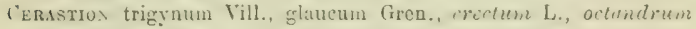
Gren., viscosum L., brachypotalum Desp., somidecandrum L., brachypetalum Desp., semidecandrum L., glutinosum Fries, pumilum Curt., aggregatum Dur., ramosissimum Boiss. (Riaeanum Desm.), vulgatum L., alpinum L., lanatum Lam., arvense L., strictım L., laricifolium Vill., latifolium L., aquaticum L......

Cenısos avicularis Moench, vulgaris Mill., corymbosus (Mabaleb

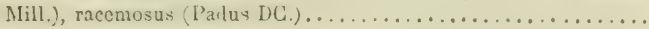

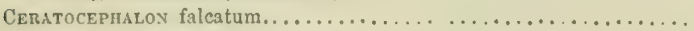

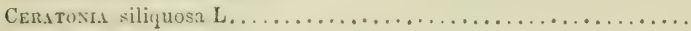

CERıtophylon submersum L., domersum L................

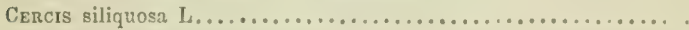

Cerinthon asperum Roth (majus Lam.), minus L, alpinum Kit.,

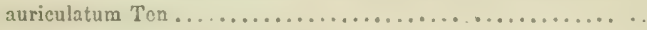

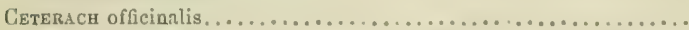

Chaenerhyloon bul'usum L., aureum L., Viilarsianum Koch, cicuturium Vill., unbrosum Jord., temulum L., nodosum Lam .......

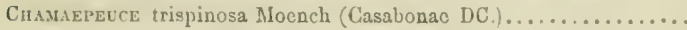

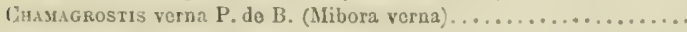

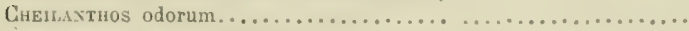

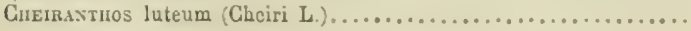




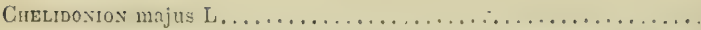

Chenopodion ambrosioideum L., botrydium L., polyspermum L., foe tidum Lam. (Vulvaria L.), ficifolium Sm., album L., opulifolium Schrad., hybriaum L., urbicum L., murale L., glaucum L.,

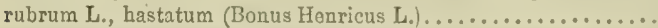

Chiroxiox pulchellum Sw., corymbosum Dulac (Centaurium DC.), linarifolium DC., spicatum Willd......................

Culorox perfoliatum L., scrotinuen Kuch, imperfoliatum L........

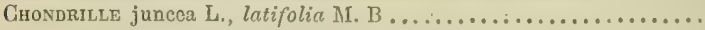

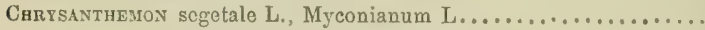

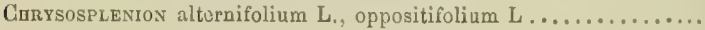

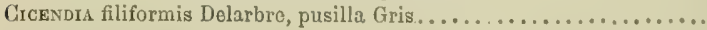

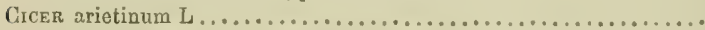

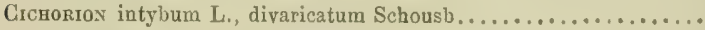

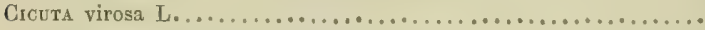

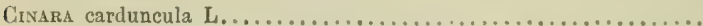

Cineraria spathulifolia DG, aurantiaca Hoppe, lanuginosa Jord., fus-

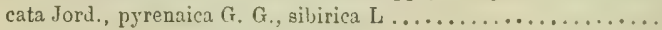

Girrcaea lutetiana L., intermedia Ebrh, alpina L..............

Crrsion lanceolatum Scop, crinitum Boiss, echinatum DC., ferox DC., odontolepidium Boiss, eriophorum Scop., palustre Scop., palustri-monspessulanum G. G., palustri-bulbosum DC., palustriacaule Hampo, palustri-oleraceum Naeg., palustri-glutinosum (palustri-erysithales) Naeg,, monspessulanum All., oleraceum Scop., oleraceo acaule Naeg,, oleraceo-rivulare Naeg., oleraceoheterophyllum. Naeg., glutinosum Lam. (Erysithales Scop.) glutinoso-acaube Michal., glutinoso-oleraceum Naeg., bulbosum DC., bulboso-oleracerm Naeg., bulboso-acaule Naog, anglicum Lob., rivulare Link, rivulari-oleraceum Naeg., rivulari acaule Naeg., spinosissimum Scop, spinosissimo-heterophyllum Naeg., spinosissimo-oleracerm Naeg., spinosissimo-acaule Naeg., glabrum DC., heterophyllum All., heterophyllo-spinosissimum Naeg., heterophyllo-acaule Naeg, acanthophyllum Arv-Touv., heterophyllo-acanthophyllum Arv.-Touv, acaule All, acauli-spinosissimum Naeg., arvense Scop ........................

Cistos umbellatus L, laurifolius L., ladanifer L., albidus L., albidocrispus Delile, orispus L., Pouzolzianus Delile, salvifolius L., rhodanensis Jord. F., microphyllus J. F., humilis J. F., apricus J. F., velutinus J. F,, platyphyllus J, F., elegans Jord. F., populifolius L., corbariensis Pourr., longifolius Lam., Jedonius Lam., monspeliensis L., monspeliensi-salvifolius (olbionsis Huct et

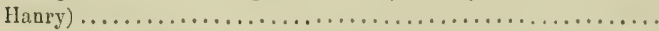

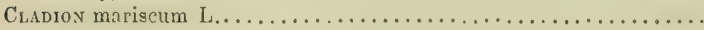

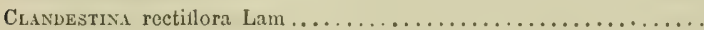

Clenratis recta $\mathrm{L}$., flammuIa $\mathrm{L}$, maritima DC., vitalba L........

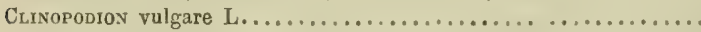

ClyPEOLA Jonthlaspi L., voyoz Jovthlaspi clypoatum........... 
CNEORON tricoccum L............................ 1:11

Cxicos benodictus L............................. 433

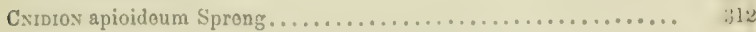

Cochlearia glastifolia L........................... 53

Colchicos autumnalo L., longifolium Cast., provinciale Loret, alpi-

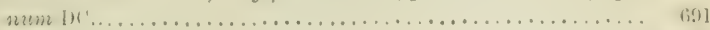

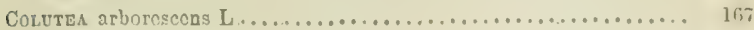

Cosaron palustro L............................. 24:3

('o)

Conopopros donudatum Koch......................... : $: * 2$

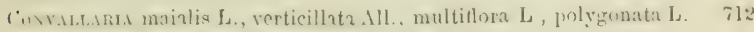

Cosvolvulus sopincola (sopium L.), soldanellus L., arvonsis L., tomontusus ('heisy, al thacoileus $\mathrm{L}$, lanupino:us J)ev, cantabricus L.,

lineatus L., tricolor L., siculus L., hirsutus Stev............

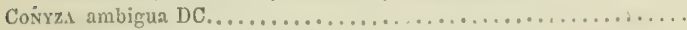

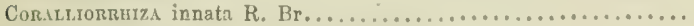

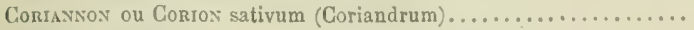

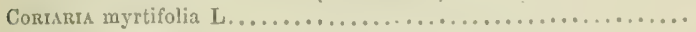

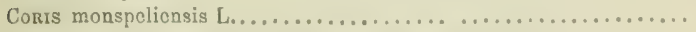

Corispermon ou mieux Coreospermos hyssopophyllum L.........

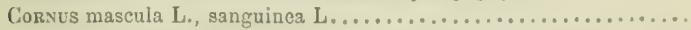

Corovilla emera L., glauca L., valentina $\mathrm{L}$, montana Scop., vaginalis Lam., minima L , coronata DC., juncea L., varia L., scorpioidea kiock.................................

Cormigrous littoralis L., telephiophylla Pourr..................

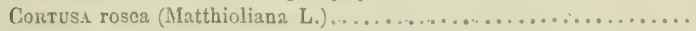

Conrdalis ou Corydillis cava Schw, et Koort., fabacea Pers, solida Sm., lutea DC., enneaphylla DC., claviculata DC............

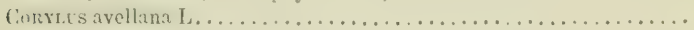

Cotone.sster pyracantha Spach, vulgaris Lindl., tomentosa Lindl....

Crataegos oxyacanthus L, oxyacanthoideus L., monogymus Jacq.,

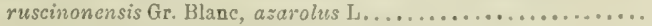

CREPIS vesicaria L., tarasacifolia Thuill., recognita Hall., bursifolia L. , erucifolia. G. G., setosa Hall., leontodontoidea All., Sufireniana Lloyd, foetida L., albida Vill., bulbosa Cass, , aurea Cass., chrysantha Froel., pracmorsa Tausch, bicnnis L., nicaeensis Balb., virens Vill, agrestis W. Kit., muralo (tectorum L.), pulchra Li, pygmaea L., lampsanoidea Froel, succisifolia Tausch, blattarifolia Vill., grandiflora Tausch, montana Monn., paludosa Moench.........

Cressa microphylla (cretica L.) ........................

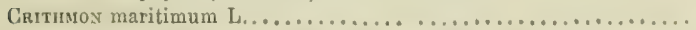
Ckocos vernus All., versicolor fawl, medius Bulb, nudiflorus Sm.,

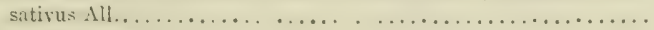

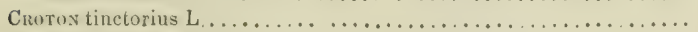

Cruclanella angustifolia $L_{\text {, }}$ latifolia $L$, maritima L............ Crupina vulgaris Cass, brachypappa Jord.................... Crypsis alopecuroidea Schrad., schoenoidea Lam., aculeata Ait..... 
Gucubalus baccifor $\mathrm{L} . \ldots \ldots \ldots \ldots \ldots \ldots \ldots \ldots \ldots \ldots \ldots \ldots \ldots \ldots$

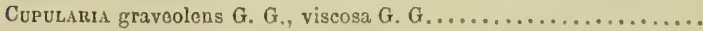

Cuscuta densiflora Soy.-Will., major DC., epithymophyta (Epithy. mum L.), Trifolii Bab. Gibs., Godroniana Desm., corymbosa

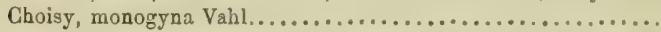

Cyclaminos (Cyclamen) europaeus L., repandus Sibth. Sm., neapoli-

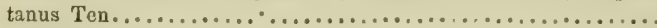

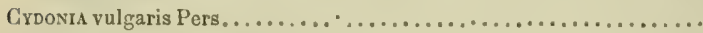

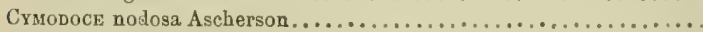

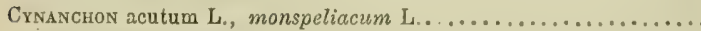

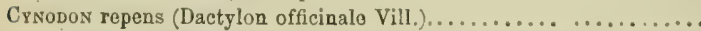

Cyxog Lossox cheirophyllum L., pictum Ait., officinale L., montanum

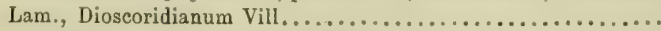

Cynosuros cristatus L., echinatus L., polybractentus Poir., aureus L.

Cypiros longus L., badius Desf, olivaris Targ.-Tozz, aureus Ten., fuscus L., schoenoideus Gris., sorotinus Rottb. (Montianus L.), flavescens L., globosus All., distachyus All.................

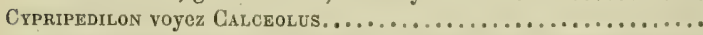

Crstoptenis fragilis Bernh., alpina Link, regia Prosl., montana Link.

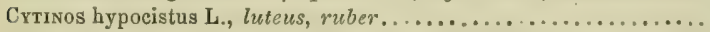

Crtisos laburnus L., alpinus Mill, sessilifolius L., nigricans L., decumbens Walp., triflorus L'Hér., biflorus L'Hér., clongatus WV. Kit., hirsutus L., capitatus Jaeq., supinus L., alpestris Thuret et Bornet, Ardoinianus Fournier, argenteus L...............

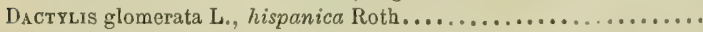

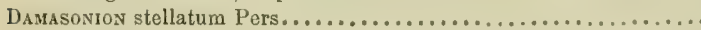

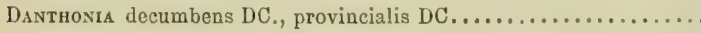

Daphe lateriflora (Mezereum L.), laureola L., alpina L., emarginata (Cneorum L.); striata Tratt., Vevlutiana G. G., orthophylla (Gni-

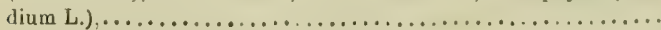

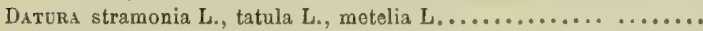

Daucos sativus (Carota L.), agrestis Jord,, exiguus Herm., mauritanicus All., maritimus Lam., serratus (7. G., Bosconianus Guss,, maximus Desf., hispidus Desf., gummifer Lam., gingidius L.,

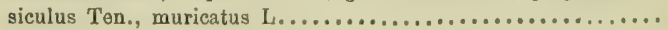

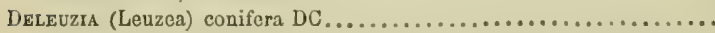

Delphinion divaricatum Dulac (Consolida L.), pubescens DC, laxiflorum (Ajacis L.), orientale Gay, peregrinum L., fissum W. Kit., elatumL., Requienianum DC., staphidium (Staphis agria L.).....

Dentaria digitata Lam., pianata $\mathrm{L}$, , bulbifera L...............

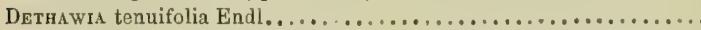

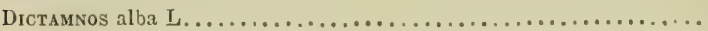

Digitalis purpurea L., parviflora All., purpureo-lutea Mey., grandi-

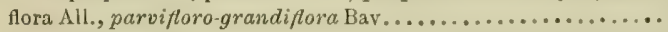

Drosanthos (Dianthus) proliferum L., velutinum Guss., armerium L., Carthusianicum L, liburnicum Bartl. et Wendl., vaginatum Chaix, atrorubens All., Seguierianum Chaix, silvaticum Hoppe, 
hirtum Vill, granitirum Jord., subacaule Vill, noglectum Lois., deltoidoum L., caosium Sm., silvestro Wulf, orophilum Jord., saxicolum J., juratense J., collivagum J., aggcricolum J., Guyetanianum J., consimile J., longicaule Ton. (Godronianum Jord.), virginoum L., pungens $G$. G. (an L.), brachyanthum Boiss., mons-

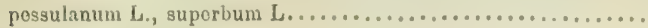

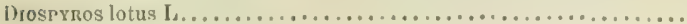

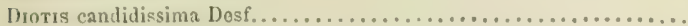

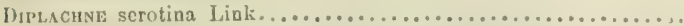

Diplotaxis humilis G. G. (pic Saint-Loup pròs Montpollior), repanda G. G., saratilis DG., tenuifolia DG., muralis DG., viminea DC.,

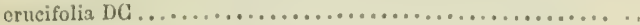

Dirsacos silvostris Mill., laciniatres L., fullonius Mill., pilosus L....

Donax arundinacous P. de B., mauritanicus Rocm. Schult.........

Doronicum plantagineum L., cordatum Lam. (Pardalianches L.), austriacum Jacq................................

Donycnion suffruticosum Vill., collinum J. F., elongatum J. F., frutescens J. F., duurulosum J. F., cincrascuns J. F., strzocladum J. F., affine J., decumbens J., gracile J., herbaceum Vill.....

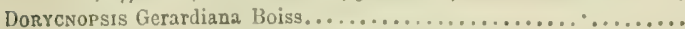

DRABe pyronaica L., aizoidea L., saxigona J., chrysantha J. F., ochroleuca J. F., trichocarpa J. F., bergesiaca J. F., alpestris J., cuspidata M. B , tomentosa Wahlb., frigida Saut., nivalis DG., flad-

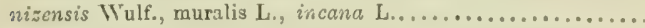

Dracostocephilos integrifolium (Ruyschianum L.), austriacum L...

Droseron rotundifolium L., longifolium L., obovatum M. Koch, inter-

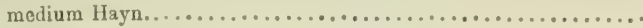

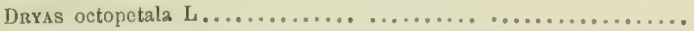

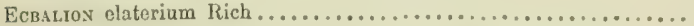

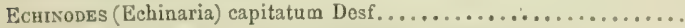

Echisor's sphaerocephala L., pauciflora Lam. (Rhytros L).... . . . .

Echinospermox lappulum Lohm, diflexum Lohm..............

Ecnson italicum L., pyramitlale Lap., albereunum Naudin et Debeaux, vulgare L., tuberculatum Link et Hoffm., parviflorum (Wierzbickianum Hab.), maritimum Willd., creticum L., plantagincum L., calycinum Viv., arenarium Guss..................

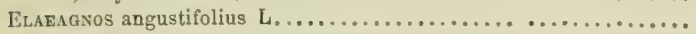

Elatine macropoda Guss, hippuroidea (Alsinastrum L.), hexandra DC., triandra Schk............................

Elysos crinitus Schreb., silvaticus Huds. (europaeus L.)...........

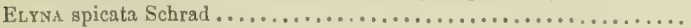

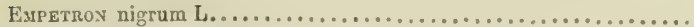

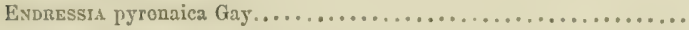

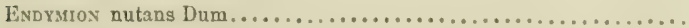

EPHEDRA rigida, helvetica C. A. Mey., nebrodensis Tin., maritima

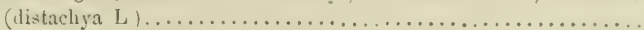

Éprobios origanophyllum Lam., alpinum L., palustre L., obscurum 
Schrob.; totragonum L., Lamyanzm Schultz, rosoum Schreb., trigonum Schr., montanum L., Duriaeanum Gay, collinum Gmel., lanceolatum Seb. Maur., parvillorum Schreb., hirsutum L., spicatum Lam, rosmarinifolium Haenke, Fleischerianum Hocbst... .

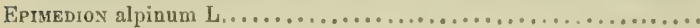

Eprpactis ensifolia Sw, lancifolia DC., rubra All., latifolia AlJ., microphylla Sw., palustris Crantz......................

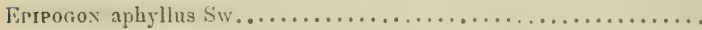

Equisetum arvense L., cburneum Roth (Telmateja Ehrh.), silvaticum L., palustre L., limosum L., hicmale L., ramosum Schl., trachyodontum Schl., variegatum Schl., umbrosum Mey...............

ERAGrostis mogalostachya Link, brachystachya (poceoidea Link),

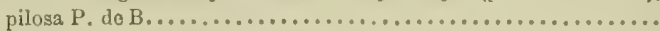

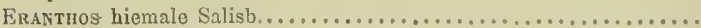

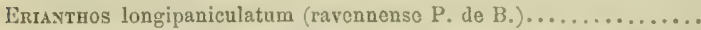
Erice mediterranea L., multiflora L., decipiens St-Am. (vagans G. G. an L.), tetralix L., cinerea L., arborea L., scoparia L., carnea L.

Erigeron canadensis L., acris L., serotimus Weihe, Droebuchensis Mill:, glandulosus(Villarsianus Bell.), al pinus L., glabratus Hoppo,

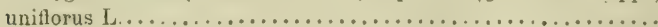

Erinacea pungons Boiss, (Costujes dans les Pyr. Or.) omis.......

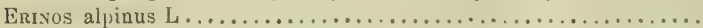
Eriophoron alpinum L., capitatum Host (Scheuchzcrianum Hoppo), vaginatum L., polystachyum L., gracile $\mathrm{Koch}$, angustifolium Roth, latifolium Hoppe.........................

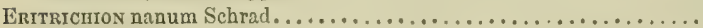

Erodion malachoideum Willd., littoreum Lem., laciniatum Cav., botrydium Bert., ciconium Willd, moschatum L'Hér., cicutarium L'Hér. triviale Jord, praetermissum, commixtum, subalbidum, pallidiflorum, bicolor, fallasc, pilosum, romanum WVilld., petraoum Willd.................................

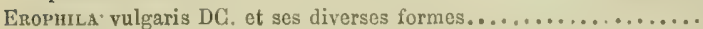

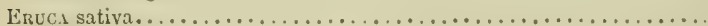
Erucastrus bracteatum G. G. (Pollichianum Schimp. Spenn.), obtu.

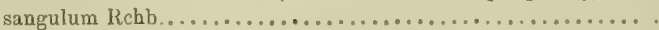

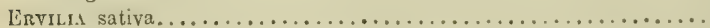

Ervus hirsutum L., tetraspermum L., pubescens DC., gracile DC ... Eryngron alpinum L., leucacanthum (Spina-alba Vill.)., amethystinum Lam. (Bourgati Gouan), csmpestre L., maritimum L .......... Errsimon cheiranthoideum L., virgatum Roth, confertum J., densiforum, glareosun, confize, cheiranthum Wallr. (Côto-d'Or), australo Gay, holveticum DC., ochroleucum DC., pumilum Gaud.,

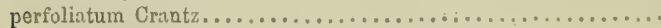

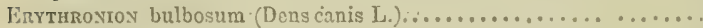

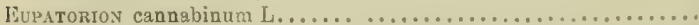
Euphorbion nummularium Lam. (Chamacsyce L.), auriculatum Lam.

(Peplis L.), helioscopium L., strictum L., achacnocarpum Guss., 
pubescons Dosf., pilosum L., palustro L., hiberaicum L. (perperam hibernum), dulco L., papillosum do Pouzolz, verrucosum Lam., flavicomum DC., spinosum L., linearifolium Lam. (Gerardianum Jacq.), saxatile Lois., acutifolium Lan. (Pityusa L.), maritimum Lam. (Paralias L.), dendroideum L., nicaeense All., esulum L., pseudocyparissium Jord., saliceticolum J., araricum J., riparium J., tenuifolium Lam., terracinum L., serraium L., aleppicum L, cyparissoideum L., exiguum L., sulcatum Delens, falcatum L., taurinense .Hl., peplum L., peploiden Gouan, biumbellatum P’oir., segetalo L., densillortin (Pinea L.), portlandicum L., silvaticum Jacq., purpureum Lam. (Characias L.),

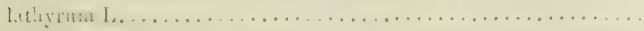

E.pur.ist. officinalis L, campestris Jord., uliginosa Duc., montana Jord., alpina Lam., hirtella Jord., puberula J., rigidula J., maialis J., cuspidata (ericetorum J.), nitidula Reut, cuspidatissina (salisburgensis Funk), cupraea J., minima Jacq..........

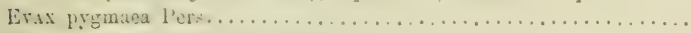
Eroxisros europaeus L., latifolius Seop ..................

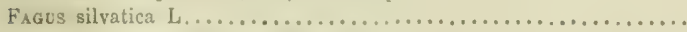

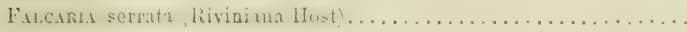

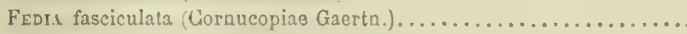
Ferula nodiflora L., glauca L., sulcata Bertol. (Ferulago I........ Festuca tenuifolia Sibth., ovina L., alpina Gaud., Halleriana All. pyrenaica Reut., duriuscula L., indigosta Boiss., violacen Gaud., rubra L, heterophylla Lam., pumila Chaix, aurala Gaud., varia Haenke, Havescens Boll., eshia Ram., pilosa Hall., nutans Ilost (Scheuchzeriana Gaud.), silvatica Vill., spadicea L., arundinacea Schreb., pratensis Huds., gigantea Vill ..................

FICART ranunculitlora Mocnch, grandiflora Rob ................

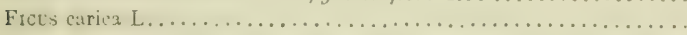

Filago spathulata P'resl., germanica L., canescens Jord., lutescens Jord., criocephala Guss., arvensis L., minima Fries, gallica L.,

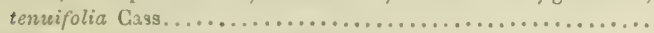

Foeviculum vulgaro Gaerta., piperitum DC..................

Fragina resea L., cullina Ehrb., silurelicole J. F., callivaga J. F., Thomasiana J. F., drymophila J. F., Hagenbachiana Lang.,

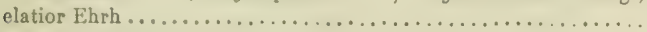

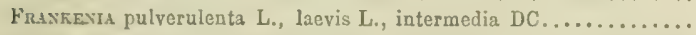

Fraxinus excolsior L., oxyphylla Bieb, oblusa G. G., rostrata Guss., bilobu G. G., parvifolia Lam., monophylla Desf., thyrsantha

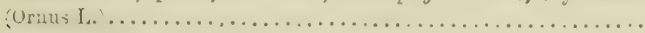

Fritiltaria meleagris L., pyrenaica L., delphinensis Gron., involu-

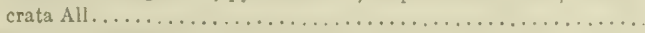

Fumana procumbens G. G., loatgipedunculata (Spachiana G. G.), laevipes Spach, viscida Spach, thymopliylla Pers..............

Fusarta capreolata L., pallidiflora Jord, spcciosa Jord., Bastardiana Bor. (muralis G. G. non Soud.), major Badarro, agraria Lag., Catal. Bassin du Rhonc. 
vagans Jord, , anatolica Boiss. (Kralikiana Jord.), officinalis G. G., densiflora DC., Vaillantiana Lois, parviflora Lam., spicata L... GsGra stenopetala Fries, lutea Schult., minima Schult., fistulosa Duby.,

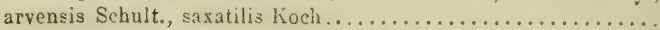

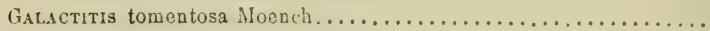

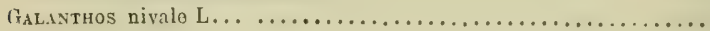

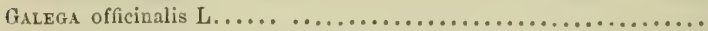

Galeopsis ladana L., lalifolia, angustifolia Ebrh., intermedia Vill., arvatica Jord., ochroleuca Lam., pyrenaica Bartl., nodosa Moench. (Tetrahit L.), praecox Jurd, pallida Jord., Reicheszbachiana Reut., bifida Boenn, glaucescens Reut., sulphurea Jord, Verlotiana Jord., alpicola Jord., leucantha Jord..................

Galiox cruciatum Scop., pedemontanum All., vernum Scop., rotundifolium L., ellipticum. Willd, boreale L., verum L., decolorans G. G., eminens G. G., purpureum L., silvaticum L, Irevigatum L., maritimum L., elatum Thuill., dumeticolum Jord., viridulum Jord., rigidum Vill., pallidulum Jord., erectum L., corrudifolium Vill., cinereum All., rubidum Jord., myrianthum Jord., Prostianum Jord., luteolum Jord., leucophaeum G. G., alpicola Jord., brachy podum Jord., lactum Jord., collinum Jord., scabridum Jord., Timeroyianum Jord, implexum Jord., intertextum Jord., papillosum Lap., silvestre Poll,, nitidulum Thuill, Fleurotianum Jord., laevs Thuill, montanum Vill, argenteum Vill, anisophyl. lum Vill., tenue Vill., Jussiaeanum Vill., pusillum L., hypnoideum Vill., pyrenaicum Gouan, helveticum Weigg., pusillum Vill., megalospermum Vill,, cometorrhizum Lap., saxatile L., palustre L., rupicola Bor., elongatum Presl., debilo Dosv., uliginosum L., setaceum Lam., divaricatum Lam., tenuicaule Jord., parisiense L., litigiosum DC., decipiens Jord,, anglicum Huds., ruricolum Jord., tenellum Jord, aparinum L., spurium L., Vaillantianum DC., tenerum SchI., tricorne L., sacharatum All., minutulum Jord., verticillatum Danth., murale All...........

Garidella nigelliformis L............................

Gastridion lendigerum Gaud., scabrum Presl.................

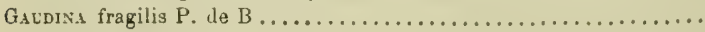

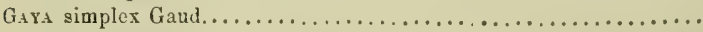

Gevista sagittalis L., pilosa L., delphinensis Verl., hunifusa Vill., tinctoria L., lasiocarpa, ovata W. Kit., cinerea DC, aspalathoidea Lam., Lobeliana DC., scorpia DC., anglica L., germanica L., hispanica L., candicans L., radiata Scop., linifolia L., horrida DC. .

Gentiane lutea L., brevifissa (Burseriana Lap.), punctata L., luteopunctata G.G., purpurea L., luteo-purpurea, purpureo-punctata, cruciata L., asclepiadea L., macrocarpophora (Pneumonanthe L.), acaulis L., latifolia G. G. (Kochiana P. S.), alpina Vill., angustifolia Vill., coriacea (Clusiana Perr. Song.), pyrenaica L., bavarica L., imbricata Schl., Rostaniana Reut., verna L., brachyphylla Vill., angulosa M. B., germanica Willd., obtusifolia Willd., 
campestris I., tenella Rottb, nivalis I., utriculosa L., ciliata L.

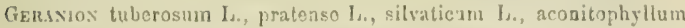
L’llör, bohomicum L., nolosum I., phroum I/, palustro I., argentcum L., sanguinoum L., columbinum L., disscctum L., pyrenaicum L., molle L., pusillum L., rotundifolium L., macror. shizum L. (Alp. Mar.), Jucidun L., Robortianum L., mediterraneum Jord., scmiglabrum J., purpureum Vill., simile Jord., modestum $\mathrm{J}$, minuiflomu $\mathrm{J}$, Villusianum $\mathrm{J}, \ldots . \ldots \ldots \ldots \ldots$

Gerontorocion (perperam Geropogon) glaber $1 . . . . \ldots \ldots \ldots \ldots$. Geus urbanum L., rivale $\mathrm{L}_{\text {., }}$ intermedium Bihrh., silvaticum Pourr. pyrenaicum IVilld, inclinatum Koch, montrnum J., reptans L.,

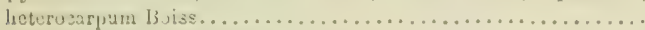

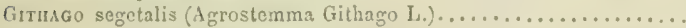
Goaprouus palustris Gand, illpricus Koch, communis I., segotalis (rawl. . . . . . . . . . . . . . . . . . . . . . . .

Guaucrox luteum Scop., corniculatem Curt..................

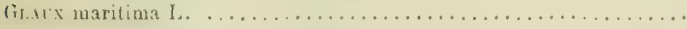

GLEchosion (Glechoma) hederaceum I.....................

Globularia vulgarig L., Wilkommiana $\mathrm{Nym}$, nudicaulis $\mathrm{I}_{\text {., }}$, cordifo.

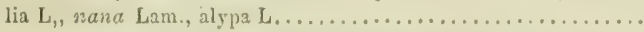

Gurceria airoidea Rchb., fluitans 1R. Br., plicata Frios, loliacca Godr., aquatica Wahlb., festuciformis Heynh., convoluta Fries, distans

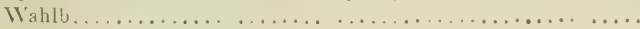

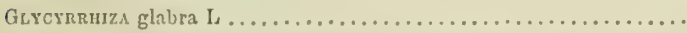

Gxisuluon luteoalbum L., silvaticum L., norvegicum Gunn., Hoppia-

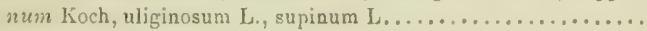

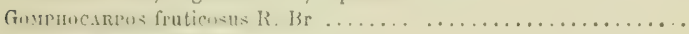

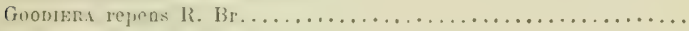

Grimutis leptophylla sw. . . . . . . . . . . . . . . . . .

Granatum puniceum (perperam I'unica Granatum L.) ............

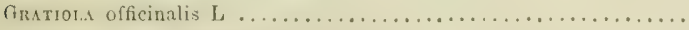

Gregonra lutea (Vitaliana Duby).......................

Grpsophilon repens L, alpestre Jord, sabaudum J. F., muralo L...

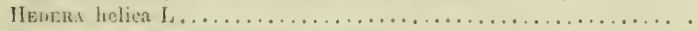

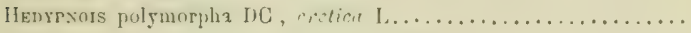

Hedrsanon obscurum L., humilo L., capitatum Desf. . . . . . . . . . . . Helantaemon niloticum Pers., salicifolium Pers., intermedium Thib., hirtum Pers, vulgare Gaortn., tomentosum Dun, obscur'um Pers., nummulariun Aill., grandiforum DC., pulverulentum DC., velutinum Jord, calcarcum Jord, pilosum Pers., celandicum DC., ulpestre DC, penicillatım Thib, canum Dun., marifolium L., guttatum Mill., tuberarium Mill. ..................

Helichryson arenarium DC., decumbons Camb, stochadense DC. et ses formes, angustifolium DC. et ses formes................ Henrotropion curopaeum L., supinum L., curassavicum L.........

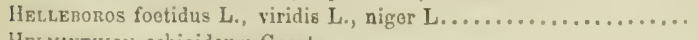

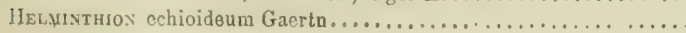


Herodes glandulosum (palustro Spach). . ................. 110

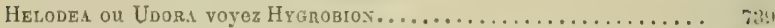

HELoscradion nodiflorum Koch, repens Koch, inundatum Koch...... $320^{\circ}$

Hemerocaldis fulva $L$, flava $L \ldots \ldots \ldots \ldots \ldots \ldots \ldots \ldots \ldots \ldots \ldots$. 70 .

Ifep.tica triloba Chaix............................ 11

Heracleox sphondylium L, Lecoqianum G. G., palmatum (Panaces

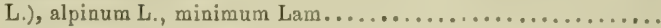

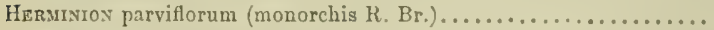

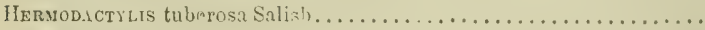

Hersiarid glabra L., hirsuta L., cinerea DC., incana Lam., latifolia

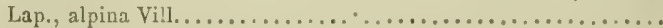

Hesperis silvestris Clus. (matronalis L.), laciniata All...........

Hieracion staticifolium All., pilosellum L., nigrescens Fries, pilosissimum Fries, virescens Fries, Saussurianum Arv. Touv., velutinum Heg. et Heer, biflorum Arv. T., caricinum Arv. T., Faurianum A. T., subrubens A. T., hypoleucum A. T., Schultesianum Schultz, bifurcum M. B., fallacinum Sch., auriculatum L., florentinum All., praealtum Vill., cymosum L., hybridum Chaix, anchusoideum A. T., glaciale Lach., Smithianum A. T., Laggerianum Schultz, alpicola Schl. (vallée de Sas, Simplon), pratense Tausch, sabinum Seb. Mauri, aurantiacum L., pumilum Lap., glaucum All., politum Fries, saxetanum Frios, chondrilloideum Vill., bupleuroideum Gm., falcatum A. T., leucophseum G. G., chlorophyllum A. T., calycinum A. T., scorzonerifolium Vill, elegans A. T., villosum L., dentatum Hoppa, dasytrichum A. T., nigritellum A. T., valdepilosum Vill., speciosum Horn., ustulatum A. T., armerioideum A. T., trichocladum A. T., leucochloum A. T., piliferum Hoppe, glanduliferum Hoppe, subnivale G. G., saxatilo Vill., intertextum A. T., olivaceum G. G., oleovirens A.T., neocorin thum Fries, composit:m Lap., ala tum Lap., cerinthoideum Fries, cordifolirm Froel, vogesiacum Moug, sonchoideum A. T., amplexicaule L., rupicola Jord., pulmonarioideum Vill., pseudocerinthum Koch, urticaceum A. T., rhombifolium A. T., ligusticum Fries, alpinum L., heterodontum A. T., Jacquinianum Vill., lanatum Vill., andryaloideum Vill, eriopsilum Jord., pictum Pers., Liottardianum Vill., Kochianum Jord., lansicum A. T., Reboudianum A. T., Ravaudianum A. T., verbascifolium Vill, thapsoideum A. T., melandrifolium A. T., floccosum A. T., lychnioideum A. T., mollitum A. T., chloropseum G. G., Pamphilianum A. T., rupestro All., sublanatum (pseudolanatum A. T.), lanatellum A. T., farinulentum Jord., leiopogonum Gren., cephalodern A. T., oligocephalum A. T., murale L. et ses formes, incisum Koch, bifidum Kit., cirritum A. T., porroctum Gren, papyraceum Gren., laevicaule Jord., virgulatum A. T., vulgatum Fries et ses formes, stelligerum Frocl., rapunculiforme A. 'T., buglossoiderun A. T., vestitum G. G., lasiophyllum Koch, pallidum Biv., pallidiforme A. T., ceratodontum A. T., nelanosper- 
mun A. T., brunolliformo A. T., Guillonianum A. T., scorpioideum A. T., caeruleum A. T., viride $A$. T., macilontum Frics, subalpinum A. T., subsecundum A. T., subviride (pscudoviride A. T.), homiplecum A. T., doronicifolium A. T', isatifolium A. T., juranum Fries, subjuranum A. T., cichoriaceum A. T., ochroleucum Schl., picroidoum Vill, lactucifolium $\Lambda$. T., cydonio phyllum Vill, prenanthoideum Vill., perfoliatum Froel., lanceolatum Vill., bifrons $\mathrm{A}$. T., symphytaceum A. T., hetcrospermam A. T., lycopophyllum Frool., tridentatum Fries et scs formes, sabandum $\mathrm{L}$. et ses formes, hirsutum Bornh., umbellatum $\mathrm{L}$. et ses formos, albidum Vill................

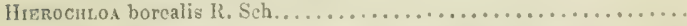

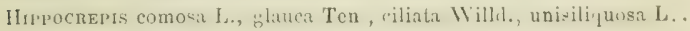

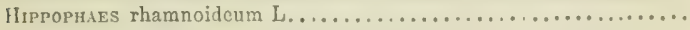

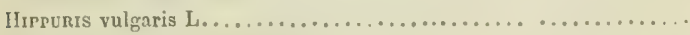
HiRschferdia adpressa Moench. . . . . . . . . . . . . . . . .

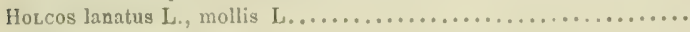

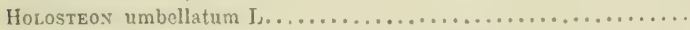

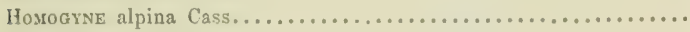

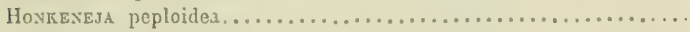
Honoces vulgare L., hexastichum $\mathrm{L}_{\text {., }}$ disticbum L., murinum L., secalinum Schreb, maritimum IVith., bulbosum L..........

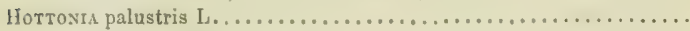

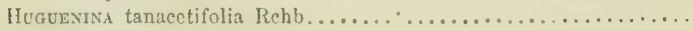

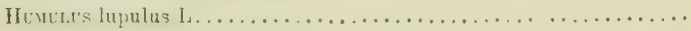
Hutcansra alpina $\mathrm{R}$. Br., petraca $\mathrm{R}$. Br., procumbens Desw....... Hracistros orientalis L., provincialis Jord., albulus Jord., amethysti-

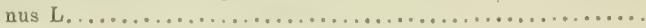

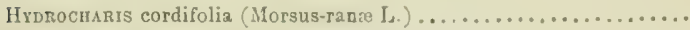

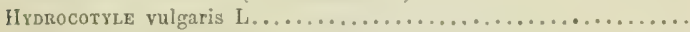
Hrgrobion canadense (Helodea canadensis Michx)..............

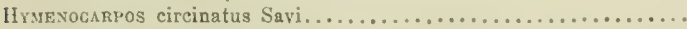
Hyoscrusos niger L., albus L., major Mill. ................

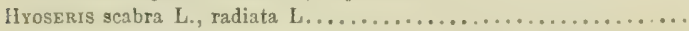
Hrpecoos procumbeas L., grandiflorum Benth, pendulum L....... Hrperrcos porforatum L., microphyllum Jord, lineolatum J., medianum J., quadrangulum L., tetrapterum Fries, humifusum L., australe Ten., linarifolium Vabl (Hérault), tomentosum L., verticillatum Lam. (Coris L.), hyssopophyllum Vill., pulchrum L., num mularium L., hirsutum L., montanum L., fimbriatura Lam. (Richerianum Vill.), ciliatum Lam, bacciferum Lam. (Androsae-

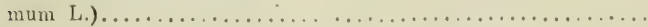

Hypochoeris glabra L., radicata L., maculata L., uniflora Vill....... Hrssopos officinalis L., beugesiacus J. F., decumbens J. F., recticaulis J. F., aristatus Godr., montanus J. F, cinerascens J. F...... IBERIS spathulata Berg. (Pyr.-Of.), Candolliana Jord., stricta Jord., aurosica Chaix, pinnata Gouan, affinis Jord., ciliata All., linifolia

ili:

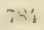

IR:

borit?

25.

35

801

ni

$3+i$

93

815

52.2

4:

(i):

5.3

708

728

$3 \%$ ?

T:3!

$13 \%$

5ับ

4i1)

32 .

108

441

618 
L., Villarsiana Jord, delphinensis Jord., umbellata L., colieno Jord, majalis Jord., Lamoltiana Jord., Violettiana Soy.-Will., Timeroyiana Jord., intermedia Guers., Prostiana Soy.-TVill., polita Jord., Garrexiana All., saxatilis L., amara L ...........

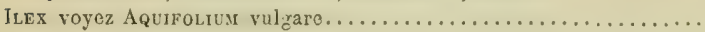

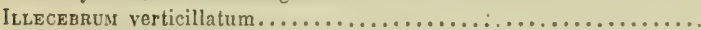

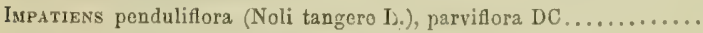

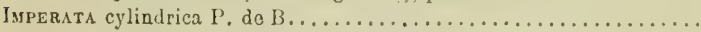

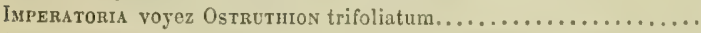
Invea helenia L., conyza DC., bifrons L., squarrosa L., hirta L., salicina L., Vaillantiana Vill, semiamplexiacaulis Reut., crithmoidea L., montana L., britannica L., helenioidea DC .............. Iris pumila Vill.; Iutescens Lam., germanica L., pallida Lam., floren. tina L., lutea Lam. (pseudacorus L.), foetidissima L, maritima Lam., graminea L., sibirica I., siphia Ehrh................ Isatis tinctoria L., cane'scens DC., hirsuta DC., alpina $\Lambda 11 . . . \ldots$.

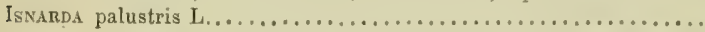
Isoetes lacustre L., setaceum Del, adspersum A. Br., reticulatum

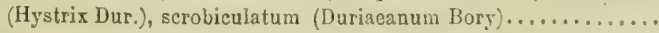

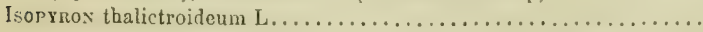
JAsrone vulgaris (montana L.), perennis Lam., Carioniana Bor,

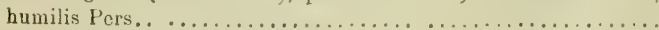

JASmLum fruticans $L$, of ficinalo $L \ldots \ldots \ldots \ldots \ldots \ldots \ldots \ldots \ldots$

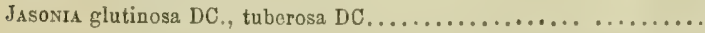
Jonthl.ASPI clypeatum (Clypeola Jonthlaspi L.), psilocarpum J. F, lapidicalum J. F., petraeum J. F., hispidulum J, F., anbiguum J. F., laevigatum J. F., microcarprm Moris..............

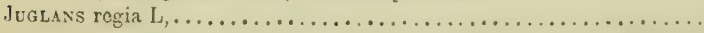
Juscus conglomeratus L., effusus L., diffusus Hoppe, glaueus Ehrh., paniculutus Hoppe, arcticus Willd, filiformis L., acutus L., ma ritimus Lam., atratus Lam. (Jacquinianus L.), triglumis L., trifidus L., pJgmacus Thuill., capitatus Weig., supinus Moench, lamprocarpus Ehrh., lagenarius Gay, striatus Schousb., acutiflorus Ehrh., anceps Lah., alpinus Vill, obtusiflorus Ehrh., squarrosus L., tenuis Willd., multillorus Desf., compressus Jacq., Gerardianus Lois, tenageius $\mathrm{L}_{\text {s. }}$, bufonius $\mathrm{L}$., fasciculatus Bert., ranavius P. S. Juniperus communis L., nana Willd, oxycedrus L., phoenicea L., lycia L., macrocarpa Sibth., sabina L., Villarsiana Jord.......

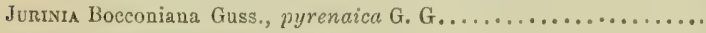
JUSSTAEA grandiflora Michx.........................

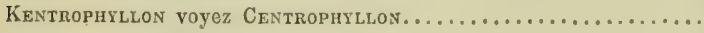

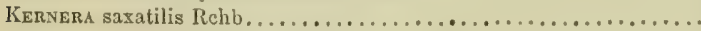

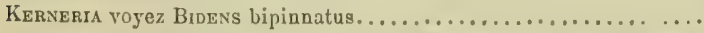
Kivautia hybrida Coult., arvensis Koch, Timcroyiana Jord., dipsacifolia Host, puberula Jord, cuspidata Jord, longifolia IV. Iit., mollis Jord., collina Req., subcanescens Jord, carpophylax Jord.

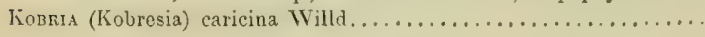


Kocur prostrata Schrad., arenaria Roth, hirsuta Nolto........... KoEl.ER. cristata l'ors., grandiflora Bert., brevifolin Reut., sctacea I'ers., valesiaca Gaud., prebesccus Parl., alpicola G. G., villosa Yors., phleoidea l'ers..........................

LAcruc. ramosissima G. G., viminca Liok, chondrillantha Bor., saligna L., scariola L., dulia Jord, virosa L., flavida Jord, sativa L., napiformis (Chairiana Vill.), muralis lires, perennis L., tenerrima Pourr. . . . . . . . . . . . . . . . . . .

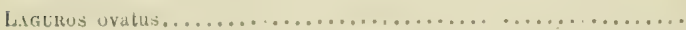

I.AxuU grandillorum Pours, amplexicaulo L., incisum Willd., pur. purcum L., maculatum L., album L., flexuosum Ton., galeobdolum

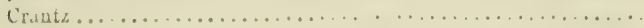

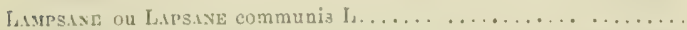
LAPPA minor DC., intermedia IRchb, major Gaerta., tomontosa Lam. Liser (Liserpitıus) latifolium L., Nostlerianum Soy.-Vill., gallicum C. Bauh., aspreticolun Jord., siler L., pruthenicum L., hirsutum

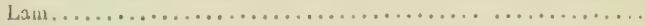

L.isidgirostis argentea DG. (Calamagrostis Link) ..............

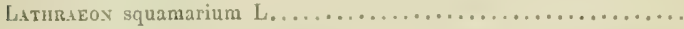

I.ATIrios clymenus L., articulatus L., ochrus DC., filipetiolatus (aphica L.), foliaceopetiolatus (Nissolia L.), pyrenaicus Jord., hirsutus L., cicerinus L., sativus L., annuus L., silvestris L, latifolius L., ensifolius Bad., heterophyllus L., cirrhosus Ser., tuberosus L., palustris L., pratensis L., angulatus L., sphaericus L., inconspicuus L., stans Vis, setifolius L............. LAurentr. Micheliana DC . . . . . . . . . . . . . . . . . .

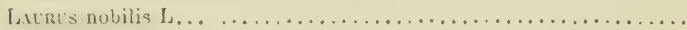
L.AFAxDULA stoechadensis L., spicata L., fragrans Jord., delphinensis Jord., latifolia Vill., erigens J. F., intermpta J. F., inclinans

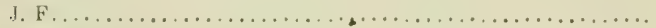

Litaterd arborea L., cretica L., olbia L., maritima Gouan, punctata

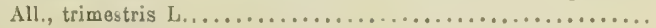

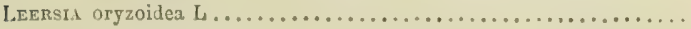

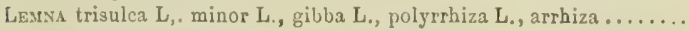
LEss esculenta Moench, nigrican Godr .................

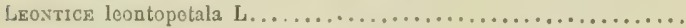

Leontodon autumnalis L., taraxacifolius Lois., pyrenaicus Gouan, protoiformis Vill., hastilis L., hispidus L., alpinus Vill., Villar-

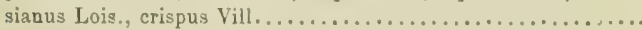
Lnovropodos alpinum Cass.........................

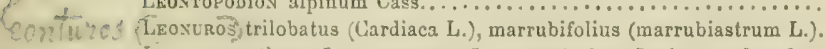
LEPIDION sativum L., cainpestre L., campicolum Jord., errabundum Jord, acceders Jord., heterophyllum Benth., hirtum Sm., ruderalo L., graminifolium L., polycladum Jord., drabophyllum

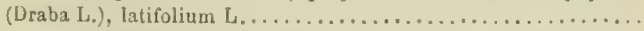
LEPTURos cylindricus Trin, incurvatus Trin., filiformis Trin........ LevcaxtueMoN vulgare I., discoideum All, pallens DC., maximum 
DC., atratum DC., graminifolium Lam., coronopophyllum G. G., palmatum Lam., corymbosum G. G., Pourretianum Timb., alpinum Lam., tomentosum G. G., parthenium G. G ............

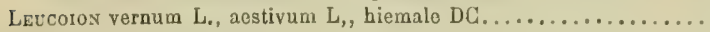

LeUzea voyez Deleuzia...............................

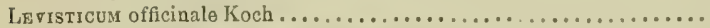

Ligosricon pyrenaeum Gouan, ferulaceum $L \ldots . . . \ldots \ldots \ldots \ldots \ldots$

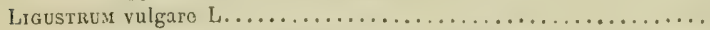

Liliuss pomponium L., pyrenaicum Gouan, martagonum L., croceum

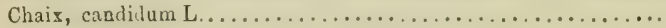

Limsanthemon (Villarsia) nymphaeoideum Link..............

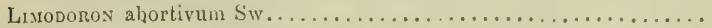

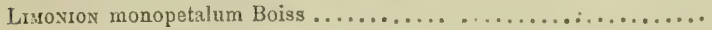

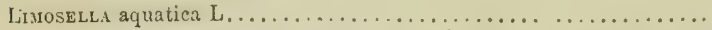

Lrvaria cymbalaria Mill., spuria Mill., elatine Desf., graeca Chav., cirrhosa Willd, vulgaris Moench, genistifolia DC., Pelliceriana Mill., arvensis Desf,, simplex DC., micrantha Spreng., spartea Hoffm. Link., chalepensis Mill, striata DG., triphylla Mill., alpina DC., petraea Jord., supina Desf., minor Desf., praetermissa Del., rubrifolia DC., origanophylla DC., villosa DC...............

LiNDERNia pyxidaria All...............................

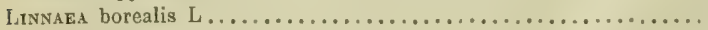

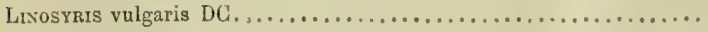

Lison nodiflorum L., campanulatum L., gallicum L., strictum L.. maritimum L., tenuifolium L., suffruticosum L., narbonense L.,

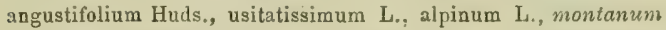
DG., (Loreyianum Jord,), Leonianum F. Schultz, catharticum L.

Liparis viridiflora (Loeseliana Rich.)....................

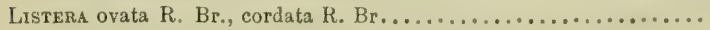

Lithospermon fruticosum L., oleifolium Jap., purpureocaeruleum L., officinale L., permixtum Jord, apulum Vahl..............

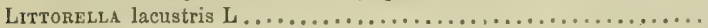

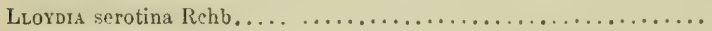

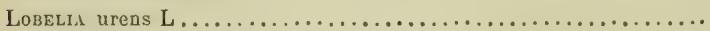

LoEfLINGIA hispanica $L \ldots \ldots \ldots \ldots \ldots \ldots \ldots \ldots \ldots \ldots \ldots \ldots \ldots$

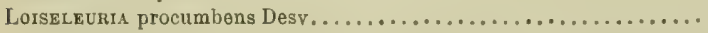

Lolius perenne L., italicum A. Br., multiflorum Lam., rigidum Gaud.,

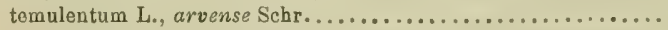

Lonicera implexa Ait., caprifolia L., etrusca Santi, periclymena L., xylostea L., nigra L., pyrenaica L., alpigena L., ca.crulea L....

Lotos rectus L., hirsutus L., hirtus J. F., prostratus J. F., parviflorus Desf., angustissimus L., hispidus Desf., conimbricensis Brot., decumbens Poir., corniculatus L., villosis Thuill, pilosus Jord., tenuis Kit., Delortianus Jord., uliginosus Schk, patulus Martr. Don., Allionianus Desy, ornithopodioideus L., edulis L..... .

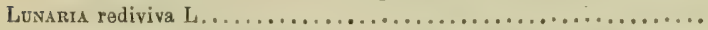
Lupinus thermus Forsk, hirsutus L., cryptanthus Shuttl, reticulatus Dosv., angustifolius $L . \ldots \ldots \ldots \ldots \ldots \ldots \ldots \ldots \ldots \ldots \ldots \ldots$ 
Luzula pilosa Willd, crecta (Forsteriana DC.), flavoscons Gaud,, maxima DC., glabrata Desv., spaulicea DC., albida DC., nivea I)C., lutea DC., campostris DC., multiflora Lej., epicata WC., pedifor-

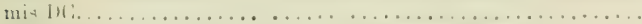

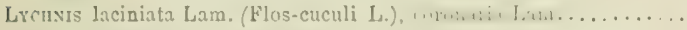

Lxcios barbarum L., ouropacum L., afrum L................

Lycopopion selaginum (Solago L.), inundatum L., annotinum L., alpinum L., complanatum L. var, chamacyparissum $\mathrm{A}$. Br.,

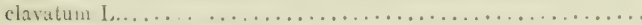

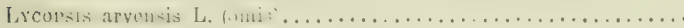

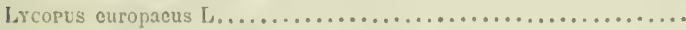

Lysimicuia thyrsantha (thyrsiflora L.), glauca Moench (Ephemerum

L.), vulgaris $L$., nummularia $L_{\text {. }}$, nemoralis $L \ldots . . . \ldots \ldots \ldots$.

Lyturos salicarium L., Gracferianum Ten., hyssopophyllum L., bibracteatum Salzm., thymophyllum L., geminitlorum Bert.....

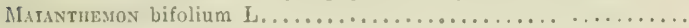

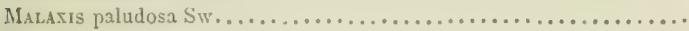

Milcolsin africana R. Br., parvillora DC., littorea R. Br., maritima

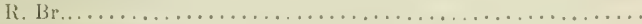

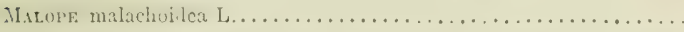

M.Alva alcea L., moschata L., Tournefortiana L., silvestris L., nicaeensis All., rotundifolia L., parvitlora L., mierocarpa Desf.........

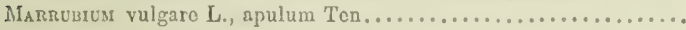

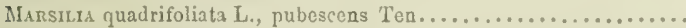

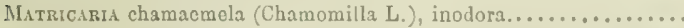

Matтurola incana R. Br., sinuata R. Br., tricuspidata R. Br., tristis

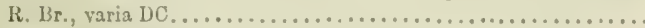

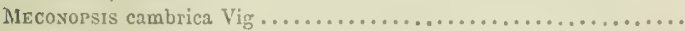

Medics (Medicago) radiuta L., lupulina L., falcata L. glomerata Balb., falcato-sativa Rchb., sativa L., scutcllata All., orbicularis All., ambigua Jord., marginata Willd, elegans Jacq., suffruticosa Ram., liocarpa Bonth., Soleircliana Duby, cylindracea DC., reticulata Benth., disciformis DC., Tenoriana DC., coronata Lam., praecox DC., polycarpa Willd., tuberculata Godr., apiculata IVilld., denticulata Willd., lappacea Lam., tricycla Godr., pentacycla Godr., ciliaris IVilld, maculata WVilld, minima Lam., marina L., littoralis Rhode, Brauniana Godr., Gerardiana WVilld., Timeroyiana Jord, Verlotiana P. S., cinerascens Jord, depressa Jord., triboloidea Lam,, murex Willd, , truncatulata Gacrtn., turbinata WVilld, muricata Benth., sphaerocarpa Bert., arborea L.

Melampyrox cristatum L., arvonse L., nemorosum L., pratenso L.,

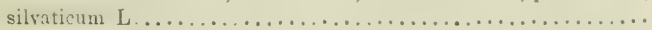

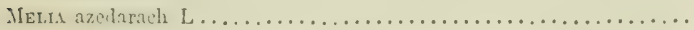

Mielica ciliata L., Bauhiniana All.; minuta L., nutans L., uniftora

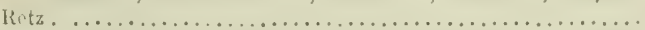

;is

$\therefore$

siv

S:3.)

(ai)

(i)

ii:i;

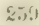

712

72

is

102

112

(ii) 2

4. $3 x$

3:?

37

$3: d$

13i;

Giai;

112

Melrlotos messanensis Desf., sulcatus Desf., infestus Guss., italicus Lam., elegans Salzm., parviflorus Desf., ncapolitanus Ten., offici-

Catal. Bassin du Rhône. 
nalis Lam., albus Lam., permixlus Jord, macrorrhizus Pers., creruleus Lam... . . . . . . . . . . . . . . . . . . . . . . .

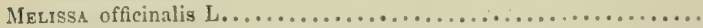

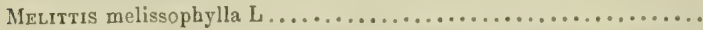

Mrstre rotundifilia L., silvestris L., viridis L., candicans Crantz, mollissima Boris.h., nemorosa Villd., aquatica L., arvensis L., agrestis Sole, parietarifolia Beck, sativa L., gentilis L., rubra Sm., piperita Huds., citrata Ehrh., odorata Sole, Nouletiana Timb., dentata Roth, Stofitziana Kerner, pulegia L., cervina L.

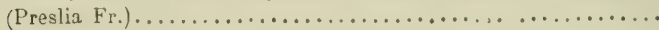

Mencintuos (Menyanthes) trifoliatum L..................... Mercurnuls perennis L., annua L., Huetiana Hanry, ambigua L.,

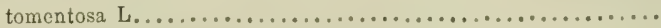

Merendera bolbocodia Ram., filifolia Camb..................

Mesembryanthemon erystallinum L., nodiflorum L..............

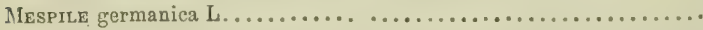
Meox athamanticum Jaeq., Mutellinum Gaertn., adonidifolium Gay. Mrcroloxchis salmantica DC., Clusiana Spach.................. Mrcroseris graeca Benth., Juliana Benth., piperella Benth.......... . Micropus erectus L., bombycinus Lag ..................... Milium caerulescens Desf., paradoxum L., multiflorum Cav., effusum

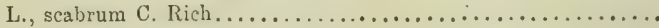
Moenringia muscosa L., dasyphylla Bruno, polygonoidea M. K, trinervia Clairv., pentandra Gay.......................

Molinaea caerulea Moench............................

Molineria minuta Parl. ...........................

Molospermon cicutarium DC ........................

Monotnopion epirhizium (Hypopithys L.)..................

MontI minor Gmel., rivularis $\mathrm{Gm} \ldots \ldots \ldots \ldots \ldots \ldots \ldots \ldots$

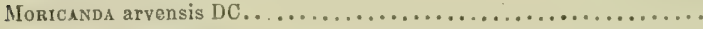

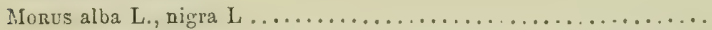

Muscari racomosum DC., negleclum Guss., botryoideum DC., como-

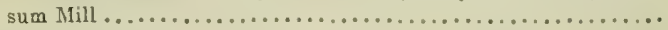

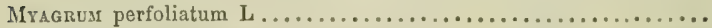

Mrosotis palustris With., lingulata Lehm.: pusilla Lois., stricta Link., versicolor Pers., lutea Balb., fallacina Jord., hispida Schlecht., intermedia Link., silvatica Hoffm., alpestris Schm.,

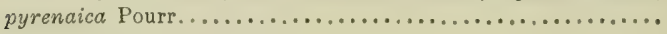

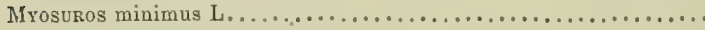

Mrrace (Myricaria) germanica) Desv......................

MrrophyLon verticillatum L., spicatum L., alterniflorum DC.....

Myrrhis odorata $L \ldots \ldots \ldots \ldots \ldots \ldots \ldots \ldots \ldots \ldots \ldots \ldots \ldots \ldots$

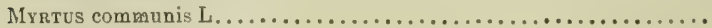

NaIAS major Roth, minor All........................

N.ıcessos bolbocodius L., grandiflorus Sal. (Pscudo Narcissus L.), major Curt., bicolor Lap., Bernardionus Hénon, incomparabilis Mill., pocticus L., biflorus Curt., juncifolius Req., junquillus, L. 
ochroleucus Lois., odorus L., chrysanthus DC., surous Lois., niveus Lois., dubius Gouan, polyanthus Lois., tizottus L. et $8 \mathrm{CS}$ formes déerites par Jordan et Fourreau...................

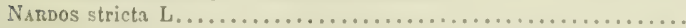

Nardunos unilateralis Boiss. (tonellus Schb.), alterniflorus (Lachena-

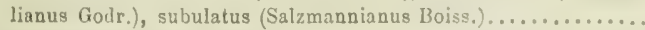

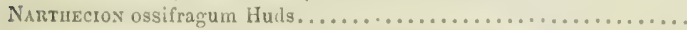

NAsturtusi officinalo R. Br, silvostro R. Br., anceps DC..........

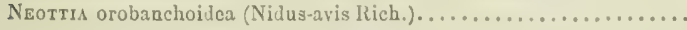

NePerd lancoolata Lam., parviflora (Nopetolla L.), cataria L., latifolia

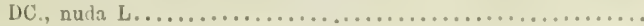

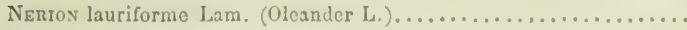

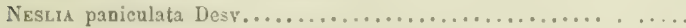

Nigella damascena L., sativa $L$, arvensis L., gallica Jord.........

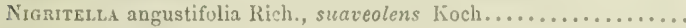

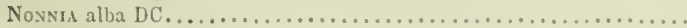

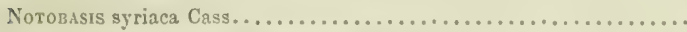

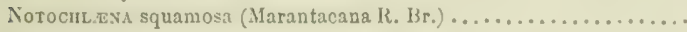

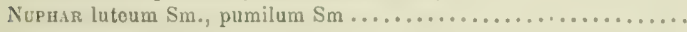

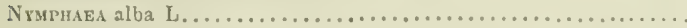

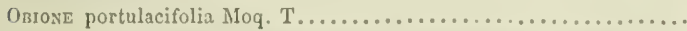

ODosrits rubra Pers., scrotina Rchb., viscosa IRchb., alpestris J. F.,

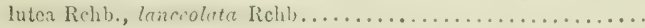

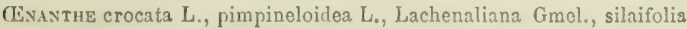
Biob., poucedanophylla Poll., fistulosa L., globulosa L. phellan-

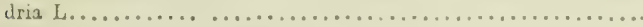

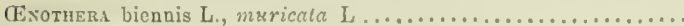

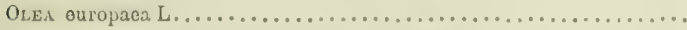

Oxphatodes linifolium Moench, vernum Mocnch................

ONobrycriss sativa Lam., montana Gaud., supina JG., saxatilis $\Lambda 11$,

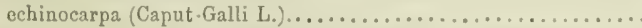

ONoxis rotudifolia L., fruticosa L., natrix L., arachnoidea Lap., ramosissima Desf., viscosa L., breviflora DC., rubescens L., cenisia L., reclinata L., campostris L., antiquissima L., procurrens Wallr., mitis Gimel. (indiqué à tort dans lo Valais sous lo nom d'allissima Lam.), parviflora Lam. (Columnaeana All.), striata Gouan, minutissima L., mitissima L., alopecuroidea L...

Oxopordon acanthium L., Delortianum Timb., tauricum Willd., illyricum $\mathrm{L}$., acaule $\mathrm{L}$, arabicum $\mathrm{L} . \ldots \ldots \ldots \ldots \ldots \ldots \ldots \ldots$

Ovosma echioideum L, arenarium W. Kit., stellulatum W. K.....

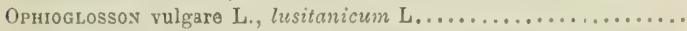

Ophrys aranifera Huds, Bertoloniana Mor., tenthredonophora WVilld. (perperam tonthredinifora), fucifora Rehb., apifera Huds., bombyliantha Link, scolopacia Cav., muscifera Huds., hirsuta (speculum Link), fusca Link, lutea Cav., alpina L.............. Opopanax altissimum Lam. (Chironium Koch)................ Orchis papilionaceus L., morio L., pictus Lois., Champagneuxianus 
Barn., ustulatus L., coreosmus (coriophorus L.), fragrans Poll., tridentatus Scop., simius Lam., galcatus Lam., fuscus Jacq., globosus L., masculus L., pallens L., provincialis Balb., palustris Jacq., laxiflorus Lam, alatus Fleury, saccatus Champ., sambucinus L., latifolius L., incarnatees L., maculatus L., bifolius L., chloranthus Cust., conopsus L., odoratissimus L., viridis Crantz,

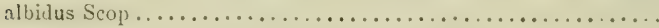

OrEochlos disticha Link, pedemontana Boiss. Reut.............

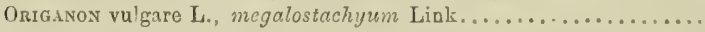
OrLAYa grandiflora Hoffm., platycarpa Koch, maritima Koch....... OnNithogalow narbonense L., sulphureum Sch., nutans L., proliferum Jord., umbollatum L. et ses formes décrites par Jordan et Four-

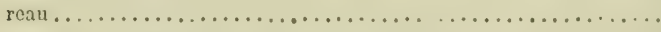

Orarthopus ebracteatus Brot., perpusillus I., compressus L........ Orobaxcue sarothamnophyta (Rapum Thuill.), Benthamiana Timb., crinita Viv,, cruenta Bert., variegata WVallr., speciosa DC., galionepiphyta (Galii Vauch.), thynosepiphyta (Epithymum DC), scabioshaerens (Scabiosae Koch), fuliginosa Reut., columbarihaorens (Columbaria Vuuch.), teucrionepiphyta (Teucrii Hol. Sch.), rhytrosepiphyta (Ritro G. G.), rubens Wallr.; silerhaerens Rap., major L., cervarihacrens (Cervariao Suard), picridipiepiphyta (Picridis Vauch.), artemisiepiphyta (Artemisias Vauch.), salvihaerens F. Sch., pubescens d'Urv, hederibaerens (Hederae Vauch.), minor Sutton, amethystea Thuill., cornua Loefl....... Ononos vernus L., variegatus Ten., luteus L., tuberosus L., niger L.,

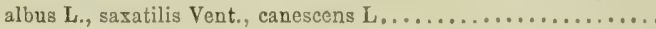

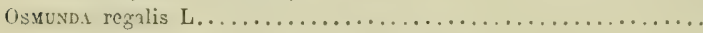

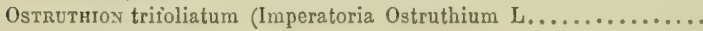

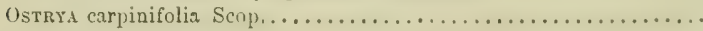

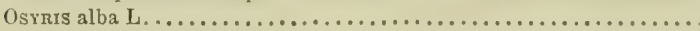

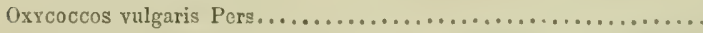

Oxrтropis campestris DG., foetida DC., Halleriana Bunge, cyanea Bieb., pyrenaica G. G., lapponica Gaud., montana DC., pilosa DC.

Pagonia corallina Retz, officinalis Retz, peregrina Mill...........

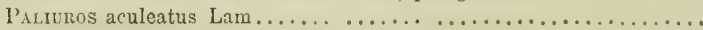

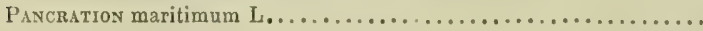

Panicus capillare L., dilatatum Poir., repens L., miliaceum L., polystachyum (Crus-Galli L.), sanguinale L., glabrum Gaud,, vagina-

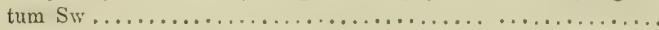

Pap.iver somniferum L., setigermon DG., hortense Hliss., thocoileum (Rhocas L.), ot les formes décritrs par M. Jordan, dubium L. et ses variétés, argcmonium L., hybridum L, alpinum L., albi

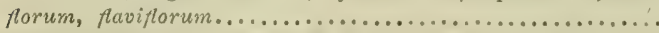
I'unietanu ofticinalis L., diffusa M. K., crecta M. K., lusitanica L. .

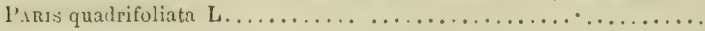

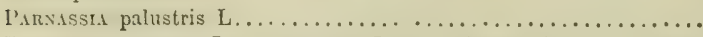
Paronxchi cymosa Lam., argentea Lam., polygonophylla DC., capi-

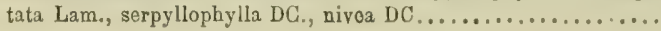


Passerivi annua Spreng., thymelea DC., tincloria Pours., eandicans

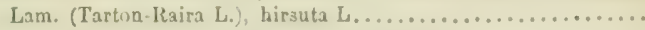

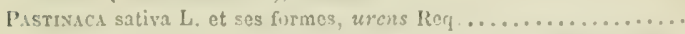

Pedicul.ars verticillata L., foliosa $L_{\text {. }}$, recutita $\mathrm{L}_{\text {., }}$ rosea $\mathrm{TVulf}$., palus. tris L., silvatica L., comosa L., incarnata Jacq., pyronaica Gay, conisia Gaud, gyroflexa Vill,, atrorubens Schl., rostrata L., Letourncuxiana Personat, tuborosa I., Barrellicriana Rchb.... Peplis portula L., erecta Req., Timeroyianc Jord................

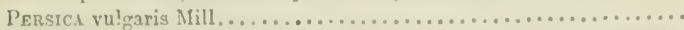

Petasites officinale Ifoench, pratense Jord., riparium J., Reuterianum J., album Gacrtn., nivcum Baumg., fragrans Prosl........

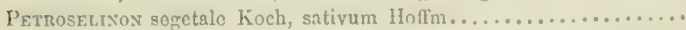

Peverdason officinale L., parisienso DC., glaucum Grud. (Cervaria Lap.), oreoselinum Moench, renetum Koch, alsaticum L., austriacum Koch, carvifolium Vill., palustre Mocnch..............

Puce alpina Wulf., frigida L., astragalina DC., australis L., Gerar-

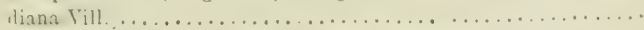

Prtıcxaroy sordidum DC., saxatilo Cass, ielonense Jord...........

Philavg tos liliastrum Lam., liliaceum (Liliago L.), ranosum Lam.,

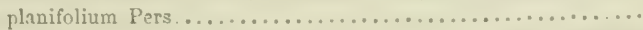

Phaldris canariensis L., brachystachya Link, minor Retz, paradosa L., crerulescens Desf., nodosa L.....................

Puisiolos rulgaris L. (quelquefois subspontané), ajouter d la pago.

Puelipaes cacrulea C. A. Ney., caesia Reut., arenaria Walp., olbicnsis Coss., lavandulacea F. Sch., ramosa C. A Moy., Mutcliana

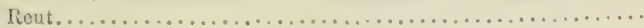

Pullzrrea angustifolia L., latifolic Duby, media L...............

Puleos pratensis L., nodosus I:, praecox Jord., serotinus J., intermedius J., phalaroideus Kool. (Boehmerianus Wib.). asper Jacq., alpinus L., commutatus Gaud, hirsutus Sut. (Aichelianus All.), arenarius $\mathrm{L}$., tenuis Schrad ........................

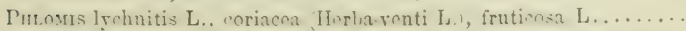

Phragutes communis Tria., giganteus Gar, mescinonensis Mab.,

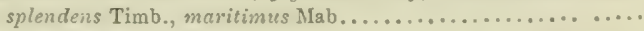

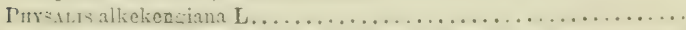

I'nrcusas paucitlorum L., glubulurifolitem Hopte, hemisphanricum L., humile Schl., Iongibracteatum (Charmclianum Vill.), orbiculare L. et ses variétćs, scorzonerifolium Vill., betonicifolium Vill., spicatum L., urticifolium Clairv. (Hallerianum All.)...........

Phitolicca decandra L..............................

Preridion vulgare Desf. ...............................

I'Icris Sprengeriana Lam., paucillura Wiild., pinnatifula Jord., stricta Jord, hieracioidea L., crepoidea Saut., pyrenaica L., Villarsiana Jord., corymbosa G. G....................

Putularra sessilis (globulifera L.), pedicellata (minuta Duricu)..... P'inpinele magna L., saxifraga L., perogrina L., leucocarpa (Tra-

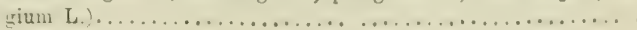




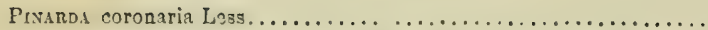

Pingurcula vulgaris L., grandiflora Lam., longifolic Ram., leptoce-

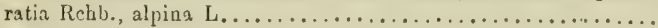

Pınus silvestris L., uncinata Ram., pumila (Pumilio Haonle), laricio Poir., pyrenaica Lap., halepensis Mill., pinea L., maritima Lam., cembra L., pectinata Lam., excelsa Lam., larix L.............

PIrola rotundifolia L., media $\mathrm{Sw}_{.}$, minor L., chlorantha Sw.,

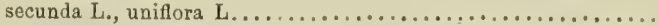

Pinus communis L., amygdaloideus Vill, malus L., acerba L.......

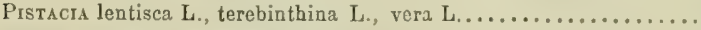

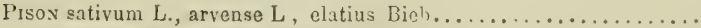

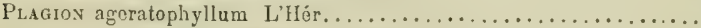

Plantago major L., intermedia Gilib, altiesima Lois. (Cornutiana Gouan), media L, brutia Ten., coronopoda L., crassifolia Forsk, maritima L., serpentina Vill., alpina L., subulata L., carinata Schr., lagopoda L., lanceolata L., Timbaliana Jord., lannginosa Koch, capitata Ten., argentea Chaix, albicans L., holostea Lam. (Bellardiana All.), fuscescens Jord., montana Lam., monosperma Pourr., psyllia L., arenaria W. Kit., cynops L.............

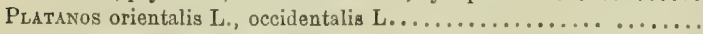

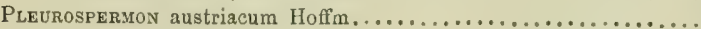

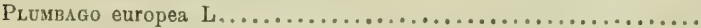

PoA annua L., supina Schr., minor Gaud., concinna Gaud., laxa Haenko, caesia Sm., nemoralis L., serotina Ehrh,, alpina L., brevifolia DC., bulbosa L., compressa L., distichophylla Gaud., pratensis L., anceps Gaud., trivialis L., silvatica Vill. (sudetica

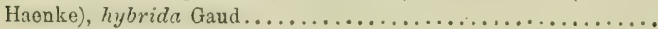

Podosperrion laciniatum DC., decumbens $G . G . . . . . . . . . . .$.

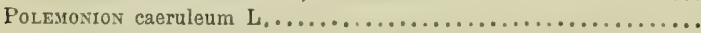

Polycarpon tetraphyllum L, alsinophyllum DC., peploideum DC...

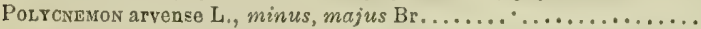

Polygald ou Polygalon rosoum Desf., vulgaro L., comosum Schk., oxypterum Rchb., Lejeunianum Bor., alpestre Koch, calcareum Schultz, alpestre Rchb., depressum Wend., amarum Jacq., austriacum Grantz, pedemontanum Perr. S., alpinum P. S., exile DC., buxifolium (Chamaobuxus L.), rupestre Pourr., monspelia-

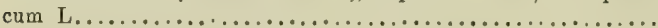

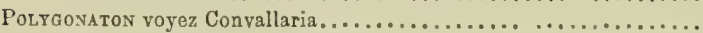

Polygosos bistortum L., viviparum L., amphibium L, lapathophyllum L., persicarium L., serrulatum Lag., nite Schr, minus Huds, hydropiperinum L., maritimum L., littorale Link, intermedium Lois., flagellare Spreng., aviculare L., arenarium W. Kit., virgatum Lois. (Bellardianum All.), convolutum (Convolvulus L.), dumoticola L, alpinum All., fagopyrum L., tataricum L.,

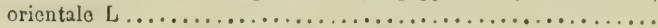

Polypodion vulgaro L., villosum Dulac (Phegopteris L.), rhaeticum L., triangularo Dulac (Dryopteris L.), calcarevm $\mathrm{Sm} . . . . . .$. . 
Potxpogon monspelionsis Desf., maritimus Willd., littoralis Sm.... Pow (1)reopteris 1..), whtusum [Mul. Filix Mas I..) cri-tatun lintl,

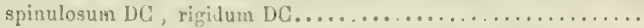

Porulus tromula $I_{\text {. }}$, alba L., canoscens Sm, virginiana Desf., nigra

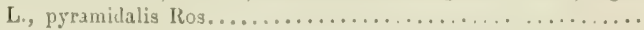

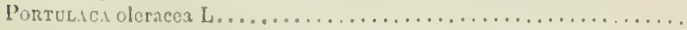

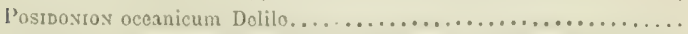

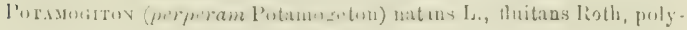
gonophyllus Pourr., rufoscens Schr., gramincus L., plantagineus Ducr., lucons L., decipiens Nolte, praelongus WVulf, perfoliatus L., crispus I., compressus L., acutifolius Link,, obtusifolius M. I., pusillus L., trichoideus Ch. Schl., pectinatus L., marinus

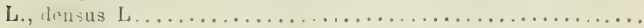

I'utcitill. fragariformis Ehrh., micrantha Ram., splendens Ram., alba L., saxifraga All., nitida L., caulescens L., petiolulata Gaud., nivalis Lap., nivea L., minima Hall., frigida Vill., grandiflora L., pedemontana Reut., subacaulis L., Clementiana Jord, cinerea Chaix, vestita Jord., opaca L., verna I., vivariensis Jord., stipularis Pourr., alpestris Hall., ambigua Gaud., aurea L., pyrenaica Ram., intermedia L. et ses formes, thuringiaca Bernh, delphinensis G. G., multiflda L., tormentilla L., orophila Timb., reptans $\mathrm{L}_{\text {, }}$ procumbens Sibth., anserina L., rupestris $L_{\text {., }}$ muricaulis Jord., supina L., argentea L. et ses formes, inclinata Vill., recta L., hirta L., fruticosa L.................

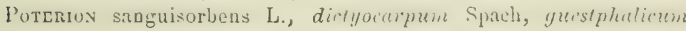
Boenn., Delortianum Jord., obscurum J., muricatum Spach, Magnolianum Spach............................

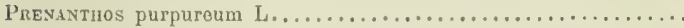

1'nisula grandiflora Lam., officinalis Jacq., suaveolens Bert., variabilis Goup., elatior Jacq., grandiflori-elatior, elatiori-grandiflora, elatiori.officinalis, intricata G. G., farinosa L., longiflora All., auriculata L., marginata Curt., viscosa Vill., pedemontana Thom., latifolia Lap., integrifolia L., Allioniana Lois.

Psimmites (Psamma) arenarium R. Sch., australe Mab...........

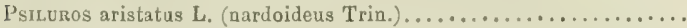

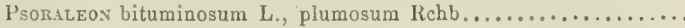

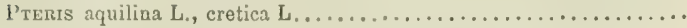

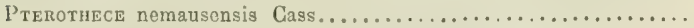

Pтrciotis heterophylla Koch, Timbaliana Jord................

Pulicaria odora Rehb., dysenterica Gaertn., sicula Moris..........

Pulmonaria silvestris, officinalis L., tuberosa Schr., azurea Boss.,

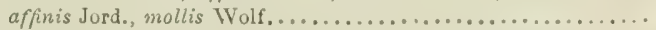

Pulsatrlla vernalis Mill., sericea (Halleriana All.), vulgaris Lob., montana Hoppe, rubra Lam., nigella Jord., propera Jord., alpioa Lois., sulphurea $\mathrm{I}_{\text {.., }}$ myrrhidophylla Vill.............

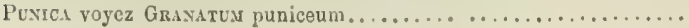


Prcnocomon (perperam Picnomon acarna Cass.) spinosum.........

Prrola voyez Prrola .................................

Pyrus voyez Pirus....................................

$41 \%$

517

248

Quercus robur L., brevipedunculata (scssiliflora Sm.), longipedun. culata (pedunculats Ehrh.), fastigiata Lam., tomentosa (Tozza Bosc), recurvisquamosa (Cerris L.), dentata (Fontanesiana Guss.), subera L., ilex L., Ausendiana G. G., coccifera L............

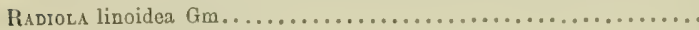

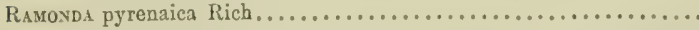

Ranunculus hederaceus L., aquatilis L., fluitans Lam., trichophyllus Chaix, divaricatus Schr., renifolius (Thora L.', alpestris L., glacialis L., dasycarpus (Seguierianus Vill.), rutifolius L., aconitophyllus L., platanophyllus L., parnassophyllus L., angustifolius DC., pyrenaeus L., graminous L., lanccolatus C. Bauh. (Flam. mula L.), longifolius C. Bauh. (Lingua L.), auricomus L., montanus WilId., gracilis Schl., aduncus G. G. (Villarsianus DC.), villosissimus (Gouanianus Willd.), acris L., vulgatus Jord., Boraeanus J., Friesianus J., Stevenianus Andrz, lanuginosus - L., palustris Sm., velutinus Ten., silvaticus Thuill., repens L., reptabundus Jord., glaber J., villosis J., bulbosus L., bulbifer Jord., albonaevis J., valdepubens J., sparssipilus J., monspeliacus L., cyclophyllus Jord., albicans J., chaerephyllus L., chaerephylloideus Jord, philonotus Ehrh., trilobus Desf., parviflorus L., ophioglossophyllus L., lateriflorus DC., nodiflorus L., arvensis L., muricatus L., apiophyllus C. Bauh. (Scelcratus L.)...... Rapistrum rugosum All., microcarpun Jord. (Linnacanum Boiss.

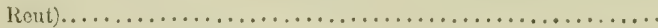

Reseda longicalycina (Phyteuma L.), lutea L., macrosperma (Jacqui-

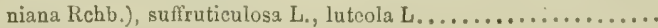

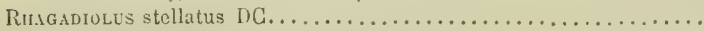

Ruamios cathartica L., silvatica Serres, saxatilis L., Villarsiana Jord., infectoria L., alpina L., pumila L., alaterna L., Clusiana

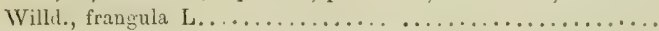

Rirapuniss sativa L., longistyla (Rhaphanistrum L.), landra Mor..... Rhapoxticus cinaroideum Less, heleniopbyllum G. G., scariosum

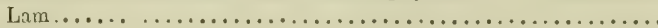

Rernanthos majus Ehrh., minus Ehrh., alpinum Baumg..........

Rhododendros ferrugineum L., hirsutum $\mathrm{L} \ldots \ldots \ldots \ldots \ldots \ldots \ldots \ldots$

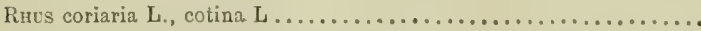

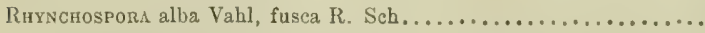

Rrbes crispum (Uva crispa L.), nigrum L., alpinum L., rubrum L,

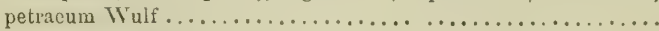

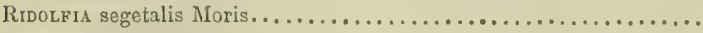

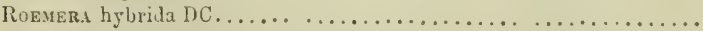

RorıPa nasturtifolia Spach, pyrenaica Spach, amphibia IBess., rusti-

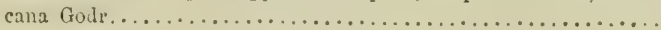

Rosa. - Section I Synstylac - sempervirens L., microphylla DG.,
6,3

102

558 
scaudens Mill., mostrala DC., ruscinonensis Déségl. - bibractoata Bast., arvonsis Huls., erronea liip., ovata l.cj...........

Section. Il Siylosae. - stylosa Dosy., symstyle Bast., lerecochroa Desv., festigiate I3ast.........................

Section III Gallicanac, - bybrila Schl, sublacvis 13oullu, arvina liroch, Polliniana Spreng. - austriaca Craatz, arenivaga Dés, subinermis Chab., geminata'Rau, l'ourracane Dés., incarnata Mill., vireseens Dés, velutiniplora Dés, et $\mathrm{O}_{z}$, mirabilis Dés., silvatica Tausch, decipiuns 13or., opacifolia Chab. - gallica L., provincialis Ait., assimilis Dés., ruralis Desf., gumila L.....

Section IV Pimpinelophyllae. - pimpinolophylla DC., Halhonetiana Crëp., consimilis Dés., sproter Des., Osanoniana Dés., mitissima Gm., reversa IV. Kit., yentilis Stern's. - myriacantha DU., Riprevibluc Dis.........................

Section V Sabiniae, - Sabiniana TVoods, coronata Crép., sabauda

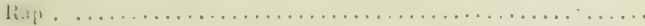

Section VI Cinnamomeae. - cinnamomca L, foecundissina Mlocnch, blanda dit............................

Section VII Alpinac. - alpina L, intercalaris Dés., adjecta Dós., monspeliaca Gouan, pendulina Ait, lagenaria Vill, pyrenaica

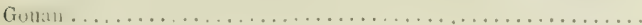

Section VIII Montanae - rubrifolia Vill, montana Chaix, salevensis Rap., Perrieriana Song., caballicensis Puget, falcata l'ug., glauca Vill., complicata Gren., intricata Gren., fugax Gren., venosa Sw., alpestris Rap., Scluztriana Rip................

Section IX Caninue, - canina L., glaucescens Desv, nitens Desv., syntrichostyla Rip., macracantha Dés., mucronulata Dés., senticosa Ach., fallens Dés., Tourangiana Dés. Rip., ramosissima Rau, globularis Franchet, montivaga Dés., spuria Pug., sphaerica Gren., aciphylla Rau - dumalis Bechst., rubescens Rip., rubelliflora IRip., squarrosa Rau, mulmundariensis Loj. medioxim. Dés., Cariotiana Chab., glaberrima Dum., oblonga Dós. Rip., lioclada (porperam cladoleia Rip.), silvulicola (silvularum Rip.), insignis Dẻs. Rip., Chaboissaeana Gren., eriostyla Rip. Dis., curticola Pug., stcnocarza Dés., villosiuscula Rip., stephanocarpa Dés. Rip., adscita Dés., macrocarpa Mér., biserrata Mér., sphaeroeidea Rip., brachypoda Dés. Rip. - andega vensis Bast., agraria Kip., Lemaitriana Rip., condenswla Pug., purpurascens Rip., vinealis Rip., Verlatiana Crép., Subertiana Rip., Rousseliana Rip., Kosinscyana Bess., firma Pug., asper. nata Lés., verticillispinosa (verlicillacantha Mér.), inconspicua Dés., Achariana Bilb., Chajertiana Dés., Timeroyiana Chab., haberiana Pug., transmota Crép., psilophylla Rau, Aunieriana Cariot, Laggeriana l'ug., Leveillaeana Boullu, scotophylla Boullu, P'ouziniana Tratt., leptocladu Boullu, Chaviniana Rapin, Wolfana do la Soie, Martiniana Gren. - dumeticola Thuill, obtusifolic Desv., urbica Lćm., ramealis Pug., semiglabra IRip.,

Catal. Bassin du Thüne. 
hemitricha Rip., trichoneura Rip., obscura Pug., globata Dés., platyphylla Rau, platyphylloidea Dés. Rip., implexa Gron., jactala Dés., sfhaerocarpa Pug., coreophylla Fries - collina Jacq., corymbophora Borkh., Deseglisiana Bor., hispidula Rip., trichoidea Rip., imitata Dés., bellavallensis Pug., approximata Dés., fallaciosa Dés., cinerea Rap., Bovernieriana Lag. de Ia Soie, Friedlanderiana Bess., macrantha Desp., alba L...........

Section X Eglanterice. - lutea Mill ....................

Section XI Rubiginosae. - tomentella Lém., similata Pug., valesiaca Lagg. Pug., nebrodensis Guss., viscida Pug., Blondaeara Rip. - trachyphylla Rau, insidiosa Rip., consanguinea Gren., Godetiana Gren., Cottetiana Pug., marginata Wallr., subolida Dés., Pregetiana Bor, nemorivaga Dés., flexuosa Rau, subflexuosa (pseudoftexuosa Ozanon), speciosa Dés., Jundzilliana Bess. sepincola Thuill., agrestis Savi, mentitc Dés., arvatica Pug., virgultosa Rip., Billetiana Pug., Seraphiniana Viv., cheriensis Dés., lugdunensis Dẻs., Jordaniana Dés., Vaillantiana Bor. rubiginosa L., comosa Rip., umbellata Lecrs, permixta Dés., septicola Dés., operta Pug., echinocarpa Rip., silvicola Dés, Rip., micrantha Sm., dimenuta Bor., lactiflora Dés, Lemaniana Bor., ladanophora Timb., subdola Dés., rotundifolia Rchb., uce.

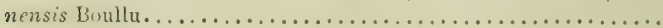

Section XII Tomentosae, - cincrascens Dum., dumosa Puy., micans Dés., marcyana Boullu, farinulenta Crép. - foetida Bast., spi. nulifolia Dematra, vestita Godet, abietina Gren., subcuspidata Crép., genevensis Pug. - tomentosa Sm., intromissa Crép., collivaga Cottet, dimorpha Bess., subglobosa Sm., tunionensis Dés., confusa Pug., annesiensis Dés., Andraionosciana Boss. mollis Sm., omissa Dés., resinosa Sternb., subresinosa Crép., Grenieviana Dés., pomifera Herm, friburgensis Dés. Pug., recondita Pug., Murithiana Pug., Gaudiniana Pug., gombensis

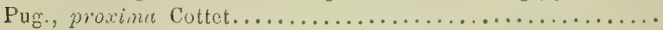

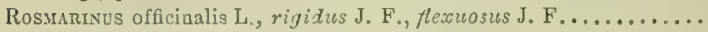

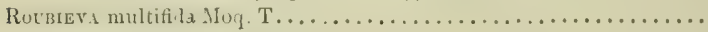

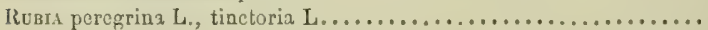
Rubus saxatilis L., idacus L., caosius L., parvulus Genev., validulus Genev., ligerinus Gonev., deltoideus Müll., degonor Müll., debilitatus Rip., serpens G. G., nemorosus Hayne, corylifolius Sm., agrestis WV. Kit., dumeticola IV. N., glandulosus Bell., calliphyllus Müll., pendulinus Müll., scaber IV. N., hirtus IV. N., Reuterianus Merc., atratus Genev., rudis WV. N., longiculpis Müll, radulus W. N., Schleicherianus IV. N., Guntherianus W. N., Mentianus WV. N., Bloxamianus Bor., hispidus Merc., piletocaulis Müll., vestitus W. N, piletostachyus G. G., leucanthemus Müll., cons. picures Müll , patulus Müll., thyrsoideus WVimr., albidus Merc., discolor WV. N., incamalus Müll., vusticanus Merc., tomentosus Borkh., specsabilis Morc., collinus DC., albidus Merc., pellitus 
Rip., cuncifulius Mere, clongatus More, vmlulatus Mere., Thipartionus Genev., fruticasus L., futigicus W. N., plicatus

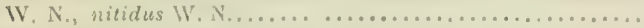

Rusex palustris Sm., lnxiflorus, densiflorus (maritimus L.), pulehnr

L., obtusifolius Is., conglomerat's Murr., sanguineus L., crispus

L., macrophyllus (Ilyclrolapathum Ifuds.), acutus L., bortensis Lam. (Pationtia L.), aquaticus L., alpinus L., bucephalophorus L., tingitanus L., scutatus L., arophyllus (arifolius All, ), amplo. xicaulis Lap., acotosus L., thyrsoideus Dosf., acctosellus L.....

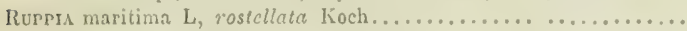

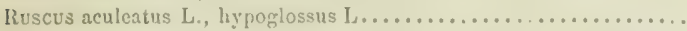

Rura montana L., augustifolia Pers., bracteosa 1)C., graveolens L...

S.crivd procumbons L., muscosa Jord., apotala I, ciliata Frios, maritima Don, subulata WVimm., Linnacana L'resl., glabra WVilld.,

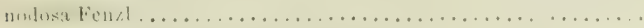

SAgița aquatica Plino (S:igittaria sngittifolia L.).. ............

SAlicornil herbacea L., fruticosa L., sarmentosa Duv. J., macrostre.

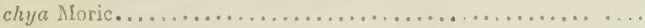

Salrx pentandra L., fragilis L., alba L., vitellina L., babylonien L., incana Schr., purpurea L., rubra IIuds., WVimmeriana G. G., daphnoidea Vill, viminalis L., Smithiana WVilld, oloifolia Vill., cinerea L., grandifolia Ser., capron L., aurita L., aurito-repens Wimm., repens L., hastata L., nigricans Sm., holvetica Vill., caesia Vill., glauca L., arbuscula L., myrsinitis L., pyrenaica Gouan, reticulata L, rotusa L., serpyllopliylla Scop, herbacea I.

S.ı.sola decumbens Lam. (Kali L.), tenuifolia Moq. T. (Tragis L..),

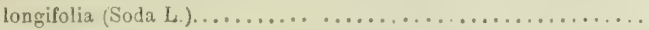

Satrid officinalis L, verticillata I., sclarca L., aethiopis L., glutinosa

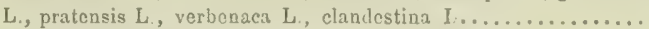

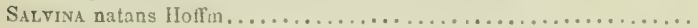

SAmbucus herbacea Dalechamps (Ebulus L.), nigra L., racemosa L...

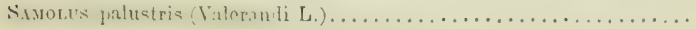

Saxguisorbi officinalis L, montana, serotina Jord.............

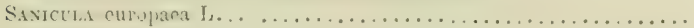

Santolins cyparissophylla (Chamacyparissus L.), incana Lam., squarrosa DC., viridis Villd., rigidula J. F., Benthamiana J. F., pectinata Lag., provincialis J. F., intricata J. F.............

Saponari. officinalis L., oeymoidea L., lutea L., vacesia L.......

SArothasinos vulgaris WVimm., arboreus Webb, purgans G. G.....

SAtureia hortensis L, montana L., brevis J. F., petraen J. F., pro.

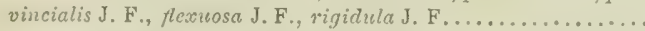
Siussuria depressa G. G., macrophylla Saut., discolor DC., alpina DC. SAxifragi stellaris L., lencanthemophylla Lap. (Clusiana Gouan), cuncifolia L., hirsuta L., rotundifolia L., refexa (Hirculus L.), aspera L, bryoidea L., aizoidea L., granulata L., bu'bifera L. pedemontana All., tridactylitis L., petraea L., Bellardiasra All. geranioidea L., ladanophora Lap., Prostiana Sternb. (pedatifida 
Sm.), olscura G. G., pentadactylis Lap., nervosa Lap., ascendens L., abigifolia (ajugifolia L.), capitata Lap., puboscens DC., groenlandica L., exarata Vill., leucantha Gaud., intricata Lap., muscoidea Wulf., moschata WVulf, androsacea L., Siguicriana Spreng., planifolia Lap., sediformis (sedoidsa L), sponhemica Gmel, hypooiden I. et ses formes, aiznonir Jacr. et ses formes, pyramidalis Lap. (Cotỵlelon I.), longifolia Lap., lingulata Bell., cochlearis Rehb., mutata L., mutato-aisoidea Reut, (mêlé auprécédent), media Gouan, luteo-purpurea Lap., diapensoider Bell., caesia L., valdensis DC., oppositifolia L., biflora All., retusa

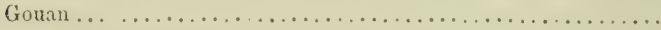

Scabrosa graminifolia L.; sericea J. F., virescens J. F., stellata L. et ses formes, ueranica L., maritima L., columbaria L., pratensis Jord., ochroleuca L., affinis Jord,, patens J., tenuisecta J., brigantiaca J., sericea J., suaveolens Desf, gramuntia $\mathrm{L}_{;}$; candicans J., lucida Vill., vestila J., alpestris J., succisa L. et sos formes. Scandix pectinata (Pecten Veneris L.), hispanica Boiss., australis L.

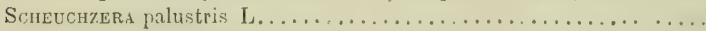

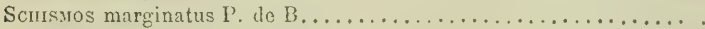

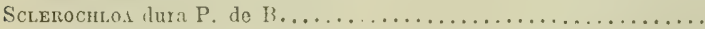
Scleropod maritima Parl., subsessilis (hemipoa Parl), rigida Gris.,

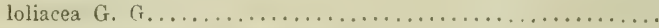

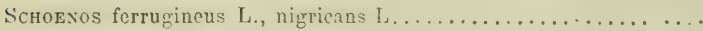

Scrlda maritima L., autumnalis L., hyacinthoidea L., amoena L., italica L., verna Huds., squamosa (Lilio-Hyacinthus L.), bifolia L.

Scrreus silvaticus L., Michelianus L., maritimus L., digynus, compressus Pers.( holoschoenus L., lacustris L., digynus (Tabernaemontanus Gm.), carinatus Sm., littoralis Schr. (Triqueter L.), Pollichianus G. G., pungens Vahl, mucronatus L., supinus L., setaceus L., filiformis Savi (Savianus Seb. M.), fluitans L., pauci florus Lightf., caespitcsus L., alpinus Schl...............

Scheranthos annuum L, uncinatum Schur, (hamosum do Pouz.), verticillatum Tausch, marginellum Rchb., bienne Reut., peren-

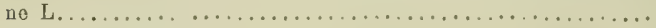

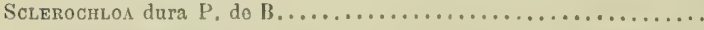

Scleropol maritima Parl, subsessilis (hemipoa Parl.), rigida Gris.,

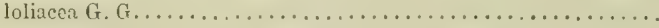

Scolopendron ofilcinale Sm., sagittatum DC. (Hemionitis Sw.).....

Scolysos maculatus $I_{d,}$ hispanicus L., grandiflorus Desf..........

Scorpiuros subvillosus L., vermiculatus $\mathrm{L} \ldots \ldots \ldots \ldots \ldots \ldots \ldots \ldots$

Scorzonera hirsuta L., purpurea L., austriaca Willd., crispa DC., humilis L., parviflora Jacq., aristata Ram., hispanica L., glastifolic Wallr., crispatule Boiss........................

Scrophubaria vornalis $L$, peregrina L., alpestris Gay, nodosa L., aquatica L., alata Gilib., lucida L., canina L. et ses formes,

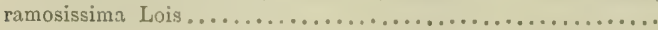

ScUteldaria alpina L., bastifolia L., galericulata $\mathrm{L}_{\text {, }}$, minor L...... 


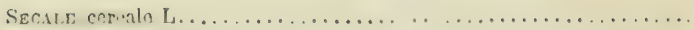

Securigera cotonilla 1 C. . . . . . . . . . . . . . . . . .

Sedur roseum Scop. (Thodiola L.), maximum Sut. (Telephium L.) ot ses formes, pupurascens lioch et ses formes, anacampserum L., stellatum L., copaeum L., alsinophyllum All, rubens I., pallidiflorma J. F., mediterraneum J. F., caespitosum I)C., atratum L., annum L., villosum L., hirsutum All, cruciatum Desf., album L., micranthum Bust., anglicum Huils., dasyphyHum I., brevifolium DC, alpestre Vill., acro L., sexangularo I.., rellexum L., rupestre L., albescens Haw., elegans Lej., aureum Virig., altissimum Poir., anopetalum DC., Verlotianum Jord, montanum I'. S., aristatum Vill., amplesicau'c L...............

Seragramla spinulosa A. Br, holvetica Spreng., denticulata Kioch..

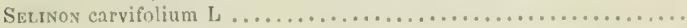

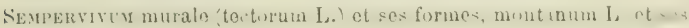
formes, arachnoideum L., globiferum L, hirtum L., Allionianum

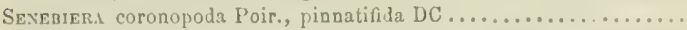

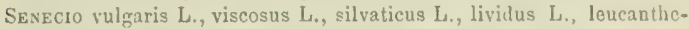
mophyllus l'oir., crassifolius WVilld., gallicus Chaix, adonidoplayllus Lois., aquaticus Huds., crraticus Bert., Jacobaeus L., nemo. rosus Jord., fosculosus Jord., crucifolius $\mathrm{L}$, calvescens Mor. Notar., cinerarius DG, leucophyllus DC., incanus L., unitlorus AlI. (cources de l'Arc en Maurienne), cordatus koch, paludosus L., Fuchsianus L., Jacquinianus Rchb., cacalioikleus Lam., car nosus Lam. (Doria L.), Tournefortianus Lap., doronicifolius

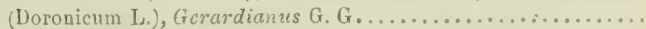
Seripias cordigera $\mathrm{L}$, longipetala Poll, brevibracteata (Lingua L.), neglecta De Not., occultata Gay......................

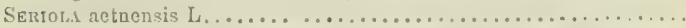

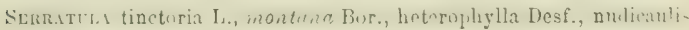
DC . . . . . . . . . . . . . . . . . . . . . . . . .

Seser tortuosum L., elatum L., montanum L., glauecsecns Jord., coloratum Ehrh., brevicaule Jord., carvifolium Vill., libanotum

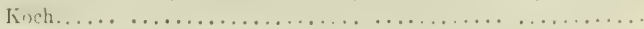

Sestera cacrulea Ard., argentea Savi.....................

Setaria glauca $\mathrm{P}$. de B., viridis $\mathrm{P}$. do B., verticillata P. de B,, ambi-

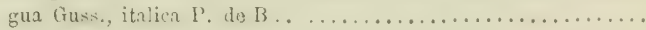

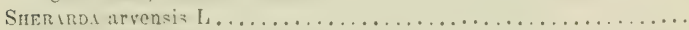

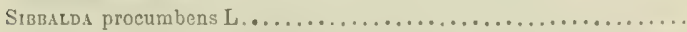

Sideraxis romana L., montana L., hirsuta L., scordioidea L., provincialis J. F., nemausensis J. F., ruscinonensis Timb., hyssopophylla L., pyrenaica Pourr., remotiflora J. F., longicaulis J. F., stricta J. F., orophila J. F., jurana J. F., integrifolia J. F..

Sil.us pratensis Bess., virescerrs Boiss ..................... Silene vesicaria Schr. (inflata Sm.), brachiata Jord., puberula Jord., glareosa J., alpina Thom., conica L., conoidea L., gallica L., agrestina J. F., occitanica J. F., modesta J. F., quinquevuluera

819 
L., myloptera J. F., jucunda J. F., cruentata J. F., nocturna L., brachypetala Rob. Cast., nicaeonsis All., vallesia L., portensis L. armeria L., inaperta L., sediformis Jacq., saxifraga L., quadriflda L., rupestris L., acaulis L., bryoidea Jord., exscapa All., cretica L, cordifolia All, muscipula Li, noctiflora L., pratensis Godr. (Lychnis dioica DC.), diurna Godr. (Lychnis silvestris Hoppe), nutans L., spathophylla Jord., italica Per1., paradoxa L., otitis Smith., pseudotitis Bess., coclestis (Lychnis CœIi-Rosa

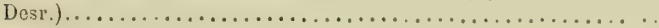

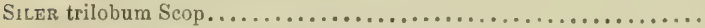

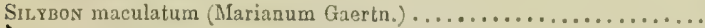
SINEPI (Sinapis) arvenso L., cheiranthum Koch, glarcosum Jord., arenosum Jord., montanum DG., album L..................

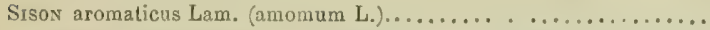
Sisymirion offlcinalo Scop., polyceratium L, supinum L., asperum L., longisiliquosum (Column.eanum Jacq. ', pannonicum Jaeq., Alliarium L, crysimastrum Lam. (Iric L,), austriacum Jacq., rupestricolum Jord., strictissimum L., parviflorum Lam. (Sophia L.),

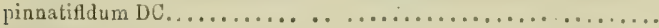

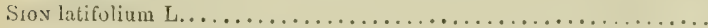

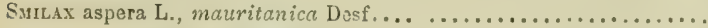

SMYRNION olusatrum L., perfoliatum L...................

SoLANUM nigrum L., minialum M. K., chlorocarpum Spenn., villosu:m

Lam., tuberosum L., dulcamarum L....................

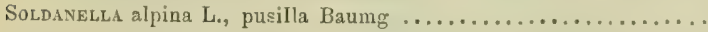

Solıdıgo virgata (Virga aurea L.), minutu Vill., patulifolia Jord., monticola Jord., lithospermophylla WVilld, glabra Desf........

Soxchos tenerrimus L., oloraceus L., asper Vill., glaucescens Jord, arvensis L., maritimus L., decorus Gast, aquatilis Pourr., palus-

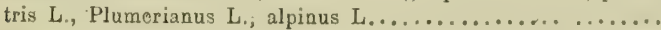
Sonbus aucuparia L., hybrida L., scandica Fries, Mougcotiana Soy.. Will, aria Crantz, torminalis Crantz, mespiloidea (Chamaemos-

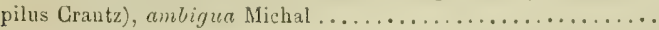

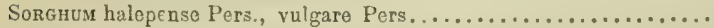
Sparganion ramosum Huds., simplex Huds., natans L., minimum

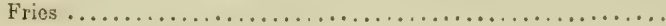

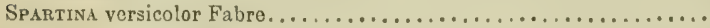

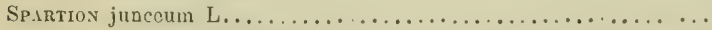
Spectlarid vulgaris (Speculum A. DC.), hylrida A. DC., falcata $\lambda$.

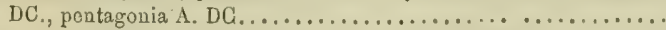

Spergura arvensis L., pentandra L., Morisoniana Bor............ Sperguharia segetalis Fenzl, rubra Pers., media Pers., marginata

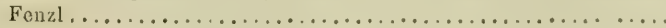

Sphewopus divaricatus Rchb. (Gouanianus Trin.)..............

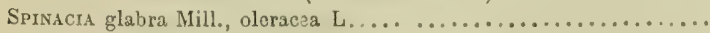
Spiraed tuberosa (filipendula L.), ulmaria L., paniculata (Aruecus L.). Spirantuos aestivale Rich., autumnalo Rich................. 
Stıcurs germanicus L., italicus Mill., heracleus All, alpinus L., silvaticus L., palustris L., palustri-silvaticus Sch., arvengis L.,

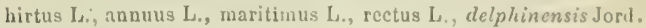

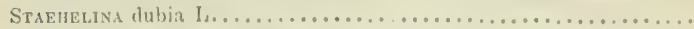

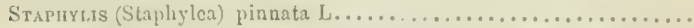

Statice sinuata L., limonia L., serotina lichb., lychnidophyllla Gir., olcifolia IVilld, confuea G. G., densiflora Gir., duriuscula Gir., Legrandiana Timb. G., minuta. L., yirgata IVilld., pubescons IVG., bollidifolia Gouan, echioidea L., dilfusa Pourr.............. Stellari nemoralis L., media Vill, neglecta Weiho, Boracana Jord., holostea L., glauca With., graminea L., uliginosa Murs........

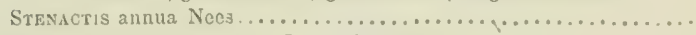
Strp.. tortilis Desf., juncea L., eapillata L., pennata L.............

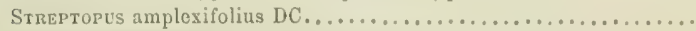

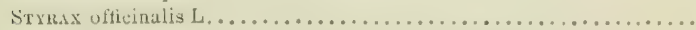
Suaeda fruticosa Forsk, maritima Ium., splendons G. G.......... Subularia aquatica L. (¿tang do Carlito I’yr. Or.) ajouter pago.......

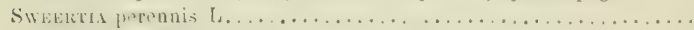
Srspiryon officinale L., tuberosum L., bulbosum Schimp.........

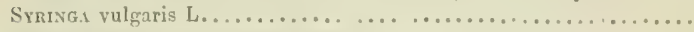

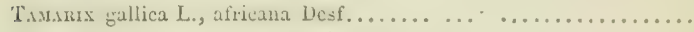

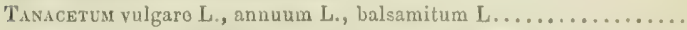

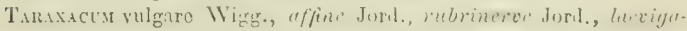
tum DC., leucospermum Jord., gymnanthum DC., obovatum DC., erythrospermum Andrz., palustre DC., udum Jord., maculatum

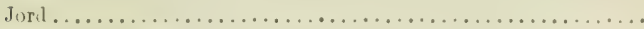

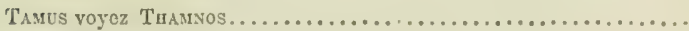

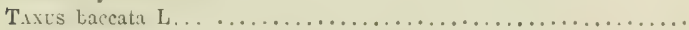

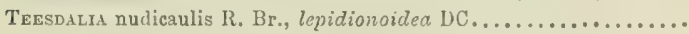

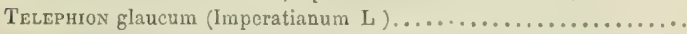

Tetragonolobos siliquosus Roth, purpurous Moench.............

Teucrios fruticans L., tripartitum (Pseudo chamaepitys L.), botrydium (Botrys L.), scordium L., scordioiderm Schrob., scorodonium L., massilionso L., chamaedryum L., lucidum L., flavum L., virescens J. F., pulverulentum J. F., marum L., pyrenaicum L., montanum L., aureum Schreb., saxicolım J. F,, polium et

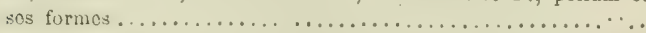

Thalictron aquilegifolium Is, alpinum L., foetidum L., odoratum

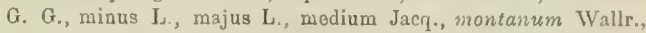
(calcarerm Jord.), monticolum Jord., expansum Jord., elegans

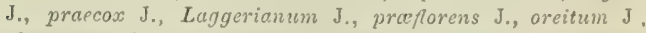
obscuratum J., collinum Wallr., glancescons Willd., brevepubens J., paradoxum Jord., (Jor Janianum Schl.), et autres formes, angustifolium L., simplex L., flavum L. et ses formes, tuberosum L.

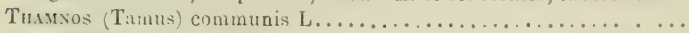

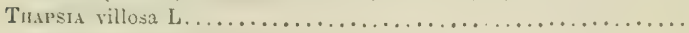

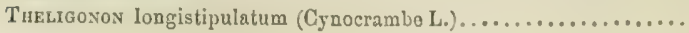


THesion alpinum L., intermedium Schr., pratense Ehrh., pyrenaicum

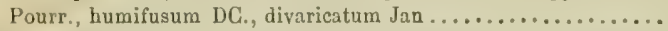
ThL.AsPi arvenso L., montanum L., Villarsicnum Jord, perfoliatum L., martiale Jord., erraticum J., inproperum J., alpestre L., Gaudinianum Jord., Lereschianum Reut., silvestre Jord., virens Jord., beugesiacum J., salticolum J., Verlotianum J., brachypetalum J., alpinum L., rotundifolium Gaud., alliaceum L. (Alp.

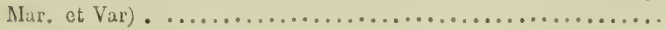

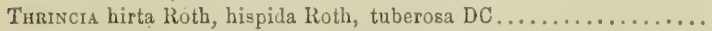
Thyмos vulgaris L., serpyllus L., chamaedryus Fries............ Truh granclifolia Ehrh., intermedia DC., parvifulia Ehrh..........

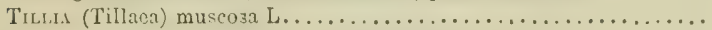

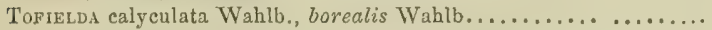
Tolprs barbata Willd., discolor J. F., virgata Bert.............. Tordulion maximum L., apulum L.................. Tonilis rubella Moench (Anthriscus Gmol.), segetalis Thuill, (helvetica Gmel.), tenuisecta Jord., heterophylla Guss., nodosa Gaertn......

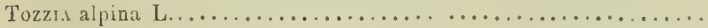
Triagopogon pratensis L., orientalis L., crocifolius L., stenophyllus Jord., porrifolius L., australis Jord,, major Jacq., dubius Vill.,

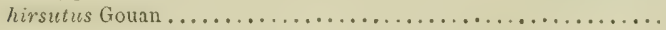

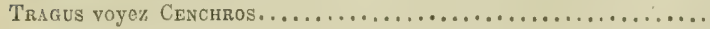

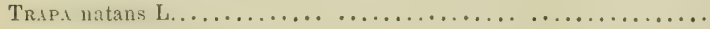

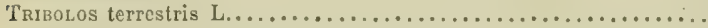
Trichonema bolbocodium Rchb., minimum Ten. (Columnaeanum Rchb.), ramiflorum Ten., subalbidum Jord, flavefolum Jord...

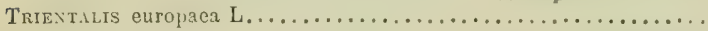

Trifolrum stellatum L., angustifolium L., incarnatum, Alolinesianum Balb., purpureum Lois., rubens L., alpestre L., hirtum All., Cherlerianum L., medium L., pratense L., pannonicum Vill., ochroleucum L., maritimum Huds, panormitanum Presl., lappaceum L., supinum Savi, ligusticum Balb, arvense $L$. et ses formes, lagopodum Pourr., saxatile All., Bocconianum Savi, dalmaticum Vis., striatum L., scabrum L., subterraneum L., fragiferum L., resupinatum L., tomentosum L., vesiculosum Savi, spumosum L., glomoratum L., suffocatum L., laevigatum Desf., montanum L, alpinum L., caespitosum Reyn. (Thalianum Vill.), pallescens Schreb, , repens L., nigrescens Viv., elegans Savi, hybridum L., Michelianum Savi, parviflorum Ehrh., angulatum WV. Kit., Perreymondianum Gr., filiforme L., agrarium L. (aureum Poll.), procumbens L., minus Relhan, patens Schr., badium Schr., spadi-

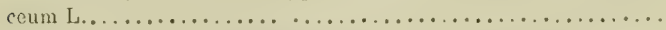

Triglochin palustre L., Barrelierianum Lois., maritimum L....... TrigonelLA graeca (Foenum graecum L.), gladiata Stev., monspeliaca L., polyceratia Benth., ornithopodioidea DC., hybrida Pourr.,

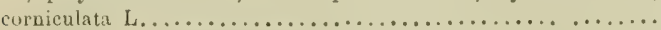

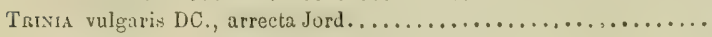


Triticus vulgare $\mathrm{L}_{\text {., }}$ turgidum L., speltum L., monococenten L., vilb)

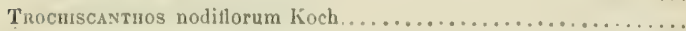

Trum.tus europarus $\mathrm{L} \ldots \ldots \ldots \ldots \ldots \ldots \ldots \ldots \ldots \ldots \ldots \ldots \ldots$

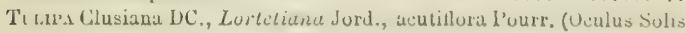

St-Am.), praceox Ten., Didieriana Jord, silvestris L., alpestris

J. F., grallica Lois., Celsiana LG., Gesnoriana L..............

Tunica sasifraga Scop, erecta J. F, xerophila J. F..............

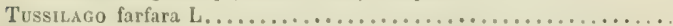

Trube latifulia L., angustifolia L., luylumases ('bah., minima Hopje.

Trrimsnus leucographus Cass..........................

Ulex curopaeus Sm., parvillorus l'ourr., provincialis Lois., nanus Sm.

Ulues campestris Sm., oflusa IVilld....................

Usbrcrces pendulinus $\mathrm{DC}_{n}$, sodiformis DC..................

Unosfermon granditlorum (Dalechampianum Desf.), picroideum Desf

UrTICA urens L., membranacea l'oir., dicica L., pilulifera $\mathrm{L}$..... . .

Utricularia vulgaris L., intermedia Hayne, neglecta Lehm., minor L.

VACcinium myrtilium L., uliginosum L., rubrum (Vitis idaea L.).....

VAILlantu muralis L., hispida L....................

Vareriana officinalis L., phu L., cordifolia (pyrenaica L.), dioica L., tuberosa L., globularifolia Ram., tripteris L., montana L, saliunca

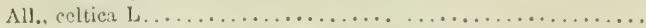

VAlentanell.t olitoria Poll., carinata Lois, auriculata DC., pumila DC., echinata DC., puberula DC., microcarpa Lois., dentata Koch et Ziz (Morisoniana DC.), truncata DC., eriocarpa Desv., coronata DC., discoidea Lois., vesicaria Moench .................

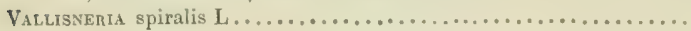

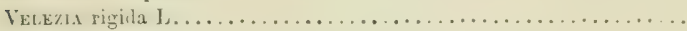

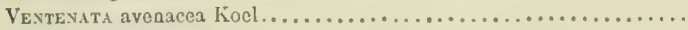

Veratrum album L., Lobelianum Berah....................

Verbascum thapsum L., montanum Schr., thapsiforme Schr., crassifo. lium Holfm. Link, phlomoideum L., australe Schr., sinuatum L. . maiale DC., pulverulentum Vill,, lycbnitum L., nigrum L., den. tatum Lap. (Chaisianum Vill.), blattarium L., virgatum With., formes bybrides...........................

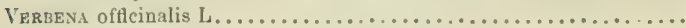

Veronica spicata L., teucriophylla (Touerium L.), prustrala L., chn maedrya L., urticifolia L., crassifolia (beccabunga L.), anagallida L., anagalloidea Guss., scutellata L., pubescens Koch.. montana L., aphylla L, officinalis L., Allioniana Vill., nummularia Gouan, fruticulosa L., saxatilis Jacq., bellidifolia L., alpina L., lilacine Townsend, serpyllopbylla L., Ponaenna Gouan, peregrina L., arvensis L., verna L., acinophylla L., triphylla L., praccox All., persica Poir., agrestis L., didyma Ten., hederifolia L., cymbalaria Bod, longiperluta Jord..........................

Vrburnos laurifolium Borkh. (Tinus L.), lentum (Lantana L.), opuli.

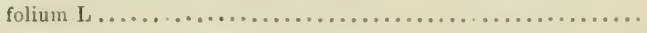

S1:

312

25

$1 ; 93$

85

368

744

413

120

672

281

445

เริ 2

521

509

350

354

357

739

89

801

642

7.33

15:?;

Catal. Bassin du Bhône. 
Vicra sativa L., cordata WVulf., angustifolia Roth, segetalis Thuill., Farsteriana Jord., Bobartiana Forst., Salliana Timb., cuneata Guss., lathjroidea L., pyrenaica Pourr., amphicarpa Dorth., peregrina L., lutea L., hybrida L., narbonensis L., bithynica L., sepincola (Sepium L.), tricolor Seb. M., pannonica Jacq., argentea Lap., onobrychioidea L., dumeticola L., pisiformis L., silvatica L., orobophylla DC., cracea L., Gerardiana Vill., tenuifolia Roth, varia Host, littoralis Salzm., atropurpurea Desf., monantha Desf., di-

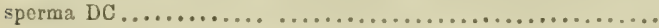

Visca minor L., major L., acutiflora Bert, difformis Pourr.......

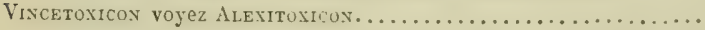

Vrola pinnata L., palustris L., hirta L., Foudrasiana Jord., Thomasiana P. S., collina Bess., propera Jord., permixta J., sciaphill a J., delphinensis J., sulphurea Cariot, Beraudiana J., virescens Jord. (alba Besser), scotophylla Jord., odorata L., sepincola J., multicaulis J., dumeticola J., floribunda J., propinqua J., consimilis J., jucunda J., suavissina J., subcarnea J., silvatica Fries., parviflora (Reichenbachiana Jord,), grandiflora(Riviniana Rchb.), nemoralis Jord., arenaria DC., canina L., mirabilis L., pumila Chaix, stagnina Kit., elatior Fries, stricta Horn., biflora L., arbcrescons L., calcarata L., flava (Zoysiana WVulf.), sudetica Willd., cenisia L., tricolor L., segetalis Jord., agrestis J., obtusifolia J., contempta J., variata J, subtilis J., alpestris J., Cariotiana J., nemausensis J., Sagotiana J., Paillouxiana J., gracilescens J.,

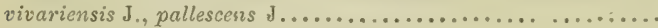

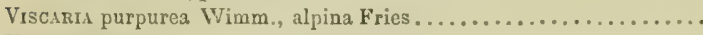

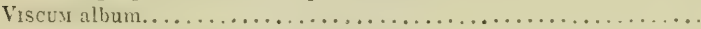

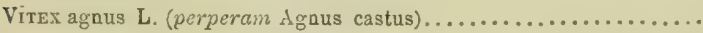

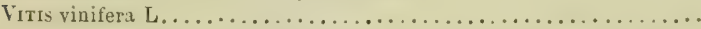
Vulpid myuroidea (pseudo-myuros Soy. Will.), sciuroidea Gm., ciliata Link, setacea Parl, geniculata Link, ligustica Link, bromoidea Rchb., incrassata Parl., puberula Guss.................

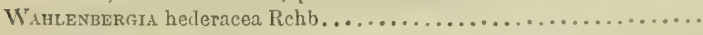
Villemetia apargioidea Cass., prenanthoidea G. G.............

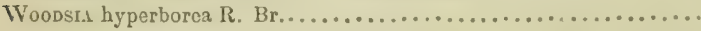
Xantho strumarium L., maerocarpum DC., italicum Mor., spino-

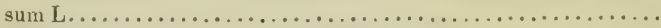

Xatarta (perperam Xatardia) scabra Meissn................... Xerantuemon annuum L., inapertum Willd, cylindraceum Sibth. Sm.

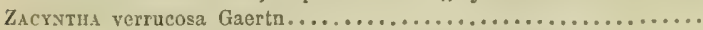
Zavichellia palustris L., terruis Reut., pedicellata Fries..........

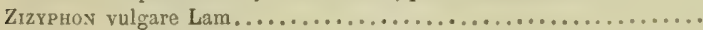

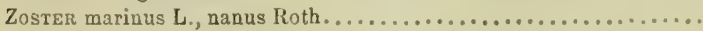








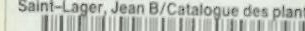

3518500107997 
